
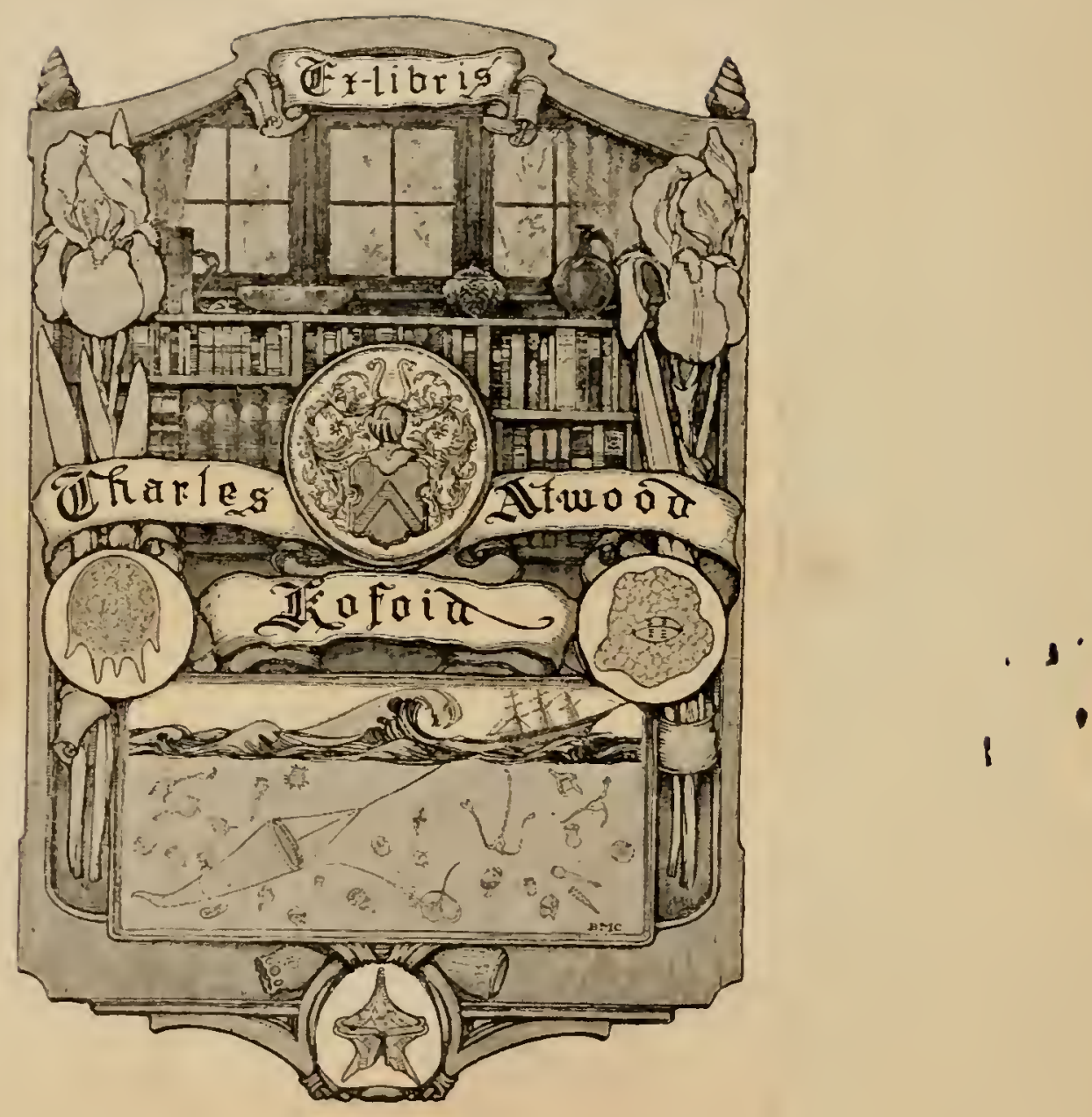


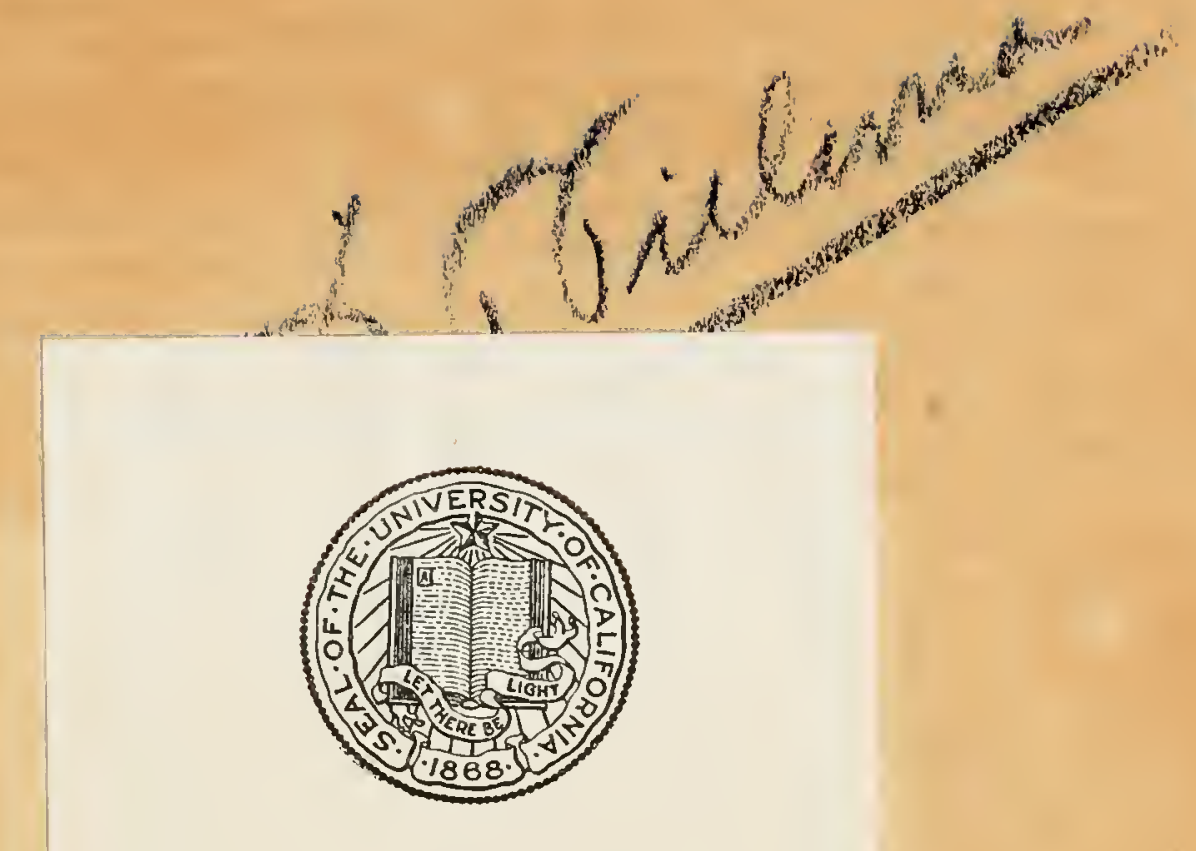

\title{
THE LIBRARY OF
} THE UNIVERSITY OF CALIFORNIA

\author{
PRESENTED BY
}

PROF. CHARLES A. KOFOID AND

MRS. PRUDENCE W. KOFOID 

ESSENTIALS OF TROPICAL MEDICINE. 



\section{ESSENTIALS OF \\ TROPICAL MEDICINE}

PYY

WALTER E. MASTERS, M.D.Brux., M.R.C.S., L.R.C.P.Lond.

Graduate of the London School of Tropical Medicime,

Fellow of the Society of Tropical Meticine and Hygiene,

Feliow of the Royal Gergraphical Society,

Gratuate and Fellow of the Rosal Instilute of Public Health.

Grainate and Mrmber of the Royal Sanitary Institule,

I.ate Menical Officer, Colomlia, South America, Lale Mellical Offeer, Belgian Congo,

Late Surgeon, Whipps Cross IIar llosfital,

Present Medical Officer, Gold Coast, IVest Africa

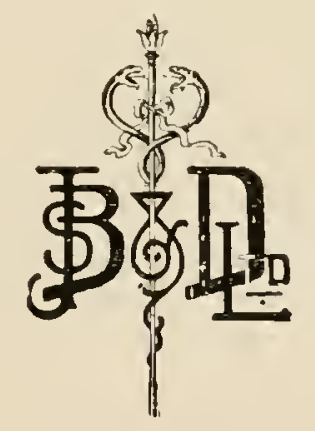

ILollooll

JOHN BALE, SONS \& IMANIELSSON, LTD.

OXFORD HOUSE

83-9I, GREAT TITCHFIELD STREET, OXFORD STREET, IV. I 



\section{PREFACE.}

Tite subject of Tropical Medicine, considered in its broadest sense, has become vaster than any other specialized branch of the Medical Sciences. The comprehensiveness of the subject is only realized when one undertakes a course of study at one of the Tropical Schools, a course particularly essential, but all too short in order to become acquainted with the rarious branches of the subject. The student finds that he must gather together his knowledge from as many imposing text-books in the three or six months of his study as he required for the whole of his qualifying examinations, a task almost impossible in the time. Also the busy tropical practitioner, who requires adequate essential information, cannot consult libraries or include large volumes in his limited equipment, and if he had them he would find it a somewhat laborious task to peruse them for the few essential facts he needed to know.

This work contains nothing new. It is not intencled to substitute any other work. It is a digest of our knowledge of Tropical Medicine, including Skin Diseases, Eye Diseases, Venoms, Iygiene and Laboratory Hints in a form handy for ready reference: the vade-mecum of the student and busy tropical practitioner.

It was commenced for the sole use of the writer, but it has been suggested that its utility would be appreciated in a wider field. In the event of another edition being required the writer would welcome any suggestions for its improvement from medical men.

For the illustrations the writer is much indebted to the authors of the following works and to the publishers through whose courtesy their loan was made possible :-

"A Handbook of the Gnats or Mosquitoes, including a Revision of the Anophelina." By Lieut.-Col. George M. Giles, M.B.Lond., F.R.C.S. ${ }^{*}$

Album of Photographs issued by the South Indian Branch of the B.M.A. Compiled by Major P. C. Gabbett, I.M.S.*

"Anwbic or Tropical Dysentery: its Complications and Treatment." By W. Carnegie Brown, M.D., M.R.C.P.*

"Beriberi." By Edward 13. V'edder, A.Mi., M.D. New York: William Wood and Co.

"Laboratory Studies in Tropical Medicine." By C. IV. Daniels and H. B. Newham.* 
"Reports of the Sleeping Sickness Commission of the Royal Society." By F. W. Motl, M.D., F.R.S.*

"Researches on Egyptian Bilharziosis." By R. T. Leiper, M.D., D.Sc.*

"Sprue and its Treatment." By W. Carnegie Brown, M.D.*

"The Animal Parasites of Man." By H. B. Fantham, M.A. Cantab.; J. W. W. Stephens, M.D.Cantab.; and F. V. Theobald, M.A.Cantab.*

"The Diseases of Warm Countries." By Dr. B. Scheube."

"Webster's Diagnostic Methods." Philadelphia: P. Blakiston's Son and Co.

"Tropical Medicine and Hygiene." By C. W. Daniels, M.B., F.R.C.P.Lond.*

"Venoms: Venomous Animals and Antivenomous SerumTherapeutics." By A. Calmette, M.D.*

W. E. M.

London, 1920. 


\section{CONTENTS.}

SECTION I. PAGI

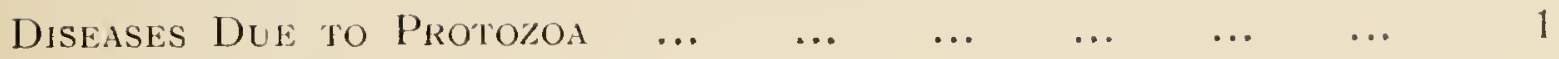

SECTION II.

Diseases DuF to BaCteria $\ldots$.

SECTION III.

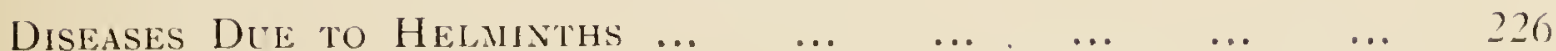

\section{SECTION IV.}

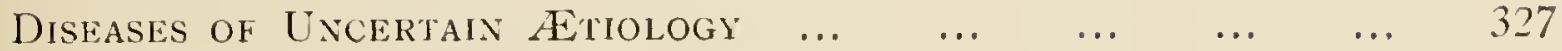

SECTION V.

Diseases Dee tro Vexoms and Porsons f..

\section{SECTION VI.}

DISEASES OF THL SKI

SECTION VII.

$\begin{array}{lllllllllll}\text { Diseases of The Eyes } & \ldots & \ldots & \ldots & \ldots & \ldots & \ldots & \ldots & 508\end{array}$

SECTION VIII.

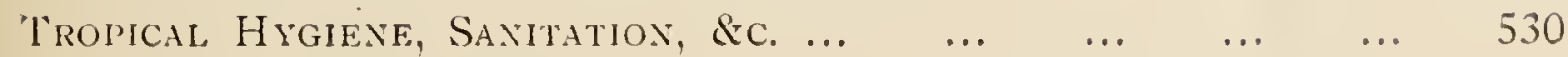

SECTION $1 X$.

LABORATORY HINTS $\quad \ldots \quad$ H

$\begin{array}{lllllllllll}\text { APPENDIX } & \ldots & \ldots & \ldots & \ldots & \ldots & \ldots & \ldots & \ldots & 667\end{array}$ 



\title{
ESSENTIALS OF TROPICAL MEDICINE.
}

\author{
SECTION I.
}

\section{DISEASES DUE TO PROTOZOA.}

INTRODUCTION TO PROTOZOOLOGI.

MODES OF TRAXSMISSION OF PROTOZOAL DISEASES.

RELAPSI IG FEVER.

SPIROCH.ETOSIS ICTEROHAMORRHAGICA (WEILS DISEASE).

NOTES ON TICKS.

FRAMBCESIA TROPICA.

THE DYSENTERIES.

AMIEBIC.

CiLIAR.

LEISHMANic. (See KaLA-AZaR.)

LaVERaNic. (See Subtertian Malaria.)

Pseudo-disfinteries. (See PSeudo-dysenteries.)

LIVER ABSCESS.

AMCEBIC ABSCESS.

TROPICAL LIVER.

THE LEISHMANIASES.

KALA-AZAR.

INFANTILE KALA-AZAR.

DERMAL LEISHMANIASIS.

Differentiation betweEx KaLa-AZar and Oriental Sore.

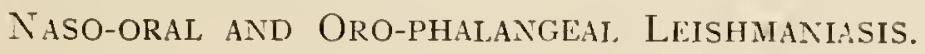

CANiNe LeishManiasis.

PSEUDO KALA-AZAR.

MALARIA.

The Malarial Mosquito.

TRYPANOSOMIASIS.

African Tripanosomiasis.

SOUTH American Trypanosomiasis.

The TSETSE Fly.

NOTES ON MAMMaLIaN TRYPANOSOMIASIS. 


\section{DISEASES DUE TO PROTOZOA.}

\section{INTRODUCTION TO PROTOZOOLOGY.}

The Protozoa are now definitely proved to be factors of the greatest importance in the causation of Tropical Diseases. It is essential that all tropical practitioners should be acquainted with the outlines at least of their life-history and comparative pathology. One only has to mention such diseases as malaria, kala-azar, trypanosomiasis, frambcesia tropica, and relapsing fever to indicate the large amount of tropical pathology coming under this head.

\section{DEFINITION.}

Protozoa are unicellular organisms, occurring singly or in clusters, either non-parasitic, parasitic on the lower animals only, parasitic during part of their existence, or parasitic in different animals during different stages of their development, and reproducing themselves-

Asexually by binary fission (schizogony);

Sexually by budding (sporogony);

Sexually by rejuvenescence by conjugation.

\section{ORIGIN OF INFECTION.}

Very probably the primitive form of protozoon had some of the characteristics of the simple amoba. This could be taken into the alimentary tract, from thence into the blood-stream, consequently facilitating blood-sucking insecta in carrying out their part in the transmission of the protozoa in question.

The blood of man may have been infected from human intestinal excreta or from infective material coming from the intestinal tract of some invertebrate.

The first form was undoubtedly non-pathogenic, pathogenicity probably beginning in bats and birds, followed some time later by pathogenicity in man, the intermediate links being lost.

Some protozoa cannot pass through the placenta, e.g., the malarial parasite, while others can pass through and infect the foetus, e.g., the spirochates and treponemata.

Many of the insect "carriers" infect their eggs, and thus hand down the infection to a new generation of blood-suckers.

The hamatozoan types may live-

In the blood-stream free, e.g., trypanosomes;

In the red blood cells, e.g., malarial parasite;

In the white blood cells, e.g., Leishman-Donovan body;

In the endothelial cells, e.g., Leishman-1)onovan body. 


\section{MORPHOLOGY.}

It may be that all protozoa have not yet been recognized, but some of those known are large, e.g., the Sarcocystida in muscle; and some are small, e.g., the Hamoprotozoa.

The protoplasm consists of-

A clear hyaline ectoplasm;

A dark granular endoplasm.

The protoplasm contains-

One or more nuclei, a complex body of chromatin and achromatic substance. It may be single or divided into two-

One controlling the function of nutrition-trophonucleus;

One controlling the function of motion-kinetonucleus, or

A macronucleus which is trophic and kinetic; and

A micronucleus which is purely reproductive.

Chromidiosomes are smaller particles of chromatin which give rise to the chromidia, intra- or extra-nuclear.

Metachromatic gramules consist of chromatic grains in process of ana- or kata-bolism.

rolutine gramules contain nucleic acid and food material for the nucleus.

Metaplasmic granules are products of cytoplastic ana- or katabolism.

Centrosomes, one or two granules outside the nucleus essentially concerned in binary fission.

Archoplasm, the clear protoplasm around the centrosome.

Rhizoplast, that portion of the flagellum which penetrates into the cytoplasm.

Vacuoli, their function may be-

Respiratory and excretory;

Digestive and excretory.

Protozoa move by pseudopodia, cilia, or flagella, or they may become quiescent and encysted.

\section{LIFE-HISTORY.}

Under favourable conditions of food, moisture, temperature, \&c., reproduction is asexual and may follow the methods of -

(I) Binary fission.

This is single division of the nucleus into two portions, followed by the cytoplasm.

(2) Gemmation.

The nucleus divides into two or more portions, which separate at the periphery, each being surrounded by a differentiated portion of the cytoplasm. 
(3) Spore Formation.

A quiescent body, the schizont, is formed. The nucleus and cytoplasm divide into a number of asexual spores known as merozoites, leaving, as a rule, a "nucleus de reliqual," or rest-body. These merozoites enter new cells in the host, where they grow into trophozoites, and complete an asexual life-cycle or the cycle of simple schizogony.

Under adverse conditions, such as scarcity of food in the host, \&c., the merozoites develop into more resisting forms or gametocytes. In these latter female and male elements are separated. Such elements, nuclear, may remain in the same cell or separate cells. Usually the male element of one fuses-zygosis-with the female element of another and forms a new individual, with a single new nucleus known as a sporont. This reproduces itself rapidly by binary fission or spore formation, the resulting spores being known as sporozoites.

(4) Parthenogenesis.

This is a form of reproduction from the female type of parasite as may take place in malaria. The female gametocyte can resist drugs in the body and lie dormant until taken up by a blood-sucking insect, when the nucleus and protoplasm will divide, one portion disappearing, while the other forms merozoites, and starts the crcle of schizogony again .

\section{PATHOGENICITY.}

The pathogenicity of protozoa is considered under the diseases caused by them.

\section{CLASSIFICATION.}

There are five groups:-

(1) The Sarcodina (Rhizopoda).

These move by a protrusion of protoplasmic processes, broad and blunt or thin and sharp. They may or may not be covered in part with shells. They multiply by budding or fission. Occasionally spores are formed.

(2) The Mastigophora (Flagellata).

These have specialized motile protoplasmic processes known as flagellum or flagella. The shape of the parasite is defined and covered with a membrane.

They multiply by longitudinal fission.

(3) Sporozoa.

(A) Telosporidia.

In these the spore formation is distinct from and later than the trophic phase of the life-cycle. 
(B) Neosporidia.

The spore formation and trophic growth proceed simultaneously.

(4) Ciliata (Infusoria).

These possess cilia throughout their whole existence or only during the early stages of their life-cycle. The cilia are formed from the ectosarc only.

Reproduction is by transverse fission and budding.

Protozoologists may decide upon a rearrangement of this classification later.

Protozoa of pathogenic significance in man may be grouped as follows :-

(I) Sarcodina.

Pathogenic amoba are dealt with under Amobic Dysentery.

The Chlamydophrys stercorea, a rhizopod, is believed to be an amoba parasitic in man during one stage of its existence. It has been found in cows, rabbits, mice and lizards.

(2) Mastigophora.

The Cercomonas hominis is a rounded or pearshaped single flagellated parasite found in normal faces, also in the excretions of diarrhea and cholera patients.

The Cercomonas vaginalis is a similar parasite not uncommonly found in the vagina of Cingalese native women.

The Tetramitus mesnili has a pear-shaped body with three flagella and a large cytostome. It may cause diarrhoa and dysentery. It has been seen in people from the Crimea, Bahama, Hawaii and West Africa.

Trichomonas hominis may be found in some diarrhoea patients.

Trichomonas raginalis has been found in Central Africa, Ceylon and Europe. Its name is descriptive. Its presence does not necessarily give rise to symptoms.

Lamblia intestinalis with its pear-shaped body carries three flagella from its broad end and one from the narrow end.

It has been found in the rabbit, cat, dog, sheep and large bowel of man. Its cysts can be found in

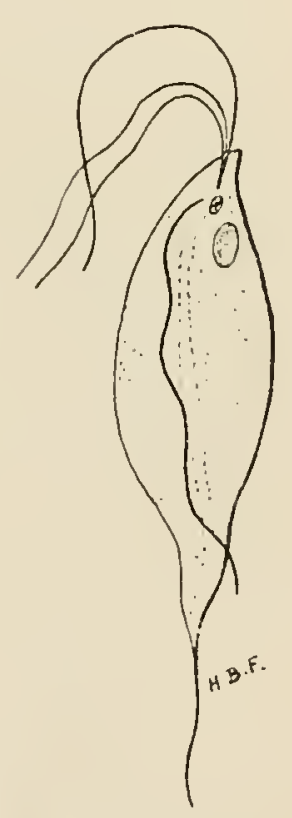

Trichomonas in. testinatis from man, showing anterior flagella, cyto. stomic depression anteriorly, undulating membrane, nuclei, and axostyle. $\times 2,500$. Original. the freces. Pathological changes are not severe. Treatment is difficult.

The Leucocytozoidre are not found in man.

The Trypanosomidx are dealt with under special headings.

The Herpetomonas (Leptomonas, Crithidia) are not found in man. 
The Leishmania are dealt with under the Leishmaniases.

The Histoplasma capsulatum is a round or oval parasite enclosed in a refractile capsule with an acentral nucleus and chromatic particles causing an acute specific disease linown as Histoplasmosis. The parasite is found in the endothelial cells of capillaries and small bloodvessels in the liver, spleen, lungs, intestine and lymphatic glands as well as in the leucocytes. It causes an irregular ferer characterized by disseminated hyaline pseudogranulomata in the fungs, splenomegaly, necrotic areas in the liver, ulceration of the large and small intestine, severe ancmia, and with it a marked leucopania.

Dr. Darling discovered it at Panama in 1906.

Nothing is known as to treatment.

Babesia has not been found in man with the doubtful exception of Babesia hominis in Spotted Fever of the Rocky Mountains (Manson, I go3).

Sambon believes that Blackwater Fever is caused by a minute babesia. See "Blackwater Fever."

The Plasmodida pathogenic to man are dealt with under Malaria.

Pathogenic Spirochates are dealt with under Relapsing Fever.

The Treponema pertenue under Frambosia tropica.

(3) Sporozoa.

(A) Telosporidia.

Coccidium oviforme (Eimeria stieda) occurring in the liver and bile ducts of rabbits has been found five times in man. In these cases there were: fever, enlarged liver and spleen, both of which contained coccidia, the infection of the spleen being probably via the bloodstream.

Hamogregarines, in all probability, never occur in man.

(B) Neosporidia.

Sarcocystis tenella bubali is common in buffalo meat in Ceylon, the parasites being known as "milk nerves," which appear as white patches lying amongst the muscle fibres of the tongue, larynx, diaphragm and skeletal muscles. The ingestion of the spores may cause: Irregular fever in man.

S. lindemanni and $S$. muris have been described in man upon several occasions. There are-Regular fever, myositis, sometimes necrosis of muscle fibres. Spores may be found in the blood, and the parasites should be searched for amongst the muscle fibres.

They are found normally in warm-blooded animals. In them the spore formation commences early and continues until a large size has been attained. The spore has two coats, a number of fibrous sectors dividing the spore, and remaining continuous with the inner coat.

No human has died from the disease. 
Sarcosporidiosis was proved in a Barbadian negro; the sporozoa were found in a portion of the biceps muscle (Darling), but the disease aborted in three weeks, leaving the patient free.

Rhinosporidium seeberi is similar to the above, but has a welldefined sporoblast. The parasite has been obtained from nasal polypi and penile papilloma (Ingram). The growth has a raspberry appearance, with white spots on a general red background. The dots are the cysts. The irritation of the parasite causes proliferation of the mucosa and sub-mucosa of the affected part. The source of the infection is unknown.

The condition is known as Rhinosporidiosis.

It has been seen in South America, India and Ceylon.

The treatment is to remove the growth and cauterize the base.

Balantidium coli and minutum cause diarrhoea and abdominal pain in man (enterocolitis). See the Dysenteries.

(4) Ciliata.

Numerous forms of ciliated protozoa existing in man but not producing any symptoms are not dealt with here.

\section{MODES OF TRMNSMISSION.}

Insects become infected with animal parasites in various ways:-

(1) Insects suck up parasites with the blood when they are feeding upon that medium. Such parasites develop and multiply in the insects and are later injected into man during the act of drawing more blood.

(2) The development of the parasite taken up with the blood may continue in the eggs of the host, which process of development is not completed until the eggs are hatched. Young ticks, having developed from an infected egg, may bite a warm-blooded host and infect him, although it has not previously fed on infected matter. This process takes place in the case of the piroplasma and spirochate.

(3) Parasites drawn up with the blood may develop in the alimentary tract of the insect and infective sporozoites may be passed with the fæces.

(4) Larve of the invertebrate host living in water may become infected directly by means of their food. These larva and their ingested parasites develop simultaneously, so that by the time maturity of the insect is reached the parasites are very numerous. Such parasites may then be passed with the freces and live independently, to enter into other larve later. Flagella may thus be transmitted. This method applies not only to protozoa, but also to metazoa and bacteria.

The following facts show briefly how the most important protozoal diseases are transmitted :- 
(I) The malarial parasites, P. vivax, P. malaria and P. falciparum have their reservoir in convalescents from primary attacks and for weeks after such attacks; the parasites leave the host, man, by blood drawn up by blood-sucking insects; the sexual stages or external development of the parasite takes place in some species or anophelines; il re-enters man with the saliva of the biting mosquito.

(2) In Yellow fever the parasite is not known, but the reservoir is the blood in the early stages of the disease; it leaves the host like the above; passes its external phase in a mosquito, Stegomyia calopus; and re-enters man by mosquito bites about ten days after feeding on infected persons, after which period the mosquitoes lave become infective.

(3) In Dengue fever the parasite is not known; its reservoir is human blood, and is carried away with the blood by the Stegomyia calopus or Culex fatigans; re-infecting man by the mosquito.

(4) In Trypanosomiasis the parasite, Trypanosome gambiense or T. rhodesiense, has its reservoir usually in man in the former instance and usually in wild game in the latter case; it leaves the lost with the blood; passes its external life in the Glossina palpalis or G. morsitans; and re-enters man eighteen days or more after the fly has fed on an infected person by biting and sucking blood in the ordinary way.

(5) In Kala-azar the parasites, Leishman-Donovan bodies, probably have their reservoir in man; leave the host in the leucocytes with the blood; pass their extra-corporal life in bed bugs, Cimex rotundatus, and re-infect man by the bites of these bugs.

(6) In Relapsing fever the parasite, Spirochate recurrentis, has its reservoir usually in man; leaves the host by the blood plasma; passes its external life probably in the Pediculus, and re-infects man by its bites.

(7) In African Tick fever the parasite, S. duttoni, has its reservoir in man or a progeny of infected ticks; leaves man in the blood and saliva; passes its external life in the Ornithodorus moubata and reenters man by its bites.

(8) In Spirochatal diseases, Yaws, Srphilis and some ulcers, the parasites, $S$. pertenuis and $S$. pallida, have their reservoir in man; leave the host by the discharges; have no external development, and re-infect man by contact usually with an abraded surface.

(9) In Amobic dysentery the parasite, Entamoba tetragena, has its reservoir in convalescents from the disease, leaving the host in the freces in an encysted form; further developing while encysted, in earth, water or dust; re-infecting man by the mouth in water, milk, food, sc., contaminated by the parasite. 


\section{DEFINITION.}

\section{RELAPSING FEVER.}

An acute infectious disease or group of diseases:-

Caused by the Spirochretes, S. recurrentis, S. novyi, S. duttoni, S. carteri.

Transmitted by bed bugs, body lice and ticks.

Characterized by sudden onset, definite course ( 1 to 7 days), rapid subsidence and indefinite relapses.

\section{HISTORICAL.}

A relapsing fever was known to Hippocrates in Thasos, which he described:

The next notice of it was by Rutty of the Dublin epiclemic in $177^{\circ}$.

In 1873 Obermeier published his discovery of the causative spirochæte during a Berlin epidemic.

In 1897 Tictin proved the bed bug as a carrier.

In I904 Philip, Ross and Milne in Uganda discovered that the tick Ornithodorus moubata was the carrier of the African Tick Fever.

Dutton and Todd in the Congo confirmed this, and proved that the parasite could pass into the egg and larva, thus infecting succeeding generations.

Napoleon's Grand Army was attacked by it in the great retreat from Moscow. The allied Armies were affected by it during the Crimean War, and both armies suffered from it in the Russo-Turkish War.

\section{DISTRIBUTION.}

Relapsing Fever is found in Europe, Asia, Africa, North, Central and South America, and probably in Australia.

The Relapsing fever of Europe is caused by $\mathrm{S}$. recurrentis or obermeieri ;

The Relapsing fever of America is caused by S. novyi;

The Relapsing fever of Asia is caused by S. duttoni;

The Relapsing fever of Africa is caused by S. carteri.

The number of the spirochætes present in the peripheral blood during infection is variable according to the type of parasite; thus in the African type the infection is light, in the European type it is heavy, and in the Indian type it is variable.

The different trpes will now be considered seriatim.

\section{AETIOLOGY.}

\section{EUROPEAN RELAPSING FEVER.}

The causative organism is $\mathrm{S}$. recurrentis (obermeieri), which is found in the peripheral blood during attacks, but is usually absent between them. Its absence from the peripheral blood during apyrexial 
periods is due to the formation of antibodies such as agglutinins and parasiticidal substances, but some spirochates resist this attempt at cure and cause a relapse when they are in sufficient numbers. Resistant types of spirochates develop during and after an attack. Spirochates are usually present in the peripheral blood from the onset of the fever until the crisis, then suddenly and totally disappear, possibly collecting in the spleen. They have never been found in the excretions or secretions. Some immunity is caused after an attack, but only for that specific spirillum. Gozony concludes that infection is possible through the damaged skin, healthy mucous membrane of the alimentary or genital tract, or possibly by the conjunctiva. It is proved that the parasite is carried by the bed bug, Cimex lectularius, and lice by direct inoculation. The spirochates multiply by longitudinal and transverse fission. They are hereditarily transmitted from adult to young lice, and are thus maintained in nature.

The infection can be transmitted from mother to foctus. On this point Nattan-Larrier, Breinl and Kinghorn conclude that-

Both $S$. recurrentis and $S$. duttoni have been proved to transmit infection to the fotus in 80 per cent. of cases, but the number of spirochretes penetrating the placenta is not great; this fact would explain the long incubation, small blood infection and short persistence of the parasite in the blood in these foctal infections, but their virulence is not attenuated.

When pregnancy is nearing the end there may be a serious illness with death of the fœilus in utero. No lesions of the uterus are necessary to facilitate the passage of the spirochretes.

Very little immunity is given to young born of an infected mother.

\section{INCUBATION.}

Two to ten days, never after the fourteenth day, sometimes on the same day.

\section{SYMPTOMATOLOGY.}

Onset.-This is usually sudden with rheumatic-like pains, headache and constipation, which sometimes precede the rigors. There is severe frontal headache, pains in the back, limbs and epigastrium; weakness, giddiness, convulsions in the young, flushed face, injected conjunctive, fever to $103^{\circ}-104^{\circ} \mathrm{F}$., pulse-rate to IIO-I20, respirations increased, sometimes nausea and vomiting.

Course.-The skin becomes yellowish, hot and moist.

There is often a rose-coloured macular eruption on the thorax, abdomen and legs for one or two days.

The temperature keeps up until the eighth day, third to fourth day in Serbian cases. 
There is thirst, nausea and romiting of yellow-green matter.

Constipation is common, but diarrhoea is rare.

The liver and spleen are enlarged and tender.

The pulse is 120 to 1 to, but not dicrotic. Respirations are $48-50$.

Red cells and $\mathrm{Hb}$. are diminished.

There is leucocytosis of the polymorphs.

Spirochates are found in the peripheral blood and engulfed in the leucocytes.

A troublesome cough is common with râles and a scanty expectoration.

Muscle and joint pains continue and cause sleeplessness.

Delirium is not uncommon, stupor is rare.

The urine is febrile and often contains a little albumin.

Sometimes herpes labialis is seen. There may be rose-coloured spots on the trunk and limbs.

Crisis.-This takes place on the sixth or serenth day, usually by rigor.

There is a violent perspiration and sometimes diarrhoea.

Epistaxis is not uncommon. There is a sudden fall of temperature, pulse-rate and respirations, then a deep sleep, followed by rapid improvement. Sometimes there is a dangerous collapse at the crisis with old people.

Intermission.-All appears to be normal, but it is the exception for the disease to end here. The patient usually desires to leare the hospital. About the fourteenth day from the onset there is a

Rclapse, with rigor and severe symptoms as before, lasting three or four days. The urine is increased, the pulse-rate goes up, and the prostration is often marked. The termination is by crisis which may end the illness. If there are more relapses, which is common, they become gradually milder in severity. The second relapse occurs about the twenty-first day from the onset, is milder, and lasts about three days as a rule.

Convalescence is sometimes slow.

\section{COMPLICATIONS.}

Bronchitis, pneumonia, dysentery, diarrhoea, hamatemesis.

Cerebral hamorrhage, conjunctival hamorrhage, iritis, iridocyclitis, corneal ulcers.

Abortion during the first relapse is common.

Nephritis, otorrhoea, neuritis, parotitis and adenitis are not uncommon.

Meningism, paresis with spasticity of lower limbs.

Recurrent myocarditis, followed always by death (van Hoof). 


\section{DIAGNOSIS.}

In the early stages one must differentiate it from Malaria, Typhoid, Typhus, Yellow fever, Seven-day fever and Influenzi. 'The following are the main points to watch :-

(1) For spirochates in the blood.

(2) Malarial parasites in the blood.

(3) Agglutination; add a drop of suspected serum to a drop containing spirochates, mix, cover with cover-glass, seal, incubate at $37^{\circ} \mathrm{C}$. for half an hour. Clumping of spirochates into non-motile masses is positive.

(4) Typhoid by absence of leucocytosis.

(5) Typhus by absence of spirochrtes, agglutination test and presence of rash.

(6) Yellow fever by black vomit

(7) Seven-day fever by slow pulse and absence of spirochates.

(8) Influenza, from a mild attack of Relapsing fever, not easy to differentiate apart from absence of spirochates.

Relapsing fever may co-exist with Malaria, Small-pox, Measles, Plague.

Diphtheria and Scurvy after famines.

\section{PROGNOSIS.}

This is good. Mortality 4 per cent. in Europe, 14 per cent. in Egypt (Sandwith).

Marked jaundice is bad.

Pregnant women usually abort.

Death may be caused by: Toxamia during the first attack; collapse during the intermission: complications intervening.

\section{TREATMENT.}

Rest in bed is essential.

Salvarsan, neosalvarsan, galyl or kindred preparations are specific, and should be given intravenously or intramuscularly; the former is better. These drugs have revolutionized the treatment of these fevers. One should begin with small doses as some patients stand them badly, say with $0^{\circ} 30 \mathrm{grm}$.

Relieve pains with salicylates, aspirin, antipyrin and quinine.

Opium or morphia may be necessary.

Vomiting with ice, champagne, hismuth mixture, morphia and codeine.

Epigastric pains with fomentations sprinkled with opium tincture. Cough by codeine and heroin or expectorant mixture. 
Constipation by laxatives and enemata.

High temperature by cool sponging.

Give appropriate feeding during the intermission.

A serum has been promised.

\section{PROPHYLAXIS.}

Keep bugs, lice and biting insects alway from the patient.

Wooden bedsteads should be abolished and iron ones substituted.

Practice the strictest cleanliness, disinfect clothing, houses, \&c.

Doctors, nurses, hospital attendants and laundry hands are very liable to attack.

\section{MORBID ANATOMY.}

There is an enlargement of the spleen, five or six times its normal size, and may weigh five or six pounds. On section it is dark coloured, soft with enlarged follicles, showing congestion and a cellular increase. There may be minute abscesses and wedge-shaped infarcts.

The liver is enlarged up to five pounds in weight, the lobules are poorly defined with cloudy swelling, fatty infiltration and leucocytic infiltration into the portal system.

The kidneys are enlarged, congested, cloudy swelling, and fatty degeneration. The stomach is congested.

The heart is soft and flabby.

The bronchi are congested, frothy mucus, lungs hypostatic congestion.

The brain is congested.

All the viscera may be stained yellow with bile.

The bone marrow is hyperamic.

Sometimes there is a submucous petechize.

\section{AMERICAN RELAPSING FEVER.}

It is present in North, Central and South America. In the two latter instances the fever is similar, but the spirochate has not yet been definitely classified.

\section{ASIATIC RELAPSING FEYER.}

The causative parasite is the $\mathrm{S}$. carteri. The carrier is unknown. The disease has long been endemic in India, commonest in the Bombay Presidency. Infected bugs can convey the disease to monkers, but Rogers suspects mosquitoes as human carriers, while Mackie favours Pediculus capitis.

The spirochates are found in the blood during the acute attack, 
becoming more numerous towards the crisis, after which they completely disappear. They are not always seen during the relapse, and may not be found at all during a typical attack. On the other hand, they have been known to be so numerous that the circulation of the blood has been hindered and a dusky lividity caused (Carter).

Rigors are usually absent in this form.

After the crisis, instead of feeling better, there is more often collapse, sometimes resembling that in a cholera patient. There is much debility.

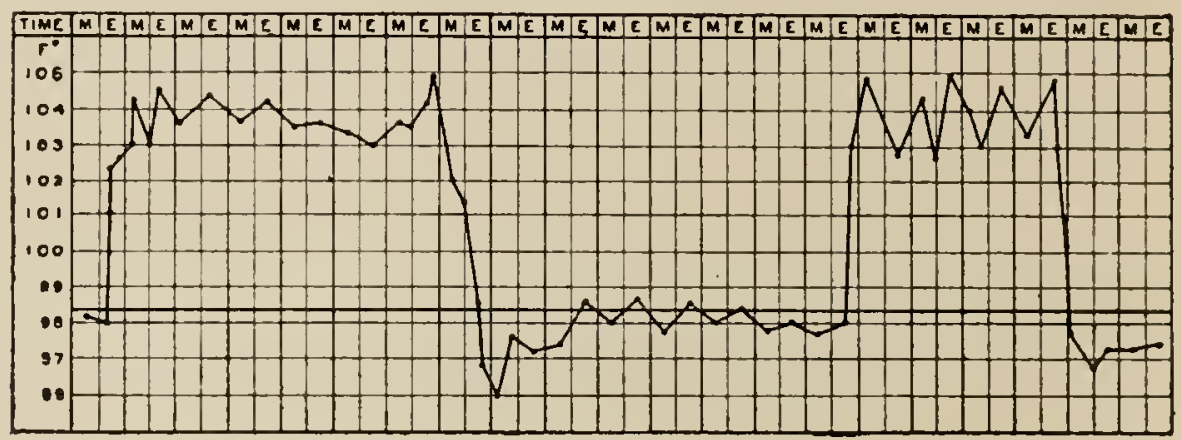

Relapsing fever. Indian.

In 16 per cent. of cases there is a sudden rise of temperature during the first intermission period when spirochates are not found in the blood.

Hamorrhage from the stomach and intestines is more common than in the other forms. Parotitis is present in 10 per cent. of cases.

Bile in the urine is scanty.

Epistaxis occurs in from 10 per cent. to is per cent. of cases.

Vomiting of bile occurs frequently, jo per cent. to So per cent.

Diarrhoea is present in ro per cent.

Maniacal symptoms are known.

The first relapse occurs about the fourteenth day from the onset, seventh day from the intermission. The second intermission may last longer than the first ten days. Second relapses are common, but the liver does not so markedly enlarge, and the crisis is not so well marked.

A third relapse may occur after a secondary intermission of ten to seventeen days.

The percentage of relapses to be expected can be seen from the following table:-

With one relapse, $49^{\circ} 2$ per cent.

With two relapses, 20 per cent.

With three relapses, 5 per cent.

With four relapses, 2 per cent.

In some cases the attacks may be more prolonged, irregular, 
ending by lysis and with marked jaundice, to per cent. to So per cent. of cases.

The mortality is higher than in the others, 18 per cent. In Bombay, 3o per cent. to 40 per cent., according to Daniels. Sudden death from heart failure is common.

Heart stimulants should be given and collapse avoided by camphor, ether, strychnine, hot bottles, blankets, \&c.

\section{AFRICAN RELAPSING FEVER (African Tick Fever).}

The causative organisms are $\mathrm{S}$. duttoni and $\mathrm{S}$. recurrentis (obermeieri) in West, East and Central Africa.

The carriers are Ornithodorus moubata and Pediculus humanus.

The disease is present in Angola, Congo State, Uganda, German Last Africa, Portuguese East Africa and the Zambesi Valley.

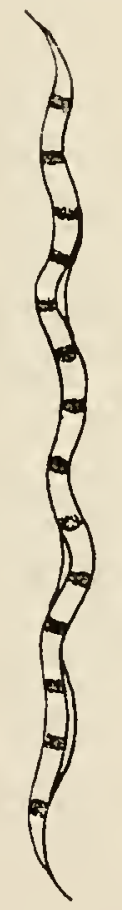

a

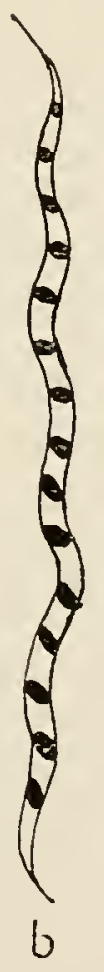

b

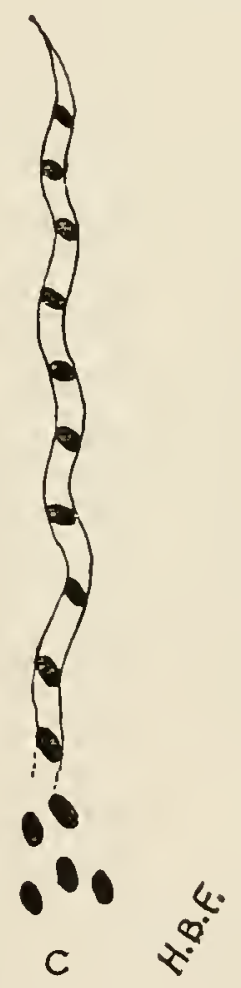

Spirocheta duttoni. $a$, blood form showing slight membrane; $b$, granules or coccoid bodies clearly formed within the organism; $c$, beginning of extrusion of coccoid bodies in the tick. (After Fantham.)

The infection is by small spirochrtal chromatin segments, which come from the lumen of the gut of the tick and contaminate the wound made by it. The chromatin segments pass into the wound as a result of the regurgitation of the gut contents or the Malpighian secretion, and also as a result of its faces contaminating the abraised surface.

Scratching after a tick bite is said to cause sufficient abrasion for the parasites to pass through the skin and cause the infection. 
There is often some local inflammatory reaction at the site of the bite. In this variety there is more romiting and diarrhœe with bloodstreaked stools.

The spirochates are very sparse in the blood, as compared with the other varieties, but heavy infections have been met with in Arabia.

On the second day of the illness there is restlessness, thirst, splenic pain, cough, the temperature has a morning fall without any relief of the symptoms, and an evening rise. There may be delirium.

The liver does not enlarge, but the spleen does.

Therè is polychromatophile degeneration of the red cells and a marked increase in the number of blood platelets.

After the third or fourth day there is a crisis preceded by a pseudocrisis.

Relapses are very common, five to eleven, lasting three days each, and spreading over a month or more. $\quad$ 1ll crises are preceded by pseudo-crises.

In the relapses, acdema of the eyelids is a common feature.

Iritis is a common complication.

There is often an abrupt violent delirium (Porot).

Some consider that there is a mild and a severe form of the disease.

The mortality is not known, but it is generally low.

A rapid fall of temperature without improvement of the simptoms. usually indicates the approach of death.

All Europeans should encamp 20 to 30 metres away from native huts, rest houses or caravans. The bed should be well above the ground, and a good mosquito net used with its lower ends well tucked under the mattress.

\section{In North AfricA.}

In North Africa the causative organism is $\mathrm{S}$. berbera, as a rule.

It is probably spread by lice. It is common in Algeria, Egypt, Tripoli and Tunis.

The surgeons of Napoleon's Army noticed the disease.

The incubation is believed to be about twelve days.

The fever reaches its height during the first twenty-four hours.

The spleen and liver enlarge, but jaundice is generally absent.

Vomiting is common, but not diarrhoea.

\section{Urethral Spirochatosis.}

Macfie found a case of acute urethritis on the Gold Coast due to spirochæte infection. The temperature was slightly raised and joint pains were troublesome.

The name S. urethræe was proposed for the parasite. 


\section{SPIROCHATOSIS ICTEROHAMORRIIAGICA (WEIL'S DISEASE).}

\section{DEFINITION.}

An infective febrile jaundice caused by $\mathrm{S}$. icterohamorrhagica and characterized by pyrexia, marked prostration, jaundice, albuminuria and hamorrhages.

\section{HISTORY.}

I 4 49, Larrey first described it. After him were several other French workers prior to Weil in 1886.

I9I 4 , Inada and Ito in Japan found the causative parasite.

I916, Martin and Pettit found the disease to be present amongst the French troops in Flanders.

\section{DISTRIBUTION.}

There are widespread epidemics in the United States.

The disease is epidemic and endemic in Japan.

It is also found in India, Africa, the Near East, and amongst the French troops in Flanders and on the Jalian front.

\section{AETIOLOGY.}

The causative organism is the $\mathrm{S}$. icterohamorrhagica.

They have been recorered from the blood and urine; from the former during the first days of the disease, and the latter from the ninth to the fifteenth day. They disappear at the end of the fifth week. They have also been found in the kidneys of the field rat, which animal is now held to be a reservoir host for the spirochetes.

An immune body has been found during the disease in the blood which is said to destroy and dissolve the spirochates. It is very difficult to find them at all in some severe cases of the disease.

Sera have been suggested for prophylaxis and treatment.

Infection is probably by infected rats, passing infected urine and fouling the water and food such as could so easily happen in the trenches in Flanders.

\section{SYMPTOMATOLOGY.}

The incubation is doubtful, probably six to eight days.

The onset is sudden.

There are: conjunctival congestion, headache, general body pains, bilious vomiting, slight swelling of lymphatic glands, gastric disturbance, epistaxis ( 17 per cent.), fever, $100^{\circ}-104^{\circ} \mathrm{F}$, lasting ten to fourteen days, jaundice (6o per cent.) - this comes on the fourth or fifth day when present-albuminuria, constipation, liver and spleen slightly enlarged. Marked general weakness and prostration. 
Herpes labialis (42 per cent.), which becomes hamorrhagic.

In severe cases there may be marked hamorthages under the skin and from the mucous membranes.

There is sometimes a secondary ferer.

Some workers describe a meningitis and claim to have found the organism in the C.S.F. (Costa and Troisier).

The attack lasts from ten days to three weeks.

The attack may be so slight that few of the above symptoms may be present, while in severe infections all may be present and sudden death caused by rectal hamorrhages.

In some cases the spirochates seem to concentrate in the duodenum. There is inflammation of the mucous membranes and of the papilla of Vater. The swelling causes obstruction of the bile flow along the common duct and jaundice results.

In some cases the swelling of the affected mucous membranes causes mechanical obstruction of the bile in the liver itself, followed by jaundice, or the swelling may be so slight that obstruction does not exist and jaundice is not seen. In any case the jaundice is not due to an increased formation of bile.

\section{PROGNOSIS.}

On the whole this is good, but matked hemorrhages are always bad.

The mortality is under 6 per cent. On the Trentino and Isonzo fronts it was only 0.55 per cent. (36r cases).

Some cases are so slight that only an inoculation of guinea-pigs will enable one to make a diagnosis at all.

\section{DIAGGOSIS.}

The spirochate can be recovered from the urine after the tenth day, but it is always difficult to find it even in centrifuged blood.

An inoculation of a guinea-pig is preferable.

Localized symptoms will exclude Gallstones and Cholecystitis.

Biood cultures and IVidal's reaction will exclude Typhoid and Paratyphoid.

The spirochates can now be grown with comparative ease, hence as the sera of convalescent patients cause clumping-agglutination - - of the spirochates, we have an important diagnostic test.

Severe forms of trench fever cannot be diagnosed from this disease in the slighter attacks when no jaundice is present.

Acute yellow atrophy is not easy to exclude in some cases because we do not know the cause of it. It is suggested that one form of it may be caused by this spirochate. 


\section{TREATMENT.}

is for Relapsing fever.

Immune horse serum, three doses of Io c.c. each in the first twentyfour hours (Inada).

Destroy all rats, especially those that are apt to foul water or food.

Much of our knowledge may have to be revised and the above modified as we learn more about the clisease.

\section{NOTES ON TICKS.}

Ticlis belong to the Order of the Acarina, of which they are the largest specimens, always visible to the naked eye, and the females invariably larger than the maies, some of the latter when engorged with blood being nearly half an inch long.

They differ from insects by having four pairs of legs and in having the three parts of the body, hhorax and head fused into one unarticulated mass.

After impregnation the female attaches herself to the host, becomes enormously distended with blood, drops off, hides herself, deposits her eggs by thousands within two to ten days after leaving the host. After two to three weeks the eggs are hatched, the forthcoming larva resembling minute moving grains of sand, with three pairs of legs, but no sexual orifice. The larva attaches itself to its vertebrate host, grows, moults, becomes a nympha with four pairs of !egs, and a pair of stigmata behind the hindermost pair of legs.

It moults thrice more and becomes adult. Then the sexes unite.

After fertilization the male dies, but the female seeks blood.

If blood cannot be found the female can fast for weeks, months or years, until blood can be found.

The two families of ticks are: (1) The Ixodida, e.g., Ornithodorus moubata.

(2) Agarsida, e.g., Argas persicus.

The former example transmits the spirochates of African Tick Fever, and the latter of Miana disease, \&c.

Ticks are transmitters of important animal diseases due to Spirochata and Babesia.

Ornithodorus moubata.

It is found throughout Tropical Africa.

The body is flattened, oval in outline, and greenish-brown in colour.

The integument is hard, leathery, covered with closely set tubercles lined by grooves. It lives in native huts, hides during the day in cracks of walls, floors, thatched roofs, and is very active during the night. 
It attacks man and beast, feeds slowly; the rictim must be asleep for it to receive a good feed which requires two to three hours.

It deposits its eggs in batches of $50,70,100$; these hatch in about

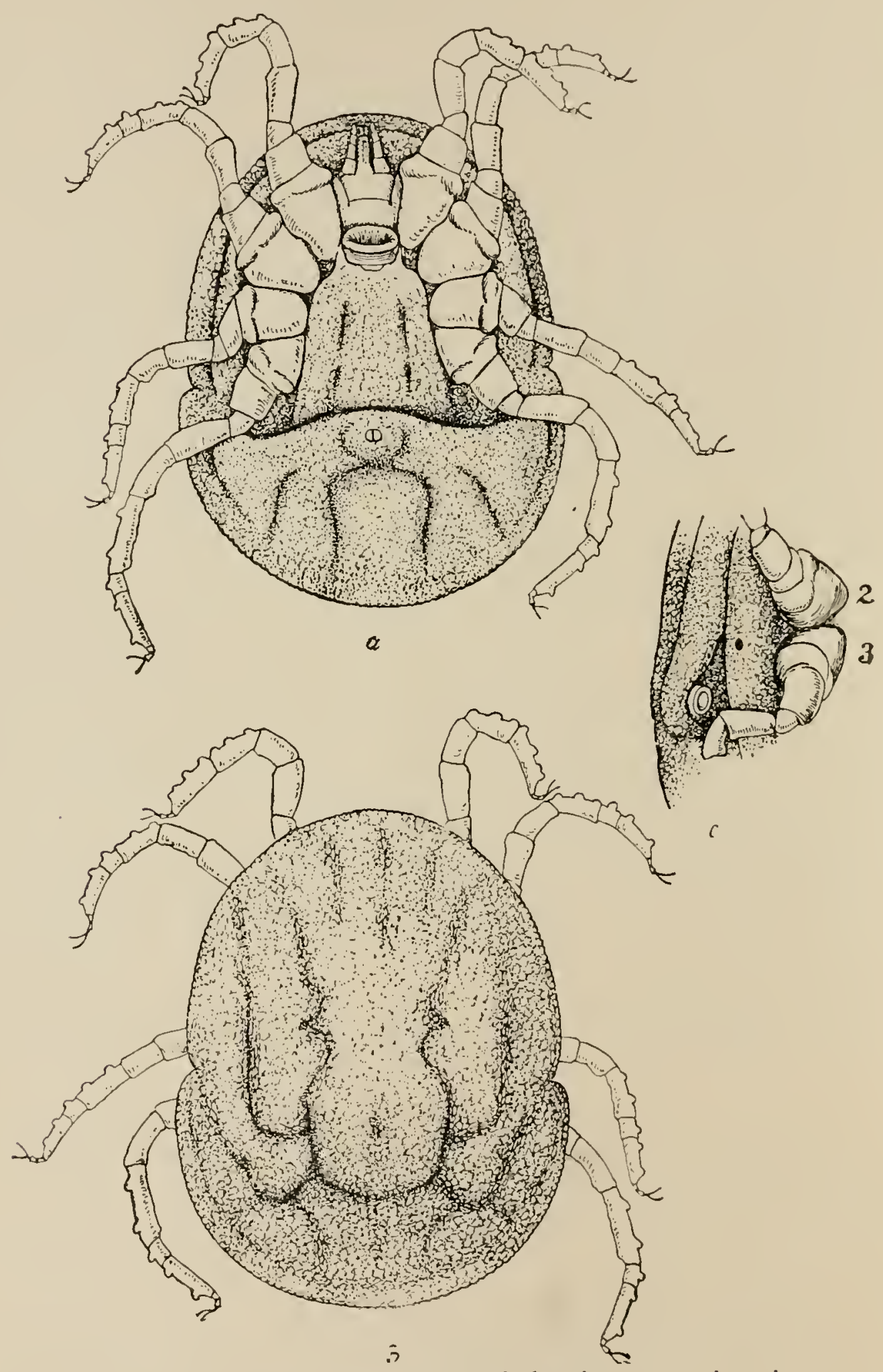

Ornithodorus moubata. $a$, Ventral aspect; $b$, dorsal aspect ; $c$, lateral aspect between second and third pair of legs.

twenty days. The larval stage is practically omitted in this variety. After about thirteen days the eggshell splits, also the larval skin, and an eight-legged nymph throws off both covers simultaneously. 
This tick is commonly found along the lines of travel, e.g., rest liouses, caravanserai, \&c. It may be carried long distances in bedding, mats, porters' cloths, \&c.

The natives protect themselves by applying cow dung to the floors and walls of their houses and by smoking the thatch.

Pyrethrum powcler is good when placed between the sheets. A little sprinkled down the backs of porters is a good measure.

A night light is a protection.

Argas persicus.

This lick is in shape a flattened oval, rellowish green or red in colour, spotted on the back with many white granulations; its legs are a pale vellow, and the whole insect is much smaller than the one described above.

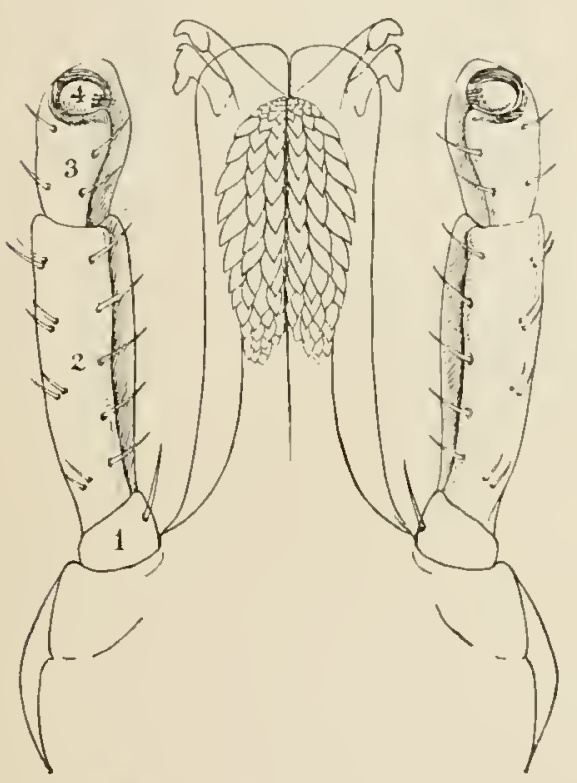

Nouth-parts of Ixodes.

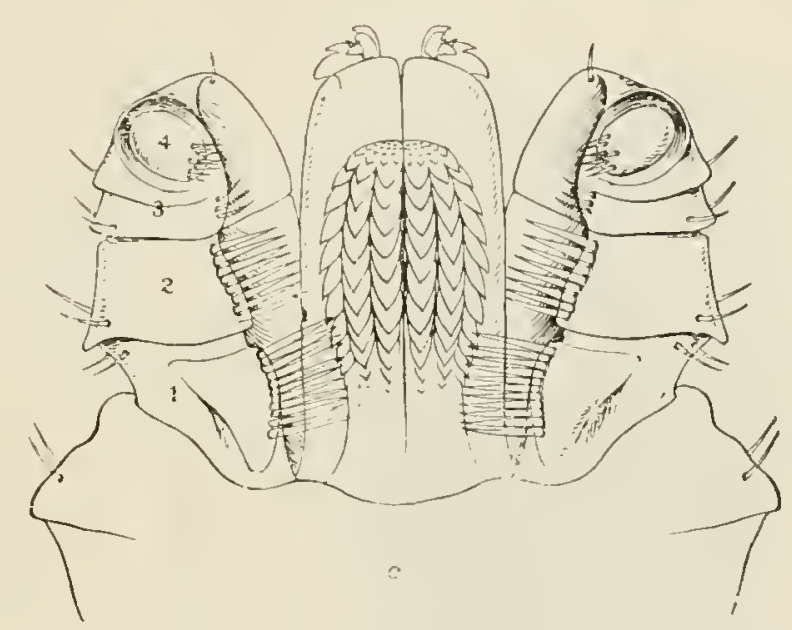

Mouth-parts of Rhipicephalus.

It is found in Persia, Syria, Turlestan, Russia, China, Algeria and Cape Colony.

It attacks poultry and humans.

Its habits are those of O. moubata.

In Persia it may so infest villages that the inhabitants may be driven out of it.

\section{FRAMBCEIA TROPICA (YAIVS).}

\section{DEFINITION.}

A tropical, specific, infectious and contagious disease due to the Treponema pertenuis, characterized by a yellow-tipped, granulomatous eruption.

\section{DISTRIBUTION.}

Originally it was purely an African disease, now it is widely spread. 
Africa.-Tripoli, Sudan, Mrest Coast, Congo State, Angola, Uganda, Rhodesia and Madagascar.

Asia.-Malay Peninsular, Assam, Tpper Burmah, Sian, Java, Batavia, Philippines and Ceylon. In Ceylon the average admitted per annum over ro years was 3,500 .

America.- West Indies, British Guiana, Venezuela, Colombia, Brazil. Some of the Southern United States.

Australu1.- North Australia, Samoa, New Iebrides, New Caledonia, Fiji, New Zealand and Tasmania.

\section{THE CAUSATIYE ÁGENT.}

The organism is a spirillum, the Treponema pertenuis (Castellani, I905), is to $20 \mu$ long, with six to twenty fine uniform coils as slown by Leishman's stain (for five minutes, then with water admixed and stained for one to four hours). It is more

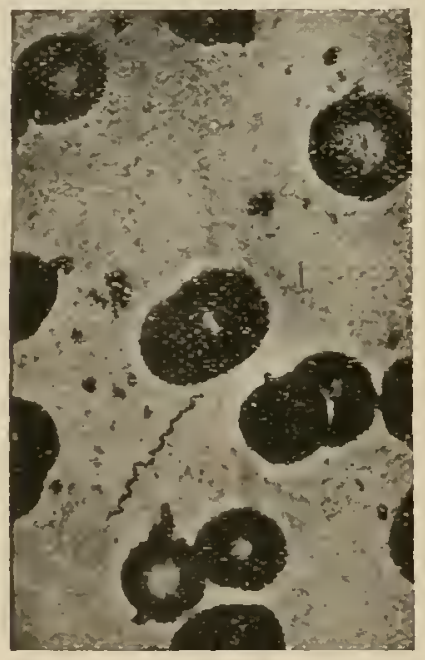

Treponema pertinue. (After Castellani and Chalmers.) difficult to stain than 'T. pallidum; the difference between the two must be based on biological tests.

Its presence is constant in the primary lesion or " mother yaw" and in the unbroken papules of the general eruption. It is also found in the spleen, Irmphatic glands and bone marrow. It has not yet been seen in the blood, but its presence is certain, because when monkers are injected with blood from an infective person it will develop typical yaw lesions. It has not been found in the C.B. fluid or the tertiary lesions.

Multiple infection of the ulcers is very speedy.

\section{HISTOPATTHOLOGY.}

The surface epithelium is greatly thicliened over the site of the papules. The corium is the site of a marked adema.

There is an infiltration by millions of plasma cells: as a matter of fact, there is nothing else (Macleod).

There is no perivascular mononuclear infiltration as in Syphilis, or any endothelial proliferation in the vessel walls.

Giant cells are absent, but they are present in syphilitic lesions.

A well-marked hyperkeratosis is seen.

The parasite is chiefly found in the epithelial layers (after Leishman's stain). 


\section{SYMPTOMATOLOGY.}

The symptoms last six to twelve months, frequently longer.

Three stages have been described:-

The Primary Stage.-The primary lesion or mother yaw.

The Secondary Stage.-The characteristic general granulomatous eruption.

The Tertiary Stage.-The deep-seated ulcerations and gummatouslike nodules.

These divisions are very arbitrary.

\section{INCUBATION.}

This is from two to nineteen weeks, but may be absent.

\section{PRODROMATA.}

Malaise, rheumatoid pains, headache and irregular temperature, but these may be absent.

\section{THE PRIMARY STAGE.}

The primary lesion occurs at the seat of inoculation, and is nearly always extragenital on the mamme or hip.

At first there is a papule the size of a split pea, in seren days it becomes moist, develops a yellow crust, other smaller ones coalescing with it. The crust breaks down, and an ulcer remains with clean-cut edges and a granulating base. This may heal, leaving a white, later, pigmented scar, or it may become a bare granulomatous mass. It is often painful at first, but is never inclurated.

The proximal glands may be enlarged, but do not suppurate.

This mother yaw may develop on an old ulcer, insect bite or wound.

The smallest abrasion is sufficient to afford an entrance to the virus.

The mother yaw is usually still present when the secondary eruption appears.

The duration of the primary yaw is a few weeks to a few months.

\section{THE SECONDARY STÁGE.}

From one to three months after the appearance of the primary lesion there may be malaise, headache, pains in the bones, joints and muscles.

Pin-head papules then appear, commonly on the limbs and face, but frequently over the whole body; these soon show a yellow crust. Some disappear, others coalesce, and others increase in size, forming large nodular masses. The yellow crusts conceal a yellowish fungoid granular mass secreting a thin, slightly purulent fluid.

These ulcerations often form rings about the mouth and anus, enclosing good skin.

After several months the secondary eruption diminishes and hyperkeratosis sets in, when the papules become hard and warty. 
Most of the lesions usually dry up within six months (three to six months in children) and disappear. In some cases the lesions may persist for years, in other cases the eruption may reappear every few months. Each granuloma lasts about two months.

The offensive sour odour given off is probably due to the secondary infection.

The eruption is seldom painful except when it attacks the palms of the hands and soles of the feet, when the pain may be considerable.

The eruption may atack the margins of the nails, when the latter may become hard, britte, thick, and finally be shed.

White patches may peel off the palms and soles, thus simulating syphilitic psoriasis.

The centre of the papules may fall out, learing a pitted appearance which may persist for years.

Fever is common and is intermittent or remittent in type, but it only occurs prior to the secondary eruption.

The cervical and inguinal lymphatic glands often enlarge, become hard, but do not suppurate.

Joints sometimes become acutely painful and swollen simulating rheumatism, but sodium salicylates are useless. As a rule no fever accompanies this condition.

Periostitis is common and often affects the digital phalanges.

Contractures of the flexors of the forearm may become permanent.

Neuritis and neuralgia pains occur frequently.

Hyperidrosis is often limited to the face, hands and feet.

Anremia is common but is not severe, from three to four millions.

\section{THE TERTIARY STAGE.}

This may not develop; some deny its existence altogether. Daniels affirms it in Fiji cases.

Gummatous nodules and deep ulcerations may follow the secondary eruption after several years. On the other hand, they may not ensue at alf.

When the skin is affected the ulcers are rounded or irregular in outline, with very thick and undermined edges and a granulating base. There may be painful nodes under the periosteum of the ribs or sternum. Some cases show a chronic periostitis which alters the shape. of the bones. Bahr has clescribed acute and chronic periostitis, osteitis, epiphysitis, synovitis, ulceration and gangosa. There may be ulcerations about the nasal bones resulting in rhinopharyngitis mutilans.

Internal affections have not yet been described. 

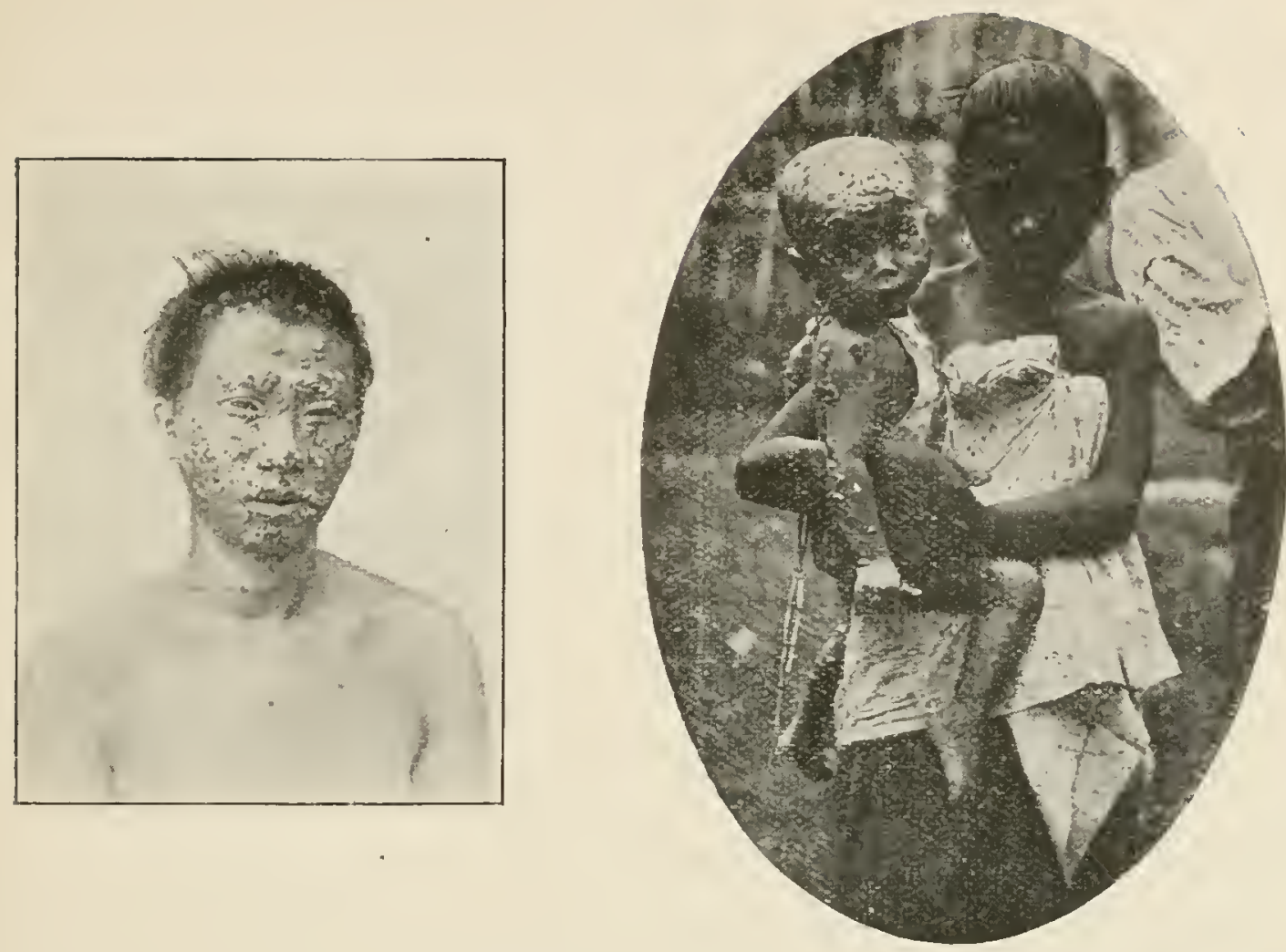

Lale Manifestations.

Iarper, in Fiji, where syphilis is said to be absent, affirms that tabes, general paralysis, aneurism and gangosa are late manifestations of yaws.

\section{COMMUNICABBILITY.}

The disease is not hereditary. Two-thirds of the patients get it before puberty. Parents are infected from their children more than children from their parents, as a result of the child suckling, when the mother will have the primary yaw on the nipple or breast and as a result of the child being carried astride the hip, when the primary yaw will appear in that position.

Infection is by direct contact; a damaged surface is necessary.

Insects may carry the disease, for they eagerly crowd on the open sores and suck the secretion which may be deposited later upon the damaged skins of other people (Robertson).

The mortality is low, 2.5 per cent. in treated cases, but the working capacity is much reduced.

Immunity is usually afforded after the first attack.

The habits of the people predispose them to the disease, the handing round of chopsticks and water-pipes from mouth to mouth and the employment of sleeping mats common to all. 


\section{DIAGNOSIS.}

From Syphilis.-Syphilitic patients may contract it and the reverse. Monkeys inoculated with yaws do not acquire immunity against syphilis.

Syphilis is worldwide in its distribution, but vaws is limited to certain tropical regions.

In Samoa, syphilis was unknown prior to is8o, but raws has been endemic there ever since the people have been known.

In British Guiana yaws has recently disappeared, but syphilis remains (Daniels).

The primary lesion is usually extragenital in yaws.

Pruritis is usually well-marked.

The eruption is nearly always papular, becoming granulomatous. It is not hereditary.

There is no thickening of the blood-ressel walls so characteristic in syphilis.

There is a marled diffuse plasma cell infiltration of the granulomata.

Giant cells are absent in yaws but present in syphilis.

The consensus of opinion goes to show that yaws is not identical with syphilis, although the morphological similarity between the causal agents of both diseases is a very strong one. The same argument applies to Tuberculosis and Leprosy.

From Verruga Peruviana.-This disease is limited to certain valleys of the Andes at an elevation of 3 feet to 10,000 feet.

It has a high mortality, io per cent. to 40 per cent.

It has a severe fever of long duration.

The eruption attacks the mucous membrane, which bleeds readily.

\section{TREATMENT.}

Salvarsan, neosalvarsan, galyl and kindred preparations act rery quickly, but they may fail to effect a cure in oid tertiary cases. Neosalvarsan is better than salvarsan because-

It dissolves more readily in water;

It gives a neutral reaction:

It is less toxic and better tolerated;

It can be given intramuscularly and intravenously.

The average single dose for men is $0.6-0.75 \mathrm{grm}$.

$$
\begin{array}{llll}
, & , \quad, \quad & \text { women, } 045-0.6 \mathrm{gmm} . \\
, & ,, & , & \text { children, } 0.15-0.3 \mathrm{grm} .
\end{array}
$$

More than $0^{\circ} 9 \mathrm{grm}$. should not be given in any case.

Intravenously.

25 c.c. of freshly distilled water are required for each $0.15 \mathrm{grm}$. Some give neosalvarsan and galyl concentrated, such as when 
$0^{\circ}+5-0^{\circ} 6 \mathrm{grm}$. is dissolved in io c.c. of sterile distilled water.

$0.75-0.9 \quad$, . 0 I5 , , , , ,

The median basilic rein is usually chosen.

Intramuscularly.

Inject into the buttocks 5 c.c. of a $\frac{1}{2}$ per cent. Solution of norocain.

Leave the cannula in situ, and after a few minutes inject the neosalvarsan through it. For each o' is grm. use 3 c.c. of freshly distilled water in making the solution.

Doses are prepared in sealed tubes containing $0^{*} 15,0^{\circ} 3,0^{\circ} 45,0^{\circ} 6$, $0^{\circ} 75$ and $0^{\circ} 9$ grim.

Add the contents of the sealed tube to a sterile saline solution $0^{\circ} 4$ per cent. at room temperature.

Well-boiled sterile tap water may be used.

Inject immediately and aseptically at a temperature not above $68^{\circ}-72^{\circ} \mathrm{F}$.

Sufficient should not be prepared for several patients at the same time. Neosalvarsan must not be reheated. If any remains over it should be throwri ariay.

Galyl has given excellent results in the writer's experience. Its low toxicity, reduced price and equal efficacy compared with salvarsan are points to be appreciated.

Intravenous treatment always gives the best results.

Give two injections eight days apart.

Potassium iodides can be given in large doses, $1^{\circ} 0-2^{\circ} \mathrm{O}$ grm. thrice daily, omitted one week in six. Continue the treatment for several months after the disease has cleared up.

Mercury is useless.

I6 c.c. of serum of yaw patients already treated with salvarsan has efficient curative properties. Boiling does not reduce its power.

The ulcers and crusts can be washed with perchloride of mercury to take away the smell and to allay the itching. They may then be dusted with iodoform.

Protargol ointment 20 per cent. has been used for ulcerative lesions.

\section{THE DYSENTERIES.}

The dysenteries include a group of diseases manifesting abdominal pain, tenesmus, small, frequent, blood-mucous stools, ultimate emaciation, and pyrexia in the bacterial variety.

\section{HISTORY.}

3,000 B.C. Hindu records carry us back to this time when dysentery was divided into acute and chronic varieties. 
The IIindu surgeon, Sushruta, wote three to four centuries B.C. that :-

"Ile falls an easy victim to internal and external diseases who drinks of, or bathes in, a pool of water which is full of poisonous worms, or is saturated with urine, or facal matter, or is defiled with the germs of vermin or decomposed animal organisms, or is covered over with the growth of aquatic plants, or is strewn orer with withered and decomposed leaves, or which in any way is rendered poisonous and contaminated, as well as he who drinks and batlies in the freshly collected water of a pool or reservoir during the rains."

Hippocrates seems to have introduced the term "dysentery" and to have differentiated it from diarrhoa.

Most ancient writers of the Near East were acquainted with it.

$53+$-1.D. there was an epidemic of dysentery in France.

S20. It occurred as an epidemic in IIungary.

IoS3-II3. It was in Germany in epidemic form, and in

1316 it risited England as an epidemic.

Wars, famine and foul drinking water were the chief allies of the various epidemics.

I216. King John died from it. Edward I (1.307) and Sir Francis Drake (I595) both were attacked by it a few days before their deaths.

ifir. The military camp at Bordeaux lost if,ooo men by it.

I53S. The first European pandemic took place, which was followed by at least six others some time later. Some of these pandemics lasted for three years.

1655. Cromwell's failure to take St. Domingo was due to dysentery. There were 1, ;oo deaths during the three weeks of the conflict among the troops and islanders. The disease decimated the same troops when they took Jamaica from the Spanish.

IS 7o. The Franco-Geman Mar lost 2,360 deaths from dysentery.

1859. The living amobe were found in the faces at Prague by T.ambl.

IS73. Lösch at St. Petersburg found and described for the first time the amoba when he called it "Amoba coli," an organism now believed to have been the Entamaba tetragena in that particular case.

1SS3. Koch differentiated the two main types of dysentery, amobic and bacillary.

IS93. Kruse and Pasquale differentiated the two types of amoba, the harmless coli and the pathogenic tetragena.

IS94. Japan had 38 .o9t deaths from the disease during the land campaign with China. They lost three from disease to one from wounds.

In the Russian War these figures were reversed. 
In I 897 they lost 22,300 from the same disease.

I899-I902. In the South African Campaign I,342 died from dysentery.

1900. In India the deaths from bowel complaints reached nearly half a million (Hirsch).

In the American Civil IV ar the Federals lost 37,79+ of their troops from dysentery and diarrhoea.

\section{CLĂSSIFICATION.}

(1) Bacterial, caused by:-

B. dysenteria of Shiga. (See section on Diseases due ta Bacteriology.)

3. dysenterize of Flexner. (See section on Diseases due to. Bacteriology.)

B. procyaneus. (See section on Diseases due to Bacteriology.) and kindred organisms.

(2) I'erminous, caused by:-

Schistosoma japonicum. (See section on Diseases due to I Ielminths.)

Schistosoma hamatobium .

Esophagostomum brumpti.

Faciolopsis buski.

Gastrodiscus hominis.

(3) Protosoal, calused by:-

Amoeba (amcebic).

Balantidium (ciliar).

Kala-azar (Leishmanic). (See article on Kala-azar.)

Malaria (Laveranic). (See article on Malaria.)

(4) Pseudo-dysenteries. (Most common in temperate climates.)

These manifest diarrhoea, blood-mucous stools, abdominal: pain and tenesmus.

The causes are:-

Intestinal tuberculosis,

Intestinal cancer and polypi,

Inflamed internal hemorrhoids,

Gummata of rectum,

Intussusception,

Errors of diet, e.g., small fish bones eaten by natives,

ptomaines, e.g., ricin,

excreta of locusts in water (Prout).

Deeks classifies the Drsenteries of Ancon Hospital thus :-

(I) Amobic dysentery, caused by E. histolytica of Schaudinn.

(2)

, , ,

E. tetragena of Viereck. 
(3) Bacillary dysentery, caused by Shiga's, Flexner's and allied bacilli.

(4) Bilharzial

Schistosuma mansoni.

(5) Balantidial Balantidium coli.

(6) Malarial

( The malarial parasite.

(S) Nephritic , Tubercle bacilli of Koch in the intestinal ulcers.

associated with acute diffuse nephritis or secondary to chronic nephritis with an acute process superadded.

(9) Diphtheric colitis, a very fatal form.

(Io) Dysentery in the course of pellagra.

(I I) , caused by the ingestion of decompusing meats or fish.

(12) , secondary to cardiac or hepatic disease.

(13) Clinical dysentery, often climatic, when no microscopic or cultural findings are successful.

\section{AMABIC DYSENTERY.}

\section{DEFINITION.}

An acute or chronic specific disease of the intestine caused by Entamoba tetragena, sometimes accompanied by E. coli, which is said to be non-pathogenic.

The disease is generally insidious in onset with a tendency to chronicity; relapses are common; there is liability to liver abscess. It may concur with other dysenteries.

\section{DISTRIBUTION.}

In Africa, Asia, America, Europe, China, India, Russia, Germany. in short, throughout the tropical world and frequently in the temperate zone.

\section{THE PARASITE.}

This is the E. tetragena, but it is not unlike the E. coli on superficial observation. The following points will assist in the description and differentiation.

\section{F. tetragenir cysts}

Size... $\quad \ldots \quad$... 8 to $30 \mu$

Shape $\quad . . \quad \ldots$ Spherical, amoeboid processes sometimes oval

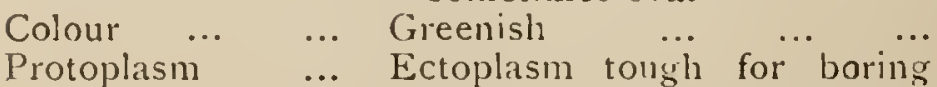

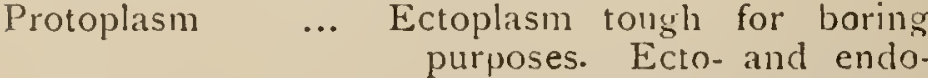
plasm casily distinguished. Ectoplasm refractile

$$
\text { E. coli cysts }
$$

Io to to $\mu$

Spherical, when resting

Opaque : greyish

Ecto- and endoplasm not easily distinguished. Ectoplasm not refractile, homogeneous 


\section{E. tetragena cysts}

Pseudopodia ... Large and easily distinguished. Consists of ecto- and endoplasm

Vacuoles ... ... $\quad$ Many

Nucleus ... ... Often absent, when present its structure is hidden. Nuclear membrane not well defined. Changes position markedly. Never more than four nuclei -lience "tetragena"

Chromidian bodies Contents ... ...

There is little chromatin Erythrocytes, bacteria, pus cells, crystals

Motility ... ... Great progressive motility ... Multiplication ... In intestine by fission and budding. Buds small, $4 \mu$ with two nuclei. (n) hard freces and outside body resistant spores are formed without encystment. These develop when ingested.

Yathogenicity ... Pathogenic, penetrates the tissues and often causes liver il)seess

\section{E. coli cysts}

Hard to distingush

Entirely ectoplasm

Never more than one. May be absent

Almost always present, with well defined nuclear men:brane and otber structures. When organism moves, nucleus retains its relative position. May be up to eight nuclei

There is much chromatin

Rarely cells or organisms

Motility limited

In intestine by binary fission and multiple fission into eight amobulac. 'These are set fiee when ingested

Never patlogenic and never seen penetrating the tissucs. Never causes liver abscess. They are limited to the large intestine and caecum.

\section{LIFE-HISTORY.}

The E. tetragena reproduces in three ways:-

(I) By binary fission, discovered by Schaudinn. The nucleus divides by amitosis into two, and then the crtoplasm splits into two equal daughter cells.

(2) By gemmation.-Found by Schaudinn to be more frequent than binary fission. The nucleus divides by amitosis into two or more daughter nuclei with a portion of the cytoplasm, which then separates off from the mother cell, the daughter cells being the smaller.

(3) By spore formation.--The chromatin becomes diffused, collects around the periphery, the nucleus disappearing. The ectoplasm forms into small knob-like processes, each containing several chromidia. These become separated off and are surrounded with a yellowish-brown envelope, forming spores ready for infecting a new host (Schaudinn). These are surrounded by a cyst wall which is broken down when ingested and the eater infected.

These E. tetragena are found in the faces, intestinal contents, débris from dysenteric ulcers, liver abscesses, mucosa and submucosa of intestinal wall. On the warm stage $t^{4}=y$ show active amoboid movement. The cyst resists drying. 
Wenyon has reproduced the disease in cats, but if it is commonly present in animals is not known.

It should not be forgotten that the dry spores can be carried by flies and wind (Vedder).

Some affirm that the presence of intestinal bacteria, e.g., paracolon bacillus, is necessary for the growth of the amobre (Lesage).

Flies are blamed as mechanical carriers of the parasite from freces to food (Wenyon).

\section{PATHOLOGY.}

Microscopic.- The spores enter the body with drinking water and food, producing young amcbre in the large bowel. These enter the

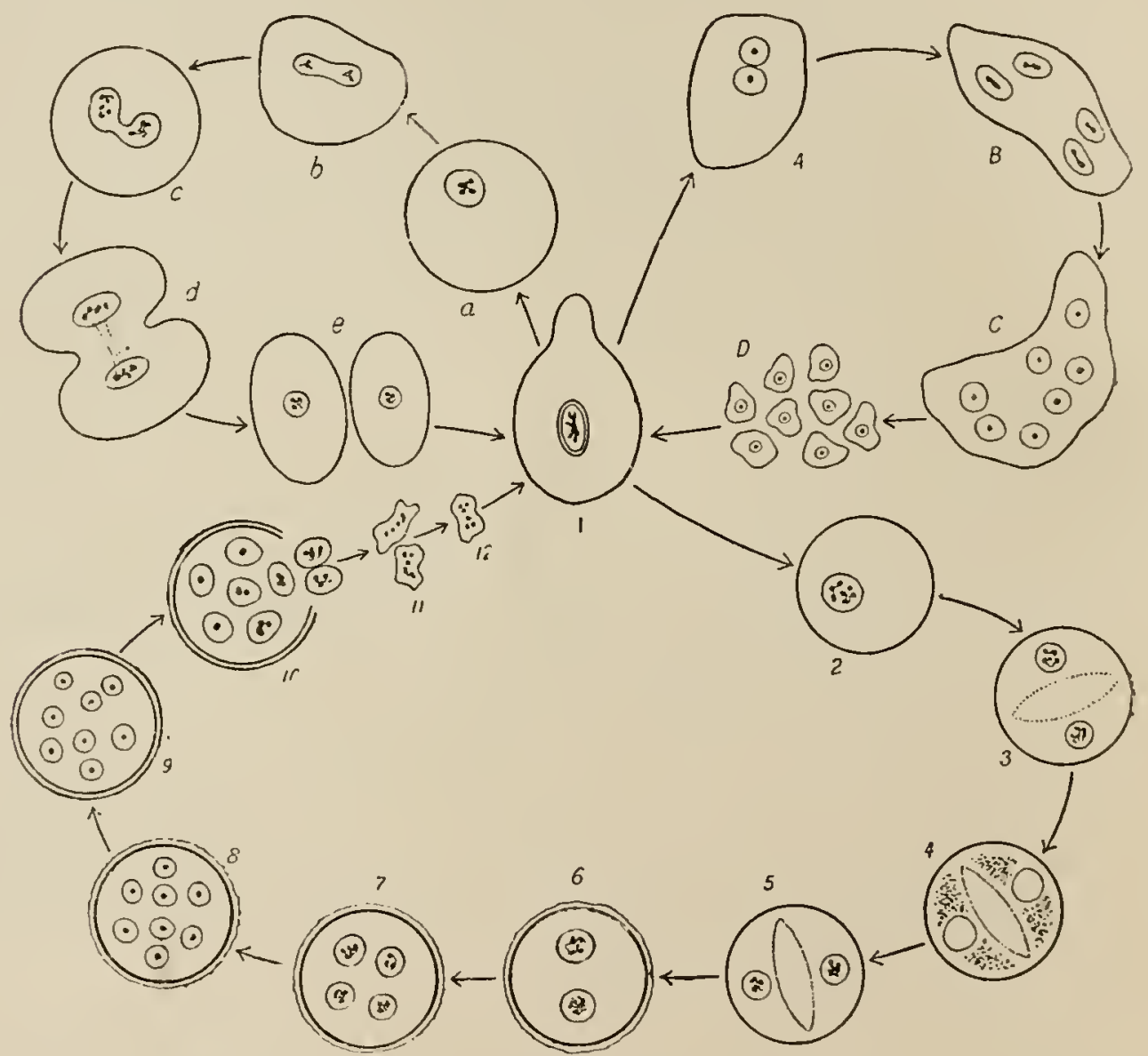

Entamciba coli: life cycle, $a-\ell$, stages in binary fission: $A-D$, schizogony, with formation of eight merozoites; $2-10$, cyst formation or sporogony, with formation of eight nucleate cysts. (After Castellani and Chalmers.)

mucosa by Lieberkiihn's follicles, entering the lymphatics, and passing to the submucosa, they feed upon the tissue cells and erythrocytes of the blood. They may destroy the tissue and enter the tributaries of the portal vein or branches of the mesenteric artery, sontetimes causing thrombosis. They find their way to the liver, causing hepatitis and liver abscess in 15 per cent. of cases.

Patches of the mucosa become oedematous and infiltrated by small 


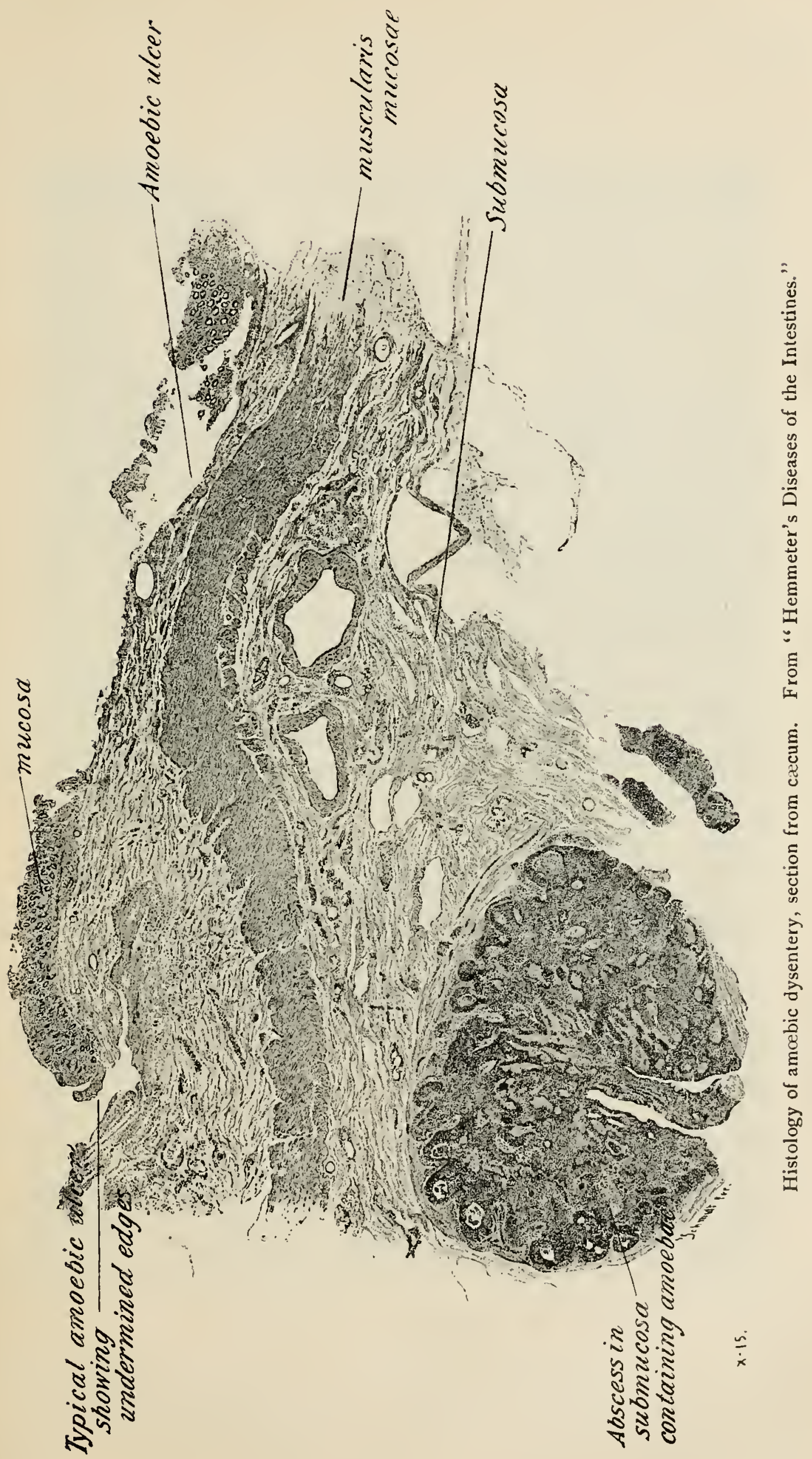


cells, a small slough is cast off and an ulcer formed, which then deepens considerably. Bacteria aid the work of ulceration.

The ulcers are rounded or oval, lying transversely with undermined edges, and may penetrate the muscular and even the peritoneal coat, perforating the same and setting up peritonitis or abscess. In some instances black scars are formed and the ulcer repaired. The blackness is due to the union of hydrogen sulphide from the bowel with iron from the blood.

Constriction, stenosis and progressive emaciation may ensue. The bowel may become gangrenous.

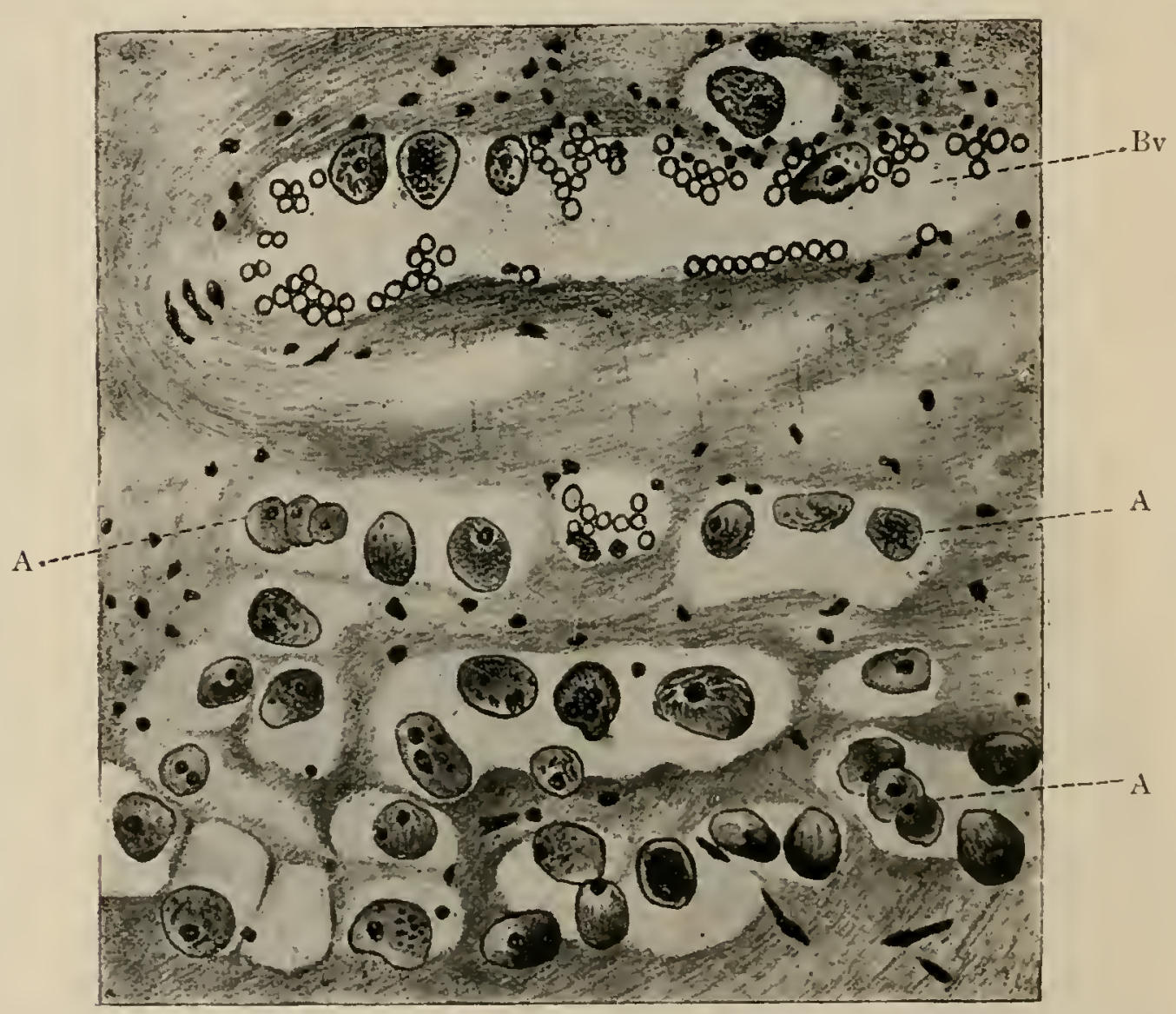

Section through wall of large intestine (of a man) close under an ulcer caused by Entameiba histolytica. A, amcebe that have penetraled partly in blood-ressels $\mathrm{Bv}$, partly in tissue of submucosa to the muscularis. Magnified. (After Harris.)

Amobre may be carried to the spleen and salivary glands, causing abscess.

Macroscopic.-Cardinal features.

Body emaciated, abdomen sunken, rigor mortis early, decomposition quickly sets in, the tissues are dry, the large intestine is contracted, thickened, and sometimes gangrenous.

Perforation and purulent peritonitis. Mesocolon congested and often adherent. The neighbouring glands are enlarged and hyperamic.

The colon is adherent externally to the surrounding structures.

The mucosa is reddened, ulcerated, infiltrated chiefly in cæecum, 


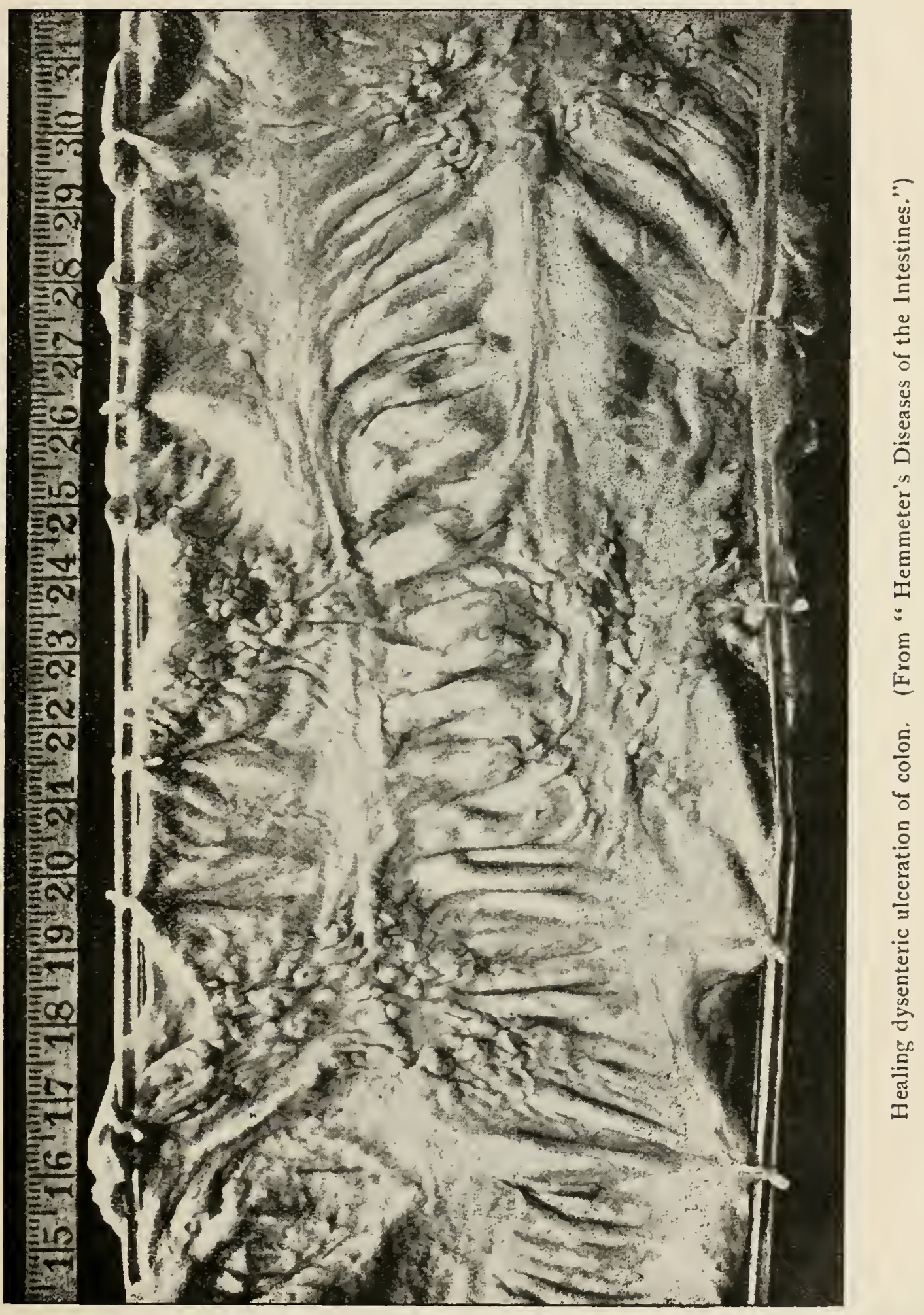


hepatic flexure and sigmoid. There are also firm nodules surrounded by dilated vessels, which later become ulcerated.

The remains of the submucosa is thickened, also the muscular coat.
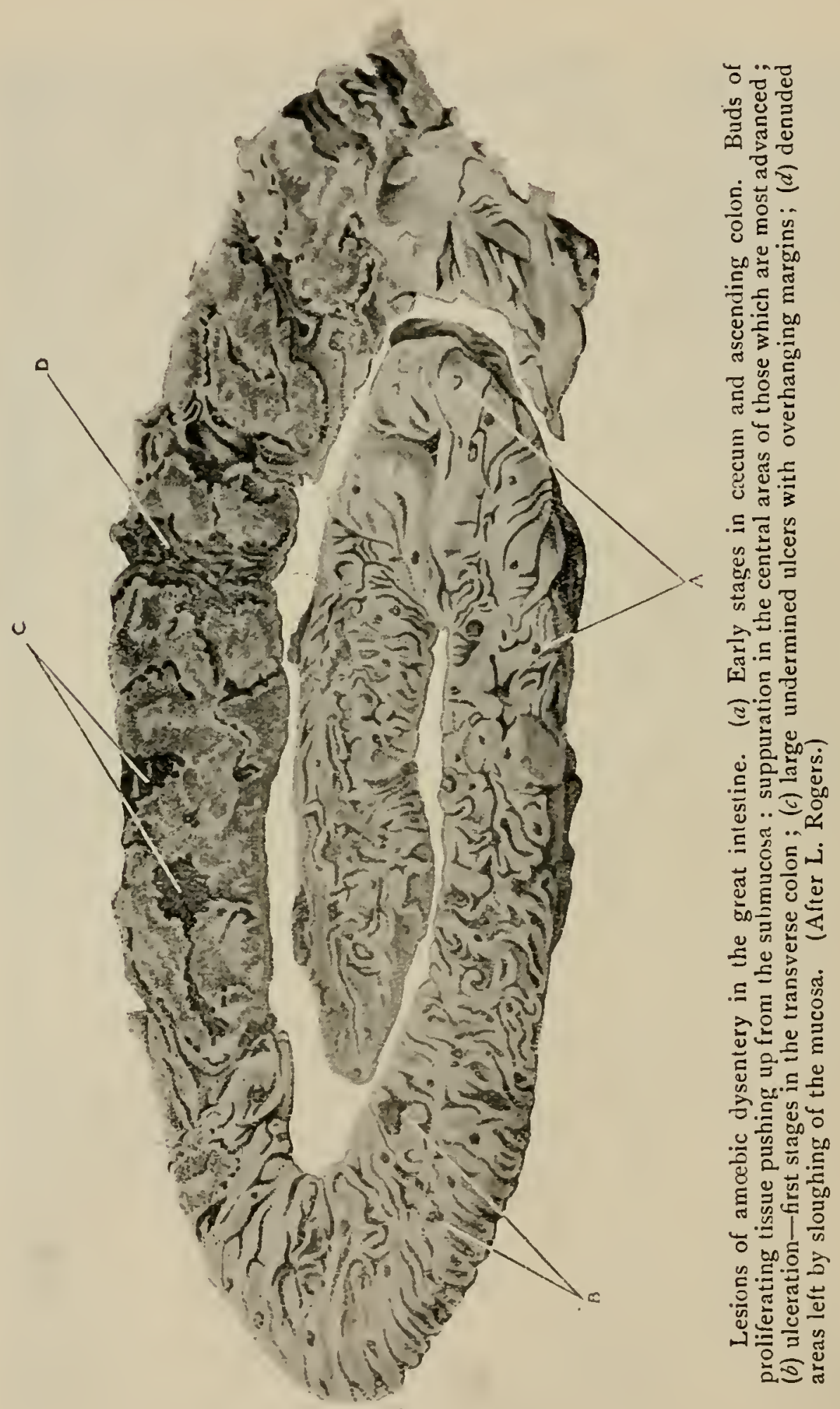

The liver is fatty, perhaps congested; there may be one or more abscesses. For details, see article on Liver Abscess.

\section{SYMPTOMATOLOGY.}

Acute amobic dysentery.

Onset is usually sudden, pain in the lower abdomen often severe, much griping and straining at stool, stools up to thirty per diem with 


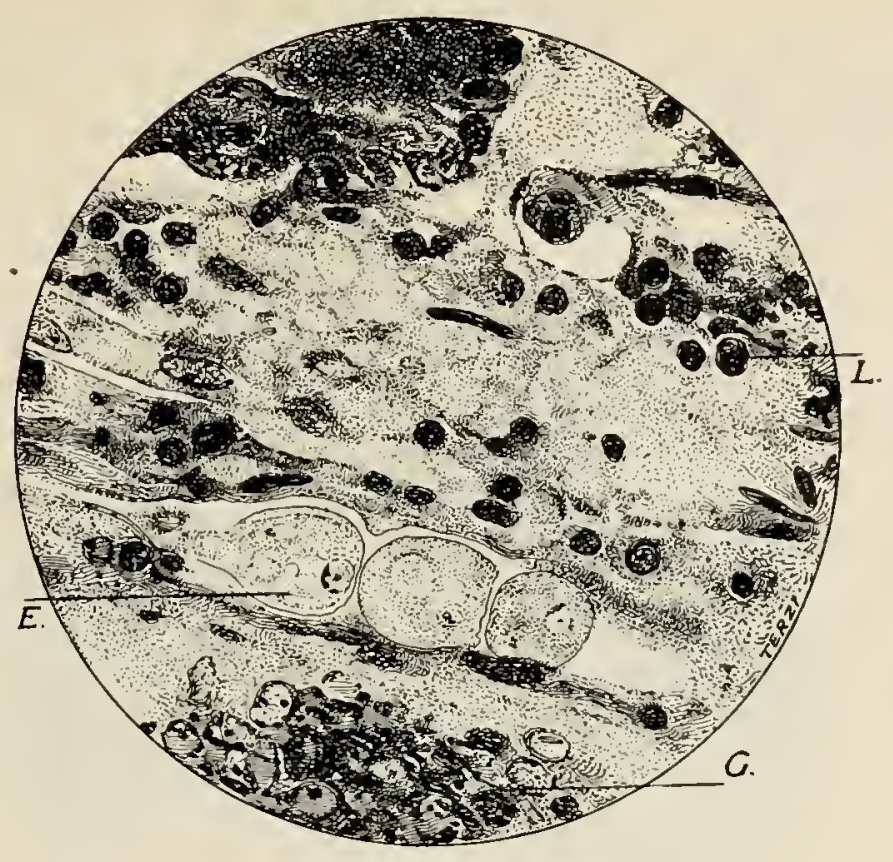

Entamaba histolytica in tissue. Section of submucosa of colon, fixed in corrosive sublimate and alcohol, and stained with nigrosin. Three entamcere may be seen following each other in a tunnel which the leader is excavating. $E$, Entamœlıa; $G$, glandular tissue (disintegrating); $L$, sniall cell infiltration. $\times 350$.

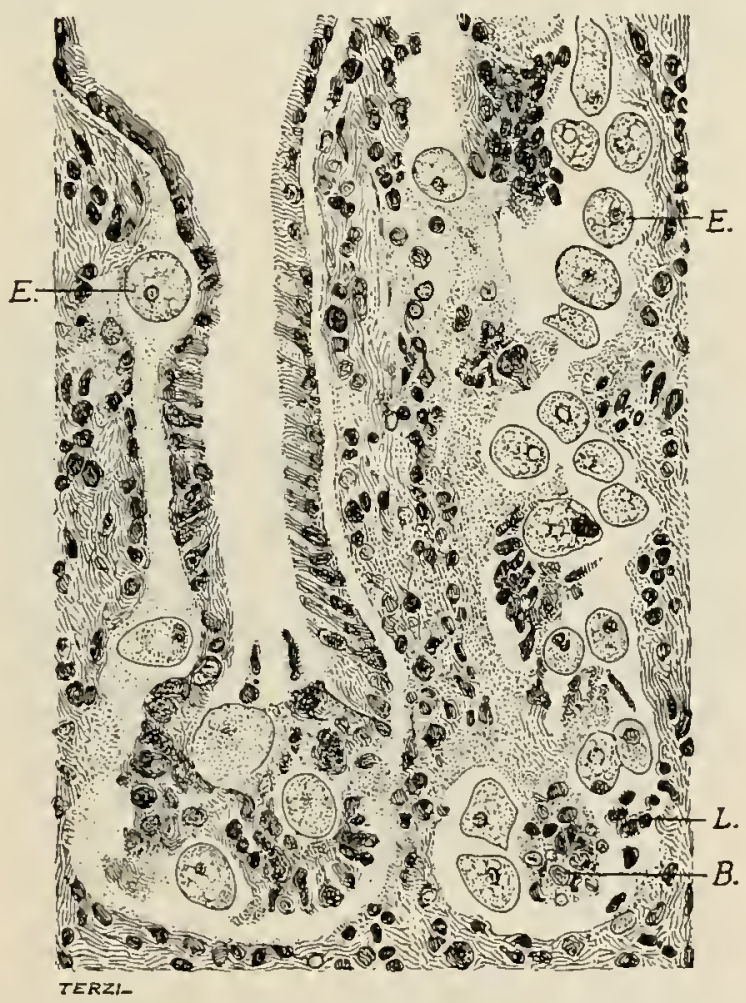

Entamceba histolytica in mucosa of colon. Invasion of the crypts of Lieberkihn from the interglandular tissue, and subsequent destruction of epithelium by the organisms. $E$, Entamcebr; $L$, small-cell infiltration; $B$, blood corpuscles and débris. $\times 300$. (After Dopter.)

blood and mucus, leucocytes, amobæ, bacteria, Charcot-Leyden crystals and bits of tissue.

Anorexia, moist furred tongue, sometimes nausea and vomiting. 
Dyspepsia, pain along line of large gut.

Pulse and respirations quickened. Red cells diminished. leucocytes and eosinophiles increased.

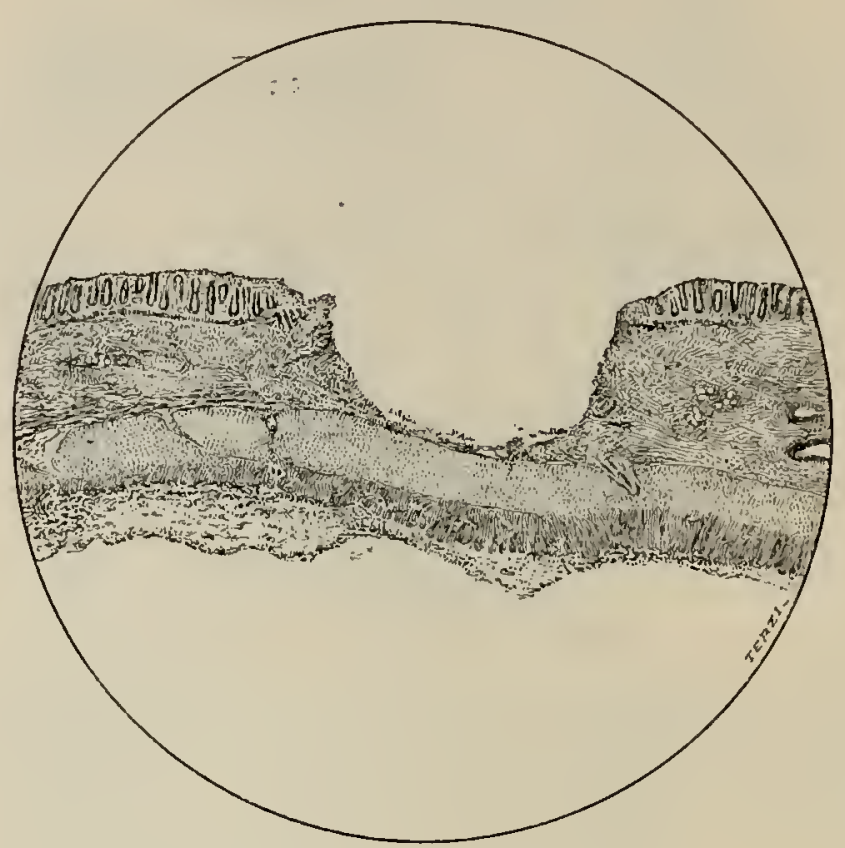

Section of intestine from a case of amœbic dysentery, showing a typical " "llcer of Harris." $\times 45$.

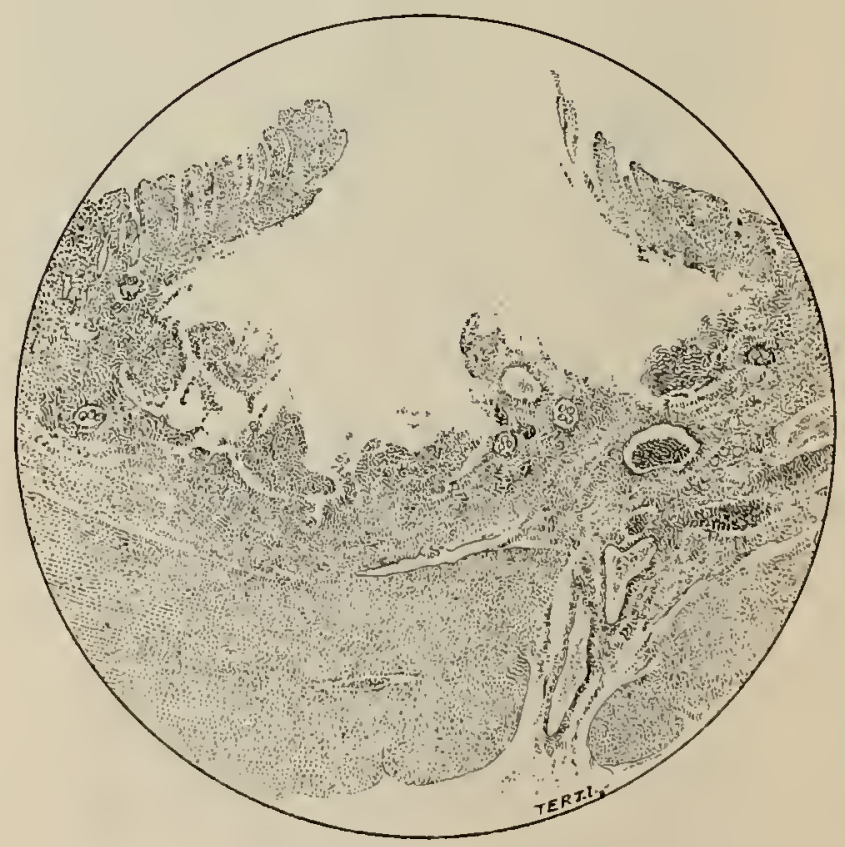

Seclion of small amobic abscess, in which the pus pushed through the muscular tissue, and collected beneath the serous coat of the peritoneum. $\times S_{5}$.

Urine diminished with some albumin and casts usually.

Remittent fever may be present or absent.

Normal temperature, absence of pain and tenderness after they have been manifested may indicate: Recovery, hamorrhage, or gangrene. The condition of the stools will indicate if the condition is improving. 
Death may result in seven to ten days from exhaustion, perforation, peritonitis and hamorrhage.

Chronic am abic dysentery.

This follows the acute, or it may be insidious in onset not preceded

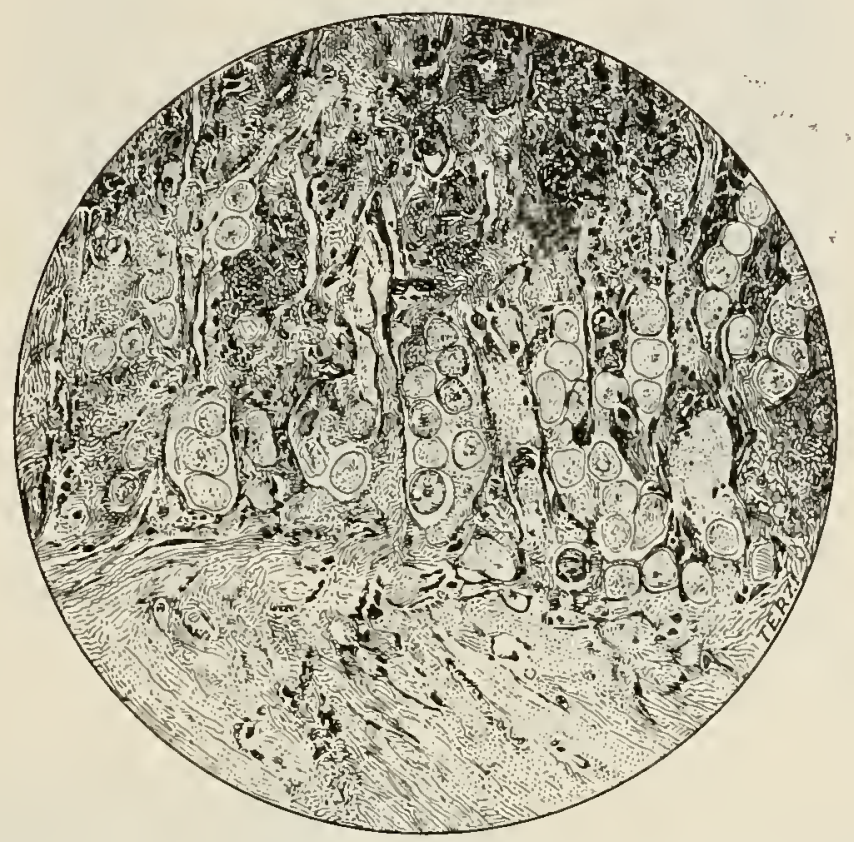

Experimentally infected cat's colon. Under the higher magrificalion, the glands of Lieberkühn are seen to be full of entamcebre, which have destroyed most of the epithelium, and are traversing the basement membrana. $\times 350$. (After Jürgens.)

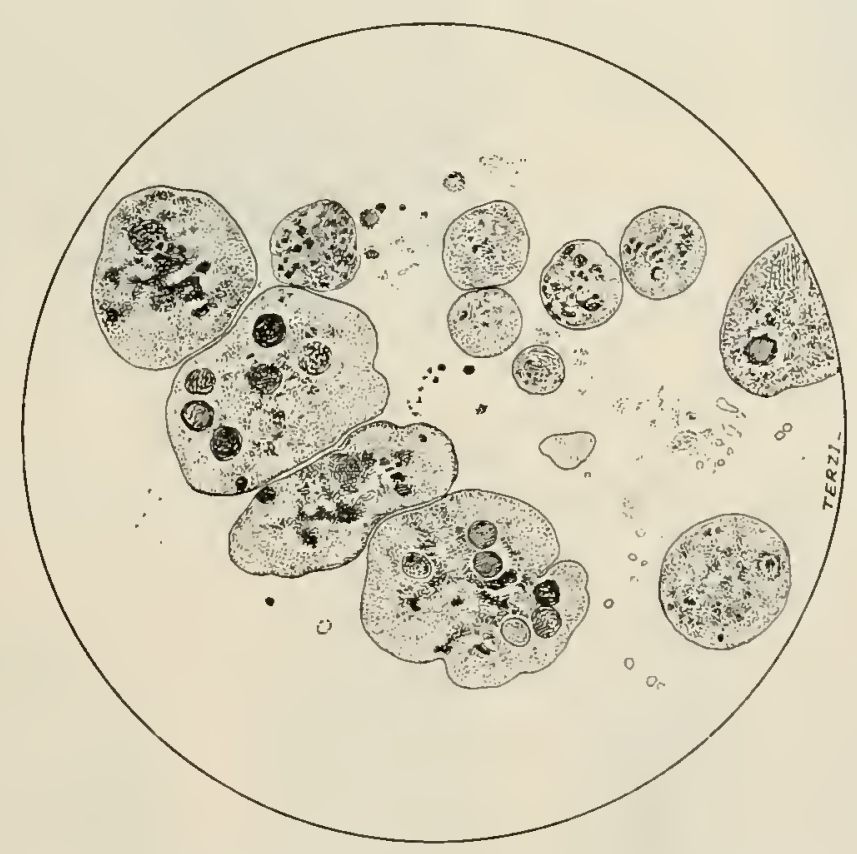

Entamaba histolytica. Organisms in dysenteric mucus. $\times 400$. The granular protoplasm occupies almost the entire cell, and is distinguished from the ectoplasm only by a slight difference in shading.

by an acute attack; there is diarrhoa, with occasional abdominal pain and passage of freculent material with much mucus and perhaps streaked with blood. In between attacks there may be constipation. Stools, which are often more frequent at night, are from twelve to four- 


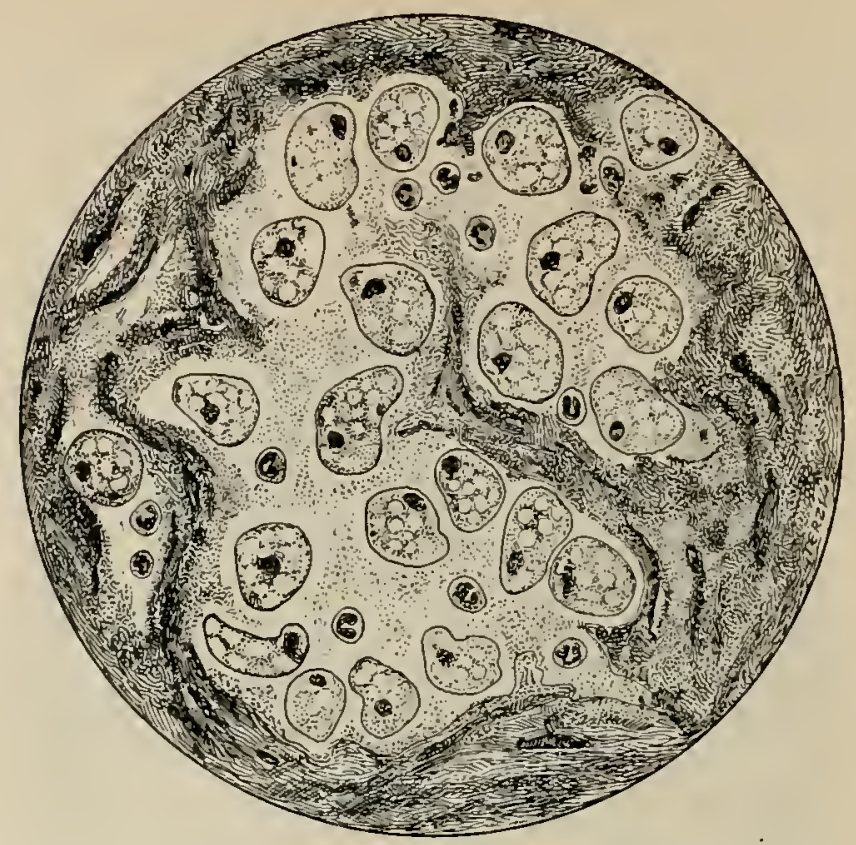

Amœbic suppuration of the brain. Section of a minute abscess. The cavity contains numerous entamobre and pus corpuscles. The nuclei of both are deeply stained. (After Legrand and Dopter.) $\times 300$.

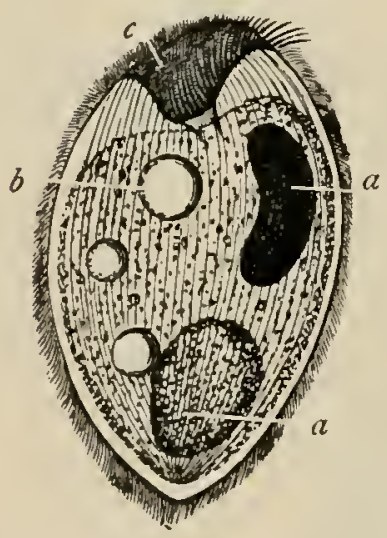

Balantidium coli. $a$, nucleus; $b$,vacuole ; $c$, peristome; $d$, bolus of food. (After Leuckart.)
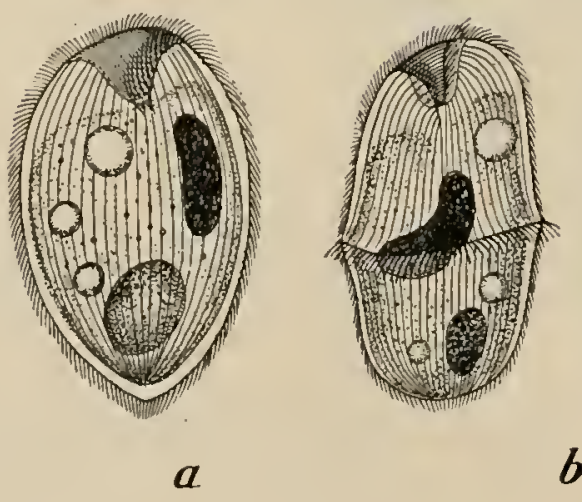

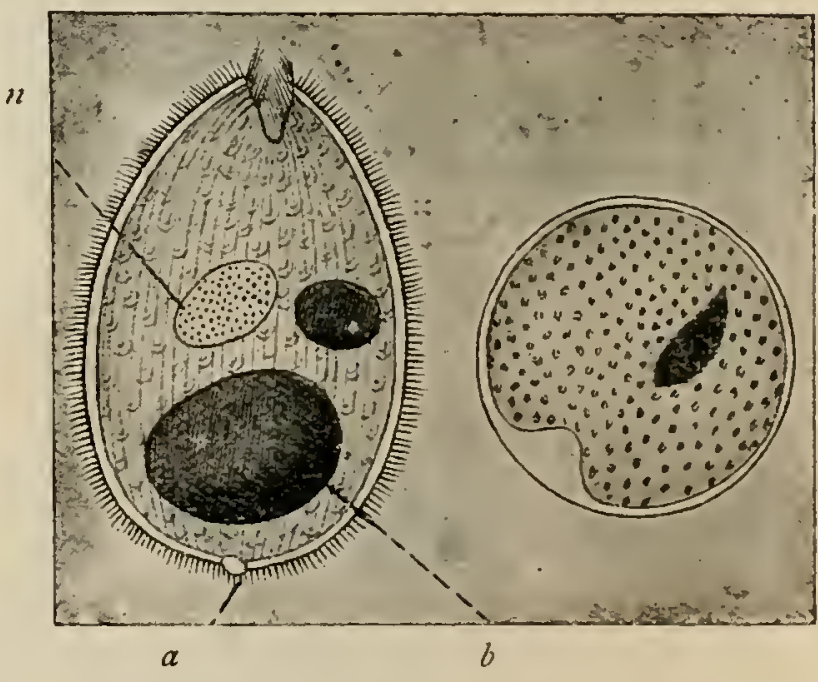

Balantidium coli, free and encysted; $a$, anus or cytopyge ; $n$, macronucleus ; $b$, bolus of food. (After Casagrandi and Barbagallo.)
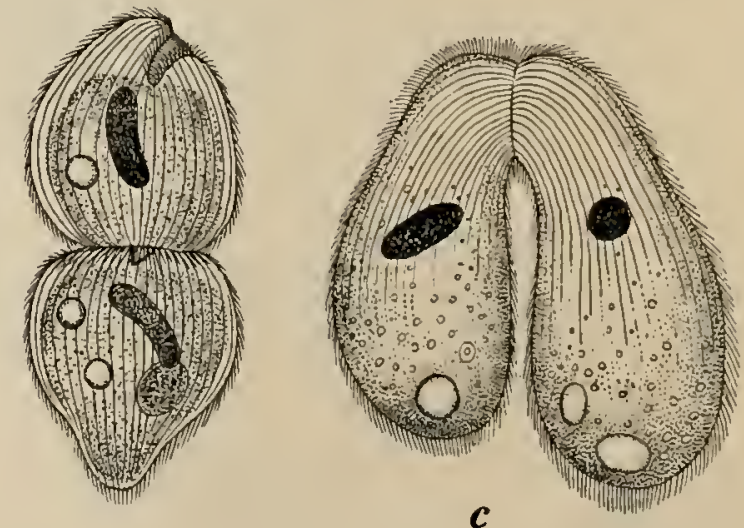

Balantidium coli. $a$, Natural condition; $b$, reproduction by binary fission ; $c$, reproduction by sexual conjugation. 
teen per diem. The condition may persist for years, resulting in marked emaciation.

Large offensive sloughs may be passed at any time.

The cysts are responsible for the spread of infection, hence carriers are a danger to a community. Certain carriers manifest no symptoms.

Before a naked-eye examination of the stools they should always be washed. Errors in diet can be more readily detected and corrected by this means.

The Amaba minuta lives on the surface of the mucous membrane, but is capable of maintaining the infection. After an acute attack caused by $A$. tetragena the $A$. minuta may lead to a relapse of the acute condition, but by itself alone it is doubtful if it causes any symptoms.

One must not forget that the amoba are not confined to the bowel.

Irinary amobiasis is not infrequent in Indians and other Asiatic races.

Because of the possible widespread distribution of the amoba in the human body some have used the term Amcebiasis in preference to Amobic Dysentery.

The stools should always be washed under the tap when one is searching for cysts, and in all dysenteric cases it should be done systematically with every stool. The washing should be continued until all traces of facal matter have disappeared. This method enables the physician to differentiate the sloughs and mucus. The diet can also be controlled and any undesirable ingested foods detected.

It is often necessary to concentrate the cysts in doubtful cases.

The method is as follows:-

(I) Shake up for half an hour about one gram of faces with 300 c.c. of normal saline until the facal mass is disintegrated and an emulsion is formed.

(2) Pour it into a separating funnel, and shake for half a minute with one-twentieth of its volume of ether or methylated spirits. The facal matter absorbs ether and becomes lighter than the water. Cysts are not affected by the ether and remain at the bottom in the saline.

(3) Draw off the saline from the separating funnel and centrifuge it for several minutes. The bottom of the tube should contain the cysts in about fifteen times more concentration than the ordinary faeces (Cropper and Row).

Colonel Wenyon has shown that the mobile stage of the pathogenic amoba is not infectious by the mouth. Indeed, "the cystic phase is the sole cause of transmission," and these cysts are able, under favourable circumstances, to live for at least a month outside the body.

\section{COMPLICATIONS.}

Hepatitis, liver abscess, gangrene of bowel, peritonitis, hamorrhage, stenosis of sigmoid, abscesses in other parts. 


\section{DIAGNOSIS.}

From B.ICTERIIL DISENTER by its chronic course, rate pyrexia, absence of toxic symptoms, complication of liver abscess and the fincling of the amodbe in the stools.

When examining the stools for amobe a little methylene blue will stain the pus and epithelial cells, but not the amoba, thus facilitating their detection and examination. It is not important in practice to diagnose $A$. tetragena from $A$. coli, if one employs the rule that if the symptoms of dysentery are present and amoba are seen in the stonls treat as for amobic dysentery.

Wenyon suggests that the stool should be examined with a little iodine tincture, so that the nuclei may be well seen with an oil immersion and No. 2 eyepiece, the condenser slightly lowered to reduce the light.

\section{Amcebic}

Nearly always chronic in its course Pyrexia rare

Toxic symptoms absent or late when no liver abscess

Liver abscess in 15 per cent. of cases

Small intestine frequently attacked

Amœbre found in the stools

\section{Bacillary}

Nearly always acute

Pyrexia common

Toxic symptoms usually present

Never liver abscess, unless multiple and small

Nearly always confined to the large intestine

Dysentery bacilli found in the stools

From Sub-tertian malurial dysentery by the absence of malarial parasites the presence of fever and its not vielding to quinine treatment.

From Ulcerative colitis and Hemorrhagic typhoid by high ferer. prostration, pain, violent delirium and the absence of entamobre. The diagnosis is often difficult.

From Helminthic infections by finding the causative parasites.

From Intestinal tuberculosis by marked tenderness in the crecal region rather than over the sigmoid flexure and the absence of parasites.

From Malignant disease of the sigmoid and rectum by the serere tenesmus, increasing difficulty to pass large-formed stools, the age of the patient and the absence of parasites.

\section{TREATMENT.}

Absolute rest in bed.

Relieve griping by morphia, hypodermically, also by enemata or suppository. Give castor oil half to one ounce ( $55-30 \mathrm{grm}$.).

Emetine hydrochloride is a specific, half a grain $\left(0^{\circ} 03 \mathrm{~cm}\right.$.) dissolved in normal saline, given wice daily until 12 grains $(0.5 \mathrm{~cm}$. have been given. It can be administered hypodcrmically; sterile tubes of the drug can be obtained ready for use; it may be given orally as "alcresta" ipecacuanha, $1 \frac{1}{2}$ grains $(0.09 \mathrm{~cm}$.) of emetine can be given 
by the mouth per diem in this way. It can also be given inlravenously, $0^{\circ}$ Io grm. dissolved in 10 c.c. of saline daily.

Io-20 per cent. of cases are not cured by emetine.

Emetine bismuth iodide in gelatine capsules, one grain $\left(0^{\circ} 06 \mathrm{~cm}.\right)$ thrice daily per os until 36 grains have been given, has now superseded the emetine hydrochloride treatment. It has cured "emetine-resistant" cases.

There are several good alternative treatments, e.g., tincture of opium, i5 minims, followed in fifteen minutes by ipecacuanha, 10-20 grains in cachet or pill, twice daily. The best Brazilian ipecacuanha should be used, as it contains 72 per cent. of emetine.

In the chronic stages, use daily intestinal irrigations of quinine bihyclrochloride, I-5, OOO to I-I,OOO, $\frac{1}{2}-1 \frac{1}{2}$ litres slowly injected through a well-vaselined rectal rubber tube.

Tannic acid is good, 5-

These could be preceded by a morphia suppository half an hour before. Later a bismuth mixture could be given, followed by a tonic. It is a good practice to give small doses of emetine later to prevent relapses.

There could, of course, be a combined treatment of emetine and ipecacuanha.

Deeks strongly advocates the bismuth-milk treatment as follows :-

Rest to increase resistance and diminish bowel morement. Generous milk diet-it is absorbed before reaching the large bowel. Saline or water irrigation thrice daily to wash away toxic products. Bismuth subnitrate, grains 180 , a heaped teaspoonful, mechanically suspended in a tumbler of water; effervescent, three hourly, day and night in severe cases, reduced as the patient improves. When the stools are few and the tongue clean give it thrice daily. The bismuth salt locks up the free and the nuclear sulphur of the bacteria in the intestine. It destroys certain organisms and influences others.

Entamoba show early degenerative changes (James), become vacuolated, swollen and fragmented. The characteristic frecal odour disappears. The entamoba usually disappear after the fourth day, no matter how serious the condition.

Too much opium favours retention of toxic products that irritate the normal mucous membrane and interfere with the production of enzymes and hinder digestion.

Crecostomy in selected cases of emaciation and exhaustion (Herrick) should be done. It prevents food residue from reaching the ulcerated colon, admits of better irrigation of the bowel, and makes possible a more varied and generous diet; but, on the other hand, convalescence is lengthened and a restorative operation is necessary later. 
Sir Patrick Manson recommends simaruba officinalis as follows :-

$$
\begin{aligned}
& \begin{array}{llllllll}
\text { Simaruba bark } & \ldots & \ldots & \ldots & \ldots & \ldots & \text { Grm. } 15 \\
\text { Pomegranate fruit rind } & \ldots & \ldots & \ldots & \ldots & , & \text { I } 5
\end{array} \\
& \text { Gum Arabic } \quad \ldots \quad \ldots, \quad \ldots \quad \ldots \quad \text {... } \quad \ldots \quad, \quad \text { I } 5 \\
& \text { Add water to one litre and boil to half its bulk." } \\
& \text { Give one ounce (30 grm.) thrice daily. }
\end{aligned}
$$

Dapter suggests a creosote rectal wash, I-Ioo, twice daily. $\mathrm{He}$ maintains that the creosote is absorbed, and acts upon the amoebæ in the tissues and in the localities where other drugs have failed to reach them.

The administration of sulphates at the same time as other treatment will assist in washing out the bowel, but patients must not be exhausted by their prolonged use.

A dry but varied diet is most generally useful.

Men, dogs and cats act as carriers without obvious symptoms, and should be treated pro bono publico.

Remark.-The Entamoba tetragena can cause a serious cystitis of the urinary bladder quite apart from any dysenteric affection (Wright).

\section{CILIAR DYSENTERY.}

(I) Balantidium coli (sometimes B. minutum) may cause troublesome intestinal disturbances, such as occasional, remittent or persistent diarrhœa, in which the parasites can be found.

Blood and mucus are present in all the severe cases.

Peritonitis may occur with or without perforation.

Extensive ulceration is found in fatal cases, most marked near the rectum, and less so in the direction of the caecum.

Sometimes there is vomiting, with odema of the face and extremities. It is chiefly confined to the large intestine.

The diagnosis can only be made by the microscope.

There is no specific treatment.

The mortality is about 30 per cent.

It is most common in the Philippines, Japan and Europe.

\section{THE PARASITE.}

It is an oval-shaped, large, ciliated infusorian, $30-200 \mu \times 20-70 \mu$.

It reproduces by division, budding and conjugation.

It may lose its cilia and become encysted.

It is a common parasite of the pig.

It is very active in liquid human stools.

It has been found post-mortem in the exudate, follicles, ulcers, muscular coat, blood-vessels and lymphatics of the intestine.

The method of infection is unknown. 


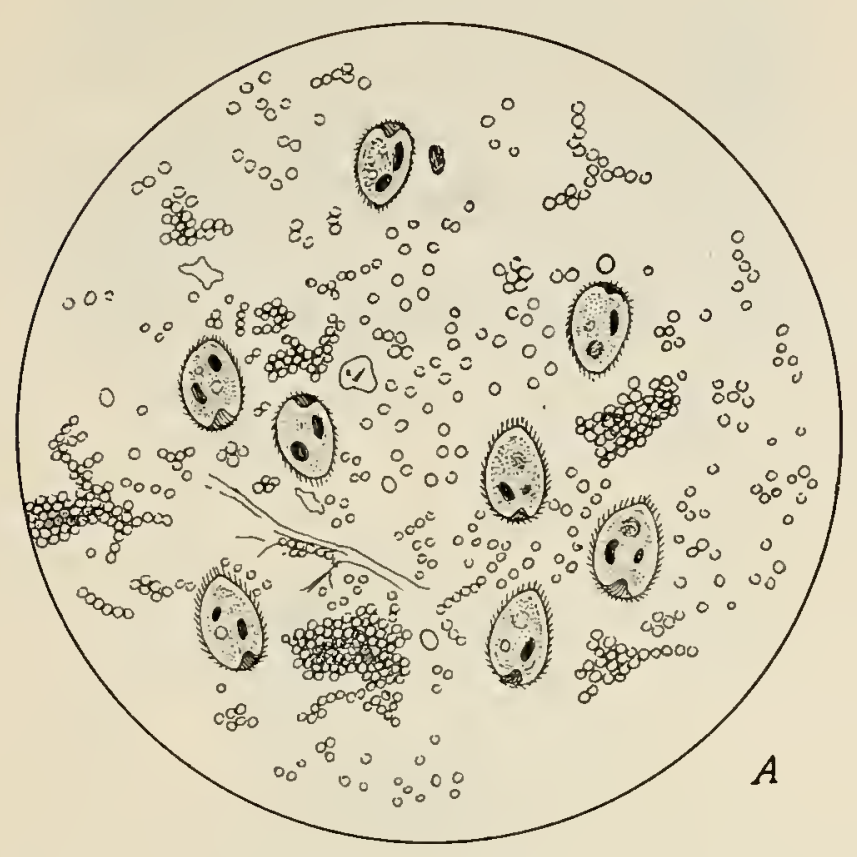

Balantidium coli. Living forms in the dejecta of Balantidian dysentery.

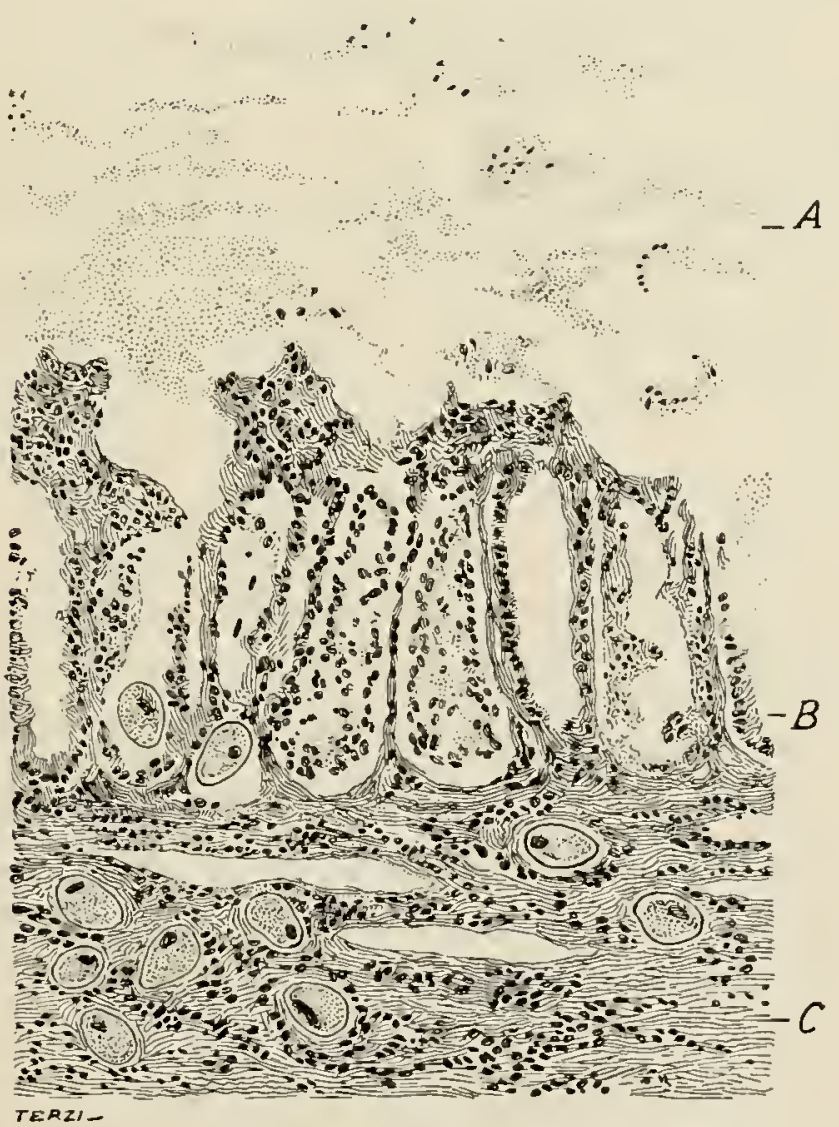

Balantidium coli. Invasion of the wall of the sigmoid flexure in a case of Balantidian dysentery. $A$, Mucus ; $B$, glandular infiltration and destruction; $C$, parasites in the connective tissue of the submucosa, with infiltration of small cells. (After Dopter.) 


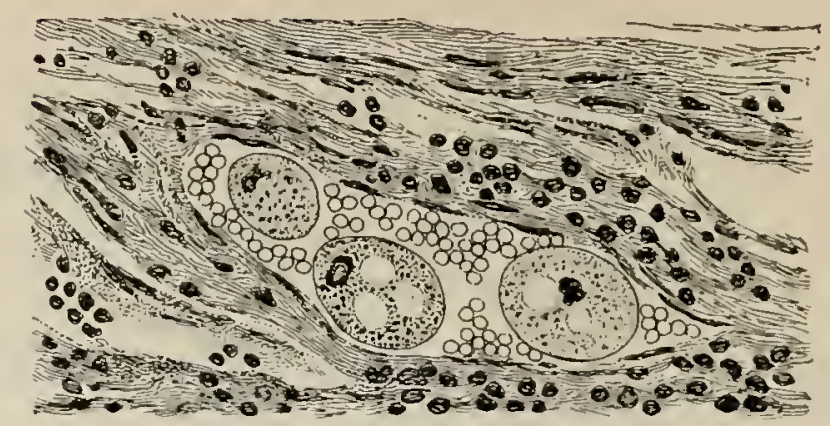

Balantidium coii. Parasites in a lymph space of the submucosa. (After Dopter.)

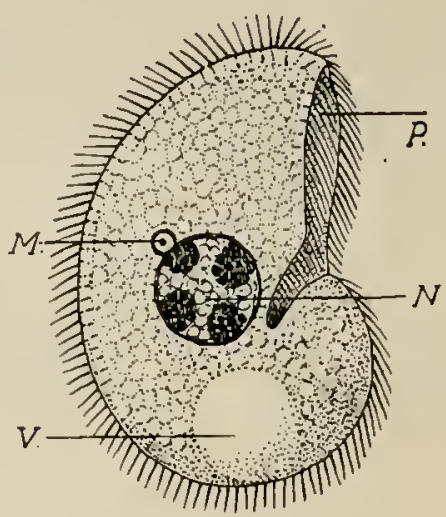

Balantidium minutum. $P$, Peristome; $N$, nucleus ; $M$, micro-nucleus; $V$, vacuole. (Afler Schaudinn.)

\section{TREATMENT.}

Castor oil, salines, intestinal irrigation with tannic acid or quinine. Emetine and ipecacuanha can be tried.

Methylene blue has been recommended, rectal injections, and by the mouth.

(2) Lamblia intestinalis is probably pathogenic, attacking principally the small intestine. It is common in India.

The tissues are not invaded, but one may have a chronic recurrent diarrhoea with abundant mucus, with bile or blood stains. There are usually intervals of relief between attacks with constipation.

The parasice, a split pear-shaped, double nucleated, protozoon with a sucking disc, can be found in the diarhoca excretions.

The disease is chronic and may last thirty years.

When searching for the parasite one should note the two flagella at the tail end. There are also three other pairs from the central nuclei. There are two nuclei, one on either side of the longitudinal dividing line; its aspect is not unlike that of a face. The parasite, about I2-is $\mu$ long, soon dies on leaving the body, but it is very persistent in the host.

Lamblia is a common parasite in rats and mice, in which it causes an enteritis. These rodents probably form a reservoir of infection for 


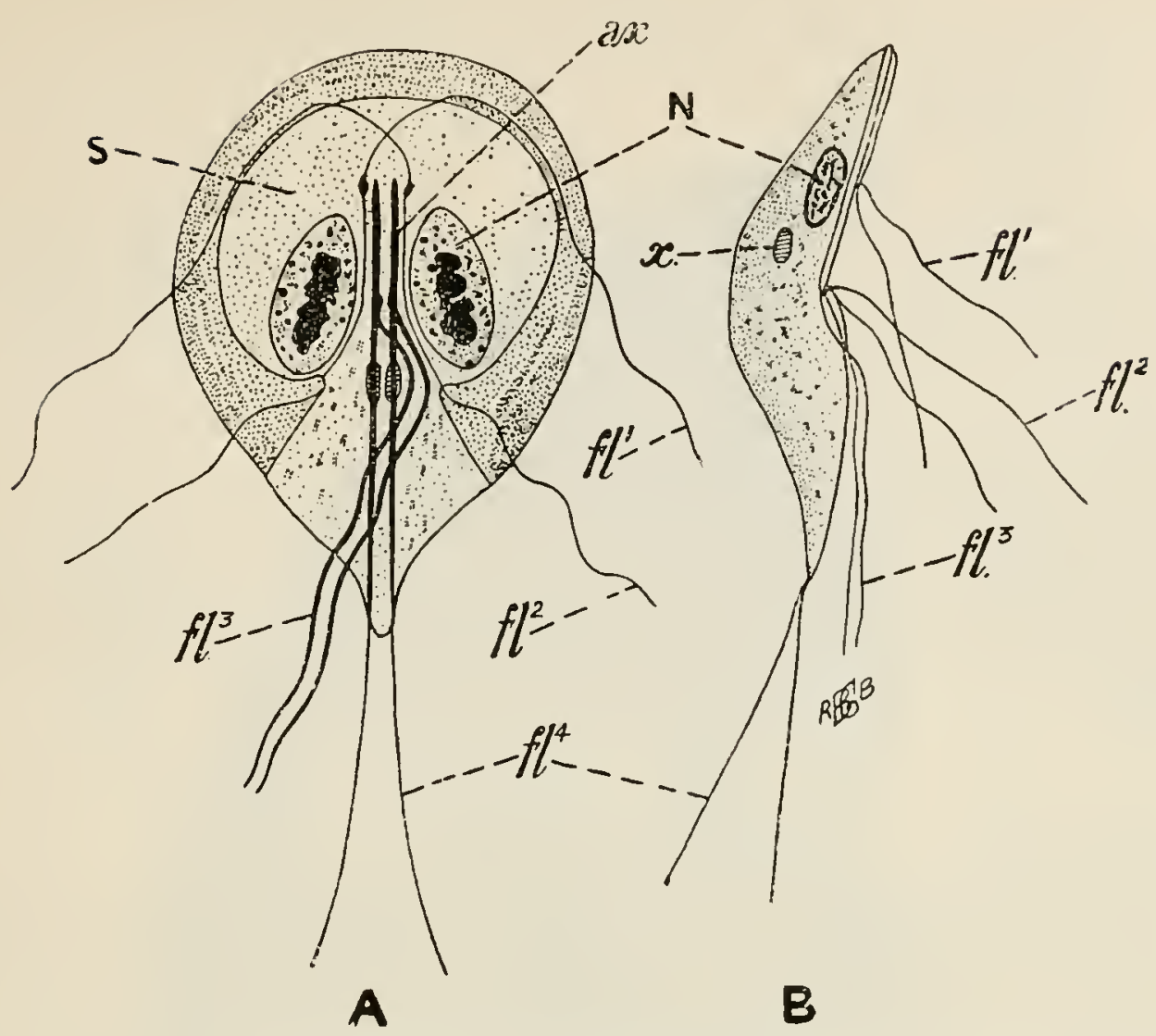

Lamblia intestinalis. A, ventral view ; $\mathrm{B}$, side view $\mathrm{N}$, one of the two nuclei ; $a x$, axostyles ; $f^{1}, f^{2}, f^{3}, f^{1}$, the four pairs of flagella; $s$, sucker-like depressed area on the ventral surface: $x$, bodies of unknown function. (After IVenyon.)

man. The cysts have been found in the faces of the common house fly, which probably acts as an insect porter (Bahr').

\section{TREATMENT.}

Free purgation and intestinal antiseptics. Enemata are not much use for the small intestine.

Metlylene blue can be tried, I-2 grains, with or without liquid paraftin, ?

Beta-naplithol has been tried.

(3) Trichomonas intestinalis is the common intestinal flagellate in man which lives in the large intestine and cacum. It is a large pearshaped body with three free flagella and one attached to the undulating membrane. Its movements are very rapid, but a sixth objective will reveal its characters. It will survive outside the bod! for days.

Some assert that it is harmless, being found usually where other organisms have deranged the intestine.

Encysted forms have not been proved.

(4) Tetramitis mesnili las been blamed for intestinal irritation, followed by diarrhoea. It somewhat resembles the trichomonas, but the latter has an undulating membrane and no large cytostome like the former. It dies in a few hours when passed in the faces, while trichomonas persists for days. It may not be pathogenic. 
(5) Less well known causative ciliar parasites are:Colpoda ciscullus. Nyctotherus faba.

\section{giganteus.}

Cercomonas, Bodo, Prowazekia are rare. Each of them possess flagella from the blunt end of organism, but they cannot be seen unti] a few days after the fæces have been passed.

They are not pathogenic.

They all probably have an amœboid stage.

(6) Coccidia, Genus Isospora, have been found in faeces of patients from Gallipoli, and are now regarded as having some pathogenic significance in man. Little is yet known of them, except in animals and birds.

\section{LIVER ABSCESS.}

\section{INCIDENCE.}

In India the native soldiers are seventeen times less liable to hepatitis than the European soldier, and twenty-five times less liable to liver abscess.

\section{YARIETIES.}

Single: These are tropical in 75 per cent. of cases, and are due to amœebæ, ascarides and gallstones.

Multiple: Usually pyæmic, sometimes coccidia.

\section{POSITION (Cantlie).}

Supra-hepatic: The pus lies between the layers of the suspensory ligament; is seldom associated with dysentery; the pus is usually sterile.

Intra-hepatic: Usually amœbic; the pus is always infected.

Sub-hepatic: Rare, and when present is not dysenteric.

\section{CAUSATION.}

Traumatism, chiefly pyogenic cocci.

Gallstones, chiefly B. coli communis.

Parasites, amobae, ascarides, hydatids, coccidia, distoma rare.

Direct extension, from right empyama, peritonitis, right perinephritis.

Typhoid, along the bile ducts from the intestine or by pylephlebitis, or directly from a focus of suppuration.

Pyæmic, along the portal vein after dysentery, gastric or duodenal ulcers, appendicitis, abscess of spleen or pancreas, after treatment for hamorrhoids, sepsis of umbilical cord, along the hepatic artery, after septic injuries of the cranium, otitis media, ulcerative endocarditis and septic diseases of the lungs. 


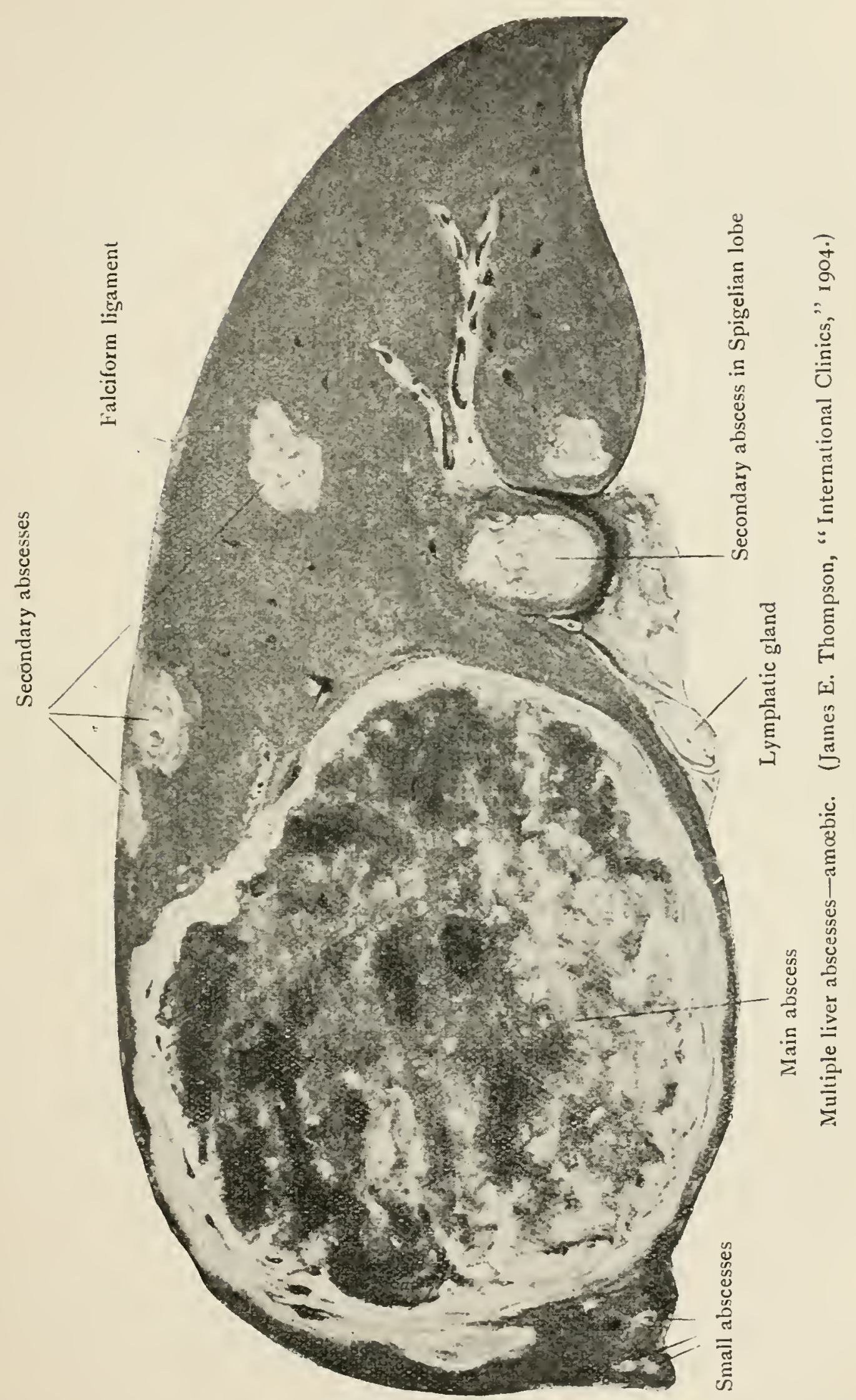




\section{AMGEBIC ABSCESS.}

\section{HISTORY.}

2 I per cent. of dysenteric autopsies in various tropical countries had liver abscess.

35 per cent. of European soldiers in India who died from dysentery had liver abscess.

$84^{\circ} 4$ per cent. of the patients at the Seamen's Hospital who had liver abscess revealed at the post-mortem evidence of dysentery.

Liver abscess is more common in Europeans than natives and in males than females.

Waring says that 65 per cent. of liver abscess cases were alcoholics.

The disease is more common between the ages of 20 and 40 , but may occur at any age.

In 90 per cent. of cases the organism is associated with or derived from dysenteric processes in the colon.

Liver abscess was operated upon in the days of Iippocrates.

\section{PATHOLOGY.}

The abscess, which is usually single, is most frequently in the right lobe of the liver. It is so frequently in the right lobe because the patient is in bed, and the right lobe being heavier than the left lies lower, and the amoba coming along the portal vein are assisted by gravity to affect the lower or right lobe. No matter how large the abscess may be it never crosses over to the other side, and in this it simulates hydatids and malignant growths.

There is no anastamosis or communication whatever between the vessels of the two sides; injection has proved this. There are really two livers arising from two distinct buds from the gut and the gallbladder lies between them (Cantlie).

An abscess may occupy half the liver, and the patient recover because the other half takes on the work, as in the case when one kidney is removed. There is no doubt that the whole liver is enlarged and congested. The right lower lobe of the lung is congested.

The abscess wall is thick, firm, ragged, and is made up of three layers :- -

(I) The outer layer is of hyperemic liver tissue with some connective tissue. In some cases a fibrous capsule may be formed from it.

(2) The middle layer is of brownish red friable liver tissue.

(3) The inner layer consists of grey necrosed tissue containing amœebre and pus cells, the latter less frequently. There is no lining membrane.

The contents of the abscess consist of a viscid chocolate-coloured 
pus, with red blood here and yellow mucus there, while a little green bile may add to the variety of colour. The pus is not readily taken up by the dressing and has a peculiar smell. The pus is usually sterile.

Under the microscope can be seen red blood cells, broken down liver tissue, large, granular, pigmented, spherical cells, leucocytes, débris, oil globules, hæmatoidin crystals, amoeba, and very rarely pyogenic cocci. Amoba are best seen four days after the operation coming through the drainage tube. They persist until the abscess has healed (Manson). The necrosis is thought to be due to the toxic action of the E. tetragena.

\section{SYMPTOMATOLOGY.}

There is nearly always a history of dysentery, but amoebic diarrhoea is not uncommon. Rogers states that 30 per cent. of his cases gave no history of dysentery, I4 per cent. of no bowel disturbance, but 98 per cent, of them revealed ulceration and scarring post mortem.

There is chill, headache, foul tongue, loss of appetite, languor and depression. Weight and fulness in the right hypochondrium, sharp stabbing pain often affecting the right shoulder and arm from the phrenic nerve and the fourth cervical to the brachial plexus.

Cough, according to the extent of the irritation of the diaphragm and pleura; there is often dyspnoea due to the base of the lung being affected.

Sweats at night, earthy tint of skin, emaciation.

Breathing rapid and shallow, chiefly on the right side, and thoracic.

Pulse, 8o-roo; hands and feet cold. Rigors sometimes.

Right rectus rigid; the left is not so. Pain on palpation.

The whole right side bulges, and the intercostal spaces are obliterated.

The liver dulness is higher than normal and arched above. It is also lower than normal.

Pleuritic friction may be heard.

The body is often bent to the right side and the right leg flexed.

Cutaneous oedema of the chest wall is common, but œedema of the feet and ascites are rare.

Vomiting may occur from gastric irritation due to pressure.

Occasionally one sees varicosity of the epigastric and hamorrhoidal veins.

Jaundice is not constant, and is but slight.

There is leucocytosis, the polymorphs going up to $74-87$ per cent.

Basal pneumonia of the right lung alone in a dysentery patient should arouse suspicions. 


\section{DIAGNOSIS.}

From Hepatitis by :-

A definite epigastric swelling compatible with liver abscess, X-ray examination and surgical exploration.

The trouble is not progressive, but often intermittent.

Chronic malaria with hepatitis may be troublesome, but the spleen also will be enlarged considerably.

Trupical liver or febrile congestion is due to diet, alcohol, \&c.

Intermittent hepatic fever due to infective cholangitis gives a history of cholelithiasis.

Syphilitic gummata by history, Wassermann and drug reaction.

Enteric, uncomplicated, shows a leucopania.

Undulant fever, Kala-azar and Tuberculosis the same.

An excess of mononuclear leucocytes is not common in liver albscess, but there is a constant excess of polymorphs, 75 -So per cent.

Double infections are common. Drugs will sometimes help, as quinine in malaria and emetine in drsenteric abscess.

The stools slould always be examined for amobas.

\section{TERMINATION.}

The abscess may run its course in from three weeks to three months. It may burst and refill or encyst and give rise to no more symptoms. 28 per cent. rupture spontaneously, and usually into the lung, peritoneum or pleura. There may be $5-10$ ounces of pus coughed up in twenty-four hours. Recovery may ensue or sudden liemoptosis result. The pus coughed up or vomited from the stomach may cease and recovery take place, or the whole series of symptoms may be repeated.

When there is spontaneous rupture the possible positions are as follows :-

Into the abdomen or intestine; here the pus causes the intestines to become matted together, but the pus is sterile. This condition should never be operated upon; the pus will be absorbed. When into the intestine the pus is passed per anum and usually missed. When it passes into the peritoneum a fatal peritonitis has been caused.

Into the stomach when the pus is romited.

Into the pleura, rare; causes emprema.

Into the lung, may bog its base, give rise to signs of pleurisy, expectoration of pus and blood; zo per cent. recover (Groves).

Into the pericardium, when it is rapidly fatal.

Into the inferior rena cava, rapidly fatal.

Into the gall-bladder, when the pus is passed per anum with possible recovery .

Externally, by the skin, when the prognosis is at its best. 
The pus may pass between the two layers of the ligamentum pulmonis and here penetrate the bronchus, but it never passes from the lung tissue into the bronchus (Cantlie). The pus is then like anchovy sauce.

The mortality of Liver Abscess is always high, 50-80 per cent.

In Algiers, 80 per cent. In the Indian Army, 57\% per cent.

\section{TREATMENT.}

Liver abscess can often be prevented if, when there are signs and symptoms of hepatitis, treatment is adopted as for amobic dysentery.

When the abscess has once formed operation is the only thing.

The starvation diet before operation should not be observed in these cases. From two to three hours before give a very nutritious and easily digestible meal, well sweetened. It prevents some of the evil effects of the chloroform poisoning dependent upon the state of the liver (Cantlie).

Always give a general anasthetic.

Insert the needle to diagnose correctly. If pus, then operate.

When the pus is in the left half of the liver, which is very rare, do not explore with a needle, nor use a trocar and cannula, but cut down and evacuate the pus in the ordinary way.

In mapping out the liver before operating remember:-

That the left margin of the liver does not coincide with the right costal margin. In a tall man it is normally above, and in a short, stout man below the costal margin, as the ribs pass outwards more transversely.

To get the lower border for that particular patient place the patient's hand (right) with the upper border at the articulation of the sternum with the ensiform cartilage and draw a line along its lower border; this is the normal lower border line of his liver. Each inch below this line means one pound of liver extra, and this is serious (Cantlie).

Examine for an egg-shaped swelling along the upper border between the right nipple and the sternum.

Remember that the pleura comes to within three fingers breadih of the lower costal margin and the lung to five fingers breadth.

When needling, it does not matter if the pleura is penetrated.

Even if the base of the lung is pierced it is not of importance, as this is congested, doing no work, and the puncture will relieve the tension.

Bleeding from the hepatic reins is good; it relieves congestion and affords comfort even if no abscess is found.

The portal vein has never been entered.

The vena cava is 42 inches away from any part of the surface of a 
normal chest, so that a needle of $3 \frac{3}{4}$ inches in length can do no harm in any case.

Do not insert the needle where the liver overlaps the stomach.

Do not forget that one lobe may be enlarged by overwork because the other is atrophied.

It is possible to miss the abscess after six punctures.

Always incise the skin first, then use the needle, or a piece of skin may block the needle. Never aim at the vena cava each time as some areas are thus missed.

To evacuate the pus a special trocar and cannula are required, that is, when the abscess is in the right half of the liver; the cannula should be $3 \frac{1}{2}$ inches long; the vena cava is $4 \frac{1}{2}$ inches distant from the surface. Push the cannula off the trocar when the end is inserted in the liver, and the liver cells are by this means pushed aside and not cut.

Do not wash the abscess cavity out with carbolic; it coagulates the blood and causes it to resemble pus.

Remove the ribs below the pleura, if necessary, and put in a tube. This tube can be of greater diameter than the cannula and inserted by stretching it upon a special rod, which is then inserted down the cannula, the cannula withdrawn, the rod liberated from the rubber tube by an appliance and withdrawn, leaving the tube in situ, exercising pressure upon the surrounding liver cells and preventing pus from leaking into the pleural cavity. Hremorrhage is also thus prevented. Do not evacuate all the pus before the tube is inserted or the abscess cavity will collapse, and there will not be a cavity in which to insert the rubber tube.

The after-treatment is all-important.

Drain the abscess by syphoning. Attach to the rubber tube a glass tube, and to this a rubber tube passing to a bucket by the bedside.

If the syphon action is excessive blood may come; then raise the bucket on to a stool, then a chair, then on to the bed, and so reduce the suction until the desired effect has been obtained.

Give emetine at the same time as for amobic dysentery.

Retain the tube in position for two to ten weeks, shortened from time to time as required, keep it in until the bile comes or the pus has all been evacuated. Then remove the syphon tube, and shorten the rubber tube as the liver pushes it out.

When bed sores arise turn the patient on to his side.

If the fever still remains there are more abscesses or the drainage has been insufficient.

If the abscess is aseptic, irrigate daily with quinine $1-1,000$, but remember that such fluids with flocculi of pus may enter the open mouths of the hepatic veins and may prove rapidly fatal.

Patients treated for I iver Abscess should never return to the tropics. 
Operation has reduced the mortality 1032 per cent.

Cantlie had 115 recoveries with 27 deatlis.

Some aspirate the abscess and inject quinine bihydrochloride, $0.50 \mathrm{grm}$. to the litre (Rogers and Wilson).

Quinine kills the amceba on the abscess wall, and the pus becomes less copious and almost serous as long as it is sterile.

Change the dressing at least twice claily; the pus tends to run by the side rather than into the dressing.

Failure of this treatment is due to:-

(I) A secondary abscess which may burst into the first cavity.

(2) Septic infection. Culture the pus organisms.

(3) Insufficient emetine.

(4) Intercurrent disease, e.g., enteric fever, \&c.

\section{TROPICAL LIVER.}

This is a distinctive disease affecting Europeans residing in the tropics. It is more prevalent generally during the early years of residence.

The foul tongue, painful liver, earthy skin, and irritable temper of such affections are well known.

The liver functions are adversely affected by heat, food, alcohol, malaria, abuse of exercise, congestion and inflammation.

\section{TREATMENT (Rogers).}

Absolute rest in bed.

External applications, such as hot fomentations on oleate of iodine.

Internal medication, e.g., salicylate of sodium, ammon. chloride, sodium sulphate, pot. iodide, ipecacuanha. Also calomel, blue pill, soda and phenalgin, followed by a saline purge.

Ipecacuanha is most useful if there is hepatitis. Give it in large doses on an empty stomach, 20-40 grains at night, preceded by chloral, 20 grains.

Ipecacuanha in keratine capsules does not act until it has reached the intestine, and hence is to be recommended.

\section{THE LEISHMANIASES.}

These will be considered under the following heads :-

(I) Kala-azar.

(2) Infantile kala-azar.

(3) Dermal Leishmaniasis (Oriental sore).

(4) Naso-oral and Oro-phalangeal Leishmaniasis.

(5) Canine Leishmaniasis.

(6) Pseudo-kala-azar (Tropical febrile splenomegaly). 


\section{DEFINITION.}

KALA-AZAR .

A chronic, specific, infective, febrile disease, caused by Leishmania donovani; characterized by irregular fever, enlargement of the spleen and liver, emaciation and anæmia, frequent hyperpigmentation of the skin, and accompanied by a heavy mortality.

\section{HISTORY.}

1869. It was noticed by British officers in India, decimating and, in some instances, depopulating numerous districts of the Garo Hills. The Garos called it "kala-azar," or the black fever.

I875. It became epidemic with a very heavy mortality.

I882. The first account of it was published by Clarke.

I889. It spread to Assam along the lines of communication.

1900. Sir William Leishman found the parasite in the spleen of a soldier at Netley, but he did not publish it until 1903, in which year Donovan found the parasite by splenic puncture during life.

1907. Patton found the parasite in the leucocytes of the peripheral blood, also that it became flagellated in the alimentary canal of bugs.

\section{DISTRIBUTION.}

It is endemic, and may become epidemic in East India, Assam, Madras and Calcuta. It is present in Arabia, Ceylon, Burma, IndoChina, China, the Yangtse valley, Sudan, Egypt, Tunis, Algeria, Sicily and Portugal.

The Indian epidemic began in the Lower Bengal at the foot of the Garo Hills, slowly advancing up the valley of the Bramaputra, travelling 100 miles in seven years.

- It followed the lines of intercourse, attacking first the larger stations. Isolated villages seemed to escape. As it spread into new villages ahead it died out in those first attacked.

It seemed to cling to a place for about six years, and then died out without apparent cause. A house would appear to retain the infection for months.

The disease terrorized the natives, those afflicted were expelled from their villages, made semi-drunken, and taken into the bush to be destroyed.

There have been sporadic cases in other parts difficult to account for, although they seemed to be typical cases of Kala-azar.

\section{ETIOLOGY.}

The disease is spread by Leishmania donorani.

The carrier blamed by Donovan is a Conorhinus.

The predisposing causes are explained by the habits of bugs thus :- 
An epidemic will travel slowly along lines of human communication.

It runs in families, the children suffering most.

The poorer sections of the community are affected most.

The dwelling becomes infected and will infect those close to it.

Neither sex nor season has any influence.
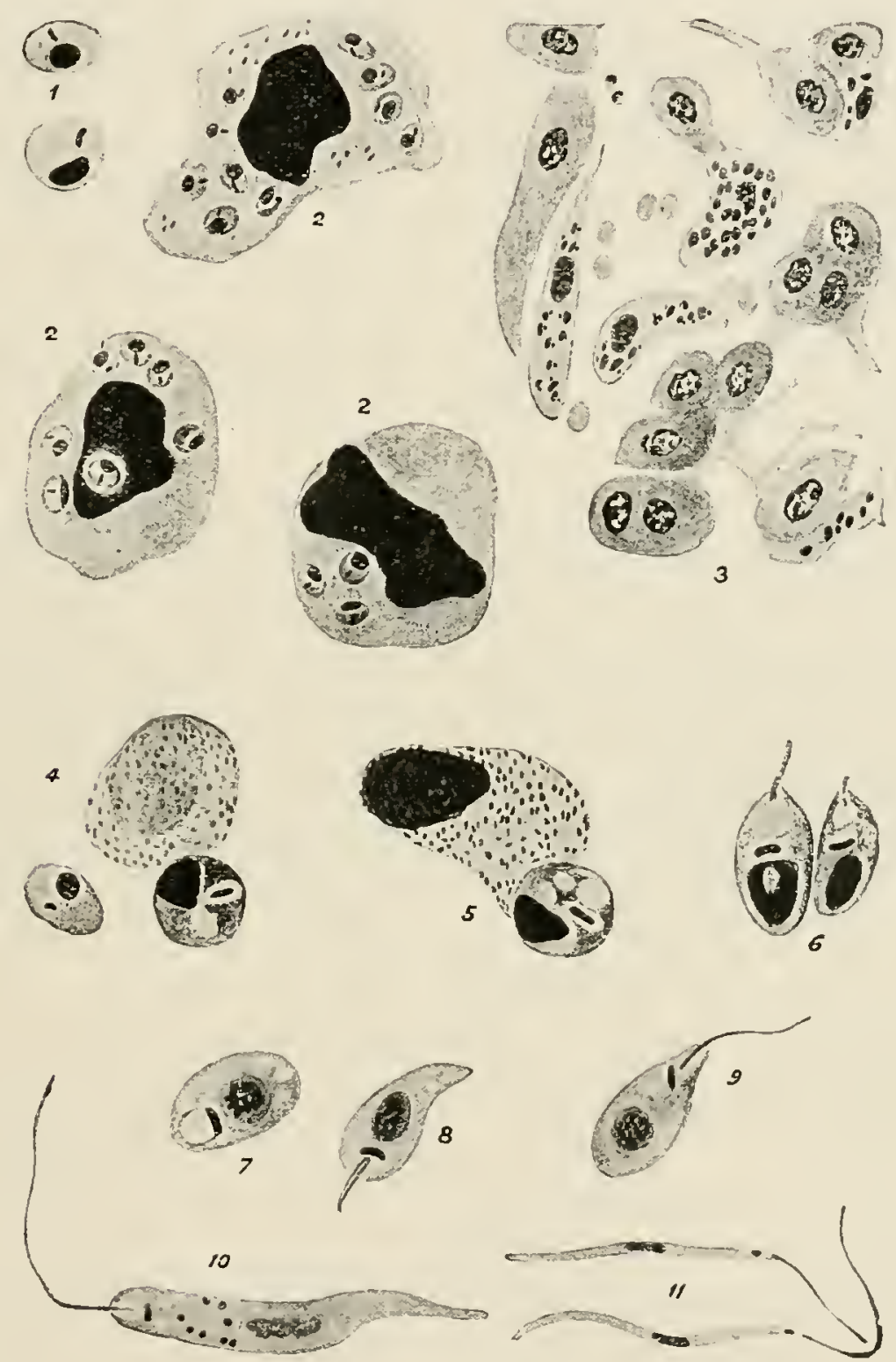

Leishmania donovani. I, Free forms, each with nucleus and rod-shaped blepharo. plast (after Christophers); 2, endothelial cell and leucocytes containing parasites (after Christophers); 3 , capillary in the liver showing endothelial cells containing parasites (after Christophers); 4, two parasites escaping from a leucocyte in the alimentary canal of the hug (after Patton); 5, further development in bug (after Patton); 6, young flaggellate forms in bug (after Patton); $7-11$, culture forms (after Leishman) ; 7,8 , 9, show development of flagellum.

\section{THE PARASITE.}

The Leishman-Donovan "body" is a small ovoid or roundish organism, 2-4 $\mu$ in diameter; when stained it shows two lilaccoloured chromatin masses of unequal size enclosed in a cytoplasm of a bluish tint.

The larger mass is the nucleus, oval and centrally placed in the 
resting stage, elongated, and situated peripherally in the pre-division stage.

The smaller mass is the muco-nucleus or blepharoplast, a short rod, perpendicular or at a tangent to the nucleus.

The parasite multiplies by simple fission, the nuclear masses always elongating and dividing before the protoplasm.

The parasites are almost always intracellular in man.

Their growth and multiplication within the cell causes the cell to enlarge and disrupt; these parasites set free enter other endothelial cells or are engulfed by leucocytes in which they may be seen in the peripheral circulation. Clusters of them may be seen, 50-200, in a structureless matrix débris of the host cells.

In cultures the parasite enlarges rapidly, becomes elongated, and acquires a flagellum at its rounded end acquired from the blepharoplast, the whole measuring $\mathrm{1} 2-20 \mu$ in length.

Attempts to transmit the parasite to vertebrates have failed.

The parasite can be cultured from the blood, even during apyrexial periods, on N.N.N. media. Ten or twelve tubes of this media should be inoculated, otherwise parasites, if scanty, may not be secured. Eight out of ten tubes may be negative. It is the flagellate form of the parasite that develops in the cultures (Cornwall and Menon).

Run a few drops of finger blood into 15 -20 c.c. of citrated saline solution, centrifuge it, inoculate N.N.N. media with the deposit of corpuscles.

Donovan believes that a biting insect carries it to man, while Manson suggests that insects might deposit it upon the wounded and abraded surfaces of man.

Once in the body it enters an endothelial cell of a capillary bloodvessel or lymphatic, and therein grows and multiplies until up to 220 may be seen in one cell (Leishman).

The liver, spleen, bone marrow and lymphatic glands may be thus affected, less so the pancreas, kidneys, suprarenals, testicles and lungs.

The enclosing cell ruptures, the liberated parasites are taken up by leucocytes, polymorphonuclears, mononuclears, and rarely eosinophiles, as a result of which process the parasites appear in the peripheral circulation, especially if a cliarthoea due to intestinal ulcers increases the polvmorphonuclears.

The further development takes place in the bug, but the complete life-cycle has still to be ascertained.

\section{INCUBATION.}

Not yet ascertained.

One case was under ten days.

It is generally considered to be from three weeks to several months. 


\section{SYMPTOMS.}

Onset is often by rigor, which may recur daily.

Initial fever is irregular, high, intermittent or continuous, showing a double remission daily on a four-hourly chart.

Vomiting is not infrequent.

Less frequently there are gastro-intestinal disturbances, or in some instances the onset is insidious.

Secondary low fever is chronic in its course, continues from two to six weeks, then gradually declines. The spleen and liver enlarge, may be painful and tender. Then there is an aprexial period, or a very mild fever. Profuse sweats are common. Rigors are now rare. Apyrexia and pyrexia may now alternate. The anemia increases.

Cachexia is now manifest. There are: asthenia, wasting of muscle with protrusion of abdomen. The ribs show, the extremities are thin,

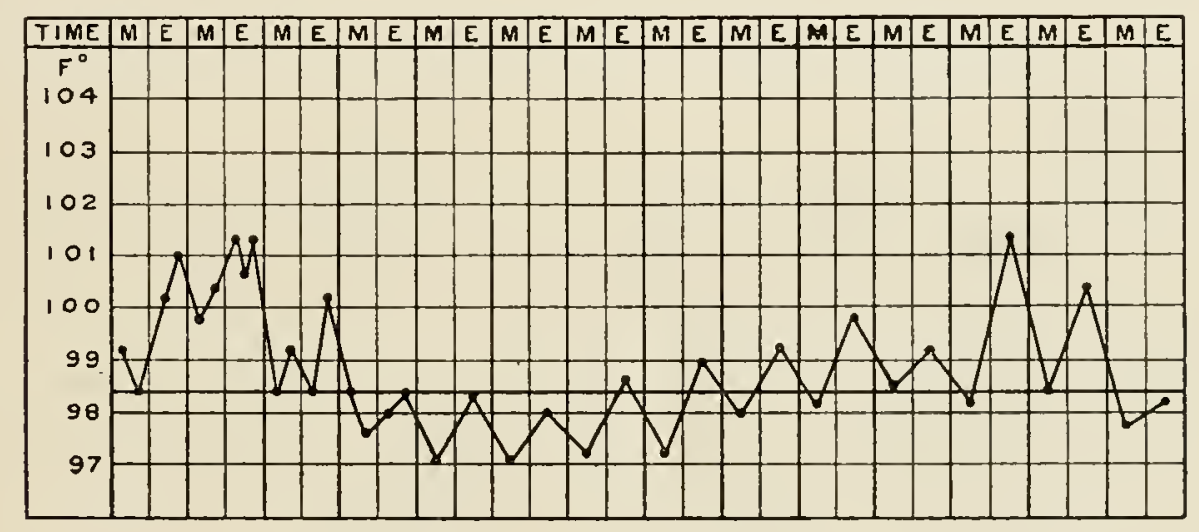

Kala-azar. Undulating type.

cheeks sunken, nose sharp and ankles puffy. There may be oedema of the legs and ascites.

The skin becomes an earthy grey colour.

The hair becomes dull, dry, brittle, and may fall out.

Epistaxis and bleeding from the gums are not uncommon.

The spleen may nearly reach the pelvis, but the liver is not so much enlarged.

Diarrhœea and dysenteric attacks are common.

Hæmorrhages may occur from anywhere-nose, gums, stomach, bowels, or under the skin.

There may be a papular eruption about the thighs. Ulcers may form.

\section{TERMINATION.}

The disease goes on for months or years, average twelve to eighteen months. 4 per cent. improve slowly, 96 per cent. die of asthenia or some intercurrent disease (Rogers). 
Dysentery carries off about go per cent. of them, then comes phthisis and pneumonia.

Usually the tongue is normal and the digestion good.

\section{THE BLOOD.}

The parasites may be found in the peripheral circulation.

There is marked ancemia, 4-2,500,00o reds per $\mathrm{cm}$. (Rogers).

Ib. is reduced in proportion.

The colour index is normal.

There is marked leucopenia, 2,ooo or less.

Polymorphonuclears and eosinophiles are reduced.

Mononuclears and lymphocytes are increased.

The reduced polys probably predispose to bacterial invasion.

Coagulability is clecreased ; exploratory punctures may set up a fatal hamorrhage.

The whites to reds vary from I to 2,000 , I to 4,000 reds instead of I to 625 .

\section{DIAGNOSIS.}

An irregular chronic fever with a very enlarged spleen and a relative mononuclear leucocytosis is probably Kala-azar, but one must find the Leishman-Donovan body to be certain.

Centrifuge the blood for the leucocytes.

To puncture the spleen is dangerous, but is practised at times.

Some artificially excite bullae for the leucocytes.

A blood examination will exclurle a leukremia.

The double daily remission of fever is important.

Banti's disease is sometimes troublesome; the microscope only will exclude it.

\section{PROGNOSIS.}

This is always bad. Moderate estimates state that jo per cent. die who have had treatment, and 96 per cent. Without it.

The increase of the polys is a good sign.

Better results may be obtained by tartar emetic, intravenously and in strong doses. The writer suggests Injectio antimonii oxide.

\section{MORBID ANATOMY.}

The body is emaciated. There is marked muscular atrophy.

There is some oedema present.

The spleen is much enlarged, it is firm, deep red in colour, the capsule thickened and congested throughout. Many parasites may be seen. The spleen sometimes weighs so ounces.

The liver is somewhat enlarged, the intra-lobular arteries are dilated, they contain macrophages full of parasites, the liver cells are 
atrophied and degenerated. Sometimes the cirrhosis is severe and no parasites are seen.

There may be ulcerations of the skin and the intestine.

There may be hamorrhages in various parts.

The bone marrow cells show numerous parasites, the yellow marrow has become red, soft and diffluent.

Intestinal ulceration is very common, parasites may be found in the walls of the ulcers and the proximal lymphatic glands.

Parasites are sometimes found in the kidney blood-ressels, but never in the secretory tubules.

\section{TREATMENT.}

Antimony tastrate or sodium antimonyl tartrate has been found most useful, given intravenously. Rogers claims to have had good results.

The following prescription is useful:-

$$
\begin{array}{llllr}
\text { R. Tartar emetic } & \ldots & \ldots & \ldots & 2 \text { grm. } \\
\text { Liq. fowleri } & \ldots & \ldots & \ldots & 6 \text { c.c. } \\
\text { Aq. dist. a. } & \ldots & \ldots & \ldots & 300 \text { c.c. }
\end{array}
$$

Of this 2 c.c. diluted with distilled water, intravenous, twice weekly.

Antimony oxide is well worth a trial.

Others have used atoxyl, 3 grains, every other day intramuscularly (Manson and Low).

Others have used quinine, 60-90 grains daily, until the temperature falls, then 20 grains daily.

Salvarsan is useless.

Other treatment is symptomatic:-

Good food and hygiene.

Calcium chloride or lactate for hamorrhages.

All other diseases in the patient should be cleared up.

Isolate the patient, disinfect infected dwellings, destroy all bugs.

$$
\text { INFANTILE KALA-AZAR. }
$$

\section{SYNONYMS.}

Leishmania anzemia.

Febrile splenic anamia.

Splenomegaly

Ponos.

\section{DEFINITION.}

A subacute or chronic specific disease resembling Kala-azar, caused by $\mathrm{L}$. infantum and occurring in childhood.

\section{DISTRIBUTION.}

Southern Europe, North Africa, Mediterranean shores and islands. 


\section{ETIOLOGY.}

The causative organism is Leishmania infantum.

The carriers blamed are Pulex irritans and P. serraticeps.

The parasite is indistinguishable from L. donovani morphologically, but the latter is not inoculable with success into dogs.

The parasite can be found in the peripheral circulation, bone marrow, spleen and liver in both children and dogs.

The organism can be cultured and subcultured indefinitely.

The disease can be reproduced in monkeys and dogs, but the latter are spontaneously infected and may form the reservoir of the virus and its ectoparasites, e.g., fleas (Basile). Wenyon doubts this.

The disease manifests itself in children usually between the ages of two and three in the springtime and early summer.

Several members of the family may be attacked.

The pathological findings are as in Kala-azar.

\section{SYMPTOMATOLOGY.}

There is an insidious onset which may coincide with an attack of diarrhcea and vomiting.

These are: irregular fever, pallor, epistaxis, apathy, with some enlargement of the spleen.

Hamorrhages from the nose, gums and under the skin.

A diarrhoic or dysenteric attack may be marked, alternating with constipation. The appetite is usually preserved.

Wasting, ancemia progressive, $1,500,000-3,000,000$ reds.

$\mathrm{Hb}$. is below 50 per cent. Leucocytes from I,500-3,00o. Mononuclears form 70 per cent. to so per cent.

Ulcerative stomatitis is not uncommon. Noma on the face or genitalia.

The spleen now enlarges enormously, bulging out the abdomen.

The pulse-rate is rapid, I20-160. Hæmic murmurs are rare.

The opsonic index is low, especially for B. coli communis.

Transient aedemas of any part may occur.

The liver is generally enlarged.

Death is from exhaustion, rarely under six months.

Second attacks are rare, hence the Jews of Baghdad at one time inoculated their children.

\section{COMPLICATIONS.}

Bronchitis, broncho-pneumonia, pleurisy, fatal dyspnoea from adema of the glottis.

Noma is common in Italy and Greece.

Otitis media is rare. 


\section{TREATMENT.}

This is very unsatisfactory.

Treat as for Kala-azar.

Splenectomy is not advised as the disease is a general disorder.

Cochran, however, claims good results from operation.

Infected dogs should be destroyed.

Common cleanliness will diminish the prevalence of fleas.

Spontaneous cure does take place at limes.

Some claim good results from salvarsan.

Antimony should be tried as for Kala-azar.

\section{Dermal Leishmaniasis (Ormental, Sore).}

\section{DEFINITION.}

A specific circumscribed ulceration of the skin caused by I. tropica.

\section{DISTRIBUTION.}

It is endemic in many tropical and sub-tropical countries.

In the Sudan they have the non-ulcerating type.

In South America it is known as "Forest yaws."

It occurs in Morocco, Tunis, Tripoli, Algeria, Sahara, Egypt, Congo, West, East and South Africa, Syria, Asia Minor, Mesopotamia, Arabia, Persia, Caucasus, Turkestan, Valley of the Indus, N.W. Provinces, Rajputana States, Crimea, Cyprus, Crete, Greece, Italy, Brazil and Guiana. It is a misnomer to call the disease "Oriental sore" with this distribution.

\section{ETIOLOGY.}

It is caused by L. tropica.

It is conveyed by direct contact between man and man through abraded surfaces or small wounds. Insects maty play a part in its transmission.

There is no general infection in dogs occurring in the same parts as is the case with Infantile Kala-azar, except perhaps in Persia (Gachet).

Laveran claims that house flies can carry the parasites mechanically and could thus infect wounds and ulcers.

Gachet blames the dog fly, e.g., Hippobosca canina.

\section{HISTOPATHOLOGY.}

There is atrophy and disappearance of the epidermis of the affected parts. There is a cellular infiltration of the corium and papillae by lymphoid cells, plasma cells, and large round cells. These latter, which are probably proliferated endothelial cells, are packed with Wright's bodies. 
The squamous epithelium is surrounded by desquamated epithelium. Crusts are formed. The lymphatic and blood ressels in the deeper layers are surrounded by lymphoid cells. There are vertical epithelial columns extending deeply down with a few cell nests and isolated masses of cell infiltration.

It is essentially an infective granuloma.

Hair, sweat glands, sebaceous glands and follicles are inraded and destroyed by granular tissue.

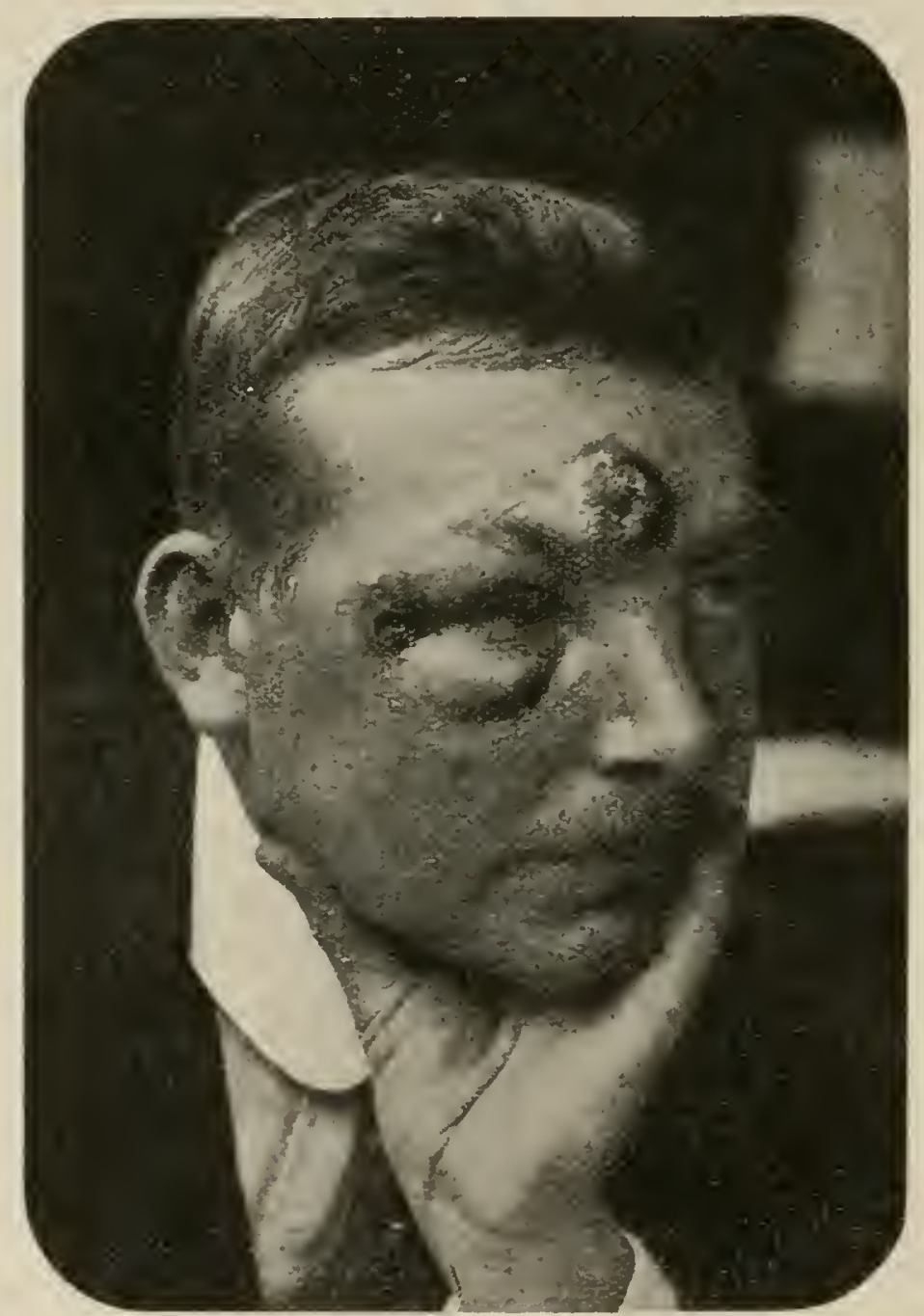

A case of Leishmaniasis from South America. (Pholograph by R. McKay.) (From the Journal of the London Sihool of Tropical Medicine.)

Polymorphonuclear cells do not predominate in the ulcers as in pyogenic ulcerations, but the mononuclears do.

\section{SYMPTOMATOLOGY.}

The incubation period is from a ferr: days io a few months.

During this period there is usually some irregular ferer.

Then several small spots appear, not unlike mosquito bites. These become red, markedly indurated, increase in size, and the surface becomes corered with scales. 
After three to four months ulceration begins with a yellowish secretion which dries hard. Some forms do not ulcerate, e.g., the Soudanese. The ulcers spread under the scab, the surrounding tissue becomes adematous, the ulcer, when the scab is removed, reveals fungating granulations. The proximal lymphatic glands do not usually enlarge.

The peripheral blood shows distinct mononucleosis.

The time of coagulation is increased.

There may be leucocytosis during the fevers, and leucopania be:ween them.

Healing begins from the sixth to welfth month by granulation commencing in the centre. A pinkish disfiguring scar remains which may readily break down again. The sore may be multiple or simple.

The ulcer may spread to the mouth or nose and may attack the mucous menibrane.

The sores are auto-inoculable by scratching.

Uncovered parts are generally attacked; the face, hands, feet, \&c., are most affected. Repeated attacks often occur.

The non-ulcerating type has been called male, and the ulcerating the female type. In some cases one patient may show both types of the condition.

The ulcers may contain pyogenic organisms.

There are no constitutional symptoms. The parasites are not found in the blood (Wenyon).

\section{DIAGNOSIS.}

In the endemic areas the diagnosis is not difficult.

The following points should be noted :-

(1) Does the patient come from an infected area?

(2) Are the nodules and ulcers on uncovered parts?

(3) Is the course of its development typical?

(4) Have the proximal glands enlarged? If so, it is probably not Leishmaniasis.

(5) Have the L. tropica been found in the scrapings?

Syphilis and Yaws are excluded by treatment.

A chancroid on the face is difficult. One should decide by the scrapings.

\section{PROGNOSIS.}

It is good as regards life.

Phagediena may cause death by secondary septicremia and pyramia.

Serious disfigurement may ensue about the face. 
The ulcers are troublesome and unsightly rather than painful and dangerous.

\section{TREATMENT.}

This is rery unsatisfactory.

Try nitrate of mercury ointment.

Remove scabs by boric acid fomentations.

Disinfect with mercurial solutions.

Apply antiseptic ointments.

All wounds should be disinfected at once with tincture of iodine.

Guard against blood-sucking insects.

Increased sanitation reduces the incidence considerably.

Dogs and camels may be reservoirs of the disease.

Tartar emetic should be tried intravenously and its ointment applied to the ulcers.

The Nile boil, Bucharest boil, \&c., have nothing to do with this disease. They are progenic in origin.

\section{Differentiation between I Kala-azar and Oriental Sore.}

\section{(After Balfour and Archibald.)}

For brevity K. A. will equal Kala-azar and O. S. Oriental Sore.

Family distribution.

K. A. An extraordinary tendency to attack a number of persons in the same family or household, this family incidence clearly pointing to house infection.

O. S. Rarely, if ever, more than one person attacked.

Age incidence.

K. A. Children most commonly attacked.

O. S. Adults the rule.

Race incidence.

K. A. Immigrants rarely attacked before eight years' residence in the East. The majority of patients seen are natives.

(). S. Immigrants attacked within a fer months to a year's residence in the East. The majority of patients seen are natives.

Seasonal incidence.

I. A. No seasonal incidence known, though it is probable that the majority of cases are infected in the cold season.

O. S. Affection first appears usually from September to April, and may last for sixteen months or more. 
Fevers typical of the disease.

K. A. Double remittent type of fever in certain cases. Double continued type. Low continued type. Frequency of different types of fever in various stages of the disease.

O. S. No fevers.

Recovery after septic complication.

K. A. Such as cancrum oris.

O. S. Septic complications no effect, as the parasite thrives best in cultures with masses of cocci and bacteria.

Effect of quinine.

K. A. Large doses in early cases have cured.

O. S. No result.

General symptoms in-

K. A. Early cases give history of rigors daily, headache, sickness. 33 per cent. of cases in hospital show diarrhœa, and 7 per cent. dysenterv.

O. S. None of these symptoms present.

Spleen and Liver.

K. A. Enormous enlargement of both viscera.

Coagulability of blood decreased.

O. S. No enlargement of riscera, and coagulability increased. Distribution of parasite.

K. A. Polynuclear leucocytes infected. Spleen, liver, bone marrow heavily infected. Intestinal ulcers and cutaneous ulcers contain the parasite.

O. S. Superficial polynuclears rarels, if ever, contain the parasites, whereas they swarm in the large mononuclear cells seen in a smear.

Cultivation and derelopment.

K. A. At $27^{\circ} \mathrm{C}$., in blood containing 10 per cent. citrate, parasites live for several days and multiply, but there is no material alteration in their form, and no increase in size.

At $22^{\circ} \mathrm{C}$., and not above $25^{\circ} \mathrm{C}$., flagellated forms appear.

Exposure to a temperature of about $25^{\circ} \mathrm{C}$. causes rapid death and degeneration of the developing forms.

Sterility of the culture tubes is of crucial importance.

Contamination with micrococci and bacteria causes rapid death and degeneration of the parasites; staplylococci the most deadly contamination for the parasite. 
O. S. At $28^{\circ} \mathrm{C}$., in infected, unheated, undiluted serum with no citrate, flagella forms appear in five days.

At $28^{\circ} \mathrm{C}$. flagella forms have been obtained.

Contamination with micrococci and certain bacteria highly favourable, as the parasite develops symbiotically with masses of these organisms.

Staphylococci the most favourable contamination. Further, the most perfect monadina forms are seen attached to large masses of staphylococci.

NASO-ORAL AND ORO-PHAL.INGE.IL LEISHM.ANI.ASIS.

There is no real difference between this and the ordinary Demal Leishmaniasis, except that the mucous membranes of the nose, mouth and pharynx are primarily attacked by L. tropica. Naso-oral Leishmaniasis is also known as Espundia. The condition is often more chronic than the dermal condition, but it is seldom produced as a result of direct extension from the skin.

It seems to be common in Peru, Brazil and Colombia.

The larynx, trachea and asophagus may be affected by the extension of the granulomatous ulcerations.

In such cases death may be due to exhaustion and amyloid disease.

There may be destruction of the nasal cartilage inducing a marlied deformity of the nose; the skin may be wedematous, with patches of hard oedema below the eyes.

The breath may be offensive.

Deglutition may be difficult.

The disease may last from twenty to thirty years, the final appearance not being unlike that of a cachectic carcinomatous patient.

The condition is much more chronic than the dermal trpe, and usually commences in the mucous membranes primarily, the slin perhaps remaining free throughout.

The microscope only will differentiate it from Tuberculosis and Blastomycosis.

An attempt must be made to destroy the initial lesion, otherwise the treatment is purely simptomatic.

\section{C.nine Leishimaniasis.}

This is mentioned only because it is affirmed by some that Leishmaniasis in dogs forms the reservoir of infective parasites in humans, especially for Infantile Kala-azar.

Pulex serraticeps, which occasionally feeds on man, can serve as a carrier of the disease because parasites develop in the fleas.

Dogs have not been infected by Indian Kala-azar. 
In dogs there is an acute and a chronic form of Kala-azar.

Acute occurs in young dogs with fever, loss of appetite, wasting, tremors, motor disturbance in the hind-quarters and diarthoea (rare).

The animal dies comatose in three to five months.

Chronic with insidious onsel, progressive ancmia, weakness, wasting, tremors, \&c.; spleen much enlarged; the disease may resemble rabies.

PSELDO-KALA-IZAR (Tropical febrile splenomegaly).

\section{DEFINITION.}

This is mentioned here because of its clinical similarity to Kala-azar.

It is a chronic, irregular, febrile disorder of unknown causation, characterized by splenic hypertrophy, gastro-intestinal disturbance and emaciation.

\section{DISTRIBUTION.}

South America, India, Ceylon, China, Egypt, Philippines, North Africá and Italy.

It can only be differentiated from Kala-azar by absence of the parasites during life and after death by repeated examination.

Spleen puncture is unsuccessful.

The febrile attack differentiates it from Banti's disease, and splenic removal does not effect a cure.

The prognosis is bad.

Arsenic and tartar emetic should be given.

$$
\text { MALARIA ("Mal aria," Italian for "bad air"). }
$$

\section{DEFINITION.}

Malaria is the term employed to indicate:-

A group of acute or chronic specific fevers or to morbid conditions arising directly therefrom.

Caused by the Sporozoal organisms Plasmodium malariz, Plasmodium vivax and Laverania malaria.

Carried by the Anophelina, in which the parasites undergo part of their life-history.

Characterized by fever, usually periodic, ancmia, enlargement of spleen, and the deposition of a peculiar pigment in the viscera and elsewhere.

Quinine is a specific treatment for most of these conditions.

\section{HISTORY.}

In 550 B.C. Hippocrates recognized and differentiated periodic fevers into quotidian, tertian, sub-tertian and quartan.

Galen, Celsus and other Romans also described these fevers accurately. 
I640 was the first advance in our knowledge of them, when the introduction of cinchona enabled a differentiation of malarial from other febrile diseases. This drug was probably first used by the Indians of Peru, and later rediscovered by R.C. priests. The Countess d'E1 Cinchon, the wife of the viceroy of Peru, sent some of it to Europe, hence its name.

I 7 I 7 . The Italians used drainage as a protective measure.

1847. Meckel described the peculiar pigmentation found post mortem in the spleen and blood of one who had died from malaria.

1854. Planer demonstrated the pigment in living finger blood from a malarial patient. It was not then known that these pigments were the parasites.

I880. Laveran established definitely the parasitic nature of the infection. Golgi demonstrated the definite correlation between the development of the parasite and the periodicity of the fever paroxysms.

I88I. Mosquito nets were first used in India.

1885. Golgi and others distinguished and differentiated species of parasites and an asexual life-cycle.

I894. Sir Patrick Manson suggested that the malarial parasite must have an extra-corporeal life and probably in a blood-sucking insect, e.g., the mosquito.

I897. McAllum saw the conjugation of living sexual forms.

Ross demonstrated malarial parasites in the stomach wall of the mosquito.

I90o. The microscope was not much used in the tropics prior to this date.

It is interesting to note that in $1763,12,000$ Frenchmen disembarked at Kourou, French Guinea. They lost io,ooo of their number by death from disease, chiefly malaria, within twelve months.

\section{AETIOLOGY.}

This will be dealt with under the heads of Predisposing and Exciting causes.

Predisposing causes.

(1) Residence in an area infected with malarial carriers (humans with gametocytes) and mosquitoes capable of transmitting the disease.

(2) Residence in an infected area with meteorological conditions of air, moisture and temperature particularly suitable for the development of the parasite, their carriers and larve. Warm, marshy places in the wet seasons are ideal. There must be an average temperature of $60^{\circ} \mathrm{F}$. at least. 
(3) Occupation in deltas of rivers, low-lying marshy districts, or working in such places as rice fields, recently deforested lands, \&c.

(4) Age. Infants are more readily attacked than adults.

(5) Racial. Native races suffer less than immigrant races in an infected country because of the partial immunity of indigenous peoples.

(6) Diminished bodily resistance and the factors that cause it, e.g., chills, starvation, over-feeding, the onset of other diseases, chronic ailments, \&c.

(7) Altitude. Mosquitoes do not thrive at high altitudes in the tropics due to the cool air and winds present there. Malarial convalescents should be sent to the hills, but at slow stages.

(8) Decomposing regetable matter. This is not indispensable, but it is a stimulating factor for fly breeding when present.

(9) Subsoil moisture. Where the subsoil moisture frequently rises high, there facilities are afforded for fly breeding, given other favourable conditions.

(ro) Rainfall. Heavy rains wash out the drains, pools, and with them the contained larve. Less heavy rains fill them only. Where water lies for several weeks in the tropics mosquito breeding is stimulated. Where there is no water, as in the Sahara desert, there is no malaria, the oasis excepted.

(I I) Strong winds. Nosquitoes fly a few feet from the ground. When wind or currents of air prevail, as from fans and punkahs, mosquitoes seek shelter.

(12) Trees. A belt of trees between breeding places and dwellings is said 10 be protective.

(13) Time of day. Mosquitoes bite most frequently just before sunrise and just after sunset and at night. In shady or dull places they will bite at any time. There are stragglers at all hours.

(I4) Disturbance of soil. This predisposes in so far as there are usually excarations in which puddles of water accumulate for mosquito breeding.

In construction of roads, railways, houses, forts, barracks, Sc., carelessness in this respect may cost the enterprise hundreds of lives, as happened at Hongkong.

Exciting.causes.

The exciting causes are P. malaria, $P$. vivax, and $I$. malaria because (Manson) :-

(1) Their occurrence in the blood is sooner or later associated with the malarial clinical phenomena. 
(2) Malarial fever is invariably associated with one of these parasites at some time of its course or another.

(3) The phases of a malarial fever bear a definite relation to the phases of the life-cycle of the particular parasite present in the blood.

(4) The characteristic pigmentation of the viscera is fully accounted for by the pigment-forming property of the parasite.

(5) Injections of malaria-infected blood will, after an incubation of twelve days, be followed by an attack of malarial fever, and similar parasites can be recovered from the blood as those injected.

(6) The administration of quinine will clear up the clinical symptoms, and cause the disappearance of the symptoms in the majority of cases.

(7) If certain species of mosquito which have fed on infected blood be dissected at intervals, the evolution of the parasite can be followed out.

(8) Mosquitoes thus fed, if kept for a week, will, on biting an uninfected man, produce, after incubation, a typical malarial attack. Infected mosquitoes from Rome sent to London were allowed to bite the son of Sir Patrick Manson. A typical malarial attack followed.

(9) If the non-immune is effectively protected against the mosquito bite he will not contract malarial ferer, though living in a malarial country.

Three factors are necessary for an attack of malarial ferer :-

(I) The malarial parasite.

(2) The necessary mosquito.

(3) The non-immune man.

If only two of these factors are present malaria cannot exist.

\section{THE PARASITE.}

Three parasites have been mentioned, but some believe there are others which cause a quotidian form of the fever, but such have not been conclusively demonstrated.

None of these parasites have ever been found free in air, water, soil or decaying regetation.

The three parasites enter the human body as sporozoites, passing from the salivary glands and the hypopharyngeal canal of the mosquito into human blood at the time of the bite.

One mosquito, when biting an infected person, may take up from $6,000-7,000$ crescents per $\mathrm{cmm}$. of blood, but they may all perish by phagocytosis in the intestine of the fly. 
When once injected into human blood the parasite again has the risk of being killed, or it may lie dormant in the spleen to develop when the resistance of the victim is reduced, or it may multiply and cause an attack almost at once.

Some days must pass between the bite of an infected mosquito and the subsequent attack of fever, because the parasites must multiply enormously and generate sufficient toxin to produce the fever. It has been estimated that 350 millions of parasites are required in the body to produce a rise in temperature of one degree $F$., hence one may find parasites in the blood when an attack is not anticipated by the patient. If but few parasites are injected by the mosquito the incubation period is lengthened; if many parasites, then it is shortened. The average time between the bite and the ferer is nine to twelve dars.

During this period there are premonitory srmptoms, such as lassitude, body pains, \&c., so that quinine taken at this time may abort the attack.

The parasites may increase at a very rapid rate, as shown by Ross, thus: Suppose that $\mathrm{I}$, ooo protospores of $P$. wivax have entered the red cells, only ten out of the fifteen to twenty spores produce, then they will increase as follows:-

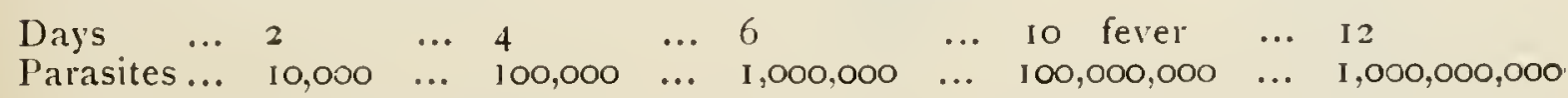

The rise of temperature is caused by the liberating of toxins when the red cell is ruptured by the sporozoites. The sexual forms take no part in producing the fever in the patient in whom they have been found.

The pigmentation of the cells and organs is due to the hamozoin liberated from the parasite as the cell ruptures.

The liberated young spores attack and enter new red cells until the majority of the red cells may be infected and a critical condition arise for the patient. In some cases, e.g., quartan, the attacks may subside owing probably to a partial immunity.

The merozoites can pass through the placenta, infect the foctus and cause congenital malaria, from which some racial immunity may be established.

The $P$. malaria lives in the circulatory blood chiefly, and sporulates there every seventy-two hours. It gives rise to general symptoms, which are known by the term quartan malarial ferer.

The $P$. vivax lives in the circulatory blood, but sporulates every forty-eight hours chiefly in the spleen, causing general symptoms known as tertian malarial fever.

L. malaric causes a stickiness of the red cells so as to cause them to adhere somewhat to the capillary walls of the organs in which they 
sporulate every thirly-six to forly-eight hours. They give rise to severe local symploms as well as general ones, the local symptoms differing as to the part attaclied, hence trouble may arise owing to a mechanical blocking of the capillaries of the brain, \&c., or to the local intense action of the toxin, or to both of these causes in one individual. There result, therefore, the pernicious manifestations of malaria, e.g., in the brain, causing coma or paralysis; in the pancreas, causing hamorhagic pancreatitis, \&c.

These symptoms are known under the term subtertian malaria.

Each type of parasite may cause irregular symptoms at first, but each settles to grow, sporulate and to rupture their host cells at the same time, due probably to the fact that the toxin liberated may kill off the immature forms.

It is to be remembered that double and triple broods are known of the same parasite in the same individual.

The parasites also have a hamolytic action, destroying the red cells, hence throwing more work upon the already overtaxed liver. This leads to excessive bile formation with diarrhoa and bile in the urine. If this detritus is excessive, the liver, not being able to convert the hæmoglobin into bile, some of it will pass through causing hamoglobinuria.

An antitoxin is formed which seems to quickly neutralize this hamolytic toxin, and may lead possibly to a cure of the disease.

Starvation diminishes the power of the body cells to manufacture sufficient of this antitoxin, hence relapses may occur.

\section{MORPHOLOGY AND HISTOLOGY.}

Each parasite has an intracorporeal or human cycle, also an extracorporeal or mosquito cycle. Some affirm that there is a latent phase álso.

\section{INTRACORPOREAL OR HUMAN CYCLE.}

This human life span is forty-eight or seventy-two hours according to the type of parasite. A few hours before a febrile paroxysm the parasites may be seen in the red cell as a pale, ill-defined disc occupying part or the whole of the red cell in the benign tertian form, distending the cell in many cases.

Scattered irregularly about this pale body are many black or reddish-black dots excreted by the parasite. These are called hamozoin (melanin).

These parasiles concentrate into sereral central blocks, around which the parasite, becoming segmented, arranges its segments or spherules. The cell ruptures, the spherule escapes into the liquor 


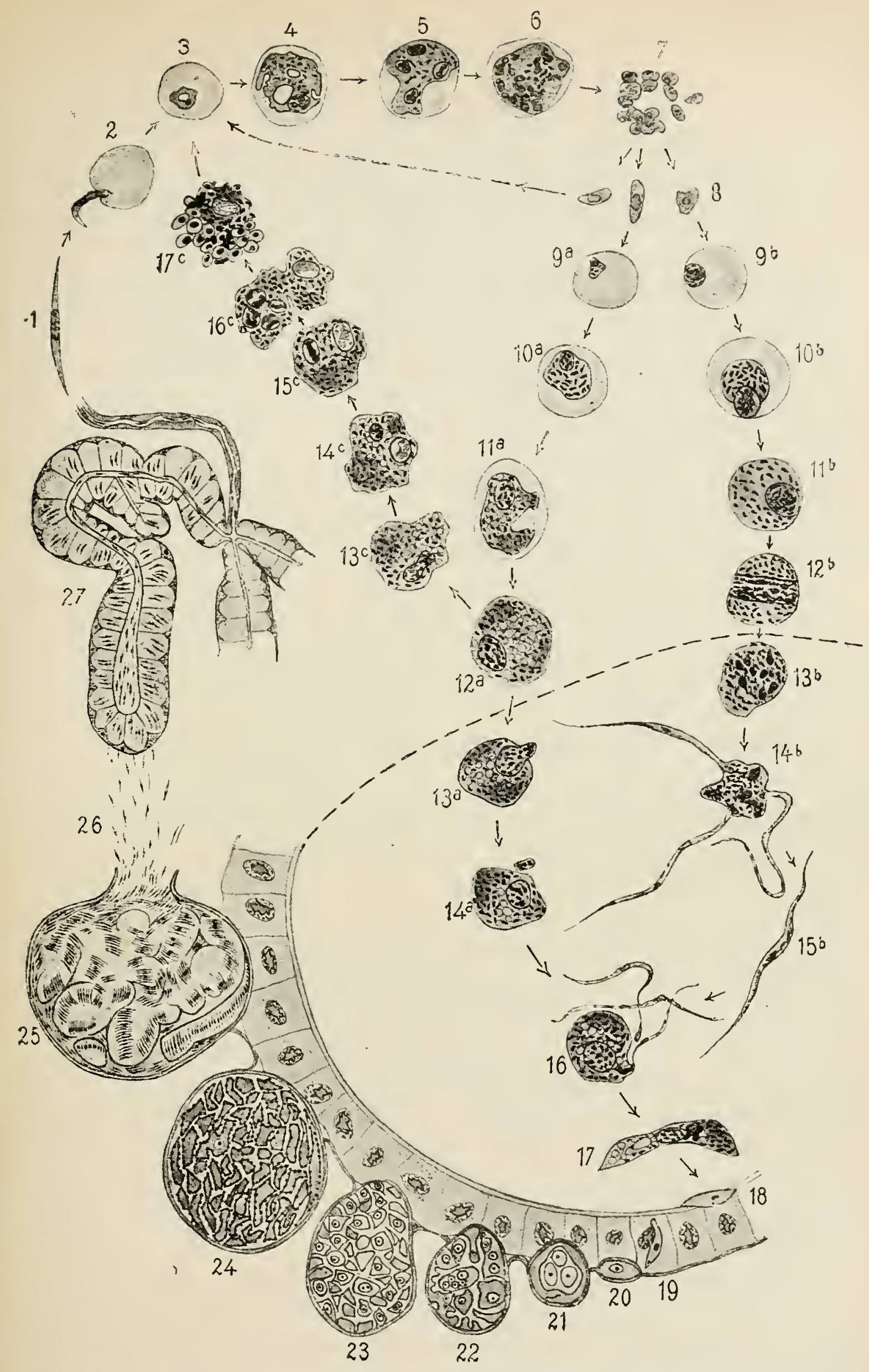

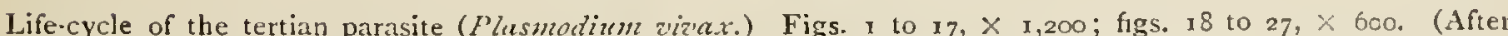
Lübe, hased on figures by schaudin and Gimsi) corpuscle ; 3,4 , growth of the parasite, now sometimes called a trophozoite; 5,6 , nuclear division in schizont ; 7, free merozoites; 8 , the merozoites which have developed making their way into blood corpuscles, (arrow pointing to the lett) and increase hy schizogony $(3-7)$; alter some duration of disease the sexual individuals appear; $9 a-12 a$, macrogametocytes; $g-12 b$, microganietocytes, both still in the blood-vessels of man. If macrogametocytes (iza) do not get into the intestine of Anoskeles they may perhaps increase parthenogenetically according to Schaudinn $(12 a ; 13 c-17 c)$. The merozoites which have arisen $(\mathrm{i} z c)$ become schizonts $3-7$. The phases shown underneath the dotted line $\left(x_{3}-1_{7}\right)$ proceed in the stomach of Anopheles. $x_{3} b$ and $x_{4} b$, formation of microgametes; $x_{3} a$ and $1_{4} a$, maturation of the nacrogametes ; 15 , microgamete; 16 , fertilization; 17 , oökinete; 18 , oükinete in the walls of the stomach; 19 , penetration of the epithelium of 1 he stomach; $20-25$, stages of sporogony on the outer surface of the intestinal и all ; 26 , migration of the sporozoites to the salivary gland ; 27 , salivary gland with sporozoites. 
sanguinis, the pigment being liberated at the same time is then taken up by phagocytes, which may also destroy many spherules. The spherules which have escaped the phagocytes attack and enter new red cells. The young parasite now shows active amoboid movements, shoots out long, retracting, slender pseudopodia, growing as it feeds upon the hamoglobin, converting the latter into parasites, protoplasm and hemozoin particles. The movements gradually cease and spherules are formed, completing the cycle. The hamozoin particles show a Brownian movement, otherwise the particles are now passive.

The young parasite contains nucleus and nucleolus, which break down and become diffused in the protoplasm, only to reappear as small scattered nucleoli around which the parasite segments itself. As long as the nucleus remains entire the hamozoin is situated at the periphery: when the nucleus becomes segmented the hamozoin becomes central.

These facts are not sufficient to describe the latency of the parasites in the body, and their recurrence causing later a typical attack of the fever. It is known that good powers of resistance and quinine farour latency, but that reduced resistance discourages it.

\section{EXTRACORPOREAL OR MOSQUITO CYCLE.}

When fresh mounted malarial blood is watched under a microscope, for ten to thirty minutes certain parasites may be seen to throw out long slender processes, one to six, giving the cell a cuttle-fish-like appearance. These flagella, or more correctly microgametes, act like spermatozoa, since this is the male form. These vigorous filaments, four to six times as long as the diameter of the body of the cell, break away, swim free in the liquor sanguinis, and are lost to sight.

These flagellated cells are derived from crescent bodies and other large parasites just prior to the concentration of hremazoin and segmentation. Such bodies are only formed seven to ten days after the onset of acute malarial symptoms. These cells may not be numerous, but they persist after all other forms of the parasite have disappeared, and may be seen from one to six weeks after all clinical symptoms have cleared up. After this time they may gradually disappear. These crescents are not affected by quinine.

Thompson has shown that gametes as individuals do not persist, but that there is an asexual cycle which produces the gametes from time to time. The individual gametes do not live very long in the peripheral circulation, probably about ten days; if they persist after that time it is because the asexual cycle has not been killed. Hence, when gametes can be found relapse is always possible and probable. Others agree with Thompson in this.

The severity of the infection does not account for the relapses; the mildest quartan will relapse time after time (James). 
Quinine given by the mouth very often does not eradicate the asexual cycle in the marrow and spleen; the residual parasites become immune; the longer the asexual cycle persists, the easier it acquires immunity against the drug, consequently large doses must be given to eradicate the disease.

The crescont body of subtertian malarial infections exhibits no amoboid movements, has a very crescentic shape, contains needleshaped hemozoin particles somewhat centrally placed, and shows a thin circular outline of the damaged red cell outside it.

Rarely twin crescents may be seen within one red cell. These cell membranes are better made out in stained specimens.

When the crescent is young the hamozoin particles are scattered.

When the crescent is mature the hamozoin particles are concentric.

When the crescent is effete the protoplasm shows vacuolation, e.g., degeneration.

The hamozoin can be dissolved by weak solutions of ammonia.

In the male the pro:oplasm is hyaline and the hamozoin loosely arranged; the protoplasm stains more deeply; the nucleus is larger.

In the female the protoplasm is faintly granular, the hamozoin more concentrated and centrally placed. The protoplasm stains faintly, the nucleus smaller.

The early forms seen in the spleen and bone marrow about the fourth day of a subtertian malarial attack are rarely seen in the peripheral blood until they are mature, which is about seven days later.

Some of the crescents outside the body become oral or spherical and throw off microgametes like the large spherical parasite; the remaining central portion breaking up is carried away by phagocrtes when the microgametes have become separated.

Just before the filaments are thrown out the agitation of the hyaline sphere becomes intense. These are the male sex cells.

The granular spheres do not give off these filaments or microgametes because they are the female sex cells.

In the parasites other than the subtertian ones the large spherical parasites act in a similar way, with the exception that they are never crescent-shaped.

Ross has shown that the blood must come in contact with the air for these changes to take place in the male sex cells. Adding a little moisture will hasten the process. Both of these factors, air and moisture, can be added by gently breathing on the slide before adjusting the cover slip, the male sex cells of course being mature.

\section{THROUGH THE ANOPHELINA.}

When mature crescents are taken up by some of the Anophelina. they become transformed into male or female sex cells, the filaments 
of the male cell break up, one filament butts and bores its way into the granular or female sex cell. Where this filament or microgamete disappears into the female cell a small minute papilla is thrown out to meet the oncoming microgamete. No second filament can enter, no matter how energetically it attacks the impregnated cell. Later this cell becomes oral, elongated lanceolate, and finally a vermicular form, the

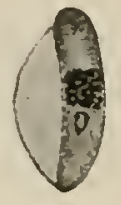

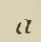

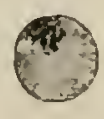

b

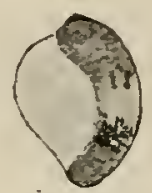

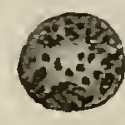

i

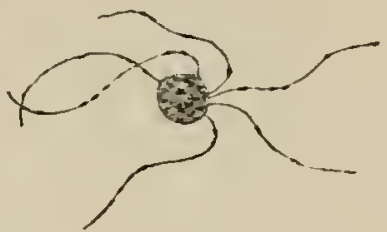

$e$

Stages of development of pernicious or malignant terlian pirasies in the intestine of Anopheles maculipennis. (After Grassi.) a, macrogamelocyte (crescent) slill attached to human blood curpuscles; $b$, macrogametocyte (sphere) half an hıur after ingestion by the musquilo; $c$, microgametocyle (cresceni) altached to the blood corpuscle; $d$, micrnganietocyte (sphere) half an hour after ingestion; the nucleus has divided several times; $e$, microgametes attached to the residual body (polymitus slage).

anterior end becomes pointed and hyaline, the posterior end broad and granular as a result of the presence of the hrmozoin. The vermicule then moves about, and is now called the Oökinet. This can. pass through a red or white cell. The oökinet now passes through the wall of the mosquito's stomach and the delicate basement membrane of the stomach wall, and lodges amongst the longitudinal and

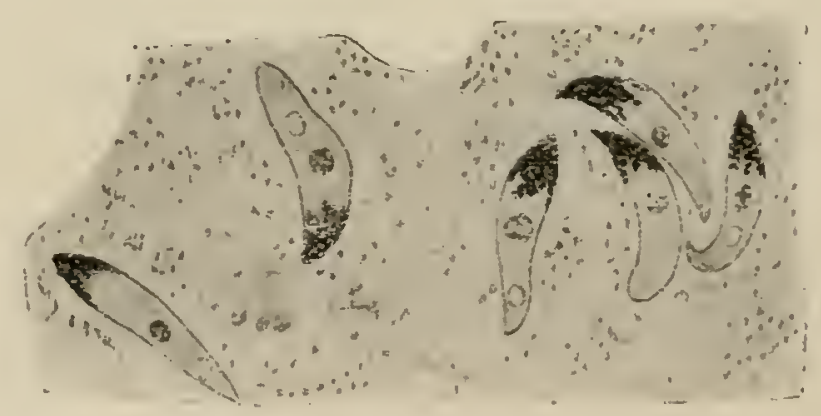

Oökinete of the malignant tertian parasite in the stomach of Anopheles maculipennis, thirty-two. hours after ingestion of blood. (After Grassi.)

transverse muscle fibres between the inner membrane and the outer coat of the stomach wall. Here it grows for a few days, becomes spherical, acquires a capsule, and by its increasing size bulges inwards the stomach wall. It is now an Ö̈cyst. Spherical daughter cells are formed within it. Around these numerous spindle-shaped nucleated bodies are formed, giving it the appearance of an apple studded with needles. The spherules disappear and the spindles remain loose in the capsule, and are now known as sporozoites. In about one week the capsule ruptures and discharges its contents into the body carity 
of the mosquito, learing there probably by the blood, and ultimately reaching the three lobed salivary glands which communicate with the base of the proboscis by means of a long duct. The parasites may be seen in the cells of the salivary glands and in the ducts. The parasite is now ready to enter man when the mosquito bites him.

\section{SUMMARY OF COMPLETE LIFE-CYCLE.}

Sporozoites enter human blood corpuscles, where they exhaust the Ilb., form pigment, become mature, and develop into either-

(I) Schizonts, which are the segmented and rosette forms.

The hamozoin concentrates, merozoites form, burst the red cell and enter fresh ones, only to begin again

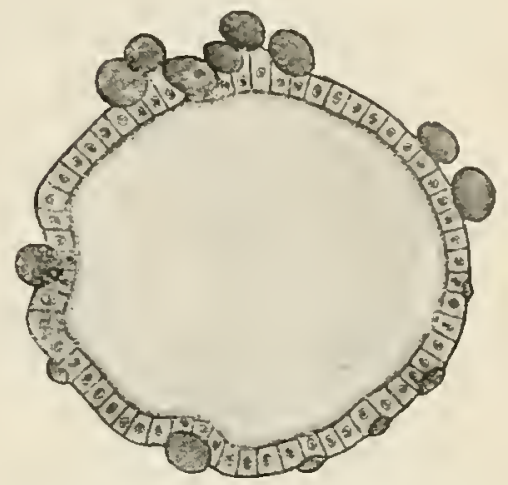

Section of the stomach of an Anopheles, wilh cysts (oöcysts) of the malignant tertian parasite. (After Grassi.) the endogenous life-cycle. This is known as the ascrual stago of schizogony.

(2) Gametes. These arecrescents in the subtertian form, spherical bodies in the tertian and quarian forms. There are male and female sexual cells, which become differentiated in the mosquito. Microgametes break off from the male and impregnate the female cell or macrogamete. This impregnated female is now the zygote. It begins to move and is now an oökinet. It rests in the stomach wall, forms a capsule, and is now an ö̈cyst. It divides into daughter cells and residua! bodies. The daughter cells

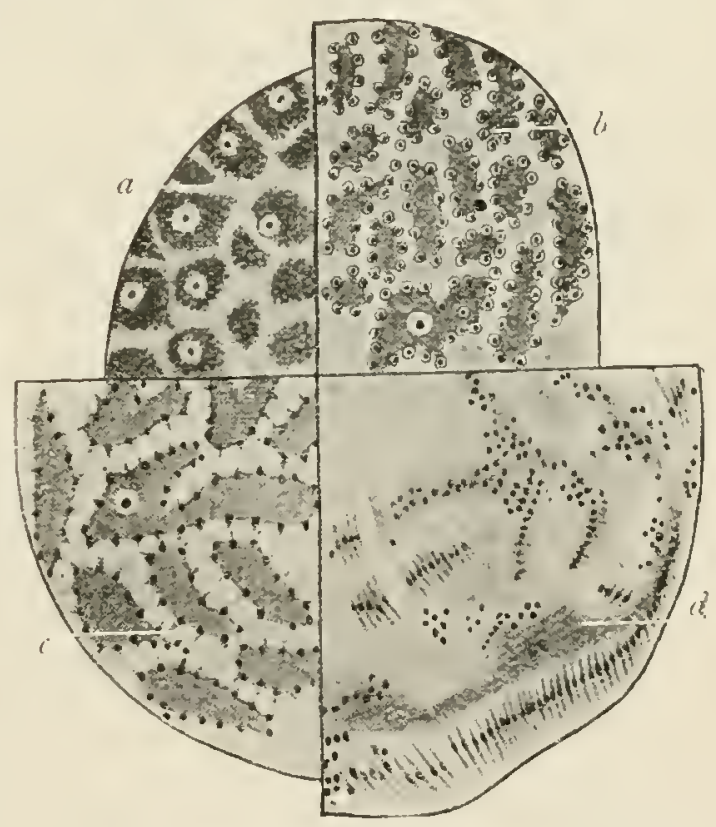

Four different sporulation stages of malarial parasites from Anopheles maculipennis, much magnified. $a-c$, of the maliguant tertian parasite; $a$, four to frour and a half days afier sucking; $b$ and $c$, five to six days afier suck. ing ; $d$, of the lerian parasite, eight days after sucking. (After Grassi.)

produce around themselves needle-like budies, perhaps 10,000 in one cyst, which become sporosoites.

The oöcyst ruptures, the sporozoites reach the body carity, and ultimately the salivary glands, and are injected into man along the proboscis. These become schizonts in his blood, and renew the endogenous cycle of sporogony.

It is suggested, but not proved, that the mosquito may live in 
vertebrates other than man, or probably the mosquito may infect its own eggs, and thus hand down the infection without the human element being present. This has not been demonstrated.

CHARACTERISTICS OF THE MALARIAL PARASITES.

(Modified after Manson and Mannaberg.)

For brevity in this statement " $Q$ " will mean Quartan, " $T$ ", Tertian, and "S-T" Subtertian.

\section{DURATION OF DEVELOPMENT.}

Q. 72 hours.

'T. 48 hours.

S-T. 36 to 48 hours.

\section{MOYEMENT.}

Q. Slight movement in the immature forms.

T. Active amoboid morement in the immature forms and also in the middle-aged forms.

S-T. Active morement; remains present in the pigmented stage.

\section{HAMOZOIN.}

Q. Coarse grains; little or no movement.

$T$. Finer grains in immature forms; often in the larger actively swarming.

\section{MAXIMUM SIZE.}

Q. The size of a red blood corpuscle.

T. Ditto, sometimes a little larger.

S-T. Half to two-thirds the size of a red cell.

\section{FORM OF SEGMENTATION.}

Q. Daisy form; the single spores roughish, with distinct nucleolus.

T. Sunflower or grape-like spores, single, round, small, nucleolus rarely seen.

S-T. Irregular heaps.

\section{NUMBER OF MEROZOITES.}

Q. 6-I2.

T. 15-20, often less.

S-T. IO-I2, rarely 15-16.

\section{FORM OF GAMETOCYTE.}

Q. A sphere.

T. A sphere.

S-T. A crescent. 


\section{ALTERATIONS IN INFECTED BLOOD CORPUSCLES.}

Q. The red blood corpuscles are little discoloured, and are not materially altered in size; some appear smaller.

T. The red corpuscles are often hypertrophied and have lost colour, it may be completely. Schiiffner's dots in deeply stained specimens.

S-T. The red blood corpuscles shrink frequently; they are dark, or may be perfectly colourless.

\section{RELATIYE NUMBER OF PARASITES SEEN IN PERIPHERAL AND YISCERAL BLOOD.}

Q. Parasites seen in peripheral circulation throughout the whole cycle, and in as great numbers as in visceral blood.

T. Parasites seen in peripheral blood throughout the whole cycle, but not in such large numbers in visceral blood.

S-T. An enormously greater number of parasites present in internal organs as compared with peripheral blood. The latter part of the cycle takes place in internal organs only.

\section{INFLUENCE OF QUININE.}

Q. Causes disappearance of parasites readily.

T. Ditto.

S-T. Causes disappearance of parasites less readily.

\section{LIABILITY OF RECURRENCE OF PARASITE AFTER LEAYING INFECTIYE REGIONS.}

Q. Liability persists for a very long period.

T. Liability persists for a long period.

S-T. After a few months less liability to recurrence.

\section{THE MOSQUITO.}

It does not follow that if there are Anopheline that there is malarial fever, e.g., England, Epping Forest in particular, harbours many of these mosquitoes, but there is no malaria in consequence because the parasite is absent. Again, if there are none of these mosquitoes there cannot be any new cases of malaria if the parasite is brought in by carriers.

If there is an attack of malarial fever where there are no mosquitoes it is because the patient has been previously bitten elsewhere.

For example, cases have arisen after weeks at sea owing to the climatic changes reducing the resistance of the patient and giving the parasites, lying in the spleen probably, a new opportunity to do mischief.

Where mosquitoes exist the temperature must be favourable for the 
development of the parasitic oöcyst in the body of the mosquito. If it is below $15^{\circ}-16^{\circ} \mathrm{C}$. no further development is said to take place. L. malariæ is said to require the highest temperature, $P$. vivax the lowest, and P. malarize will develop at low or medium temperatures. These differences may account for the unequal distribution of the parasites and for their lesser danger in sub-tropical and temperate climates.

For details of the habits, $\&$ c., of mosquitoes, see article at the end of the present one.

\section{MAN.}

Man is the third essential factor for the spread of malaria; without him, as far as is known, malaria cannot exist.

The native population in the tropics forms the great reservoir of parasites, but Europeans may also carry large numbers of gametocytes.

A new district should always be investigated with regard to malaria and the following facts ascertained:-

(I) The diagnosis of the causative parasite.

(2) The population of the area under consideration.

(3) The average number of infected persons, ascertained by the spleen rate.

(4) The average number of persons having gametocytes in the blood.

(5) The species of Anophelinæ and their breeding places.

(6) The species which carry the parasite.

(7) The species in which the parasite is found.

(8) The number of Anophelines in the infected area.

\section{DISTRIBUTION AND CLIMATOLOGY.}

Malaria is most prevalent in the region of the Equator, $63^{\circ} \mathrm{N}$.$35^{\circ} \mathrm{S}$.

Regions that were once deadly may become salubrious, e.g., England, Germany, Austria-Hungary. On the contrary, countries once free may become infected, e.g., Mauritius, which was said to be infected during the sixties of last century by the introduction of carrying mosquitoes (Ross).

The West Coast of Africa, from Senegal to the Congo, is perhaps the worst malarial country in the world.

India is heavily infected, especially about the swampy lands at the foot of the IImalayas.

It is also found in the following countries: Borneo, Arabia, Syria, Siam, China, Western Australia, New Guinea, Solomon Islands, Bismarck Archipelago, Central America, West Indies, Mexican Gulf, the north of South America, Brazil, Paraguay, Bolivia, Colombia, 
Peru, Chili, many of the Southern States of North America, Russia, Italy, Greece, Turkey, Austria-llungary, Southern France, even amongst the soldiers of the present War. In Switzerland, along the Rhine in Germany. Some parts of Sweden.

For malarial epidemics there must be malarial parasites, carrying mosquitoes, man, heat and moisture.

High altitudes and strong winds diminish its prevalence.

Malaria does not exist in :-

The Seychelles, Indian Ocean, Fiji, Society and Friendly Islands, Southern Pacific, Barbados and St. Helena in the Atlantic.

Where malaria is prevalent children tend to become immune about the twelfth year (Ross).

\section{PATHOLOGY.}

The febrile phenomena are explained by various malarial products :-

(1) Toxins that act on the heart controlling centre.

(2) Hamolytic toxins.

(3) Toxins acting on the innervation.

Blood serum taken before a rigor and passed through a Berkefeld filter will, when injected into a healthy man, cause a febrile paroxysm resembling malaria (Daniels).

The heat output during the cold stage of an attack is diminished, but the causative metabolic changes are but little known.

The urine is increased during this stage, probably due to the contraction of cutaneous vessels and increased B.P. internally. The specific gravity is raised as a result of the increased metabolism caused by the toxins. The colour is dark and the acidity increased. Nitrogen is in excess owing to urea. The salts are all increased, except phosphates, which are diminished during an attack, and increased during the intermission, therefore phosphoric acid is retained during the attack. The excretion of iron is increased in the hremozoin and hæmosiderin. The increase commences just after the attack.

There may be a considerable quantity of urobilin excreted, and the indigo blue may be increased. To test for urobilin :--

Add to urine equal parts of $\mathrm{ZnSo}_{4}$ solution $\left(\mathrm{ZnSO}_{4}\right.$ Io, absolute alcohol, 9o). Shake the mixture. Add a few drops of lugol, stir and filter. Fluorescence equals urobilin.

The diazo reaction is present in 5.5 per cent. of cases.

During the intermission the quantity of urine is diminished, but the nitrogen keeps up.

During convalescence there is polyuria with low specific gravity. especially in subtertian cases. 
The faces show increased excretion of bile and iron, both from blood destruction. Stercobilin is increased.

The sweat has a peculiar odour and is toxic to rabbits.

Goose skin may be caused by some malarial toxin acting on unstriped muscle, affecting the erector pilorum.

Hamozoin is the black dust-like grains of pigment formed from hæmoglobin and distributed over the body after the rupture of the red cells. Phagocytes remove it to the connective tissue, e.g., the liver and spleen. It is soluble in alkalies, entirely and rapidly in ammonium sulphide, but not in water, alcohol or acids. It contains an organic compound of iron which will not give the Berlin blue reaction. Eventually it disappears from the body, but how is not known.

It is peculiar to diseases caused by malarial parasites except melanotic tumours, but in the latter instances it is never seen in the blood-vessels. Intravascular black pigment is pathognomonic of malaria. It is most abundant in the splenic vein and lymphatic glands at the hilum of the liver.

Hcemosiderin is a yellow pigment found as yellow granules in the parenchyma cells of the liver, spleen, kidneys, pancreas, bone-marrow, capillary epithelium, and less frequently in the leucocytes.

It contains an inorganic compound of iron which gives the Berlin blue reaction (ammonium sulphide and double cyanide of iron and potassium). It is insoluble in alkalies, acids and alcohol (Manson).

It is not peculiar to malarial diseases, but may occur in any where there has been marked blood destruction, as in hæmoglobinuria, pernicious anæmia, extensive burns and toxic poisoning by such agents as potassium chlorate and pyrogallic acid.

The liver converts the free hamoglobin into bile pigment, which, when in excess, overflows, as it were, causing bilious diarrhoea and vomiting; consequently polycholia is a constant feature of malaria. The yellow tinging of the skin and scleræ may be due to free hæmoglobin and not to cholæmia as a result of bile absorption as once commonly believed (Manson). This yellow pigment is probably precipitated hamoglobin, stored up and waiting to be worked off by a busy liver. Should the hamoglobin go beyond this it overflows, and then we have haemoglobinuria.

The red cells.- The body (I $50 \mathrm{lbs}$.) contains about 25,000,000,000,000 red cells. To examine all these under the microscope at the quickest rate would take over $\mathrm{I}$, ooo years (Ross).

In severe infections 12 per cent. are infected, i.e., 2,000,000,000,000.

If only i in I0o,ooo corpuscles are infected, i.e., 250,000,000, there will be but little manifested of the disease (Ross). 
Daniels says that $600,000,000$ red cells could be infected without the victim having "fever." It has been estimated that 300,000,000 are required to produce each degree $\mathrm{F}$. rise in the body temperature due to a malarial attack. Anything under this number has been called "latent malaria."

The different pictures caused by the various malarial parasites will be given in a table later.

The marked blood destruction during an attack, especially in the subtertian variety, is considerable, but it is made up very quickly during the intermission, so that the number of red cells may be almost normal by the time of the second paroxysm in tertian and quartan cases. Unaffected red cells become pale, vary in shape (poikilocytosis), sometimes with megaloblasts (polychromatophilia), basic and granular degeneration with normoblasts.

Oligocythamia is always present. If I per cent. of the red cells is attacked and contains a parasite, we should expect a reduction of I per cent. of total red cells. If every twentieth cell, then 5 per cent. of the total destroyed, and so on, but it is not so. Only after one paroxysm of fever the drop may be from 5 per cent. to Io per cent., and after some one pernicious attack $\frac{1}{2}$ to one million per c.c. of blood will be destroyed, and if the pernicious attacks continue the count may be about one million instead of $5^{\frac{1}{2}}$ millions.

The $\mathrm{Hb}$. value of the remaining corpuscles goes down to per cent., 20 per cent. to 50 per cent. below normal.

The volume of blood is also diminished as demonstrated post mortem.

The venous system, apart from that of the portal system, spleen and liver, may be markedly empty. Blood may not flow freely from the pricked finger. The writer's blood during a pernicious attack in Colombia was so viscid and blackish that hæmocytometer pipettes were repeatedly blocked and no count could be made.

Anæmia during first attacks is marked, but rapidly replaced.

Anæmia during the following attack is less marked and less rapidly replaced.

The white cells. The leucocytes are at first increased during the rigor, 8,000 to 30,000 in some cases, but they markedly diminish. causing a leucoprenia with a ratio of $\mathrm{I}-500$ or 600 . In pernicious malaria there may be leucocytosis. Mononuclears are more marked during the leucopænia, and persist for some time after the fever has subsided. It is a useful factor for diagnosis when the patient is seen after the attack. The increase is relative and absolute: 15 per cent. increase suggests malaria. It is also present in other protozoal infections. 
Phagocytosis is carried on more by the large mononuclear elements and transitional leucocytes than by the polys. A phagocyte may engulf a red cell and its contained parasite.

Vacuolization and a reduced staining power have been noticed.

Myelocytes have sometimes been found in subtertian cases : $\mathrm{Da}$ Costa).

\section{MORBID ANATOMY.}

(I) In acute malaria of $P$. malarice.

The parasite does not especially accumulate in any one organ.

The spleen is enlarged, not soft or very melanotic.

The liver and bone-marrow may be markedly melanotic.

There are parasites in the spleen and the blood, but not in the brain.

(2) In acute malaria of $P$. vivax.

Death may be caused in rare cases by coma, hæmoglobinuria with pigmentation of the bone-marrow, spleen and liver. The spleen is enlarged. Its contained blood shows large numbers of parasites.

The kidneys and colon are inflamed.

The endothelial cells of the brain are swollen and contain pigment (Ewing).

(3) In the acute malaria of L. malaria.

The organ chosen for sporulation suffers most, and produces the symptoms giving the character of that particular type of the disease. The organ attacked principally may be the brain, intestine, heart or pancreas.

\section{Macroscopic.}

The body is pale, yellowish in white races; the heart muscle pale and flabby; ecchymoses sometimes in the epi- and endo-cardium.

The lungs may be normal or congested, rarely sclerosed.

The liver is enlarged, dark brown to slate colour on section, soft and congested.

The gall-bladder is full of dark bile.

The spleen is enlarged, capsule tense; on section, deep brown to black in colour.

The stomach and intestines are not affected, except in choleraic forms, when the mucous membrane is congested (except about Peyer's patches and the solitary glands) with blood-stained contents, flakes of mucous and dark pigmentation due to hæmozoin readily demonstrated by the microscope.

The lymphatic glands are swollen.

The pancreas is normal or in a condition of hæmorrhagic pancreatitis.

The suprarenals are congested.

The kidneys are normal or congested, with punctiform hamorrhages in the pelvis, and cloudy swelling in the parenchyma. 
The bone-marrow is hyperemic and chocolate-brown in colour.

The brain is normal, or in the cerebral type of the disease one finds œedema, hyperamia of leptomeninges; brown or black pigmentation of cortex and punctiform hamorrhages in the white matter under the cortex.

The spinal canal is similar to the brain.

The retina may show numerous hæmorrhages.

Microscopic.

Parasites are found in the blood of the heart, spleen, bone-marrow, capillaries of brain, intestine, pancreas, \&c., but the types are not differentiated clearly after death. They shrink considerably. The mononuclears show pigment granules. The polys show phagocytosis in a small degree. Macrophages may be seen from the internal organs containing parasites and red cells.

The heart muscle is loaded with hæmosiderin and the capillaries with parasites.

The lungs contain parasites in all stages of derelopment with pigmented macrophages and leucocytes. The pneumococcus is alwars present, and perhaps forms a double infection.

The capillaries of the liver are enlarged and swollen by the endothelial cells loaded with pigment, and filled with blood cells containing parasites and leucocytes with pigment.

The perivascular lymphatics are swollen.

The liver cells are compressed between dilated capillaries and contain hæmosiderin and bile pigment. Rarely there are localized patches of necrosis.

The portal canals are infiltrated with red cells containing parasites.

The red cells of the spleen show schizonts and crescents.

The leucocytes and macrophages show pigment.

The kidneys show pigmentation of the capillary walls of the glomeruli. Parasites are rare here, but plentiful between the tubules. Phagocytes with pigment and parasites are not uncommonly seen here. The cells of the glomeruli degenerate and are cast off into the capsule: the epithelial cells of the convoluted tubules degenerate and are cast off into the lumen.

The suprarenals show irregular areas of vasodilatation, full of red cells, many of which contain parasites.

The same may be said of the capillaries of the abdominal fat.

The bone-marrow is chocolate-coloured in the small bones and brownish red in the long ones. It is soft, diffluent, contains sporulating parasites and crescents.

The brain in cerebral cases has the capillaries full of sporulating parasites, mononuclear elements, macrophages containing dead para- 
sites. Nissl's bodies may be damaged and disappear or degenerate only. There may be degeneration of the neurofibrils. The punctiform hæmorrhages are due to the diapedesis of apparently normal red cells.

(4) In the chronic malaria of $P$. vivax.

(There are no records of post-mortem findings in chronic malaria due to P. malarix.)

Those of $P$. vivax are :-

Enlarged spleen, which is firm, dark, containing parasites and pigment in the endothelial cells about the Malpighian bodies. Some hremosiderin .

The liver revealed nothing macroscopical, but microscopically pigment could be seen collected in the large intracellular masses in the portal canals. The macrophages contained pigment.

The bone-marrow was slightly pigmented, showing a few endothelial cells with black pigment.

In one case (Ewing) the patient was ill for twelve months, and died from endocarditis three months after the last malarial attack.

(5) In chronic malaria due to L. malarice.

The spleen, liver and bone-marrow are the parts always affected.

Spleen, enlarged, firm, slaty colour, depending upon the amount of contained pigment, signs of old perisplenitis, e.g., thickened capsule; Malpighian bodies stand out boldly, as they are enlarged and non-pigmented.

The capillaries are dilated, and are separated by splenic pulp or connective tissue containing giant cells.

The pigment may be scattered diffusely, but is generally collected around follicles or extracellular, and contained in lymphatics of arterioles or septa.

Liver, large, hard, pigment not always present, seen at times gathered around the periphery of lobules; later the pigment is perivascular and finally disappears. The capillaries or lymph spaces are dilated, and by their pressure cause atrophy of the liver cells; the hepatic cells may be seen repairing the damage done.

Bone marrow, usually pigmented and of a chocolate colour in the small bones, reddish in the long ones. The change of colour is due to replacement of the fat by vascular tissue.

(6) In malarial cachexia.

It may follow a few acute attacks, or more commonly coma as a sequel to chronic malaria.

Anæmia is marked. Cidema of the ankles and abdomen frequent.

The spleen is enormously enlarged, the liver also.

The bone-marrow is yellow, sclerotic or gelatinous. 
Parasites may be found or may be absent.

Sometimes there are amyloid changes in the kidneys with parenchymatous nephritis, associated more rarely with amyloid changes in the intestine, spleen and liver.

\section{SYMPTOMATOLOGY.}

A typical febrile malarial attack.

In all types of malarial fever there are definite attacks every twentyfour, forty-eight, or seventy-two hours. Each attack has four stages, followed by an interval of apyrexia.

(I) Premonitory stage.

This is sometimes absent.

When present it lasts from a few hours to a few days.

There are: Lassitude, aching of bones, headache, anorexia, yawning, sometimes vomiting.

Two-thirds of actual agues take place between midnight and midday, most other febrile diseases from midday to midnight (Manson).

(2) Cold stage.

This lasts about an hour.

Teeth chatter, patient shivers and shakes from head to foot.

Wraps himself in blankets to keep himself warm.

Vomiting may be distressing.

The features are drawn and the skin blue, but the body temperature may be several degrees above normal and rapidly rising.

The urine is abundant and passed frequently. Urea is increased Young children may have convulsions.

Then follows the--

(3) Hot stage.

This lasts four hours.

There are waves of warmth, intense heat.

Blankets are thrown off ; there is a desire for more air.

The face is flushed, pulse rapid, full and bounding.

Headache intense, vomiting frequent, respirations hurried.

Conjunctivæ injected, sometimes erythematous rash.

Skin dry and burning. Body temperature $40^{\circ} 6^{\circ}$ C. $-4^{\circ} 0^{\circ} \mathrm{C}$. (104 $106^{\circ} \mathrm{F}$.).

Urine scanty, loaded with urea and chlorides. Phosphates diminished.

Bile pigment present in urine.

Then follows the-

(4) Sweating stage.

This last from two to four hours.

There is profuse perspiration, clothes and bedding are soaked. 
Fever rapidly declines. The urine is as in the hot stage.

Distressing symptoms abate, relief comes, patient sleeps readily.

Temperature may fall to normal or sub-normal.

Apart from weakness the patient feels well.

The whole attack lasts from six to ten hours, but may vary much .

A similar attack takes place one, two or three days later, unless quinine is given in the interval.

The spleen enlarges temporarily, but remains slightly larger after each attack. In frequent attacks the spleen may be enlarged enormously, permanently and accompanied by a cachectic state.

The most pernicious fevers, so common in Africa, are most atypical; the patient may reach a serious condition before a doctor is consulted.

The rise of temperature is caused by the discharge of malarial toxin, when the red cells rupture, and not because of the parasites being liberated in the serum. Between attacks the young parasites are growing to maturity in fresh red cells to cause another rise of temperature, when the latter are ruptured.

There are continued and remittent types of malarial fevers, when parasites of all stages may be met with in the red cells.

\section{CLASSIFICATION.}

Acute Malaria.

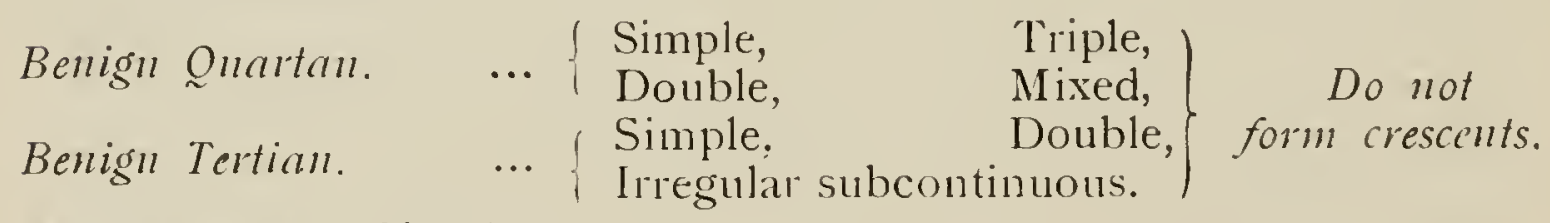

Malignant or Simple,

Subtertian. Double,

(Crescents.) Irregular,

Remittent, $\quad \cdots\left\{\begin{array}{l}\text { typhoid. } \\ \text { adynamic. } \\ \text { bilious. }\end{array}\right.$

Pernicious with general symptoms, e.g.,
Algide,
Diaphoretic,
Hæmorrhagic,
Scarlatiniform.

Pernicious with local symptoms, e.g.,

Cardiac,

Pulmonary .

Pulmonic,

Pleuritic.

Gastrointestinal,

Choleraic,

Dysenteric,

Hæmorrhagic pancreatitis,

Peritonitic. 


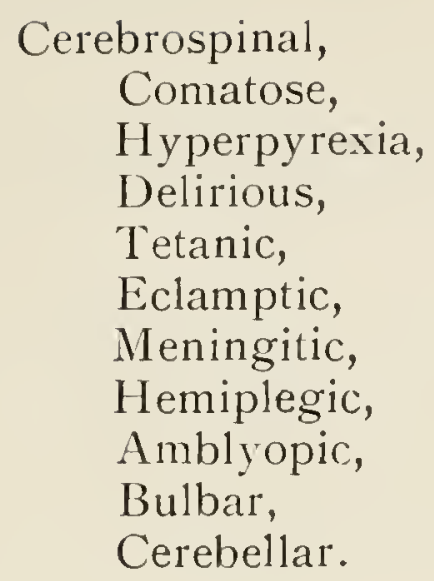

Chronic Malaria.

Cachexia .

\section{CLINICAL VARIETIES.}

Simple Quartan Fever (Benign).

The parasite is $\mathrm{P}$. malariæe.

Its cycle is seventy-two hours.

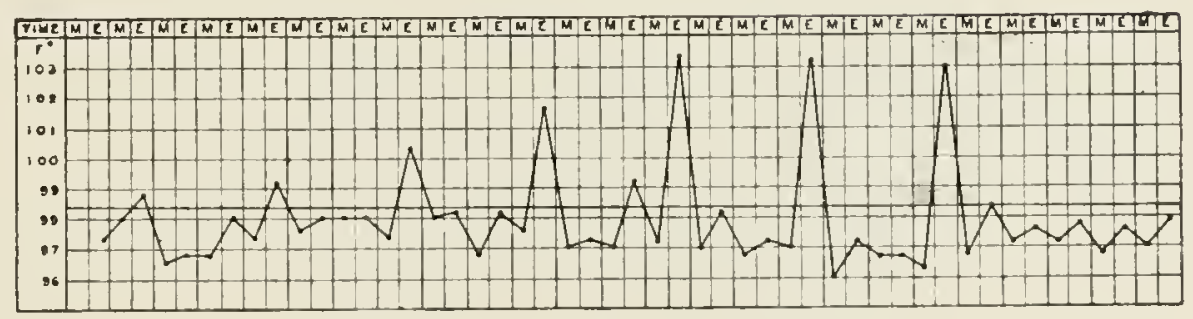

Quartan fever.

At first the parasite is a small, round, clear speck in the invaded red cell. Its amoboid movement is feeble.

When it becomes pigmented all amoboid movement ceases.

The hæmozoin it carries is large in amount, coarse in grain, and is seen as short rods.

The segmented or mature parasite has eight to ten merozoites, symmetrically arranged "daisy" fashion around the block of black hæmozoin.

A shining nucleolus can be seen readily in the centre of each pearshaped segment.

The gametocyte or sexual form is a spherical pigmented body like an ordinary parasite that has escaped whole from the red cell.

The hæmozoin granules may show very active amœeboid movement.

The enclosing red cell is not enlarged.

When mature the parasite completely fills the cell, often without any ring of hæmoglobin, but as red cells vary in size in these fevers, a quartan parasite in a large erythrocyte may resemble a tertian parasite in certain stages of its growth. 
It is supposed that some parasites degenerate, neither segmenting nor forming gametes.

The various stages of growth and the segmented "daisy" body are more frequently seen in the peripheral blood than those of other varieties.

This fever was formerly common in England.

It is more common in temperate than tropical climates.

Cachexia is not so common with this as other malarial fevers.

The individual attacks are very amenable to quinine, but the parasites are more difficult to kill off than those of other varieties (Manson).

If untreated the fever is supposed to exhaust itself and die out, but spontaneous cure is rare.

Double, Triple and Mixed Quartan Fevers.

These occur when there are two or three families of the same kind

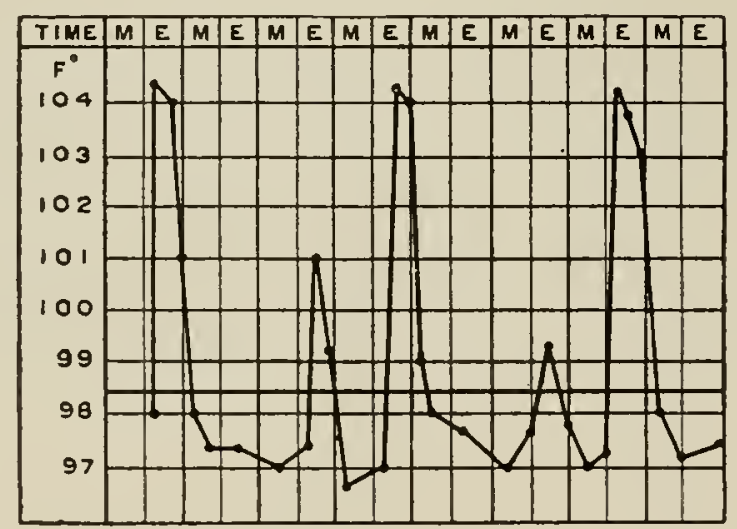

Double quartan.

of parasite present. There is a daily fever. The condition is diagnosed by the microscope only. The rises of temperature may be unequal for each family of parasites present, but when there are other varieties of malarial parasites the condition is known as a mixed one, and greater differences of temperature variation may be noticed.

Major Donovan records one case in which he found the three varieties of malarial parasites. This seems to favour Laveran's view that the malarial parasites constitute a single species with three varieties, a view not widely held.

Simple Benign Tertian Fever.

The parasite is $P$. vivax.

The cycle is forty-eight hours.

The very young form is indistinguishable from that of the quartan, but later one sees-

Much more amœboid movement. 
The change of form and position of the parasite in the corpuscle from time to time.

That it pushes out and retracts pseudopodia.

The movement is suspended when the concentration of hæmozoin is accomplished.

The hremozoin particles are finer than quartan and have more active and incessant movement.

There is hypertrophy and marked-decolorization of the invaded red cell, which may be twice its normal size.

The rim of hamoglobin becomes almost colourless.

With deep Leishman's stain the Hb. shows chromatophile dots,

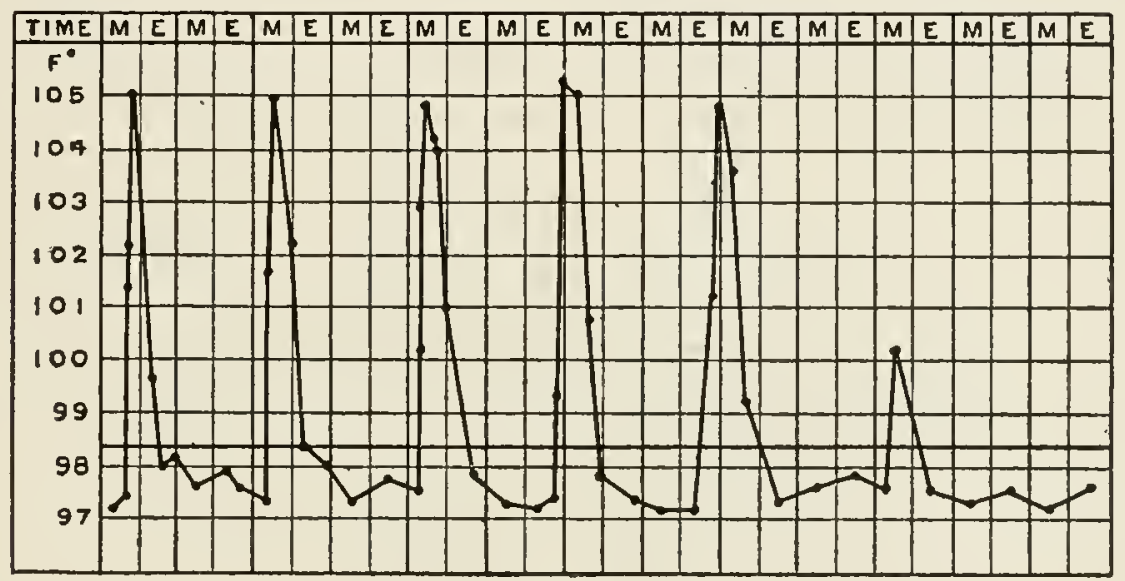

Simple benign tertian.

"Schüfner's" dots, which are not shown by quartan or sub-tertian parasites.

The segmented body has sixteen to twenty spherules, like a cluster of grapes and not daisy fashion. One or two particles of pigment can be seen among the spherules. These latter are smaller, smoother and rounder than those of the quartan.

The segmented parasite is called a "rosette" body.

As in the quartan the gametocyte is a spherical body, but much larger than the quartan.

This parasite is the commonest, and occurs in tropical and temperate climates.

The fever resembles that of the quartan type.

Spontaneous cure is more likely than in the quartan type.

The resulting anæmia is more readily recovered from than that of quartan malaria.

Double Benign Tertian Fever.

This is the same as the above, but there are two families of parasites maturing on different days. The two attacks may not be equal in severity. 


\section{Irregular Subcontinuous Benign Tertian Fever.}

Parasites may mature at different times of the same day, thus producing a somewhat continuous fever with remission and exacerbations. In this respect it may resemble subtertian fever.

Irregularities may also be caused by a mixed infection of other types of malarial parasites.

\section{Simple Subtertian Malarial Fever.}

The parasite is Laverania malariz.

The cycle is probably twenty-four to forty-eight hours.

These are crescent-forming, and all are malignant.

The subtertian parasite is much smaller than the tertian or quartan, occupying as it does half to three-quarters of the red cell.

The early unpigmented stage is difficult to see.

The amoboid movements are very active at first.

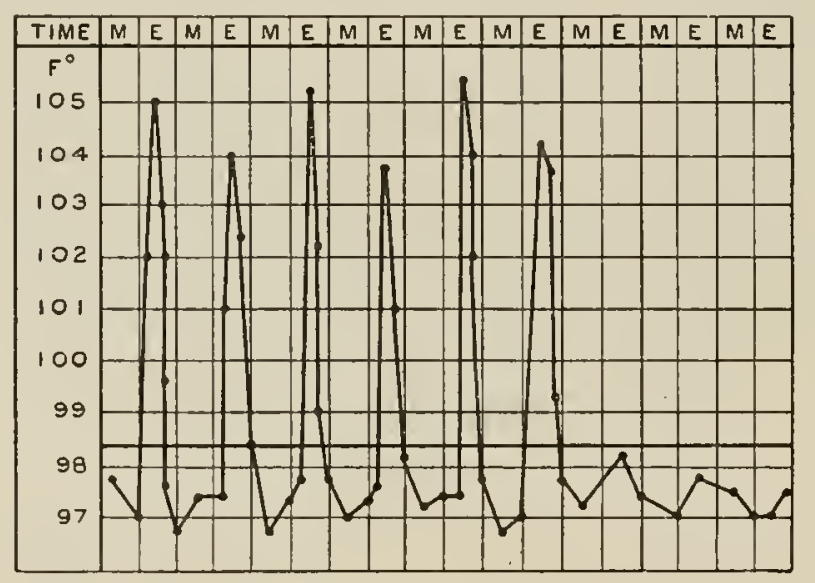

Double tertian.

The parasite settles down later and forms small colourless rings.

Multiple infection of individual cells is common, much more than in other types, owing perhaps to the enormous number present at the same time.

The invaded corpuscle seems to be filtered out by the capillaries and small arteries of the deeper viscera and bone-marrow; hence few are seen in the peripheral blood.

The segmented form is very rarely seen in the blood.

It has ten to twelve segments.

The invaded cells shrink, become darker, sometimes crenated or folded, when they are known as "brassy" bodies, when the parasites may be made out as small minute pale rings.

The gametocyte is crescent-shaped, and in this is unique.

A week may elapse after the small intracorpuscular parasites have been seen before crescents are formed, probably not until the pabulum has been exhausted by swarms of endogenous parasites, so that the 
Essentials of Tropical Medicine.

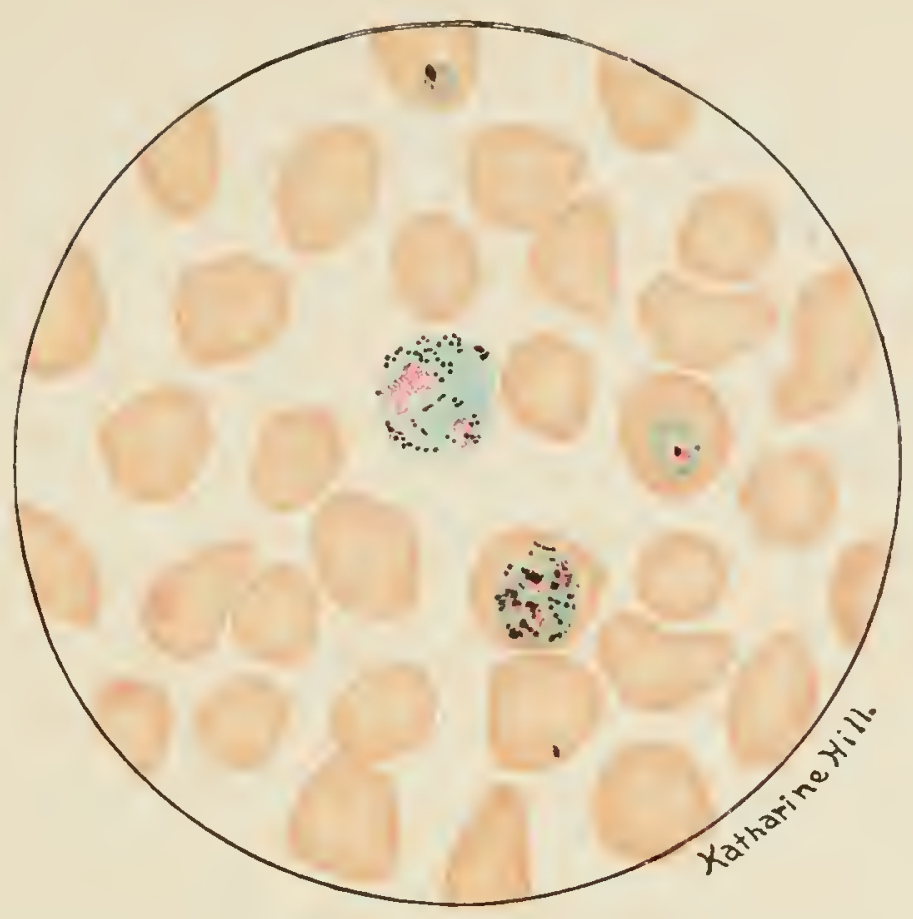

Tertian Matarial Parasite. (Wrigits's Stain.) (From Webster's Diagnostic Methods.)

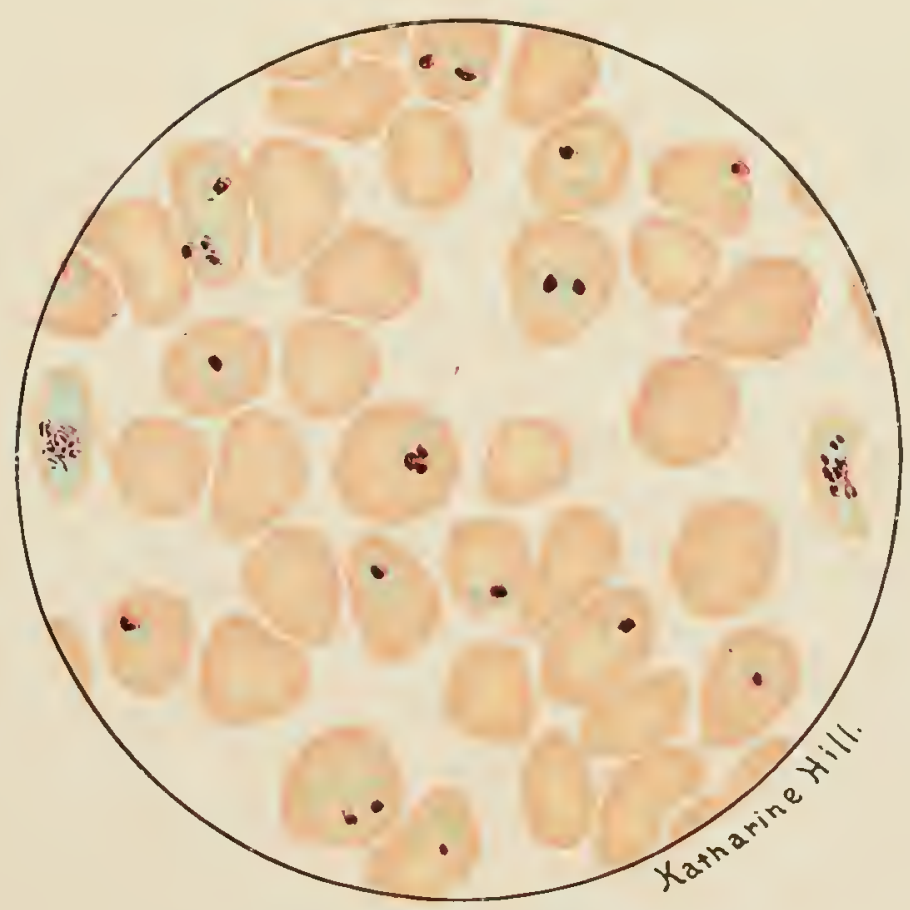

Estivo-Autumal Parasite. (Wright's Stain.) (From W'ebst er s Diagnostic Methods.) 
crescent provides for life and growth elsewhere, e.g., in the mosquito. These persist in the circulation, in spite of heroic quinine treatment, from two to six weeks, after all other parasites have disappeared.

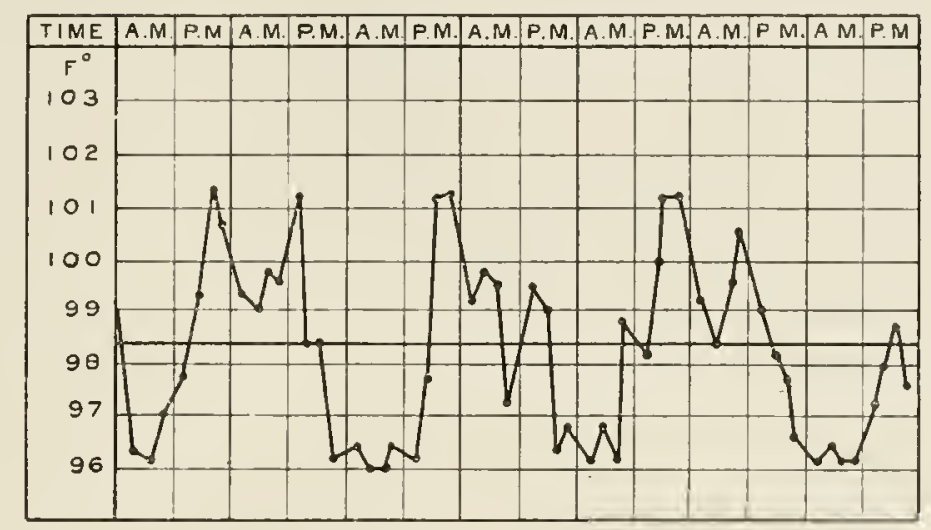

Subtertian malaria. Periodicity still definite.

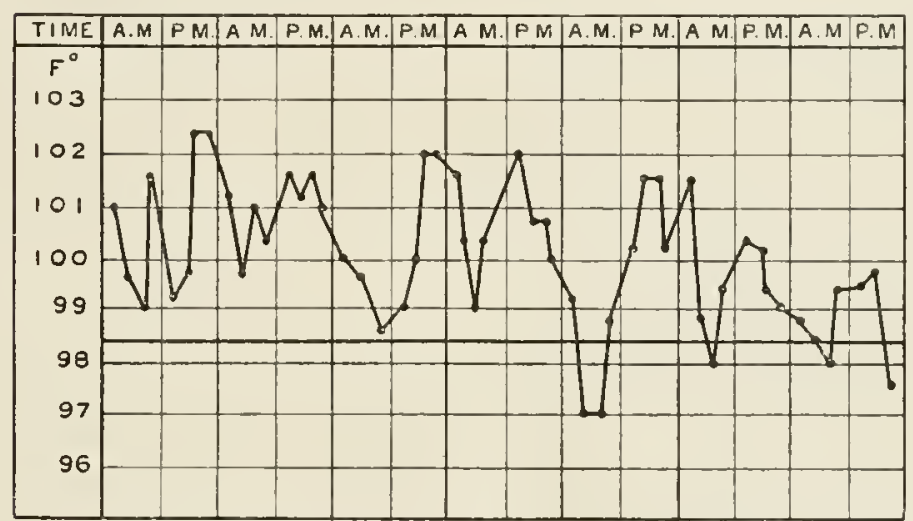

Subtertian malaria. Periodicity indefinite.

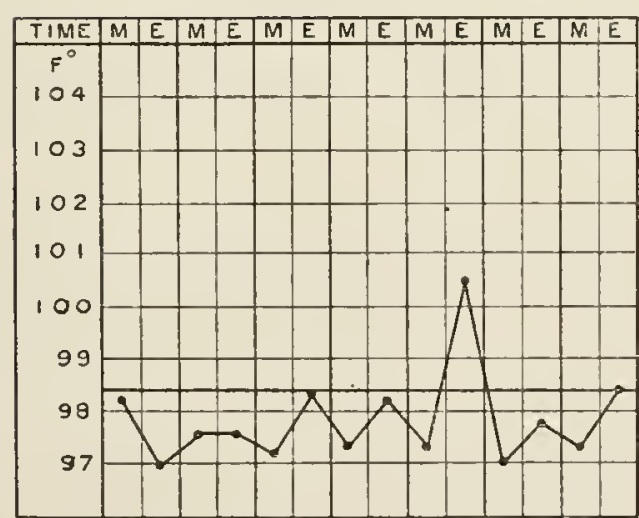

Subtertian malaria (untreated). Slight fever only. No definite periodicity. Diagnosis based on blood examination.

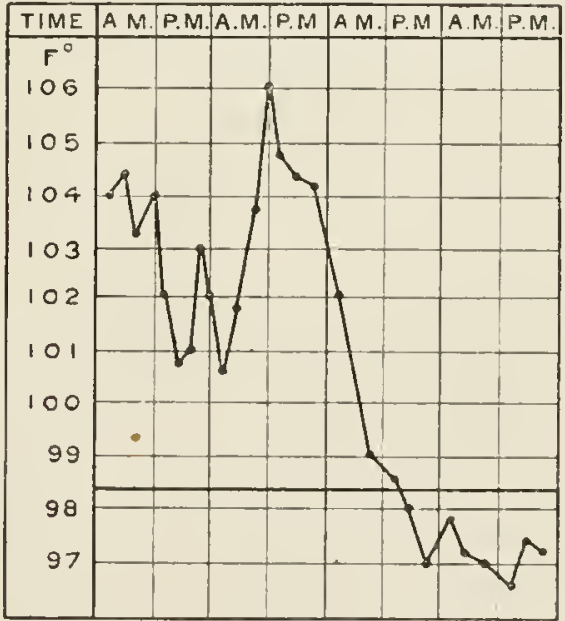

Severe subtertian malaria (treated).

Quinine given very early may stop their formation, but later it has no effect upon them. The presence of crescents is usually marked with cachexia.

It is common in all tropical regions. In the sub-tropics it occurs 
in the late summer and late autumn, and hence is called "æstivoautumnal" fever.

It has been noted that crescents can rarely be found in the African subtertian fevers, but when these patients get to Europe the parasites can more frequently be found (Manson).

The fevers produced by the subtertian parasite are very irregular, the rigor is less marked, the pyrexial stage more prolonged, while vomiting, body pains, intestinal disturbances and depression are more marked. Relapses are much more frequent. There may be a false crisis preceding the true one. There is a rapid destruction of corpuscles, followed by a marked cachexia. Grave symptoms may arise at any time.

Double Subtertian Fever.

This is caused by two broods of the Laverania malariæ parasite, and has symptoms in common with the preceding form.

Irregular Subtertian Fever.

This is caused by several broods of $\mathrm{L}$. malariæ sporulating at different times, causing an irregular fever. It is common on the West Coast of Africa.

Remittent Subtertian Fever.

An ordinary attack may be prolonged or two attacks may be continuous, the second commencing before the first has concluded.

These may be serious at any time or may become pernicious.

There may be sleeplessness, restlessness or delirium.

The liver and spleen are enlarged and tender.

There is slight dilatation of the right side of the heart.

Unless drastically treated the fever may resemble-

(I) Typhoid fever in its clinical manifestations, with low delirium, prostration, dry tongue, liver and spleen much enlarged, and melanæmia;

(2) Bilious fever, with vomiting, diarrhoea, constipation, jaundice, due probably to the absorption of modified hæmoglobin, e.g., hæmaphein; the liver is much enlarged. There is much blood destruction, much bile formation, and sometimes coma and death.

(3) Adynamic fever, with tendency to hamorrhages, local gangrene, hæmoglobinuria, great weakness, nervous depression, muscular and cardiac debility, profound and rapid blood deterioration, icterus, leucocytosis and melanamia.

The hæmoglobinuria must be differentiated from blackwater fever proper. The former complicates a subtertian attack.

It has been shown that L. malariæ produces an hæmolysin in such quantities that it cannot be kept in check by the anti-hæmolysin, which results in dissolved blood being excreted by the kidneys. Jaundice is more rare in this condition than in ordinary blackwater fever. 


\section{PERNICIOUS SUBTERTIAN FEVER.}

This type is caused by certain viscera being selected by the parasite for sporulation. The consequent symptoms have local as well as general manifestations owing to that particular viscus being affected not only by the toxins, but because of its impaired nutrition, for the capillaries may be completely blocked by parasites, red cells, swollen endothelial cells, leucocytes loaded with pigment, free pigment and merozuites. These fevers are accompanied by a high mortality.

Tre shall now deal with them seriutim.

(1) Algid Pernicious Subtertian Fever.

The condition of collapse is marked, e.g., sharp nose, sunken cheeks, lips and extremities cyanotic.

The pulse is small, soft, frequent. Respirations are laboured.

The slin is cold and clammy.

There is severe thirst.

The patient may die in a few hours.

(2) Diuphoretic P.S.F.

The sweating of the fourth stage is excessive, a pool of perspiration may fall on the floor beneath the bed.

Exhaustion and collapse may follow.

(3) Homorrhagic P.S.F.

There may be hamorrhages from the skin, mucous membrane of the bronchi, intestines, stomach, or generative organs during the attack, but not during the intermission.

A grave anamia may follow with : hheady pulse, coma, delirium, conrulsions and death.

This variety is rare.

(4) Scarlatiniform P.S.F.

There is a diffuse scarlatiniform rash with desquamation of the horny layer and erythema of the fauces.

A typhoidal state may carry off the patient.

(.) Cerebrospinal P.S.F.

The symptomatic manifestations may resemble:-

(a) Coma.-There is a sharp onset, with weakness, sleepiness, headache, disturbance of vision, stupor or delirium, ending in coma. The pupils may be contracted. The speech is scanning in character. There may be hæmorrhages under the skin and into the retina.

The urine and motions may be passed involuntarily.

The heart is dilated, pulse slow, becomes quick and thready before death.

In fatal cases, the tongue becomes dry and the patient colder and colder. 
There may be improvement for twenty-four hours with relapse and death. Some cases recover.

(b) Hyperpyrexia.-The temperature may rise to $107^{\circ}-108^{\circ} \mathrm{F}$. There may be brief mania, followed by muttering delirium, unconsciousness, coma and death in one or a few hours.

It is due to embolism by malarial parasites, swollen cells, débris, \&c., blocking the capillaries supplying the thermic centre.

(c) Delirium.-There may be delirium with sudden onset, followed by exhaustion, coma and death. All are fatal. The fever is usually high. It may be mistaken for thermic fever.

(d) Tetanus.-Some delirious patients may show trismus, contraction of limbs, opisthotonos, retraction of abdomen, and conjugate deviation of the eyes.

There may be recovery or death with hyperpyrexia.

(e) Eclampsia.-Children may have convulsions, stupor, coma and death.

(f) Meningitis.-It occurs in young persons and children. There are: vomiting, slow pulse, headache, retraction of head, rigidity of neck, convulsions, hyperzesthesia, fever, irregular pulse, coma and death.

(g) Hemiplegia.-There may be all the signs and symptoms of hemiplegia with paralyses. There may be aphasia, often resulting in permanent psychical disturbances, loss of memory, \&c. This is due to the blocking of the capillaries supplying Broca's convolution (embolism).

(h) Amblyopia.-Blindness is sometimes complained of, is usually only temporary lasting for several days, but in some cases it persists, when there may be found optic neuritis, peripapillary oedema, extravasation of leucocytes, plugging of retinal or choroidal vessels by parasites, pigment, leucocytes and consequent multiple hamorrhages in the fundus (Poncet).

In quinine amblyopia (see p. 524) there is retinal anamia due to toxic spasm of the arterioles and the amblyopia is more persistent. The discs are white and the vessels shrunken, while no inflammatory symptoms are present.

In the malarial condition one eye only may be affected, and the condition may clear up quickly under quinine treatment. The pupils react to light, but they do not react in quinine amblyopia.

(i) Bulbar.-There is difficulty of speech and deglutition with 
facial paralysis or paresis, increased tendon reflexes, ataxiá and romiting.

(j) Cerebellar.-There is cerebellar ataxia, severe headache, vomiting, \&c.

(6) Gastrointestinal P.S.F.

The symptoms may resemble-

(a) Cholera.-There is vomiting, abdominal pain, severe diarrhoea, the stools are loose, profuse and numerous, painful cramps of legs.

Collapse may ensue with sunken eyes, cyanosis, clammy skin, hiccough, severe thirst, scanty urine, delirium, coma and death.

Occasionally a case recovers.

The condition may be mistaken easily for true cholera, but the stools usually contain some bile (not rice water-like).

The blood also will show indications of malaria.

This manifestation is seen in the Punjab.

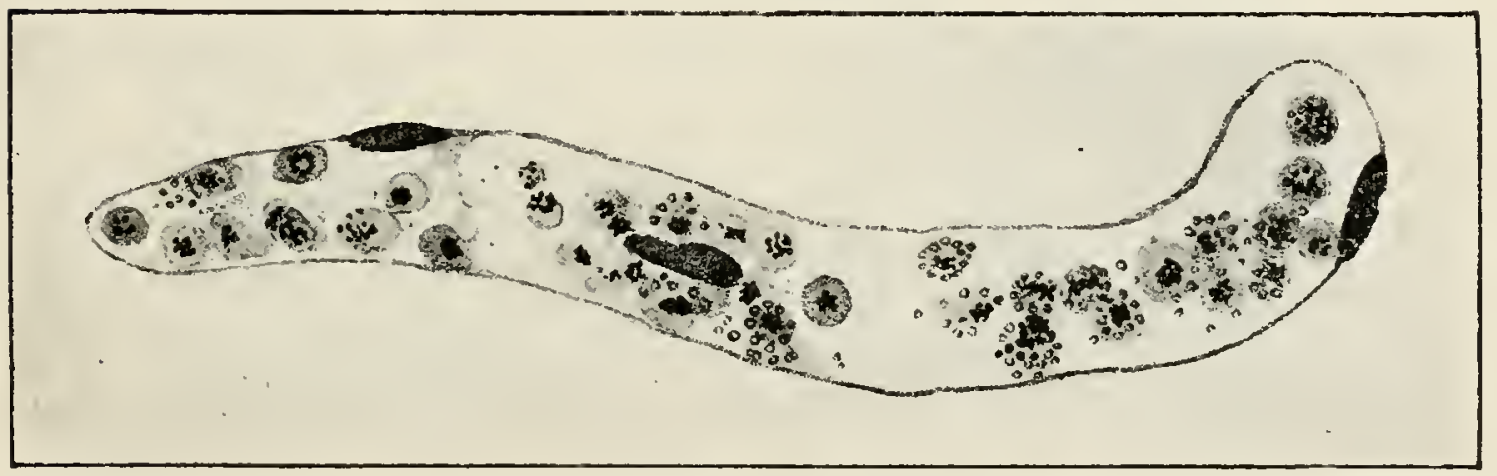

Parasites in capillary from intestine.

(b) Dysentery.-The fever is usually high.

The stools contain blood and mucus tinged with bile.

There is great distress and prostration.

The pulse is small and rapid.

Quinine may result in rapid recovery.

(c) Hemorrhagic Pancreatitis.

The diagnosis is made after death.

It is seen in Ceylon.

There is an acute onset with epigastric pain.

There may be signs of partial intestinal obstruction with marked collapse.

(d) Peritonitis.-This may be simulated when the suprarenal glands are affected in particular.

(7) Cardiac P.S.F.

The right heart is enlarged, there is severe cardiac pain, and romiting of blood. 
The patient passes into an algid condition and dies.

The capillaries of the heart may contain blood in a condition of stasis which may cause the death of the patient, especially in those with fatty heart and pericardial adhesions.

Chronic alcoholic subjects and beriberi victims run grave risks in exposing themselves to malarial infection (Daniels).

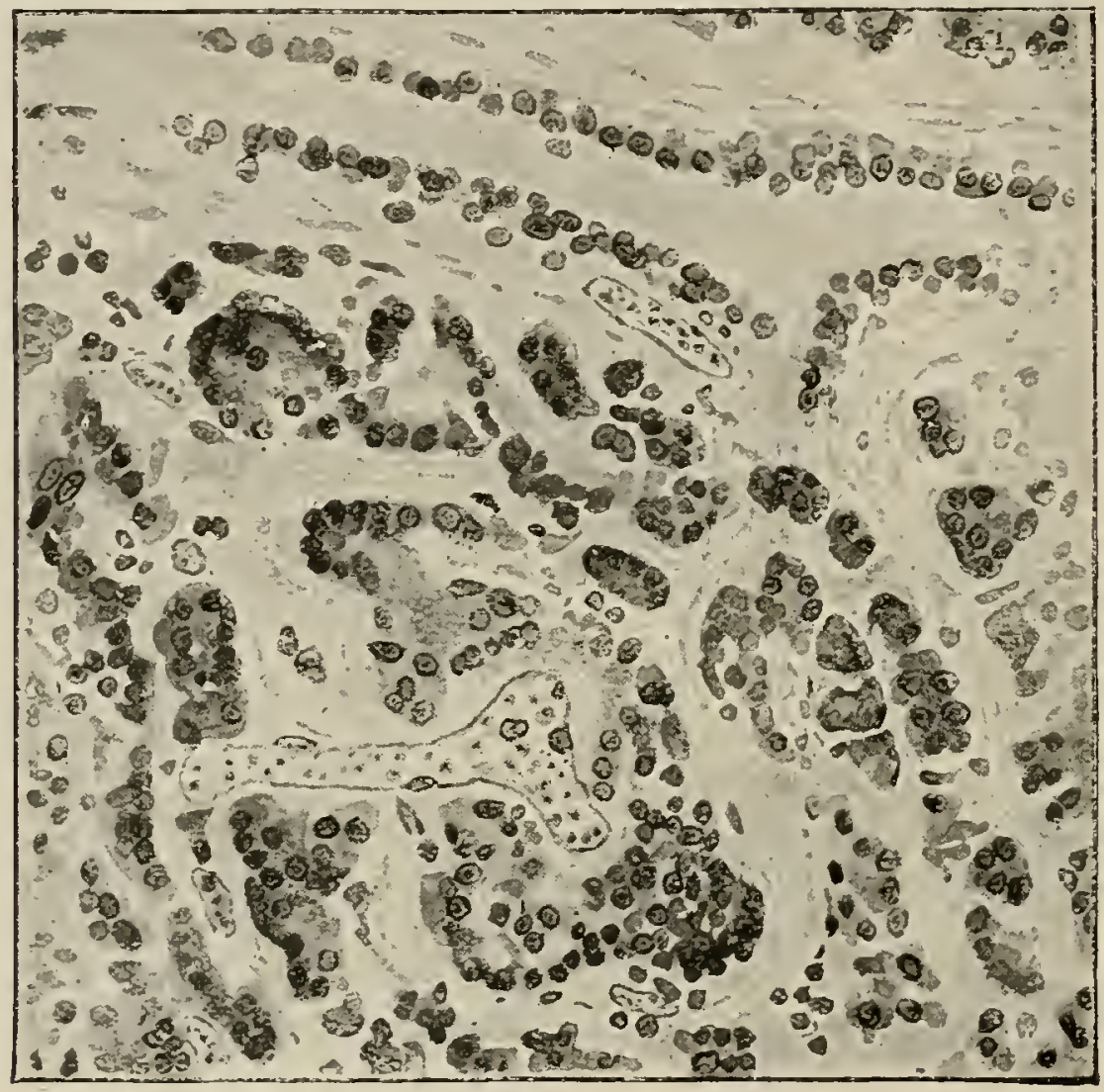

Parasites in capillaries of pancreas.

(8) Pulmonary P.S.F.

Resembling-

(a) Pncumonia.-There is blood expectoration, in the red cells of which are seen malarial parasites. There is dyspnœa and cough. The symptoms are worse during the fever than during the remission.

The blood is usually full of the parasites.

The pneumococcus complicating the fever may be the cause of this manifestation.

(b) Pleurisy. - There is sharp pricking pain, dry cough, friction sounds, which are worse during the febrile attack.

Apart from all these varieties of subtertian manifestations, the testicles may be selected as the point of attack with the corresponding symptoms. The appendix may also be chosen with its symptoms.

Dry gangrene of the feet and legs may be caused, malarial endarteritis (Tomaselli). 


\section{CHRONIC MALARIA.}

The causative organism is usually Laverania malarice, but either of the malarial parasites may cause it.

The numerous symptoms are often slight but frequent. There are :-

Repeated small fevers, enlargement of spleen and liver, pigmentation of slin and mucosa, odema of feet, anamia which is often marked, hamorrhages which make operations dangerous, multiple neuritis, skin eruptions, sinovitis, palpitation, dizziness, bronchial and intestinal disturbances, morning diarhoea, fleeting pneumonias and headaches.

Sometimes the fevers are absent, such not being necessary for the condition.

The spleen may become enormous in size, firm but painless. It is very friable, and may be ruptured by slight blows or even spontaneously.

Vigorous games and corporal punishment should be withheld from such.

The liver enlarges from chronic hepatitis. There is hypertrophy of the intralobular connective tissue which leads to hypertrophic or atrophic cirrhosis. This incurable condition leads to portal obstruction and ascites. The hamosiderin may be in blocks as large as blood corpuscles. The term "Siderosis" is then applied to this condition.

The secondary ancmia may be profound with loss of red cells, and $\mathrm{Hb}$, with a large increase of the mononuclear elements.

Abortion and sterility are common effects of this anamia.

The condition described above is known as "Malarial cachexia."

If the acute conditions were adequately treated with quinine this condition could hardly arise.

Unfortunately malarial relapses are exceedingly common even when quinine has been administered and without reinfection. Some of the parasites, lying dormant, wake up later and cause a relapse for some unknown reason, unless it is because the resistance of the patient is diminished at that time.

Recurrences may often occur after several years. Malarial parasites have been found in the blood four years after leaving a malarious district. Apparent recurrences in malarial districts are usually reinfections.

Latent malaria is a term given to a condition when malarial parasites are found in the blood and there are no symptoms present.

The parasite is usually of the subtertian variety.

Masked malaria is a term used when there may be neuralgias, 
cerebral abscess, chronic diarrhoea, $\& c$., when the malarial parasite is found, and the condition clears up on the administration of quinine.

Congenital malaria is doubted, but one case is on record where a malarial mother gave birth to a child, in the umbilical cord of which parasites were found. After death they were also found in the heart blood. The parasites were identical with those in the maternal blood and the placenta. Bignami and others have failed to find parasites in similar cases.

Juvenile malaria, when chronic, causes stunted growth and retarded puberty.

\section{SEQUELE AND COMPLICATIONS.}

The sequelae, such as neuritis, neuralgia, anamia, cirrhosis of the liver and of the spleen, with traumatic rupture of the same, have already been mentioned, but to these may be added mental modification, insanity, tinnitus aurium, vertigo, deafness, anosmia and loss of taste.

Malaria may be complicated by-

Typhoid fever, to which condition the term of typho-malaria has been applied. There is no hybrid or distinct disease, but the one complicating the other, there being two distinct causative organisms present.

Amabic dysentery may be present, but one must not forget that malaria itself can cause dysentery.

Lobar pneumonia is not uncommon. The low form in chronic malaria is of a deadly type. The possibility of a malarial pneumonia has not yet been decided.

Nephritis may be present and is due to irritation by the malarial toxins. It is common in children (Daniels).

\section{DIAGNOSIS.}

Decide positively and treat accordingly if :-

(I) The fever has a typical quartan or tertian periodicity.

(2) When malarial parasites are found in the blood.

(3) When hamozoin is found in mononuclear leucocytes.

Spleen puncture for diagnosis is not devoid of risk.

A quartan or benign tertian will yield to quinine within four days, but not necessarily so a subtertian attack.

One must differentiate malaria from :-

Typhoid Fever, Insolation, Liver Abscess, Kala-azar, Undulant Fever, Influenza, Yellow Fever, Dengue, and Seven-day Fever.

A mononuclear increase in a differential blood count is useful, but it may indicate a past malarial attack or pneumonia, sepsis, or an acute 
hepatitis. The latter will mask it by producing an increase of other leucocytes. Children commonly have mononuclear increase without any disease (Daniels).

\section{PROGNOSIS.}

The mortality amongst natives is usually low.

In Europeans resident in the tropics it is high, Africa in particular. Simple tertian and quartan parasites give the best prognosis.

Subtertian is always grave; pernicious symptoms deadly.

People unable to take quinine should not be allowed to reside in the tropics, e.g., malarial countries.

Complications always make the outlook more serious, especially typhoid fever and pneumonia.

Syphilis in a malarial patient will not yield to anti-syphilitic treatment until the malaria has been cleared up.

Ross gives us the following interesting figures for India:-

The mortality is 5 per 1 , ooo living, or I, I50,00o per annum.

It is greater than the mortality of cholera and dysentery combined.

A quarter to one-half of all tropical sickness is due to malaria.

Malaria tends to abound in the most fertile countries, hence very malarial countries cannot be prosperous.

\section{TREATMENT.}

For the patient to "fight the fever" himself by his own will-power is a marked error of judgment, and an unjustifiable risk for the patient to take.

The indications are :-

(1) To kill the parasites.

(2) To aid the excretion of the toxins.

(3) To relieve the symptoms of the patient.

Quinine is a specific if the right kind is given and a sufficient quantity used. Quinine is slightly cumulative; the maximum is eliminated in four to twelve hours, nearly all of it in thirty-six hours. It can be recovered from the urine within fifteen minutes from its administration.

The sulphate is soluble $\mathrm{r}$ in 800 of water and must be dissolved in mineral acids. It is the cheapest kind, but is not to be recommended. In the early days of quinine treatment, when the Chinese patients of Sir Patrick Manson passed the quinine given as the sulphate in pills, the pills were collected by Chinese physicians, washed, and sold again to fever patients.

The hydrochloride is soluble $I$ in 40 .

The bisulphate is soluble $\mathrm{I}$ in $1 \mathrm{I}$.

The bihydrochloride is soluble $\mathrm{I}$ in $\mathrm{I}$. 
The bihydrobromide is readily soluble in water, and is given to those with deafness or headache or idiosyncrasy towards quinine.

Quinine valerianate is given to nervous people.

f Quinine tannate is not so bitter and can be made up chocolatecoated for children.

( Euquinine is very expensive. It is useful for women and children.

Solubility and Equivalent Value of Salts of Quinine (after Manson).

\section{Name of salt}

Sulphate

Sulphate ... $\quad$..

Hydrorhloride
Bihydrochloride

Hydrobromide $\cdots$

Bihydrobromide .

$60^{\circ} 0$

Phophate $\cdots \quad \ldots \quad \ldots \quad$... $59^{\circ}$ I

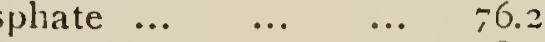

Lactiate $\quad \ldots \quad \ldots . \quad \ldots \quad 78 \cdot 2$

Salicylate $\quad . . \quad \ldots \quad \ldots \quad$... $70^{\circ}$ I

Hydrochloro-sulphate $\quad . . \quad 74^{\circ} .3$

$\begin{array}{llll}\text { Valerianate ... } & \ldots & \ldots & 73^{\circ} \circ\end{array}$

$\begin{array}{lllll}\text { Arseniate } \quad . . & \ldots & \ldots & 69^{\circ} 4\end{array}$

Tannate $\quad . . \quad \ldots \quad \ldots \quad 20^{\circ}$
Percentage of the alkaloid in the salt

Solubility in water

Amount equiv-

alent in value to one of quinsulphate

\begin{tabular}{|c|c|c|c|c|}
\hline In & $800 \mathrm{pr}$ & arts & $\ldots$ & 1.00 \\
\hline ," & 40 & $"$ & $\ldots$ & $\cdot 90$ \\
\hline " & I & , & $\ldots$ & $1 \cdot 02$ \\
\hline ", & 45 & "9 & ... & $\cdot 96$ \\
\hline, & 7 & , & $\ldots$ & $1 \cdot 23$ \\
\hline ,9 & II & ", & $\ldots$ & $1 \cdot 24$ \\
\hline , & 420 &, & .. & ${ }^{\circ} 96$ \\
\hline, & 10 & , & $\ldots$ & $\cdot 94$ \\
\hline, & 225 & , & $\ldots$ & $1 \cdot 05$ \\
\hline ", & 2 & $"$ & $\cdots$ & .99 \\
\hline , & I IO & , & $\ldots$ & 1.01 \\
\hline \multirow{2}{*}{\multicolumn{3}{|c|}{ slightly }} & $\cdots$ & 1.06 \\
\hline & & & $\cdots$ & $3^{.67}$ \\
\hline
\end{tabular}

If ingested quinine, especially the sulphates, are not dissolved by the acid gastric juices of the stomach it is probably not dissolved at all, as this is not permitted by the alkaline juices of the intestines. Such quinine is then excreted practically unchanged.

In small doses quinine is a vascular tonic, but in large doses it is a cardiac depressant.

Most of it is oxidized in the tissues. The kidney excretes it as quinine dihydroxyl.

It may cause cerebral congestion, manifesting itself as tinnitus, deafness, headaches, \&c. It may cause constriction of the retinal arteries, followed by temporary amblyopia or degeneration of the retinal ganglion cells, followed by permanent amblyopia. Various kinds of eruptions have been reported from the use of quinine.

Ten grains of quinine bihydrobromide contains bromine equal to that in 3 grains of pot. brom., hence if this quinine is used prophylactically for long periods mental depression may be expected from the quinine and bromide.

\section{MODE OF ADMINISTRATION.}

Under all circumstances it is best given after food, as then there is more gastric juice, the acidity of which facilitates its solubility.

(i) By the mouth.

The bisulphate or bihydrochloride are to be recommended if the expense is not a consideration. They can be taken as powders, 
solutions, capsules, cachets, tabloids, tablets, or pills. They may be plain, sugar, or chocolate-coated. The sugar-coated ones are not to be recommended unless they can be used quickly, as the sugar hardens in the tropics and hinders the solution of the drug. In some cases it is useful, as it can be given to patients unknown to them.

Tablets or tabloids are compact and most useful for rork involving much transport. For hospital use the sulphate, dissolved in mineral acids and flavoured, is cheapest. Many tropical residents take 5 grains daily as a prophylactic, or I5 grains ( $\mathrm{I}$ grm.) twice weekly.

A regular habit is to be encouraged.

For malarial attacks, 5-ro and even 20 grains t.d.s. has been taken. Darling recommends heroic does; such were absolutely: necessary in the writer's experience when in Colombia.

One should wait until the temperature has begun to fall before administering the quinine, except in chronic cases, because the headache is increased, and the quinine cannot kill the young parasites until the red cells enclosing them are ruptured.

When headaches and tinnitus aurium are common, pot. brom., 5-10 grains, or dilute hydrobromic acid should be administered with each large dose of quinine.

In old Blackwater cases one should proceed carefully with $\frac{1}{2}$-grain doses.

If the fever is not being mastered, inhalation of amyl nitrite may dislodge the parasites from the capillaries so that the quinine can get at them.

Children stand quinine well by the mouth. They must have sufficient, say a child under one year, from $\frac{1}{2}-1 \frac{7}{2}$ grains six times daily. A child aged 3-10 years old 2-3 grains six times daily.

After fever in adults give 15 grains ( 1 grm.) daily for a week, then Io grains for two weeks, followed by $5^{-10}$ grains for a month.

Iron and arsenic should be given.

In patients with weak hearts digitalis should be given.

(2) By the rectum.

Useful in gastric disturbance. One should double the ordinary doses one would give by the mouth, say $20-30$ grains, in $10-12$ ounces of water or warm saline. The writer when in extremis received 40 grains at night, followed by 40 grains the next morning.

This method is most useful for comatose patients. Use a large catheter attached to a glass syringe or rubber tube with funnel. Give it slowly.

Continue by the mouth as soon as possible.

(3) By the muscle.

Subcutaneous injections should be abandoned. The writer has a 
scar resulting from a subcutaneous injection of quinine the size of half-a-crown that he will carry to the grave. The necrotic ulcer required four months to heal.

Intramuscular injections are useful when there are gastric disturbances and serious subtertian attacks. Painful spots may remain for months at the point of each injection. I have seen a case of wrist drop following an injection of quinine in the upper arm, the needle having penetrated the musculo-spirial nerve, not to mention the consequent necrosis caused by the quinine itself. One of my patients had been previously treated by quinine injections in both buttocks (even when there had not been any romiting), and had been partially paralysed in both legs owing to permanent damage having been done to both sciatic nerves.

For intramuscular injections the bihydrochloride should be dissolved in normal sterile saline solution, so $\mathrm{grm}$. of quinine in $\mathrm{s}$ o $\mathrm{grm}$. of saline, given I-Ioth at a time (Bacelli).

Giemsa's formula is not supposed to be painful in administration.

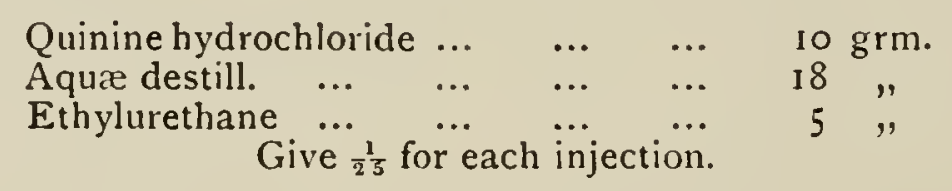

Herme:ically sealed vials can be purchased ready for use.

The gluteal-region is chosen usually for the injection along a line drawn from the posterior-iliac spine to the ischial tuberosity.

It is doubtful if intramuscular injections are sufficiently rapid in their action for severe and acute cases. There is always some local damage done and absorption would be slow in consequence, during the process of which the patient might die.

(4) In the vein.

In pernicious cases no time can be lost, hence the intravenous method has its uses.

An injection of ether should precede that of the quinine if the pulse is bad. A superficial vein about the elbow should be chosen. I 5 grains (I'oo grm.) should be given at a time, well diluted in 20 c.c. of saline, and injected slowly. It should be repeated if necessary, but after the first injection there may be sufficient recovery to continue quinine treatment by the mouth.

Quinine should be given with care in pregnancy as it may cause abortion, but untreated malarial attaclis are more likely to produce it.

The administration of quinine may precipitate an attack of hamoglobinuria. 5 grains has caused this (Sandwith).

Fever cases, during the acute attack, must have-

Rest in bed, light diet, blankets for cold stage, hot bottles, hot 
drinks to acid perspiration. In hot stage, vinegar or its equivalent to the forehead; for headache, aspirin can be used but in severe cases heart failure may be caused by it. Caffeine is good in small doses, 2-3 grains. In the sweating stage remove all blankets, damp. clothing and bedding, then administer tepid sponging, iced soda water, \&c. For-

Tomiting: iced champagne; mustard leaf to pit of stomach ; chloroform and morphine mixture, stomach lavage.

Hiccough: mustard leaf, codeine, morphia.

Cough: codeine and morphia.

Constipation: calomel, 1-3 grains, followed by saline.

Hyperpyrexia: cold sponging, cold packing, cold baths, cold enemata. If the temperature is kept within limits for a few hours the quinine will have a chance to act.

Algidity: salines given hypodermically, intramuscularly, intravenously or per rectum. Warm applications to body, oxygen inhalations, ether and strychnine.

Hcmorrhages: calcium lactate, adrenalin, salines.

Big spleen: counter-irritants, saline aperients, quinine, iron and arsenic.

Convalescence: Give quinine for three months, iron and arsenic for two months. If the attack has been very serious send patient to a cool climate.

\section{PROPHYLAXIS.}

It is most essential that all the scientific methods of prophylaxis should be carried out. By them the death-rate, case incidence, illness and mortality from complications, as well as the disease and the expense to employers of labour, are enormously decreased.

The work of Colonel Gorgas and his Sanitary Staff at Panama for the abolition of Yellow Fever and the enormous decrease of Malaria is too well known to be repeated here. This work was made possible by the sanitarian. The money spent in such work is insignificant when compared with the work achieved and the lives saved.

Prophylactic measures against Malaria may be summarized under two heads :-

(1) Protection against mosquito bites.

(2) Mosquito reduction.

These will now be dealt with.

\section{(A) PRotection AgAinst mosquito Bites.}

(1) Mosquito nets must be used invariably, twenty-five strands to the inch, which will keep out the Simulida also. The nets should be tucked under the mattress and not allowed to fall loosely on the floor. 
It should be kept in good repair. Each net will last one year in travel, and about two years stationary. When the strands are becoming rotten the nets should be destroyed. A net with small holes or not tucked under the mattress is worse than not having one at all.

(2) Portable mosquito-proof rooms could be used, and are extremely useful for doing night work or reading in mosquito districts. They are essential in some districts when one wishes to work in peace during the daytime when flies are a pest.

(3) Fixed mosquito-proof rooms can be used which are more stable for permanent residence. Copper gauze wire can be used fixed to timbers. Portable ones on frames are also made with copper wire, but they are subject to much damage in constant transit.

Some steamers trading between Liverpool and the Amazon are now screened against mosquitoes. The sea air may be injurious to the copper gauze.

(4) Mosquito proofing of windows and doors excludes flies, moths, glare of the sun, damp exhalation after heavy tropical showers, and allows the breeze to enter unimpeded. Mosquito wire is of tinned iron, copper or brass, the prices being as two is to three.

(5) Mosquito proofing of verandahs. This is more expensive, but in selected cases gives good results. It obscures light and seems to retain the heat.

(6) Hands and feet can be protected by mosquito boots, glores, \&c.

(7) Medicine. All exposed to mosquito bites should take 5 grains of quinine daily. To keep off mosquitoes employ eucalyptus, petroleum, camphor bags about. neck, \&c.

Natives like the mixture of "Camber-green oil," with which they paint their bodies. It is made up of Citronella oil, I $\frac{1}{2}$; Kerosene, I ; Cocoanut oil, 2.

(8) Constant movement will prevent mosquitoes from settling nearly as frequently as they otherwise would.

(9) Fans and punkas. These creating a breeze are useful in houses or on verandahs. They can be driven by hand or electricity.

\section{(B) MOSQUITO REDUCTION.}

(I) By killing adults in houses with a small hand net.

(2) Fumigation for the same purpose. This method is much used in Italy and the States. Sulphur is generally used.

R. Powdered sulphur, 2 lbs. per I,ooo cub. feet.

Moisten with methylated spirits.

Place in shallow pan, supported on brick, in a large flat basin of water in a sealed room.

Leave for three hours after ignited, and then ventilate rcom well.

Pyrethrum powder may also be used, 3 lbs. per i, ooo cub. ft.

Sulphur affects brass work and steel goods. 
Camphor and carbolic acid can be used, equal parts, 4 ounces dissolved by gentle heat, per r, ooo cub. ft. Only about half an hour is required.

(3) By natural enemies, e.g., bats, birds, lizards, dragon flies, fishes as Girardinus poeciloides (millions), tadpoles. All such should be bred and protected. Such means, however, are not always best.

It may be much cheaper and better to remove the water altogether than to maintain " natural enemies."

(4) Dealing with the larve.-All small receptacles for water should be removed or filled with lime or given a surface film of kerosene, if such are used for table legs to keep away ants, \&c.

Larger quantities of water and small streams can be covered with a thin surface film of crude kerosene which does not permit the larva to reach the surface for air. It requires half an ounce per square yard every seven days to be effective.

Wherever possible destroy and do not treat mosquito breeding places. Small pools should certainly be drained. Holes in rocks and trees should be filled with rubble and cement. Plants which breed them, as pineapples, bananas, \&c., should be removed from near dwellings. Culicines breed in palm trees and sugar cane. Larvicides, such as copper sulphate, \&c., can be used in water, which rapidly kills off the larre, but it becomes poisonous for other animals.

(5) Screening of breeding places is sometimes necessary, such as of water tubs, wells, cesspits, \&c. Catte should be watered at certain parts only and the hoof impressions levelled.

(6) Drainage.-Open channels should be cut in the soil and concreted. Drains could be of stone and gravel. Local material available will help one to decide. The subsoil should be drained by pipes. As much as possible of the surrounding land should be suitably cultivated.

(7) Trees and bush.-Eucalyptus trees are not worth while. Many trees should not be allowed to remain near to houses as they attract and give out moisture, exclude breeze and increase heat.

All bush and undergrowth within 200 yards of all villages, houses and dwellings and all grass within this area above 2 inches in height should be cut down. A mosquito will not be able to cross such a zone without destruction by sun or wind.

(\$) Houses should be built several feet above the ground, upon sites high and dry, well away from coolie lines and native villages. Houses should be lofty-of stone, if possible, or brick-with good light and ventilation, which mosquitoes do not like. Whitened walls are good. Curtains and fancy decorations are bad.

As to the height of the site, one may remember that the temperature of the air tends to fall ${ }^{\circ}$ F. every 300 feet above sea-level. Malaria 
tends to diminish at 5oo-1, ooo feet high, but under suitable conditions it may be found 5 ,ooo feet high.

(9) Rice should not be cultivated within five miles of towns in Italy because of the swampy nature of the ground. In India, however, where this law would mean famines it could not be enforced.

Professor Pearson has worked out an interesting point on mosquito reduction. He affirms that if mosquito propagation is suppressed within the circular area of a mile-

The mosquito density at the centre will be 3 per cent.

At a quarter of a mile from the centre i 8 per cent.

At the periphery 75 per cent. of the density surrounding that area.

Sir Ronald Ross's work at Ismailia reduced malarial cases from 1,551 in 1902 to 37 in 1905 , the latter being all relapses. The cost of the work was $2-3$ francs per head of the population.

Every malarial district before being opened up should be systematically surveyed with regard to malarial propagation, and the work commenced at once on given lines.

Celli has shown us some interesting figures on the

Relative Values of Malarial. Prophylaxis.

Method of prophylaxis

Percentage of infection
None

33
Protection om mosquito alone Quinine and
protection
from mosquito

Hence quinine alone will prevent 13 per cent. of cases, and

Quinine plus mosquito protection will prevent $31 \cdot 25$ per cent. of cases.

\section{FOR NEW INFECTIONS ARE REQUIRED :-}

(I) That an infected person with gametocytes is living in or near to the locality.

(2) That an Anopheles, capable of carying such gametocytes, sucks up sufficient blood from the infected person.

(3) That this mosquito lives for a week or more under suitable conditions.

(4) That it then bites a person non-immune and not protected by quinine.

(5) Infected persons and carrying flies must be sufficiently numerous. If there are many flies and few infected persons the chances of becoming infected are greater than if there were few flies and many infected persons.

The following facts may be of interest :--

Spleen Rate.

Within 300 yards of jungle, undrained, it was ti per cent. 
Within 1,000 yards it diminished to 2.6 per cent. (Watson).

At the edge it was 59 per cent.

Fry found it to be 23 per cent. at 200 yards away and nil at one mile distance.

Mosquitoes fly readily for a quarter of a mile, but half a mile appears to be beyond the normal distance of flight. A gentle breeze will carry them a distance of one and a half miles.

100,000 mosquitoes per 100 yards square was once worked out to be an average number present.

Anopheles are not hungry for twelve to twenty-four hours after being hatched out or after laying eggs.

On an average only one out of forty-eight of all females ever have the chance of carrying malaria even in a malarial district (Ross).

The cost of a new hospital would often suffice to prevent more cases of sickness than are treated in it, and the cost of invaliding would often suffice to prevent the disease which caused it.

\section{THE MALARIAL MOSQUITO.}

The knowledge of these mosquitoes is so extensive that special works must be consulted for details. A résumé only of the outstanding facts can be attempted here.

The four great families of the Nematocera, order Diptera, which are of interest to the medical officer are the-

(1) Culicidæ, or mosquitoes.

(2) Psychodidx or moth-like midges, containing the notorious Phlebotomus.

(3) Chironomida, or midges.

(4) Simulidiæ, all the species of which are extremely bloodthirsty.

\section{FAMILY CULICIDA.}

They are distinguished from the midge-like flies by:-

(1) The venation. The costal vein runs all round the edge of the wing.

, first vein is very long.

,, second bifurcates.

, third is very short and goes off the second at right angles.

, fourth both bifurcate.

, fifth

,, sixth is simple and short.

(2) The close fringe of scales on the posterior border of the wings. 
(3) The projecting proboscis of extraordinary length.

There are two sub-families: (a) Corethrina.

(b) Culicinae.

\section{SUB-FAMILY CULICINÆ.}

Genus Anopheles.

The Sub-genera are important :--.

Anopheles (sensus restrictu).

Myzorhynchus.

Arribalzagia.

Christya.

Myzomyia.

Pyretophorus.

Nyssorhynchus.

Neocellia.

Cellia.

Chagasia.

All known carriers are included in the Genus Anopheles, of which there are about Ioo species universally distributed, but chiefly in the Tropics. Every medical officer should examine the stomach and salivary glands of mosquitoes caught in his district for malarial parasites in order to ascertain the species prevalent there.

For the dissection of mosquitoes, see p. 637.

The known malarial carriers in the different countries are:-

(After Alcock.)

\section{IN EUROPE.}

Anopheles maculipennis.

,, bifurcatus.

," ("Pyretophorus") superpictus.

, (Myzorhynchus) pseudopictus.

\section{IN SOUTHERN ASIA.}

Anopheles (Myzomỵia) lis:onii.

\begin{tabular}{|c|c|}
\hline ", & ,, culicifacies. \\
\hline "' & (Myzorhynchus) ba \\
\hline ," & $\begin{array}{cl}\text { "' } & \text { sinensis. } \\
\text { (Nyssorhynchus) fuligiosus. }\end{array}$ \\
\hline ", & obaldii. \\
\hline$"$ & $\begin{array}{c}(" \text { Neocellia ") } \\
\text { stephansii. } \\
\text { willmorii. }\end{array}$ \\
\hline
\end{tabular}

\section{IN TROPICAL AFRICA.}

Anopheles (Myzomyia) funestus.

, ("Pyretophorus ") costalis. 
Anopheles (Myzorhynchus) mauritanus.

,", paludis.

IN AUSTRALIA.

Anopheles (Nyssorhynchus) annulipes.

\section{IN NORTH AMERICA.}

Anopheles maculipennis.

," bifurcatus.

\section{IN TROPICAL AMERICA.}

Anopheles (Myzomyia) lutzii.

$$
\text { , ("Cellia") argyrotarsus. }
$$

IN THE UNITED STATES (Von Ezdorf).

Anopheles allumanus.

$\begin{array}{ll}, " & \text { argyrotarsus. } \\ , & \text { crucians. } \\ , & \text { intermedium. } \\ , & \text { quadrimaculatus. } \\ , & \text { pseudomaculipes. } \\ , & \text { pseudomaculipennis. } \\ , & \text { tarsimaculata. }\end{array}$

The following species are known to carry the larva of Filaria bancrofti:-

A nopheles maculipennis.

, (Myzomyia) rossii.

,, (Myzorhynchus) nigerrimus.

," , minutus.

., (Myzomyia) funestus.

" ("Pyretophorus") costalis. The Malarial parasite also.

, ("Cellia") argyrotarsus.

\section{SUMMARY OF FACTS RELATING TO THE ANOPHELES} ('un $\omega \phi \in \lambda \eta_{i}=$ unprofitable, harmful).

\section{CULICID $Æ$.}

Adults.-The head is small, hemispherical, has a distinct neck, brown cheeks covered with scales, used for differentiation of species.

Eyes reniform; no cœlli.

Antennæ long, slender, 14-15 segments, first globose; others carry whorls of hairs, wispy in female, thick and bottle-brush-like in male.

Labium, in the majority, is long and slender, covered with scales; ends in pair of small stiffish labella, bent when piercing.

Maxillary palps, covered with scales, show specific and sexual 

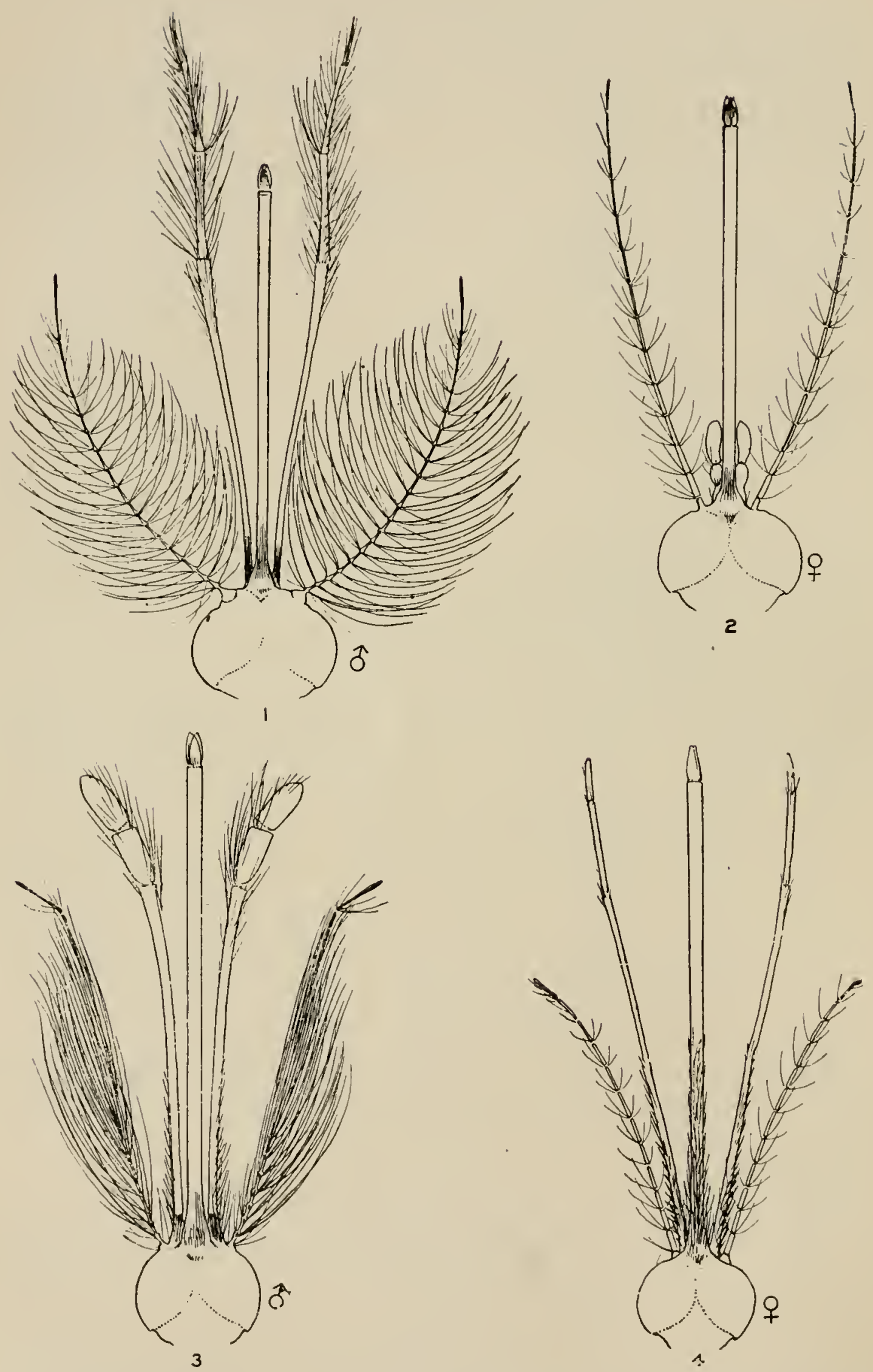

I, Culicine, male; 2, Culicine, female; 3, Anopheline, male; 4, Anopheline, fenale. 
differentiation; may be longer than proboscis; clubbed in male Anopheles.

The parts ensheathed in proboscis are long, slender, piercing organs except in the Corethrina, consisting of :-

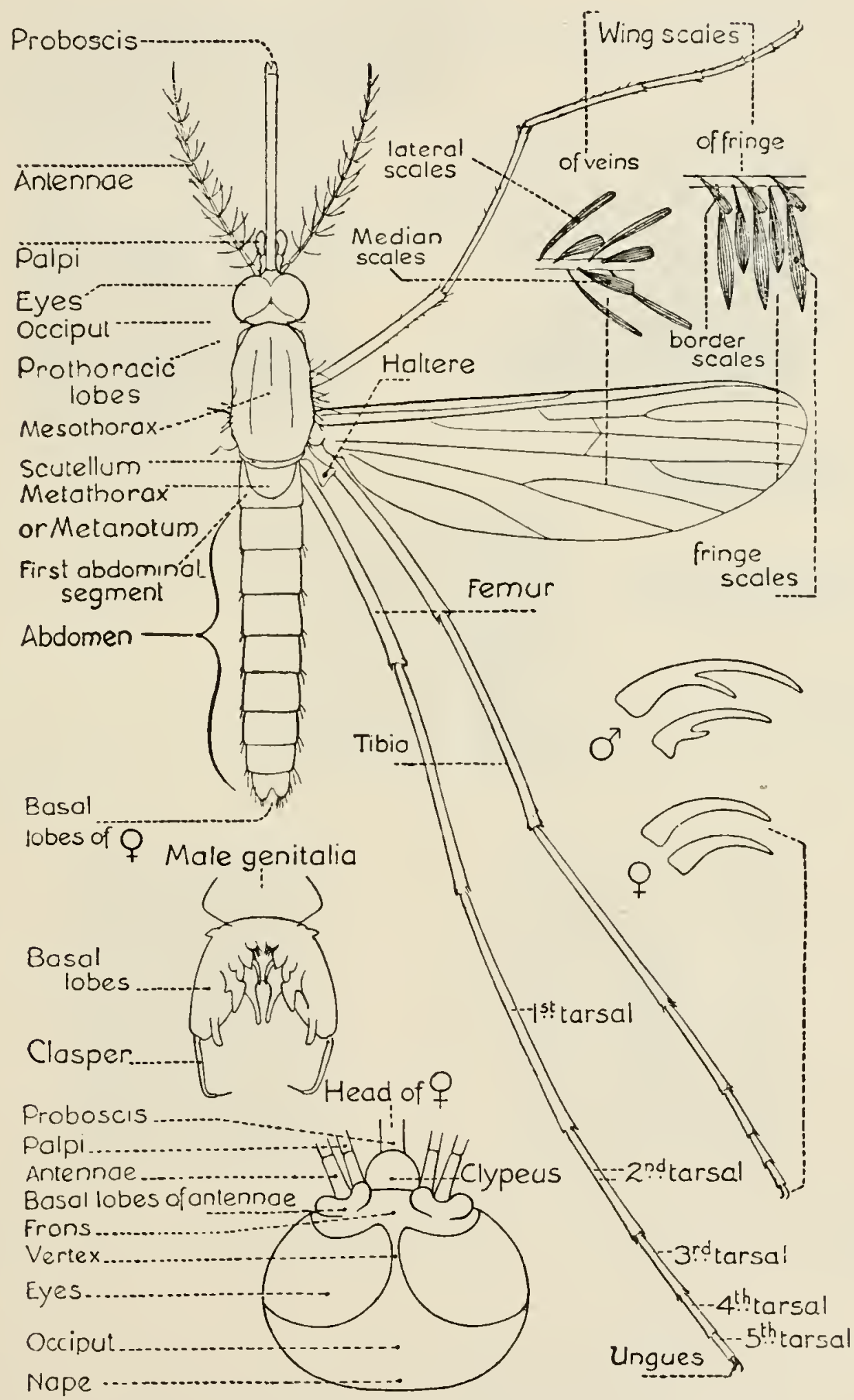

Diagram showing the structure of a typical mosquito. (Theobaid.)

(1) A stout-pointed epipharynx, grooved ventrally.

(2) A slender hypopharynx, which is itself an efferent tube for the saliva; forms when applied to the epipharynx a groove known as the afferent suctorial tube. 
(3) A pair of slender mandibles expanded and very finely serrated at the tip. (Absent in the males.)

(4) A similar pair of maxillae coarsely serrated at tip. (Absent in the males.)

Sometimes the mouth parts of the males, which only live on juices, appear to consist solely of the epipharynx and labium
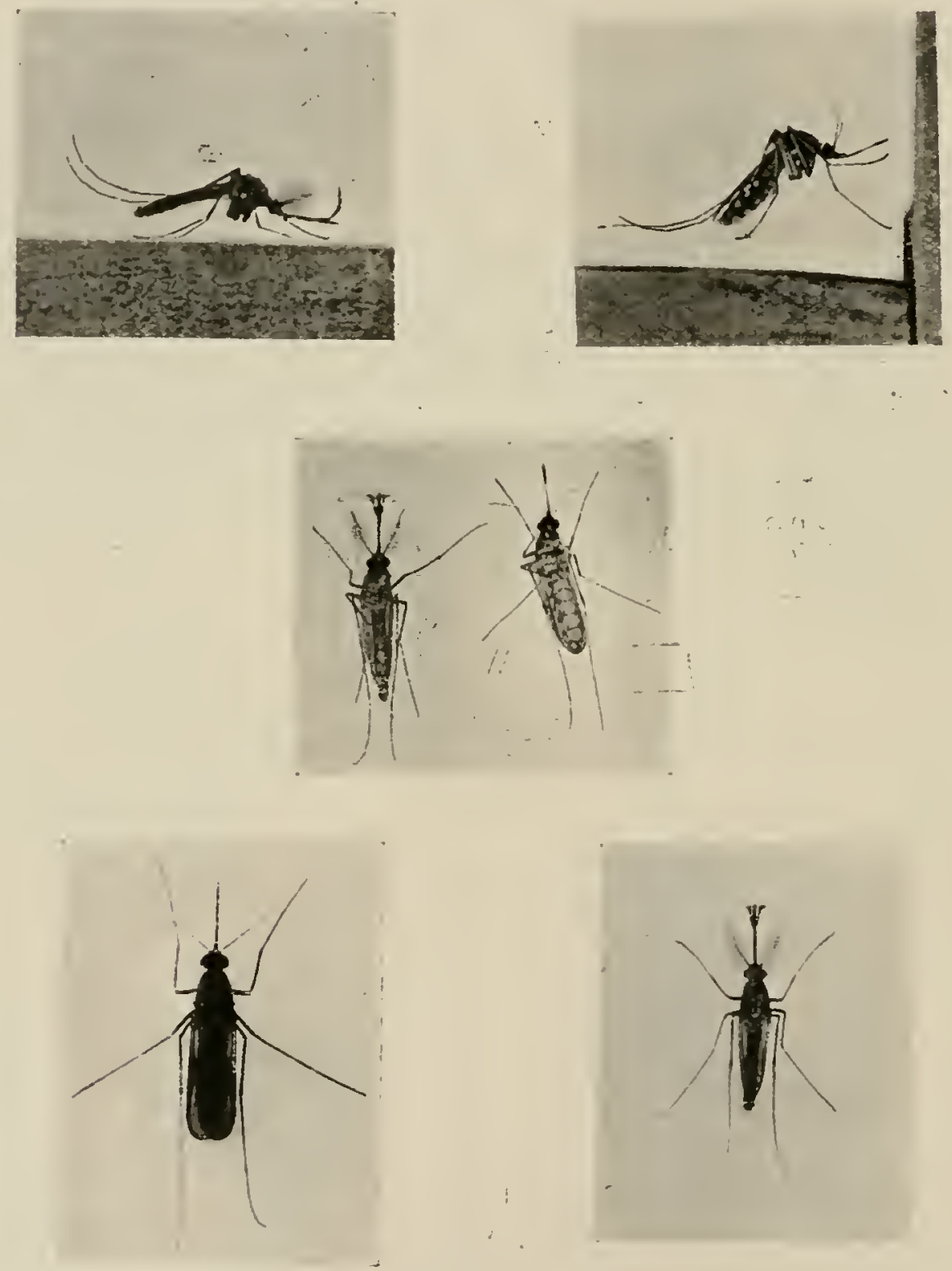

Photographs of living mosquitoes, C. impellcns, Walk.). Above $\delta$ and $q$ in profile; in the middle ventral aspects of the same; and beneath $q$ and $\delta$ dorsal views. About twice natural size.

Thorax is covered with scales or hairs.

Scutellum is narrow, not lobed in Anopheles, and is overlapped by the menatonum, which is bare.

Abdomen is long, narrow, scales or hairs, nine segments, last bilobed, complicated in the male with a pair of chitinous clasping hooks.

The wings are as described above; all the veins are clad with scales, lanceolate in shape for Anopheles. 
The halteres (balancers) are plainly visible.

The legs are long, slender; fifth segment carries pair of claws.

In order to spot the male from the female readily use the antenna as the chief guide.
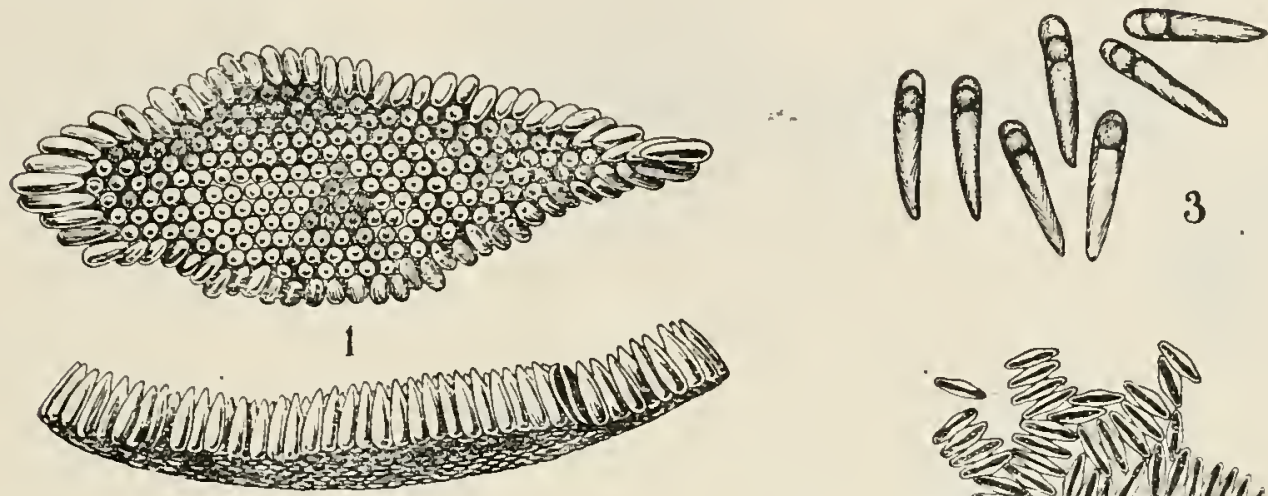

2
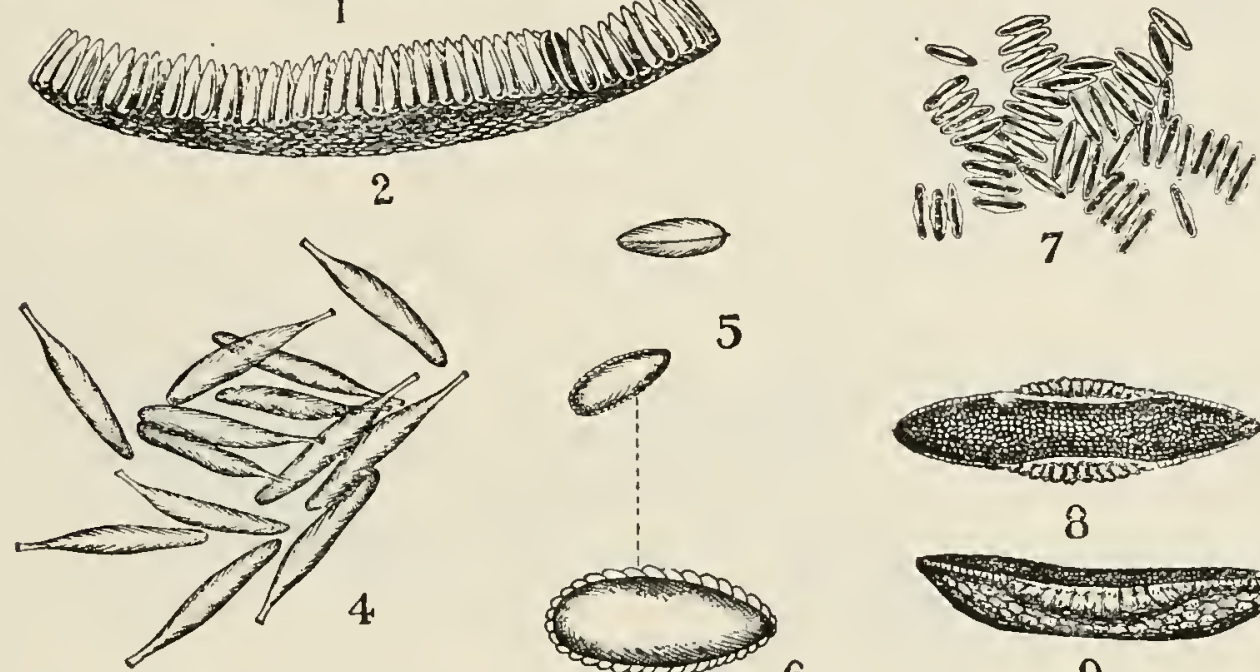

7

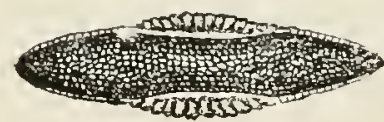

8

6

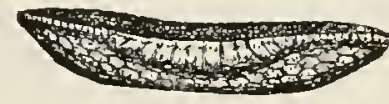

9

Various forms of Mosquito Eggs.-(I) Egg-boat of Culex, seen from above; (2) the same, side view (after Sambon); (3) separate Culex eggs; (4) eggs of Panoplites (after Daniels); (5) eggs of Stegomyia; (6) the same more highly magnified (after Theobald); (7) groups of Anopholes eggs, as they float on the water (after Sambon); (8) egg of Anopheles maculipenmis, showing lateral floats, seen from above, $\times 30$ diams.; (9) the same, viewed laterally (after Nuttall).

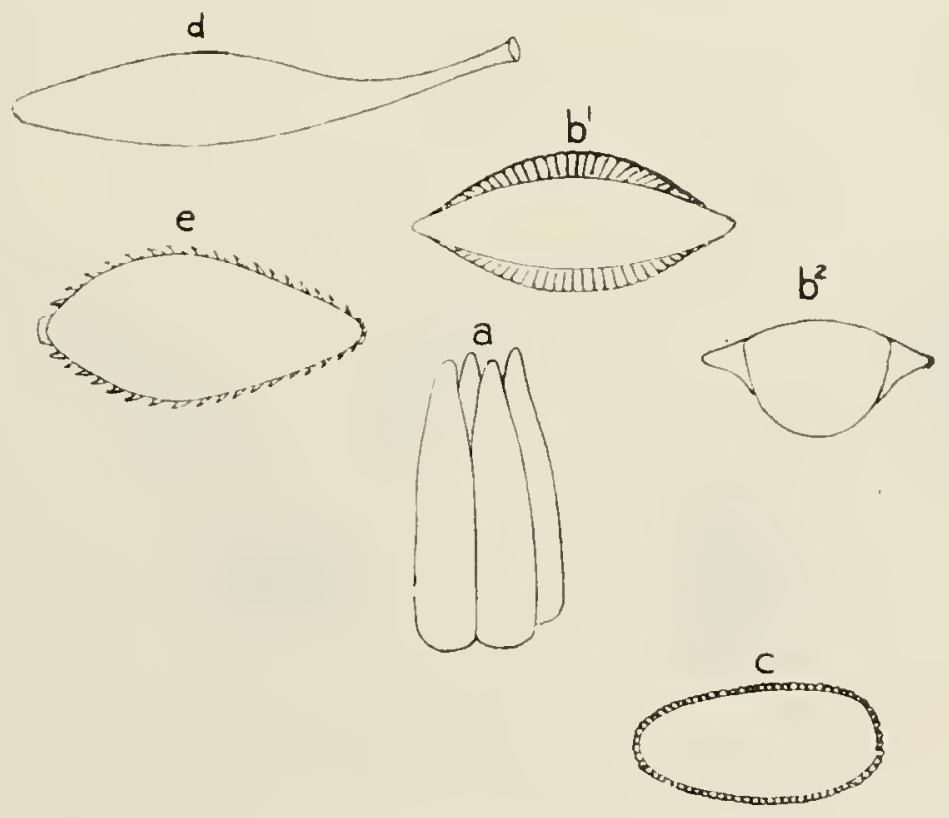

$a$, eggs of Culex ; $b^{1} b^{2}$, eggs of Anopheles ; $c$, egg of Stegomyia ; $d$, egg of Treniorhynchus; $e$, egg of Psorophora.

For the differentiation of species see a special work. Eggs.-These are laid on the surface of the waterIf as a sheet of jelly-like frog's spawn they are of the Coretinra. 


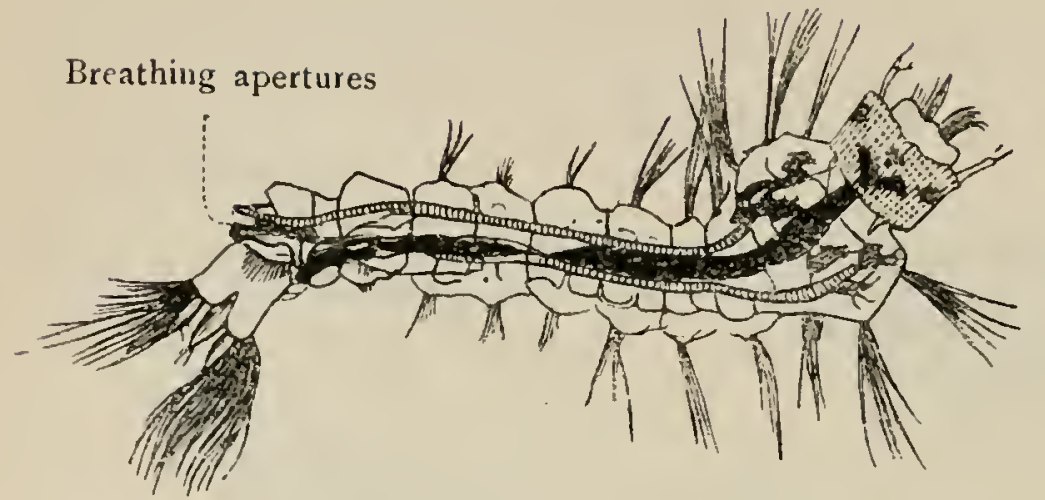

The anterior parts are shown in the prone position and the hinder ends in profile, owing to the tody being twisted by the pressure of the cover-glass.

(Anopheles rossii, Giles ; larva)

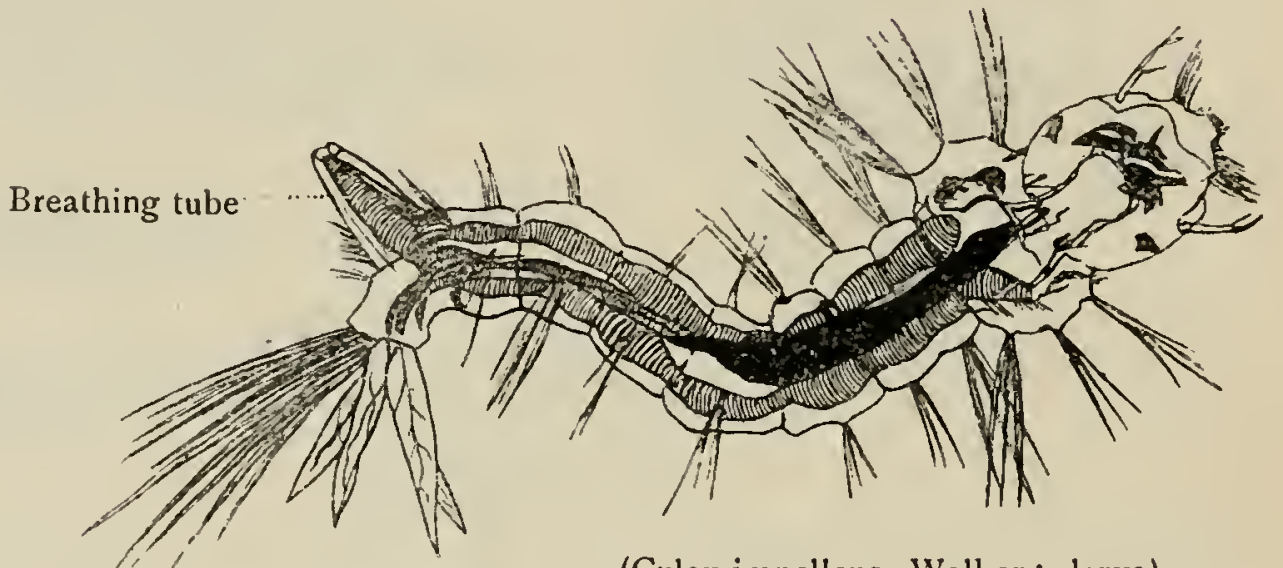

(Culex impellens, Walker ; larva)

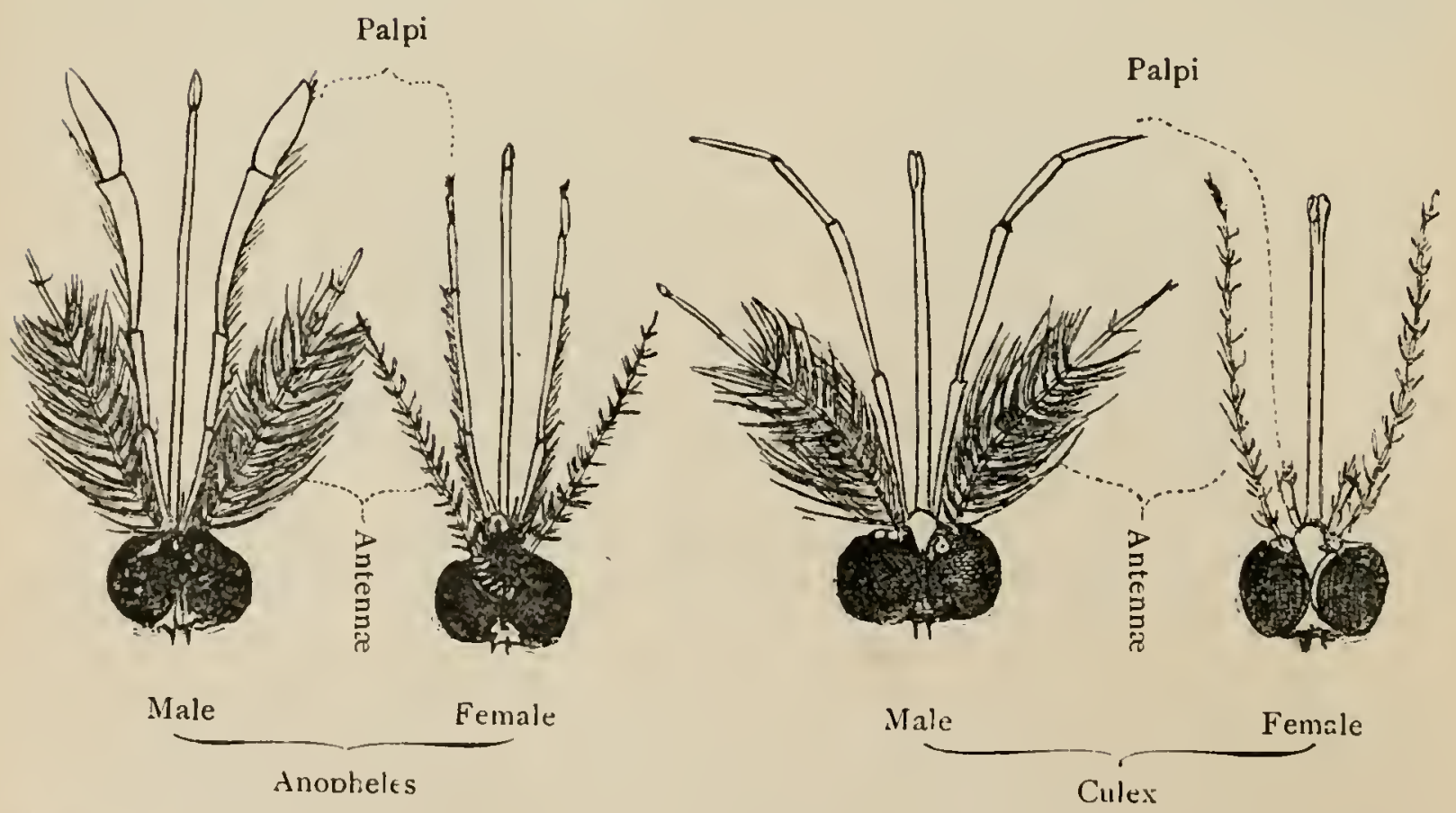


If in groups or rafts they are of the Culex.

If singly they are of the Anopheles.

Each egg is oval, one end blunter than the other, with a pigmented chitinous shell. It has a very fine external membrane which facilitates the floating of the eggs.

They hatch in twenty-four hours in a hot tropical season.

Larva.--All must live in water and can be found in collections almost anywhere, even in sea water, but not as a rule in rivers where the current is strong. They are very active, and live principally on algæ and similar vegetable matter.

The head is a rounded chitinous capsule with well-developed appendages.

The eyes are irregular masses of pigment in the younger, and distinctly faceted in the older ones.

The antennie are long.

The clypeus is prominent, to which are attached the "mouthbrushes," which have a rotatory motion to sweep food to the mouth.

The thorax has three segments, distinguished from the abdomen only by an arrangement of hairs, which are in tufts, single-branched or feathered.

The abdomen is elongate, soft, with nine segments sometimes beset with stiff hairs like the woolly-bear caterpillar, but usually hairs are lateral and on the free edge of the last segment. On the dorsum of the eighth segment the breathing organs open by two independent orifices in Anopheles, or one breathing tube of varying length in others. This latter has a valve and carries spines.

The intestine opens at the free end of the ninth segment, which carries bunches or whisks of hairs and four tapering tracheal gills.

In repose some larve hang with the head downwards with the tip of the breathing tube at the surface for air, but the larva of the Anopheles groups lie horizontally to the surface.

Larva become fully grown in about one week, but in cold climates may remain unchanged during the winter.

They moult several times and feed continuously.

Pupa.-They must live in water.

They are active, but do not feed.

In appearance they are like tiny lobsters.

The head and thorax form one mass.

Two ear-like breathing trumpets come from the back of the head mass for breathing.

The curved abdominal ninth segment ends in a pair of large bladelike fins.

On the first segment there are two tufts of hairs, fan-like, to catch 
the surface film and to bring the pupa into a good position for breathing.

The pupa matures in two days in hot weather.

When the adult is about to emerge the pupa straightens its back, the skin of the cephalothorax bursts along the back, out of which opening the adult appears. It rests for a few minutes and then flies away.

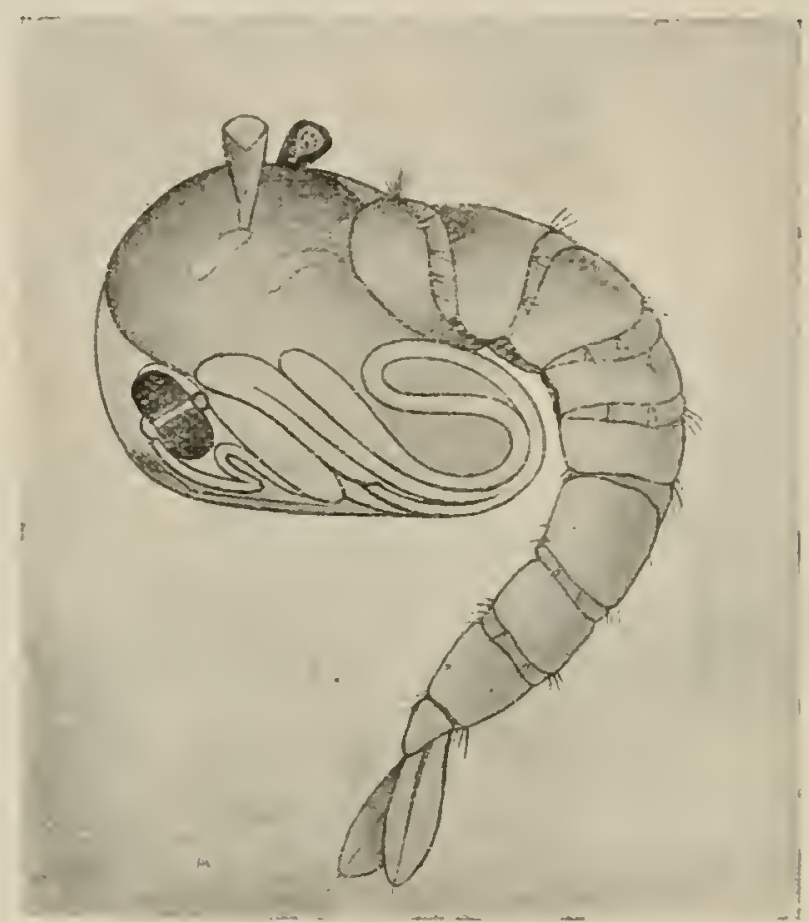

Pupa of Anopheles maculitennis, Meig. Enlarged. (After Grassi.)

Soon after a female is hatched it probably becomes fertilized; some maintain that it takes blood first; oviposition follows the blood meal, and takes place in the early morning.

The time of development is (average):-

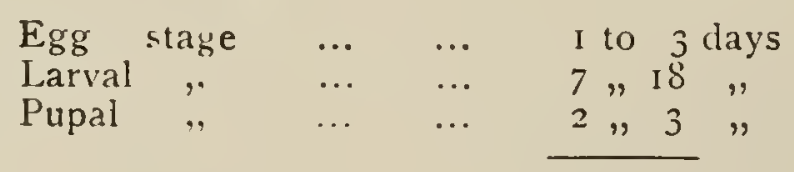

Io to 24 days, or longer

\section{BIONOMICS.}

The imago emerges from the pupa during the late afternoon when the female is ready for fertilizing the male. The latter sex usually predominates numerically.

The female alone bites man and animals in order to obtain blood, which forms rich food for the eggs, but much more rarely they will feed on juices, the Culicina more than the Anophelina.

The mouth parts of the female alone are adapted for piercing.

It is during this act that malarial sporozoites are injected into the 
human body from the infected mosquitoes. They pass down the hypopharynx or salivary tube, while blood and malarial gametes pass up the labial tube to the mouth.

They feed mostly at night, but may do so at any time of the day.

They become blown out with blood, then retire to digest it.

Next morning the female flies to the nearest water to lay her eggs; she may travel half a mile for this if it is necessary.

The Anopheles prefer clean water with weeds, the Culicina any kind.

The Anopheles eggs may be found in the back-eddies of streams, margins of lakes, large wells, puddles, in broken bottles, shells, holes of rocks and trees, and in plants like bamboos, pineapples, \&c.

At night the female seeks blood again.

The larva are cannibals, living on each other as well as on alga.

These larva must be attacked by the medical officer everywhere, or he can only protect himself with nets and drugs.

In the dry season mosquito-larva can astivate and wait for more suitable conditions.

The adult lives for about four weeks, not counting hibernating periods.

One adult mosquito can give off 200,000,000 in four months (Ficalbi).

Adulis may be transported great distances in ships, railway carriages, but it is by accident and not by choice.

The mosquito engorges herself with blood in one minute.

She begins to secrete the liquor sanguinis almost at once, this being the refuse of digestion.

The peculiar buzzing noise so well known to tropical residents is due to the vibration of its proboscis, and has no relation to the ring movements (Darling).

Darling affirms that Anopheline females will suck blood at any time and do not wait for fecundation.

Ross says that the female sucks blood after fecundation, also that the male only lives for a few days.

Darling says that the male lives fifteen to nineteen days, e.g., as long as the female when suitable food is available, such as sliced banana, \&c.

Mosquitoes are not confined in their attacks for blond upon man, for they are known to attack invertebrates, e.g., insects, and also young fish.

Possibly a quarter of the total number of anopheles may succeed in biting human beings once. A third of these may live for a further ten days, of which only a quarter may succeed in biting again. 
That is, only one out of every forty-eight mosquitoes can ever have a chance of carrying malarial parasites (Ross).

The Mansoni titillans has now been found to be the most numerous of all mosquitoes in the Panama Canal Zone. It has habits which enable it to escape both in the larval and pupal stage all larvicidal efforts, as these stages are passed beneath the water. This is rendered possible by breathing tubes being adapted for piercing the rootlets of the Pistia stratiotes and thus obtaining their supply of oxygen. The roots are sometimes several feet long, and to these the larve are attached. Not all plants, however, contain them, other conditions being necessary.

The adult mosquito has long been known in the Canal Zone, but the larvæ and pupæe have not before been found.

\section{TRYPANOSOMIASIS.}

(1) African Trypanosomiasis.

(2) South American Trypanosomiasis.

(3) Notes on Mammalian Trypanosomiasis.

\section{AFRICAN TRYPANOSOMIASIS.}

\section{DEFINITION.}

An acute specific infection caused by :-

( I) Trypanosome gambiense, carried by Glossina palpalis.

(2) Trypanosome rhodesiense, carried by Glossina morsitans.

Other species of trypanosomes may cause the disease.

The morbid manifestations are: irregular chronic fever, fleeting skin eruptions, local œdena, adenitis, physical and mental lethargy, mania, eye lesions, tenderness of muscles and bones.

\section{HISTORY.}

The "surra" of India has been ascribed to the bites of certain blood-sucking flies by the natives from time immemorial.

1724. John Atkins in "The Navy Surgeon" described the sleeping distemper common among the negroes of the Guinea Coast, seen by him in $\mathrm{I}_{72 \mathrm{I}}$, but not published until I 724 .

1803. Winterbottom described it as he saw it on the West Coast near Sierra Leone. Slave dealers would not buy slaves with enlarged cervical glands.

I 846 . Trypanosomes first found in fishes.

1879. Lewis found the rat trypanosome ( $T$. lewisi) in Bombay.

I880. Evans found the T. evansi in horses of India at Madras. 
1849. Clarke saw trypanosomiasis on the Gold Coatst.

r869. Guerin found it in Martinique in the negro slaves imported from Africa.

I89o. Nepveu found the trypanosome in the blood of man in Algeria while searching for malaria. This was the first time it had been found in man, and it was accidental (Manson). Its significance was not then known.

i 89 . The first case was brought to London under Sir Stephen Mackienzie.

I895. Bruce showed that "nagana" was due to T. brucei.

I900. Two human cases were brought to London under Sir Patrick Manson. The morbid anatomy of these cases was studied in detail by Dr. Mott.

I90I. Ford and Dutton found $T$. gambiense in the blood of patients on the Gambia.

1902 and 3. Castellani found trypanosomes in the cerebrospinal fluid of patients in Uganda.

1903. Sir David Bruce and Nabarro showed that Trypanosomiasis was spread by Glossina palpalis, an hypothesis already formed by Sambon and Brompt on epidemiological grounds.

1905. Thomas introduced "atoxyl" for treatment.

I907. A bureau was founded in London for the study of the disease. Ehrlich found that trypanosomes may becone atorylresistant. Plimmer and Thomas introduced tartar emetic associated with atoxyl for treatment.

r9ı. Stephens and Fantham created a new species, e.g., T. rhodesiense.

1912. Kinghorn and York showed that G. morsitans was a transmitting agent for $T$. rhodesiense. Livingstone (1841-187.3) gave arsenic to horses for nagana.

Manson had used liquor arsenicalis.

\section{DISTRIBUTION.}

It was first noticed on the West Coast of Sierra Leone. It was imported from this endemic centre to the West Indies from time to time, where it soon died out.

In ${ }^{8} 82$ it existed from Senegal to Loando and the islands of the Gulf of Guinea. It was much wider spread at this time, but it was not known to civilization.

In I 898 it was kinown on the Upper Niger.

The disease was probably spread in Africa as follows:-

In I 886 Stanley took some Congolese natives across Africa to the Victoria Nile. Some of these natives settled to the west of Lake Albert 
Nyanza and in Uganda. There is little doubt that men of Stanley"s expedition and Emin Pasha's followers carried the disease with them across Africa into Busoga and Uganda. The former place was linown to be infected in 1896, and the latter extensively infected by 1900. The western shores of Victoria Nyanza were found infected in 190i; the eastern shores and German East.Africa in rgor; Dutton and Todd found it widely disseminated in the Congo State 1904-1905, spreading along trade routes. These writers stated that the disease would spread into Eastern Rhodesia, which has since come true. It has spread upwards from Uganda to Wadelai on the Victoria Nile since 1904, and had invaded the Lado before 1908. It was recognized in the Sudan, Bahr-el-Ghazal province, in 1909 .

Its present boundaries (1919) are:-

On the IVest Coast from St. I.ouis in Senegal to Mossamedes in Angola, up to Timbuctoo on the Niger, throughout the Congo into Uganda, Rhodesia, Southern Nyasaland and Portuguese East Ifrica.

From L'ganda and Busera southwards to German Fast Africa and Lake Tanganyika, and northwards to the Bahr-el-Ghazal province.

It may spread to Arabia as tsetse flies exist there.

Of course it may also spread to co-existing lands wherever tsetse flies prevail.

\section{ETIOLOGY.}

The known causative organisms are T. gambiense, Igo2, and T. rhodesiense, 19ı0. The Uganda strains may differ from the others, an hypothesis suggested by the difference of its virulence.

The trypanosomes of animal trypanosomiasis may cause it. Professor Lanfranchi, who has never left Europe, was accidentally infected with a supposed laboratory strain of $\mathrm{T}$. brucei, causing irregular febrile attacks, general debility, and large patches of cutaneous adema over a period of two years.

T. gambiense probably lives in antelopes chiefly.

T. rhodesiense in hartebeest, waterhogs and domestic pigs.

These animals and man form the reservoir from which man is infected.

It is not known if Glossina infect their eggs.

Trypanosomes are known to develop more rapidly at high temperatures.

The trypanosomes are injected at the same time as the Glossina palpalis and morsitans suck blood.

Koch has suggested that it may be also transmitted by sexual intercourse.

Some authorities blame mosquitoes also. 
Minchin considers that encysted forms from fly droppings may be ingested and cause infection.

It is fairly conclusive that Tabanides convey it mechanically from beast to beast in herds of cattle.

Race does not affect infection.

Occupation predisposes in so far as there may be exposure to the flies near their breeding places or cover areas. Ilence those working along the shores of rivers and lakes of infected regions are exposed to the bites of infected Glossina, usually palpalis, while porters marching through the bush are exposed to the bites of G. morsitans.

\section{THE PARASITE.}

As seen in fresh blood the trypanosome is an active wriggling organism with a laterally compressed spindle-shaped body, provided with a delicate undulating membrane. Fringing the dorsal edge of the trypanosome and terminating in a free whip-like filament is the flagellum.

Stained specimens show a nucleus, somewhat central, and a minute deeply staining chromatic mass, the blepharoplast, which is nearer to one pole, e.g., the thicker or aflagellum or anterior end of the organism. Near to the blepharoplast is a small nonstaining area, viz., the vacuole. The thickened free border of the undulating membrane springs from the blepharoplast at one end, and is continued as the
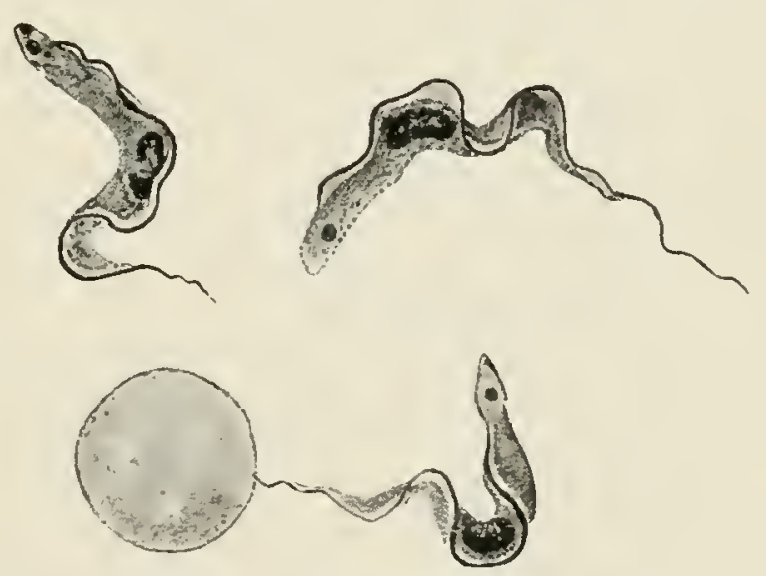

Trypanosoma gambiense. $\times$ I,7oo.

(After Dutton.) flagellum at the other.

In some places the cytoplasm is homogeneous, while in others it is faintly granular when stained.

There is great diversion in the dimensions of the parasite in body, nucleus and flagellum.

Multiplication is by longitudinal dirision, commencing at the blepharoplast, which elongates and then divides. This is followed by the duplication of the thickened margin of the undulating membrane and longitudinal division of the whole body. The flagellum does not divide.

The complete separation of the wo halves along the posterior border does not take place until the new flagellum has been formed. When separation takes place it is postero-anteriorly.

Sexual differences have not been decided upon. 


\section{HABITAT.}

Parasites occur in the blood, lymphatic glands, cerebrospinal fluid and probably in the fluid of the serous cavities, which facts point to the lymphatic system as being the most important habitat.

It may be very difficult to find the parasites in the peripheral blood. When present they may be more readily found during a febrile attack.

T. gambiense has not yet been cultivated on artificial media, but it can be readily communicated to monkeys, dogs, rats, guinea-pigs and other animals.

The rat trypanosome, $T$. lewisi, has been cultivated through many generations.

During adverse conditions, such as increasing scarcity of nutriment, lowering of temperature, addition of chemical solutions and the influx of sera from non-susceptible animals, the trypanosomes tend to agglutinate, e.g., to congregate in bunches, with their extremities (anterior) directed towards the centre.

One bunch may contain 100 individuals. The parasites may disperse again, apparently unaltered and uninjured.

In cultures, trypanosomes may assume a spherical form and lose their flagellum.

The time required for the trypanosome to remain in the fly before transmitting the infection is probably $20-47$ days (Manson).

\section{CLASSIFICATION.}

(After Bruce, Iectures before Royal College of Physicians, 1915.)

Classification is based upon :-

(1) Morphology'

(2) Pathogenic action of animals. \&

(3) Mode of development in tsetse flies.

Cultivation is of no assistance at present.

Inoculation experiments and serum diagnosis are not helpful for diagnosis on the field.

(I) Morphology.

In fresh films one ascertains the general appearance of the trypanosomes and the kind of movement. Some vibrate about one spot, others hurl themselves about the field with great power and velocity.

Stained specimens give more exact details as to length, breadth, cell contents, nucleus, micronucleus, undulating membranes and flagellum.

(2) Pathogenic action on animals.

The passage of a trypanosome through a series of animals of the same species exalts their virulence towards that animal, e.g., the wild strain of the nagana trypanosome which kills a rat in 20-30 days, but 
if passed through rats for many generations the rat will be killed by them in two days.

The passage of trypanosomes through different species will lower their virulence, e.g., some wild trypanosomes will kill monkeys in a few days, but if passed first through a goat, an attempt to infect the monkey fails, hence its passage through the goat lowers the virulence of trypanosomes for monkeys.

Care is necessary in adopting this as a means of differentiation.

It has been observed that-

$T$. brucei is more virulent for laboratory animals than $T$. gambiense. Some are deadly to horses and cattle, and are harmless to dogs, monkeys and rabbits. Others show a preference for domestic pigs.

(3) Mode of Development in Tsetse Flies.

All the trypanosomes pathogenic to man and domestic animals in Africa, with the exception of two northern species, pass through a specific cycle of development in the tsetse flies. The mode of development in the tsetse is different for different species, and this feature may be used for differentiation. North African species have not been found capable of developing in tsetse. It may be that from disuse they have lost the faculty, if ever they had it.

\section{CENTRAL AFRICAN PATHOGENIC TRYPANOSOMES.}

Group $(A)$ : Trypanosome Brucei Group.

Synonyms: T. rhodesiense, T. ugandie. The question of identity of $\mathrm{T}$. rhodesiense and $\mathrm{T}$. brucei is still sub judice.

The differentiation of the groups is according to the development of the trypanosome in the tsetse. The microscope will decide to which group the trypanosomes will belong.

General features of all in this group.

All are more or less polymorphic.

They vary much in size and shape.

The cytoplasm contains numerous dark-staining granules.

The kinetonucleus is small and situated at some distance from the posterior extremity.

The undulating membrane is well developed and has bold folds.

They affect many species of animals, including man, cattle, horses. dogs, \&c.

T. brucei and T. gambiense develop in the tsetse in the same way, at first in the intestine, then in the salivary glands. There they completely develop into infective forms. No other group invades the salivary glands.

(I) Trypanosoma brucei. 
It is the most widely distributed pathogenic trypanosome of Central ifricá.

It causes nagana amongst domestic animals.

In Nyasaland it causes trypanosomiasis in man.

It is rapidly fatal to man and domestic animals.

It is carried by the Glossina morsitans and pallidipes.

(2) T. gambiense.

Synonym: 'T. nigeriense.

It cáluses trypanosomiasis in man.

Cattle and antelopes form the reservoir for the virus without causing symptoms in them.

This trypanosome is less fatal to man than $\mathrm{T}$. rhodesiense. Some recover.

It is carried by the $\mathrm{G}$. palpalis.

(3) T. evansi.

Synonym : T. soudanense.

It causes surra in horses and mules, camels and cattle in India, Philippines, Mauritius, North Africa, \&c.

It is transmitted by the bites of fleas, flies and (?) by eating infected meat.

(4) T. equiperdum.

It causes dourine or mal de coit in horses in Europe, India, North America and North Africa.

It is spread by coitus.

It is always fatal from two to eighteen months.

Group (B): Trypanosoma Pecorum Group.

General features of all in this group:-

All are small and monomorphic.

The cytoplasm is non-granular.

The kinetonucleus is prominent, sub-terminal, and often seems to project beyond the margin.

The undulating membrane is fairly well developed.

Development begins in the intestine, and later passes to the proboscis and salivary duct or hypopharynx, where they complete their development and become infective.

(I) T. pecorum.

Synonyms: ' $\mathrm{l}$. confusum, T. nanum.

It causes an important trypanosomiasis in cattle.

It is carried by 'T. morsitans, in which its development takes place.

It is also carried mechanically by Tabanidre (?).

(2) $T$. simice.

Synonym : T. ignotum.

It rapidly kills domestic pigs. 
Its flagellum is uncertain.

Its reservoir is the warthog.

Group $(C)$ : Trypanosoma Vivax Group.

General features of all in this group:-

They are monomorphic.

Their movements are extremely rapid.

Their posterior extremity is enlarged.

The cytoplasm is clear and hyaline.

The kinetonucleus is large and terminal.

The undulating membrane is but little developed and simple.

The trypanosome only affects horses, cattle, goats and sheep.

It does not affect dogs, rabbits and pigs.

Development begins in the labial cavity of the proboscis, later in the salivary duct or hypopharynx. No part takes place in the intestinal tract.
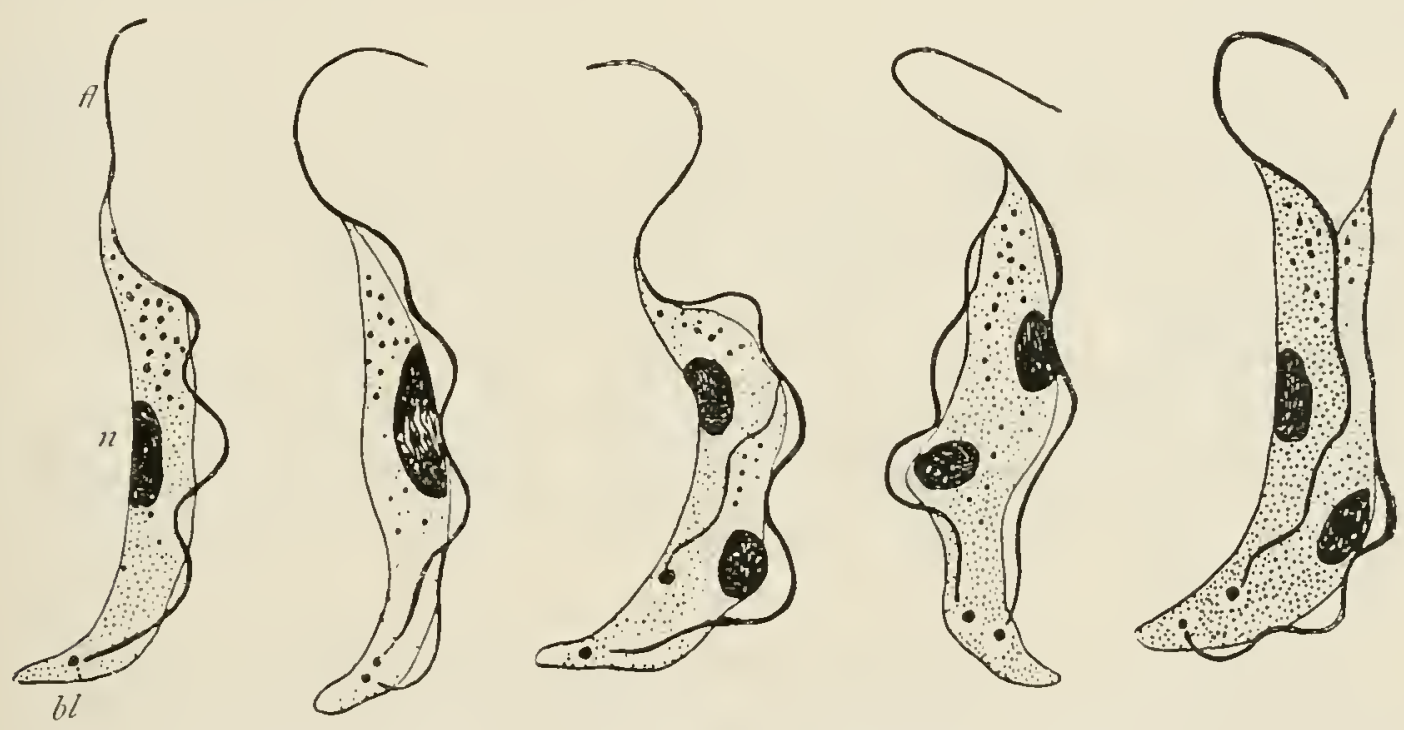

Trypanosoma bruce i in division. $n$, nucleus; $b l$, blepharoplast ; $f$, flagellum. $\times 2,000$. (After Laveran and Mesnil.)

(I) T. vivax.

Synonym : T. cazalboui.

It is very active.

It is widely distributed.

It attaclis horses, cattle, sheep and goats only.

It is fatal to cattle in Uganda.

(2) T. capra.

It affects cattle, sheep and goats only.

It is more hearily built than the above.

It is found in Nyasaland and L. Tanganyika only.

(3) T. uniforme.

It is as $T$. vivax, but smaller.

It is only found in Uganda. 
These trypanosomes will now be dealt with a litte more in detail.

T. brucei (rhodesiense).

History.

It causes nagana in domestic animals.

It causes Trypanosomiasis in man of a virulent type.

It was the first pathogenic trypanosome discovered in Central or South Africa. It was found by Sir David Bruce in North Zululand, 1895.

Stephens, in roog, was examining a supposed gambiense at Liverpool when he noticed different characters to those expected, hence he called it $T$. rhodesiense. The parasite was obtained from a patient who had been resident in Northern Rhodesia and was suffering from Trypanosomiasis. In this way the Congo and the Rhodesian types of the disease were differentiated for the first time.

It has been decided for the present that $\mathrm{T}$. brucei and $\mathrm{T}$. rhodesiense are identical.

\section{Distribution.}

T. brucei and nagana are widely distributed from the Sudan on the north to Zululand on the south, from Gambia on the west to Zanzibar on the east. It may be that the $T$. togolense of Togoland and the T. uganda of Uganda are the same species.

Morphology.

In the $T$. brucei there is a greater diversity of shape than is found among species of other groups; the short, stumpy forms have no free flagellum, while the long forms are slender and have a well-marked free flagellum.

They are actively motile, but do not move far from one place.

The protoplasm of many trypanosomes show granules, especially at the anterior end.

The nucleus is oval in the slender and round in the stumpy forms. It is frequently placed far back in the body of the organism, more so in the short forms.

The kinetonucleus is small, round, and about $1 \cdot 4-2 \mu$ from the posterior extremity.

The flagellum averages $5^{\cdot S} \mu$, but is absent in stumpy forms.

Animal Susceptibility.

It attacks man, horses, mules, donkeys, oxen, goats, sheep, monkeys and dogss. Birds, crocodiles, lizards and frogs are not affected by it.

One trypanosome may begin the infection as well as a thousand.

Infection can take place very easi!y through the skin; the slightest puncture or scratch suffices. Infection probably takes place also through the mucous membrane. I'ndoubtedly infection is most frequently by the isetse bite. 
The disease runs a fairly rapid course in man, killing him in three to four months. Horses, donkeys and mules die in about thirty-eight days.

In the ox it is more chronic and some recover.

It is fatal to horses ats in man. No case infected has been known to recover, and remain free from the disease for a year.

Goats, sheep, monkeys, dogs, rabbits, guinea-pigs and rats nearly all die. Amongst these animals, three recovered out of 318 infected.

It is highly probable that the Trypanosome of man in Nyasaland and of nagana are not separate species.

\section{Carriers.}

Glossina morsitans and pallidipes are the carriers of nagana.

About one per 500 of tsetses are infected with the trypanosome.

The infected tsetses are equally numerous all the year round.

If man is bitten by one tsetse in the nagana area it is 500 to $I$ against his taking the disease.

For its development in the tsetse see the development of $\mathrm{T}$. gambiense, which is identical with $\mathrm{T}$. brucei in this respect.

Reservoir.

The big game of the fly country are very heavily infected, more than 32 per cent. These form the reservoir.

The other trypanosome species pathogenic to domestic animals were found as follows: $T$. pecorum, $I 4^{\circ}+$ per cent.; $T$. simia, $I^{\circ} 7$ per cent.; T. caprae, $11^{\circ} \mathrm{I}$ per cent. of all the game examined. Consequently all such game should be exterminated (Bruce) in those areas.

T. gambiense.

History.

It was first seen in the blood of man in 1901 .

It was found by Dr. Ford and described by Dr. Dutton, but it was not then associated with Trypanosomiasis.

The next year Dutton and Todd were sent out to investigate the trypanosome at Bathurst. They reported that in their opinion the blacks were immune to the disease, but that they acted as reservoirs for the more susceptible whites.

The real danger was ascertained by the Royal Society Commission in Uganda, 1903 .

\section{Distribution.}

North.-From St. Louis, at the mouth of the River Senegal, to the Bahr-el-Ghazal district in the Egyptian Soudan.

East.-Down to the eastern shore of Victoria Nyanza.

South.-To the southern end of Lake Tanganyika, the River Luapula in Northern Rhodesia, and Donguela in Portuguese WVest Africa. 


\section{Morphology.}

It much resembles the nagana parasite.

There is great variation between the long and short forms, but among the short furms there is an absence of the blunt-ended ones.

The protoplasm contains many chromatin granules.

There are no posterior nuclear forms.

The kineto nucleus is small, round, from $1.8-I^{\prime} \cdot 1 \mu$ from the posterior extremity.

The undulating membrane is well developed and has bold folds.

The flagellum is long and free, except in the short forms, where it is absent.

The length of the organism is about the same as the T. brucei.

No difference can be ascertained by the microscope in blood preparations between $T$. gambiense and $T$. brucei.
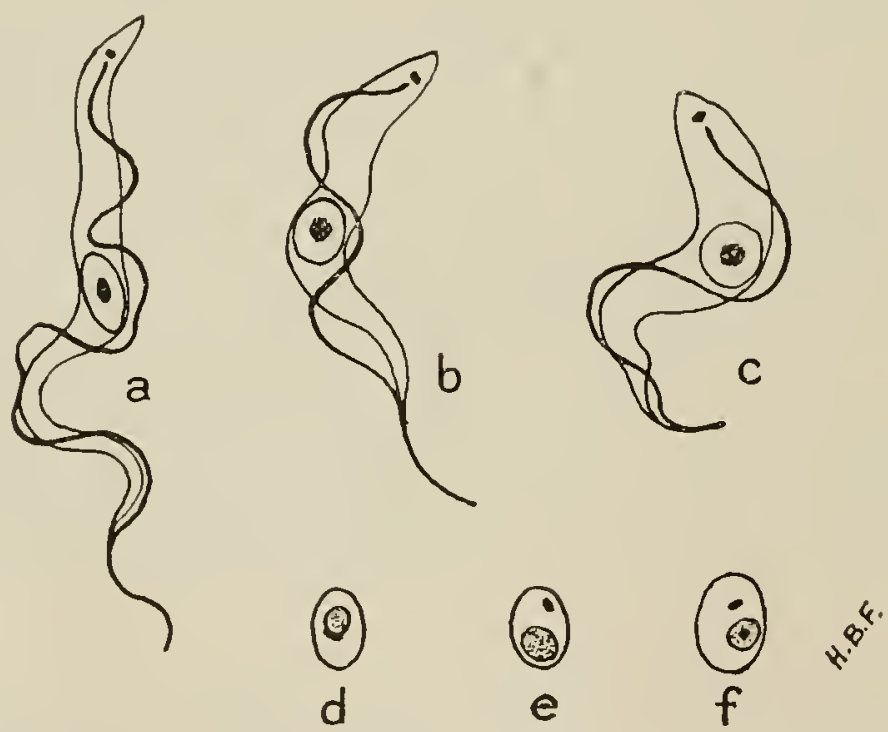

Trypanosoma gambiense. Development in vertebrate host. $a$, long, slender, $b$, intermediate and $c$, short, stumpy forms, found in the blood: $d, e, f$, non-flagellate, latent forms from internal organs. $\times 2,000$. (Original. From preparations by Fantham.)

\section{Animal Susceptibility.}

It is difficult to infect experimental animals from infected human blood, and in this it differs much from T. brucei.

The rat is the least refractory, but its virulence can be increased by passing it through several of the same species.

The virulence of both these trypanosomes is illustrated by the table below :-

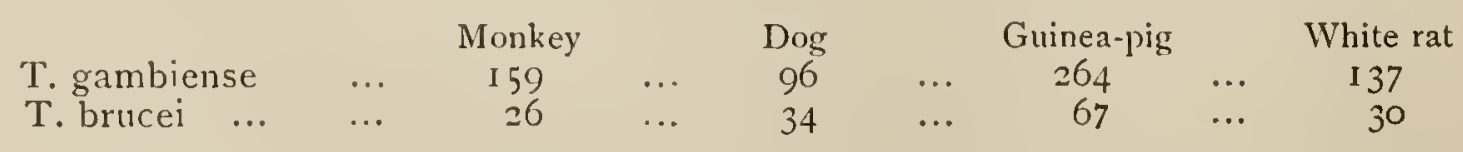

hence $\mathrm{T}$. gambiense is much more chronic than $\mathrm{T}$. brucei, and this test is best for the differentiation of the species. 


\section{Carriers.}

The Glossina palpalis. It inhabits wonded shores of rivers and lakes.

Although the shores of Lake Victoria Nyanza, the banks of the Nile and other rivers swarmed with these tsetses before i $\$ g \$$, they only became infected with trypanosomes about this time. Consequently one concludes that the infection was brought by Emir Pasha's men from the Belgian Congo.

Thirty per cent. of the natives working for the Government (one month yearly, at Entebbe in Uganda in lieu of hut tax) were infected with these trypanosomes in 1903 . The hut-ax labourers were removed from the lake shores, and one year later the flies infected with this

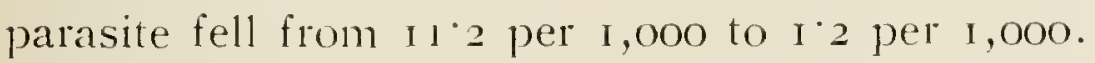

In I9I2, as the lake shores became more deserted, it had fallen to $0^{\circ}$ It per $\mathrm{I}, 000$. Hence man in the vicinity means more flies infected.

A fly may remain infective for sereral months.

Man is by no means the only source of the virus.

Tsetses can convey the infection for some fifty days after the fly has fed on an infected animal (Kleine).

An infected fly may and does infect susceptible creatures bitten on the first bite.

Cycle of Development.

The cycle of development of the $T$. gambiense in the $G$. palpalis is as follows:-

Laboratory flies when fed on infected animals become infective in an average of thirty-six days, extremes twenty-seven to fifty-three days.

Of those tsetses allowed to feed on infective animals, only o 50 per cent become infective.

When in test-tubes the trypanosomes seem to die off, but after twenty days a resistant strain appears which soon multiplies by myriads.

Many flies become infected, but few become infective, e.g., trypanosomes partially develop in many, but go on to completion in but few tsetses. It was found that 2 per cent. became infected, but only 0.5 per cent. infective.

For the first three to four days after the Glossince have fed on infected blood, trypanosomes are found in all, these being the trypanosomes originally ingested with the blood. In six to seven days, when digestion is completed, most have disappeared from the tsetse, except in from 2 per cent. to 8 per cent. of those infected. In the remainder, say about 5 per cent., the trypanosomes develop, increase, and fill the whole of the gut, fore, mid and hind gut, with swarms of multiplication forms. 
This infection may continue for the rest of the life of the fly.

One case retained its infection for ninety-six days, but some lose their infectivity and become harmless.

Trypanosomes are never found in the proboscis except immediately after a meal.

The greatest development is found in the fore, mid and hind gut, first degenerate blood forms, then comes a dominant type, a long, broad form with a narrow, simple, undulating membrane and a very short flagellum, if one at all. This appears to rise from a pinkcoloured body near the kineto nucleus, a condition or phase never seen in ordinary blood trypanosomes. There are masses of these at all times in all parts of the gut. When the blood supply runs low these degenerate and disappear. When there is fresh blood supply they multiply and increase at an astounding rate, hence there are at the same time trypanosomes in all phases of their development which beggars description.

Trypanosomes appear in the salivary glands on the twenty-fifth day and remain present there. They arrive there by way of the proboscis and salivary duct or hypopharynx. Trypanosomes have never been found in the body cavity. As soon as the trypanosomes reach the salivary gland they revert to the original blood forms and become infective.

Conclusions.

Trypanosomes taken into the alimentary canal of tșetse flies retain their shape and infectivity for some eighteen hours.

They then degenerate and lose their power of infectivity.

They disappear in the majority of cases within five to six days.

In a small percentage of flies, the female and the male, the trypanosomes retain their position, multiply, swarm in the gut, but do not resemble the original trypanosomes.

After some twenty days the trypanosomes reach the salivary crland, resume their original blood form, and regain their infectivity.

\section{Reservoir.}

When men were numerous about the shores of Lake Victoria Nyanza, 20 per cent. to 50 per cent. of the natives were infected. (The epidemic there has since died out.) Months after the natives had been removed the flies were still infective. Animals were then suspected and examined. Cattle were found heavily infected, but showed no sign of the disease, but healthy susceptible animals could be infected from them by tsetses. The same can be said of antelopes. Animals not showing the trypanosomes microscopically would infect susceptible animals. Five vears after the removal of the population from about certain parts of the shores of Lake Nyanza antelopes were still carrying 
infective trypanosomes. Should this district become repopulated the epidemic undoubtedly would come back again.

Groups $(B)$ and $(C)$.

None of these attacks man, hence little will be said about them.

Group $(B)$.

T. pecorum.

Morphology.

It causes the most important trypanosome disease of domestic animals in Central Africa.

It is the smallest of all pathogenic trypanosomes, being $9-$ I $8 \mu$ long. It is short and stout.

The contents of the cell are homogeneous.

The nucleus is oval and centrally placed.

The kineto nucleus is small, round, situated towards the posterior extremity, and may appear to project beneath the edge of the organism.

The undulating membrane is simple. There is no free flage!'um.

Pathogenicity.

It is a disease of herds, horses, donkeys, oxen, goats, sheep and pigs. Some attacked recover.

It readily loses its virulence by passage through certain other animals. If a trypanosome, infective to the monkey or dog, \&c., passes through a goat, it loses its power of infectivity for the monkey and $\operatorname{dog}, \& c$.

$T$. nanum is really a strain of $T$. pecorum.

It is not so rapidly fatal to horses, donkeys and mules.

In Nyasaland two-thirds of the cattle were lost and seven-eighths of the goats.

Carriers.

The Glossina morsitans. The Tabanida.

$4^{\circ} 6$ per 1, ooo tsetses were infected by the $T$. pecorum.

Once a herd is infected, then ordinary cattle or buffalo flies-the Tabanida, may spread it. These flies come and go in swarms, during which visits they are real pests to cattle.

The cattle feed at the hottest time of the day and crowd for selfprotection, hence conditions are very favourable for mechanical transmission. When diseased members of a herd are removed, the disease seems to cease when the Tabanida and not the Glossina morsitans are present.

Between Igo8-1913 some 2,500 to 3,000 head of cattle died in the Barotse Reserve in Northern Rhodesia, though no Glossina morsitans was present.

The mortality begins in February and ceases in June each vear. 


\section{Development.}

At first this takes place in the gut of the tsetse, then in the labial cavity of the proboscis, later in the salivary duct, but, be it noted, not in the salivary gland. In the salivary duct it reverts to its original form and becomes infective.

The cycle requires nineteen to fifty-three days for the development of infectivity. The intestinal forms cannot be distinguished from other intestinal developmental forms of pathogenic trypanosomes.

Thousands of infective forms can be obtained by a biting fly attempting to bite one's finger through a watch glass, when the saliva will reveal them.

Reservoir.

$1+4$ per cent of animals examined were infected. These were:-

Eland, 60 per cent. ; Koodoo, 66 per cent.; Bush buck, zo per cent.; Buffalo, 22 per cent.

$T$. brucei were not found in any of the animals examined.

T. simice.

This is very virulent towards monkeys and clomestic pigs, killing them in a few days. It is harmless to oxen, antelopes, dogs and smaller experimental animals. It affects goats and sheep.

It is like $T$. pecorum, in that its virulence soon becomes modified.

The warthog is the only member of wild game yet found to harbour it. Io per cent. of the thirty examined were found to be infected.

The trypanosome is $14-24 \mu$ long.

The long undulating body frequently extends in a straight line.

The cytoplasm is clear.

It is very fatal to domestic pigs, killing them off in 5.3 days.

It kills monkeys in $10 \cdot 8$ days, goats and sheep in $46 \cdot 6$ days.

3.4 per $\mathrm{I}$, ooo flies were infective after experiments.

Its development in the tsetse is as that of $\mathrm{T}$. pecorum.

\section{Group $(C)$.}

The size only separates the species of this group.

T. virax.

It causes the most important cattle disease in U'ganda.

It is widely distributed in Central Africa. It has been reported from the Soudan, Senegal and Northern Rhodesia.

Its marked activity during life distinguishes it.

It only affects horses, cattle, goats and sheep. Other animals are refractory.

It has been found in the bush buck.

Tsetse flies were found infected by it.

T. uniforme.

As above, but smaller. 
Plate A.

Mott.

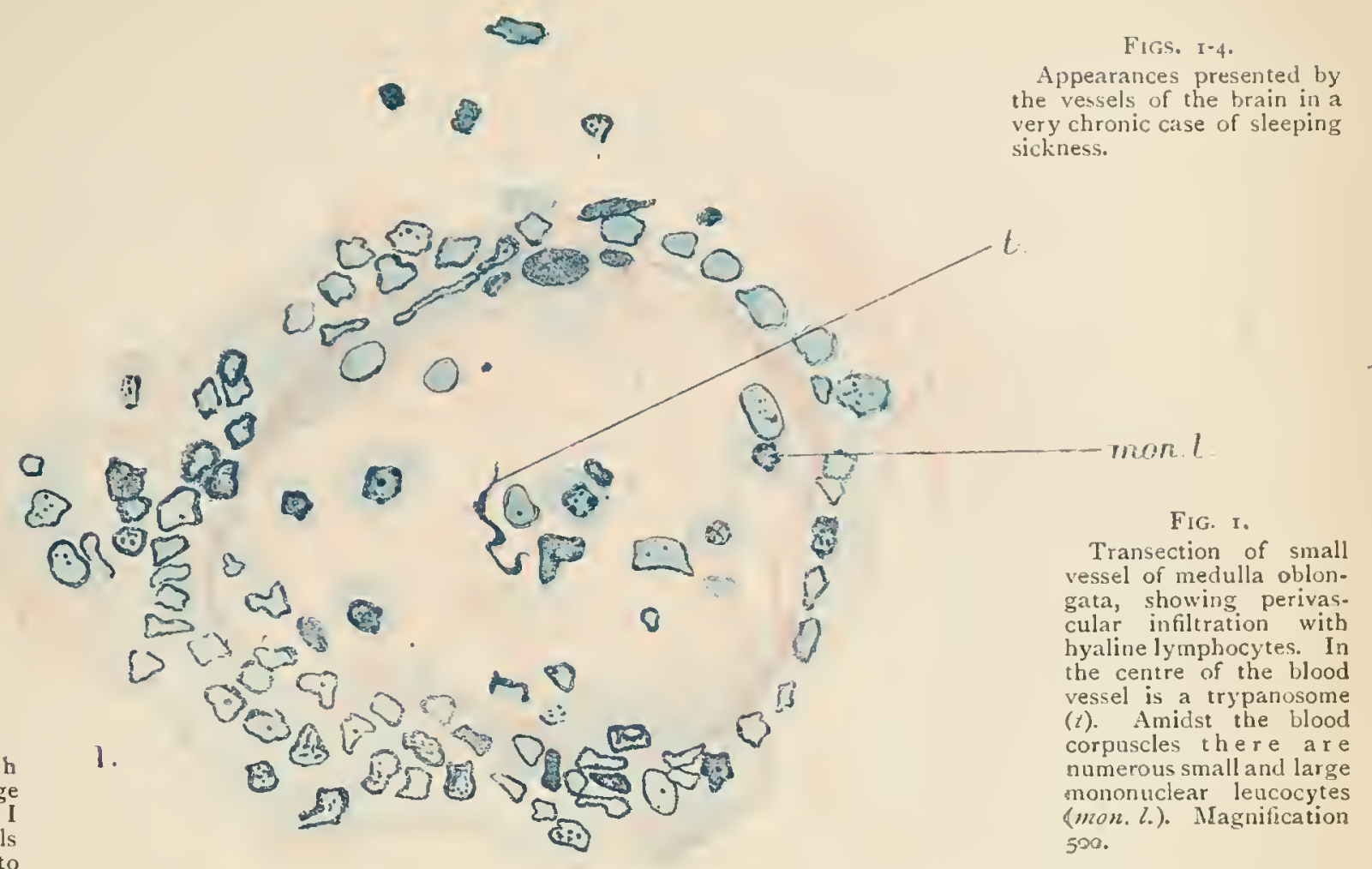

Small vessel, with plasma cells $(p)$ and large granule cells, which I have termed morular cells $(m)$. They correspond to Körnchen Zellen of Alzbeiner. Magnifica. tion 500.

FiG. 3 . Small vessel dividing ing nuclear proliferan ing of the endothelial cells in the neighbourhood are plasma cells $(\not p)$, lym. phucytes (l), and glia cells $(g)$. Magnification 500.

3.
कิ
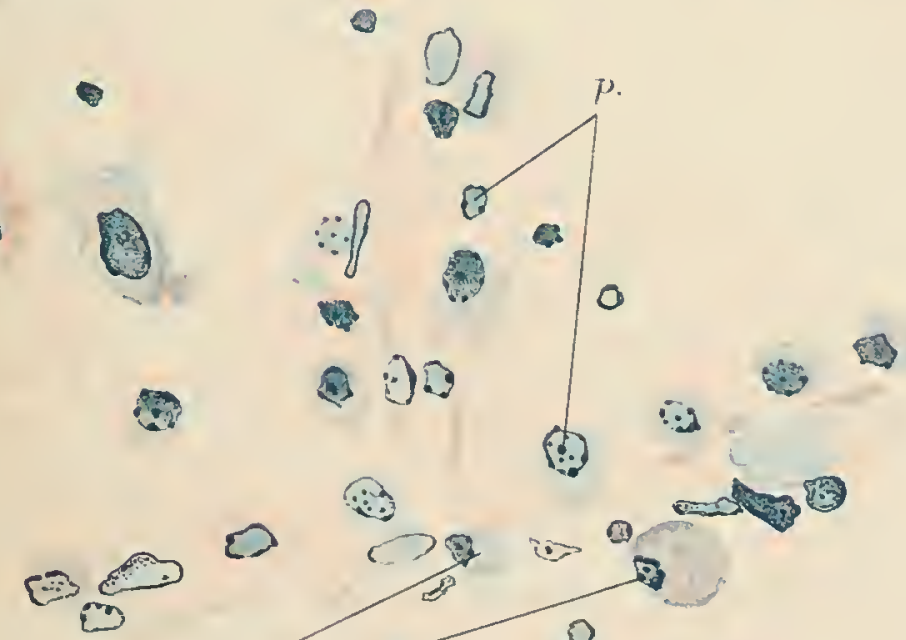

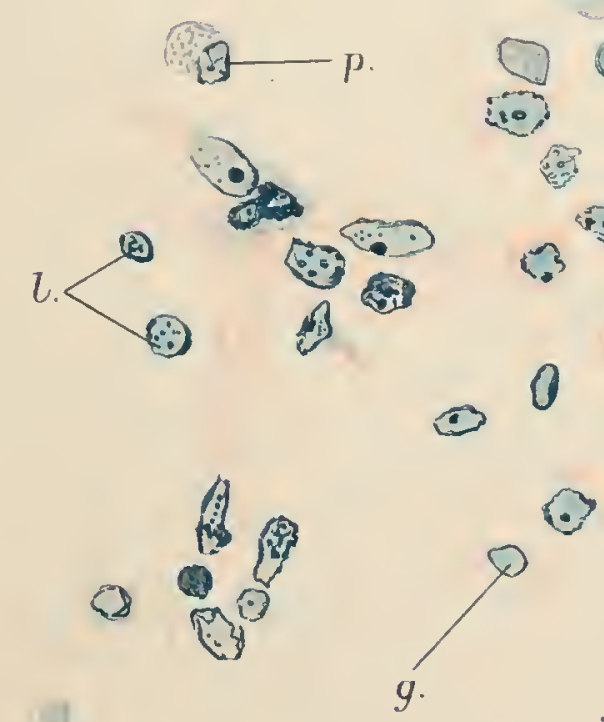

06

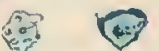

Appearances presented by very chronic case of sleeping ckness.

Transection of smal ata, showing perivas cular infiltration with ymphocytes. In centre of the blood (t). Amidst the blood corpuscles there are mononuclear leucocytes 500.

י



Mott.

FIG. I.

Three large glia cells (y). their branches ending in a network around and upon a small vessel; lymphocytes (l), and plasma cells $(\phi)$ are seen scatrered about. Magnification 500 .

FiG. 2.

Small vessel, showing endothelial nuclei proliferated, and three plasma cells. Magnification 500.

$g$

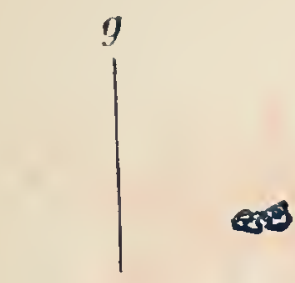

1.

Fic. 3 .
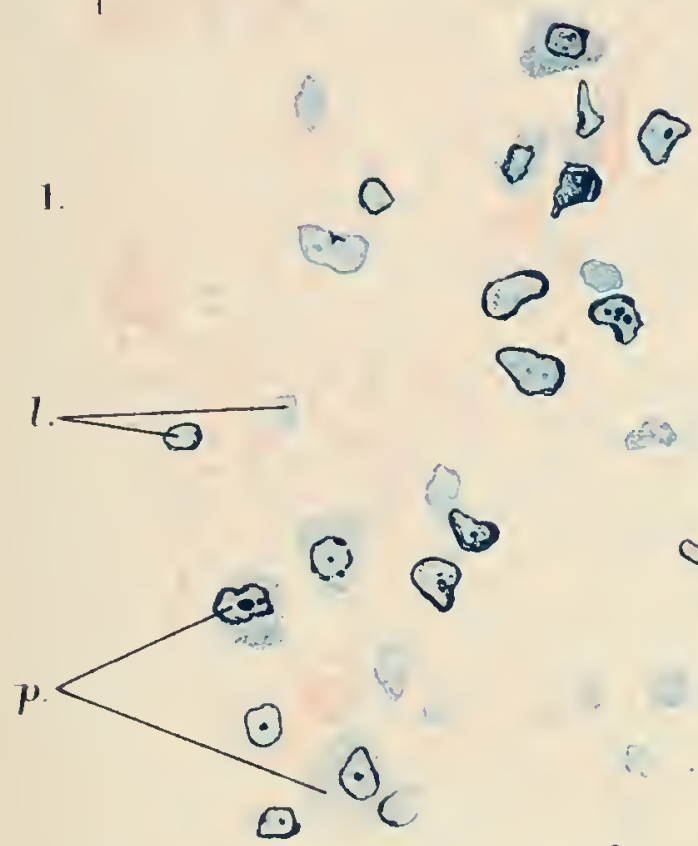

is
禹

0

Q<smiles>[CH]=C</smiles>

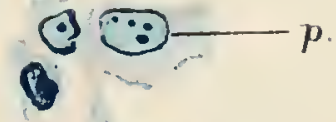

aro

-

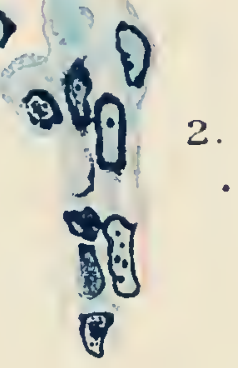

A transection of a vessel in a very chronic case of sleeping sickness, show. ing marked perivascular infiltration. Magnification 250.

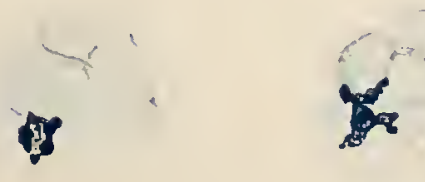

5.

FIG. 5 .
FIG. 4.

Active proliferating young glia cells found in great numbers in sleeping sickness t issues. The

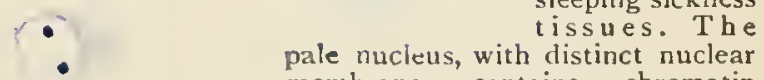
membrane, contains chromatin granules, with an arrangement in. dicating mitosis. Surrounding the nucleus is the pink-stained cytoplasm, with a tendency to form star. ike processes. Magnification 500.
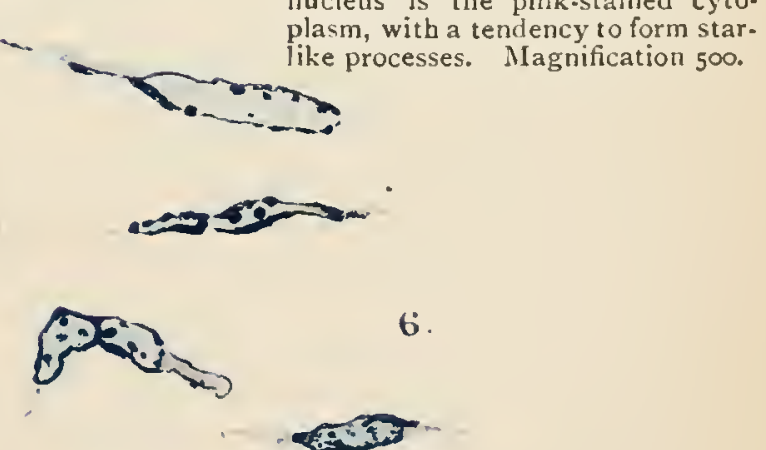

6.

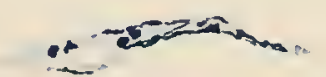

Two large morular cells from a very chronic case of sleeping sickness. Magnification 500.

FIG. 6.

Rod cells (Stïbchen Zellen) are rarely met with, although occasionally appearances like fig. 6 are seen. Magnification 500. 



\section{Plate C.}

FIG. I.

Transection of cervical nerve close to the spinal ganglion, showing an infection of the sheath of the nerve by diplo-streptococci. The adjacent lymphatic glauds showed points of suppuration. Magnifcation 200. (a) The micro-organisms, magnified 500. Case 69, L. L.

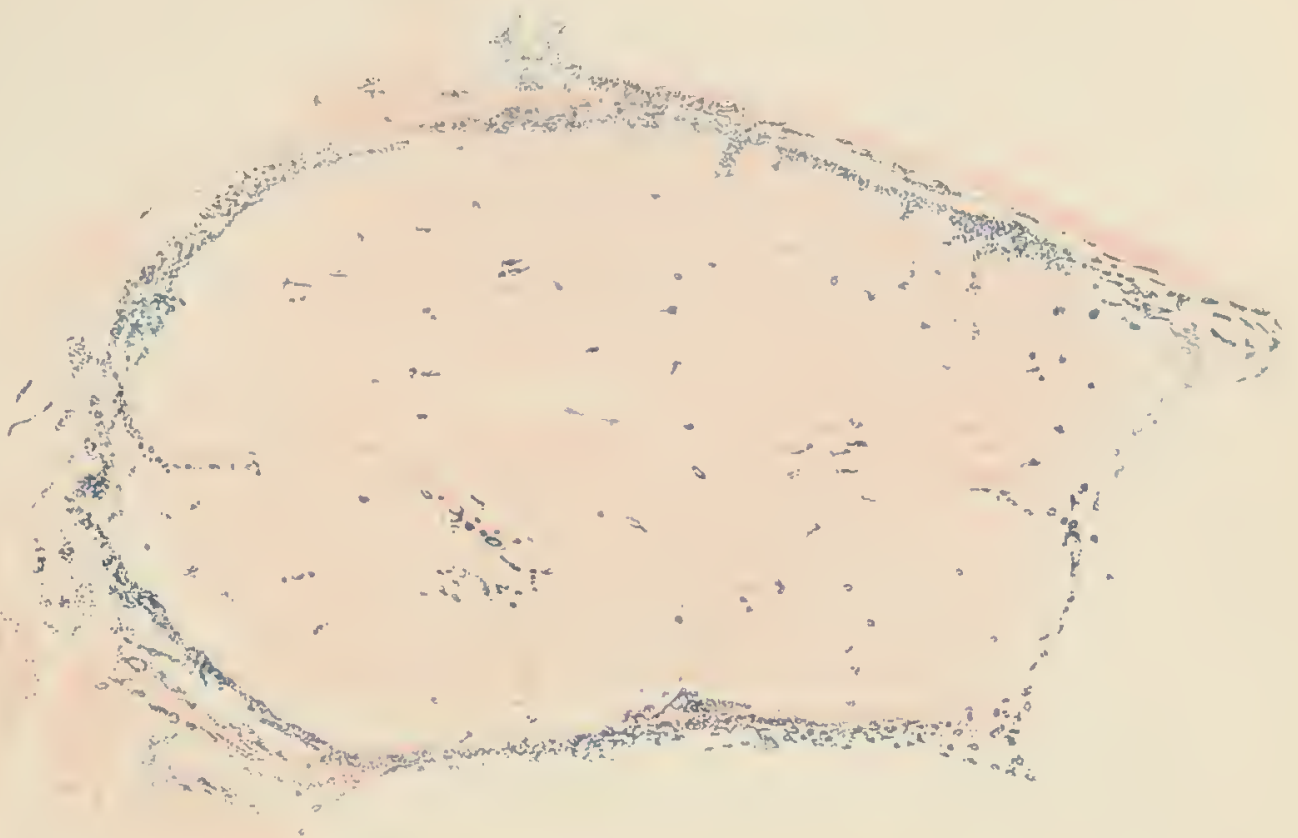

Fig. 2.
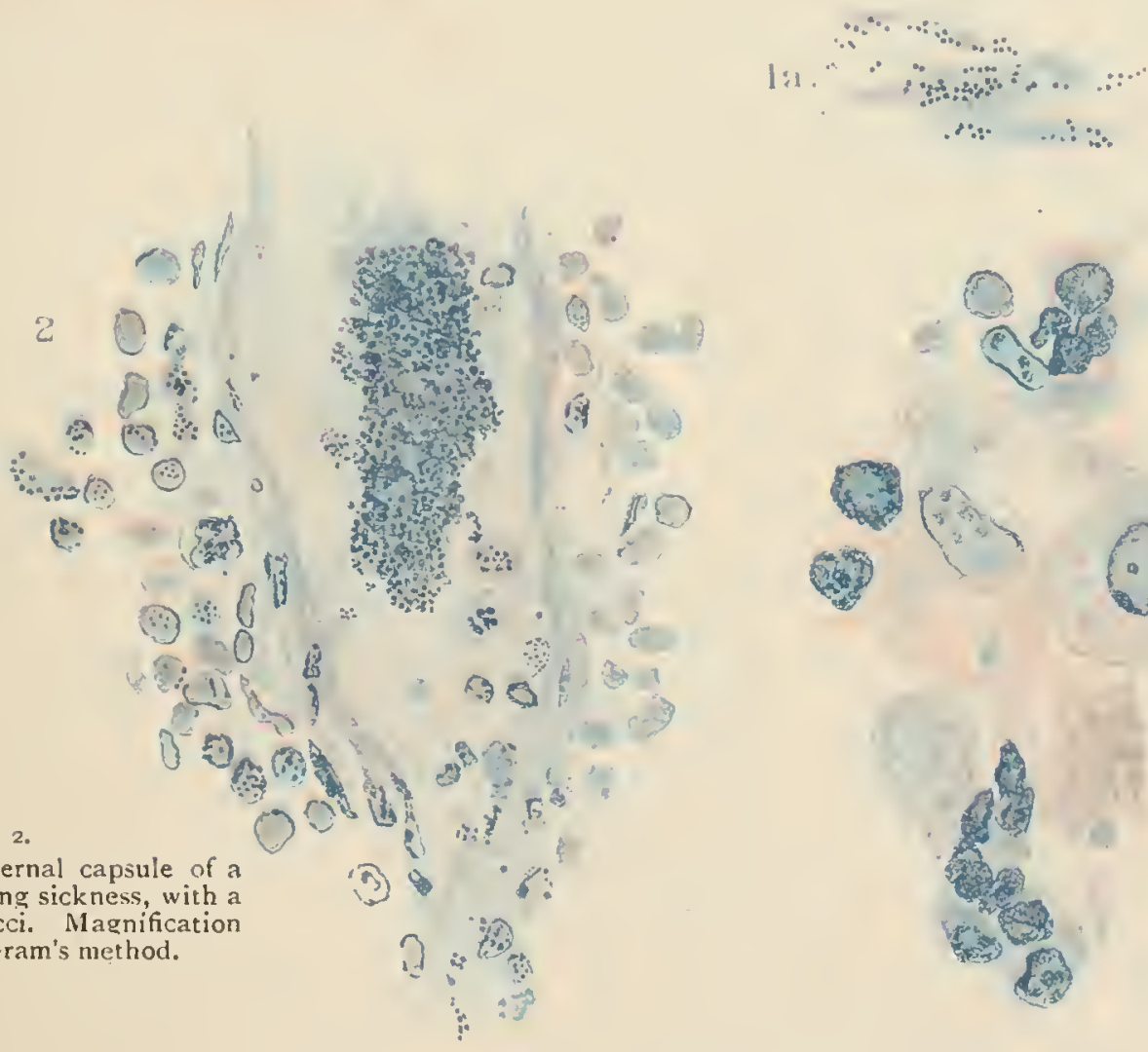

Vessel of the internal capsule of a case of acute sleeping sickness, with a large plug of cocci. Magnification 5oo. Stained by Gram's method.

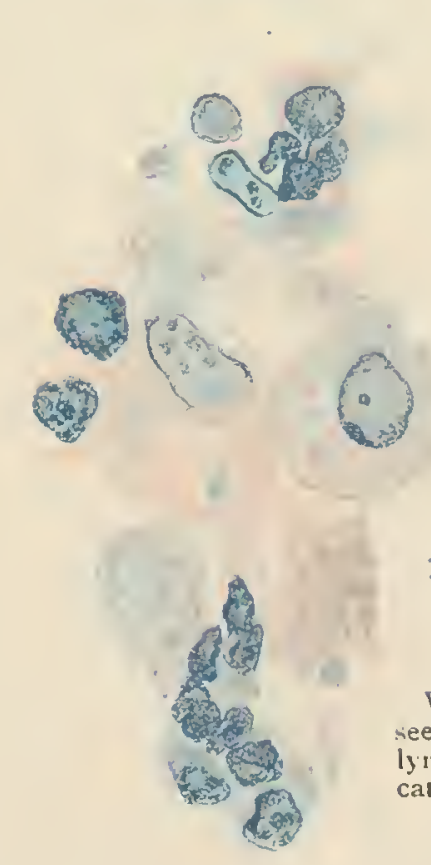

FIG. 3.

Various degenerated cells een in section of sterile yimphatic gland. Magniti cation $\mathrm{r}, 000$. Leishman stain.

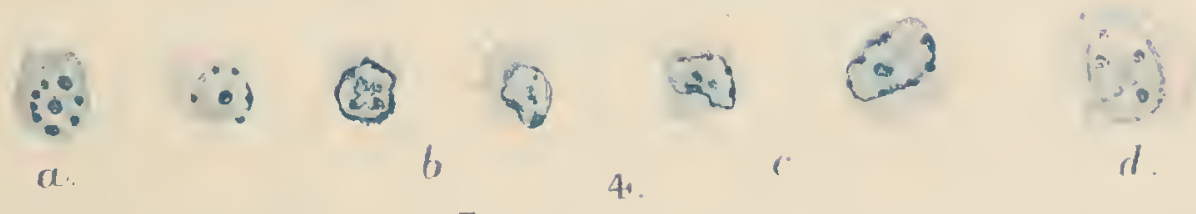

FIG. 4 .

Lymphocytes and their transition to plasma cells $\alpha, c ; d$, degenerate 1 p'asma cell seen in section of lymphatic gland. Magnification I,ooo. Leishman stain. 

FIG. I.

Thread - like bodies and granules de eply stained, seen in section of lym. phatic gland. probably altered and degenerated trypanosomes. trypanosomes.
Mlagnification s,000.
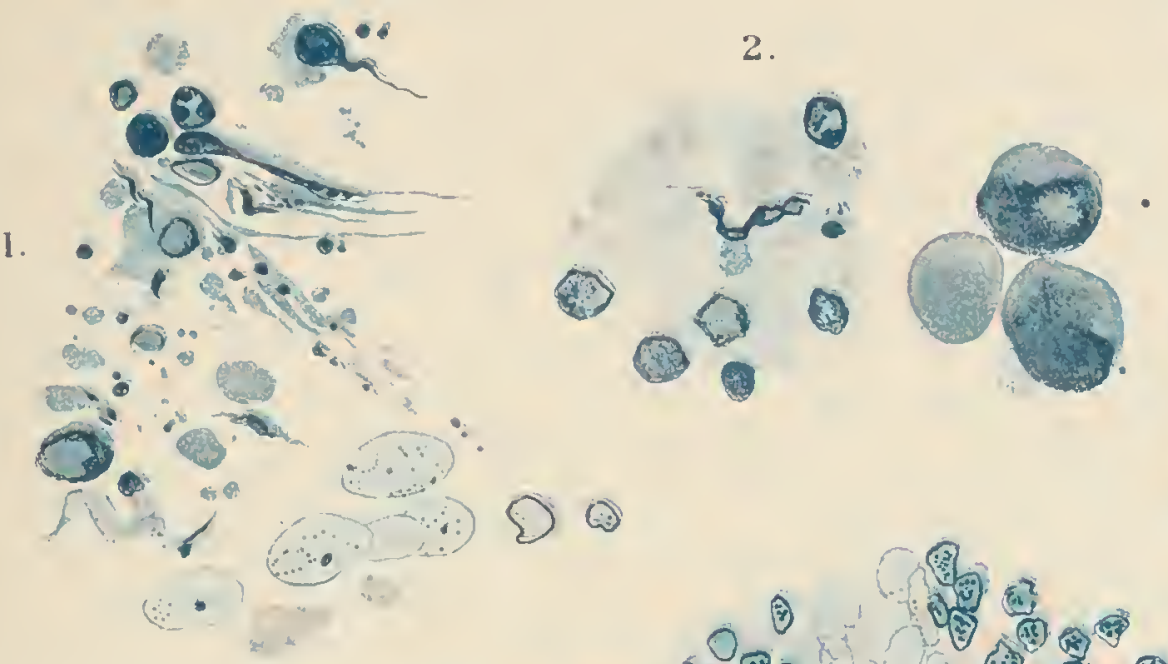

$\therefore \quad:$

Q

FiG. 2.

Trypanosome in a lymplatic gland section amidst disintegrated cell products. Figs. 3 and 4 (Plate IV.), and figs. $I$ and 2 (Plate V.), are drawings made from the same sections, $5 \mu$ in thickness, stained with Leishman's stain and prepared from an enlarged cervical gland removed during life from a case (Bara Risgallab) of trypanosome fever, before symptoms of sleeping sickness had occurred. Nagnification 1,000 .
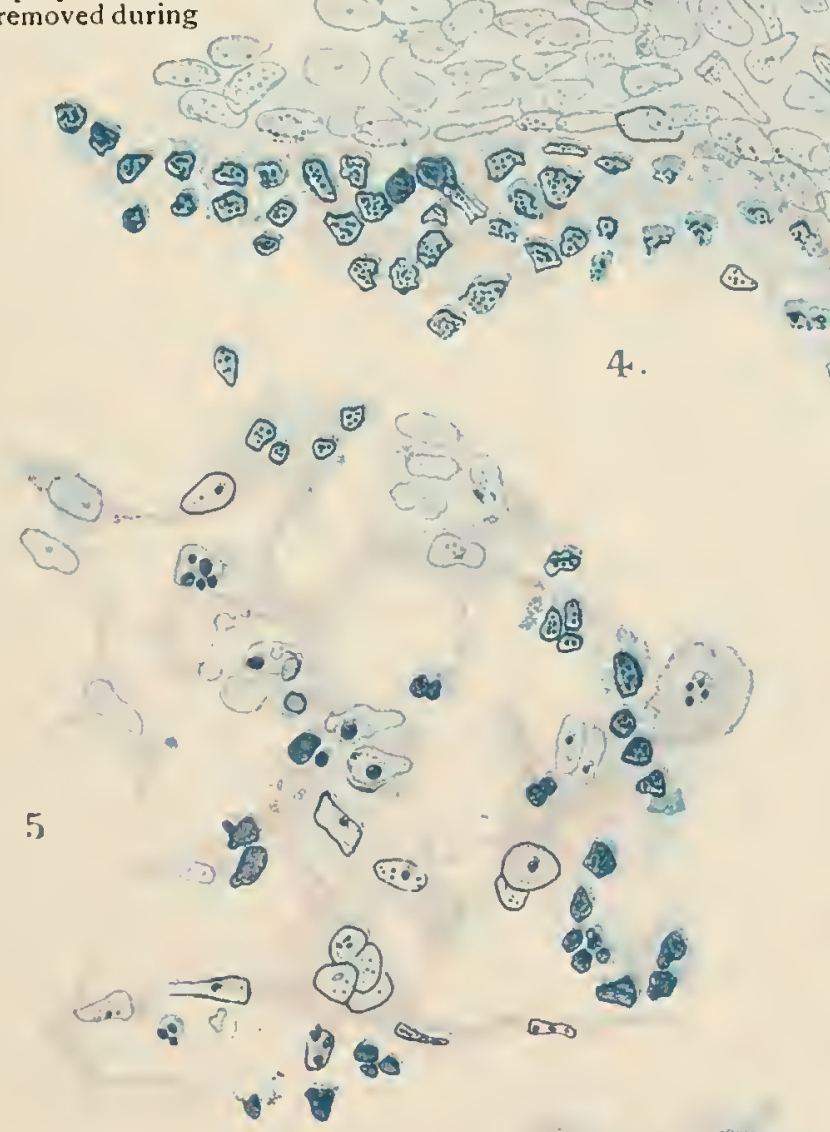

FIG. 5.

Proliferation of the connective tissue cells of the reticulum of a lymph sinus; marked proliferation of the nuclei of the endothelial cells seen. This chronic change closely accords with the change observed in the perivascular lymph spaces of the central nervous system. Magnification 500 .

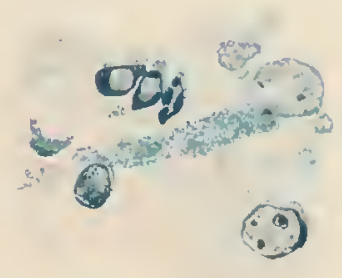

FIG. 4 .

Section of lymphatic gland from a recently fatal case of sleeping sickness in a European. The glands in this case were not much en. larged. There is a very marked proliferation of the endothelial nuclei. Magnification 520.

FIG. 6.

Various granules and products of cell (and try. panosome? degeneration seen in the perivascular infiltration of the central nervous system in sleeping sickness. Magnification I,000. 

Fü. 1.

Longitudinal section of vesss' of brain of ox that died of Jinga infection. Trypanosomes in various modified shapes are seen. Some of these may be amneboid forms of trypanosomes; probably some are trypanosomes which lave been attacked by leucocytes. Magnification 500. Stained in bulk-methylene blue and eosine.

\section{FIG. 2.}

Small vessel of the medulla oblongata of rabbit inoculated with Surra. The animal died three montlss after infection. Shows a plasmodial mass in the centre and trypanosones in a whorl near by. Magnification 1,ons). Polychrome.

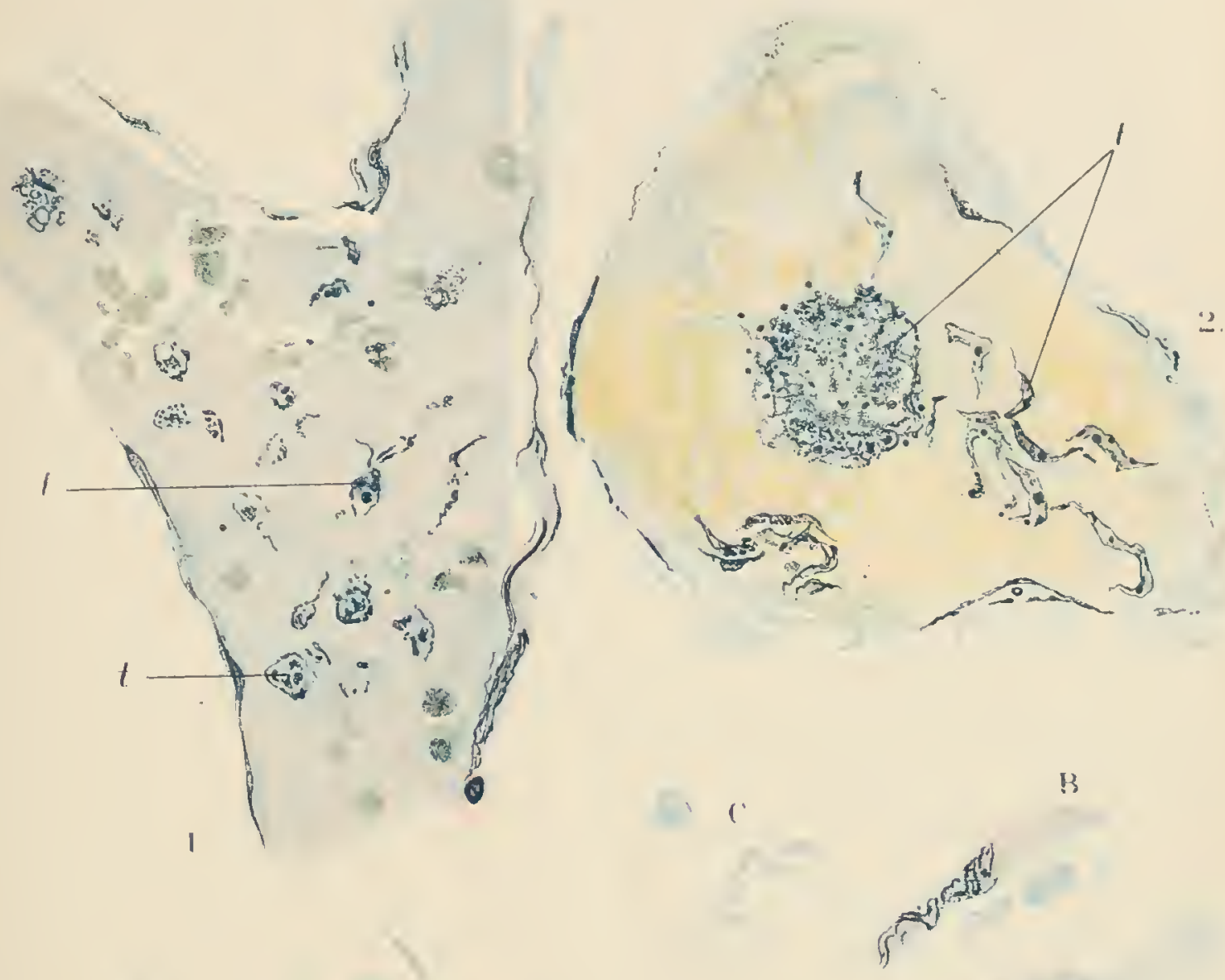

FiG. 3 .

Nerve cells of above, showing chromotalysis, ancl a small vessel with the trypanosomes $(t)$ coiled up, blocking it. Magnification $\mathrm{t}, 00 \%$. Polycbrome.

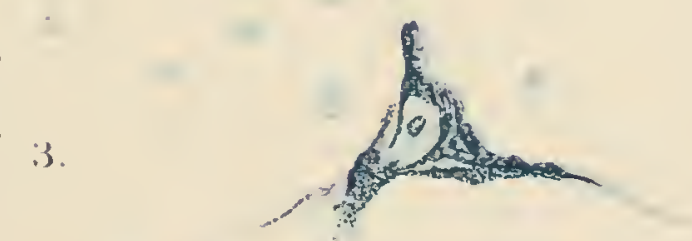

迹
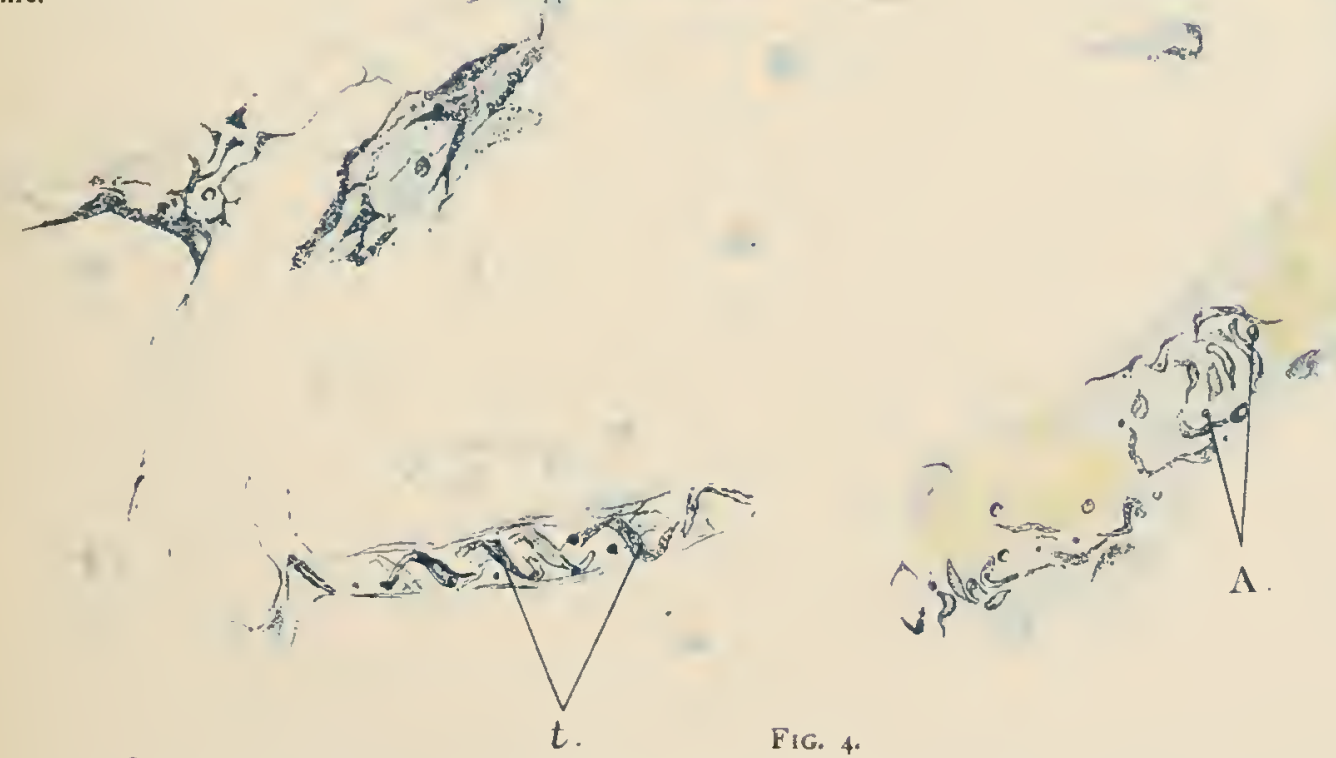

Fig. 4.

Somewhat similar appearances as in fig. 2, seen in longitudinal section of vessel. Numbers of chromatin ring, probably macro-nuclei (A); (B) capillary blocked by trypanosomes: (C) trypanosomes in the tissue; (D) ganglion cell, showing marked chromolytic changes, prolıahly due to capillary obstruction. The nucleus is swollen and clear, the body of the cell shrivelled, and there is an abence of Nissl granules. Magnification r, ooo. Romanowsky. 

It is only found in L'ganda.

It is carried by Glossina palpalis.

Its reservoir is in wild game near the lake shore.

T. capre.

It has only been reported from Lake Tanganyika and Nyasaland.

It only affects cattle, sheep and goats.

It is very active during life, and measures $18-32 \mu$ long.

It is more heavily built than the vivax; its posterior half is swollen and the end blunt. The anterior end is narrow and pointed. The cytoplasm is clear. The nucleus is centrally placed.

The kinetonucleus is large, round, and close to the posterior extremity.

The undulating membrane is better developed than in the T. vivax, and has bolder folds and a well-marked free flagellum.

It kills goats in 56.5 days, sheep in 15 days, and rats in 2 days.

The carrier is Glossina morsitans.

3.5 per cent. per 1 , ooo flies examined were infected.

Its derelopment is limited to the proboscis of the tsetse fly.

Its reservoir is the water buck, reed buck, $11^{\prime} 1$ per cent. of which were infected.

\section{PATHOLOGY.}

The outslanding feature is a chronic inflammation of the lymph system, probably caused by uxins of trypanosomes. Trypanosomes enter the lymph-stream, blood-stream, and the cerebrospinal fluid.

There is polyadenitis.

The heart and other organs show lymphatic infiltration.

There is fever in the early stage, followed by meningo-encephalitis and meningo-myelitis, proliferation of neuroglia, and lymphocyic accumulations around the ressels.

These two processes compress the ressels and lessen the blood supply to the brain and cord, which is followed by malnutrition, cerebral changes, and the sleeping sickness manifestations.

The trypanosomes cannot pass through the placenta.

An ultimate secondary infection by streptococci and pneumococci is common.

\section{MACROSCOPIC.}

There is usually emaciation, but the writer has lost many who have been particularly fat.

There may be dry desquamation of the skin to exfoliation. One of my cases was called "the serpent" because she shed her skin from time to time.

There is polyadenitis in the neck, groins and all lymphatic glands. 
The cerebrospinal fluid is increased. The base of the brain is pale, the dura mater sometimes adherent to the bone, the gyri of the brain may be flattened. The pia arachnoid is thickened in places, sometimes adherent to the brain.

The brain substance is sometimes firmer than usual, but may be soft and cedematous.

There is usually congestion of the brain. The ventricular fluid is increased.

The findings of the cord are similar. Sometimes there are hæmorrhages also.

The cauda equina may be surrounded by gelatinous tissue.

Sometimes there is ascites and pericardial fluid in excess.

The lungs may show pneumonic changes.

\section{MICROSCOPIC.}

Mott did not find it easy tc differentiate true trypanosome findings from those due to terminal affections caused by diplococci, streptococci and colon bacilli, but the following were generally agreed upon:-

Cerebrospinal system.

A round-celled infiltration surrounding the vessels of the pia arachnoid of the brain and cord, best seen in the membranes where there is excess of cerebrospinal fluid, and in the brain around the vessels of the medulla, pons, cerebellum, and those entering the base.

There is a growth in size, and then a proliferation of the neuroglia elements.

Round cells are found later in the meshes of this proliferated glia, which are :-

(I) Lymphocytes.

(2) Plasma cells of Marschalko, having a nucleus at one end of the cell, probably derived from lymphocytes.

(3) Morula cells of Mott, large, round or oval cells with an eccentric blue nucleus, probably degenerate cells of Marschalko.

(4) Some mononuclear leucocytes.

(5) A few polymorphonuclear leucocytes.

The lymphocytes are probably produced by proliferation of endothelial cells about the perivascular lymphatic space.

The ependyma of the lateral ventricles may show proliferation and dense fibrous formation.

There is atrophy of the dendrons and diminution of the Nissl bodies, and alteration of the nucleus, which becomes large, clear and eccentric. The changes are more marked in the cerebral cortex and medulla than in the cord.

The cells of the posterior spinal ganglion show chromatolysis. 
The central canal of the cord may be dilated, but more often it is oceluded by cell proliferation.

Sometimes there is hamorrhage of the cord ressels.

These changes were not noted in a cured Trypanosomiasis case which died later from another disease.

The lymphatic glands.

Trypanosomes are often found in them.

The lymphatic cells often change into cells of Marschalko. These then change into morula cells.

Endothelial cells of a lymphatic sinus proliferate, become phagocytic, containing lymphocytes, red cells and chromatin particles.

The gland is congested.

The fibrous tissue of the capsule and septa proliferate and become thickened.

When the inflammation subsides, the gland tissue becomes less vascular, firm, hard and full of dense fibrous tissue.

Secondary infection may occur and abscesses result.

The lungs.

These are hyperamic often.

They may show pneumonic complications.

The heart.

There is small-celled infiltration in all its lavers.

Sometimes it shows hemorrhages.

The liver and spleen.

There is a thickening of the capsule.

The spleen is congested and the trabecula thickened.

The bone marrow.

It is very cellular, vessels congested, hamorrhages.

\section{SYMPTOMATOLOGY.}

There are three stages:-

(I) Incubation.

(2) Febrile or glandular.

(3) Cerebral.

In all three the clinical manifestations are irregular in degree and duration.

\section{INCUBATION.}

The time in man is not known. Probably two to three weeks, or it may be less than ten days. Some individuals have not shown any symptoms after being infected for five years. I had several patients who had carried trypanosomes for two vears and showed no other manifestations of the disease al all. 
The local irritation of the tsetse bite may be so slight as not to be observed, but in some there is a raised itching patch which may last for several day's.

\section{SUMMARY OF SYMPTOMS.}

To aid the memory these may be summarized as follows: Polyadenitis, fever, erythema, paresis, lachycardia, somnolence, adema, enlarged spleen, aniemia, headache, epileptiform fits, tremors, change of character, itching, hyperasthesia, incontinence of faces and urine, enlarged liver, eye symptoms, delusions, insomnia, vomiting, melancholia, epistaxis, feet pains, paresis of lower extremities, dermatographia, painful swelling of ankle, sexual impotence, hemiplegia, giddiness, deafness, loss of hair, phlebitis, facial paralysis and orchitis.

\section{FEBRILE OR GLANDULAR STAGE.}

This stage is known to some as the Trypanosome Fever Stage.

The manifestations in Europeans are much more frequent than in natives, and may be considered as follows:-

Fever.-There are attacks of fever unaffected by quinine, lasting for about one week and recurring at intervals, or the fever may not recur, or may be so slight as not to be noticed, or it may be taken as a "touch of malaria."

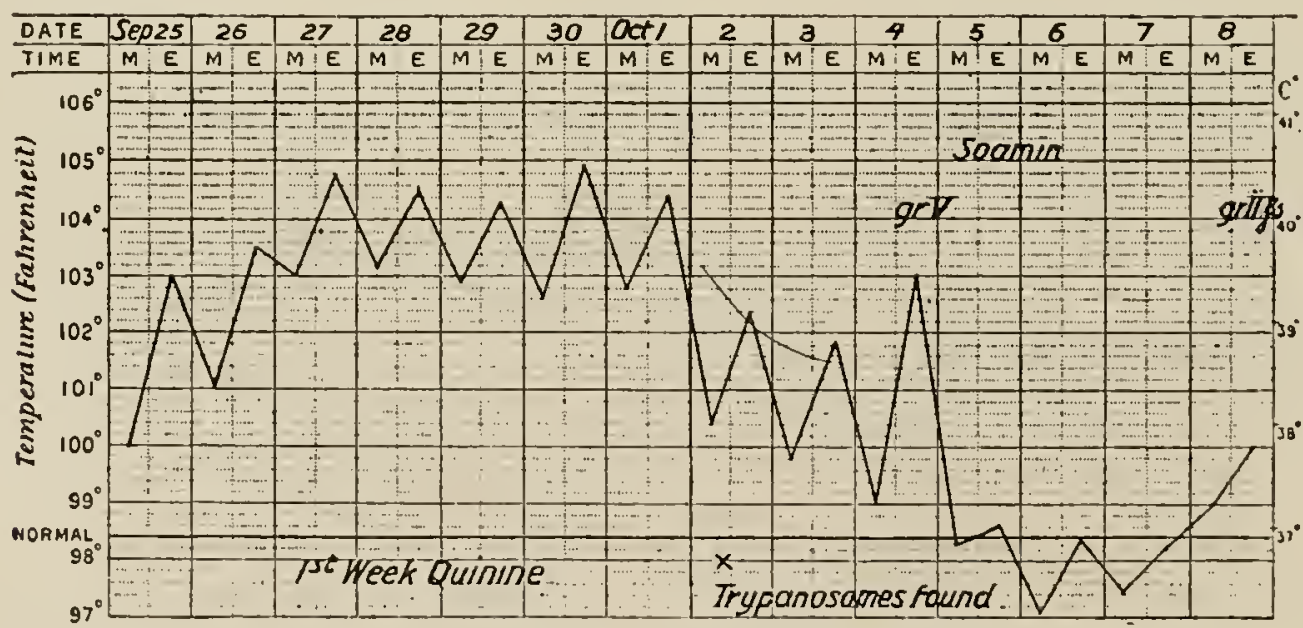

Chart I, I909, Onset of disease and marked temporary effect of soamin in the second week.

The pulse-rate and respirations are increased. The pulse-rate may be very rapid during the febrile attacks and during the apyrexial period also. The spleen and liver enlarge, but it is difficult to exclude malaria.

Neuralgic pains and headaches are common.

Erythematous Eruptions.-Pinkish patches, irregular in position and outline clear in the centre, may be horseshoe shape or circular; 


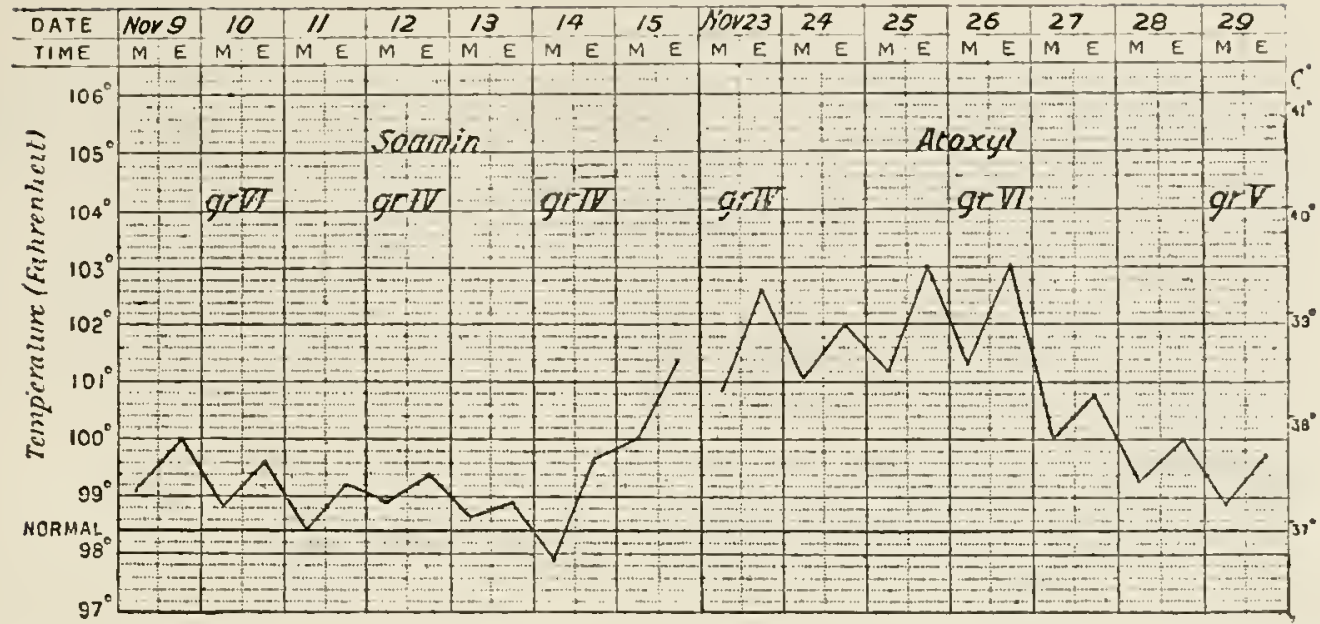

Chart II, I909. Third and fifth weeks. Imperfect control of temperalure by soamin and atoxyl in large doses.

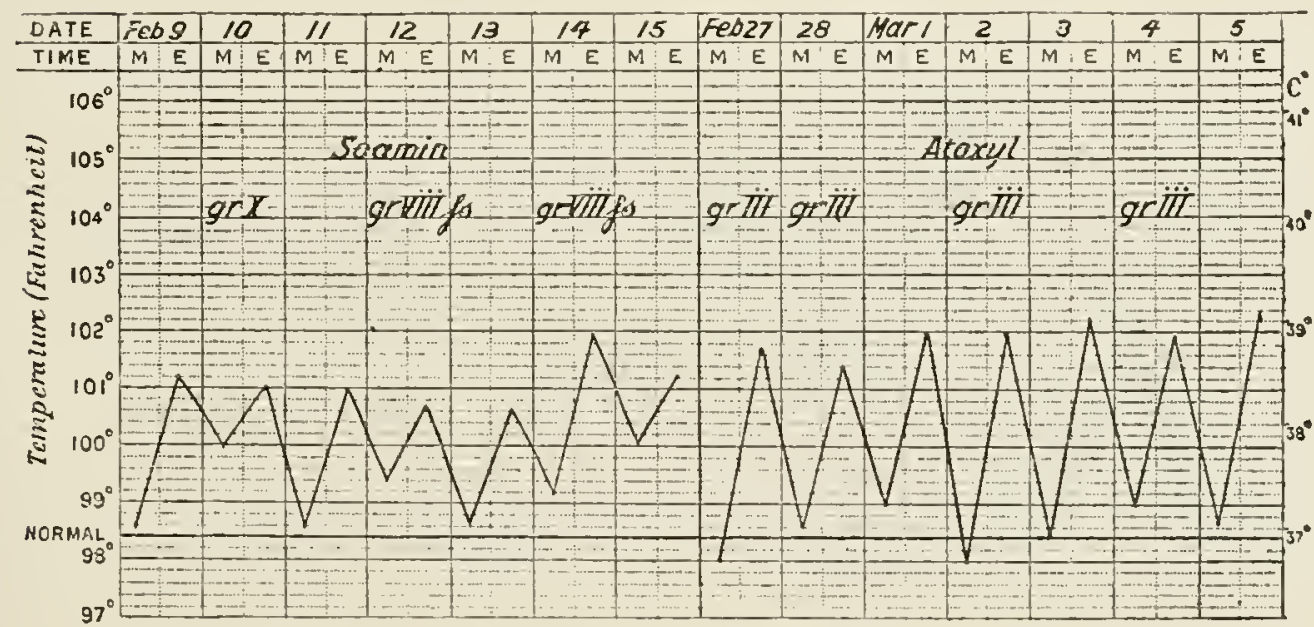

Chart III, I9Io. Two other periods some three months later, showing absence of any marked beneficial effect from these drugs. Trypanosomes were usually to be found.

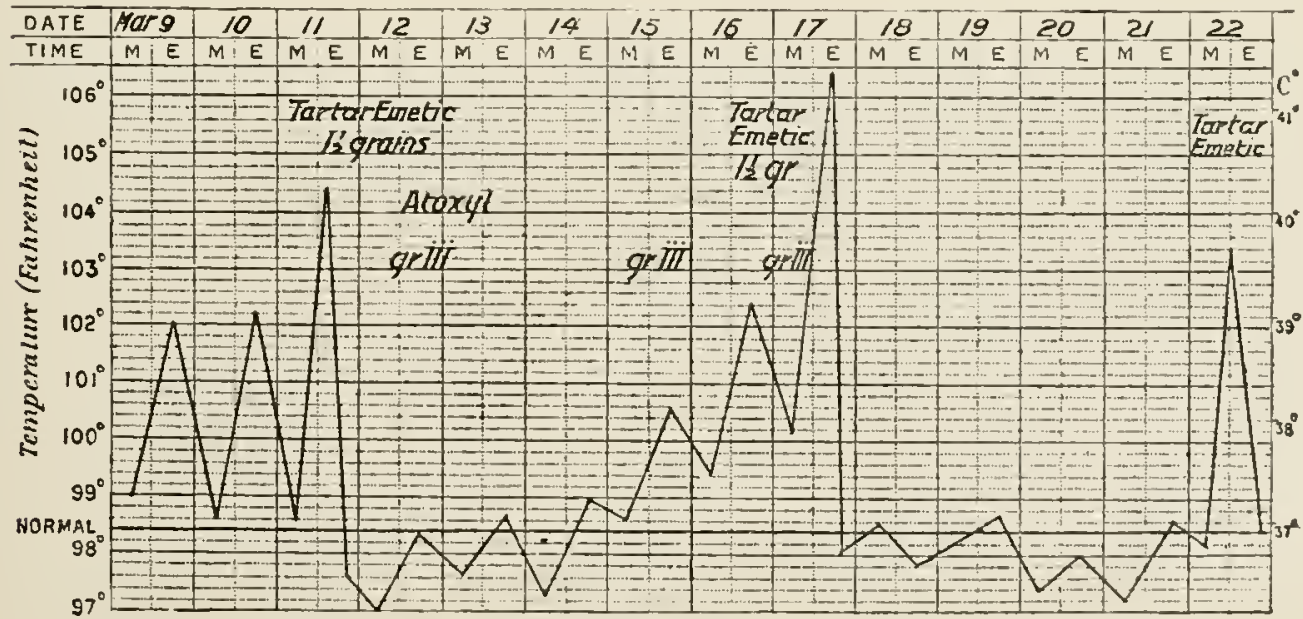

Chart IV, 1910. Marked temporary effect of intravenous injections of tartar emetic. The trypanosomes, which before the injection were 3 to 200 leucocytes, were not found for three days, but became as numerous as before in six days. The severity of the reaction is indicated by the temperature. 
they may appear anywhere, but are usually on the trunk or thighs. There may be solid infiltrated patches and rubeloid spots.

A mottled and bluish appearance of the rounk is very important. When once seen it is never forgotten, and could be seldom mistaken.

Rarely a vesiculo-papular eruption is seen.

There may be patches of painful local oedema about the hands or feet readily mistaken for theumatism. Many of these eruptions last for a few days and reappear elsewhere. They are known as Trypanides.

\section{LYMPHATIC GLANDS.}

Enlarged, especially in the neck, posterior triangle; this is Winterbottom's sign. They are soft at first, elastic, and later become hard and fibrous. They vary in size from a split pea to an almond. Few become larger than this.

Decp Hypercesthesia.-This is known as Kerandel's sign from a physician who contracted the disease and described the condition. It is general, and the pain on contact is intense. The slightest pressure feels like a bruise and is exceedingly painful.

A puncture with an hypodermic needle feels like a "red-hot poker" in bad cases. In accidental knock by a walking stick gives excruciating pain. There is deep bone pain as distinct from deep muscle pain. It is felt over the ribs, extremities, about the eyeballs, face and head, so that wearing a helmet is painful. The leg pains have been mistaken for beriberi.

Localised Gidema.-One ankle may swell for a time and then the swelling may disappear. There may be adematous patches over the temple region or on the thigh. Later there is a more extensive aedema.

Asthenia and Ancmia.--The anemia may be marked and progressive. Wreakness is considerable, although no wasting may be present.

Eyc Lesions.-Dr. Daniels has called attention to these, and describes them as toxic irido-cyclitis, conjunctivitis, iritis, lieratitis, retinal changes, photophobia and deep odema of the lower eyelids. with deep pain of the eyeball. They were rarely present in my rases.

Orchitis is present in some cases.

This febrile stage may go on for years or it may be cured, either spontaneously or by treatment or both, except in Rhodesian cases.

The appetite is usually good. Wasting is absent unti] the sleeping sickness stage.

The symptoms vary much in different individuals, no one showing all of them except in very few instances. A few of my European cases chosen at random will indicate this point.

Case (1).-In trypanosome area for eight years wilhout leave 
(Central Africa). Fever almost constantly for months past, did not take quinine, marked anamia, weakness marked, pallor, spleen nearly reached iliac crest, liver slightly enlarged, malaria also present, pains in legs and calves excruciating, worse at night, exhausted because no sleep, aspirin, bromides, trional, opiates useless. Sent to me as beriberi. No trypanosomes in blood, no auto-agglutination, no eruptions, no eye lesions, pains in legs, with polyadenitis chief symptoms. Sent home and diagnosis confirmed.

Case (2).-In trypanosome area, Central Africa, three years; some fever, probably malarial, trypanosomes found in blood, some autoagglutination, polyadenitis, no other disease present, strong man at work and refused treatment, as no symptoms when first diagnosed. Given soamin intramuscularly and sent home.

Case (3).-History exactly as above. A very stout, strong man. Given tartar emetic intravenously and Fowler's solution by the mouth, and sent to Europe. Dr. Daniels writes some months later that he does not find any symptoms of the disease in him.

Case (4).-In trypanosome area six months, Central Africa; an ideal man, physically and mentally, prior to attack; marked weakness, stubborn fever would not yield to quinine, frequent micturition for six months, sleeplessness, nervous prostration marked, admitted to hospital unconscious the day after the fever commenced. Temperature remained up for five days, in spite of quinine 30 grains daily, no malarial parasites in blood, no trypanosomes found after forty-two examinations, no auto-agglutination, polyadenitis marked. Long convalescence, prolonged weakness, memory poor, headaches, marked mottling of chest, back and thighs, purple colour, disappeared on pressure, deep pains of legs and muscles excruciating, then over body, face, head, agonizing pain on the slightest touch. Erythematous patches, fleeting, red, not raised, massage impossible, worse at night, as if "someone was twisting his legs off at the ankle," opiates of little use.

All the typical symptoms were present, except trypanosomes, autoagglutination and eye iesions.

Was given Tartar emetic intravenously ; ditto Galyl; Soamin by the mouth and intramuscularly. Temperature normal for ensuing eight months; when discharged all symptoms disappeared except slight leg pains.

Natives may show some of these symptoms, but in my cases (over 4,0oo) there were no eye lesions, except oedema and one persistent double conjunctivitis not otherwise accounted for.

Deep pain is very rare, fever also, but a dry, scaly eruption is fairly common. Headaches, delusions, hysteria and mania are common. Edema of all parts common. Trypanosomes rare and auto-aggutina- 
tion very common in fresh blood films. There is often tremor of the tongue and fingers.

They decline treatment as a rule in this stage because treatment is more painful than the disease.

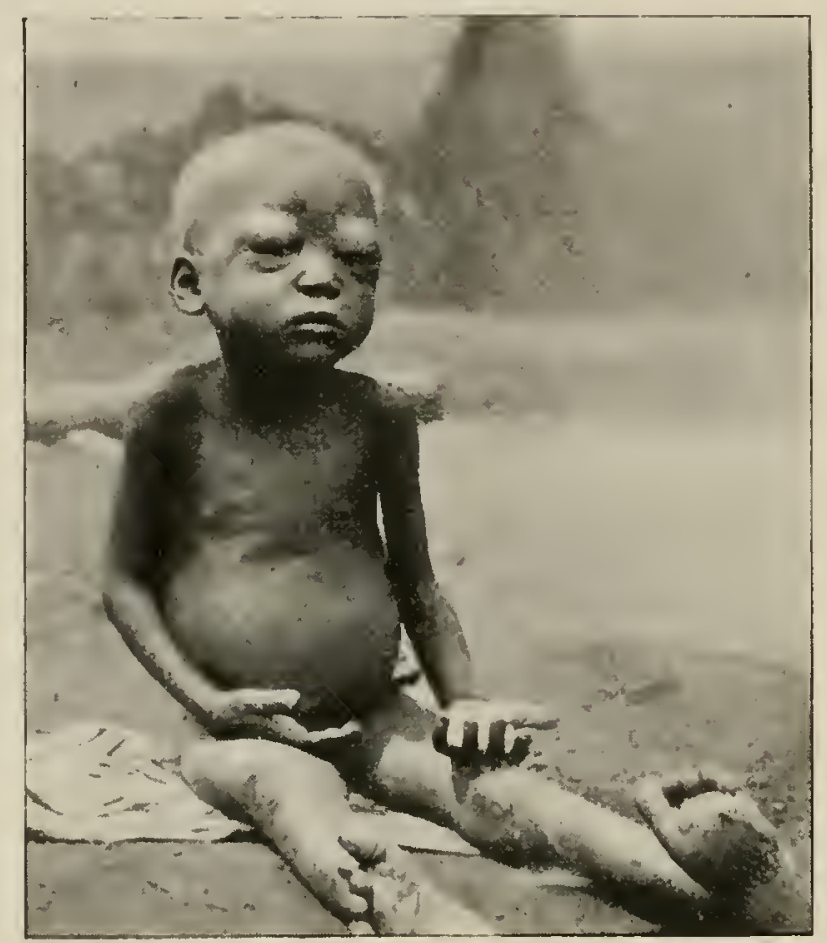

Child with trypanosomiasis showing oedematous swellings of the eyelids.

\section{THE CEREBRAL STAGE.}

In the Sleeping Sickness stage death is inevitable, but when the former stage ends and this one begins is not accurately known.

This stage lasts from a few weeks in acute cases to a few months in chronic cases. Some have lived for years, and have then died from some intercurrent disease.

One notices a change in the habits of the patients, the disposition is modified more often for the worse, they become apathetic, dull, there is disinclination for exertion, they become careless, dirty, find it difficult to walk, become indifferent to most things, and forget to masticate the food that lies in their mouths.

Sleep is often excessive, but it is more frequently a lethargic condition that is manifested. There are fine tremors of the tongue, hands, arms, legs, and even of the abdomen. There is a peculiar gait, a difficulty in raising the feet from the ground, he shuffles along or throws his feet outwards in walling.

There is no paralysis as a rule; the superficial reflexes are normal, the deep reflexes are increased and then lost. Delusions and mania are fairly common. Romberg's sign is sometimes present. Later 
there may be rigidity of the neck and legs, a tendency to permanent flexure of the legs, on the thighs, and abdomen. One case of mine could not bend his legs at all, and danced with them quite stiff all day and night, except when he was put down prostrate by attendants. He could not rise again himself, but he continued to dance when he was put in the upright position.

IIyperasthesia is not common. They often wound themselves against sticks, \&c.; ulcers form which are difficult to heal owing to their reduced condition and their dirty habits.

Daily fever is common when there are pneumonic symptoms, at night up to $100^{\circ}-104^{\circ} \mathrm{F}$., falling to subnormal in the morning.

There may not be any fever at all, or the temperature may be subnormal. There are no rigors or sweating in fever cases, but the pulse is unusually quick, 90-I4O, and is out of all proportion to the temperature. It is, as a rule, regular, readily compressible, and small. Respirations are increased. There may be Cheyne-Stokes breathing before death. Congestion and adema of the lungs with pneumonic patches are common before death.

Digestion is good. Sometimes there is constipation, but more often diarrhoea. Unclean feeding may account for it, e.g., fungi, baked clay, \&c. The usual intestinal flora can be found.

The blood shows trypanosomes, auto-agglutination, mononucleosis, and sometimes polyleucocytosis before death.

The acidity of the blood is said to be increased, or probably better, the alkalinity is diminished, which is probably due to amino acids secreted by trypanosomes, and produced by the action of the amino acids upon serum proteids.

Sexual desire does not necessarily cease, neither does menstruation, but they may disappear in some cases.

The lymphatic glands become fibrosed, smaller and harder.

The skin is usually dry and rough, but may be normal.

Emaciation is common, but not the rule. Muscular weakness is very marked, tremors are pronounced, saliva dribbles from the mouth, urine and freces are passed involuntarily, bedsores form, the pulse cannot be felt at the wrist, the temperature is subnormal, coma, and death.

Perverted appetite is common; they will sometimes eat earth when good food is at their side. One case died from intestinal obstruction, and $2 \frac{1}{2}$ pounds of clay lumps were found in the intestines. Many of the people, however, are earth eaters when in normal health.

Theft is a common propensity, but it is certainly more marked when this disease is present.

Mania is common. One case was of a boy who attempted to drown 
himself. He was very fat, but he slept twenty-two hours out of twentyfour. He recovered under treatment, so that he slept but ten hours daily and went to work again. Some cases are very violent, and it is impossible to inject them intravenously.

Edema of the eyelids, face, legs, arms, penis, scrotum and abdominal wall is fairly common in the final stages. Ascites may also supervene, also an increase of the pericardial fluid.

Irido-cyclitis and other eye troubles were so rare in my cases as to be ignorable. My district was 4,800 sq. miles in extent, with a population of from 100,000-200,000, of whom 75 per cent. were infected with the disease.

Some cases die from the disease without any enlargement of the lymphatic glands, and others survive when they have become markedly enlarged.

\section{COMPLICATIONS.}

Pneumonia is common, and is one of the common terminal phases. Laryngitis, oedema of the glottis, and iritis have been recorded.

Amobic dysentery is fairly common, and usually causes death when present.

Cerebrospinal meningitis due to streptococci, pneumococci, or meningitis is not uncommon.

Epileptiform symptoms are frequent.

Helminthiasis is common, but it may not give rise to any troublesome symptoms. Filaria may be present.

Malaria is common in some districts and rare in others.

Jiggers and resulting "tattered toes" are almost universal. A considerable amount of septic absorption may result from ulcers caused by them.

\section{DIAGNOSIS.}

The finding of the trypanosome is conclusive.

Always suspect Trypanosomiasis when fevers are not controlled by large doses of quinine in a trypanosome district.

Look for erythema in Europeans. There will be a rapid pulse during the apyretic periods. Asthenia without wasting should arouse suspicions. Deep hyperesthesia; Kerandal's symptom is common in Europeans. Tremor of the tongue; Low and Castellani's symptom should be looked for.

Cervical adenitis, Winterbottom's sign, is common and important.

Search the blood for trypanosomes, auto-agglutination, mononucleosis, but remember that auto-agglutination is also present in Filariasis, Malaria, Syphilis and Yaws. 
In searching for trypanosomes, the following methods are available :-

( I) The peripheral blood, fresh blood films. Stained blood better for details of parasite, but less frequently successful.

(2) Scarification of the epidermis. Examine resulting lymph for the trypanosomes. Not very successful.

(3) Citrated blood, centrifuged repeatedly. Examine the third sediment. The results are better.

(4) Centrifuge citrated blood in small tubes and examine the leuco. cytic layer. Dutton and Todd's method.

(5) Puncture the glands and examine the juice. The glands are often small and natives object to the puncture. A valuable method.

(6) Centrifuge cerebro-spinal huid for i 5 minutes, examine sediment fresh and stained. It is negative in febrile but positive in sleeping sickness stage. A valuable method, introduced by Castellani.

(7) Inoculate susceptible animals. Use 20 c.c. of blood or cerebrospinal fluid and inject it into monkeys or guinea-pigs, dogs or white rats. Monkeys and white rats are soonest killed in positive cases. See p. 132.

\section{PROGNOSIS.}

It is always serious.

It is curable in the febrile stage but not in the sleeping sickness stage. All Rhodesian cases, febrile and later stages have remained uncured hitherto. There is one queried case of recovery diagnosed in May, 1913. Of 500 trypanosome cases invalided to England, 300 are already dead.

Improvement is always possible except near the end.

With treatment, life is prolonged; without it death is almost certain.

The Congo gambiense strain of trypanosome is the least virulent.

The Uganda gambiense strain of trypanosome is the more virulent.

The Rhodesian gambiense strain of trypanosome is the most virulent.

Death is inevitable in the sleeping sickness stage.

Whole villages have been depopulated by it in Senegambia, Angola, Uganda and the Congo. The populations of the islands of Lake Victoria Nyanza have been annihilated by it.

\section{TREATMENT.}

Nothing need be said here concerning good hygienic conditions, the exclusion of accompanying diseases, \&c.

There is no specific for the disease.

Soamin has not realized the hopes of its inventor. 
The chief drugs now relied upon are antimony oxide, tartar emetic and arsenic.

Arsenical preparations.

Liquor arsenicalis has cleared up some cases. It was used before Soamin was known. It should be given well diluted in increasing doses, from 5-15 minims and omitted every fifth week.

Salvarsan and Neosalvarsan. These are not more useful than Soamin and much more costly.

Atoxyl. This is as Soamin, but more toxic, causing optic atrophy, gastro-intestinal inflammation, and peripheral neuritus. It has now been replaced by Soamin.

Atoxylate of Mercury. Less satisfactory than atoxyl.

Soamin. The best arsenical preparation to use.

The methods of giving it are various. By the mouth, by the rein and intramuscular. Examples:-

3 grains daily per os, omit every fifth week.

2-3 grains intramuscular every third day for at least two rears. (Manson.)

$7 \frac{1}{2}$ grains intramuscularly every fifth day (Broden).

$7 \frac{1}{2}$ grains intramuscularly two successive days every ten days for ten months (Koch).

The writer has given $7 \frac{1}{2}$ grains (0.50 grm.) of soamin every fifth day for two years without clearing trypanosomes from the lymphatic glands in the majority. The drug was always suspended on manifestations of arsenical poisoning. Thousands of injections were given following this method, but were abandoned for combined treatment as being unsatisfactory. Soamin will cause the disappearance in some cases of the trypanosomes, but we cannot rest contented with it.

Orpiment. An organic salt of arsenic used for the lower animals. Not any use in human trypanosomiasis.

Galyl. This has proved useful in some cases with tartar emetic given between doses of galyl. Useful in European cases.

Antimony preparations.

The following are new preparations which have not yet been sufficiently tried. They are: Antileutin, antimony and ammonio-potassiotartras, antimony aniline tartrate, and antimony-sodium tartrate.

Tartar emetic is the best known and most ridely used.

All tartar emetic preparations should be preceded by an injection of caffeine; as tartar emetic lowers the blood pressure. I have given thousands of doses of tartar emetic intravenously without any heart srmptoms of importance.

Tartar emetic is too painful for intramuscular use. It was thus first used and abandoned by Sir Patrick Manson in the early days. 
It can be given by the mouth or rectum, 1-2 grains in large quantities of water, say $1.2-2$ pints, every third day.

Tartar emetic is perhaps best given by the vein, $\frac{1}{2}-1$ grains every third day. Method of administration.

Ĺse a 20-c.c. Record syringe. Prepare a solution of tartar emetic so that o c.c. contain ? or more grains of the drug. Apply a tourniquet to the arm so as to distend the veins, paint the spot with iodine, draw into the syringe so c.c. of the drug, fill syringe with boiling water, attach rubber connecting tube, expel the air, give syringe to assistant to hold, take needle and plunge it into the distended vein, allow a few drops of blood to run through to ensure that it is well in the rein, attach syringe holding piston end upwards so that any air bubble will be at the upper end, unfasten tourniquet, inject the fiud leaving one c.c. in the syringe, withdraw needle and empty syringe through the needle so as to expel any drop of coagulated blood that may be in the lumen, put the whole in a solution of antiseptic and it is ready again for use. A litule iodine and bandage on the site of the injection is sufficient. It is not necessary to dissect out the veins except in rery few cases. With plenty of assistants one can give 40 injections in an hour in this way. The reactions which follow tartar emetic are always marked. Young infected natives cannot take more than $\frac{1}{2}-1$ grain at a time no matter for how long the drug has been taken. Soamin was given with tartar emetic intravenously but toxic symptoms were marked and it was abandoned.

After giring more than 6,ooo injections of arsenic and tartar emetic the method and drugs found by me to be most useful up to the present were :-

A combined treatment of :-

One grain (0.06 grm.) of tartar emetic intravenously every third day, every second day in selected cases.

I-2 grains ( $0^{\circ} 06-0^{\circ}$ I 2 grm.) tartar emetic by the mouth in a mixture containing glycerin, sodium bicarbonate, and chloroform water.

1 $1 \underline{\underline{I}}$ grains $\left(0^{\circ} 7 \mathrm{~g} \mathrm{grm}\right.$.) soamin intramuscularly once weekly. Omit treatment every fifth week or on the appearance of toxic symptoms.

Arsenic alone tends to produce arsenic-fast trypanosomes. One must give heroic doses of whatever drugs one uses. Ascertain the idiosyncrasy of the patient towards the drugs and administer them just short of toxic symptoms, omiting them every fifth week.

Solutions of mercury, formalin and collargol have been tried separately and together, intravenously, but without success. Many natives would rather die than have intramuscular injections of any kind. They prefer intravenous injections in spite of the after reaction. 
Attempts have been made to procure a curative and immunizing serum, but without success for man.

It is interesting to note that the physician, Kerandal, claims as his cure intravenous injections of tartar emetic given in four series of daily injections of $\mathrm{IO} \mathrm{cm}$. each, the number of doses in each series being $\mathrm{I} 7$, I5, I5 and Is respectively, or 55 in all, equal to $550 \mathrm{grm}$. of tartar emetic. His weakness disappeared as "if by enchantment" after the first course of injections. Atoxyl, of which he took jo grm. in all, did not appear to affect the course of the disease.

\section{PROPHYLAXIS.}

What has been said about sanitary measures for the prevention of mosquito breeding need not be repeated here.

In whatever work is undertaken the Government must co-operate.

There should be medical inspection of all the villages on the boundary of the fly area. Natives must co-operate also, and this is the most difficult task. If they do not volunteer to do so they must be forced. Some suggest that the infected should be isolated and treated, but this is neither practicable nor possible in the District du Kwango of the Belgian Congo, where about 70 per cent. of the total population is infected. To adopt such regulations would mean the closing down of all important worlis in that district, and the natives would be far worse off than before.

Fly-free areas should be chosen or prepared, new villages built and maintained, plantations prepared and maintained, all infected villages to be destroyed and the inhabitants removed to the new ones, the infected peoples treated drastically, and those in the last stage removed to lazarets. This is a gigantic task, and is beyond the purse of the Government or any private enterprise in Central Africa. It should be worked as far as possible. The bush should be cleared and planted for 300 metres around every village, and from the water's edge for 100 metres. Then a new generation of individuals would arise, free from the disease, capable of increasing their kind and developing their country under guidance and control.

Fly catching is impracticable in most districts to be of any real good.

The extermination of big game is, in my opinion, unadvisable. Wherever man may go some animals will accompany him, and these will become carriers as well as man.

The legs of Europeans should always be protected with puttees or leggings when passing through fly districts.

European residences should be well away from native quarters.

Quinine does not seem to be a prophylactic in any way. 


\section{NOTES ON THE GLOSSINA.}

The glossina are limited to Africa and the shores of the Arabian Gulf. Formerly they existed in North America.

The Genus contains ten species, which may be distinguished as follows :-

\section{(After Manson.)}

\section{GENUS GLOSSINE.}

Large species, length of body at least I $\mathrm{mm}$.

G. longipennis. Thorax with four sharply defined dark-brown oval spots.

G. pusca. Thorax without spots.

Small species, length of body rarely reaching i $\mathrm{mm}$.

Hind tarsi black.

Antennæ black.

Two last joints of front tarsi black.

G. palpalis. Abdomen not very marked.

G. tachinoides. Abdomen conspicuously marked.

All joints of front tarsi yellow.

G. bocayei.

Antenna yellow.

G. pallicera.

Two last joints of hind tarsi black.

Front and middle tarsi yellow.

$G$. pallidipes.

Front and middle tarsi black.

Abdominal stripe one-third of seg-

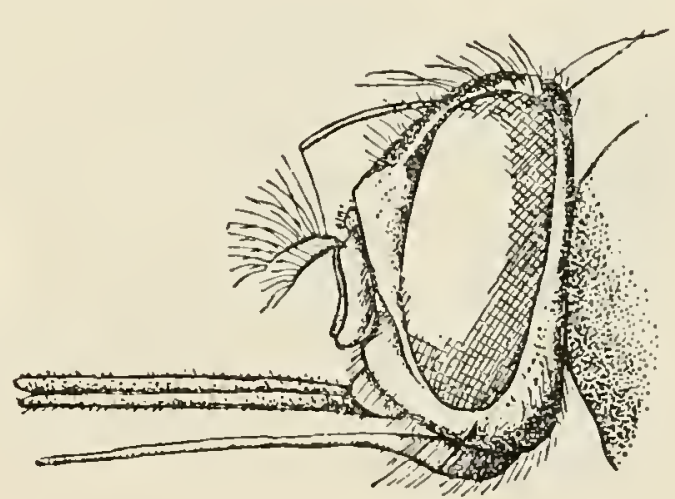

Head of Giossina longipalpis, Wied. (After Griinberg.) ment.

G. morsitans.

Abdominal stripe one-sixth of segment.

G. longipalpis.

They are ordinary looking flies about half an inch long. A strong proboscis stands out horizontally. Both sexes suck blood. The wings are long and close over each other when at rest like blades of a pair of scissors.

The dorsal aspect of the abdomen is marked by five or more distinct bands. The mouth parts consist of the labrum, labium, hypopharynx or terminal duct of the salivary gland. The labrum and labium together form a suctorial tube for blood and is called the labial cavity. The salivary glands are long convoluted organs lying chiefly in the abdominal segment. 
Probably all the species are capable of carying trypanosomes. We will give a few features of the two most important.

\section{GLOSSINA MORSITANS.}

This species was known first as a result of the writings of Livingstone and Cummings.

\section{Habits.}

It inhabits dry thorny scrub, which covers large areas of Central Africa. It is not found along the river banks or other water courses; it dislikes water. It requires shade and is never found on the open plains exposed to the tropical sun. It begins to bite soon after sunrise on a fine day, usually disappears at mid-day, comes out again towards sunset and is then very active.

In rain and wind they will remain hidden.

They are most numerous towards the end of the rains.

\section{Food.}

Of 5 oo flies examined in Nyasaland the intestines contained mammalian blood, chiefly antelope's, in 57 per cent. of cases.

They never suck fruit juices like the mosquitoes.

They feed once during $30-70$ hours.

When feeding, the fly fully distends itself in 1 to I minute after it has punctured the skin. A male can absorb I'3 times its body weight of blood and a female $\cdot 6$ times its weight. In two or three minutes after the feed, minute drops of digestive fluids are exudated from the anus, a process which continues at the rate of 2 per minute for 20 minutes. Some hours later a thick brown excrement follows and the abdomen becomes marliedly reduced in size.

Reproduction.

All Glossina are pupiparous and in this differ from most of the Diptera. The female produces one white-yellowish larva once in ten days. This is retained in the oviduct until fully grown. When born it creeps into the soil or amongst the leaves and debris and within a few hours has changed to a hard, black puparium. The adult fly emerges in about $23-60$ days according to temperature. The female generally chooses the under surface of a tree for this purpose, generally near a native path or a game track so as to facilitate the mother's obtaining food for her young and herself.

\section{GLOSSIN E PALPALIS.}

These are widely distributed. They are lovers of water and are to be found frequenting the edges of rivers, lakes, ponds, water holes in banks where there is clear water and plenty of shade, thick undergrowth with high trees and dense jungle.

They are never found on open beaches backed by grassy plains even 
with scrub near to the water. They are not found amongst the reed beds or papyrus swamps such as exist in the L'ganda river valleys. They are never found away from water except where they have followed man or beast a mile or so for his blood.

\section{Habits.}

Their bites are as those of the G. morsituns. They fly rapidly, settle quickly on the skin and soon ingest enough blood to fill them out perhaps without a prick laving been felt.

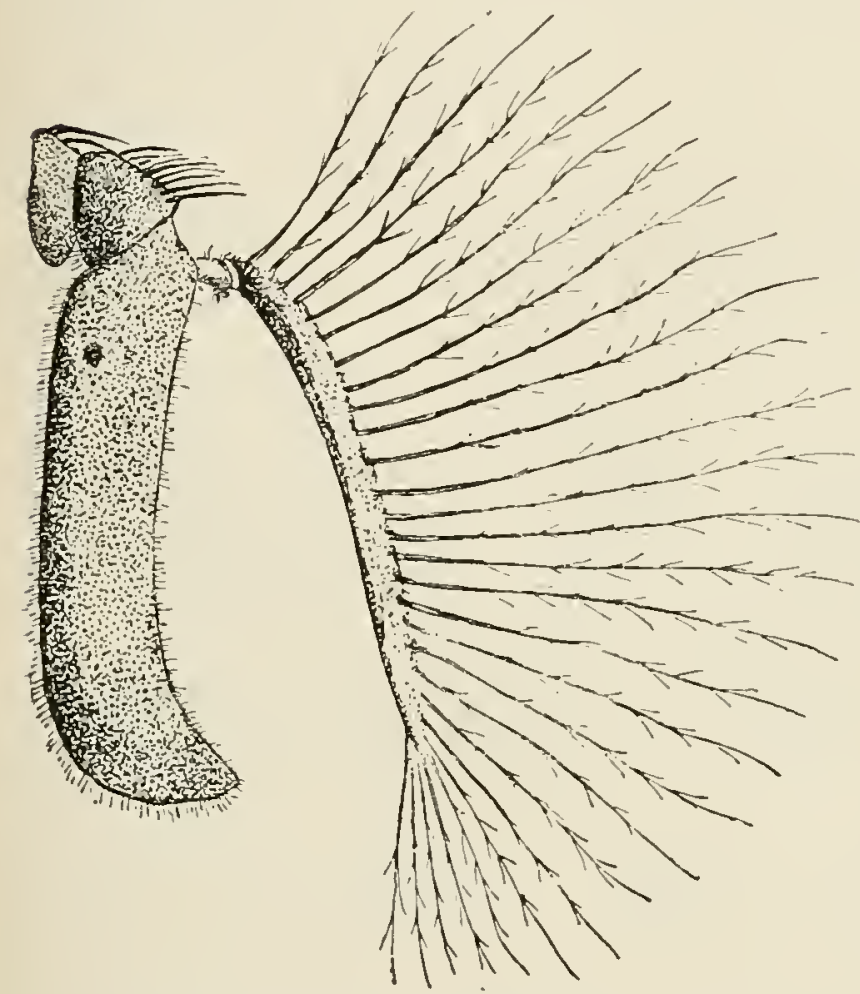

Antenna of Glossina fallidipes, male. (After Austen.)

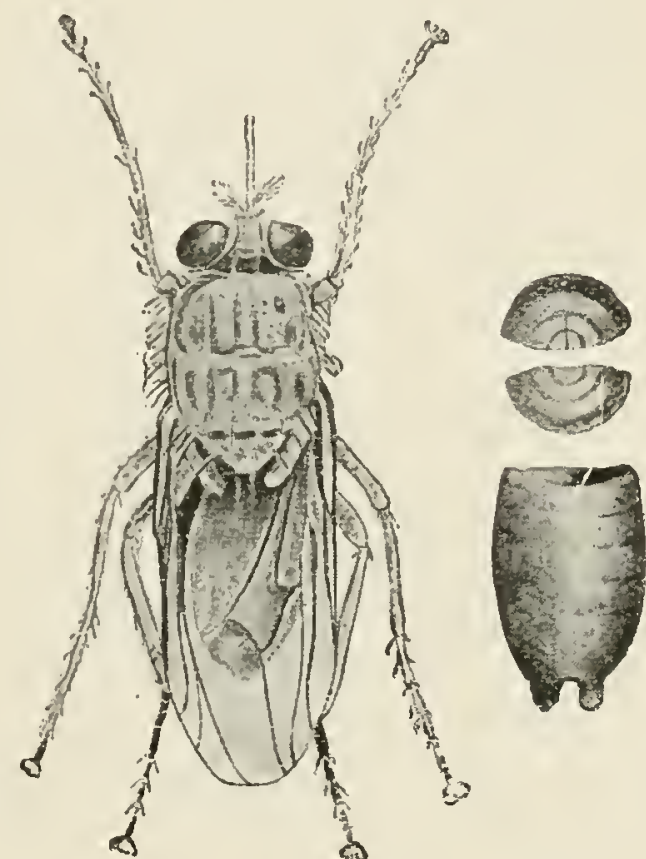

Glossina palpalis and puparium. (After Brumpt.)

Feeding.

When a $G$. palpalis has fed on an infected animal it becomes infective on an average of 34 days from the feed. 1t remains infective for about 75 dars.

Food.

In populated districts man supplies sufficient blood but in captivity or at any time they will feed on hot or cold blooded animals, birds or reptiles.

\section{Reproduction.}

Similar to G. morsitans.

Sandy banks banked by a belt of vegetation are often chosen. The pupa are found one to two inches below the surface of the ground amongst or near to vegetation.

There is no hereditary transmission of trypanosomes in the tissue, all trypanosomes are obtained from the animals they feed upon. 


\section{SOUTH AMERICAN TRYPANOSOMIASIS.}

\section{HISTORY, \&c.}

This disease is caused by Trypanosoma crusi discovered by Chagas in I 909 .

It is spread by the bug Lamus megistus and allied forms.

Its reservoir is probably in the Armadillo, Dasypus novencinctus.

The bugs live in the burrows of the armadillo.

The disease is only known amongst the poorer inhabitants of the State of Minas in Brazil where it appears to attack the whole population.

\section{AETIOLOGY.}

The Trypanosoma cruzi has a large kineto nucleus.

It is found in domestic animals and man.

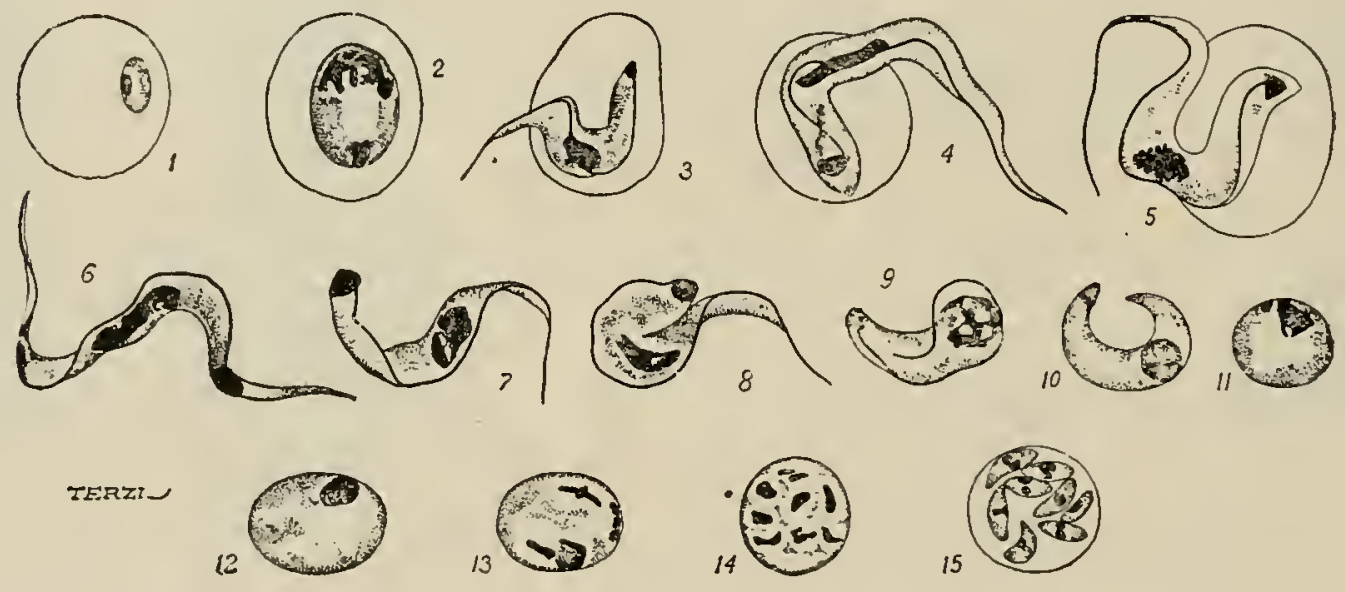

Trypanosoma cruzi. Schizogony. I, merozoite in rej blood corpuscle; 2, parasite totally enclosed in red cell, no flagellum or undulating membrane; 3-5, parasites partially enclosed in red cell; 6,7 , parasites in human blood; $8.1 \%$, parasites in lungs of the monkey, Callithrix; 12, 13, initial forms of schizogony ; 14, 15, schizogony in the lungs of Callithrix. (After Chagas.)

Three forms of the trypanosome are seen in man :-

(1) With a large nucleus, loose chromatin and a terminal kineto nucleus.

(2) A narrower form with an oval nucleus and dense chromatin.

(3) A form with an elongated nucleus.

The trypanosome undergoes multiplication in one of three ways:-

(I) Schisogony.-This takes place in the capillaries of the lungs of the callithrix where it loses its flagellum and undulating membrane, it curves itself and forms a disc which divides into eight merozoites. These enter the red cells and become adult trypanosomes. Later they leave the red cells and live free in the blood-stream. Schizogony probably differentiates sexual forms and lays the basis for infection of invertebrate hosts. 
(2) Sporogony.-This takes place in the bug Lamus magistus.

Sexual and asexual forms develop, the final stage of development being found in the salivary glands when they are ready to infect a new vertebrate host. The development in the gut requires about eight days.

(3) Agamony.-This is an asexual form of reproduction and takes place in the cells of the various organs of the callithrix, i.e., in the hypertrophied endothelial cells of the lungs, in the cardiac muscle, in striped muscle and in the neuroglia of the central nervous system. In these situations it appears as a rounded body but without flagellum or undulating membrane.

The trypanosome is not known to undergo any longitudinal division in the peripheral blood or the internal organs of the host.

The trypanosome is easily cultivated on blood agar.

The Lamus is infective to vertebrates, 8-10 days after an infective feed and remains infective for a long time.

\section{PATHOLOGY.}

The trypanosome enters the body cells, especially of the muscle, more frequently about the back and extremities. In these cells it assumes a Leishmania-like form without flagellum but with a tropho-kineto nucleus. Division is by binary fission, the two daughter cells dilating the mother cell, or more correctly, the cyst membrane which ruptures, liberating the two enclosed forms. The parasites now become flagellates and move about. Local inflammation now sets in with the presence of trypanosomes in the blood. There may also be a production and liberation of toxins because the liver undergoes fatty degeneration.

Different organs may be attacked in this way, the local symptoms and pathological changes being in accordance with the organ or organs selected.

\section{P. M.: MACROSCOPIC.}

There is serous effusion into the abdomen.

The liver is enlarged with fatty degeneration.

The spleen is enlarged, hyperæmic and soft.

The mesenteric glands are enlarged, hyperamic and soft.

There is serious effusion into the pleural cavities.

The same condition affects the pericardial sac, there may also be hremorrhagic pericarditis.

The heart may be much enlarged, there may be intense myocarditis.

The lymphatic glands are swollen and hyperæmic.

The thyroid gland is enlarged.

The dura mater is congested. There may be signs of leptomenin- 
gitis, encephalomeningitis, adhesions between the leptomeninges and the cerebral cortex.

The cerebro-spinal fluid is increased.

A general myxocdematous condition is usually present under the skin.

\section{MICROSCOPICALLY.}

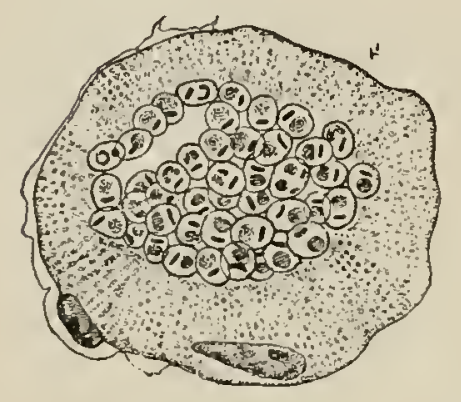

Trypanosoma cruzi. Transverse section of a strialed muscle containing rounded forms of the parasite in the central portion. $\times$ I, 000 approx. (After Vianna.)

\section{SYMPTOMATOLOGY.}

Acute.-There is a violent attack of fever in a child or a newcomer.

The fever shows a morning remission and an evening rise.

The thyroid gland enlarges.

The face becomes odematous with characteristic crepitations.

The lymphatic glands enlarge, especially in the neck.

There may be fugitive aedemas of any part.

The spleen enlarges and becomes painful.

The same takes place with the liver.

Sometimes there are signs of meningitis.

Sometimes there is albumin in the urine.

The fever passes off to recur at intervals.

During the attack trypanosomes are found in the blood.

The child eventually dies, recovers or the condition becomes:-

Chronic.-There is marked thyroiditis accompanied by loss of hair. Hypertrophy of the lymphatic glands continues. There is a dull expression, a bluish-bronze tinge to the skin, tachycardia, intestinal and nervous disorders, convulsions.

Chagus says that the chronic stage may show group symptoms a1s :-

(I) Pseudo-myxodematous.

There is hypertrophy of the lateral lobes of the thyroid gland in 
PATHOLOGICAL MAMMALIAN TRIPANOSOMIASIS 157 quite young but not in older children. A peculiar bronze colour of the skin probably due to the invasion of the suprarenal capsule. The lymphatic glands enlarge in the neck, axilla, groin and the parotid also.

(2) Myxodematous.

The thyroid gland atrophies with the usual classical symptoms of myxadema, e.g., rough skin, loss of hair, firm aedema, arrest of mental development in younger children, mental degeneration of older ones. There may be inflammatory eye troubles.

(3) Cardiac.

There is arrhythmia, alarrlythmia, extra systole, sinus irregularities.

(4) Nervous.

Spastic paralysis in the legs.

Athetosis in the arms.

Aphasia, pseudo-bulbar paralysis, suprabulbar paralysis.

(5) Chronic with acute and sub-acute exacerbations.

Preponderance of fever.

This variety causes a heavy mortality.

The parasites are rarely found in the blood.

It has a long history.

It usually occurs in patients showing marked enlargement of the thyroid gland. Infantilisms may be sequele of the disease.

\section{TREATMENT.}

As for African Trypanosomiasis.

Also treat for hyperthryoidxe and symptomatically.

\section{PROPHYLAXIS.}

Aim at the prevention of the bites of Lamus magistus.

\section{NOTES ON PATHOLOGICAL MAMMALIAN TR Y PANOSOMIASIS.}

\section{TRYPANOSOME EVANSI.}

It causes surra in horses, mules, camels and cattle.

It occurs in India, Burmah, Indo-China, Java, Philippines, Mauritius and North Africa.

The symptoms are: fever, emaciation, adema of the extremities and of the ventral surface, eye lesions, great muscular weakness, paralysis and death.

The treatment is by arsenic in big doses.

It is transmitted by the bites of flies and fleas. Some suggest by the ingestion of infected meat also.

\section{TRYPANOSOME BRUCEI.}

It causes nagana (weakness).

It occurs in Zuzuland, North Transvaal, Pretoria to Nyasaland, 
the basin of the Limpopo, German East Africa, British East Africa and Uganda.

The carrier is Glossina morsitans and the Tabanidæ.

The trypanosome gives rise to:-

An acute disease in mice, rats, dogs, monkeys and cats.

A subacute disease in rabbits, guinea-pigs, equines and pigs.

A chronic disease in cattle, goats, geese and fowls.

It is fatal to horses, asses and dogs.

The symptoms are: fever, infiltration of coagulable $1 \mathrm{ymph}$ in the subcutaneous tissues of the neck, abdomen and extremities, rapid destruction of the red cells, extreme emaciation, and often blindness.

The treatment is by arsenic.

\section{TRYPANOSOME EQUINUM.}

It causes mal de caderas, a disease of the hind quarters in horses and dogs in South America.

It is spread by the ingestion of the infected meat of dogs, also by fleas. Some blame the Tabanida and Stomoxys.

It is very fatal to horses.

The symptoms are: rapid weakness, fever, paralysed hind quarters, staggering gait, stumbles and falls. Albuminuria, hæmaturia, eruption on the neck, shoulders and hind quarters not uncommon. Conjunctivitis and chemosis. There are serous exudations into the serous cavities and the spinal canal.

A mare will die in two months after the paralysis begins.

\section{TRYPANOSOME EQUIPERDUM.}

It causes dourine or mal du coit in horses.

It occurs in Europe, India, North Africa, and North America.

It is spread by coitus between stallion and mare, not by flies, hence the trypanosome is capable of penetrating a healthy mucous membrane.

The incubation is eleven to twenty days.

The symptoms are: edema of the genitals, which is painless; it is not inflammatory; there is some fever; this lasts one month, when weakness and emaciation begin.

An eruption comes in about forty to forty-five days, in circular areas, with oedema about the flanks, hind quarters, neck, shoulders and thighs. It is often transient, and lasts about one week in all.

There is synovial engorgement of the joints and tendon sheaths.

There is enlargement of the lymphatic glands, particularly of the inguinal glands.

Anæmia and paralysis then set in. The mucosa are pale, and marked emaciation is rapid. There are superficial abscesses, nonhealing in character, conjunctivitis, ulcerative keratitis. 


\section{ESSENTIALS OF TROPICAL MEDICINE.}

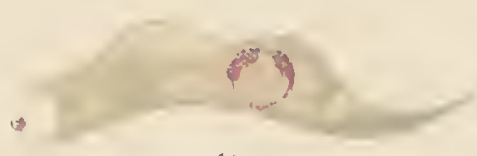

(a) $T$. lezvisii. The antiflagellar end is sharply pointed, the centrosome usually at some little distance from the end of the body, and the nucleus is always in the Aagellar half of the borly. Size 25 to $30 \mu$

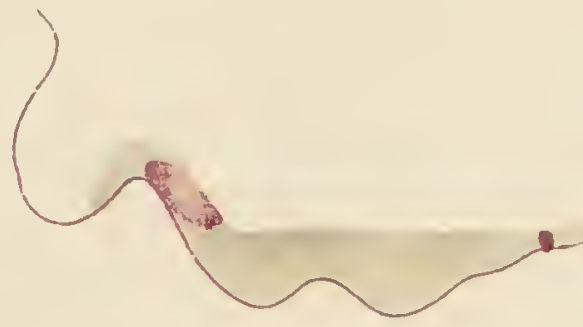

t.

(b) T. gambiense. The antiflagellar end is usually bluntly rounded, the centrosome close to that end, and the nucleus at the middle of the body. Size 20 to $25 \mu$.

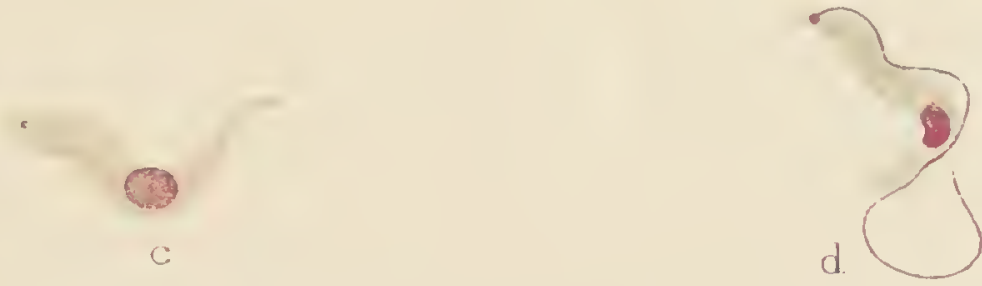

(z) T. nanum. Characterized by its small size, being only about $14 \mu$ in length. The centrozome is small and the nucleus is round and in the centre of the body.

(d) $T$ : theileri. Characterized by its large size, being some $6_{5} \mu$ in length.

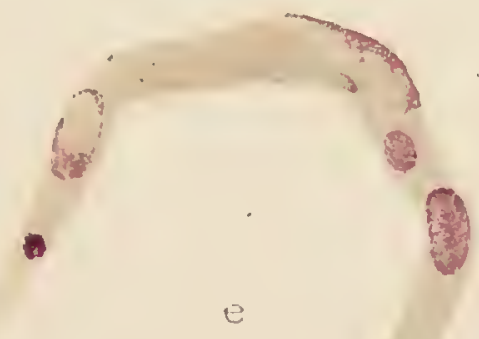

(e) $T$. dimorbhor. The imprtant characteristics of this type are the broad body, the short free portion of the flagellum, the close application of the undulatung membrane to the body and the presence of at vacuole between the centrosume and nucleus.

$$
\text { Bale \& Dirnolsisuri, td. del.et hth }
$$

Face page 158. 

Micturition is difficult; the urine is thick.

Sensibility is diminished, paralysis comes on due to softening of the cord. The animal dies in two to eighteen months from the onset.

The disease is always fatal.

The condition may be more acute than as described, with death after a few days from the eruption from acute paralysis.

TRYPANOSOME CAZALBOUI (T. vivax of Bruce).

It causes disease in horses and cattle in West Africa.

It is carried by Glossince.

TRYPANOSOME SOUDANESE (T. evansi of Bruce).

It causes tahaga in dromedaries and zausfana in horses.

The former disease occurs in the Upper Niger, and the latter in Southern Algeria.

It is carried by the Tabanida.

\section{TRYPANOSOME HIPPICUM.}

It causes murrina amongst mules in North America.

It is spread by flies which suck wounds and by coitus.

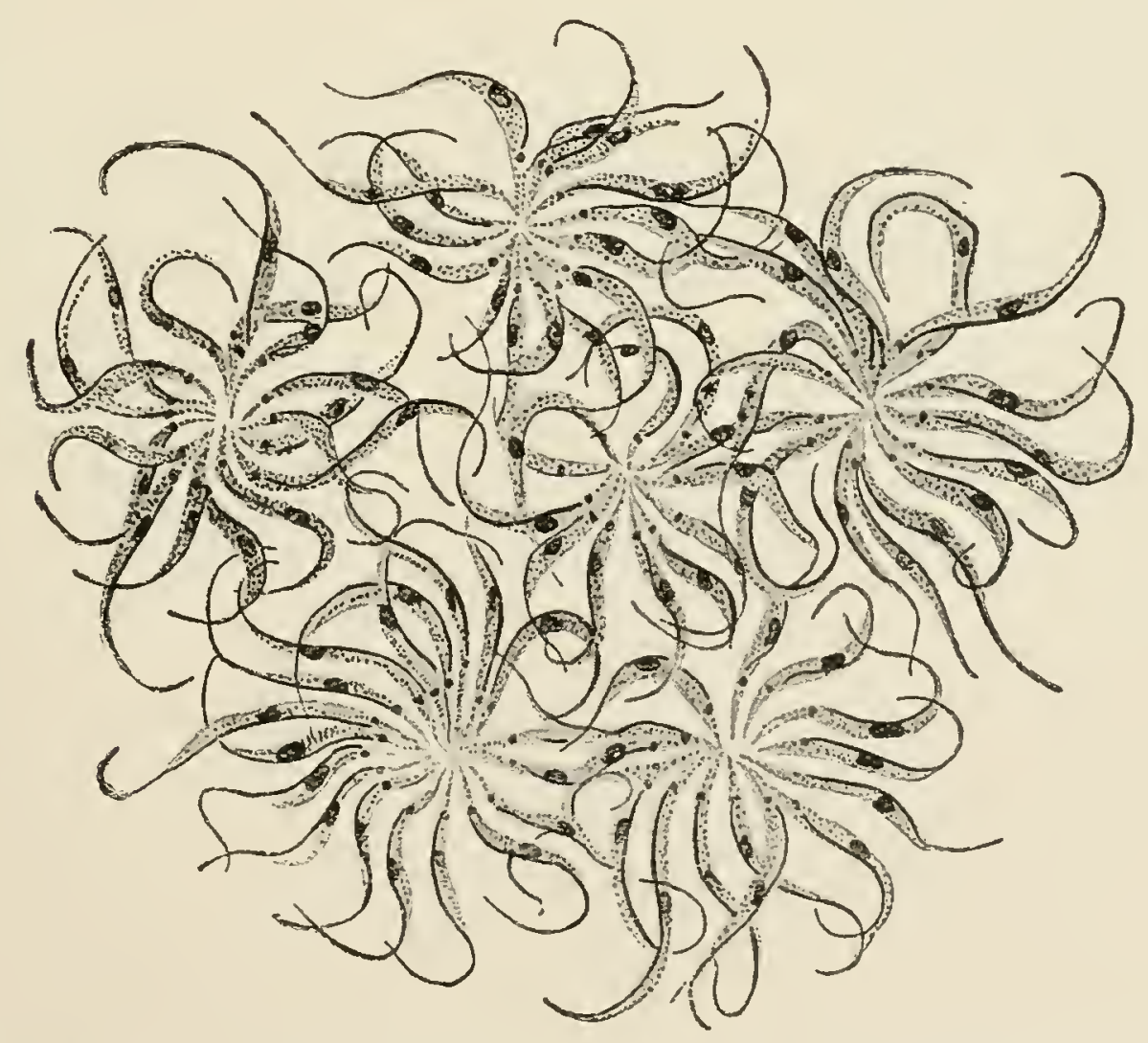

Trypanosoma lewisi. Multiplication rosettes. $\times$ I,000. (After Laveran and Mesnil.)

\section{TRYPANOSOME CONGOLENSE.}

It causes trypanosomiasis amongst horses, cattle, goats, sheep and dromedaries in French and Belgian Congo, N.E. Rhodesia.

It is carried by the Glossinæ. 


\section{TRYPANOSOME DIMORPHON.}

This attacks cattle, dogs, sheep, pigs and goats in several parts of Africa. It is carried by the Glossina palpalis.

In the horse there is loss of vigour, in two or three weeks there is fever, the next month there is marked weakness, abdominal swelling, the testicles hang down and are odematous, the coat is staring, the whole appearance is apathetic. There is fluid in the serous cavities. Death takes place in about one year.

There is hypertrophy of the glands, fatty liver and congested lungs.

Probably the $T$. pecorum of Bruce includes the $T$. dimorphon, congolense and confusum of the above descriptions. The point is still sub judice. 


\section{SECTION 11.}

\section{DISEASES DUE TO BACTERIA.}

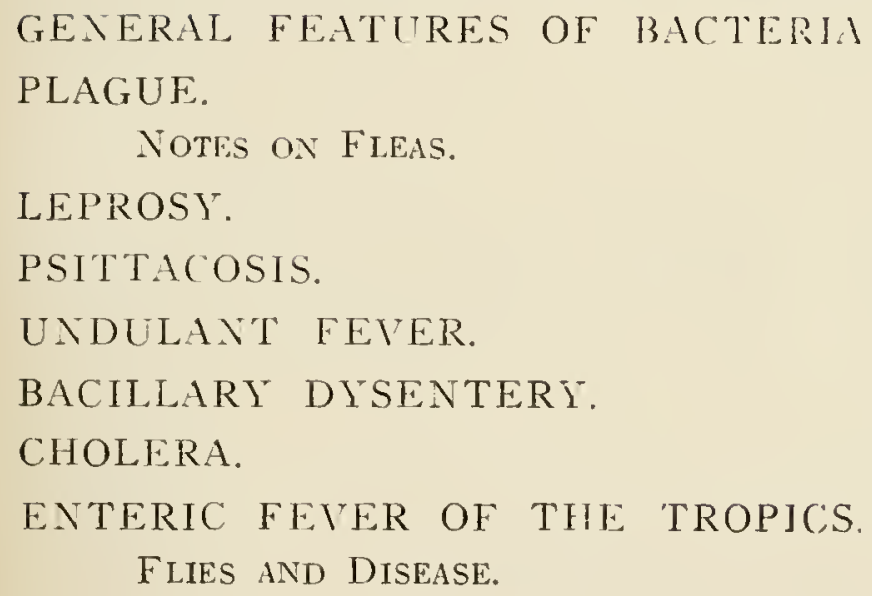




\section{DISEASES DUE TO BACTERIA.}

GENERAL FEATURES OF BACTERIA.

(After E. W. Hey Groves.)

\section{GENERAL CHARACTERS.}

Fission fungi. Devoid of chlorophyl. Unicellular vegetable organisms. Consist of protoplasm surrounded by a cell wall.

\section{REPRODUCTION.}

(a) By simple fission.--The common method.

(b) By Spore formation.-Only found in a few bacilli.

Never found in common pyogenic bacteria.

Occurs, e.g., in B. anthracis, B. tetani, B. oedematis maligni. Spores are formed when conditions for bacterial life are unfavourable. They have a thick capsule, and are very strongly resistant to desiccation, heat, or antiseptics.

(c) By budding, e.g., yeast.

\section{LOCOMOTION.}

Passive.-By Brownian movement, physical or chemical currents.

Active.-By flagelle, i.e., actively contractile protoplasmic processes.

Found in B. typhosus and B. coli.

\section{SHAPE.}

Cocci. Round; never produce spores. Gram +

Staphylococci; arranged irregularly.

Diplococci; arranged in pairs.

Streptococci; arranged in chains.

Tetracocci; arranged at four corners of a square.

Sarcina: B. arranged at eight corners of a cube.

Bacilli. Rod shaped, often spore-bearing. Gram + and -

Streptobacilli; chains of bacilli.

Leptothrix; long threads.

Spirilla; spirally curved.

\section{PHYSIOLOGY.}

They require organic proteids for food because they have no chlorophyl.

Parasites obtain it from living animals or plants.

Saprophytes obtain it from dead tissues.

Facultative parasites ordinarily live on dead tissues, but can also grow in living, e.g., B. proteus. 
Facultative saprophytes ordinarily live in living tissues, but can with difficulty be cultivated outside, e.g., gonococci.

Conditions necessary for bacterial existence or activity.

Proteid food in an assimilable form. NVater. Temperature at or about blood heat. Darliness. Oxygen or its absence.

Acrobes, e.g., pyogenic cocci, require oxygen.

Anacrobes, e.g., B. tetani, cannot live in oxygen. But they can often live in company with other bacteria which absorb oxygen even in the presence of air; e.g., B. tetani can grow in a surface wound if it is contaminated by cocci.

Conditions causing a suspension of bacterial activity or growoth.

Absence of pabulum. Dryness. Cold. Sunlight.

Conditions causing bacterial death.

Heat at or below boiling point quickly kills all except spores.

Spores require temperature of $250^{\circ} \mathrm{F}$. or long boiling to kill.

Chemical reagents, e.g., carbolic acid, mercury salts, etc., must be of a certain strength; act for a certain time and come into intimate contact.

In all conditions in which the living tissues are more potent than the bacteria.

Products of bacterial activity when growing in living tissues.

Acids or alkalies, e.g., B. coli produces an acid.

Gases (rare), e.g., B. coli and B. oedematis maligni.

Pigments (rare), e.g., B. pyocyaneus.

Aromatics. Indol, phenol. Alcohols.

Ferments. Diastase, curdling ferment, proteolytic ferment.

Ptomaines. Crystallizable alkaloids.

Toxins. The specific poisons, probably albumoses.

(a) Intracellular toxins, e.g., tuberculosis. The toxin remains closely associated with the bacillus, and only acts in its neighbourhood.

(b) Extracellular toxins, e.g., diphtheria, tetanus.

The toxin is found in the body fluids, and acts at a distance from the bacteria.

\section{THEORIES OF IMMUNITY.}

The tissues acquire two methods of defence.

(1) They kill the bacteria ; antibacterial action.

(2) They neutralize the toxins; antitoxic action.

Antitoxic action is an actual chemical antithesis to the toxin produced in the blood by infection.

This is a proteid, and is called antitoxin: it can be made to neutralize the toxin outside the body.

Best exemplified in diphtheria and tetanus. 
An:i-bacterial action is brought about by two agencies :-

(I) Phagocytosis. The leucocytes digest the bacteria.

(2) Action of anti-bacterial substances in the blood serum which kill and dissolve the bacteria outside the leucocytes.

Bacteriolysins. Exemplified in anti-streptococcus, anti-cholera, anti-plague, anti-pneumococcus, and anti-typhoid sera.

Phagocytes. Is increased by the action of a substance called an opsonin produced by the action of the bacteria and the toxins in the blood.

Opsonic Index is the proportion of bacteria devoured by leucocytes in an infected blood to those devoured by leucocytes in normal blood.

Directly after an injection of a raccine (bacteria of toxins) the opsonic index falls = negative opsonic phase. During this time a further dose of vaccine does harm.

After a few days the opsonic index rises = positive opsonic phase. During this time a further dose of vaccine increases the phagocytosis.

\section{DISTRIBUTION.}

In air and water. Plentiful in proportion to warmth and proximity to organic life.

In earth. Always abundant near the surface.

In animals (apart from disease).

On and in the skin, hair follicles, and sweat glands.

Mouth, especially round the teeth.

Whole alimentary canal. Most numerous at the end of the small and beginning of the large intestine.

Lower parts of the urethra, vagina and nose.

\section{INFECTION.}

The invasion of the living tissues by bacteria in such a manner as to produce local or general disease.

\section{CONDITIONS NECESSARY FOR INFECTION,}

(1) Dose of bacteria. Bacteria must be introduced in a certain number, or they will be destroyed before they produce any effect.

(2) Virulence.-This varies enormously naturally.

May be increased artificially: By passage through successive animals.

May be decreased artificially : By cultivation outside the body, especially if done under unfavourable conditions.

\$3) Pathogenicity.-Only certain kinds of bacteria are capable of producing disease in certain kinds of animals. 
(4) Susceptibilit! of the host-i.e., a rulnerability of the living tissues by the bacteria.

Increased by: Any constitutional depression, cold or wet, starvation, youth of the patient, alcoholism, narcotics and constitutional diseases, e.g., Bright's disease or diabetes.

(5) Entrance of the bacteria into the tissues, provided by:(a) A wound or abrasion or ulcer. (b) An inflamed or injured part, e.g., an inflamed tonsil or a sprained joint. (c) Profound constitutional depression, allowing the normally present bacteria to enter and multiply in the tissues.

\section{IMMUNITY.}

Is a condition of absolute or relative insusceptibility of the individual 10 infection after invasion by virulent pathogenic bacteria. There are two varieties:-

Natural Immunity.-Racial or individual.

Acquired Immunity.-Aclive immunity; passive immunity.

Natural Immunily.- Nature of the condition is not known. No antitoxic or antibacterial substance can be separated from the blood.

Is lessened by cold or wet, starvation, youth, hamorrhage, alcohol and poisons.

Actioc Immunity.-Caused by a previous infection, either as the natural or modified clisease.

Inrolves an actual illness. Takes time for its development. Lasts many years.

Is brought about by:--

(a) Previous attack.

(b) Giving the disease, e.g., old-fashioned inoculation of small-pox.

(c) Injection of attenuated virus, e.g., vaccination for small-pox, anthrax (in cattle), hydrophobia.

(d) Injection of dead bacteria, e.g., plague, typhoid, tuberculosis.

(e) Injection of toxins, e.g., in the animals which provide the antitoxins of diphtheria and tetanus.

Passive Immunity.-Caused by injecting the blood of an activelyimmunized animal. Inrolves no illness. Follows immediately on injection. Only lasts a few months.

Is most successful against extracellular toxins, e.g., diphtheria and tetanus.

\section{DEFINITION.}

\section{PI.AGLE.}

A septicamia caused by the Bacillus pestis, spread amongst men and other animals by heas, characterized by fever, rapid course, anc? high mortality. 


\section{HISTORICAL FEATURES.}

There is a coin of Lucius Severus showing Asculapius, God of Medicine, with the feet on a dead rat and a nude human figure in terror by his side.

The Greeks worshipped a rat-killing Apollo who was reputed to bring and remove plague epidemics.

Ir 40 B.c. the Bible records an epidemic in the country of the Philistines which produced buboes in man and killed rats (mice of the field), when 50,070 men died ( 1 Sam. v. 4).

7 IO B.C. perhaps Sennacherib's army was attacked by a similar pest (2 Iings xix. 35).

542 A.D. there was an outbreak in Pelusium, a great Egyptian market, whence it spread to Byzantium, the City of the World, into Asia, North Africa, Western Europe, Ireland, lasting in epidemic form about 200 years.

IIth to Ifth century it was pandemic, declining and leaving Western Europe in the i th century.

I403 A.D. the Venetians erected the first lazaretto for the isolation of the sick by a quarantine of forty days, also disinfecting clothing and merchandise. A yellow flag was to be hoisted by ships coming from a foreign port, and both crew and passengers inspected.

The sacred Hindoo book, the "Bhagavat Purana," believed to be Soo rears old, speaks of plague in India. It advises the vacation of the house wherein dead rats were found. It is now endemic in India. There were epidemics:-

I825, in Delhi.

${ }_{1} \delta_{3} 6$ in Rajputana and in Rohilcund, the Pali Plague, at the same time.

I736-1 809 it was introduced into China by Mohammedans returning from Mecca. Since endemic in Junnan, it has spread southwards, reaching,

I867, Pakhoi.

I894, in June, Kitasato found the Bacillus pestis in Hong-Kong.

I 896 it spread from China to Bombay, hence over India.

$189 \&$ the pandemic spread from India to Madagascar and Mauritius. I899, Malay States, Philippines, Australia, United States, South America.

I9oo, Capetown, Glasgow.

1907, almost world-wide.

Tripoli and Uganda are endemic areas in Africa.

rgog, in Manchuria, the pneumonic form was prevalent. The hot season of the tropics and the winter of the Temperate Zone are deleterious to its spread. The bacilli rapidly disappear from the stomach 
of the flea above $85^{\circ} \mathrm{F}$; a at $10^{\circ} \mathrm{F}$. they are virulent. Ifigh temperature causes adult fleas to refrain from laying eggs and prevents larve from developing. Below $50^{\circ} \mathrm{F}$. fleas do not become infected when sucking blood.

1896-1905. During these ten years in Bombay, I, 154,613 people died of plague. Hygienic conditions have forced plague back from Europe, where it was once more prevalent than in the tropics.

From I 896 to I9I 2 plague in India killed $7,000,000$, or about 7 per minute.

I664-I679, the last time plague was in England, 7o,ooo people died in London.

The effect made upon English national life by this attack was most marked. Every English hedgerow is a reminder of plague; they mark the change in land tenure which followed the Black Death. The loss of labour by the plague drove out rilleinage and serfdom, after which it became necessary to define the fields. From that period dates the emancipation of the English labouring classes.

Plague helped to lill the textile industries of the Eastern Counties, and laid the foundations of the modern prosperity of Lancashire and Yorkshire.

It facilitated the growth of English literature. Up to the time of the plague French was the principal language of the schools and the wealthy, but so many teachers died in the epidemic that a new race of English teachers arose.

I 802 , Whyte communicated it to himself, and died while conducting an experiment.

${ }_{1} \delta_{35}$, at Cairo, two condemned criminals were inoculated, but recovered.

I $89 \&$, in a Vienna laboratory, the pneumonic type was contracted from cultures. There was no epidemic present.

Plague does not spread well in sanitary districts.

It is not nearly as contagious as small-pox and scarlet fever.

Igro-IgII. The Plague in China was of the pneumonic type, the most severe. The great majority of the natives and Europeans attacked died, about $+6,000$ in all. The epidemic was brought by the hunters of the marmot, which is an animal susceptible to epizootic plague.

The disease was spread by the hunters when they returned home.

No instance of plague was found amongst the rats examined.

Rats are undoubtedly to blame in India and elsewhere. Their extermination is a problem. At Tokio, during five years, there were 4,80o,00o rats killed at a considerable financial outlay, but even then there was not any reduction noticed in the rat population. 
The isolated cases of pneumonic plague which occur in Siam do not appear to have any tendency to cause extension of the disease, and persons coming into intimate contact with such patients do not become infected. The Manchurian experience was very different (Manuad).

1914. During the plague in New Orleans, there was one quarter where, although the people were living over a hotbed of infection, no human case ever occurred. It is suggested that the rat population was so large that human blood did not present any temptation to the rat fleas, as their natural hosts, the rats, provided ample food (Akin).

1916. There was plague in Bristol during July in humans and rats. Active measures eradicated it. Eight out of seventy-four rats caught in the rag factory were infected. The 200 tons of rags were then destroyed by fire, an excellent means of disinfection.

This is the twelfth British seaport in which plague has occurred within the past fifteen years, apart from a sharp pneumonic attack in Essex about ten years ago, when a few people died and several varieties of animals were found infected.

History shows that plague is wont to visit a country once in 300 years; the last epidemic occurred in Britain some 300 years ago; another is due now (Cantlie).

\section{THE CAUUSATIYE BACILLUS.}

The B. pestis of Kitasato and Jerson is found in the initial cutaneous vesicle, buboes, spleen, blood and sputum in pneumonic cases.

Monkeys, rats, guinea-pigs have been successfully inoculated with typical symptoms.

Bovines and equines give local reaction only.

Canines, birds and reptiles are apparently immune.

Human plague is an offshoot of rat plague.

In glucose the bacillus forms acid, but not gas.

In lactose it gives no reaction.

In broth with cocoa-nut oil it forms flocculent tapering masses.

Haffkine's stalactite growth, very delicate and very readily broken off.

The pseudo-tuberculosis bacillus is much the same; inoculating guinea-pigs is required to differentiate them; this kills in three weeks, the plague bacillus in three days.

B. pestis is a short, oval rod with rounded ends, capsulated singly, but in cultures they grow in chains. It does not form spores. No true motility. Stains readily with basic aniline stains. Gramnegative; grows on ordinary media at body heat; flourishes best in 


$$
8
$$



oxygen; readily lilled by heat; strong resistance against cold. Gains entrance through skin abrasions, more often flea bites.

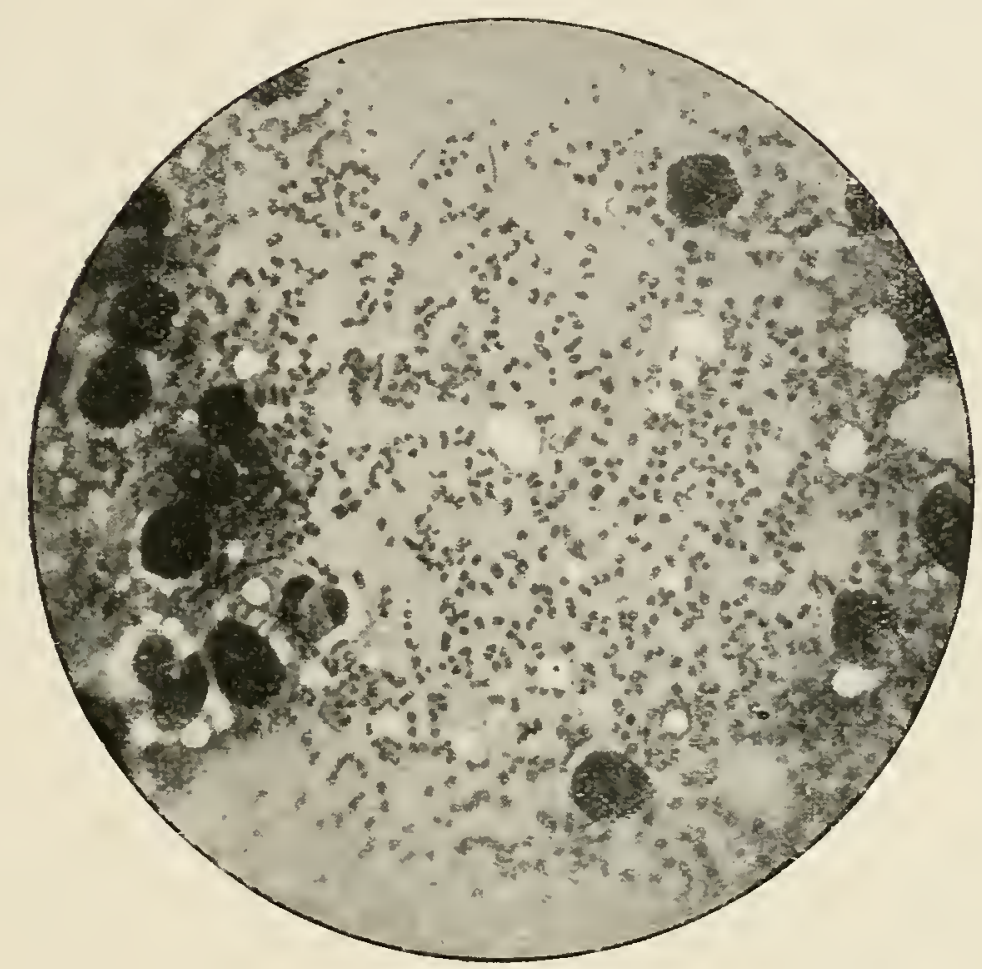

Bacilius pestis. Smear from gland juice, I/12 oil immersion.

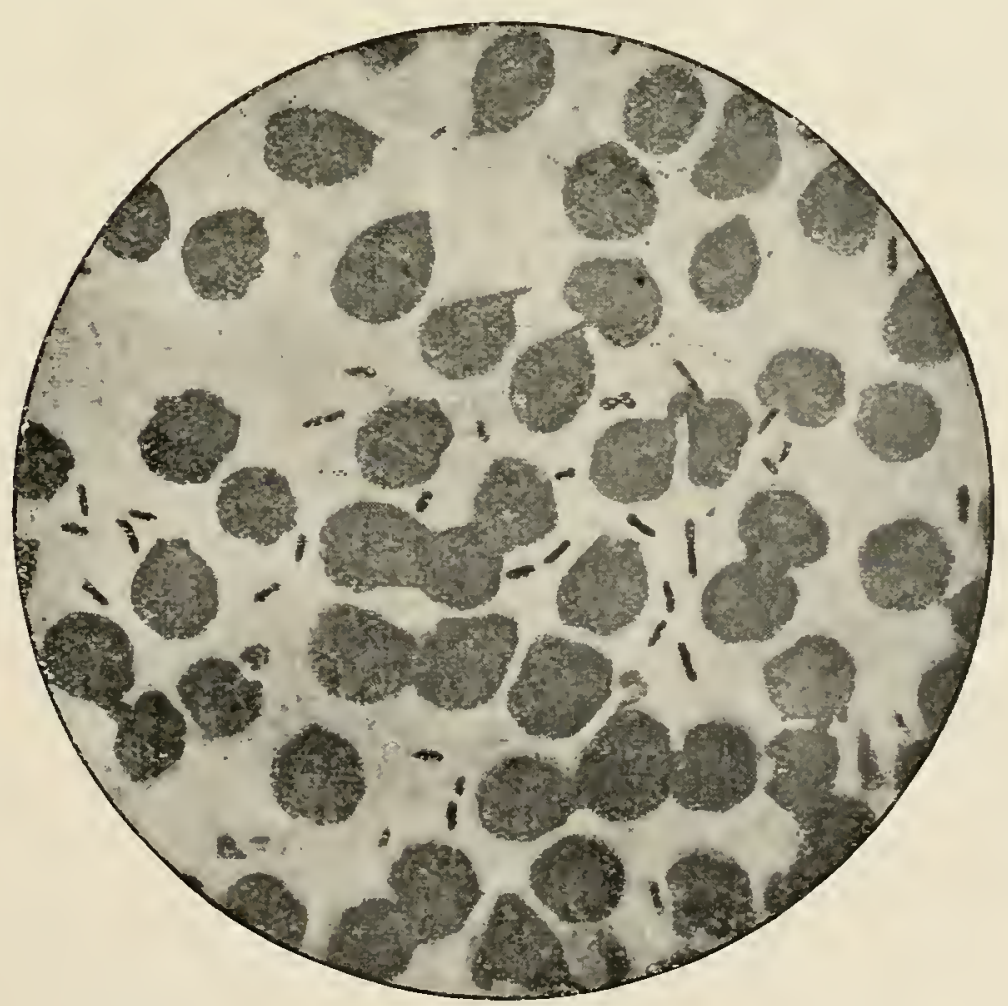

Bacillus pestis. Blood film, 1/12 oil immersion.

\section{PLAGUE EN RATS.}

Acute and chronic.

In the acute type bubo is found in the neck or axilla in $8_{5}$ per cent. of infected rats. There is subsequent congestion, purplish-red 
muscles, waxy, mottled, or finely granular liver, hamorrhages, and pleural effusion. Bacilli in bubo, spleen and blood.

In the chronic Iype.-Encapsulated caseous foci, abscesses with bacilli in viscera, but it does not spread the disease.

Temperature largely affects the prevalence of the disease.

In Bombay, from Desember to May, 1, 766 infected rats were found in one reek during which a search was made.

In the cooler season, from June to November, they are not so prevalent, there being only trenty to thirty found during the week's search.

All rats are not equally infected. Epimys norvegicus and E. rattus are the principal species. The former carries twice the number of fleas, and infection is more rapidly spread by it.

E. rattus is more common in Bombay, lives in houses, less seldom in stables and outhouses. Breeds all the year, and averages a family of five.

E. norregicus lives outside houses as a rule, in sewers, drains and stables. It is never found above the third floor of a house. It breeds all the year, with an average family of eight.

One species infect the other solely by the flea. Infected urine, faces, air, soil and food will not give plague to a healthy rat.

The flea cannot jump more than + inches. If rats are suspended above this height from infected fleas ther do not become infected, hence the disease is not air-borne.

Infected fleas by biting heallhy rats produced the disease in 55 per cent.

The blood of the plague rat contains $160,000,000$ bacteria per c.c.

The stomach of a flea holds $0.5 \mathrm{~cm}$. of blood.

Hence a gorged flea can receive 5,000 bacilli.

They escape solely in the faces of the flea. Multiplication of bacilli takes place in the stomach and intestines of the flea.

Infected fleas transmit disease in from seven to fifteen days.

Take spleen smears and examine for $B$. pestis.

Do a Gram stain; if positive, the organisn is not plague.

Then grow cultures and inoculate guinea-pigs.

In spleen snears there may be clumps of bacteria from lymphatics and ressels. No other bipolar staining organisms give this.

Plague in domestic animals in Bombay either does not occur or has little significance.

Some Indian rats harbour B. pestis without symptoms, but if injected into other animis i: lills them.

Young rats suckled by infected mothers are not infected if fleas are absent. 
Cold dead rats are harmless, as the flea has forsaken them for another warm host.

The Indian Musk Rat, Crocidura coerulea, is highly resistant to plague. Only one rat was found to be naturally infected with plague by the Indian Plague Commission (Kerandel).

\section{THE CARRIER.}

The flea most common in rats is Zenopsylla cheopis, but others, as Ceratophyllus fasciatus and Pulex irritans, are capable.

Infection by the flea is due to faecal infection of the proboscis or of the wound made by it, which transmits the bacilli to the skin.

Humans are infected by the 7 . cheopis from the Epimys rattus.

It is found on humans after cases of plague in man.

Plague infection may persist in fleas for one or two months in the cool weather, and subsequently give rise to an epizootic (Bacot).

$P$. restimenti is capable of transmitting plague infection also (Swellengrebel).

Air, lowever, does carry the bacillus in the pneumonic type from the sputum. The bubonic or septicamia is not spread from man to man, but from rats to man.

Bugs can also act as carriers, C. rotundatus.

In California the ground squirrel. Citellus beecheyi, is subject to plague, and its common flea, C. acutus, spreads it from one to another and will bite man. In Manchuria plague started among those who handled the Tarabagan (Arctomys bola); no infected rats were found. The epidemic was pneumonic.

\section{PATHOLOGY.}

The site of the flea bite is sometimes marked by a vesicle, the contents containing $B$. pestis in large numbers; the bacilli travel by the lymphatics to the nearest glands; some pass to the thoracic duct, thence to the blood-stream, and so cause a septicamia. More ofter they remain and multiply in the lymph glands and the peripheral lymph sinuses. Their toxins cause cell degeneration, periglandular serous infiltration, and later, degeneration of the walls of blood-vessels and hamorliage. The lymphatics are matted by the exudation, femoral, inguinal, axillary, iliac and cervical. The groin glands are infected most, as they drain the largest skin area. The bacilli may get direct to the blood-stream by injuring the veins in the primary bubo. Lymphatic glands also become affected after the infection has become a septicemia. Also the lung causing bronchitis and secondary pneumonia, spleen, liver, kidneys, skin and other organs.

The sputum and saliva can be infected forty-eight days after the 
temperature is normal, and abscesses may contain bacilli ten weeks after.

The chemical pathology is unknown.

In epidemic pneumonic plague the primary infection is in the bronchi from inhalation, setting up peribronchial inflammation.

Lobular and lobar pneumonia develop quickly, and then a septicæmia.

There is no evidence that it has ever started in the great intestinal tract, though many bacilli must have been swallowed.

P.M.

Post-mortem rise of temperature is not uncommon. Decomposition early.

(I) The skin about buboes shows hamorrhage resulting from toxins acting on endothelial cells of vessels. The hamorrhages are petechial or diffuse and contain bacilli. Tesicles, pustules, so-called carbuncles may be seen.

(2) Glands.-The primary bubo shows periglandular infiltation. Glands matted, greyish, soft centre, numerous hamorrhagic spots or large hiemorrhages.

The secondary buboes (those infected by drawing from the primary gland) are degenerate glands, no oedema, endo- and peri-glandular haemorrhages.

The tertiary buboes (those infected after it has become a septicamia) are hard, hyperamic and hamorrhagic.

(3) Muscles, especially those of abdominal walls, are hamorhagic.

(4) Viscera.-Spleen enlarged two to three times the normal size, congested, hamorrhagic, bacilli very numerous, necrotic areas, dotted with pin points.

Liver enlarged, somewhat mottled, hamorrhagic, cloudy swelling, fatty degeneration.

Lungs, some bronchitis, and often patches of secondary broncho-pneumonia.

Effusion into pleural sacs common with hamorrhage.

R. heart dilated, fatty degeneration, cloudy swelling, hamorrhage.

Stomach hyperæmic, hamorrhage, solitary glands and Peyer's patches swollen, mesenteric glands markedly so.

For sections stain with strong carbol thionin, stop its action with oil of cloves and remove the stain therewith.

\section{SYMPTOMATOLOGY.}

Incubation.-Two to ten days, average three days.

Prodromal symptoms.-General malaise, headache, gastrointestinal clisturbance, glandular pain, chilliness, giddiness, mental dulness. 
Onset.- Sudden, temperature on third day rises to $103^{\circ}-104^{\circ} \mathrm{F}$., and falls on the sixth or seventh day (remember pneumonia). Pulse 130, respirations 30-45. Marked prostration, vomiting, diarrhoea, general body pains, rigor and staggering gait. In a few hours after the onset eyes become bloodshot, nostrils dilated, and temperature very irregular; if recovery it may fall by lysis, or if no recovery it falls rapidly to subnormal, and rises rapidly to $107^{\circ} \mathrm{F}$, and death supervenes. Thirst is marked. Tongue furred at first, later sordes on tongue, teeth, and lips.

Abdomen not tender unless there are enlarged abdominal glands. Spleen always enlarged. Liver often.

Cardiac dilatation important.

Pulse may rise to 180 and become thready.

Coagulation of blood diminished. Bacilli can be grown from the blood in many of the cases. Red cells and Hb. increased, leuco-

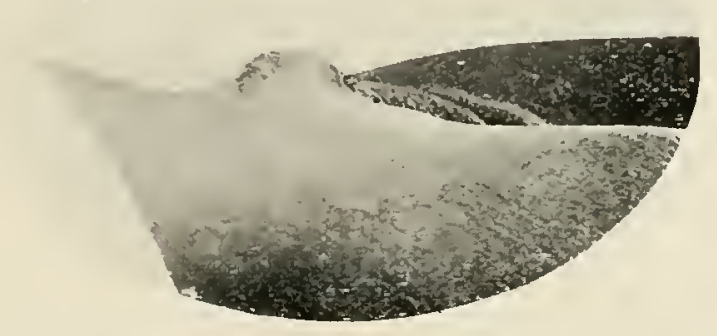

Inguinal plague bubo. (From Prolessor Simpson's collection.)

cytosis is marked 90,000-100,000, but leucopænia may ensue in septicæmic forms.

Breathing rapid, B.S. harsh with moist râles, signs of lobar pneumonia may occur, sputum is then hrmorrhagic and full of bacilli.

Buboes, seen in groin, axillix and neck, are painful. The legs may be drawn up, or the head bent over because of them, when seen in the first twenty-four hours. These glands may resolve or suppurate.

Skin hot and dry, with resicles, pustules, necrotic areas, petechix, ecchymosis, epistaxis, hematemesis, malæna, hæmaturia.

Urine diminished. Specific gravity high; bacilli may be found.

Anuria usually before death.

Pregnant women always abort.

Weakness, wild delirium, coma, death third to fifth day.

If recovery, temperature resolves by lysis, pulse rate is diminished and convalescence begins. Death may still ensue from cardiac failure. suppuration, septic infection, or secondary hacmorrhage. 


\section{YARIETIES.}

(I) Ambulatory Type, the mildest form. - The symptoms can be looked upon as the prodromal symptoms, aggravated somewhat.

(2) Bubonic Type.-The bubo is usually in the groin. The fever maximum is reached on the fourth or fifth day, and lasts till the seventh or tenth day, falling by lysis, and rising again if suppuration sets in.

(3) Septicamic Type. Onset sudden, temperature high, very rapid pulse, marked prostration, severe vomiting, diarhora, hemorrluge, death in one to three days.

Children may be playing in the street in the morning, haemorrhage in the afternoon, and dead next morning.

(4) Pneumonic Type.

(i) Primary pneumonia.

No prodromata; incubation two to five days.

Cough and dyspnoea within twenty-four hours; bloody expectoration with bacteria second to third day.

Conjunctiva injected, respiration rapid, dyspnoea, cyanosis. Spleen not usually palpable.

Very fatal.

(2) Secondary pneumonia (after buboes).

As general symptoms.

MORTALITY varies. Apart from mild attacks it is 60-95 per cent.

Chinese $93^{\circ} 4$ per cent.

Japanese 6 o per cent.

European $18 \cdot 2$ per cent.

Of known and controllable cases in hospitals, jails, \&c., 35-45 per cent.

\section{TREATMENT.}

Bed, good nursing, fresh air.

Fever with sponging and cold applications.

Ieart by digitalis, strophanthus and strychnine.

Restlessness; opiates, chloral, potassium bromide.

Haemorrhage; calcium chloride or lactate.

Constipation; calomel and saline.

Pneumonia; the ordinary treatment.

Kidneys, much fluid to keep them flushed.

Diet; broths, milk, stimulants usually.

Diarrhoea ; ice pills and morphia, hydrocyanic acid, salol. drain.

Buboes. Glycerine and belladonna, when suppurating incise and 


\section{PUBLIC PROPHYLAXIS.}

Institute a sanitary service in an infected country with collectors, bacteriologists, \&c.

Port sanitary authority to investigate and disinfect ships.

Isolate as far as possible.

Quarantine five dars.

Medical inspection of passengers by rail, road and river.

Disinfection of clothing, and merchandise by Clayton's method.

Rats to be caught and examined.

Recognition of early cases, investigations of sudden deaths.

House to house visitation by M. O.

Village houses burned. Kill rats by $S()_{2}, S\left(O_{3}\right.$, Danyz's rirus on bread; encourage cats. Wrork from the outside of a circle. Cover hands with oil of aniseed as rats know if humans lave touched food set to catch them. Phospliorus paste, with glucose to prevent combustion, with lard as a basis. Traps; two to 100 of the population (in proportion).

Prophylaxis as suggested by Haffkine.

(1) Discovery and notification of persons attacked with disease.

(2) Isolation of those attacked.

(3) Certain precautions with regard to the disposal of the dead.

(4) Segregation of those who have come in contact with the sick or dead.

(5) Institution of cordons round infected areas, or

(6) Placing in quarantine arrivals from infected places. Detaining the sick and suspected, liberating the remainder.

(7) Or examine travellers, isolate sick and suspected and let others free under surveillance.

These are always difficult, often impracticable, and achieve little.

Second method. Within the affected area.

Destroy or disinfect houses, furniture, clothing, bedding, carriages, goods, warehouses, grains and other stores, garbage, drains and streets.

Outside the infected area.

Refusal to admit carts, trains and ships with goods from infecied places; or refuse to admit only certain goods; or inspection of trains, carts, ships with measures by which these and the goods they convey, as well as the belongings of travellers, are rendered harmless.

The expense and difficulty of such is enormous.

Third method.

(1) Destruction and leeping away of rats by poisoning, trapping, tar and sulphuric acid mixture, or by the domestic cat. 
(2) Structural alterations of dwellings and stores, demolition of insanitary buildings, prompl disposal of garbage, periodical inspection of stores having drainage with riew of keeping down rats.

(3) Destruction and dispersion of fleas by petroleum, \&c.

(4) Fumigation of houses against rats and fleas.

(5) Obligation of ships from infected regions to anclior allay from the shore.

(6) Provide mechanical meáns for preventing ráts from landing along mooring cables and gangways.

(7) Fumigation of ships arriving with plague patients, plague rats or suspected bedding.

Such would cause a dislocation of traffic and an outcry, formidable to face; the result is that plague is still spread.

If men are honest, rats are not and regulations are broken down.

\section{PRIYATE PROPHYLAXIS.}

Yersin's serum, from a horse inoculated with fresh agar cultures; give 20-200 c.c. intravenously and subcutaneously near the buboes. There may be urticaria and pleurodynia for three days.

For Yersin's serum to have any appreciable effect on the course of. the disease, the first injection must be given within forty-eight hours from the appearance of symptoms.

Lustig and Galcottis' serum, from a horse injected with vaccine. Both these are supposed to stimulate phagocytosis and are not bactericidal.

Lustig and Galeottis' vaccine; shake agar cultures with I per cent. of caustic potash, and after two hours add $0^{\circ} 5$ per cent. acetic acid, and thus obtain a precipitate of nucleo-proteids.

Subcutaneous or intramuscular 2-3 milligrammes.

Haffkine's Prophylactic Taccine.-Grow bacteria on broth and butter fat 4 to 6 weeks; stalactite growths form.

Sterilize at $65^{\circ}-70^{\circ} \mathrm{C}$. for one hour.

Decant into bottles with a little carbolic acid.

Dose.-3 c.c. for an adult man. Subcutaneously about deltoid.

There is a local and general reaction arising in a few hours and disappearing in 1 to 3 days. Reaction may be severe or mild.

This protects from 2 to 12 months and is used much in India. Good results.

It requires to to 4 days to produce sufficient immunity; during that time resistance is diminished. In a Punjab experiment in I903 the mortality was 60 per cent.; in the inoculated it was 23 per cent.

\section{ATTENUATED CULTURES.}

Strong.-Intramuscular injection of one whole twenty-four hours agar slant of living virulent culture. The reaction is not excessive. 
Opsonic index rises markedly ten days later.

Guinea-pigs, mortality 66.6 per cent.; inoculated 16.6 per cent.

Klein's protective inoculation prepared from the organs of dead animals of plague; he sars:-

(I) It requires ten to iwelve days to prepare it; Haffkine's requires four to six weeks.

(2) A large amount can be prepared of uniform strength.

(3) Its efficacy is easily standardized by injection into the rat.

(4) Being dry and sterile it can be preserved without antiseptic and kept unaltered.

(5) That its injection into the rat protects certainly many weeks.

(6) Cost of its preparation is much less than Haffkine's. This has not yet been tried on man.

Masks of flannel cloth to cover the head tied about neck with window of sheet celloidin are advisable.

\section{NOTES ON FLEAS. (Order Siphonaptera.)}

Fleas are active parasites of mammals and birds. They usually have a preference for one host, and attack others with some reluctance.

They act as carriers or necessary intermediaries, e.g.-

(I) The common dog flea and the common European rat flea harbour the cysticercus of certain tapeworms of their respective hosts.

(2) The trypanosome of the rat passes through certain stages of its developmental cycle in the European rat flea.

(3) They are carriers of the plague bacillus among rats and other rodents, and from these to man.

Pulex (Xenopsylla) cheopis most commonly infests house rats in the tropics.

The Pulex irritans (the human flea) and others are capable of carrying the plague bacillus.

\section{EXTERNAL APPEARANCE.}

Fleas are laterally compressed, wingless insects, with two piercing, sucking mouth parts. The head is broadly articulated to the thorax; all three segments of the thorax are distinctly independent. Eyes may or may not be present; when present they are simple and not compound as in the house fly. A comb of teeth is found on the edge of the cheek or on the lower edge of the head in most species.

The antenna, two basal segments and a club with nine rings, lic behind and above the eyes. Bristles are seen irregularly placed about the head and thorax. They have a pair of longitudinally grooved, serrated, needle-like mandibles, which form an efferent tube for saliva, 
and an unpaired bristle which, opposing itself to these, forms an afferent tube for sucking blood.

The three pairs of legs with their paired claws are long and strong.

The abdomen has ten segments; the last three are modified for sexual purposes.

The male has on the ninth segment large claspers and a large complex penis.

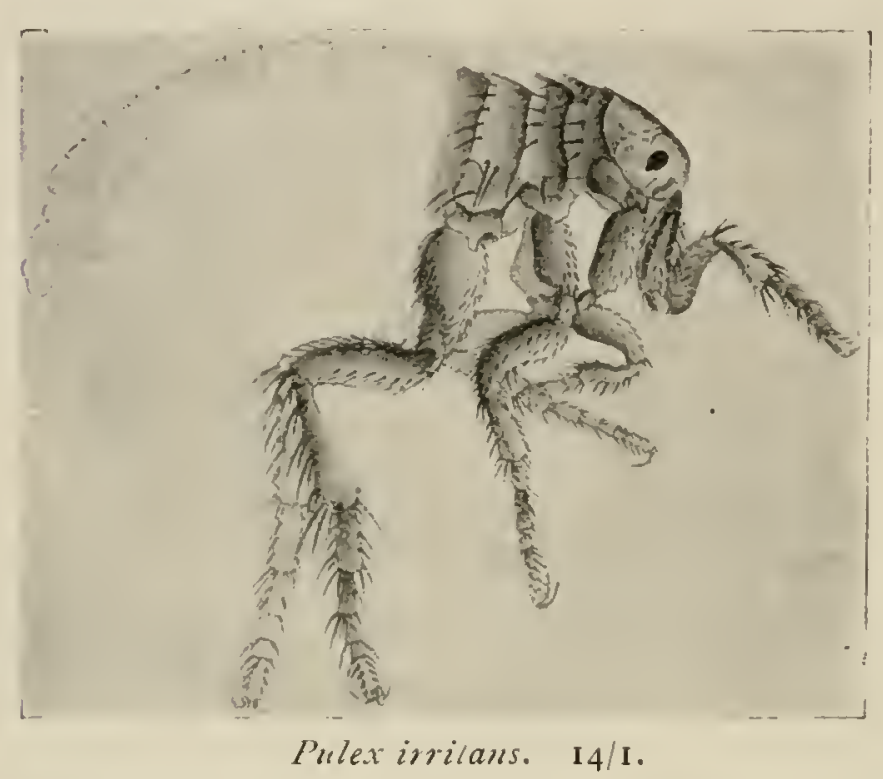

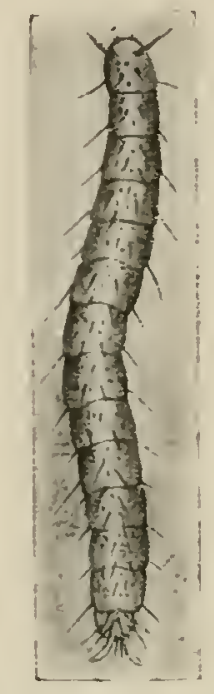

Larva of flea. Enlarged. (After Railliet.)

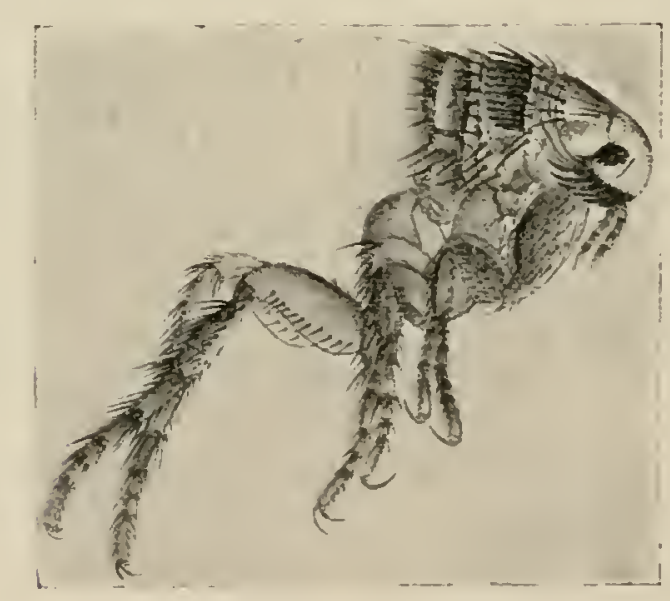

Pulex serraticeps. 22/I.

\section{ALIMENTARY TRACT.}

In the head is an elastic pharynx acting like a suction pump; its cavity, when expanded by the contraction of certain muscles, forms a vacuum.

The stomach is very distensible, the rectum is large. They carry two pairs of salivary glands.

\section{REPRODUCTION.}

The eggs are large, dropped casually in places where domestic animals sleep, hatching in two to fourteen days according to tempera- 
ture. The larve are active, white, hairy maggots with a head and thirteen segments, the last carrying a pair of hooks. They eat organic matter from dust, and spin a cocoon in two weeks or longer, in which they pupate.

The cocoon coated with dust contains a hump-backed pupa which can develop in two weeks.

There are two main families :-

Pulicida, which contains Pulex irritans, the common human flea, and Xenopsylla cheopis, the tropical rat flea.

Sarcopsyllide, which contain the Dermatophilus penetrans or jigger.

Indigenous to South America, but carried to the Gold Coast, East Africa, and Bombay.

It flourishes best in sandy places. It attacks man and other animals, including the pig. Both sexes suck blood, but the pregnant female embeds herself in the skin, often under the bed of a toe-nail, and in this inflamed pocket lays her eggs. Pain and inflammation result, leading to indolent ulceration and even gangrene.

\section{TO DESTROY FLEAS.}

Close the room, spray completely with 5 per cent. solution of formalin, or sprinkle copiously with powdered naphthalin, and leave closed for two days.

Then burn all sweepings. Wash floor with formalin or lysol.

Ants eat the larve and pupa.

For personal use, use quinine, quassia, sulphur, petroleum, which are repellent to fleas.

\section{DEFINITION.}

\section{LEPROSY .}

A chronic infective disease caused by Hansen's bacillus, with characteristic lesions of the skin, mucosa and nerves.

\section{HISTORY.}

Perhaps brought from Egypt to Italy by Pompey's troops, disseminated throughout Europe by the Roman legions. The returning Crusaders (rog8) increased it.

Lepers were isolated in Europe in the $13^{\text {th }}$ century. They were compelled to wear a special dress, to use clappers when on the roads, and to point with a stick to articles in the market which they wished to buy. They were forbidden to drink from the public fountains, or eat with healthy people. They were officially dead, and the burial service was read over them as soon as they were diagnosed as lepers. I.epers gradually diminished from this time onwards. 
The Spaniards perhaps took the disease to America.

In the 16 th century the imported West African slaves carried it to the West Indies, the Chinese to the Sandwich Islands, Colombia, New Zealand, \&c.

It was carried to South Africa by the Dutch from Java in 1756, and by the East Indian troops in the I $9^{\text {th }}$ century.

Hansen discovered a bacillus in $187 \mathrm{I}$, and associated it with the causation of the lepra lesion in 1877 .

The method of infection is unknown.

\section{DISTRIBUTION.}

Present in Ireland in 432 A.D., and in England in 950 A.D.

The last British leper died in the Shetland Isles in 1798.

Present in Iceland, Spain, Italy, Balkans, Crete, Cyprus, Norway, Sweden and Greece. Common throughout Asia, Ceylon, India, Australia (Queensland and New South Wales). The Sandwich Islands in I89 had one in thirty infected, I,500 in all. It is present in the United States, Mexico, Central America, Colombia, West Indies, Venezuela, Guiana, Brazil, Africa and Egypt, which was probably its original home.

In India, in IS9I, there was one leper in every 2,000 people, ro5,000 in all.

In New Caledonia there were no lepers in 1865 . A Chinese known leper went there, and later in I 888 there were 4 ,ooo lepers.

In the $13^{\text {th }}$ century there were 19,000 leper houses in Christendom, according to the Benedictine monk, Mathew Paris.

In France there were 2,000 and in England 95, besides smaller pest houses in Ireland and Scotland (Sir James Simpson).

I 890 , there were I, Ioo lepers in Norway.

I906, there were only 500 there.

I9I5, there were 40 lepers in the United Kingdom (Dr. George Pernet).

\section{THE BACILLUS.}

Hansen's Bacillus lapræ resembles T.B. in size, shape, and staining reaction. Stain as for T.B., but use a weaker solution of $\mathrm{H}_{2} \mathrm{SO}_{4}$, 5 per cent., in decolorizing. For films and thin sections use the $\mathrm{H}_{2} \mathrm{SO}_{4}$ solution for fifteen seconds.

It has been found in nasal secretion, tears, saliva, sputum, milk, semen, urethral and vaginal secretions, fæeces, skin scales, extra- and intra-cellular in liver, spleen, slands, and breaking-down tubercles.

It has not been found in earth, dust, air, water or food.

It is doubtful if it has ever been cultivated.

It is acid-fast, but stains more readily than T.B. with cold, weak 
carbol fuchsin, and is more readily decolorized, is motile, and easily obtained. It is numerous in nodular and scarce in anæsthetic forms.

Not found in muscle, bone or cartilage.

Apes have been inoculated (Nicolli).

\section{HOW SPREAD.}

It cannot arise de novo, hence it must be carried by one or more of the following: air, soil, water, plants, insects, beasts, food, or man. It has only been found in human tissue.

Perhaps contagious. Benson, an Irishman, acquired leprosy in the West Indies, and returned to Ireland. He died from it in eleven months. His brother had lived and slept with him, and later wore his clothes; in four years he, too, was a typical leper. He had never been out of the British Isles.

Note its spread throughout Europe, its rapid spread in the Sandwich Islands. In twenty years Soo lepers were isolated, and one-tenth of the population was affected.

Father Damien went as a missionary from Belgium to the Sandwich Isles in 1873 , and died of leprosy in 1889 .

Ten per cent. of the children of lepers become lepers.

The success after isolation supports the contagion theory.

Against contagion.-The attendants of Hendela Leper Asylum of Ceylon have not contracted it.

There is no proof that sexual intercourse spreads it.

A child has never been born a leper.

It is present among people who do not eat fish.

All possible means of spread have their supporters.

It may in all possibility be spread by insects, ? bed bug.

As lepers are sterile early, if it is hereditary, leprosy would soon die out.

The fish theory is not now held by many. If it is a good medium it should be good for cultivating the bacilli, but it is not. In Abyssinia there are 8,00o lepers who rarely, if ever, eat fish.

\section{PATHOLOGY.}

How and where it enters the body is unknown. Perhaps by the nasal mucous membrane (McLeod).

They form colonies in the lymph spaces, and later are disseminated by blood and lymph vessels.

Few diseases show so many bacilli.

Plasma cells surround capillary vessels and lymph capillaries, which are much dilated.

The Leproma.-Iarge lepra cells containing masses of bacilli. 
A smooth white and brown glistening nodule, hard from fibrosis or soft from degeneration, well supplied with vessels. No true giant cells. The bacilli do not invade the surface epithelium, nor the cutis beneath this, nor sweat glands, nor hair sheaths superficial to the opening of the sebaceous glands.

In section one sees the epithelium normal, but with no interpapillary processes.

Then larger cells of connective tissue free from bacilli.

Then lepra cells, plasma cells, connective tissue cells, vessels with little connective tissue, vessels much thickened, sometimes with the lumen obliterated, and then lymph spaces dilated and filled with bacilli surrounded by mucus forming the "globi."

Beneath this is normal connective tissue.

The leproma may break down the skin and ulcerate, disseminating the bacilli in the discharge.

Bacilli may enter the nerves, causing hyperplasia of the coats, forming thickened leproma about the vasa nervorum, and later, in the perineurium and endoneurium.

These press on the nerve fibres, causing degeneration of the neurilemma, and destruction of the nerve fibres, which become converted into connective tissue.

The bacilli may attack the anterior cornua of spinal cord.

Any organ may be the site of leprotic lesions.

\section{P.M.}

Skin tubercles may or may not be ulcerated. Pigmented patches may be seen. Liver enlarged, bacilli infiltrate portal systems, seen as yellow dots. Spleen also. Bacilli are never found in the liver cells themselves.

Ovaries and testes may show infiltration and fibrosis of interstitial tissue, which destroys sexual elements and causes the sterility so marked in lepers.

The lymphatic glands are infiltrated and enlarged.

The ulnar, median, peroneal, post-tibial and great auricular nerves are affected most.

Posterior sclerosis of the cord and meningitis are not uncommon.

Trophic changes in joints and perforating ulcers.

Lungs may be affected, more solid than in T.B., caseate less frequently, less prone to be destroyed.

\section{SYMPTOMATOLOGY.}

Incubation not known; perhaps two or three years at least.

Begins usually in subjects from ro to 13 years of age, rarely after 40.

Sometimes children are lepers first and parents later. 
Blotches and patches for years may have been disregarded.

Epistaxis, frontal headache, intermittent fever, numbness, abnormal, local or general sweating without sufficient cause.

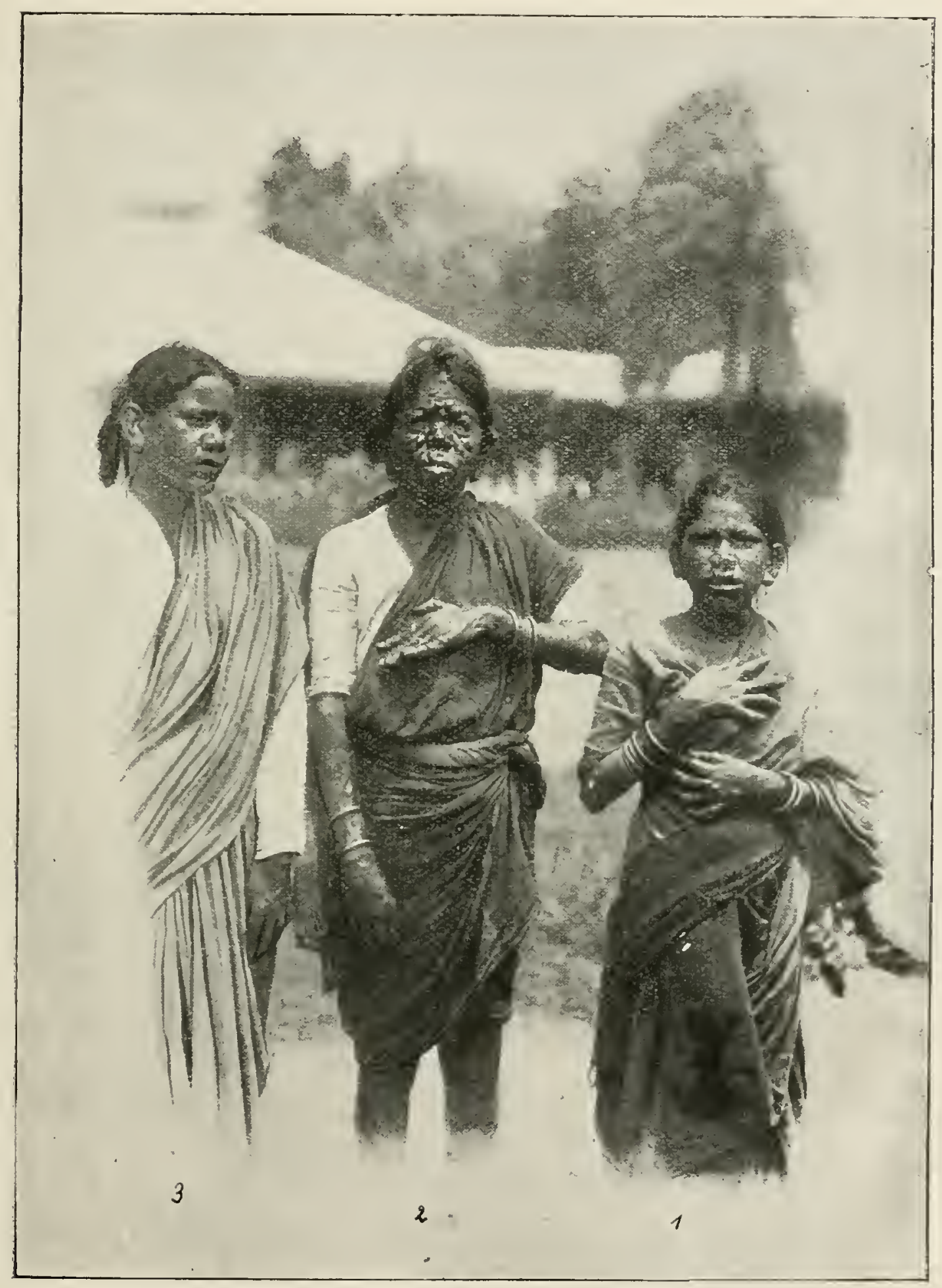

(I and 3) Hindoo girls: (2) Hindoo woman with nodular leprosy.

These may be seen at intervals for several years. The point of entry is perhaps the nose. I 28 out of 153 lepers had the bacilli in the nose first. After general dissemination the bacilli settle in the skin and nerves, giving us the tubercular, anasthetic or mixed leprosy. 
(I) Tubercular Variety.

A severe fever accompanied by an erythematous, diffuse or macular eruption on the face and limbs. This may subside or macules may develop into tubercles.

This is repeated, glands enlarge, fever may sometimes be absent -

Tubercles and nodules come first, usually on the face and forehead, cheeks, alie of nose, lobules or ears, lips and chin. They soon alter the appearance of the patient. The tubercles are the size of split peas, dirty pink or dark brown, resembling sebaceous follicles. Beard, moustache, and eyebrows drop out early. Complete madarosis is not uncommon .

Paralysis of the frontalis muscles is sometimes seen.

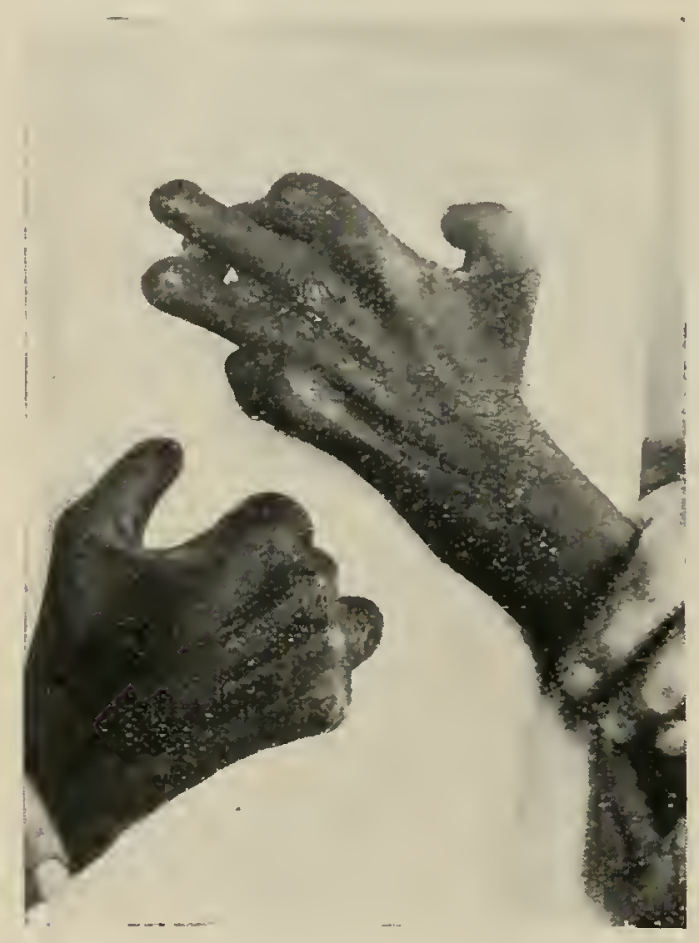

Deformities in anesthetic leprosy.

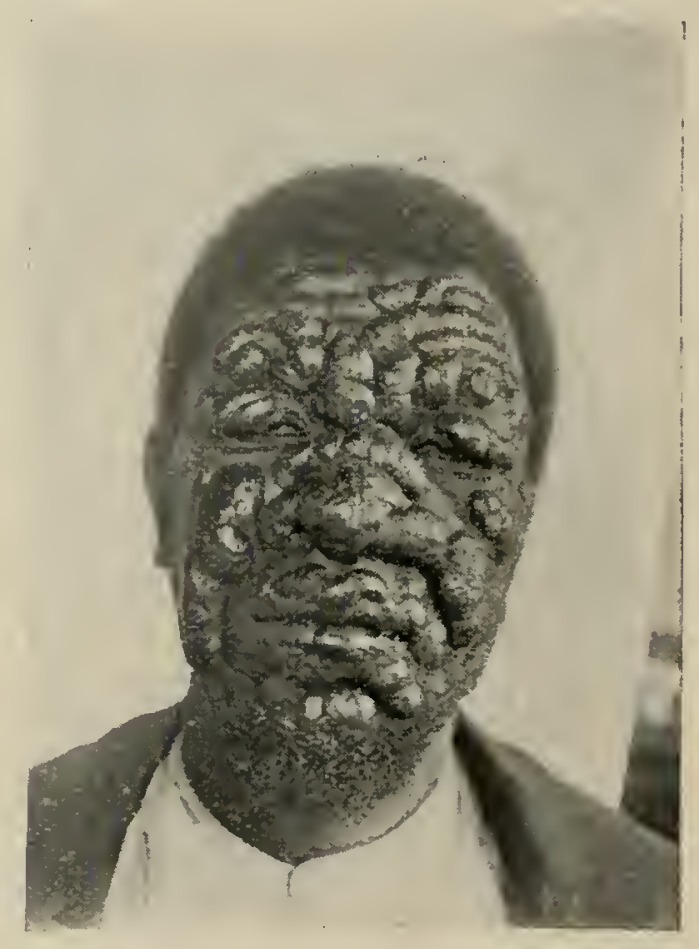

Tubercular leprosy. Marked case.

About the cye.

Eyelids show diffuse or nodular lepromata, these may ulcerate and destroy the eyelids.

Conjunctiox may become infiltrated, ectropion, zerophthalmia.

Episclera infiltrated external to corneo-sclerotic junction.

Small spots on cornea = "keratitis punctata leprosa."

Uveal tract is infiltrated, anterior and posterior iritis may occur; nodules may form in the ciliary body or near the canal of Fontana, causing irido-cyclitis, or irido-choroiditis.

About the nose.

Nasal mucosa shows nodules, occlusion may ensue with ulceration and destruction of the nose, disfiguration from cicatrization. Sense of smell is lost. 


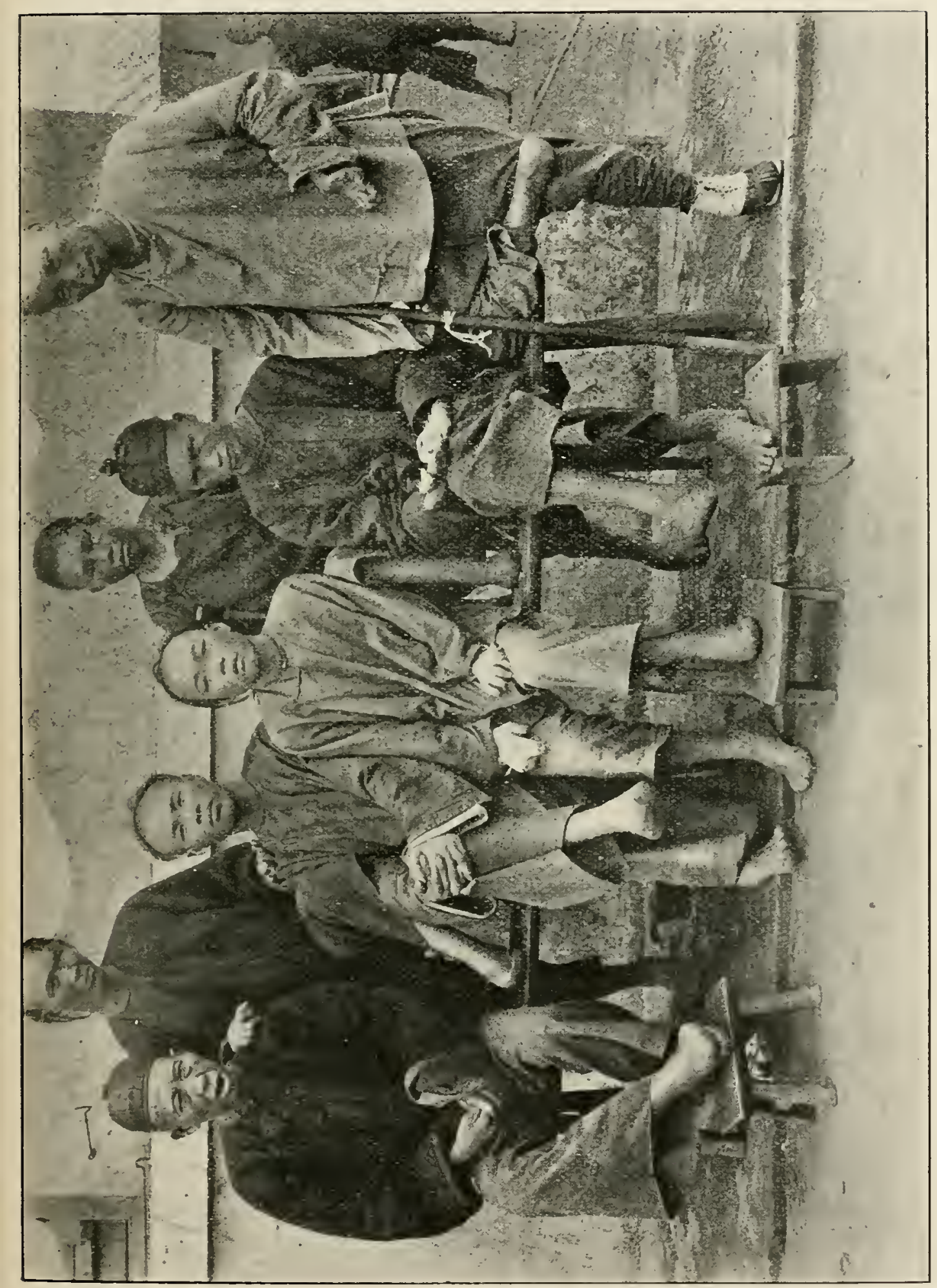

焉 


\section{About the mouth.}

Tongue, mouth and pharynx may be affected, mastication and deglutition difficult; when in larynx the voice becomes raucous, respiration impeded. Lips become paralysed, salira dribbles, gums retract, teeth fall out, taste is lost.

About the skin.

A goat-like odour. Tubercles on hands, arms and legs which may ulcerate.

Submaxillary, cervical and femoral glands may be enlarged and may suppurate.

Testes fibrous, menstruation irregular and may cease.

Fingers and toes atrophy, ulcerate, drop off, then phalanges and metacarpals likewise.

Blood.

Nil diagnostic. No marked or consistent changes.

Nerves may be attacked and add their corresponding signs and symptoms.

Ulcerations may cicatrize and produce deformities or suppurate and produce amyloidosis or become phagedænic causing septic poisoning, gangrene of fingers or toes. Nephritis is common.

\section{(2) ANAESTHETIC VARIETY.}

Infiltration is chiefly in the nerves; fibres irritated at first, then destroyed. Shooting pains of ulnar and peroneal. There are sensory disturbances as burning, numbness, formication; rasomotor disturbances as flushings, glossy skin; and motor disturbances as facial twitchings of muscles.

A macular eruption, flat red spots appear without any general disturbances, some being pigmented. Spots increase in size, centres become pale, the peripheries usually are raised, marked with papules or vesicles, or dry whitish scales. Such areas may coalesce forming patches half an inch to six inches in diameter.

Within these, the skin is anasthetic, hairs fall out, does not sweat.

Wrinkles and scales appear.

Hyperæsthetic outer border may look like a big ringworm.

The disease may become quiescent and fade at any time.

Nerves.-Ulnar nerve thickened, felt behind internal condyle, great auricular felt over sternomastoid. Peroneal below head and fibula, \&c.

With destruction of nerve, hyperesthesia ends and anæsthesia begins.

Noticed first usually along ulnar side of hand and forearm.

It may be patchy or in distribution of nerve. 
Paralyses of interossei of hands (main-en-griffe), thenar and hypothenar eminences, also of forearm causing "drop wrist."

Plantar muscles of toes, peronei, extensors- "drop foot" with an internal twist.

Reflexes increased, later diminished, muscles waste, R.D. distinct.

Trophic lesions, whitlows on fingers occur, necrosis of phalanges, or simple absorption of bone, the nail persisting.

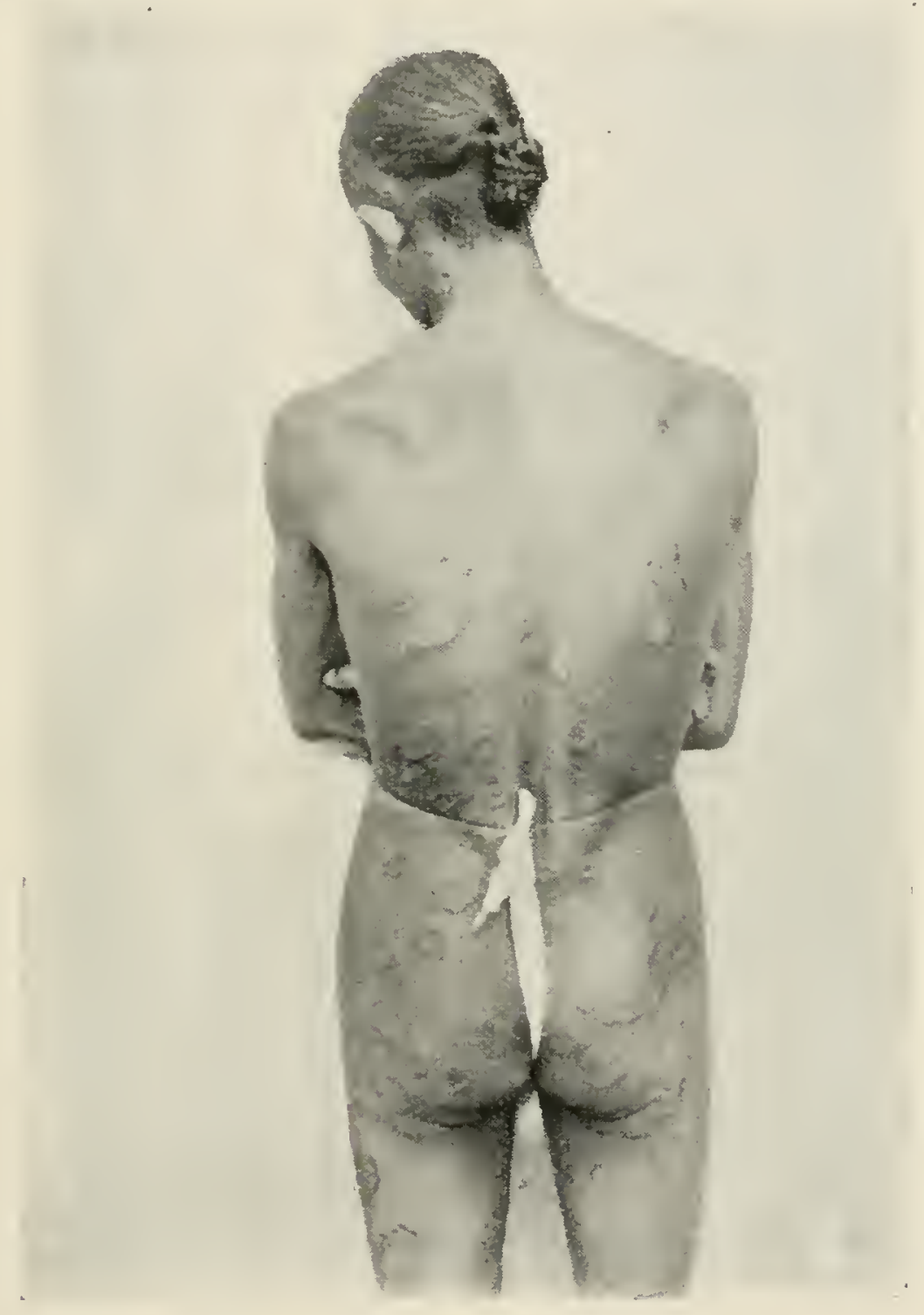

Maculz in anæsthetic leprosy.

Also on lower extremities there are bulle varying in size from a pea to that of an egg. Perforating ulcers.

Fissures in digits of hands, toes, dry gangrene, or bones of fingers may soften and become osteomalacic.

Eye is more often damaged by secondary infection, the result of the absence of lachrymal secretion and lagophthalmus.

Secretion of tears is diminished, xerophthalmia with posterior or total symblepharon, while desiccation and destruction of cornea may 
result. Secondary infection leads to keratitis, onyx, hypopyon, iritis, irido-cyclitis and total destruction.

Sterility is not so frequent.

\section{(3) MIXED VARIETY.}

Some tubercular cases develop nerve leprosy.

Some nerve cases develop tubercular leprosy.

Some advance equally.

Diagnosis.

Bacilli always present in the nodular variety. In early cases look in the nasal secretions.

In the anasthetic form the bacilli are more difficult to find.

\section{PROGNOSIS.}

Cure is exceptional. Periods of apparent cessation not uncommon. Tubercular or mixed, 2 to 2 years' duration.

Anasthetic form, 6 to 20 years' duration.

Intercurrent disease often kills. Death is seldom from the disease itself.

\section{TREATMENT.}

None specific. Fresh air, good food, isolation, removal of children, light work, amusement, rural colonies.

It is a wise procedure to emigrate the children of lepers; 160 of such emigrated to Canada and not one has become a leper (Hansen).

Surgical treatment as required for ulcers, whitlows, \&c.

Chaulmoogra oil, obtained by cold expression from the seeds of gynocardia, some species. It is easily adulterated and often substituted. Rub into patches, also to be given internally 11 5-Io to 11 .30-40 t.d.s. in capsules or in pill with tragacanth and soap or in emulsion.

Limes remove the taste of the oil.

Rogers prescribes gynocardic acid, also known as chaulmoogric acid, and believed by some to be the active principle of the oil. This latter contains almost certainly useless palmitic acid.

Dr. Sandwith recommends the use of this oil by hypodermic injection.

Hot baths may be tried.

Strychnine for nerve cases gr. $\frac{1}{30}$ t.d.s.

Omit treatment every seventh week and use ichthyol ointment 5 per cent.

$\mathrm{X}$ rays have been tried, Finsen and radium.

$\mathrm{Hg}$. perchloride subcutaneously early every other day is useful.

"Nastin," a neutral fat from Streptothrix leproides, combined with benzoyl chlorine and made into ampoules with sterile olive oil, 0`0005 to 0.0002 gramme of nastin, once weelily can be tried. 
Leproline (McLeod), good results, but too early to say as to ultimate utility.

Rhizophora Mangle used much in Cuba. (Nicholson and Padilla.)

Lepromata disappear definitely fifth to seventh month of treatment, they suppurate and scar or are absorbed, encysted and calcified.

Ulcers heal by eighth month and anæsthetic patches disappear.

In the ninth month the eyelashes and eyebrows grow again.

Patient is cured in a year.

Subcutaneous injection of iodoform. 30 per cent. emulsion in alive oil. Inject 2-8 c.c. daily, first near the lesions.

Paint with the tincture externally.

The iodine may disinfect the body, or some bacilli having been killed, these set free anti-bodies which destroy the rest.

Intravenous injection of iodoform. Place $\frac{1}{2}$ grain tablet of iodoform powdered, in hypodermic syringe.

Draw therein $m$ iii of liquid paraffin and $\|$ vii of ether.

Seal needle with lanolin to prevent evaporation of ether.

Shake till iodoform dissolves. Inject it aseptically into the mediobasilic or other superficial vein, given twice weekly at first, gradually increased to gr. I five times weekly.

The nodules four to six at a time can also be injected $m x$ to $\| x x x$

\section{PSIT'TACOSIS.}

\section{DEFINITION.}

A fatal specific epizootic among parrots, capable of spreading a severe febrile condition to man.

\section{ETIOLOGY.}

In 1892, 500 parrots were shipped from South America for Paris. 300 parrots died en route from enteritis.

200 parrots were sold in two lots after arrival in Paris.

In 26 days an epidemic of psittacosis broke out, affecting 49 persons of which 16 died. Several persons in the same house were attacked. There have been other small outbreaks since.

The organism is a bacillus of the paracoli group, closely related to, but distinct from, the B. typhosis of Ebert and is pathogenic for parrots and other birds.

The feathers become infected with frecal matter. These are cleaned by the bird's tongue and thus infection is spread among them.

Humans caressing the birds are infected by the bill or through some slight wound or contamination of food. It rarely spreads from man to man. 


\section{PATHOLOGY.}

In parrots an enteritis is caused, in man a septicæmia often complicated by lobular pneumonia.

There is congestion and degeneration of the liver, kidneys, lungs and heart.

\section{SYMPTOMATOLOGY.}

Incubation 7 to 12 days.

Onset sudden, or insidious, may be much like typhoid.

Temperature $102^{\circ}-\mathrm{IO}_{4}^{\circ} \mathrm{F}$. Pulse $110-120$, quick respirations, cough.

Signs and symptoms of lobular pneumonia. Tongue dry and furred.

Spleen enlarged. Diarrhoea or constipation. Rose coloured spots on skin, patient dull and apathetic.

If no pneumonia, patient recovers in 15 to 20 days.

If there is pneumonia the mortality is $35-40$ per cent.

\section{TREATMENT.}

Adapt treatment as for typhoid fever and pneumonia.

Quarantine of two weeks should be enforced for imported parrots. The dead (infected) burnt with their cages.

Parrots should not be fed by hand or caressed.

\section{UNDULANT FEVER. (MALTA FEVER.)}

\section{DEFINITION.}

A chronic infectious disease of low mortality caused by the Micrococcus melitensis of Bruce and closely allied germs, characterized by a prolonged irregular remittent fever and enlargement of the spleen.

\section{DISTRIBUTION.}

The endemic areas are the coasts and islands of the Mediterranean, Italy, France, Greece, and also India, especially the Punjab. It also exists in S. Africa, Uganda and the Sudan, China, the Philippine and Fiji Islands. N. and S. America and the West Indies. Common between ten to thirty years of age in both sexes, mostly in the warmer periods of the year.

\section{AETIOLOGY.}

The cause is M. melitensis (M. paramelitensis; M. pseudomelitensis. The two latter differ from the former in agglutination and absorption tests, causing para-undulant fever).

The organism is a small, flagellated micrococcus, grows on agar and bouillon, feebly on gelatine without liquefaction. Stains with basic aniline dyes. Gram negative. May often be cultivated from the 
blood, sometimes from the urine, occasionally from the faces. It can be found in the spleen, kidneys, liver, lymphatic and salivary glands, blood, bile, faeces, urine, milk, ? alimentary tract. It can live in dust for two months and in water for one month. It has been found in mosquitoes, but they are not proven infective agents. Monkeys have been infected and the germ recovered.

The organism has been found by agglutination in $5^{\circ}$ per cent. of Maltese goats examined and has been obtained from the milk of io per cent.

Milk is the chief medium by which it is carried to man even though but a little in tea is taken. Hence the rich suffer most and hospital patients about the Mediterranean in particular.

Captain Joshua Nicholson took sixty-five Maltese goats on board in 1905. An epidemic of undulant fever followed. Nearly all who trank of the milk suffered.

Perhaps goats become infected by drinking human urine when lacking salt: mules, asses, horses, cows, rabbits and fowls can carry it without being obriously diseased.

Modes of Infection:-

(I) By the alimentary canal (common).

(2) By the respiratory system (rare).

(3) By the cutaneous (very rare. Flies not proved).

(4) By sexual intercourse (possible).

\section{PATHOLOGY.}

I general septicamia is caused by the germ entering the bloodstream by the alimentary mucose.

The spleen is enlarged and congested.

Perhaps an attack produces immunity.

The organisms produce hamolysins, the body reacting by producing agglutinins lasting four to ten years, also a specific serum which can be used for immunization.

\section{P. M.}

Spleen enlarged, 10 to $4+$ ounces, congested, sometimes soft and friable.

Liver enlarged, congested, cloudy swelling or glomerular nephritis. The whole intestine congested, colon sometimes ulcerated.

I.ungs congested especially at the bases.

\section{SYMPTOMATOLOGY.}

Incubation six to fourteen days.

Onset insidious, headache, sleeplessness, anorexia, general muscular pains, perhaps vomiting and constipation for about a week. 

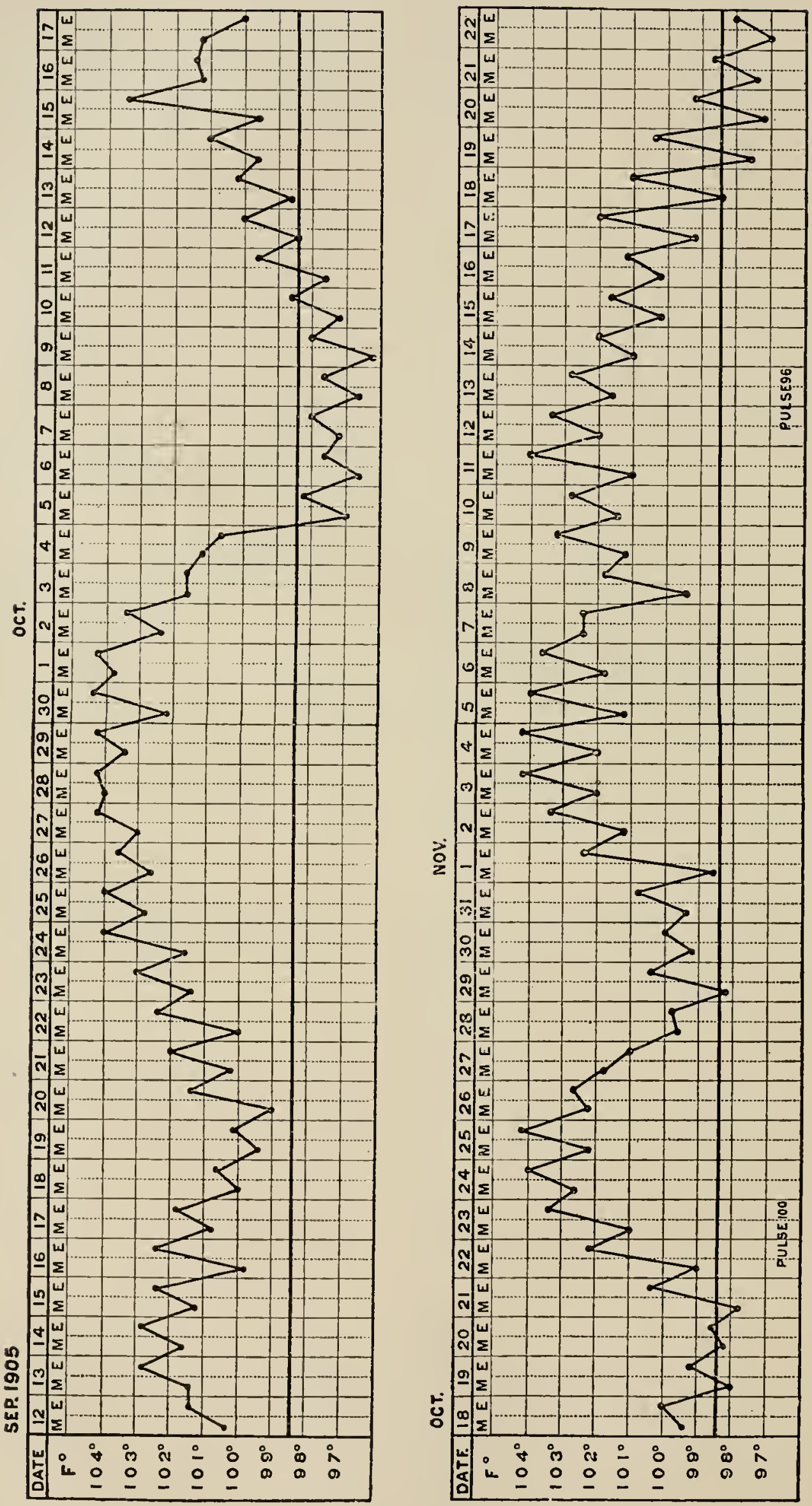

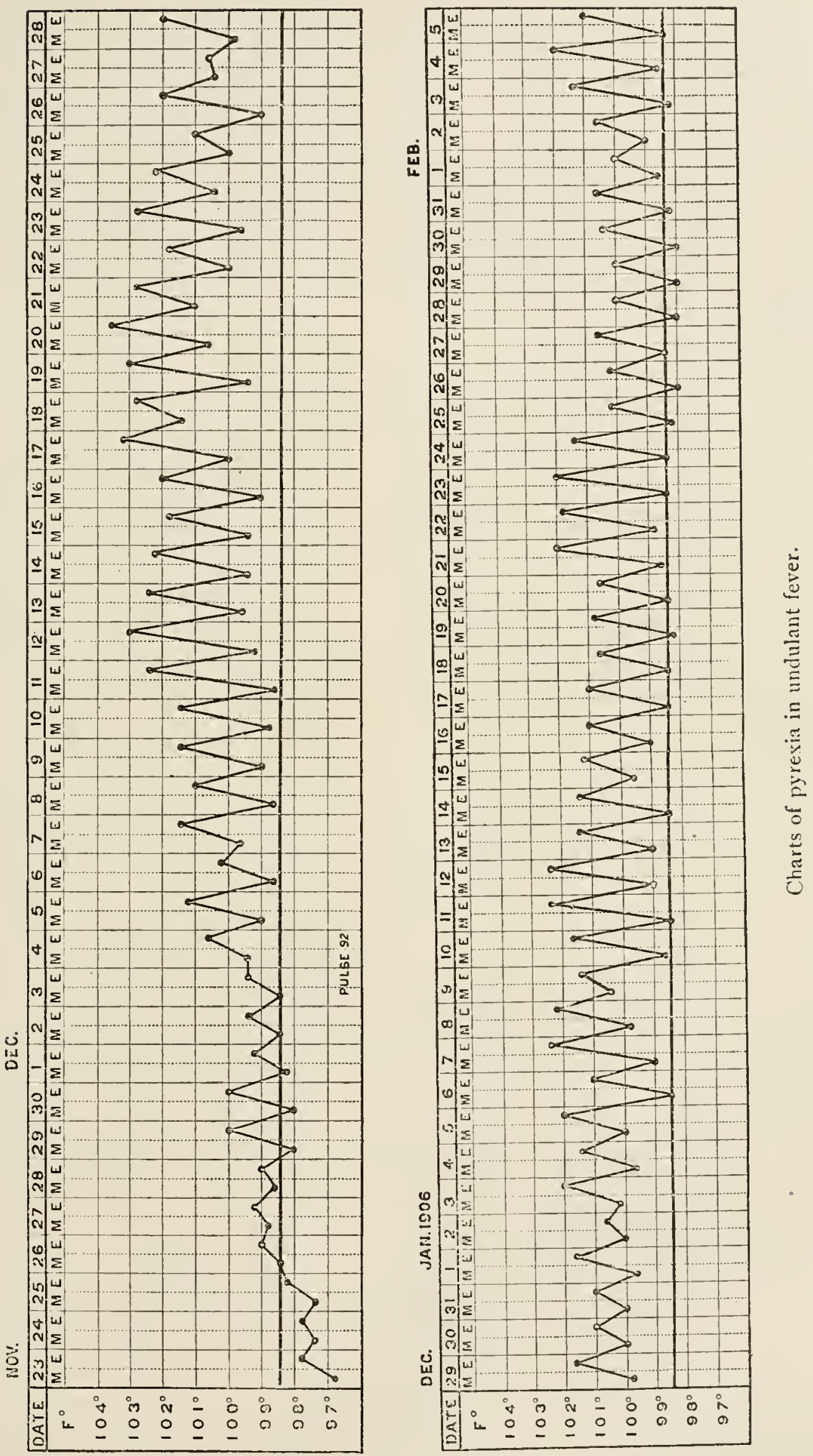
The temperature goes up somewhat ladder-like to about Io ${ }^{\circ} \mathrm{F}$., pulse $80-90$.

Tongue coated, edges red, slightly congested fauces.

Some bronchial catarrh, tender epigastrium and enlarged spleen.

This continues for about two weeks, the temperature remaining between $103^{\circ}-105^{\circ} \mathrm{F}$. and then declines with general improvement. In about two days a relapse occurs. This subsides and another follows and these relapses and remissions may last months, during which the temperature is undulating, a marked rise in the morning and a fall in the evening.

The patient becomes more ancemic and wasted. Dyspepsia sets in, constipation or diarrhoea reveals irritation of the intestines. Throat sore, gums spongy, spleen painful, lung bronchitic, heart excitable with hamic murmurs, dienching sweats, disagreeable odour, crops of sudamina after the hird week, big joints painful and swollen, orchitis, parotitis, a general nervous disorder shown by insomnia, hysterical emotion, photophobia, delirium, hallucinations are troublesome. A rapid anamia is commion.

The red cells show 20-40 per cent. loss with some poikilocytosis, Hb. low.

Decrease of polymorphonuclears, increase of mononuclears up to So per cent.

Leucocyte count normal. Phagocytosis and bactericidal power of blood diminished. Agglutination test; diluted $\mathrm{I}$ in $5^{\circ}$ to $\mathrm{I}$ in $\mathrm{I} 5 \mathrm{O}$, it lists two to seren years. Course of disease 20 to 300 days, average 90.

Convalescence long and slow, perhaps six months.

Many soldiers and sailors are permanently invalided.

Mortality low, about 2 per cent.

Symptoms arising in connection with the genital organs are not unknown, such as ovarian pains, dysmenorrhoea, anenorrhœea, menorrliagia, \&c. The M. melitensis has been frequently known to remain for long periods in the vaginal mucous membrane and contagion during intercourse is quite possible. Mammitis, like orchitis, is a not rare complication.

The influence of the fever upon a pregnant woman may vary very much according to the period of gestation, and when the woman is infected. Abortion and premature labour are faroured during the fever when it is high.

In some well authenticated cases it has been shown that M. melitensis can pass through the placenta and infants born at term generally show signs of infection and are weakly.

Whether or not the infant acquires the disease by drinking the infected milk of a nursing mother is a debatable point. Generally the 
child appears to have a congenital immunity as early infection is rare, according to the experience of some. Still it would be advisable to interdict the woman, whose mammary secretion was infected, from suckling her infant.

It is worth knowing too, that horses, mules, cows, and sheep may all become naturally infected, that milk products may harbour the germ, and that the latter is fairly resistant outside the body.

The malignant types cause death from hyperpyrexia or the typhoid state during the second or third weeks.

The intermittent types last from six weelis to six months without serious symptoms.

The ambulatory types are dangerous as carriers of disease.

\section{COMPLICATIONS.}

Intestinal ulcers, hemorrhage, persistent vomiting, diarrhoea, hyperpyrexia, pneumonia, pleural effusion, cardiac failure, orchitis, parotitis, arthritis, neuralgia.

\section{DIAGNOSTIC FEATURES.}

The prolonged undulant fever.

The profuse sweatings.

The arthritic symptoms.

The agglutination test.

Exclude typhoid by a Widal reaction (see p. 611).

Exclude malaria by blood parasites.

Exclude kala-azar by spleen puncture.

\section{TREATMENT.}

Entirely symptomatic. General principles liold good.

The utmost nursing care is needed. Be careful to change the clothes after sweating. The heart and lungs must be carefully watched.

Vaccine and yeast therapy can be tried.

Give I00-500 millions of autogenous germs as a vaccine.

Give yeast, dr. ii, twice daily.

Avoid goat's milk in endemic areas or sterilize it before using.

Leave the district as soon as possible.

\section{PROPHYLAXIS.}

This is all important. In Gibraltar this fever disappeared after the goats were removed. In Malta the case incidence amongst the British troops was reduced from $643 \mathrm{in} 1905$, to $7 \mathrm{in} 1907$ and $\mathrm{I}$ in 1910.

(I) General measures. Notification, isolation, disinfection, sterilization of water and milk, good hygiene. 
(2) Personal measures. Cleanliness, vaccine, care in nursing mothers.

(3) Veterinary measures. Examinations of blood and milk of all goats and other suspected animals.

Slaughter of infected animals.

Proplylactic raccines to all goats.

Prevention of the importation of infected animals.

\section{BACILLARY DYSENTERY.}

\section{DEFINITION.}

Acute or chronic, sporadic, endemic or epidemic, specific infective intestinal disorders caused by several varieties of bacteria, associated with tenesmus, abdominal pain, frequent mucous and blood stools.

\section{DISTRIBUTION.}

Universal, but especially in warm climates and where sanitation is defective as in jails and asylums, during famines and war-time.

In temperate climates the dysentery bacillus causes the infantile diarrhoeas. There seems more prevalence at the end of a dry season, perhaps because of the infection of water by flies.

\section{ETIOLOGY.}

There are numerous types of true dysentery bacilli and of pseudodysenteric bacilli. There are three main types:-

(1) Bacillus dysenteria of Shiga-Kruse, which does not ferment mannite.

(2) Bacillus dysenterize of Flexner, which ferments mannite, forming an acid in it. These bacilli are found in the dejecta, and can be scraped from the bowel post mortem. Agglutination will help in their differentiation.

(3) The Bacillus of IIiss. They belong to the Coli group, and the members thereof have the following characteristics:-

They are Gram-negative, non-sporing, non-resistant, none liquefy gelatine, all attack glucose, all change the reaction of milk, all form creamy growths on agar.

For specific claracteristics, see p. 6r9.

The infection is by faces, flies, dust, soiled clothes, contaminated water or soil. Convalescents act as carriers. I I per cent. of persons in an epidemic were found to be carriers.

The bacilli may live in the bowel, producing no symptoms until the patient's resistance is lowered, when the typical disease may show itself. After the first few days other bacteria may overcrowd the dysentery bacillus so as to make its isolation difficult. 


\section{PATHOLOGY.}

The bacilli are ingested with food or water and proceed to the intestine, where they grow and multiply. There they form toxins, one acting upon the lower bowel and the other on the nervous system. These are absorbed by the blood. The first is excreted into the lower bowel and causes:-

(I) In acute cases.

An exudation of lymph into the submucosa and mucosa.

This coagulates and is invaded by cells.

The capillaries are pressed upon and the blood supply cut off for small areas.

Coagulative necrosis and thrombosis of vessels ensues; the mucosa and the muscularis mucosa are destroyed.

This forms a fibrous or diphtheroid membrane on the ridges first.

This membrane contains micro-organisms and leucocytes.

The membrane separates off in flakes, leaving ulcers.

These, at first superficial, become deeper and larger.

The peritoneum becomes adematous and mixed infection is common.

The condition can be summed up thus :-

(I) Intense hyperamia, the mucosa is swollen, corrugated and hamorrhagic.

(2) Necrosis and fibrinous exudation with pseudo-membrane formation.

(3) Ulceration, at summit of folds, extending deeply and laterally.

The ulcers heal by scar formation, and become pigmented as a result of the $\mathrm{H}_{2} \mathrm{~S}$ of the bowel acting upon the iron of the blood.

The other toxin causes peripheral neuritis.

Septicæmia is very seldom caused.

(2) In chronic cases.

The mucosa and the whole gut is thickened and granular.

The ulcers have thickened edges.

Scarring causes strictures of the bowel with dilatation above.

A typical ulcer :-

(1) Site, on the ridges of the large intestines.

(2) Edges, irregular and thickened.

(3) Base, may be formed of any of the coats.

(4) Result, tendency to contract on healing.

These descriptions will also indicate the post-mortem findings.

There would be adhesions and signs of peritonitis, and parts of the sut may present a black friable mass. 


\section{SYMPTOMATOLOGY.}

There is an endless variety in character, urgency and significance.

The nearer the lesion to the rectum the more urgent is the tenesmus.

The nearer the cæcum the more griping. There is never liver abscess.

We will group them thus:-

Acute, Chronic, and Infantile.

(I) Acute bacterial dysentery.

Incubation twenty-four hours to six days.

Onset.-Sudden, abdominal pain about the umbilical region first.

Incessant call to stool, griping, tenesmus, very small stools of slimy mucus, and later blood-stained. 50-200 in twenty-four hours. Passage of a stool gives no relief.

Slight or moderate fever, $99^{\circ}-103^{\circ} \mathrm{F}$. Great thirst, foul tongue, dry skin. Anus becomes painful, prolapse not uncommon. Blood in stools, rapid exhaustion, sleeplessness, restlessness.

Vomiting is not common. There may be constipation of the upper bowel with diarrhoea of the lower, recognized by distension.

The typical dysenteric motions have no facal odour.

The pulse is quickened, especially if much blood is passed per rectum, and may become irregular.

Bad signs are :-

Irregular and rapid pulse, subnormal temperature, hiccough, diminished number of stools in midst of attack during the second or third week.

Good signs are :-

Motions more fæculent, less numerous, disappearance of blood and mucus, less pain and tenesmus.

Cases have been known to improve much, and then with a slight fæculent diarrhoea the patient has died within a few hours from gangrenous dysentery, or with the latter type there may be thin, watery, dirty, foul stools, with sloughs of every shape and colour.

Profuse hamorrhage is always possible.

Others may commence with rigors, and the temperature may keep high, $103^{\circ}-105^{\circ} \mathrm{F}$, with mental symptoms as stupidity, sleeplessness. delirium, abscesses, carbuncles and bed-sores, simulating somewhat enteric fever. Death may ensue in a few days from exhaustion, toxæmia or hyperpyrexia.

The stools.

In an average case they consist of odourless gelatinous blood and extremely tenacious mucus which adheres to the pan.

In three to four days they become of a more purulent nature, yellow in colour, and contain less blood. Later bile is seen, which indicates 
that the contraction of the lower part of the large gut has passed off. Fæcal matter may then be passed. A stool of glairy mucus, like sputum, is usually a catarthal inflammation due to the I' bacillus.

In the most acute fulminating varieties the stools contain much blood, and have been compared to " meat washings."

When necrosis of the mucous membrane has taken place the siools may be choleraic, but may contain dark green sloughs of mucosa (Bahr).

(2) Chronic bacterial dysentery.

Insidious onset or may ensue after an acute attack. The bowel is not healthy, and any dietetic error, chill, fatigue, or indulgence will cause the symptoms to appear.

The stools are loose, five to ten per diem, partly facal, much mucus, rarely blood, like "frog's spawn " or " boiled sago." The tongue is glazed red, anæmia and emaciation are progressive, cachexia is apparent.

The digestion is impaired, all functions are below par resulting from anzemia and toxamia. Fermentation produces much intestinal gas.

These symptoms may subside and recur at intervals.

The disease may continue until death from exhaustion or some intercurrent disease, or it may clear up spontaneously.

If no blood is passed it is commonly dysenteric diarrhcea.

(3) Dysenteric infantile diarrhioa.

This is an extremely common disease in the tropics.

Flexner's bacillus commonly, and Shiga's bacillus rarely, are believed to be the exciting agents.

The symptoms resemble those of the enteric type of chronic dysentery. It begins by vomiting and a temperature of $103^{\circ}-105^{\circ} \mathrm{F}$.

The abdomen is distended and tender.

The stools are frecal, but often green with blood and mucus.

The fever is remittent. There may be repeated attacks.

Death may be from exhaustion or convulsions.

Reovery may take place, but the convalescence is always slow.

\section{COMPLICATIONS.}

Peritonitis with or without perforation (rare).

Pleurisy, pericarditis, endocarditis.

Arthritis, tenosinovitis, perhaps the result of secondary infection.

Anzemia and dropsy.

Peripheral neuritis, frequent in mild forms; one nerve only may be involved.

Iritis, toxzemia, vields to atropine. 
Parotitis, abscesses, rarely pyamia.

Hiemorrhage in gangrenous cases.

Appendicitis may set in.

Typhoid is a most serious complication and has occurred.

\section{SEQUELÆ.}

Stenosis of the large bowel due to cicatricial contraction of the healing ulcers.

Constipation and diarrhoea intermitting result of above condition.

Colic and romiting, meteorism.

\section{DIAGNOSIS.}

Inflamed hamorrhoids can be seen and felt.

Syphilis of the rectum can be diagnosed by the Wassermann reaction and excluded by " 606 " injections.

Rectal carcinoma can be excluded by the history, examination with finger and microscope.

Intussusception is very sudden in onset, the abdomen is not tense, a sausage-shaped tumour is felt. Operation will discover and correct it.

Malarial dysentery parasites will be seen in the spleen by puncture and smear.

Kala-azar dysentery parasites will be seen in the spleen puncture and smear.

Amobic and balantidic dysentery by microscopic examination of faeces.

A bacteriological examination of the faces should be carried out.

The agglutination reaction should be tried with the cultures prepared from the faces and other known organisms.

\section{PROGNOSIS.}

Mild acute attacks; good recovery the rule.

Severe acute attacks; poor, mortality high.

Gangrenous acute attacks; bad, nearly all die.

12-25 per cent. in all classes together die.

The lower down the bowel the better the prognosis.

The higher up the bowel the worse the prognosis.

Early prophylactic serum improves the prognosis.

New arrivals in the tropics suffer more than native residents.

In Japan, the mortality was reduced from 35 per cent. to 9 per cent. by serotherapy.

\section{TREATMENT.}

(I) Acute attacks.

Rest in bed. Bland diet.

Use of urine bottle and bed pan. Constipation must be overcome, no matter how long the patient has been passing mucus. Damp 
cotton-wool instead of sanitary paper should be used. Stools to be saved for physician's inspection. Protection of stools from flies.

Disinfection of stools with carbolic acid.

Give castor oil $\tilde{5}$ ss with opium tincture, 10 minims; much opium causes constipation and distension of gut and flatus.

In a few hours give astringents :-

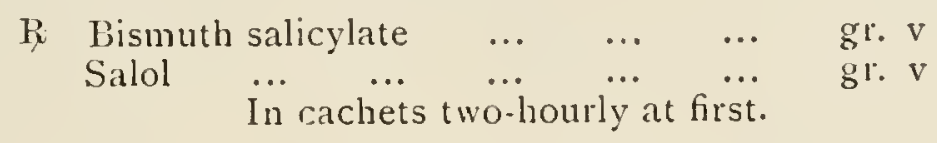

High rectal enema, boric acid I in 5oo; normal saline gr. V to $\tilde{5} \mathrm{i}$ are well borne in mild cases.

In severer cases give a polyvalent or other serum (Shiga) as soon as possible.

(1) In mild cases ro c.c. only.

(2) In medium cases ro c.c. twice, six hours between.

(3) In severe cases 10 c.c. twice daily for three days, or to-So c.c. intravenously first day, 60 c.c. second day, 60 c.c. third day.

This hastens the cure, ameliorates the symptoms, and reduces the mortality.

In connection with the rapid preparation of serum, Professor Flexner has hastened the process considerably. In summarizing his work, he says:-

Antidysenteric serum can be safely prepared in the horse by the method of three successive intravenous injections of living cultures with intervening rest periods of seven days.

When this method of immunization is employed, the specific antibodies responsible for agglutination and protection appear early and rise rapidly.

By inoculating alternately living dysentery bacilli belonging to the Shiga and Flexner groups a polyvalent serum of high titre may be prepared.

A polyvalent serum so produced should be suitable for the therapeutic treatment of acute bacillary dysentery, irrespective of the particular strain or strains of the dysentery bacillus causing the infection.

An effective antidysenteric serum suitable for therapeutic employment in man can be prepared in the horse in about ten weeks.

Calcium chloride or lactate gr. I 5 to $\mathrm{gr}^{\mathrm{r}}$. 30 is good.

Salines are useful, especially when sera are not available or are not rery successful. Stop on the third day if no improvement, otherwise continue:-

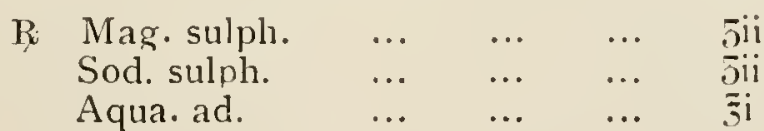

$5 i$ two hourly until two days after motions are faculent. 
Latudanum fomentations to abdomen for pain.

Suppositories of morphia or cocaine for tenesmus.

Or hypodermic injection of morphia gr. $\frac{1}{4}$.

Reduce prolapse and apply hazeline or gall and opium ointment.

Rectal tubes will permit gas to escape and allow of lavage, but pain often contra-indicates this.

After acute stage bismuth subnitrate gr. IO-20, two-hourly.

Rectal enemata of boric or tannic acid.

Milk and fruit good.

Calomel, $\frac{1}{4}$ gr., hourly, has been tried.

Yellow santonin in olive oil and sour milk have their advocates.

Appendicostomy will permit the washing out of the bowel from above in gangrenous cases.

(2) Chronic attacks.

Treatment as for mild cases sometimes suffices.

Diet.-The bowel must have rest.

Albumin water and whey only at first. Then diluted milk and barley water with sodium bicarbonate.

Thin arrowroot, malted milk.

Chicken broth and milk puddings later.

Lightly boiled eggs and chicken.

In severe cases give small quantities two-hourly.

Food must be well masticated later.

Avoid much brandy, strong meat essences, and fruit.

High bowel irrigation of silver nitrate with buttocks raised.

Solution, ? $?_{2}^{-1} \mathrm{gr}$. to the ounce. This gives the best results of all astringents. Precede with two pints of I per cent. sodium bicarbonate to remove mucus. Sera should be tried.

Tannigen, gr. v, three to six times daily, or

Tannalbin, gr. xv, three to six times daily have given good results.

Liquid paraffin is always good, soothing the bowel, breaking up the scybalous masses, and is mildly antiseptic.

Pilula plumbi cum opii, gr. iv, four-hourly.

Autogenous vaccines can be tried.

Appendicostomy is preferred by some.

(3) Infantile diarrhoea.

Castor oil 5i-5ii, followed by calomel gr. $\frac{7}{6}$, or grey powder gr. $\frac{1}{4}$, each hour until six doses have been given in a child of two years.

Later tannigen gr. iii or tannabin gr. v, three-hourly.

Sera are good when bacilli are diagnosed, 5-10 c.c. twice daily.

Treat convulsions by bromides, gr. $\mathrm{x}$ in enemata. General principles hold good. 


\section{CHOLERA.}

\section{DEFINITION.}

An acute, specific, endemic or epidemic disease, caused by Kroch's Vibrio cholere, ${ }_{1} S_{3}$, and characterized by violent purging, rice-water stools, romiting, muscular cramps, suppression of urine, collapse and high mortality.

\section{DISTRIBUTION.}

The name is of Greek origin, and was perhaps applied because the riolent watery stools resembled water rushing from a spout.

It has been known in India since the most ancient times.

Ahmed Shah's army was decimated by it in $1+38$. There were several outbreaks after this in India, Burmah and Ceylon. The first great epidemic outside India was from 1817 to $182 \%$, when China was infected.

Although cholera is not an endemic disease in Europe, its manifestations in the 19th and 2oth centuries killed millions of its people. There have been nine invasions of Europe in a little orer so vears. The chief centre of attack was about the Caspian Sea in Russia. All the invasions probably came from India by indirect routes. The endemic centres of Java, Indo-China and the Philippines were probably not implicated. An excellent summary of these attacks is given by Professor W. J. Simpson as follows:-

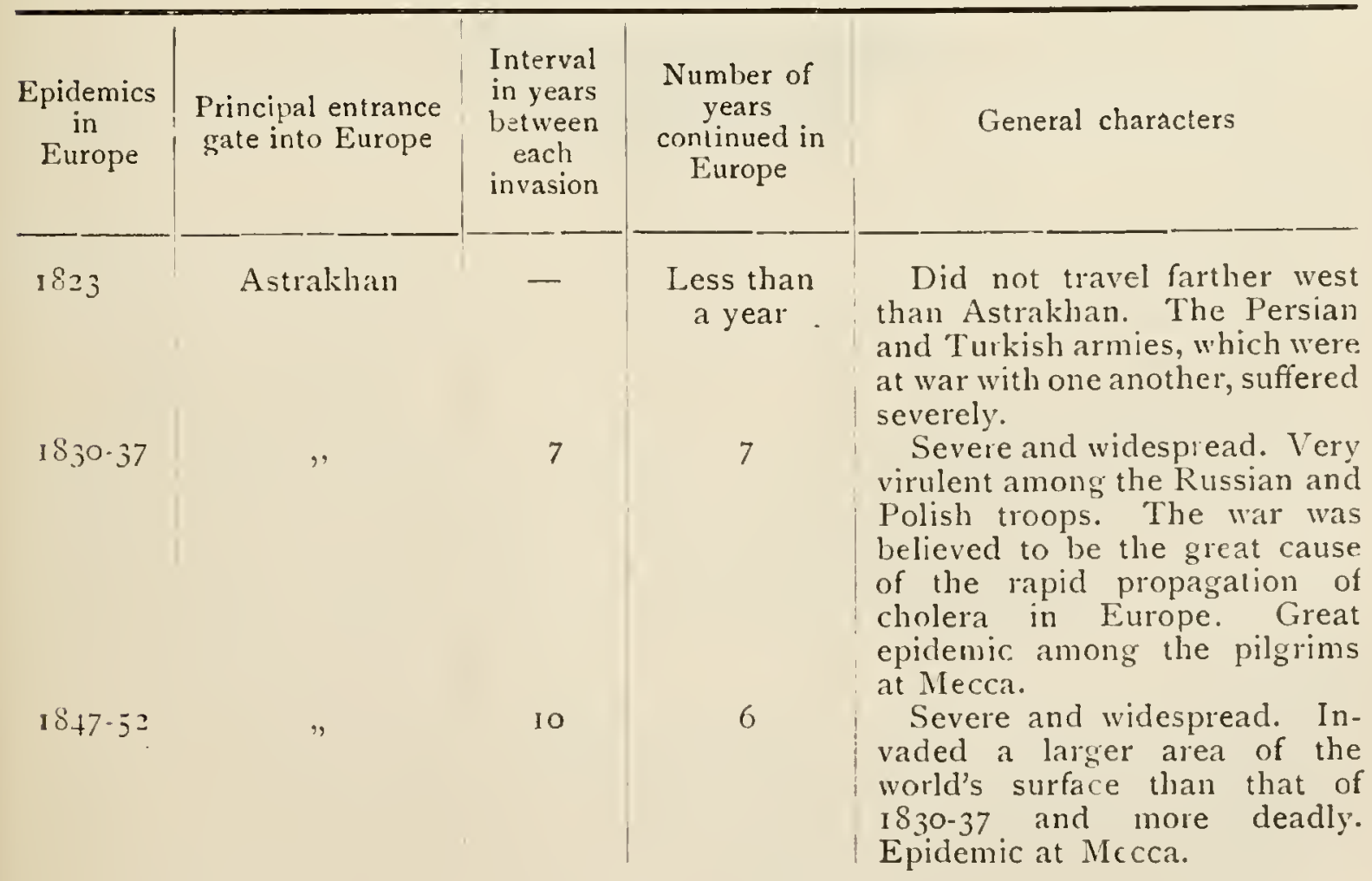




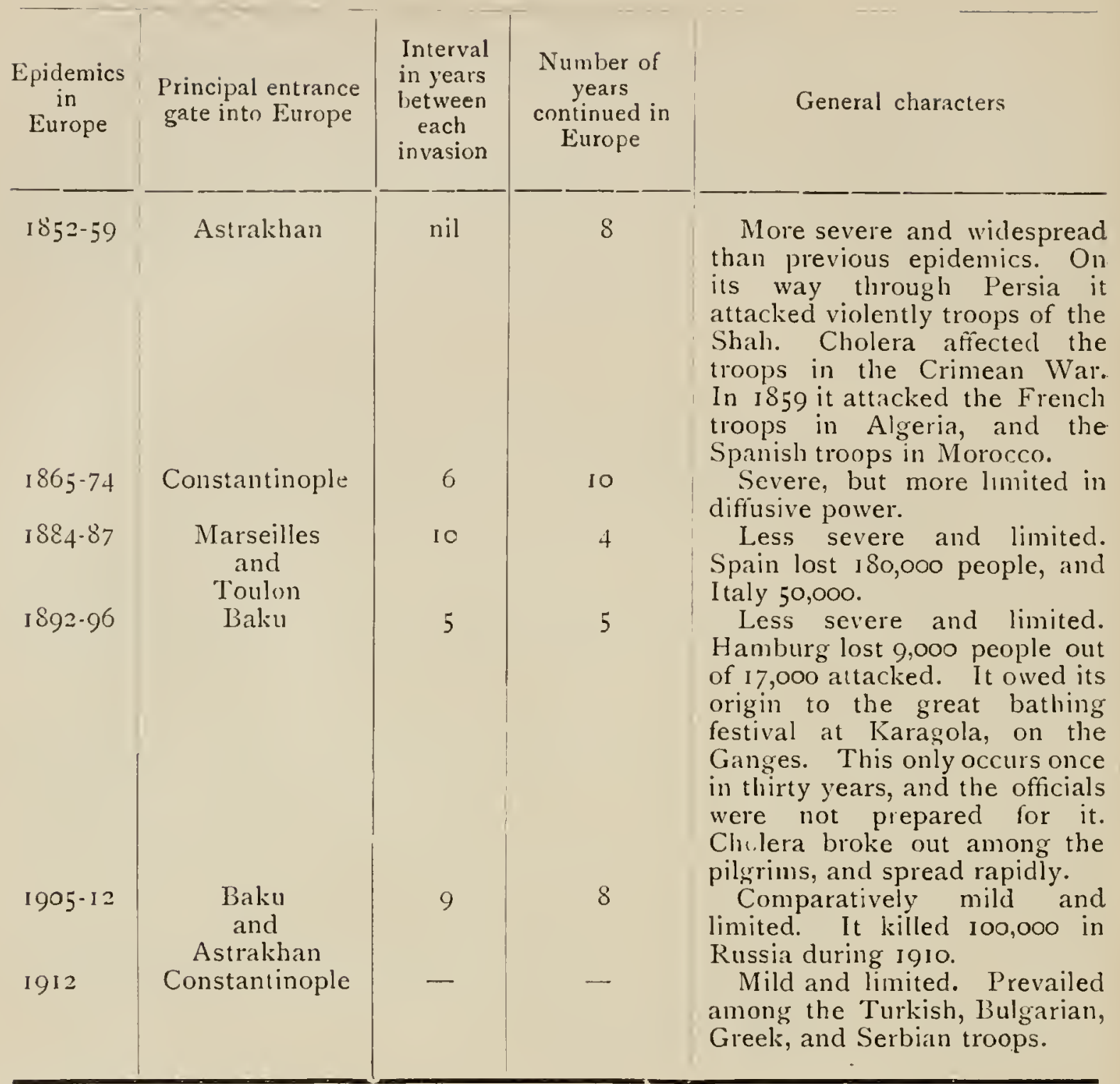

The relation of cholera epidemics to wars is all-important.

It is of interest to know that during the Napoleonic wars the disease was not known to Europe, so that these were free from this devastating malady .

(I) In the Egyptian War of I882-3 the civil population lost 50,000 from cholera, but the troops were little affected by it.

(2) Cholera was virulent during the Russo-Polish War of 1831 .

(3) After the Crimean War came the infection of all Europe.

(4) After the Balkan War its distribution was widespread. Both the French and British armies and fleets suffered. There were probably about 18,000 cases behind the Chataldja lines, whence it was conveyed to the Bulgarian army, and later to the Greek and Serbian armies.

(5) The Great European War of I9I4:-

At the commencement of the War cholera prevailed inRussia, the Province of Podalia.

Hungary, 53 different centres. 
Austria, 39 different centres.

Germany, 10 different centres.

Turkey, 2 different centres.

Persia and Arabia are seldom free from the scourge.

In 19I5 the Russians took cholera to their Austrian guards and to the civil population. There were then 30,000 cases with 16,000 deaths in Austria, and 5,000 cases with 3,000 deaths in Hungary.

During the same year there were 147 ,000 deaths in India, of which 68,000 were in Bengal.

Tropical and South Africa, the Azores, the Pacific Islands have not yet been infected. The principal endemic centre is lower Bengal.

It always adopts channels of human inter-communication; ships, rivers, roads, railways, producing a widespread epidemic in places of bad sanitation.

\section{ETIOLOGY.}

The existing cause is Koch's comma bacillus.

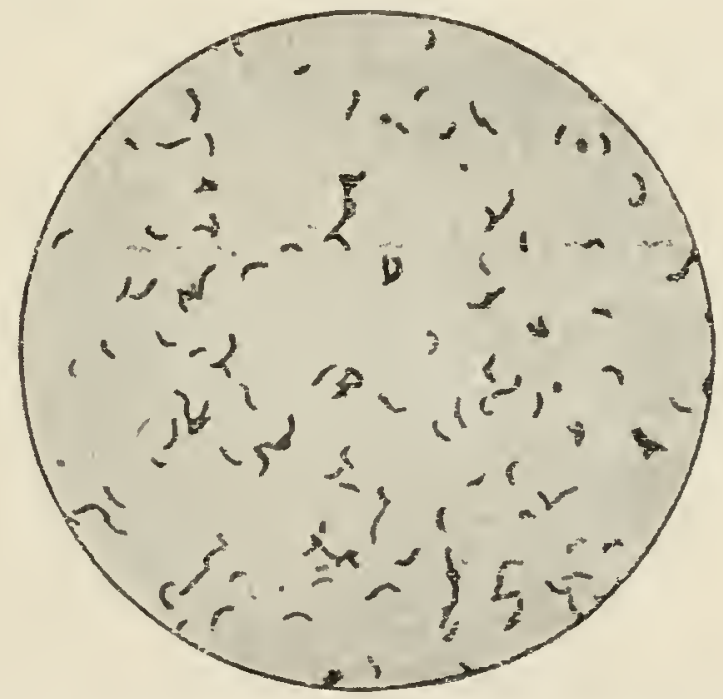

Cholera spirilla. (From Pitfield's " Bacteriology.")

Predisposing causes are low-lying areas, contaminated soils, unripe fruit, lowered vitality from any cause, as seen in gaols.

infection is by-

(I) Direct contact. A medical man examined a patient suffering from cholera, then had his lunch. Patient and doctor were both dead in twenty-four hours. Care is required in performing autopsies.

(2) Fomites form a common means of infection.

(3) Water is the medium.

The comma bacillus passes out with the faces, and as it lives and multiplies in water for 16.3 days or more, a place having a poor sanitary system would have all the conditions favourable for an epidemic.

Hampstead, a cholera-free place in I 854 , was infected by a lady and 
her servant who drank water taken to them from the broad Street well, for which they had a special liking.

The Hamburg and Altona instance is well known. Both towns used water from the river Elbe for drinking purposes. The former tornspeople did not filter their water; an epidemic of cholera broke out, and there were 8 ,ooo deaths, or $13^{\circ} 4$ per cent. of the population.

The latter people filtered their water, and during the epidemic they only had 328 deaths, $2^{\circ}$ I per cent. of the population.

(4) Milk is especially dangerous, as it affords a good growing medium.

(5) Carricrs are extremely dangerous; though not obriously ill themselves, they carry and give out virulent comma bacilli in their frcal excretions.

(6) The bacillus vibrio has been found upon and within the bodies of flies, hence these should be considered an important means of transmission. Ants, too, should be looked upon with suspicion.

(7) Green vegetables eaten raw, such as lettuces, watercress and tomatoes are liable to become infected. In the East vegetable gardens are often contaminated with human frecal matter.

For details of the organism, see p. 62 I.

\section{PATHOLOGY.}

The vibrio has a local habitat, living and growing in the small intestinal mucosa, epithelial cells, glands and lumen giving off in all probability an endocellular toxin which, when set free and absorbed, causes the general symptoms. This toxin causes the blood elements to enter the blood thus: First water, then inorganic salts, especially sodium chloride, later phosphates and potassium salts, and still later organic substances. This produces a great concentration of blood ( $073-10-8)$ and an increase of hb. and red cells to 8 , ooo, ooo per c.mm., also of white cells up to $60,000 \mathrm{c} . \mathrm{mm}$.

The alkalinity of the blood is decreased and the coagulative porer altered.

This concentration of the blood causes a fall in the blood pressure, shown by the feeble compressible pulse (50-So $13 m$. of $\mathrm{Hg}$ ).

The result of this low blood pressure is that the urine is scanty, high sp. gi'., albumin, casts and much indican.

Perhaps the blood pours out bactericidal substances.

\section{POST MORTEM.}

Rigor mortis marked and early. Rise of temperature and muscular movements after death not uncommon. Tissues dry. Blood thick, may be tarry.

Stomach empty, vessels injected. 
Small intestine contains greyish grumous material, consisting of food particles, epithelial cells, red and white corpuscles, and microorganisms. Mucosa hyperamic and swollen, sometimes ecchymosis.

Salivary and mesenteric glands enlarged.

Liver congested, loaded with blood, ducts full of bile.

Spleen shrivelled and small.

Kidneys enlarged, tubules blocked with granular débris, cloudy swelling.

R. heart dilated. V'enous system congested with dark, thick, tarry blood.

L. heart empty. Arterial system empty.

Lungs dry, anamic and collapsed.

Urinary bladder empty and contracted.

The vibrios are usually confined to the intestines, but they have been found in the liver, kidney and heart.

\section{SYMPTOMATOLOGY.}

Incubation three hours to six days.

Typical form.

Onset sudden as a rule; sometimes malaise precedes it.

There are three stages.

(1) Evacuation stage.

Diarhoea with or without colicky pains. Loose faculent motions, followed quickly by the typical rice-water stools containing numerous white flakes of mucus, epithelial cells and vibrios.

Vomiting early, food first, then watery fluid with bile and sometimes blood, then rice-water material.

(2) Algide stage.

Always much thirst, sometimes hiccough, urine diminishes and may cease.

Subcutaneous tissues give up their fluid and the facies alter, e.g., nose sharp, cheek-bones prominent, eyes sunken, skin of fingers wrinkled.

B.P. falls, pulse weak and rapid, cyanosis, dyspnoea, husky roice.

Painful muscular cramps, in calf, arm and abdomen especially.

Reflexes diminish. Mind clear, patient apathetic between cramps.

Skin cold, axillary temperature lowered, rectal raised.

Pulse runs, heart weak and irregular, urine suppressed, temperature subnormal.

Comatose and death in twelve to thirty-six hours.

(3) Reaction stage.

Improvement may commence at any stage. The symptoms gradually disappear and indications of normal health return. 
Convalescence may be rapid, or a secondary febrile condition sets in and lasts two days to two weeks.

The status typhosus is not uncommon, especially just before death.

Raised temperature, dry brown tongue, low muttering delirium, tremblings, toxic rashes, erythematous, papular or hemorrhagic in nature.

Other forms may show predominance of purgation symptoms.

Other forms may show predominance of cramps.

Others may show signs of collapse and die before diarrhoea and romiting sets in.

Other forms may show nothing but vibrios in faces.

Cases are usually worst at the commencement of an epidemic.

The case mortality averages about 50 per cent.

A coagulum in the $R$. heart or spasm of the pulmonary ressel refusing to transmit the thickened blood may cause sudden death.

\section{COMPLICATIONS.}

Hyperpyrexia is rare. Abortion the result of contraction (cramp) of uterus possible.

Secondary infections account for most complications as pneumonia, enteritis, nephritis, parotitis, corneal ulceration. There may be errors of refraction.

\section{DIAGNOSIS.}

Easy in epidemic, difficult in sporadic cases.

An extremely careful bacteriological examination is necessary to detect the true Vibrio choleræ of Koch. In dispatching material for diagnosis, collect and sample a piece of bowel ligatured into a boiled glass bottle well stoppered. No antiseptic to be used.

Methods of diagnosis :-

(1) Make ordinary films of the white flakes. Stain with diluted carbol fuchsin ( $\mathrm{I}$ in 50 ) ro minutes, or with Löffler's blue 5 minutes.

If a fish-in-stream arrangement of curved rods be seen cholera can be strongly suspected.

(2) Smear the surface of a lactose-agar plate (MacConkey's media) with a rice-like flake, using a bent sterile glass rod. Jnoculate with the same rod not recharged two more plates and inoculate at $35^{\circ} \mathrm{C}$.

If small, delicate, yellowish, roundish dots appear within twelve to eighteen hours, cholera vibrio can be suspected.

Examine the germs of a colony, if very active, comma-like or straight, if it agglutinates with anticholera serum in a dilution down to $\mathrm{I}$ in 4,000 , it is in all probability cholera vibrio. 
(3) Mix directly a drop of the stools with immune serum. If agglutination suspect cholera vibrios (Dunbar). This is useful only when the vibrios are very numerous.

(4) Inoculate four tubes of peptone water with sample of stuol $\frac{1}{4}$ c.c., $\frac{1}{2}$ c.c., I c.c., 2 c.c., respectively.

A scum on the surface of the medium in S-ro hours is suspicious of cholera. Examine scum for comma vibrio, inoculate plates with the germs to confirm. Add to peptone water medium a few drops of pure $\mathrm{H}_{2} \mathrm{SO}_{4}$. In true cholera the indol reaction is present in S-10 hours.

(5) Inoculate the surface of three serum tubes with the sample and incubate at $35^{\circ} \mathrm{C}$. If no liquefaction in 16 hours, it excludes cholera.

If liquefaction in 16 hours, exclude cholera by plating out, \&c. If liquefaction then, the vibrio may be present, plate out and further examine.

(6) Inoculate a sedimentative tube of peptone water and immune serum with the faccal sample. Incubate at $37^{\circ} \mathrm{C}$. for three to seven hours.

If true cholera, numerous small flocculi of agglutinating vibrios, at first suspended, sink to the bottom (Bandi).

(7) Inoculate with farcal sample media of pure bile mixed with 3 per cent. of a 10 per cent. solution of $\mathrm{Na}_{2} \mathrm{CO}_{3}$.

Incubate at $37^{\circ} \mathrm{C}$. Plate and further examine.

In epidemics due to cholera nostras, mushroom poisoning, bacterial food poisoning, trichinosis, pernicious malarial fever, all have a low mortality as compared with cholera. If an epidemic has over $5^{\circ}$ per cent. mortality it is cholera.

\section{TREATMENT.}

The indications are:-

To destroy and remove the ribrios.

To neutralize the toxins.

To prevent secondary infections.

To relieve the symptoms.

$\mathrm{B} \subset \mathrm{d}$ at once under the best hygienic conditions.

Warmth essential. Water to drink in sips. Turpentine stupes to abdomen. No food during acute attack. Hot, strong coffee, good.

Rogers' method.

Calcium permanganate water (gr. i to vi to a pint) ad lib.

Permanganate pills :-

Potassium permanganate gr. ii.

Kaolin and vaseline as required.

To be taken every $\frac{1}{4}$ hour for two hours, then one pill half-hourly. 
Those vomited being replaced. Continue until the stools are green and less copious, which can be expected in twelve to twenty-four hours.

In mild cases give one four-hourly, in severe continue as above.

Pills old and hard are useless.

In collapse, hot bottles and when pulse fails open median basilic vein with Rogers' special silver cannula inserted and hypertonic saline solution given until B.P. returns.

Sodium chloride gr. cxx.

Calcium chloride gr. iv.

Potassium chloride gr. vi.

Water to a pint.

Boil for 15 minutes to sterilize.

Inject at a temperature varied according to rectal temperature.

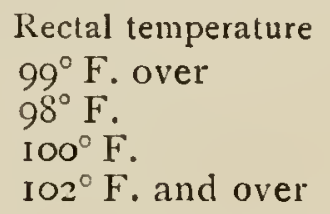

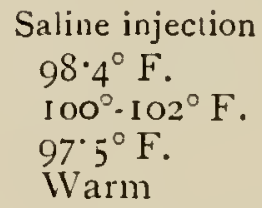

Warm

If blood pressure falls below $70 \mathrm{~mm}$. of $\mathrm{Hg}$ give intravenous saline at once. Give it in cases of restlessness, cyanosis, cramps, black blood as shown by pin prick.

Stop the injection in cases of respiratory or other distress.

Leave the cannula in situ as it may be needed to repeat the injections. Warm rectal injections can be given.

If no severe cramps, strychnine hypodermically.

If suppression, dry-cup over each loin.

In the stage of reaction give :-

Bland foods and avoid meat extracts also.

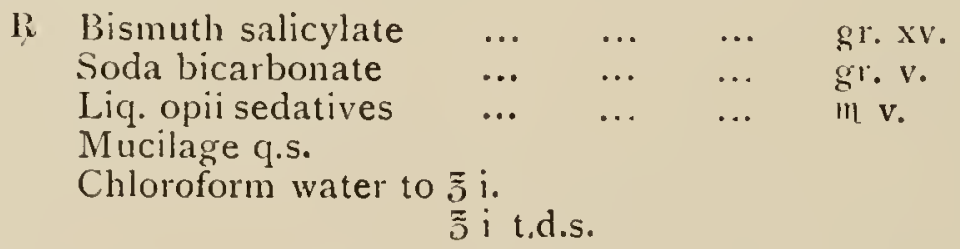

For persistent romiting give :-

(I) Cocaine gr. $\frac{1}{8}$ dissolved in $5 i$ of water.

(2) Mist pepsini co. et bismuth ro minims, half-hourly for four doses.

(3) Two drops of tinct. iodine in water.

For cramps: massage, morphia, inhalations of mixture of chloroform and oxygen.

For delirium: bromides, with tincture of hyoscyamus.

For prostration; strychnine or camphor in ether.

Watch for uremia.

Opium eaters all die. 
Rogers gives some very interesting figures comparing the results of the old methods with the new as follows:-

Oln System, $1895-1905$

NEW SYSTEM, 1913-19I5

Age Cases Deaths Percent. Cases Deaths Percat.

Reduction mortality per cent.

To 5 years!

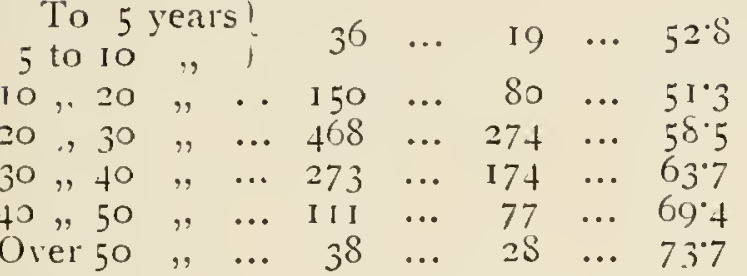

$\left.\begin{array}{rrrrc}22 & \ldots & 8 & \ldots & 36 \cdot 3 \\ 24 & \ldots & 2 & \ldots & 8 \cdot 33 \\ 121 & \ldots & 17 & \ldots & 14 \cdot 0 \\ 197 & \ldots & 44 & \ldots & 22 \cdot 3 \\ 134 & \ldots & 28 & \ldots & 20 \cdot 9 \\ 43 & \ldots & 16 & \ldots & 365 \\ 23 & \ldots & 11 & \ldots & 47 \cdot 8\end{array}\right\}$

... $6 \mathrm{I}^{\circ} \mathrm{O}$

... $73^{\circ} \mathrm{O}$

... $64^{\circ} \mathrm{C}$

... $67^{\circ} \mathrm{O}$

... $47^{\circ} \mathrm{O}$

... $35^{\circ} \mathrm{O}$

The remaining mortality was due to collapse, uramia, pneumonia, heart failure and exhaustion, hyperpyrexia, septic complications.

The total mortality is reduced by his treatment to 20 per cent. or one-third of the former rate.

Cox claims better results still as a result of using isotonic saline instead of hypertonic saline. Cox, who has treated over 2,ooo cases, gives his figures as follows:-

INDIA

Rogers, 1909-10

Number of cases, $\mathrm{IO}_{3}$

Average amount transfused, $4 \frac{1}{2}$ pints

Mortality, 32 per cent.

\section{ChINA}

Collupse cases only

Number of cases, 666

Average amount transfused, 13 pints

Mortality, I 8.8 per cent.

In certain cases Cox gives hypertonic saline for the first eight pints and then continues with the isotonic salines.

Rogers denounces opium in any case.

Cox has found it very useful in cases of collapse.

Cox continues: An adequate reaction proportionate to the degree of collapse must be looked for and assisted. This condition is never attained until the infusion reaction rigor (which occurs approximately when five pints have been infused) is well passed. The continuance of the saline infusion after the occurrence of the reaction rigor up to 8 -ro pints will procure the following benefits :-

(I) Dilution of the blood.

(2) Elevation of the blood pressure, with re-establishment of suppressed renal flow.

(3) Elimination of the endotoxins from the blood and later from the stools, thus obviating the onset of the febrile reaction stage of cholera, the remainder of the illness being usually apyrexial.

No apprehension need be felt at a rise of temperature to $\operatorname{Ioz}^{\circ} \mathrm{F}$. during the infusion, in fact, this degree of temperature oscillation I consider the most favourable for cessation of infusion of less than six 
pints; the temperature of the saline in the cistern should be lowered and the infusion continued.

That the reaction induced by the saline infusion may, to some extent, be due to a toxiemia induced by the endotoxins of water-borne bacilli used in the infusion is possible; that it entirely accounts for the reaction rigor I do not believe. In any case, it will be interesting to see to what extent the phenomena of the infusion reaction will be modified by filtration previous to boiling.

\section{PROPHYLAXIS (Castellani).}

(I) Public.

(r) Protection of the frontiers, regular inspection, quarantine stations.

(2) A central Cholera Board, with a full staff and apparatus for bacteriological work, disinfection, \&c.

(3) Instruction of the populace by pamphlets.

(4) Ilouse-to-house search for cases.

(5) Search for carriers and sources of infection.

(6) Distribution of medicines and disinfectants.

(7) Provision of medical aid to be readily obtained by all.

(8) Crusade against house-flies.

(II) Private.

(1) Personal cleanliness.

(2) Avoidance of foods liable to be contaminated.

(3) Avoidance of foods liable to cause diarrhoea.

(4) Filtration and boiling of all water for drinking and cooking. The regular cleansing of all filters and water receptacles.

(5) Boiling of milk and protection against flies.

(6) Clean sanitary dwellings.

(7) Anti-cholera vaccination. Ilaffkine's protects for six to fourteen months.

(8) Immediate application for medical aid in the event of any diarrhoeal illness.

Professor Simpson, working at Calcutta, was inoculating some civilians with I-Taffkine's vaccine of living cultures, when an epidemic broke out four days afterwards with the following interesting results :-

The 654 uninoculated had 7 I deaths, or 10.86 per cent.

The 4 I 2 inoculated had I 2 deaths, or $2^{\circ} 99$ per cent.

Not only so, but for the ensuing twelve months the inoculated were almost free from the disease, while cases were cropping up all the time among the uninoculated, the ratio of the mortality being as 3 is to $\mathbf{I}$.

Kitasato in Japan, and Castellani in Ceylon, used devitalized vaccines, but Simpson used living cultures. 
During the Balkan War, 1913, Greece used devitalized vaccines with interesting results:-

Those with two vaccinations, 91,22+ soldiers had 644 cases, or $\mathrm{I}-\mathrm{I}, \mathrm{OOO}$.

Those with one raccination, I 4,613 soldiers had 6 IS cases, or $42-1,000$.

The non-inoculated, 8,968 soldiers had $83+$ cases, or 93-1,000.

Those not raccinated had fourteen times more cholera cases than those vaccinated twice.

When the raccinations were completed cholera disappeared as if by enchantment, sporadic cases only arising from newly arrived, noninoculated soldiers.

Equally goot results were obtained from the civil population.

One has to remember that the type of the disease was not a very virulent one. Epidemics of cholera vary much in their mortality from 9o to 20 per cent.

Carriers are always troublesome. A convalescent may carry the vibrios for three weeks, some up to one year. The stools should be free on two examinations before the patient is discharged. At present there is no satisfactory way of clearing them out of the gall-bladder.

\section{ENTERIC FEVER IN THE TROPICS.}

\section{DEFINITION.}

A specific infectious fever, three varieties caused by:-

(1) Bacillus typhosus (Ebert) causes typical typhoid fever.

(2) Bacillus paratyphosus A causes paratyphoid fever $A$.

(3) Bacillus paratyphosus B causes paratyphoid fever B accompanied by diarrhora, pea-soup stools, rose coloured rash and running a prolonged course of about 2 I days.

Typhoid fever has long existed in the tropics but was previously concealed under the term of "remittent fever," $8 \mathrm{c}$. It is now wellknown to exist in many if not most tropical countries, occurring in Europeans and natives. It is alarmingly prevalent among young soldiers and civilians in the East, especially during their first three years' residence.

\section{ETIOLOGY.}

Caused by the above organisms.

It attacks both sexes usually between 15 and 25 years, but no age is exempt.

Infection is conveyed by:-

( I) Contaminated water, ice or milk.

(2) Foods contaminated by filies. 
(3) Uncooked vegetables grown on infected soils.

(4) Shell-fish grown on polluted beds.

(5) Direct contact with the soiled linen, stools and urine, typhoid abscesses of the patient.

(6) Contaminated soil resulting from defective drains and cesspools. The soil may be dry and be blown on to food or swallowed in the dust.

(7) Typhoid carriers.

\section{(1) F ECAL CARRIERS.}

The proportion of female carriers to the male is as five to one.

As women have gall-stones more frequently than men, and as the gall bladder is a habitat of the typhoid bacillus it is not to be wondered at that symptoms of gall-stones occur in 14 per cent. of cases after enteric fever. The bacilli may be passed down the gall bladder to the intestines and expelled in the faces without the patient being obviously ill, yet such bacilli may be very virulent and cause cleath to persons infected by them. The discharge of the bacilli appears to be intermittent. It is stated that women are more commonly carriers than men because their resisting powers are reduced by the decrease in the alexins of the blood during the menstrual and puerperal periods.

\section{(2) FLY CARRIERS.}

Infection by the house-fly (Musca domestica) and the fruit-fly (Drosophila ampelaphila) is most important because the fly takes up the bacilli into the alimentary canal in which the bacilli increase in number and virulence. (See habitats of house-fly, \&c., p. 224.)

The fly is a known foul feeder. After settling on faecal material it goes on to human food, regurgitates a little fluid from its crop to dissolve the food substances and thus infects them. It also defaecates frequently. The term "autumnal fever" has been given to epidemics which are in the time of the greatest prevalence of the flies.

\section{(3) URINARY CARRIERS.}

In such cases the habitat is the renal pelvis which is usually chronically inflamed. It may also live in the urinary bladder and other processes of the urinary tract. The bacilli pass directly into the urinary stream and are thus excreted.

Freal and urinary carriers may infect direc:ly the air, food, food utensils, drinking water and fomites, which in turn infect the victim.

B. paratyphosus B, commonly met with in Europe, lives often in the gall bladder and infects the faces. It has not been found in the urine. It has been found in the common house-fly. 
B. paratyphosus $A$ is prevalent in India and China. Several outbreaks have been traced to facal contamination.

Epidemics may be due to the seasonal prevalence of flies, distribution of milk, an infected well or stream, the seasonal use of sliell-fish, eating of fried fish so common in the East End of London.

For a description of the typhosus bacillus, see p. 619.

Europeans newly entering the tropics should be particularly careful. In Bengal 50 per cent. of the cases in Europeans occurred within one year of their arrival from Europe.

Rogers noticed that in India $+1 \cdot 67$ per cent. of the cases were in children under is years of age.

Natives are asserted to be somewhat immune to the disease.

Roberts in explaining this gives some interesting comparisons.

The gut of the native is longer, more muscular and thicker than that of the European, also Peyer's patches are less in evidence.

Habitat and diet also are important predisposing factors.

The Native

Food cold and dry.

Bulky, coarse, much waste.

Vegetabie grains, cereals, pulses ; Jarge cellulose content; low protein and fat content.

Food partially cooked, plain and monotonous. fasts.

Meals infrequent, tıvice daily, long

Mastication generally good.

Life and work in the open air.

Frecal evacuations twice daily, large, I0-12 ounces.

Strain on stomach and large bowel.

The majority eat to live.
The European

Hot and fluicl.

Concentrated and soluble.

Animal food, with high protein and fat content.

Thoroughly cooked, well mixed, and varied.

Meals frequent, 4-5 daily.

Faulty in the extreme.

Sedentary and indoors.

Small, 5-6 ounces, constipation com mon, purgatives.

Strain on stomach and small intestine

More live to eat.

\section{PATHOLOGY.}

The clisease is a septicamia produced by the invasion of the intestinal lymphoid tissue by bacilli which have entered by the mouth. In this tissue they multiply, many passing by the lympliatics to the abdominal lymphatic glands and the spleen increasing rapidly in each. In the blood they are destroyed if few in number and their toxins neutralized. If it does not proceed beyond 1his an ambulatory or abortive attack is produced.

When the bacilli are more numerous but destroyed by bacteriolysis there may not be sufficient antitoxin to neutralize their toxins, then the fever begins. Perhaps the antitoxin is formed irregularly and so accounts for the intermittent nature of the disease and the relapse in the tropics. The endotoxin causes the endothelial cells to swell so that the small lymph channels in the liver are blocked and patches of focal necrosis formed. 
While the bacilli may be found in any part of the body they prefer the lymplatic tissue. When a clump blocks a cutaneous iymphatic a rose-coloured macular papule appears which explains the characteristic rash.

Autoinfection may possibly explain some cases of second and third attacks, the bacilli of the gall-bladder or other habitat injecting anew the general system of the patient.

The gradual onset is the result possibly of the struggle between the antitoxins and the bacterial toxins. The rare sudden onset may be due to lowered resistance of the patient.

Immunity is partial, sometimes complete for the particular bacilli causing the attack, but not against other varieties.

\section{P.M.}

Bowels distended with gas. Marked congestion of the mucous membrane about ileo-cacal junction.

Mesenteric glands enlarged and congested.

Spleen and at times the liver also enlarged and congested. The former is friable and dark red in colour.

Perforation may be found.

The heart soft, flabby and friable.

The voluntary muscles undergo granular degeneration.

Peyer's patches are affected according to the stage reached.

First Weck:-

Swelling, the result of leucocytic infiltration. The submucous coat is also inrolved, and sometimes the muscular coat.

Second IVeck:-

Sloughing, the surfaces are abraded, the sloughs are bile-stained.

Third Week:- .

Separation, the sloughs come away, leaving an ulcerated surface, with undermined edges in the long axis of the bowel having its base of muscularis mucose, infiltrated mucosa or peritoneum.

Fourth IVeck:--

Scarring, the ulcers granulate up, the scar forming and contracting.

The solitary glands may pass through the same process.

The ileum and jejunum are usually affected.

The bile is usually light coloured, watery, but may be inspissated.

Kidneys enlarged, both cortex and medulla congested, fatty degeneration, rarely small abscesses.

There may be an inflammatory condition of part or whole of the respiratory tract. Hamorrhagic infarcts, pyamic abscesses and purulent infarcts may be seen. Venous thrombosis is not uncommon.

The bone marrow is generally congested. 
Arthritis of joints and abscesses in various parts; sequela may be found.

\section{SYMPTOMATOLOGY.}

Incubation three to twenty-three days, arerage ten days.

Onset insidious, headache, sometimes epistaxis, increasing weakness.

As the condition is a septicamia any part may be affected, causing pleurisy, romiting, or other symptoms tending to obscure the diagnosis.

The disease is usually ushered in by some other ferer, as malaria.

First Week:-

The temperature rises "staircase" manner, going up two degrees in the evening and falling one degree in the morning (not always) up to about $\mathrm{IO}_{4}{ }^{\mathrm{O}} \mathrm{F}$.

The pulse is slow and often dicrotic.

The skin.-Cheeks flushed. Skin hot and dry, sometimes intermittent sweating. A bluish mottling on abdominal wall. Often yellow coloration of palms and soles.

The tongue.- White moist fur on dorsum, tip and edges are red. Mouth dry, thirst marked.

The abdomen is distended, tenderness in the right iliac fossa, and spleen is enlarged. Abdominal reflex is diminished or lost. Vague general pains.

Exhaustion increases, patient becomes apathetic, drowsy, eyes bright; sleeplessness and romiting are not uncommon.

The stools.-Diarrhora in 20 per cent. of cases, becomes ochreyellow (pea-soup) in appearance, but the bowels may be confined throughout.

Bacilli may be cultivated from the blood, but specific agglutination is not available.

Second TVeek:-

The syrmptoms above are aggravated, but headache may disappear.

The temperature remains high. Mild delirium is not uncommon.

Rash appears from seventh to twelfth day in 70 per cent. of cases on abdomen or chest in crops; arms and legs may also be affected. The isolated maculopapules which disappear on pressure are slighty elevated, rose-red, $+\mathrm{mm}$. in diameter, last three to five days, leave a yellow stain. They may continue to end of third reek.

Tendency to hamorrhage (10 per cent. of cases) is marked, and danger of bowel perforation possible.

The specific agglutination reaction may now be obtained.

The diazo-reaction of the urine is now also possible. 
The bacilli are not so numerous in the peripheral circulation, but more plentiful in the spleen, faces, urine (33 per cent. of cases), and rose spots.

Liver and gall-bladder symptoms may set in.

B.P. diminished, dicrotism perhaps has disappeared.

Cold extremities. Vomiting rare.

The typhoid state may set in, lips and teeth being covered with dark brown scales (sordes), tongue dry, furred, fissured, pharynx inflamed or ulcerated. More or less meteorism always present, and may be very troublesome. Reduction in red cells, Hb. and leucocytes shown, but the mononuclear leucocytes are increased. Coagulability of blood reduced.

\section{Third Week:-}

Temperature falls by lysis to normal about the twenty-first day, and with this symptoms may subside and convalescence begin. In some cases profound symptoms may appear. The heart is weak and rapid. The lungs congested, fatal. Epistaxis possible. Perforation not uncommon (3 per cent. of cases). Subsiltus tendinum often present. Emaciation increases. Liver clulness encroached upon by the tympanites and breathing becomes thoracic. Urine may be suppressed. Peritonitis or collapse may cause death.

Fourth Treek:-

Temperature becomes normal; convalescence begins.

Watch for relapses.

In severe cases the status typhosus may continue; the heart may fail, for the myocarditis is considerable.

The varieties are according to the severity of the symptoms, ambulatory, abortive, mild, typical, severe and masked. Hamorrhagic cases have been known, when there has been bleeding from the mucous membrane, tongue, gums, bladder and intestines, also purpuric eruptions.

In Ceylon the disease caused by B. paratyphosus, viz., Paratyphoid fever, is indistinguishable from Typhoid fever, though it generally runs a milder course. The intestinal ulcers are identical with those of typhoid. Cases of mixed infection are not rare.

\section{COMPLICATIONS.}

Malaria very important.

Hamorthage from bowel, nose and mucous surfaces.

Perforation may occur in mild cases.

Peritonitis, with or without perforation. 
Meteorism or tympanitis.

Thrombosis, usual of femoral rein, embolism possible.

Veuritis, bronchitis and pneumonia.

Early typhoid state or cardiac failure.

Bed sores and boils. Alopecia.

Typhoidal arthritis, typhoidal spondylitis, ascending myelitis.

Periostitis. Inflammation of thyroid. Iritis, orbital cellulitis.

Relapses during the third and fourth week after the temperature has been normal are not uncommon.

Signs of perforation.-Sudden and severe pain, rapid and extreme distension of the abdomen, absence of abdominal respiration, tympanic percussion note, absence of hepatic and splenic dulness. Shock, rapid pulse and respiration, fluid in abdomen. Collapse.

\section{DIÄGNOSIS.}

In typical cases easy. The insidious onset, dicrotic pulse, medium enlargement of spleen, rose rash, leucopenia; if all are present it scarcely permits of mistake.

In the tropics the course is more often atypical.

Malarial fever will modify the temperature chart, alter the appearance of the rash, and easily throw one off his guard.

Bacteriological diagnosis is therefore all-important, as seen in :-

(1) The agglutination test (Widal). Important. See p. 6i I.

(2) Hamocultures. Important. See p. 623.

(3) The bacilli in the stools and urine.

(4) Spleen puncture gives good results, but should not be a routine procedure.

(5) Ophthalmo-reaction and cuti-reaction, similar as for tuberculosis.

(6) Subcutaneous inoculation of dead cultures and Vincent's splenic diagnosis.

(7) Complement fixation.

With regard to the W'idal reaction (see p.611), the three strains of bacilli should each be tried failing agglutination of one of them.

It is absent during the first week in any case, and may be in exceptional cases during the whole period. On the contrary, if a positive result is obtained, one must remember that a former attack would give it for months or years after it was over. It may be possible in vaccinated persons.

It has been found present in jaundice, Weil's disease, and puerperal fever.

The rapid differentiation of the bacilli of the typhoid group by means of test papers has been derised by Hollande and Beuverie. 
For the differentiation of the Bacilus typhosus, B. paratyphosus A and $\mathrm{B}$ and $\mathrm{B}$. coli four test papers are used as follows :-

(I) A piece of filter paper is dipped into a 1 per cent. solution of silver nitrate, and is then quickly dried; it is then dipped into a ro per cent. Solution of collodion in equal parts of alcohol and ether, drained and dried in the dark.

(2) A filter paper is dipped into a I per cent. solution of neutral red containing 10 per cent. glucose, and then dried and impregnated with collodion as before.

(3) A filter paper is dipped into a ro per cent. solution of lead subacetic and dried, as before, with collodion.

(4) A paper is dipped into the following solution : $0^{\circ} 2 \mathrm{grm}$. litmus,

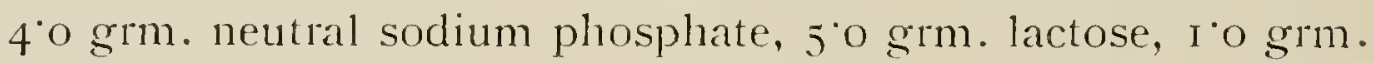
sodium bicarbonate, 50 c.c. distilled water; it is then dried and again treated with collodion.

Portions of these prepared papers are then dropped into peptone. broth tubes, which are then autoclaved at i $S^{\circ} \mathrm{C}$. for twenty minutes.

Results.

(I) With the silver nitrate paper a growth of $B$. coli is obtained in about twelve hours, of the three other bacteria in about two to three days.

(2) With the neutral red paper, the red colour of the paper and broth are unchanged by $B$. typhosus, the paper remains at the bottom of the tube, and there is no gas formation.

With the three other organisms the colour changes to. canary-yellow, and gas is formed between the collodion and the paper, and the latter then floats to the surface.

(3) Lead acetate paper is blackened after about twenty hours, except in the case of $B$. paratyphosus $A$, in which no change occurs.

(4) With the litmus lactose paper the lilac colour of the paper and broth is clischarged after about twenty hours, but in three days. the colour returns in the case of $B$. paratyphosus $B$.

In using hemocultures there are two methods:-

( I) Dilution method.

Take 2.5 c.c. of blood from the median basilic aseptically, drop it into a large sterile flask at once containing 200 to 300 c.c. of faintly alkaline broth.

Incubate at $37^{\circ} \mathrm{C}$.

In twelve to twenty-four hours, in positive cases, the broth becomes: cloudy and shows a germ growth. Then test this in various media.

The agglutination test should also be done.

(2) Bile enrichment method.

Take blood as above or from the finger tip. 
Drop it into a mixture of oxbile go c.c., glycerine ro c.c., and peptone 2 grm., distributed into small flasks each containing 20 c.c. of the medium.

Incubate and test as above.

Ehrlich and Diaso Reaction.

Generally present in typhoid during the second and third week.

It may be absent in very mild cases.

Very active tuberculosis gives it fairly constantly.

Measles gives it more frequently than German measles.

If it is present it is not diagnostic of typhoid, but may be used for confirmation, especially if the other diseases can be excluded.

Prepare two fresh solutions :-

(I) A saturated solution of sulphanilic acid in 5 per cent. HCl.

(2) A $2 \frac{1}{2}$ per cent. Solution of sodium nitrite.

To 5 c.c. of urine, add an equal quantity of solution.

(1) Then add a few drops of solution (2) and shake till frothy.

Add ammonia till alkaline.

If the liquid is port-wine colour and the froth is red, the reaction is positive.

In examining the faces use Conradi-Drigalski medium.

The malachite green solution inhibits the coli growth and permits that of the typhoid bacillus.

In cxamining water, pass it through a filter; the organisms remain on the filter candle; brush these off into the above medium.

Alum will bring down a precipitate entangling the organisms, which can then be treated as above.

The typhoid bacillus is seldom found in the water that has caused the disease, because fourteen days have elapsed since the patient partook of it and the organisms would have gone.

\section{DIFFERENTIAL DIAGNOSIS.}

In malaria, examine the blood for the parasites.

In relapsing fever, the onset is sudden and the blood will show the parasite.

In undulant fever, do an agglutination test; cumulative evidence is important.

In yellow fever by sudden onset, albuminuria and black romit.

In typhus fever by sudden onset, mental symptoms appear early.

In acute miliary tuberculosis, irregular fever, pulse and respiration rapid, and is not dicrotic.

In psittacosis, the specific bacilli can be isolated from the blood and sick parrots will be about.

In ulcerative endocarditis, by the murmurs and bacteriological examination. 
In paratyphoid fever $A$ and $B$ the agglutination test and cultural characteristics have to be relied on .

One must not forget that typhoid and another disease may co-exist.

The mortality in the tropics is about $20-25$ per cent.

\section{TREATMENT.}

(1) General management.

Patient to be put on a wire-woven mattress with a soft horsehair mattress placed upon it, two blankets, then the sheet with a draw sheet, and its waterproof covering in the centre.

A mosquito net must be provided.

The room must be large, as empty as possible, and well ventilated.

All motions and urine and fomites sterilized at once in carbolic.

A day and a night nurse well skilled and vaccinated are essential.

The patient to be sponged all over twice daily with tepid water.

Gently rub irritated parts with rectified spirits, and then dust with boric or starch powder.

Mouthwash necessary. Bed pan and urine bottle to be used all the time.

(2) Diet.

No solid food is the safest rule.

Milk, beef-tea, chicken broth, albumin water from white of eggs.

Plasmon, barley water, malted milk, whey, junket and weak tea.

Water ad lib., neither hot nor cold.

(3) Drugs.

The less the better as a rule. Simple enema for constipation.

Urotropine gr. $x$ t.d.s. in second week to disinfect urinary tract.

(4) Serotherapy.

Chantemesse's anti-typhoid serum has good results.

The Wright-Leishman's vaccine from forty-eight hours culture, in three doses 5 oo millions of bacilli, ten days later 1, ooo million, ten days later another $\mathrm{I}$, ooo million. This is used as a prophylactic.

Leishman had 19,000 cases under observation in India with the following results :-

9,00o were inoculated. Case incidence, 53 per cent. Case mortality, 9 per cent.

Io, ooo were not inoculated. Case incidence, $30^{\circ} 4$ per cent. Case mortality, 17 per cent.

The immunity lasts about one year. If the whole Indian army was inoculated, I, ooo cases of enteric ferer and 200 deaths would be aroided each year.

Mixed vaccines can be obtained for the three stains. 
SPECIAL SYMPTOMS.

(I) Tympanitis. Fomentations, turpentine stupes, turpentine I5 minims every three hours. Hypodermic injection of eserine $\mathrm{gr}^{2} \cdot \frac{1}{50}$.

(2) Hamorrhage. Stop all food, drink sips of water only, ricebag to abdomen, raise bedclothes on a cradle. Give simple enema. Morphial useful if one is sure that perforation has not occurred.

Calcium lactate gr. xr t.d.s. Lead acetate.

(3) Perforation. Laparotomy as soon as possible. Salines per rectum, 2-6 litres per diem for a week.

(4) Diarrhoea. Tannalbin gr. x-xii t.d.s.

An enema with gr. $r$ of Dover's powder also.

Avoid bismuth, as it obscures traces of blood.

(5) Cholecystitis. Lrotropine gr. $x$ t.d.s., anti-typhoidal vaccination or surgical treatment.

(6) Hyperpyrexia. Sponging, bath immersion $75^{\circ}-85^{\circ} \mathrm{F}$. No antipyretics.

( 7 ) Delirium. Bromides, lumbar puncture.

(8) Cardiac failure. Digitalis and its preparations, or camphor and ether, strychnine and salines.

(9) Phlebitis. Wrap leg in cotton-wool after applying ichthyol in lanoline (2 per cent.).

(10) Bedsores. Harden skin with spirit lotion and disinfect $\mathrm{H}_{2} \mathrm{O}_{2}$ twice daily; dust with zinc oxide powder.

(I 1) Abscesses. Incise and drain.

(I 2) Bone lesions. Supports as necessary; operative treatment.

\section{FLIES AND DISEASE.}

\section{FLIES ARE DANGEROUS TO HEALTH.}

(I) By carrying pathogenic organisms directly from infected matter upon which they have settled to a wound abrasion, or absorbent mucous membrane surface. Hence perhaps spreading disease as anthrax, ophthalmia, yaws and tropical sore.

(2) By feeding on infected matter and then carrying that infection by means of regurgitation or defaecation upon a receptive surface as above. The germs passing through the flies' intestines with undiminished virulence or possibly multiplying therein. Staphylococcus pyogenes and the tubercle bacillus has thus been found.

(3) By carrying the germs as above and infecting the food and drink of man. Hence diseases can be disseminated as typhoid fever and cholera, perhaps of infantile diarrhoea and dysentery. They also carry the eggs of parasitic worms. 
(4) By blood-sucking flies biting infected vertebrates sucking up the pathogenic organisms and injecting them later into the body of a healthy individual as the hamamoba of malaria, the trypansome of sleeping sickness, the filariae of filariasis and the unknown organism of yellow fever.

(5) By biting flies causing local irritations and thus predisposing 10 septic infection.

(6) By the adult flies laying their eggs in wounds and the natural orifices of the body like the green-bottle and the notorious screwworm fly, the larva later burrowing their way into the tissues and causing even mortal damage.

(7) By the flies laying eggs on the food and the larva damaging the victims' health resulting from their presence in the intestine.

The ignorant and apathetic slum population suffer most, especially in crowded bazars and markets where offal is plentiful and sanitation bad.

\section{HABITS, \&c., OF THE MUSCA DOMESTICA (House-fly).}

Order.-Diptera ( = with two wings).

Suborder.-Cyclorrapha (=-a circle; - suture). The imago escapes from the puparium through a circular slit at the anterior end.

Scction (2).-G. schizophora (= a cleft; = I wear). In these adults the frontal suture is always well defined.

Subsction (I).-Muscoidea.

Family.-Muscida, including house-flies and tsetse-flies.

Genus.-Musca domestica. Important in carrying typhoid ferer; gS per cent. of flies infecting houses are Musca domestica. These common flies are world-wide. Small, dull-coloured, blackish-grey thorax, yellowish abdomen, with a median dark stripe. Arista feathered dorsally and ventrally, proboscis soft, retractile and ends in a large fleshy labella. The fourth longitudinal vein bends forward at an abrupt angle so as to nearly close the first posterior cell. In the female the eyes are wide apart, in the male they are set close together.

In hot countries they breed all the year round.

In cooler countries they breed summer and autumn and then die out except for a few sheltered ones. These survivors and new ones which have hibernated, emerging from pupa soon increase their kind in warm weather.

The female lays I 20-I 50 eggs in a batch in any moist rubbish heap, stable sweepings, vegetable debris, house refuse, \&c. The white, shiny, sticky eggs hatch in twenty-four hours in warm weather.

The larva or maggot is whitish, cylindrical, tapers anteriorly, has twelve segments and a very minute head. It has a pair of large, black 
mandibles for tearing and burrowing into food material. At the opposite end the main tracheal runks open by three slits. It feeds on decomposing refuse.

It is full-grown in live days in the tropics, in England one to eight weeks.

The larva then becomes barrel shaped, skin hard, becomes dark in colour, forms the pupa, this stage lasting about five days.

Period of development in the tropics about five days.

Period of development in England about two to ten weeks.

An adult fly lives from three to sixteen weeks.

\section{TO SUPPRESS THEM.}

House refuse, street sweepings and all rubbish 10 be burnt or deposited several miles away from human dwellings and leeward of prevalent winds.

Iatrine refuse should be buried bevond reach of the flies.

Horse dung required for agricultural or other purposes should be dumped at a distance, treated with quicklime, moved about with a fork frequently so that the birds may get at the larre. Carts conveying refuse must not drip their contents on the highway. Some enemies of the fly should be encouraged, as: fungi, e.g., Empusa musca, spiders, centipedes, larve of beetles, ants, wasps, toads, lizards and rats.

The use of gauze and fly-papers adds to one's comfort but are useless for suppressing flies.

\section{HOW THEY INFECT MAN.}

The mouth parts of this fly are so made that they cannot get solid particles into their mouths. 'They taste all foods by pressing their oral lobes upon them. If such food is satisfactory a little fluid is regurgitated on to it from its crop in the attempt to dissolve some of the food substance so that it can pass along the minute pseudo-trachea. They pour upon the substance, later eaten by man, numerous typhoid bacilli from infected faeces and thus infect a new victim by the food, sugar, bread, milk, meat or edge of the cup, \&c. Not only so, but they defacate about fifty times in twenty-four hours and the typhoid bacilli pass out perhaps in an enhanced condition of virulence and infect the food materials, water and utensils of the human host. These were very instrumental in spreading typhoid fever during the Boer War, IgorI9O2.

The blue-bottle or blow-fly is of the same family (Genus Calliphora) and has similar morphology and life history.

The fruit-fly Drosophila ampelophila is also a musca with a similar history. 
SECTION. III.

\section{DISEASES DUE TO HELMINTHS.}

GENERAL RENARKS.

DISTRIBUTION'.

THEIR PATHOGENIC ACTION.

NOMENCLAT URE.

THE GROUPING OF HELMINTHS.

I. - Trenatodes Pathogenic to Man.

General Morphology, Life-Histori, \&c.

Classification.

The More Pathogenic Varieties.

Paragonimilasis (ENDEAIIC Henoptosis).

SCHISTOSOMIASIS.

II.-Cestodes Pathogenic to Max.

General Morphology, Life-Histori, \&C.

Classirication.

The Pathogenic Species Seriatim.

The TREATMENT OF TAPEWORMS.

ili.-Nematodes Pathogenic to Man.

General Morphologi; \&c.

THE LESS IMPORTANT SPECIES.

STRONGILOIDES STERCORALIS.

GNathostoma SPINIGERUM.

THE FILARID压.

Historical Notes.

Filaria bancroeti, MORPhIOLOGY, Life-History，\&C

The Diagnosis of Microfilaria.

THE Filariases.

DisTRIBUTION.

PATHOI.OGI.

Clinical Varieties.

FILARIAL LYMPHANGITIS.

, ORCHITIS AND HydROCEI.F.

". IJMPHANGIECTASIS.

Abscesses.

PhlemeCtasis

," VARICOSE LYMIPH GLANINS.

Filarial C HILOUS Extrayasations.

CHYLURIA AND LYMPHURIA.

CHYLOUS AND LYMPHATIC DIARRHEA.

CHYLOCEIE OR LYMIPHOCELE.

Chyious Ascites. 
ELEPHANTIASIS.

Clinical Varieties.

ELEPHANTIASIS OF THE LEG.

$\begin{array}{lll}, & , & \text { SCROTUM. } \\ , & , & \text { VULVA. } \\ , & , & \text { BREAST. } \\ , & , & \text { ARM. } \\ , & , & \text { SCALP. } \\ \text {, } & \text { ", } & \text { LOCALIZED AREAS. }\end{array}$

Notes on Filarial Carriers.

CULEx Fatigans.

Stegomyia Calopus.

LOASIS.

Chrysops DIMIDIATA ANI SILACEA.

Calabar Swellings.

VOLVULOSIS.

Dracontiasis.

TRICHOCEPHALIASIS.

Trichiniasis.

ASCARIASIS.

OXYURIASIS.

ANKYLOSTOMIASIS.

THE MODE OF INFECTION BY HELMINTHS.

THE PRESERVATION AND EXAMINATION OF HELMINTHS. HIRUDINIASIS.

POROCEPHALOSIS. 


\section{DISEASES DUE TO HELMINTHS.}

\section{GINERAL REMARIS.}

The animals causing disease in man are from three kingdoms, viz.: Animal, Protista or Intermediate, and Vegetable.

We are concerned here with the former or Animal kingdom, which for convenience is divided into the :-

Sub-kingdom r, Protozoa, and the Sub-kingdom 2, Metazoa.

The Protozoa are dealt with in another section.

All Metazoa are multicellular, free-living, or parasitic animals, having groups of cells to perform definite functions and are sometimes referred to as the Animal kingdom in a restricted sense.

The parasitic metazoa may be ectoparasites as insects, mosquitoes, \&c., or endoparasites as helminths.

The Metazoa parasitic for man are classified thus:--

Phylum (I) Platyhelmia.

, (2) Nemathelmia.

, (3) Annulata.

, (4) Arthropoda.

In this section we shall deal with those Platyhelmia and Nemathelmia pathogenic to man. These are principally to be found among the Trematodes (Flukes), Cestodes (Tapeworms), and Nematodes (Threadworms).

\section{DISTRIBUTION.}

Some helminths are found everywhere, such as the Ascaris lumbricoides, Oxyuris vermicularis, Trichocephalus dispar and others.

Others are prevalent where dogs and sheep abound, as the Tania echinococcus in Australia; Flukes are found chiefly in Isia, Filaria rolvulus in West Africa, and the Gastrodiscus in Assam.

The ankylostomes are restricted to warm climates where they can obtain plenty of moisture, or to warm temperatures in other countries in the presence of moisture as in the Cornwall mines.

\section{Factors controlling the Distribution (Leiper).}

(I) General sanitary measures and personal hygiene.

The latter measure thoroughly carried out would stamp out Oxyuris in six reeks. The eggs are laid about the buttocks, re-infection taking place by the eggs getting in the finger nails.

(2) The preparation of food.

Trichinosis is common where fresh-water fish and insufficiently cooked food are eaten. 
(3) The disposal of excreta.

Failure in this measure causes apparent epidemics of ankylostomiasis.

(4) Religion.

Tania solium is carried in pork and is practically unknown in Mohammedan countries.

(5) Temperature.

If the temperature is not sufficiently high, ankylostomes cannot develop. Neteorological conditions must be favourable for the growth and development of the parasite and its host.

(6) The control of the water supply.

The Guinea-worm is common where the water in the village pond is used for washing purposes at one side and drinking purposes at the other. In China, men defaecate into ponds, the fishes consume the excreta, while at the other side are men fishing and devouring the results of their sport, becoming infected consequently with Clonorchis.

(7) Carriers.

Soldiers from the Boer War came lome with bilharzial hamaturia and infected others in England in this way.

Some pigmies from Central Africa came to london bringing with them Necator americanus.

(S) Domesticated animals.

Where sheep-rearing is common, the Echinococcus prevails as in Australia.

(9) The distribution of the intermediate host.

Snails and slugs are necessary for Flukes.

The Bothriocephalus cannot spread without special species of fish.

Trematodes require an intermediate host for their development.

\section{Theil pathogenic action varies according to:-}

(1) The sisc of the worm which may cause mechanical injury as the Ascaris when blocking ducts, \&c., causing thereby appendicitis, retention of pancreatic fluid, \&c.

(2) The position of the worm which will alter the symptoms and the seriousness of the infection. A paragonimus in the lung, a fluke in the liver, or a hamatobia in the bladder give a more varied set of symptoms than a Guinea-morm in the subcutaneous tissue of the leg.

(3) The number present, as when several lumbricoides form a coil and cause intestinal obstruction.

(4) The condition of the host. Ascarides may be harmless in a 
healthy individual, but when in a dysenteric or typhoid intestine their presence is more serious.

(5) The bacterial infection induced by them. The Trichuris trichiura is harmless in itself but can introduce bacteria into the mucous membrane of the appendix, \&c., and set up an inflammatory condition. Helminths are usually covered externally by bacteria, and their intestines may contain a most extensive microbic flora.

(6) Their selective action. Ankylostomes are always found in the small intestine. They will pass through the skin, veins, heart, lungs, bronchi, trachea, esophagus and stomach but will not rest anywhere until they have reached the small intestine. Perhaps there is a special secretion there which they prefer. Again, trematodes select out their own special mollusc. If trematode embryos are placed in a tank with various molluses the embryos will be attracted by one particular variety only. They will even prefer the fluid in which their special mollusc has been placed and removed, ignoring all other fluids that are the same in every other respect.

The condition produced may be the result of :-

A mechanical erosion of tissue.

The absorption of food materials ingested.

Toxæmic poisoning.

Fibrosis, eosinophilia, and secondary anemia.

The retention of essential glandular secretion.

\section{NOMENCLATURE.}

There has been and is still much confusion in the naming of parasites. A close study must be made of the literature of any supposed new species before names are applied.

As a guide to those who may require them, a set of rules has been drawn up to regulate the giving of names, and are known as :--

The International Rules of Nomenclature.

(1) The Language Rule.

The scientific names of animals must be Latin or latinized, of treated as such.

The generic name has a capital, the specific name a small letter. The generic name must be one word and used in the singular. The sub-genus, when one is present, can be inserted in brackets, with a capital letter between the genus and the specific name.

The family name ends in "idæ," the sub-family in "inæ." The author of a scientific name is the one who first publishes 
that name. One man may describe the creature found but another may name it; this latter person is the author.

(2) The Rule of Priority.

The first name griven must be adopted by all unless rendered invalid. Hence Trichina was found to have been applied to an insect some 50 years before it was applied to the new worm and consequently was changed to "Trichinela."

Different spelling can be a different name.

(3) The Rule of Homonyms.

When two distinct genera and species of animals receive the same name that name applied first must stand.

(4) The Rule of Appropriateness.

No name is allowed to be clianged simply because it is inappropriate. An author cannot reject the name he has once given.

\section{THE GROUPING OF HELMINTHS.}

Helmintlis occurring in the lumman intestine belong to two distinct groups of the animal kingdom, viz. :-

(1) Platyhelmia, (2) Namathelmia.

\section{Platyhelmia.}

These are flat and leaf-like. 'There is no body cavity.

The mouth is at the anterior end, or near to it, and is on the ventral surface. It is simple, forked or branched, but always blind.

There is no hind gut or anus.

The muscular fibres are angular, longitudinal, and circular.

The excretory system is highly complicated and consists of at branched set of tubules discharging at or near the postero-centra] surface.

The sexes are not separate with two exceptions, the important one being the Bilharzia hanatobium.

There are two classes of Platyhelminths, Trematoda and Cestoda.

Cestodes are really proliferating trematodes. The latter consist of a single segment, the former of many. The latter have an alimentary canal, the former none.

For classification see next page.

The species will be described according to their pathogenicity in man under their respective headings.

\section{Nemathelmia.}

These are bilaterally symmetrically thread-like with a distinct cavity between the body wall and the gut in which the genital organs freely float.

The alimentary canal, when present, is a straight tube from mouth to anus, or it may be absent. 

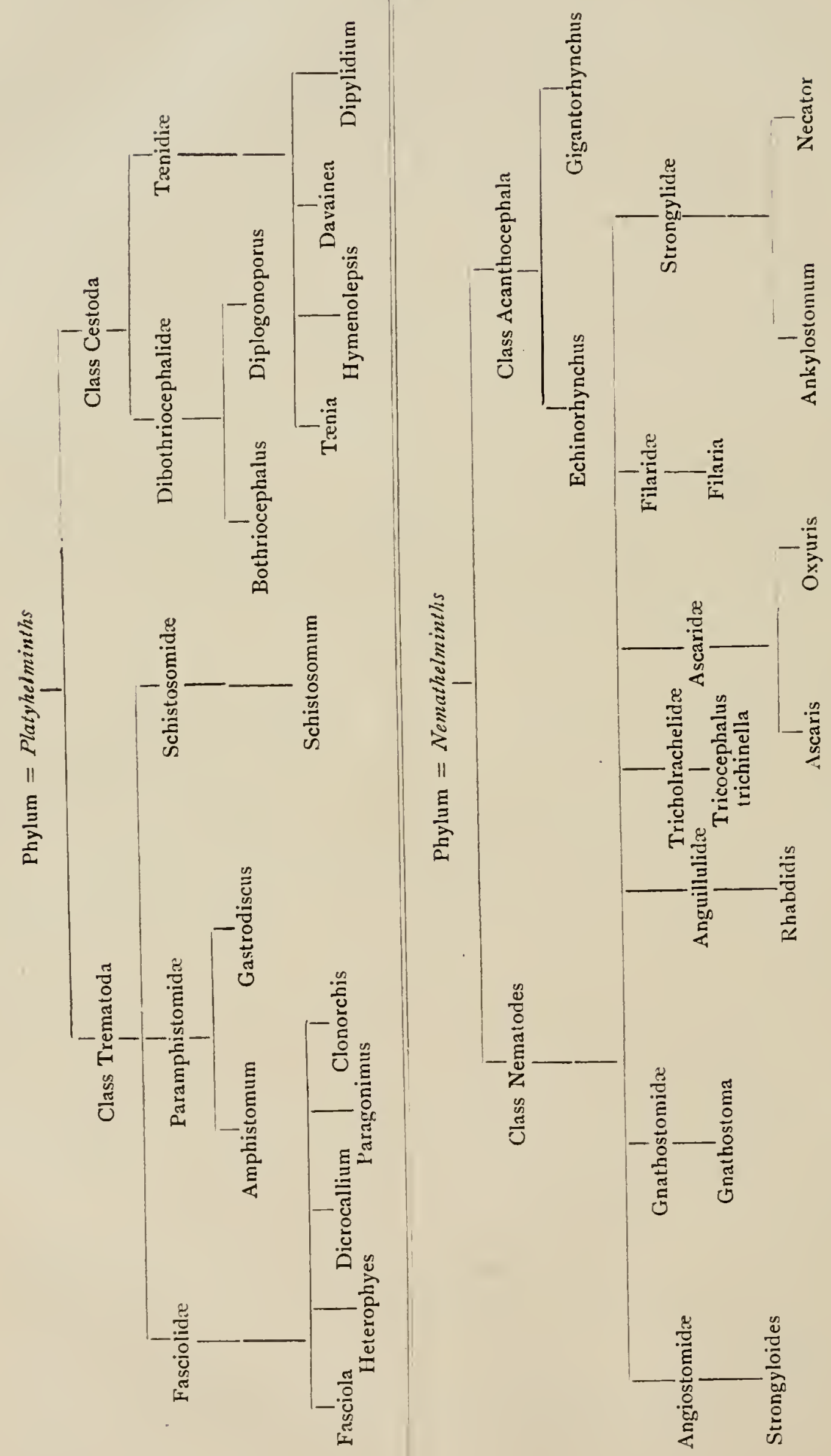


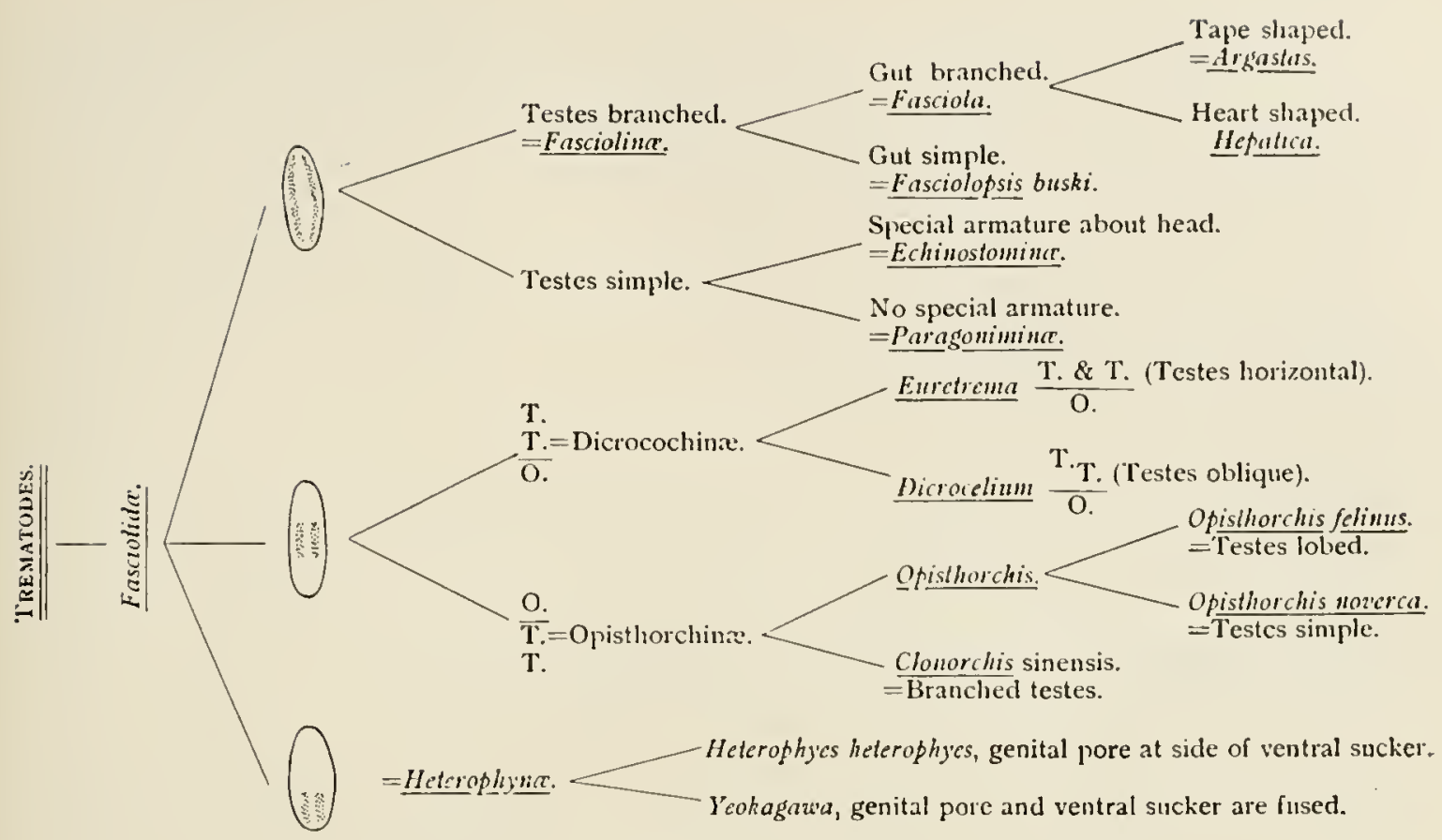

N.B. The dots in the diagram show the position of the eggs.

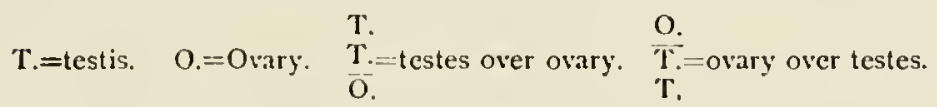

Fasciolide. Points of Differentiation.

\begin{tabular}{|c|c|c|c|c|c|c|}
\hline Name & Size & Ca:ca & Testes & Yolk Glands & Cirrus & Habitat \\
\hline Fasciola ... & $\begin{array}{l}\text { Large, } \\
\text { leaf like }\end{array}$ & Bıanched & $\begin{array}{l}\text { Branched } \\
\text { posterior }\end{array}$ & Full length & Present & Liver \\
\hline Echinostoma & $\begin{array}{l}\text { Very small, } \\
\text { narrow }\end{array}$ & $\begin{array}{l}\text { Not branched, } \\
\text { long }\end{array}$ & $\begin{array}{l}\text { Bilobed } \\
\text { posterior }\end{array}$ & $\begin{array}{l}\text { Posterior, } \\
\text { half }\end{array}$ & $\begin{array}{c}\text { Well } \\
\text { developed }\end{array}$ & $\begin{array}{c}\text { Small } \\
\text { intestines }\end{array}$ \\
\hline Fasciolopsis & Large & $\begin{array}{l}\text { Not branched, } \\
\text { long and wavy }\end{array}$ & $\begin{array}{l}\text { Branched } \\
\text { posterior }\end{array}$ & Full length & Very long & $\begin{array}{c}\text { Small } \\
\text { intestines }\end{array}$ \\
\hline Opisthorchis & $\begin{array}{l}\text { Long and } \\
\text { flat }\end{array}$ & $\begin{array}{l}\text { Not branched, } \\
\text { long }\end{array}$ & $\begin{array}{l}\text { Lobed } \\
\text { posterior }\end{array}$ & $\begin{array}{l}\text { Middle, } \\
\text { third }\end{array}$ & $\mathrm{Nil}$ & Liver \\
\hline Clonorchis... & $\begin{array}{c}\text { Long and } \\
\text { narrow, } \\
\text { pigmented }\end{array}$ & $\begin{array}{l}\text { Not branched, } \\
\text { long }\end{array}$ & $\begin{array}{l}\text { Branched } \\
\text { posterior }\end{array}$ & $\begin{array}{l}\text { Middle, } \\
\text { third }\end{array}$ & Nil & Liver \\
\hline Paragonimus & Broad, thick & $\begin{array}{l}\text { Not branched, } \\
\text { long }\end{array}$ & $\begin{array}{l}\text { Simple } \\
\text { posterior }\end{array}$ & $\begin{array}{l}\text { Whole } \\
\text { length }\end{array}$ & Nil & Lungs, \&c. \\
\hline Heterophyes & Small & $\begin{array}{l}\text { Not branched, } \\
\text { long }\end{array}$ & Simple & $\begin{array}{c}\text { Posterior, } \\
\text { half }\end{array}$ & Nil & $\begin{array}{c}\text { Small } \\
\text { intestines }\end{array}$ \\
\hline Dicrocœelium & Medium & $\begin{array}{l}\text { Not branched, } \\
\text { short }\end{array}$ & Simple & $\begin{array}{l}\text { Middle, } \\
\text { third }\end{array}$ & Present & Liver \\
\hline
\end{tabular}


The muscular fibres are longitudinal. There are no cilia.

The excretory system consists of a simple bilateral symmetrical system of tubules opening into a single excretory pore on the ventral surface of the body in the middle line.

The sexes are separate.

The Nematoda always have an alimentary canal. Nearly all those found in man are from this class.

The Nematomorpha have an alimentary canal which atrophies in the adult.

The Acanthocephala have no alimentary canal.

Nemathelmia pathogenic to man will be dealt with later.

\section{THE PLATYHELMIA HAYE THREE CLASSES.}

Class (I) The Turbellaria. Flat worms, ciliated, free-living.

They are not important for man.

Class (2) The Trematoda. Flat worms with an alimentary canal. They are parasitic to man.

Class (3) The Castoida. Flat worms without an alimentary canal. They are parasitic to man.

\section{1.-TREMATODA PATHOGENIC TO MAN.}

\section{THE MORPHOLOGY.}

Their average length is $\frac{7}{2}$ in., but their extremes are $I^{\cdot} 25$ to 3 in.

They are leaf-like or tongue-shaped, rarely cylindrical.

There are two suckers, one ventral and one oral, which fix the parasites to the lining of the intestine or other organ.

The mouth lies in the oral sucker.

The aesophagus may be long or short, and often has unicellular salivary glands.

The intestine is branched or simple and encls blindly.

There is no anus.

The testes may be branched, or simple, or lobed.

The ovary may be branched, simple, or lobed, and is usually in front of the testes.

These characteristics are used to differentiate the species as shown in the preceding table of the Fasciolida.

The genital pore is usually near the rentral sucker.

The cirrus pouch lies behind the genital pore and is present in some as a muscular collar which, when it contracts, inverts by its spines the canal they surround and thus gives an erectile organ which comes out of the genital pore.

The yolk glands, also used to differentiate the species, are arranged in one of three ways, viz. :-

In the middle third of the body placed laterally.

In the posterior third of the body placed laterally. 
In the whole length of the body placed laterally.

They are always small and numerous.

Laurer's canal is on the dorsum and is for the escape of yolk food when it is in excess, thus preventing the uterus being blocked with yolk (Leiper).

\section{THE LIFE-HISTORY.}

This is taken from a known typical example, viz.: Fasciola hepatica.

The ovum passes along the ovarian duct from the ovary and in this tube is fertilized and surrounded by yolk cells.

The shell gland then secretes a shell around the mass which then passes to the uterus and escapes in the freces of the parent worm. The egg thus passed has al lid. (Except the Schistomida.)

The ovum segments and grows, using up the yolk and forms a Miracidium. This escapes in a few weeks from the lid of the egg into the water. It has two eve spots, anterior papillat and cilia by which means it swims about. It now grows and develops an alimentary canal. By means of its anterior papille it enters the cavity of some snail, otherwise it dies in about eight hours. The special organs are now lost. 'The organism grows and a sporocyst is formed. Cellular differentiation again takes place.

The organism now has a cuticular lining, under which is a muscular layer lined with epithelium internally, forming thus a cavity which answers as an alimentary canal. This form is the redia. It now forces its way out of the cyst and wanders about the snail. It has a ridge or collar about its anterior end, and two stumpy protuberances posteriorly to aid locomotion.

Cells now bud off from the inner side of the body wall forming the cercaria. This is something like a young Fasciola with a tail.

These cell groups pass out from the redia by the genital pore, then leave the snail, swim about in the water, become encysted on grass and water weeds, and are then devoured by sheep, \&c. In the process of ingestion they lose their cyst, travel to the bile ducts, and there develop into mature flukes in about six weeks. It will be noticed that many flukes develop from one egg.

\section{HABITAT.}

They occur in the liver, intestines, lungs and urinary tract of man.

Dogs, pigs, cats and cattle are also infected.

In sheep they cause what is commonly known as "sheep-rot."

They may not cause obvious symptoms in man, but there may be irritation of any of the above organs by the eggs or the parasites themselves.

Sometimes they cause serious diseases in man. 


\section{TREATMENT.}

In cases of diarrhoea of obscure causation examine the freces, urine and sputum microscopically.

Be careful about domesticated animals; they are often a source of infection.

All green regetables should be cooked.

Avoid the so-called edible snail.

For the specific treatment see the following paragraphs.

\section{THE CLASSIFICATION OF TREMATODES.}

There are three important families:-

(I) Fasciolida, having two testes.

(2) Schistosomida, having one testis.

(3) Paraphistomide, having two testes.

For other details see previous tables.

\section{THE PATHOGENIC VARIETIES.}

These will now be dealt with scriatim.

Watsonius watsoni causes diarrho:a and anemia.

It lives in the small intestine and inflames the mucosa.

It has two pharyngeal pouches.

The oesophagus divides into two long intestinal caca arranged laterally.

The testes are lobulated and lie the one behind the other in the mid-line.

The ovary lies behind the testes.

Treat with eucalyptus and chloroform as in Anliylostomiasis.

Fasciola hepatica has usually been found in the liver of man, but it has been found also in the blood-ressels, in the cranial cavity, and about the feet, forming there superficial abscesses.

It is the common liver fluke of herbivorous animals.

It is very common in Africa, Burmah, Eggpt and other parts.

It is very widely spread. It is the cause of the dreaded "sheep rot."

The intestine divides into two principal lateral crea, which in turur give off many branched cæeca.

The two testes are much branched and lie behind each other in the mid-line.

In front of the ventral sucker is a cirrus pouch.

The ovary is tubular and branched. It lies above and in front of the testes.

These worms are situated in the bile ducts and cannot be dislodged. 

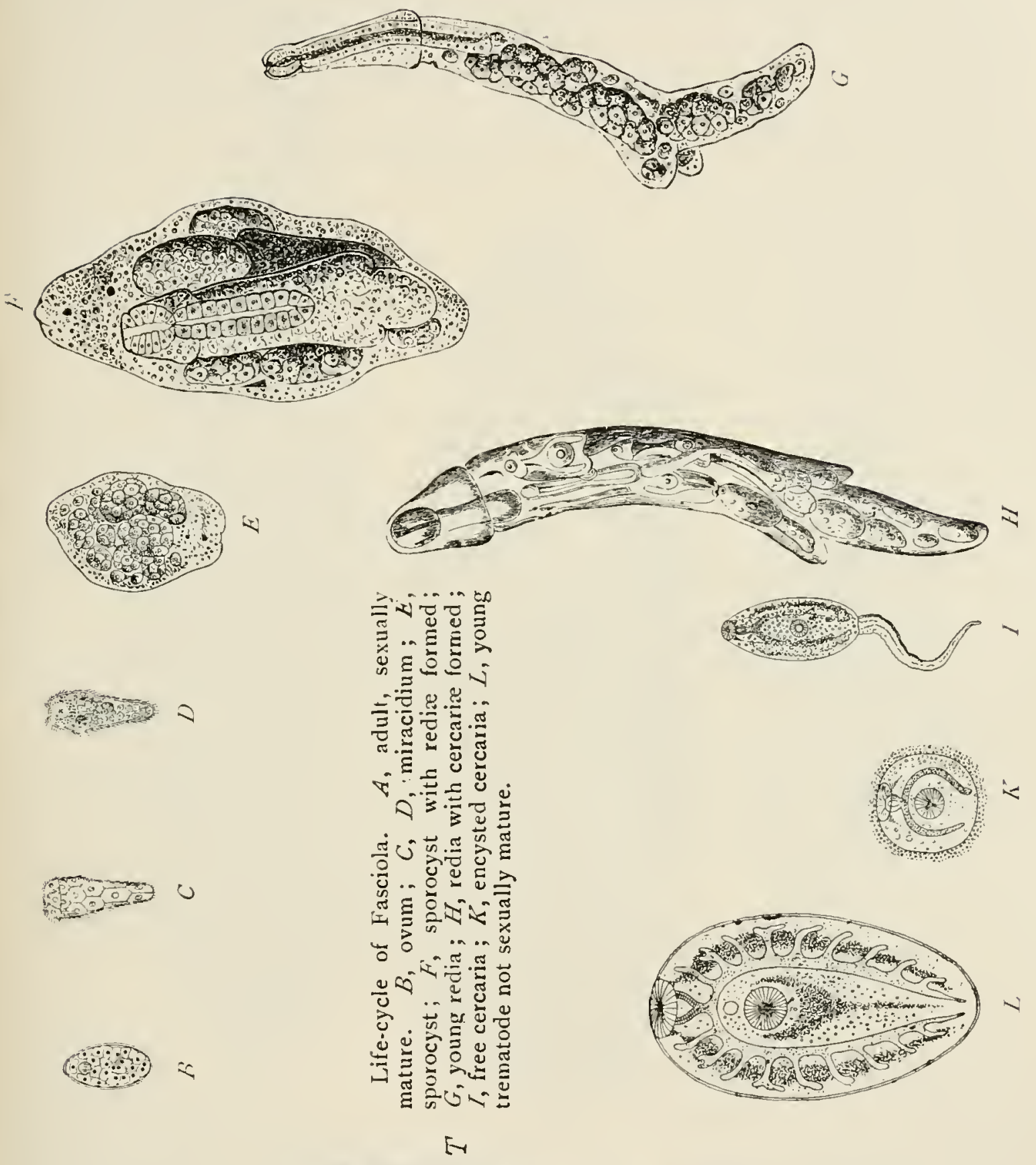

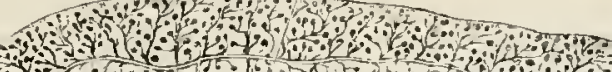

E

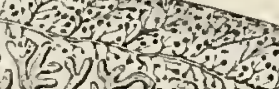

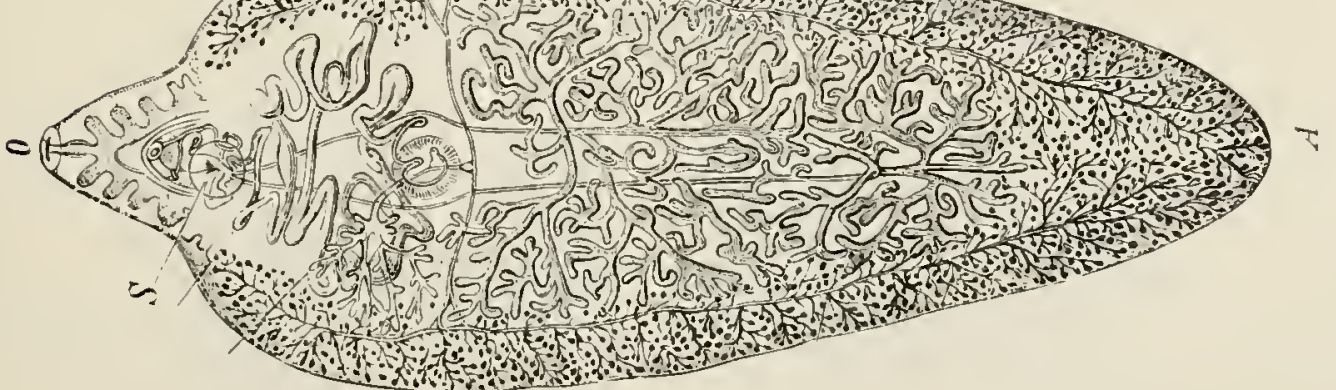


Fasciolopsis buski, a large trematode, 24 to $70 \mathrm{~mm}$. long, is not uncommon in men and pigs.

It is prevalent in the south of China and other places.

The intestinal creca which extend to the posterior are not branched.

The cirrus is very long, being one quarter the length of the worm.

The testes are branched and are situated below the ovary.

The worms cause dysentery and diarrhoea.

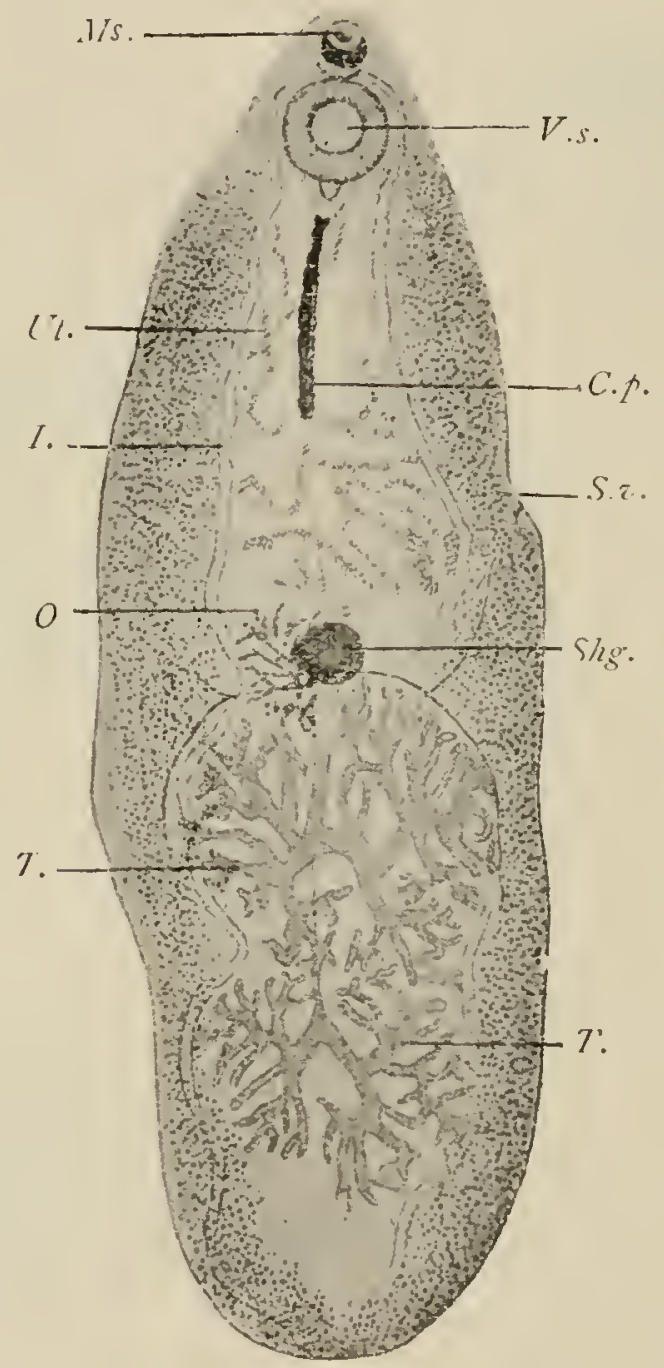

Fasciolopsis buski, Lank, $V . s .$, ventral sucker; $C_{. p}$. , cirrus pouch; $I_{\text {., intestin } t}$ fork; S.v., vitellaria: T., testes; $O$., ovary; Ms., sucker; Shg., shell gland; Ul., uterus. Magnified. (After Odhner.)

Opisthorchis felineus has been found in the bile ducts of man, causing inflammation thereof, with atrophy of the liver, jaundice and ascites.

The eggs containing a ciliated miracidium are expelled in the faces.

These parasites are more commonly found in the gall-bladder and bile-ducts of dogs and cats.

The intestinal cæca are unbranched.

The testes are lobulated and are situated the one behind the other. 
Clonorchis cndemicus has been found in the liver of man.

It causes an enlargement of the liver with diarrhoca.

The intestinal caeca are unbranched.

The yolk cells are arranged laterally in the middle third.

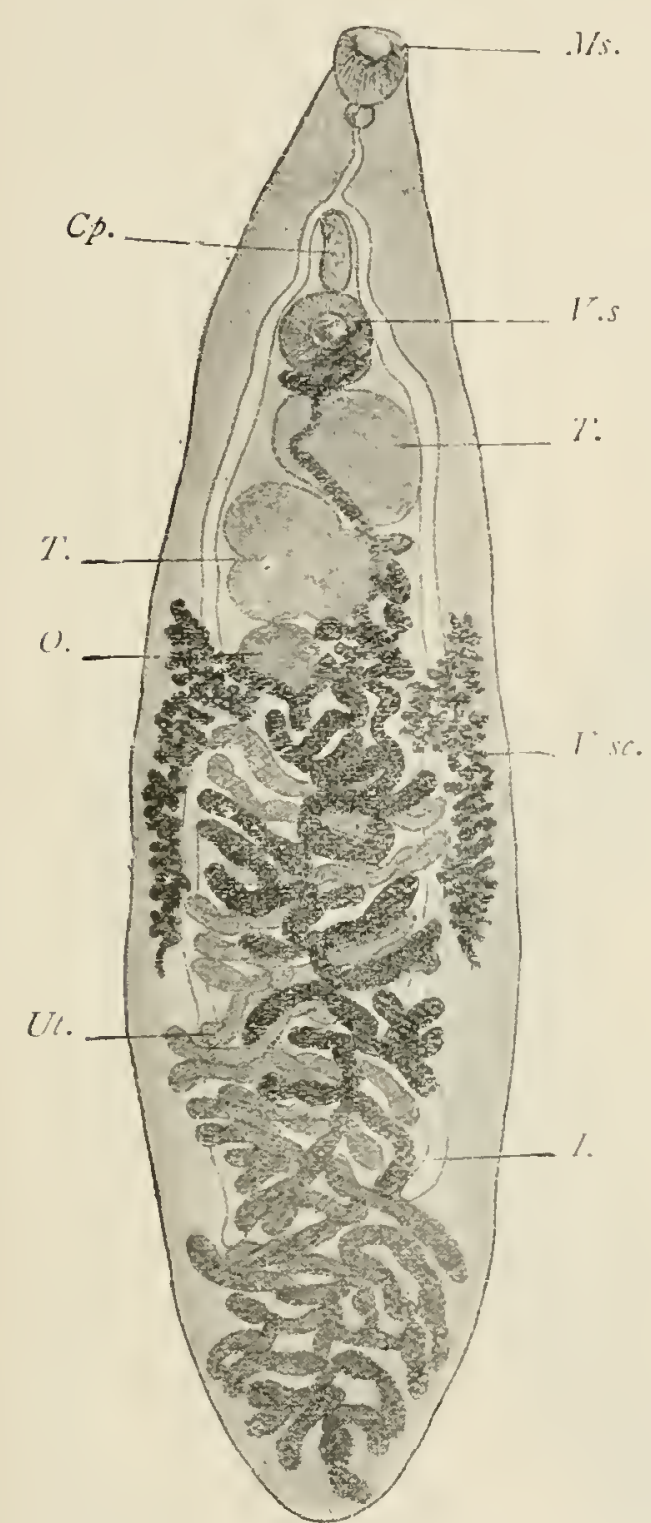

Dicrocclium lanceatum, Stil. and Iass. I5/1. V.s., ventral sucker; $C p$., cirrhus pouch; $I$. , intestinal bifurcations; $V_{\text {. }} s$. , vitelline sacs; $T$., teslicles; $O$., ovarium ; iIs., oral sucker; Ut., uterus.

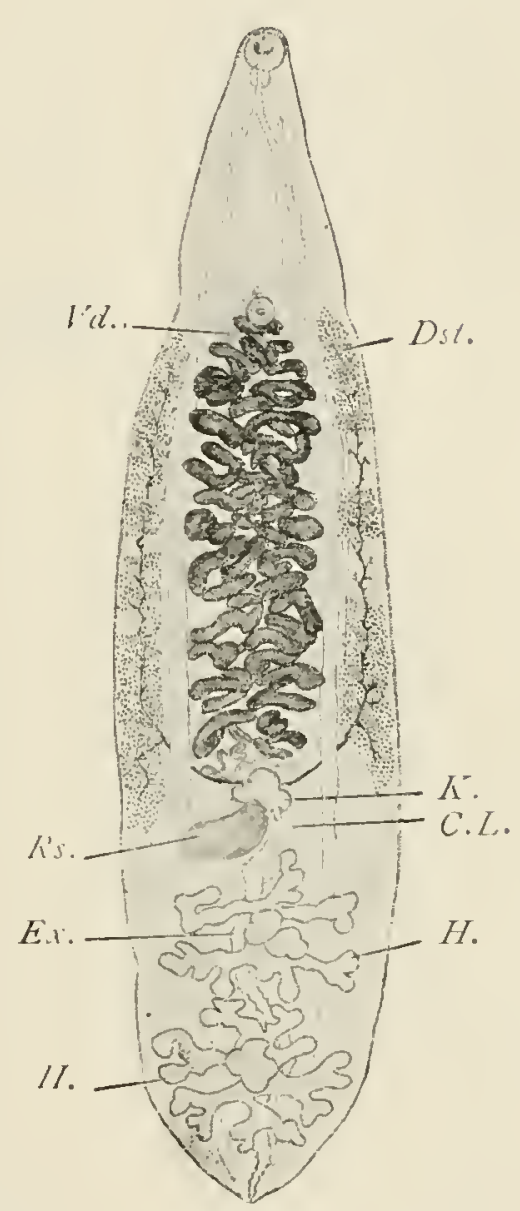

Clonorchis sinensis. C.L., Laurer's canal; Dst., vilellaria ; $E x$., excretory bladder; $H$., testes ; K., ovary; R.s., receptaculum seminis; $l \cdot d$., terminal sectiun of vas deferen:. Magnified $4 \frac{7}{2}$ times. (After Looss.)

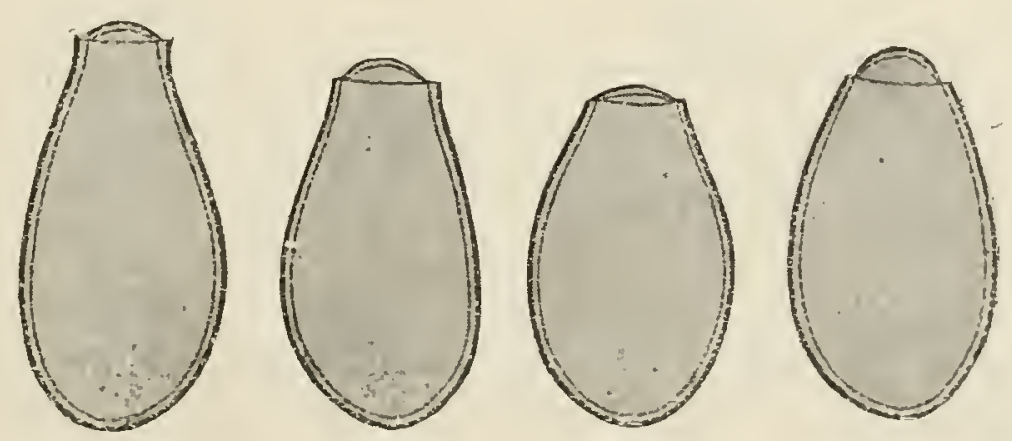

Ova of Clonorchis sinensis. The knobs on the ends of the eggs are not shown. 9co/I. (After Lcoss.) 
The testes are much branched and are situated behind the uterus.

The ovary is trilobate.

Paragonimus westermanii and the schistosoma are important pathological trematodes, causing diseases in man known as Paragonimiasis and Schistosomiasis.

These will now be dealt with in more detail.

PARAGONIMI ISIS (Endemic IIamoptysis).

\section{DEFINITION.}

The chronic, local or general infection of man by l'. Westermanii producing characteristic crstic lesions.

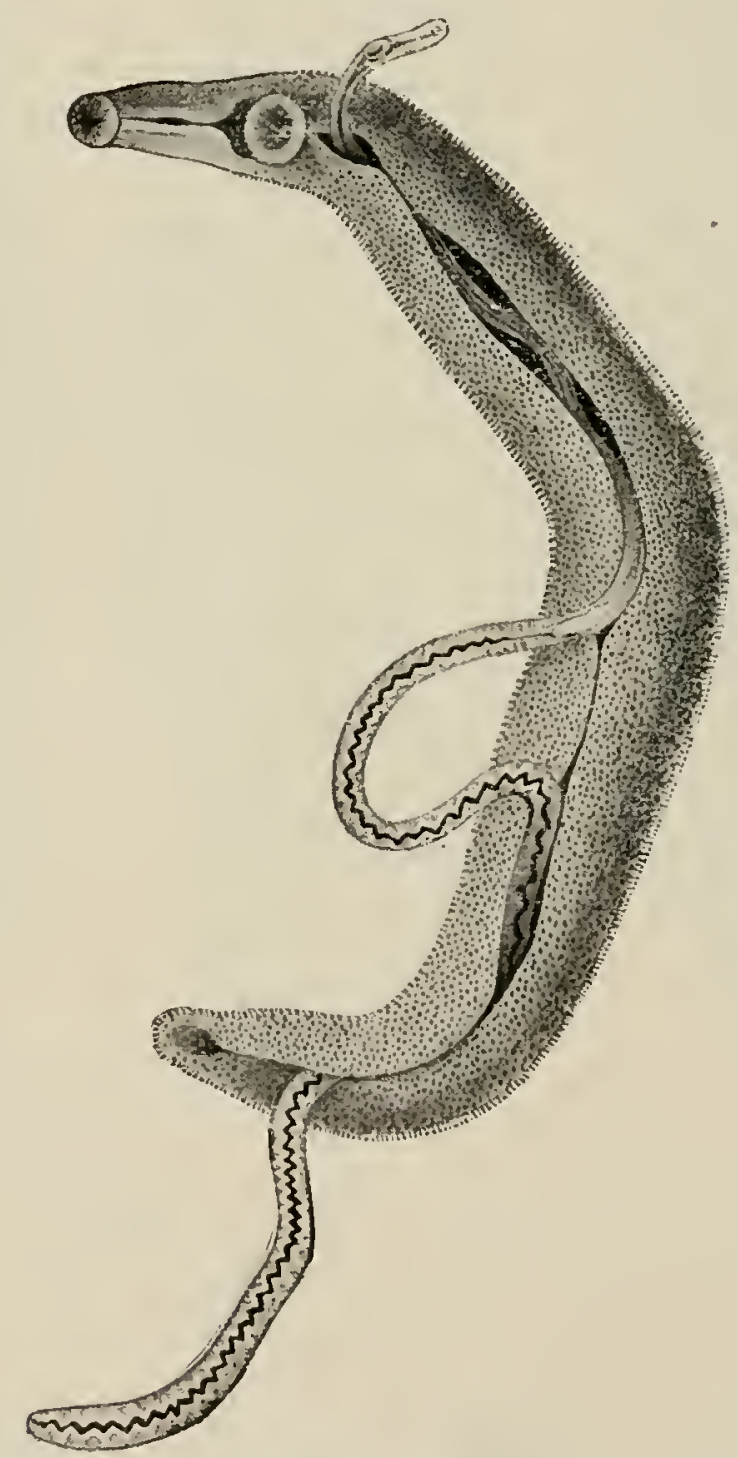

Schistosoma|ha'matobium, Bil. : male carrying the female in the canalis gynacophorus. I2/I. (After Looss.)

\section{DISTRIBUTION.}

In China, Korea, Japan, Formosa, Philippines, Sumatra.

\section{THE PARASITE.}

This is the P. westermanii. 


\section{MORPHOLOGY.}

A greyish leaf-like parasite quarter of an inch long.

The oesophagus is short, the two lateral caca are wavy but not branched.

The testes are simple, one at either side of the mid-line arranged diagonally.

The yolk glands are situated in the full length laterally.

There is no cirrus or cirrus pouch.

Their life history is not known.

They infect man, cats, dogs and pigs.

The true host is the tiger cat.

Man may be infected through food or drink, and hence the worms are distributed to other parts through the lymphatics. This is, however, not yet proved.

The eggs are yellowish with a thick shell.

\section{PATHOLOGY.}

The lesions have been classified thus (Musgrave) :-

(I) The suppurating lesion.

(2) The tubercle-like lesion.

(3) The ulcerating lesion.

These may occur in the skin, bronchial or intestinal musosa, or in the bile duct.

At first the connective tissue seems to be infiltrated with eggs, forming a cirrhosis or a round-celled infiltration with eosinophiles.

An abscess may form containing caseous matter, or an ulcer develops.

If this occurs in the liver cirrhotic changes take place.

If it occurs in the serous membrane there is an adhesive inflammation with brown patches of eggs.

If it occurs in a solid organ, a small focal cyst forms surrounded by fibrous tissue.

The abscess, cyst or ulcer contains degenerated cells, blood, eggs and parasites.

The fibrous lesion spreads in a star-shaped manner with small cysts in their centre. These reach the cutaneous or muscular layer and so open into the skin, bronchus, intestine, or the bile duct, causing there an ulcer. These ulcers may become secondarily infected. The ulcers sometimes heal by scarring.

Post mortem, these typical lesions may be found in muscle, lungs, serous membrane, spleen, pancreas, intestine, bladder, epididymis, prostate, and the choroid plexus of the brain.

The body is often emaciated and anæmic. 
Ulcers sometimes form in the axillae or groin, from which can be found an infected mass to the glands.

The lungs may show diffuse cirrhosis, bronchiectatic cavities, pneumonia and caseous abscesses.

\section{SYMPTOMATOLOGY.}

In a general infection there are fever, enlarged lymphatic glands, muscular pains and cutaneous ulcers.

In a thoracic infection one has cough, purulent or bloody sputum with eggs, characteristic Leyden crystals. There may be the physical signs of broncho-pneumonia, a pleural effusion, serous or purulent. The sputum may be of a red viscid gummy consistency. The cough is more troublesome in the morning than at night.

In an abdominal infection there is a dull general pain with tenderness. There may be the signs and symptoms of diarrhœea, appendicitis, hepatic cirrhosis, \&c.

In a cerebral infection there are the signs and symptoms of epilepsy which may be Jacksonian.

\section{DIAGNOSIS.}

This rests mainly upon finding the eggs in an endemic area.

\section{TREATMENT.}

This is symptomatic.

Remove the patient from the endemic area.

Pot. iodides may be tried.

Give creosote for the cough and to reduce the number of eggs.

\section{DEFINITION.}

\section{SCHISTOSOMIASIS.}

A chronic endemic disease of the urinary tract and rectum; causing hamaturia, cystitis and dysenteric symptoms.

The causative parasites are :-

(1) Schistosoma hamatobium.

(2) ,, japonicum.

(3) , mansoni.

In all these trematodes the sexes are separate.

\section{(I) INFECTION WITH S. HAMATOBIUM.}

\section{DISTRIBUTION.}

Egypt, South Africa, Asia, India, Syria, Mesopotamia, and the West Indies, Madagascar, Mauritius and South America.

In Egypt the infection occurs in the early winter months after the floods. 50 per cent. of the people are infected. 
Calcified eggs have been found in mummies of the 2oth dynasty $1250-1000$ B.C.

\section{THE PARASITE.}

S. hamatobium, it is a bisexual trematode.

\section{MORPHOLOGY.}

The Male.-This is from 12 to $14 \mathrm{~mm}$. long by $\mathrm{I} \mathrm{mm}$. broad. It is thin and flat, the lateral margins may be turned inwards forming the gynacophoric canal in which the female lies, thus giving to the male a filiform appearance.

The cuticle is bossed with short spines to enable it to cling to the walls of the vessels and travel against the blood-stream. There is no

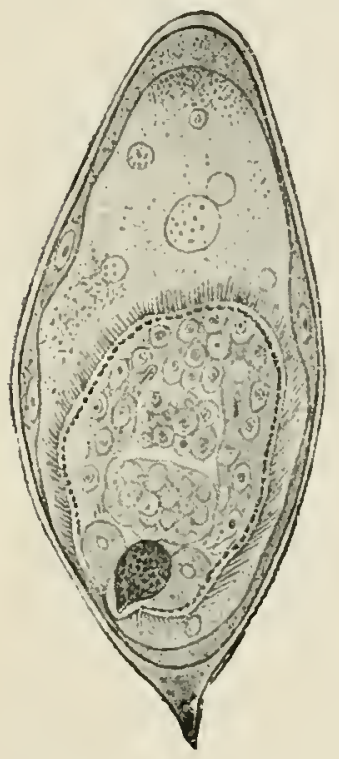

Ovum of Schistosoma hamalobium, Bilh., with miracidium, which has turned its anterior end towards the posterior end of the egg. $275 / \mathrm{I}$. (After Looss.)

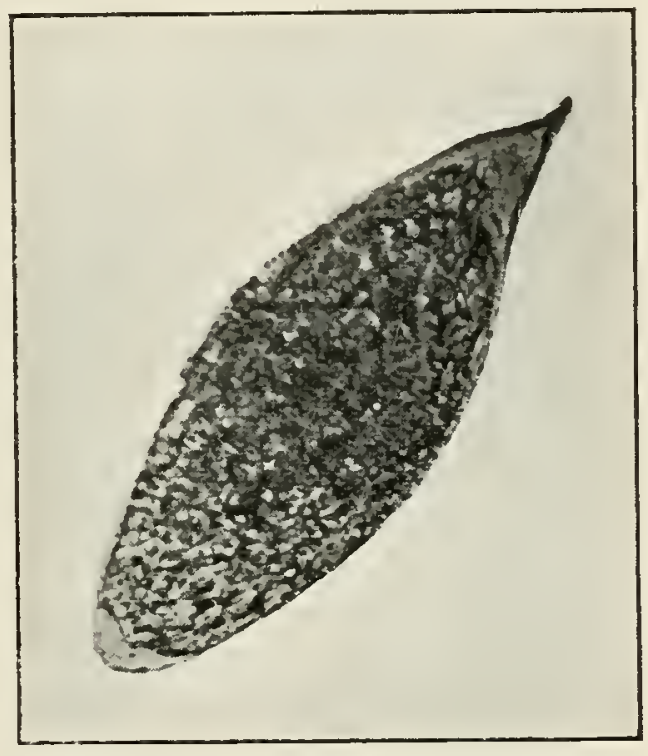

Ovum of Schistosomun hamatobium, with terminal spine. (Not found in Chına.) The patient was in the African mounted police. (By William Pepper, Philadelphia.)

pharynx, but the oesophagus is long with two caca which unite behind the testes into a median trunk.

The excretory pore is postero-dorsally situated.

There are four to five testes.

The Female.-This is $20 \mathrm{~mm}$. long by $0^{\circ} 25 \mathrm{~mm}$. thick. It is a long, thin trematode with a smooth cuticle except about the sucker and tail-end, where there are large spines.

The alimentary canal is as in the male.

The uterus ends in a genital pore just behind the ventral sucker.

The eggs are large, $0^{\circ} 8$ by $0^{\circ} 06 \mathrm{~mm}$., oval, yellowish, with a thin shell, slightly transparent, no lid, and a terminal spine at the posterior end. 


\section{THE LIFE HISTORY.}

The young immature male and female are only found in the portal system from whence they go to the portal vein. Here they pair and then pass to the liver.

The male carries the female down along the portal and the inferior mesenteric reins to the bladder. Ilere oviposition takes place and the eggs are deposited in the superficial capillaries. From this position they gradually work their way to the surface and enter the bladder itself. Some of the eggs, however, become calcified in the process.

If the eggs are taken from the urine and examined they will be seen

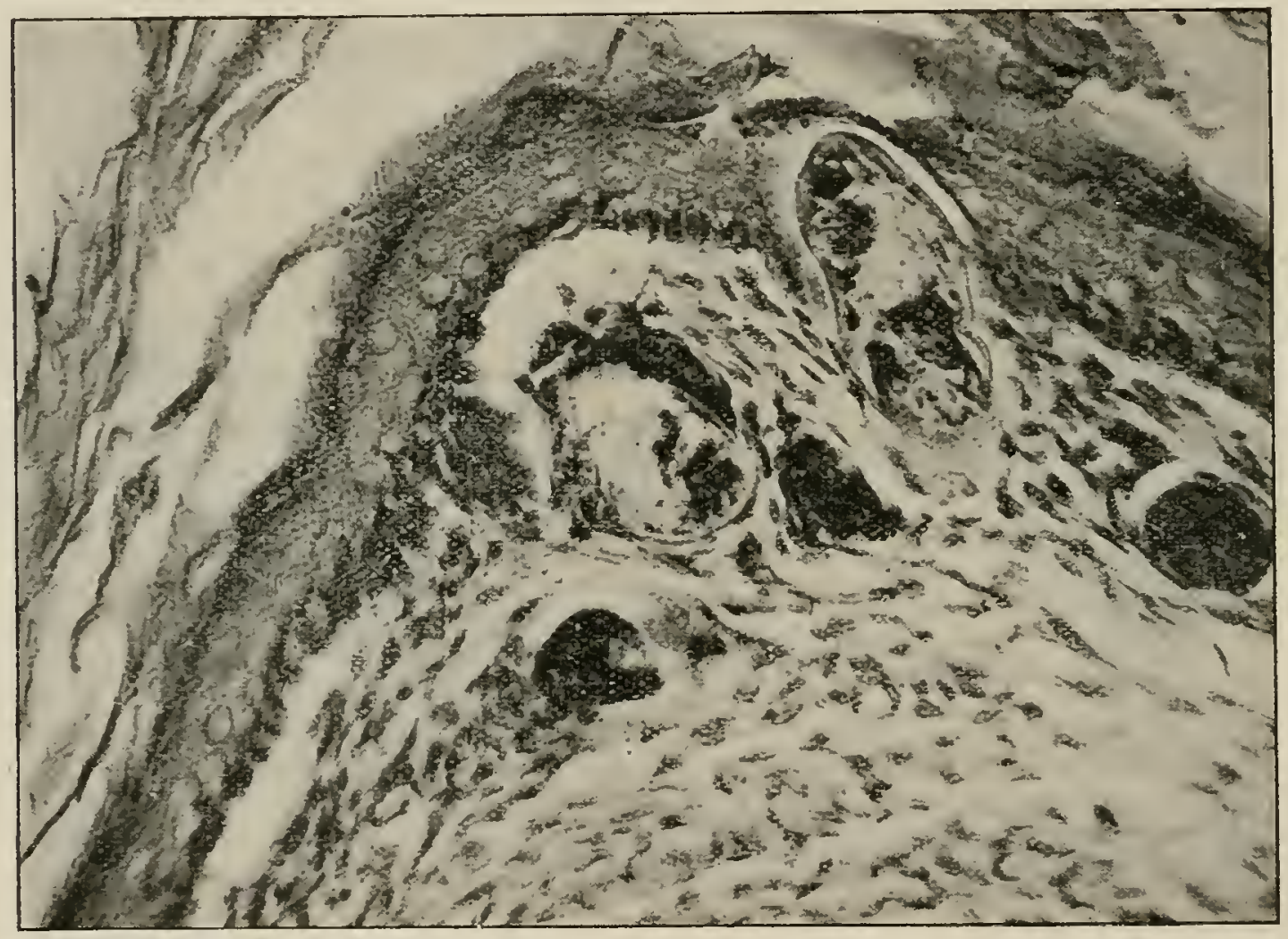

Micrograms of sections of skin of a newly-born mouse which had been immersed for half an hour in water containing large numbers of Bilharzia cercariæ.

to contain a living miracidium which can be liberated if the egg is broken while under the microscope.

The eggs must pass to water at once or the miracidium dies.

It does not live more than thirty to forty hours even when it is deposited in water, but must be again taken up by man.

No other host except man has been found to carry on the infection. How he becomes infected has not yet been proved.

The infection is by ingestion, also probably they enter the skin while people are working in the mud fields or bathing in the muddy pools infected by the urine of diseased persons.

The eggs may work their way to the rectal veins and mucous membrane, and then be passed out in the faces. 
Others believe that the life-cycle, like that given of a typical trematode (p. 235), is necessary.

The African natives believe that it gets along the urethra while they are bathing; and tie twine around the penis to prevent the infection. Bathing does seem to account for many infections and drinking impure water for others. There is no evidence for direct contagion.

\section{PATHOLOGY.}

The irritation set up by the eggs causes a round-celled infiltration. The epithelium proliferates, and flattened projections are formed which

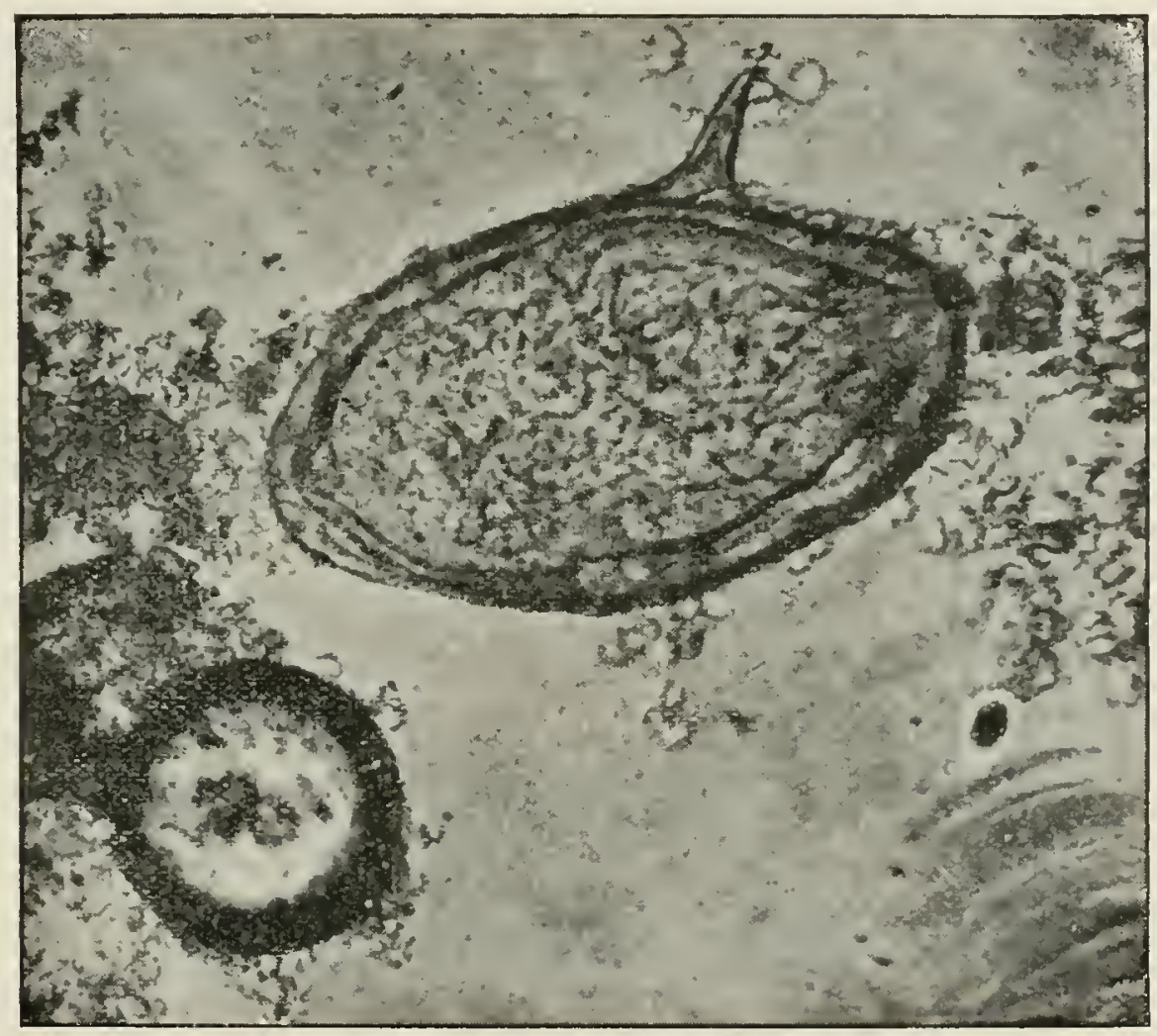

Ovum of Schistosomum hematobium. For comparison with Schistosomum japonicum and Schistosomum mansoni. This parasite has not yet been reported in China, and is probably nol found there. (By Williain Pepper, Philadelphia.)

feel sandy to the louch; these may go on to form vesicles and ulcers in which eggs can be found. The eggs can escape without ulceration.

The bladder wall is much thickened.

The pelvis of the kidneys and the meatus urinarius may also be affected.

Turner has found the eggs in the lungs.

\section{P.M.}

The bladder mucosa is much thickened with bilharzial tissue, covered with adherent mucus containing many eggs.

Vesicles are most frequently found around the trigone, containing a whitish fluid and eggs. Papillomata may be formed. 
These bleed readily and cause hæmaturia.

Many eggs become surrounded by connective tissue and calcify, forming sandy patches, and cause atrophy of the mucosa.

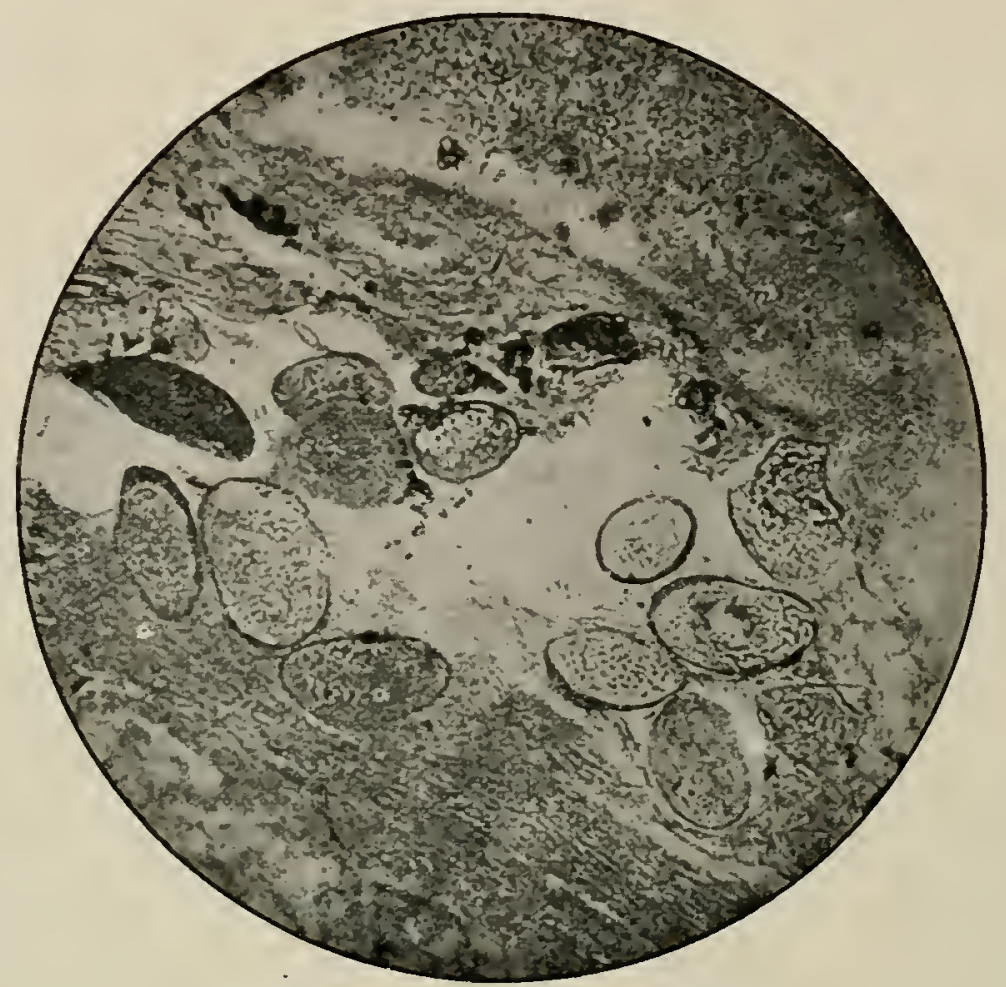

Ova of Schistosomum japonicum in biliary ducts, $\frac{1}{3}$ objective. (By Bell and Sutton, Hongkong.)

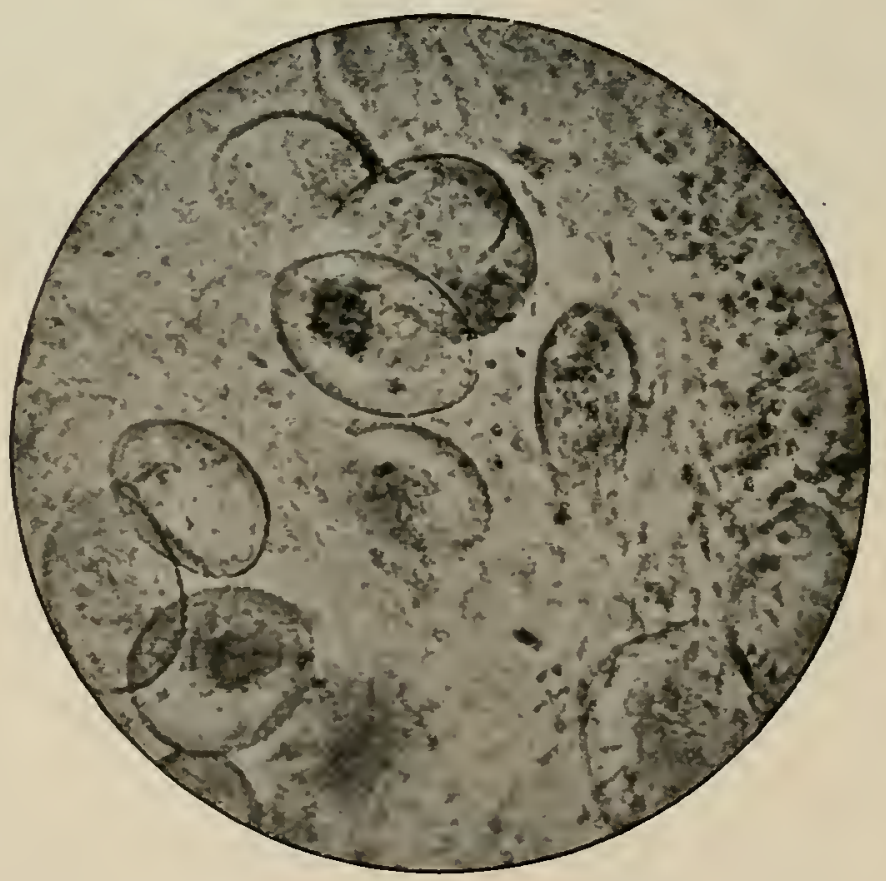

Section of intestine, showing ova of Schislosomum japonicum. $\times 250$. (By J. Bell, Hongkong.)

The urine collects in the bladder depressions, decomposes, phosphates form, and a further phosphatic incrustation lines the bladder wall more or less in patches. 
The ureteric orifices may be occluded, causing retention of urine and hypertrophy of the ureters with dilatation, thus making ascending
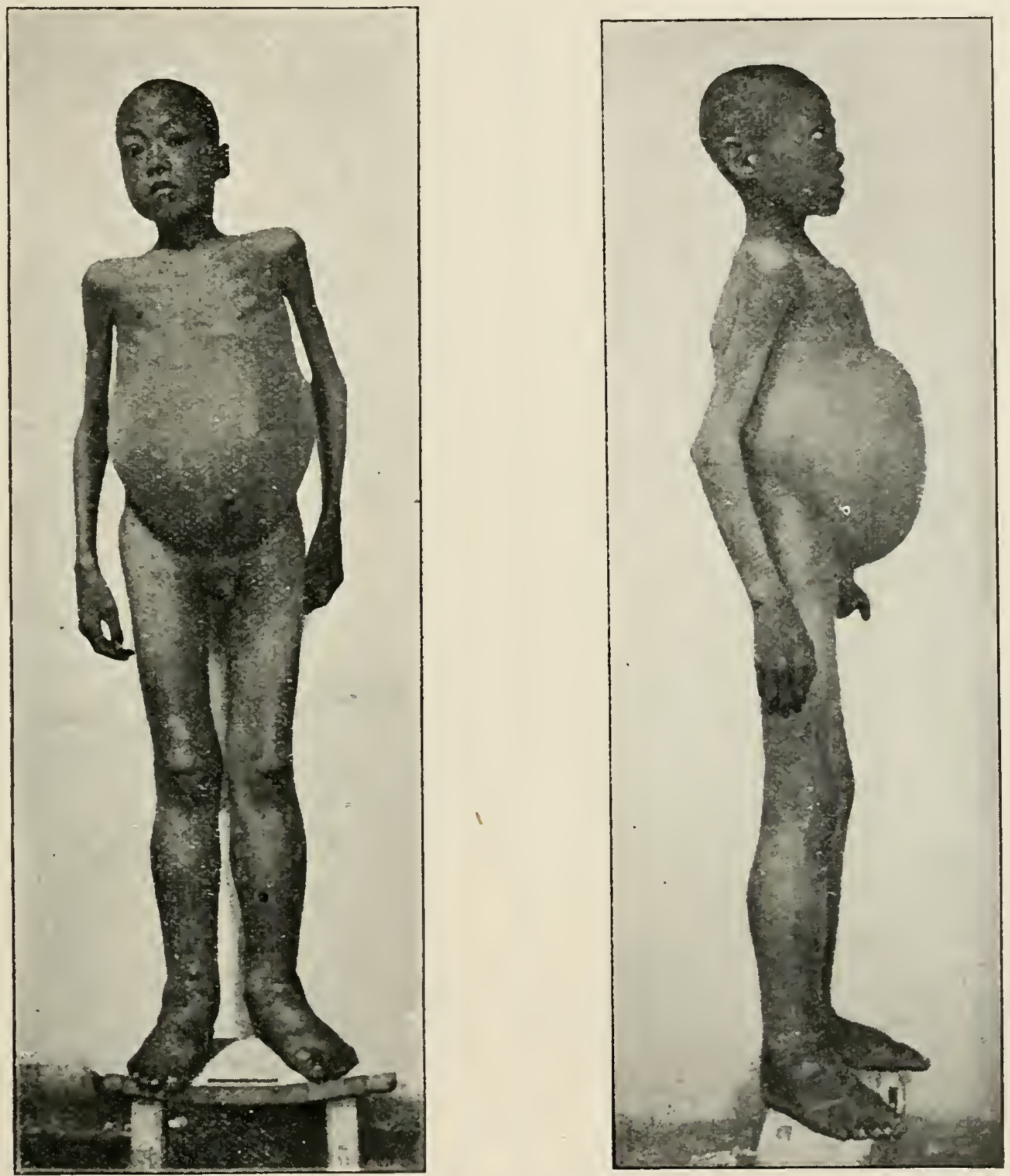

Case of Schistosomum japonicum. Severe infection of three years' duration. Ova very abundant in stools. After tapping, a large mass of glands forming a tumour as big as the two fists together could be felt in the cæcal region. Liver dulness was diminished. After tapping, by pushing the hand well up under the ribs, the liver surface could be felt to be bossed. Spleen not enlarged.

Age twenty-one. The patient left the hospital a few days after the photo was taken, and looked as if death was imminent. In hospital he was tapped four times, but the abdomen filled up to as great a degree within ten days of each tapping.

This illustrates what one might call the final stage of schistosomum disease, when the previously enlarged liver shrinks with cirrhosis. The only other photos I have seen published were thuse of Dr. Peak's cases, but I think they illustrated an earlier stage of the infection (earlier, or perhaps less severe). (Notes, case and photos by J. A. Thomson, Hankow.)

septic infection easy. Calculi may form in the bladder. There may be interstitial nephritis. 
Bilharzial tissue may form in any part of the urinary tract.

It may spread to the rectum and abdominal wall with sinuses, and may affect the prostate.

\section{SYMPTOMATOLOGY.}

After the Transvaal War, many of the soldiers took it with them to England, India, and to other places.

Incubation (?) from three to six months (Sandwith).

The symptoms may be absent or considerable, according to the amount of the infection.

There are: frequency of micturition, burning pain about the urethra and perinaeum, straining after micturition. Hæmaturia at the end of micturition at first (endemic hamaturia).

The urine centrifuged shows the ova.

There may be pain in the back, gluteal region, and down the legs.

The urine is foul-smelling, decomposes, is alkaline, turbid, and contains pus, phosphates, blood and ova.

Cystitis sets in and the other symptoms increase.

There is no rest day or night.

A calculus may form to add to the misery. The prostate enlarges.

A urinary fistula may form above the pubis.

Pyonephrosis may develop, the kidneys enlarge, and septicamia set in. Prostatic inflammation predisposes to new growths.

Stricture is not uncommon. In females there is vaginitis with papillomatous masses about the vulva. The uterus and ovaries have been found infected.

Severe hamorrhage may ensue from the large vessels.

There may be cirrhosis of the kidneys with high blood-pressure.

When the spinal cord is attacked the symptoms may imitate tabes dorsalis or disseminated sclerosis.

\section{TREATMENT.}

Most cases cease to discharge eggs within five years of leaving the endemic area.

There is no specific drug for the parent worm.

Give male fern. in v, t.d.s. for a long time.

Give bladder washes of silver nitrate I in 10,000 at first, or quinine 4 per cent., or adrenalin in saline.

Helminthol, grm. i, t.d.s. may be tried.

Urotropin, salol, benzoic acid, buchu, and hyoscyamus all have their advocates. Good results are claimed for emetine.

Christopherson claims that antimony tartrate intravenously will cure. In vesicle infections inject along the dorsal vein of the penis.

Much water and fluids should be given to act mechanically. 
Surgical treatment for calculus and fistula as required.

All stimulants and excesses must be avoided.

Prohibit micturition and defacation into water; boil and filter all drinking water.

The mortality is low. Amongst affected Europeans removed from the endemic area less than I per cent. die.

The recent Bilharzia Mission to Egypt, 1915, consisting of Drs. Leiper, R. P. Cochin, J. G. Thompson, has thrown much valuable light upon this subject. A few points are as follows:-

The incubation of the disease is probably one to two months, and is not as long as previously thought.

The absence of a pharynx in the cercaria is the one reliable character by which Bilharzia cercaria can be distinguished from other distomas.

Storage of water for thirty-six hours will destroy all cercarize. If this was carried out with regard to the Cairo water supply alone, probably the 10,000 children now infected annually would be spared.

The extension of perennial irrigation in Egypt appears to have encouraged the spread of the disease.

Of 625 men who became infected in South Africa, 359 were still on the list in I9I1, exclusive of those permanently pensioned. The total cost to the State was about $\mathcal{E} 10,000$ per annum (Leiper).

The conclusions arrived at by the Mission are of interest as contrasted with those of Loos. They are shown thus:-

CONCLUSIONS BASED ON THE LOOS HyPOTHESIS.

(1) All transient collections of water, such as those resulting from occasional showers of rain, road waterings and domestic waste, are dangerous if freshly contaminated.

(2) Large bodies of water, such as the Nile canals, marshes and birkets, are little liable to be infective.

(3) All water in a given area would automatically become safe in 30 hours if the native infected population were removed.

(4) Infected troops would be liable to reinfect themselves, to spread the disease among other troops, and to convey the disease to any part of the world.
CONCIUSIONS BASED ON THE RESUlTS OF THE PRESENT INQUIRY.

(1) Transient collections of water are quite safe after recent contamination.

(2) All permanent collections of water, such as the Nile, canals, marshes and birkets, are potentially dangerous, depending on the presence of the essential intermediary host.

(3) The removal of infected persons from a given area would have no effect, at least for some months, in reducing the liability to infection, as the intermediate hosts discharge infective agents for a prolonged period.

(4) Infected troops cannot reinfect themselves or spread the disease directly to others. They could only convey the disease to others in those parts of the world where a local mollusc could efficiently act as carrier. 
(5) Infection only takes place through the skin.

(6) Infection in towns is due to contact with recently contaminated moist earth or water.

(7) Eradication depends upon education and complete sanitary control throughout the country. The sustained co-operation of the affected individual is essential.
(5) Infection actually takes place both by the mouth and through the skin. Recently contaminated moist earth or water is not infective.

(6) Infection in towns is acquired from unfiltered water which is still supplied, even in Cairo, in addition to filtered water, and is delivered by a separate system of pipes.

(7) Eradication can be effected without the co-operation of infected individuals by destroying the mollusc and intermediaries.

The practical conclusions are as follows :-

That unfiltered water taken from canals, ditches, or birkets would be rendered safe:-

(I) If kept beyond the survival period of the cercaria, i.e., fortyeight hours.

(2) If heated to $5^{\circ} \mathrm{C}$, a temperature at which the cercaria is immediately killed.

(3) If previously treated with those chemicals that are lethal to the cercaria.

The use of tabloids of sodium bisulphate, I 6 grains to a pint of water, giving a dilution of $\mathrm{I}-567$, is recommended.

The following points should be attended to :-

(I) Personal contact of any kind with unfiltered water is dangerous. The surface of the water is the most likely to be infective as the cercariæ congregate there. An intake pipe should always be led, therefore, to the centre of the stream, and should draw the water from near the bottom and at a place where there is little or no vegetation.

(2) It is essential in drawing water for storage, in order to destroy the bilharzia cercaria, that no infective mollusc be admitted. This can be ensured by screening the intake pipe with gauze having about sixteen meshes to the inch. The common mosquito gauze or prosphorbronze wire gauze is very serviceable.

(3) The water in the wells and "sakias" may be regarded as much safer than that from other sources. Hitherto molluscs have not been found in these wells.

(4) Shallow barrel sand filters are open to suspicion. It has been found experimentally that after fifteen minutes cercaria succeed in passing in large and increasing numbers through four inches of desert sand.

(5) Although the reproductive activity of bilharzia in molluscs is probably most intense during the summer months, the occurrence of mature cercariae in infected molluscs in February shows that there is a certain liability to the infection throughout the year. 
(2) INFECTION WITH S. JAPONICUM.

\section{DISTRIBUTION.}

This is known in Japan as Katayama disease from a Japanese town in which it was common. The parasite is similar to $\mathrm{S}$. hrematobium.

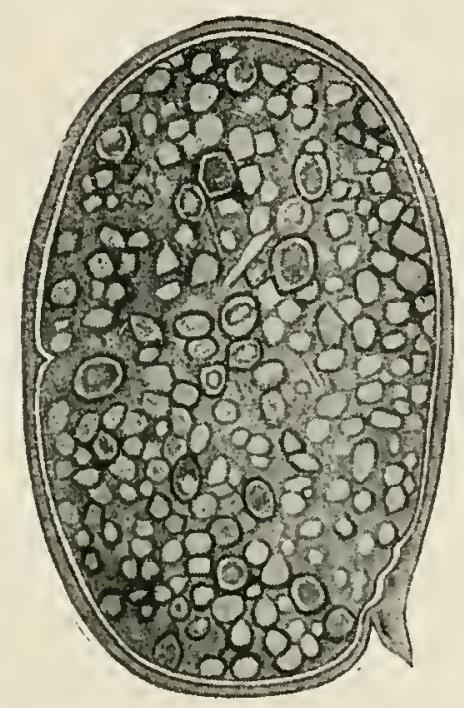

Schistosoma jafonicum : from dog. Uterine egg. $\times c$. Soo. (After Katsurada.)

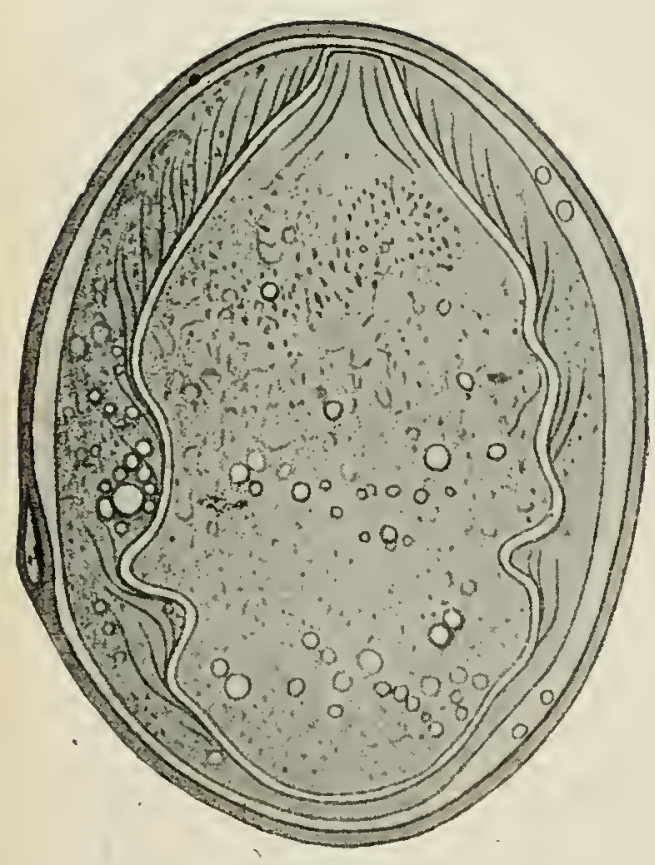

Schistosoma japonicum : from dog. $\times$ c. 80o. (After Katsurada).

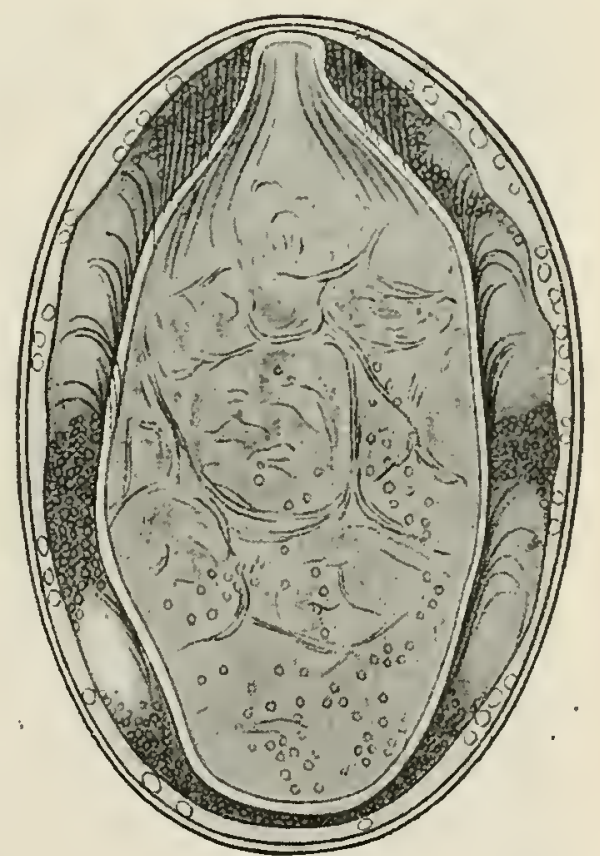

Schistosoma japonicum: from dog. Egg from freces. $\times$ c. 800 . (After Katsurada.)

It is found in Japan, China, and in the Philippines.

In China the cases are only found in the low-lying districts.

\section{THE PARASITE.}

The $\mathrm{S}$. japonicum is introduced by the skin or mouth from polluted water. 
Males only have been found to be infected, and these were usually farmers and boatmen from the ages of youth to 54 .

The parasite differs from $\mathrm{S}$. hamatobium in but one or two small features, viz.: it is smaller, the male has no tubercles, the eggs are oval, yellowish, without an operculum, and no definite spine, but Leiper has found a small protuberance in 75 per cent. of those examined.

Some suppose that the parasites feed on blood, and others that they produce a toxin that causes a progressive ancmia in man.

The life-history is unknown. Perhaps some fresh-water mollusc or crustacean is necessary.

\section{PATHOLOGY.}

The blood-stream distributes the eggs, which are carried onwards until they lodge as emboli in various orgars, especially in the alimentary canal and liver, where bilharzial tissue is formed, causing hamorrhagic enteritis.

\section{P.M.}

On opening the abdomen there are signs of chronic peritonitis.

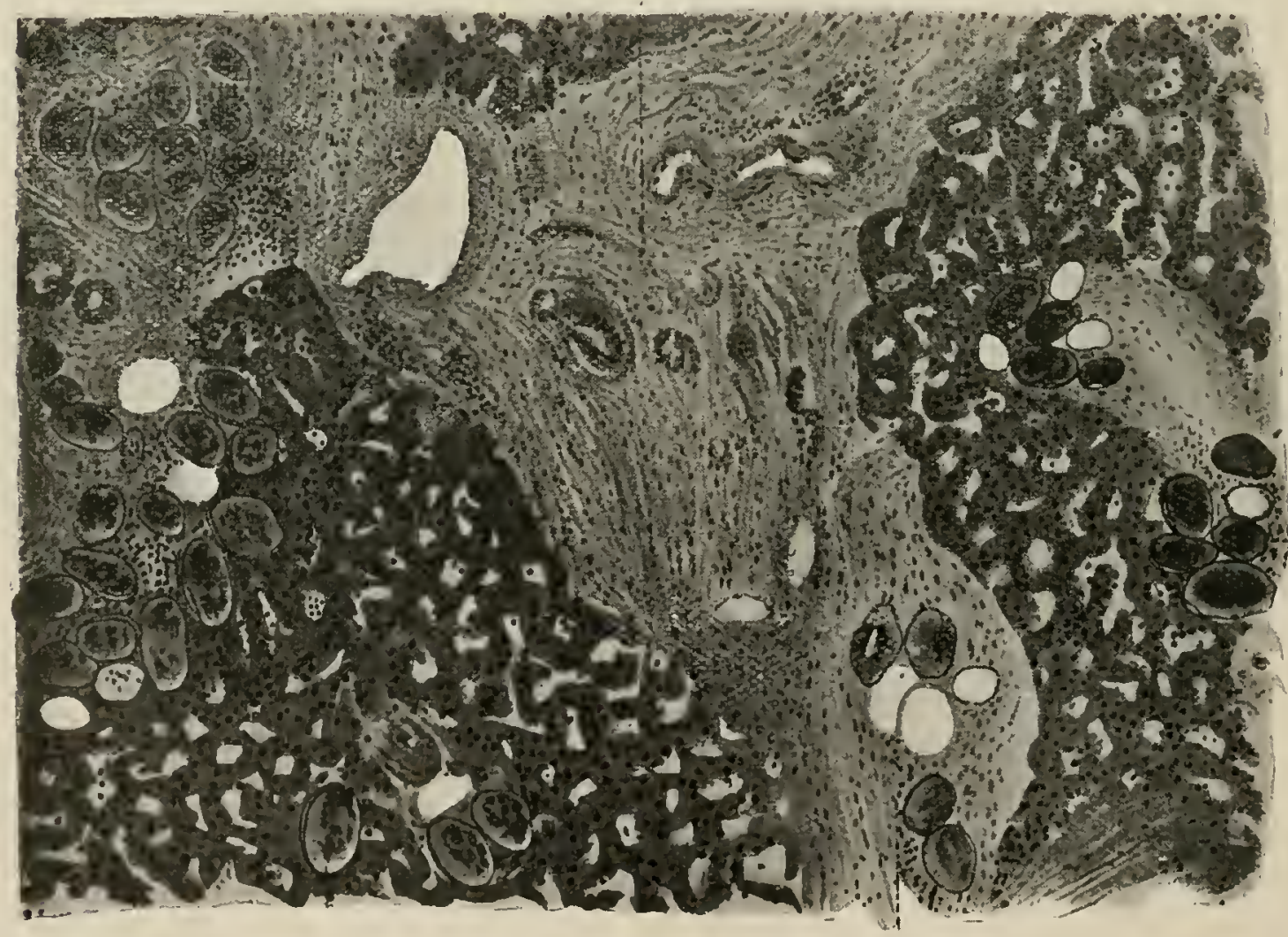

Schistosoma japonicum: liver showing eggs in the intra-and inter-lobular connective tissue. $\quad x$ c. So. (After Katsurada.)

'The liver is small, cirrhotic, with nodular surface. Glisson's capsule is thickened, and is found to contain numerous ova.

The intestine is thickened, mucous membrane swollen, hyperæmic, friable, with patches of ulceration and necrosis, sometimes papillomata. 
The recto-vesical pouch is almost obliterated.

The outer coats of the intestine are very tough, almost cartilaginous. The rectum may be three-quarters of an inch thick and adherent to the

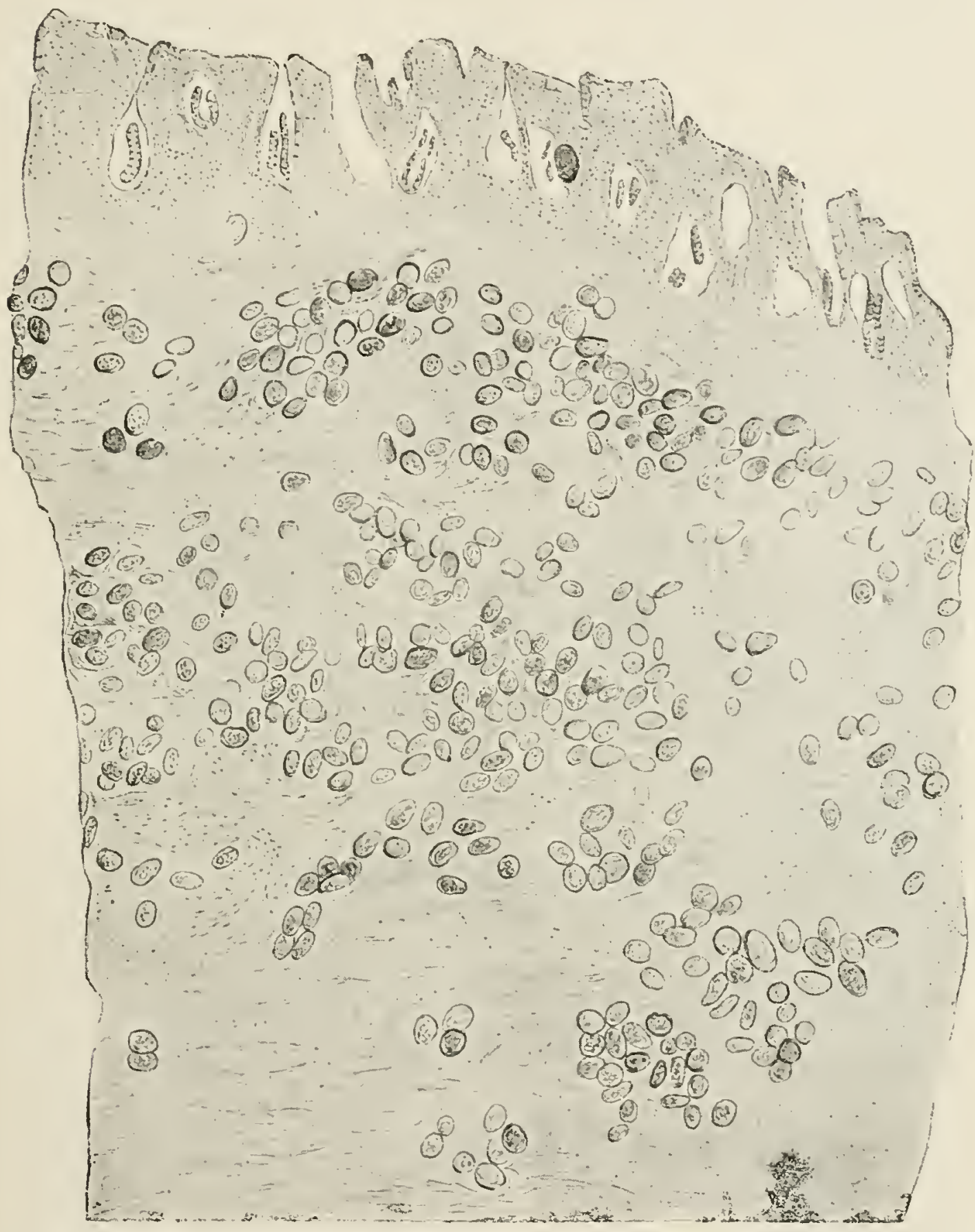

Schistosoma japonicum: section through the gut of a Chinaman showing eggs. $\times 58$. (After Catto.)

bladder. The mucous membrane of this organ is not usually affected as in the case of S. hæmatobium.

There may be hamorrhagic infiltration of the dura mater, with wedge-shaped sclerosed areas of brain matter (Katayama).

The eggs may be found in the mesentery, mesenteric glands, mucous membrane of the gall-bladder, pancreas, pylorus, lungs and brain. 
The adult worms are found in the portal system, especially at the bifurcation of the smaller mesenteric ressels.

\section{SYMPTOMATOLOGY.}

These are very irregular.

They may be nil or slight with urticarial rashes and eosinophilia.

There may be cough with scanty expectoration, diminished breath sounds, and crepitant râles.

During the ensuing months these may increase and be accompanied by diarrhœa, with ova in the fæces, enlargement and tenderness of the liver, with general abdominal pains.

The hypogastrium shrinks and the epigastrium enlarges.

There is dyspepsia and the passage of undigested food materials.

The liver may then shrink and the spleen enlarge, followed by ascites, ancemia, emaciation, eosinoplilia, which latter may reach 50 per cent.

If the lungs are affected there are signs of bronchitis or of bronchopneumonia.

If the brain is affected there are signs of Jacksonian epilepsy.

The knee-jerlis in these cases are increased.

The urinary tract is usually normal.

There may be a muddy complexion.

\section{DIAGNOSIS.}

Look for a chronic enlargement of the liver and spleen with ascites, chronic diarrhoea, and marked eosinophilia from 10 per cent. to 50 per cent.

Look for ova in the stools containing a miracidium.

\section{PROGNOSIS.}

Death nearly always ensues.

Intercurrent disease is usually the immediate cause.

\section{TREATMENT.}

This is as for S. liematobium.

\section{(3) INFECTION WITH S. MANSONI.}

This parasite is held to resemble that of $\mathrm{S}$. hamatobium, but some helminthologists will not accept that it is a distinct species at all.

\section{THE THEORY OF SAMBON.}

In 1903 Manson found eggs with a lateral spine which had attacked the rectum. He suggested that it came from a new species of worm. 
In 1907 Sambon formed a new species of it and called it S. mansoni. His reasons were that:-

(I) Lateral-spined eggs are never found in the urine or bladder, but only in the faces, rectum and liver.

(2) The egg is oval with a lateral spine; that of S. hamatobium is more oblong with a terminal spine; and $\mathrm{S}$. japonicum is more rounded with a very small lateral spine in 75 per cent. of those examined (or, as some declare, with no spine at all).

The average size of the $\mathrm{S}$. mansoni egg is 150 by $65 \mu$ with a straight terminal spine.

The average size of the $\mathrm{S}$. hamatobium egg is $115 \mathrm{by} 5^{8} \mu$ with a thick lateral spine.

The average size of the $\mathrm{S}$. japonicum egg is 82 by $68 \mu$ without a spine.

(3) The females differ in the genital tract, and the uterus contains lateral-spined ova.

(4) The geographical distribution is different.

S. hæmatobium only is found at the Cape.

S. mansoni only is found in the West Indies and S. America.

\section{THE THEORY OF LOOS.}

Loos disagrees with the above saying that the lateral-spined eggs are the products of unfertilized females. The females not being embraced by the male cannot withstand the blood-stream and become carried into side channels, finding their way into the bowel. These unfertilized females only shed laterally-spined ova which are passed in the faces.

Again he says that lateral-spined ova are frequently found in the fæces. He declares that there are no differences between the female generative organs.

The straight-spined ova have been found in the urine in the West Indies.

As man is probably the intermediate host, there can be no limit to the S. hæmatobium.

In 1911 Looss modified his riew, and stated that the eggs develop pathogenically.

There are many supporters of Sambon's theory.

It is agreed that lateral-spined ova cause :-

\section{INTESTINAL SCHISTOSOMIASIS.}

\section{DISTRIBUTION.}

It is found in Egypt, Central Africa, Uganda, South Africa, Belgian Congo, America, and the West Indies. 


\section{PATHOLOGY.}

The rorms live in the portal vein and capillaries of the bowel, where they lay their eggs. The eggs burst the capillary, enter the nucosa, cause cellular infiltation, thickening, mucus-covered papillomata and ulcers. A dense fibrous infiltration of the peritoneum results.

Large polypoid masses may protrude through the anus.

A fistula may be caused in the subcutaneous tissue about the sacrum and coccyx.

The ova reach the liver by the blood-stream, and there form a patch of fibrosis or an abscess.

Gallstones may form around the ova of the bladder.

The pancreas, spleen, urethra and vagina may be infected.

A chronic interstitial pneumonia may be caused by ova reaching the lungs, and from thence may enter the heart and the general circulation.

\section{SYMPTOMATOLOGY.}

Sometimes nil in slight infections.

There may be symptoms of chronic dysentery, abdominal pain, blood, mucus, and ova in the stools.

Prolapse of the rectum or of papillomatous growth is not uncommon, which is sometimes mistaken for hæmorrhoids.

There is great agony from tenesmus when at stool.

The prolapse, inflamed and painful, is the source of great discomfort.

In cases where there is more fibrosis there is ditriroea and constipation.

Tumours are sometimes found about the cæcum or colon, which are hard, movable, and are elongated in the long axis of the bowel.

These may increase during the following months or years.

Emaciation and attacks of colic are common.

The liver may be enlarged and cirrhotic, also the spleen.

The urine may be normal.

\section{PROGNOSIS.}

This is always unfavourable when the symptoms are marked.

The patient must be moved from the endemic area, if improvement is to be expected.

\section{TREATMENT.}

Give male fern.

Treat on general lines as for S. hrematobium.

Whitehead's operation may be done for the treatment of many rectal polypia.

Irrigation of the intestine can be tried with sodium hypochlorite, ro in $\mathrm{I}, 000$, or of tannic acid, 3 in $\mathrm{r}, 000$. 


\section{II.-CESTODA PATHOGENIC TO MAN (Human Tapeworms).}

$$
\text { (Kestos = a girdle.) }
$$

\section{GENERAL REMARKS.}

Cestoda are Platyhelmia which differ from Trematodes in that:-

(1) There is no alimentary system, the food being absorbed through the cuticle.

(2) There is a simple fixative apparatus at the anterior extremity of the body.

(3) The parasites are divided into segments, each segment being a distinct hermaphrodite animal.

(4) They are longer and flatter, varying from $\frac{1}{4}$ to 24 inches long.

The adult inhabits the intestine, and the larval form usually inlabits some other host.

Every vital function is subordinate to the development of ova.

They have been known for centuries.

Moses forbade the Israelites to eat pigs and similar animals because parasites were then known to exist in them.

Aristotle recognized the proglottides of tapeworms.

\section{MORPHOLOGY.}

Cestodaria with only one segment and not important. Cestoda having a scolex and segments.

We are concerned with these latter.

The true cestodes have a tape-like whitish segmented body.

The anterior end has a scolex attached to the intestinal wall, the segments or proglottides follow and increase in size from the head downwards. The head attaches itself to the mucous membrane of the intestinal tract by means of muscular suckers. There may also be one or more circular rows of hooks situated upon the most anterior part of the head or rostrum. Each segment contains male and female sexual organs. The thick cuticle contains lime salts in abundance. Among the cellular elements are calcareous corpuscles, 3-30 $\mu$ in diameter, which are characteristic of the cestodes, having perhaps a protective or skeletal function.

There is an outer layer of longitudinal and an inner of transverse muscle fibre which encloses the parenchyma.

There is an excretory system of anastomizing capillaries passing into collecting tubules which open to the exterior on the last proglottis.

The nervous system consists of one ganglion and two nerve cords.

The male generative organs reach maturity first. The vas deferens from the follicular testes enters the cirrus pouch and terminates near the vaginal orifice at the genital atrium. 
Two ovaries develop, connected by a common oviduct which joins the spermatic duct. This latter is then joined in turn by the common duct of the yolk gland and the ducts of the shell gland, then it passes on to the uterus. This latter is usually a blind tube but it may be open by a special aperture.

When the uterus is full of eggs it fills up the proglottis, and the male organs atrophy and disappear. Hence :-

In the first segments no sexual organs are seen.

In the slightly mature segments the male organs are made out first.

In the more mature segments the female organs develop as the male organs diminsh.

In the fully mature segments the male organs have disappeared.

\section{THE LIFE HISTORY.}

The mature proglottis can fertilize itself or another segment.

The spermatozoa pass along the spermatic duct, meet the ovum and fertilize it. The ovum then receives its yolk and shell, and passing to the uterus escapes by the uterine orifice, when there is one, or when the proglottis is destroyed.

The egg is oval, yellowish with or without an operculum.

Two membranes are formed, one near the shell, the other near the embryo. The shell and the outer envelope are lost and in the freces is seen the embryophore in its inner envelope with three pairs of hooks. This is the Onchosphere.

Some species are ciliated. This onchosphere is then taken up by a new host of a different class, the envelope is then cast off, the hooks burrow their way to suitable tissues, after which its hooks are lost and a bladder-like cyst is formed. In the walls of this cyst develops a scolex. This cyst is called the

\section{CYSTICERCUS.}

From Onchosphere to Cysticercus requires two to six months.

This infected tissue is ingested by another animal, the cyst is dissolved and the scolex develops in a few weeks to an adult tapeworm, which can live for about a year.

They obtain food by osmosis from the intestine.

\section{CLASSIFICATION OF CESTODA.}

Order (I) Pseudophyllidea. The scolex may or may not be armed with two groove-like suckers. It has three genital orifices.

Order (2) Tetraphyllidea. These parasites are not found in man. 
Order (3) Cycloplyllidea. The scolex has four suckers and the anterior end in a rostellum with or without hooks. 'The segmentation is distinct. There is no uterine orifice. The eggs are without lids.

(Brder (4) Diphyllidea. These are very rarely parasitic in man.

()rder (5) Trypanorhyncea. Not parasitic in man.

The sub-families, genera and species of Orders ( 1 ) and (3) found in man are given in the following table.

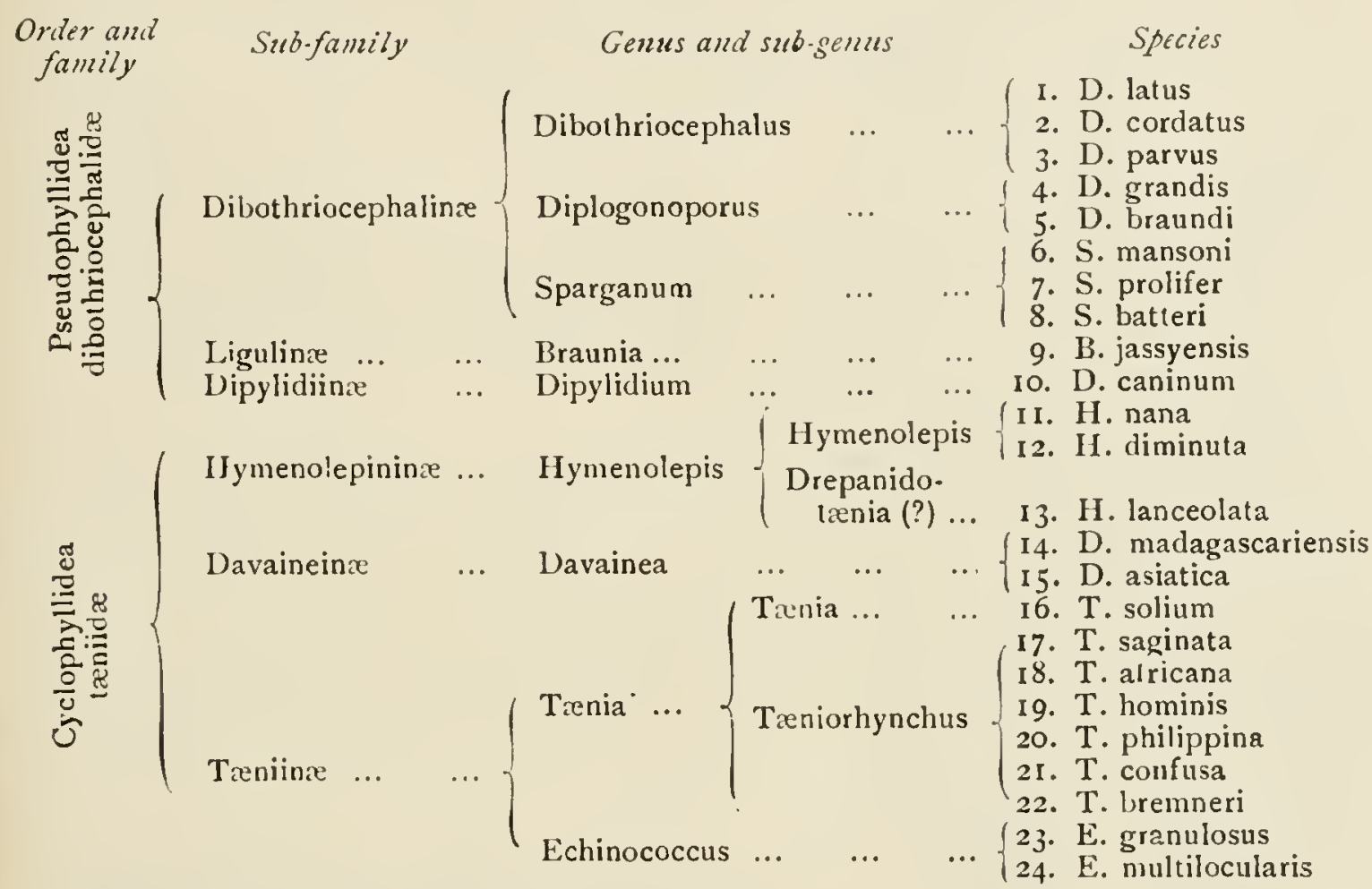

\section{MAN IS THE HOST FOR :-}

T. solium, saginata, africana, hominis, philippina, confusca and also 'T. bremneri.

Dibothriocephalus parrus and Ilymenolepis nana.

Davainea madagascariensis and asiatica.

Rarely Dibothriocephalus latus which is usually in the dog.

Rarely Dibothriocephalus cordatus which is usually in the seal and walrus.

Rarely Hymenolepis diminuta which is usually in the rat and mouse.

Rarely Drepanidotania lanceolata which is usually in ducks and geese.

\section{CYSTS MAY DEVELOP IN MAN FROM}

\section{'T. solium.}

Echinococcus granulosus and multilocularis.

Larval forms of Sparganum.

? Cysticercus of $T$. saginata. 
THE FOLLOWING LIST OF TAPEWORMS FOUND IN MAN will be useful for reference.

Table of Tapeworms Found in Man. (After Daniels). Platyhelminths

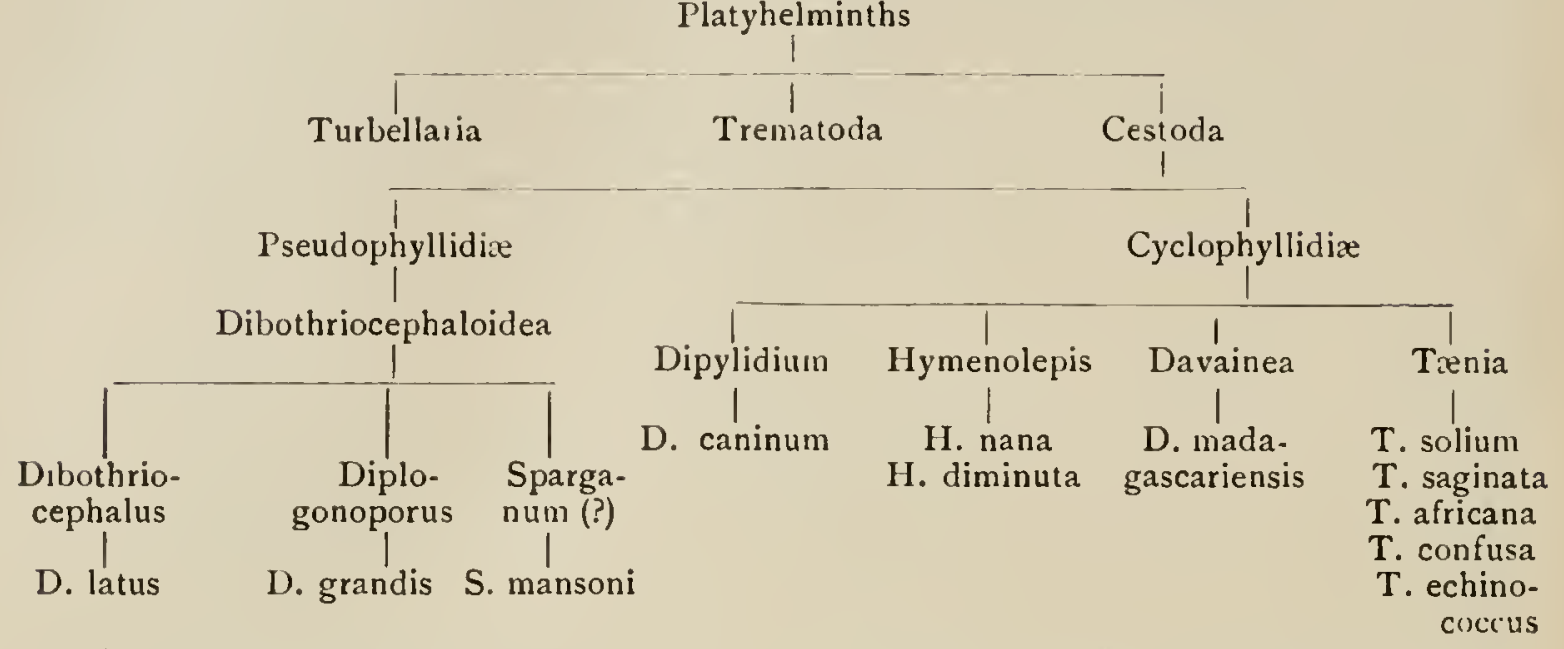

Only the important species found in man will be dealt with.

\section{DIBOTHRIOCEPHALUS LATUS.}

This is the only important helminth of the first Order.

It occurs in man, dogs, cats and foxes.

It is nine metres long and has 3,00o to 4,00o segments.

It has two deep laterally placed suckers.

The proglottides are very broad, ro to $20 \mathrm{~mm}$, and are broader than long.
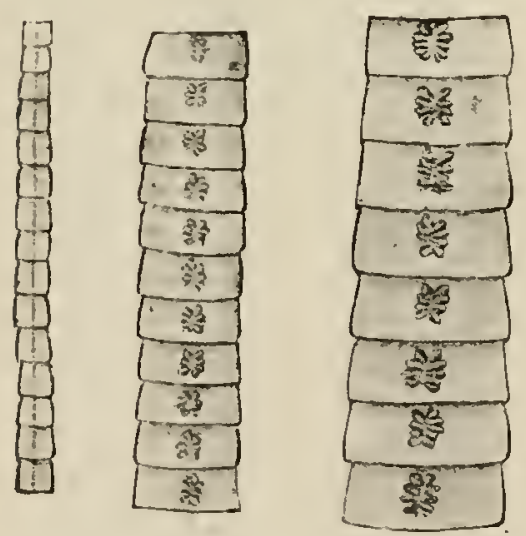

Various chain of segments of Dibothriocephalus latues, show. ing the central uterine rosette. (Natural size.)

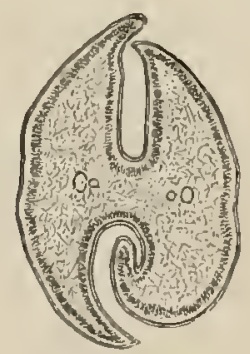

Transverse section of the head of Dibothriocepha'zus la'us. $30 / \mathrm{I}$.

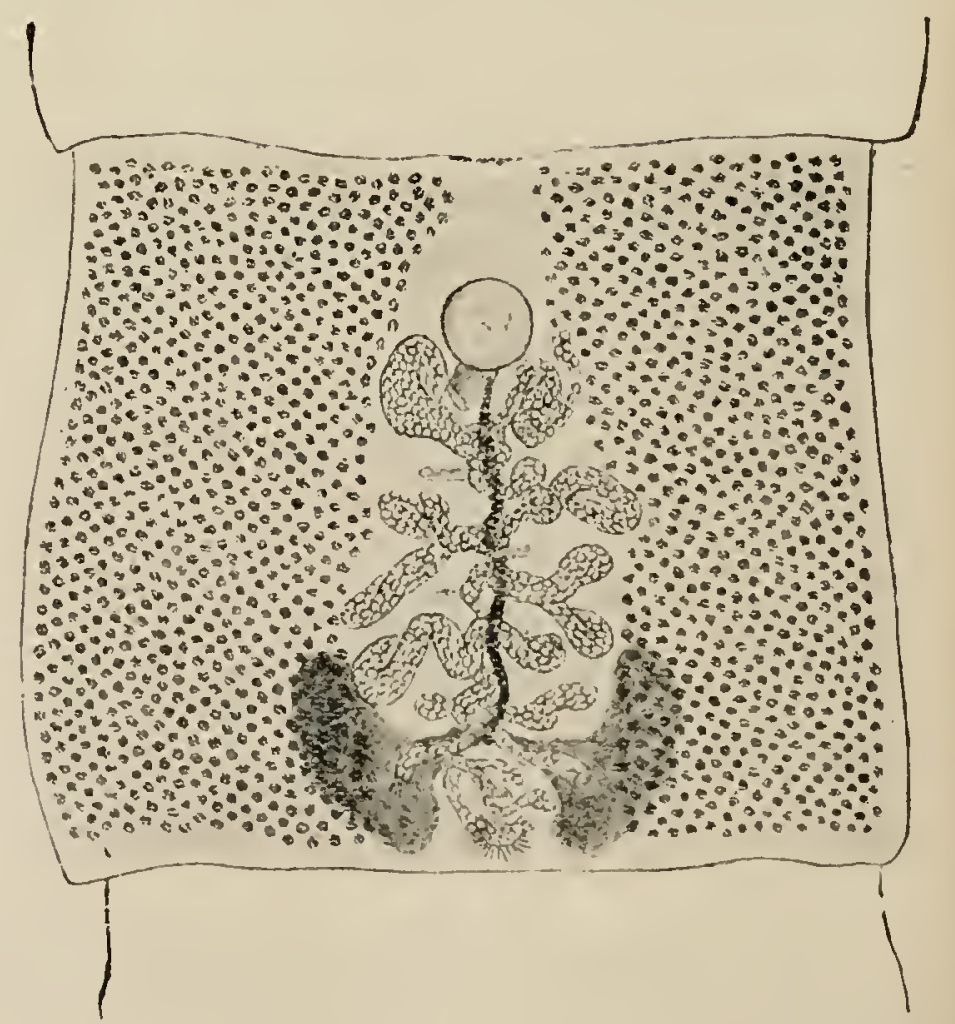

Fairly mature proglottis of Dibothriocephalus latus. The vilellaria are at the sides; the uterus, filled with eggs, is in the middle, al-o the vagina (the daık stripe passing almost straight from the front to the back), and the vas deferens (almust hidden by the uterus). Abuve in the centre is the cirrus sac, and helow the shell gland and ovary are seen. I5/I. (From a stained preparalion.) 


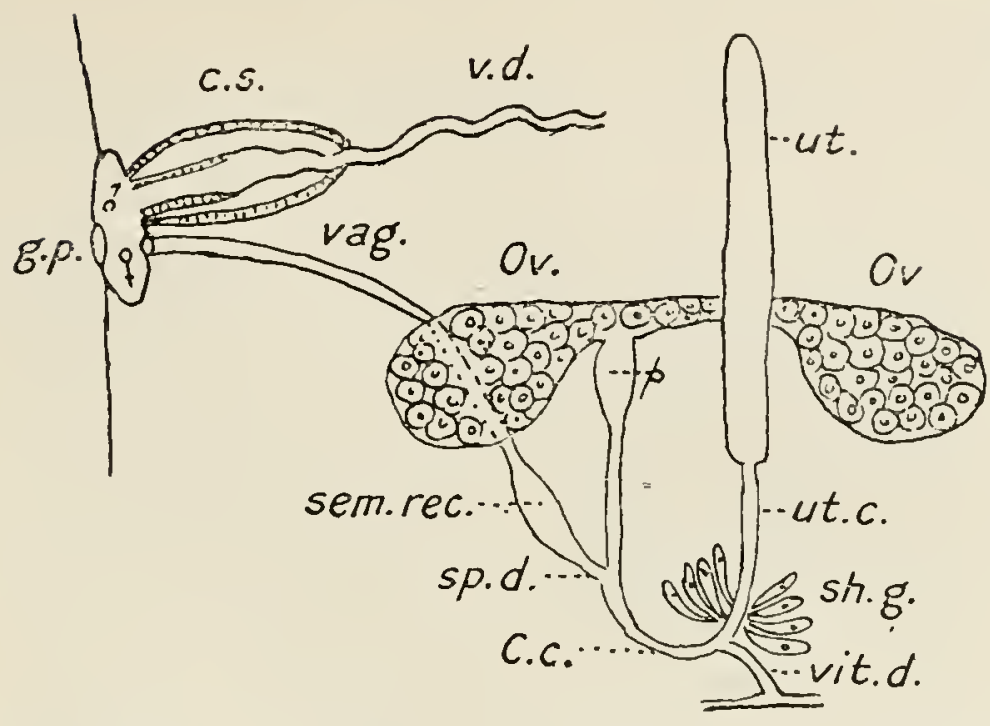

Diagram of genitalia of a Cestode. $s . p$. genital pore: $q \delta$, male and female ducts opening into genital sinus; $c . s$. , cirrus sac; $v . d$. , coiled vas deferens ("outer seminal vesicle" ; vag., vagina; sem. rec., seminal receptacle ; sp.d., spermatic duct; $C_{. c}$. , fertilization canal; vit. $d$. , vitelline duct; sh. $g_{.}$, shell gland; wt.c., uterine canal; ut., uterus; $O_{v}$, ovary; $p .$, pumping organ. (Stephens.)

The vas deferens opens dorsally and the vaginal opening lies immediately behind it. The rosette uterus has four to six convolutions and has its separate opening.

The six-hooked onchosphere passes into the fish.

When the fish is eaten, adult worms are formed from the onchosphere, and the eggs are passed into the faces in twenty-four days.

The egg has an operculum, this is the only exception. The operculum is, of course, common in trematodes.

The onchosphere has no sucker.

The parasite is found in Switzerland, N.E. Europe, and Japan.

The infection by it produces a severe anamia with fever and a quick pulse.

\section{SPARGANUM.}

This is a term applied to the larval stages of Dibothriocephalidæe, which are too premature for their genera to be known.

S. prolifer is I to $12 \mathrm{~mm}$. long by $2.5 \mathrm{~mm}$. broad. The head is motile and can be invaginated. An apical depression serves as a sucker. It can multiply by transverse fission. No genitalia are seen. The adult is unknown.

The parasite produces subcutaneous nodules in the fascia of muscles and the abdominal cavity of man. There may be an universal acnelike itching eruption.

A parasitic cyst usually contains one or two worms, a watery, jelly-like or opaque substance. The worms may be encapsulated in the cysts and may thus survive in man for years. 


\section{DIPYLIDIUM CANINUM.}

This parasite is found in the dog and cat but very rarely in man and children. It is 15 to $40 \mathrm{~m}$. long by $2 \mathrm{~mm}$. broad.

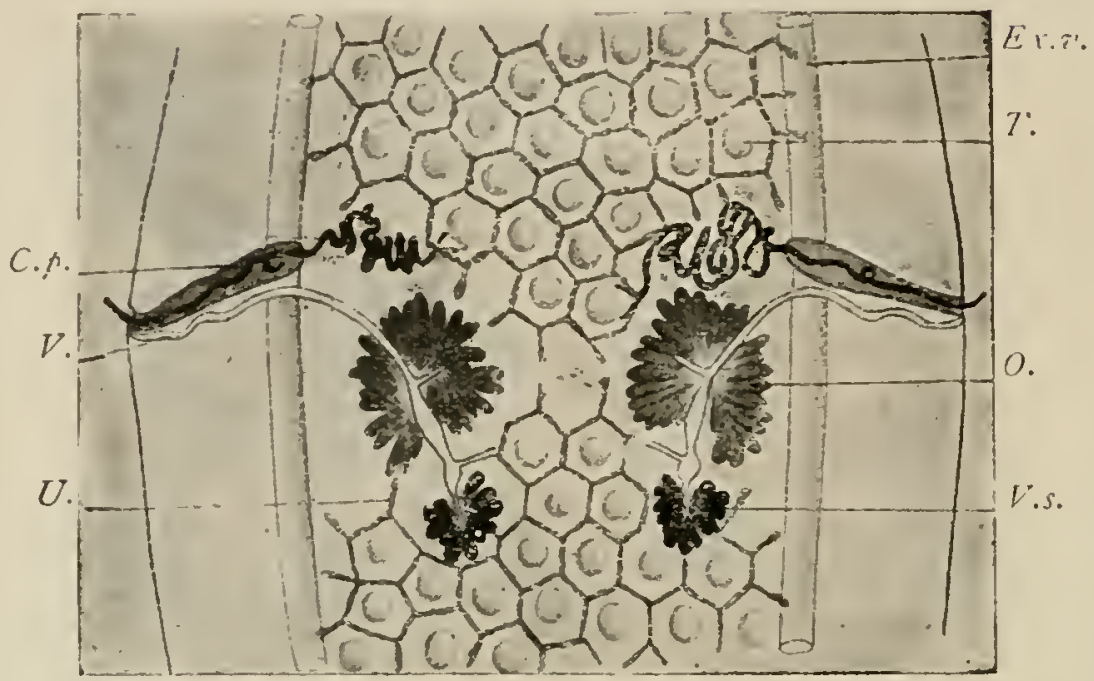

Dipylidium caninum: central portion of a proglottis. C.p., cirrus sac; $I^{*}$.s., vitellaria; $E x$. $v$., excretory vessels; $T$., testicles laying in the meshes of the uterine reticulum which laterally forms pouches; $O$., ovary; $U$., reticulum of uterus; $V_{.}$, vagina and seminal receptacle (below ovary). Magnified. (After Neumann and Railliet.)

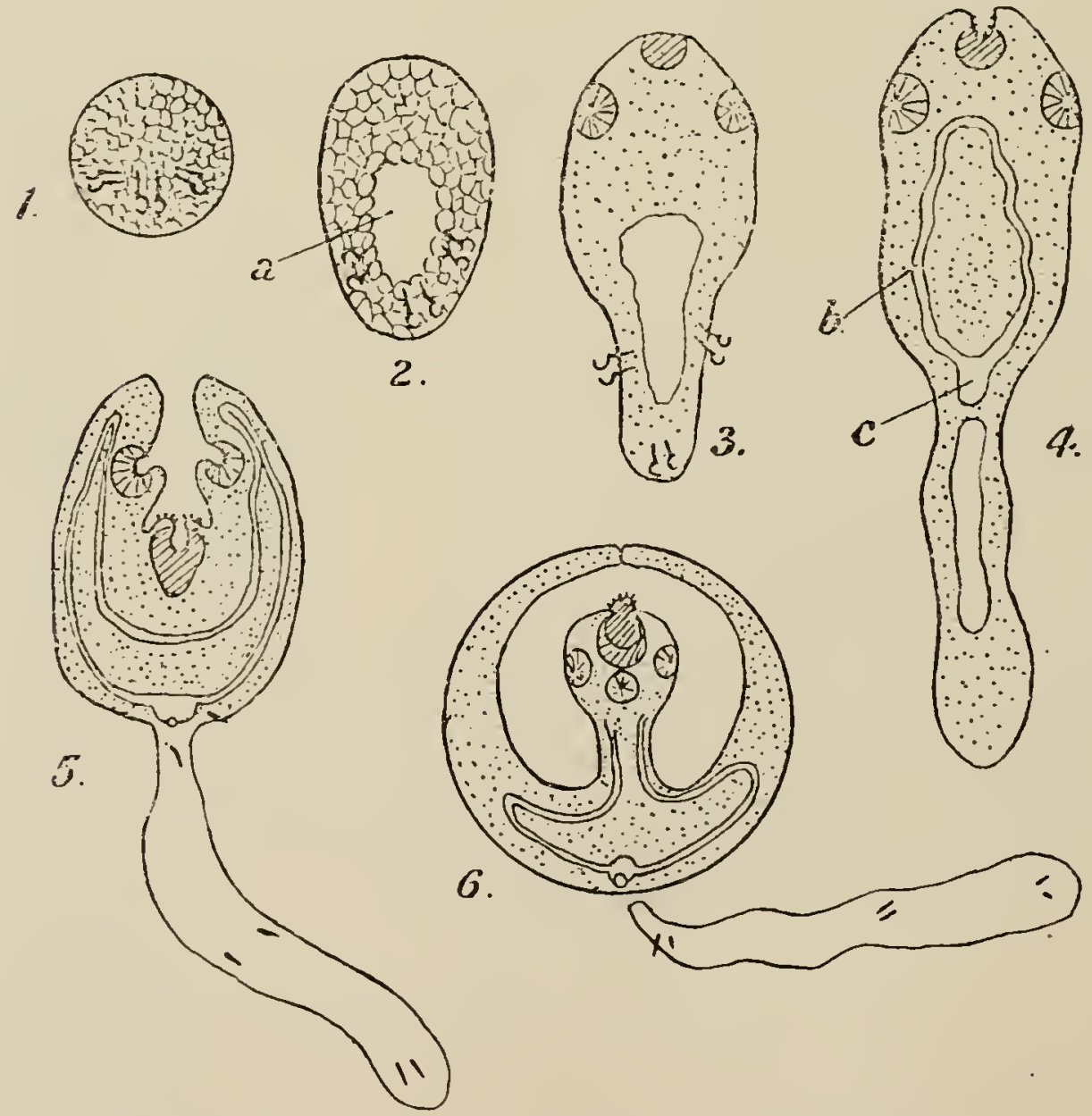

Dipylidium caninum : development of embryo. I, solid hexacanth embryo; 2 , primitive lacuna $(a)$ in the embryo; 3 , elongation of hinder part, rudiments of sucker and rostellum appearing ; 4, "body" and "tail" distinct, $(b)$ and $(c)$ excretory system ; 5, fore-body inv:ginates into hind-body, excretory bladder has a pore; 6 , tail has dropped off; scolex growing up into secundary cavity formed by fore-body; the primitive cavity has been absorbed at stage 4. (After Renham, Gras,i and Rovelli.) 
The scolex has a typical rostellum with three to four rings of hooks and four unarmed suckers. The genitalia are double with spores on each side. Ripe proglottides get into the fur where eggs infect the dog and cat, louse or flea in which cysticercoids develop. The dog bites the insect, gets the crsticercoid on its tongue and later licks man, thus infecting him. Cats may infect milk when drinking from a household vessel and children thus become infected.

\section{HYMENOLEPIS DIMINUTA.}

This parasite is conmon in rats and mice, rarely in children.

The cysticercus is found in the larva and the imago of the mealmoth, earwig, and beetle.

The adult is 20 to $60 \mathrm{~cm}$. long by $3.5 \mathrm{~mm}$. broad.

It has an unarmed rostellum with four elliptical suckers.

\section{HYMENOLEPIS NANA.}

This is the dwarf tapeworm of man. It is widely spread in Egypt, Europe, America, Siam and Japan.

Ten per cent of the children of Sicily are affected.

It is 10 to $15 \mathrm{~mm}$. long by $0.6 \mathrm{~mm}$. broad. The smallest tapeworm in man. It has a rostellum with one ring of twenty-four to thirty hooks, and also has a rather long neck. It has 150 proglottides. The genital pore is marginal. The mature uterus contains about thirty eggs. The cysticercus stage has not yet been found; perhaps it is passed in some insect.

The symptoms in children may be nil or slight.

In bad cases there is intestinal irritation and anamia.

Infected children should be isolated as infection spreads rapidly.

Male fern should be given.

There may be up to sereral thousand in one individual.

\section{DĀYÃ INEA MADDĂGÁSCARIENSIS.}

This is found in children, but is not very important.

It is 25 to $30 \mathrm{~cm}$. long by $\mathrm{I}^{\circ}+\mathrm{mm}$. broad. The scolex has four suckers and a rostellum with ninety hooks.

The proglottides number from 500 to 700 , and are longer than broad.

The genital pores are unilateral, and are situated near the proximal corner.

The uterus is a many-coiled tube which, when mature, uncoils, loses its wall, and the eggs, then free, become surrounded by cells which form capsules for themselves. 300 to 400 of these capsules may exist in one proglottis in transverse rows. 


\section{TAENIA SOLIUM.}

The uterus consists of a central stem, with seven to ten lateral branches on either side, each again branching.

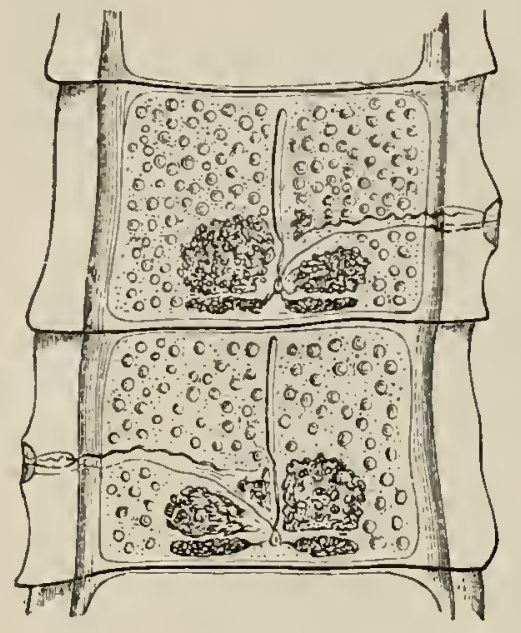

Two fairly mature proglottids of Tienia solizm, showing ovaries (one bi-lobed), vitellaria, central uterine slem, cirrus and vas deferens (above), vagina (below), testes (scattered), longitudinal and transverse ex. cretory vessels

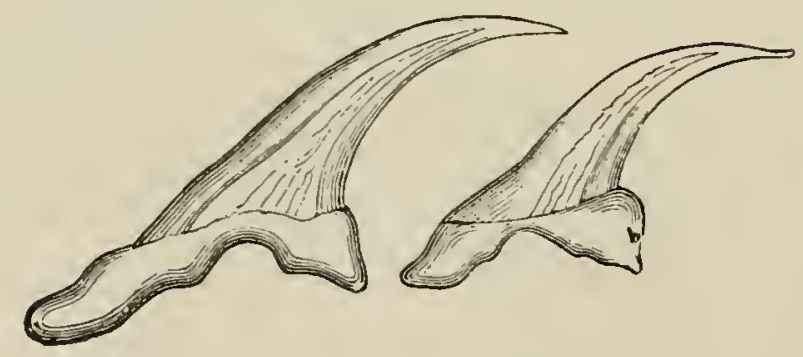

Large and small hooks of Tienta solizm. $280 / 1$. (After Lfuckart.)

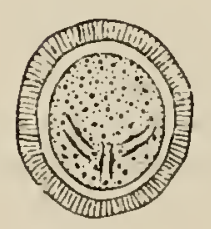

22
Trenia solizm. 2I, egg with external membrane; 22, without (embryophore). (After Leuckart.)

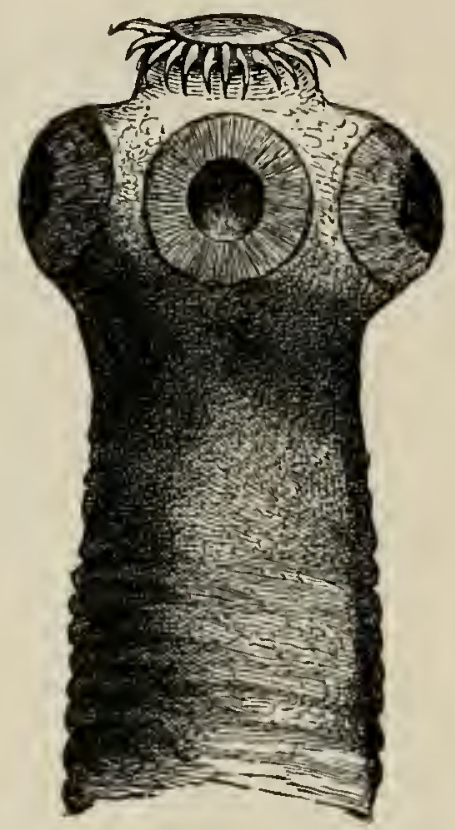

Head of Tenia solium. 45/I.

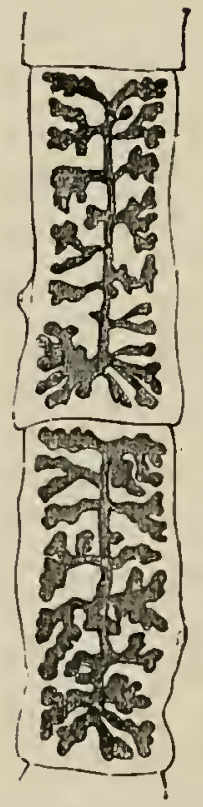

Two mature proglottids of Trenia solium with fully developed uterus. $2 / I$.

This parasite is found wherever men and pigs exist.

It is 2 to 3 metres long, it has reached $S$ metres, and is about $6 \mathrm{~mm}$. broad. The scolex has a rostellum, armed with a double row of hooks twenty-two to thirty-two in number. There are four hemispherical suckers. 
The proglottides number from 800 to 900 , and are longer than broad.

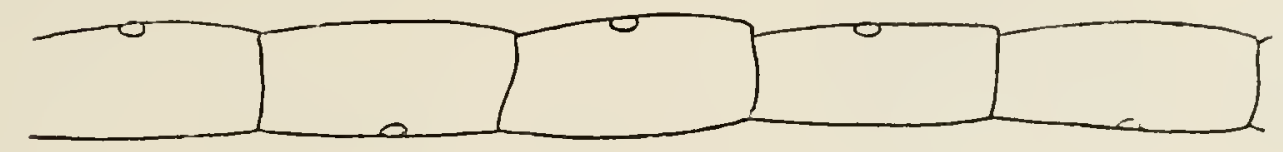

A

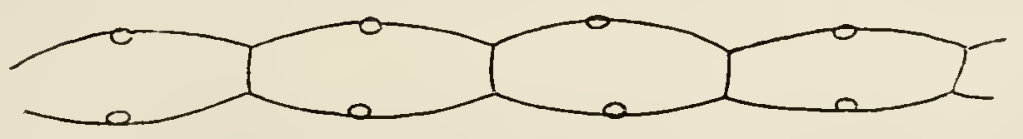

B

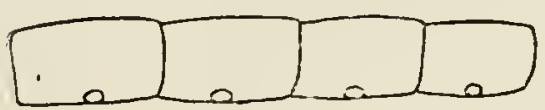

C

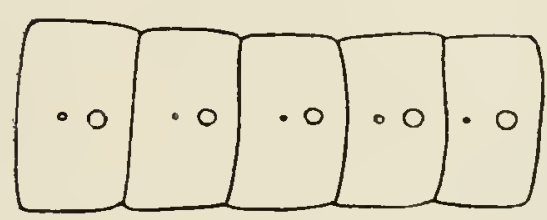

E

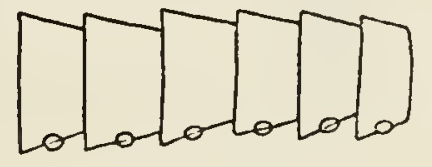

D

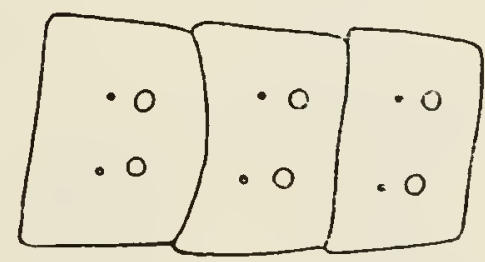

$\mathbf{F}$

A, Truna saginata; B, Dipylidiun ; C, Davainea; D, Hymenolepis ; F, Bothriocephalus F, Diplogonoforus grandis.

The genital pore is lateral and irregularly alternate.

Adults live solely in the human intestine and may exist there for years.

The cysticercus develops in the flesh of the pig which, if eaten undercooked, infects man. This is popularly known as " measly pork."

Rarely is man infected with the Cysticercus. cellulosa which, when present, appears in the brain, eye, muscle, liver and lung. One or more of these organs. This infection is caused by contaminated water or food.

The adult causes colicky pains and diarrhoea alternating with constipation. There are, emaciation and ancmia. The greater darger is infection by the Cysticercus.

\section{TAENIA SAGINATA.}

Perhaps this is the largest taperrorm in man. It is called the unarmed "beef-rorm."

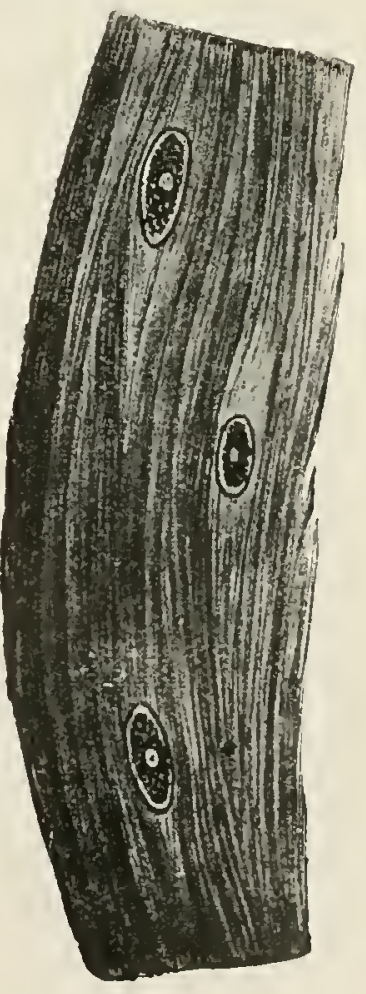

A piece of the muscie of the ox, with three specimens of Cysticercus bovis. Natural size. (After Ostertag.)

The adult only occurs in man and the cystic stage in cattle. It is common in Africa and Asia. 
The parasite is 4 to 10 metres long by $4.7 \mathrm{~mm}$. broad.

There are four hemispherical suckers, often pigmented. It has a sucker instead of a rostellum.

The proglottides number about i,ooo.

The genital pore is lateral and placed irregularly.

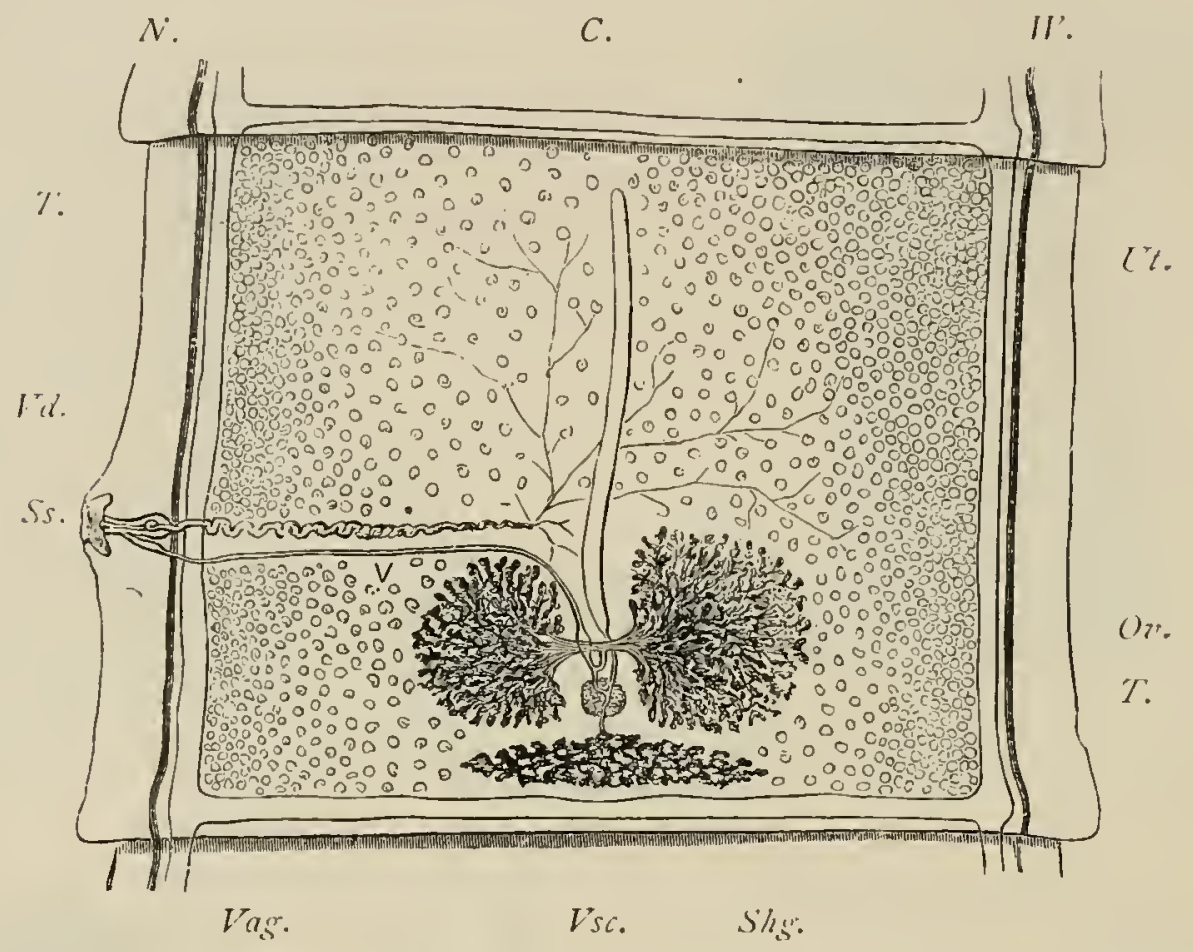

Proglottis of Tania saginala, Goeze, showing genitalia. $C$., transverse excretory canal; $N$., lateral longitudinal nerve; $W$. , longitudinal excretory canal ; $T$., testicles scattered throughout the proglotis ; Ut., opposite the central uterine stem (a closed sac); $S s$, genital pore leading into the genital sinus; above the cirrus and coiled vas deferens $(V . d$.$) , below the vagina (V a g$.$) , bearing near$ its termination a dilatation, the seminal receptacie; $V_{s c}$., the triangular vitellarium, and above it $(S h g$.$) the shell gland; leading from this to the uterus is seen$ the short uterine canal, on either side of this the two-lobed ovary $(0 \%$.). $10 / 1$.

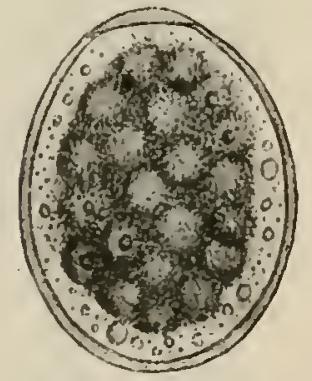

Egg of Diplogonoporus grandis, showing the morula surrounded by yolk cells and granules. 440/1. (After Kurimoto.)

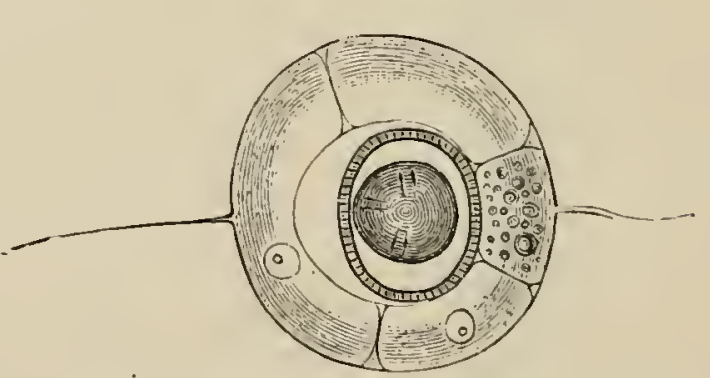

Uterine egg of Tienia saginata, G. Uterine shell with filaments; the oncosphere with embryonal shell (embryophore) in the centre. 500/1. (After Leuckart.)

Cattle take up the embryophore in contaminated food or water which then develops in their muscles as the Cysticercus bovis.

The muscles of the tongue and of mastication are chiefly affected. Man is infected by eating insufficiently cooked infected meat.

The cystic stage is very rare in man. 
The anamia is more severe than that caused by $T$. solium.

The parasite is said to be more difficult to kill.

It is common in Great Britain.

It has no rostellum and no hooklets.

The uterus has 20 to 30 lateral processes on either side, but these do not again branch as in $\mathrm{T}$. solium.

As many as 300 immature parasites have been found in one pound of the psoas muscle of an infected beast.

\section{ECHINOCOCCUS GRANULOSUS.}

This causes the cystic form of hydatid disease.

Hydatid disease has been known since the days of Hippocrates.

The cysticercoil form onl! occurs in man. 然

\section{DISTRIBUTION.}

In Iceland, Australia, Europe, and New 7ealand; rarely in India and South Africa, Egypt and the Philippines.

The adult lives in the intestine of dogs, jackals, and wolves.

The cysticercus lives in man, sheep, ox, and pig.

\section{MORPHOLOGY.}

It is about 2.5 to $6 \mathrm{~mm}$. long. It has a scolex and three segments.

The last proglottis, when mature, equals all the others in size.

The scolex has four suckers. The rostellum has a double row of twenty-eight to fifty hooks. The genital pore is alternate.

The mature uterus has only lateral protuberances, the eggs may form in heaps.

\section{THE LIFE CYCLE.}

Man is infected with the onchospheres when petting a dog, or by ingesting contaminated food. From the stomach the para-

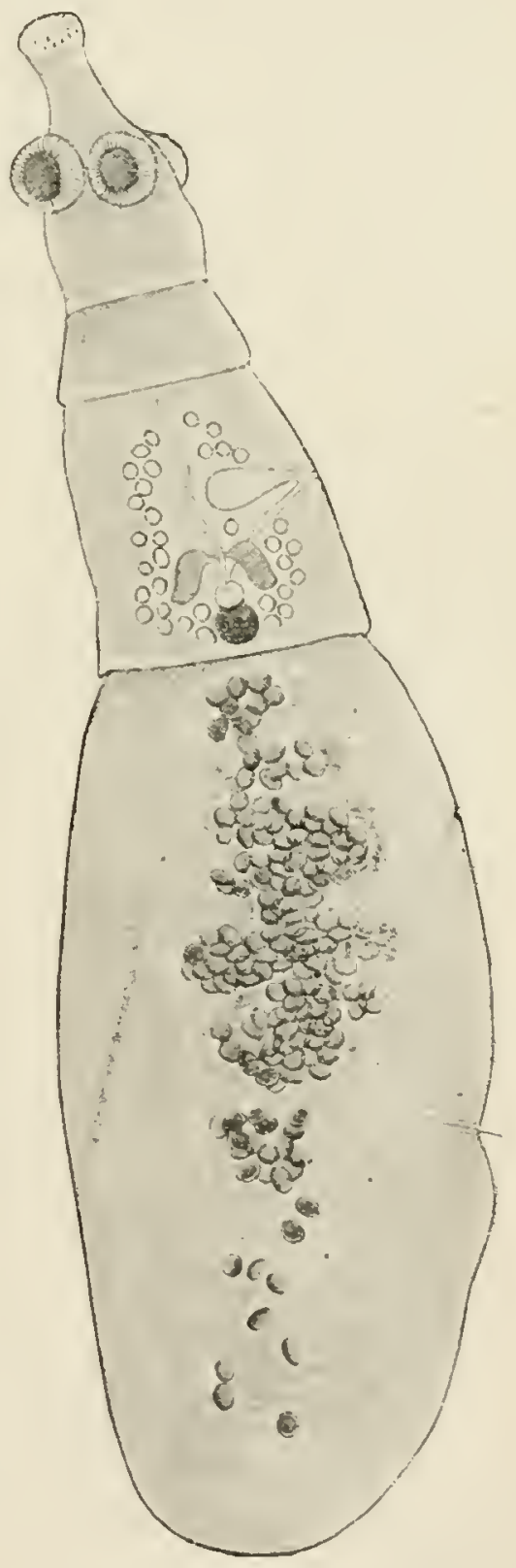

Ticnia echinococcus: the cirrus sac, the vagina, uterus, ovary, shell gland and vitellarium, and the lesticles at the sides are recognizable in the second progloltis; the posterior proglottis shows the uterus partly filled with eggs, as well as the cirrus sac and the vagina. 501 . 
site bores its way to the liver or other organs, grows and forms a cyst of three layers:-

(I) A thin layer or endocyst with granular, nulceated cells.

(2) A thick outer layer or ectocyst which is laminated and opaque.

(3) A connective tissue layer about the whole.

The inner layer of cells in cattle forms numerous hollow brood capsules called the E. cysticus sterilis, which have the endocyst external and the ectocyst internal. Of these brood capsules, heads develop called E. cysticus fertilis, but these more commonly occur in domesticated animals.

In man daughter-cysts appear between the strata of the cuticle into which endocystic cells have found their way and formed cysts.

These latter may grow and escape internally or externally. If externally they lie between the capsule and the mother-cyst and form E. hydatidosus exingenus, or if internally they fall into the mothercyst and are then known as E. hydatidosus endogenus. These daughter-cysts may remain sterile or produce brood capsules and heads which are then called grand-daughter-cysts. The wall of the mother-cyst may disappear and leave the daughter-cysts free.

\section{PATHOGENICITY.}

The cysts grow slowly so that the organs have time to accommodate themselves somewhat to the changes. If the organ cannot expand the symptoms are early and marked. If the cyst ruptures the fluid will cause urticarial symptoms, rigors, local pain, and tenderness.

The cystic fluid is toxic and a sudden discharge of a large cyst into the abdominal cavity may be so toxic as to kill, acting upon the heart like prussic acid.

It may rupture into the lung and drown the patient.

The cysts may become fatty, cheesy, and calcified. They maty become secondarily infected.

\section{ECHINOCOCCUS MULTILOCULARIS.}

This causes the multilocular form of hydatid disease.

\section{DISTRIBUTION.}

South Germany, Switzerland, Austria, Russia, and Serbia.

It is absent in Iceland, Australia, and the European districts where the cystic form is met with.

It occurs in animals and man.

\section{MORPHOLOGY.}

The cysts are composed of parenchymatous tissue internally and externally. There is no third outer fibrous coat.

These produce not only scolices but immature amoboid embryos 
which enlarge the crst and may enter the blood-stream and cause metastases.

The amoboid embryo is a multiple, folded, chitinous resicle which forms a toxin detrimental to the host.

It is stated that ripe proglottides develop directly from the cyst because it is capable of producing living embryos with or without a capsule.

The scolices are often destroyed by phagocytes from direct contact with the host's tissue.

Feeding experiments have failed.

\section{PATHOGENICITY.}

A primary tumour is formed in the liver, brain, spleen, kidney, adrenals with metastases in different organs.

The symptoms rary according to the organ affected.

There may be anemia, emaciation and wealiness leading to prema ture cleath.

\section{THE TREATMENT OF TAPEWORMS.}

\section{(1) PRELIMINARY.}

When the patient is not in hospital.

Give a brisk saline purge.

No food to be allowed for twelve hours.

Then give one to one and a half drachms of freshly prepared oil of male fern in gelatin capsules or as an emulsion.

Follow this with one ounce of castor oil in two hours.

\section{(2) THOROUGH.}

When the patient is treated in hospital.

Absolute rest in bed.

Light diet for two days.

Remove the mucus from the intestine and about the worm by giving during this period:-

Ammonium chloride, so grains thrice daily, \&c.

Small doses of podophyllin each morning.

At the end of the second day omit all food after 4 p.m., and give at that hour one ounce of Epsom salts in warm water.

On the third morning at 7,8 , and 9 a.m. respectively, give half a drachm of the freshly prepared oil of male fern each time.

At ro a.m. give :-

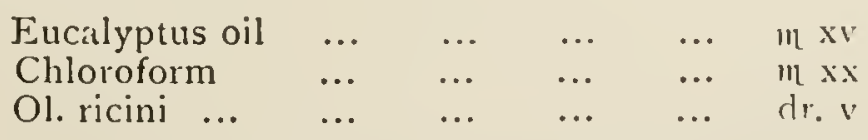

No movement by the patient is allowed during this treatment. 
No food to be given until a motion has been passed.

Fluid foods are to be given for the remainder of that day.

Preserve and examine all stools for the scolex.

The treatment for Echinococcus is largely symptomatic.

\section{(3) PROPHYLACTIC.}

Keep cattle from all facal matter.

Irge absolute cleanliness with regard to food and water.

lork and beef meat should always be well cooked.

\section{III.-NEMATODES PATHOGENIC TO MAN.} Metazoa.

Nematodes belong to the Nemathelminths, Phylum (2) of the

Their study is most important as Filariasis and Ankylostomiasis are among the diseases caused by these helminths.

\section{MORPHOLOGY.}

They are white filiform worms covered with a cuticle, usually tapering at both ends and enclosing a large undivided body cavity.

There is a mouth at the anterior end with two to six lips leading to a thick muscular oesophagus which may be armed with teeth.

The intestine is a flattened tube leading to a short rectum or anus.

The excretory pore is in the mid-ventral line behind the mouth, the pore being the outlet for two excretory tubes which unite to discharge there.

The nervous system is a circum-asophageal ring with six anterior and six posterior nerve trunks.

The sexes are separate.

The male genital organs, testes, vas deferens, vesicula seminales and ductus ejaculatorius are modifications of one tube which opens close to the anus. There are also spines and papillae.

The female genital organs, ovary, oviduct and uterus are modifications of two much-coiled tubes. These unite forming the vagina which opens in the ventral mid-line in front of the anus.

The parasites appear to live upon intestinal juices. Some enter the villi. Their life histories differ and are dealt with separately.

\section{THE PRESERYATION AND EXAMINATION OF NEMATODES.}

See end of present section, p. $32 \mathrm{I}$.

The most important of these Nematodes will now be dealt with seriatim.

\section{STRONGYLOIDES STERCORALIS (Family Angiostomidæ).}

The adult is very small, $2 \cdot 2 \mathrm{~mm}$. by $34 \mathrm{~mm}$. long.

There are four lips to the mouth. 
The asophagus is one quarter the length of the whole body.

The anus is just in front of the tail tip. The sex is doubtful. It is perhaps an hermaphrodite.

It is found in the duodenum and jejunum, deep in the mucosa.

The parasite is widely distributed.

The ova are $54 \mu$ by $32 \mu$ and occur in the faces during attacks of diarrhoa. The ova are normally deposited in the mucosa, here the embryos hatch, reach the lumen of the gut and pass out in the faeces. They grow into male and female adults on reaching the water. These conjugate and the females then lay eggs. Free living embryos develop from these which moult and become filariform embryos which will penetrate the horny layer of the skin and reach the corium (Mozocchi). They pass, via the lungs, trachea and aesophagus to the small intestine (Fülleborn). They may also pass directly by the blood stream. In the small intestine they burrow into Lieberkihn's follicles and lay their eggs.

These parasites cause an intestinal catarh.

They are the only worms that pass embryos in fresh freces.

\section{GNATHOSTOMA SPINIGERUM (Family Gnathostomidæ).}

The male, about ro $\mathrm{mm}$. long, has two fleshy lips guarding the mouth.

The cuticle in front of the neck has eight transverse rows of hooks pointing backwards.

There are two unequal spicules.

These parasites are very rarely found in man. Only two specimens are known.

They wander about the connective tissue and form subcutaneous tumours.

\section{THE FILARIDA.}

Diseases caused by Filaria are called Filariases, manifestations of which include F. lymphangitis, orchitis, lymphangiectasis, abscesses, phlebectasis, varicose lymphatic glands, chylous extravasations and elephantiasis, Loasis, Calabar Swellings, and Volvulosis.

These will be dealt with seriatim.

\section{THE FILARIASES.}

\section{HISTORICAL NOTES.}

Elephantiasis was noticed by the ancient Indian writers.

Celsus first used the term to indicate leprosy, and all writers since have repeated the error.

Arabian physicians described it in the ninth and tenth centuries. Hillary in r 750 clearly differentiated it from leprosy. 
Chapoton in 1812 described Hamatochyluria in Mauritius.

Demarquáy in ${ } 86_{3}$ found a Microfilaria in a hydrocele at Paris.

Wucherer in r $\$ 66$ found a Microfilaria in urine, in Egypt, but neither at the time recognized its importance.

Lewis in 1868 found the Microfilaria in the blood and lymph of persons with elephantiasis of the leg and assumed it to be the cause.

Bancroft in 18,6 found adult females in a lymphatic abscess of the arm, and in a hydrocele of the cord. He afterwards called it F. bancrofti.

Manson in 18 - 8 found the disseminating mosquito of the F. bancrofti as well as the periodicity of the Microfilaria, and he also showed that the worms retired to the large ressels of the lungs during the day.

Only about ten adult filaria have been collected.

These parasites are non-bursate nematodes with a simple mouth and a limited number of genital papilla. The spicules are dissimilar in shape and size.

These nematodes always live in the connective tissue, lymphatic ressels and in the blood-stream, but nerer in the intestine.

When they leave the human body they pass at once into some intermediate host. They do not pass into water or any other media. The exception to this is the F. dracunculosis.

The skin of these nematodes may be :-

(1) Smooth, as in F. bancrofti, perstans and demarquayi.

(2) Striated, as in Dirofilaria mag.

(3) Bossed as in Loa loa.

(4) Corrugated transversely as in F. rolvulus.

\section{FILARIA BANCROFTI.}

\section{MORPHOLOGY.}

It is a long, whitish, filiform worm, with a smooth cuticle and a globular head, with an unarmed lipless mouth. Only one complete specimen has ever been found of the adult.

The tail of this worm incurves when dead. The male measures $36.6 \mathrm{~mm}$. long by $0^{\circ} \mathrm{r} 2 \mathrm{~mm}$. thick. The anus, close to the posterior end, is guarded by two projecting lips.

There are six pairs of papilla, three pre-anal, and three post-anal. There are two curved retractile spicules, $0.6 \mathrm{~mm}$. and $0.2 \mathrm{~mm}$. long.

The female measures $76 \mathrm{~mm}$. to roo $\mathrm{mm}$. long by o'is thick.

The rulra is close to the anterior end and opens into a single ragina, from which two uterine tubules run almost the whole length of the body. These contain eggs and embryos. 


\section{THE LIFE-HISTORY.}

The Adult.-The males and females, from three to four inches long, may be found coiled together in the lymphatic glands, or in the lymphatic vessels of the scrotum, arm, leg, epididymis, testicle and hydroceles

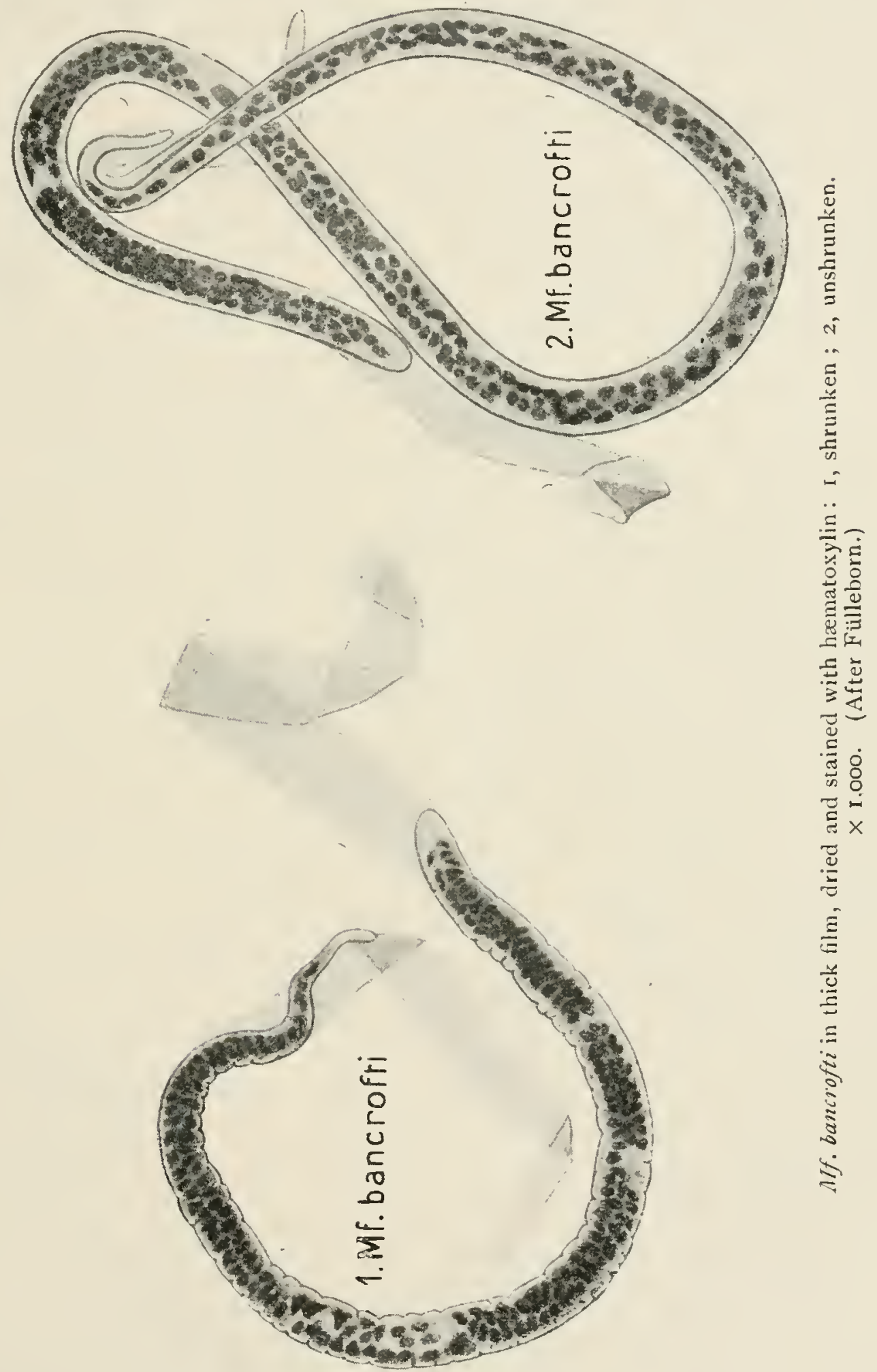

of the cord; rarely in the lymphatics of the pelris and abdomen.

The males are less numerous than the females.

The Ooum.-The intra-nterine egg, $50 \mu$ by $40 \mu$, containing a coiled-up embryo, becomes elongated by the embryonic morements. 
The embryo is set free and is discharged from the genital pore. It is then free in the lymph stream in which the adult female happens to be.

The Embryo.--The embryo or Microfilaria nocturna passes from the lymph stream to the thoracic duct, the right heart and lungs to the peripheral circulation where it is to be found at night.

These Microfilaria wriggle about in the blood-stream, moving the blood cells to and fro, but without making progress. They are about 300 by $8 \mu$, with rounded anterior and tapering posterior ends. They are enclused in a sheath, longer than itself, inside of which it darts to and fro. Were it not for these sheaths the embryo might use its anterior spine to escape through the wall of the blood-vessel, and so aroid the mosquito that transmits it.

A thick proboscis carries the spine, which can be covered by a retractile and protractile six-lobed prepuce.

The dermo-muscular transversely striated body encloses many granules which are wanting in definite parts of the parasite thus-

(i) In the anterior extremity of the body (not the sheath).

(2) In a transverse spot, $2 I^{\circ} 5$ per cent. of the whole length from the anterior extremity.

(3) In a $V$-shaped spot, 30 per cent. of the whole length from the anterior extremity.

(4) In a median line, the centre, 63 per cent. of the whole length from the anterior extremity.

(5) In an oval spot (tail spot), 85 per cent. of the whole length from the anterior extremity.

(6) In a central spot, $9{ }^{\circ} 5$ per cent. of the whole length from the anterior extremity.

The significance of these granules is not known.

Its Habits in Man.- They are to be found in the peripheral blood ar night from 5 p.m., increasing to midnight, then until 8 a.m. diminishing. During the day they live in the lungs, large thoracic vessels, carotid artery, heart, muscle and kidney.

If man sleeps during the day instead of at night the periodicity will be reversed, but not completely, hence sleep may have something to do with its periodicity.

The common carrier, Culex fatigans, bites at night, but the carrier in Fiji, Stegomyia pseudo-scutellaris, bites in the daytime, and Bahr says that there the Microfilaria are found in the blood during the daytime also, so that the periodicity is controlled by the habits of the more efficient insect host. How the Microfilaria maintain their position against the blood-stream has not been shown. One blood film may show as many as 500 embryos. It has been calculated that there may be $45,000,000$ in a fully grown man. 
The number of adults necessary to produce them is not known.

One Filaria can live for several years in the human body, and a Microfilaria can live in the blood-stream for several months (Bancroft).

Its Habits in the Mosquito. - The mosquito sucks infected blood; the Microfilaria passes to the stomach, where the sheath is fixed by the surrounding hæmoglobin and also altered by the gastric juice.

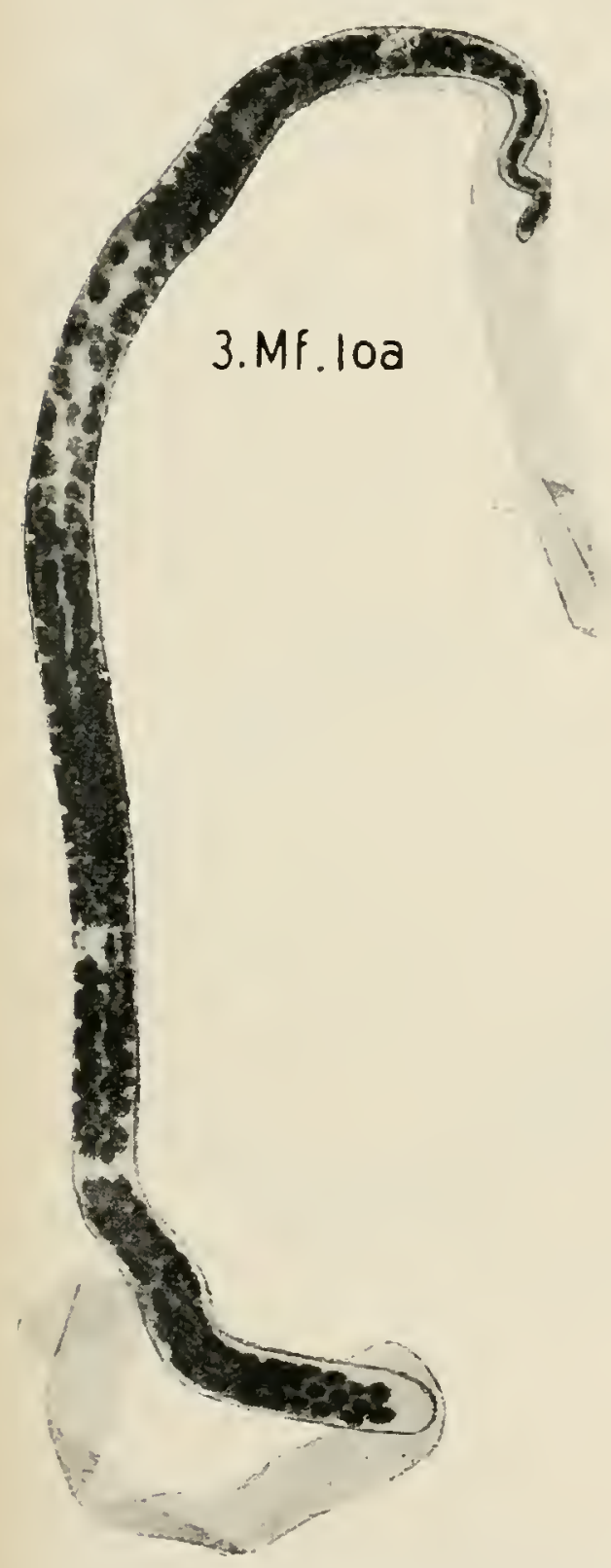

Mf. Loa: in thick film, dried and stained with hæmatxylin. $\times$ I, ,oo. (After Fülleborn.)

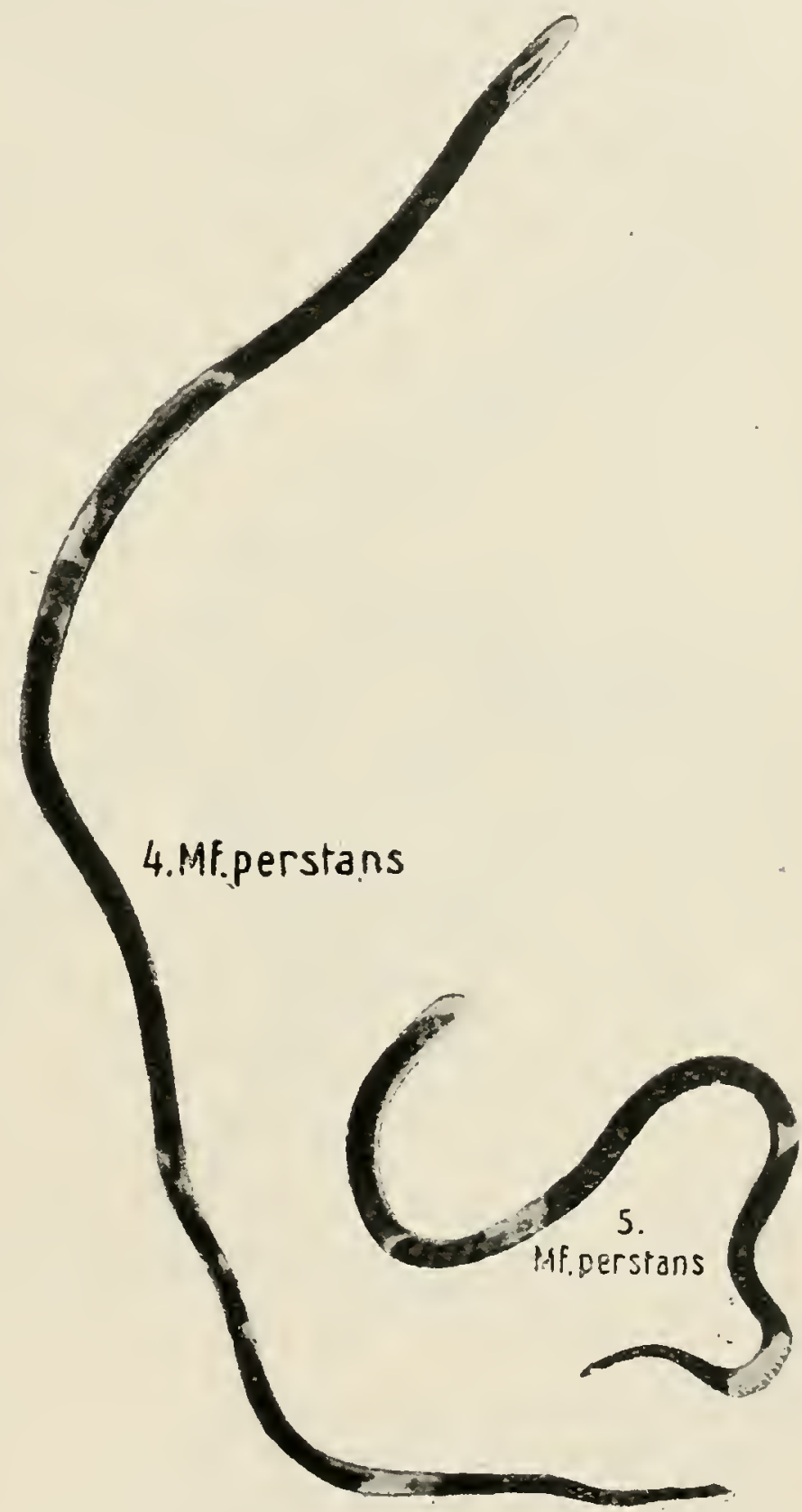

Mf. perstans in thick film, dried and stained with hrmatoxylin ; 4, unshrunken; 5, shrunken. $\times 1,000$. (After Fillleborn.)

As a result of these changes the embryo is able to rupture the sheath with its spine and to escape. The stomach wall is then pierced and the embryo passes to the thoracic muscles, where it grows considerably during sixteen to twenty days. In this position they develop four lips, an alimentary canal and a three-lobed tail. The position is now changed, and the embryo finds its way to the prothorax, head and 
labium. Here the parasites wait, and when an animal, usually man, is bitten, the Microfilaria passes through Dutton's membrane, and enters the wound made by the proboscis of the insect. When a fly is fed upon vegetable matter, however, the Microfilarice will not pass through. From the wound the embryo passes into the lymphatics, where it becomes sexually mature, fecundation is affected, and new generations of larva are poured into the lymphatic stream. These then pass to the thoracic duct as described above. The embryos are often harmless; it is the injured parent worm and the immature products of conception that are dangerous. A man's blood may

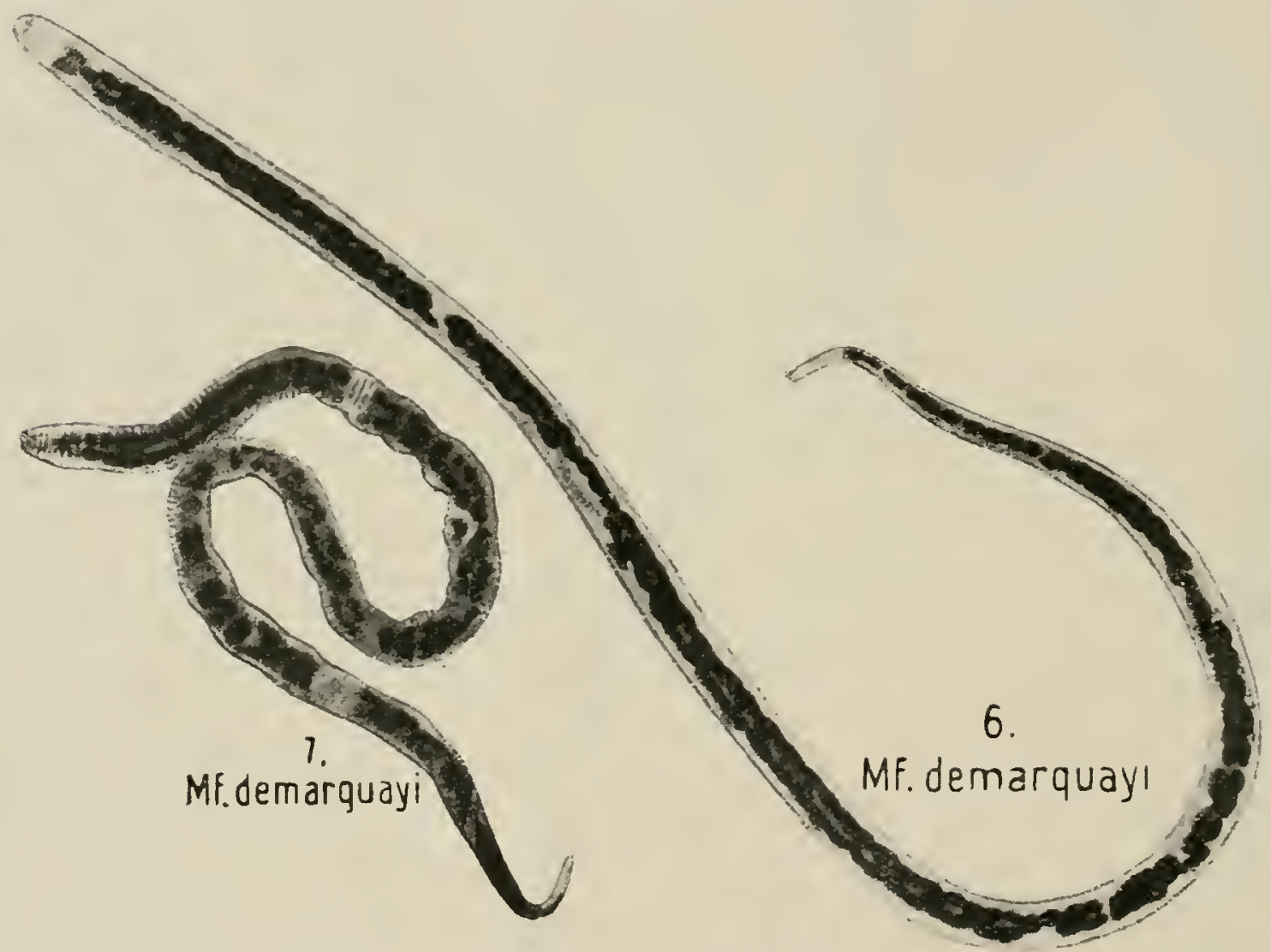

Mf. demarquayi in thick film, dried and stained with hrmatoxylin. 6, unshrunken; 7, shrunken. $\times 1,000$. (After Fiilleborn.)

swarm with the embryos, and yet he may show neither signs nor symptoms.

To demonstrate the Microfilaria.-Take an ordinary blood slide at night, place it at once in weak fuchsin, four minims to one ounce of water, leave it for one hour, and examine it wet without a cover-glass. To partly decolorize use weak acetic acid.

To fix, stain with methylene blue for thirty seconds and examine it wet.

The hremoglobin is washed out, and the only coloured objects are the leucocytes and the embryos.

To keep them alive for six or seven days, ring a cover-glass with raseline and place it upon the fresh specimen in the ordinary way. 
The Diagnosis of Microfilaria.

\begin{tabular}{|c|c|c|c|}
\hline MF. BANCROFTI (nocturna) & MF. LOA (diurna) & MF. PERSTANS & MF. DEMARQUAY \\
\hline $\begin{array}{l}\text { Length } 0.317 \times 0.0084 \mathrm{~mm} \text {. } \\
V \text { spot is } 090 \mathrm{~mm} \text {. from } \\
\text { the head }\end{array}$ & $\begin{array}{l}0.245 \times 0.0075 \mathrm{~mm} . \ldots \\
\mathrm{V} \text { spot is o.060 mm. } \\
\text { from the head }\end{array}$ & $0.195 \times 0.0045 \mathrm{~mm}$. & $0.21 \times 0.005 \mathrm{~mm}$ \\
\hline $\begin{array}{l}\text { Seen in the peripheral } \\
\text { blood at night }\end{array}$ & $\begin{array}{l}\text { Seen in the peri- } \\
\text { pheral blood in the } \\
\text { daytime }\end{array}$ & $\begin{array}{l}\text { (Adults in con- } \\
\text { nective tissue, } \\
\text { pancreas, be- } \\
\text { hind pericar- } \\
\text { dium and aorta) }\end{array}$ & $\begin{array}{l}\text { (Adults seen in } \\
\text { conn ective } \\
\text { tissue and root } \\
\text { of mesentery) }\end{array}$ \\
\hline Many & Few present & & \\
\hline ersible $\quad .$. & $\begin{array}{l}\text { Periodicity not rever- } \\
\text { sible }\end{array}$ & No periodicity ... & No periodicity \\
\hline $\begin{array}{l}\text { Curved when dead } \\
\text { Sheath smaller when dead }\end{array}$ & $\begin{array}{l}\text { Straight when dead } \\
\text { Sheath longer when } \\
\text { dead }\end{array}$ & & \\
\hline $\begin{array}{l}\text { Nuclear core does not end } \\
\text { abruptly }\end{array}$ & $\begin{array}{l}\text { Nuclear core ends } \\
\text { abruptly }\end{array}$ & & \\
\hline $\begin{array}{l}\text { Nuclei small, definite, } \\
\text { stain well }\end{array}$ & $\begin{array}{l}\text { Nuclei large, hazy, } \\
\text { stain poorly }\end{array}$ & & \\
\hline Tail straight and pointed & $\begin{array}{l}\text { Tail curved and } \\
\text { pointed }\end{array}$ & $\begin{array}{l}\text { Tail blunted and } \\
\text { truncated }\end{array}$ & $\begin{array}{l}\text { T a il sharpely } \\
\text { pointed. } \\
\text { Femalehasbul- } \\
\text { bous cutaneous } \\
\text { expansion at } \\
\text { the tip }\end{array}$ \\
\hline $\begin{array}{l}\text { re does not go } \\
\text { tremities }\end{array}$ & ditto & $\begin{array}{l}\text { Nuclear core goes } \\
\text { to tip of tail }\end{array}$ & $\begin{array}{l}\text { Nuclearcore does } \\
\text { not reach ex- } \\
\text { tremities }\end{array}$ \\
\hline $\begin{array}{l}\text { Has a pale area about the } \\
V \text { spot }\end{array}$ & ditto & & \\
\hline $\begin{array}{ll}\text { Transversely striated } & \ldots \\
\text { Enclosed in sheath } & \ldots\end{array}$ & $\begin{array}{l}\text { ditto } \\
\text { ditto }\end{array}$ & No sheath & No sheath \\
\hline $\begin{array}{l}\text { Adults found in lymphatic } \\
\text { system }\end{array}$ & $\begin{array}{l}\text { Adults found in } \\
\text { connective tissue } \\
\text { usually }\end{array}$ & $\begin{array}{l}\text { Adults in connec. } \\
\text { tive tissue }\end{array}$ & $\begin{array}{l}\text { Adults in connec- } \\
\text { tive tissue }\end{array}$ \\
\hline In most tropical regions & In Vest Africa & $\begin{array}{c}\text { In Africa and } \\
\text { British Guiana }\end{array}$ & In West Indies \\
\hline
\end{tabular}

The Microfilaria volvulus has no sheath, is sharply pointed, and does not look unlike the Mf. bancrofti out of its sheath. They are not seen in the blood-stream.

\section{The Diagnosis of Microfilaria.}

This is not always easy. The points of value are, according to Daniels :-

(I) The presence or absence of a loose sheath.

(2) The periodicity-that is, the times when the embryos arc present in the blood-stream.

(3) The character of the termination at the narrow end.

(4) The nuclear matter and how it terminates anteriorly.

(5) The dimensions of the embryo, dead and alive.

(6) The position assumed by the worm after death.

For the differential points the reader is referred to the table above. 


\section{THE FILARIASES.}

\section{THE CHIEF CAUSATIVE PARASITE.}

This is the Filaria bancrofti as described above.

Its position among the Nematodes can be seen at a glance by refering to the table on p. 232 .

\section{DISTRIBUTION.}

Asia is perhaps the endemic home of the disease. From thence it probably passed to Africa, from thence to America, from the Southern United States downwards.

It is also present in Australasia.

It is more common along the sea coasts and the banks of large rivers.

High air temperatures and considerable atmospheric humidity seem to assist its spread.

Bahr concludes that at one time or other every Fijian is the subject of filariasis.

50 per cent. of the Samoans are infected.

33 per cent. of the people of Cochin in India carry it.

\section{KNOWN CARRIERS OF THE WORM.}

Culex fatigans.

Stegomyia pseudo-scutellaris.

Mansonia uniformis.

,, pseudo-titillans.

Pyretophorus costalis.

Myzomyia rossii.

Myzorhynchus nigerrimus.

$$
\text { ", minutus. }
$$

Cellia albimana.

The worm is capable of undergoing part of its development in certain other mosquitoes.

\section{PATHOLOGY.}

The parent worm may not produce any ill-effects, but-

(I) If it obstructs a lymph trunk the mechanical pressure of the retained lymph damages the tributary channels, or

(2) If the parent is injured abortion may ensue, when oval eggs instead of elongated embryos are passed, and these eggs being thicker than the embryo are liable to block up the lymph channel of the skin or lymphatic gland.

Heavy infections with parent worms have not yet been described.

It must be remembered, however, that few post-mortems have been recorded. The adults are difficult to find. When there are varicose lymphatics or lymphatic glands with clear lymph, look for the obstruc- 
tion below the junction of the lacteals with the receptaculum chyli, but when the fluid is milky the obstruction is beyond this point.

Obstruction may be caused by :-

(1) A coiled-up mass of worms behind a valve or dilated sinus.

(2) A single worm in a dilated lymphatic, the draining gland being probably blocked by aborted ova.

(3) Constant irritation caused by worms may lead to the permanent blocking of a main lympl channel.

There is often much fibrosis of the glands, when the lymphatics can be seen collected in clusters between tissue strands. This condition is accompanied by an excessive number of eosinophile cells in the glandular substance (Bahr).

The clinical manifestations are according to the site of the obstruction. This may be anterior or posterior to the junction of the lacteals with the receptaculum chyli.

(I) When the obstruction is anterior to the junction of the lacteals with the receptaculum chyli there are chylous extravasations. The thoracic duct may be blocked which results in the engorgement of the lymphatics of the renal, lumbar and the pelvic regions with dilatation of the lacteals.

If the engorged vessels of the urinary tract rupture, chyluria results.

If some blood vessels rupture also, as is common, then one has hamato-chyluria, the milky opacity being due to the large amount of protein and not to fat.

If the engorged vessels of the abdomen rupture, one has chylous ascites.

If the engorged ressels of the tunica vaginalis rupture, one has chylocele.

(2) When the obstruction is posterior to the junction of the lacteals with the receptaculum chyli, one finds ordinary ascites, hydrocele lymph scrotum and the varicose groin glands.

The resistance of the tissues is lowered by this condition and inflammation is readily set up which may go on to abscess and elephantiasis.

\section{The causes of Elephantiasis can be grouped as follows:-}

(i) As described above.

(2) An injury to the female worm ma cause abortion and conseqnent obstruction of the lymphatics by the aborted eggs. The normal microfilaria although 250 to $300 \mu$ long is only 7 to $8 \mu$ in breadth, while the aborted eggs are 50 by $34 \mu$, hence it can be readily understood that eggs 34 in their smallest diameter would completely block lrmphatic vessels along which microfilaria 7 to $8 \mu$ in diameter could travel.

Lymph stasis causes inflammation and hypertrophy of the connective. tissue with excess of lymph and hence the limb enlarges (Manson). 
(3) The cundition may be caused by a blockage of lymph channels of the diseased area as a result of the frequent and prolonged invasion of the adult filaria. The microfilaria may not reach the blood but they may die in the gland. The periodic discharge of these microfilaria may be a factor in producing lymphangitis, orchitis and funiculitis, the parent worm dying as a result of these inflammatory attacks (Bahr).

In these conditions the blood is usually normal.

There is often secondary ancmia in hamato-chyluria and diarthoea.

Sometimes there is leucocytosis during the febrile period.

The eosinophiles at times may be increased in blood examinations.

\section{P.M.}

The findings are according to the variety of the lesion.

In Lymphangitis the lymphatic vessels are enlarged and inflamed with cyst-like dilatations and the walls are thickened and fibrous. Abscesses, varying in size, may contain dead worms. These dead worms maly become calcified or the calcareous cleposit may be gradually a)sorbed until only minute yellow spots remain. The worms, calcified or living, are surrounded by eosinophilic cells in large numbers.

Filarial abscesses have been found in the gastrocnemius muscle, popliteal space, groin, quadriceps muscle, over the internal condyle of the knee, in the axilla, latissimus clorsi and the serratus magnus muscles, and in the subcutaneous tissue.

In the abscesses are also found Staphylococcus pyogenes aureus and Streptococci pyogenes.

In Lymphatic varicosity the vessels are much dilated with thickened walls. The glands are dilated, the true gland tissue disappears leaving a fibrous sac with numerous septa.

In Elephantiasis, the vessels are dilated and thickened in early cases. There is a round celled infiltration into the connective tissue of the part, leading later to the hypertrophy of the connective tissue of the skin and fasciae, this latter including that of muscles, vessels and nerves.

All the tissues are sodden with lymph.

The muscles show fatty degeneration.

The bones are thickened or perhaps covered with osteophytes or more rarely are atrophied and still more rarely are invaded by caseous abscesses.

\section{CLINICAI, VARIE'TIES.}

\section{(1) FILARIAL LYMPHANGITIS.}

\section{DEFINITION.}

An inflammation of the lymphatic ressels in any part of the body caused by Filaria bancrofti. 


\section{SYMPTOMATOLOGY.}

A shivering lit, temperature $101^{\circ}$ to IO4 $\mathrm{F}$, vomiting, headache, red ocdema of skin which is sometimes painful, the vessels are like a red line under the skin. The temperature falls in two days accompanied by an erysipelatous eruption which may last several days longer. The lymphatic ressels return to their normal condition, rarely they are left as a hard cord with the adult filaria in the lumen.

\section{TREATMENT.}

Rest in bed, mild purge, aspirin for the headache, lead and opium lotion locally, later ichthyol and lanolin ointment.

Evacuate the abscess when necessary.

\section{(2) FILARIAL ORCHITIS AND HYDROCELE.}

\section{SYMPTOMATOLOGY.}

Pain in the testes, ferer, pains in the back, lower abdomen and groins.

There is often bilious romiting. The testicle enlarges, is tender and painful, effusion into the tunica vaginalis of lymph or chyle.

This effusion may become coagulated and persist, or it may form a filarial hydrocele which may be absorbed or require tapping.

\section{TREATMENT.}

Rest in bed, saline purges, lead and opium lotion.

\section{(3) FILARIAL LYMPHANGIECTASIS.}

\section{DEFINITION.}

A dilatation of the lymphatic ressels from obstruction due to Filaria bancrofti.

\section{SYMPTOMATOLOGY.}

It can occur anywhere but it is commonly found in the scrotum, spermatic cord and extremities.

When it occurs in the scrotum there is fever, redness, swelling, pain. After the ferer is over the scrotum may remain swollen and elastic. It may be smooth or rugose, or it may show vesicles which on rupturing show lymph or chyle will filarial embryos or very rarely eggs. The discharge of lymph may be so much in twenty-four hours as to cause marked exhaustion, the condition requiring operation.

An attack usually ends in diaphoresis.

When it occurs in the spermatic cord there is pain, swelling in the testes, cord and lower abdomen. Fever is less frequent than in the above cases. Later a swelling may appear along the cord which dis- 
appears when the patient lies down, and reappears when he is standing. The vessels are softer, less tortuous and more pouch-like than in true varicocele.

When it occurs in the leg there is fever, enlargement of glands, a soft swelling in the groin which disappears on the patient lying down and reappears on standing. It does not give any impulse on coughing.

\section{TREATMENT.}

Antiseptic dusting powder. Lse a suspensory bandage.

If the patient is exhausted from discharged lymph remore some of the distended tissue and tie the dilated ressels.

Elephantiasis or chyluria may result.

\section{(4) FILARIĀL ABSCESSES.}

These may occur in the thorax and the retroperitoneal lissue.

In the latter case the symptoms may be those of peritonitis.

The dead body of the parent worm is usually absorbed but if. it acts as an irritant an abscess may arise.

\section{(5) FILARIAL PHLEBECTASIS (F. varix).}

This consists of masses of enlarged superficial veins which may occur in the axilla, groin or other regions.

\section{(6) VARICOSE LYMPHATIC GLANDS.}

These occur chiefly in the groin, the inguinal and femoral glands, less often in the axillary and the lumbar (P.M.) regions.

The glands enlarge after each fever. 'They form soft elastic swellings, easily movable, at first covered with normal skin, but later they become fixed to the deep fascia.

The fluid from an exploratory puncture shows at times microfilariz, and always lymph or chyle. The glands are usually small, but they may become very large. They are seldom painful.

\section{TREATMENT.}

Remove if necessary.

The condition is difficult to eradicate completely.

Radium may be tried.

\section{(7) CHYLOUS EXTRAYASATIONS.}

Lymph or cliyle may be extravasated by dilated lymph or lacteal vessels into the-

(I) Urinary passage causing chyluria and lymphuria.

(2) Bowel passages causing chylous and lymphatic diarrhoea.

(3) Tunica vaginalis causing chylocele. 
(4) Peritoneum causing chylous ascites.

These four conditions will now be dealt with scriatim.

(I) Chyluria and Lymphuria.

The dilated lymphatic ressels of the urinary tract rupture owing to obstruction in or below the thoracic ducts and may discharge :-

Chyle, when the condition is called chyluria.

Chyle plus blood, hence hamato-chyluria.

Lymph, hence lymphuria.

Lymplı plus blood, hence haemato-lymphuria.

The only difference between lymph and chyle is that the latter, which is derived from the lacteals, contains 65 per cent. of fat, while lymph contains about 35 per cent. of fat.

\section{SYMPTOMATOLOGY.}

The onset is usually abrupt, with vague pains in the perineum, thighs and back, rarely ferer; more often the passage of milky or bloody urine is the first sign.

(A) The urine may pass out without efforl.

The urine generally clears, then milky or bloody urine occurs intermittently for years, less frequently it is continuous, and may last days, months, or even years. Exertion or emotion may increase the symptoms or precipitate another attack.

(B) The urine may clot into a semi-transparent, gelatinous, pinky mass in the bladder, causing severe pain and strangury.

There may be considerable pain in passing small clots, efforts lasting some hours.

On standing the urine separates into three layers:-

(I) A creamy upper layer of fat.

(2) A whitish fluid with a floating coagulum.

(3) A reddish sediment.

This sediment contains fat and oil globules, fatty granular matter, a coagulum of fibrin entangling microfilaria, free microfilaria, white corpuscles, especially lymph cells, red cells, and calcium oxalate crystals.

The specific gravity is Ior 5 to 1020.

The reaction is acid.

To estimate the fat, shake with ether, separate, evaporate the ether, and weigh the fat. This latter is from 0.6 to $3^{\circ} 3$ per cent.

Albumin is present, 0.6 to 0.9 per cent. in the resultant urine. If the fat is not in excess, it is lymphuria or hemato-lymphuria if blood is present.

The amount of fat and blood may vary in the same patient. 


\section{TREATMENT.}

This is symptomatic.

Rest in bed.

Salol and urotropine.

A non-irritating diet. Very little fat.

Gentle purgation.

Astringent injections and bladder washes are of little use.

Quinine should be given.

The X-rays can be tried.

If clots persist in the bladder, anashetize the patient, wash out the bladder with an aspirator or open and drain.

(2) Chylous and Lymph Diarrhoea.

This condition is rare.

The treatment is symptomatic.

(3) Chylocele or Lymphocele.

This condition is often preceded by fever and orchitis.

It may develop gradually, the tunica vaginalis filling up with an opaque fluid which may contain microfilaria.

The tumours never become very large, but they may require tapping.

(4) Chylous Ascites.

This is common in animals, but rare in man.

Two cases only have been recorded.

There has been traumatic rupture of the receptaculm chyli when it has been necessary to cut down and repair the wound.

In such cases the intestines are matted together from the exuded lymph, but there are no indications for removing the adhesions, as they are sterile and will resolve.

\section{DEFINITION.}

(8) ELEPHANTIASIS.

A chronic inflammatory hypertrophy induced by lymph stasis in fibrous connective tissue, occurring usually in the skin and subcutaneous tissues of the leg, scrotum, vulva, arm and breast.

\section{CLINICAL YARIETIES.}

\section{(1) Elephantiasis of the Leg.}

The lower extremities are affected in 97 per cent. of all cases.

\section{SYMPTOMATOLOGY.}

There are attacks of filarial lymphangitis, dermatitis and cellulitis. The leg swells and partially resolves after each attack. The limb permanently enlarges, the natural folds of parts, such as the anklejoint, are exaggerated, the dorsum of the foot becomes swollen, while 
in the deep sulci of the folds epithelial débris, sebum and sweat accumulate, resulting in a foul discharge, followed sometimes by ulceration.

The skin becomes dark, hard and thickened. Roughened bosses or warty elerations are common (verrucose variety).

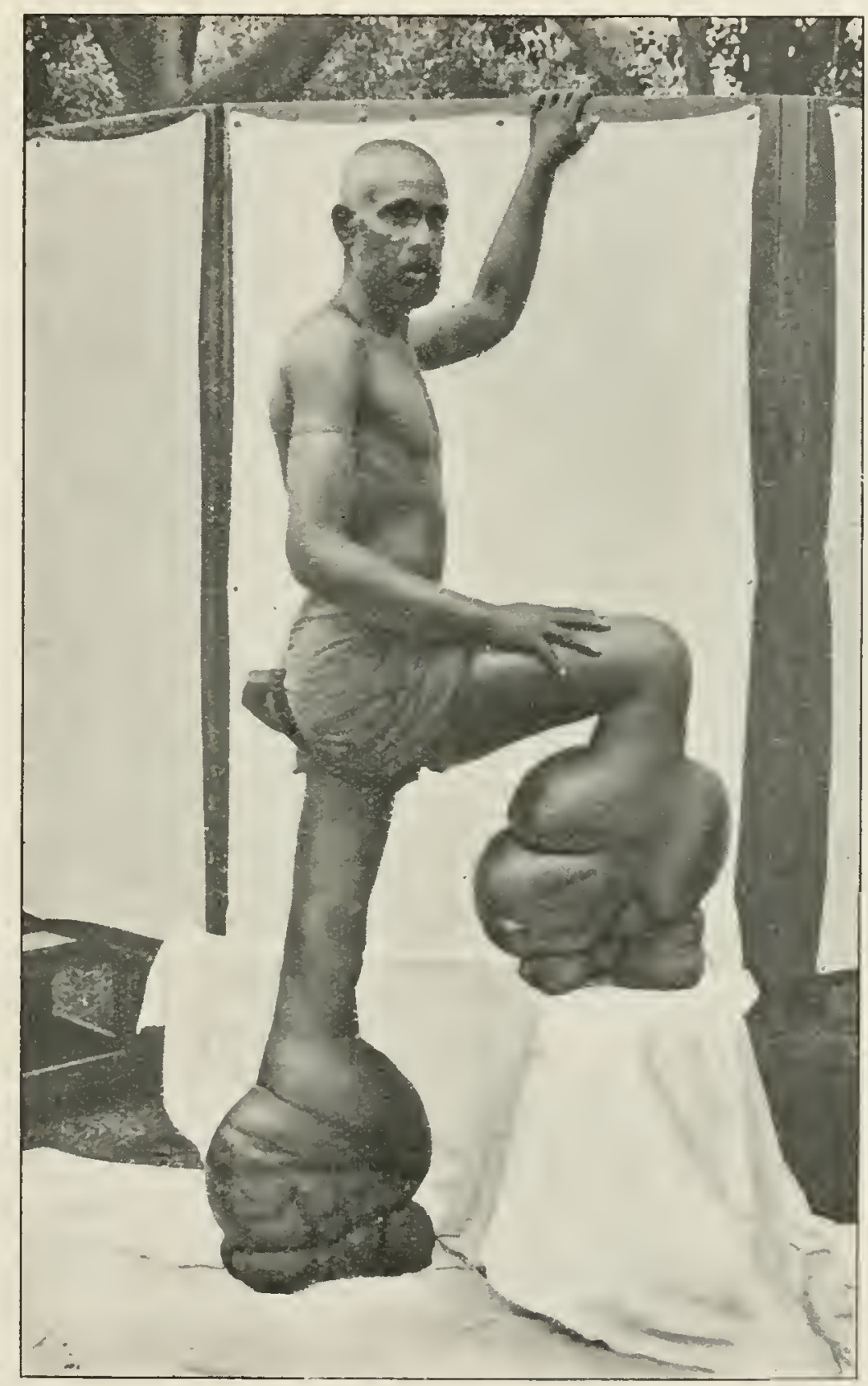

Elephantiasis of the legs. Before operation.

There is malnutrition of the part, followed by the shedding of hairs and nails, the latter being rough and thick.

Perspiration is diminished. The cutaneous sensibility is reduced. Ulcers may occur anywhere. The thigh may become implicated. The febrile attaclis may cease at any time, learing the leg enlarged even to immense proportions.

This condition may also result without any fever. 


\section{TREATMENT.}

Rest in bed. Methodical massage and bandaging, the latter with flannel or rubber. The pressure must be evenly distributed.

Avoid minor injuries. Highly laced boots are contraindicated.

Give fibrolysin or thiosinamin injections, 2 to 4 c.c. daily, into the gluteal region for three to six months, omitting every seventh day.

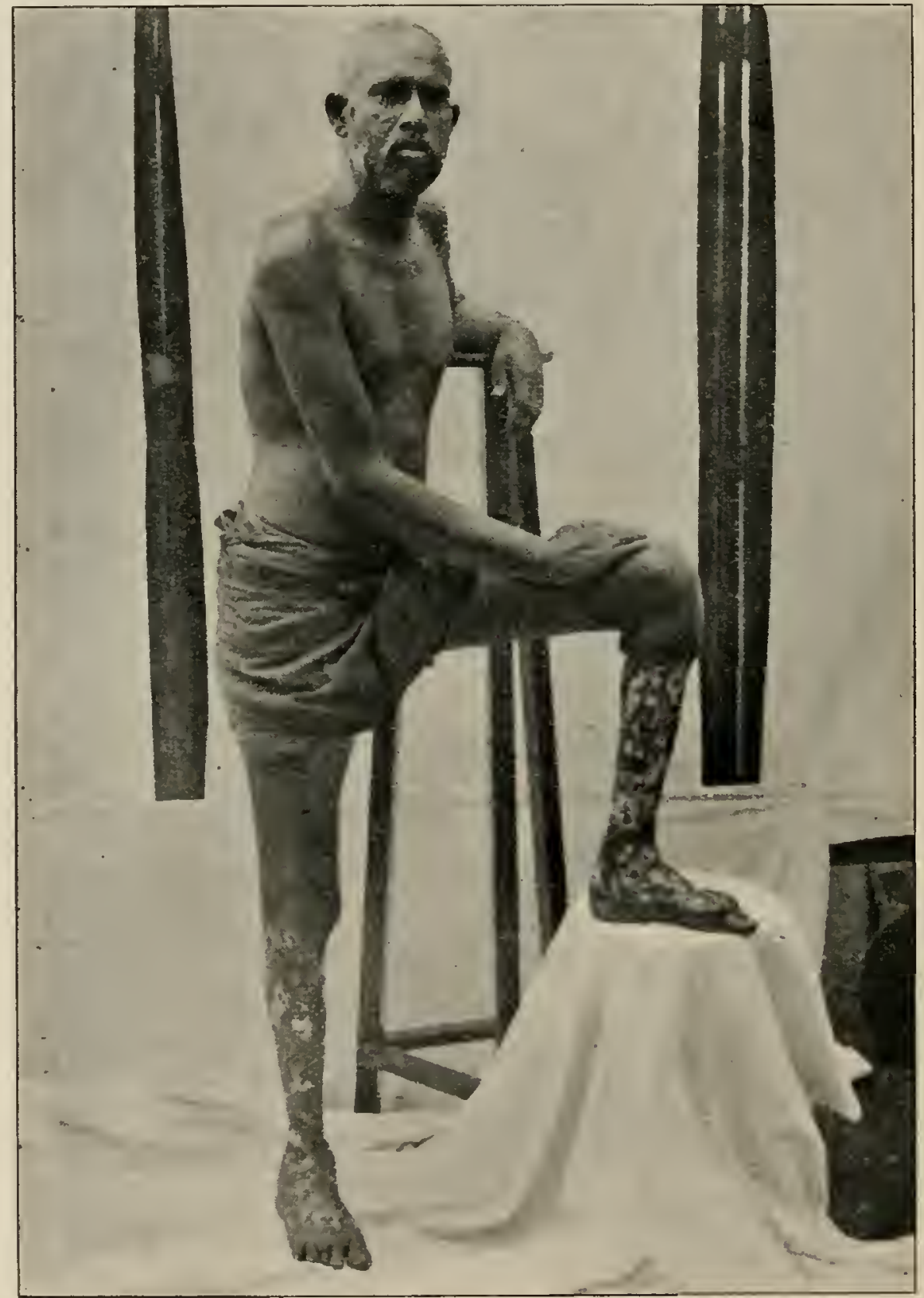

Elephantiasis of the legs. After operation.

Elevate the limb. Never expose it to the sun.

To remove horny masses, employ a spirit lotion of resorcin and salicylic acid.

After six months remove long strips of redundant skin and subcutaneous tissue. 
Poor results are oblained in acute, but better in chronic cases. Some have tapped the affected parts with Southey's tubes, others have cut out long square masses and have sutured up the cavity, while others have inserted fine threads to form the bases of new lymph channels (Lymphangioplasty).

All improvements in consequence of these measures are more or

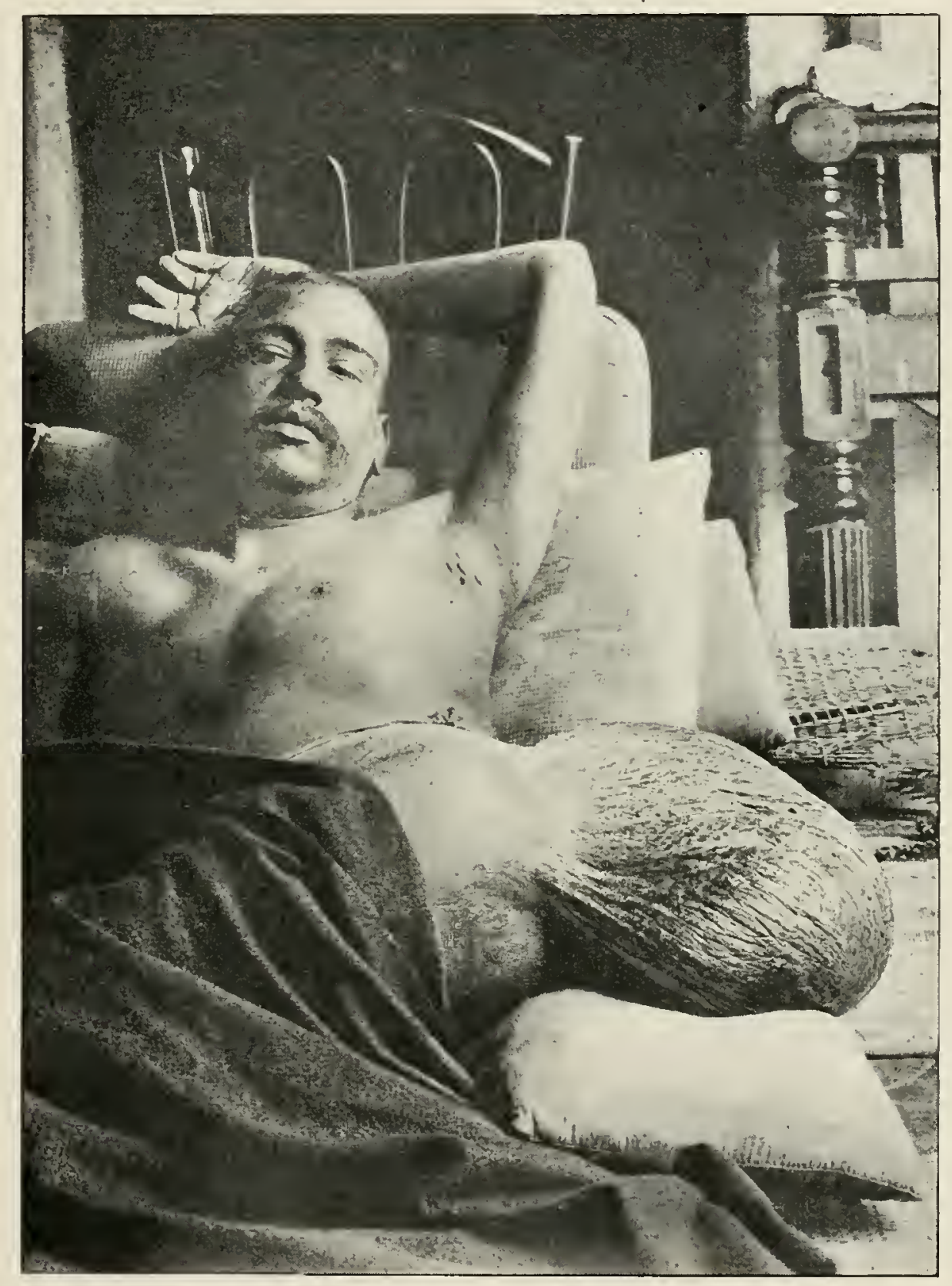

Elephantoid tumour of the buttock.

less slight and transitory. Final and permanent results of all surgical measures are disappointing.

\section{A QUERIED POINT.}

Some still ask: "Is elephantiasis arabum caused by the filaria, as these are not usually present in the blood or elsewhere?"

Sir Patrick Manson answers: "Yes," for the following reasons :- 
(1) The distribution of Filaria bancrofti and elephantiasis arabum correspond.

(2) Varix and elephantiasis arabum occur in the same districts and inclividuals.

(3) As varix is proved, elephantiasis arabum is inferred to be filárial.

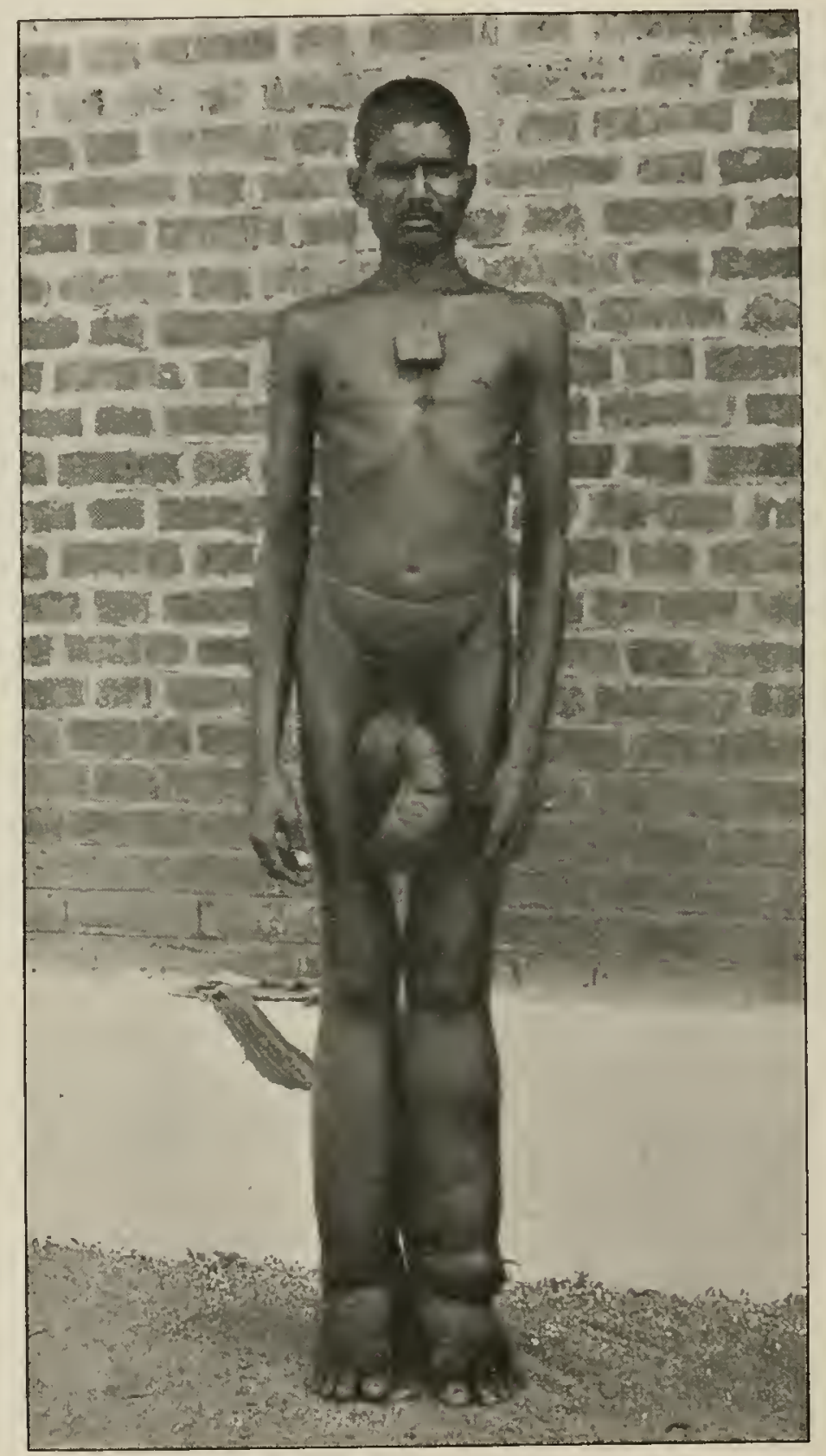

Elephantiasis of the prepuce (and legs).

(4) Lymph scrotum often terminates in elephantiasis scrotum.

(5) When the latter is removed there may follow elephantiasis of the leg.

(6) Elephantiasis and varix are both diseases of the lymphatics.

(7) Both are accompanied by recurring lymphangitis.

The worm is not found because it may have died after aborting the ova, causing embolism of the lymphatics. There then follow: $1 \mathrm{mph}$ stasis, reduced resistance of tissue, repeated mild sepsis and imperfect 
absorption of inflammatory products, the natural result being the hypertrophy of the limb.

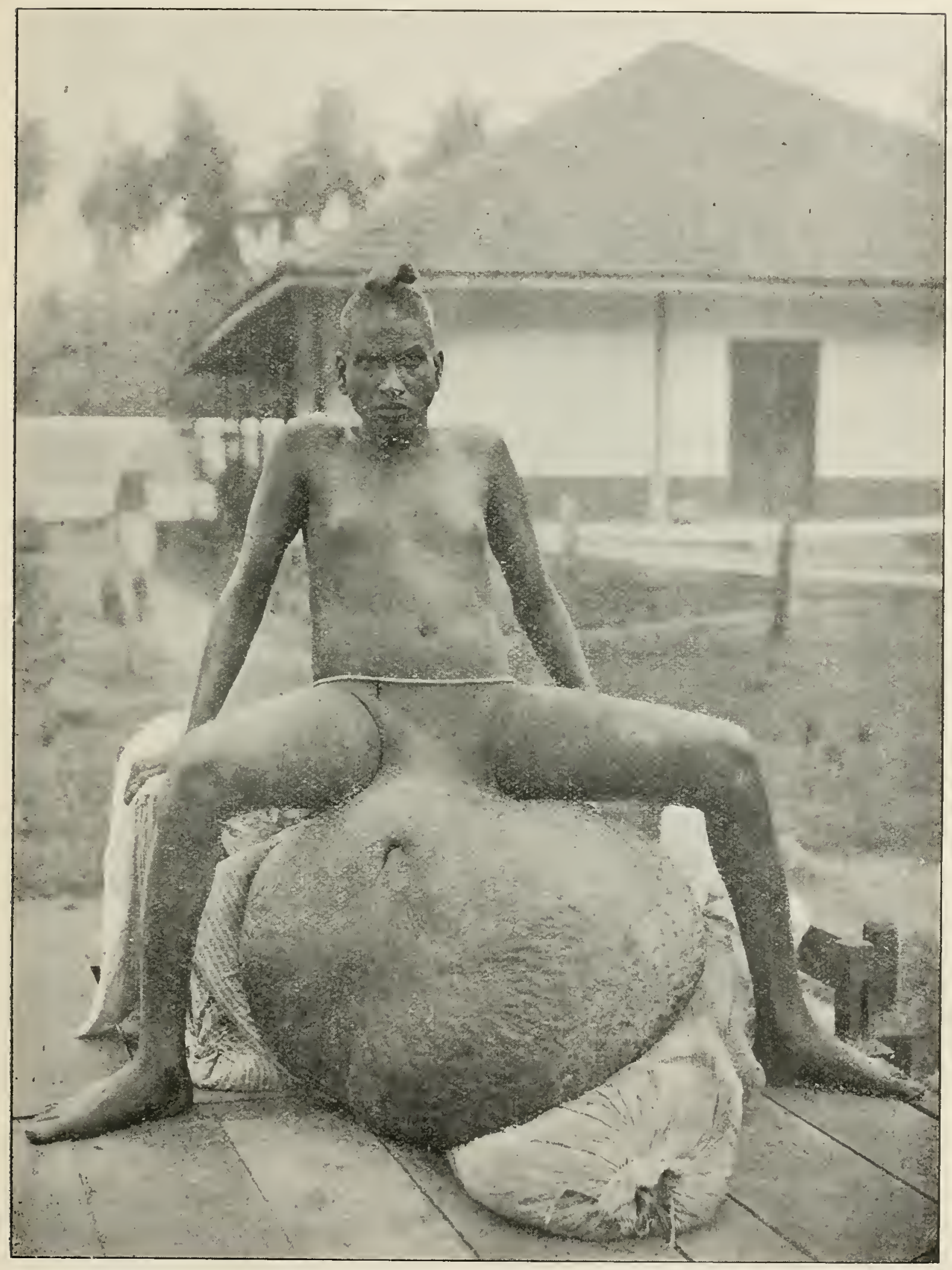

Elephantiasis scrotum. Twenty-eight years duration.

Elephantiasis may occur four to ten years after microfilaria have been seen in the blood. 
There may be sexually mature living filaria in the glands when none of the embryos have passed into the blood (Bahr).

(2) Elephantiasis of the Scrotum.

\section{SYMPTOMATOLOGY.}

A gradual enlargement with or without recurring erysipelatous attacks, erythema and fever. A large triangular mass is formed, base

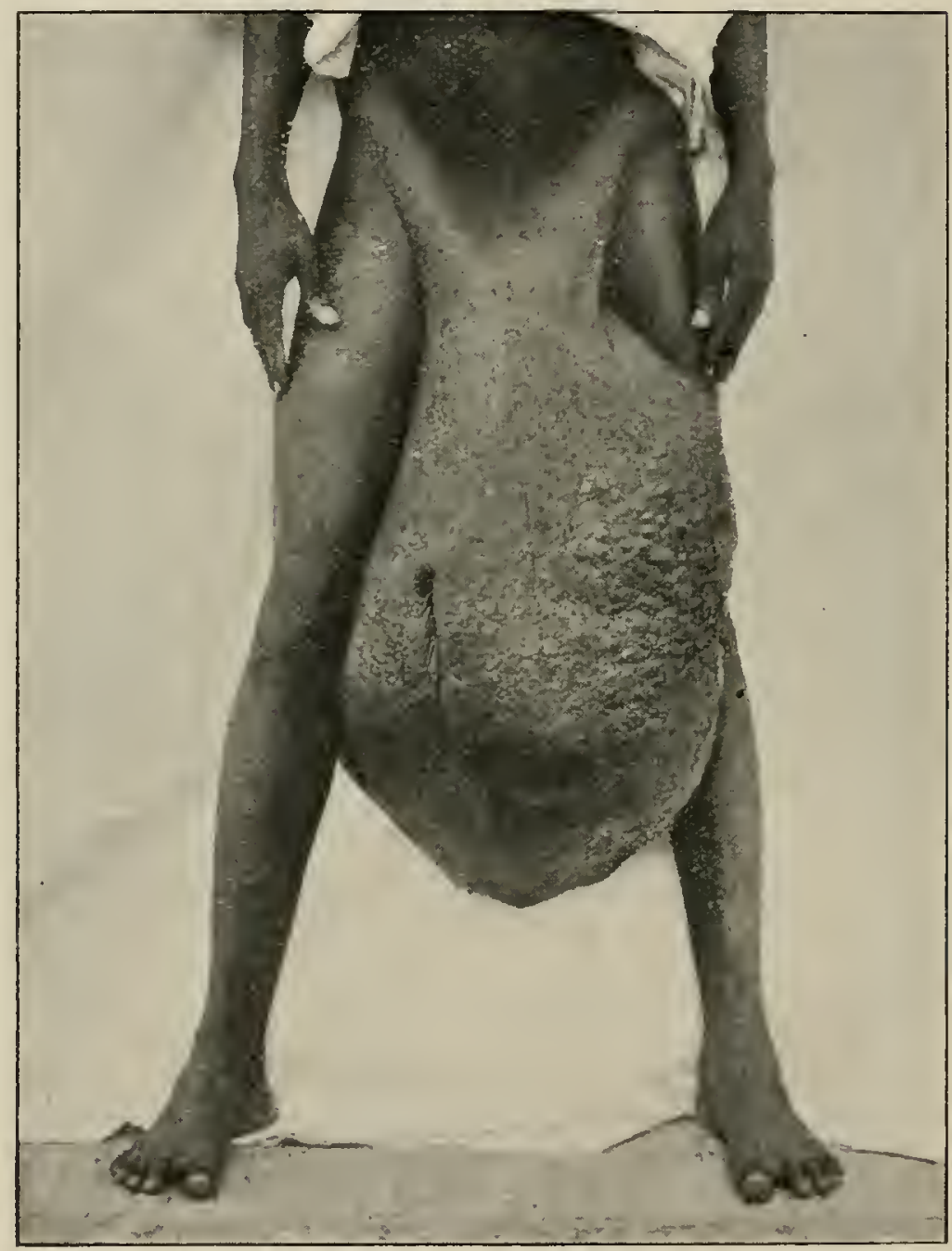

Elephantiasis. Scrotum.

downwards. On its anterior and superior aspect a canal is formed by an inverted prepuce leading to the penis.

This organ is buried in the tumour. The testicles are above and behind, and are usually surrounded by hydroceles.

The base of the tumour may be ulcerated.

A tumour has weighed 224 pounds; so to 20 pounds is common.

Tumours weighing 40 to $5^{\circ}$ pounds are not infrequent.

A localized groin tumour may weigh ro to 20 pounds.

Elephantiasis of the penis alone also occurs. 


\section{TREATMENT.}

Rest, especially during the acute phases.

Cleanliness. Avoid minor injuries. Massage.

Exercise the enlarged glands.

The removal of the mass has a mortality of 5 per cent. The operation is carried out as follows:-

Put the patient to bed for at least two days, clean the skin carefully, suspend the mass in order to drain it. Place a figure of eight elastic

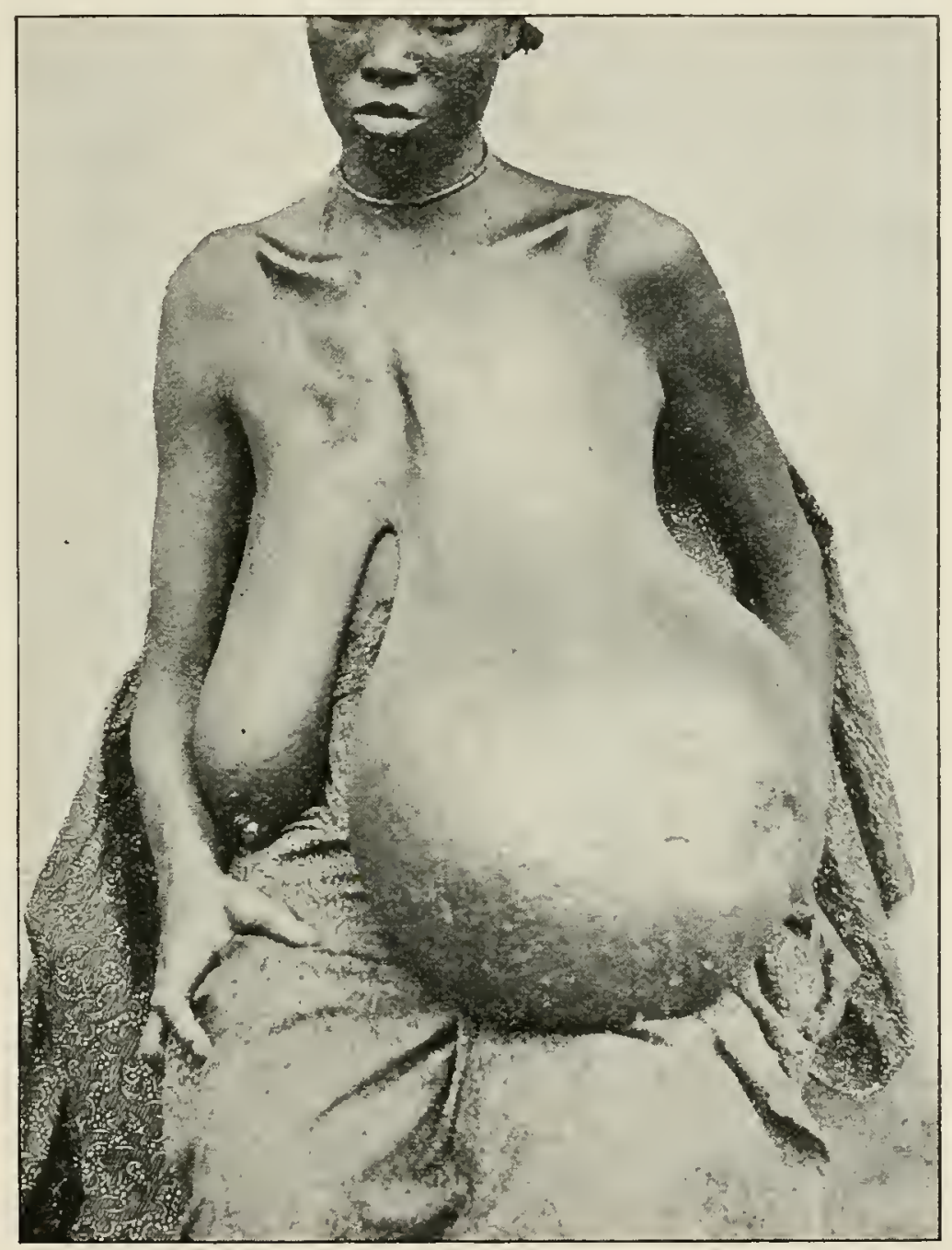

Elephantiasis. Breast. IVest Coast of Africa.

bandage around the pelvis and the neck of the tumour. Select good skin for the flaps if possible. Turn the tumour upwards and incise for flaps in front of the anus. Then cut out the anterior flaps from the pubes downwards in the mid-line, ligaturing the vessels as required. Cut down the penile canal with scissors and dissect out the testicles and penis. Pass a catheter as soon as possible; it will serve as a guide to the urethra and penis. Dissect out and separate the mass, loosen the tourniquet, and ligature any vessels. Puncture or otherwise open all hydroceles, invert the sac, remove the redundant tissue, and stitch 
the inverted sac around the testicles. Suture skin flaps over testicles and penis like the letter $\mathrm{T}$ or $\mathrm{Y}$ with the penis at the junction. Drain the lower portion of the wound. Make skin flaps for the penis if possible; if not possible, then cover the raw area with oil protective and allow it to granulate. Dress aseptically.

Skin grafting should be done early where it is necessary.

The functions of the organs are retained and restored.

It will facilitate repair to loosen the skin from the thighs with the

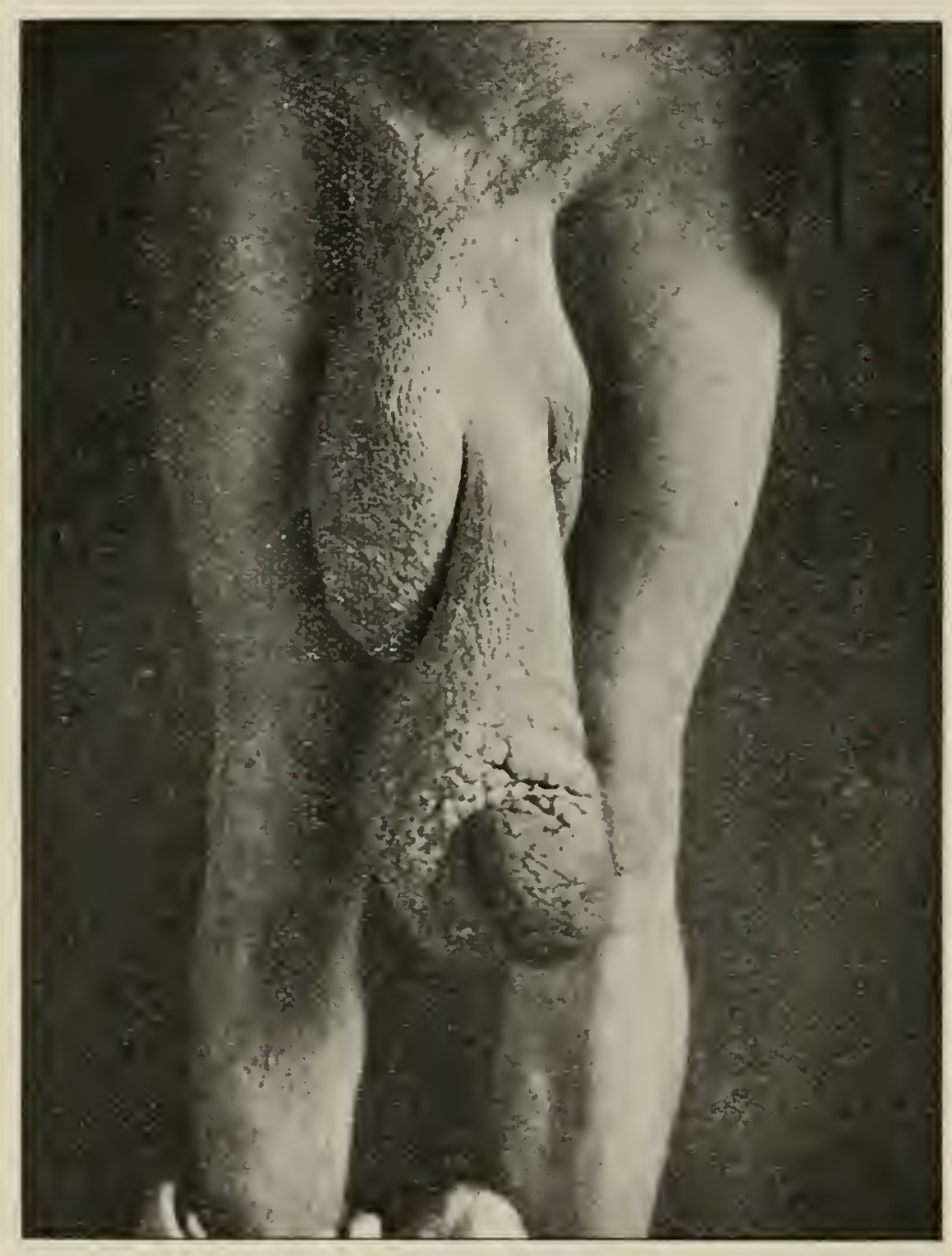

Elephantiasis. Penis. European some years after leaving Egypt, from photo. by Dr. Hunt.

fingers and draw it inwards if there is insufficient skin. Turn prepuce back to cover the anterior part of the penis. The skin around the penis, though inverted, is ustrally healthy and should be retained.

Complications are hæmorrhage and septiciemia, also sometimes difficulty in copulation, as the scar tissue may bind the penis down.

\section{(3) Elephantiasis of the Vulva.}

The labia majora and clitoris are sometimes affected, but it is a rare condition. 
These tumours may weigh from eight to twelve pounds.

Remove the mass aseptically.

(4) Elephantiasis of the Breast.

The condition is rare, but when present the tumour may grow to a large size. One or both breasts may be affected.

Remove the tumour.

\section{(5) Elephantiasis of the Arm.}

This is a rare condition, but may develop as in the leg, affecting one or both upper extremities. The muscles are not affected. Treat as for the leg.

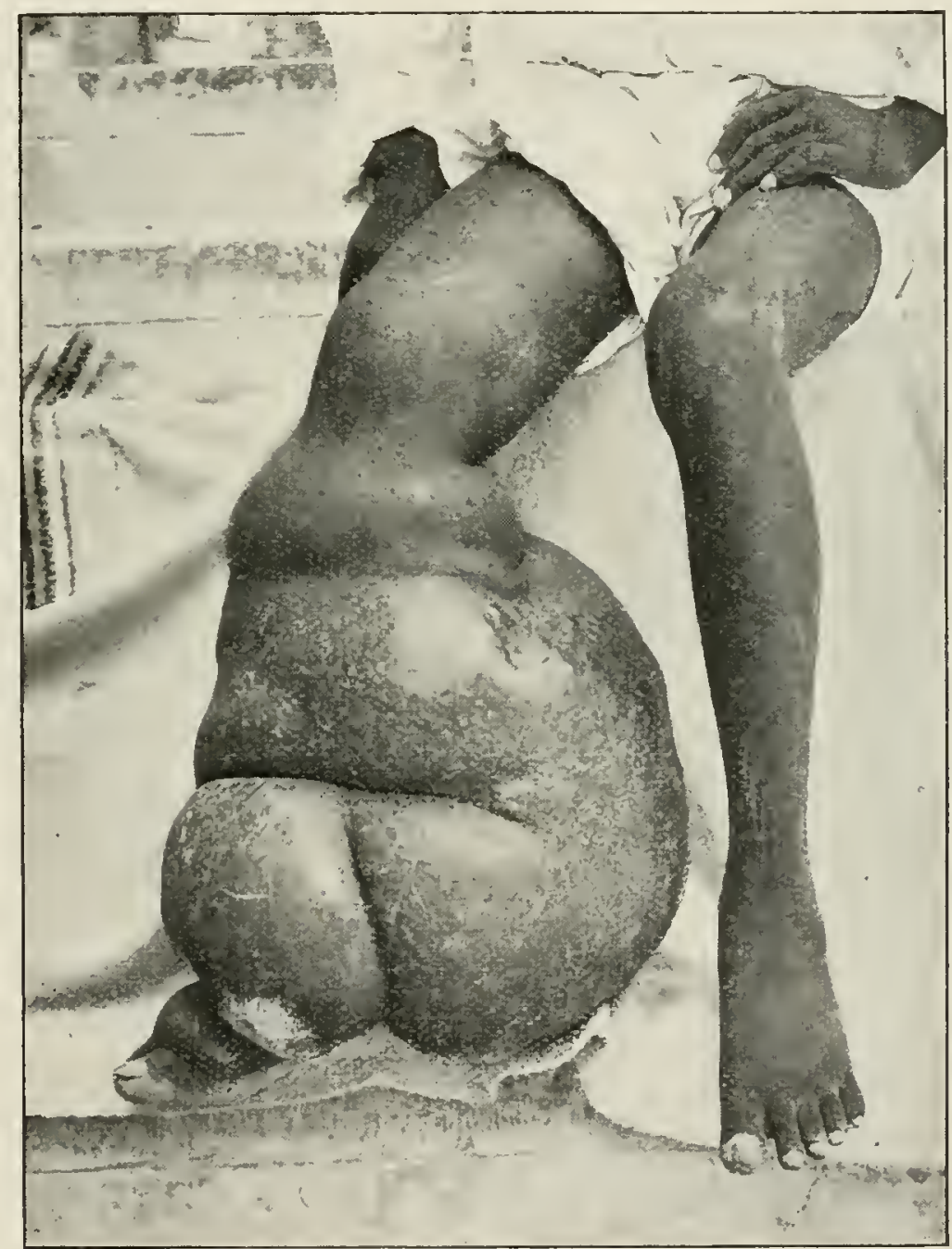

Elephantiasis. Leg. India.

(6) Elephantiasis of the Scalp.

The whole scalp becomes very much thickened, and numerous folds and deep furrows are formed.

Treatment is symptomatic.

(7) Elephantiasis of Localized Areas.

Pendulous masses of filarial origin are said to have been found. They may be single or multiple and should be removed. 
Double conjunctivitis discharging chyle has been recorded, filaria also being present (Lewis).

Acute synovitis of the knee associated with filaria is possible (Maitland).

\section{NOTES ON FILARIAL CARRIERS.}

CULEX FATIGANS (Culex= gnat).

This fly of the sub-family Culicina is one of the carriers of filaria, and possibly also of the dengue parasite.

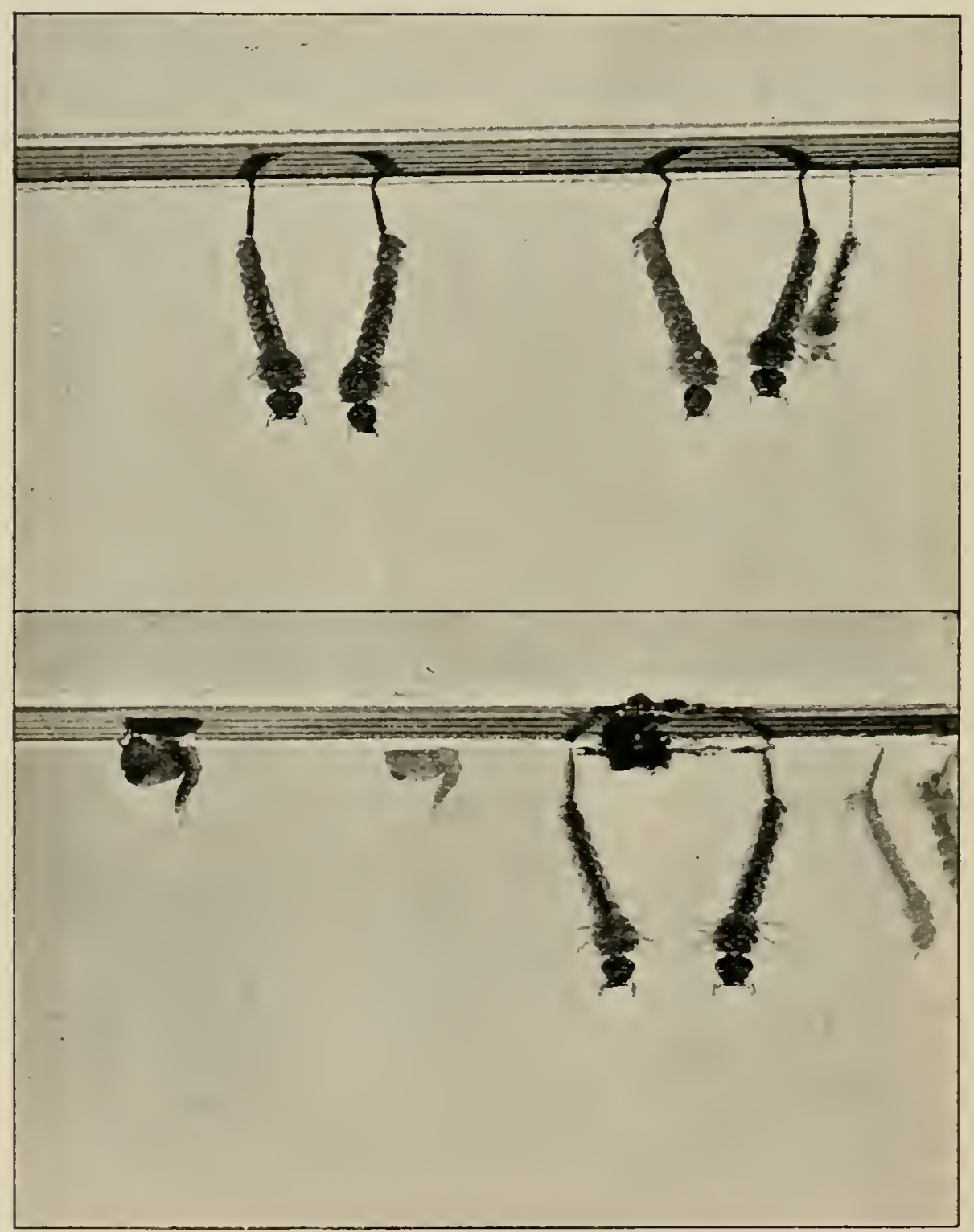

Larve and pupe of C. fatigans, Wied., photographed from life, about twice natural size.

\section{MORPHOLOGY.}

The palpi are longer than the proboscis in the male, and one-sixth the length of the proboscis in the female.

The larva has a breathing tube. The lateral thoracic and abdominal hairs are not feathered. The head is covered with narrow curved and upright forked scales on the dorsum. The scutellum is covered with 
narrow curved scales, and the wings with long, lateral, slender scales on the veins.

It is of a brownish colour, with a broad white and pale yellow band across each abdominal segment; the ventral surface is pale.

The legs are brown except for a small pale "knee" spot at the tip of the femora.

The larva may be found in any water near to human dwellings or in sewage. They have a long, slender breathing tube two and a half times the length and wice the breadth of the slender pointed tracheal gills.

The antenna carry a fan-like tuft of twenty long loairs.

Habits.-They breed in water-butts, drains, and any temporary

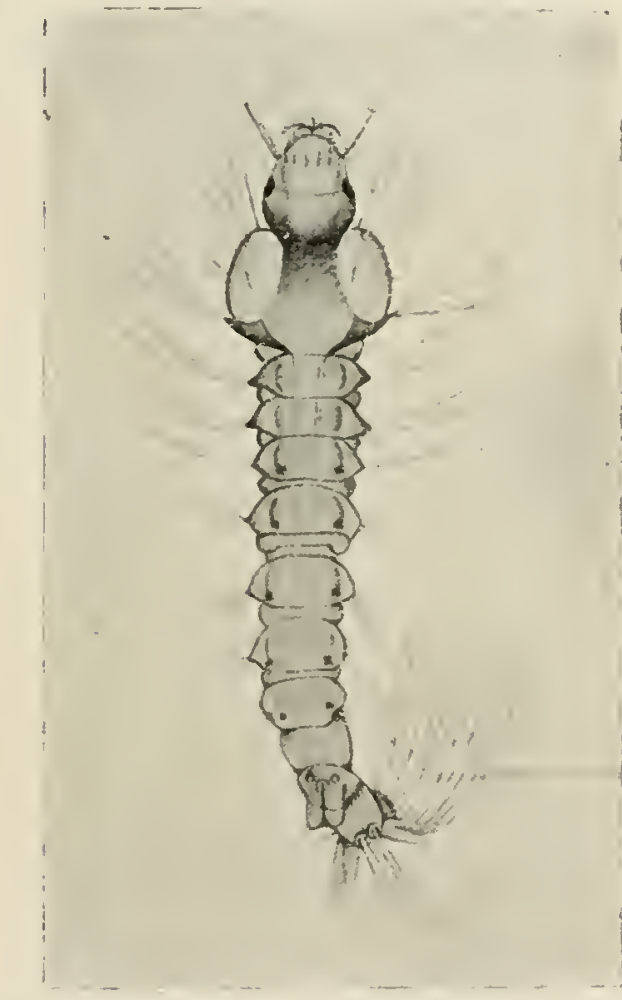

Larva of Anopheles maculipennis, Fabr. Enlarged. (After Grassi.)

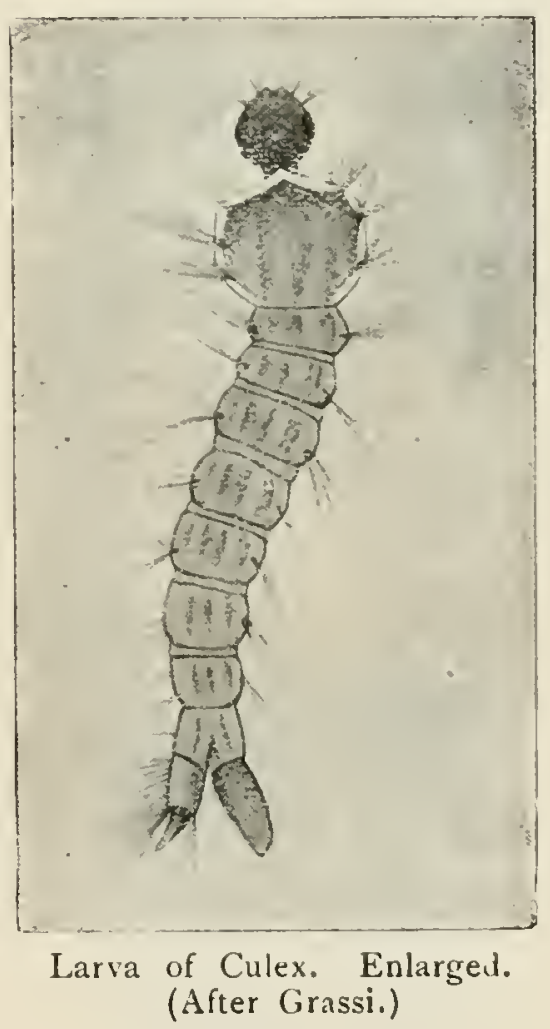

(After Grassi.)

collection of water. They are the commonest mosquitoes in the Tropics.

\section{DEFINITION.}

\section{I:OASIS.}

An inflammatory condition of the superficial connective tissue or conjunctiva caused by the Loa loa.

\section{DISTRIBUTION.}

In Africa, chiefly in Angola, Congo, and on the West Coast, where it is endemic. It is very common in Old Calabar and the Cameroons.

Perhaps it causes the Calabar swellings.

It has been imported to the West Indies. 
It seems to have a restricted geographical range.

Europeans are often infected.

\section{CAUSATIVE PARASITE.}

That a filaria has occurred in the eye has been known for four centuries. The Loa loa has been found in the ocular and palpebral conjunctiva, crossing the bridge of the nose, under the skin over the sterno-mastoid, scalp, fingers, penis, \&c.

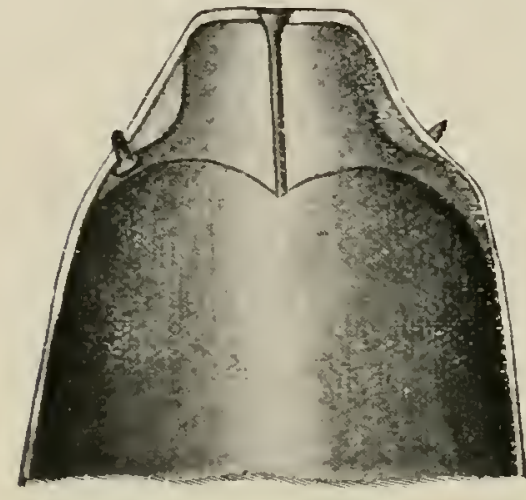

Loa loa: the anterior end of the male, magnified. (After R. Blanchard.)

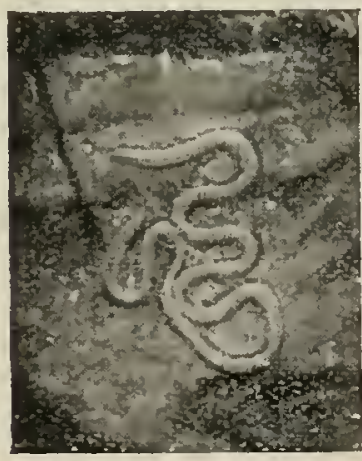

Loa loa in situ. Natural size. (Afier Fuilleborn and Rodenwaldt.)

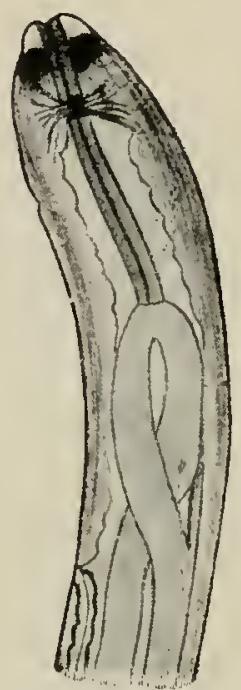

Loa loa: anterior portion of the female as far as vulva. (After Looss).

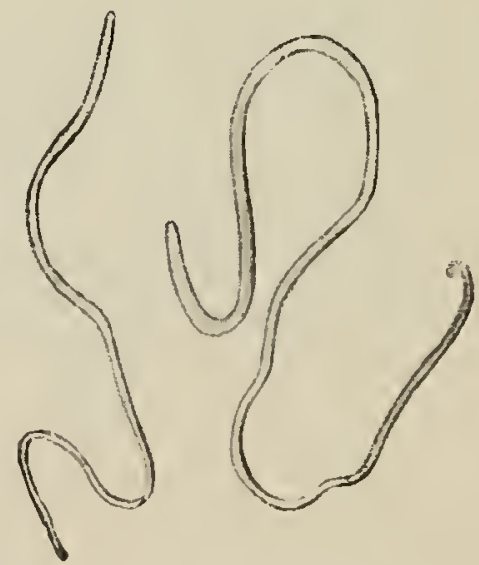

Loa loa: male on the left, female on the right. $\times 2$. (After Looss.)

It has rarely been found in the anterior chamber of the eye.

\section{MORPHOLOGY.}

The male is a thin, white, semi-transparent worm, 25 to $34 \mathrm{~mm}$. long by $0_{4}^{\circ} \mathrm{mm}$. thick, tapering at each extremity with a head like a truncated cone, and the tail incurved with a rounded tip.

It has transverse corrugations of the cuticle with a wavy outline, wide apart in the females and close in the males. 

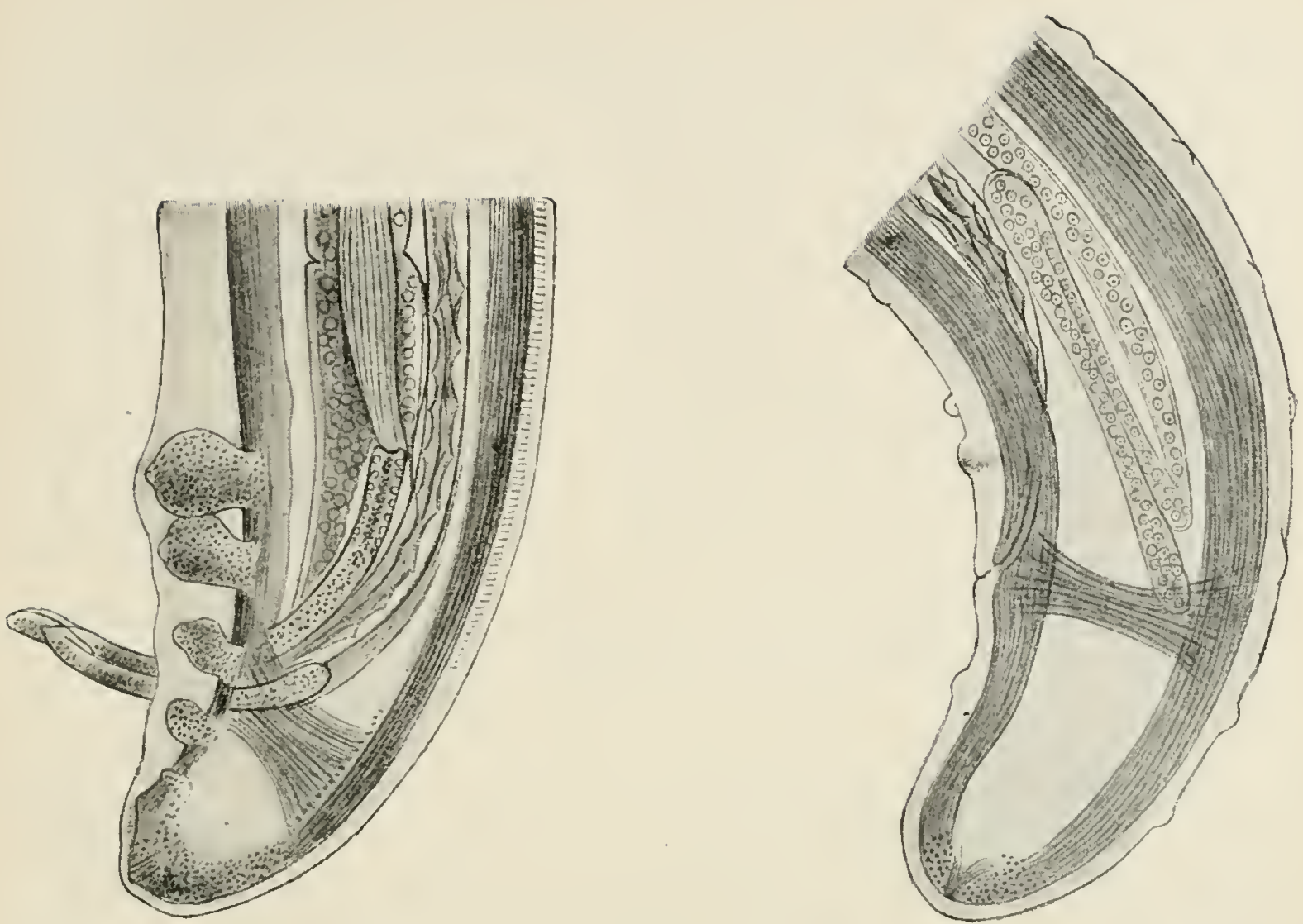

Loa loa: on the left, the hind end of a male; on the right, of a female. Note the cuticular bosses shown in the figure of the female. $\times 2 S_{5}$. (After Looss.)

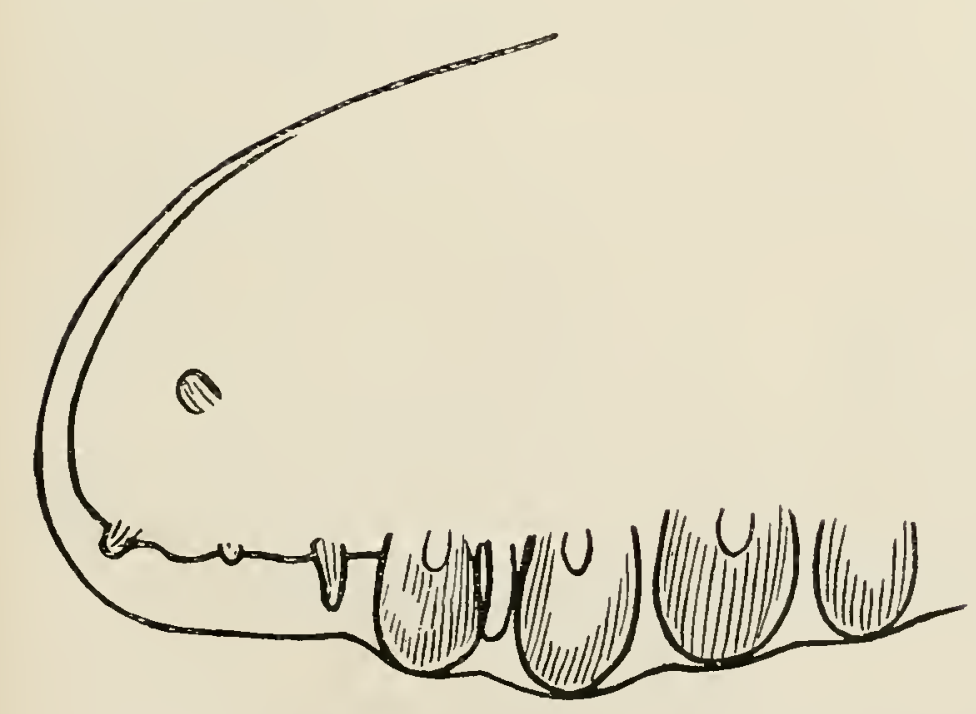

Loa loa: lateral view of tail of male showing papillee. (After Lane and Leiper.)
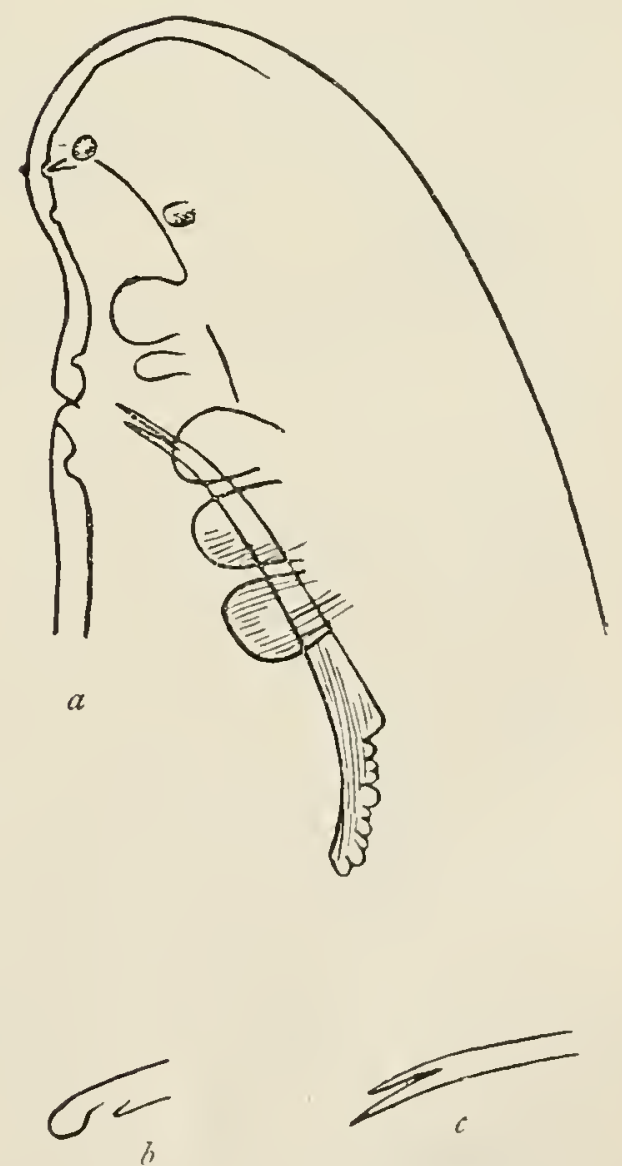

Loa loa. a, ventrolateral aspect of tail showing papillre and one spicule; $b$ and $c$, terminations of the two spicules. (After Leiper.) 
The anus is So $\mu$ from the tip of the tail. There are three pairs of pre-anal and wo pairs of post-anal papillæ.

The worm carries two spicules of unequal length; the larger is traversed by a fine canal (Penel). There are rounded bosses scattered over the cuticle averaging $30 \mu$ in height.

The mouth is terminal, small, unarmed, with a powerful muscular cone.

The oesophagus is short and is not bulbous.

The excretory pore is $0.65 \mathrm{~mm}$. from the anterior end.

The worm has a tubular testis and vas deferens filled with spherical spermatozoa, which organ terminates in a vesicula seminalis near to the base of the spicules.

The female is 44 to $63 \mathrm{~mm}$. long by $0_{4} 4 \mathrm{~mm}$. thick. The vulva is $2.5 \mathrm{~mm}$. from the anterior end.

\section{LIFE-HISTORY.}

The unsegmented egg is 32 by $17 \mu$ ।

The morula stage is 40 by $25 \mu$.

In viterus.

The rolled-up embryo is 50 by $25 \mu$.)

The embryo approaches the vulva, unrolls and elongates itself inside the egg membrane before it is extruded; the embryo then measures 250 by $6 \mu$. The embryo now escapes into the lymph stream and blood current, where it is known as Microfilaria diurna. In the circulation it enlarges to 298 by $7.5 \mu$, perhaps by osmosis. the microfilaria do not completely fill their sheath.

\section{HABITS.}

They are seen in the peripheral blood during the day, but not at night. They have no relation to sleep as the Microfilaria bancrofti. The curves are irregular; in the Mf. bancrofti they are graceful. The microfilarice when found in the peripheral blood are never in such numbers as the Mf. bancrofti.

The Mf. diurna requires from three to four years in the human body before it reaches maturity; they then live for fifteen years or more.

The immature forms are usually found in children, but the mature forms in adults.

The rate of progress in the human body is supposed to be one inch in two minutes. Warmth such as that from a fire or the direct sun will attract them to the surface.

The worm becomes cretified when dead.

\section{CARRIER.}

This is the Chrysops dimidiata and silacea (Leiper).

It is one of the Tabanidæ with hind tibias armed with spurs. 
It has a short proboscis, the antennae are longer than the head, the wings have a black band running across them, the eyes are golden green during life, marked with purplish spots. The wings are half open when resting.

The female bites very severely, causing pain and sometimes inflammation with odema.

The eggs are deposited upright on leaves and plant stems near water in a single layer. They are whitish or brown in colour.

The larve and pupce are not unlike those of the Tabanus.

The complete life-history is unknown.

\section{SYMPTOMATOLOGY.}

These are often nil. When the worm is under the conjunctiva there are piercing pains, uncertain vision, and swelling of the eyelids.

There may be some general itching.

They may cause painful swellings in various parts, but these are exceptional. The swellings do not suppurate and subside in a few days.

The blood may show a marked eosinophilia up to jo per cent. of the total leucocytes.

\section{TREATMENT.}

This is symptomatic.

\section{DEFINITION.}

\section{CALABAR SWELIINGS.}

Calabar swellings are smooth, temporary, slightly raised tumours about the head, ankles and feet, probably caused by Loa loa and other filariæ.

\section{DISTRIBUTION.}

On the West Coast of Africa only, especially about Southern Nigeria and the Cameroons.

\section{SYMPTOMATOLOGY.}

It is not certain as to how the lumps originate, but the most likely theories are :-

(I) That they are the result of the expulsion of microfilarize from the uterus of the parent worm. This would explain their evanescent nature (Manson).

(2) That they are due to the expulsion of the waste products of the worms.

The swellings are often painless, the size of half a goose's egg.

Some of them disappear quickly, others gradually during two to three days. 
There may be nausea, headache, slight pruritus, with some eosinophilia up to 50 per cent. of the total leucocytes (Stephens).

The swellings do not pit on pressure, neither do they suppurate.

They may develop singly, one coming as a former one declines.

\section{TREATMENT.}

Apply ichthyol ointment or 5 per cent. liq. plumbi to the swellings.

\section{VOLVULOSIS.}

The parasite, Onchocera volvulus, (F. volvulus) was first found in tumours of the scalp and chest of a negro on the Gold Coast by a medical missionar!.

\section{DISTRIBUTION.}

Sierra Leone, Gold Coast, Dahomey, Nigeria, Cameroons, and the Congo.

\section{MORPHOLOGY.}

The male $F$. volvulus is a whitish, filiform, sheathless embryo as found in the microfilarial form, $30 \mathrm{~mm}$. by $0^{*} 14 \mathrm{~mm}$., with slightly attenuated ends and transversely striated cuticle. The tail is strongly incurved. There are nine pairs of papillac about the anus (Brumpt).

It carries two unequal spicules $177 \mu$ and $82 \mu$ long.

They are found in the axillae, popliteal space, elbow, sub-occipital region and intercostal spaces. They are common about the chest.

They are not painful and are easily enucleated.

They do not suppurate. Eggs and embryos may be found in the swellings when incised.

The female is $65 \mathrm{~mm}$. by $0^{\circ} 36 \mathrm{~mm}$. The vulva is $0^{\circ} 76 \mathrm{~mm}$. from the anterior end.

\section{LIFE-HISTORY.}

The worm lies in a lymphatic vessel which becomes inflamed and causes a condensation of the adjacent connective tissue, in which become embedded both males and females. In a central lymphatic space the posterior end of the male and the anterior end of the female project, and in this way copulation takes place. Later the embryos are passed into this space. These latter are from $25^{\circ} \mu$ to $300 \mu$ long by $5 \mu$ to $6 \mu$ thick, with pointed tails and no sheath. The microfilaria have not been seen in the blood.

The adults may live for years in the human body.

Their complete history and carrier is not known.

\section{PATHOGENICITY.}

Lymphangitis and perilymphangitis without or with fever, leaving a small tumour. 


\section{DRACONTIASIS.}

\section{DISTRIBUTION.}

Tropical Africa. In the Sudan it is to be found at Gallabat, Gedaref, Upper White Nile, Kordofan and the Bahr-el-Ghazal Province. India, Persia, Turkestan, Arabia and a few places in South imerica where it has been carried from Africa, such as at British Guiana.

It is also present in the Fiji Islands.

It has been seen in Europe.

In parts of the Deccan at certain seasons $5^{0}$ per cent. are infected.

In parts of the West Coast at certain seasons 95 per cent. are infected.

The disease has been reported in horses, oxen, dogs, \&c.

\section{THE PARASITE.}

Dracunculus medinensis. (Guinea worm.)

Perhaps this was the fiery serpent of Moses as he knew the method of twisting the creature about a stick.

The host is man.

The intermediate host is the Cyclops quadricornis.

This worm is the largest of human filaria, its average length being about 40 inches with extremes of 12 to 70 inches.

The femate, of which we know the most, is a long, white worm with a hooked tail and a large uterus which occupies nearly the whole of the body cavity. The embryos when ready are evacuated from it at the anterior end near to the mouth. The uterus protrudes through the head parts, rupturing them (Leiper), and when about one inch of it has passed the sac fills with opaque fluid and ruptures. When a little water is added the motionless embryonic forms uncoil themselves and swim about.

\section{LIFE-HISTORY.}

The young are discharged into fresh water soon after the parent has broken the overlaying skin and before sepsis has set in. These young forms are taken into the body cavity of a cyclops where in about five weeks they will develop into mature larva. These are taken into the human stonach with drinking water or vegetables, where the $0^{\circ} 2$ per cent. of $\mathrm{HCl}$ kills the cyclops and at the same time stirs up the larva to such activity that they break through the cyclops at the mouth, anus and genital opening. They then swim about in the gastric fluid after which they penetrate the gastric wall and so reach the mesenteric connective tissue. Here they copulate and the males die. The preg- 


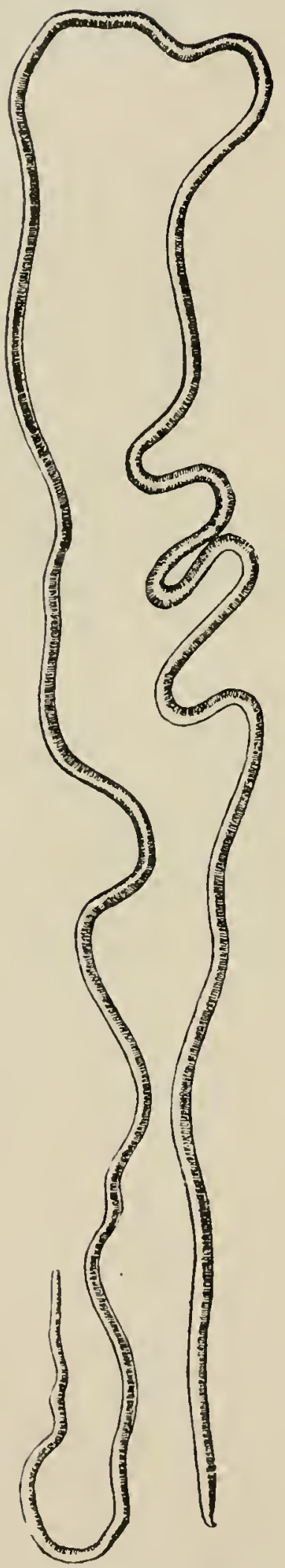

Guinea worm, Dracun. culus medinensis. (After Leuckart.)

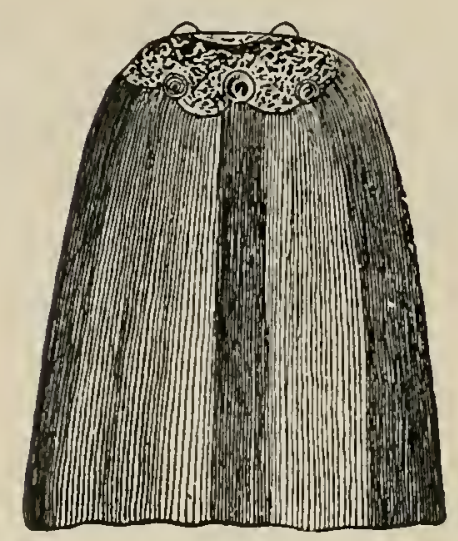

Anterior extremity of Guinea worm, showing dorsal and ven. iral lips, one lateral and two submedian papille and the lateıal line. (After Lenckart.)

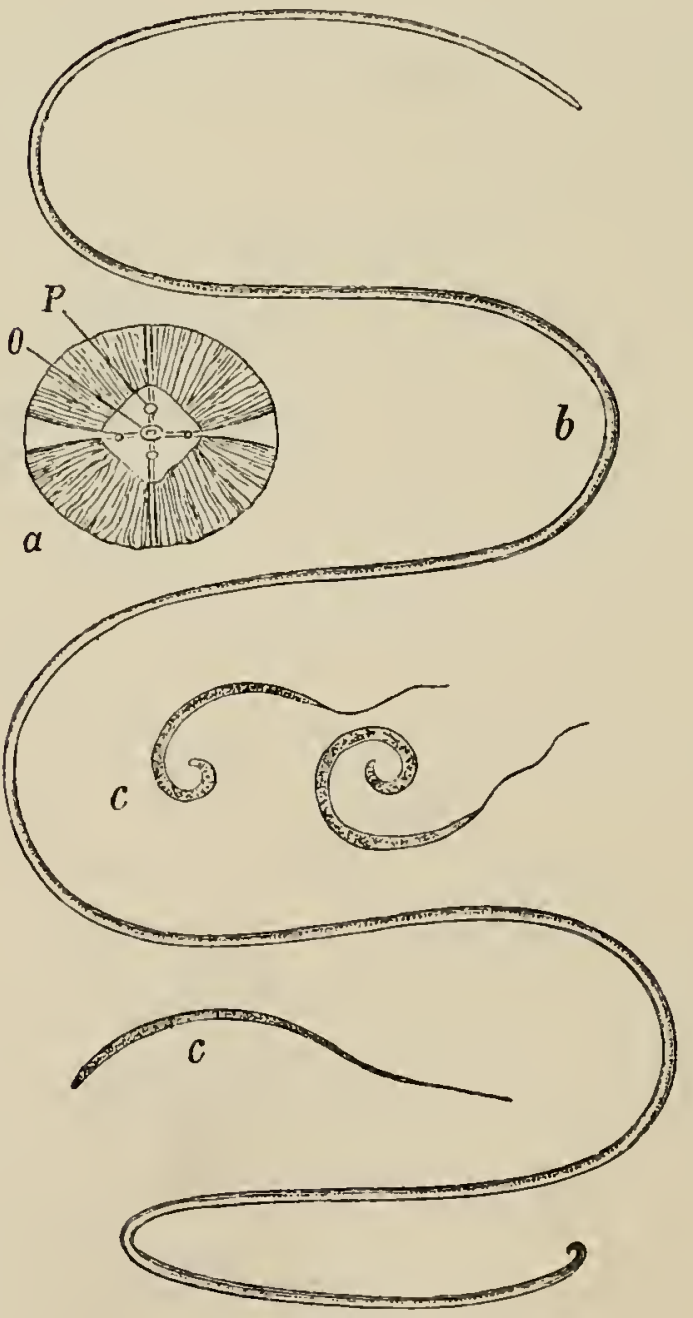

Diacunculus medinensis. $a$, anterior extremity seen end on; $O$, mouth; $P$, papilla ; $b$, female reduced more than half; $c$, larvæ enlarged. (After Claus.) 


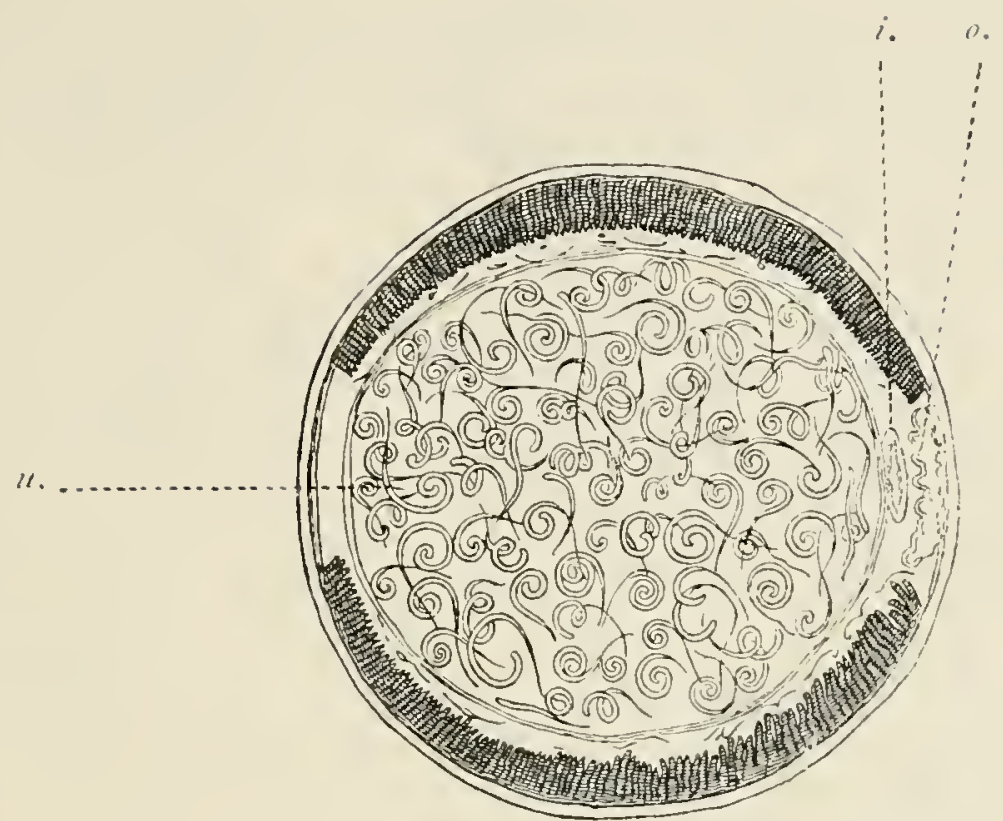

Transverse section of female Guinea worm; $u$, uterus containing embryos; $i$. intestinal canal!; o. ovary. (After Leuckart.)
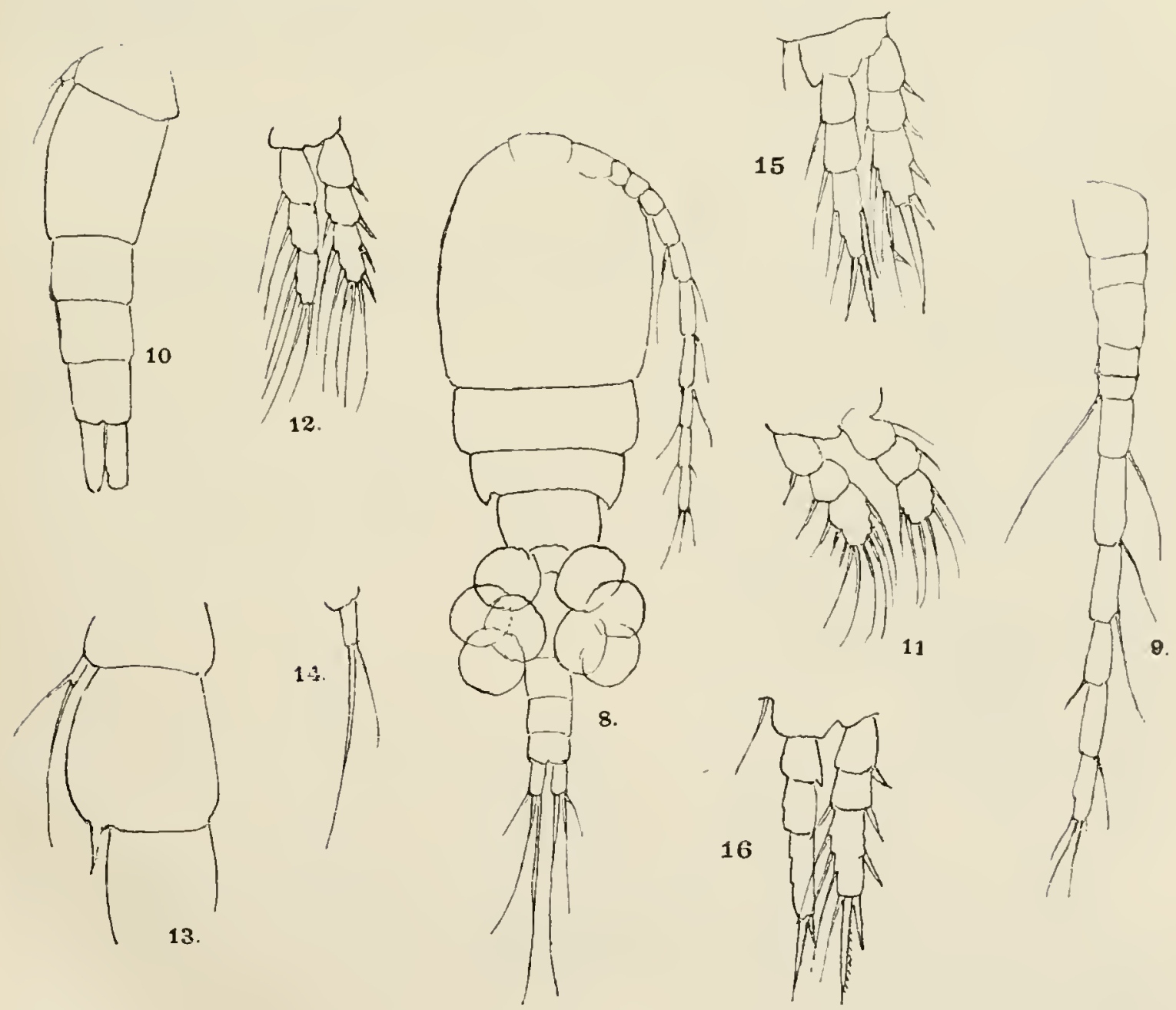

Cyclops virescens, 9.8 , Female, ventral view, $\times 120 ; 9$, anterior antennæ, $\times 240$; I0, urosome and last thoracic segment, $\times 240$; 11 , foot of first pair, $\times 320$; 12,15 , I6, foot of second, third and fourth pairs, $\times 240 ; 14$, foot of fifth pair, $\times 440$; I3, last thoracic segment and first segment of urosome of male, $\times 240$. 
nant female then seltles down ultimately in a place where it can deposit its young in or close to water. The parts chosen are:-

(r) The anlile or lower leg in $8_{5}$ per cent. of cases.

(2) The backs of water carriers in India (doubtful).

(3) Rarely about the knee joints.

The life span of a parent worm is about ten to twelve months.

Therefore, the embryos are discharged into the water where they must be taken up by an intermediate host, e.g., cyclops in which they develop into mature larva in five weeks but in which they can survive for 4 days. The larve are then swallowed by man, or as some assert, they enter the skin of the subject while he is bathing or standing in the water or mud. The mature worms form in about one year and they can persist for ten to twelve months during which time they discharge their embryos.

The life cycle is broken :--

(1) By death of the embryos as a result of sepsis of the round.

(2) By death of the embryos when there is no water near at hand.

(3) If no cyclops is present in the water or if the cyclops dies or is not ingested by man.

(4) If the larva are immature when ingested or fail to escape from the body cavity of the cyclops.

(5) If there are not the two sexes in the embryos and if the female is not impregnated while wandering through the connective tissue.

\section{PATHOLOGICAL LESION.}

A small bulla on the surface of the skin about the external malleolus marks the anterior end of the female worm. The bulla bursts, the uterus prolapses, the young are discharged and a portion of the worm is often extruded, which the natives twist around a stick in an attempt to extract it. This area is very liable to become septic.

\section{SYMPTOMATOLOGY.}

An ulcer appears as above. Urticarial eruptions are not uncommon.

Rigors and fever may occur. Eosinophilia may go up to 13 per cent.

Synovitis and arthritis are rare. The writer extracted one from over the internal tuberosity of the knee after synovitis. The synovitis trouble cleared up when the worm had been removed.

\section{TREATMENT.}

The worm lies zig-zag and even though much may be removed the posterior end may have remained in the same place, the worm has only been straightened in the majority of attempts to remove it by twisting it around a stick. 
Douche the part, where the uterus presents, with cold water, when it will empty its uterus. The worm will then be absorbed, emerge or in any case it will be easier to extract. The douching may be required several times daily over two to three weeks.

If the worm is visible or palpable under the skin several incisions can be made and the worm removed in parts.

Some inject perchloride solution, I in I,ooo, but they are painful and not very successful.

The native method is often one of straightening and not of extracting.

Treat the ulcer on ordinary lines.

\section{PROPHYLAXIS.}

Carefully boil and filter all drinking water. A fine handkerchief is sufficient.

Steam should be passed into wells to kill the cyclops present.

Aroid drinking water used for bathing purposes.

Improved sanitation will cause its disappearance.

\section{TRICHOCEPHALIASIS.}

This is an infection of the intestine by Trichocephalus trichiurus or whipworm.

It is cosmopolitan in its distribution.

\section{THE PARASITE.}

It belongs to the family Trichotrachelidx.

The male is 40 to $45 \mathrm{~mm}$. long with a spirally coiled posterior end.

Its spicule, 2 to $5 \mathrm{~mm}$. long, lies in a retractile pouch.

The female is a little longer than the male.

The egg, unsegmented as it appears in the freces, is brown, oval, thick-shelled with a pale area at each pole. Several months are necessary before the embryo matures.

No intermediate host is necessary.

The embryo is ingested inside its shell taken with either food or drink, human hands or flies acting as

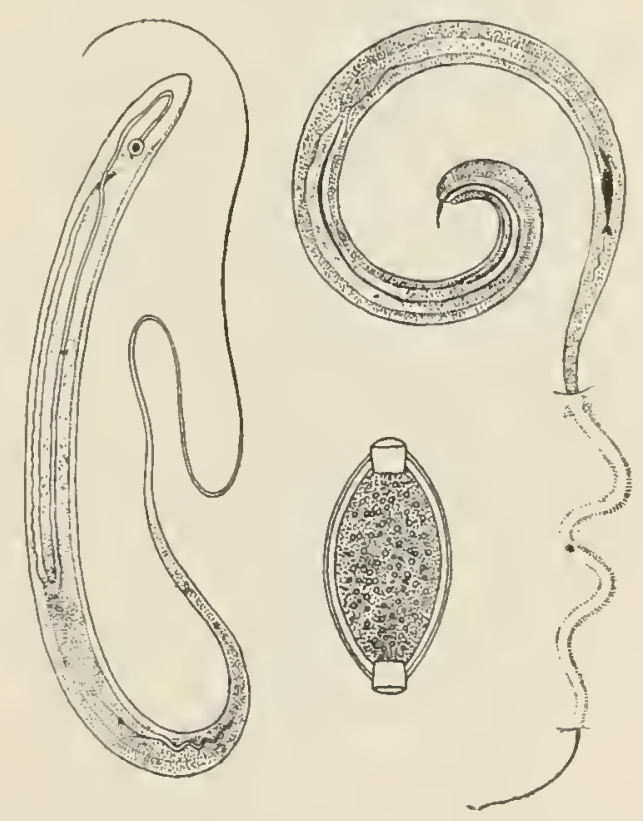

Trichuris trachinra: on the left, male; on the right, female with the anterior extremity embedded in the mucous membrane of the intestine; below, egg. carriers. The gastric juice dissolves the shell and in about four weeks the embryo reaches maturity in the intestine.

The worm can live for years in moist earth. 


\section{PATHOGENICITY.}

The worm is usually harmless although some affirm that it causes appendicitis at times; this, however, is very cloubtful.

Diarrhoa in children living in insanitary conditions is more common.

Perhaps the head is buried in the mucous membrane which makes their ejection more difficult and thus disposing to bacterial infection at the site of the wound. No ecchrmoses are seen at the point of fixation.

The eggs can infest utensils from dust long dried and hence the infection is not always due to frecal contamination.

\section{TREATMENT.}

When there are symptoms present treat as for ankylostomiasis.

\section{TRICHINIASIS.}

This is an infection of the gastro-intestinal and muscular system by the nematode Triohinella spiralis of the family Trichinellida.

The condition caused by the infection is of considerable importance as shown by the fact that in llamburg alone 100 inspectors were employed to examine pork, the carcases numbering from 25,000 to 30,000 per month. This work cost $\$ 25,000$ annually.

\section{DISTRIBUTION.}

It is common in Germany and in some parts of America, India and China. It is rare in England and France.

\section{THE PARASITE. LIFE-HISTORY, \&C.}

Two mammalian hosts are necessary for its development which need not be of different species, but no intermediate host is required.

The cysts are found usually in human or pig's muscle and appear as minute white specks which are the encysted larre. Their long axis lies in that of the muscle fibre. They measure foo by $250 \mu$.

The cyst membrane is formed by inflamed connective tissue.

The larve may thus live for years but may become calcified and die.

They are chiefly found in the muscles of the diaphragm, larynx, tongue, abdominal and intercostal regions.

These cysts are ingested alive with the muscle fibre unless severe cooking has destroyed them. The gastric juice dissolves the cyst wall and the parasites emerge and pass to the intestine where they become sexually mature, reaching 1 to $3 \mathrm{~mm}$. in length. Copulation takes place about two days after the cysts have been ingested, after which the males die. The females grow, penetrate the mucous membrane of the bowel and reach the lymph spaces and there deposit living larra.

One adult female may give rise to i, 500 larræ. 
The female lives from about five to seven weeks doing this.

The larvæ are carried by the blood and lymph stream all over the
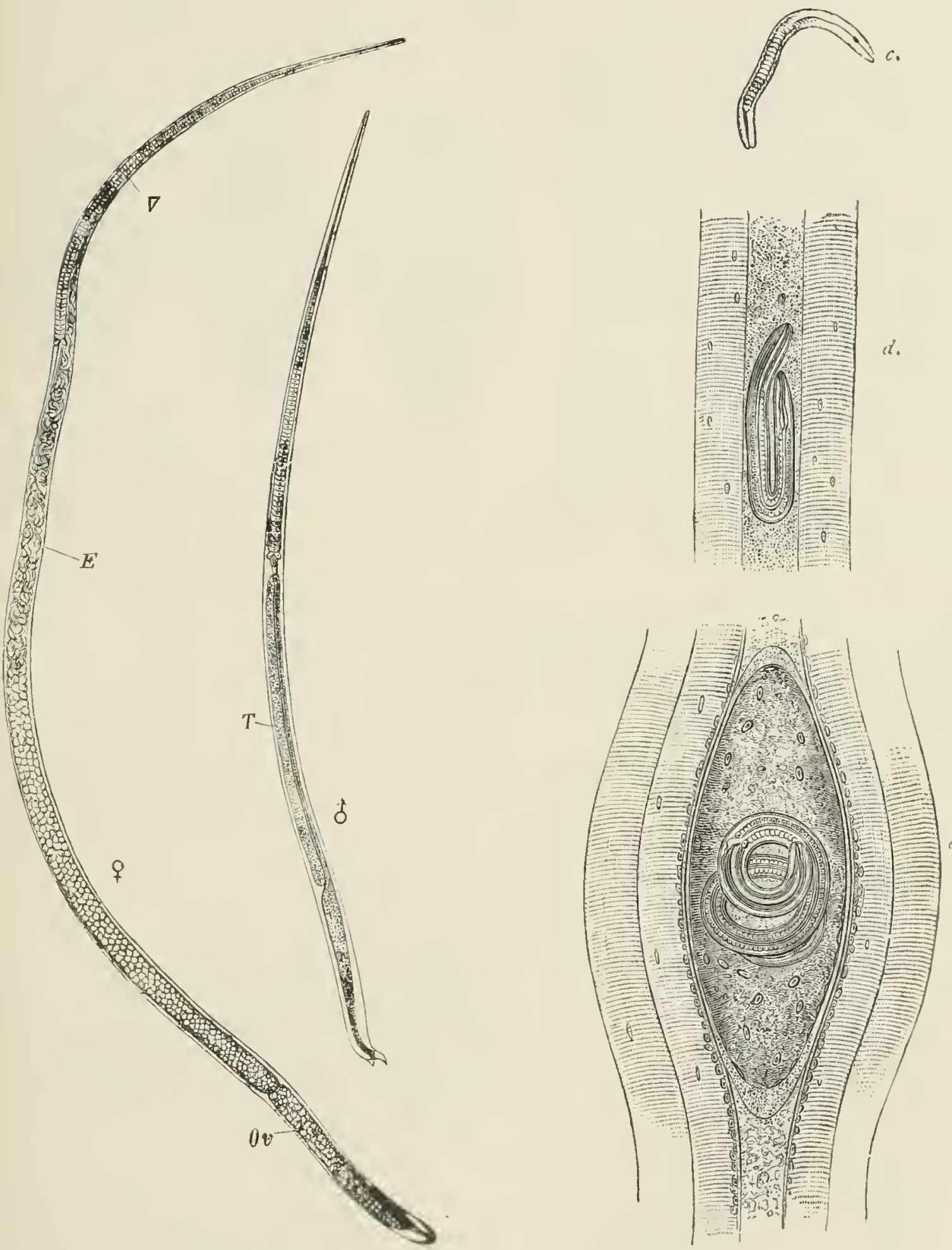

Trichinella spiralis. F, mature female: E. embryos; $I$, vulva ; Ou, ovary. o, mature male: $T$, testes. $c$ newly born larva. $d$. larva in the muscles. encapsuled larva in the muscles. Magnified. (After Claus.)

body. They then leave the capillaries, burrow their way into the tissues and in from nine to ten days become encapsuled in the muscles.

The muscle fibre infected degenerates, becomes inflamed, and a cyst is formed from the inflammatory tissue. 
The cyst resists a temperature of $80^{\circ} \mathrm{C}$. or smoking, pickling and freezing.

The adult worm has an anterior part thin and whip-like, the

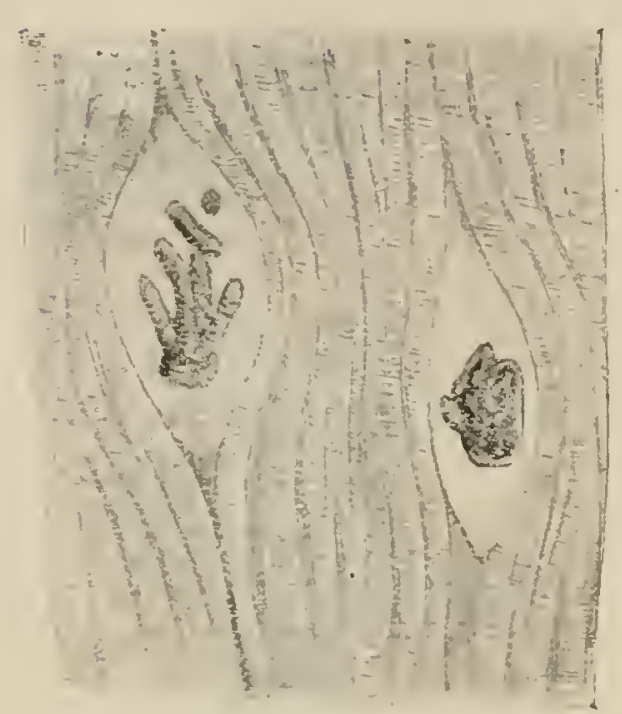

Calcified Trichintia in the muscular system of a pig; the capsu'es are not calcified. (After Ostertag.)
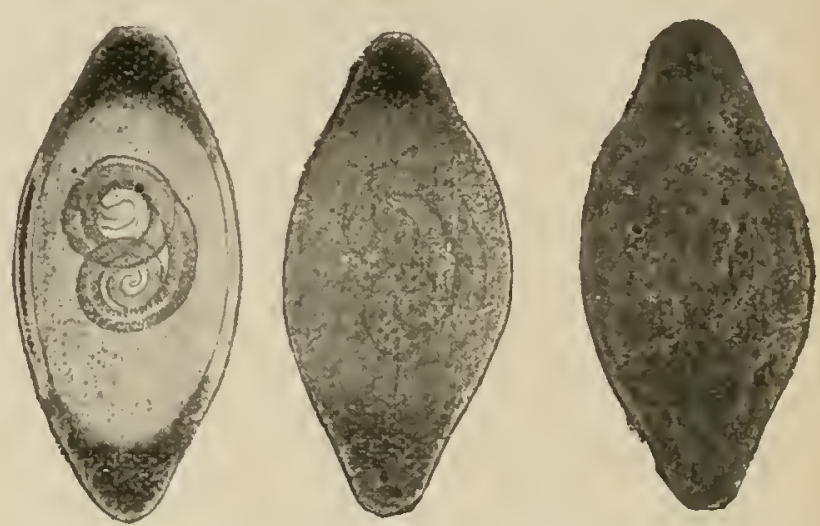

Vaisous phases of the calcification of Trichinella of the muscles, which starts at the poles of the capsule.

posterior is thick and carries the genitalia, but there is not the abrupt transition as in the Trichocephalus.

There are two cone-like appendages. The female vulva is placed far forwards.

This worm is really a parasite of the black rat (Epimys rattus) and the sewer rat (Epimys norvegicus).

\section{SYMPTOMATOLOGY.}

There are: irregular fever not unlike typhoid at times, some general abdominal pain and tenderness with agonizing pains as the larva burrow through the muscle, which pain lasts intermittently for from five to seven weeks. When the muscles of respiration, mastication or deglutition are invaded the essential functions are rendered difficult.

When the female has finished depositing embryos and all have become encysted the symptoms clear up.

The eosinophilia is very high. The larve are not found in the blood.

There is often diarrhœa, raised temperature and cedematous swellings.

Fatal peritonitis may occur in a heavy infection.

\section{TREATMENT.}

Anthelmintics are useless.

The treatment is symptomatic. 


\section{PROPHXLAXIS.}

All rats near to pigsties must be destroyed.

Pigs must be kept away from the offal of slaughter houses.

Meat should be cooked at a temperature of $62^{\circ}$ to $70^{\circ} \mathrm{C}$. in all parts.

In some sausages the pork is uncooked. Such pork should be examined, passed and marked by an inspector qualified for the work. The parts affected chiefly are the tongue, laryngeal muscles, diaphragm and abdominal muscles. To search for the cysts press a small portion of the muscle fibre between two translucent glasses or plates when the larva are more readily seen.

The larva are not killed by freezing, and hence frozen meat may be infected.

\section{DEFINITION.}

\section{ASCARIASIS.}

This is an infection usually with the Iscaris lumbricoides or round worm, rarely with the Toxascaris canis or Belascaris.

The two latter are smaller and are more commonly found in cats and dogs.

\section{DISTRIBUTION.}

In all tropical and sub-tropical climates.

The A. lumbricoides is parasitic only in man. When the eggs are passed externally their development is limited until re-introduced into man.

\section{THE PARASITE. LIFE-HISTORY.}

The eggs are laid in the small intestine and measure from $50 \mu$ to $70 \mu$ by $40 \mu$ to $50 \mu$. They have a thick yellow transparent shell coated
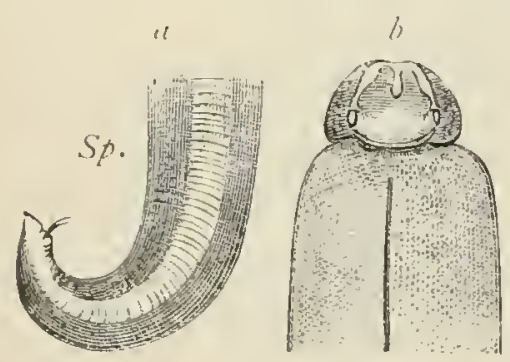

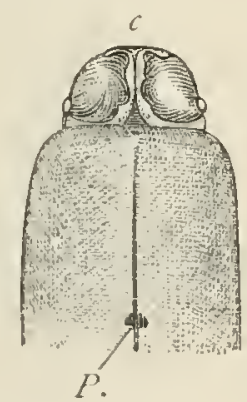

Ascaris lumbricozdes. $a$, posterior extremity of the male with the spicules protruding from the orifice of the cloaca $(S p) ;$.$b , anterior extremity$ from the dorsal surface, the two lobes of the pulp of the lip separated by the "saddle"; $c$, anterior extremity from the ventral surface; $P$., excretory pore. (From Claus.)

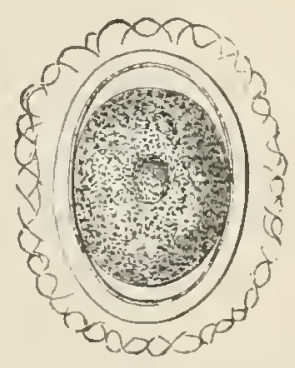

Ovum of Asiarls lumbricoides, with shell and albuminous envelope. $400 / 1$.

irregularly with albumin and stained a brownish rellow by facal material. This outer covering may be removed during the passage through the intestine. The shell affords high resistance to changes in temperature and moisture. The contents are granular but do not divide into nuclei as in the egg of the ankylostome. 

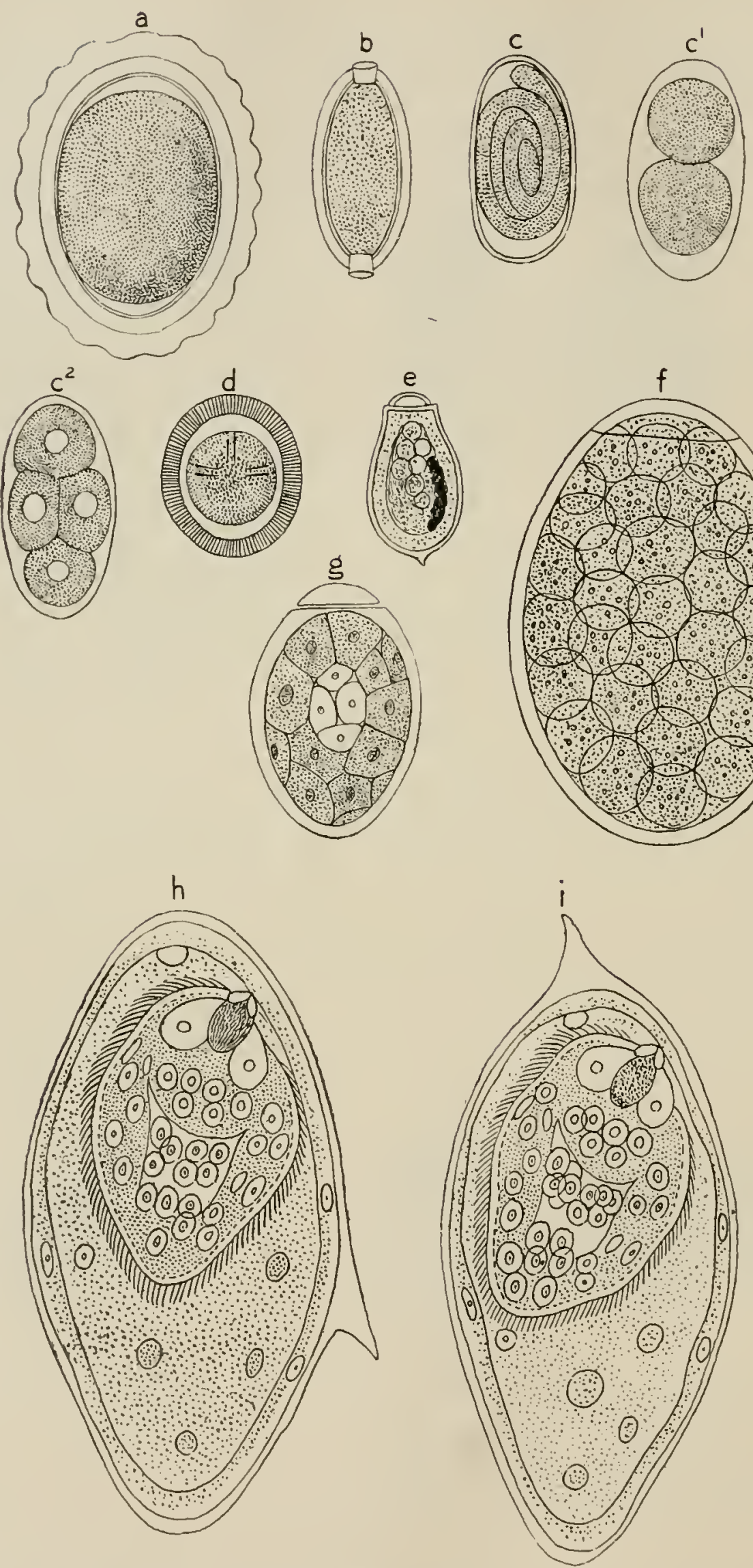

a, Ascaris lumbricondes; b, Trichocephalus dispar; $c$, Oryuris a'ermicularis; $c^{1}, i^{2}$, Ankylostomum duodenale; $d$, oncusphere of Cestode; $e, f, g$, various Fasciolidip; h, Schistosomum (?) hematohium ifrom freces); i, Schistosomum hiematohium (Irom urine). 
In warm and moist soil a spiril embryo is seen in one month, but requires to be ingested by man before hatching and developing into the adult form.

The source of infection is by polluted vegetables, fruit, water, and soiled hands. The eggs pass unaltered through the alimentary canal of the house-fly, hence house-flies are carriers (Stiles).

The eggs hatch out in the small bowel where the adults remain as spindle-shaped yellowish worms, the male 15 to $25 \mathrm{~cm}$. Iong by $3 \mathrm{~mm}$. thick. The female is from 20 to fo $\mathrm{cm}$. long. They look very like the ordinary ground worms. The male carries two spicules posteriorly, and there are about seventy papilla around the cloaca.

The vulva of the female is at the junction of the anterior and middle thirds of the body.

The eggs are usually in the small intestine but have been seen post mortem in the stomach, osophagus, nose, larynx, trachea and bronchi, more rarely in the liver, pancreatic duct and vermiform appendix.

\section{SYMPTOMATOLOGY.}

Symptoms are often nil.

Skin eruptions and irritation may be present.

If many are present there may be signs of toxæmia or intestinal obstruction. Odd worms may wander in many places, when the symptoms are according to the mechanical obstruction caused, as when one passes into the stomach, romiting may set in,

Passes into the appendix, inflammation of that organ arises,

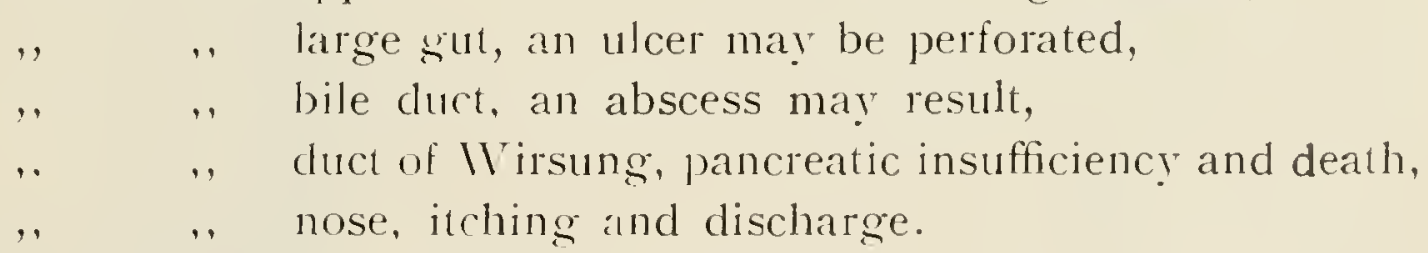

They have caused obstruction, perforation, peritonitis and death (Prasad).

In searching the freces for eggs one must remember that:-

The eggs may have an albuminous coating, or

They may be decorticated, or

They may be unfertilized, or

They may have become embryos, each stage presenting a different picture.

\section{TREATMENT.}

Give santonin, grains 1 to 3 , each morning for three day's.

Accompany this by calomel in equal doses.

For children give one-sixth of a grain of each for each year of life for three days.

Repeat the treatment after one week if the eggs are still present.

Santonin in excess may cause yellow blindness. 


\section{THE PARÁSITE.}

\section{OXYURIASIS.}

The Oxyuris vermicularis or thread-worm is world-wide in its distribution and occurs only in man.

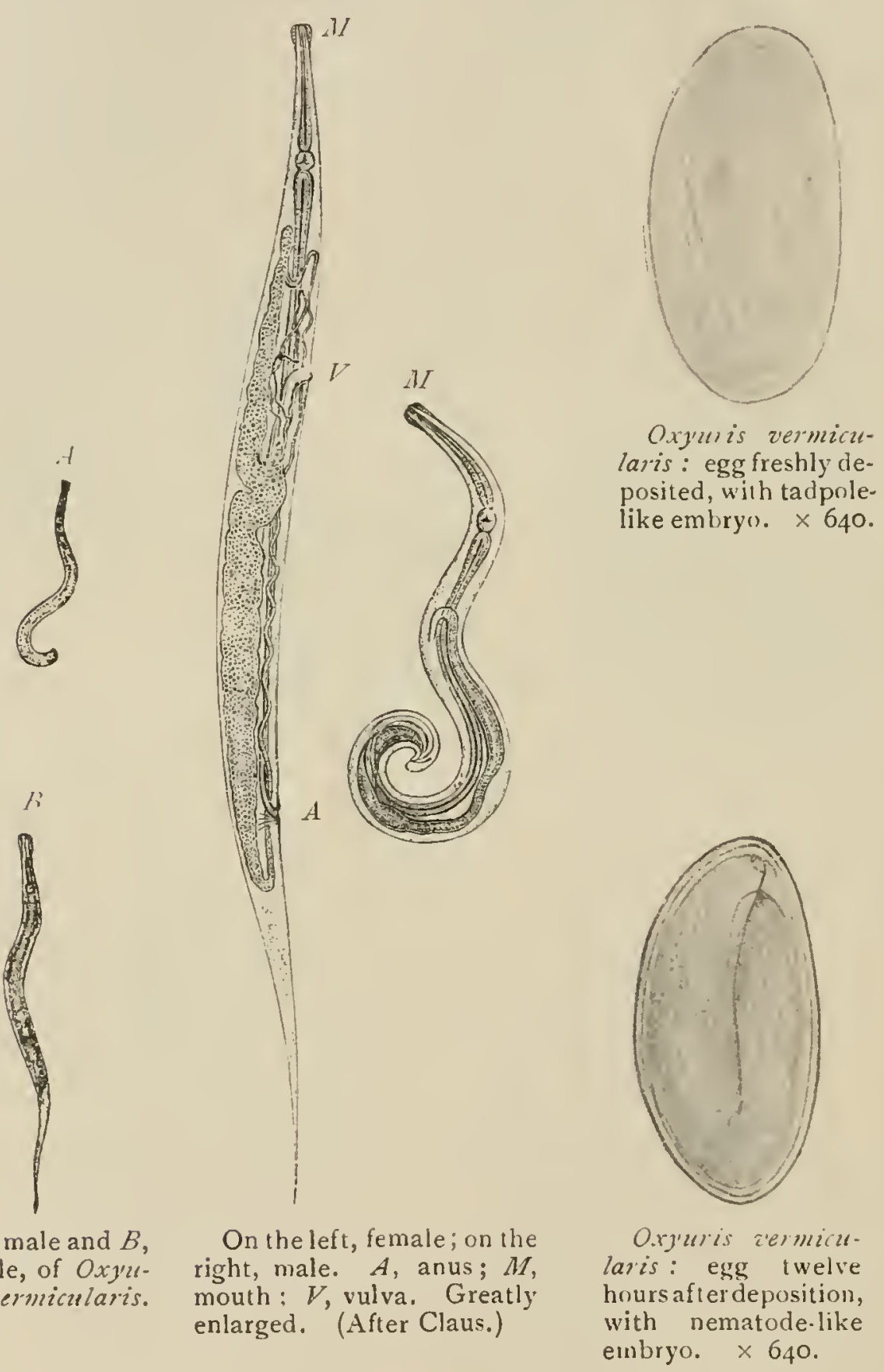

The male is 3 to $5 \mathrm{~mm}$. long and the female 9 to $11 \mathrm{~mm}$. long.

They live in the lower part of the small intestine but the gravid females migrate to the cæcum, colon and anus. The males die after copulation.

The eggs are given off in the gut and measure 50 by $20 \mu$.

They have a thin shell and contain a well developed embryo when the egg is passed in the fæces. These eggs escape and are re-intro- 
duced upon fruits and vegetables. The egg capsule is dissolved in the stomach, the embryo passes to the small intestine, grows and becomes mature in fourteen days and then copulation takes place.

The adult worm is a fine, minute, thread-like, round worm with a long bulbous oesophagus and transversely striated cuticle. The male is coiled up at the posterior end and carries one spicule.

The female is straight and the posterior end tapers to a point.

\section{SYMPTOMATOLOGY.}

Irritation about the anus is common.

One often meets with nocturnal enuresis and insomnia.

Entero-colitis and slight fever are rare.

rasal irritation is uncommon.

The worms may cause vaginitis in girls and masturbation in both sexes.

\section{TREATMENT.}

To kill the young forms use santonin and calomel as for Ascaris.

To kill the gravicl females give rectal injections of quassia, alum, one drachm to one pint, or of salt, two drachms to one pint.

For rectal irritation or vaginitis give diluted mercurial ointment, one in four. Iodoform or napthalin suppositories may be used.

\section{DEFINITION.}

This is a toxamia passing to a progressive anxmia caused by the Ankylostome duodenale and the Necator americanus associated with fatty degeneration of the heart, liver, kidneys, and of the intestinal mucosa.

\section{DISTRIBUTION.}

The clisease is found wherever there is moisture and a suitable temperature for the growth of the parasite.

It is present in America, Ifrica, Isia, Australia, New Guinea, Fiji, and in the mines of Cornwall and Belgium.

It was taken to the United States by African slaves.

In Porto Rico 70 per cent. of the people are infected and 30 per cent. of the death rate is due to this cause.

In the Army of the Southern States 6 per cent. were carriers.

In the mills of the Southern States 12.6 per cent. were carriers.

In Java 97 per cent. of the population is infected.

\section{THE PARÁSITE.}

This is the Ankylostome duodenale. (Also the Necator americanus as it gives rise to similar symptoms.) 
The disease was perhaps known and treated bv the ancient Egyptians $1,55^{\circ}$ years B.C.
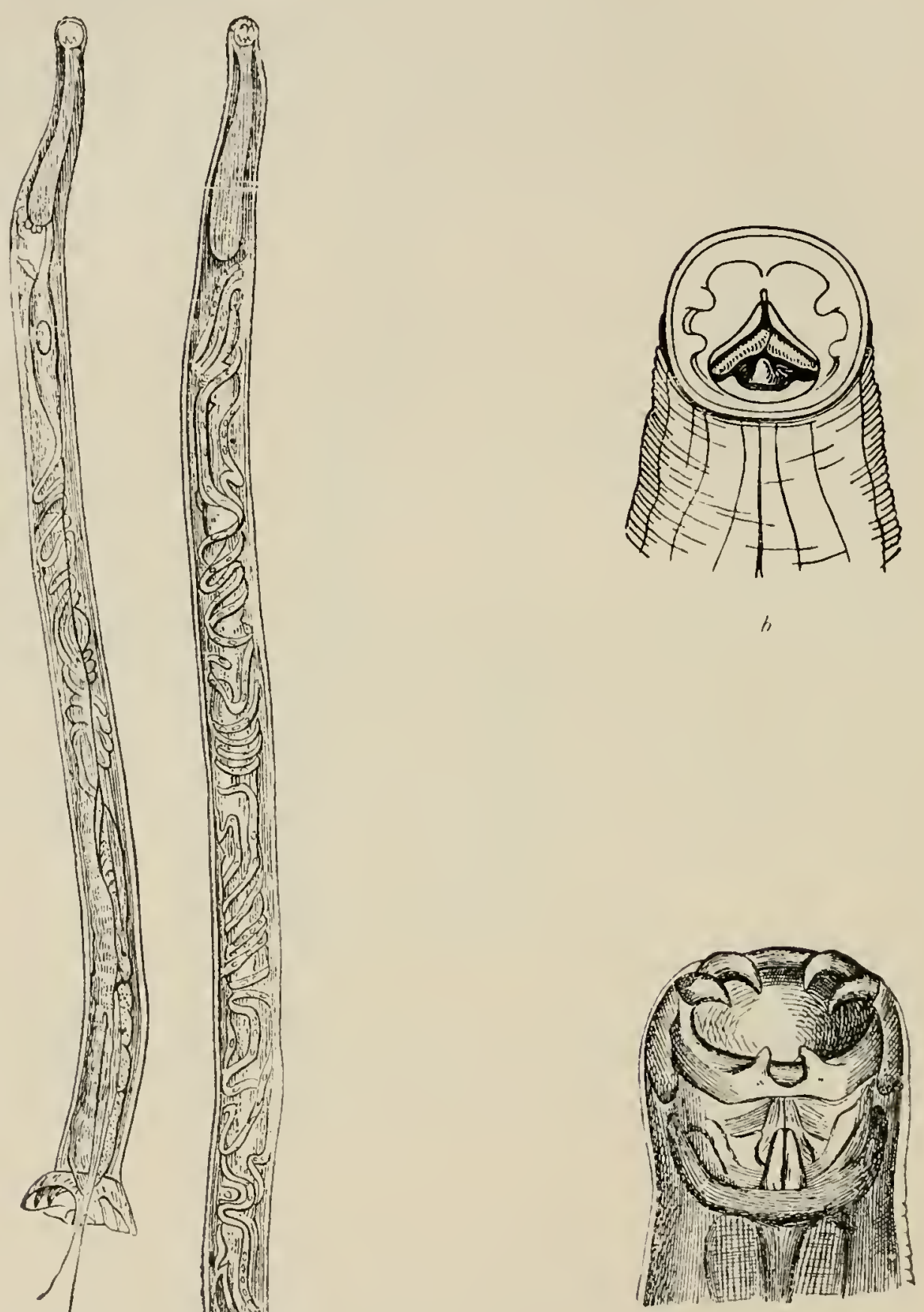

$m, f$, male and female Ankylostomes: $a$, head of Ankylostomum duoienale; $b$, head of Necator americanus.

It was rediscovered by Dubini in Mlalan, ..1. 1838.

The parasite is a white cylindrical worm living in the upper intestine, the jejunum chiefly, retaining its position with its four hooks and two conical teeth, which are carried about the mouth. 
The disease was perhaps known and treated by the ancient Esyptians $1,5,50$ years B.C.

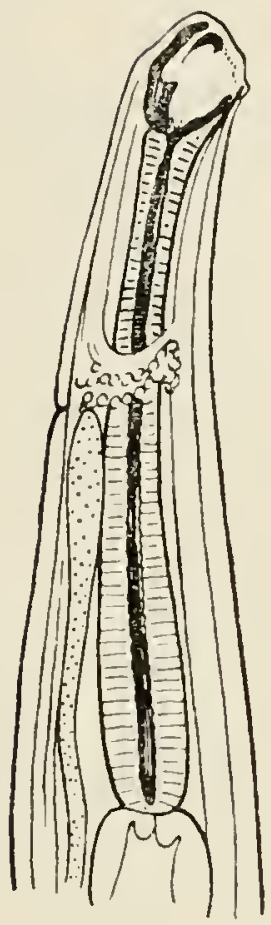

A

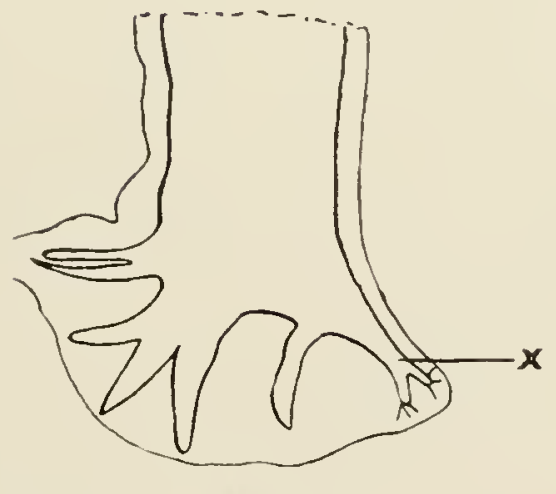

A

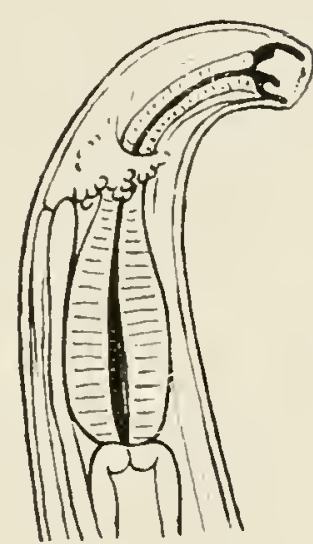

B

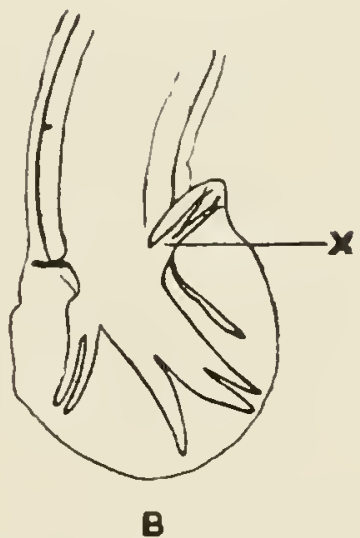

$A, A$, head and tail of male, Ansylostoma dnotenale; B, B, head and tail of male Necator americanus; $\mathrm{x}, \mathrm{x}$, dorsal rays.
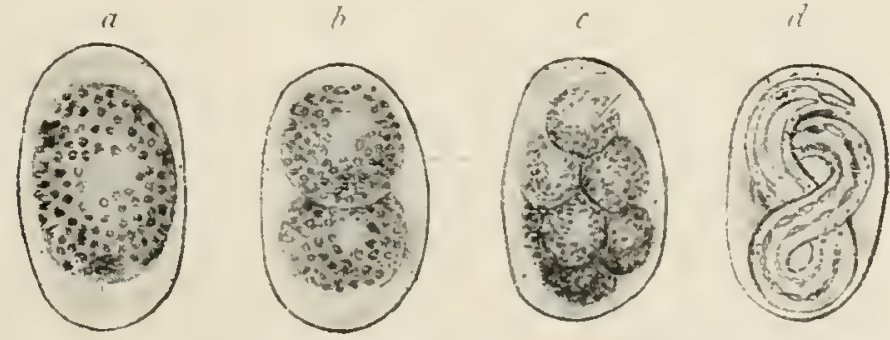

Ancylostoma duodenale: eggs in different stages of development. $a$ to $c$, in fresh freces; $d$, conlaining a larva, only in old freces. $\times 336$. (After Looss.)

Two long and slender spicules can often be seen projecting through the opening of this bursa. 
The female, I2 to $13 \mathrm{~mm}$. long, has the vulva at the junction of the middle and hinder parts of the body, so that when copulation takes place the worm looks like the Greek letter gamma $\gamma$.

\section{LIFE-HISTORY.}

The adults live in the jejunum, where the females lay their eggs. These eggs are colourless, with a thin, plain double shell, the cellular contents being separated from the shell by a clear space. During its passage through the intestine the cellular mass divides into two, then four parts, and is usually seen in this form in the faces. With sufficient air, water and leat the embryo forms in twenty-four hours, escapes from the shell, and begins to feed on fæecal material. Larve measure from 200 to $250 \mu$ by is to i $7 \mu$.

Ecdysis is completed in four to six days in the tropics; the larvæ then ceases to grow. It can remain alive in this condition for months, living on the food in its own cells. The larva may be quite active, swinming or climbing to any other suitable place.

It enters the hair follicles of man, causing " ground itch," and may even penetrate gloves, paper and boots in order to reach the skin. Thence it passes to the lymphatics and venous blood-vessels. Some of them reach the right heart and the lungs, whence they pass from the capillaries to the alveoli along the bronchioles, bronchi, trachea and larynx to the oesophagus, stomach and intestines, where they remain. This occupies from seven to ten days, during which time several ecdyses take place. In eight days these larvæ, 3 to $5 \mathrm{~mm}$. long, attain maturity. In fourteen to sixteen days copulation has taken place, and the eggs are seen in the freces.

The whole cycle takes eight to ten weeks.

Sambon says that when the larva are in the lungs they pass from the pulmonary capillaries to the pulmonary veins into the general blood-stream, and hence into the mucosa of the jejunum, which they pierce, and so enter the lumen of the bowel.

He believes that those seen by Loos in the respiratory tract are stray ones. Perhaps both are correct, but experience has shown that the great majority pass as I oos has described. Some may enter directly by the mouth, as when miners eat their food with soiled hands.

In order to grow the larvæ, place a thin layer of fæces in a Petri dish and a piece of moistend blotting-paper on the top. The worms will grow in two to three days and come upon the top of the blottingpaper, where they can be well examined.

Keep the paper moist; later breathe on the paper; this will stimulate movement.

For mounting them, embed in glycerin and egg-white smear, treat 
with absolute alcohol, Wash out glyerine, and stain with hamatin and eosin.

\section{SYMPTOMATOLOGY.}

\section{Clinical Course.}

Papules or vesicles may form where llue larvæ enter, perhaps due to the pus cocci introduced. This is known as "ground itch" or "bunches." A leucocytosis and an eosinophilia can be seen under the microscope before other symptoms are manifest. The first symptoms generally observed are, however, pallor, weakness, breathlessness, cedema, general or local, emaciation perhaps concealed by the oedema. Flatulence, dyspepsia, nausea, rumiting, epigastric pain, constipation or diarrhoa; later in the final stages there may be a general anasarca.

One finds the ova in the stools with, sometimes, blood and mucus, also Charcot-Leyden crystals.

In some cases there is a perverted appetite, perhaps due to mental disturbance (geophag:).

Impotence is common, 68 per cent. of Sandwith's cases.

Puberty is delayed in both sexes. It arrests growth and development generally.

Lemann noticed that infantilism was present in young hookworm patients of the Southern United States. He finds that the disease produces :-

(I) A general retardation of skeletal changes.

(2) A general retardation of growth, symmetrical and harmonious.

(3) I failure of derelopment of the genitals as well as the absence of secondary sexual characteristics.

(4) A general slowness and dullness.

\section{THE BLOOD EXAMINATION.}

The rolume of blood is increased, causing dilatation of the heart and hemic bruits at the apex. Caagulation is diminished, the blood readily spreading over the skin when punctured. The blood condition predisposes to palpitation, and a quick, thready, dicrotic and intermittent pulse.

The red cells are markedly diminished; one patient had but 930,000 and recovered. The hamoglobin may be as low as is per cent.

There are many changes in the red cells, but they are more uniform than in pernicious anæmia. One finds poikilocytes, megalocytes, normoblasts and megaloblasts. Polychromasia is not infrequent. The eosinophilia is from io per cent. to $5^{\circ}$ per cent., sometimes wellmarked in children, but may be absent in adults (Low).

The leucocytes, total count, vary from 20,000 to 50,000 per $\mathrm{cm}$. 
The disease is often fatal during parturition and during operative treatment. Sometimes a low fever is present, but this is the exception.

The ancmic condition is probably caused by a toxin excreted by the worms whicls passes into the circulation through the wound made in the mucosa of the jejunum, logether with the actual blood lost at the site of the bites. The condition is aggravated undoubtedly by septic absorption from the same foci.

Post mortem, yellow pigments and iron granules are found deposited in the liver and kidney cells in bad cases.

\section{COMPLICATIONS.}

Albuminuria, present in so per cent. of Sandwith's cases.

General aedema may be present.

Any intercurrent disease, as dysentery, which is common.

Pregnancy or operative procedure are serious complications. The former cases die of heart failure just before the child is born.

There is a tendency to post-partum hamorrhage.

Cardiac failure is common.

\section{PROPHYLAXIS.}

The ankylostome embros, when passed under good conditions for them, can survire and derelop as free living larve araiting access to man in order to complete their development, hence prophylaxis is important and ma! be summarized thus:-

\section{(1) Educational.}

The instruction of rich and poor with regard to methods of infection, symptoms, treatment and prophylaxis.

(2) Public.

Search for and treat carriers, anamic people and those with skin eruptions.

The provision of sanitary conveniences which should be kept in good condition.

(3) Private.

Protection of the feet by boots, \&c.

The proper cleaning of hands before meals.

Immediate treatment of eruption on hands and feet.

\section{TREATMENT.}

The indications are to expel the worms and to aid blood formation. There are four main lines of treatment as follows:-

\section{(1) Thymol Treatment.}

Put the patient in bed on starvation diet for two days.

Exclude all other possible ailments. 
Alcohol is strictly forbidden as it dissolves the thymol and may produce a fatal result.

Some sive stryctinine, but this is very soluble with thymol and in any case should not be given by mouth.

Give a saline purge at the end of the second day.

All food prohibited during the thymol treatment.

Give thymol, one to two grams next morning.

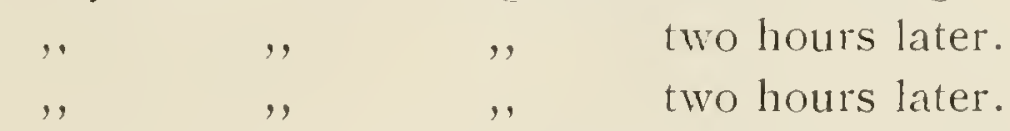

Give a saline aperient four lours after the last close of thymol.

Castor oil must not be given as thymol is soluble in it.

Sandwith recommends strychnine injections before the treatment.

Do not give thymol to patients with fatty hearts, visceral disease or with an ancmia below 1, 500,000 red cells per $\mathrm{cm}$.

\section{(2) The Eucalyptus Treatment.}

The preliminary two days as above.

Give a saline purge at 6 p.m.

Next morning at 7 a.m. and 7.30 a.m. give the following mixture :-

$\begin{array}{lllllllll}\mathrm{R} & \text { Olei eucalypti } & \ldots & \ldots & \ldots & \ldots & \ldots & 15 & \text { minims }\end{array}$

$\begin{array}{llllllll}\text { Chloroformis } & \ldots & \ldots & \ldots & \ldots & \ldots & 22 & \end{array}$

$\begin{array}{lllllll}\text { Olei ricini } & \ldots & \ldots & \ldots & \ldots & \ldots & \text { half a drachm }\end{array}$

This amount to be given on each occasion.

For children divide the whole dose into three parts and give each part twenty minutes later than the preceding. The total amount being one half that of the aclult.

\section{(3) The Seta-naphthol Treatment.}

The preliminary treatment as before.

The beta-naphthol must be fresh and kept cool.

It is a popular remedy with the French.

$\begin{array}{llllll}\text { P. } & \text { Beta-naphthol, finely-powdered } & \ldots & \ldots & \ldots & 30 \text { grains } \\ \text { Mucilaginis tragacanthre } & \ldots & \ldots & \ldots & \ldots & \text { I drachm } \\ \text { Aquie menthre piperitze } & \ldots & \ldots & \ldots & \ldots & 5 \text { drachms }\end{array}$

Give a purge overnight.

At 6 a.m. give full dose as above.

At $S$ a.m. repeat.

At io a.m. repeat.

A1 12 noon give a saline purge.

(4) The Oleum Chenopodii Treatment (Day and Ferguson).

Preliminary treatment as above.

Give the abnve oil in three doses of 16 drops on sugar at two hour intervals.

Give castor oil purge $17 \mathrm{grm}$. with chloroform $3 \mathrm{grm}$. 
This oil has a disagreeable flavour and is expensive.

In any of these methods three repeated treatments may be necessary, in which cases allow an interval of four to seven days.

In some cases it is difficult to clear out all the worms even with the repeated courses.

The anamia should be treated on general lines.

Iron, grains zo or more should be given daily.

Reduced iron, ferric perchloride, ferrous sulphate, iron carbonate (Blaud), and the ammonia citrate are useful in the order named.

Arsenic and digitalis can also be given.

\section{Points of Difference Between the Ankylostome duodenale and}

THE NECATOR AMERICANUS.

\section{A. duodenale}

The worm is thicker and longer

The bend of the neck is slow

The mouth capsule is large and thick

The cesophagus is long and cubular

The teeth are small and shallow, with a large brownish pear-shaped hook

There are two separate spicules in the male

The dorsal ray of the bursa has three prongs

\section{Table indicating BRiefly the Mode of Infection by Helminths.}

(After Daniels.)

\begin{tabular}{|c|c|}
\hline WORM & LEAVE MA.N \\
\hline $\begin{array}{l}\text { Ascaris lum- } \\
\text { bricoides } \\
\text { and Tricho- } \\
\text { cephalus } \\
\text { dispar }\end{array}$ & $\begin{array}{l}\text { As eggs with un- } \\
\text { developed con- } \\
\text { tents }\end{array}$ \\
\hline $\begin{array}{l}\text { Oxyuris ver- } \\
\text { micularis }\end{array}$ & $\begin{array}{c}\text { Adults leave rec- } \\
\text { tum and deposit } \\
\text { eggs on skin }\end{array}$ \\
\hline $\begin{array}{l}\text { Strongyloides } \\
\text { intestinalis }\end{array}$ & $\begin{array}{l}\text { Embryos passed } \\
\text { with faeces }\end{array}$ \\
\hline $\begin{array}{l}\text { Ankylostome } \\
\text { duodenale } \\
\text { and Necator } \\
\text { americanus }\end{array}$ & $\begin{array}{r}\text { Eggs; division of } \\
\text { egg contents } \\
\text { has commenced }\end{array}$ \\
\hline $\begin{array}{l}\text { Trichina spir- } \\
\text { alis }\end{array}$ & $\begin{array}{l}\text { Embryos develop } \\
\text { to their limit in } \\
\text { the muscle of } \\
\text { the host }\end{array}$ \\
\hline F. bancrofti... & $\begin{array}{l}\text { Embryos ab- } \\
\text { stracted with } \\
\text { blood by mos- } \\
\text { quitoes }\end{array}$ \\
\hline
\end{tabular}

\section{N. americanus}

The worm is thinner and shorter

The neck bend is sharp

The mouth cansule is small and thin

The oesophagus is bent and bulbed

The teeth are large pointed, spheroidal and colourless

There is one spicule in the male

The dorsal ray of the bursa has two prongs

\section{RE-ENTER MAN}

Development of egg contents change into em. bryo in water or on earth

Eggs undergo little or no further development, as embryo is already formed

Develop, become sexually mature, and multiply sexually

Embryos hatch out and develop to a considerable extent in moist eartl

Only in animals eating the infected flesh

Embryos develop in mos. quito, into larvæ with an alimentary canal
Eggs containing embryos are sivallowed, usually with food

Eggs containing embryos are swallowed-usually reintroduced into the same patient

Enter man with food, or in any substance contaminated with infected earth, or through the skin

Embryos re-introduced by the mouth, probably in food contaminated with earth. Also enter through the unbroken skil:

Usually from eating pork containing encysted larva

Larve discharged by mos. quicoes on man and enter through the un. broken skin 


\begin{tabular}{|c|c|c|c|}
\hline WORM & LEAVE MAN & LIFE OUTSIDE MAN & Re-Enter Man \\
\hline F. loa & $\begin{array}{l}\text { By Chrysops dim- } \\
\text { idiata }\end{array}$ & $\begin{array}{l}\text { Similar development in } \\
\text { salivary glands of } \\
\text { Chrysops }\end{array}$ & \\
\hline F. medinensis & $\begin{array}{l}\text { Embryos dischar- } \\
\text { ged into water }\end{array}$ & $\begin{array}{l}\text { Embryos swallowed? by } \\
\text { chrysops and there } \\
\text { develop to a certain } \\
\text { extent }\end{array}$ & $\begin{array}{l}\text { Cyclops containing devel- } \\
\text { oped larva sivallowed } \\
\text { by man }\end{array}$ \\
\hline I. saginata ... & Oncospheres & $\begin{array}{l}\text { In muscle of cattle (Cys. } \\
\text { ticercus bovis) }\end{array}$ & $\begin{array}{l}\text { Eating beef containing } \\
\text { Cysticercus bovis }\end{array}$ \\
\hline T. solium $\quad .$. & Oncospheres & $\begin{array}{l}\text { In muscle of pig (Cys. } \\
\text { ticercus cellulosa) }\end{array}$ & $\begin{array}{c}\text { Eating pork containing } \\
\text { Cysticercus cellulosa }\end{array}$ \\
\hline $\begin{array}{l}\text { T. echinococ- } \\
\text { cus }\end{array}$ & $\begin{array}{l}\text { "Hydatid cysts." } \\
\text { Does not leave } \\
\text { man, who is an } \\
\text { accidental host }\end{array}$ & $\begin{array}{l}\text { Adult worm in intestines } \\
\text { of dogs, \&c., who derive } \\
\text { infection from food, } \\
\text { offal from slaughter- } \\
\text { houses, \&c. }\end{array}$ & $\begin{array}{l}\text { By swallowing oncosph- } \\
\text { eres from freces of } \\
\text { infected dogs }\end{array}$ \\
\hline $\begin{array}{l}\text { Trematodes, } \\
\text { both Fascio- } \\
\text { lida and } \\
\text { Paramphis- } \\
\text { tomidae }\end{array}$ & $\begin{array}{l}\text { Eggs with unde- } \\
\text { veloped ovum } \\
\text { in faeces, or in } \\
\text { one instance in } \\
\text { sputum }\end{array}$ & $\begin{array}{l}\text { Ovum develops in water, } \\
\text { embryo enters molluscs } \\
\text { or fish and there multi- } \\
\text { plies asexually, produ- } \\
\text { cing ultimately young } \\
\text { flules, cercaria, encys- } \\
\text { ted on vegetables or } \\
\text { free swimming in water }\end{array}$ & $\begin{array}{l}\text { Swallowed by man on } \\
\text { vegetables or in water } \\
\text { or in undercooked fish }\end{array}$ \\
\hline Schistomidx & $\begin{array}{l}\text { Eggs with unde- } \\
\text { veloped ciliated } \\
\text { embryo, mira- } \\
\text { cidium, with } \\
\text { urine or faeces }\end{array}$ & $\begin{array}{l}\text { Embryos escape from egg } \\
\text { when urine or faeces } \\
\text { are diluted. Further } \\
\text { development, if any, } \\
\text { outside man, is un- } \\
\text { lnnown }\end{array}$ & $\begin{array}{l}\text { Probably enters through } \\
\text { the slin during bathing }\end{array}$ \\
\hline
\end{tabular}

\section{THE PRESERVATION AND EXAMINATION OF HELMINTHS. (After Daniels.)}

SMALL NEMATODES.

Preservation.

(1) Shake up the live worms in a I per cent. salt solution, to remove inucus.

(2) Kill by dropping into boiling zo per cent. spirit and allow to cool.

(3) Transfer to fresh zo per cent. spirit for storage.

\section{For Clearing and Mounting.}

(1) After treating with stages 1 and 2 as above, transfer to a mixture composed of 70 per cent. spirit, 95 parts with 5 parts of pure glycerine.

(2) Evaporate in a water or paraffin bath until all the alcohol has gone.

(3) Mount in glycerin jelly .

(4) Ring with gold size.

\section{Examination without Mounting.}

(1) Take from the jo per cent. spirit and place in methylated spirit. 
(2) Transfer to colourless cold tar creosote, allow to clear, and examine in that fluid.

After the examination is concluded pass through methylated spirit back to zo per cent. spirit as before to store.

\section{LARGE NEMATODES.}

These are treated in the same way as small ones except that they cannot be cleared and mounted by the glycerine method.

\section{SMALL TREMATODES.}

\section{Preseryation.}

(1) Place alive in a test tube one-third full if I per cent. Saline, and shake vigorously.

(2) Add to the test tube rapidly an equal quantity of saturated solution of sublimate.

(3) Shalie vigorously for three minutes.

(4) Transfer to jo per cent. spirit to store.

\section{Examination without Mounting.}

As for small nematodes.

In order to make stained and mounted specimens :-

(1) Transfer from 70 per cent. spirit to a s per cent. solution of alum to which is added a litle hamatoxylin, until the whole is a light claret colour. The hamalum solution (hamatin, $2.5 \mathrm{grm}$., absolute alcohol 50 c.c., alum 5o grm. or to saturation, water 1,000 c.c.) diluted with distilled water gives good results, and so does a weak solution of carmine, but in that case longer staining is required. Leave in this one to four days.

(2) Decolorize slightly with $\frac{1}{2}$ per cent. acid water.

(3) Wash well.

(4) Dehydrate with spirit and oil of cloves.

(5) Pass through zylol and mount in balsam.

\section{LARGE TREMATODES.}

\section{Preservation.}

(I) Drop alive in a s per cent. Saline and shake vigorously.

(2) Add formalin (commercial) to this to make about a ro per cent. solution, and shake vigorously until death occurs.

The specimens can be kept in ro per cent. formalin.

\section{Examination.}

This is best done by embedding and cutting sections.

\section{To mount Specimens whole.}

(1) Press between two slides whilst alive, and drop into zo per cent. spirit.

(2) Stain and clear as in small specimens. 


\section{CESTODES.}

\section{Preservation.}

(I) Shatie gently in 1 per cent. saline.

(2) Add formalin to this to make a 10 per cent. Solution and shake gently until they die.

The specimens can be stored in ro per cent. formalin.

\section{To stain and mount Segments.}

(I) Place allive in weak glycerin, faintly coloured with carmine, and leave until stained, or dilute hamalum may be used.

(2) Press between two slides and drop into methylated spirit. They should remain in this for twenty-four hours.

(3) Remove the pressure and place the segments in fresh methylated spirit for an hour or so.

(4) Clear in oil of cloves.

(5) Pass through zylol and mount in balsam.

Creosote may be used to clear instead of oil of clores.

\section{The Preservation of Helminth Ova in Fæces.}

\section{Method (1).}

Alcohol, jo per cent., with 5 per cent. glycerine added, is raised to boiling point.

Fluid faces, or faeces made fluid by dilution with saline, are poured into the boiling alcohol, stirred, and then set aside to inspissate in a warm place. When the faces have become a sticky mass, sufficient pure glycerine is added to make a soft paste (Leiper).

\section{Method (2).}

To fluid faces add equal parts of Langeron's lactophenol. 'This latter consists of carbolic acid one part, lactic acid one part, glycerin two parts, and water one part.

Shake rigorously.

This mixture will form a pasty jelly and will keep indefinitely.

A permanent preparation can be made at any time by stirring a small portion into a loop of melted glycerin jelly on a slide, a cover glass is placed on the jelly, and when this is set the preparation is completed by sealing with gold size (Leiper).

\section{DEFINITION.}

\section{HIRLDINIASIS.}

An invasion of the mouth, nose, pharynx and larynx, or an affection of the skin by leeches, a group of unsegmented worms allied to Chatopoda. 


\section{ETIOLOGY.}

Leeches are troublesome chiefly in the Near East, Ceylon, Philippine Islands, Japan, Australia and the Asiatic and the American jungle.

There are many species but few readily attack man.

\section{There are the :-}

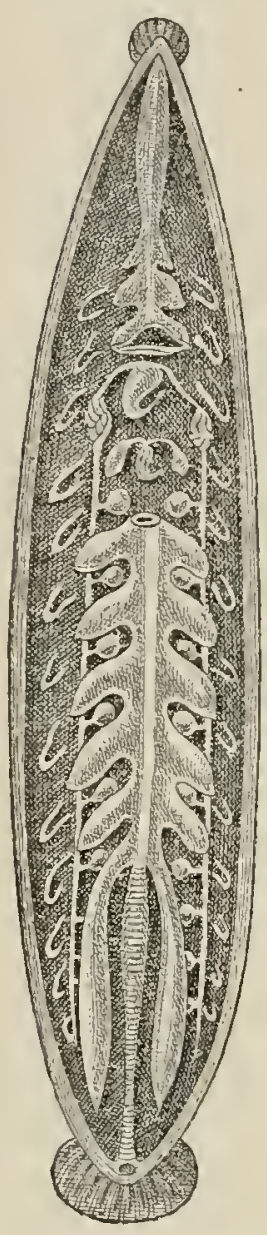

The internal organs of the leech. The creature has been opened from the dorsal surface, and part of the intestine has been removed. The testicles, with vas deferens, may be seen between the blind ducts of the intestine; beyond these on either side the segmental organs. The female genital organs are in front of the most anterior pair of lesticles. (After Kennel.)

(1) Land leeches or Hemadipsa. These live in the damp jungle country, as the Hiemadipsa ceylonica of Ceylon. They rest upon twigs and leaves awaiting the presence of some mammal to attack.

They are very active and are able to pass through very narrow orifices such as the lace-hole of a boot. Their bite is painless and the first indication of their presence may be that the boot is full of blood. In such cases there is often some local inflammation and sloughing.

(2) Water leeches, or Limnatis. These live in springs like the Limnatis nilotica and are troublesome to man when he drinks the spring water. The thirsty traveller at clusk is usually the victim.

The salivary glands of these parasites open into the mouth and secret a fluid which prevents the coagulation of the blood

When it has gorged itself it drops off but the bleeding continues.

The pharynx of the leech has a powerful muscular sac which by contracting and dilating forms a sucking pump.

The blood is stored up in the crop and its cæcal diverticula and only a little is used daily for food.

\section{SYMPTOMATOLOGY.}

This is according to the variety and position of the leech.

There may be bleeding from the nasal or buccal carities which may indicate that the leech or leeches are anywhere in the respiratory tract to the trachea and the deglutition tract to the stomach.

There may be irritating cough and dyspnœa. Bleeding from the skin with land leeches. Anamia.

\section{TREATMENT.}

Saline solution will loosen them when it is possible to apply it.

Saline solution upon the clothing or boots of jungle travellers is 
protective. A "dip in the briny" with one's clothes on before commencing a march is sufficient in most cases.

Cotton wool soaked in 30 per cent. cocaine applied to the parasite will paralyse it and cause it to drop off, but if the leech is in the larynx the patient must be held with the head downwards or the leech will dron into the trachea.

Leeches should not be removed forcibly.

The gastric juice is said to kill them.

Leeches are not known to carry disease.

\section{POROCEPHALOSIS.}

There are twenty known species of Porocephalus, two of which occur in man. They belong to the parasitic Arachnoidea and Iinguatulida .

They have elongated rermiform bodies and a continuous coelom.

The female is 9 to $\mathrm{I} 2 \mathrm{~cm}$. long, the male 3 to $+5 \mathrm{~cm}$. long by 2 to $4 \mathrm{~mm}$. broad, with twenty-six to thirty rings.

They are commonly found in the nasal cavities and lungs of prthons and snakes. The eggs probably pass from these reptiles into water and thence into man. These eggs develop into larve and nympha which may then become encysted in the lungs or the liver of man, or they may move freely in the small intestine and eren, at times, enter the peritoneal carity.

The P. armillatus and the P. moniliformis affect man. They are a bright lemon yellow colour when fresh.

\section{DISTRIBUTION.}

The P. armillatus is found in Ifrica only. Chiefly in Egrpt, West Coast and the Congo.

The P. moniliformis is found in Jara, Philippines and India.

\section{PATHOLOGY.}

The larve are found coiled up in crsts of the liver and lungs.

Here they become nymphr, leare the crsts and wander about the body .

They may pass into the lungs causing bronchitis or bronchopneumonia.

Or into the peritoneal cavity causing peritonitis.

Or into the intestinal tract causing diarhoea.

\section{SYMPTOMATOLOGY.}

The distinctive symptoms are not appreciated until they become terminal. Then one has emaciation and weakness associated with an 
attack of bronchitis or pleurisy. There may be cavities in the lungs and offensive sputum which may contain the parasites. One patient was known to expectorate from 75 to roo of these parasites. The liver is usually enlarged.

The condition has been mistaken for phthisis.

There is no known effective treatment. 


\section{DISEASES OF UNCERTAIN FETIOLOGY.}

PELLAGRA.

BERIBERI.

EPIDEMIC DROPST.

HILL DIARRHCEA.

SPRUE.

PHLEBOTOMUS FEVER.

VERRUGA PERUVIANA.

YELLOW FEVER.

NOTES ON THE STEGOMYIA CALOPUS (FASCIATA).

SPOTTED FEVER OF THE ROCKY MOUNTAINS.

DENGUE FEVER.

TYPHUS FEVER.

HAMOGLOBINURIA.

FAVISM.

HEATSTROKE.

SOME UNCLASSIFIED FEVERS AS:-

JAPANESE RIVER FEVER.

NASHa FEVER.

Pigmentary FEver.

ARCHIBALD'S FEVER.

Hyperpyrexial FEVER.

BaCillus Asiaticus FEVER.

Double Continued Fever.

The Macular Fever of Tunisia.

LOW Intermittent NoN-malarial Fever.

RAT-BITE FEVER.

FIVE-DAY FEVER.

TRENCH FEVER.

THE EXANTHEMATA.

TROPICAL HAY FEVER.

TROPICAL NEURASTHENIA.

ENDEMIC PERIPHERAL FEVER.

ENDEMIC PARALITIC VERTIGO.

LATAH.

AMOK.

ENDEMIC FUNICULITIS.

EPIDEMIC GANGRENOUS RECTITIS. 
INFANTILE BILIARY CIRRHOSIS,

GOITRE.

CLIMATIC BUBO.

GOUNDOU.

BIG HEEL.

AINHUM.

GANGOSA.

CHAPPA.

PONOS.

TROPICAL LIVER.

BOOMERANG LEG.

WHITMORE'S DISEASE.

ONYALIA. -

AFEBRILE SPLENOMEGALY 


\title{
DISEASES OF UNCERTAIN AETIOLOGY.
}

\author{
PELLAGRA.
}

(Pellagra-rough skin; "pelle," the Italian word for skin, "agra" dneaning rough.)

\section{DEFINITION.}

A chronic endemic disease of unknown causation with cutaneous, gastro-intestinal and nerrous symptoms which usually recur in the spring and autumn.

\section{DISTRIBUTION.}

Perhaps Francis of Assisi suffered from it.

It was well known by medical men to be ridespread in laly at the beginning of the eighteenth century.

Casal discovered it in Spain in 1735.

Also present in Portugal and France.

Some of Napoleon's soldiers became infected in Italy.

It is still very prevalent in Austria and Hungary.

Roumania has 200,ooo cases (Babés).

Reported in the British Isles in I860. In Scotland and the Shetlands in Igog.

It is also found in Egypt, West Indies, Mexico, North American Indians, India, Straits Settlements, Philippine and Sandwich Islands; Africa, Europe, Asia, Oceania, America, and perhaps is world-ivide.

It is found chiefly in country places and along certain streams, less seldom in towns.

Attacks most frequently appear during the late springtime and the autumn. It tends to disappear in the winter.

None are exempt. Females are attacked to males as two is to five.

Jews are rarely affected.

It is to be noted that those engaged in agriculture appear to be affected most.

\section{AETIOLOGY.}

The chief theories are:-

(i) The Deficiency Theory.

(2) The Maize Theory.

(3) The Parasitic Theory.

(4) The Mineral Acidosis Theory.

(1) The Deficiency Theory.

The lack of some nitrogenous complex in the food.

When steam-milled mealie bread alone was supplied to prisoners 
in the Victoria jail in Rhodesia the disease broke out; when meat, vegetables and rice replaced the mealie the epidemic ceased, and when hand-milled maize was obtained the improvement was "immediate and almost magical"' (Nightingale).

\section{BuT}

Pellagra may occur in people having the best of suitable food.

\section{(2) The Maize Theory.}

Pro. (1) The history of maize production and consumption.

Maize is native to America, where it has been found in the graves of the Incas. Columbus or his followers introduced it to Europe, but it did not grow well in the British Isles, which therefore remained free from the disease until the nineteenth century, when maize was imported in larger quantities and used in the preparation of cakes, porridge, pop-corn and whiskey.

It grew well in Spain, Italy and France, where Pellagra appeared.

(2) In the Nile delta there is plenty of maize and plenty of pellagra, but higher up the Nile millet is used instead of maize, and pellagra is found less frequently.

(3) In Colombia it is said to be found only in people who take the drink made from fermented maize called "chicha."

(4) Maize is found more or less all orer the world, so is pellagra.

Con. (1) There are entire districts in Italy and Brazil where the people have never lived on maize, but where pellagra is prevalent. Other districts where bad maize is used almost exclusively there is no pellagra.

(2) Relapses occur in jails over long periods, five to fifteen years, where maize is rigidly excluded.

(3) Pellagra was present in Europe before maize was introduced, e.g., the attack of Francis of Assisi, before the time of Columbus.

(4) Prophylactic and curative measures based on the maize theory have yielded very little result. Progressive symptoms continue after absolute prohibition of the cereal (Alberto Cencelli).

\section{Yarious Maize Theories.}

The Photodynamic Theory (Raubitscheli).

Pro. An exclusive maize diet proves deleterious to white mice and guinea-pigs if such are exposed to the sun. 
Other cereals do the same. 11 is maintained that photodynamic substances are introduced by cereals into the blood. These with sunlight produce toxins and cause pellagra symptoms. In darkness mice have been cured.

Cox. Hirschfelder searched the blood of severe cases for the photodynamic substance, but failed to find any.

Pellagra occurs in jet-black negroes, whose pigment protects them considerably from the sun's rays.

The Deficiency Theory.

Pro. Insufficient protein in maize food.

Cox. Many live on rice and potatoes which contain less nitrogen ratio than maize.

The Toxicily Theory.

Some claim that there is an-

Individual susceptibility to maize intoxication.

Others that there are toxins produced in the spring by the germination of maize.

Others that there are toxins generated in the bowel by aid of the B. coli.

Others that there are alkaloidal and other poisons in fermenting maize.

\section{The Infectivity Theory.}

The most popular view. The maize is cultivated and harvested under unfarourable conditions, and stored in damp places and becomes mouldy. Ilirsch states that after a bad season cases increase.

This infection may be :-

(I) Fungi as Pernicious glaucum, Aspergillus, \&c., the fungi or their toxins.

(2) Bacteria as B. solanacearum, B. subtilis, B. pellagræ.

\section{(3) The Parasitic Theory.}

This is supported by the Illinois Commission.

A. Vegetable Parasite, e.g., Tizzoni's streptobacillus.

He claims to have found it in the blood, cerebrospinal fluid, and viscera of pellagrins after death. Others refute this.

B. Animal Parasites.

(1) A slender nematode worm (Filarida alessandrina) in drinking water.

(2) A phase of amobic dysentery (Long).

(3) A protozoan infection spread by a biting fly (Sambon). 
PRO. Sambon supports his view on the basis of its being protozcan because :-

(1) There is a mononucleosis present in the blood.

(2) There are long intervals of quiescence followed by relapse.

(3) There is a constant and characteristic topographical distribution.

(4) The symptoms, course, duration, and morbid anatomy are similar to those found in other parasitic diseases.

(5) Sambon found a spirochate in a bulla on the hand of a severe pellagra case.

Con. No parasite has yet been found.

Fluids from pellagrins have not reproduced the clisease when injected into other animals.

A biting fly is blamed because:-

It is a disease of rural districts essentially.

It is in some way related to moving water.

It has a definite seasonal influence, spring and autumn, which coincides with the appearance of certain flies.

It affects labourers in the field.

It is not contagious (Goldberger).

Young children and whole families often are readily affected in an endemic centre.

Sambon suggests one of the simulida because:-

Their larve live in running water.

They have two seasons, spring and autumn, when they attack man and beast in swarms.

BUT

They are not found in the Nile, where pellagra is common. The lllinois Commission does not agree on this point.

\section{( $\left.\Psi_{1}\right)$ The Mineral Acidosis Theory.}

The findings of Professor Alessandrini and Scala of the Pellagra Commission of Rome may be summarized thus :-

Pellagra is strictly localized and limited to areas where the water drunk has almost exclusively been in contact with clay.

Clay is silicate of alumina.

The action of rain water upon this produces by hydrolysis silicic acid and hydrate of alumina which pass into the water in a colloidal form.

A colloidal silica-alumina is formed, some as a deposit and some remaining in suspension. 
The ingestion of this latter causes a retention of salts in the tissues resulting in their excessive fixation which, when liberated in excess, causes the pathological lesions of pellagra.

They affirm to have considerably improved, if not to have cured, cases by musculat or subcutaneous injections of neutral citrate of sodium.

Daily injections of a 10 per cent. Solution of trisodic citrate were given. The acidosis is said to be neutralized by it.

\section{PATHOLOGY.}

There is an infiltration of the true skin and aedema of the connective tissue. The rete Malpighii is infltrated with cells, the stratum corneum is thickened and shows hyperkeratosis.

A blood examination shows a reduction of the red cells.

Fatty degeneration and characteristic deep pigmentation of all viscera. Trasting of all muscles. Intestinal mucous membrane hyperamic, sometimes ulcerated. Liver and spleen at:ophied.

Brain and cord show chronic leptomeningitis with much thickening, a perivascular cellular infiltration with pigmentation and degeneration of nerve filaments. Ventricles distended. Brain shrunlien.

Lateral columns and crossed pyramidal tracts affected, mid-dorsal region most marked. The condition is not unlike G.P.I.

\section{SYMPTOMATOLOGY.}

Incubation not known, but the disease has occurred in a child three months old. There is no support for heredity.

The skin eruption has occurred in a healthy person two months after visiting a pellagrous area.

Onset, insidious. A sunburn may appear on the back of hands or feet, face and neck. It burns and may blister. Perhaps sore mouth, giddiness, muscular weakness. In a few weeks the redness fades and pigmentation occurs; the skin may be thickened. In a few days there is desquamation of these areas, the underlying skin being apparently normal.

Autumn comes and the patient gets better. The next spring, or several seasons later, it recurs surely and more severely; dermatitis and bulla may appear; inflammation of the whole mouth, tongue protruded with difficulty, edge may have a greyish membrane, there is enlargement of the parotid gland, dyspepsia, abdominal pains, diarrhoea, or even dysentery. Marked vertigo, the patient may fall, weak, trembling in head, arms and legs, melancholic, highly emotional, patient may seek to drown himself.

He has burning pains at night and may go to the river to relieve them. Someone pulls him out of the water and reports that he was. 
seeking to drown himself. He has abdominal pains, and thinking that the cook has been poisoning him, he seeks to kill the cook and finds himself in an asylum. There is reason in his madness.

Winter comes and with it relief, but the skin does not become normal. With repeated attacks the skin changes are marked, the mind

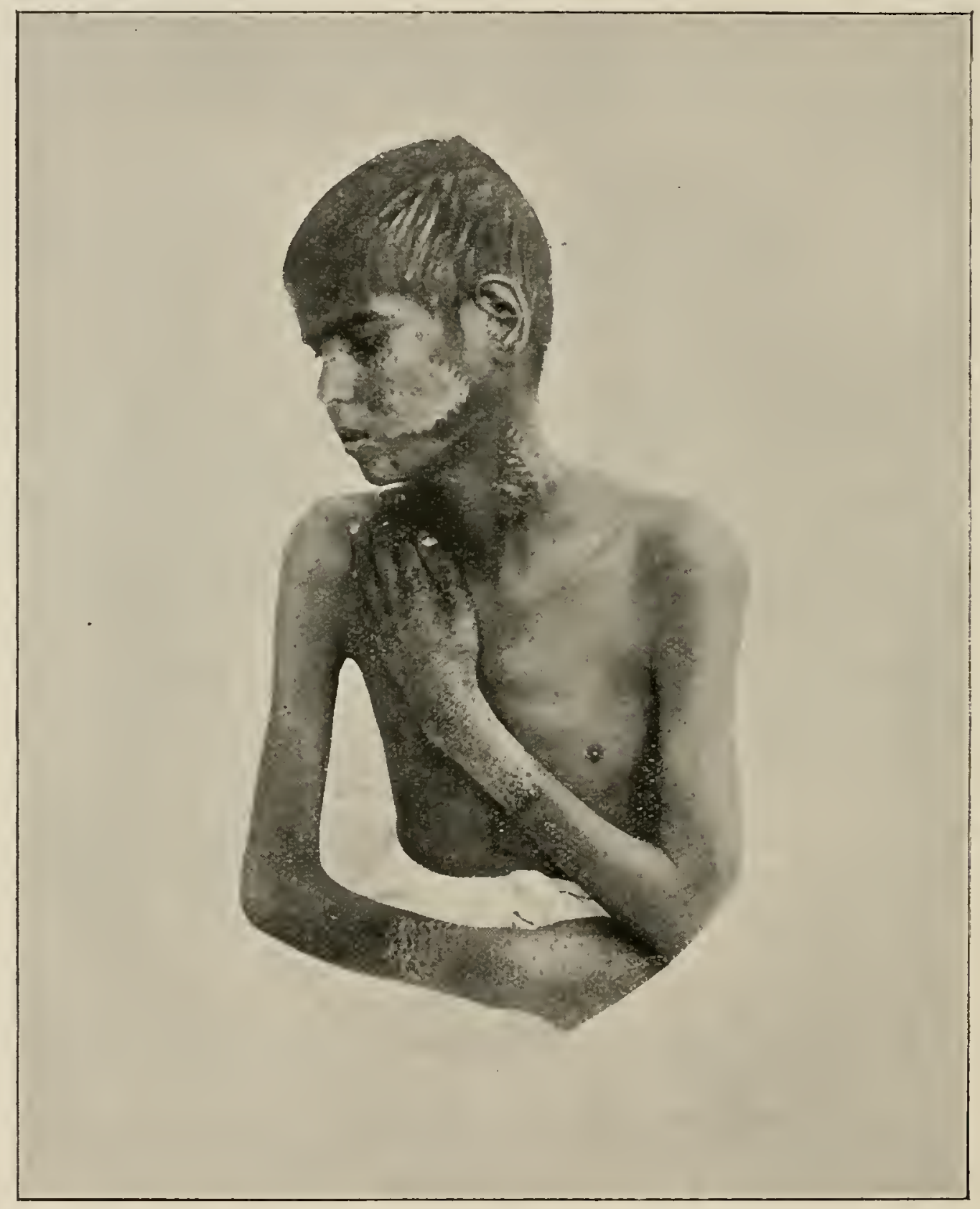

Pellagra in Egypt. Photograph by F. M. Sandwith, M.D., M.R.C.P.

becomes permanently affected, melancholic or maniacal symptoms set in. Pain about the back at either side of the dorsal spine, over spinal nerves in dorsal and lumbar regions.

K. J.'s plus later. Patient walks with his legs apart, may fall backwards or forwards

Facies of anxiety and mental worry, sleeplessness, discontent, and mental depression or excitement, and irritability or stupidity. 
There is often loss of memory. He may refuse food. Dementia may develop.

There may be ptosis, hemianopsia, diplopia, amblycpia and mydriasis.

In extreme cases the bladder is paralysed, there is profuse diarrhoea, dropsy and death. This latter in five to fifteen years from onset.

Very rarely there are acute cases lasting only a few months.

Skin eruption shows congestion, inflammation, thickening and pigmentation of a light sepia tint. Atrophic wasting.

The tongue sheds its epithelium and is known as Sandwith's bald tongue (also present in sprue and scurvy).

Blood pressure is low. Pulse-rate increased, extremities cold, skin often bluish.

Sexual power is diminished. Saltish taste in mouth common.

Temperature but little altered. Emaciation marked.

Complications and terminal affections are naturally numerous.

To sum up:-

(1) Cutaneous signs are: a remitting erythema and dermatitis of the exposed parts. The skin loses its elasticity.

(2) Gastro-intestinal signs are: salivation, stomatitis, aesophagitis, dyspepsia due to hyperchlorhydria, nausea, vomiting, diarrhoca, dysentery symptoms and constipation. Proctitis with anal excoriations.

(3) Mental symptoms are: vertigo, melancholia, myasthenia, tremblings, curious delusions, irritability.

(4) Genital symptoms are: intense vaginitis, a sero-sanguineous discharge with excoriation of surrounding parts. Mucous membrane a scarlet red like that of the mouth.

(5) Nephritic symptoms are: acute or chronic nephritis in many cases, albumin, and casts in varying proportions.

\section{DIAGNOSIS.}

From acute dermatitis as erythema solare, \&c., by the gastrointestinal and nervous symptoms.

From chronic dermatitis as eczema by pigmentation, peculiar localization to the exposed parts chiefly.

Ichthyosis is all over the body and congenital.

Gastro-intestinal symptoms as sprue by their not having an eruption.

\section{PROGNOSIS.}

Be always guarded. Early mild cases may recover if removed and placed in good suitable surroundings. 
Cases have relapsed after two to fifteen years.

It is not easy to say when a person is free from the disease, once they have been attacked by it. It is better to speak of recovery from an attack than a cure of the disease.

Recovery is more frequent and more permanent in children than adults.

The deatli-rate in the initial attack is $15^{\circ} 8$ per cent. ( 1, I So cases).

The death-rate in the recurrent attack is ${ }^{2} 2^{\circ} 3$ per cent. ( 1,1 So cases).

It is higher in Black than White races (Siler, Garrison and MacNeal).

Low blood pressure may cause death after exertion.

Typhoid complications have a high mortality.

Complications such as ankylostomiasis are always unfarourable.

\section{TREATMENT.}

There is none specific.

Protect from the sunlight.

Use soothing ointments.

Diet important, fresh fruit and fruit juice, meat broths, miik.

Avoid starchy foods.

Dilute nitric acid 1510,30 minims in four ounces of water, thrice daily, one hour before meals.

A ro per cent. Solution of trisodic citrate, intramuscular or subcutaneously.

Atoxyl 3 grains daily, intramuscularly, are good.

Liq. arsenicalis in small doses can be tried.

Radio-active serum injections are on trial.

Later one can give eggs, meats, fish, green vegetables and fresh fruit with dilute nitric acid continuously.

Remore the patient to a non-pellagrous area.

The Italian Government prohibits the sale of bad maize.

The line of treatment largely depends on the causative view held by the practitioner.

\section{DEFINITION.}

\section{BERIBERI.}

An acute or chronic, endemic or sporadic disease of unknown causation; characterized by a degeneration of peripheral nerves associated with gastric disturbances, nedema and cardiac failure.

\section{DISTRIBUTION.}

Eastern Asia, Japan, China, Philippines, Indo-China, Java, and Malaya, also in other parts frequently where Chinese and Indian 
coolies are imported. It has been reported in the Congo (Van den Branden).

$\mathrm{It}$ is the scourge of many mines and plantations in the Malay and Eastern Archipelago.

\section{HISTORY.}

Beriberi probably attacked the Roman army in Arabia (24 B.C.).

In the Straits Settlements and Malaya (population I,250,000) there have occurred 150 ,000 cases with 30 ,ooo deaths in the Government IIospital during the last twenty years (Bradden).

In the Japanese navy, prior to 1884,33 per cent. of the sailors were continuously disabled by it.

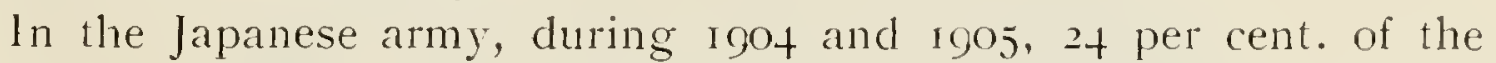
total sick and wounded were its victims.

The Government of Malaya pays $f_{10,000}$ per annum for direct hospital cases.

Labourers, sailors, soldiers and prisoners are most frequently attacked.

It is common in large cities, and overcrowding favours its spread.

It is very common amongst Mohammedans, who do not take alcohol.

\section{ETIOLOGY.}

It is a deficiency disease. It tends to be racial in young adults between fifteen and thirty, but no age is exempt.

Disturbances of the soil and high atmospheric pressure are predisposing factors. The exciting cause is unknown, but theories are legion. The chief are:-

(1) Arsenical Poisoning (Ross, I900). It is based largely upon finding arsenic in the hair of recent beriberi patients. Herzog failed to find it in ten patients.

(2) Oxalate Poisoning (Treutlein). Then oxalates were given in the food of fowls beriberi symptoms set in. The acid was supposed to remove the calcium salts and so predispose to nerve degeneration.

Excess of calcium salts was found in the urine of beriberi patients.

(3) Carbon Dioxide Poisoning (Ashmead). The result of excessive inhalation of carbon dioxide. Overcrowding alone, however, does not cause it. 
(4) Food Poisoning.

Ichthyotoxismus from eating bad fish, but those not eating fish have developed it.

Sitotoximus due to eating rice without the husks or when stale or badly kept. Some blame a parasite living in the husk which is ingested when the rice is insufficiently cooked, but it has appeared where the people never eat rice.

$a$
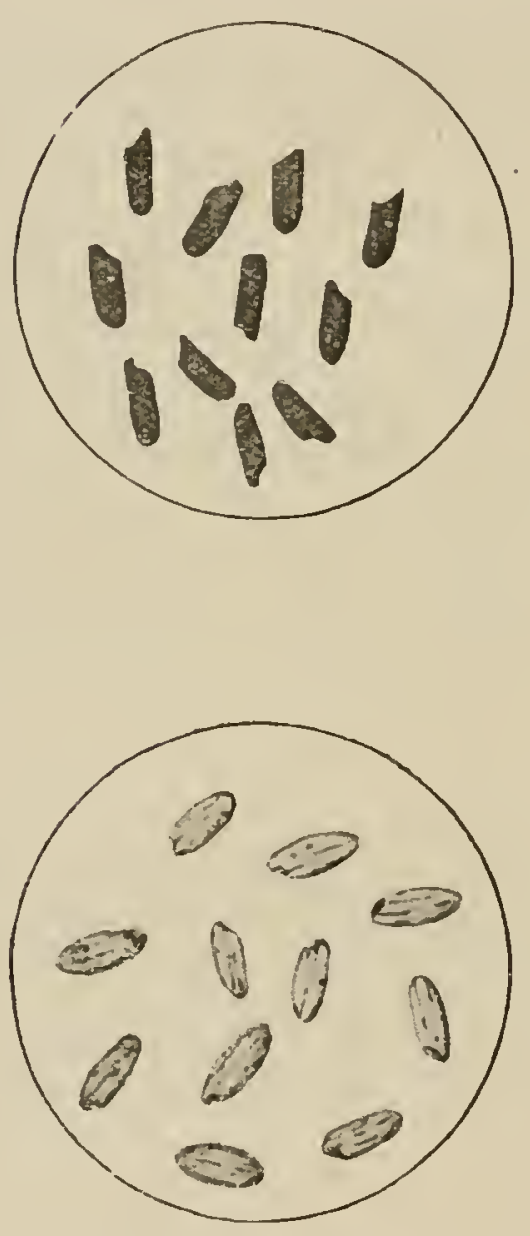
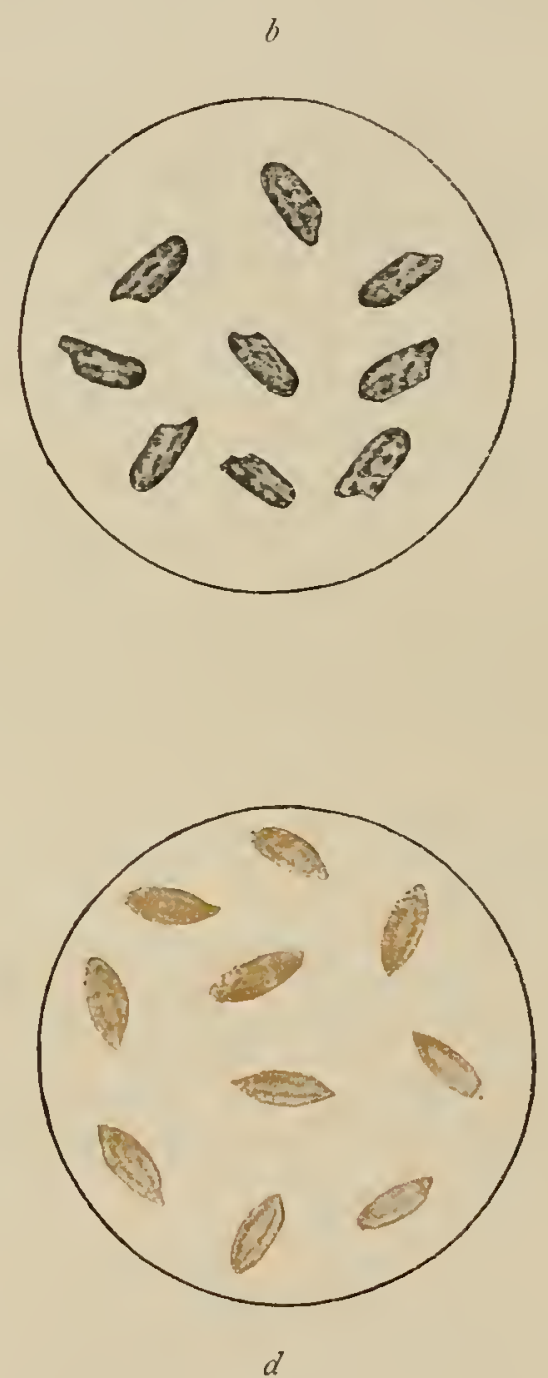

a, a highly milled or polished rice slained by Grams iodine solution. The starch, being completely exposed, stains uniformly a dark blue. This rice will produce beriberi.

$b$, an undermilled rice stained in the same way. The unstained areas show where the adherent pericarp has protected the starch from the action of the iodine. This rice will probably not produce beriberi.

$c$, a sample of undermilled rice that contains still more pericarp. The use of this rice will surely prevent the development of beriberi.

d, the unhusked grain or "palay." When the husks are removed by hand pounding, a rice like $c$ or $b$ is produced.

(5) Food Deficiency. This is the riew held most widely.

Deficienl nitrogen. The peripheral layers of the rice, lost in milling, contain a vitamine substance. This is necessary for 
the metabolism of nerve tissue, and if not supplied in food, beriberi appears (Funk).

Increase in the food of nitrogen for Japanese sailors was followed by less beriberi.

Deficient fat. An epidemic at Chaudabum was supposed to be stopped by an increase of fat in the food.

Deficient phosphorus. Normal rice contains 04 per cent. of ? pentoxide. If less it is a dangerous rice (Fraser).

Deficient iegctables with an infection. The disease disap-

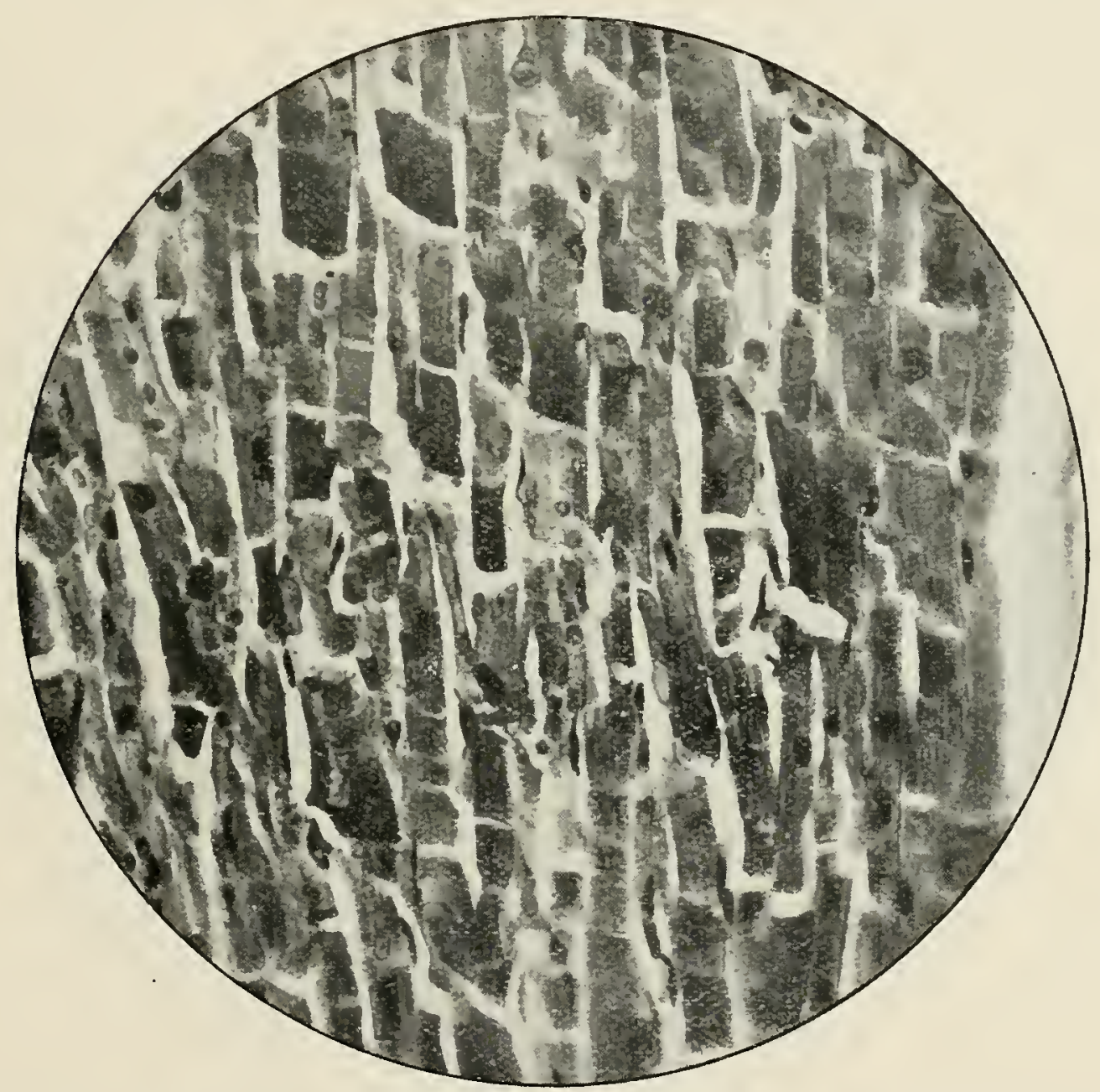

Section of myocardium from case of beriberi, showing segmentation and fragmentation.

peared when a sufficiency of vegetables, chiefly potatoes, was given (Fales).

The deficiency causes the micro-organism to flourish (Fales). Fletcher concludes that uncured rice is either directly or indirectly a cause of beriberi, the actual cause being either :-

A poison contained in the rice.

Protein deficiency.

Bad nutrition resulting from uncured rice rendering the patient liable to invasion by the specific organism. 
(6) An Intoxication (Manson). Caused by some germ living outside the body, in the soil, house or ship occupied by human beings under certain conditions of temperature and moisture; the toxin inhaled or swallowed causes the disease.

Evidence. When patients are removed from an endemic area they begin to recover.

The germ may be carried from place to place.

The same ship redevelops the disease annually on reaching the Tropics.

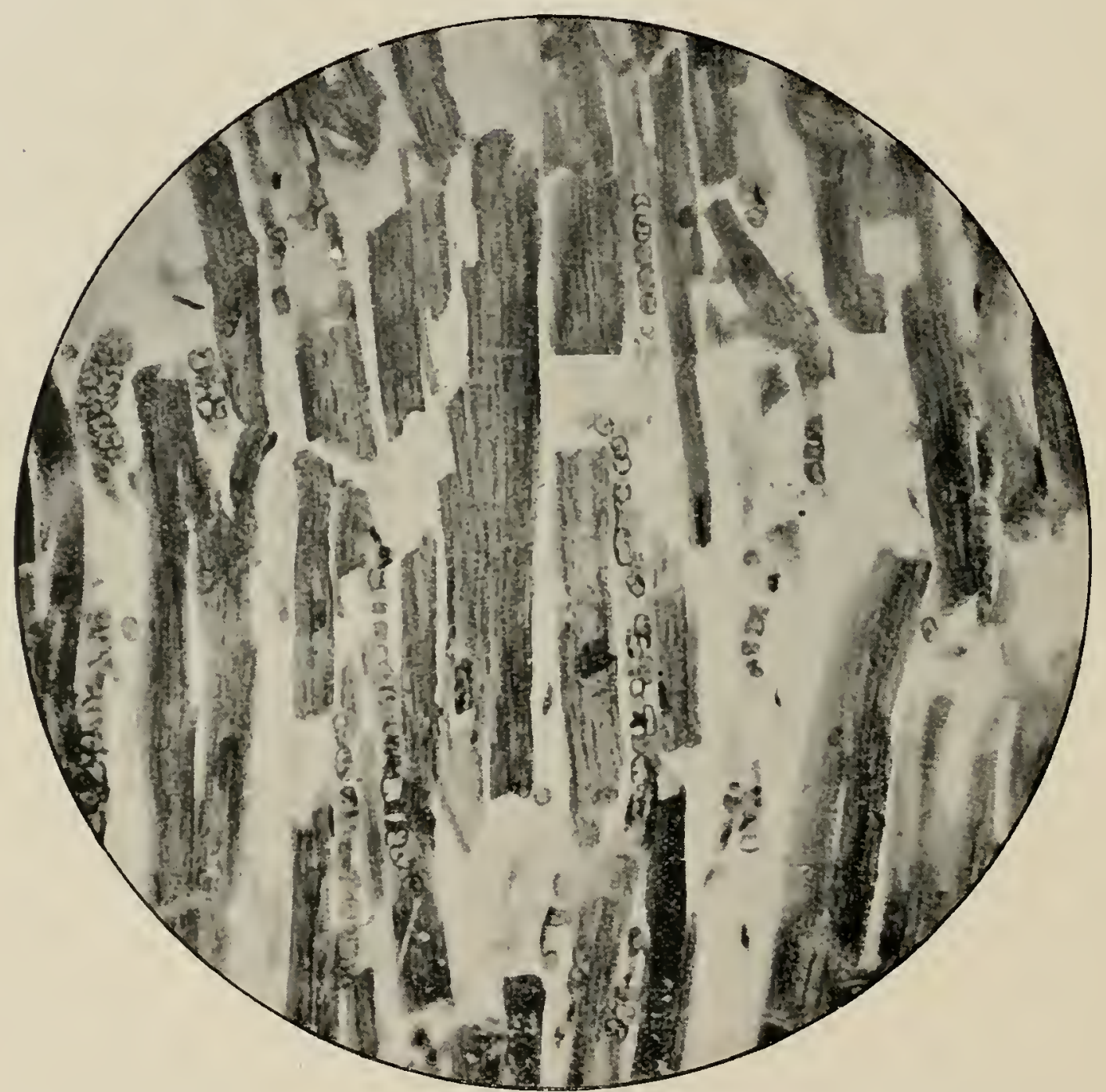

Section of myocardium from a case of acute wet beriberi, showing more advanced fragmentation. (From article by Herzog, and plate lent by the courtesy of The Philippine Tournal of Science.)

(7) An Autointoxication (Deurch).

This condition is asserted to be produced by substances formed in the body.

(8) Parasitic Causes (Sambon).

The parasite in some way enters the body.

Insects such as cockroaches may carry it.

The infection is not by the fæces (Daniels), nor by air or water. 
Perhaps an intermediate host, such as lice, is necessary (Daniels).

It may be the I'. capitis as such have a tendency to cling to one race.

Amongst the suspected parasites are :-

Various protozoa, nemathelminths, bacilli, fungi, cocci, and a specific bacillus causing duodenitis, the symptoms of this latter arising from the absorption of the toxin as in diphtheria (Hamilton IVright). Daniels denies duodenitis and thinks that perhaps a protozoan may yet be found.

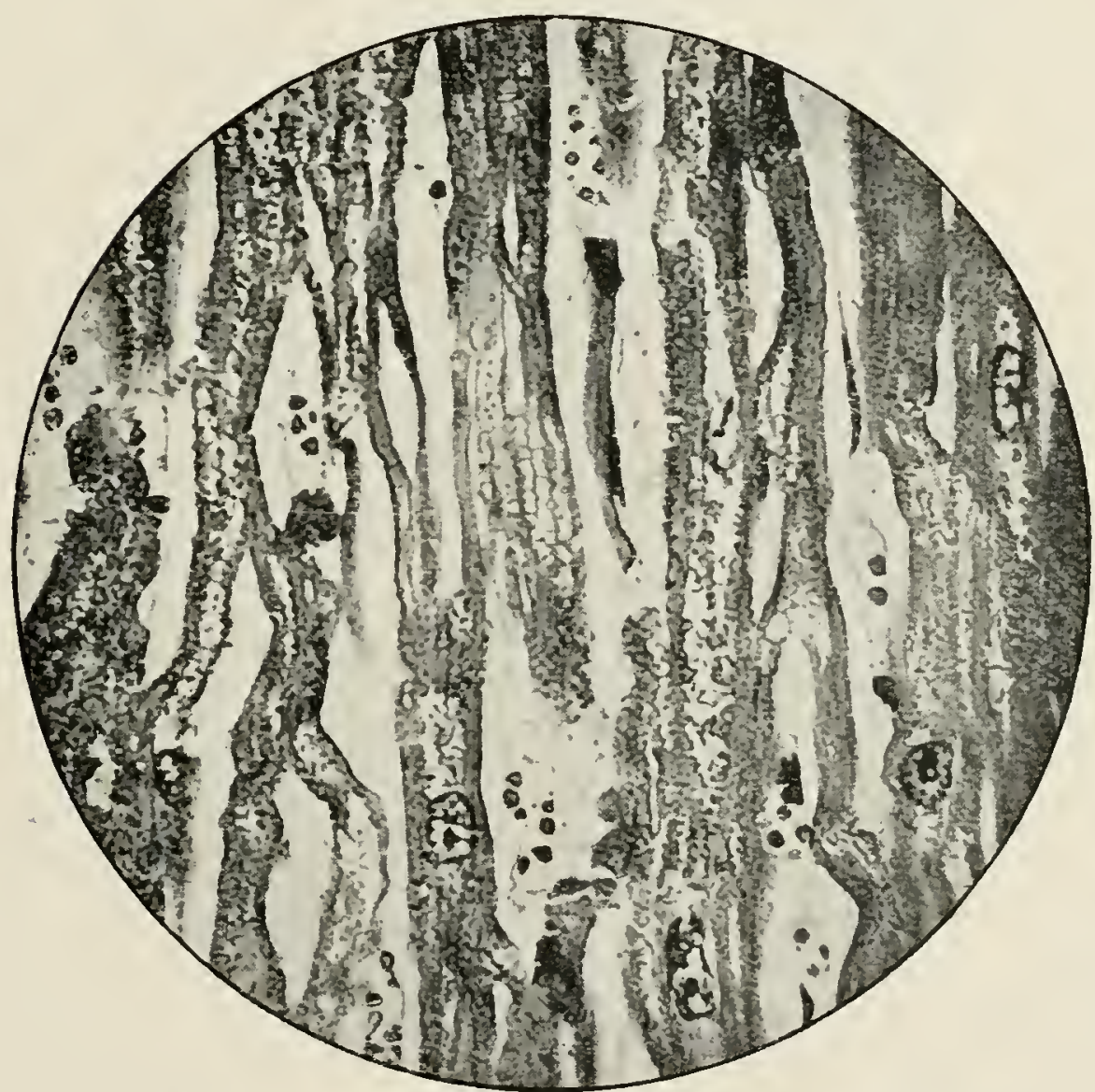

Section of myocardium from a case of sub-acute wet beriberi, hæmatoxylin-eosin strain, showing loss of striation, vacuolation and granular degeneration. (From article by Herzog, and plate lent by the courtesy of The Phalippine /ournal of Science.)

\section{PATHOLOGY.}

Blood taken from a beriberi patient and injected into a cat produced a fall in B.P., with dilatation of the gastric vessels, also those of the intestines and liver. The right heart was dilated and there was general venous engorgement.

\section{P. M.}

There is always some odema in acute cases. Often froth at the mouth. The body is either swollen with dropsy or emaciated. 


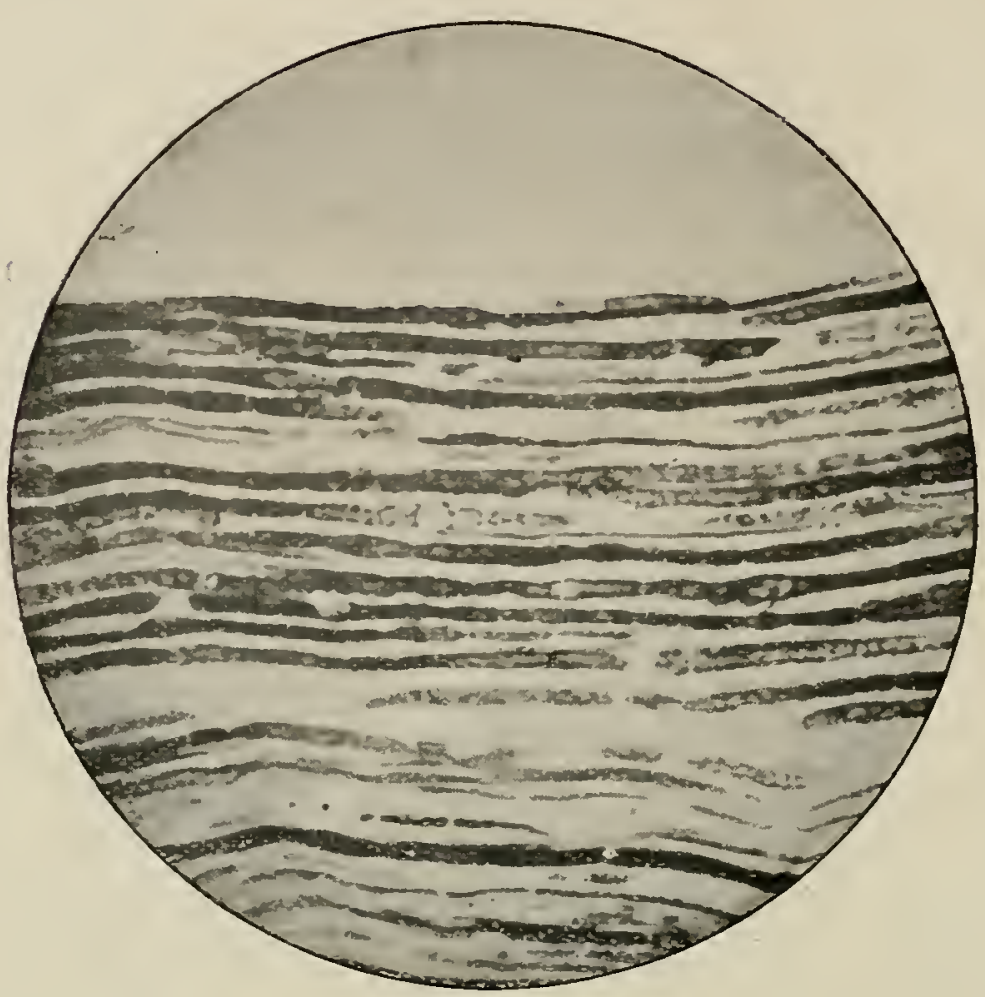

Longitudinal section of popliteal nerve from a case of acute beriberi. Weigert-I'al stain, showing different degrees of degeneration of the medullary sheath. (From Herzog, plate lent through the courtesy of The Philippine Foumal of Science.)

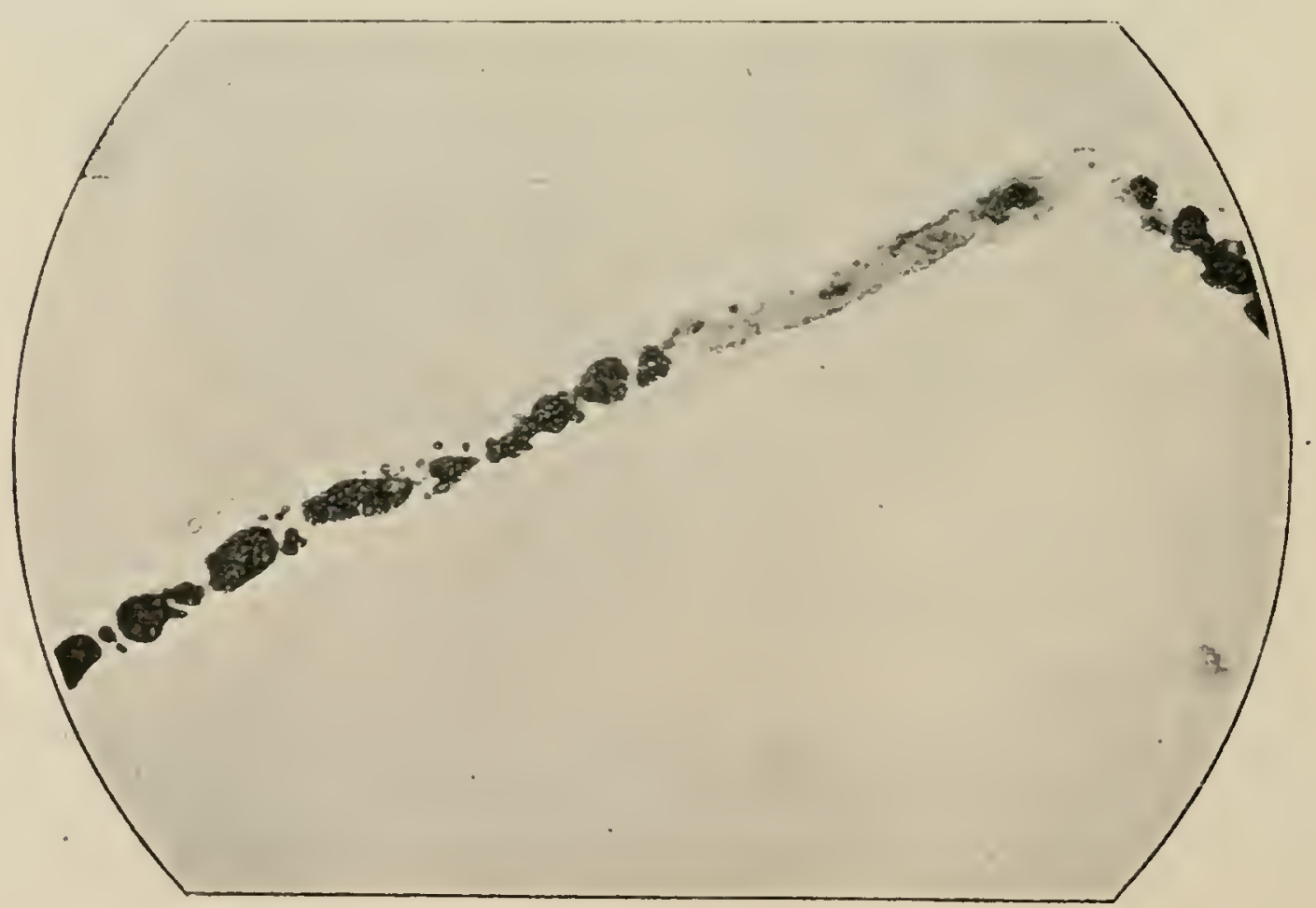

Fibre from sciatic nerve of fowl suffering from polyneuritis (Marchi method), showing advanced degeneration. (From Vedder and Clark, and plate lent through the courtesy of The Philippine, fournal of Science.) 
The subcutaneous tissues are usually oedematous.

The veins are filled with dark fluid blood.

There is serous fluid in the abdomen, chest and pericardium.

The mucous membrane of the trachea and larynx may be oedematous with the lumen full of fluid.

The lungs are congested and odematous.

The right heart is dilated and fatty degeneration is present.

The muscles show atrophied and normal fibres side by side.

There is nerve degeneration of the bulbar nuclei, thoracic ganglia, vagal nerve endings and peripheral nerves.

The congested liver sometimes shows hamorrhagic patches.

The kidneys show cloudy swelling and sometimes hamorrhages.

The meninges are hyperamic with an increase of fluid in the ventricles. There is marked degeneration in the bulb.

Thus summing up one finds:-

Degeneration of nerves.

Secondary degeneration of muscles including the heart. Excess of blood in the right heart and veins.

Serous effusions into the cavities and cellular tissue.

Oedema of the lungs.

\section{VARIETIES.}

Atrophic, Dry or Paralytic.

Hypertrophic, Wet or Dropsical.

Mixed.

Infantile.

The incubation is unknown.

\section{(1) The Atrophic, Dry or Paralytic Variety.}

Insidious onset, malaise, dull pain in epigastrium, tenderness on pressure, headache, palpitation, dyspnoea, subcutaneous cedema, K. J.'s slightly increased, sense of heaviness in the legs, difficulty in walking, K. J.'s disappear gradually. After the first week there is usually a peculiar high stepping gait, tender calves, " pins and needles," anæsthesia and paræsthesia.

The anterior tibial and peroneal muscles are chiefly affected. Sometimes the forearms are affected with diminished grip and wrist drop. There is no tremor but the patient is often too weak to hold a bowl of rice. The sphincters are normal.

There is wasting of muscles, the paralysis ascends from the calf to the thigh, gluteal region, arms, abdomen, diaphragm, intercostals and larynx.

There is loss of sense of heat, pain and cold, but the areas do 


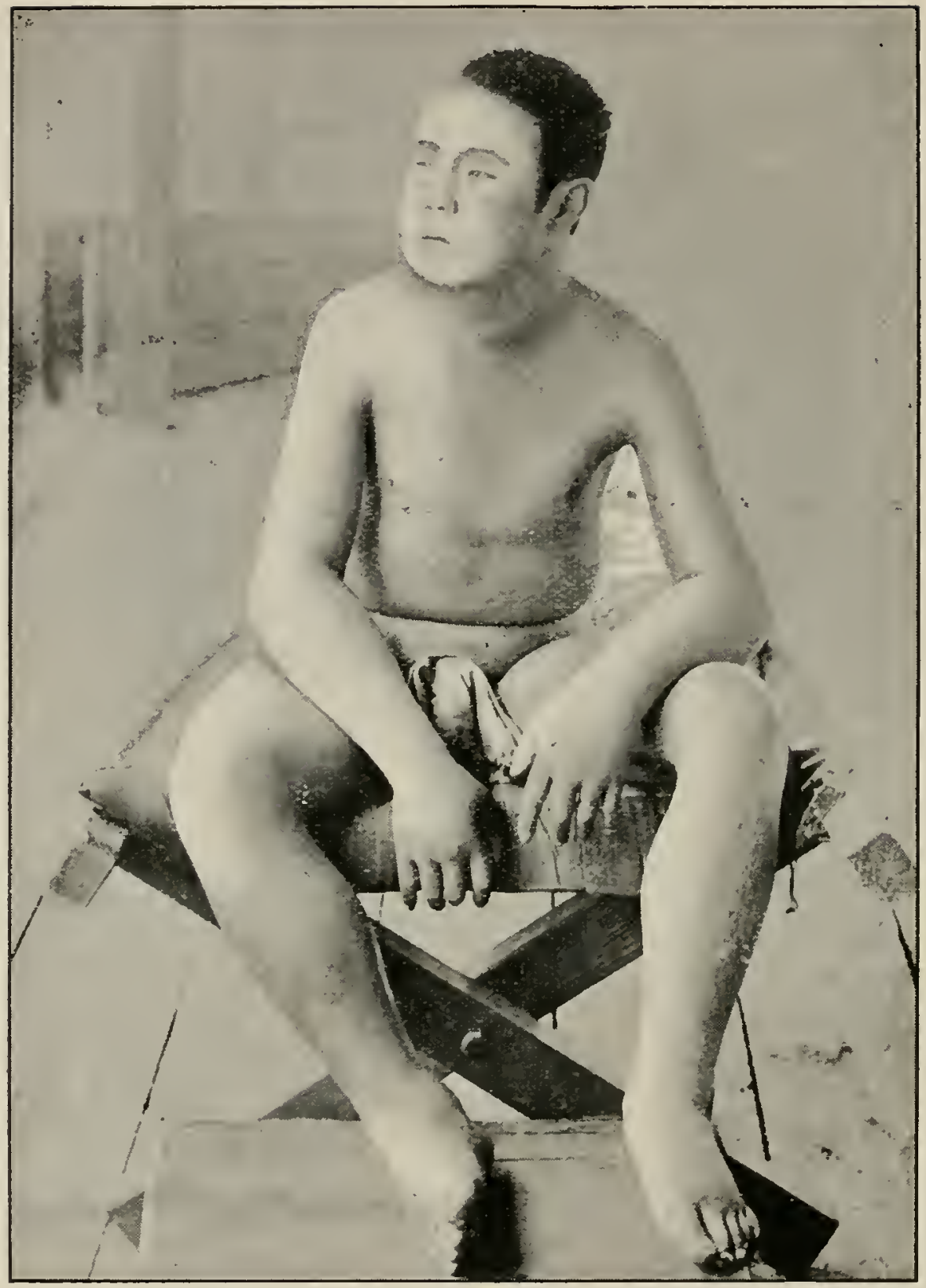

Beriberi : œdematous variety. Japanese immigrant in Fiji.

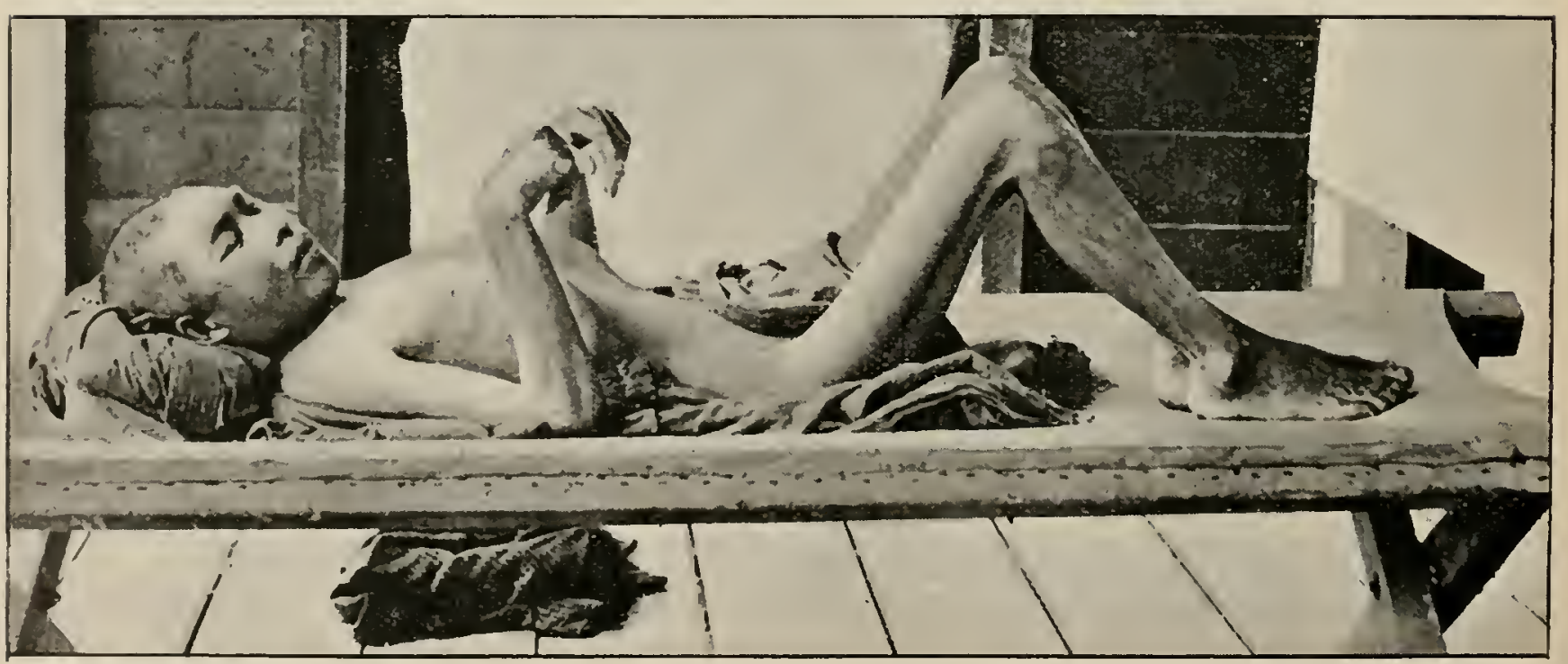

Beriberi : atrophic variety, showing muscular atrophy and drop-wrist. Japanese immigrant in Fiji. Photographs by Henry Noble Joynt, Labosa, Fiji. 
not correspond with the segmental or nerve areas. It is very patchy.

Diffuse apex beat, dilated right heart, loud systolic murmur, reduplication of the pulmonary second sound, palpitation and epigastric pulsation.

The pulse rate is increased and the tension diminished.

With increasing oedema the urine is diminished, when the cedema is clearing up the urine is increased. Urea and chlorides are diminished, phosphates and indican increased.

There is cutaneous adema of the legs, scrotum, abdominal wall, face and arms, with exudation into the abdoninal cavity, pleural sacs and pericardium.

Digestion is fair but a good meal increases the epigastric distress. The bowels are often constipated.

The temperature is normal or sub-normal.

The patient may remain like this for weeks or months, sometimes better, sometimes worse; he may recover with or without paralytic deformities or he may die suddenly.

If fever occurs it is a complication, usually T. B., malaria or dysentery.

One attack predisposes to another. The patient may die at any time during the attack.

There is lymphocytosis 30 to 68 per cent.

\section{(2) The Hypertrophic, Wet or Edematous Variety.}

This variety is usually somewhat acute.

There is nausea, epigastric pain, clean tongue, throat congested, cardiac oppression, epigastric pulsation, palpitation, dyspnoca, pulse rate much increased by the slightest exertion, hemic murmurs, right heart dilates, urine diminishes, there is effusion into the cavities of the pericardium, pleura and peritoneum.

The oedema is somewhat localized and fugitive. It is firmer than in Bright's disease.

There are patches of anasthesia and hyperasthesia in the course of the anterior tibial and musculo-cutaneous nerves.

The paralysis may be slight or extensive.

The heart becomes more embarrassed, the lungs engorged, the nostrils dilate, the mouth opens in the struggle for breath, the pupils dilate, the face becomes cyanosed, the extremities cold, the patient becomes unconscious and dies. This may take place in a few hours.

Some account for the adtma by suggesting that the patho- 
logical condition of the nerves will not permit of the regulation of transudation and absorption of fluid in the connective tissues.

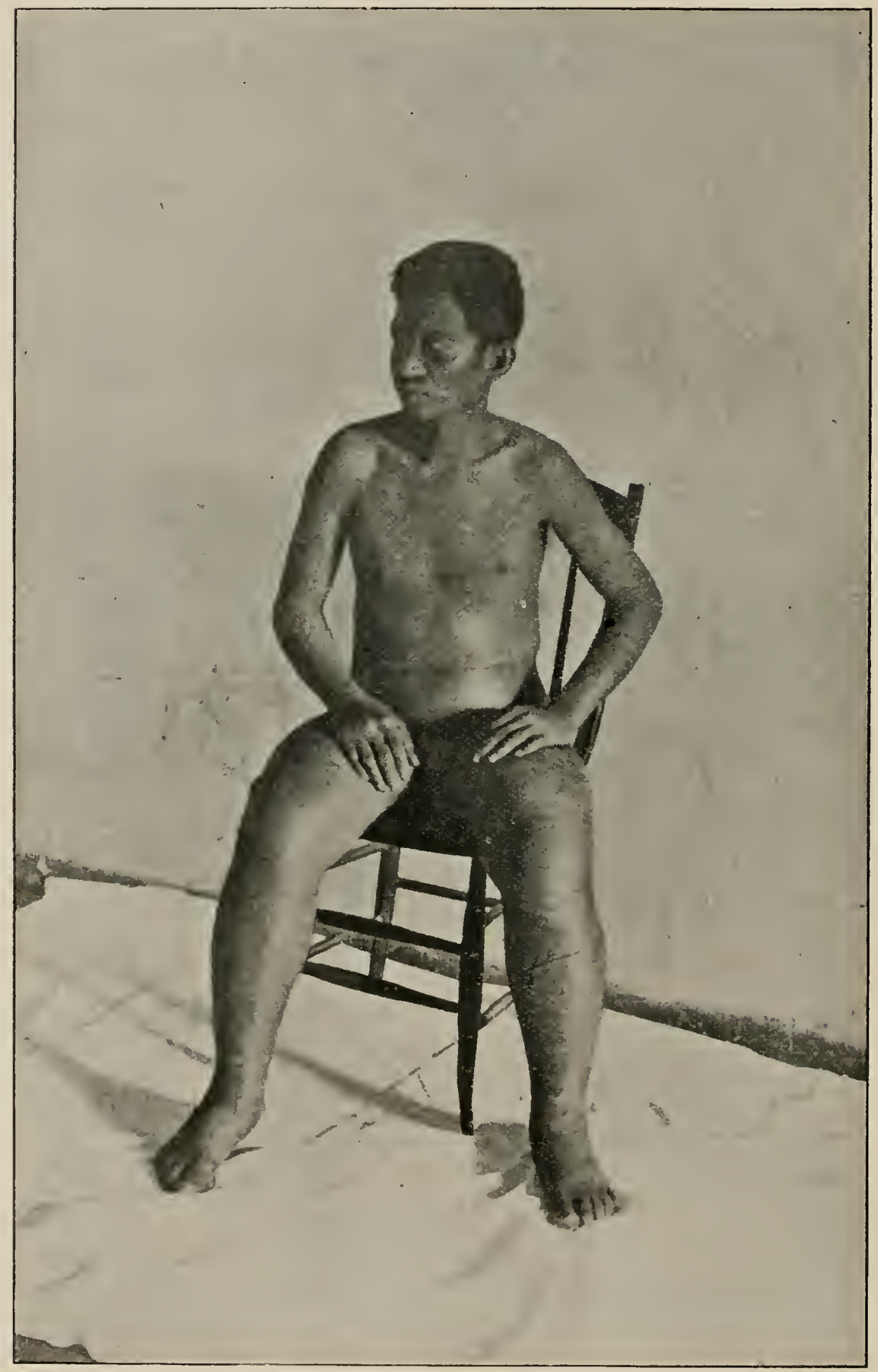

Acute wet beriberi. Great cedema of legs and feet. (From Herzog; plate lent through the courtesy of The Philippine Journal of Scitnce.)

(3) Mixed Variety.

There is odema over the shin, feet, flanks, sternum, and the root of the neck. Some hyperasthesia, paresis and ataxia. 
The K. J.'s are diminished. Heart as before. The general health is unaffected.

Some appear almost normal, others lie like logs.

Some appear to be dying one day and seem well the next.

Some are like skeletons while others are bloated with dropsy, and again others have just enough dropsy to hide the muscular wasting.

The laryngeal muscles are more often affected so that coughing is difficult. A profound diuresis converts a bloated victim into a skeleton in one day.

\section{(₫) Infantile Yariety.}

This is seen in the Philippines in the infants of beriberi mothers.

They have a defective diet and suffer from oedema, dyspepsia and cyanosis. They often die suddenly.

It causes 56 per cent. of the infantile mortality in the Philippines

P.M. shows degeneration of peripheral nerves.

Children seem to improve when weaned, suggesting that the cause may be due to cessation of absorption of milk.

\section{DIAGÑOSIS.}

\section{Look for :-}

Increase followed by loss of K. J.'s.

Patches of anasthesia or hyperasthesia on the legs.

Pain on handling the calf muscles.

Subcutaneous aedema, usually of the leg first.

There is no albumin in the urine and no fever.

In alcoholic neuritis, there is a history of habit and general trembling.

In arsenical neuritis, there is abdominal pain and diarrhoea.

In lead neuritis, there is colic and blue line on the gums.

In dropsies due to heart there is history of rheumatism or other fevers.

\begin{tabular}{|c|c|c|}
\hline , &, , & kidneys there is albumin in the urine and casts. \\
\hline & ", & ankylostomiasis there are ova in the fæces. \\
\hline , &, & $\begin{array}{l}\text { epidemic dropsy there is fever and no anæs- } \\
\text { thesia or paralysis. }\end{array}$ \\
\hline & ," & myelitis one finds relaxation of sphincters. \\
\hline & ", & $\begin{array}{l}\text { pellagra there are skin eruptions and mei } \\
\text { changes. }\end{array}$ \\
\hline
\end{tabular}

Scurvy should be excluded by the spongy and bleeding gums. 
In diagnosing the condition from epidemic dropsy note :-

(I) The Resemblances and (2) The Differences.

\section{(1) The Resemblances.}

There are epidemics in both diseases.

The K. J.'s are altered.

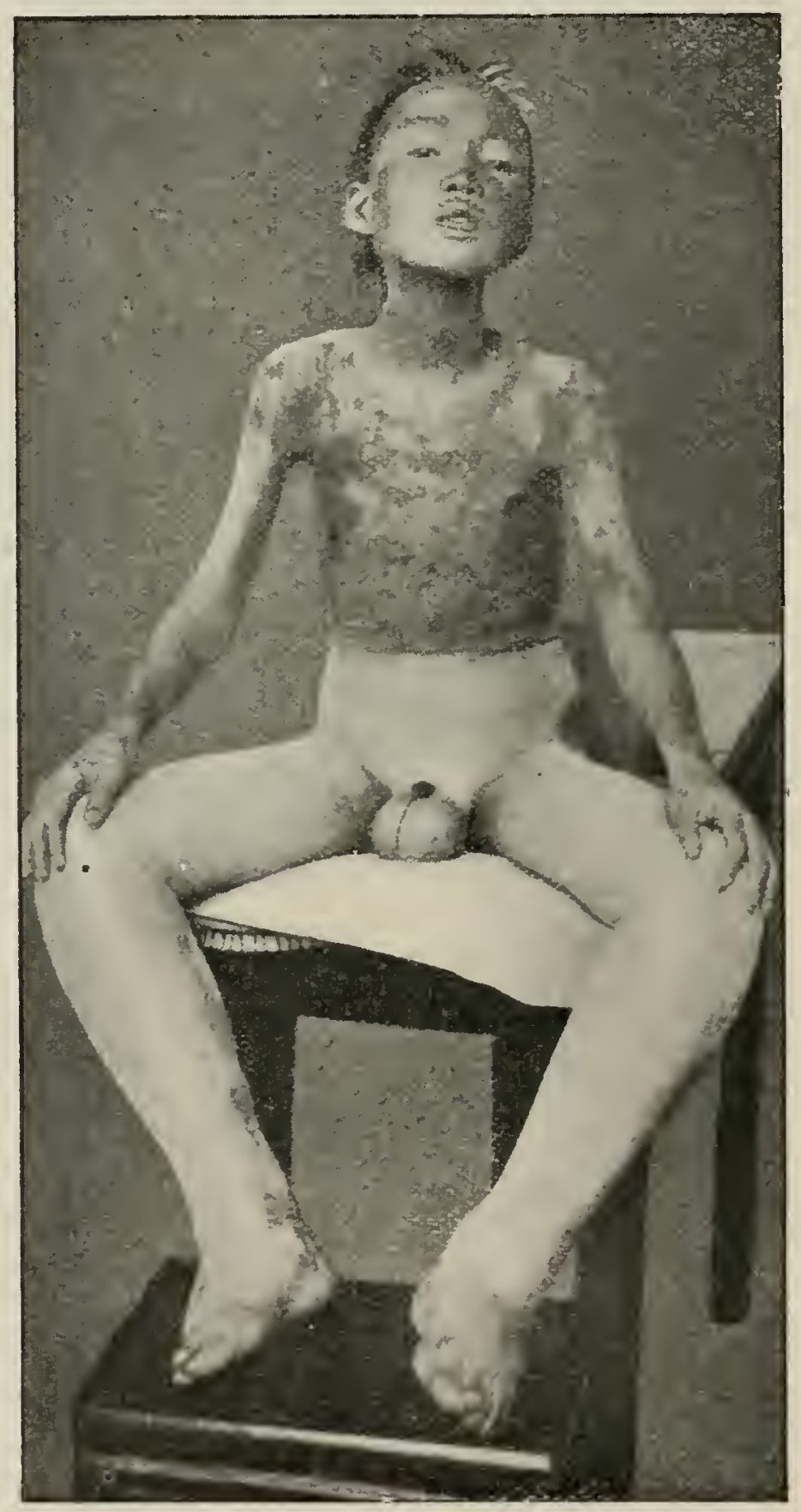

Wet beriberi. General cedema below the waist. Patient shows lypical facial expression of air hunger. Taken on admission one week before death from heart failure. (By Jefferys.)

There is a dropsical condition.

There is some cardiac disturbance.

Some disturbed cutaneous sensation.

Some hyperasthesia in both.

Death with dyspncea or orthopncea. 
(2) The Differences.

Beriberi

(I) K. J.'s painful for 24 hours, then disappear in 95 per cent. of cases

(2) Anæsthesia marked and not linited to dropsical areas

(3) True paralyses even when no swelling is present

(4) Hyperæsthesia is found in muscles on deep pressure

(5) Death is more sudden and more distressing, often in cases that have not been obviously bad

(6) There is no anæmia

(7) Initial fever, dry skin, rashes, slight desquamation, not common

8) There is peripheral neuritis but the central nervous system is not affected

\section{PROGNOSIS.}

This varies considerably and is according to the country and the type of the disease.

In Java the mortality is 2 to 6 per cent.

In Sumatra the mortality is 60 to 70 per cent.

Cardiac failure is always bad. To move much is fatal.

Vomiting is a bad sign as it indicates that the vagus is involved.

This latter feature is also manifested by the marked dilatation of the stomach and embarrassment of the heart.

Death may be due to dilatation of the heart and syncope, hydrothorax, pericardial effusion or paralysis of the diaphragm.

The dropsical variety is more serious and uncertain than the paralytic.

\section{TREATMENT.}

This is purely symptomatic.

Absolute rest in bed is essential.

Digitalis or strophanthus is good.

Amyl nitrite or trini trini should be handy.

Venesection, eight ounces to relieve the right heart when necessary.

Oxygen during dyspnoea.

For paralyses, strychnine, massage and electricity to prevent atrophy and cramps.

Removal from the supposed site of infection as soon as possible.

Atropine, one I50th to one 5oth grain, hypodermically, or tinclure of belladonna for dyspnoea (Braddon).

Plenty of good nourishment, in small quantities.

Full diet plus one drachm of yeast and $200 \mathrm{grm}$. of beans daily (Simpson). 
Yeast can be obtained as-

Export yeast, of which give 2 ounces daily.

Royal yeast cakes of 15 grm. each, give six daily.

Fresh yeast from local bakeries, 250 c.c. daily.

Pour into the yeast some boiling milk, stir it into a thin cream, add more hot milk and sugar to make it more palatable.

It can be flavoured with essence of lemon.

Foods should be given, as the patient improves, in the order mentioned, viz.: Pea soup, eggs, fresh milk, brain, liver, sweetbread, kidneys, peas, beans, lentils, porridge, brown bread, meat, and fresh lemon-juice the whole time.

An extract of the outer husk of rice after polishing daily, to infants (Vedder), in twenty-drop doses, two hourly.

Eliminate rice from the diet as a rule.

Good hygienic surroundings are essential. Little water is necessary.

Disinfect the supposed infected house.

\section{DEFINITION.}

\section{EPIDEMIC DROPSY.}

A specific endemic disease, lasting from three to six weeks with fever, profound anæmia and the sudden appearance of anasarca.

\section{DISTRIBUTION.}

In Calcutta, Assam, Daka, Mauritius.

It disappears as the hot weather comes.

In Mauritius, one tenth of the population was attacked with a mortality of 2 to 3 per cent. 729 died during the epidemic.

In Calcutta the attack was more limited, but it had a mortality of 20 to 40 per cent.

Europeans in these localities were not affected.

\section{ATIOLOGY.}

Both sexes of all ages are equally susceptible.

The disease may remain latent, breaking out later.

It is not very contagious if at all.

How it is transmitted has not been ascertained.

The causation is unknown.

\section{SYMPTOMS.}

In Mauritius the epidemic was introduced by diarrhœa and vomiting.

There is itching and burning of the soles of the feet.

Oedema was invariably present, the legs being involved first. 
It may affect the whole body.

Fever to $\mathrm{IO2}^{\circ} \mathrm{F}$. is usually present at some time during the attack. Aching of muscles, bones and joints comes on early as a rule.

Anæsthesia is not consistent and muscular paresis is rare.

An eruption, erythema of the face and rubeola of the trunk and limbs was seen in Mauritius but less so in Calcutta. It comes seven days after the odema appears and goes in ten to fourteen days.

Cardiac dilatation with the concomitant symptoms was common in Calcutta. Pleural and pericardiac effusions, oedema of the lungs with their corresponding signs and symptoms were common in all but mild cases. Anæmia, wasting and prostration was marked.

\section{DIAGNOSIS.}

See "Differences" in Beriberi, p. 349.

\section{TREATMENT.}

This is symptomatic.

Diuretics, diaphoretics and saline purges to remove the fluid.

Tapping may be necessary to relieve the lungs or the heart.

For cardiac weakness, digitalis or strophanthus.

For attacks of orthopncea, nitrites and trini trini.

Iron and arsenic during convalescence.

\section{HILL DIARRHOA.}

\section{DEFINITION.}

A gastro-intestinal catarrh occurring in high altitudes in the tropirs, with liquid, pale, frothy morning stools.

It is somewhat epidemic in the Indian Hill Stations.

\section{ETIOLOGY.}

Causation unknown.

Perhaps it is an infection, because good sanitation and good water diminishes its frequency.

THEORIES. A diminished atmospheric pressure (Crombie). Irritation by mica in the water (Duncan). Fxcal contamination of water.

Exposure to cold.

Functional digestive disturbance in the low tempirlatures of high altitudes (Bahr).

\section{PATHOLOGY.}

The mucous membrane of the stomach and bowels is congestci, there is also a proliferation of lymphoid and fibrous tissue.

There is a thick layer of mucus but no ulceration.

The tongue is seldom affected. 


\section{SYMPTOMS.}

It begins soon after arrival on the hills from the plains.

The onset is sudden with abdominal pain in the early morning and the passage of stools followed by relief. This is repeated next morning.

The stomach feels to be much distended, there is gurgling.

The patient is better on returning to the plains. In Ceylon, however, there are similar symptoms with patients when on the plains (Castellani).

The tongue and mouth are never involved.

It has a very low mortality.

\section{TREATMENT.}

The patient must avoid hills over 6,ooo feet high

Rest in bed. Warm clothing.

Liquor hydrarg. perchlor. I to $1 \frac{1}{2}$ drachms, fifteen minutes before each meal. Pepsin, 12 to 15 grains, two hours after meals.

Milk diet diluted as necessary.

\section{SPRUE (PSILOSIS).}

\section{DEFINITION.}

It is a chronic catarhal inflammation of the alimentary canal; characterized by large, pale, frothy motions; ulceration of the tongue and mouth with digestive disturbances.

\section{DISTRIBUTION.}

Asia, Burmah, India, China, Japan, Fiji; West Indies, South America, rarely in West Africa and Europe. Probably world-wide in tropical and sub-tropical countries. It is a regional as opposed to a climatic disease.

\section{EETIOLOGY.}

The causation is unknown.

Some of the suggested causes are: climate, food, helminths, bacteria, fungi, protozoa and a symptom-complex. Fungi are often found in the mouth and the intestines of patients, the commonest being the genera Monilia and Saccharomyces. They are perhaps secondary infections causing the frothy diarrhoa, because sodi-bicarb. will clear out the fungi, when the frothy stools will cease, but the disease continues.

Many fungi grow badly in alkaline media. 
Any depressing influence, intestinal irritation, or an exhausting malady will predispose to the disease. It is generally seen in old tropical residents during or after being in the tropics.

It may be a specific infection falling upon over-stimulated glands owing to certain meteorological conditions (Manson).

Intestinal fermentative changes, bacterial or parasitic in origin, may cause it in those long resident in hot climates (Cantlie).

It is a disease of the European in the tropics.

Natives are very ratrely attacked.

Both sexes of all ages and classes are subject to it.

There is some evidence of its being a direct infection.

Inoculation of sprue tongues, scrapings and stools injected into animals have failed (Bahr).

Bahr, a recent worker, submits evidence of its being a blastomycotic infection which may be summed up as follows:-

(I) Yeast cells and mycelial elements are found intracellularly in tongue lesions early, but not later, when the inflammation has subsided.

(2) Yeasts are the only organisms found in the deep layers of the tongue.

(3) The desquamation of epithelial cells and subacute inflammation of the tongue and osophagus are changes one could expect from yeast infection.

(4) Yeasts were found in intestinal mucus of sprue, but not in other cases of chronic diarrhoea.

(5) The stools are such as one would expect in yeasts.

(6) Its relapsing nature is compatible with the life-history of the Blastomyces.

(7) The sprue yeast is apparently identical with the thrush fungus.

(8) Wasting, andemia, and degeneration of hepatic capillaries and endothelium, the symptoms of sprue, are reproduced by intravenous injections of broth cultures of a pathological veast. in animals.

(9) Similar sprue symptoms are found in infants, the subjects of thrush infections.

(Io) The scattered portions of the digestive tract affected by this fungus would best explain the varying clinical manifestations of sprue.

(I ) The local tropical conditions favour a luxuriant growth of fungi.

Dold in examining sprue stools for yeast found that-

Normal stools yielded yeasts in 7.5 per cent. of cases.

Diarrhnea stools (not sprue) in 16 per cent. of cases. 
Fæces from cases of sprue in $92^{\circ} \mathrm{I}$ per cent. of cases.

Either Blastomycetes or Oïdia or both were present in the latter.

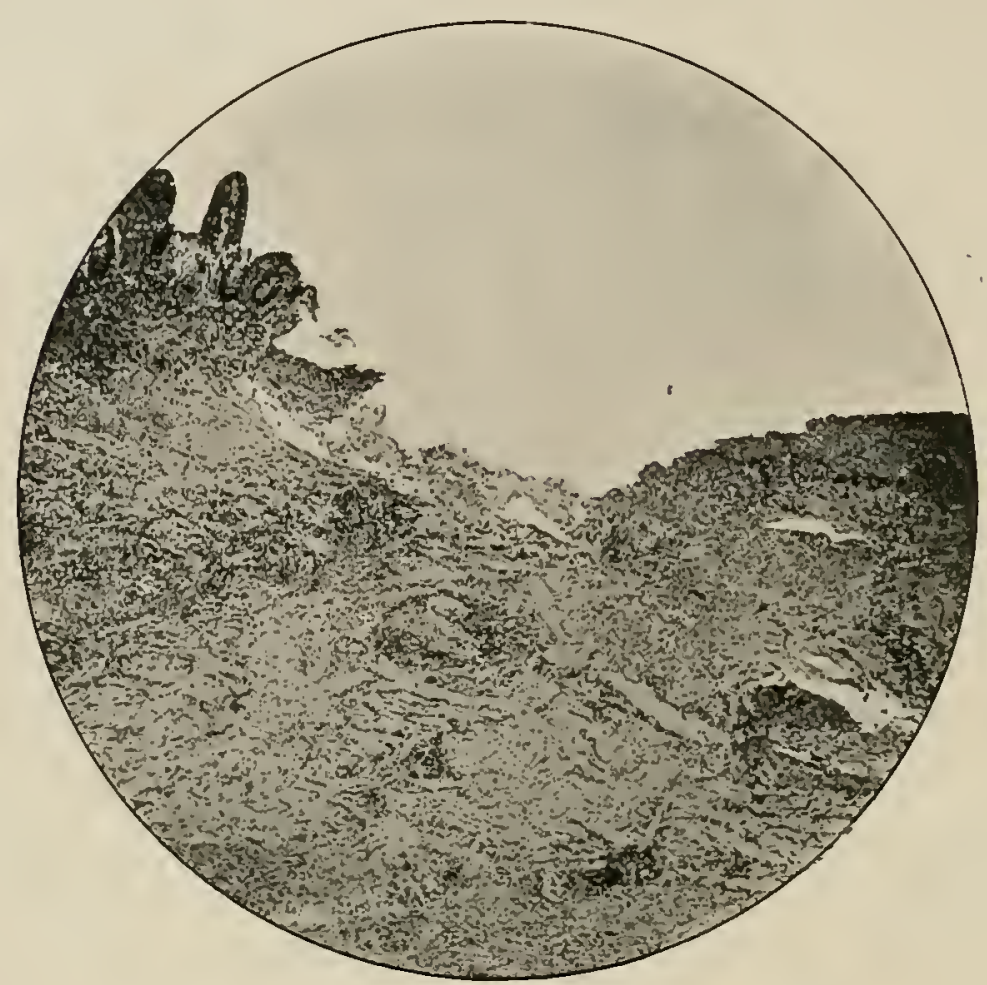

Sprue. "Small" ulcer of intestine. $\times 55$.

Dense infiltration of submucosa by embryonic cell growth. Invasion of villi and crypts of Iieberkiihn with subsequent destruction of mucosa.

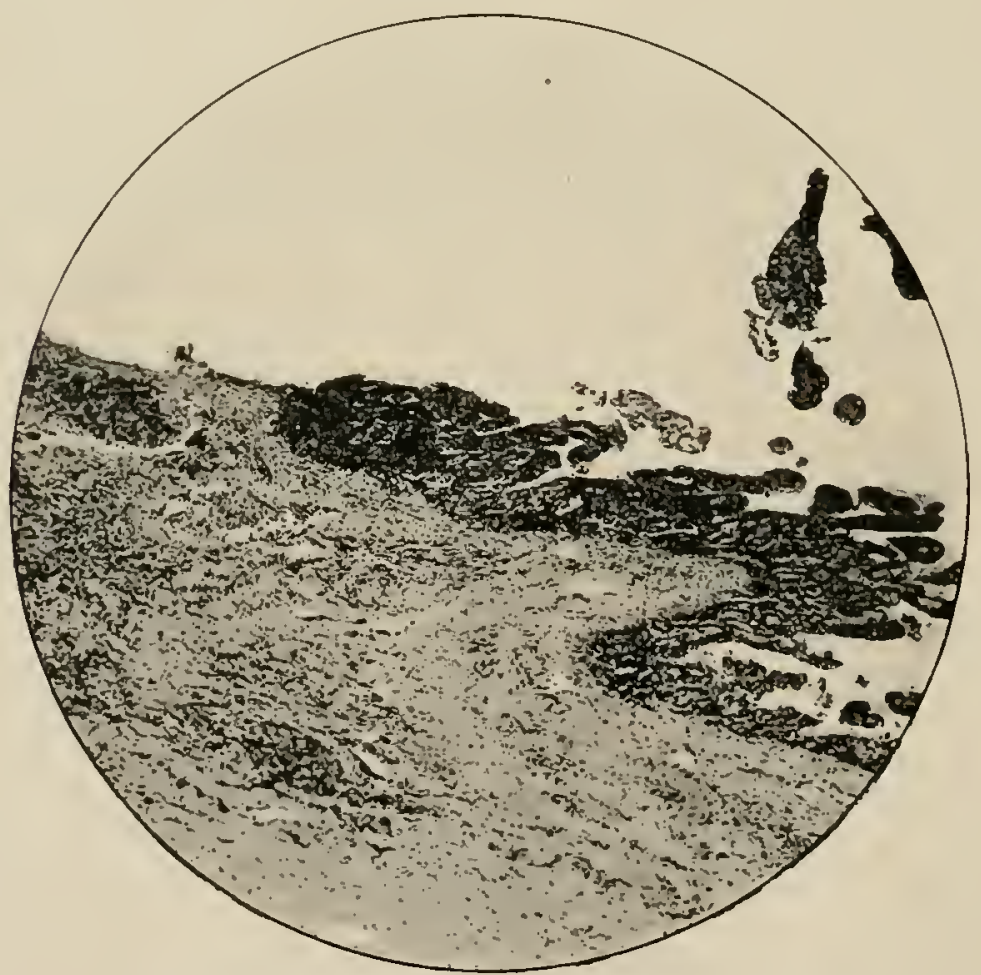

Sprue. Edge of "small" ulcer. $\times 55$.

Destruction of mucosa by infiltration, and subsequent shedding of epithelial structures.

They gave acid and gas reactions on all kinds of sugars, which explains the acid reaction and the gaseous character of the sprue stool. 
The liver is diminished in size due to carbohydrates being quickly decomposed in the digestive tract by the specific orginisms of sprue,

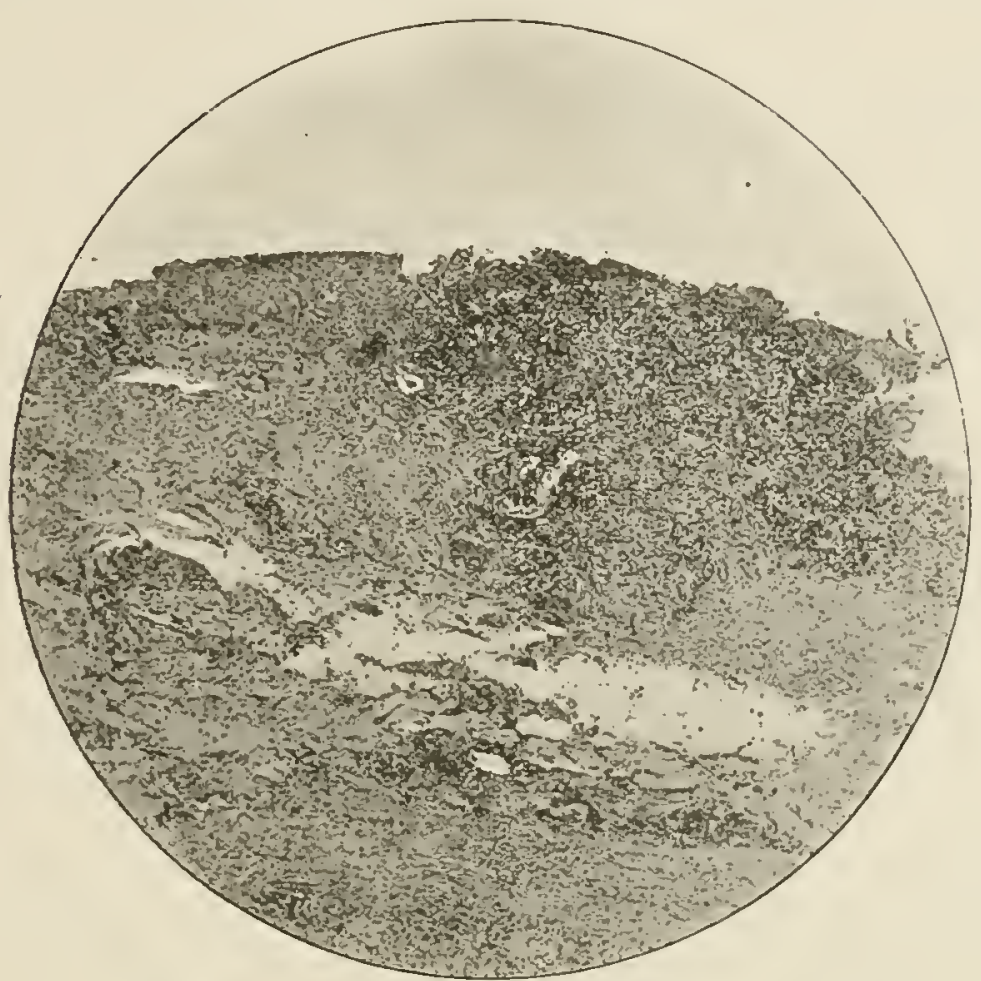

Sprue. Base oi small ulcer of intestine. $\times 55$.

The mucosa has been shed, and the dense infiltration of the submucosa is causing it to break down and come away as a necrotic slough. The lymphatic vessels in the centre of the infiltrated portion are full of pus.

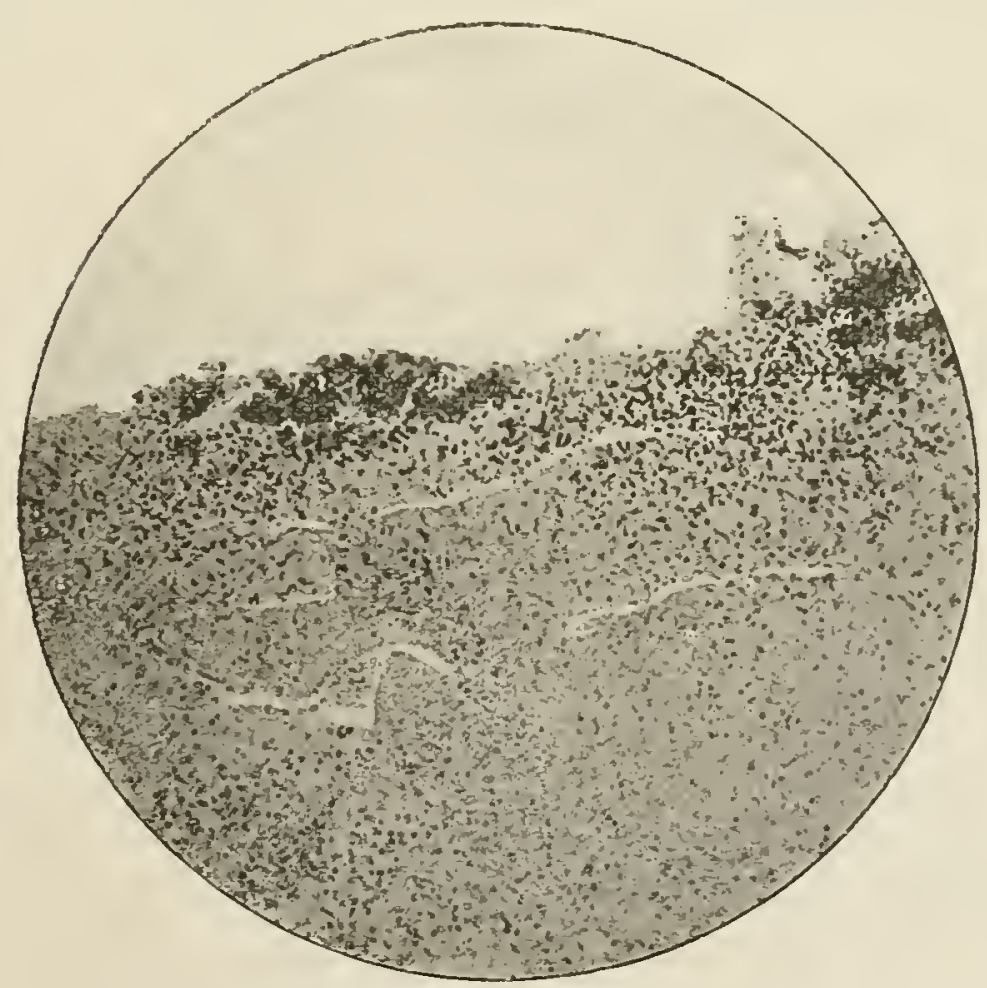

Sprue. Base of small ulcer in intestine. $\times 350$.

Dense infltration and formation of pus in superficial layers.

leaving little or none to be absorbed and deposited in the liver as glycogen . 


\section{BuT}

The thrush fungus, Monilia albicans, is a terminal though uncommon infection in other chronic wasting diseases as phthisis, cancer, diabetes, \&c.

Gastro-intestinal infections have been reported in the temperate zone.

\section{PATHOLOGY.}

One finds desquamation of the mucosa of the gastro-intestinal tract with atrophic changes of mucosa, glands, pancreas and liver.

There is fatty degeneration of the tongue; inclusive elements in the epithelial cells is a well-known feature. There may be large structureless cells, keratohyalin or other cells containing granular agglomerations, cell degenerations or inclusions.

The gastro-intestinal tract shows submucous congestion, congestion, thrombosis, exuclation of hamoglobin, round-celled infiltration and necrosis of the mucosa. Digestion and absorption of food is hindered, but the absorption of poisons facilitated. The toxins damage the liver and a toxamia results. The liver is congested and enlarged, or more often atrophied and small, the result of hyper-activity and early exhaustion, which accounts for the early bilious stools and later the pale frothy motions.

On opening the intestines-

The upper third contains normal faeces of a yellow colour due to bilirubin.

The middle third contains white or grey faces due to alteration of bile pigments into chromogens.

The lower third contains green fieces (Vaughan Harley).

The blood picture in the last stages of severe cases may resemble that of pernicious anæmia. Chromatin granules are commonly seen enclosed in red cells.

\section{P.M.}

Body emaciated, wasting of all organs, absence of fat. Skin hangs in loose folds, aedema of ankles. Tongue shows patches of infiltration in the connective tissue, resicles, small ulcers, filiform papillae atrophied and fungiform papille swollen. Pillars of fauces and tonsils show sub-epithelial inflammation, suppuration and ulceration.

Esophagus inflamed, mucous membrane attenuated in patches.

Stomach: mucous membrane pale, atrophied, rough and cirrhotic.

Small intestine: mucous membrane somewhat eroded. I.arge intestine ulcerated.

Peritoneum thickened, chronically inflamed. 
Bowel wall rery thin, sometimes like tissue paper. Mucous coat atrophied, submucous coat has fibrosed areas with dirty grey tenacious mucus. The villi and glands destroyed. Erosions are most marked in the colon.

In the swollen endothelial cells of the venules of the spleen are certain hyaline bodies, probably of a degenerative nature not found in other cliseases, perhaps produced in response to the extensive yeast infection found in sprue. These reasts are small, Gram-positive, and have no affinity for fuchsin. They are not Russell's bodies (Bahr).

\section{SYMPTOMATOLOGY.}

Insidious onset. Sore mouth, dyspepsia, diarthoea, tongue inflamed but clean with small vesicles, ulcers and bare patches, ditto on palate, pillars of fauces, inside of cheeks, acid salivation.

Warm and spiced foods, hot, acid, or alcoholic drinks cause pain.

Mucus accumulates about the mouth. Though hungry the patient eats little.

There is a burning pain behind the sternum on swallowing; the neck and thorax are emaciated, the abdomen is distended with gas, and the ankles with odema.

There is discomfort and distension after meals, acid eructations, and sometimes romiting.

In the early morning there are several copious, painless, grey offensive, frothy stools.

The faces contain mucus, epithelial débris, many bacteria and fungi.

The nitrogen and fat are increased (undigested food and falty acids).

Often the hydrochloric acid of the sastric juice is diminished or absent (Manson). 'The same applies to the intestinal digestive juice.

The blood coagulates slowly. There is anamia, I,ooo,ooo2,000,000 reds.

The colour index is low. leucopenia to 3,000 .

Depression and irritability; tongue glazed, small and fissured, is more like cartilage.

Pulse slow and feeble. Emaciation.

An acute diarrhoa or cardiac failure may cause death.

The symptoms are often intermittent, but steadily growing from bad to worse. The disease may be mild or virulent, liable to sudden remissions and latent periods.

A clenuded tongue makes mastication difficult.

.. ocsophagus makes swallowing painful.

., stomach causes dyspepsia.

., intestine causes diarrhnea. 
The stools are pale because of-

(1) The farinaceous diet.

(2) A colourless reduction-product of hydrobilirubin.

(3) The abnormal percentage of contained fat.

The stools are large owing to

(1) The lack of absorption of carbohydrates, \&c.

(2) The lack of digestion

The acid reaction is due to lactic and butyric acids (not to $\mathrm{HCl}$ ), and the absence of pancreatic ferments.

Cammidge's urinary reaction is negative (Bahr).

\section{DIAGNOSIS.}

An irregularly, chronic, pale, frothy, morning diarrhoea with ulceration of the mouth and flatulent dyspepsia in a tropical resident is in all probability sprue.

In Stomatitis the characteristic stouls of sprue are not present.

In Thrush, look for the fungi Monilia in the white patches of the tongue. This condition, however, may complicate sprue.

In Hill Diarrhoea the mouth symptoms are often absent. There is a history of living at an high altitude.

In Chronic Dysentery the mouth symptoms and pale stools are absent, while blood and mucus is passed. There is usually no griping with sprue. Look for amobe and the dysentery bacillus.

In Chronic Pancreatitis the mouth is normal, there is much fat in the stools, and there are Cammidge's crystals in the urine.

Remember that the denuded epithelium of the tongue is only seen in Sprue, Pellagra and Scurvy.

\section{TREATMENT.}

The patient must assist the practitioner. His co-operation is essential.

Absolute rest in bed. Avoid chills. Remove all supposed causes. Clear the bowel contents with castor oil.

The diet is all-important.

\section{Milk Diet.}

The milk must be free from contamination and adulteration.

If it contains much fat dilute it with whey made with the juice of limes. Sterilize the milk.

If the attack is severe begin with whey only, ad libitum, $;$ to $S$ pints daily.

Milk, sipped, three to four pints daily as soon as possible.

As the symptoms improve increase the milk gradually by half a pint daily until seven pints are being taken. Let it be distributed over twelve meals daily. 
For six meels there should be no other food.

The patient may then get up.

If the symptoms do not improve reduce the amount of milk and return to whey. Starvation diets are dangerous.

Improvements are noted by the stools becoming brown and the mouth troubles diminishing.

Should the milk cause romiting add sodi-bicarb. or sod. citrate or a little lime water.

Relieve constipation by enemata.

In addition to the milk some recommend fruit, such as strawberies, apples or bananas.

Eggs in milli can then be given, chicken broth, Benger's food, fish, biscuits and potatoes.

Relapses are common. Give a purge at once and begin with milk.

Some give more fruit than milk. Fruit which is not acid should be given, such as peaches, pears and bananas.

\section{It is necessary to ayoid:-}

Dark meats, most regetables, spiced foods, cold or hot drinks, all indigestible substances, all alcoholic drinks and smoking.

\section{Meat Diet.}

Six ounces of raw meat juice with a little dilute hydrochloric acid and salt.

Give one teaspoonful each fifteen minutes, rapidly increased when possible. Then meat, free from fat, cartilage or fibrous matter, take 2 lbs. with 2 ounces of fresh suet, mince and pound, cook, and give the whole in six separate meals daily.

A little water or tea with lime juice instead of milk should be taken twice daily for six weeks. "(The Salisbury cure.)

In seven days introduce fruit, chicken, eggs and fish.

Some add curdled milk.

\section{Cantlie's Treatment.}

Put the patient to bed. Apply hot wet packs from the nipples to the groin for two hours morning and evening.

A binder around the body.

Three meat meals daily of 5 ounces of meat with beef-tea, calves'foot jelly or plain jelly and salt to taste, two-hourly.

Castor oil, $1 \frac{7}{2}$ ounces, each morning for three mornings.

Santonin, 3 grains, morning and evening.

Strawberries, 3 lbs. to 4 lbs. daily between meals.

When the stools are fairly solid, about the fourth day, add poached egg and minced chiclien. Tegetable marrow later, and 
thin slices of bread baked in an oven for twenty minutes.

Give milli every fourth day for wenty-four hours.

\section{Drugs.}

Astringents are dangerous, antiseptics useless.

Yellow santonin, 3 to 5 grains, twice daily in olive oil.

If the santonin is white expose to the sun for a few days.

Ipecacuanlia, 20 grains daily, for three days in severe cases (Cantlie).

To be discontinued when the stools are brown and free from odour.

Paint the mouth with glycerine and borax; for pain, spray or paint on 2 to 5 per cent. cocaine or stovaine.

Give morphia for painful aesophagus.

Adrenalin, 5 to ro minims ( 1 in 1,000), gives immediate relief.

For muscular pain, massage and pilocarpine, $1 / 10$ th $\mathrm{gr}$. to $\mathrm{r} / 5^{\text {th }} \mathrm{gr}$. thrice daily.

For acute diarrhoea, opium or lead and opium pills.

Send the patient to a temperate zone as soon as possible.

Alkalies diminish, acids increase the srmptoms.

Cantlie sars that milk clots in the acid fermentation, the patient absorbing only the whey.

Emetine and streptococcal vaccines can be tried, the latter prepared from the mouth lesions (Rogers).

Good results have been obtained with the following medicinal remedies :-

Large doses of pancreatin, 2 grm. daily, dilute hydrochloric acid, calcium carbonate and a little tannic acid. The pancreatin was continued for months with good effects while being taken, and slight relapses when it was omitted.

Some claim good results from Monilia raccines. Six injections of the emulsion, eight to fourteen days apart, the primary dose of 0.05 C.c. increasing to a final dose of 1 to c.c.

Of 62 patients thus treated, 49 were cured, I 2 improved, and I died (Michel).

\section{PHIEBOTOMLS FEVER (THREE-DAY FEVER).}

\section{DEFINITION.}

An acute specific three-day ferer of unknown causation, spread by the Phlebotomus papatasii.

\section{DISTRIBUTION.}

Around the Mediteranean and Adriatic seas, India, Egrpt and South America.

".'It agrees with the distribution of the P. papatasii. 
It is not found on the heights. It confines itself chiefly to inhabited places (Doerr).

It is a disease of the summer. 'The fly dies out in winter, but perhaps the infection is continued by the egg.

\section{ETIOLOGY.}

An unknown virus is infective during the first and up to the end of the second day of the fever only.

The Pasteur-Chamberland filter keeps back the virus.

The carrier, 1'. papatasii, is not infective until one week after it has itself become infected, hence some organism develops in the fly.

The infection nlay be transmissible to the young broods.

An antibody is probably produced. Serum from convalescent patients may neutralize infected serum.

\section{SYMPTOMS.}

Incubation one to seren days.

Sudden onset, as a rule, like most fevers with chilliness, severe frontal headache, pain about the eyeballs, general body pains, injected conjunctiva.

Skin hot and dry, temperature rises rapidly to rof ${ }^{\circ} \mathrm{F}$. in twentyfour hours, pulse 100 to 116 , often slow. Ocular movements painful. Patient irritable, sleeplessness, appetite lost, taste impaired, epigastric pain. Diarhara sometimes, congested mouth and throat. Pain in joints, knee and elbow especially. Sensation of burning in the palms and soles. Leucopenia + ,ooo to 5,00o. Sometimes a general erythema. Temperature falls in thirty-six to forty-eight hours, slowly with (often) epistaxis, sweating, romiting, diarrhoea and weakness.

Convalescence is long. No death has been recorded.

Relapses are common. Perhaps there are no reinfections.

\section{DIAGNOSIS.}

The short duration, three days, is about the only diagnostic feature.

From Influensa by there being no catarrhal symptoms with Threeday fever.

From Dengue by the short duration only.

From Malaria by there being no parasites.

From Malta fever by the duration, sudden onset, absence of organisms in the blood and negative agglutination test.

\section{TREATMENT.}

Rest in bed. Saline purges. Aspirin. Tonics later. Prophylaxis as in Malaria. 


\section{THE PARASITE.}

Family : Psychodicle.

Sub-family : Phlebotominæ.

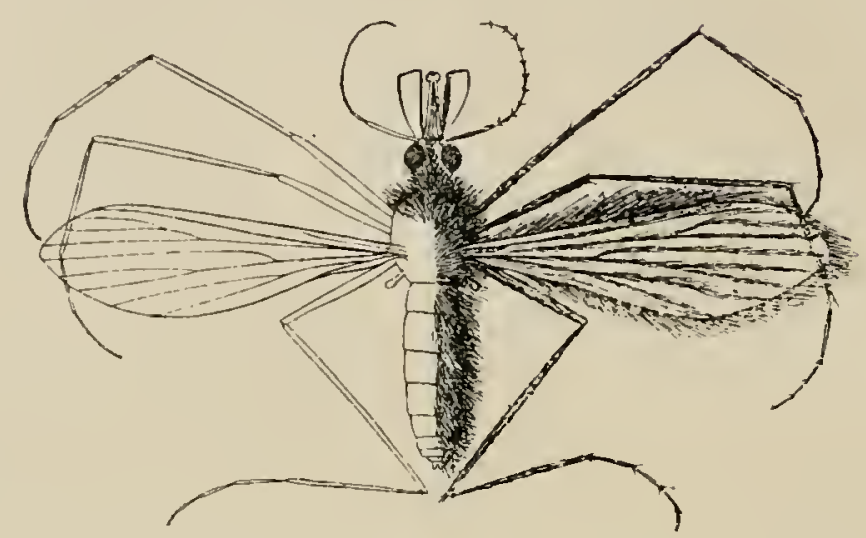

An owl midge, Phlebolomus sp. Greatly enlarged.

(From Giles's "Gnats or Mosquitoes."

Genus : Phlebotomus.

Species: P. papatasii.

\section{Characters.}

Small wings, hairy, second long vein twice forked. Legs long, slender, densely covered with scales. Abdomen of ten segments, the last carrying the genitalia. The oesophagus divicles into two, one to the sucking tube, the other to the mid-gut.

\section{Life-History.}

The female after fertilization sucks blood, lays zo to So eggs, singly, in damp places such as fissures in stones and bricks. They hatch in four to six days in warm, twelve to fourteen days in cold weather.

The larvæ have no eyes, well developed mouth parts, body of twelve segments, spiracles on the first and penultimate segments.

Two very long bristles on two tubercles on the ultimate segment.

This is characteristic. These are as long as the body. Larval stage lasts two to fourteen days. Pupe motionless, wrinkled brown skin on the last three segments which fixes it to stones in clark crevices.

The pupal stage lasts eight to twenty-eight days.

The whole time required is from one to two months.

\section{Habits.}

Nocturnal, attracted by light. During the clay it is found in shady places, latrines, under stones, \&c. The female only bites.

The fly passes through the ordirary mosquito net.

Spider webs are useless as they do not catch them.

The bite is shar 3 , it leaves a rose red papule set in a reddish macula. It itches for days.

Sunlight drives the fly away. It is not a house-fly. 


\section{DEFINITION.}

\section{VERRLGA PERUVIANA.}

It is a chronic, endemic, non-contagious disease of unknown origin, resembling yaws. It is characterized by irregular fever, associated with theumatoid pains, ancmia and granulomata of the skin, mucous membrane and riscera.

\section{HISTORY.}

It was in South America before the Spaniards. It was recorded there in 1543 .

In $188_{5}$. D. A. Carrion, a student of medicine at Lima, desiring to know if a fever then present was related to verruga, vaccinated both arms with the blood from a rerruga tumour. In twenty-one days symptoms set in and he died of the ferer five weeks later. Consequently it is sometimes known as Carrion's fever.

\section{DISTRIBUTION.}

It is confined to certain provinces in South America. Peru is the most endemic area. It is prevalent on the western slopes of the Andes and Ecuador, Bolivia, and the northern parts of Chili.

It is found in narrow valleys, along tributaries of rivers and small streams. The disease only occurs in summer when rivers are in flood and insects abound. Drinking water is blamed by some of the people.

\section{ATIOLOGY.}

The causative organism is unknown. Perhaps it is some bloodsucking animal that carries it such as the arachnoid.

As a rule one attack confers immunity.

Strangers visiting an endemic centre are very prone to it.

Newly-born infants may acquire the disease.

In some respects it resembles raws, but unlike that disease, it attacks both domestic animals and man.

\section{PATHOLOGY.}

Some doubt the identification of Carrion's fever with nodular fever.

The two diseases have not yet been proved to be identical.

The disease is said to commonly occur among animals, \&c., as horses, mules, asses, dogs, and also fowls.

Post mortem, there is marked pallor of the bodr, odema and dropsy, hypostasis of the lungs, enlargement of the liver and spleen, hyperamia of the bone marrow. Then there are the verrugas of the slin and other parts of the body.

HISTOPATHOLOGY. A kind of neoplasm begins about a capillary vessel, the areolar tissue reacting through some perivascular irritant.

The connective tissue fibres swell, embracing embryonic connective 
tissue cells. The interareolar spaces contain polymorphs and macrophages. A non-ulcerative skin lesion shows the cells of the surface epithelium swollen and distended with glycogen. The papillary layer disappears, the dermis is infiltrated with round cells, there are numerous small vessels which may become cavernous, hence the possibility of much hemorrhage.

The subcuaneous faty tissue is always inflamed.

Ulcerations farour secondary infections.

\section{SYMPTOMATOLOGY.}

Incubation is unknown. Perhaps eight to forty days.

Prodromata, malaise, lassitude and depression. Gradual ancemia, rheumatoid pains, local or general, sometimes morning fever which may rise to $104^{\circ} \mathrm{F}$., insomnia, and sometimes dehirum. Pulse soft and rapid. The fever terminates in about twelve hours with sweating and cessation of pains. The liver and lymphatic glands are enlarged.

There is constipation as a rule but some have diarrhoa.

There is a great destruction of red cells to 900,000 per cim.

The $\mathrm{HB}$ is increased.

There is always a marked leucocytosis, 20,000. The polymorphs being about 75 per cent. The bone marrow shows excess of normoblasts and neutrophile myelocytes.

\section{SEVERE TYPE (Carrion's Fever).}

The fever is marked, and is accompanied by severe pains and diarrhoea. The urine is acid, dark, scanty, high specific gravity, never blood, rarely albumin, reduces Fehling's solution. The anæmia is profound to 500,000 in extreme cases (Monge).

Death in from two to three weeks.

\section{MILD TYPE.}

The febrile stage is from one to eight months, the skin itches, the eruption is on the face, neck, arms, legs, conjunctiva, about and within the mouth. Small erythematuus spots, sometimes with small resicles, rarely with bulla. The erythema develops into a papular eruption about the size of split peas. These are discrete, red, firm, small warts, liable to bleed, which may also occur on the mucous membrane and viscera. Some larger nodules may appear to be free under the skin, then adhere, ulcerate, bleed, then become large, red, fungating masses, chiefly about the elbows and knees.

There may not be any healthy area of skin left. What there is becomes adematous, the fever declines with the presence of the eruption, general symptoms abate, the patient feels better but the anamia is marked and hamorrhage from the nodules may cause death. 
Verrugas occurring in the larnyx cause dyspnoea.

\begin{tabular}{|c|c|c|}
\hline , & , & bronchi cause bronchitis. \\
\hline ", & ', & lungs cause pneumonia. \\
\hline ", & $"$, & pleurat cause pleurisy. \\
\hline ", & ", & nose cause epistaxis and occlusion. \\
\hline ", & , & nesophagus catuse dysphagia. \\
\hline ", & ,' & intestines cause blood and diarrhoca. \\
\hline ", & ", & meninges cause cerebral symptoms. \\
\hline ' & "' & \\
\hline
\end{tabular}

In four to six months there are several crops each preceded by atacks of fever. The eruption then disappears, nodules dry up without scarring, ulcers cicatrize. The patient is convalescent but anæmic and weak. The eosinophiles may disappear.

\section{ATYPICAL TYPE.}

Sometimes the eruption is less ypical and somewhat localized or it may develop and disappear, the general srmptoms returning later and are then more serious.

Some cases show but little fever.

\section{PROGNOSIS.}

An early general eruption is a good sign.

A mild eruption with anamia is bad.

Subcutaneous nodules with Carrion's fever, bad.

Mortality 10 to fo per cent.

The disappearance of a poor eruption with a return of the fever and the pains are bad.

\section{TREATMENT.}

There is nothing specific known.

Remove the patient from the endemic area to warm country districts. Try arsenic, atoxyl, arsenobillon.

Aroid chills and cold baths.

Drugs are useless in the febrile stage.

Give tonics during the convalescence.

Treat Carrion's fever as typhoid.

\section{DEFINITION.}

\section{YELLOW FEVER.}

It is an acute specific, endemic and epidemic fever, characterized by two paroxysms of fever separated by an intermission; albuminuria, jaundice, black vomit and hamorrhage.

The Stegomyia calopus carries the virus.

\section{DISTRIBUTION.}

Yellow fever is supposed to have attacked the troops of Columbus 
in 1495, in the island of St. Domingo. These Spanish troops carried it to America.

$\mathrm{It}$ is endemic on the east coast of Mexico, Central and South America as far as Rio de Janeiro, and the Antilles. Also in Guatemala, Spanish Honduras, Nicaragua, Costa Rica, Labrador, French Guiana, Dutch Guiana, Ecuador and along the rivers Magdalena, Orinoco and Amazon.

The true home centre is Central America and the West Indies.

It was formerly common but now is extinct on the Isthmus of Panama.

Since 1905, eleven cases have been. detected and isolated by the Canal Zone authorities.

These cases came from Cartagena, Guayaquil and Buenaventura.

The epidemic of 1635 to 1690 affected Guadeloupe, Cuba, Jamaica, San Domingo, Martinique and Vera Cruz.

West Africa has probably been a second endemic centre since 1520, when the Cape Verd Islands and the Gulf of Benin had an epidemic.

From these endemic centres ships carry the disease to $46^{\circ} 5^{6} \mathrm{~N}$., and $34^{\circ} 54^{\prime} \mathrm{S}$. Towards the North the disease becomes milder but towards the South it may become yery severe.

England, Swansea, was attacked in 1865 but it died out.

France was attacked in 1861 .

Madrid in Spain, in 1878 .

The distribution seems to coincide with that of the mosquito, S. calopus.

This is supported by the fact that :-

The disease is endemic in warm climates.

It increases in the summer and disappears during the winter in temperate climates.

It is carried by ships.

It affects low-lying parts of the coast, especially the insanitary districts.

An atmospheric temperature of over $75^{\circ} \mathrm{F}$. is required for its active propagation. Towns upon the sea coast, banks of rivers and near deltas are often infected.

The disease follows lines of communication.

In Rio de Janeiro visitors maintain the disease.

Long residents in an infected district tend to become immune.

\section{AETIOLOGY.}

The causative organism is an ultra-microscopic, non-filtrable virus, because :-

o. I c.c. of infected blood will reproduce the disease in a nonimmune person. 
Diluted blood passed through a Pasteur-Chamberland filter will cause the disease when injected intravenously into a non-immune. Time is required for the rirus to develop in man and the mosquito before it is infective, three days in man and twelve days in the mosquito, hence it cannot be a chemical substance.

If infected serum is diluted, the virus becomes more and not less potent after time has been allowed for growth.

The virulence of the blood is destroyed at $55^{\circ} \mathrm{C}$.

The S. calopus is absolutely necessary for its spread.

Contact with the bedding, fomites and excretions of an infected person will not cause it. The virus may only live in man and the S. calopus.

The higher apes, guinea-pigs and fowls are suspected.

The fly must go twelve days after feeding upon an infected patient before its bite will reproduce the disease in man, but these flies are then infective for life.

According to French physicians the virus can be transmitted by the mosquito to the first and second generations. These cannot infect man until they have been adult flies for thirteen days.

The adult mosquito can live for about five months and lays seven batches of eggs. The fly, before her first egg-laying, is immature and bites at any time but is harmless. Afterwards she is strictly nocturnal.

\section{PATHOLOGY.}

The Liver.-The liver cells swell, pressing upon the bile capillaries obstructing the bile, causing hepatogenous jaundice, staining the skin and tissues yellow and passing out in the urine.

The swollen liver cells also block the intralobular capillaries causing congestion of all viscera drained by the portal vein, especially the pyloric region and the duodenum. This impaired circulation predisposes to secondary infection.

Portions of liver are so degenerate that the urea function is impaired and a universal toxic condition due to the resulting ammonicmia sets in. Hamorrhages are caused as a result of the damaged epithelial lining of the capillaries.

The Blood.-A few normoblasts may be present, otherwise the number and shape of the red cells are normal. The loss of hb. is marked after the third or fourth day. The specific gravity of the blood falls, its coagulation is diminished.

Haemoglobinamia often occurs before death.

Ammonizemia is present in bad cases.

The polymorphs are slightly increased, 3,200 to 20,000 per cm.

The Urine.--Atbumin on the second day which increases rapidly. 
Bile appears about the fifth or sixth day. Red cells may be present, leucocytes are rare. Casts, hyaline, granular and epithelial appear in turn.

Lrea is diminished in bad cases.

The diazo reaction is usually absent.

The Yomit. First white, consisting of mucus; acid and colourless, or it may be blood-stained.

Then red, consisting chiefly of bright, red blood.

Later black, consisting of red cells, débris, fat, epithelial cells, micro-organisms and hydrochloric acid in traces. The $\mathrm{HCl}$ converts the hb. into acid hamatin giving the black colour.

\section{The Immunity.}

Racial immunity, so-called, in endemic regions is really due to their having had mild attacks in childhood.

Acquired immunity usually is lifelong, and is given by a typical attack.

Relative immunity is acquired by receiving an inoculation of infected blood serum, heated for five minutes to $55^{\circ} \mathrm{C}$.

It has been shown conclusively that negroes of the West Coast are not immune to yellow fever but are as liable as whites,

\section{$B U T$}

the practitioners of Guayaquil recognize the natural immunity of very young children and have presented evidence in support of their views (O'Brien). This appears to pass off with age, during which transient periodic mild attacks give an acquired immunity.

\section{P. M.}

The skin is yellow and stained with hamorrhages.

The buccal mucosa is fissured, the mouth may contain blood.

The liver is yellowish brown, hemorrhagic, cells swollen and fatty.

The gall-bladder contains inspissated bile, perhaps mixed with blood.

The spleen is congested and soft, normal in size.

The gastro-intestinal tract is full of black tarry blood.

The mesenteric glands are sometimes enlarged.

The kidneys are congested, not enlarged.

The urinary tubules show fatty degeneration with granular débris in the urine.

The urinary bladder is usually empty.

The pericardium may show effusions and the heart ecchymoses.

The pleura may show haemorrhages; the lungs are often congested.

The uterine mucosa are congested and the organ may contain blood.

The meninges are congested and may show hamorrhagic spots 


\section{SYMPTOMATOLOGY.}

Incubation.-Two to seren days.

Prodromata usually absent. Malaise may be present for several dàs.

The First. Ferer. - This comes on the first or second day, severe frontal headache, sometimes rigors, pain in back and limbs, face and skin flushed, eyes injected and painful, temperature rises rapidly to ${ }_{10} 3^{\circ} \mathrm{F}$. or higher, respirations quick and laboured, pulse increases from 100 to 120 , full and bounding. Vomiting with epigastric pain, constipation, urine diminished with high specific gravity, acid reaction, albuminuria on second day, insomnia.

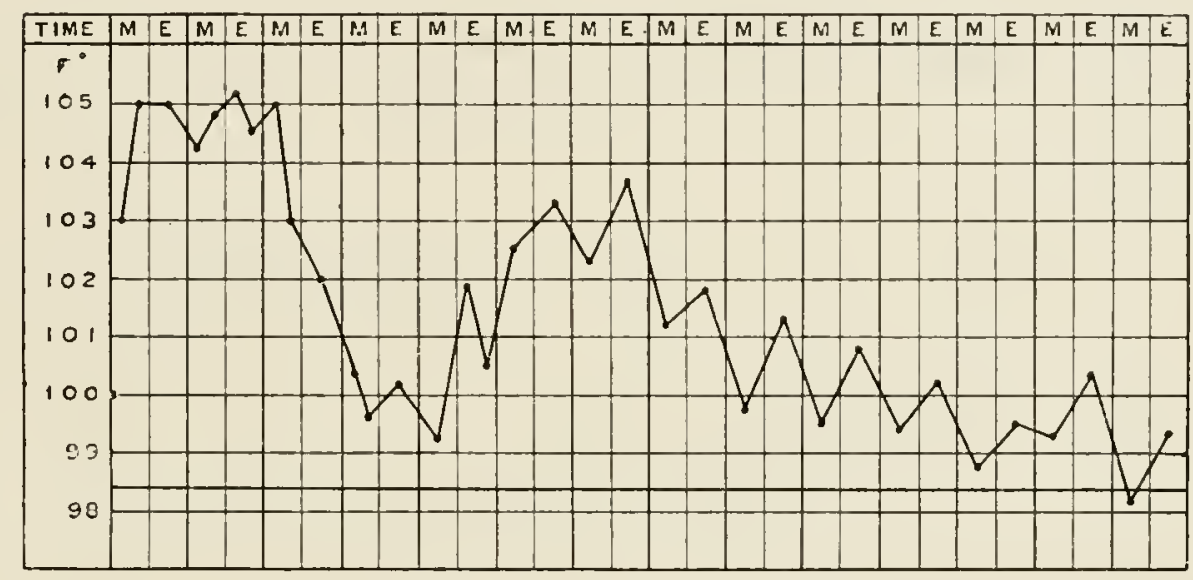

Yellow fever. Severe attack.

The Crisis.-This occurs about the end of the second day. The temperature falls suddenly to normal or subnormal with sweating, the pain and the flush both disappear, the patient feels better and sleeps. well. The urine increases. A mild abortive case may recover at this stage.

The Second Feicr. This occurs on the second to the fourth day. A few hours after the temperature has fallen 10 its lowest and the crisis. has taken place the temperature begins to rise again steadily until it reaches $104^{\circ}$ or $105^{\circ} \mathrm{F}$. But the pulse decreases to sixty or forty beats per minute (Faget's sign). The skin becomes jaundiced, and it deepens with the days. The romiting and the epigastric pains return with thirst and prostration. Tongue dry, furred with red tip and edges. Hamorrhages everywhere, romit black, malaena, epistaxis, subcutaneously, from the mouth, \&c. The urine diminishes, albumin increases. There may be anuria. Restlessness and delirium are often present.

The Tormination.-If recovery, then, after about four days, the temperature declines, sweating commences, more urine is passed, albumin diminishes, romiting ceases, sleep is usual, and convalescence sets in. 
Recovery is rapid.

If fatal, then jaundice deepens, bleeding becomes worse, urine is suppressed, subsultus tendinum is seen, coma and convulsions precede the fatal issue. Death may ensue in an acute attack, preceded by a rapid rise of temperature.

Relapses may occur at any time within two or three weels of the attack, and are predisposed to by dietetic errors.

\section{PROGNOSIS.}

As a rule convalescence, when it has set in, is not protracted or complicated. Pre-existing heart or kidney affections are bad.

Congestion of the lungs, abscesses, gangrene and intussusception are dangerous complications.

Dysentery and hepatitis are common sequelex and add to the gravity of the case.

In the United States the mortality is 10 to 25 per cent.

In West Africa and the endenic area the mortality is 45 to So per sent.

In children the mortality is low.

Anuria, hyperpyrexia, black vomit, malena are all bad omens.

All cases are serious, especially for alcoholics.

Abortion is the rule.

\section{DIAGNOSIS.}

Early cases are alway's difficult.

Typical attacks are easy.

Mild attacks in a non-endemic area are difficult.

It is all-important to diagnose early.

Look for albuminuria, Faget's sign, jaundice, and the black vomit.

Dengue shows a preliminary rash, leukæmia, no albuminuria, and Faget's sign is absent.

Subtertian malaria shows the blood plasmodium and absence of Faget's sign.

Blackwater fever shows a mononuclear increase with destroved hb. in the urine.

Relapsing ferer shows blood parasites and a leucocytosis.

\section{TREATMENT.}

The indications are:-

(1) To prevent the spread of the infection.

(2) To eliminate the toxins rapidly.

(3) To alleviate the symptoms.

-IVe will deal with preventive measures last. 


\section{To eliminate the Toxins rapidly.}

By the Borel.-Give smill doses of calomel to disinfect the alimentary tract. Follow this with doses of sodium sulphate unt il the bowels are well opened. Afterwards give enemata, twice daily, of sodium sulphate, $1 \frac{1}{2}$ drachms to I pint.

By the Skin.-Wrap the patient in warm blankets, but not so as to oppress the patient with them. Precede this by a hot mustard bath. By these measures the skin will be stimulated and the headache relieved.

By the Kidneys.-To stimulate the kidneys and dilute the toxins 1ise Sternberg's treatment :-

R. Sodii bicarbonatis ... $\quad \ldots \quad \ldots \quad \ldots \quad$ grains 1 jo Hydrargyri perchlor: $\quad \ldots \quad \ldots \quad \ldots, \quad \ldots \quad \frac{1}{3}$ $\begin{array}{lllllll}\text { Aqua } & \cdots & \ldots & \ldots & \ldots & \text { ad } 60 \text { ounces }\end{array}$ Give $\dddot{1} \frac{1}{2}$ ounces hourly

or, a drink of sodium bircarbonate and fresh limejuice made neutral with sodium sulphate if necessary. This may be administered per rectum.

\section{To alleviate the Symptoms.}

Relieve pain by aspirin or phenacetin, never opium.

Apply hot fomentations for severe lumbar pain.

Warm mustard bath for increasing the urine.

Do not give food during the first few days.

Prevent romiting by sips of champagne, iced if possible.

Put plasters to pit of stomach.

Treat hyperpyrexia by cold sponging.

Bleeding should be treated by calcium lactate or adrenalin injection or ergotin.

Anuria by hot fomentations to the loins, cupping, lot-air baths.

Cardiac insufficiency by strychnine, camphor, or ether.

Weakness should be met, after three days, with milk, lime water, bast water, crean, lemon jelly, olive oil by mouth or skin.

After three days of normal temperature, chicken broth, custards, \&c.

Aroid strong alcoholic stimulants and meat extracts.

Be very careful during remissions not to give food.

Always increase diet with great caution.

\section{PROPHYLACTIC MEĀSURES.}

To spread the disease three factors are necessary:-

An infected person from the first to the third day of the disease.

The presence of non-immune persons.

The presence of the Stegomyia calopus (fasciata).

To spread the infection the fly must bite the patient infected during the first three days. Twelve days must elapse for the development of the virus in the mosquito; then it can bite and infect a non-immune 
person for the rest of its days, and may also hand down the virus to the first and second generation, these latter spreading the disease fourteen days after becoming adults.

An infected ship should be anchored a quarter of a mile from the shore or other ships as the fly is not likely to cross this distance.

The quarantine slould be five to seven days because the incubation is up to seven days.

Place the sick in mosquito-proof rooms, mesh twenty strands tor one inch, and quarantine the remainder of the crew or members of the household.

Disinfect the ship by a Clayton's disinfector.

As the flies are quiet between 9 a.m. and 3 p.m. visits can then be made without risk.

Houses should be fumigated by sulphur dioxide or prrethrum powder, burnt in the proportion of $2 \mathrm{lbs}$. to 1 , ooo cubic feet of space.

The breeding places of the flies should be destroyed.

\section{BOYCE'S SUMMARY.}

(A) When the ferer is endemic:-

(1) Segregation of non-immunes.

(2) Screening of bed, verandah, or, if possible, the house: completely.

(3) Systematic mosquito destruction by :-

Removal of breeding places.

Screening of water cisterns.

Oiling of waters not screened or drained.

The infliction of fines for non-observance of sanitary rules.

Drainage and bush cleaning.

(4) Education.

(5) Quarantine administration.

(B) When yellow fever has already broken out :-

(1) Removal of non-immunes from the infected area and deflection of the traffic from this area.

(2) Isolation of all cases, sure and suspected.

(3) Provision for isolation of contacts.

(4) Early notification.

(5) Fumigation.

(6) Emergency mosquito measures :-

Removal of receptacles, oiling, screening, and draining-

( 7 ) Education by lectures, meetings and pamphlets.

(8) General organization of the medical forces. 


\section{NOTES ON THE YELLOW FEVER MOSQUTTO, STEGOMYIA CALOPLS (FASCIATA).

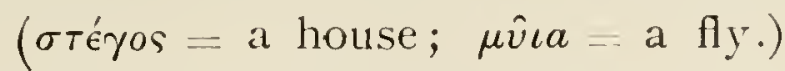

The S. calopus belongs to the Culicales of the sub-family, Culicinæ.

On account of its wide distribution, domestic habits, vital tenacity of its eggs, the different infections it is capable of carrying, yellow fever in particular, it is one of the most formidable insects in existence. They are small black mosquitoes, with bright whitish markings on head or thorax, and bright whitish transverse marks on abdomen and legs. They are common in all tropical and sub-tropical climates between $40^{\circ} \mathrm{N}$. and $40^{\circ} \mathrm{S}$. from sea-level to nearly 3 , ooo feet high.

\section{THE ADULT FEMALE.}

The head is black with white markings not unlike a crown in shape; the palpi are short, black, tipped with white. The scutum is brown, with a lyre-shaped set of marks thereon. The scutellum is white. The abdomen is blackish brown, with white transverse bands across the dorsum and on the sides of the segments. The femora are black with a white tip; the tibia black, the tarsus black with two to five white bands. It has the common mosquito wing, with the third and fifth long vein forked.

The adult male is similar, but has long palpi, and does not bite man.

\section{HABITS.}

A house-haunting mosquito always.

Perhaps the commonest mosquito on board ship.

It bites in the daytime as well as at night.

It breeds close to human liabitations.

Its eggs are laid in anything that holds water-a sardine tin or water-barrel.

\section{EGGS.}

Almost black, laid in seven batches, they stick closely together, but not in rafts. They are very resistant; if kept dry they will remain fertile for six months.

The yellow-fever virus is transmitted to a second generation.

\section{LARYA.}

Dark in colour, has a short, stout breathing tube and rery large tracheal gills. Laterally, on the eighth abdominal segment is a single row of eight to nine scales like miniature arrow heads and a wisp of three small hairs. It can remain submerged for a long time, and can live in filthy water aided by its large tracheal gills. 


\section{SPOTTED FEVER OF TIE ROCKY MOUNTAINS.}

\section{DEFINITION.}

An acute endemic, non-contagious, febrile disorder, with a petechial or papuric eruption following the bites of infected ticks. It is found in valleys, in sharply defined areas, and occurs at definite times and seasons.

\section{DISTRIBUTION.}

The United States only.

In Montana it is found along the Bitter Root valley, at Rock Creek, and Bridger.

In Idaho throughout the entire valley of the Snake River and its tributaries.

In Wyoming at Cody and Meeteeste.

In Nevada, in the north only, along the valley of the Quinn River.

In Oregon towards Idaho in the east only.

The fever occurs in those seasons when the ticks (D. andersoni) are most prevalent.

The elevation is in all cases between 3,000 and 4,000 feet above sea-level.

\section{AETIOLOGY.}

The causative parasite is stated to be localized in the endothelial cells and smooth muscle fibres (Wolback). Its exact nature is not known.

The organism is said to be present in the blood-ressels of man, monkeys, rabbits, guinea-pigs and ticks.

The virus cannot be separated from the blood cells by washing, neither will it pass through a Berkefeld filter.

One attack produces immunity.

The virus can be acquired and transmitted by the larve, nymph, male and female adults of $\mathrm{D}$. andersoni.

Other ticks are also suspected.

Men are more frequently attacked than women, commonly from fifteen to fifty years of age. Hence with out-door work there is greater risk of infection.

Jack rabbits and deer mice are important hosts of the carrier ticks as well as domestic animals.

\section{PATHOLOGY.}

Post mortem, rigor mortis is well marked. Petechice are found on the skin.

Tick bites may be risible. Liver and spleen are congested, 
enlarged and soft. Kidneys are conges:ed, sometimes with subcapsular hamorrhages.

Microscopically one finds capillary congestion of the viscera with excess of leucocytes. Extravasation into and pigmentation of the skin.

Acute parenchymatous degeneration of heart muscle, spleen, liver and kidneys.

\section{SYMPTOMATOLOGY.}

Incubation.-Two to seven days.

Prodromata.-Pains radiating from irritating tick bites, malaise, nausea.

Onset.-Fairly sudden with chill, severe headache, pains in back, temperature $103^{\circ}$ to $104^{\circ} \mathrm{F}$. , furred tongue, dried skin, congested conjunctiva, dry cough, epistaxis, febrile urine.

Course.- Temperature gradually rises to $105^{\circ}$ to $107^{\circ} \mathrm{F}$. from the sixth to the twelfth day.

There is an eruption about the third day on the wrists and ankles, covering the body in two days.

The macules, I to $5 \mathrm{~mm}$. in diameter are not raised, at first they disappear on pressure, then become permanent and later petechial, sixth day. There is also a dusky red mottling of the skin.

In very mild cases the eruption may be absent.

The pulse is 110 to 150 , becomes weaker, often dicrotic and may be irregular. There is a slight reduction of the red cells and hb. with a slight increase of leucocytes.

In severe cases odema of the face and limbs may be present.

Constipation is troublesome.

There may be mild bronchitis with a slight increase in respiration.

The urine may show a trace of albumin with a few granular casts or it may be suppressed in bad cases.

From the fifth to the twelfth day the typhoid state may lead to death.

If not, then lysis about the eighteenth day and convalescence.

\section{COMPLICATIONS.}

Pneumonia is common.

Gangrene of the fingers and toes and the skin of the penis and scrotum may occur.

Nephritis, cardiac weakness and meningitis are not unknown.

\section{DIAGNOSIS.}

Typhoid fever may be troublesome. It has, however, a more insidious onset, the nature and commencement of the rash are different, there are intestinal symptoms and a WVidal reaction can be done. 
Typhus fever ends by crisis, otherwise they are almost indistinguishable.

Two varieties of the same organism are possibly the causes of both diseases.

Japanese river fever may be very similar, but one should look for one or more eschars near the genitals or the axilla in this disease.

The eruption, which begins on the face, does not become petechial.

\section{PROGNOSIS.}

In Montana the mortality is about 90 per cent. and is always high.

In Idaho the mortality is about 2.5 per cent., always low.

The outlook is good when the eruption is not general and poorly defined.

\section{TREATMENT.}

Remove the tick by applying ammonia, turpentine, lierosene or carbolized vaseline.

Cauterize the bite with pure carbolic acid.

Cold applications, Dover's powders, \&c., for the headache.

Cold sponging for hyperpyrexia.

Cardiac stimulants may be necessary.

Avoid the districts where the disease is prevalent.

Destroy the ticks and their breeding places as far as possible.

In Montana they practise the following measures:-

(1) The reclamation and cultivation of arable land.

(2) The burning over of the foot-hills.

(3) The killing of wild animals.

(4) Hand picking and the dipping of domestic animals in arsenical dips.

(5) Sheep grazing.

(6) The destruction of ground squirrels by the use of carbon bisulphide pumps.

\section{DEFINITION.}

DENGUE.

An acute specific, infective, non-contagious fever of unknown causation, spread by the bite of the Culex fatigans and Stegomyia fasciata, characterized by two febrile paroxysms, the pulse varying directly with the temperature, and a marked leucopenia.

\section{DISTRIBUTION.}

In tropical and sub-tropical zones where the Culex fatigans abounds.

In Egypt, Arabia, Persia, India, Burmah, Indo-China, China, North America, Spain, Peru, West Indies, West Africa, Fiji Islands, Western Australia, Ceylon. 
An epidemic or pandemic takes place about every twenty years (Manson).

Often 75 per cent. of the population are attaclied.

It prefers the coast line, deltas and valleys of great rivers, but there are many exceptions to this.

In IS7o to s 873 it spread all over India.

\section{ETIOLOGY.}

An unknown organism living in the blood is the cause.

An intravenous injection of 20 c.c. of dengue-fever blood, filtered or unfiltered, produces, after an incubation of two to three days, the typical symptoms of the disease.

This organism passes through a filter which will retain M. melitensis, the filtrate producing the fever.

Some people appear to have natural immunity against the disease.

An attack produces a temporary acquired immunity.

IIot countries suffer most. Cold limits the disease.

\section{SYMPTOMATOLOGY.}

Incubation, two to six days.

Prodromata are usually absent.

Onset is sudden, introduced often by some severe cold, extreme fatigue, deep flushing of the face, a shivering in man, convulsions or delirium in children.

Initial fever commences with a rapidly rising temperature, $103^{\circ}$ to ro6 $6^{\circ}$ F., pulse quickens, go to 140 , buccal mucosa becomes congested, conjunctive injected, while occasionally vomiting and diarrhœea are present.

Severe pains in the head, eveballs, lumbar region and legs.

Joints when moved actively cause agonizing pain, but when moved passively are almost painless, hence the pain is really caused by the adjoining muscles. Very rarely are the joints red and swollen.

The patient may be unable to walk because of the pains.

Gastric disturbance and romiting may occur.

The lymphatic glands are not enlarged.

Insomnia and delirium are not infrequent.

Leucopenia is almost constant, 3, SoO per c.mm.

The small lymphocytes are increased and the polymorphonuclears decreased. Otherwise the blood is normal.

Intermission or remission then occurs from the second to the fourth day as a rule. The temperature falls by crisis, accompanied by profuse perspiration, diuresis, diarrhoea, and sometimes epistaxis. The latter relieves the headache at once. The pains almost disappear and the patient may be able to resume his work. 
In some cases the temperature remits only to $100^{\circ}$ or $102^{\circ} \mathrm{F}$, and the symptoms abate only.

Terminal fever and cruption. This occurs about the fifth day, temperature rises to $102^{\circ}$ or $103^{\circ} \mathrm{F}$., the pains return and are severe, a rash appears on the palms and the backs of the hands, elbows and knees, rapidly spreading to the trunk and legs. This rash may be:-

(I) A measly eruption, small circular dusky patches.

(2) A scarlatiniform eruption, numerous bright red, small papules forming larger patchy areas.

(3) A mixed type or abortive only.

The eruption lasts two to eight days followed by a furfuraceous desquamation lasting two or three weeks.

The second temperature falls by crisis about the sixth day.

The pains may come back again at intervals for weeks afterwards.

Convalescence may be quick and permanent or protracted and complicated.

The seven-day ferer of Rogers is held by some to be a variety of Dengue.

The mortality is almost nil ( $\mathrm{O}^{\circ} \mathrm{I}$ per cent).

\section{SEQUEL A AND COMPLICATIONS.}

Relapses are not uncommon.

Pains in the joints and muscles.

Hæmorrhage from any of the mucous membranes.

Hyperpyrexia is rare, so also are pleurisy, pericarditis, orchitis, endocarditis and meningitis.

\section{DIAGNOSIS.}

Look for sudden onset, severe muscular pain, intermission on the third day, and rash on the fifth or sixth day.

Yellow fever has its low pulse with a high fever, jaundice and hæmatemesis.

Influenza has catarhal symptoms, and rash is absent.

Scarlet fever has sore throat and enlarged cervical glands.

Measles has catarrhal symptoms, but no severe muscular pains.

Rheumatic fever has swollen joints.

Small-pox will be difficult until the eruption is seen.

Early enteric cases are not easy to distinguish; the cause of the fever will decicle it.

\section{TREATMENT.}

It is useless to attempt to cut the fever short.

Cool applications to the forehead for lieadache.

Dover's powder or morphia for the pain. 
Stimulants should be aroided.

Purgatives and emetics tend to increase the muscular pair.

Massage. Electricity and iodides can be applied.

Change of air and place are necessary.

\section{TYPHUS FEVER.}

\section{DEFINITION.}

An acute specific contagious fever of unknown causation, characterized by sudden onset, marked nervous symptoms, macular eruption terminating by crisis.

\section{DISTRIBUTION.}

It is a disease of temperate and cold climates, but in the tropics it may occur in high altitudes and during cool seasons.

It is present in Nubial, Egypt, Tripoli and Morocco, Asia Minor and Persia, and from thence to India.

It is also found in North China and Hiogo, Japan.

It is endemic in Mexico and common in Peru and North Chili.

It is prevalent among the Ballian States and Austria, who lost 30,000 of 50,000 cases.

It has been present in Ireland for centuries.

In Egypt, I9'I4-I9I5, there were 23,855 cases with 6, I.32 deaths.

\section{ETIOLOGY.}

The specific organism is unknown. It exists in the blood, which is virulent from the third to the tenth day of the attack (Nuttall).

The disease has been transmitted to apes by typhus blood.

Human body lice carry the causative organism.

It is associated with dirt and filth.

\section{SYMPTOMATOLOGY.}

Incubation about twelve days.

Onset sudden, frontal headache, pains in body, sometimes in chest, temperature rises rapidly, $101^{\circ}$ to $\mathrm{r}_{4}{ }^{\circ} \mathrm{F}$., pulse quick, Ioo to I 20 , face flushed, appearance dull, heary and pathetic.

Tongue thickly furred, rapid exhaustion, a mousy odour from the skin. Delirium sometimes early.

Rash on abdomen or chest about the fifth day. At first a transient erythema, then a macular, measly eruption, followed by the petechial form. The rash is mild or absent in 20 per cent. of cases. When present it covers the whole body quickly, except the face, palms and soles.

The typhoid state comes on rapidly with low muttering delirium.

The pupil, before contracted, is now dilated. 
The urine may be detained.

Paralysis of the sphincters or grave complications terminate the attack.

About the thirteenth or fourteenth day the fever terminates by crisis, profuse sweating, critical diarrhoea and diuresis, with excess of urates.

Convalescence then begins.

Second attacks are rare.

\section{COMPLICATIONS.}

Bronchitis, Broncho-pneumonia, Retention of urine, Hyperpyrexia, Parotid bubo, Pyæmic abscesses, Thrombosis of femoral vein, Bed sores, Gangrene of extremities, Relapsing, fever and Acute miliary tuberculosis.

\section{TREATMENT.}

Good air and plenty of it is essential for this disease.

Shield the eyes from excessive light.

Liquid and bland foods should be given as they can be assimilated.

All noise in the sick room should be avoided.

Strychnine, digitalin, \&c., are necessary for the heart.

Hyoscine, bromides, or morphine may be required for nerve symptoms.

20 c.c. intravenously of the serum from patients who have been convalescent twelve days have been useful in some cases; repeated if necessary.

All clothing must be thoroughly disinfected at once.

All hair must be shaved off and the whole body thoroughty cleansed. No lice, no typhus.

\section{HEMOGLOBINURIC FEVER (Blackwater Fever). \\ DEFINITION.}

An acute fever of uncertain causation characterized by excessive blood destruction, jaundice, hæmoglobinuria, diminution and suppression of urine.

\section{DISTRIBUTION.}

Most commonly in tropical Africa and parts of India.

It is less commonly found in South Italy, Sicily, Greece, Asia Minor, Southern Russia, China, Siam, Malay Peninsula and the Southern regions of the United States. Central and South America and Brazil in particular, West and East Indies, and Polynesia.

It is extremely rare in the Roman Campagna, the classic land of malaria.

Its endemicity is said to be limited to low swampy grounds. 
It appears to have some dependence upon meterological conditions. It attacked the Chinese labourers when building the Congo railway. It attacked the labourers constructing the Corinth canal.

It attacked the Chinese army at Kwangsi like a plague.

It attacked the prisoners in Sardinia in 1885 .

\section{AETIOLOGY.}

Theories.- These are numerous. The chief are as follows:-

(I) A manifestation of an active malarial infection.

(2) The result of quinine poisoning.

(3) That it is due to a specific organism.

(4) That it is a condition brought about by previous malarial infection.

(1) That it is a Manifestation of an active Malarial Infection.

Pro. It has been pointed out :-

(I) That where the malarial mortality is high, blackwater fever is not uncommon, but it is scarce where the malarial mortality is low.

(2) That people attacked with blackwater fever had previously suffered from malaria and had usually been attacked several times.

(3) That the day preceding the hamoglobinuria the patient's blood contained parasites in $95^{\circ} 6$ per cent. of cases, while the day after the attack they were seen in only $I 7^{\circ} I$ per cent. of cases (Stephens).

CON :-

(4) That there is found a monoleucocytosis.

(I) That it has occurred in people who have never had malaria.

(2) That it is uninfluenced by quinine.

(3) That in Panama hamoglobinuria is rare, but acute malarial infection very common. This view is not widely held. Most seem to agree that acute malaria may be the exciting factor, but that some other agent is necessary.

(4) That blackwater is rare in some districts where malaria is most rife.

(2) That it is the Result of Quinine Poisoning (Koch).

This view is not widely held because-

Quinine administered to patients without previous malaria has never caused hamoglobinuric fever, no matter how great the dose.

Hamoglobinuric fever has occurred when no quinine has been administered. 
The taking of quinine does sometimes determine blackwater, but much more often it does not.

Blackwater fever was known to Europe by Hippocrates and others long before cinchona bark was imported.

Some assert that quinine will improve and in some cases cure the condition.

\section{(3) That it is due to a Specific Organism.}

Sambon in 1898 proposed a Babesia.

Many parasites have been described since. The idea perhaps arose because of a somewhat similar disease in animals caused by a piroplasmosis. The similarity unfortunately is only superficial for :-

With homoglobinuria in animals-

It depends upon infection by a definite organism.

That organism is present in numbers proportional to the severity of the disease.

Its insect host is well known.

No previous illness is necessary or usual before the disease is manifested.

If the animal recovers, immunity for it is established.

All non-immune animals suffer in proportion to their exposure to the infection.

The symptoms are progressive as a rule, little blood at first, but more later.

There is no natural immunity.

With hamoglobinuria in man-

No organism except the malarial parasite is constantly found.

Where malarial fever is absent hæmoglobinuria is absent also. Those races relatively immune to malaria are relatively immune to blackwater.

One attack of hremoglobinuria does not produce immunity, but perhaps increasingly tends to predispose to another attack.

The writer had a patient with three attacks within eighteen months, each one increasingly dangerous.

Relapses are not uncommon.

Most blood is usually passed early; the amount diminishes with the disease.

That it is not in the least infective is against its being of parasitic origin.

Hrmoglobinuria is so acute that the parasite, if one there is, must be numerous and possessing high powers of vitality. There may be long intervals between two cases in the same district. If a parasite, how does it survive? 
(4) That it is a Condition brought about by a previous Malarial

\section{Infection.}

A case of hamoglobinuria usually gives a history of one or more attacks of malaria. Small doses of quinine in some such Central African patients is sufficient to precipitate the disease (Sandwith).

The subtertian parasite seems to be usually the parasite of the malaria. This accounts for the statement that malaria (benign tertian or quartan) may be prevalent, but no hamoglobinuria.

The Ancon Hospital Records show that malaria complicates practically every other disease, but neither clinically nor at autopsy has blackwater fever complicated any infectious disease other than malaria. In not one case of blackwater can evidence of malaria, either remote or immediately prior, be excluded (Deeks and James).

The hamoglobinuria seems to be a hamolytic toxic action.

Subtertian malaria las a strong toxic action, as evidenced by the cytolytic action upon the kidney cells and the parenchymatous focal neurosis of other viscera. Hamolysis is closely allied to cytolysis, in the former case the cells of choice being the erythrocytes. 25 per cent. of the red cells may be destroyed as a result of one paroxysm of subtertian malaria. Although the life of a red cell is perhaps only four weeks and the whole is regenerated every twenty-eight days, the vascular system could not long stand the strain.

The writer had a case that gave 850,000 red cells per cubic millimetre the second day of the fever.

In studying 230 cases of hamoglobinuric ferer at Ancon Hospital, Deeks and James write their views thus :-

An hamolysis has two groups, a combining and a destructive group. The first must unite with the cell before the latter can destroy it. The exciting factors are :-

(i) Renewed malarial paroxysms to produce sufficient toxin to overwhelm the cells.

(2) Then a lowering of body resistance.

(3) The administration of quinine which may act in either of two ways :-

By depressing the vital processes of the body, or

By acting as the toxophore radical of the hamolysis.

No regularity of results following the administration of quinine can be predicted. Hamoglobinuria has followed the taking of I grain of quinine, in others it has not resulted after taking 30 grains over several days, but it does not occur except in those who have had a previous attack of malaria.

Forty-two thousand cases admitted for diseases other than malaria did not develop one single case of blackwater. $94^{\circ} 6$ per cent. (246) of 
blackwater patients showed one or more previous attacks of malaria. In 230 cases subtertian parasites were found in $76^{\circ} 4$ per cent. of cases, and benign tertian in $23^{\circ} 6$ per cent. of cases.

Most of the cases develop after twelve months' residence in the tropics. After three to four years' residence in an endemic region the hamoglobinuric rate diminishes, hence length of residence appears to be a factor. Deeks and James sum up their investigations thus :-

(1) Hzemoglobinuric fever is a manifestation of malarial toxicity, for the most part brought about by repeated attacks of malaria.

(2) It may appear coincidentally with an acute malarial paroxysm.

(3) It may be determined by any depressing influence.

(4) It may be induced by the administrations of quinine.

(5) Neither quinine alone, nor malarial infection alone, causes hamoglobinuria, but one or both of these, plus the toxin eventuated during the course of one or more malarial attacks.

(6) Syphilis is a predisposing factor, because of its influence in the production of malarial recrudescences.

(7) The treatment varies with the condition present.

(8) To ensure against the recurrent attacks of malaria, with the subsequent production of hæmoglobinuric fever, it is necessary to raise the patient's resistance to a maximum, and to eradicate the malaria by a thorough course of treatment with quinine.

There is no proof that it has spread from one country to another; on the contrary, the disease manifests itself when certain conditions relative to the epidemiology of malaria and to that of no other disease are present.

The conditions are:-

(I) The presence of a population non-immune against malaria.

(2) The prevalence of malaria in such quantity as to produce an almost continuous infection in this population.

(3) A large proportion of subtertian malaria, because the amount of blackwater fever is in direct proportion to the intensity of this variety.

(4) The neglect of prompt and continued administration of quinine, especially in primary attacks, to persons non-immune against malaria.

In every locality, without exception, where these conditions obtain, hæmoglobinuric fever is found (Deeks and James).

\section{PATHOLOGY.}

Christophers and Bentley, working on the malarial theory, put forward the following views:-

(I) Laverania malarize, the subtertian parasite, acts upon the endo- 
thelial cells of the blood capillaries, stimulating them to excessive destruction of red blood cells by phagocytosis.

(2) This phagocytosis results in the production of an auto-
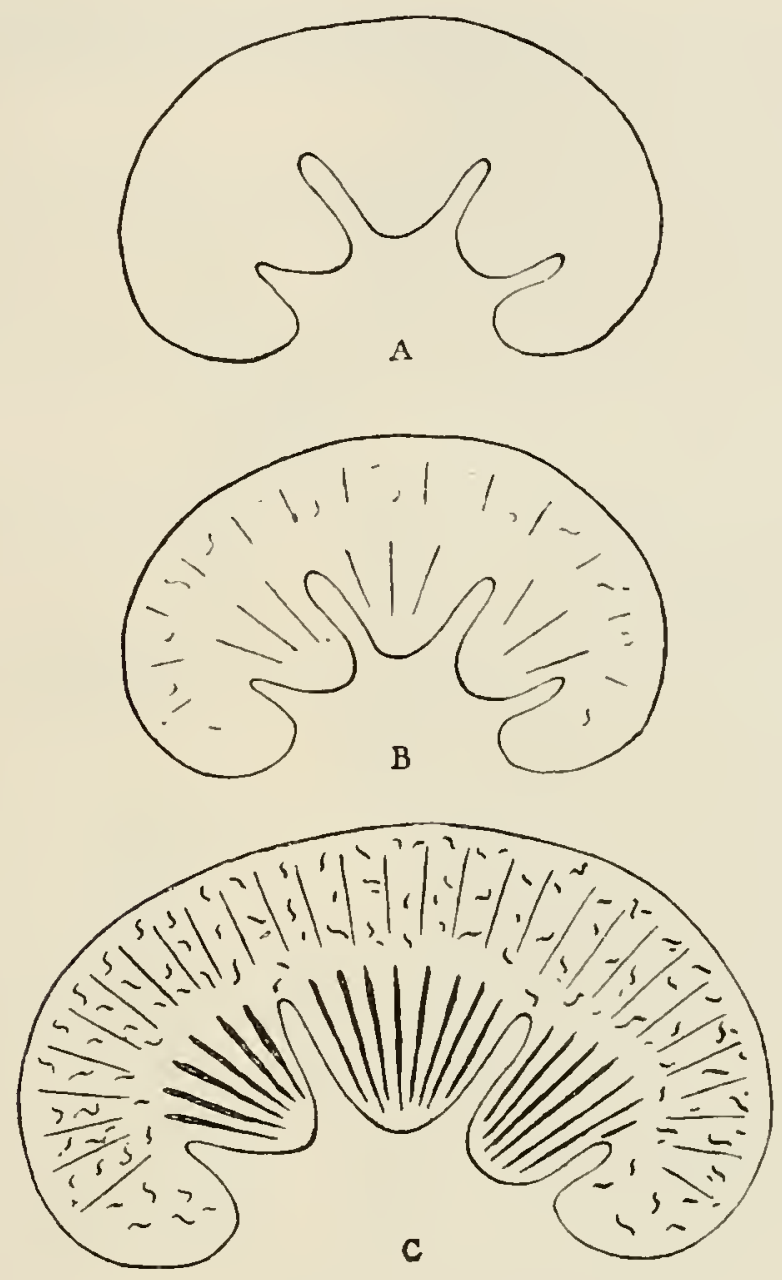

Diagrammatic representation of the condition of the kidney in health (A), during experimental hremoglobinuria of rabbit (B), and in suppression of urine following upon blackwater fever (C). In B small casts are present in some of the uriniferous and collecting tubules; in $\mathrm{C}$ large casts are present in many of the uriniferous and collecting tubulcs. In the latter, considerable enlargement of the kidney is also observed. For the sake of comparison $B$ is represented as tri-papillary instead of being uni-papillary, as in the rabbit.-(From the "Annals of Tropical Mledicine and Hyoiene," Liverpool University, by permission.)

hæmolysin retained in the endothelial cells until set free by some exciting cause as chill, over-exertion, \&c.

(3) The hamolysin is then suddenly set free and destroys the red cells by solution in the plasma, principally in the blood of the liver, kidney and spleen.

(4) This lysæmia produces hæmoglobinæmia, quickly appearing in the urine as oxyhamoglobin, which may become methhæmoglobin on standing.

(5) Some of it, the liver probably converts into bile; the remainder passes out in the urine.

(6) Large coarsely granular casts in the ducts of Bertini mechanically block the tubules and cause suppression of urine (Barrett). 
Plahn believes it to be the result of nervous inhibition glomerular secretion. Others suppose it is the result of 1

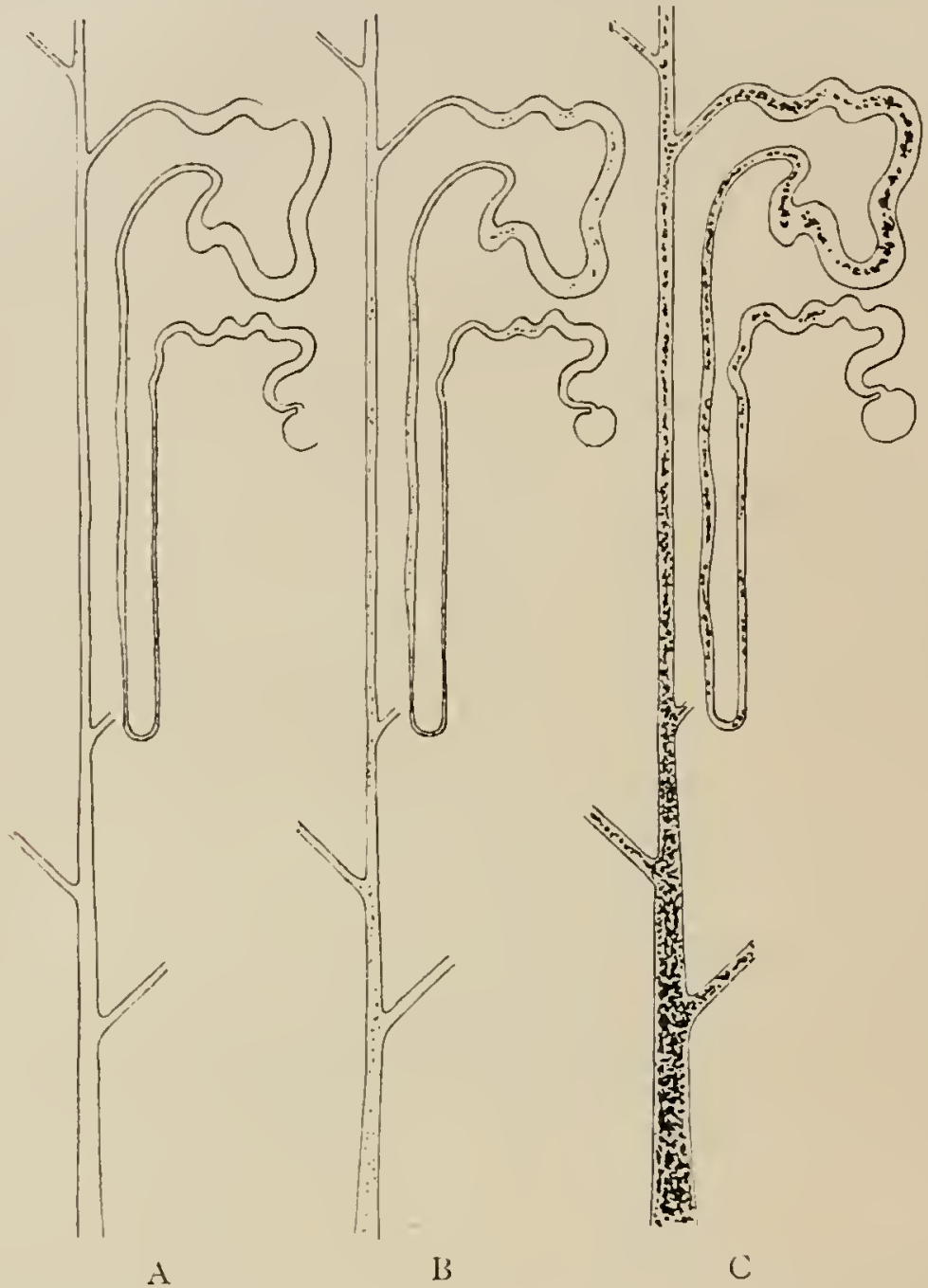

Diagrammatic representation of a renal tulule. (A) normal; (B) commencing d hemoglobinuria : (C) sufficient deposit to be washed on and cause obstruction of $t$ the pyramid. (After J. O. Wakelin Barratt and Warrington Yorke.)-From the of Tropical Medicine and Hygiene," Liverpool University, by permission.

caused by the disease. The improvement of the conditi the B.P. is raised, as when after salines are giren, supl mechanical theory.

\section{P.M.}

The cardinal features are:-

Jaundice of the tissues.

Liquid blood.

Swollen and congested kidneys.

The kidneys are enlarged, dark red, degenerated tubular epi the tubules block with granular material.

The liver is enlarged, the gall-bladder full of inspissated bi of liver necrosis with thrombi in the sub-lobular reins, and hæen in the cells.

The bone-marrow is yellowish, fluid, or gelatinous. 
BLOOD-SPECTRA COMPARED WITH SPECTRUM OF ARGAND-LAMP.

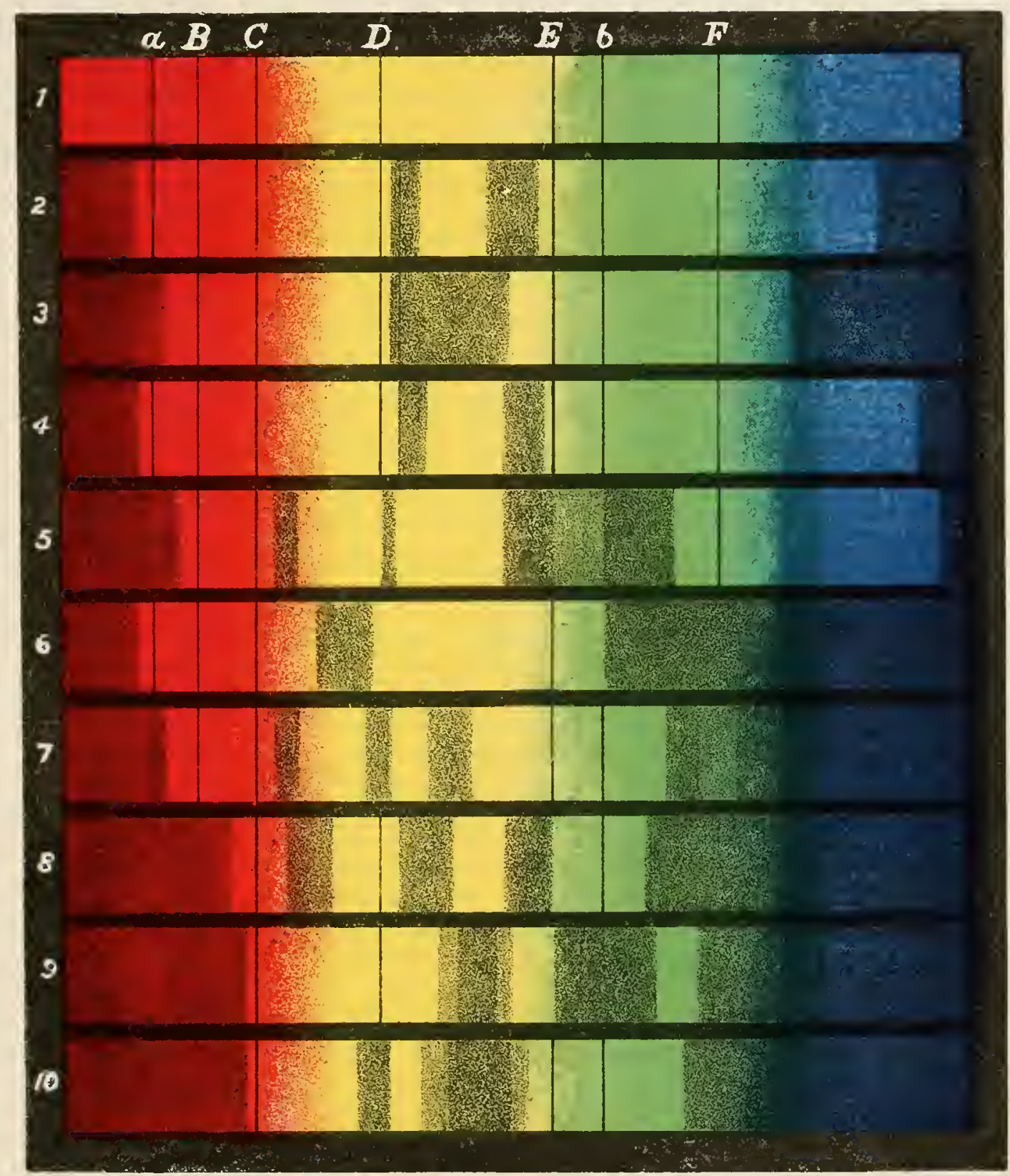

1 Spectrum of Argand-lamp with Fraunhofer lines in position.

2 Spectrum of Oxyhæemoglobin in diluted blood.

3 Spectrum of reduced Hæemoglobin.

4 Spectrum of carbonic oxide Hæmoglobin.

5 Spectrum of acid Hrematin in etherial solution.

6 Spectrum of alkaline Hrematin.

7 Spectrum of Chloroform extract of acidulated Ox-bile.

8 Spectrum of Methæmoglobin (alkaline.)

OSpectrum of Hremochromogen.

10 Spectrum of Hæmatoporphyrin.

Most of the above Spectra have been drawn from observations by Mr. W. LEPRAIK, F.C.S. 


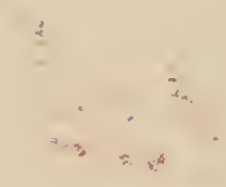


The blood is thin, watery, diminished tonicity, red cells and hb. much reduced showing degenerative changes. Malarial parasites or pigment may be seen.

There is leucocytosis during the fever, leucopenia with a mononuclear increase afterwards.

The urine is yellow, dark red, blackish. Diluted, the spectroscope shows oxy- or meth-hanoglobin, low specilic gravity, a dark brown granular deposit from destroyed blood cells. When boiled and allowed to stand for some time a bright purple colour develops (Plhen's reaction). The urine does not decompose for some time.

Albumin is pentiful. Phosphates are diminished. Crobilin can be seen with the spectroscope after acidulating the urine with acetic acid and extracting with amyl alcohol.

\section{SYMPTOMATOLOGY.}

As a rule the patient has had recurrent malarial attacks, and has taken quinine spasmodically or not at all. White people are more prone to it than coloured.

Prodromata are often absent. When present there may be lassitude, general pains, loss of appetite, restlessness, yellow tinging of skin and conjunctiva.

Course: shivering fits, headache, severe pains in back, hiccough, nausea, retching, romiting food, then green bile. Excessive thirst, constipation, urgent desire to micturate. Liver and spleen tender, skin hot and dry, yellow skin and conjunctiva. Temperature rises rapidly to $103^{\circ}-104^{\circ} \mathrm{F}$., pulse $\mathrm{IOO}-\mathrm{I} 20$, small, regular, readily compressible. Blackish urine with burning sensation of the urethra.

After a few hours perspiration sets in, temperature falls to $100^{\circ} \mathrm{F}$., the urine clears, convalescence begins, or in a severe case romiting increases, temperature again rises, diarrhnea sets in with bilious motions and blood. Some recover, others die of exhaustion, hyperpyrexia, coma, anuria, and uramia as a result of this or successive attacks.

In any cases of recovery convalescence is much protracted.

There is always grave ancemia.

The mortality varies from + per cent. to 50 per cent.

The figures on the IVest Coast are of interest, as compared with those of East Africa and Nyasaland :-

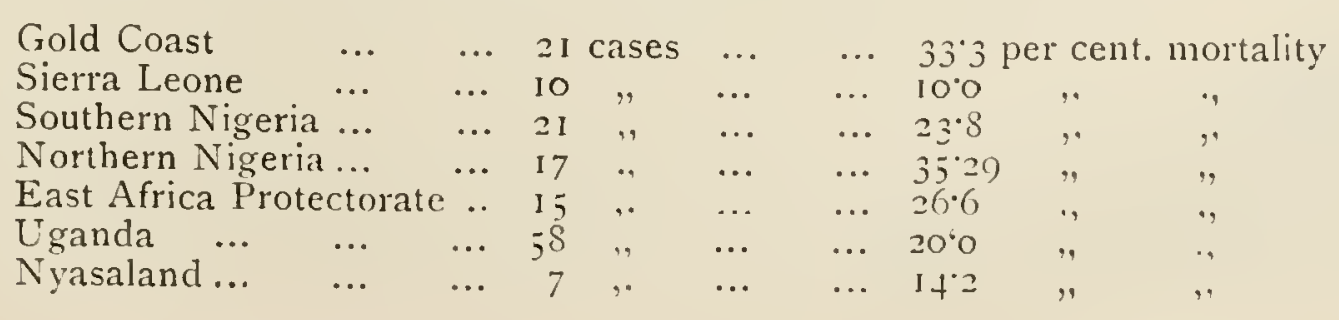


The urine is dark brown, acid, separates on standing into two layers, the upper clear or port-wine colour, the lower brownish grey with hyaline, and hib. tube casts and débris. Red cells are few or absent.

Epithelial cells may be seen.

\section{TREATMENT.}

Rest in bed is absolutely necessary with the best nursing available. The patient must not move if the attack is severe.

All kinds and plenty of bland fluids as the stomach will retain them. Calomel followed by mag. sulph. can be given with care.

Hot, moist applications over the stomach and loins and hot-air baths are always good.

If parasites are present quinine is indicated, but the results must be watched. If the condition is aggravated withdraw it for a few days, and then repeat it.

The chief essential indications are:-

(1) To flush the kidneys.

(2) To raise the blood-pressure.

(3) To strengthen the heart.

Give digitalin, ergotin and morphia for the vomiting when present. Normal salines, and plenty of them, must be given one way or other.

These can be given per rectum with a rubber catheter well inserted;

Or, and also subcutaneously, 8 to 16 ounces every three hours;

Or, and also intravenously (Rogers), four pints at a time.

Some claim good results from salvarsan given in two pints of saline (Burkitt).

Burkitt and MacGilchrist agree that the cause may be due to increased alkalinity of the blood, in consequence of which the former administered potassium and sodium bicarbonates with success, giving them in I drachm doses hourly in plenty of water.

When the danger is past give some mild ferruginous preparation, followed by Fowler's solution.

Syphilis when present must be treated as soon as possible.

The patient should leave the district early and advised not to return.

The saline treatment is all-important. Fluid must be passed into the circulation. It is useless to give pilocarpine when the blood has little fluid to give up and the kidneys already exhausted by the lack of it.

All urine must be carefully measured and the time noted when passed.

If the amount is decreasing put in more fluid by mouth, rectum, subcutaneously or intravenously, until the amount increases to the 
normal, but an excess of fluid injected and none excreted may so overtax the heart, alieady weak, as to cause sudden death.

A red cell count should be done every other day, and the move. ments of the patient, such as sitting up and using the bed-pan, \&c., must be controlled thereby. To attempt to walk too early may cause sudden death.

Exercises should be graduated and permitted according to the red cell regeneration, this being a much safer guide than the feelings of the patient or the temperature chart. One patient may regenerate in much less time than another.

\section{FAVISM.}

\section{DEFINITION.}

An acute febrile anæemia with icterus and hæmoglobinuria.

\section{DISTRIBUTION.}

It occurs in Sardinia, and perhaps other Mediterranean ports.

\section{ETIOLOGY.}

Sufferers blame fresh beans, raw or cooked, and even the smell of bean flowers when in blossom. It usually occurs in the season when the bean ripens.

\section{SYMPTOMATOLOGY.}

A few hours after ingestion of beans or exposure to the scent of the flowers an acute febrile anæmia develops, and the red cells may be rapidly reduced to $2,000,000$ per $\mathrm{c} . \mathrm{mm}$. and the hb. to 20 per cent. There is icterus; the liver may be tender to pressure; the urine contains free hb., urobilin and indican. The blood-serum, however, is clear and does not contain any hb.

Sometimes there is bilious vomiting and diarrhoea.

Children usually die, adults recover.

The blood quickly regenerates.

Malaria and quinine can be excluded from the causative factors.

Little is known about it at present.

\section{TREATMENT.}

As for hæmoglobinuria.

\section{HEAT SYNCOPE AND HEAT STROKE.} thus :-

Of this disease there are two groups, which may be classified

(I) Heat syncope, an exhaustion with little or no fever, but with some cardiac failure as a rule. 
(2) Heat stroke: (a) Direct, with high body temperature after exposure to the sun (= sunstroke).

(b) Indirect, with high body temperature but exposure to the sum is not necessary.

The difference between heat syncope and heat stroke can be tabulated thus (Bram):-

\section{Heat Syncope}

(I) History of exposure to excessive heat, usually indoors

(2) Onset gradual, no conrulsions or paralysis, but prodromata as headache, dizziness, nausea, \&c.

(3) Rarely, loss of consciousness

(4) Skin cool, pale and clammy

(5) Conjunctivx pale, pupils clilated or normal

(6) Temperature normal, sulmormal or slightly elevated $100^{\circ}-102^{\circ} \mathrm{F}$.

(7) Pulse weak and thready

(8) Respiration shallow and sighing

(9) Course greater in duration, prognosis favourable, usually terminating in recovery in a few days

(io) Recovery usually uninterrupted and complete

(II) Usually debilitated by disease

\section{Heat Stroke}

History of direct exposure to the sun's rays

Onset sudden, often with convulsions or paralysis

Usually complete unconsciousness

Skin hot, dry, flushed

Injected conjunctivæe, contracted pupils

Temperature very high, $105^{\circ}-113^{\circ} \mathrm{F}$.

Pulse high and bounding

Respirations irregular, often CheyneSiokes in type

Course brief, with guarded prognosis, terminating in death or recovery in from a few hours to a day or more

Complications and sequelie not uncommon

Previously in normal health

\section{(1) HEAT SYNCOPE.}

\section{DEFINITION.}

Faintness or fainting as a result of exposure to a high atmospheric temperature associated with humidity and bad ventilation in persons whose bodies have been debilitated by disease.

\section{ETIOLOGY.}

The two necessary factors are:-..

(1) High wet bulb temperatures.

(2) Abnormal bodily health.

This latter includes any organic disease, especially chronic alcoholism which causes fatty degeneration of the heart's muscle, allowing dilatation under conditions of moist hot air in badly ventilated places. Unsuitable clothing and vigorous exercise also predispose. Alcoholism is the most potent factor.

\section{SYMPTOMATOLOGY.}

The patient may be in a badly ventilated building as a theatre or church, in a crowd, or wearing unsuitable clothing as soldiers on the march, or unduly fatty. The patient becomes giddy, staggers and falts. There is pallor, small pulse, dilated pupils, shallow breathing, 
cold skin, temperature normal or sub-normal, partial or complete unconsciousness. Headache is common.

Death is the exception.

There are usually no after symptoms.

\section{TREATMENT.}

Open air, apply cold water to the face, loosen the clothing about the chest. Ammonia salts to the nose. Brandy to drink. Injection of strychnine when necessary.

Bed, hot bottles and stimulants if the temperature is sub-normal.

\section{(2) HEAT STROKE (Sunstroke).}

\section{(A) Direct.}

\section{DEFINITION.}

A febrile condition resulting from exposure to the direct rays of the sun accompanied by pulmonary congestion, convulsions, coma, recovery or death.

\section{ETIOLOGY.}

A dog shaved and exposed to the direct rays of the sun, dies. If it is covered with coloured glass, nothing happens.

This variety is common in Persia and India, but may occur anywhere if the temperature is high enough.

The theories as to the causation are :-

(I) Caloric, a hyperpyrexial condition.

(2) Actinic, due to the sun's chemical rays.

(3) Microbic, due to some germ (Sambon).

(4) Toxic, due to poisons generated by the excessive heat.

Manson's classification is as follows:-

(a) Superheating of the blood.

(b) Paralysis of the thermic centres causing :-

(I) Excessive production of heat.

(2) Retention of body heat.

(3) Expansion of cerebro-spinal fluid causing pressure on the brain .

(4) Vasomotor paralysis.

(5) Paresis of heart ganglia.

(6) Excess of carbon dioxide in the blood.

(7) Coagulation of the myosin.

(8) Suppression of sweat.

(9) Deficient serosity of the blood.

The thick skull and black skin of negroes are protective. 
Puntoni, who has done recent work on this subject, concludes thus :-

The lissues which compose the cranium from the dura mater to the skin, are diathermal both to the yellow-red and to the violet-ultraviolet rays, but they absorb the red and green-blue rays.

Sunstroke is brought about by the action of the violet-ultraviolet rays upon the meninges and the cerebral substance.

At the same time, one must not completely forget the red-yellow rays, which facilitate the photo-chemical action of the violet-ultraviolet rays by means of the heat which they produce.

With regard to prophylaxis against sunstroke, it is well to protect the susceptible parts (head, neck and spine) with green clothing, which absorbs the violet-ultraviolet rays as well as the red-yellow, letting pass the green rays only, which are harmless because they are arrested altogether by the tissues of the cranium.

Since in practice it is necessary to combine the prophylaxis against sunstroke with that against heat stroke, it is advisable to cover the green, heat-absorbing clothing with white material, which is well known to possess the greatest reflecting properties.

The eyes should be protected by means of coloured glasses; these again, for the above reasons, should be green.

\section{SYMPTOMATOLOGY.}

The patients may be affected in various ways under different conditions, thus :-

(1) One may have been marching long in the sun, with tunic fastened up to the throat, when there are headache, convulsions, and not infrequently incontinence of urine.

(2) Or the patient may perspire profusely, turn pallid with rapid respirations and fall partly unconscious.

(3) Or excessive thirst may be experienced followed by coma.

(4) Or a headache may become more and more severe, with intolerance to light; this may be followed by partial coma, with recovery or death. If recovery there is often headache for six or eight weeks.

(5) Or after prolonged exposure to the sun, a hot dry skin, established intolerance to light, sound and movement.

There may be vomiting.

Recovery may be :-

(1) Sudden and complete.

(2) Or prolonged over several days or weeks, then clearing up.

(3). Or persisting and leaving some permanent trouble as impaired memory, partial blindness or deafness, insanity, epilepsy. 
It appears to be a form of meningitis.

The temperature rises suddenly as a rule, $104^{\circ}, 107^{\circ}$, I I $0^{\circ} \mathrm{F}$.

There may be frequent desires to micturate.

\section{PATHOLOGY.}

Post mortem, there may be little found.

There may be a post-mortem rise of temperature, or a raised temperature maintained for some time after death. When the temperature has been known to fall after death it has never been known to rise again after any disease.

The lungs are congested. The vessels in the meninges are congested. There may be minute hamorrhages in the brain substance. There is a coagulative necrosis of the nerve cells.

\section{(B) Indirect.}

This is much the same as the direct but exposure to the sun is not necessary. It may come on at night.

There may be prodromata.

Sambon says that it has a distinct geographical distribution, and is caused by some microbic agency.

Newcomers are more liable to it.

Long resiclence produces a relative immunity.

The predisposing causes are as before mentioned.

It is most prevalent in the hottest season.

It may become epidemic at times.

It has a definitive course, peculiar lesions, tends to terminate by crisis and thus behaves like a specific fever.

Some authorities affirm that this is the same as the direct form.

\section{THE MORTALITY OF HEAT STROKE.}

Early judicious treatment is very effective.

The mortality is 15 per cent. to 20 per cent.

It is stated that among the English troops in India one in four die.

During the Great War, 1,026 men were passing some hours in a train in India. There were 1,36 cases of heat stroke with 15 deaths.

Overcrowding was blamed chiefly.

Sir Victor Horsley died from heat stroke while on active service in Mesopotamia.

\section{DIAGNOSIS.}

Diagnose from A, E, I, O, U, and sometimes $\mathrm{D}$ and $\mathrm{T}$.

$A$ lcoholic poisoning.

$E$ pileptic conditions.

I ntracranial causes such as meningitis, cerebral hamorrhage, thrombosis, embolism, gummata, and all tumours. 
$O$ pium poisoning.

$U$ ræmic conditions.

$D$ iabetic conditions.

$T$ ropical diseases, malaria, sleeping sickness, \&c.

\section{TREATMENT.}

Remove the patient to a cool place and loosen clothing.

Application of cold water and cold sponging.

Ice is essential for hyperpyrexia.

Put the patient upon a rubber stretcher covered with a sheet and the head raised, a sheet also covering the patient.

Place ice on sheet for two minutes and put the patient between blankets in bed.

Give tincture of digitalis, 30 minims.

If the patient is plethoric, bleed from the median basilic vein

Cease treatment when the temperature is down to $102^{\circ}, 103^{\circ} \mathrm{F}$. in the rectum.

Later apply hot bottles and stimulants.

Avoid strychnine as convulsions are not uncommon.

Artificial respiration may be necessary for an hour or longer.

Antipyretics are dangerous.

Relapses are common.

Salines and bromides may be useful.

Urge total abstinence.

Blue glasses and helmets should always be worn afterwards.

Spinal pads and neck shields are preventive measures.

\section{SOME UNCLASSIFIED FEVERS.}

\section{(1) JAPANESE RIVER FEVER (Tsutsugamushi Disease).}

\section{DEFINITION.}

An acute endemic febrile disorder caused by the bite of the larval form of the mite Leptus akamushi, associated with a small local necrotic area, enlarged proximal glands, and an exanthematous eruption.

\section{DISTRIBUTION.}

The Island of Nippon (Japan), in the Akita and Nugata districts.

The causative mite appears in the areas flooded by rivers a few weeks after the waters have subsided. Because of them the districts have been almost abandoned.

\section{AETIOLOGY.}

This is unknown.

Bacterial, protozoal and chemical theories have been advanced. 
An attack does not confer immunity.

Poor people of reduced resistance entering the infected lands from July to October may contract the disease.

\section{SYMPTOMATOLOGY.}

Incubation is four to ten days.

Prodromata, nothing definite, sometimes malaise and giddiness.

Course. It lasts about fourteen days. Rigors, frontal headache, temperature IOI $^{\circ}$ to $\mathrm{IO}^{\circ} \mathrm{F}$.

Tender enlarged lymphatic glands nearest to the bite of the mite; a vesicle or blackish necrotic patch indicates the latter.

Temperature rises to $105^{\circ} \mathrm{F}$. The necrotic area is thrown off leaving a circular red punched-out ulcer.

The sixth to seventh day a large red papular (2 to $5 \mathrm{~mm}$.) eruption occurs on the face and then spreads over the body with a somewhat macular appearance. The rash does not itch.

The gums may become spongy and bleed.

Respirations are increased, B. S. harsh, rhonchi all orer chest.

The conjunctivæ are injected. There is lachrymation.

Hyperasthesia is common and is present all over the body.

Lysis begins about the fourteenth day and lasts about one week.

In bad cases, coma, hyperprexia, cardiac failure or pulmonary cedema may carry off the patient.

\section{MORTALITY.}

About 30 per cent. die. It increases with age. $12^{\circ} 5$ per cent. during the first ten years of life and 57 per cent. in the seventh decade.

\section{TREATMENT.}

This is symptomatic.

\section{(2) PIGMENTARY FEVER (Cobb).}

Occurs in Bengal during the hottest months.

Sudden onset; the temperature rises to $103^{\circ}-104^{\circ} \mathrm{F}$. Headache, nausea, vomiting, and a peculiar pigmentation of the nose and cheek.

The fever lasts eight to ten days. Little is known about it.

\section{(3) HYPERPYREXIAL FEYER.}

Seen on the West Coast of Africa and Ceylon.

Onset like malarial fever with sweating, but no parasites are seen.

The second day the patient feels almost normal.

The third day the temperature begins to rise and reaches from $104^{\circ}-107^{\circ} \mathrm{F}$. , at which it tends to remain.

Drugs are useless. 
Cold baths reduce the temperature temporarily during the first six or seven days.

The temperature then rises to $110^{\circ} \mathrm{F}$. about the eighth day.

Delirium sets in, coma and death in 50 per cent. of cases.

Others recover. The temperature remains at about ${ }_{0} 5^{\circ} \mathrm{F}$. for three weeks, and falls to normal about the sixth week.

Little is known about the atiology, \&c.

The viscera, blood and urine appear to be normal.

\section{(4) DOUBLE CONTINUED FEVER.}

This is seen in China. Onset insidious. Temperature gradually rises to $104^{\circ} \mathrm{F}$, remitting three degrees daily; pulse slow, tongue moist and red, constipation. After ten to fifteen days the temperature falls to normal by lysis.

After a lapse of two to seven days the fever is repeated and lasts about ten days. The anzemia is considerable. Convalescence is prolonged.

The mortality is low. Quinine is useless.

\section{(5) LOW INTERMITTENT NON-MALARIAL FEVER.}

This is seen in India, China, Siam and Ceylon.

The onset is insidious, or it may follow a mild form of influenza.

The fever is remittent between $98^{\circ} 4^{\circ}$ and $\mathrm{IO}^{\circ} \mathrm{F}$.

Chilliness is felt daily between Io and I 2 'clock.

The eosinophilic cells are increased even when worms are absent.

The course of the fever is long, some over five months.

Nothing else abnormal is found. The patient should be sent to another climate.

\section{(6) NASHA FEVER.}

It is seen in Bengal from April to August annually.

It is rarely present in the cold weather.

The onset is sudden with chill, high fever, hyperamia, swelling of the nasal mucosa, pains in head, neck and shoulders.

The face is flushed, the pupils contracted.

There is a papular eruption, some bronchial symptoms.

The fever is remittent and disappears in three to five days, when the nasal swelling subsides. Relapses may occur between the first and fourth week after the attack. Some may be severe, terminating in delirium, coma and death.

Give saline purgatives, mild diaphoretics, spray nose with iced water.

Apply tannin, ro per cent., and cocaine solution to the nasal mucosae. 


\section{DEFINITION.}

\section{(7) ARCHIBALD'S FEYER.}

An unclassified septicamia in the Anglo-Egyptian Sudan caused by a bacillus of the B. cloaca group.

\section{SYMPTOMATOLOGY.}

The temperature rises $101^{\circ}-103^{\circ} \mathrm{F}$, drowsiness, sometimes delirium, dry furred tongue, sometimes the spleen is slightly enlarged.

The temperature falls to normal in about one week, after which a long remittent ferer continues.

Pneumonia, abscesses, femoral thrombosis may set in.

Treatment is symptomatic.

\section{(8) BACILLUS ASIATICUS FEYER.}

This is seen in Ceylon.

It is caused by B. asiaticus, wwo varieties.

The disease is a septicamia.

There is a long low remittent fever, sometimes intermittent with marlied abdominal pains, but no diarrhoea.

The specific bacillus can be recovered from the faces.

The agglutination test can be used.

Autogenous vaccines may be injected.

\section{(9) THE MACULAR FEYER OF TUNISIA.}

\section{DEFINITION.}

An acute febrile disorder characterized by a macular eruption on the abdomen, palms and soles, often persisting for several days after the temperature has become normal.

\section{SYMPTOMATTOLOGY.}

The onset is sudden, rigors, ferer, joint pains, injected conjunctivæ, nausea, romiting, constipation and insomnia.

The rash comes from the second to the fourth day, red spots distributed as above.

There is lymphocytosis, 35 per cent.

The fever lasts two weeks.

The rash fades without desquamation. Perhaps this fever is related to typhus fever.

\section{(10) FIYE-DAY FEVER (YOLHYNIA FEYER).}

The findings and views of different observers are very conflicting

The causative organism is unknown.

Lice have been blamed as the carriers.

The course is mild, the prognosis good, and complications are rare.

The peculiar features are its periodicity and an increase of the leuco- 
cytes as the fever subsides. Bone cells are seen in the blood. A mild eruption is seen in some cases.

Quinine and salvarsan are said to be useless.

Collargol is said to act as a specific when given, io c.c. of a I per cent. solution three doses intravenously. This is said to cure (Heyden).

\section{DEFINITION.}

\section{(11) RAT-BITE FEVER.}

An acute infectious disease, probably caused by spirochætes characterized by a local ulcer, followed by constitutional disturbances of pyrexia, eruption and body pains.

\section{DISTRIBUTION.}

It is found in Japan, England, the United States, and Italy.

\section{AETIOLOGY.}

Futaki discovered spirochætes in the blood and tissues of patients in 1916. The organism was pathogenic to guinea-pigs.

Spirochrtes have been found in the suprarenals and kidneys of people dead of the disease.

At first the organism circulates in the blood; then, when immune bodies are formed, they are found in the kidneys. The antibodies thus formed act upon the causative spirochæetes, producing degenerate forms. In this particular the disease simulates Spirochætosis icterohæmorrhagica.

The xtiology seems now to be established, so that upon confirmation of the above findings it will be necessary to include this disease in another section of this work.

\section{SYMPTOMATOLOGY.}

The incubation is one to three weeks.

The onset is sudden. An ulcer forms seven to fifteen days later at the site of the bite which had previously healed. The proximal glands are swollen.

There is a papular purple eruption on the body in some cases, pains about the joints and muscles, pyrexia to $\mathrm{IO}^{\circ} \mathrm{F}$., and sometimes delirium.

After a few days the fever abates and the patient feels better; then it recurs. These relapses are rather characteristic of the disease.

Each attack leaves the patient somewhat better, but it may be months or years before the disease has run its contse.

The prognosis is good and complications rare.

The mortality is about $10^{\circ} 5$ per cent.

\section{TREATMENT.}

Arsenical treatment is the rule-as for Relapsing fever. 

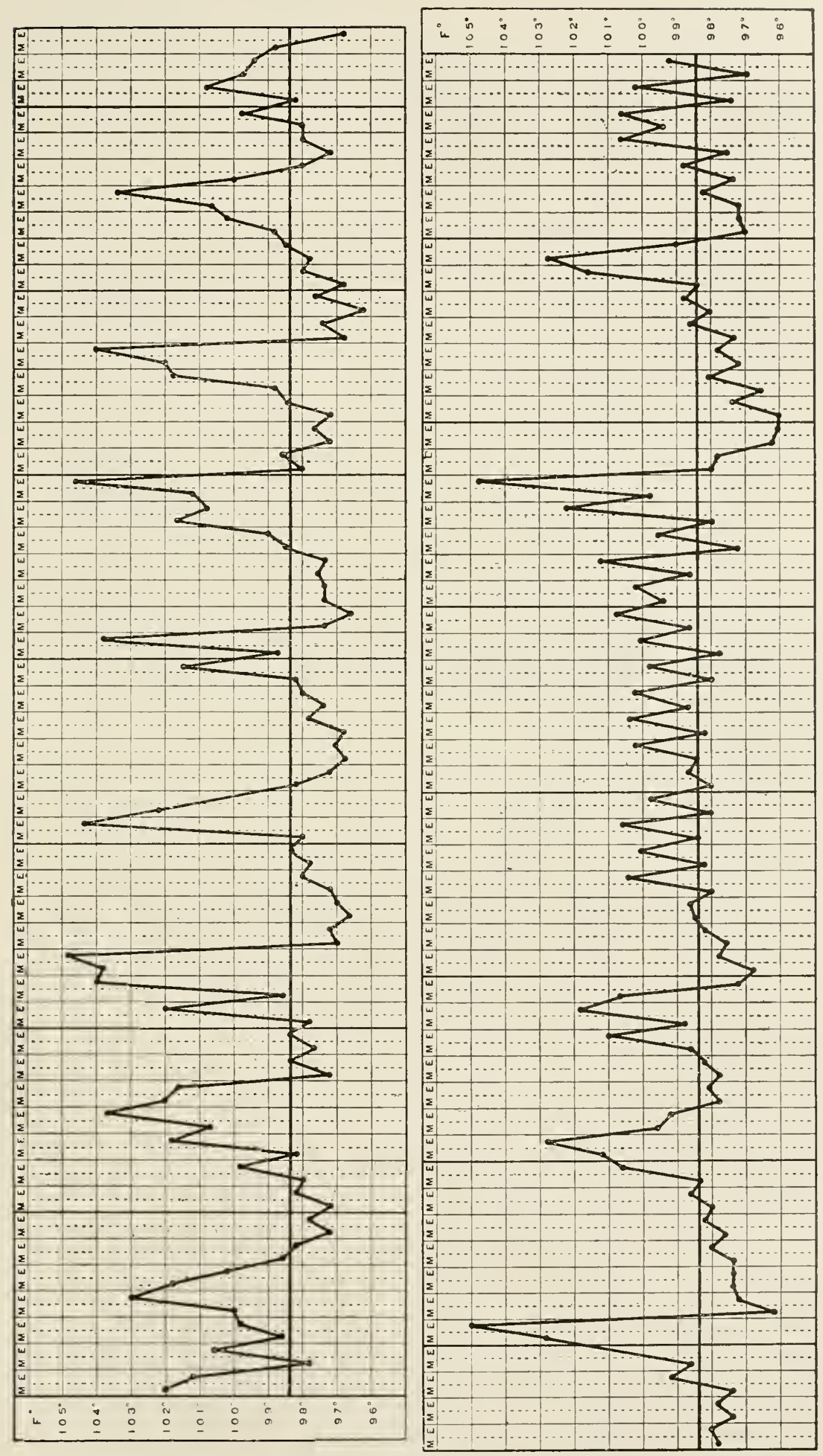


\section{DEFINITION.}

(12) TRENCH FEVER.

It is a specific infectious disease caused by a resistant filterable virus in the blood plasma, transmitted by lice.

\section{DISTRIBUTION.}

Flanders, Mesopotamia, Salonica, and Italy.

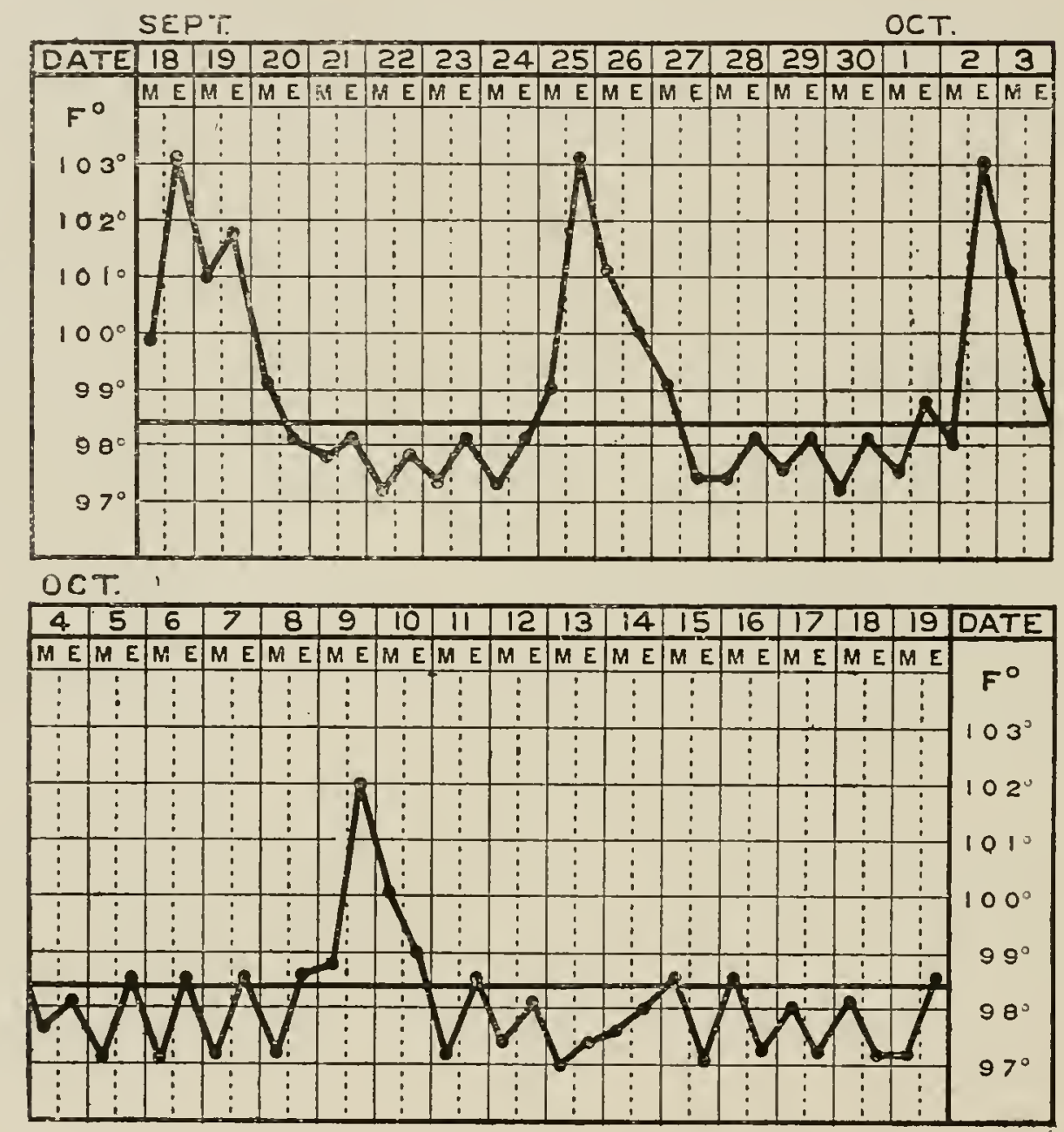

\section{ETIOLOGY.}

Trench fever.

The causative organism is present in the blood plasma, and is transmitted naturally by the louse.

The blood is always infective in the early stages.

The organism is not filterable. Spirochætes have been blamed.

The virus has been recovered from the urine and from mixed sputum and saliva, but not from the freces.

\section{SYMPTOMATOLOGY.}

The incubation period is fourteen to thirty davs, or in some cases shorter.

The onset is sudden, with headache, dizziness, pains in the legs (shin pains), back, and behind the eyeballs, pyrexia $103^{\circ}-104^{\circ} \mathrm{F}$., 
which becomes relapsing in one-half of the cases, erythematous spots in 70 to so per cent. of cases, enlargement of the spleen, and, slight albuminuria.

The fever may be but one sharp rise, or it may oscillate for four to six days, or relapse over six to seven weels, with intervals of five to seven days. Some patients have had some fever for periods of forty to sixty days with only slight remissions.

There are three to fire, or eren seren relapses.

Recovery is the rule.

\section{TREATMENT.}

This is symptomatic. Arsenical preparations as for Relapsing fever can be tried.

All lice should be destroyed and clothing sterilized.

Sputum cups and special urine bottles should be reserved for patients and their contents sterilized.

\section{THE EXANTHEMATA.}

As the majority of these are described in general text-books of medicine a few remarks only will be necessary.

Scarlet fever may be introduced into the tropics, but does not spread there.

Measles is endemic in most tropical countries and may give rise to severe epidemics.

Chicken-pox is usually mild, but it may be severe. It more often attacks adults in the tropics. It is common in India from February to March.

Yariola has long been present in India and Africa. It was perhaps introduced into China from India about the third century B.C.

An African mummy las been found with a cutaneous eruption belonging probably to the twenty-eighth dynasty (I200-1100 B.c.). How it came to Africa and India is not known. It is common all over the tropics to-day.

"Alastrim." - Perhaps this is a mild variola that does not breed true.

This acute specific fever resembles a mild form of variola.

It is widely distributed in the tropics.

Jenner's vaccination is generally held to be protective.

It differs from chicken-pox because of-

(I) Confluence of the vesicles in certain cases.

(2) Its frequency amongst adults.

(3) Partial protection by Jenner's vaccine. 
It differs from typical variola because of -

(1) Its low mortality, I to 2 per cent.

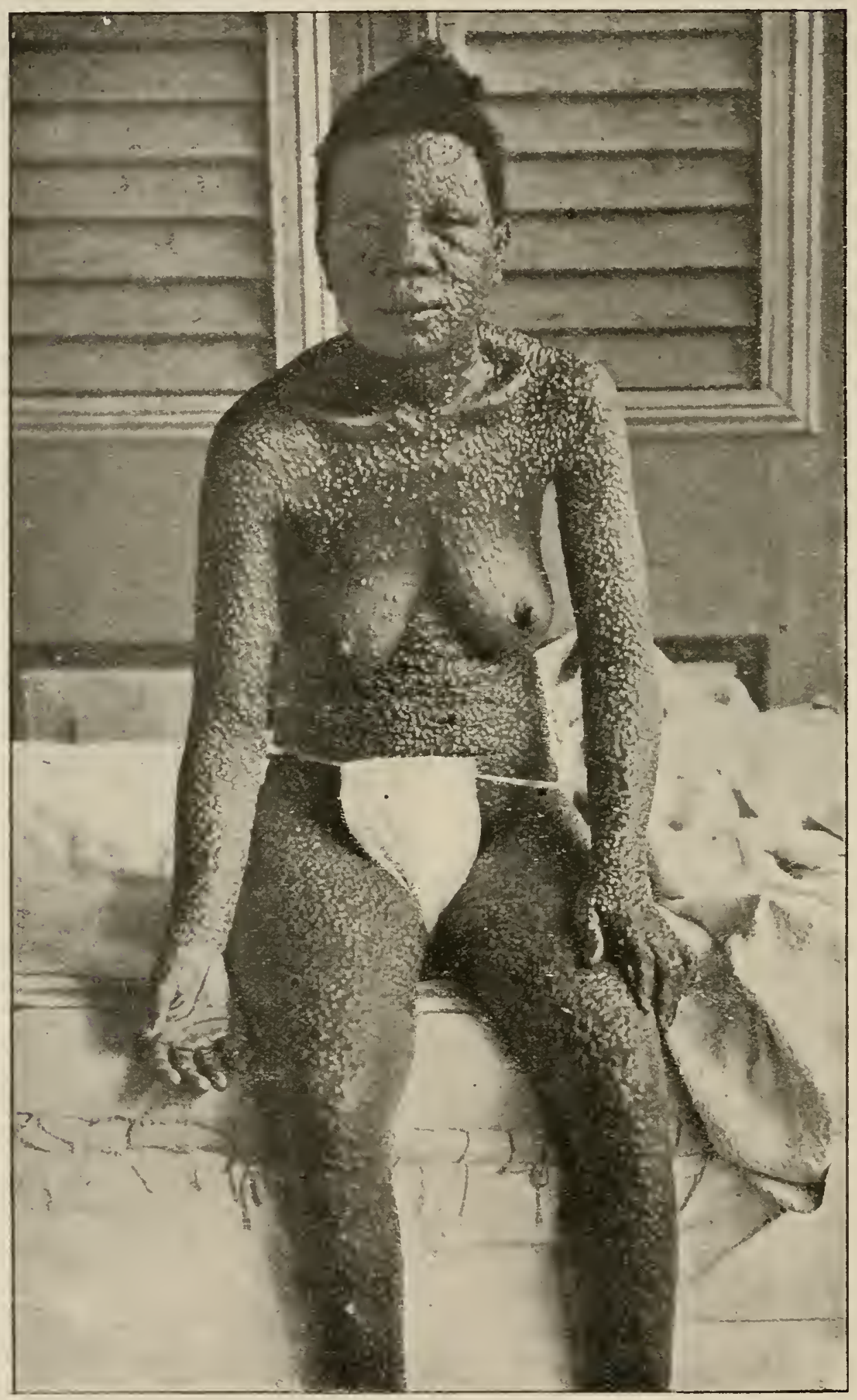

"Varioloid varicella," Trinidad. From a photo by Dr. Scheult.

(2) It is less severe in children than in adults; it is often found amongst babies.

(3) There is no secondary fever in children.

(4) The disease can occur after recent successful vaccination.

(5) Vaccination can be successful shortly after an attack of alastrim. 
(6) Some assert that small-pox does not render immunity from alastrim.

The onset is sudden, with severe pains, high fever, vomiting, sometimes delirium. The rash appears on the third day, the temperature is reduced and the symptoms disappear, and the patient feels well again.

Secondary fever is very rare, about the eighth day when present.

Papules become pustules and scales. These fall off with some pigmentation, but very little permanent scarring.

Yesicular Fever begins like chicken-pox and ends lilie small-pox.

The symptoms are in accordance with these two diseases.

The diagnosis is not easy, but the important points to note are:-

There is no true pustulation with scarring as in small-pox.

And Jenner's vaccination is successful during convalescence.

In chiclien-pox the resicles are larger and leare crusts.

In alastrim the eruption is often confluent and pustular.

The prognosis is good.

Papular Fever.-This resembles measles, but it has no catarhal symptoms.

The onset is insidious.

The prodromata are malaise, depression, rheumatoid pains in back.

Course.-The rash appears on the third or fourth day suddenly all over the bods, morbiliform, bright red, pin-head papules and red macula.

The face is not much affected.

There are papules in the mouth.

A slight fever lasts one to two days.

There is no enlargement of glands.

The eruption lasts two to five days. There is no desquamation, and there are no marks left. The rash itches.

Aspirin relieves the pains and calamine lotion the itching.

\section{Hæmorrhagic Febrile Gastro-enteritis of Children.}

It is seen in Guadeloupe.

It somewhat resembles yellow fever.

The onset is sudden with vomiting, followed by febrile biliousness.

There is a remittent fever, slight icterus, obstinate constipation, and later, black vomit.

After two to five days, improvement sets in or the child may die.

Convalescence is prolonged.

The prognosis is grave.

The treatment is simptomatic. 


\section{DISEASES OF UNKNOWN AETIOLOGY AFFECTING THE RESPIRATORY SYSTEM.}

\section{(1) TROPICAL HAY FEVER (Rhinitis spastica vasomotoria).}

Adults are attaclied. There are violent fits of sneezing, lasting from two minutes to two hours. There is an excess of nasal secretion.

Iachrymation, conjunctive injected, eyelids swollen.

These attacks may recur the same day or over several weeks.

There may be accompanying asthmatical symptoms.

The mucous membrane over the turbinate bones becomes swollen and may cause nasal obstruction.

A spray of cocaine, I per cent., and adrenalin locally is useful.

Atropine and strychnine pills may be tried.

A change of climate may clear up the malady.

\section{(2) GANGOSA (Rhino-pharyngitis mutilans).}

\section{DEFINITION.}

An ulcerative condition affecting the palate, nose, pharynx, larynx and skin, destroying cartilage and bone, thus producing permanent deformity.

\section{DISTRIBUTION.}

It appears to be endemic in the Batanes Islands, where some hold it to be the early stage of leprosy.

It is also found in the Ladrone Islands, Guam, Caroline Islands, Fiji, Murray Island, Panama, British Guiana, Ceylon, Nevis, Dominica and Italy.

Dr. Anton claims that it is a blastomycosis due to a specific parasite, the Cryptococcus mutilans. This requires confirmation.

\section{SYMPTOMATOLOGY.}

A sore throat is complained of, caused by a nodule on the palate or at the back of the pharynx, or on the posterior pillar of the fauces. This becomes a superficial ulcer covered by a greyish slough.

This ulcer spreads, destroying the adjacent tissue, soft and hard alike, so that in time the skin falls in allowing the nasal and buccal cavities to form one aperture.

It may then affect the face without or the larynx within.

Vision may be destroyed. The odour given off is most offensive.

Ulcers may appear on other parts of the skin which is uncovered by clothing.

Ugly deformities are caused by the scarring and contraction.

A chronic osteitis may set in simulating that of syphilis. 
It may go on from ten to thirty-five years, progressively or intermittently.

Wassermann's reaction is always positive.

As a rule the general health is little affected.

It is rarely fatal, and all tend to recover ultimately.

\section{DIAGNOSIS.}

There are neither signs nor symptoms of syphilis (Johnson does not agree).

The spirochate cannot be found and iodicles are useless.

There are no leprosy nodules nor anasthetic patches.

Hansen's bacillus has not been found.

Its histological characters are not those of epithelioma.

There are no metastases and its course is protracted.

Tuberculosis is excluded by the absence of Koch's bacillus and other symptoms.

Guinea-pigs cannot be inoculated successfully.

\section{TREATMENT.}

Nothing will stop its onward course.

Application of iodine tincture or the actual cautery can be tried.

Wash the foul ulcers with potassium permanganate or peroxide of hydrogen.

Segregation is essential for stamping out the disease.

\section{SOME NERVOUS DISTURBANCES IN THE TROPICS.}

\section{(1) TROPICAL NEURASTHENIA.}

This is a condition somewhat common in white people, and the better class coloured people residing in the tropics, especially where there is a moist warm atmosphere.

There are many predisposing causes, such as malarial fever, or other illness, overwork and alcoholism.

Perhaps 30 per cent. to $5^{\circ}$ per cent. of white residents become semiinvalids from this malady.

Palpitation with heat flushes is usually present. Phobia, especially with regard to diseases, is a common feature. Sleeplessness and irritability are common.

The condition is the cause of many clerical errors and inaccuracies which lead to numerous petty worries and manifestations of bad temper.

The patient should have a change of work and climate.

Neither sedatives nor tonics have been much use in the writer's experience. 


\section{(2) ENDEMiC PARALYTIC YeRTigo.}

This is seen in France, Switzerland, Shikoku, Japan.

The retiology is unknown.

An attack begins with the blurring of objects, ptosis, paralysis of the internal rectus causing diplopia, photophobia, affection of colour vision, hyperamia of the optic disc.

Speech is disturbed, mastication difficult through paresis, and later paralysis of the muscles that operate those functions.

The head falls forward as the neck and shoulder muscles are affected. The posture may be stooping.

Weakness of arms, hands and legs is not uncommonly seen.

The attack usually lasts ten to fifteen minutes.

Between attacks the patient may be perfectly well.

The disease is never fatal.

Iodides and arsenic should be given.

Remove the patient to a healthy climate and district.

\section{(3) ENDEMIC PERIPHERAL NEURITIS.}

This is seen upon the Central African plateau, 5,000 to 6,000 feet high.

It is said to affect 3 per cent. 105 per cent. of the Bantu people.

There are shooting or pricking pains in the forearms and legs; accompanied by numbness, with an erythematous rash and local swelling of the affected area.

Cold and damp increase the symptoms.

Heat and dryness diminish them.

The gait is peculiar, the patient haring the appearance of walking upon his toes and heels.

The whole body may be affected.

The disease may spontaneously clear up or it may last for years.

\section{(4) LÂTÁH.}

This is seen in the Malay Peninsula, Jara, Sumatra, Siam, Burmah, Philippines, Siberia and North America.

ETIOLOGY (Abraham).

Auditory, an unexpected noise behind a person.

Visual, some unlooked-for movement.

Tactile, an unexpected sudden touch.

\section{SYMPTOMATOLOGY.}

The action seen, noise heard or words uttered may be repeated, with the addition often of foul language. 
The patient is conscious, but cannot apparently control his actions. 'the hand spontaneously rises to the head.

There is a loss of memory of what has taken place.

The actions may be impulsive or mimetic.

It is not unlike the "jumpers" or "jerlis" seen in Europe during the Middle Ages.

It is sometimes present during religious revivals of to-day.

It has a medico-legal aspect of considerable importance.

These subjects are often teased, irritated and made worse.

Auto suggestion can be tried.

Determination on the part of the patient is essential.

(5) ÂMOK (= an impulse to murder).

This is a term applied to a condition, partly physical, partly psychical, following a period of depression.

They are capable of self-violence and of wounding and lisiling others.

Memory fails them as to what they have done.

The attack is followed by a condition of stupor.

It is seen in Malaysia, Trinidad, India and Liberia.

The exciting causes are excessive smoking of Cannabis indica, or opium, or some strong emotion, such as anger, fear or sorrow.

After this the patient broods over his wrongs for days or weeks, then complains of the appearance of clevils, and goes forth to kill them with his flame-shaped knife or his gun.

The attack lasts but a short time.

\section{DEFINITION.}

ENDEMIC FUNICULITIS.

An acute specilic suppurative inflammation of the spermatic cord of uncertain atiology. The theories of causation are legion.

\section{DISTRIBUTION.}

It is seen in Southern India, Egrpt, Mest Indies, Ceylon.

\section{PATHOLOGY.}

The whole cord is wholly inflamed, infiltrated and swollen.

It may be $3 \frac{1}{2}$ inches in circumference.

On incising it transversely, yellow pus exudes from the veins of the pampiniform plexus and the vas deferens.

The inflammation also attacks the epididrmis.

There is usually some odema and a little clear fluid in the tunica vaginalis. 
The microscope shows the reins of the pampiniform plexus dilated with pus cells and thrombi; the coats of the veins and the ras deferens are infiltrated by small cells.

\section{SYMPTOMATOLOGY.}

The onset is sudden. The patient after a day of hard rork feels tured, takes a bath, and has a shivering fit and a rise of temperature. There may be romiting.

There is pain along the cord and epididymis.

The condition becomes rapidly worse.

On the third day there may be continuous romiting and some hiccough.

The temperature is about I $^{\circ} \mathrm{F}$., the pulse small and rapid.

The cord is swollen, hard and tender.

The skin over it is normal.

The condition is usually unilateral.

There are no signs of gonorhosa or trauma.

If left, a septicamic condition sets in, the slin becomes jaundiced, cutaneous hamorrhages, the fever rises and the pulse quickens, the hiccough is worse, and death follows in a few days.

Spontaneous recovery is the exception.

\section{TREATMENT.}

The only effective treatment is prompt surgical interference.

The inflamed cord should be excised as high as affected.

The testis loses its generative function in all but the mildest cases.

\section{EPIDEMIC GANGRENOLS RECTITIS. (Ackers.) DEFINITION.}

A very rapidly fatal and spreading phagedanic disease about the anus and the lower part of the colon, confined to the natives of the northern provinces of South America, Fiji and other islands of the South Pacific.

It occurs amongst the children of poor natives and also in animals.

\section{SYMPTOMATOLOGY.}

There is anal itching, tendency to frequent defacation, inflammation of the rectal mucous membrane, the latter dereloping in about three days, followed by acute dysenteric symptoms, mucus, blood, sometimes bile with much tenesmus.

There is some fever, thirst and anorexia.

A semi-liquid, slimy, foetid, blood-stained, greenish stool comes away. 
All food is refused. The thirst becomes intense. fatal.

Prolapse is common. Gangrene sets in, spreads rapidy, and is

\section{TREATMENT.}

As practised by the natives.

For animals.

An enema of strong lemon-juice mixed with diluted white rum (aguadiente) thrice daily.

Dust the anus with wood ashes.

An oil purgative is given.

This appears to be successful in early cases.

For children.

Give an enema of juice from the stalks and leaves of Spigelia anthelmentica (Pasote).

A decoction of the same is given by mouth thrice daily.

(This mixture is also given as an anthelmintic.)

A portion of roasied lemon inserted into the rectum once or twice daily.

It is said to be caused by children chewing the green tender staks of unripe maize.

\section{INFANTILE BITIARY CIRRHOSIS.}

This is a rapidly fatal disease attacking young native children in Calcutta and some other large Indian towns.

\section{AETIOLOGY.}

Children are attacked in their first year during dentition as a rule. They are rarely attacked from the first to the third year.

Hindoo children in Calcutta had a mortality of 1,616 (1891-1893), and Mahommedan children in the same city had so dead during the same period.

The children of the rich suffer more than those of the poor.

It tends to run in families, and attacks them as the young are born. Nearly if not all of those attacked die.

Alcohol, syphilis, and malaria have been excluded.

The cause is unknown.

Some suggest that it is a variety of kala-azar.

\section{SYMPTOMATOLOGY.}

The onset is insidious. The liver enlarges even to the umbilicus.

There is nausea, sometimes vomiting, sallow slin, constipation, irritability and anorexia. Low fever sets in with jaundice, pale stools and dark urine. 
There may be oedema of the feet and hands with ascites.

Death from cholamia ensues in a few weeks to a few months.

\section{PATHOLOGY.}

The biliary cirrhosis seems to be the result of some gastric irritant acting upon the liver cells. The cells at first become degenerate, then the intercellular connective tissue is increased, followed by that of the portal sheaths.

There is a marked attempt to form new bile ducts between the hepatic cells (Gibbons).

\section{TREATMENT.}

Early removal from the district has been suggested.

A change of wet nurse and infant foods could be tried.

\section{GOITRE.}

In the Tropics this condition does not appear to be associated with any geographical or geological condition .

Many authorities believe it to be of parasitic origin, and suspect an amceba which is ingested with water.

A new-comer to an endemic district notices that after a few weeks his neck begins to swell gradually. At times it may be stationary, at others progressive until a permanent hypertrophy results.

The whole thyroid gland is usually affected.

Later it may undergo cystic or adenomatous changes.

Removal to another non-endemic district will cause it to diminish in size and to clear up in early cases.

Salol and thrmol are sometimes given.

\section{CLIMATIC BUBO.}

\section{DEFINITION.}

A painful gradual enlargement of the inguinal lymphatic glands with fever of uncertain atiology.

\section{DISTRIBUTION.}

The East Coast of Africa, West Indies, Straits Settlements, China, Madagascar, Chille, Uganda, and many other places.

\section{AETIOLOGY.}

Hewlett has isolated a bacillus not decolorized by Gram and not unlike the $B$. pestis.

Cantlie believes it to be a form of attenuated plague, pestis minor, Hewlitt's isolation supporting it.

Others hold that the disease is a distinct entity. 


\section{PATHOLOGY.}

The gland capsule is thickened.

The lymphocytes are in great excess.

Hamorrhagic foci may be seen.

There are numerous typical plasma cells that are absent in plague.

The retractile cells of Recklinghausen are absent, but present in plague.

\section{SYMPTOMATOLOGY.}

The onset is gradual.

Several days of malaise precede slight fevér and pain in one or other or both inguinal glands.

The inguinal glands are enlarged, hard and tender.

These may become as large as a goose's egg.

As a rule they do not suppurate.

Lymphangitis is absent.

Aspiration yields a little sterile fluid, but no pus.

The fever is low and irregular, about $102^{\circ} \mathrm{F}$.

The disease may last a few days, weeks, or months.

The general health is good.

\section{TREATMENT.}

This is symptomatic. Rest in bed. Lead lotion, ichthyol and belladonna ointment, and mild aperients.

Incise when sepsis is present.

\section{GOUNDOU.}

This condition is a bony, bilateral, symmetrical swelling situated on either side of the root of the nose. It is rarely unilateral.

Some observers assert that it is a general condition that affects the other bones of the skeleton also.

It occurs in young people of native races and is commonest in the West Indies. The writer has seen it in the Kasai basin, Central Africa and on the Gold Coast.

The condition has been reported in an European (Cantlie).

The atiology is unknown.

\section{PATHOLOGY.}

Outside the bony swelling is a thin layer of compact bone, all the remaining mass being spongy bone.

The covering periosteum strips off easily and shows no sign of inflammation.

The growth may be attached to the nasal bone, the nasal process of the superior maxilla, or to the maxilla itself.

The skin orer the tumour is always freely movable. 


\section{SYMPTOMATOLOGY.}

Gradual onset, pain in the nose, bloody discharge lasting six to eight months. As this disappears a swelling appears and increases in size.

The vision may be interfered with.

The deformity is sometimes hideous in the extreme.

There may be polypoid-like excrescences protruding from the nasal mucous membrane.

The growth may stop at any time.

\section{TREATMENT.}

Surgical remoral is easy and effective.

Other treatment is symptomatic.

\section{BIG IIEEL.}

This is at condition seen on the Gold Coast and Formosa.

The etiology is obscure.

The attaclis come in the wet season with sudden onset and fever.

There is great tenderness over the os calcis so as to prevent walling or sleeping.

In three to seven days the bone enlarges and increases progressively for two weeks or so, during which time the pain becomes less and the fever disappears.

After a month the bone ceases to grow and remains like this for one or two months, when the patient is able to walk with some degree of comfort.

The growth then gradually diminishes, but never wholly disappears. It usually affects both sides.

The ankle-joint is never affected, and the tarsal bones but seldom.

The bone should be trephined or gouged down to afford relief.

\section{DEFINITION.}

$$
\text { AINHUM ( }=\text { to saw or to cut). }
$$

A chronic dystrophy of the fifth (rarely other) toes in native races, characterized by the formation of a furrow at the digito-plantar fold, which extends and deepens until the toe is severed.

It is seen in many parts of the Tropics.

\section{PATHOLOGY.}

There is irritation of the cutaneous epithelium causing an internal proliferation, extending to the cutis, injuring the rasomotor nerves, causing spasm of the ressels, endarteritis obliterans, fibrosis of the cutis and rarefying osteitis which separates the digit. 
No organisms can be found.

! The source of the irritation: why lhis particular spot should be chosen, and why a fibrotic ring is caused, has not yet been made clear.

\section{SYMPTOMATOLOGY.}

There is no general disturbance.

It begins on the inner side of the digito-plantar furrow.

An ulcer may form that causes pain.

The condition may last two to fifteen years until the loe is severed.

In 10 per cent. of cases the fourth toe is affected.

\section{TREATMENT.}

No known treatment is of any avail.

Some divide the fibriotic ring early so as to save the toe, but the condition usually progresses.

\section{CIAPPA.}

This is seen in Lagos.

There are severe pains in the muscles and the joints of the extremities.

After a few months the pain increases and the joints swell.

Nodules, subcutaneous, the size of a goose egg appear in different parts of the body. The skin over them ulcerates, later exposing a fatty base. There may be one or many nodules.

When ulcerating they may coalesce.

Some of the nodules are absorbed without ulceration.

The ulcers may last for years.

The joints may become permanenty disorganized and the bones affected.

It may be a tertiary phase of yaws (Manson). Mercury and iodides are useless.

Scraping and antiseptics are good measures.

Neosalvarsán could be tried.

$$
\text { PONOS (= pain). }
$$

This disease, affecting young children of Spezzia and Hydra in the Grecian Achipelago, resembles infantile biliary cirrhosis of Indian towns in that-

It is confined to very young children.

It is endemic in particular districts.

It tends to run in families.

It is invariably associated with the disease of an abdominal riscus. 


\section{SYMPTOMATOLOGY.}

1t affects children of the rich and poor alike.

The onset is rather sudden in the first year of life.

Sallow tint of skin and irregular fever are present.

The spleen enlarges much and is tender, hence the name "ponos" $=$ pain.

Prostration is marked and emaciation progressive.

Late in the disease odema and ascites set in.

Hamorrhages from the gums and from other organs are not uncommon .

Bronchitis, pneumonia, dysentery, peritonitis and meningitis are complications to be looked for.

The disease lasts from two months to two years.

A pronounced leucopenia has been found.

Some suggest because of this that it may be of protozoal origin, related to kala-azar.

\section{TREATMENT.}

Nothing specific is known.

\section{TROPICAL LIVER.}

A condition of hepatic congestion brought about during the first two years' residence of Europeans in the Tropics.

Chills, changes and excesses of food and drink cause hyperamia of the liver, congestion, blood stasis and diminution of the function of that organ.

\section{SYMPTOMATOLOGY.}

There is frontal headache, malaise, nausea, dyspepsia, constipation and pale-coloured stools.

The liver is slightly enlarged and tender, slight icterus, diminished urine, irritability, slight fever to $100^{\circ} \mathrm{F}$.

\section{TREATMENT.}

Rest in bed in acute cases.

Calomel, 2 giains, followed by saline purges.

Hot fomentations to liver region are good. chills.

Bland diet, no alcohol or spiced foods, moderate exercise, avoid

The following mixture has been found useful :-

$$
\begin{aligned}
& \begin{array}{llllllll}
\text { R. Ammon. carb. } & \ldots & \ldots & \ldots & \ldots & \text { grains } 2-5 \\
\text { Sod. bicarb. } & \ldots & \ldots & \ldots & \ldots & \ldots & & 20
\end{array} \\
& \begin{array}{llllllll}
\text { Citr c acid } & \ldots & \ldots & \ldots & \ldots & \ldots & , & 20
\end{array} \\
& \text { One ounce thrice daily. } \quad \cdots \quad, \quad 20
\end{aligned}
$$


BOOMERANG I.EG.

This is a bowing forwards of the bones of the legs occurring im young adults during the period of growth. It is of unknown causation.

\section{DISTRIBUTION.}

It occurs among the natives of IVestern Australia, Northern Territory, North Queensland, Torres Straits and British New Guinea.

\section{ETIOLOGY.}

The post-mortem appearance suggests a chronic osteomyelitis of both tibia. It begins as a rarefying osteitis, passing on to a condensing osteitis, metaplasia of the fibrosed marow, and a new periosteal formation. Syphilis and tuberculosis can be excluded as causative factors.

\section{SYMPTOMATOLOGY.}

There are tenderness and pain in the tibia and fibula, the bones may become soft and bent until they gradually assume the boomerang curve with the convexity outwards.

The patient becomes flat-footed and wallis with a shuffling gait.

The subcutaneous tissues become inflamed and tender.

There is some febrile disturbance.

The symptoms gradually disappear, the bones resume their former hardness, but the deformity remains.

\section{WHITMIORE'S DISEASE.}

\section{DEFINITION.}

A septicamic infection of insidious onset, characterized by nodules and abscesses resembling those of gianders in the lungs and viscera, occurring in morplino-maniacs usually and those addicted to the cocaine habit.

A bacillus has been found, but not yet definiteiy classified.

\section{DISTRIBUTION.}

Rangoon.

\section{ETIOLOGY.}

It occurs in the adult males of the poorer classes. It has a close relation to the hypodermic syringe.

The isolated bacillus has been differentiated from the $B$. mallei.

The diagnosis is usually made at the autopsy.

\section{SYMPTOMATOLOGY.}

It is insidious in onset, usually occurring in broken-down morphine or cocaine victims. There is cough with pain, irregular pyrexia, furred tongue, slight ulceration of the tonsils, broncho-pneumonic signs and 
symptoms, palpable spleen, subcutaneous abscesses, sometimes oedema of the arms or legs.

The characteristic lesions are "nodules" of the lungs, liver and kiclneys, the former resembling patches of broncho-pneumonia.

The abscess may be subcutaneous or intramuscular, or in the lymph glands, liver or spleen.

The mesenteric glands suppurate, the sigmoid colon becomes ulcerated; petechize have been seen in the endocardium.

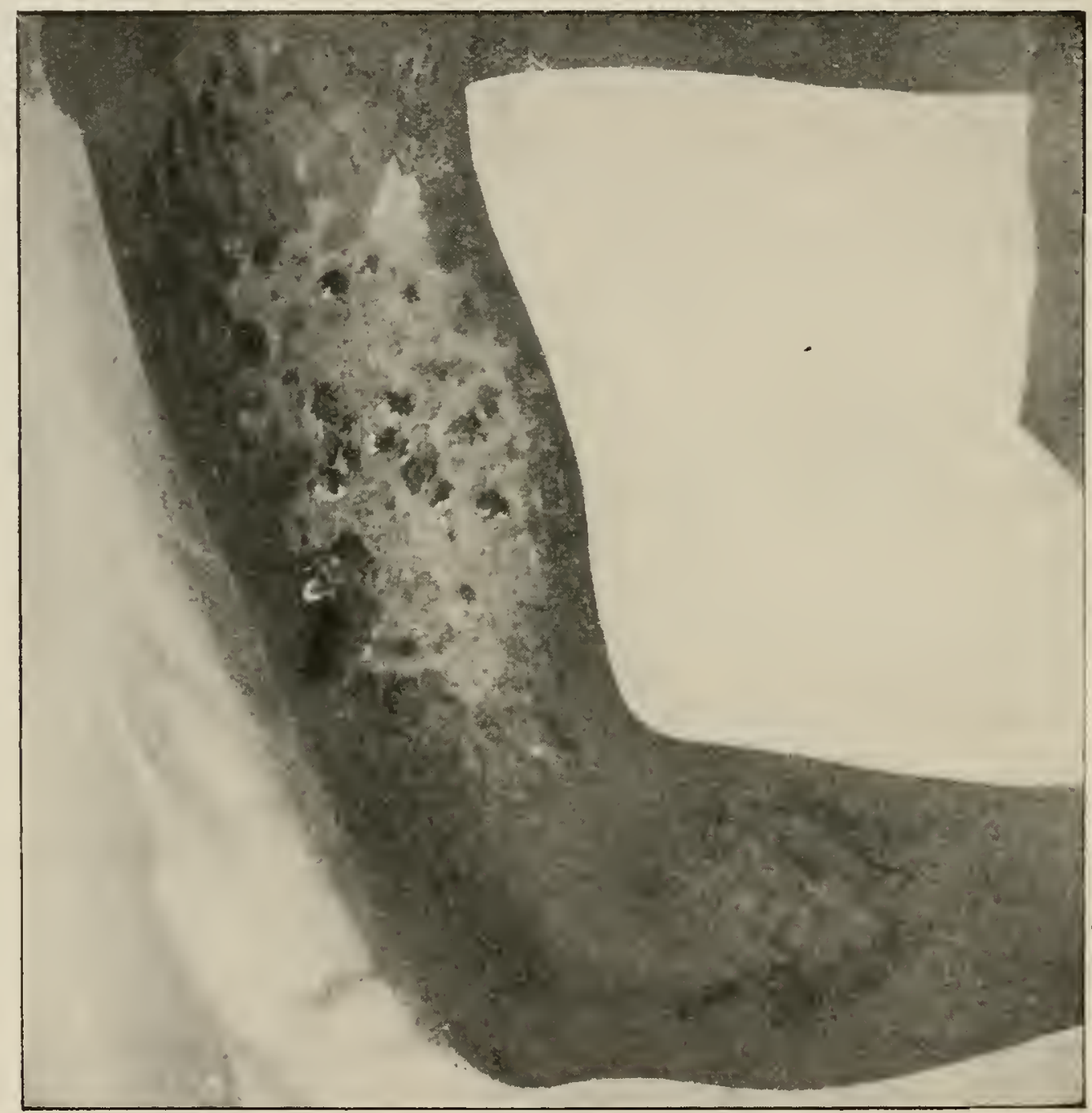

Arm infected and scarred from morphine injections. Both arms were in the saine condition. (By Jefferys.)

Diarrhoea has been noted.

The duration of the disease is from one to three months.

The disease is also known as Morphine Injector's Septicæmia.

\section{DEFINITION.}

\section{ONYALAI.}

An acute infectious disease of unknown causation, characterized by the appearance of bullie containin is blood, on the surface of the body, the tongue, soft palate, or buccal mucous membrane. 


\section{ETIOLOGY.}

Mense thinks that it may be some kind of poisoning, perhaps due to some species of the Euphorbiacea.

The disease was first described by lale Massey in Angola, 1904, later on the Portuguese Wrest Coast, East Africal and the Congo.

\section{SYMPTOMÁTOLOGY.}

The onset is sudden.

There are lassitude, a dazed appearance, tender parotids, congestion of conjunctive, slight fever, numbness and pain in various parts of the body. Bulla appear on the tongue, in the mouth and pharynx, aesophagus, stomach and bowels.

The tongue is swollen and painful. There may be romiting of blood, and diarrhoea has been noted.

Hamaturia and cerebral hamorrhage have been described with the usual signs. There may be hamorrhages in the riscera.

The bulla also occur on the skin.

The disease may recur several times.

\section{TREATMENT.}

Arsenic in full doses should be tried.

Massey recommends large doses of sodium bicarbonate and cod. liver oil.

\section{AFERRILE SPLENOMEGALY.}

This disease is also known as Pseudo-Banti's Disease.

\section{DEFINITION.}

A chronic afebrile disorder characterized by splenomegaly and severe anamia.

\section{DISTRIBUTION.}

Tropical Africa, Ceylon, India, and probably elsewhere.

\section{SYMPTOMATOLOGY.}

The onset is insidious. It attacks children and adults alike.

There is a painless enlargement of the spleen without enlargement of the liver or other organs.

There is a severe anamia, but no parasites are found.

It has been differentiated from other tropical maladies.

\section{TREATMENT.}

This is symptomatic. Iron and arsenic are useful. 


\section{SECTION V.}

\section{DISEASES DUE TO VENOMS AND POISONS.}

INTRODUCTORY REMARKS.

REPTILES.

General Features.

CLASSIFICATION.

Distribution and Pieculiarities.

The Venom Apparatus.

The Characters of THE V'ENOM.

THE VARIETIES OF VeNom.

The SrMptoms of SNake Bite.

The Treatuent of SNake Bite.

Prophylactic MiEasures.

FISHES.

TOADS AND SALAMANDERS.

ANEMONES, CORALS AND JELLY'FISHES.

HELMINTHS.

SCORPIONS AND SPIDERS, MITES AND TICKS.

CENTIPEDES.

LICE AND BUGS.

BEES AND WASPS.

ANTS AND CATERPILLARS.

MOSQUITOES.

BEETLES, SNAILS AND LIZARDS.

POISONS TAKEN ACCIDENTALLY BY MAN.

ACKEE POISONING. THE VOMITING SICKNESS OF JAMAICA.

POISONS USED IN ORDEALS, FIGHTING, FISHING, HUNTING. 


\section{DISEASES DUE TO VENOMS AND POISONS.}

\section{INTRODUCTORY REMARKS.}

T'enoms are useful :-

(1) To keep off enemies by discharging the renom into the surrounding medium, e.g., toads, salamanders.

(2) To aid nourishment by the animal mixing the venom with its own digestive juices, e.g., snakes.

(3) To serve as a means of attack or defence by inoculating the venom, e.g., snakes, scorpions, \&c.

Animals are venomous only when they are capable of inoculating their venom. Among these, reptiles form the greater part.

I Iumanity has held strange and contradictory ideas with regard to reptiles all down the ages. For example:--

In Genesis the serpent represented the Evil One.

In Greece it symbolized wisdon and prudence.

In Egypt it was the protector of crops and represented immortality.

In Rome its presence caused epidemics to cease.

In India the living Naja represents happiness and prosperity, the dead reptile, terrible calamity.

There is an average of 25 ,ooo deaths per annum from snake-bite in the Indian Peninsula alone.

300,000 vipers have been killed in Haute-Saône, France, during twenty-seven years (Calmette).

It is interesting to note that the blood also of vipers, toads and salamanders is toxic and that the eggs too carry the renom.

\section{REPTILES.}

\section{GENERAL FEATURES.}

A thick epidermis covering the skin is detached or moulted three or four times yearly in all except a few species of snakes, and these retain a few rings at the tip of the tail as in the rattlesnake, by means of which it makes the noise so characteristic of it. This moult is preceded by several weeks of torpor during which the reptile does not eat. The epidermis becomes dull, dry and wrinkled, later cracking at the mouth after which the reptile awakes, shakes itself free from the remainder and goes once more in search of food.

Their coloration, governed generally by mimicry, is not of much value for diagnostic purposes as it may be modified several times during the life of the same reptile. 
The scales and shield (the latter are simply large scales that do not overlap) serve to differentiate the species. The ventral scales, moved by the ends of the ribs, serve the purpose of locomotion. The tongue

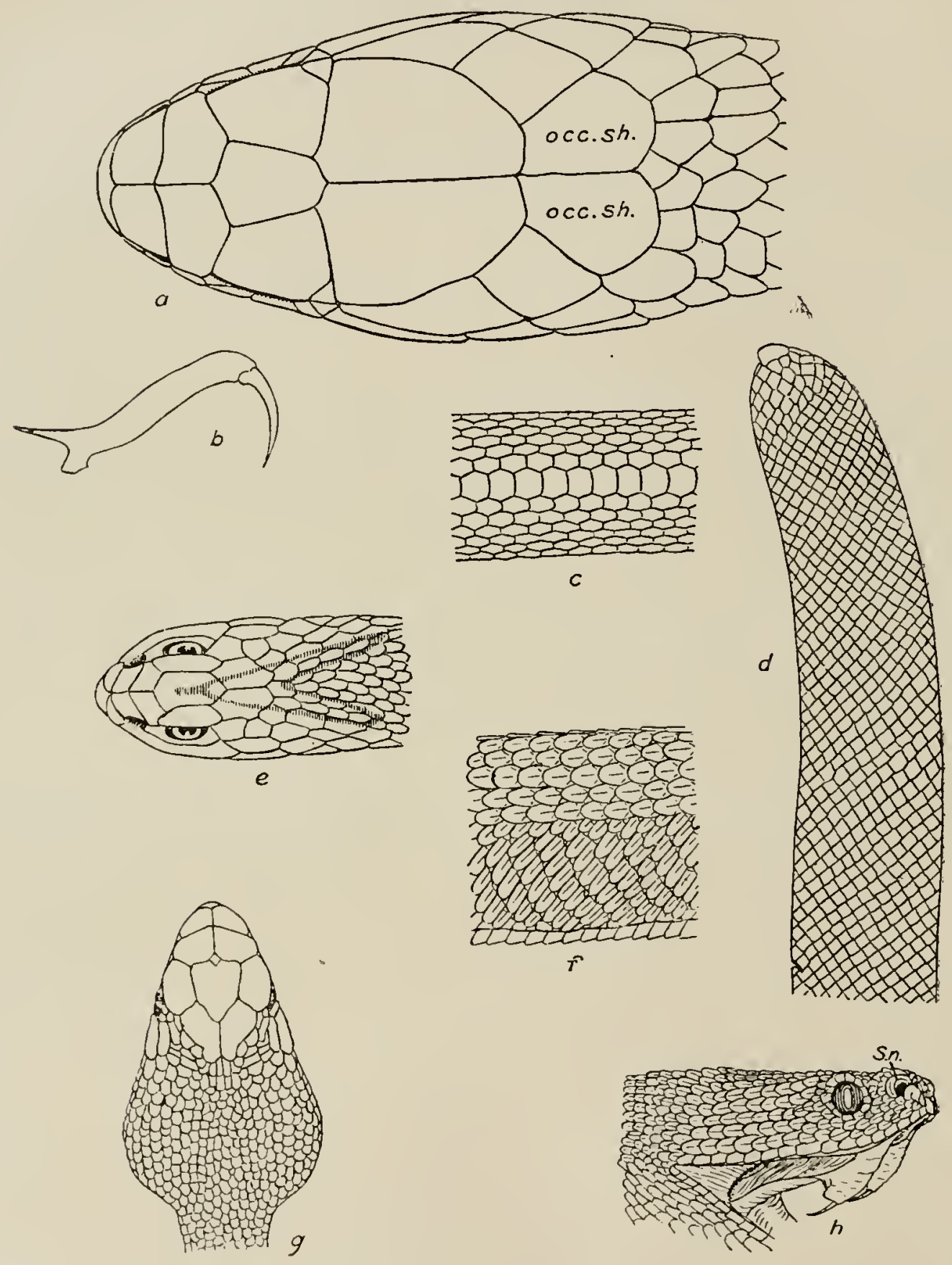

a, head of Naja bungarus, showing occipital shields; b, maxilla of Dendraspis; $c$, Bunsarus candidus, showing enlarged vertebral scales; $d$, laterally compressed tail of sєa-snake ; $e$, head of Causus rhombealus, showing large shields; $f$, Cerastes, showing oblique lateral scales; $\therefore$, head of Ancistrodon, showing large shields; $h$, head of Bilis, showing supra-nasal shield and small scales betu een nasal and rostral shields.

is long, very retractile, forked at the tip, is capable of considerable protrusion but is harmless. Some large lizards also possess a similar organ.

The eyes have no eye-lids; the cloacal orifice is transverse; the mouth is most extensile. This latter feature is permitted largely owing 
to the lower jaw being attached to the upper by means of elastic digaments which permit of considerable distension.

The teeth are used for holding and poisoning the victim in some
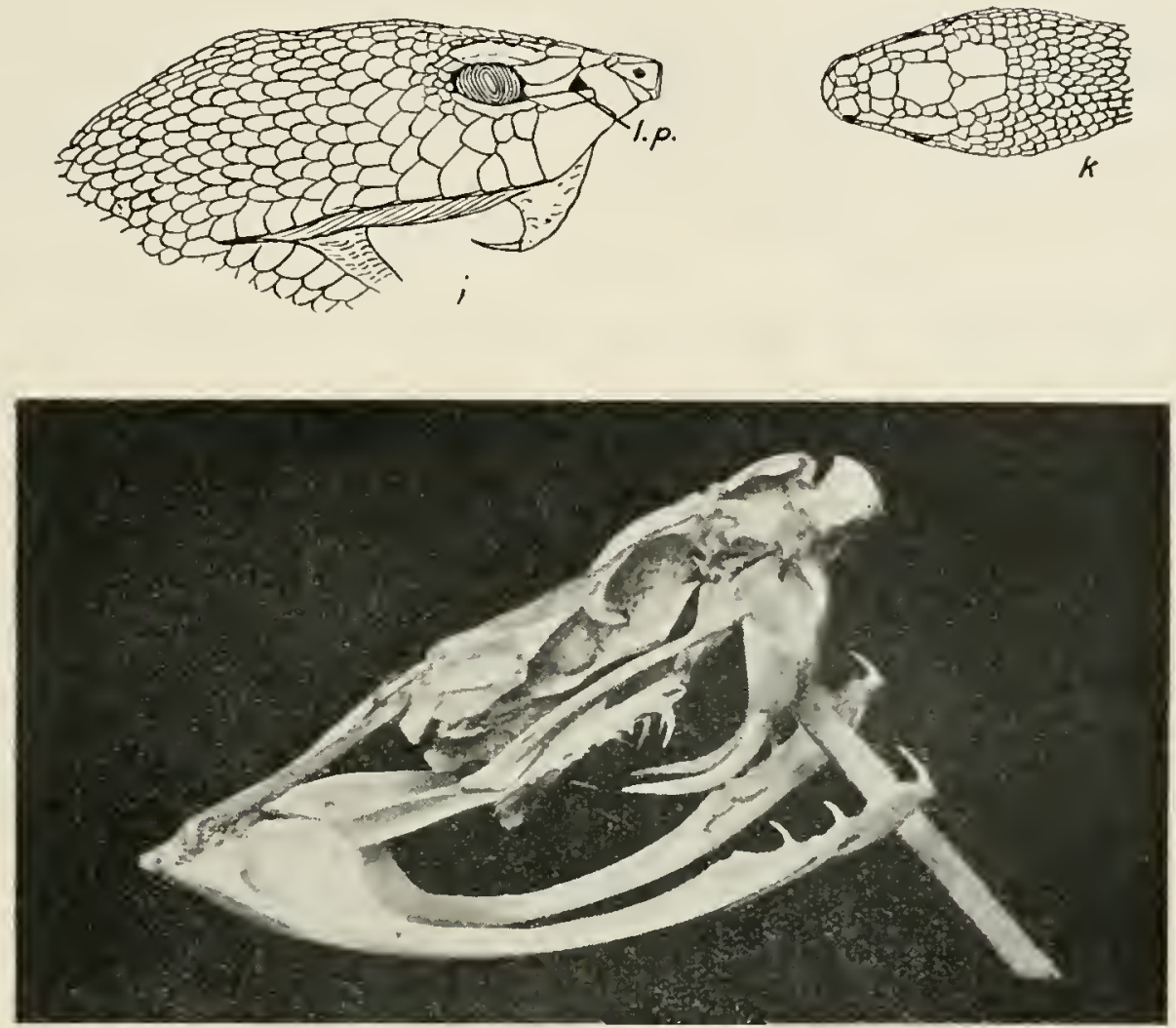

$l$

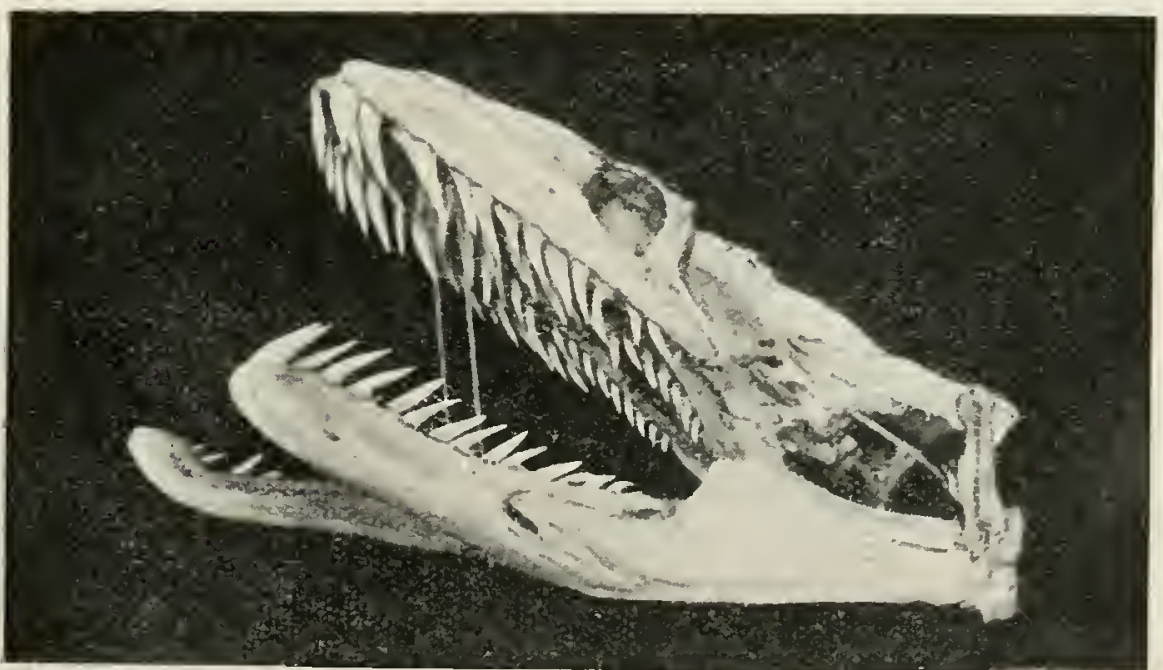

$m$

$i$, Head of Lachesis, showing loreal pit (l. p.) ; $k$, head of Vipera berus, showing shields and scales; $l$, skull of Crotalus, showing oblique maxilla and long transpalatine; $m$, skull of Python, showing inter alia absence of mandibulaı symphysis.

instances but not for mastication. The victim is swallowed whole, even though it be three times the diameter of the snake, the mouth parts are drawn over it like a glove until the whole has disappeared. Deglu- 
tition is slow and painful, but digestion is powerful and rapid, so that the bones themselves are dissolved.

There are some lizards that possess snake-like features, but on

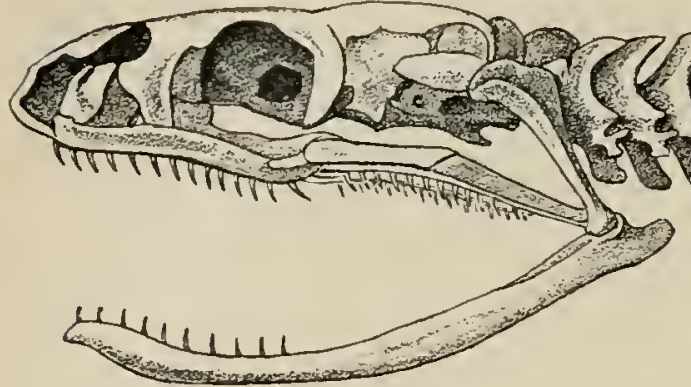

$a$

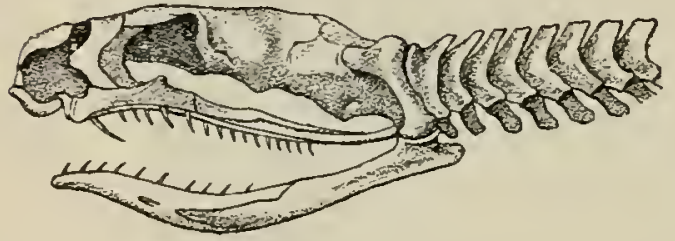

$c$

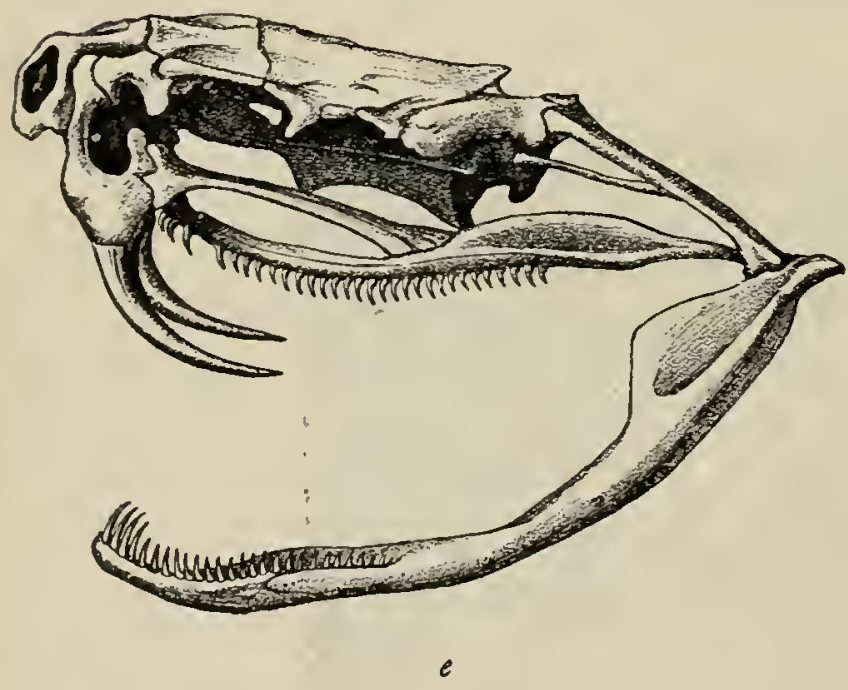

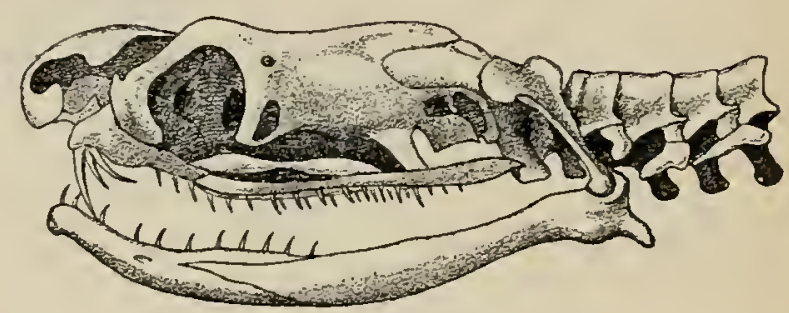

$b$

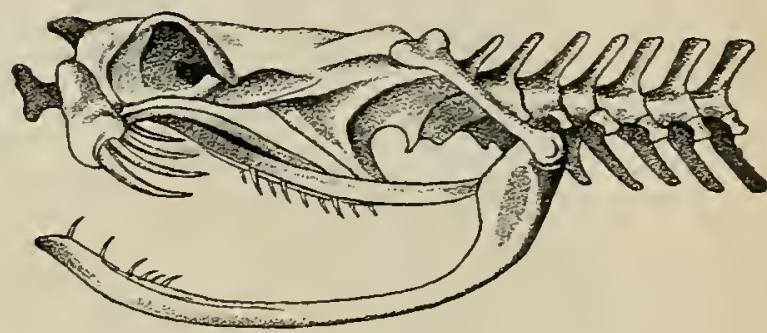

$d$

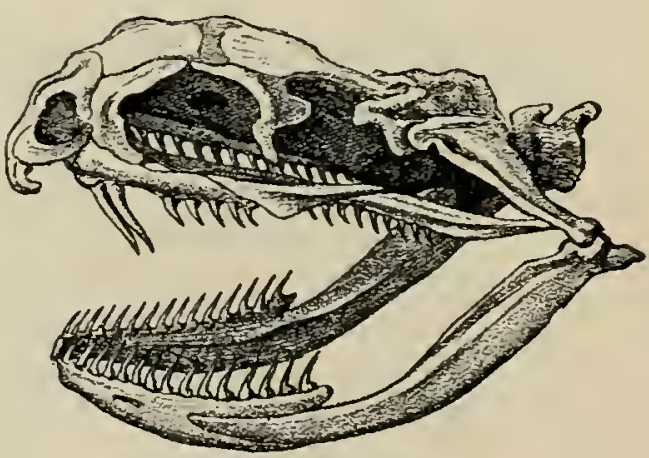

$f$

a, Cranial skeleton of one of the non-poisonous Colubridu (Ptyas mucosus); $b$, cranial skeleton of one of the poisonous Colubride (Naja tripudians); c, cranial skeleton of one of the poisonous Colubrida (Bungarus fasciatus); $d$, cranial slieleton of one of the Viperide (Vipera russellii); e, cranial skeleton of one of the Viperidie Crotalina (Crotalus durussis); $f$, cranial skeleton of one of the Colubrida Hydrophiince (Hydrophis pelamis).

examination it will be found that they do not have extensile jaws, but they do have eyelids and an external ear, which latter organ snakes. do not possess.

For details see a larger work. 


\section{CLASSIFICATION.}

The Reptilia include two orders of importance to the tropical medical officer, viz.: The Ophidia and the Lacertilia. The former is all important as it contains the venomous reptiles. The Ophidia contains two important families, viz.: The Colubrida and the Viperida.

\section{(I) The Colubridæ.}

These form nine-tenths of the order Ophidia. All snakes other than pythons and vipers belong to it. There are three groups:-

(I) Aglypha. These have solid ungrooved teeth. Their saliva is toxic to rats and mice but not to man. Among them are the common British snake (Tropidonatus natrix), the North American water

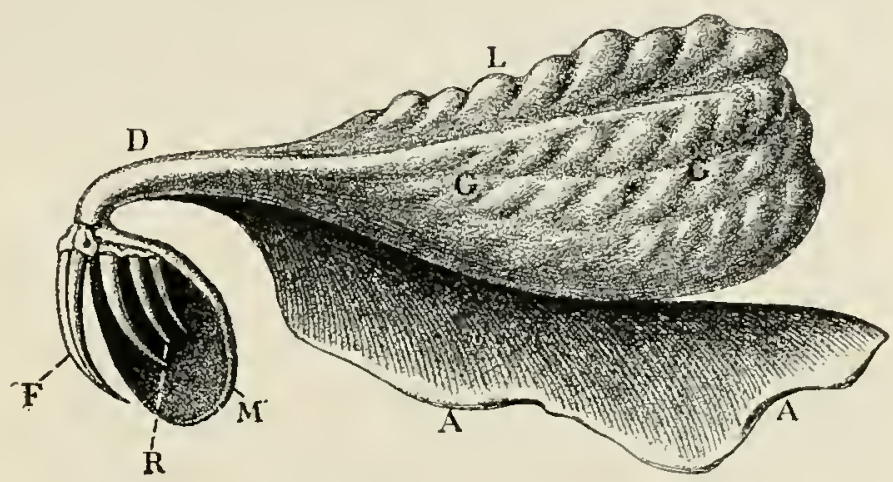

Poison-gland and fangs of a venomous snake (Naja tripudians, Colubride). (Natural size.)

$L$, Lobe of the gland; D, poison-duct; F, fang attached to the maxillary bone; $G \mathrm{G}$, gland ; $M$, capsule of mucous membrane surrounding the fangs; $R$, reserve fangs; A A, muscular fascia covering the gland. (After Sir Joseph Fayrer.)

mocassin (T. fasciatus), the Indian rat snake (Zamenis mucosus), and the African egg-eating snake (Dasypeltis scabra).

(2) Opisthoglypha. (óm $\pi \sigma \theta \epsilon \nu=$ behind; $\gamma \lambda \iota \phi \eta=a$ groove). These have one or more of the hinder teeth in the upper jaw grooved. As their fangs are situated at the back part of the mouth they are not well developed and are not very harmful to man. Among them are the Indian tree snakes (Dipsadomorphina), the Indian egg-eating snakes and water snakes (Homalapsina).

(3) Proteroglypha ( $\pi \rho o \tau \epsilon \rho v=$ before).-These have the front upper teeth well developed and grooved anteriorly, connected with ducts leading from well-developed poison glands. All are dangerous to man and animals. There are three sub-families :-

(a) Elapince. These live on land and have a cylindrical tail. They happen to be the only poisonous snakes known to Australia. Among them are :-

The Cobra-di-capello, or the snake with the hood, which when 


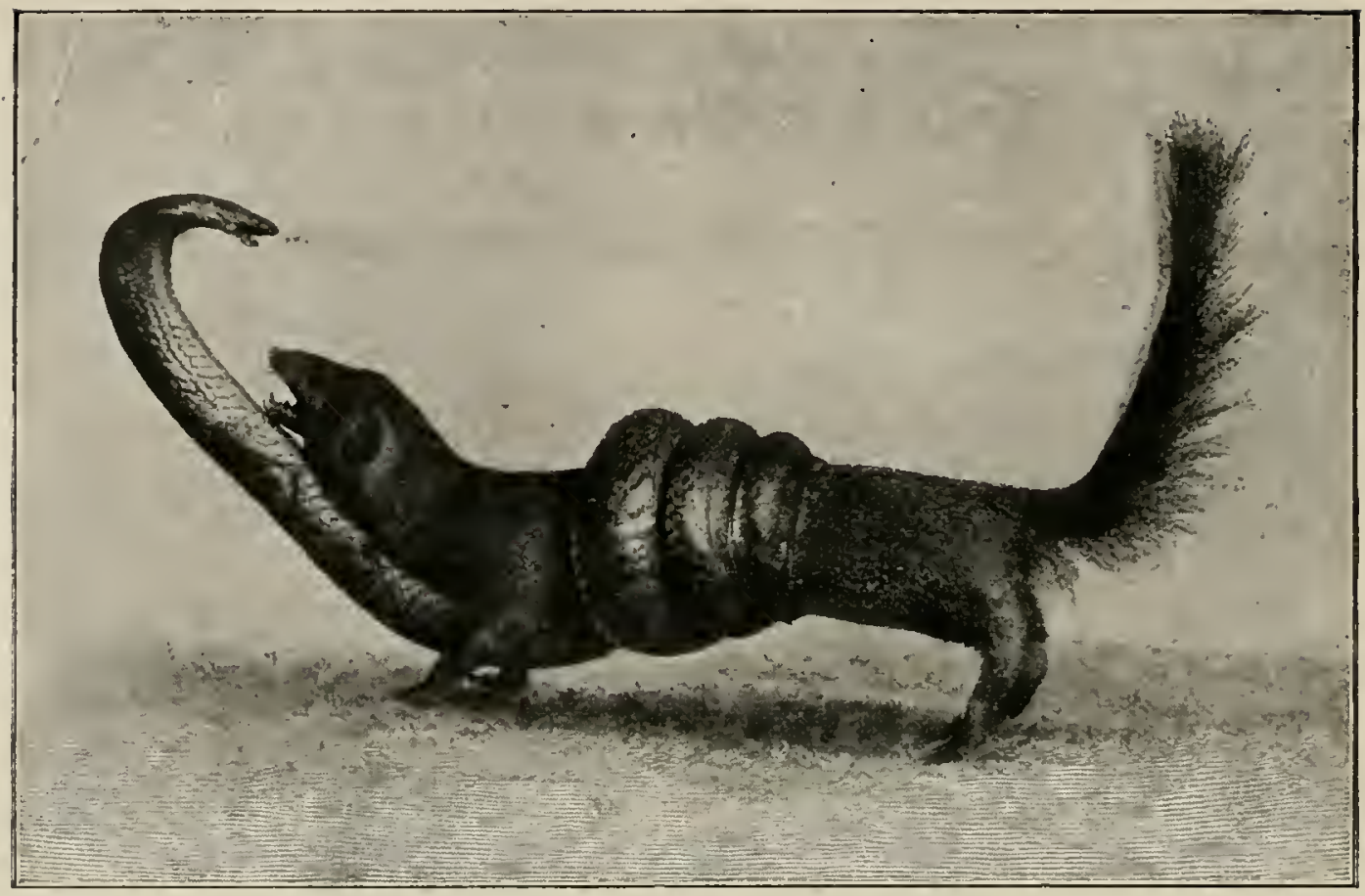

Mongoose seized by a cobra.

(For this illustration I am indebted to the kindness of M. Claine, late French Consul at Rangoon.)

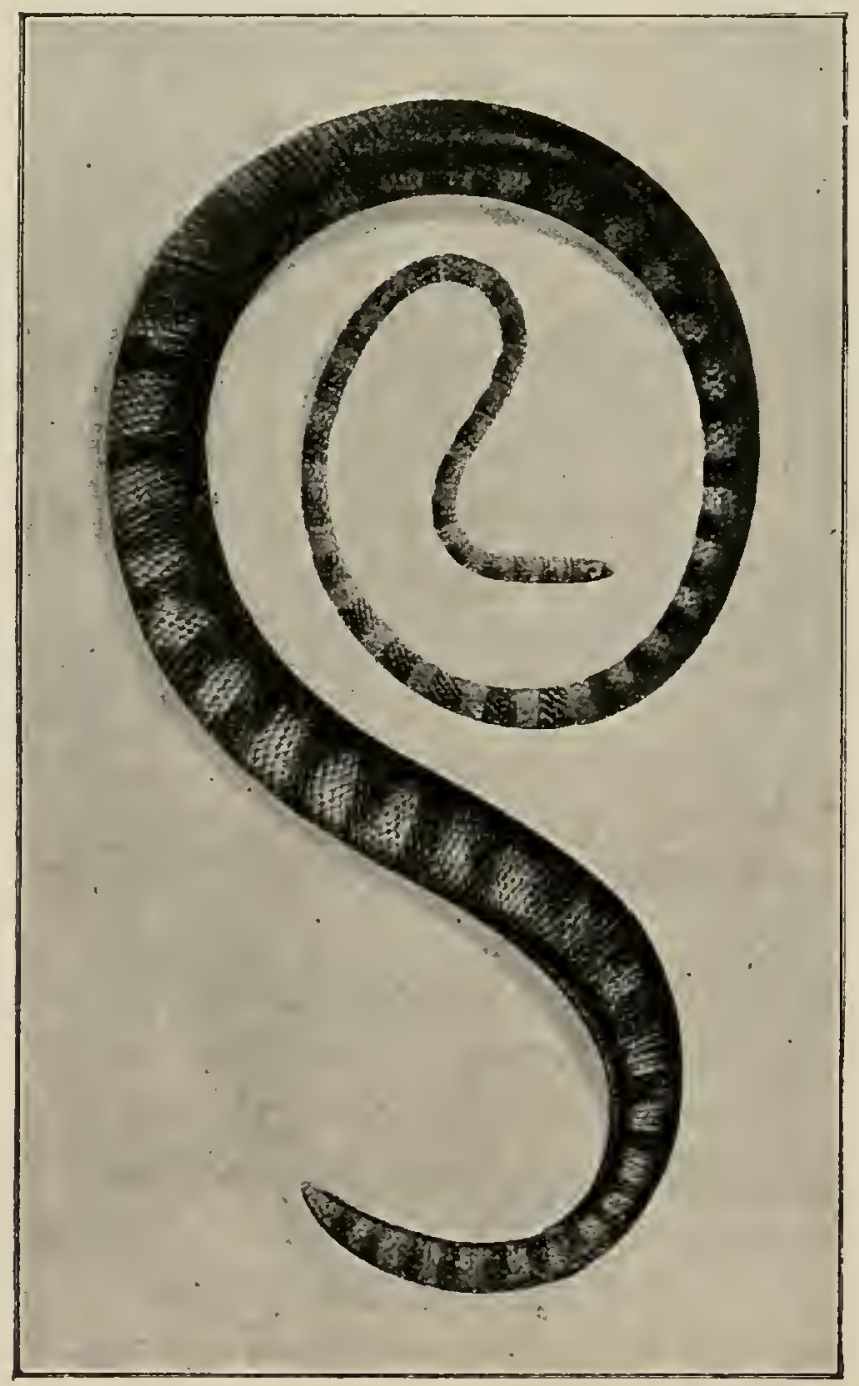

Hydrophis coronatus. (After Sir Joseph Fayrer.) 
excited, expands the skin behind the head by throwing outwards the cervical ribs.

The Naja tripudians, with the spot or spectacles on its hood.

The $N$. haje or Cleopatra's asp.

The $N$. bungarus or large king cobra.

The Bunbarus, some having yellow and black bands.

The Acanthophis artarcticus, the death adder with spines on its tail.

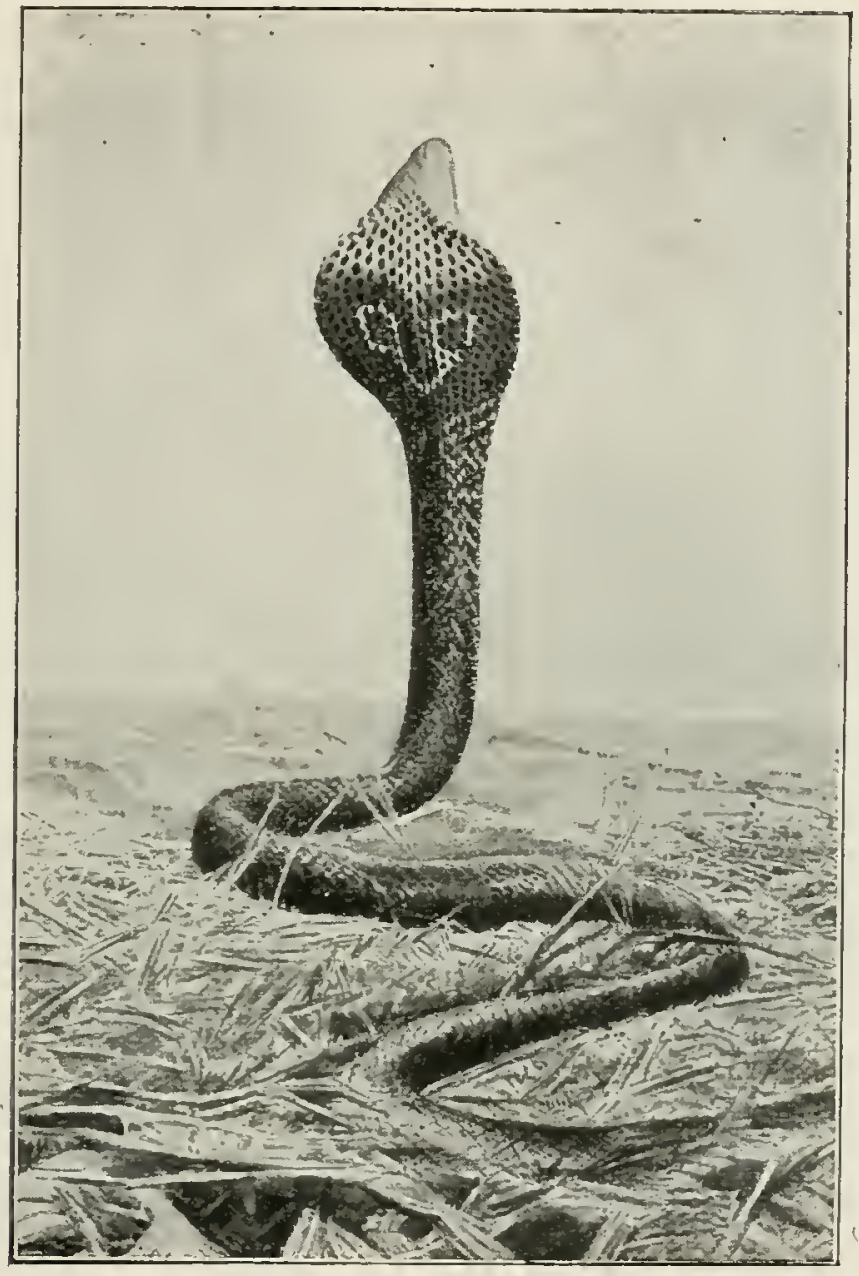

Naja tripudians (cobra-di-capello) on the defensive, preparing to strike.

The Elaps corallinus, the coral snake with black, red and yellow bands.

The Sepedons or hooded snakes, which are supposed to "spit" or throw their venom some distance and so cause severe conjunctivitis. They are found in Africa, not in Europe or America.

(b) Hydrophince. These all live in the sea with the exception of a few that have been accidentally cut off in small lakes. They have the tail flattened for swimming, and also in order to attach themselves to corals while feeding. They travel in companies, usually on the surface of the water, but they can take in large air reserves and dive to great depths. They are very dangerous to fishermen, especially in 
the Indian Ocean. Their fangs are small, but the venom, though but little is injected, is the most potent known. It is chiefly neurotoxic.

The Genera are: Distera, Acalyptus, Hydrophis, Enhydrina, Hydrelaps, Hydrus, Thalassaphis, Enhydris, Platurus and Aipysurus.

\section{(II) Viperidæ.}

These have a triangular head, short neck, stout body, stumpy tail, and tubular poison glands situated anteriorly, which are provided with

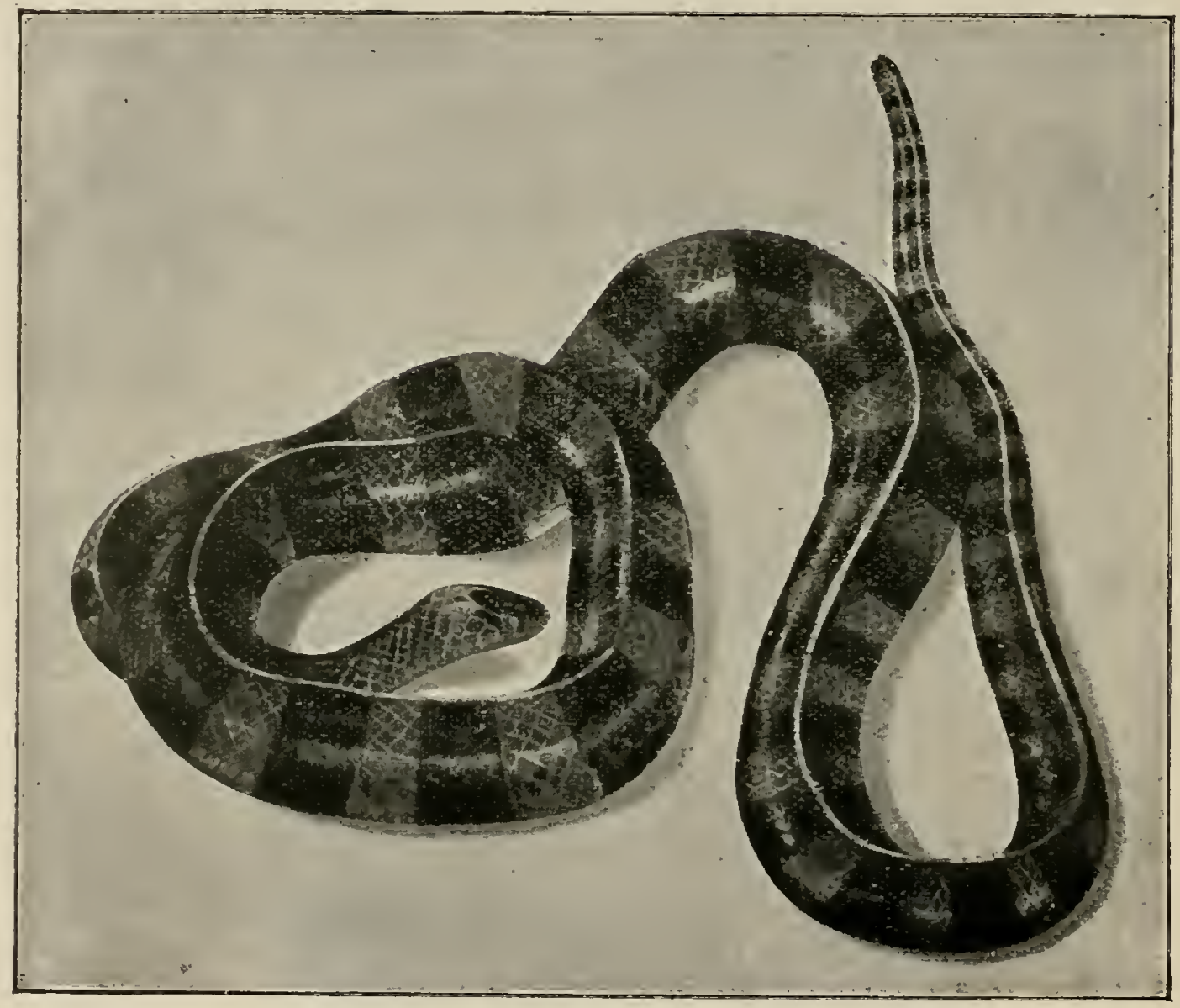

Bungarus fasciatus (India). (After Sir Joseph Fayrer.)

fangs having a large foramen for the venom. There are izo species, some of which live in trees, but all are dangerous to man. There are two groups :-

(I) Crotaline, with a deep sensory pit between the eye and the nostril on each side. These include rattlesnakes, such as :-

Crotalus, with many small scales on the top of the head.

Sisturus, with nine large scales on the top of the head.

Ancistrodon.

(2) Viperine, having no sensory pit as the Crotalinæ. Amongst them are:-

Vipera berus, the English adder.

Vipera russelli, the handsome Indian viper. 
Bitis arietans, the puff-adder of the Gold Coast.

Cerastes cornutus, the horned viper of North Africa.

Echis carinatus, the viper of the Pyramids.

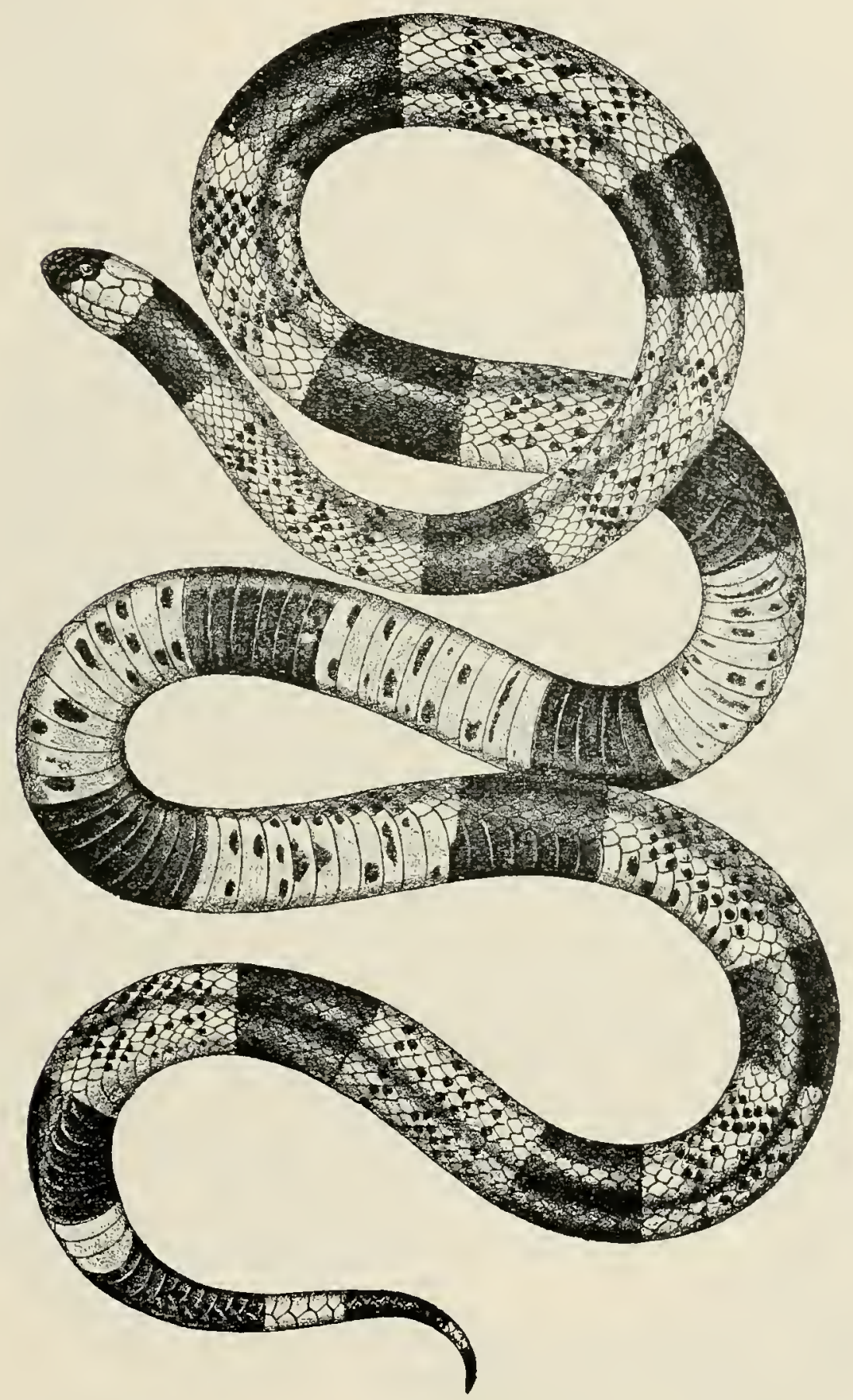

Elaps fulvius (the harlequin snake, or coral snake). (After L. Stejneger.)

\section{DISTRIBUTION AND PECULIARITIES.}

In Asia are the Elapinæ, Hydrophinæ, Viperinæ, and Crotalinæ. In Africa are the Elapinæ and Viperinæ.

In Australia and Oceania are the Elapina and Hydrophinæ. In America are the Elapinæ and Crotalinæ. 
In Asia.-Here all kinds are represented.

(I) Elapince.

The naja has a venom chiefly neurotoxic.

The bungarus has a single row of scales on the back. The $\mathrm{N}$. candidus seldom exceeds 4 feet in length, is blue-black in

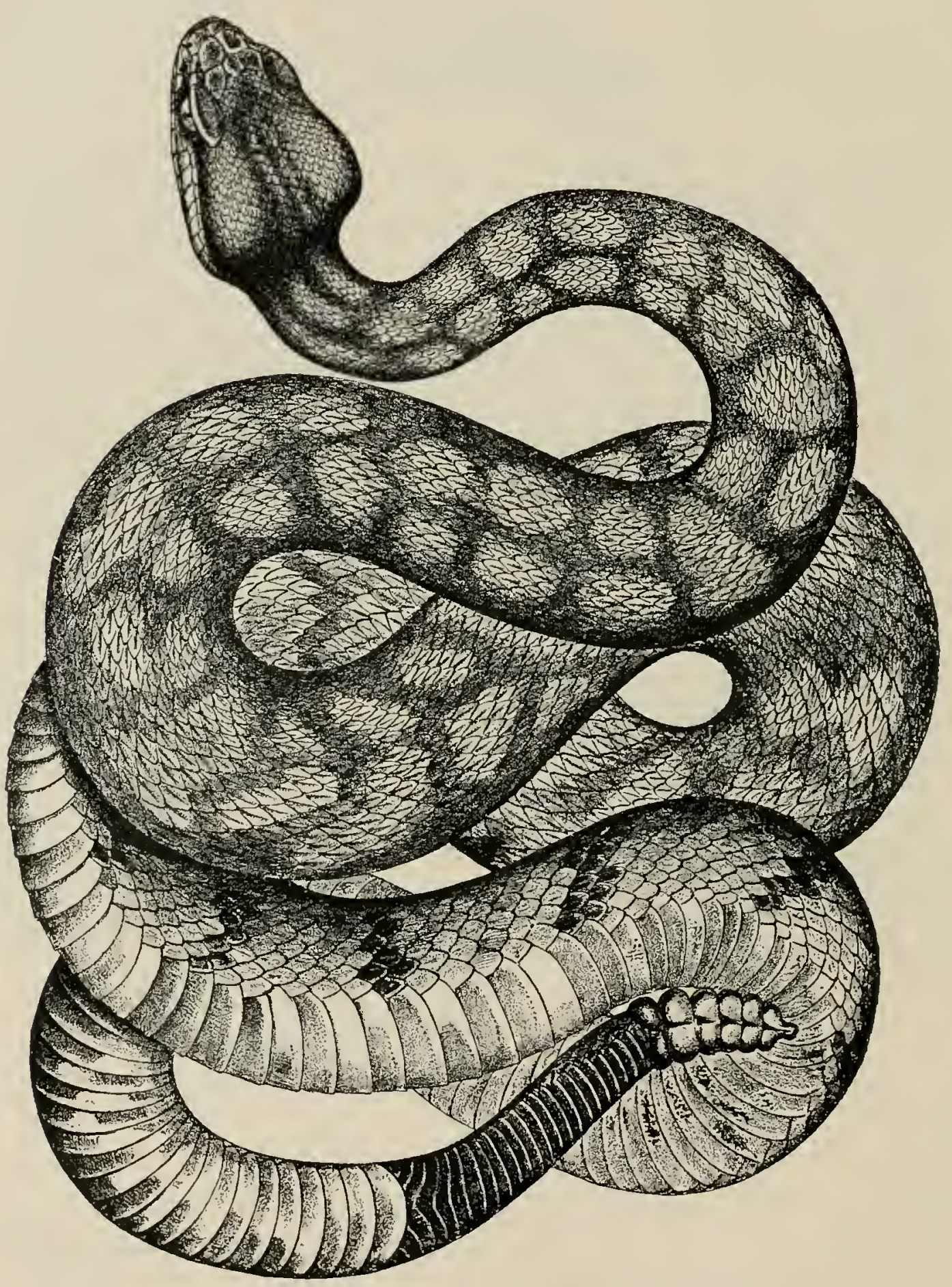

Crotalus terrificus (dog-faced rattlesnake, cascavella in Brazil). (After Stejneger.)

colour with white or yellow bands. Its fang leaves practically no mark behind. Its venom is neurotoxic. The natives affirm that it breathes upon them while they are asleep and thus kills them.

The $\mathrm{N}$. fasciatus has alternate broad rings of bluish-black and 
canary-yellow. On section it is almost triangular in shape. It has a stumpy tail, and for this reason it has been misnamed the two-headed snake. It may drop on to one's head from above

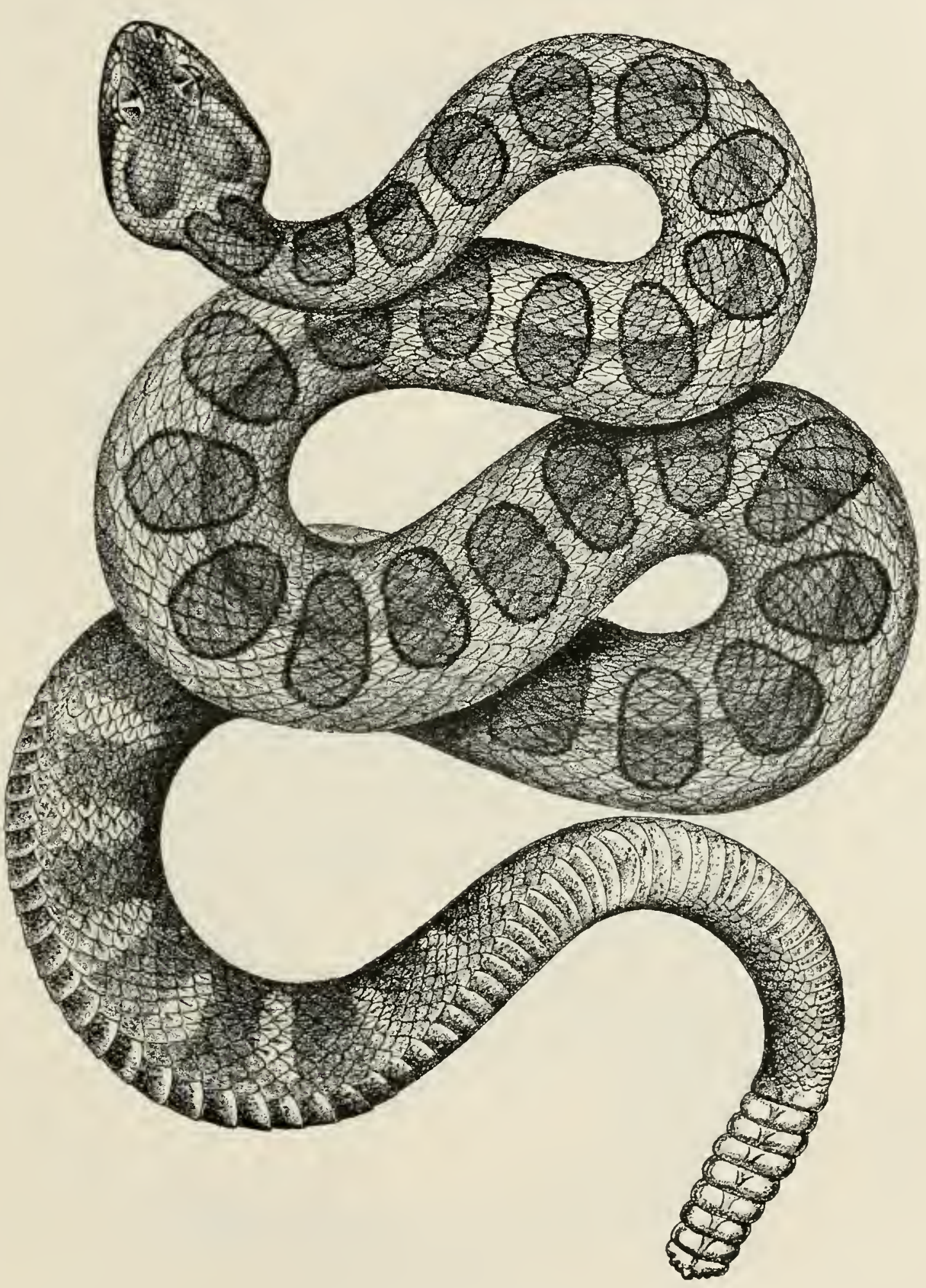

Crotalus confluentus (Pacific or mottled rattlesnake). (After Baird and Stejneger.)

at any time. The $N$. bungarus, or king cobra, has killed taking an average over eight years, i9,880 persons and 2, 100 cattle per annum. In I 889 it killed 22,480 persons and 3.793 cattle. 
The hemibungarus, calloptus and adenophis will seldom attack one.

They must be looked for in the jungle. They are a cherry. red colour. Little is known about their venom.

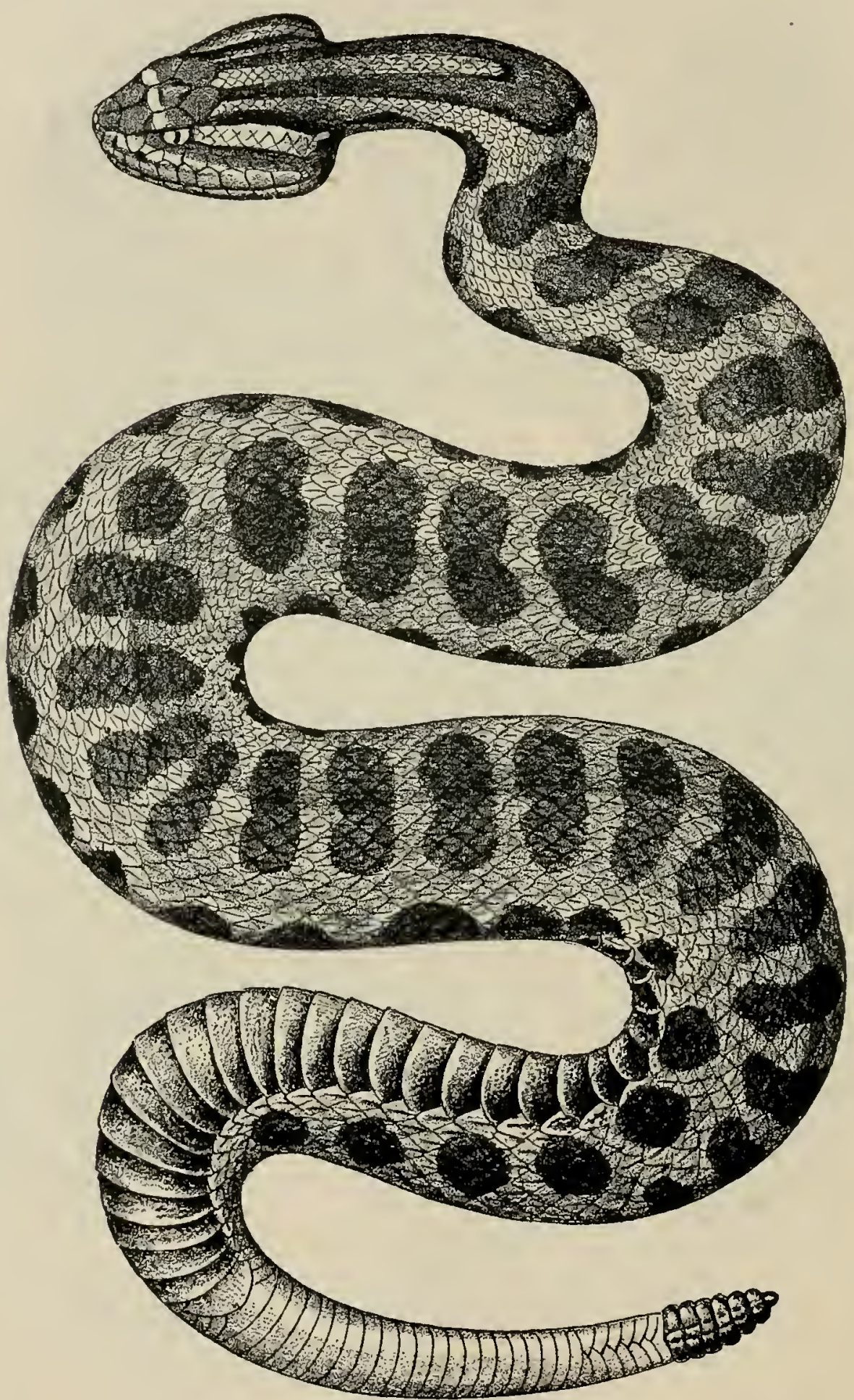

Sistrurus catenatus (prairie ratllesnake, or Massasanga). (After Holbrook and Stejneger.)

(2) Hydrophince.-These have already been described.

(3) Viperina.

The nasal and rostral shield are in contact or separated by one shield. 
Russell's viper is common, a reddish-brown snake with a series of three black lozenged-shaped marlss along its body.

The bitis is also common. It is described under "Africa."

The echis is seen in dry desert tracts, commonly in India.

The scales on the flanks are much smaller than those on the back, and are at an oblique angle, toothed and ribbed not unlike

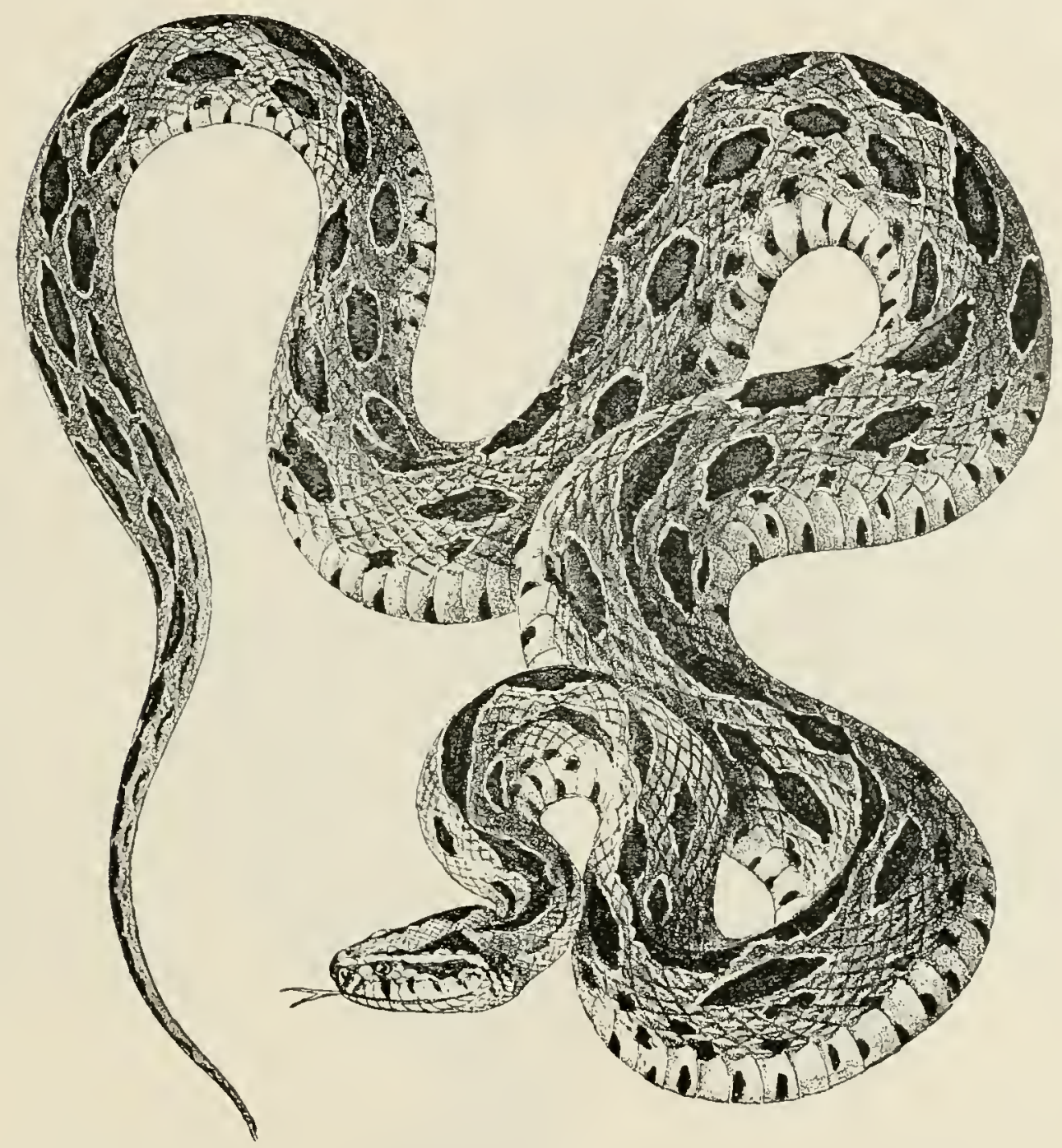

Vipera russellii (syn., Vipera elegans; daboia, or Russell's viper). India. (After Sir Joseph Fayrer.)

a spear point. They are aggressive, and their venom is highly hamolytic in action.

(4) Crotalina.

These are found in America and are mentioned later.

\section{In Africa.}

The Elapinze and Viperinæ are plentiful. Hydrophyna are found at times, but are accidental.

(I) Elapince.

The Naja and its species are known by their hoods and their erectile ribs. The scales are smooth. The $N$. haje is the 
common cobra of North Africa; the N. flava of South Africa; the N. milanolanta and N. nigrocollis of Central Africa.

The Sepedon is found only in Africa. The scales have a keel or midrib. It grows to a large size.

The Dentraspis is common. It lives in trees, and is 7 to 8 feet long, but is not as thick as the cobra.

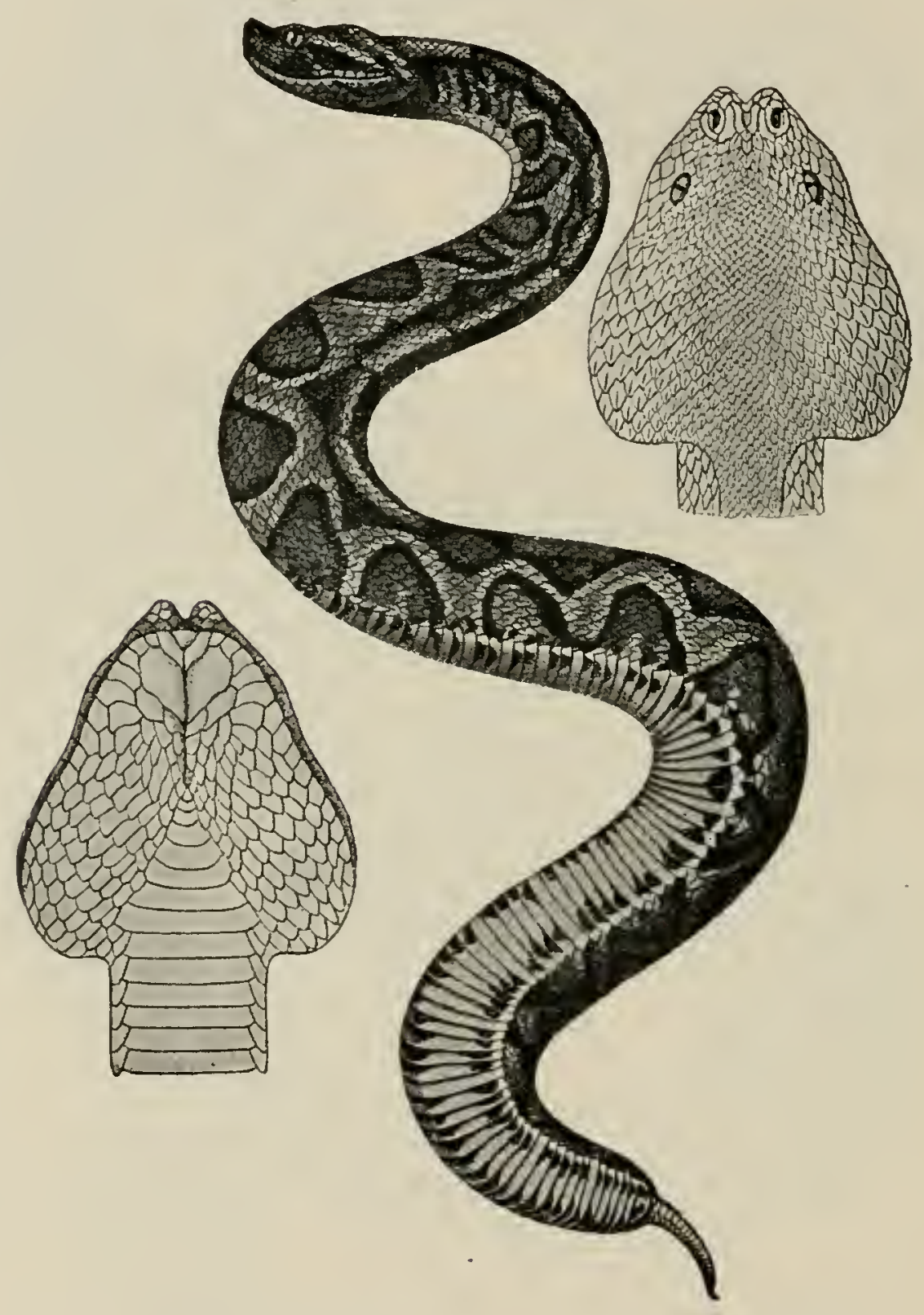

Bitis arietans (the puff-adder). (After Duméril and Bibron.)

The Elapechis is distributed over Africa south of the Sahara.

It has a very short tail. I,ittle is known of its venom.

(2) Viperince.

The Vipera is widely distributed.

The Bitis or puff-adder is common. It has a short thick body and carries enormous poison fangs, often more than I inch in length. Between the rostellum and nasal shield there are numerous small scales. The "pocket" above the nasal 
aperture is protected by a semicircular shield. The venom is hremolytic, and a good bite is usually fatal.

The Bitis arietans is pretty, with $\mathrm{V}$ markings down the back.

The nostrils are vertical and not lateral.

The Echis abounds here as elsewhere.

The Serastis is all over the Mediterranean region. They differ from the Echis by having a ridge along each side of the abdominal shields.

The Atheris differs from the above by the scales on the flanks

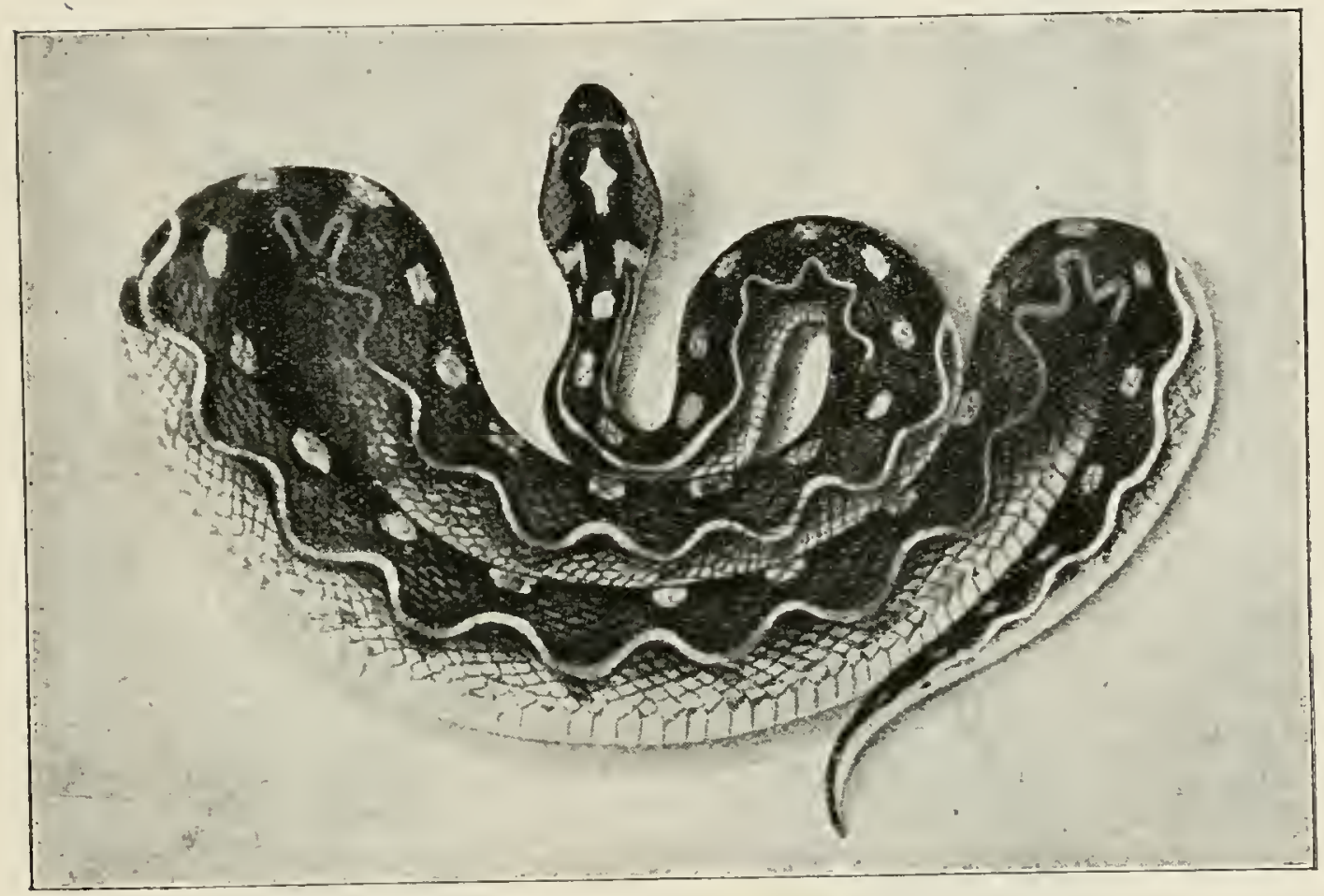

Echis carinatus. India. (After Sir Joseph Fayrer.)

being smaller and not serrated. They are of a greenish tint and live in trees.

The Causus is found in all parts of tropical Africa. The crown of the head is covered with large shields. There is often a neat broad arrow-head marking on the dorsum of its head. It is usually brownish in colour. The venom is strongly hamolytic.

\section{In Australia.}

(I) Hydrophince are plentiful.

(2) Elapince are numerous, and here are to be found twice the number of species possessed by any other country. Australia may be looked upon as the headquarters of this family. Some are not unlike vipers. 


\section{In America.}

Hydrophince are accidental.

(I) Elapince.

There is only one genus but many species. The venom of

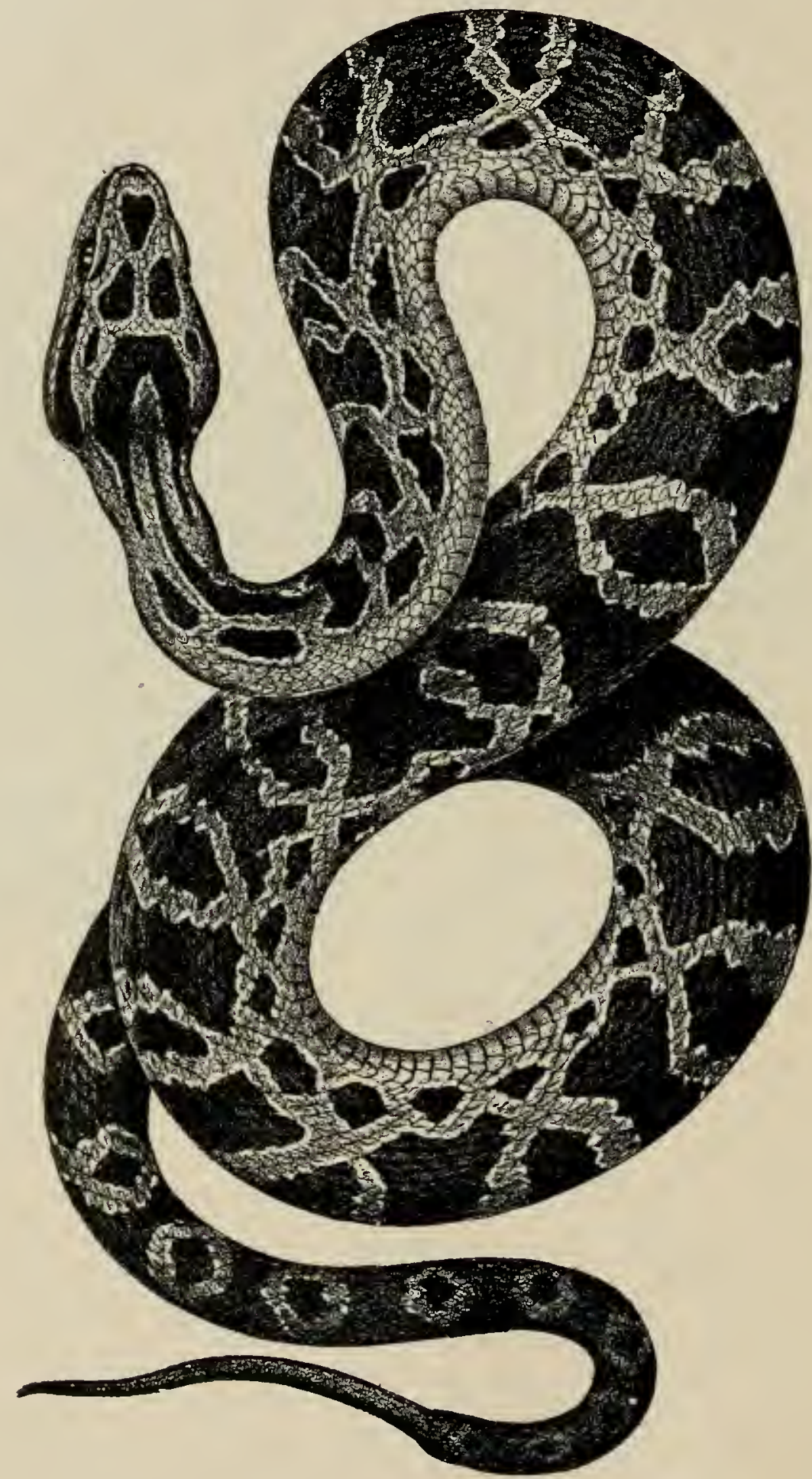

Lachesis neuwiedii (known as the urutic in Brazil). (After Lacerda.)

the Elapis is very deadly. They are beautifully coloured, broad rings of black and red alternately; there may also be narrower yellow rings between these. 
(2) Crotalince.

These are very abundant. A large sensory pit leading down to the maxilla will help in the diagnosis.

The Lachesis is a jungle snake, and has its head covered by small scales, never shields. It is also seen in Asia.

The L. mutis is very deadly. It attains to 14 feet in length and is very aggressive. It has enormous fangs. The tail is short and covered by pointed scales, and not shields.

The Fer de Lance is also very deadly.

The Ancistrodon is a fatal reptile, but its venom has not been much studied. The crown of the head is covered by large shields which are in contact with each other. It is also present in India and Southern Asia.

The Crotalus or rattlesnake only exists in America. Its bite is exceedingly dangerous. Pigs are their sworn enemies and eagerly devour them. Their resistance may be due to the thick layer of subcutaneous adipose tissue, which is but slightly vascular and slowly absorptive.

\section{THE VENOM APPARATUS.}

There are some non-poisonous snakes that possess poison glands, but the means of inoculating the venom are absent. It is used in digesting its prey.

The venom gland of poisonous reptiles resembles the modified parotid of adult persons. The venom gland is situated at the sides of the head and behind the eyes. It is covered by the masseter muscle, which when contracted presses upon the gland and forces out its poisonous contents. In some snakes the gland is like a long ribbon passing along one-third of the body underneath the skin. The gland is always covered by a tough fibrous capsule attached to the articulation of the lower jaw. When the animal bites, this capsule is pressed upon and the venom is forced along the duct. The duct is situated just above the line of the lip to a point below the eye, and ends in a small papilla or "pocket" of loose mucous membrane into which the base of the fang is inserted. There is one fang at each side in action at the same time, but reserve ones are always ready, and should one be broken it can readily be substituted. The venom runs along a groove on the anterior surface of the tooth or fang, or down a canal formed by the sides of the groove coalescing. The duct does not come directly to this groove, but terminates in the papilla or small sac described. Vipers have the longest fangs, nearly I inch in length. The fang projects downwards and backwards in the closed mouth and is firmly attached to the alveolar process. Owing to the free mobility of the cranial bones the tooth can be made "erectile" for piercing. 
In striking the body is coiled, the tail projecting, the head and neck raised a few inches, the tongue darts to and fro, air passes forcibly through the narrow glottis, causing hissing, the muscles contract, the snake straightens, the head suddenly darts forwards and upwards as required, the thrust being one-third to one-half of its body length. The jaws are widely separated, the head bent back, the superior maxillary bone is rotated forwards upon its lachrymal articulation, and thus erects the fangs. The jaws are approximated, the fangs enter the victim, the head is drawn violently backwards, and the teeth are driven deeply into the flesh. The lower jaw presses upwards and so squeezes fluid from the gland along the duct, and injects the venom forcibly into the wound at the same time as it is being enlarged. In disentangling itself the fang may be lost, but this is of little moment as others soon arrive. Unless the fangs become erectile no wound is made and the venom will flow upon the skin, doing no harm.

It is very doubtful if any snakes "spit" venom. The amount that can be injected varies with the species of reptile; its previous condition of full or impaired nutrition; its nearness to the moulting period; and according to the pressure applied to the gland.

Also by captivity, interference of clothing, failure of the snake to close its jaws, deficient elevation of the fangs, malapposition of the orifices of the poison duct and the fang, and the rapid movement of the victim attacked, thus shaking off the snake before it had time to shut its jaws.

Sea snakes inject but a few drops of venom which are very deadly. The Indian cobra can inject I drachm. Snakes in captivity always yield less venom. The Naja haje can produce about $0.36 \mathrm{grm}$. of liquid in 100 days, I $\mathrm{grm}$. of which yields about $0.336 \mathrm{grm}$. of dry venom.

Acton and Knowles have done some excellent work on this subject in India. In their results their unit is as far as possible expressed in milligrammes of desiccated venom. The yicld of venom was ascertained to be for the-

\begin{tabular}{|c|c|c|c|c|c|c|c|c|c|}
\hline Common cobra & $(50$ & ser & ons) & mean & yield & $\cdots$ & & & $\mathrm{mg}$ \\
\hline Common krait & $(48$ & $"$ & $\eta)$ & $"$ & $"$ & $\cdots$ & ... & 8 & \\
\hline Banded krait & (27 & $"$ & $")$ & $"$ & $"$ & $\cdots$ & $\cdots$ & 64 & $" 1$ \\
\hline Russell's viper & $(6$ & $"$ & $,)$, & $\because$ & ", & $\ldots$ & $\ldots$ & 108 & \\
\hline Echis carinatus & $(57$ & $"$ & $")$ & $"$ & 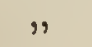 & $\cdots$ & $\ldots$ & I 8 & \\
\hline Lachesis gramineu & (15 & $"$ & $n)$ & , & $"$ & $\ldots$ & $\ldots$ & 30 & " \\
\hline
\end{tabular}

The minimum lethal dose for man was estimated ingeniously. They took the common cobra bite and studied all cases from classical writers of fatal bites, from the study of which they concluded that $13^{\circ} 6 \mathrm{mgm}$. Would just fail to kill a man, hence $15 \mathrm{mgm}$. would just be fatal. 
Having in this way formed a standard minimum lethal dose of cobra renom for man, viz., I5 $\mathrm{mgm}$, they carried out experiments on monkeys, using the first figure as a control, giving the desiccated

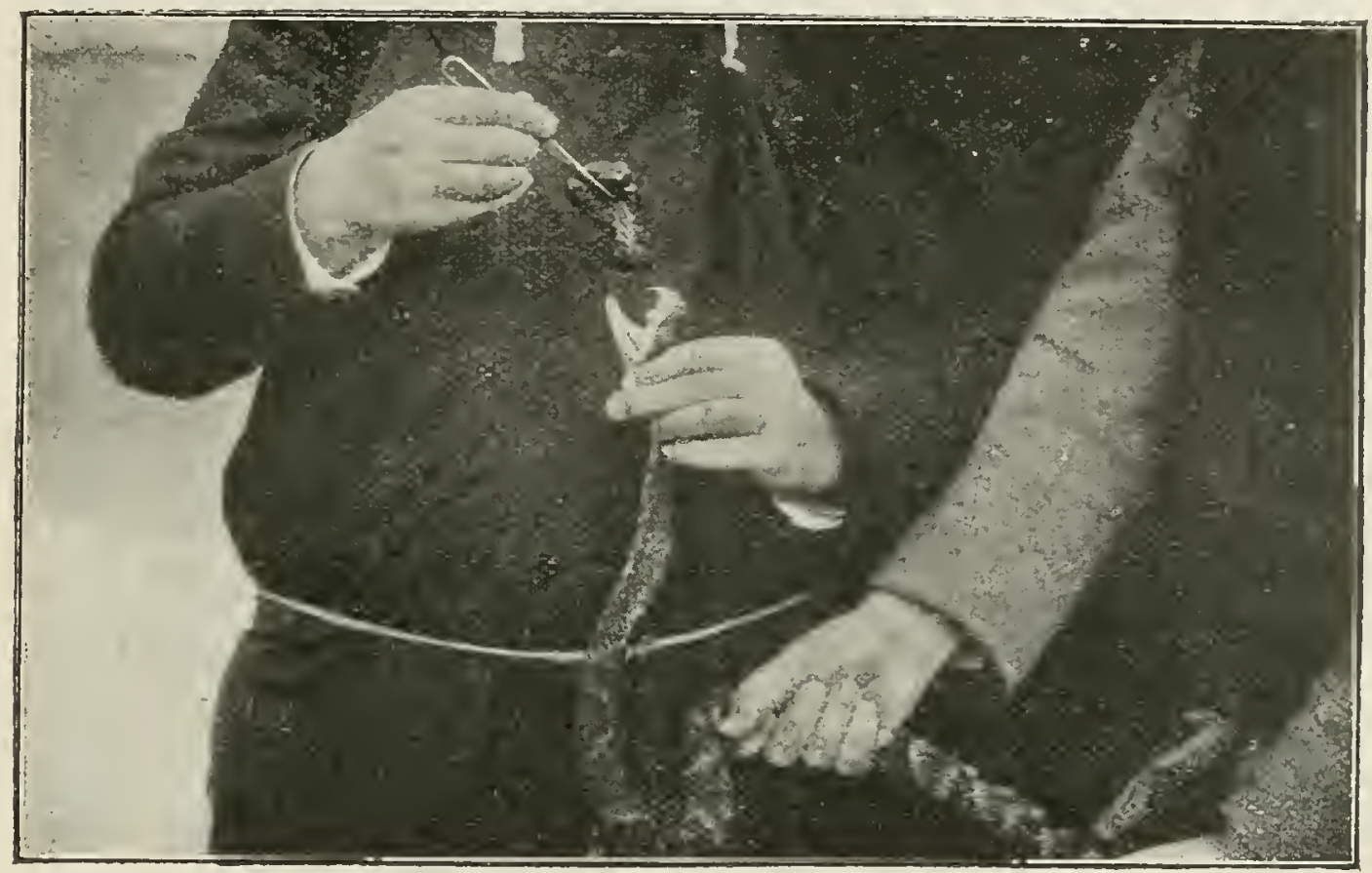

Feeding a poisonous snake, first stage.

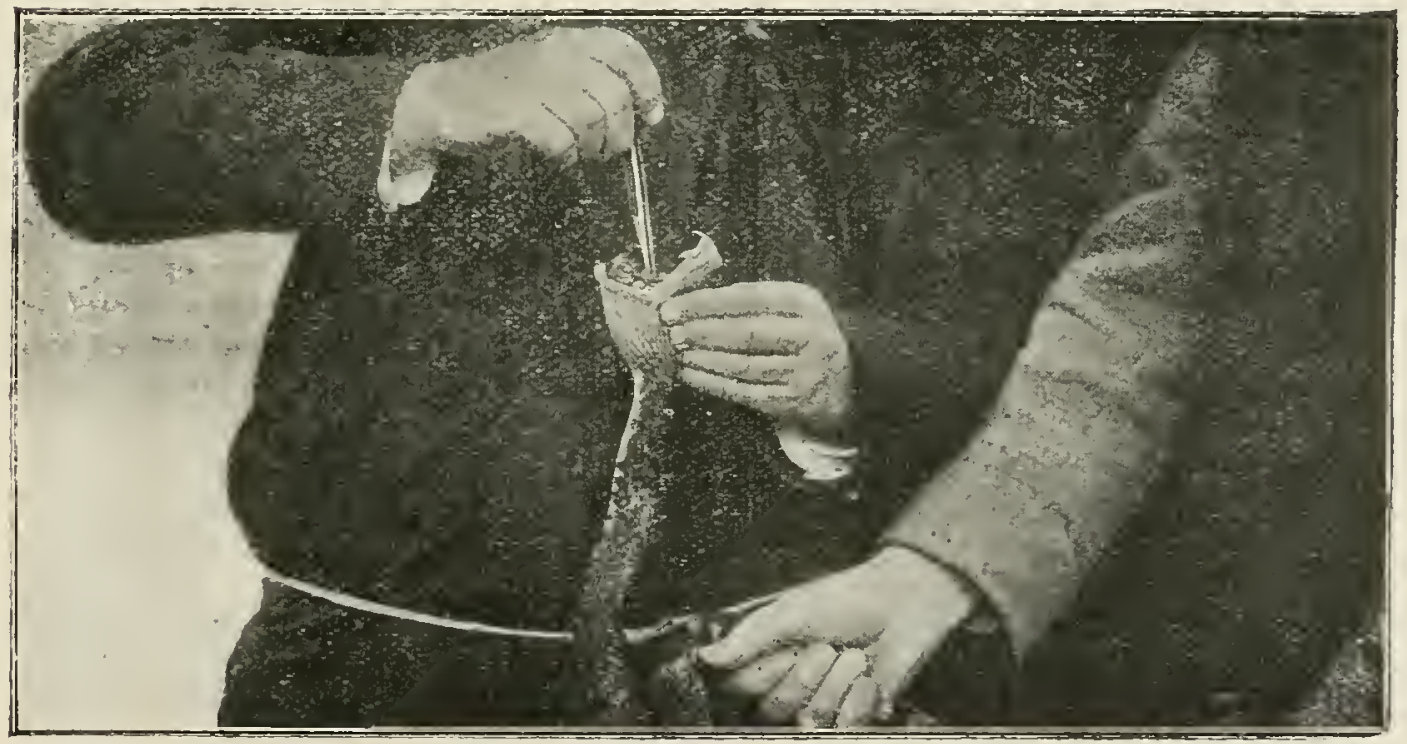

Feeding a poisonous snake, second stage.

renom subcutaneously. On this basis the minimum lethal dose for other reptiles was:-

Common cobra, 15

Naja tripudians, I5

" bungarus, 12
Bungarus candidus, I

...,$\quad$ fasciatus, 10

Viper russelli, 42
Echis carinatus, 5

Lachesis gramineus, probably more than any one snake could shed at any one tirne.

They then estimated how much renom several species of snakes could inject at one bite. This they estimated by obtaining the venom 
from the snakes in question, and estimating two-thirds of the quantity obtained as the amount which could be injected at once. The results were:-

$\begin{array}{lllllll}\text { Naja tripudians } & \ldots & \ldots & \ldots & 2 \text { I } ~ & \text { mgm. of desiccated venom } \\ \text { Bungarus candidus } & \ldots & \ldots & \ldots & 5 & , & , \\ \quad \text { fasciatus } & \ldots & \ldots & \ldots & 42 & , & , \\ \text { Vipera russelli } & \ldots & \ldots & \ldots & 72 & , & , \\ \text { Echis carinatus } & \ldots & \ldots & \ldots & 12 & , & , \\ \text { Lachesis gramineus } & \ldots & \ldots & \ldots & 14 & , & ,\end{array}$

This was when the snakes were healthy, in full natural vigour, not exhausted, roused from hibernation or in captivity.

\section{THE CHARACTERS OF SNAKE YENOM.}

Collect the venom by chloroforming the snake and squeezing the venom into a ressel. Dry it quickly in a desiccator over sulphuric acid. When dry it will remain potent for years. In the fluid form it is transparent, slightly yellowish, odourless, acid in reaction, bitter to the taste, syrupy, of a specific gravity 1030-1060, readily soluble in water or saline, rich in soluble albumins which are its toxins. All its toxic substances are precipitated by absolute alcohol. The venom is destroyed by strong acids, alkalies, potassium permanganate, boiling or by anything that "kills" ordinary albumin. Gastric juice destroys that of the Colubrine and pancreatic juice that of the Viperinæ. When dry it is not unlike iodoform in appearance. It can decompose and change its colour. Light causes it to deteriorate when in the fluid state, but it does not affect it when dry to any great extent. Fluid venom may readily be contaminated by micro-organisms of all kinds. Glycerine in equal parts is a good preservative. Its toxicity is increased during starvation, and in dry climates in the same snake species. The effects vary with the amount injected, the species of snake, the site of the injection, viz., skin, muscle, or blood-stream, and other factors.

\section{THE YARIETIES OF YENOM.}

Snake venoms are very complex liquids, each containing some of the following active principles:-

(1) Neurotoxins.

Some act on the rasomotor centre.

Others act on the respiratory centre.

Others on the nerve endings in muscle, especially of the phrenics.

(2) Agglutinins.

(3) Cytolysins.

Some are hamolysins.

Others are leucolysins.

Others are hemorrhagins.

Others are simple cytolysins. 


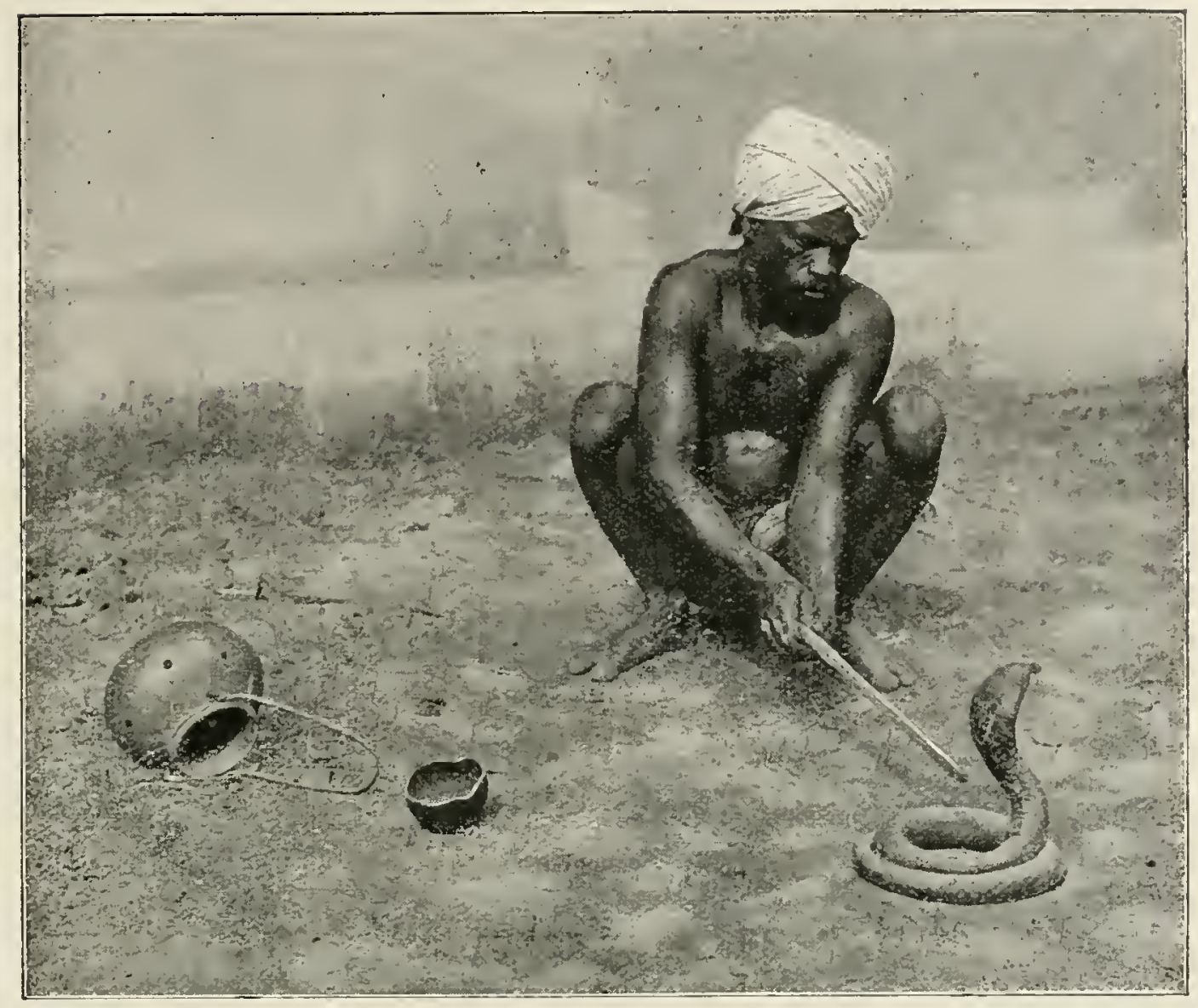

Catching a cobra-di-capello (Naja tripudians), first stage.

(At the French settlement of Pondicherry, in India.)

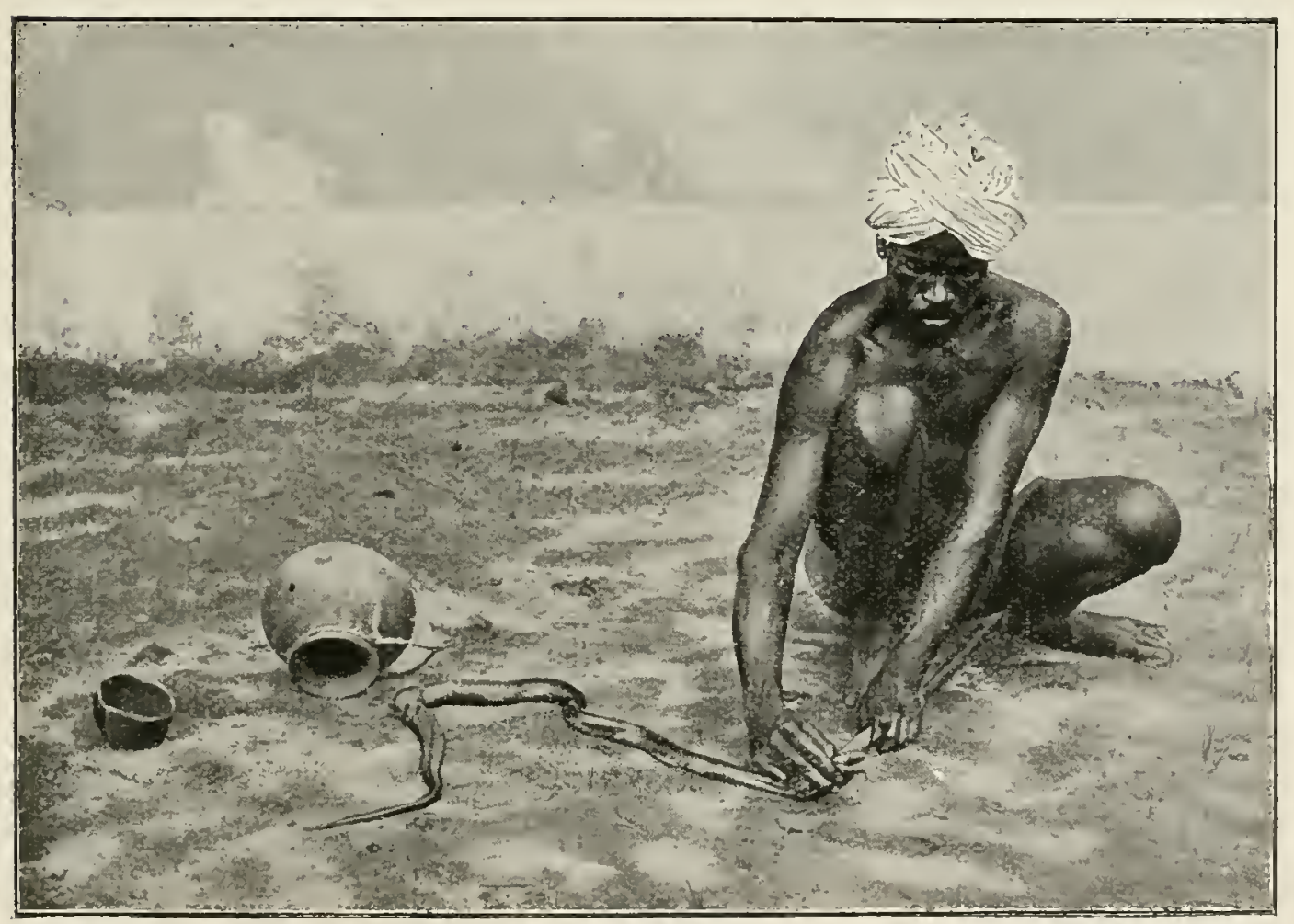

Catching a cobra-di-capello (Naja tripudians), second stage.

(At the French settlement of Pondicherry, in India.) 
(4) Intihamolysins.

(5) Antibacterial substances.

(6) A tibrin ferment.

(7) An antifibrin ferment.

(8) A proteolytic ferment.

(9) A cardiac lonic.

Numbers (1), (, 3 ) and (6) are the most imporant.

No one snake rields all these varieties of renom.

\section{To analyse Snake Veriom (Martin).}

(I) Dialysing and filtering it through a gelatine filter, supported in the pores of a Pasteur-Chamberland filter. Fibrin ferments and hamorthagins do not dialyse.

(2) Heat from $70^{\circ}$ tr $100^{\circ} \mathrm{C}$. Some neurotoxins and hamolysins will not be affected.

(3) Digest with red blond cells and serum, previously heated to $5^{\circ} \mathrm{C}$. The hamolysins are thus separated from the neurotoxins. The ervthrocytes remove the hamblysins; the heating removes the hamorrhagin, so that the neurotoxin alone remains.

Neurotoxins are very important. The renom of the Colubrina contains much. It unites with the nerve cells, atlacks the respiratory centre in the medulla, reducing the. respirations in number and amplitude to cessation, and paralyses the phrenic end plates in the diaphragm. The circulation may be kept going for some time after cessation of breathing if artificial respiration be resorted to.

The renom of some snakes, howerer, such as liper russelli. Bitis arietans, Crotalus horridus, and Lachechis anamallensis, contains a neurotoxin that acts upon the vasomotor centre of the medulla affecting the blood-pressure. When the nerve cells are attacked the Nissl granules break up in:o a fine dust-like deposit, the nucleus remains central but indistinct. In sudden death no change is noted. The cells are, as a rule, unequally effected.

Agglutinins.-These are destroyed by heating to $75^{\circ}-80^{\circ} \mathrm{C}$. White and red cells may be agglutinated. To see this reaction, add a 10 per cent. solution of dried renom in normal saline to washed corpuscles suspended in normal saline. The cellular outline may be modified.

\section{Cytolysins are of four groups:-}

Hremolysins. - I 2 per cent. Solution of the Yiper berus renom and a $0^{\circ} \mathrm{I}$ per cent. solution of Pseudechis venom destroys red cells in eighteen to twenty-four hours. These also remove the antibactericidal properties of the blond in most instances, but not in the case of the Necturus. The same renom that acts upon human red cells may not act upon those of other animals; this feature depends solely upon the 
amount of fally acids contaned in the corpuscles. If plentiful the red cells are readily affected, but if searce they are not so readily destroyed.

Leucolysins.-Weir Mitchell and Reichert notice that the mobility of the leucocytes absolutely ceased in Crocalus poisoning. The white cells become stationary, gramular and swollen, their outlines beconing indistinct and then disappear. Tise lrmphocyes are alfected last. Cobra poisuning is rery vigorous in this respect.

Hamorthagin has the power of so damaging the ressel walls as to form actual rents therein, permiting the blood to escape from them. The destruction is due to a cyolytic action upon the endothelial cells of the ressels and not to a bursting of the capillary wall.

Other cytolysins act upon the cells of the liver, kidney, testicle and ovary, causing lysis of same. The cells of the kidney tubules are necrotic and detached, filling the lumen. There are congestion and interstitial hæmorhages.

Antihamolysins are contaned in sume riper venoms which, if added to red cells in a certain concentration, will not allow hamolysis to take place. Perhaps it is due to precipitating the outer layer of hamoglobin.

Antibactericidal substances have been suspected because animals killed by snake renom rapidly decompose. It has been shown that the serum complements are fixed, and hence the bactericidal properties of normal sera are destroyed.

Fibrin ferment exists in the venom of the Viperina and Colubrida. Vascular choting is caused, conrulsions and sudden death resulting.

Antifibrin ferment is found in cobrat and allied renoms.

Proteolytic fermenls were suspected because the muscle fibres at the site of the bite were rapidly softened by crotalus renon. Citatine is liquetied by it. The muscle libres become necrotic and degenerate with a local polymorphonuclear infiltration.

A Cardiac and lascular tonic is indicated by the stimulation of the heart and the continuous circulation after the cessation of respiration, if artificial respiration is continued in cases resulting from the inoculation of cobra renom.

Exactly how it has been produced has not been demonstrated.

\section{THE SYMPTOMS OF SNAKE BITE.}

Colubrine Venom, e.g., Xaja tripudians (aphidismus - snake poisoning).

On Animals.-Paralysis of tongue, larynx, pharynx and all roluntary movement, salivation, cessation of respiration, lachrymation, mucous discharge from nose, respiratory passages and stomach, local inflammation, pain, engorgement, effusion, suppuration and sloughing. 
There is hamolysis, reduced coagulability and hamaturia. If much venom is injected there is not sufficient time for some of these to appear, such as suppuration, \&c.

On Man.-local pain, two small puncture wounds three-quarters of an inch apart exuding blood-stained fluid. Pain increases, spreads up the limb; patient feels intoxicated, loses control of legs with paralysis of jaw and other muscles; often profuse salivation and inability to speak or swallow. Pulse and respiration increase, respiration then decreases and stops. The heart continues to beat for a short time longer. If recovery, much urine is passed.

P.M.-Rigor mortis marked, blood fluid (coagulated in animals), parotids swollen, pia mater engorged, muscles a dirty red, lungs congested, bronchi contain a thin frothy fluid, right heart distended with blood, liver congested and clark, bladder contracted, fatty degeneration of liver and kidney, necrosis of renal and hepatic epithelium, round-celled infiltration of bile ducts.

The symptoms and post-mortem findings vary slightly with the species of Colubrine and the amount of venom injected.

Elapine Venom.-In Australia, where Elapines are common, the symptoms are: local swelling, and pain with constitutional symptoms in from one-half to two hours. Weakness of legs, prostration, vomiting, heart weakens, extremities become cold and skin blanched. Respiration is slowed, coma intervenes, sensations are diminished, pupils dilate, convulsions sometimes, and death. The heart continues after respiration has ceased.

The Elaps fulvius, the harlequin snake of the Southern States of America, causes great local pain and drowsiness in one hour, unconsciousness, collapse and death in twenty-four hours. If recovery, dinger is not passed until the fifth day, as the symptoms tend to recur.

Hydrophine Venom.-Almost purely neurotic. This differs from the Colubrine effects as follows: It is more toxic, less hamolytic, and hence causes less stained local effusion, and does not affect the coagulability of the blood. Symptoms are delayed, then there is stiffness, suffocation, spasms, romiting, convulsions, and death after a day or two. Local reaction slight.

Viperine Venom. - That of Russell's riper is the most fatal; a dog bitten may drop dead in from twenty to forty seconds. The cause of death is intravascular, the clotting is most marked in the portal vein. More chronic cases show much local oedema, extensive subcutaneous hamorrhages, sloughing of this area (or resolution), or abscess formation or spreading gangrene. There is rapid emaciation, profound anamia, hamaturia and melana. In these cases the blood is cherryred and will not clot; there is bleeding everywhere. The leucocytes are useless. Secondary septicamia is to be expected. 
In Man.-Local pain, swelling, lividity. Rapid swelling and spreading decoloration, excitement, thready pulse, hurried respirations, and later, stertorous breathing. Cold universal sweat, the swelling reaches the trunk, convulsions, and death from failure of the circulation ensue.

P.M.-Meninges and lungs congested.

The Bitis arietans or puff adder is the same in kind but less severe.

The Echis is very poisonous; its effects are much as the Colubrine.

Crotaline Yenom, e.g., Crotalus horridus.

Local aedema, swelling, infiltration of incoagulable blood, necrosis, suppuration, sloughing. Fall in B.P. accompanied by raso-dilatation of the portal system, respiration gradually ceases due to the failure of the circulation. The phrenics are not paralysed, and the respiratory centre is not primarily affected. There are hamorrhages into the periand endo-cardium, peritoneum, and pleura but not into the nervous system. There is no primary stimulation.

Few persons die of snake bite compared with the number alleged to have been bitten by renomous reptiles. 30 per cent. has been suggested.

\section{THE TREATMENT OF SNAKE BITE.}

The indications are :-

(1) To prevent the absorption of venom.

(2) To neutralize the venom absorbed.

(3) To aid the excretion of the renom.

(4) To maintain the strength of the patient.

(5) To treat the symptoms as they arise.

(6) To prevent as far as possible secondary infection.

\section{(1) To Prevent the Ábsorption of Venom.}

Stop the flow of blood and lympl from the bitten area by ligature.

This must be slackened for two seconds at intervals and must be applied for half to one hour. This allows time:-

For the dilution of the venom absorbed already.

For the neutralizing process to commence.

And permits of a gradual absorption only from the affected area.

Open the poisoned area freely for the application of antidotes and the local excretion of renom; this to be along the vessels in particular. The bite of the Echis, however, usually bleeds so freely that the difficulty is in controlling it.

Pour into the wound a strong solution of potassium permanganate and wash it well into the wound, attempting thus to neutralize some of the renom locally. Use crystals if there is no water available. Some find I per cent. chromic acid or hypochlorite of lime useful. 
Apply boric acid fomentations, frequently repeated, followed by a mild antiseptic dressing when the wound heals.

Suck the wound. Gastric and pancreatic juice neutralizes most venoms.

Care should be exercised that there are no abrasions about the mouth or lips. With some renoms the operation is not alvays free from risk.

\section{(2) To neutralize the Yenom absorbed.}

This includes the attempt to procure an acquired immunity, so at few remarks upon the subject of immunity as applied to snake renoms will not be superfluous.

Natural Immunity.

The mongoose and some hedgehogs are believed to be immune to cobra bite. Pigs are less acutely affected owing to a thick but slighty vascular layer about the body. In South America there is a harmless Colubrine, the mussurana, that fights, conquers, and eats the largest renomous reptiles in that continent without any obvious symptoms. Perhaps this reptile could be used therapeutically.

Acquired Immunity.

The natives of Bushmanland, Namaqualand and Damaraland are said to drink snake renom for the sake of its protection.

Some snake-charmers appear to be immune against certain species.

These individuals often extract the poison fangs preceding their performances, but there are some who undoubtedly go through it with the cobra poison fangs intact. It is asserted that some raccinate themselves by allowing young cobras to bite them from time to time. Nevertheless, accidents sometimes happen and the best of them may die in the calling. They certainly exercise great slill in the attempt to avoid being bitten.

The repeated inoculation of venom heated to $80^{\circ} \mathrm{C}$. produces some resistance in animals (Calmette). Calmette's serum is active against cobra-renom but not against Russell's viper, Bungarus, or Echis. The varieties of pure sera now in the marliet are numerous and are to be used for cases of poisoning by snakes whose names are indicated in the description of the serum.

(1) Lamb's pura Naja tripudians Merrem serum.

(2) Lamb's pura Vipera russelli Shaw serum.

(3) Tidswell's pura Notechis scutatus serum.

(4) Noguchi's pura crotalus serum. 
(5) Noguchis pura Ancistroden piscirorus serum.

(6) Brazil's pura Crotalus horridus serum.

(7) Kitajima's pura Lachesis I Iallow serum.

(8) Polyralent sera, not very efficacious.

Sera can be so made as to remain unimpaired for two years in all climates. They can be given prophylactically and curatively in the latter instance as soon as the species of snake is known or suspected. They all degenerate rapidly if not kept in a dark cool place. They must be used in large doses, Calmette recommends 10 c.c. subcutaneously and Martin, Lamb, Ioo c.c. intravenously. There is a wide difference of opinion on the dosage question.

\section{(3) To aid the Excretion of the Venom.}

The kidney is said to excrete most of the renom that leaves the body. The urine of a poisoned $\operatorname{dog}$ is said to have killed an injected pigeon in twenty-four hours.

The salivary and mammary glands also play some small part in its excretion. The condition is usually so acute and the toxin so destructive that there is but little opportunity for stimulating the excretory organs.

Salines can be given and sweating encouraged.

\section{(4) To Maintain the Strength of the Patient.}

Sirgchnine can be griven when there is but little neurotoxin in the renom.

Calfeine and adrenalin injections are indicated.

Salines intrarenously, per rectum and subcutaneously.

Ammonia and ether are good.

Apply a binder to the abdomen and bandages to the legs.

II armth is necessary.

Energetic morements are exhausting.

Alcohol as a rule reduces the activity of the antivenene.

\section{(5) Symptomatic Treatment.}

Give calcium lactate in large closes for riper renom.

Bromides, chloral hydrate and chloroform may be useful for restlessness, spasms and convulsions.

Morphia for the pain.

Artificial respiration when such is failing.

\section{(6) To Prevent Secondary Infection.}

Treat on antiseptic lines with much care and patience, especially in viper cases.

Acton and Knowles in working on this subject tell us:--

That ligature is efficacious. 
Simple amputation of the digit immediately when the toe or finger only is bitten.

Local injections of 10 c.c. of 5 per cent. solutions of potassium, zinc or calcium permanganate are good. Or of iodine trichloride or of gold chloride, which is the best of all.

Local injections must be given at once and after ligature, to be of any use. They do not affect that poison already absorbed.

Incision and excision of the wound, electrolysis, irradiation, cauterization, bleeding, perfusion, \&c., are all ineffective.

Intravenous injections of potassium permanganate are useless and dangerous.

The neurotoxin of cobra venom is absorbed quickly but fixed slowly and not firmly.

The venom of Russell's viper is absorbed slowly and fixed firmly.

Hence delay in giving antivenene is more dangerous in viper than cobra bite.

The union of venom and antivenene in vitro is instantaneous, hence in cobra bite half-hour doses are almost as good as one whole dose at once, but for viper bite give the whole dose at once in one dose, 50-Ioo c.c. intravenously or roO-200 c.c. intraperitoneally.

Acton and Knowles conclude:-

(I) Apply a firm ligature immediately.

(2) Impregnate the whole area of the bite with a hypodermic injection of a strong solution of gold chloride.

(3) Inject from roo to 200 c.c. of antivenene intravenously, if the biting snake is suspected to be a cobra or Russell's viper.

If symptoms of renom intoxication come on, further and even larger doses of antivenene should be given intravenously.

With sera concentrated ten times, a dose of 20 to 60 c.c. should save every case of cobra bite.

Prophylactic Measures.

When crossing jungle or grassy districts wear strong boots and puttees or leggings.

Carry a stick when travelling; it is often useful in striking down snakes.

Then doubtful look upon all snakes as dangerous.

Carry a lantern when walking after dark.

Always carry a little permanganate in case of need.

Never sleep upon the ground.

Cover drain pipes with gratings.

Keep the gardens free from long grass. 
Enemies of snakes should be encouraged. The Mussurana, or Oxyrhopus cloelia, is a hamless colubrine, which is said to feed exclusively on other snakes and to be addicted particularly to the venomous kinds, overcoming and swallowing them in a most heroic manner. 'This would probably be a good variety for the preparation of $\operatorname{sera}($ Alcock).

\section{YENOMOUS AND POISONOUS FISHES.}

Tropical and temperate seas abound in poisonous fishes about which little is known. Pending further knowledge we classify them thus :-

(I) Fishes which poison by their bite.

(2) Fishes which poison by their spines connected with poison glands.

(3) Fishes which poison after their consumption as food.

The former two are venomous, but their flesh can be eaten with impunity. The latter is poisonous and produces its effects when eaten, ¿.g., lamprey.

The genus Cattus is only venomous during the spawning season.

All these venoms are alike in kind, but differ in degree.

The effects are: local pain, swelling, suppuration, spreading gangrene, excitation, insensibility and paralysis.

The treatment is for snake-bite, with the exception of the antivenene.

(1) Fishes which poison by their Bite.

The type is the genus Murena. All have powerful teeth. They abound in the Tropical Atlantic and Mediterranean. The poisom pouch, capable of holding up to $\frac{1}{2}$ c.c., lies above the covering of the palate, and is lined by epithelial cells. There are three or four strong curved conical teeth with an anterior convexity. These teeth are enclosed in a sheath formed by the mucosa of the palate. The poison collects between the teeth and flows down into the wound.

\section{(2) Fishes which poison by their Spines connected to Poison Glands.}

The poison glands are generally placed at the base of the dorsal or anal fins or under spines on the operculum.

The glands communicate with one or more rays of fins.

The barbs may be:-

Grooved, the muco-membranous sheath converting this into a canal which must be ruptured before excreting externally.

Canalized by channels which lead to apertures near its tip. If these barbs enter the skin after being trodden upon, the poison is 
pressed mechanically into the wound. 'The pain is very severe and death sometimes results. Some poisomous lishes ause palpitation, fever, delirium, romiting and syncope.

\section{(3) Fishes which poison aiter their Consumption as Food.}

The most dangerous are those living amongst coral reefs. Those that are bright coloured are known to cause a severe reaction.

There are several species belonging to the genus Tetrodon, which are sometimes taken by the Japanese with suicidal intent.

The poison symptoms they call "fuguismus." The poison appears to lie in the ovaries and testicles, which contain :-

Tetrodonic acid, a white waxy body.

Tetrodin, a c̈rystalline base.

Symptoms.-.These arise within three to fifteen minutes after eating one roe. Gastric unrest, abdominal pain, burning about the fauces, nausea, severe headache, collapse and fainting; paralysis of the heart or respiration may cause death.

The mortality is high (68 per cent.).

Treatment.-Evacuate contents of stomach with an emetic.

Give stimulants such as strychnine.

\section{YENOMOUS TOADS AND SALAMANDERS.}

These belong to the Amphibia. The poisons found in their parotid glands and slin are :-

In Toads. (1) Bufotalin, an acid.

(2) Bufonin, a neutral body, less active than the above. They are but slightly toxic to man, irritating the mucous membrane, and especially the conjunctiva. Small animals are severely affected.

In Salamanders. (1) Salamandarin, an inorganic base.

(2) Salamandaridin, an alkaloid.

Neither are important.

There is a creamy culaneous secretion, toxic to many animals, the chemical nature of which is but little known.

\section{VENOMOUS ANEMONES, CORALS AND JELLY FISHES.}

These belong to the Cinelenterata. They sting by means of a specialized cell (enidoblast) which encloses a nematocyst, a long, hollow, spirally coiled filament, lying in toxic fluid. When irritated the cell sac ruptures, the coil filament is ejected, making a small wound in the animal attacked, and thus prepares a place for the poison. 
Anemones (Komularia).- Their stings cause itching, burning and skin eruptions. Those of the Mediterranean affecting the naked sponge divers cause a febrile attack with thirst, pains in the back and limbs, and a sloughing ulcer locally. Fishermen use them for poisoning animals, which die in a few minutes.

The poisons are :-

(1) Thalassin, causing redness, congestion, pruritis and sneezing.

(2) Congestion, more toxic, but somerhat antagonistic to the above.

Apply pure fat immediately to the infected site. A lager of grease vere the stin is an efficiont protection.

Corals (Millapora).- These produce local pain and erythema, followed by papules, pustules and desquamation. They abound in Malaya.

Jelly Fishes (Trachynedusa). - Thuse of the Mediteranean camse local redness, swelling and urticaria. Those of the Tropics are more severe, with agonizing pains and collapse.

Give stimulants internally and dilute ammonia locally.

\section{TOXIC HELMINTHS.}

These, such as Dibothrocephalus latus, Tania saginata, Tanta ecchinococcus, Ascarides and Ankylostomes are dealt with in the section "Diseases due to llelminths."

\section{YENOMOUS SCORPIONS AND SPIDERS, MITES AND TICKS.}

These belong to the Araclinida.

Scorpions (Scorpionida) abound in the Tropics; are much dreaded.

The last abchominal segment carries a curved spine which in attacking is brought forwards over the body, penetrating the rictim's skin, and thus making a wound into which the poison is injected. On either side of the spine is an opening through which the venom is discharged.

The toxins of different species differ in kind and degree, causing from local redness, pain and swelling in Europe to severe pain, extensive swelling, vomiting, fainting, muscular cramps and death in the Tropics.

Two hundred persons are said to die annually near Durango in Mexico from scorpion stings.

The mortality in children is 60 per cent. (Wilson). Death is rare in adults because of the sreater dilution of the poison.

In Africa stings are common, but death is rare. 
Scorpions live under stones, the bark of trees, in sand and houses, which latter they leave at dusk.

They seize their prey with their large six-jointed pedipalpi, holding it close to the mouth with the small three-jointed chelicera, bringing over the tail to sting their victim if necessary, leaving a spine in the body of their prey until the poison compressed from the bilobed gland along the duct to the spinal opening has acted well.

The venom causes agglutination of red cells leading to thrombosis, hamorrhage due to destruction in the capillary walls, coagulation and hamolysis (Jousset de Bellesme). It also contains a neurotoxin. In some respects it simulates cobra renom.

Treat as for medium cases of snake bite.

For the pain cocaine can be injected close to the sting.

Spiders (Aranea).-These grow to a large size in the Tropics.

All genera appear to be toxic. The poison gland lies at the basal joint of the chelicera, ensheathed in connective tissue. The duct runs forward from the gland to the distal hook-shaped joint, upon the apex of which it opens.

The effects of the renom resemble those of scorpions, but the general symptoms are often more delayed and may be of a tetanoid nature, lasting about ten days, as with the bite of Latrodectus mactans.

With the L. scolis of New \%ealand there may be stiffness of the jaw and mouth, difficulty in swallowing and speaking, slow pulse, slow respiration, and death or recovery in about six weeks. It may be confused with tetanus at first and typhoid later.

The Theridium lugubre may cause death in a few days. Many common spiders only cause a local reaction.

The Maoris will burn down the hut if a single person has been bitten in order to destroy the spider. They believe that its death is absolutely necessary for the recovery of the patient.

Treat as for scorpion bites.

Ticks (Ixodia). - These cause severe effects apart from their being carriers of babesia or spirochata.

The skin is pierced by the teeth on the digits of its chelicera. The digit is mored forwards and turned outwards, enlarging the wound, bringing the hypostome into position, fixing the tick by means of its curved teeth. The salivary gland at the same time pours a toxic secretion into the wound.

The Argas persicus, A. reflexus, Ornithodorus moubata cause local pain and swelling. The O. turicata has been known to cause general numbness and swelling, romiting, diarrhoca, urticaria, profuse sweating, rigors, feres and headache. An ulcer may form locally. 
The Ixodes ricinus causes a severe dermatitis, a pustular eruption, also abscesses with oedema. Lymphangitis and fever may ensue as a complication.

Remove the tick by covering it with oil so that it cannot breathe, then it will detach itself. Cauterize a bad wound. Apply sodi. bicarb. solution or menthol ointment.

\section{THE VENOM OF CENTIPEDES.}

These belong to the order Chilopoda.

They have a uniformly segmented trunk with a pair of appendages to each of its twenty-one segments. They live under stones and in shady places.

The appendages of the first segment are modified to form jaws, at the base of which the poison gland lies. The duct opens at the apex of the claw; thus a bite shows two minute wounds.

The venom is for the purpose of killing insects and larre. It causes itching and intense pain which rapidly affects the whole limb. The area of the bite turns black and lymphangitis may ensue. There may be romiting, irregular pulse, dizziness and headache. Adults recover in twenty-four hours, some children die.

Bathe the part in ammonia solution, I in 5 .

Apply fomentations. Administer opium.

\section{THE YENOM OF LICE AND BUGS.}

The local itching is well known. Their renom is of little importance.

The part they play in the dissemination of disease is discussed in other sections.

\section{THE YENOM OF BEES ĀND WASPS.}

The last abdominal segment of the bee contains a chitinous sheath in which the two barbs lie. I long, bilobed, tubular-coiled poison gland is connected by a cluct to the barbs at one end and ramifies amongst the intestinal contents at the other. One part of the poison gland contains an acid, the other an alkaline renom. The acid reaction may be due to formic acid. The peculiar aromatic smell is due to volatile substances. The toxin is not albuminous and is thought to have an organic base. The amount extracted from the hind parts of two bees in I c.c. of water will kill a mouse or sparrow. The eggs of bees, like those of the viper and toad, contain the specific venom. 
Bee renom contains :-

(I) Inflammatory poisons, perhaps from the acid gland.

(2) Neurotoxins, convulsive and narcotic, the former perhaps from the alkaline gland.

(3) Hamolysins.

Symploms.-Bee keepers are usually partially immune, but the immunity is temporary. Local inflammation and reaction is painful.

Sometimes faintness, vomiting, cold extremities and delirium appear.

A few have died as a result. Clema and shock may be marked. Gidema of the glottis is not uncommon when stings are about the throat.

The srmptoms usually disappear in twenty-four hours.

The dangers are with children and old people, especially when the stings are numerous.

Treatment.- Meak solutions of ammonia answer well. Carbolic I in 20 should be applied at once. Strohnine, immonia and ether where much shock is present.

\section{THE VENOM OF ANTS AND CATERPILLARS.}

Ants (Formicide).- Though small, ants are wonderfully organized and capable of controlling the animal world in the Tropics. That which is dead is removed by them; snakes will flee at their approach. and man does not relish a struggle with these aggressive creatures.

Many of them possess a poison apparatus analogous to that of bees.

The venom contains formic acid.

Symptoms.-Local inflammation, faintness, shivering and temporary paralysis. Dried ants are used by natives in preparing poison for arrows. Apply ammonia solution, carbolic ( 1 in 20), or camphor.

Caterpillars (Lepidoptera).- These cause a skin eruption and faintness.

The tiger-moth caterpillar produces a very painful angry eruption.

The caterpillar of Porthesia chrysorrhoea from North America causes its barbed hairs to penetrate the skin and to inject a venom that causes necrosis of the epidermal cells and the formation of small vesicles. Severe urticarial dermatitis may arise due to contact, the hairs remaining in the skin. Some people are mole sensitive to then than others.

Clear awa! the hairs with soda bicarbonate lotion (2 per cent.).

Apply ichthyol ointment (io per cent.). 


\section{THE YENOM OF MOSQUITOES.}

The females only (Culicida) attack man.

The mandibles and maxilla pierce and cut like spear points and saws.

An irritating substance is injected from the salivary glands, the nature of which has not been ascertained. The part itches, becomes red,-a weal may result; secondary infection is a common occurrence as a result of scratching them.

\section{YENOMOUS BEETLES, SNAKES AND LIZARDS.}

These beetles (Coleoptera) and their larve irritate by means of stiff hairs. Pain, inflammation and sloughing results. In the Solomon lslands there is a venomous snake of unknown species which possesses tubular barbed teeth. These are connected with a poison gland which sometimes causes severe wounds in man.

There are two lizards celebrated for their poisonous bites, the Trachyderma horribum of Mexico and the Heloderma suspectum of the United States. The former when irritated discharges a white, gelatinous salivary fluid from its mouth which is asserted to produce toxic symptoms. The creature may reach to nearly five feet in length.

The poison of the latter is passed along grooved teeth. Its effects are similar to an average amount of cobra venom, and should be treated as for that condition.

\section{POISONS TAKEN ACCIDENTALLY BY MAN.}

Poisoning by fish has been described.

Sitotoxismus is a term given to regetable food poisoning.

Ergotism is not important in the Tropics.

Lathyrismus is an intoxication caused by the ingestion of Lathyrus satireus and similar species. It exists in India. People who eat vetches in time of famine are liable to be attacked. The disease appears to be due to eating bread composed of flour of the seeds of Lathyrus belonging to the Order Leguminose.

If poverty causes an outbreak it is usually endemic.

If famines cause an outbreak it is usually epidemic.

Many toxic substances have been separated.

The incubation is not known.

The onset is insidious, pain in the back, weakness in the legs and spastic paraplegia manifest themselves. Girdle sensations, dragging feet and wasting of the muscles soon follow. The deep reflexes are increased, ankle clonus is present, muscular electrical reaction diminished, but the reaction of degeneration is absent. Incontinence of 
urine and impotence are common. The mind is normal. There is no gangrene ats in ergotism and no dropsy or heart symptoms as in beriberi. Give some food, warm clothing, bromides and counter irritants to the spine.

Lolismus is an intoxication due to the ingestion in bread of the seeds of Lolium temulentum. It is present in some parts of India and Europe. There are giddiness, trembling of arms, legs, and tongue, impaiment of rision, dilated pupils, prostration and romiting.

It is selclom fatal.

Give a castor oil purge and stimulants.

Paspalismus. - This is the result of eating bread made from the seeds of Paspaium scrobiculatum. Sometimes fatal.

Symptoms and treatment as above.

Fungi are said to play some part in its production.

Atripliasmus.- A similar disease confined to China.

The poor eat the seeds of Atriplex serrata, which grows wild in the courts, gardens and on the walls of Pekin. A parasite eaten with the weed may be the cause of the disease.

The srmptoms begin ten to twenty-four hours after its consumption.

The finger-tips and the dorsum of the hands become painful, swollen, cyanosed and cold. This condition spreads to the forearm.

The face and evelids itch and become swollen, the nose is cyanosed and cold. Sensibility to touch is diminished, to heat increased, bulle and ulcers may appear on these parts, and gangrenous patches are possible. The general health is but little affected.

The treatment is symptomatic. With Raynaud's disease there is no swelling.

Tapioca is obtained from the cassava root (Manihot utilissima). The bitter variety contains hydrocyanic acid in its milky juice, and is poisonous until it is roasted, when the volatile acid is driven out. The juice should be expressed and the root cooked before eating.

Nutmegs are poisonous if taken in large doses.

Castor Oil Seeds (Euphorbiacex) cause burning of the throat and abdomen, romiting and collapse. There may be diarhoca.

These seeds will kill in about forty-six hours.

Give emetics, stimulants and morphia.

Manchineel Tree Fruit (Hippomane mancinella, Euphorbiacea).-These fruits look very like apples and have a delightful fragrance.

They are common on the Grenadine Islands, and sailors have met their deaths mistaking the fruit for that of apples. There is gastrointestinal inflammation, dilated pupils, bullae on the skin. 
Kreotoximus is a gastro-intestinal disturbance which may simulate cholera, and is produced by the ingestion of semi-decomposed animal foods. The bacillus of Gärtner and the paratyphoid bacillus give rise to ptomaines causing the symptoms.

Emetics, purgatives, stimulants and salines are indicated, with a course later of intestinal antiseptics as salol or calomel.

\section{ACKEE POISONING.}

\section{The Vomiting Sickness of Jamaica. (After Scott.)}

\section{DEFINITION.}

A localized, epidemic, acute toxamia probably due to the ingestion and absorption of toxins from the ackee fruit, Blighia sapida.

It occurs in young adults and children in Jamaica and is accompanied by a heary mortality.

\section{AETIOLOGY.}

The disease, which is prevalent in the cooler months, November to March or April, seems to be caused by the ingestion of the ackee fruit that has fallen unripe from the tree. The epidemic nature of the disease coincides with the main ackee season.

Its localized peculiarity may be due to this fruit not being plentifully grown elsewhere, or that it is not in other places used as a food.

If the dose taken is small and vomiting takes place at once the poison is rejected and recovery may follow.

The children are chiefly affected because they are given "potwater" in which the ackee fruit has been boiled, a water solution of the toxin being most dangerous; also it is due to the children, more than adults, picking up the fallen unripe fruit and eating it.

The fruit is poisonous if it is picked up from a decayed, bruised, or broken branch; if forced open and not opened naturally on the tree and other conditions.

\section{SYMPTOMATOLOGY.}

These can be summed up under three heads:-

(I) Vomiting.-The patient, almost invariably a child, has discomfort or pain in the stomach. It romits once or twice or four times. Recovery may follow if all the toxin is rejected, or, on the contrary. the child may be fairly well or sleep, if at night, for several hours. Then there may be another attack of romiting, probably cerebral in. origin, of frothy mucus, followed by watery or bile-stained fluid. A few cases do not vomit at all.

The former is known as Initial romiting, and the latter as. 
Secondary romiting. Very few indeed recover who reach the second stage.

(2) Convulsions. - In a few minutes there are convulsions, varying in given cases from twitching movements to definite massive convulsions; single clonic muscular spasms to marked epileptoid clonic contractions.

(3) Coma.-The coma is deep and fatal. The conjunctival reflex is absent.

The temperature is usually normal or up to IOI $^{\circ} \mathrm{F}$. The pulse 90-100. The respirations 26-30.

There is no delirium.

Recovery or death is very rapid, even in one hour.

The average time from the onset of symptoms to death is $12^{\circ} 7$ hours.

The final picture is probably due to rapid absorption of toxins from the empty stomach.

\section{PATHOLOGY.}

There is hyperamia of most of the organs, including the meninges, with cedema of the suppurating tissue.

There are small extravasations in the kidneys, adrenals, lymph glands, spleen and lungs.

Microbiotic changes in the kidney tubules, pancreas and liver.

Fatty changes in many organs, especially the liver, kidneys, pancreatic cells, heart muscle, and the large Betz cells in the brain.

\section{MORTALITY.}

So-go per cent. of those attacked die.

\section{PROPHYLAXIS.}

To prohibit the fruits of Blighia sapida to be used as food.

To educate the people as to its dangers.

If not sufficient, then to request powers to destroy all the trees in the island.

\section{POISONS USED FOR POISON ORDEALS, FIGHTING, FISHING, HUNTING.}

The poison ordeal once prevalent in England is common in Africa.

The local witch doctor prepares a mixture more or less poisonous, which is given to a person "suspected" of some crime. The ingredients of many of these preparations are unknown, but poisons from the Loganiaceal, Apocyanaceal, Leguminose and Salanaceal are commonly used. In West Africa the sliced root bark of Strychnos icaja or decoction of strophanthus is used. If after drinking a 
decoction the victim can jump over a stick or micturate upon a bananat leaf he is declared innocent. This is seldom possible. The Calabar bean, Physostigma venenosum (Leguminosa) is often used. If the victim vomits he is innocent, if not he is guilty and dies.

Arrow poisons vary with the different countries in which they are employed. They are usually only known to the witch doctors, chiefs and fetish priests. Snake renom, scorpions, spiders and beetles pulped and well mixed with regetable poisons are used. Vegetable poisons are important.

Some act upon the heart and muscles like digitalis. peripheral nerve endings like curari.

," peripheral nerve endings like curari.
," nerrous system and heart like aconite and
strychnine.
, , system like snake renoms.

To treat an arrow wound, apply a tourniquet to the limb, proximal to the wound. Remove the arrow by passing a hollow tube (bamboo) over the barb and withdrawing it, or folding the skin as close to the point as possible, push the barb through, divide the shaft, withdraw the barbed end forwards and the feathered end backwards. Cup and wash the wound with strong permanganate. Give stimulants generally and fomentations locally. Strophanthus is much used in Central Africa (Livingstone).

It causes death in from ten to fifteent minutes.

Fish poisons are used all over the world by natives. The bark and leaves of trees and shrubs are thrown into the water. The fish are rendered unconscious by the poison, and, floating to the surface, are readily captured. The varieties are numerous. Some of the active principles are strychnine, hydrocyanic acid, piscidin, a non-nitrogenous substance called derrid and pachyrhizid.

Denis root will kill fish in a dilution of $\mathrm{I}$ in 25,000 of water, and

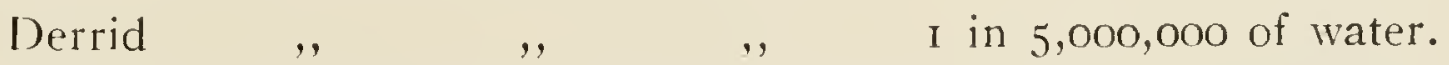

Poisons for animals are: The root of Aram montanum for tigers in India.

Aconite for elephants in India.

Tylophora fasciculata, \&c., for rats in Africa.

Dianella nemorosa $\mathrm{L}$. for rats in Malacca. 


\section{SECTION VI.}

\section{DISEASES OF THE SKIN.}

\section{INTRODUCTION.}

The skin diseases of temperate climates are prevalent in the Tropics with few exceptions, but because of differently coloured skins their diagnosis is often somewhat difficult, and clinical characters alone are insufficient for their discrimination.

The skins of Tropical races differ from those of temperate climates in thickness, pigmentation, sebum, sweat and hair.

The skin of the African negro, and to a less extent that of the Indian races, is somewhat thicker than that of the European, thus affording a good protection for his poorly clad body in the rough-andtumble life he lives.

The pigmentation is marked and characteristic of tropical natives. It is present at birth, giving the baby a muddy appearance, then darkens rapidly during the first few weeks, and slowly until puberty. Perhaps this is a provision of Providence for protecting them against the sun's rays.

The sebaceous glands are more highly developed, the secretion giving a more glossy appearance to the skin and a characteristic odour so readily noticed by the new-comer to the Tropics. This excess of sebum is also protective, as fat is a bad conductor of heat.

The secretion of sweat is also in excess. It evaporates readily and keeps the skin cool. Profuse sweating may also be the result of nerve exhaustion or of a blood toxamia calling for the elimination of toxins.

The hair is poorly developed. It is smooth in Indian races and curly in African natives, the woolly nature being due to the spiral hair follicles of the scalp. Hair growths about the face are, as a rule, thin and shaggy. The lanugo hair is but poorly developed.

Pathological conditions of the skin undoubtedly have predisposing factors in the climatic influences of the Tropics.

A hot dry climate causes rapid evaporation of sweat and a dry " cracked" skin, easily injured, while a moist heat causes damp sebaceous deposits favourable for the development of skin parasites and a softening of the skin which facilitates disease. 
The sun's rays cause lyperamia and lieatstrokes, the former leading up to subcutaneous adema and vesication in those frequently exposed, resulting in a thickening of the skin and a deposition of pigment. This condition is seen in pellagrous patients. Cold cream and vaseline are good for sumburn, lead and opium lotions are soothing. Helmets made of pith or rubber so as to protect the head and the nape of the neck should be worn from sunrise to sunset as a routine thing.

Flannel and cholera belts are often irritating and predispose to prickly heat. Silk or silk and cotton clothing next to the skin is efficacious.

Alcohol is often taken owing to increased thirst and to a feeling of exhaustion so common at the close of the day. This causes increased perspiration and predisposes to prickly heat; it is not necessary and should be avoided; in any case moderation is essential.

A daily tepid bath is necessary for skin cleansing. 111 attempts at the classification of skin diseases are at present provisional and at the best incomplete. Dermatologists are still taking certain varieties from the "Eczema" group and giving to them a definite place in the dermatological " pigeon-hole."

We feel that it will suit our purpose best to deal with the most prevalent tropical skin diseases alphabetically.

\section{ACANTHOKERATODERMIA.}

This is a term used by Chalmers to include "hyperkeratoses" accompanied by " acanthosis."

There are thickened areas of epidermis which may break down or become fissured, affecting the soles and palms.

This condition is accompanied by hyperkeratosis subungualis.

The thick horny layer is largely composed of cornified and modified prickle cells.

\section{ACARIÁSIS.}

This is an eruption affecting the whole body caused by the Pediculoides ventricosus, the "Acarien" of barley. "The eruption may simulate urticaria or scabies, and is characterized by papules of the face, neck, trunk and limbs, or sometimes by urticarial patches with a small vesicle in the centre.

The itching is intense and lasts longer than the eruption.

The symptoms may come on a few hours after handling infected barley, but it clears up itself within twelve hours if not scratched.

Swelling of the neck and face is rare.

The condition has been seen in Algeria (Sergent and Alary).

Sulphur preparations are indicated. 


\section{A CNITIS.}

An uncommon papulo-pustular eruption, probably caused by a poison generated under the influence of sunlight by cutaneous cells sensitized to the tubercular virus, and attactied by the products of an intestinal auto-inoculation, the whole process being anaphylactic in nature (Chalmers and Martin).

The papules appear about the head, neck, face, dorsum of the hands and on parts exposed to light.

The condition has been differentiated from Acne vulgaris, Acne varioliformis, Lupus vulgaris, Erythema induratum, Dermatitis nodosa rubra and Drug eruptions.

Von Pirquet's reaction was positive in C'halmers's case.

Salol, salines and calomel are indicaled.

There should be early protection against auto-intoxication.

\section{ACRODERMATOSIS.}

This is probably an impetiginous affection of the hands and feet due to staphylococci or streptococci, as seen in poor children living under insanitary conditions.

\section{ACRODERMATITIS CHRONICĀ ATROPHICANS.}

A general condition of inflammation and infiltration of the skin followed by atrophy. Its aetiology is unknown. It differs from idiopathic atrophy by the inflammatory stage.

\section{ACRODERMATITIS VESICULOSA TROPICA.}

A rare disease of unknown causation. Both hands are affected, the skin is glossy and tense, contains small vesicles with clear fluid. The fingers taper, there is pain and hyperesthesia. The hands are useless for work. The condition lasts from several months to several years and ma! clear up spontaneously.

Give ichulyol, internally, 3 grains, t.d.s., and externally as an ointment, 2-5 per cent.

\section{ALBINISM.}

A partial or complete congenital absence of pigment in the shin. iris, choroid and hair. The condition is generally hereditary and is more common in coloured peoples. It is endemic in some tropical regions. There is intolerance to light, and hence contracted pupils. The hair is white, fine and silly; the iris rose-coloured, and the pupil appears to be a pinkish-red. 


\section{ANGIO-FIBROMA CONTĂGIOSUM TROPICUM.}

A disorder of unknown causation found only in Southern Brazil.

After an incubation of fifteen to twenty-five days there is an eruption of red papules developing rapidly to the size of peas or almonds.

The face, neck, axilla and genitals are most commonly affected, also the mucosa. The papules bleed readily. "There is no ferer. They clear up spontaneously without any scar.

\section{ASPERGILLOSIS.}

A chronic fungus affection of the hairs of the beard and moustache by the Aspergillus barbe. Penicillium barba is also commonly found at the same time.

Shave the hair and use turpentine, followed by carbolic, tar, or sulphur soap.

\section{BARCOO ROT.}

This is a condition closely allied to, if not identical with, the "Veldt sore" of Africa. There are blebs in an inflammatory area containing thin pus, which arise on the backs of the hands and wrists which develop into shallow ulcers. There is a preponderance of staphylococci in the blebs and early affected hairs.

Exposure and exhaustion predispose.

Epilation of the affected hairs and ordinary antiseptic treatment under good conditions will soon clear up the condition.

\section{BIOTRIPSIS (Life-wear).}

A general atrophic condition due to old age (idiopathic atrophy).

\section{BLASTOMYCOSIS.}

Blastomycetic dermatitis is a chronic (five to ten rears) inflammatory disease of fungus origin caused by the genera, Saccharomyces, Cryptococcus, Coccidiodides and Monilia.

\section{SYMPTOMATOLOGY.}

A reddish maculo-papule appears, becomes infected and suppurates.

The papules become ulcerated nodules, which spread in extent and depth, producing a raw and ulcerated mass. The dorsum of the hand and the front of the leg are usually attacked first, but any part may be affected. The alae nasi and the lips may be destroyed. The disease is not confined to the skin, but may affect the internal organs.

The disease may last for years and then clear up spontaneously, learing a soft smooth scar.

There is very little pain and the lymphatic glands are not enlarged. On close examination numerous miliary abscesses are seen in which the parasites, epithelioid and giant cells can be found. 


\section{DIAGNOSIS.}

It closely resembles tuberculosis of the skin, and may be mistaken for the vegetating forms of syphilis.

The diagnosis rests upon finding the fungi in the minute abscesses.

\section{TREATMENT.}

Potassium iodides in large doses, 1 gramme, t.d.s., and increased until the disease disappears.

Röntgen rays, where available, can be applied with benefit.

Local applications of sublimate and iodine tincture are useful.

For visceral and general infection there is no successful treatment.

\section{BOILS.}

Tropical boils are very common, and are usually severe and resistant. Auto-inoculation should be closely guarded against.

Conditions of lowered resistance and diabetes predispose to them, but the immediate agents are pyogenic organisms, chiefly the slapliylococcus.

\section{TREATMENT.}

The condition frequently requires that the patient shall be invalided in order to raise his powers of resistance.

Treat any constitutional disorder and avoid poulticing.

Touch new boils with tincture of iodine, wash old ones with sublimate. One drop of pure carbolic acid in a small drilled hole will often abort the abscess.

Prepare and administer an autogenous vaccine.

Aroid opening boils early.

\section{BOTRYOMYCOSIS HOMINIS.}

A condition of fungating granulomas occurring as a sequel to wounds which have been infected with cocci. They grow slowly until about the size of a cherry and are pedunculated. They occur in clusters. Some authorities affirm that the condition is caused by Botryonyces ascoformans, others blame an amoba-like organism.

\section{BROMIDROSIS.}

Perhaps each indiviclual has his own peculiar offensive odour when perspiring excessively, probably due to the increased sebaceous matter secreted. Lack of cleanliness ahways makes the condition worse, but in some cases marked cleanliness will not remove the odour completely. Some of the great variety of scents may be used to counteract it. The clothing should be changed frequently.

Alcohol should be aroided. 


\section{CHIEROPOMPHOLYX.}

This disease, often better known as Pompholix or Dysidrosis, consists of sago-like vesicles rising from the prickly layer of the skin affecting symmetrically the inter-digital parts of the hands and feet. Their advent is accompanied by intense itching and burning, but they are not surrounded by any inflammatory zone. These vesicles may run together, forming bulle containing clear fluid which may dry up and scar over. The whole of the extremities may be attacked, clusters forming early about the sweat glands and often pressing upon them.

The attack lasts about two weeks, but recurrence is common.

To scratch them may cause a troublesome eczema.

The cause is unknown. Some affirm that it is a vasomotor neurosis.

\section{TREATMENT.}

Treat as for pruritis. Aroid all that promotes excessive sweating. Prick the vesicles with a sterile needle and bathe them frequently in sublimate lotion. Cotton wool placed between the toes aids the process.

\section{CHLOÁSMA.}

This disorder is an anomaly of pigmentation of the exposed parts. The varieties are classified according to the known causative agents, such as :-

Chloasma solare, due to exposure to the sun, glacial glare and other powerful light.

, caloricum, following exposure to heat.

, traumaticum, following mechanical irritation.

, due to irritating drugs.

, malaricum, in cases of chronic malaria.

, symptomaticum, or better perhaps, C. uterinum, found in some cases of pregnancy and diseases of the female genital organs.

Hyperpigmentation is seen in many diseases, as Tuberculosis, Syphilis, Leprosy, Diabetes, Addison's disease, Kala-azar, \&c.

\section{COOLIE ITCH.}

A general contagious itching eruption of unknown causation affecting the extremities, and rarely the whole body. The eruption resembles scabies, but no Acarus scabiei can be found.

Sulphur and naphthol ointments, 3 to ro per cent., should be used.

\section{COPRA ITCH.}

A troublesome dermatitis caused by an Acarus-like parasite of the Tyroglyphus, which swarms in copra and affects people handling it. The mite does not burrow into the skin. The disease lasts as long as 
the subject handles copra. Beta-naphthol ointment, 5 per cent. to io per cent., can be used, but the mite leaves the skin at night time in any case.

\section{CORDYLOBIASIS.}

The invasion of the skin by the larve of Cordylobia anthropophaga. The larval excrement forms the black centre in the small elevated area.

\section{CRAW-CRAW (Nodular Dermatitis).}

African natives use the above term for most skin eruptions, but it is most likely more truly applicable to a condition of hard papules or papulo-pustules, of various shapes and sizes, often exfoliated at the top, irregularly distributed over the extremities, more rarely over the whole body, and occurring chiefly in West Africa. Pyogenic infection is common.

The lymphatic glands draining the area are affected. Some cases clear up spontaneously, others may last for months and even years.

The cause is unknown, but some blame a microfilaria.

\section{TREATMENT.}

The treatment found most useful is to open the papules or pustules, remove the crusts, scrape the ulcers, scrub them with sublimate solution, dust with boric powder, and cover them with vaseline on lint, leaving the dressing on for one week (Emily).

Internal medication is not efficacious.

Some use a 2 per cent. salicylate lotion, followed by 5 per cent. to 10 per cent. naphthol ointment.

\section{CREEPING DISEASE OR ERUPTION.}

An eruption characterized by a raised red line up to one inch in width, which lengthens at the rate of from one to several inches daily in a sinuous or straight line, the commencement of the line fading as. the other end progresses. There is much pruritus.

Larva of the genus Gastrophilus and other genera have been found in several cases, but in others parasites were absent.

The disease may last for months or years.

Treatment is difficult. Some give subcutaneous injections of cocaine and chloroform. Treat as for pruritus.

\section{DERMATITIS BULLOSA PLANTARIS.}

A recurring condition of blebs on the soles of the feet, first described by Cantlie. The blebs form in the wet season and disappear in the dry, the condition recurring annually for ten to twenty years, and sometimes after the patient has returned to Europe.

The blebs burst, and scaly flakes of skin form over the soles of the feet and between the toes. There is intense itching. 
Treatmen: is difficult. A warm carbolic 2 per cent. foot bath daily can be tried, followed by some antiseptic dusting powder, such as zinc oxide.

Iodide is useful. Ointments are not to be used as the parts should be kept dry. Moist applications should be avoided.

\section{DERMATITIS CUPOLIFORMIS.}

A nodulo-ulcerative condition, chiefly affecting Europeans, seen in Ceylon and described by Castellani.

First one notices several small dusky spots about the lower extremities, some disappear, while others become hard, raised, infiltrated sometimes without resication or pustulation. Each may vary in size from a cherry to a pea, the centre becoming cupoliform. Later there is ulcer formation. These ulcers are painful, slow healing, requiring about twelve months, and leaving hyperpigmented spots.

A Gram-positive streptococcus, S. tropicalis, is said to be the causative agent.

The condition is very resistant to treatment. Autogenous vaccines give the best results. Antiseptic lotions, followed by Peruvian balsam, can be tried.

\section{DERMATITIS EXFOLIATIYA (Pityriasis rubra).}

This is an inflammatory condition of the skin, characterized by universal redness without infiltration or thickening, but accompanied by profuse desquamation, the flakes varying in size with the various parts of the body. It may follow other skin diseases or be independent. The onset is sudden; the eruption rapidly becomes general; the tint, bright scarlet in Europe, is white or brownish in tropical climes; the scales are arranged like slates on a roof and are never clumped into crusts. Itching is usually absent, but occasionally may be profound.

The disease is rare. The causation is obscure.

\section{TREATMENT.}

Antimony is useful in acute cases, arsenic in chronic ones.

Chills are liable to become dangerous.

The following local applications will be found useful :-

Diluted liquor picis carbonis.

Very weak creoline.

Carbolized oil, I in 20.

R. Glycerine of sub-acetate of lead $\quad \ldots \quad$ I oz. $\begin{array}{lllll}\text { Pure glycerine } & \ldots & \ldots & \ldots & \text { I },\end{array}$

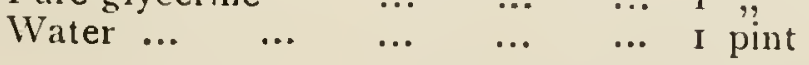
Apply to the whole body on lint. 


\section{DERMATITIS MACROGYRATA.}

A condition of large persistent, scaly, crusty gyrations upon the palms met with in Southern India. The cause is unknown.

Treatment has not been found efficacious.

\section{DERMATITIS NODOSA RUBRA.}

A general eruption of large, hard, angry red papules without umbilication or scales. The pruritus is almost unbearable.

The disease may last six to twelve months and then clear up spontaneously.

It resembles the papular stage of small-pox, but there is no fever and no scarring. The causation is unknown.

Salicylic-alcoholic lotions, 2 per cent., are useful. Internal medication has not improved the condition.

\section{DERMATITIS RIMOSA OF THE TOES.}

A condition of severe pruritus, followed by the formation of fissures between the toes. The condition is difficuit to cure.

Warm carbolic foot baths and zinc oxide powder will assist much.

\section{DERMATITIS SOLARIS.}

This is an advanced condition of erythema solare.

Avoid exposure to the sun.

\section{DERMATITIS VENENATA.}

An inflammatory condition of the skin caused by contact with certain poisonous plants and timbers.

The following classification has been suggested by Castellani:-

(1) The Rhus Group.

Plants in which the poisonous principle is contained in a clear watery fluid, e.g., poisonous ivy, the varnish tree, parsnips, \&c.

(2) The Euphorbia Group.

Plants in which the poisonous principle is contained in a thick milky fluid, e.g., the manchineel tree and all Euphorbias.

(3) The Urtica Group.

Plants in which the poisonous principle is contained in the fluid of special hairs, e.g., nettles, primroses, \&c.

(4) The Fectona Group.

Dried hard woods, the dust of which contains the poisonous principle, e.g., teak, satin-wood, ebony, rosewood, \&c.

For treatment lead lotion and calamine lotion should be applied.

\section{DERMATOPHILIASIS.}

This is a local skin disease due to invasion by the pregnant female jigger, Dermatophilus penetrans. 
Its original home was the West Indies and Tropical America $\left(30^{\circ} \mathrm{N}\right.$. to $30^{\circ} \mathrm{S}$.). It was brought to Loanda, West Africa, from Rio de Janeiro about I872. Stanley's expedition probably carried it across Africa to the East Coast, 1895 , thence to 1ndia, IS99, by the th Bombay Infantry.

These parasites attack man, wild and domestic animals, especially the pig. They live in dry sandy soil; when impregnated the female burrows into the skin of a warm-blooded animal, preferably in man about the feet; the abdonen now swells enormously until it is as large as a small pea, the posterior segment blocking the opening through which the eggs are expelled; the eggs become larra, cocoons, pupa and imagos in eiglit to ten dars.

The symptoms are itching, burning and throbbing, pyogenic infection, suppuration and ulceration when the parasite is thrown out.

Complications, such as phagedana, gangrene and tetanus, may result as with all unclean wounds.

\section{TREATMENT.}

Keep the floors free from dust. Avoid walking barefooted, dust socks and boots with iodoform, choose healthy "steward" boys, use cyllin upon the floors, extract the parasite with a clean needle when first felt, and touch the site with tincture of iodine.

\section{DYSIDROSIS EXFOLIATIYA.}

A coninuous and excessive perspiration of certain parts, usually the palms, resulting in flaky exfoliation of the epidermis.

A trip to the hills will usually clear up the condition.

\section{DEFINITION.}

\section{ECZEMA.}

A catarrhal inflammation of the skin, originating without visible external irritation, characterized by serous exudation in some stages of its evolution (Morris).

\section{SYMPTOMATOLOGY.}

It seems to rise spontaneously; has no regional limitations; has no refinite course, and too often defies all attempts of the medical attendant to clear it up. There are erythematous lesions, papules, resicles which when ruptured discharge a secretion that stiffens linen, later crustation and desquamation may predominate in turn. Itching is often unbearable, but constitutional disturbances are slight. Scratching, which it is exceedingly difficult to resist, may lead to pyogenic infection and ulceration with enlargement of the proximal lymphatic glands. 


\section{VARIETIES.}

Seborrhocic eczema, based upon excessive seborrhocic secretion (Hydrosis oleosa). The Staphylococcus epidermidis albus may contribute to the cause.

Sweat eczema, based upon excessive secretion of sweat, acting as a softening and irritating agent to the skin.

Follicular eczema, based upon an inflammatory condition of the hair follicles, possibly of microbic origin.

Nervous eczema, based upon reflex irritation of the skin after nervous shock, mental strain, and after dentition.

Most eczemas are of microbic origin, the organisms proliferating when there is some slight abnormality of skin function, the process then extending by auto-inoculation and reflex irritation.

\section{TREATMENT.}

The less drugging the better. Avoid arsenical preparations.

In selected cases antimony (rinum), calomel and opium for nervous cases are useful.

When and where possible send to a cool climate.

Locally, remove crusts and scales with weak solution of sodium bicarbonate; relieve itching by warm solutions of boric acid, followed by dry boric powder with starch; protect from the air by creams and salves; use sulphur for the seborrhoic varieties.

\section{ERYSIPELAS.}

This form of streptucoccal cellulitis and dermatilis is not uncommon. Liq. ferri perchlor. fort. and Liq. arsenicalis internally with ichthyol ointment externally will be found efficacious.

In resistant cases diphtheritic anti-serum has proved useful.

\section{ERYTHEMA SOLARE.}

Europeans, during the early years of tropical open-air life, are subject to this erythema caused by the sun's violet ray's. There is some desquamation and pigmentation.

Vaseline is useful, but sensitive skins should be protected by sun umbrellas as a troublesome dermatitis may ensue.

\section{ERYTHÁSMÁ.}

This consists of brownish red scaly patches in the genito-crural region or elsewhere caused by the Microsporon minutissimum.

Wash with soap and water, apply linamentum terebene; later, resorcin and salicylic ointments.

\section{GANGOSA.}

See article on Gangosa, section on "Diseases of Lncertain - Titiology." 


\section{GRANULOMA INGUINALE.}

Serpiginous ulceration of the genitalia is widely spread in its distribution. It is a chronic vascular granulona, probably of protozoal origin, attacking the external genitals of both sexes, and spreading to the inguinal region and perinaum. It does not tend to suppurate or caseate.

The condition is painful, disfiguring, contagious, and exceedingly difficult to cure. It may by becoming adematous simulate elephantiasis in its early stages. It occurs between puberty and 45 years of age. The general health is but slightly affected. Scarring may lead to ultimate healing.

\section{TREATMENT.}

Radiotherapy has been found effective.

Excision and scraping in later stages are helpful.

Neosalvarsan has its advocates. Tartar emetic may be useful.

Iodides in large doses can be tried.

\section{GRAIN ITCH.}

An itching eruption of weals and vesicles, some of which become pustules, affecting the neck, chest, abdomen and back, found in workmen who handle new straw mattresses which are infected with the Pediculoides ventricosus. The hands, strangely enough, nearly always escape.

\section{TREATMENT.}

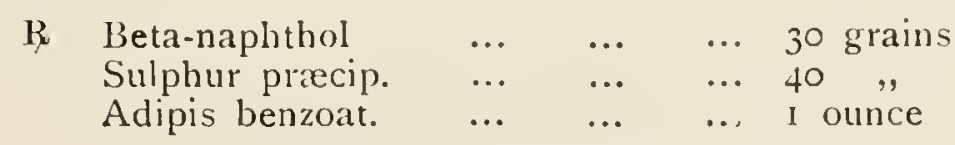

\section{HAIR AFFECTIONS.}

These are caused as a result of :-

(A) Inflammation with subsequent atrophy of the hair follicle, leading to increase or decrease of the hair pigment, atrophy, and total destruction of the hair.

(B) Concretions formed on the hair shaft.

(C) Parasitic diseases such as ringworm. These are dealt with in another place.

(1) Alopecia.

This condition is usually due to one of the following causes:A senile change which is sometimes congenital, at other times syphilitic (second stage).

A febrile or any other general disease interfering with nutrition. 
Any pressure of the temporal arteries or veins of the scalp by hard unventilated hats.

Profuse perspiration with bacterial invasion and decomposition of the hair.

Chronic seborrhœea, and a

Neurosis when it is local and follows the track of some nerve.

\section{TREATMENT.}

To remove the cause, e.g., clear up the syphilis, treat the febrile or general disorder, remove the pressure on the vessels, correct the nutrition, \&c.

Stimulate the blood supply of the scalp locally with mild irritant.

Nourish the follicles externally by ointments.

External Irritants.-There are endless varieties. A good one is:-

$\mathrm{R}$ Borate of soda $\ldots \quad \ldots \quad \ldots \quad \ldots \quad \ldots \quad$ ro drachms

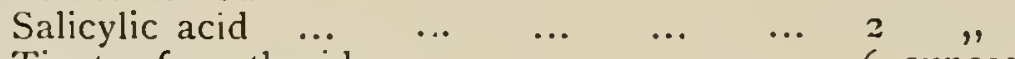

$\begin{array}{lllll}\text { Tinct. of cantharides ... } & \ldots & \ldots & \ldots & 6 \text { ounces }\end{array}$

$\begin{array}{lllllll}\text { Bay } r u m . . . & \ldots & \ldots & \ldots & \ldots & \ldots & 25 \\ \text { drachms }\end{array}$

$\begin{array}{lllllll}\text { Rose water } & \ldots & \ldots & \ldots & \ldots & \ldots & \text { IO }\end{array}$

Boiling water to $\mathrm{I} \frac{1}{2}$ pints.

Nourishing Ointments.-A good one is :-

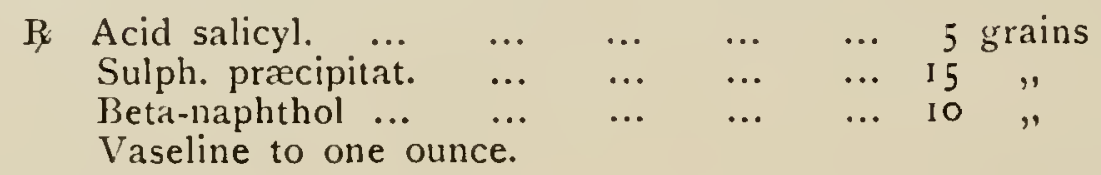

(2) Canities.

A senile change usually causes greyness, but the condition of grey hairs may be congenital, the result of disease, nervous shock, or long-continued nervous exhaustion.

In elderly persons, treatment other than dyeing is useless, while in younger persons cure is very doubtful.

Black hair may result from pilocarpine injections given for any reason.

Aniline dyes will cause a deep red brown colour of the hair in some dye workers.

Green hair is caused by copper amongst copper smelters.

Blue hair is caused amongst workers in cobalt mines.

For the treatment of grey hair in young persons the following can be tried: Tinct. of jaborandi, internally, i 5 minims t.d.s., or pilocarpine hydrochlorate, hypodermically, gr. $\frac{1}{1} \frac{1}{0}$ to .

\section{(3) Dermatitis Papillaris Capillitii.}

An inflammatory process, commencing on the back of the neck and spreading upwards. The papules form large rasp- 
berry-like vegetations, which bleed readily and give off an offensive odour and discharge. These regetations are characteristic of the disease. Abscesses may form.

Later the vegetations shrink and heal spontaneously by scarring.

(4) Folliculitis Decalvans.

A chronic folliculitis of the scalp leading to cicatrization and baldness. Use anti-parasitic ointments and stimulating lotions.

\section{(5) Leptothrix.}

Concretions upon the hairs of the axillary and scrotal regions. The hairs are very brittle and break readily.

The origin is bacterial. Use cleanliness and anti-bacterial ointments.

\section{(6) Monilethrix.}

Beaded hair affecting almost all the hairy parts of the body. The pigment is collected in the nodes, the hairs breaking off at the narrow parts. Young people are generally affected. It is probably due to atrophic changes at periodic intervals.

A modification of this condition is known as "bayonet hair."

(7) Oxergrowth $=$ hirsuties.

This condition is usually associated with some anomaly of dentition, insanity, disorder of menstruation, barrenness, severe illness, local irritation and heredity.

Treatment is of no avail permanently apart from electrolysis, by which means isolated large hairs are removed with the hair follicles. Local remedies simply scour off the hairs like pumice-stone applications.

\section{(8) Piedra.}

Small, black, gritty concretions that rattle on combing the hair. They are closely packed pigmented spore-like bodies, due in all probability to the Trichosporon giganteum. It is common in Colombian women.

\section{(9) Pseudopelade.}

Irregularly shaped patches of baldness which coalesce, cicatrize and destroy the follicles. Adults with coarse, dark, wiry hair are usually the victims. Treatment is of little avail.

(10) Tinea Nodosa.

A nodular concretion caused by fungus spores affecting the beard and moustache.

Shave and apply antiparasitic remedies. 
(11) Trichomycosis Capillitii.

Similar to Leptothrix, but due to a different organism.

(12) Trichorrhexis Nodosa.

Small bead-like whitish swellings resembling "nits" on the hair shaft causing the cortex to split, while the medulla remains unbroken. The condition is probably due to a diplococcus.

\section{TREATMENT.}

Epilation of diseased hairs, or

Frequent shaving and anti-parasitic remedies.

\section{HERPES.}

A cluster of transparent resicles the size of a pin's head to a pea, numbering from two to twenty, seated on an erythematous patch and surrounded by an inflammatory zone.

There are two types, Herpes febrilis and Herpes zoster (Shingles).

The former is designated IIerpes labialis, facialis or progenitalis, according to its position. It is symptomatic of febrile disorders, but typhoid fever is a noted exception.

Herpes zoster is nervous in origin, and follows the distribution of one or more of the posterior spinal roots on the skin. These nerves correspond with those nerves that become tender in risceral disturbances.

\section{TREATMENT.}

Menthol or collodion externally and morphia internally may be required; otherwise for all herpes treat the general disorder and apply anti-pruritic lotions and ointments locally.

\section{HYPERIDROSIS.}

General or local excessive perspiration.

It is often associated with priclily heat.

Do not try to check the excessive sweating, a physiological action, by belladonna, but add cyllin to the daily bath, and dust the body with antiseptic powder. Change the clothing frequently.

\section{ICHTHYOSIS ( $\prec \theta \hat{v} s=a$ fish).}

A dry, rough, scaly and sometimes warty skin of uncertain causation, usually congenital, but rarely acquired.

\section{YARIETIES.}

(1) Ichthyosis Follicularis.

Horny skin, patches of baldness, hair replaced in certain areas by pin-head papules which are surmounted by horny spines.

It is non-inflammatory. 
(2) Ichthyosis Hystrix (

A rare condition. It consists of longitudinal patches on the limbs and transverse patches on the trunk, corresponding with the distribution of the cutaneous nerves and associated with a general xerodermia. Such ridges may rise half an inch above the surrounding skin.

\section{TREATMENT.}

Cure is exceptional. Improvement has followed thyroid extract, soft soap and warm baths with vigorous friction, lanoline or salicylic acid inunctions in the last variety. Some patches may require excision or scraping.

(3) Ichthyosis Simplex.

Extreme scaliness, may be horny like the hide of a crocodile and of a similar colour. The nails break easily. There is slight improvement in the summer. There is much itching, great sensitiveness to cold, and a tendency to "chap" deeply.

(4) Keratosis Pilaris (Xerodermia).

This is the commonest form. The skin feels to be hard like a nutmeg grater, hence the name. There are dry skin, imperfect development of hair, and changes in the sebaceous and sweat glands.

Give inunctions of myelocene; also castor oil, followed by mag. sulph. and plain water enema.

\section{IMPETIGO.}

A pustular eruption caused by scratching, followed by inoculation of streptococci and staphylococci. The proximate lymphatic glands enlarge and may suppurate. The condition is contagious.

Remove scabs by carbolized oil or carbolic acid lotion, $\mathrm{I}$ in roo.

Apply mercurial and other antiparasitic ointments.

Vaccines may be required.

Mag. sulph, and tonics are useful.

\section{INTERTRIGO SACCHAROMYCETICA.}

A rare inflammatory condition attacking the scrotocrural and axillary regions caused by a Saccharomyces.

Antiparasitic lotions and inunctions will clear it up.

\section{JUXTA-ARTICULAR NODES.}

Multiple subarticular movable nodules about the elbow and kneejoints usually, probably caused by the fungus Nocardia carougeaui.

The writer has seen many cases in the Choco, Colombian Republic, in some of which twenty-four nodules could be counted about the joints mentioned, many of them being the size of a walnut.

Excision is advisable when they interfere with articular movements. 


\section{KAPOSI'S DISEASE (Xerodermia pigmentosum).}

A rare formation of numerous tumours, running a malignant course, and ultimately causing death by exhaustion.

The condition commences as "frecliles," which develops into a warty condition, and later into ulcerating fungoid masses, destroying all approximate tissue. The tumours are epitheliomatous in structure, but there are no secondaries.

The disease commences early in life as a rule, and requires years for its development.

Avoid the sun's rays. Excise patches and tumours. Try X-rays and electrotherapy.

\section{KERATOMA PLANTARE SULCATUM.}

A thickening of the epidermis of the heels and soles with deep furrows, at the base of which a whitish fungus can be found.

Walking is often painful.

The only known efficacious remedies are preparations of salicylic ointment, plaster and collodion.

\section{LEUCODERMA.}

A patchy, non-pigmented, white or pinkish condition of the skin; chronic and incurable; found in coloured races. The patches are irregularly shaped and distributed; local or universal, but without any modification of the skin functions. The condition is chiefly of nervous origin, but occasionally fungi, burns, or injuries mark its onset. The hair over the patches is whitish.

Exposure to the sun causes a burning sensation over such areas, and patients often complain of weakness or giddiness, making them unfit for any tropical outdoor work.

Some have found arsenical preparations useful.

The patches may be darkened by silver nitrate, potassium permanganate, or tattooing.

\section{LICHEN CONYEX.}

A chronic, pruriginous, papular, non-inflammatory eruption, pinkish in natives and reddish in Europeans, leaving no excess of pigmentation on healing. The disease lasts three to nine months and may recur.

There is no scaling, and the convex papules never show " plugs."

There may be spontaneous healing. The general health is not affected.

Internal remedies have no effect.

Externally use a salicylic-alcoholic lotion, 2 per cent., followed by naplithol ointment, 5 per cent. 


\section{LICHEN PLANUS.}

The term "lichen" does not mean much more than "papular," thus lichen simplex is really a phase of eczema, and lichen tropicus the same as prickly heat, $\& \mathrm{c}$. The word should be restricted to eruptions that are papular throughout and of a persistent typical form.

Lichen planus consists of a violet or reddish eruption of small, irregularly shaped papules, flat on the top and sometimes umbilicated, carrying a small scale in the centre. It is probably of an angioneurotic origin. The papules may group themselves in lines, curves, or rings. Vesicles seldom, and pustules never, appear in adults. The mucous membrane may be affected, but the accompanying skin condition will assist the diagnosis. In acute cases there is much depression and irritability.

Arsenic is useful given in increasing doses over long periods, but improvement is not to be expected until after the sixth week of treatment. Intestinal antiseptics are useful. Morris uses the following:-

R Liq. hydrarg. perchlor. $\quad \ldots \quad$ I ounce

Potass. iodid. $\quad \ldots \quad \ldots \quad 40$ grains

Decoct. sarsae. co. ... ... $\quad$ S ounces

Take one ounce thrice daily.

Externally :-

$\begin{array}{ccccc}\text { R Corros. sublimate } & \ldots & \ldots & \text { r.00 } \\ \begin{array}{c}\text { Carbolic acid } \\ \text { Simple ointment }\end{array} & \ldots & \ldots & 20^{\circ} 00 & , \\ \text { Apply twice daily. } & \end{array}$

\section{LICHEN TROPICUS.}

See Prickly Heat.

\section{LUPUS ERYTHEMATOSUS.}

An inflammatory condition with cellular infiltration, terminating in atrophy of the affected skin. It is often symmetrical, and commences by primary spots having a red or pinkish elevated thickened edge and a depressed centre. The redness fades on pressure.

The face is most commonly attacked in the "butterfly area."

The disease lasts from ten to twenty years, when it clears up.

Patients are most frequently women from 25 to 45 years of age.

Any slight wound causing a disturbance in the local circulation, such as a mosquito bite, will mark the site and time for its onset.

To differentiate it from Lupus vulgaris :-

\section{Lupus erythematosus.}

Begins as small red spots.

No ulcriation.

Always superficial

Develops after puberty.

\section{Lupus vulgaris.}

Begins as soft apple-jelly nodules. Ulceration frequent.

Cartilage may be affected.

Develops usually before puberty. 


\section{TREATMENT.}

Avoid stimulants, irritants and sea air.

Examine for auto-inoculation, and deal with it when found.

If intestine is affected give salol, ichthyol, \&c.

Tincture of iodine externally is often good.

For chronic cases, carbon-dioxide snow, ionization with zinc or copper, high-frequency current and Finsen light.

\section{MONGOLIAN SPOTS.}

Mulberry coloured, smooth, non-elevated spots, occurring usually about the sacral region, the colour not disappearing on pressure.

It is of unknown origin, and is found chiefly amongst the Chinese, Koreans, Japanese, Malays, Italians and Europeans. The spots may be single or multiple, one to six or more. They appear at birth, and spontaneously disappear about the third or fourth year.

No treatment is necessary.

\section{MOSSY FOOT.}

A dense, warty, rascular, painful condition of the foot, occurring in masses, seen chiefly amongst the Amazonians. It is of uncertain origin and of chronic course. Cranston Low suggests that it is Tuberculosis cutis verrucosa. The masses may be half to three-quarters of an inch high.

\section{THE MUCOUS MEMBRANE.}

Pathological skin conditions affecting the mucous membrane secondarily are dealt with elsewhere. Disorders arising more or less primarily are dealt with here.

Black Tongue (Hairy tongue).

A dark brown or blackish hyperkeratosis, usually in front of the rircumvallate papillæ, commencing in the mid-line, found usually in adult males, persisting for weeks and perhaps years, and then spontaneously disappearing.

Treatment, apart from mouth washes, is unnecessary.

\section{Cheilitis Exfoliatixa.}

A persistent exfoliation, recurring, of the vermilion of the lips, accompanied by numbness or burning pain, probably caused by the habit of gnawing and picking the lips in neurotic individuals. Radium may clear up the condition.

\section{Cheilitis Glandularis.}

A chronic inflammation of the lower lip with enlargement of the mucous glands, dilatation of the follicular openings, the formation of fistulous tracts which discharge a viscid secretion, sometimes fixing the lips together during the night. 
There is often a previous catarh of the mouth and pharynx.

Silver nitrate caustics, potassium chlorate gargle, and potassium iodide internally are most successful.

\section{Fordyce's Disease.}

Small whitish bodies affecting the lips and mouth, accompanied by a feeling of stiffness. The condition tends to become permanent. Treatment is discouraging. Caustics and scraping may be tried.

\section{Glossitis Areata Exfoliativa.}

Small, greyish, well-defined, slightly elevated spots near the tip of the tongue, desquamation in the centre and spreading at the periphery, sometimes forming circles or festoons suggesting the "langue géographique.",

It usually occurs in children, and is frequently confined to families. Its origin is unknown.

Treatment is disappointing.

\section{Grooved Tongue.}

Single or multiple grooves on the dorsum of the tongue in which food particles accumulate requiring the constant use of a mild antiseptic mouthwash.

The condition is often congenital and confined to families.

Treatment is useless.

\section{Ichthyosis Glossæ.}

A condition of leucokeratosis of the tongue resembling tylosis (keratosis of the palms and soles).

\section{Kraurosis Yulyæ.}

Atrophy of the vulva, with stenosis of the orifice occurring:-

(I) After the menopause,

(2) As a sequel of oöphorectomy,

(3) In sterile young women (rare).

Treatment is surgical when necessary.

\section{Leucoplakia of the Tongue.}

It may be Syphilitic, Parasyphilitic, or Idiopathic.

Predisposing factors are: clay-pipes, tobacco-smoke, bad dentures, ragged tooth stumps.

The ulcerating fissures may become the seat of epithelioma.

Always exclude syphilis. Caustics make the condition worse.

Hot fluids and spices are to be avoided.

Radium can be tried. Excision may be necessary.

Leucoplakia may also affect the penis, vulva and vagina.

Use X-rays, radium and excision where necessary. 


\section{Perlèche.}

A chronic stomatitis usually affecting the commissures; commonly seen in children. The fissure divides the two patches into equal parts at the commissure. The child frequently licks its lips, hence the term " pour lécher," perlèche.

The condition is probably caused by a streptococcus and is infectious. It lasts two to four weeks and then spontaneously clears up.

Relapse is common.

Cauterize with copper sulphate and apply zinc oxide ointment.

\section{MYCETOMA (Madura Foot).}

Madura was a district in India where the disease was very prevalent.

It is an invasion of the hand, but more usually the foot, less frequently other parts of the body, by fungi belonging to the following genera: Aspergillus, Sterigmatocystis, Madurella, Indiella, Mono-

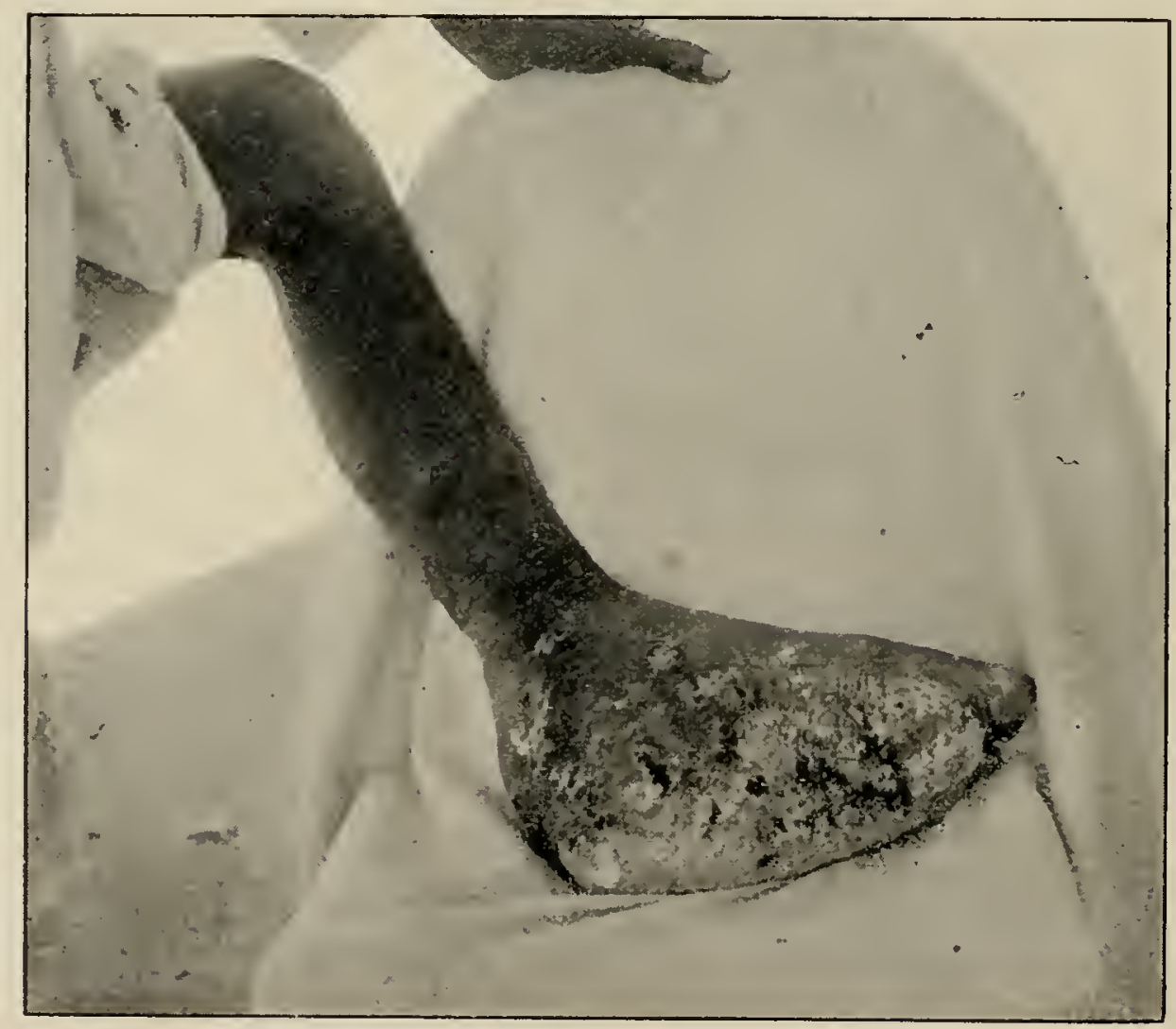

Madura foot.

sporium, Sporotrichum and Nocardia. These produce enlargement and deformity of the part, the condition being characterized by an oily degeneration and tissue fusion with cyst-like cavities and sinuses.

The disease is chronic and progressive, terminating in death by exhaustion if not removed surgically.

It is common in India, Asia, Africa, Europe and America. 


\section{AETIOLOGY AND PATHOLOGY (Castellani).}

The causal fungi belong to the genera numerated above, the saprophytic life of which is quite unknown, except that of Nocardia bovis, which lives in cereals. Barefooted natives are usually affected, the fungus entering in at some wound about the foot, the wound heal-

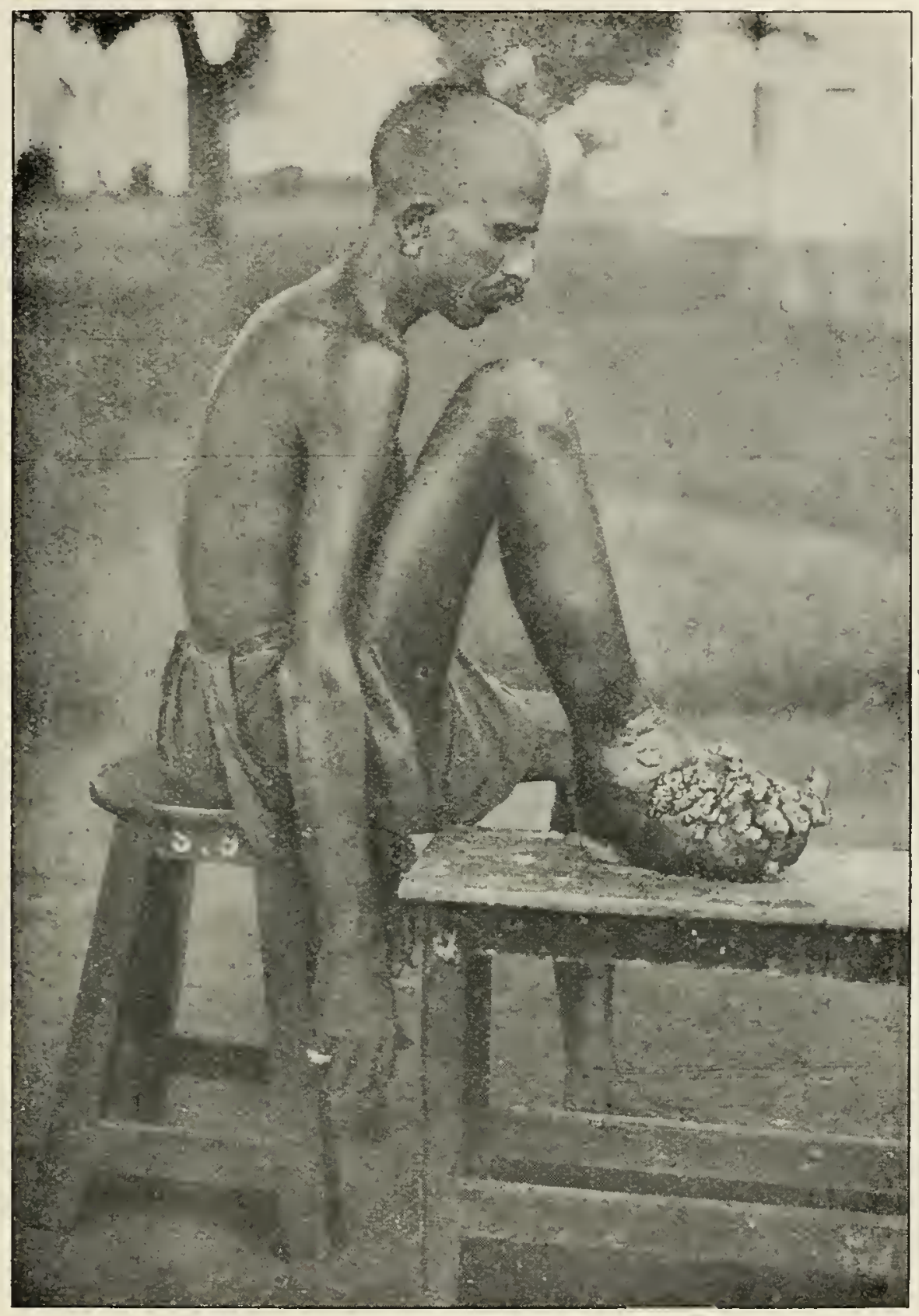

Mycetoma of the foot.

ing, but enclosing the fungus which grows slowly within, without there being any real attempt at repair by the body except for some lymphocytic infiltration and the formation of some granulation tissue. Fibrous tissue is formed about the fungus; endarteritis and periarteritis cut off the blood supply; the cellular exudate becomes oily, often foul, discharges at the surface through sinuses, the exudate carrying with it some of the parasites. As Nature thus tries to throw 
off the fungus, the latter forms special club-shaped persistent hyphre which asexually propagate the disease in the part. Leucocytes then attempt to get rid of them by engulfing the parasites and succeed in carrying them to other parts, where the leucocyte is killed by the enclosed parasite, which latter again reproduces the disease where it may happen to be.

When the fungus is surrounded by pus, a mass of débris contain-

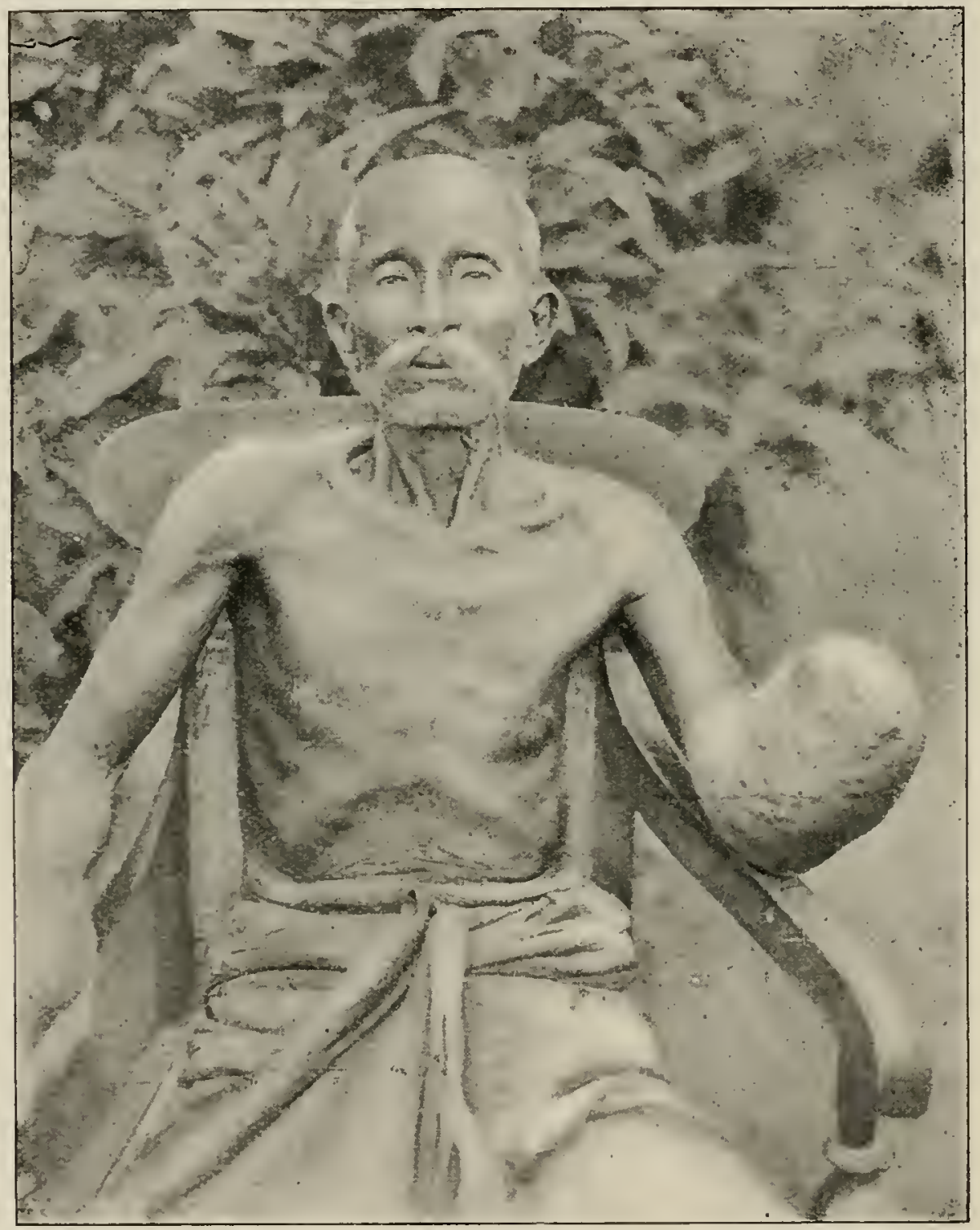

Mycetoma of the hand.

ing granules-yellow, black or pinlish-is formed and exuded. The tissue adjoining, muscle, tendon, nerve and bone, degenerate and break down into débris; the body is drained and exhausted: emaciation and cachexia set in, and some intercurrent disease ends the scene.

\section{SYMPTOMATOLOGY.}

A small wound, usually in the foot, heals, and a small swelling is noticed which is painless. After one month or so the swelling softens, ruptures, and discharges an oily blood-stained exudate containing 
minute coloured granules about the size of fish roe. The whole foot is much enlarged, especially in thickness, its shape is lost, anatomical points are obliterated, multiple sinuses reach the surface and discharge through their sinuses with distinctive odour. As the foot enlarges the leg atrophies from disuse, thus making the foot enlargement the more noticeable. Pain is usually absent; locomotion is difficult; the slin sensibility is normal; pitting is not obtained, but the tissue is elastic.

There is no spontaneous healing, and after eight to twelve years of exhaustion, diarrhoea often carries off the patient.

\section{YARIETIES.}

(A) The Melanoid Group.

(I) Carter's black mycetoma.

Parasite, Madurella mycetoma, Laveran, 1902.

The sclerotia are small, hard and blackish.

The parasite throws off a blackish débris.

(2) Bouffard's black mycetoma.

Parasite, Aspergillus bouffardi, Brumpt, 1905.

The grains are larger and more elastic than the above.

Curetting may cure this condition.

(3) Nicolle and Pinoy's black mycetoma.

Parasite, Madurella tozeuri, Nicolle, sgos.

(4) Link's black mycetoma.

Parasite, Penicillium glaucum, Link, I9r5.

(B) The Ochroid Group.

(1) Carter's white mycetoma.

Parasite, Nocardia madure, Vincent, I 894.

The sclerotia are whitish, mulberry-like and spherical.

(2) Actinomycotic white mycetoma.

Parasite, Nocardia bovis and israeli, I 872 and 1896.

The sclerotia are yellowish, sulphur colour and soft.

It destroys bones and forms metastases througlout the body.

(3) Nicolle's white mycetoma.

Parasite, Sterigmatocystis nidulans, Eidans, ${ }^{8} 88_{3}$.

The sclerotia are large, like peas, and smooth.

It attacks bone.

(4) Brumpt's white mycetoma.

Parasite, Indiella mansoni, Igo6.

The sclerotia are small and white, occurring as hard beanshaped granules.

(5) Reynier's white mycetoma.

Parasite, Indiella rejieri, I905.

The small whice sclerotia occur in soft coiled masses. 
(6) Bouffard's white mycetoma.

Parasite, Indiella somaliensis, 1905 .

The sclerotia occur as hard, small, spherical and polyhedral grains.

It destroys bone.

( $)$ Musgrave and Clegg's white mycetoma.

Parasite, Nocardia asteroides, 1890.

The sclerotia are first oval, then rary in shape.

It invades the medulla of bone.

(C) The Red Group.

Pelletier's red mycetoma.

Parasite, Nocardia pelletieri, Igo6.

It may attack other parts of the body.

It occurs in Africa.

TREATMENT.

Amputation is the most certain cure, well above the anlile or wrist, rarely less than this.

Scraping and cauterizing with potassium iodides internally may be successful in some cases.

\section{MYIASIS.}

The invasion of the skin or other parts by the larva of flies.

(1) Dermatobia cyaniventris (Estridx).

This is found in Tropical America, and is present in cattle, pigs, dogs, monkeys, birds, and rarely in man.

There is local pain, itching, swelling, odema with a central sinus, in which the larva may be seen from time to time.

The larva may attack any part.

(2) Cordylobia anthropophaga (Muscida).

It is common in Africa, where it is known as Tambu fly disease. In Europeans the thighs and buttocks are usually affected, the source of infection probably being the latrines.

In natives, the forearms, axillae and head are more commonly attacked. The inflamed area is about three-quarters of an inch in diameter.

Extract the larva and treat antiseptically. Some require a long time to heal.

(3) Chrysomyia macellaria (Screw-worm).

Common in America.

The insect lays a mass of 300 to 400 eggs on the surface of wounds, in the ears and nasal fossa of persons sleeping in the open air. Offensive discharges will attract the flies. 
The larva hatch in a few hours, and are about three-quarters of an inch long, with twelve segments carrying circles of spirally arranged spines giving a screw-like appearance. These burrow into and devour mucous membrane, muscle, cartilage, periosteum, bone, and may even reach the brain and cause death.

Neglected, the patients die; treated, they live.

Injections of chloroform, carbolic acid, turpentine, \&c., are necessary.

The frontal sinuses, antrum, and other bony cavities may require opening up.

(4) Auchmeromyia luteola.

A keen blood-sucker, the Congo Floor Maggot, common in Africa.

The larva has eleven segments, at the posterior end of each are three short limbs provided with spines directed backwards; laterally each segment bears two or more protuberances with a small spine. The anterior segment bears the mouth and two black hooks, each surrounded by minute teeth. After feeding, the semitransparent body appears red to about the fifth segment as a result of the blood taken. The adult fly corresponds in colour with the smoke-stained straw of native houses; it rests under the roof; is silent; lays its eggs in the cracks of the mud floors, under native mats, and in soft moist earth.

They feed at night. Those sleeping on beds and raised platforms escape as a rule.

\section{NODULAR LIPOMATOSIS.}

Innocent fatty, subcutaneous nodules, felt under the skin, which may at time be mistaken for enlarged lymphatic glands.

No treatment is necessary.

\section{PEDICULOSIS.}

A condition, exceedingly common in the Tropics, resulting from the bites of Pediculus humanis, P. corporis and Phthirius pubis.

The latter is less common among races that constantly shave the pubic region.

\section{PATHOLOGY.}

The mouth parts of the parasite consist of a double tube, one inside the other. The outer chitinous tube or proboscis is a fused labrum and labium armed with minute curved hooklets.

The inner membranous or suctorial tube consists of maxillæ and mandibles. 
When biting the proboscis is inserted into a sweat duct, the small hooklets everted and the skin thus gripped; the suctorial tube is then protruded until it reaches blood, the salivary glands at the same time inject their renom causing the pruritus; the pharyngeal pump draws blood, the parasite falls off leaving a minute red spot and marked itching. This area is vigorously scratched and pyogenic infection may follow. This condition, when constant with some thicliening of the skin and pigmentation, is known as "vagabond's disease."

The parasites secrete themselves in the folds of the clothing, especially about the seams, and later attack the hairy parts.

The eggs are attached to the hairs.

\section{TREATMENT.}

Shave off the hair and apply calamine lotion, or

Soak the hair in petroleum, wash with soap and water every twentyfour hours, or

Apply sulphur and resorcin ointment, wash with soap and water, soak in acetic acid, 25 per cent., comb with a tooth-comb to remove the eggs, and wash again.

All clothing must pass through steam sterilizers.

Antiseptic baths and calamine lotions are always useful.

\section{PEMPHIGUS CONTÁGIOSUS (Pyosis mansoni).}

A non-febrile, highly contagious disease of warm countries, characterized by large vesicles or bulla without inflammation or ulceration. The fluid is clear, then turbid; the blister ruptures; exfoliation of about one inch of skin takes place, and a pinkish scar forms. It may attack any part of the body, but in adults the axille and groin regions are usually selected.

The causative organism has not been proven; it is probably a pyogenic organism.

Keep the parts clean and dry. Use dusting powder of boric acid, zinc oxide and starch, equal parts. Mercuric lotions should be used.

Iron, arsenic and strychnine internally.

\section{PYOSIS CORLETTI (1915).}

An epidemic eruption of bulla amongst English soldiers in Khartoum, probably identical with Corlett's "Impetigo contagiosa bullosa" in American soldiers.

Bullae arise from apparently sound skin without fever or pruritus. and spread over the whole body and limbs.

A diplococcus (Amococcus mollis) is always found.

The soles are not attacked, there are no crusty lesions, and streptococci are absent. It does not select moist folds of skin, and is not surrounded by an inflammatory zone. 
Autogenous raccines and local antiseptics cure the condition.

Isolation is necessary to stop the epidemic.

\section{PENICILLIOSIS.}

The hairs of the moustache, beard, or axillac are attacked by the fungi, Penicillium barba, and Aspergillus barba = Aspergillosis.

The condition tends to become chronic. The diagnosis is easy.

The treatment is as for pediculosis.

\section{PHAGED ENA (Tropical Sloughing Phagedæna).}

It is a rapidly spreading gangrenous condition of the skin and subcutaneous tissues, forming a large sloughing sore which ultimately ceases to spread, and cicatrizes or remains as a chronic ulcer. Men, physically depressed from other causes, are generally attacked, such as slaves, prisoners, soldiers, pioneers, \&c.

Any small injury or ulcer may develop into one.

There is a large bleb which ruptures, exposing a foul, moist slough, which extends in all directions. The deeper structures are generally spared, but deformity always results if the victim lives.

Sapramia and exhaustion or the rupture of a large blood-vessel will carry off the patient. Pyogenic organisms have been found.

The bacillus of Vincent and an associated spirillum are commonly found.

Correct the general health, give nourishing fresh food and vegetables, opium and general tonics.

Cauterize the part under chloroform, irrigate continuously until healing takes place.

Consider the patient as infective and isolate him.

\section{PIEDRA.}

A mycotic disease of the hair found in South America, especially Colombia, consisting of one or more hard small nodosities on the hair caused by Trichosporon giganteum. The affected hairs are bent, twisted and matted together. The nodosities are groups of spores around the hair, learing the latter intact.

They can be seen with a microscope after washing with ether and soaking in liq. potass.

Bathe the hair, sponge with salicylic acid in absolute alcohol, 5 per cent.

This is good for the long hair of women.

Otherwise treat as for Pediculosis.

\section{PINTÁ (Carate).}

A group of allied epiphytic diseases of the skin common in Tropical America, characterized by pigmented areas of skin, varying in tint, 
and caused by more than twenty species of the fungi: Aspergillus, Penicillium, Monilia and Montoyella.

It is contagious, but Europeans are rarely attacked.

The Aztecs of Peru prayed about it centuries before the Spanish conquest.

\section{SYMPTOMS.}

There is desquamation and itching of the patches, the first of which may be red, white, blue or black, the varying tints being due perhaps to the variety of the fungus.

When the scalp is affected the hair turns grey, becomes thin and falls out. One person may show different tints, but the patches themselves do not change in colour.

The superficial type is usually blue or black and spreads rapidly. The deep type is usually red or white and spreads slowly, and is more difficult to cure.

\section{TREATMENT.}

Destroy all old clothes. Absolute cleanliness.

Apply chrysophanic acid, sulphur, resorcin, liniment of iodine or other epiphyticides.

\section{PITYRIASIS.}

\section{P. versicolor.}

See Tinea versicolor.

\section{P. rubra.}

See Dermatitis exfoliativa.

\section{PRICKLY HEAT.}

A papular or papulo-vesicular eruption with marked pruritus connected with profuse sweating so common in all hot climates.

Anything resulting in perspiration in hot climates may cause it.

The theories of its causation are as follows:-

(I) Sodden cells of sweat glands swell and obstruct the orifice of the sweat duct (Pollitzer).

(2) Acute distension of sebaceous glands by their own secretion (Pearse).

(3) An infective disease caused by a very minute but active amœba (Durham).

Numbers (I) and (2) may both be present, and infection is not at all improbable through scratching.

The disorder is not serious in robust people, but to weaklings it may be serious from loss of sleep, \&c.

\section{TREATMENT.}

Avoid all causes of excessive perspiration.

Avoid copious drinks and all alcoholics. 
Avoid warm and irritating clothing and close rooms; silk clothing may be necessary .

Apply spirit lotion to the skin; antiseptic baths; antiseptic dusting powders, e.g., boric acid, zinc oxide and starch, equal parts.

Calamine lotion is soothing.

Keep the bowels active with salines.

For spirit lotion use :-

$$
\begin{aligned}
& \text { R Acid. salicyl... } \quad \ldots \quad \text { I drachm } \\
& \text { Spirit. rect. ... } \quad \ldots \quad 8 \text { ounces }
\end{aligned}
$$

Followed by clusting as above.

\section{PSORIASIS.}

A chronic recurring, dry, patchy eruption of unknown origin, occurring most frequently about the elbows and knees, characterized by silvery scales.

The general health is but slightly affected.

It is usually seen in young adults.

It is frequent among Europeans but rare among tropical races.

Irritation of the skin should be avoided. Arsenic should be given internally. Fowler's solution is most useful, 3 minims, t.d.s., increased gradually when tolerated, to minims Io, t.d.s.

Locally: Remove scales with salicylate acid spirit 6 per cent. and treat when there is marked hyperamia present with ung. hydrarg. ammon. or chrysarobin, 10 to 20 grains to one ounce.

Drewer's ointment is useful :-

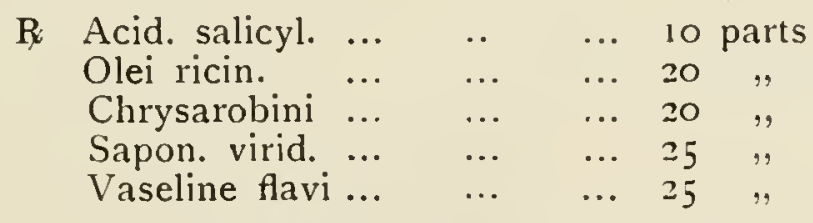

\section{PYOSIS PALMARIS.}

There are numerous discrete conical white pustules on the palms, containing the ordinary pyogenic cocci, but not surrounded by any marked inflammation. The cause is unknown, and treatment is not encouraging according to Castellani.

\section{SCABIES.}

Ten days or more after the female acarus (Scabiei hominis) has entered the skin a small vesicle appears usually between the fingers or toes, occurring amongst dirty people and those working amongst such.

There is much itching, especially at night; secondary infection is common .

Sulphur ointment, vigorously rubbed in every few hours for several days, followed by an antiseptic bath, clears up the condition.

The clothes should be boiled and sterilized. 
Balsam of Peru is also fatal to the acarus. Paint the part affected and leave it on for one night.

\section{SEBORRHEA.}

This condition is common amongst Europeans in the Tropics.

It occurs especially about the chest.

Sulphur ointment about 5 per cent. will clear it up.

\section{SKIN PIGMENTS.}

Skin pigment is a mixture of black, yellow and red pigments.

A "white" skin may arerage a mixture of pigments as follows: black, 8 ; yellow, 9; red, 50; white or absence of pigment, 33 per cent.

A "black" skin may arerage a mixture as follows: black, 68; yellow, 2; red, 26; white or absence of pigment, 4 per cent.

These tints can be obtained by blending them on a rotatory disc.

\section{SPOROTRICHOSIS.}

There are subcutaneous nodules which soften in two to three months, forming abscesses discharging a grevish rellow pus.

The disease is caused by eight species or more of the fungus Sporotrichum. This fungus is readily cultured on Sabouraud's glucose, this being the best means of differentiating the disease from Tubercutosis, Syphilis, Glanders and Blastomycosis.

The condition is rery chronic and requires vigorous treatment.

Puncture the nodules in the early stages and inject I per cent. of iodine solution. Give iodides internally in all cases in large doses.

The disease rapidly disappears under iodine treatment.

Apply antiseptic dressings to ulcers. Operative surgical measures are not necessary.

\section{SYPHILIS.}

This is very prevalent in all its phases. It should not be confused with framboria tropica. Treat as in liurope.

\section{TATTOOING (tattoo = marking).}

The formation of indelible skin marlis resulting from the insertion of pigments into slight or deep rounds. The term "tattoo" is also applied to the slin furrows or "keloid" so commonly seen in African natives. Tattooing is usually intended to be a permanent form of decoration, an advancement on the "painting" of uncivilized peoples. Extensive tattooing is very painful and requires years to complete claborate clesigns as little can be done at a time.

Sometimes several weeks are required for wounds to heal; blood poisoning and even death has resulted from the process.

The designs seen amongst Europeans and Asiatics probably come from the Japanese, amongst whom it was introduced over 300 years ago. Charcoal and ranilla are the most common pigments. 


\section{TREATMENT.}

Small marlis can be excised. Blistering and Finsen light has been used. Carbon dioxide snow leaves a scar. When gunpowder is used paint with di-iodide of ammonium, followed by dilute $\mathrm{HCl}$.

Persistent application of dilute acids and dilute alkalies may be effectual, but it is a tedious and painful process, eventually leaving a scar.

Only a prolonged dermatitis will remove extensive tattooing.

\section{TINEA (Ringworm).}

A contagious inflammatory infection of the skin affecting the hair, nails and, rarely, mucous membrane, caused by one of the fungi to be enumerated later.

Ringworm, which is derived from animals, is usually more inflammatory than that of human origin.

The appearance of the lesion varies according to the part affected, as -

(1) The hairy parts, e.g., the scalp = tinea tonsurans, so-called because it resembles in oulline the priestly tonsure; tinea barba, sycosis and palpebralis.

(2) The non-hairy parts, e.g., the body = tinea circinata, the nails = onychomycosis, the mucous membranes of the mouth, vulva, inguinal, perineal and gluteal regions $=T$. marginata (eczema marginatum).

There are two families of ringworm fungi :-

(I) Trichophytons (large spores) ( $\theta_{\rho} \xi \xi=$ hair, $\phi v \tau o v=$ fungus).

(2) Microsporons (small spores).

Of these there are many species of clinical interest which will be dealt with seriatim later.

\section{DIFFERENCES BETWEEN}

\section{Trichophytons}

Large-spored tinea

Square or oblong

Arranged in regular chains

Regularly jointed, short, mycelium

Fungus first attacks root and grows upwards, breaking the hair off short and

Small spored tinea

Round or ovoid

No definite arrangement

Mycelium irregularly jointed, curved and branching

Fungus lies about the hair as a visible greyish sheath, destroys the shaft and eats down to the root

The lesion commences in the epidermis in both cases.

The Endothrix tinea develops exclusively within the hair structure, Endo-ectothrix tinea develops within the hair. 


\section{EPITOME OF ETIOLOGY (Morris).}

Tinea tonsurans is mostly due to $M$. audouini and to the endothrix trichophytons, but the scalp, and especially the juvenile scalp, may be attacked by a great many of the fungi of both families.

The beard is attacked by trichophytons only.

The hairless skin is seldom affected by microsporons.

The nails are rarely involved; in such cases the parasite is the $\mathrm{T}$. acuminatum or $\mathrm{T}$. violaceum.

Adults are scarcely ever attacked except by endo-ectothrix trichophytons, which group is also responsible for much of the ringworm of the hairless parts.

\section{YARIETIES.}

(1) T. alba.

Parasite, T. macfadyeni.

Attacks the arms, legs and any part of the body.

Lesions are white, oval or irregular patches, margins slightly elevated and sometimes dotted with papules.

It is a form of dhobie itch.

Treatment.-Chrysarobin ointment, $2^{\prime} 5$ per cent., and tincture of iodine.

(2) T. albigena.

Parasite, T. albicans.

Attacks the palms and soles especially, also the nails, forearms and legs.

Lesions, bullæ which rupture, followed by a profuse keratosis until the palms and soles thicken considerably.

White patches may develop on the leg and arm.

Treatment as above.

(3) T. barbæ (Sycosis).

Parasite, endo-ectothrix trichophytons from animals or children, shaving brushes, \&c., less often.

$T$. acuminatium, violaceum and nodoformans.

Attacks the beard area, mostly confined to young male adults. Lesions, itching, chronic, scaly eruption, sometimes gyrated or pustular. In simple cases the hair is not damaged; in severe cases the hair is permanently destroyed.

Treatment.-Perseverance is essential for a cure.

X-rays where possible if there is no suppuration.

Epilation with forceps.

Chrysarobin and sulphur ointment.

All barber's instruments to be sterilized thoroughly each time they are used. 


\section{(4) T. capitis (tonsurans).}

Parasite, Trichophytons violaceum, T. sudanense, T. polygenum, T. exsiccatum, rarely also Microsporons.

Incubation under two weeks.

Attacks the scalp.

Lesions, patches increase in size surrounded by an erythematous zone, sometimes vesicles appear, the patch is studded by broken hair stumps and débris at the roots, making the epidermis to resemble "goose-skin."

A serious suppurative variety is known as "kerion," when the pus in the follicles loosens the hairs, but sloughing never occurs. The patches usually remain bald.

Treatment.-X-rays, epilation by forceps, turpentine oil, tincture of iodine and weak chrysarobin ointment, 2 per cent.

(5) T. circinata (Ringworm of the Body).

Parasite, usually Trichophytons, rarely Microsporons.

Attacks the face, neck, hands and wrists most frequently.

Lesions, a small red spot enlarged in a ring form and may reach a considerable size, or several small coalesce and form festooned patterns. Sometimes the diagnosis is not easy but the presence of the fungus, when found, is conclusive.

Treatment, remove superficial epidermis by iodine or liquor epispasticus. This may destroy all the fungi. It is best to follow it up with ointment as :-

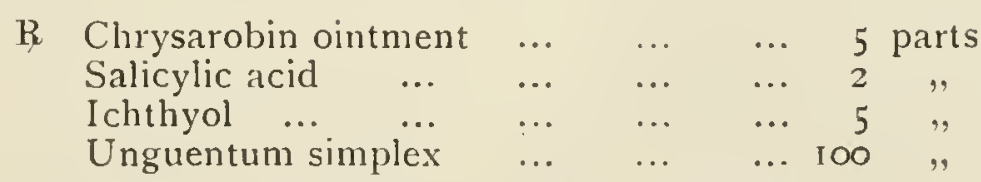

(6) T. cruris (Eczema marginatum, Dhobie Itch).

Parasite, Epidermophyton inguinale, peculiar to man, widely distributed in the tropical and sub-tropical countries. The term "dhobie itch" is loosely used in the East for epiphytic skin diseases which are supposed to be contracted from clothes returned by the dhobie or washerman.

Attacks pubic, anal, perineal, axillary and other regions where skin folds are in contact, e.g., interdigital folds. It never attacks hair but remains in the stratum corneum.

Lesions have a broad, well-defined margin, scaly and papular. They may assume an eczematoid character. Pruritus is con. siderable and pyogenic infection common.

Treatment :-

$\begin{array}{lllll}\text { R. Chrysarobin } & \ldots & \ldots & \ldots & 20 \text { grains } \\ \text { Ichthyol ... } & \ldots & \ldots & \ldots & 20 \\ \text { Ungent. zinc oxide } & \ldots & \ldots & 4 \text { drachms } \\ \text { Acid salicylic. } & \ldots & \ldots & \ldots & \text { \& grains } \\ \text { Vaseline ... } & \ldots & \ldots & \ldots & \text { I ounce }\end{array}$

Apply morning and evening for fourteen days. 
(7) T. decalvans.

An anomalous form of microsporusis of the scalp or bald ringworm. The hair falls out, leaving a bare patch in a place previously unaffected or on an ordinary ringworm area. The scalp is affected and the lesion resembles alopecia areata.

(8) T. flaya.

Parasite, Malassezia tropica (Castellani, 1905).

It is very common in all tropical countries.

The mycelial threads are thick with numerous swellings and constrictions. The spores are rounded or oval and have a double contour, and often run into clusters. In chronic cases the fungus is not so readily found. It is common amongst the Russian poor.

Attacks face, neck, chest and abdomen.

Lesions begin as tiny spots, slowly enlarging, the tint varying from deep orange yellow to deep canary yellow. There is no itching or desquamation. It tends to be very chronic.

These "beauty" spots are highly appreciated by some natives. Treatment.-Patience and perseverance are necessary for months.

A cold climate hastens the process of cure.

Apply turpentine daily. For exposed parts use :-

$$
\begin{aligned}
& \text { R Resorcin } \ldots \quad \ldots \quad \ldots \text { I drachm } \\
& \text { Salicylic acid } \quad \ldots \quad \ldots \text { Io grains }
\end{aligned}
$$

For covered parts use :-

Chrysarobin 20 per cent., or tincture of iodine.

(9) T. imbricata.

A form of body ringworm formerly peculiar to the East, but now rapidly spreading in damp equable climates of $70^{\circ} \mathrm{F}$. to $90^{\circ} \mathrm{F}$.

It is produced by a trichopliyton, and characterized by a concentric arrangement of closely set rings of scaling epidermis (Manson). It is not known in Africa.

Parasite, Endodermophyton concentricum and E. indicum (Castellani).

Incubation, nine dars.

Attacks the whole body, but aroids hairy parts, especially the scalp. The nails may be affected.

Lesions commence as spots and develop into concentric rings of tissue-páper-like scales about $\frac{1}{s}$ inch apart and having one free edge. Itching is generally intense, but the general health is unaffected. The condition tends to become chronic without spontaneous recorery. Relapses are common. 
Treatment.- Strong iodine liniment or :-

$$
\begin{array}{lllll}
\text { R. Resorcin ... } & \ldots & \ldots & 2 \text { drachms } \\
\text { Tinct. benz. co. } & \ldots & \ldots & 1 \text { ounce }
\end{array}
$$

Or, Chrysarobin ointment 5 per cent., but it is necessary to examine the urine and watch the patient.

Continue for weeks. Improvement is frequently followed by relapse.

(10) T. intersecta.

Parasite, Endodermophyton castellani, found on the inner surface of the scales; the mycelia are abundant, but spores are rare. The former are long, straight, articulated threads without fructifications.

Attacks the arms, legs, chest and back.

Lesions commence as small, round, elevated, itcly patches, with minute black papules about the border. Several may coalesce. They are at first tense, then shrivelled, cracked with white lines on the dark surface. The cracks deepen and the scales are formed.

Treatment.-Apply sulphur and chrysarobin ointments.

(11) T. nigra.

Parasite, Cladosporium mansoni, abundant in the lesions.

The mycelia are short, bent, often banana-shaped with large spores.

T. flava may co-exist with it.

Attacks any part of the body except the face, usually on the neck and chest.

Lesion is black, lustreless, with but little pruritus.

Treatment.-Easily curable except on the palms.

Small patches yield to to per cent. formalin.

Large patches to salicylic-alcohol lotion, 2 per cent.

Later apply resorcin ointment.

(12) T. nigro-circinata.

Parasite, Trichophyton ceylonese. It has large spores, not numerous, having a double contour.

Attacks the neck and scrotum.

Lesion, black rings with thick elevated margins.

Treatment.-Spontaneous healing is common, leaving dark patches.

Apply tincture of iodine.

(13) T. nodosa (Morris).

A nodular concretion containing a fungus, and its spores affecting the hair of whiskers, beard and moustache. The affected hairs split and break. It is not to be confused with piedra due to $\%$. giganteum.

Cut the hair short and use antiparasitic remedies. 
(14) T. palpebralis.

A rare form of ringworm that attacks the eyebrows usually by spreading from the face.

(15) T. sabouraudi tropicalis.

Parasite, Trichophyton blanchardi; cultures of it cannot be grown.

Attacks the legs.

Lesions commence by erythematous patches covered with small pityriasic squamæ, later there is a circinatiform appearance with a dark brown base. Pruritus is marked. In chronic cases there is thickening of the skin.

Treatment.-A cold climate causes it to disappear.

Apply chrysarobin ointment, 2 per cent. to 4 per cent.

(16) T. ungium.

Parasite, Epidermophytons, Trychophyton and Endodermophytons.

Attacks the nails of the fingers and toes.

Lesions cause discoloration of the nails, brittleness, roughness and splitting of the free border.

Treatment is difficult.

Soften the nails by rubbing in liquor potassium.

Apply wet dressings of hyposulphite of soda or

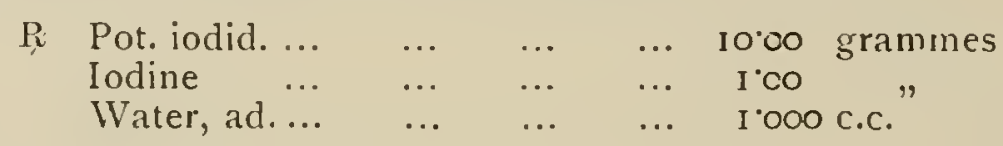

If the accompanying condition is due to $T$. imbricata, use resorcin in tincture of benzoin, $I$ in 16 .

(17) T. versicolor (Pityriasis versicolor).

Parasite, Microsporon furfur; cultures are grown with difficulty. The double contour spores are found in clusters.

Attacks the trunk usually, less frequently the arms and face.

Lesions are rounded, slightly raised, scaly patches with a well-defined border, often fused, of a brownish tint, sometimes black. Pruritus is marked.

Treatment.--Spontaneous cure ultimately.

Wash with soft soap and warm water.

Apply tincture of iodine.

Naphthol ointment, $\mathrm{I}$ in 39, or sulphur ointment, 1 in 20 , are efficacious.

\section{TRICHOMYCOSIS.}

A nodular affection of the hair, generally of the axillary regions, caused by a Nocardia which closely resembles, but is not identical with Leptothrix. The affected hairs have soft thickenings of yellow, black or red colour, easily remored from the hair. 
Formalin in spirit will clear it up, 1 in 30 .

Apply sulphur ointment at night, 4 per cent.

The principal nodular concretions of the hair are:-

(1) Nodular trichomycosis.

(2) Piedra (trichosporosis tropica).

(3) Tinea nodosa.

\section{TRICHONOCARDIASIS (Nodules on the Hairs).}

See above remarks.

\section{TUMOURS.}

\section{(1) Benign epithelial tumours.}

Epithelial moles are frequent.

Hyperkeratosis is common in all barefooted tribes.

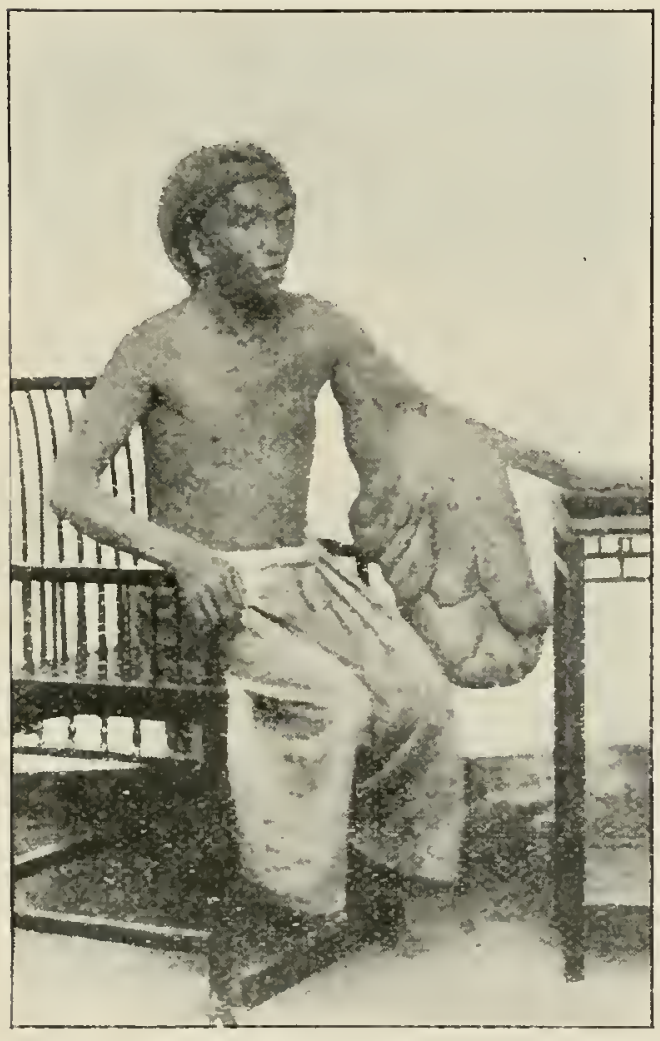

Molluscum fiturosum

Before operation.

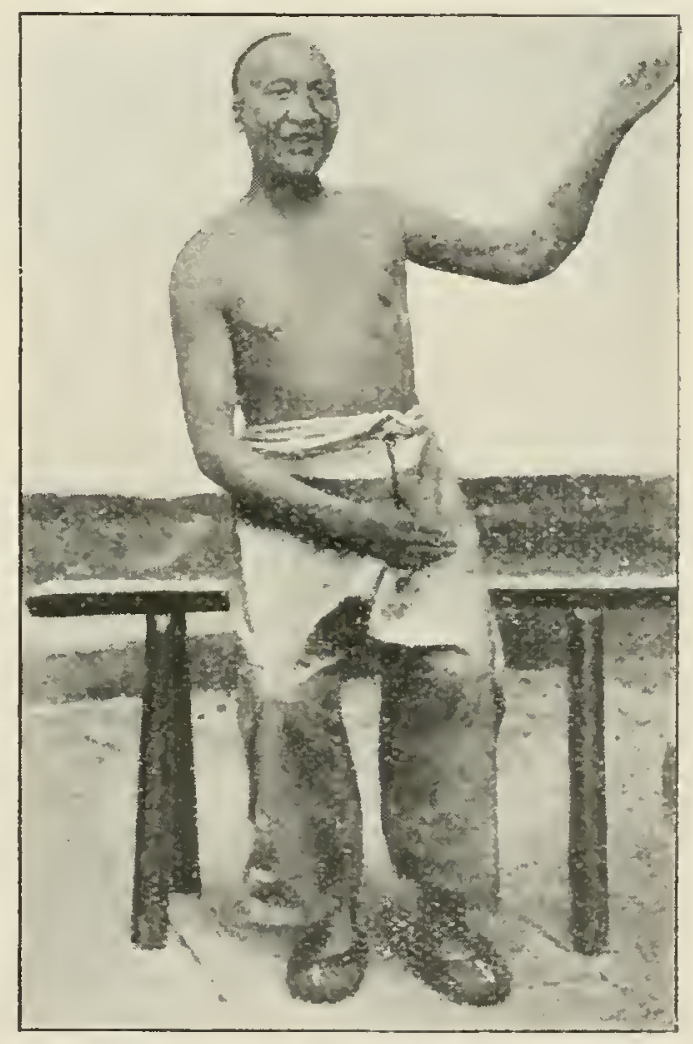

(By C. C. Elliott, Paoning.)

After operation.

Molluscum contagiosum is not infrequentiy met with.

Verruce are extremely common.

Warts are very common.

(2) Malignant epithelial tumours.

Melanotic carcinoma is rare, but has been seen in half-castes.

Paget's disease of the nipple is rare.

Rodent ulcer is rarely seen. 


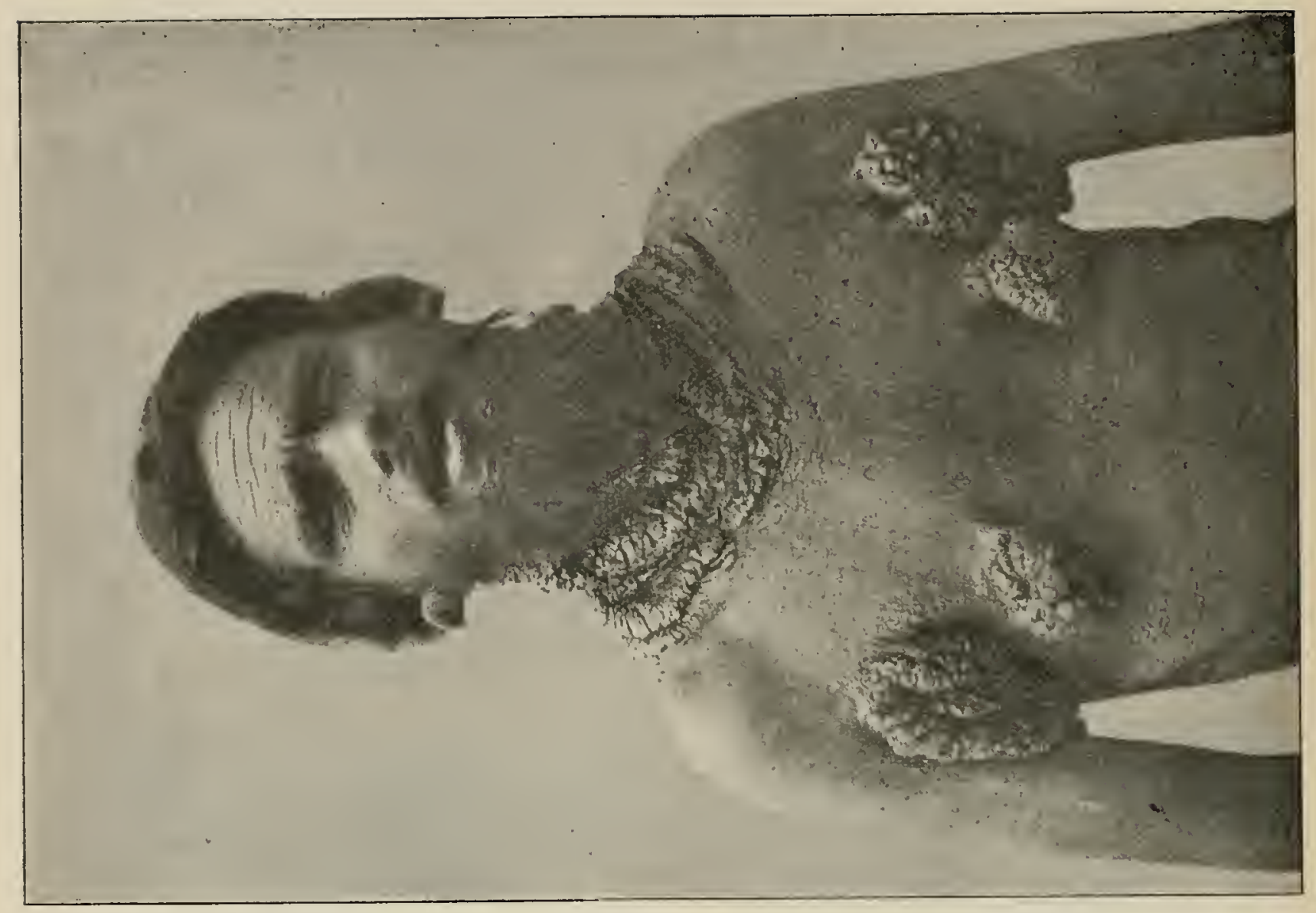

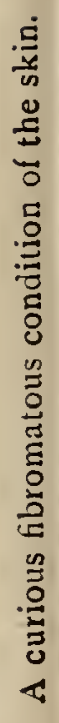

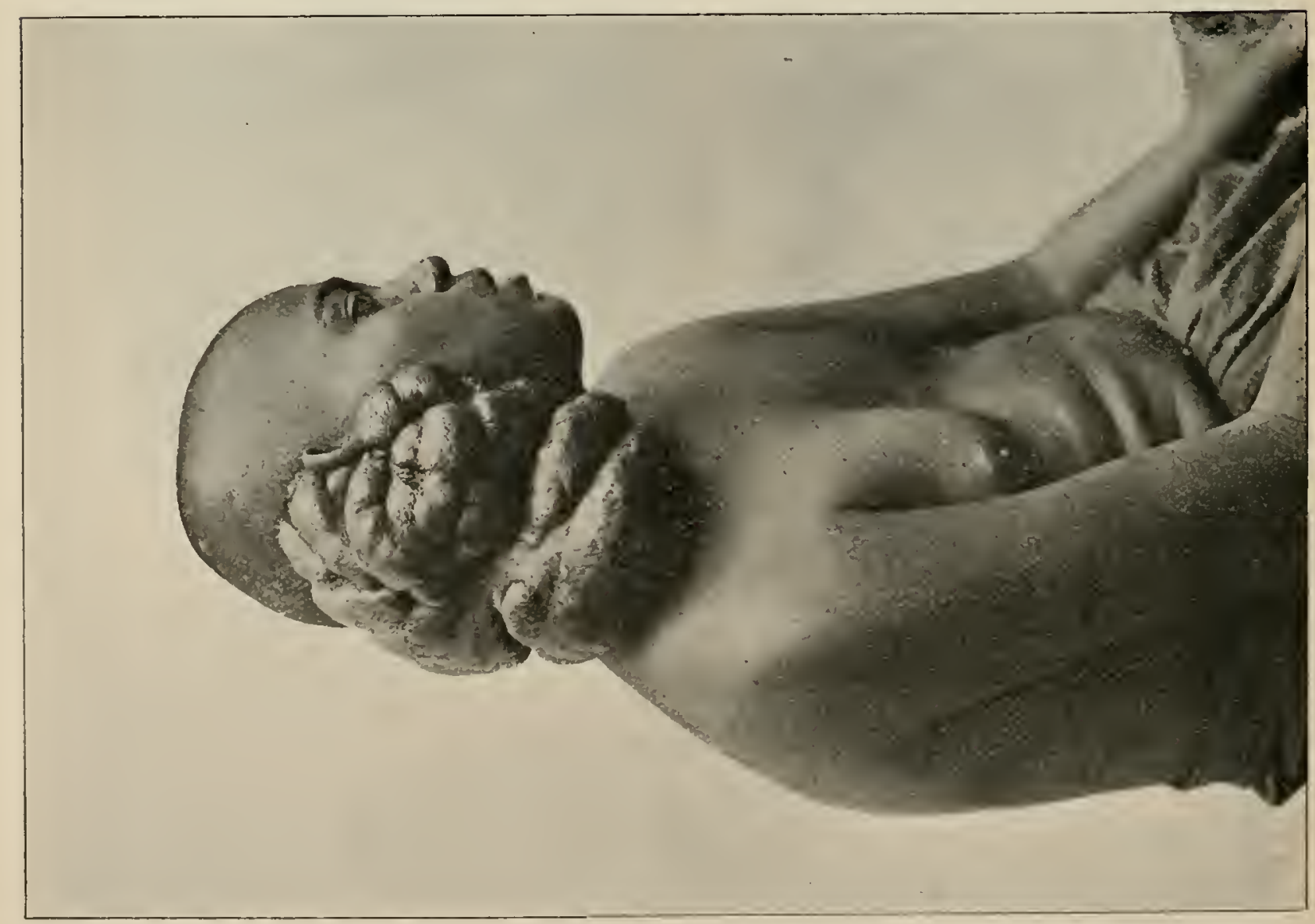

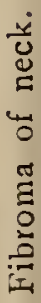




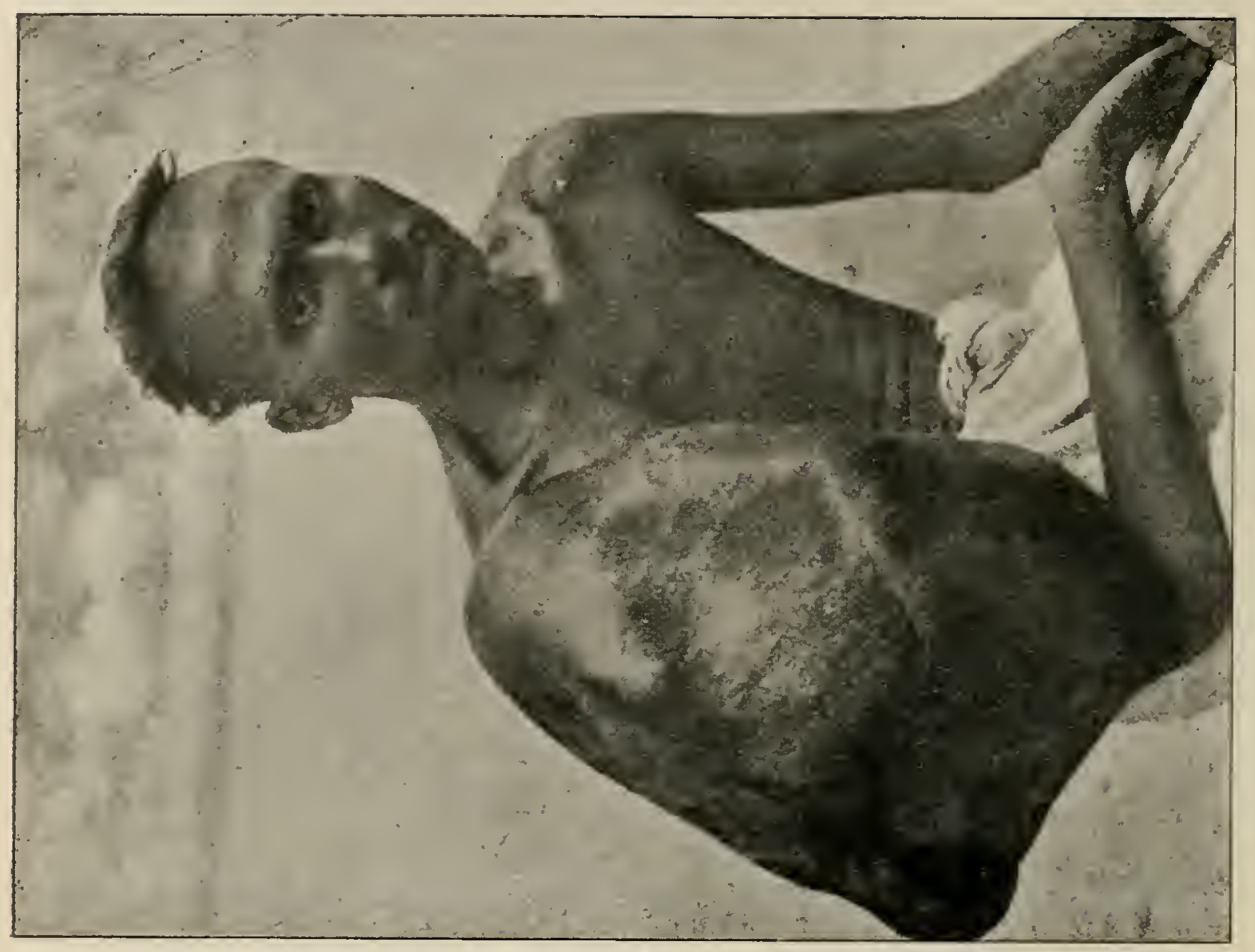

I

- 屯े

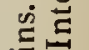

ती

$\dot{\Delta} \dot{0}=$

\%

\%

造芯

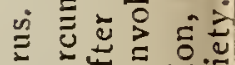

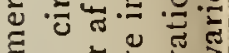

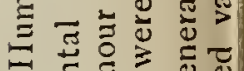

- .0

气

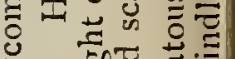

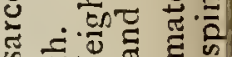

就击

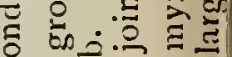

U

๘ँ

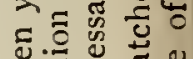

屯.

일

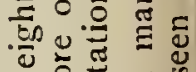

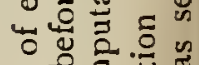

글 है

它.

记䒕 प्ठठ

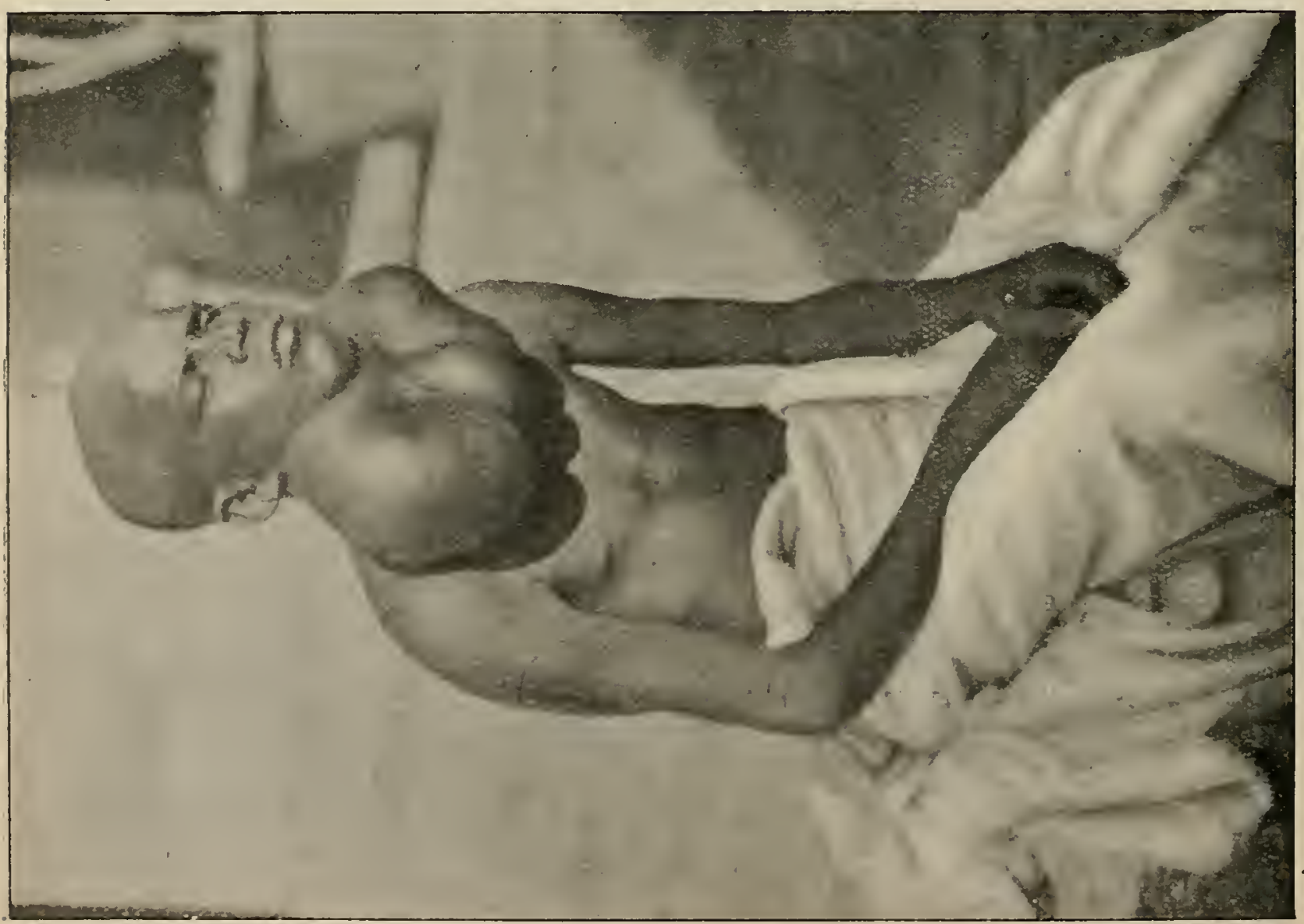

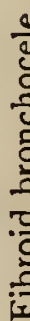



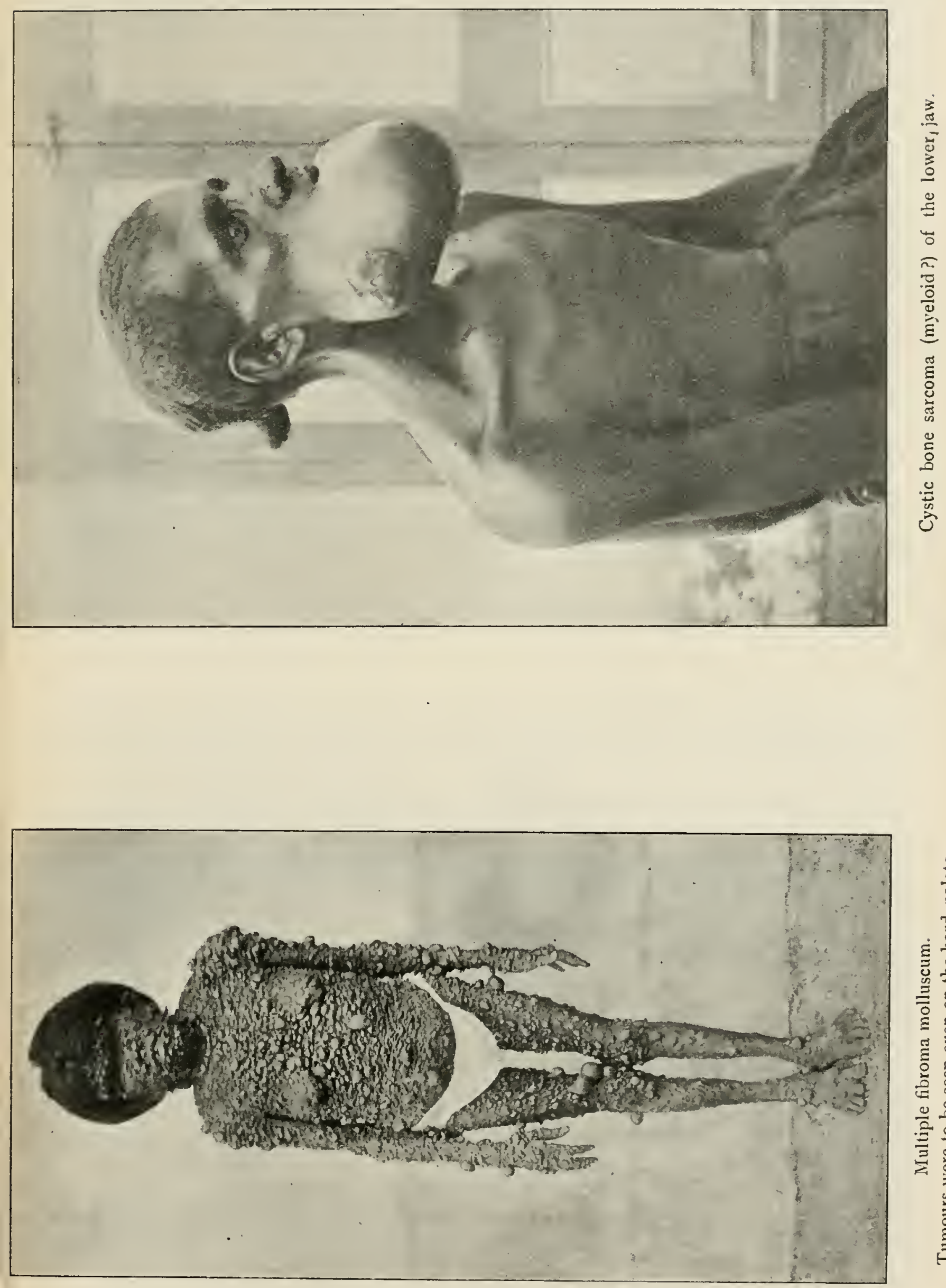

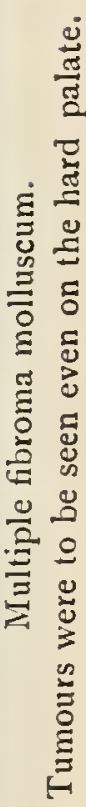




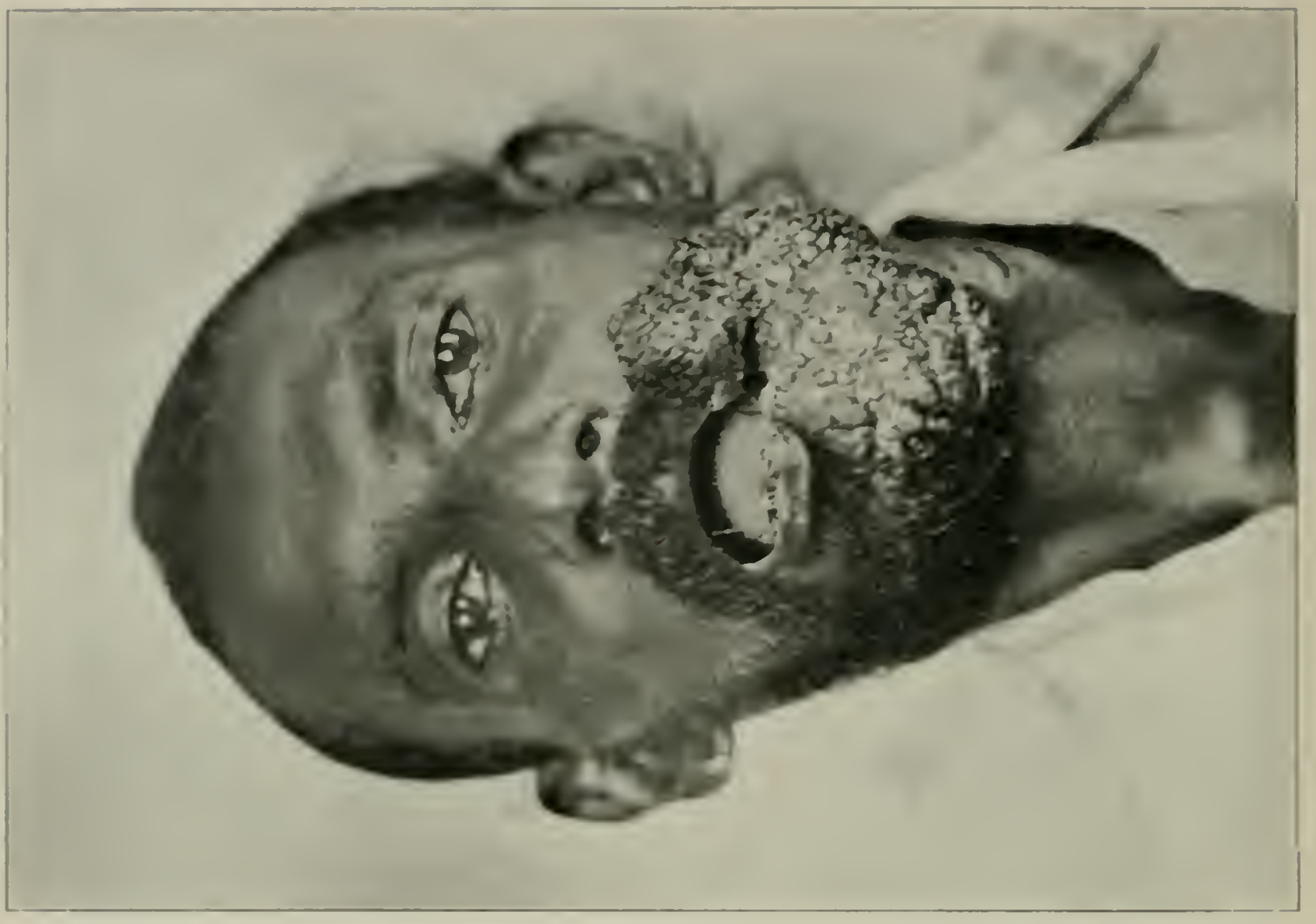

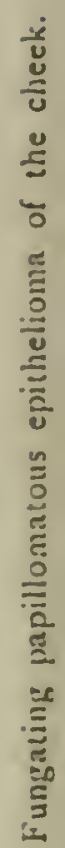

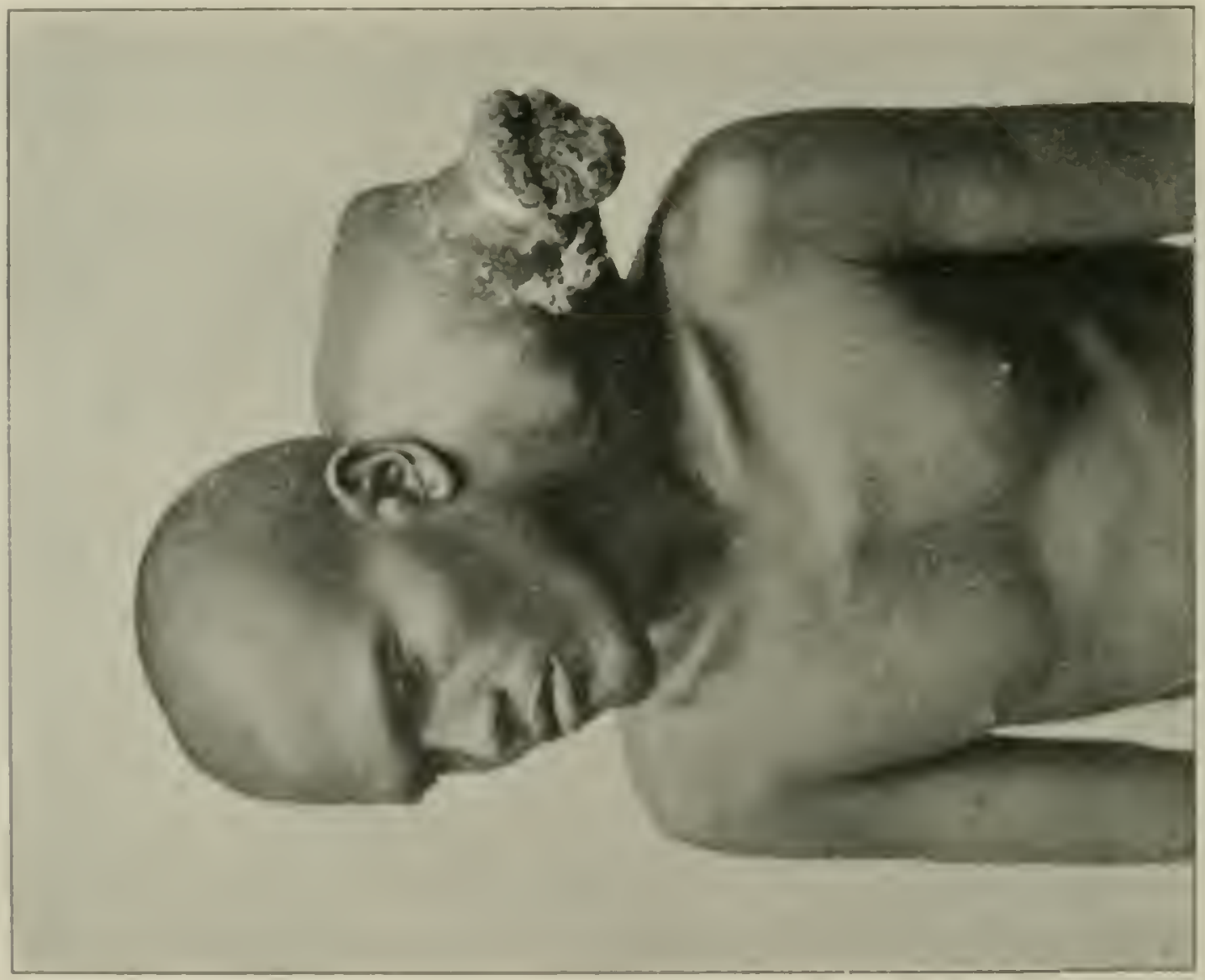

蒾 
(3) Benign connective tissue tumours.

Angromala ale fátrly common.

Fibroma molluscum or neurofibrosis of Recklinghausen, common.

Fibroma pendulum and fo single are common.

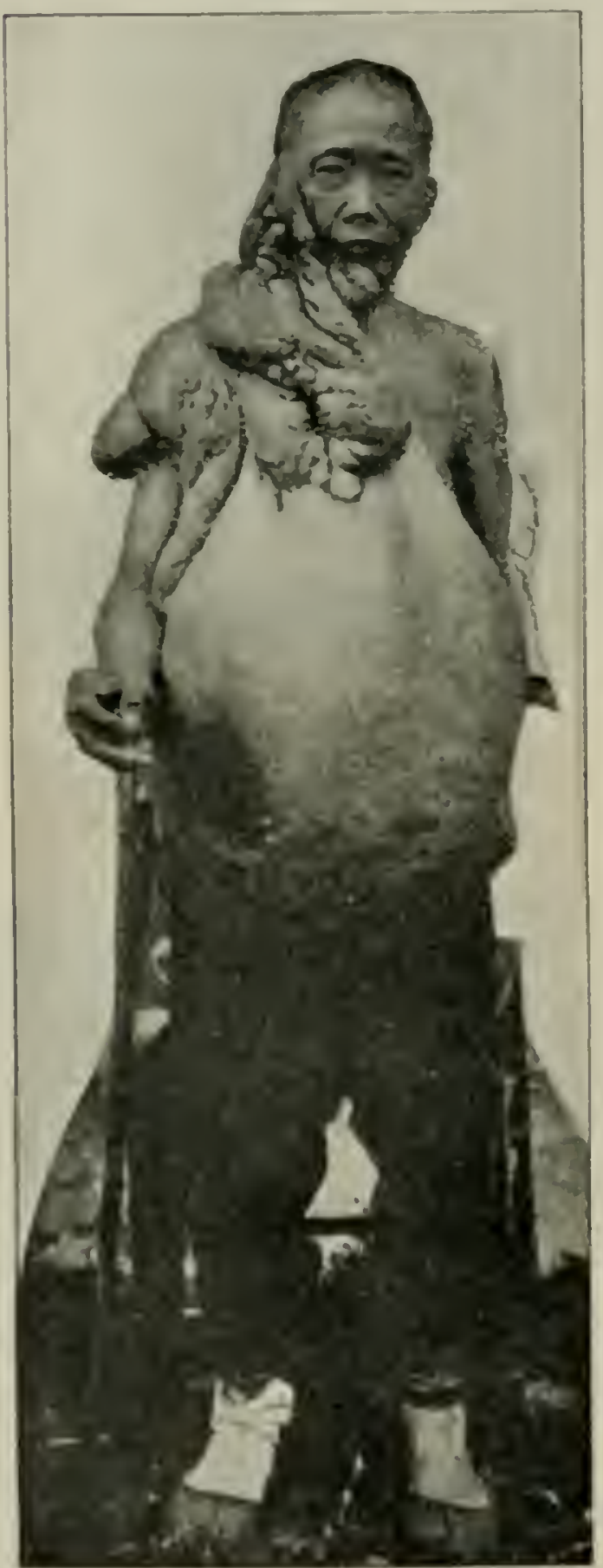

Molluscum fibrosum.

(By M. Mackenzic, Foochow.)

biasomortexis or psendoxanthomal hats been seen in half-caistes.

Cheloid is extremely common: it follows tallooing in Africantl natives and hypodemic injections in Chinese.

l. ymphangiomata are very frequent.

Multiple telangiectasis somewhat common.

Mromata are rare.

Xambloma diabeticormm is common in India and Ceylon.

Santhoma planum and tuberosum are fairly frequent.

(4.) Malignant connective tissue tumours.

I. ymplioderma perniciosum has been seen.

Mreosis fungoides lias been seen in a half-caste.

Angio-sarcoma is to be found in lle Cameroons.

Multiple sarcomatosis is rare.

The author has seen carcinoma of the cervix and of the pyloris in Colombia, South America, and carcinoma of the orars in Central Africa.

\section{ULCERATIONS.}

The majority of tropical ulceralions are the result of some trivial scratch or bruise, but as a result of neglect, underfeeding, moistness of the skin, expresure and liability to secondary infections, ulcers of enormous dimensions mar develop. In some cases the writer has seen both tibia and fibula of the same leg exposed on two sides by ulcerations, the ressels and merres remaining isolated like strings p!ainly visible. 
Various methods have been employed for the naming of ulcers asLocal names, e.g., veldt sore.

Anatomical names, e.g., interdigital.

Age, e.g., infantile.

General disease, e.g., leprotic or elephantoid ulcers.

Local pathogenic condition, e.g., varicose ulcer, ulcerating granuloma.

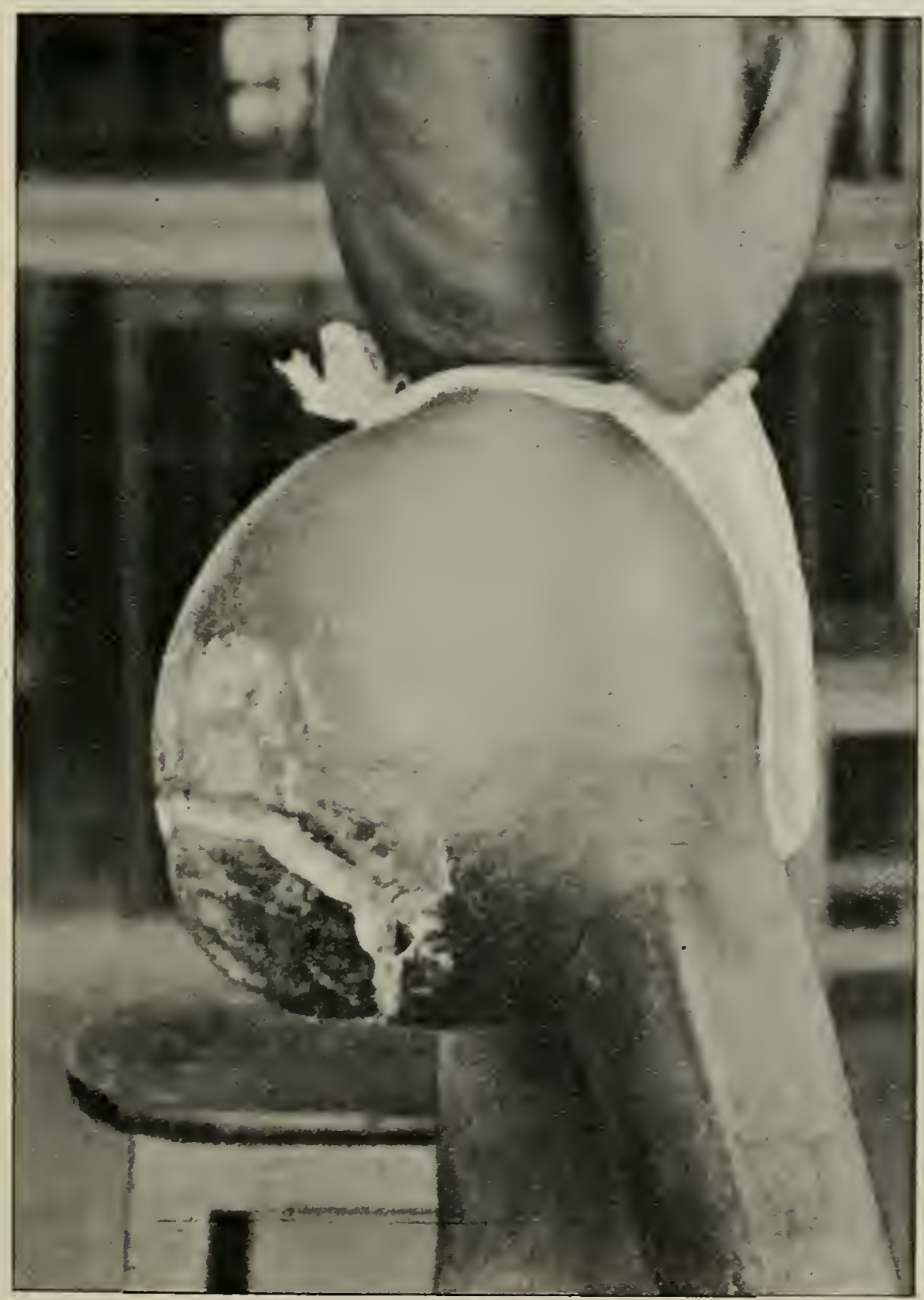

Sarcoma of the Buttock.

It will suit our purpose to use the alphabetical classification of common terms employed.

Blastomycotic, see Blastomycosis.

Cancerous, same as in temperate climes.

Diphtheroid, has grey or blackish re-forming membrane, usually on the legs. 
Elephantoid, see Elephantiasis.

Frambesial, see Frambosia tropica.

Gangosa, see Gangosa.

Glanders, same as in temperate climates.

Infantile, usually multiple and not severe.

Interdigital, treat as for septic ulcers.

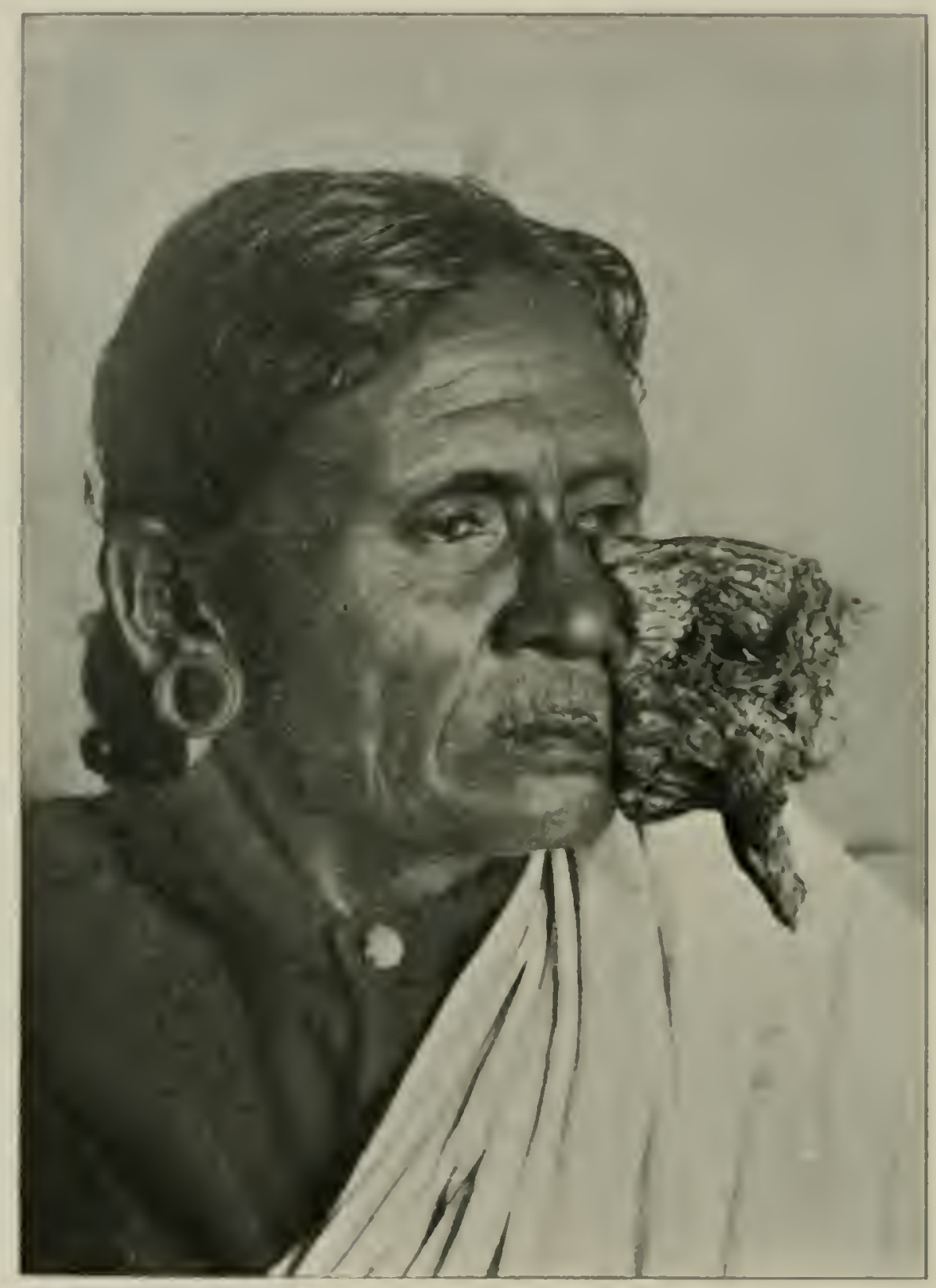

Ilorny growth from the cheek.

('robably a sebaceous horn.)

Leprotic, see l.eprosy.

Oriental sore and espundia, see leeishmantasis.

Septic, antiseptic treatment.

Syphilitic, treat the constitutional disease.

Tubercular, treat the tubercular disease.

Tropical, grenerally chronic and sloughing: spirochates and Leishman-Donovan bodies have been found in them. 
Ulcerating granuloma, see article on.

Varicose, common among young rickshaw coolies.

Veldt sore, a septic ulcer common during the Boer War.

Ulcerations which are manifestations of general diseases must be treated accordingly; ulcers of local origin are dealt with according to their severity by antiseptic lotions, powders, ointments, cauterizations and other operations.

Fomentations to clean them up, assisted by petroleum. washings if necessary, followed by cleansing lotions of corrosive sublimate, lysol, cyllin, potassium permanganate, oxygen peroxide, boric lotion, eusol, followed by antiseptic dusting powders such as iodoform, I; talc, I; boric acid, 2.

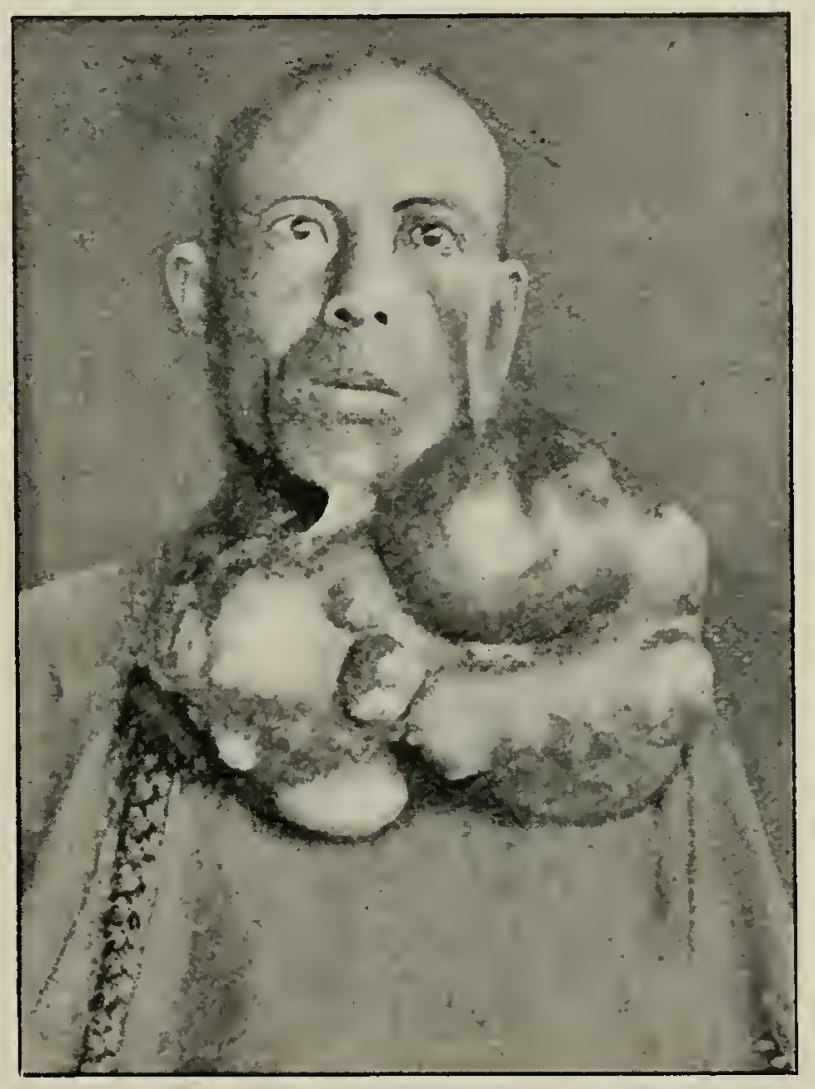

Chondroma. (By P. B. Cousland, Swatow.)

Some may require cauterization by silver nitrate, pure carbolic, or the actual cautery; others may require scraping and cauterizing under anzsthetic; some may require excision, others X-ray treatment.

Bier's passive congestion has not proved useful in the author's experience.

Rest, nourishing food, correction of other disorders and the best hygienic conditions will hasten the process.

\section{VESICULAR DERMATITIS.}

An epidemic dermatitis occurring at Leopoldville, Congo, caused by a small beetle of the genus Polderus. There may be vesicles on an erythematous patch or on long streaks with inflamed borders. 
The intial eryhemat of wenty-four to forty-eight hours is followed by the resicles which soon become mucopurulent, persisting for four or five days, and ending in despuamation. Europeans are affected on the uncovered parts.

The beetle is crushed by the victim and the secretions rabbed into skin (Rodhain and Iloussiau).

\section{XANTHODERMA AREATUM.}

Clusters of yellow spots about the lower part of the legs which coalesce, forming a large, irregular, unraised patch. The disease is purely local and not of a serious mature, although it is chronic.

Resorcin ointment assists the condition. 


\section{SECTION VII.}

\section{DISEASES OF THE EYE.}

GENEREAL REMARKS.

BLINDNESS.

LOCAL DISEASES OF THE EYE.

CATARACT.

OCULAR MANIFESTATIONS OF GENERAL DISEASES.

THE TREATMENT OF EYE DISEASES. 


\title{
DISEASES OF THE EYE.
}

\author{
GENERAIREMARKS.
}

11F do non propuse an consider the subject in detail, but only 10 mention those disorders of the eve that are more or less confined to the Tropics or are met with in an ascravated form or with greater frequency lhan elsewhere. For example, quinine amblyopia and the

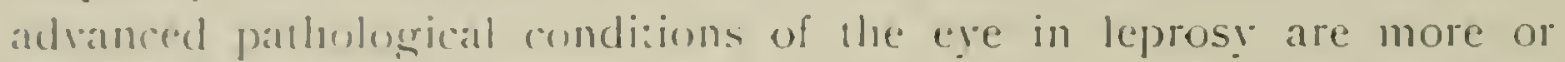
less confined :o the Tropies, and conjunctiritis is more common than in any ohlor part of the workd, due :o the filthy dwellings and labits, the prevalence of dust and fl! carriers, and the higher temperature favourable for bacterial development.

\section{BI.INDNESS.}

filindness is rery common in the Tropics.

In India (ngor) there were (welve blind men and welve blind women in every 10,(x)o idulss of each sex, while for the same period and number here were in lingland eight blind men and seren blind women.

The causes are often duc to the neglect of slight injuries followed l) seprice infertion.

1)r. Vell has clascified the causes of blindness thus :-

(1) Congenital, c.e.s. syphilitic affections of the optic nerve, retina and choruid, malformations of the corelall.

(2) Local Eye Diseases, e.g.e opluthalmia neonatorum, purulent ophthalmia in adults, catarrhal and gramular ophthalmia, ulceration of the corneal, severe paralyses of the ocular muscles, glancomat.

(3) Traumatisms, ".... injuries from sticks and horns, exposure to sunlicrht, rolipse blindness, niglu blindness.

(4) Constitutional Diseases, ("... syphilis, malignant umours, leprosy, wherculosis, yphoid fever.

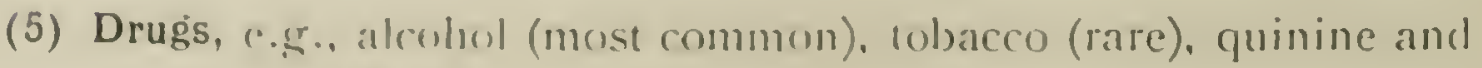
opium (rery rare). 


\section{LOCAL DISEASES OF THE EYE.}

\section{(1) Traumatisms.}

Burns and scalds. The cornea may be injured and slough. Enucleation may be necessary. If by lime and acids there is often symblepharon. To treat such cases insert a neutral oil, as castor or olive oil.

Foreign bodies such as pieces of steel. Apply 2 per cent. cocaine and remove the particle. If allowed to remain in the eye it may become encased in fibrous tissue and followed by vitreous degeneration and opacity, gross pigmentation of the choroid, iritis and optic atrophy.

Flies may carry micro-organisms and cause conjunctivitis.

Abrasions of the cornea from inverted eyelashes or scratches from baby's finger nails, \&c.

Contusions and concussion injuries (black-eye). Such may be followed by mydriasis, rupture of the ball, dislocation of the lens, hamorrhage into the anterior chamber, vitreous or retina, rupture of the retina or choroid, opacity of the lens, pigmentary changes in the retina, some optic atrophy, detachment of the iris; sympathetic irritation and inflammation may follow.

\section{(2) Exposures.}

(a) To bright light.

Eclipse blindness is an affection of the macular region of the retina from exposure to direct sunlight. The changes are probably of a chemical nature brought about by the ultra-violet rays.

Night blindness occurs in badly nourished individuals from exposure to indirect sunlight unaccompanied by any ophthalmic changes. The condition may be due to failure of the visual purple which has been bleached by the bright light. Bright light reflected from the snow will also cause blindness.

Erythropsia. A red vision due to bright reflected light. It usually occurs in persons whose lenses have been removed. The cause is uncertain, it is possibly due to hyperamia of the retina.

Cataract. This is common where there is much reflected light. Arc lights will cause it. The condition is common in glass-blowers.

Sponge-fisher's Asthenopsia. This is an hyperasthetic condition caused by the scintillation of sunlight upon the water. 
l.ighlning, log means of ils ultra-viuled rays, ma! result in hlindness through :-

The heat ra!s producing burns.

Elexerulytic aterion.

Cumcussion camsing a disturbance of the circulation.

Sunlight is also said to callse a spring callarrh which disappears in atummn and winter.

(b) Ilind and Dus:. These will ratuse:-

l'ingureule, a yellow thickening of the unter conjunctivit opposite en the palpebral lissure.

Plerygrum, an extension of a triangular vascular fold of conjunctiva spreading to the cornea.

Ophthalmic irritation and microbic infection.

\section{(3) Habits, \&c.}

Serosis amemer debilinated children is bery eommon, especially in the liast, where famine, cholera and dysentery are preralement.

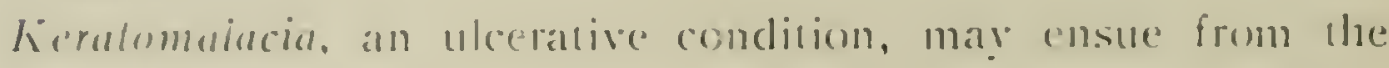
same caluse.

Calaract in India is chichly senile, allhough diabetes is common andongst regetarians.

Conorrhoul ionjuncliatis is common as a resule of unclean habits. Inflammatury conditions arise as a result of pilgrims bathing in infected water.

Toric amblyopie is aused by excessive smoling and drinking. as also is misty rision. There is a central colour scotoma for red and seren especially. There must be immediate cessition of the causaltive fatctor.

\section{(4) Insects and Parasites.}

(a) Insicis.

Sings cause adema of the lids and conjunctiva.

frribuing secretions are aroublesome, e.g., the eye-fly of India, the greatiant uf Senegal. \&c.

lediculis pubis ma! weur on the eyelashes.

Sircopteri invade the free edges of the eyelids by burrowing bencith the slin. It is not uncemmon in San Domingo. latrax mate he deposited in at aboul the eye by Musca romitoria, Dermatobia moxalis and raniventris, Sarcoplasca magnificat.

llairs or horns may lind a resting place here. Ophthadmia molosi is due to the hairs of $130 \mathrm{mb}$ ) rubi.

Infreque discharge is not uncommonly carried to the eve as b! Mlusca domeslica.

Jrriating pollen ma! catuse resicular eruption. 
(b) Animal parasites.

Filaria loa may cause trouble beneath the skin of the eyelids and conjunctive as in West Africa.

Filaria oculi humani may insert itself in the eyeball.

Cysts containing Paragonimus westermanii have occurred in the eyelids and orbit.

Dermanyssus gallina in the cornea.

Demodex folliculorum in the Meibomian glands.

Cysticercus cellulosa in the choroid, vitreous, retina or ciliary region.

Amongst other parasites that have been 'found troublesome are: Agamodistomum ophthalmobium, Monastomum lentis, Sparganum mansoni and Filaria palpebralis.

(c) Fungi.

These play an important part in local eye diseases as the following list will show.

Ocular blastomycosis is calused by Crypiococcus dermatitis and various Saccharomyces. Papules, pustules and an ulcerative warty condition of the eyelids is produced.

Ocular tinea is caused by the Microsporon lansomum and the Trichophyton tonsurans. There are crusts and pustules about the cilia.

Ocular aspergillosis is caused by Aspergillus fumigatus. There are pain, abscess and ulcer formation.

Ocular nocardiasis is cáused by Nocardia bovis, $N$. israeli, $\mathrm{N}$. foersteri, $\mathrm{N}$. dassonvillei, all which produce actinomycotic conjunctivitis, corneal ulcers, miliary metastases in the choroid and lachrymal concretions.

Ocular moniliasis by Monilia albicans, the organism of "thrush" and perhaps of sprue.

Ocular genosporosis by Genospora grapliii (rare).

Ocular sporotrichosis by Sporotrichum beurmanni infecting the eyelids, conjunctiva, lachrymal sac and iris.

\section{(5) Local eye conditions not previously dealt with.}

Congenital defects are not uncommon, such as anophthalmos, microphthalmos, coloboma, albinism and errors of refraction. Conjunclivitis.

(a) Conjunctivitis catarralis.

Acute: this is caused by the Koch-Weels bacillus and is nearly always severe, also by the Morax-Axenfeld bacillus which causes a mild type, also by the pneumococcus and staphylococcus less commonly. This latter may occur in epidemics. 
Chronic, catused bै wind, dus, evestrain, alcoholic excesses, foreign bodies and hac Mbrax-. Irenfeld bacillus.

liolliculat, this variely oceurs in epidomics. It may be bacterial and alcute ur nun-batcterial and rhronic.

There are small round palle granules the size of a pin's head found chiefly about lhe inferion formis. In trachoma the gramules alle latger and in the superior fornix.

(b) Comorrharal Comjuncliailis.

This is met will in babies and aduls. A streptococcus ma! ilso cause a similar condition in children.

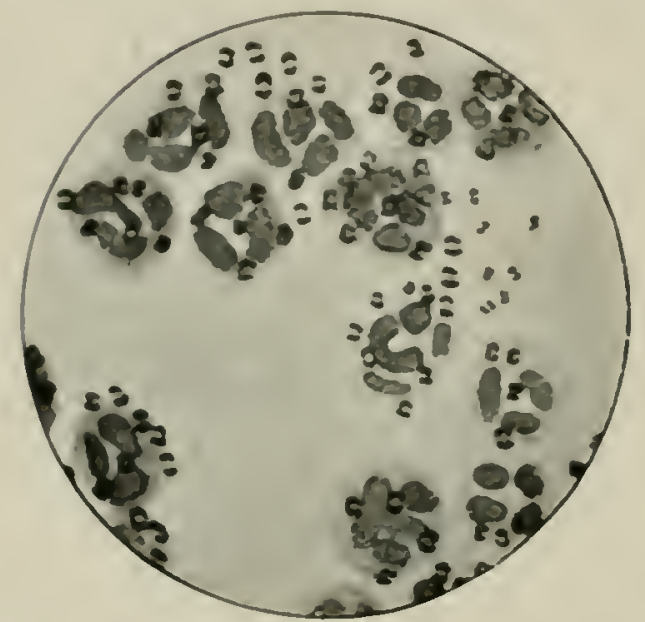

Gonococcus. $\times 950$.

(By. Hansell and Sweet.)

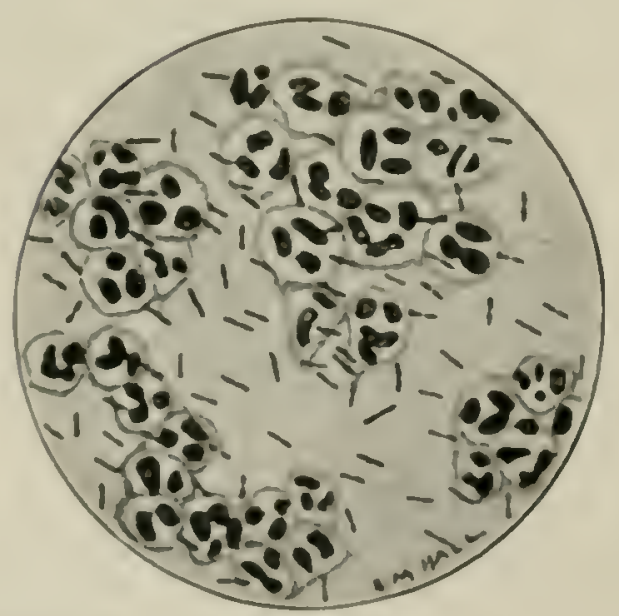

Bacillus lacunalis Morax-Avenfeld. $\times 950$. (Bj) Hansell and Sweel.)

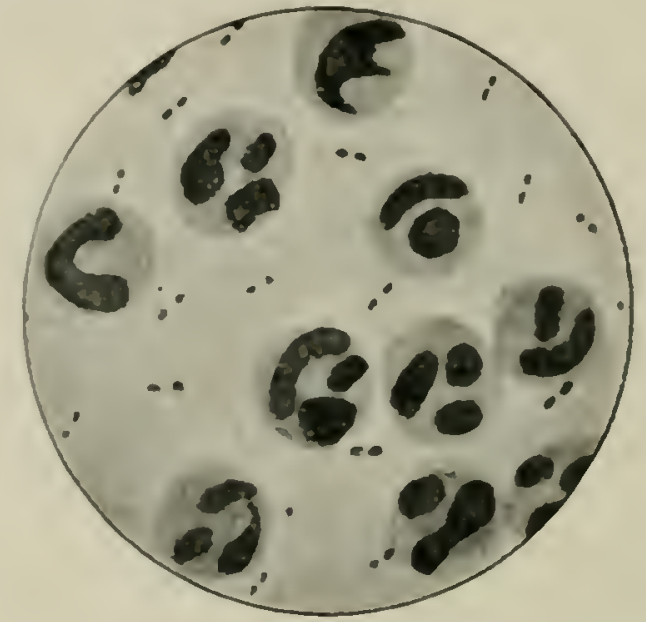

Pneumococcus. $\times 950$. (By Hansell and Sweel.)

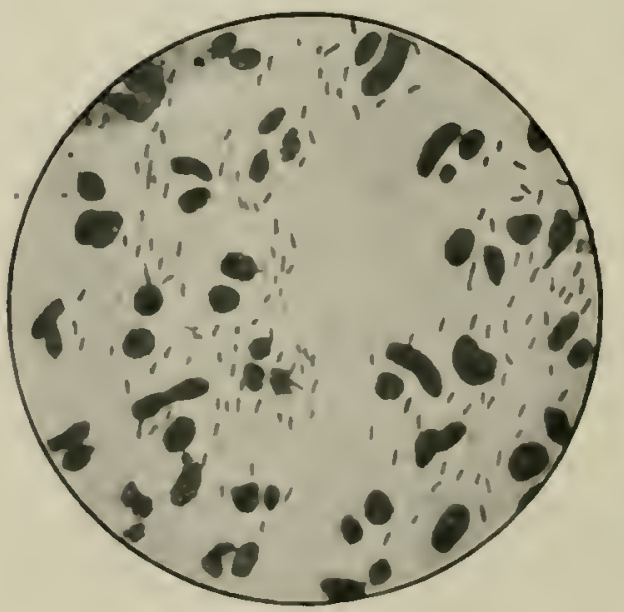

Koch-Weeks bacillus. $\times 950$. (By Hansell and Sweet.)

(c) Cranular Conjunclirilis or Trachoma.

Seventy per cent. of the chitdren in Hong-kong are infected.

It is also common in India, Japan, South America, North Ifrical, especiall! in Rig!pt. South Africa and other parts. 


\section{EETIOLOGY.}

Trachoma is found among the poorer classes. Certain races seem predisposed to it, as the Jews from Russia, the Germans, Austrians and the poorer population of the Balkan States.

Some affirm that the causative organism is a fungus, Microsporon wachomatosum, others affirm that it arises from chronic urethritis and raginitis, the organisms being carried by towels, hands, \&c., to the eyes. Cell inclusions of a chlamyzoan nature have been found in these discharges and also in the trachomatous nodules. The disease is important because of its disastrous complications and sequela.

\section{DEFINITION.}

An acute or chronic inflammation of long duration, accompanied by hypertrophy of the conjunctive and the formation of "granules" with subsequent cicatricial changes.

\section{SYMPTOMATOLOGY.}

Photophobia, lachrymation, itching and burning sensation, pain, risual disturbance, swelling of the eyelids, narrowing of the palpebril fissure, drooping of the upper lid from weight, swelling and stiffness; muco-purulent discharge, marked in recent, scanty in chronic cases. The conjunctiva of the arsus and fornix is reddened, thickened and uneven on account of the hypertrophy and granules. These granules are rounded collections of lymph corpuscles in a connective tissue reticulum. Papillary, granular and a mixed form are recognized. Cicatrical changes occur which may cure the trachoma but which unfortunately give rise to the sequela. The disease often manifests intermissions and exacerbations. Relapses are common and treatment is often discontinued too soon.

\section{COMPLICATIONS AND SEQUELE.}

Pannus is common. It consists of a newly-formed vascular issue often covering the upper part of the cornea, the vessels springing from the conjunctival vessels at the limbus. The entire cornea may be covered. The condition is not merely due to mechanical irritation but also to changes like those of the conjunctiva. Iritis and corneal ulcers may be present, the latter having opacities interfering with the vision.

Corneal opacities when chronic leave a permanent layer of connective tissue causing the opacity.

Cicatrization leads to :-

Trichiasis: the cilia are turned backwards and may touch the cornea. 
Entropion which is more pronounced in the upper lid. The distorted rilia ma! caluse corneal uleer. The edge of the lid is morned inwards.

lictropion $1 \mathrm{~h}$ hen the edge of the lid is everted and the lower lid is usmally affected. It is the result of the cicatrization of the comjunetiva and the hypertrophy and contraction of the micularis muscle.

Symblephaton is less common. The fornix is obliterated and the rye morements limiled.
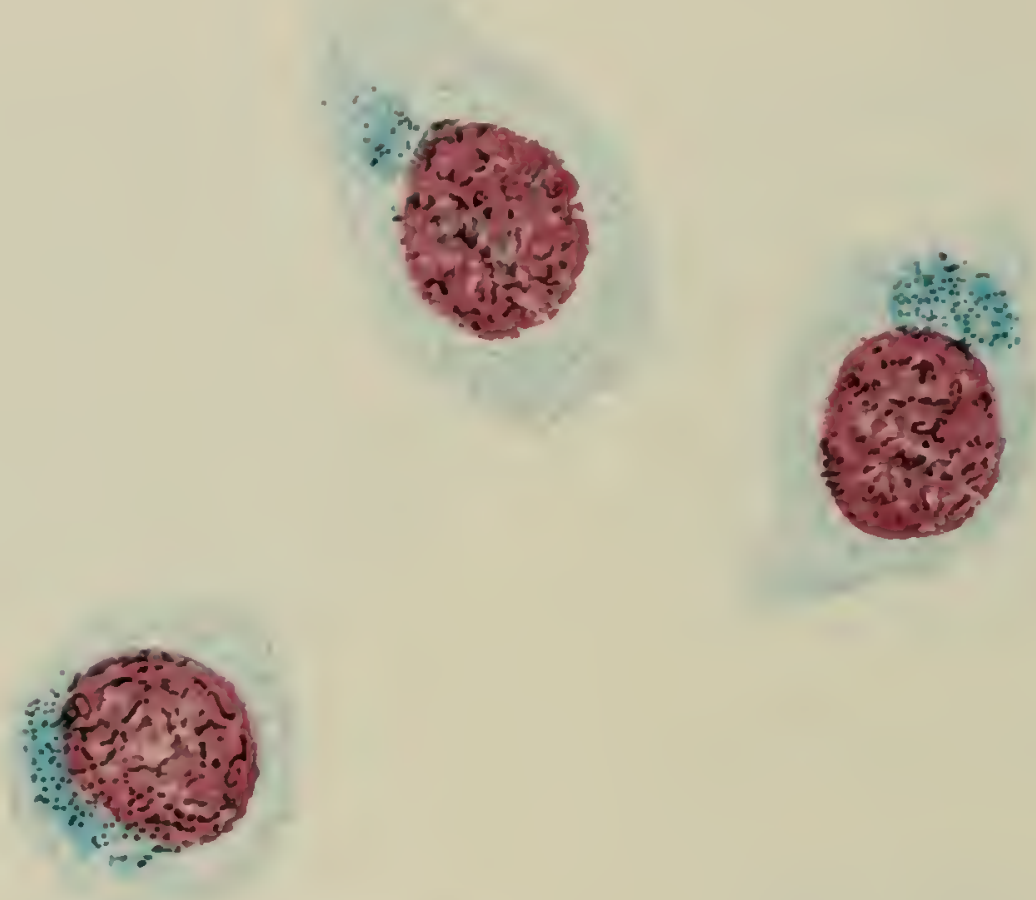

Katharine Hill.

Trachoina bodies of Prowazek-Greeff. (Giemsa stain.) Courlesy of Dr. Brown Pusey. (From "IVebster's Diagnostic Methods.")

Slaphyloma is rare, and when present is of the cornea.

Cerosis is a dry scaly contrateded condition of the comjuncliva wilh changes of the cornea in severe catses due to loss of the secreling powers of the conjunctivite.

\section{TREATMENT.}

For local application use eopper sulphate pencil once daily followed by an eye wash of wam horic acid and iced compresses. This can be pusmed for monilis.

Silver nitrate, 1 to 2 per cent. solution is gond when there is much discharge. follewed hy silline eye washes. 
Glycerine of tannin, 5 to 25 per cent., and alum stick are also used. Carbon dioxide snow applied once fornightly is good but it requires cocaine for its application.

Surgical remedies are:-

Expression, perhaps the most popular. Use Knapp's roller forceps for squeezing out the granulations. Wash afterwards with mercury bichloride. General anasthesia is usually required.

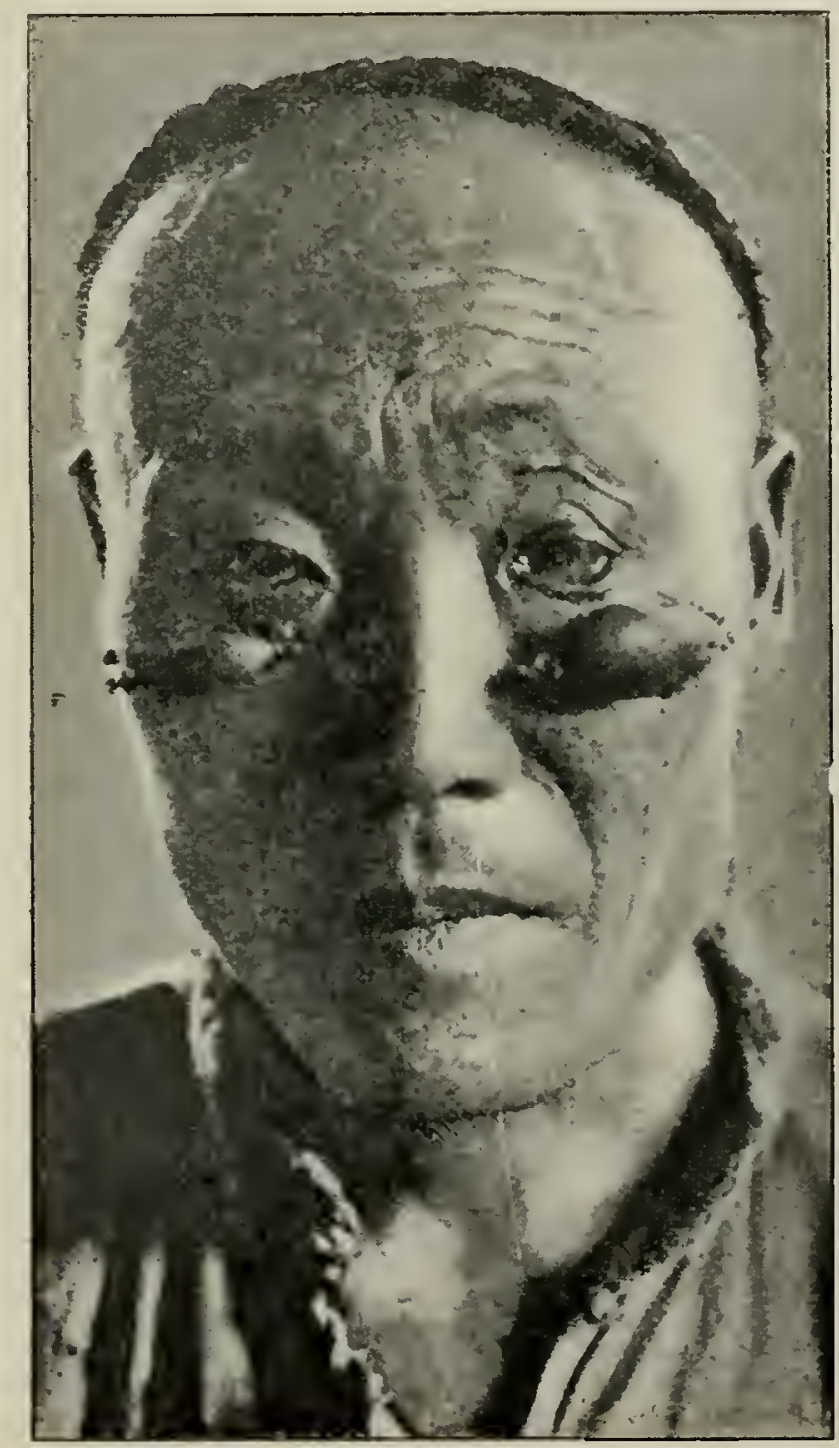

Native Chinese operation for lower-lid entropion, pinching the skin between two small sticks. In this case the compression was not complete and two curious tumours resulted. (By Jeffreys.)

Grattage, which consists of scrubbing the granulations with a stiff tooth brush, followed by an eye wash of mercury bichloride with or without previous scarification.

Excision, to remove the conjunctival fold containing the granules. The conjunctiva is often injured in this process. Curetting is popular with some surgeons. 


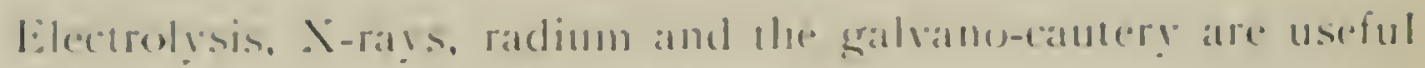
in the lainds of skilled operaturs.

Whaterer the treatment it must be combinued until every trace of hepertrophy has disapproared.

Propherlaxis is most importame and necessary warning with appropriate advice must be griven wh these concerned.

In epidemic of tratehoma is a serioms malter.

(d) Spring Catarrh or Cominnclicitis astialis.

This variety, which resembles trachoma, occuss in the spring and summer. The comjumetiva may be corered with flattened papillax of a blusish linge.

The intermittent and recurrent nature of the disease with the reappearance of the granules will differentiate this from trachuma. The atoulog! is molinown. The condition mat last for geatrs and then clear up. There is no known cure and the treatment is stomptomatic.

(c) Epilheliosis desquamativa or Samoan eye disease.

"There is a milli! secretion from trachma-like bodies. Some of these are intracellular and form a cell inclusion of small granules or "elementary" budies" which stain violet-red with Giemsats stain. The extracellulat bodies are those that are more mature. True gramules do not develop. The conjunctiva atrophies. The disease is common in Samoa ats in indicated by its mame. The suggested parasite is a chlam!dozoon, 1,yco\%onn at roplicans.

(f) Phlyctenular conjunctivitis and kcratitis.

This is fairly common in China. There are small reddened projections (phlyctenula) consisting of accumulated limphoid cells which soffen at their apices forming a small ulcer. "They mat oceur on the conjunctive or the cornea. Sometimes they appear in crops, each phlyctenule from two dats 10 two weelis.

Relapses are common. "This disease is found in children of so-called scrofulous diathesis and seems to be caused by some constifutional or dietetic errot. For lreatment, dust with calomel daily or use yellow oxide of mercury, i to 2 per cent.

(orneal ulcers are not uneommons.

Treat with the actual cantery and atropine.

()ne per cent. xeroform wintmont, moisi warm compresses. hedrogen peroxide. formalin i in fo. \&

Cocane is necessary for the actual catuery.

Perforations require iridectom!. 


\section{CATARACT.}

This is an opacity of the lens or its capsule.

\section{NOMENCLATURE.}

If the opacity commences about the centre of the lens it is a nuclear cataract, if about the periphery it is a cortical cataract. The former tend to develop in old age, the latter during middle life.

The hard variety are senile, common after the forty-fifth year and can be extracted, but the soft may occur from childbirth to the thirtieth year and require needling, \&c. All cataracts occurring under the thirtieth year are soft.

Cataracts are primary when independent of any other ocular condition.

," , secondary when other ocular disturbances are present.

, = lenticular when in the substance of the lens.

," , capsular when affecting the capsule.

," ,, capsulo-lenticular when involving both.

," , stationary when they remain incomplete.

,. ., progressiz' when they spread and tend to affect the whole lens.

", " congenital when they are due to faulty intrauterine development or inflammation.

These include the anterior and posterior polar, lamellar and sometimes the complete cataract.

, , traumatic when the capsule is pierced or ruptured by a foreign body or by a simple concussion allowing the lens to absorb the aqueous humour.

We shall follow a classification of May and Worth and deal with each seriatim.

A. Stationary Cataracts.

(I) Anterior polar.

(2) Posterior polar.

(3) Lamellar.

(4) Uncommon forms.

B. Progressive Cataracts.

(1) Senile, cortical and nuclear.

(2) Congenital, juvenile.

(3) Traumatic.

(4) Catarrhal eye complications.

(5) After cataract. 


\section{A. (1) Anterior Polay or Pyramidal Cataract.}

It is an epacing situaned an He anterion pole of the lens beneath he capsule. It maly be congenital or acquired.

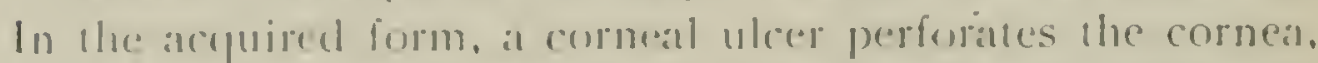
irritales ale lens, producing a probliferation of the sub(apsular rpithelium. Interference with vision is slighe The treatment is ustally nil.

"1he anteries rommber is restorred later.

A. (2) Posterior Polar Cataract.

Contrenilal, this is rombal and due to the remains of the hrabid artery which appuars as a black dot upon the red fundus reflex.

So tratment is required.

Acquired, his is comical and stellate in shape. It develops in ronnoction wilh high mropia. chorniditis, diseases of the vitreous and retinitis pigmentosat. These remain stationary for lears and may become complete. Vision is impared and treatument useless. Deal with the accompanving condition.

\section{A. (3) Lamellar or Zonular Cataract.}

These are concrenital or infantile. They are most commonly seen in children and usually affece both eyes, heing associated with rickess, al seneral disorder.

11 appoars as a grey disc-like opacily surounded or being surrounded hy a clear transparent portion of the pupil. It is most dense at the matgin of the disc. The visual interference may be slight on severe.

Iridectomy can always be done and the rision improved.

The porion chesen for iridecemy should be downmards and inwards. In the roung discission can be pratised, in chler people extraction of the lens is done.

\section{A. (4) Uncommon Forms.}

All comcenital ance somewhat uncommon.

Interference with the vision is slight. There may be:a coulral white opacity in the lens;

a fusiform, spindle-shaped upacity strething from the anterior to the posterior pole.

a punclule cataract having mumerous small white dots distribued throughous the lens.

\section{B. (1) Senile Cataract.}

This is the most common form of cataract.

Both cyes are commonly affected, one following the other. 
The nucleus remains transparent, months or years may be required for the ripening to be completed, it may become stationary at any stage.

J The Incipient Stage.

There are streaks of opacity from the cortex to the centre of the lens where the streaks become narrower. Less rarely there are dots or a general haziness.

2. The Srolling Stage.

The lens absorbs fluid, swells, pushes the iris forward and so reduces the depth of the anterior chamber. The cataract becomes stellate in appearance.

3. The Mature Stage.

The lens loses most of its fluid, shrinks, is completely opaque, the anterior chamber is normal in depth, the entire lens may be a hard "black cataract." It is now easily separable from the capsule and is "ripe," ready for removal by extraction.

4. The Hypercemic Stage.

The mature stage continues or irregular spots may be apparent. The lens may lose more fluid and may become a "shrunken" cataract, or soft and milky, and the nucleus sinks into the fluid which then equals "Morgagnian" cataract. Lime salts (chalky cataract) or cholesterin may be deposited.

The anterior capsule may become thickened and opaque (capsulo-lenticular cataract).

\section{TREATMENT.}

Extraction is the only means available, medicinal treatment is useless. Atropine may temporarily assist vision in some cases.

Removal of both cataracts at the same time should never be carried out.

Artificial ripening may be resorted to so as to hasten the process by incising the cornea, allowing the aqueous humour to escape and the cornea to fall against the lens, but such procedure is not free from risk.

\section{When is a Senile Cataract ready for Operation (?). Tests.}

(1) Examine by focal illumination.

If the opacity comes forward to the iris, so that the latter throws no shadow on the lens, then the cataract is probably mature.

(2) Cover one eye and throw a strong light suddenly upon the one to be tested; the pupil should then contract. 
(3) The patient mus have aroud perereption of light; that is to say, he must apprectatle the corering of the eye.

(4) Hie peripheral vision slould be groud.

The position of a lighted candle when placed in rarying positions in a dark room shoukd be appreciated by the patient "leen looking stratight forward.

Fixtratchion ran be carriced out with or without iridectom! as follum: :-

(1) The Corneal Siction.

Ciratine with a fell drops of adrenalin are necessary, rarely seneral anasthesia. The patient looks downwards, fixation forceps are applied, the Grafe hnife enters the cornea, with the cutting edge upwards, a linife's breadth above the horizontal meridian, and the corneal hap completed. The linife must not be twisted, the aqueous humour must not esiape, and the iris must not be cul.

(2) The Iridectomy.

The conjunctival flap is turned forwards on to the cornea, cured iris forceps are inserted and grasp the upper mallgin of the pupil. which is gently drawn out and a small portion removed with scissors.

(i) Opening of the Capsule.

The cystotome is introduced flatwise into the anterior chamber, its print turned towards the capsule, which is gently cut. The incision may be T-shaped, A-shaped, or +-shaped.

(t) Delienery of the Cularact.

Cintle pressure is exercised upon the lower part of the cornea twwards the centre of the globe with the back of the spoon; the section opens and the lens presents.

(5) The Ilound Toilet.

Sirolie the corneat upwards to remore ing cortical remnants and bloud-cluts. The edges of the section must be clear.

The lids must not touch the wound. The coloboma is natrened out with a spatula; adjust the flap, insert a drop of atropine and close the lids.

(6) 'The Dressings, E्c.

Cover with gatum, both eyes, and add cotton wool soaked in antiseptic and lix with a binocular bandage.

The patient must remain upon his back, quiet, anodynes are best griven, straining is to be aroided, the wound examined and atropine instilled daily, the iris excised if prolapsed; liberate the unoperated eye on the fifth day when the patient ma!y sit (u). In wo weeks smolied glasses are worn. 
Later, in four weeks, the hypermetropia is about ro D., with some astigmatism of 2 to $3 \mathrm{D}$. due to the incision. Give therefore a convex spherical lens of ro D. with a convex cylinder of 2 to $3 \mathrm{D}$. for distant vision, with an additional convex sphere of 3 to $4 \mathrm{D}$. for reading.

Previous errors of refraction will modify this correcting lens.

Some operators omit the iridectomy, thus avoiding prolapse of the iris, but the capsule is more liable to be left in the wound.

Soft and traumatic cataracts are evacuated by tearing the capsule with a cystotome.

In India many surgeons remove the cataract in its capsule. In such cases the suspensory ligament is ruptured, capsulotomy omitted, and the lens expressed by corneal pressure with a squint hook. The grave risk of the loss of vitreous is the chief danger.

Complications of extraction include :-

Loss of vitreous, lens dislocation, wounding prolapse of the iris, incomplete evacuation, intraocular hæmorrhage, striated keratitis, glaucoma, iritis, iridocyclitis, cyclitis, suppuration and panophthalmitis.

\section{B. (2) Congenital and Juvenile Complete Cataract.}

These are uncommon; always soft; bluish white with a pearly lustre in one or both eyes. The eye may be otherwise normal, or there may be other eye complications.

There is often a history of heredity, convulsions and other mal-intrauterine developments.

Treatment is that of discission (needling).

This should be done early, and needs to be repeated a number of times. If the patient is young give a general anasthetic, if older a local ancsthetic is sufficient.

Dilate the pupil, introduce the speculum, steady the eye with fixation forceps, divide the cornea and the lens capsule, making two superficial cross cuts, break up the lens matter by rotating a needle so that the lens matter will be absorbed.

Repeat this after several weelis. The discission must be deeper each time, and finally include the posterior lens capsule.

Three operations are usually required.

Keep the pupil dilated with atropine.

\section{B. (3) Traumatic Cataract.}

This is the result of a wound involving the lens capsule, or more rarely after concussion. Swelling and cloudiness 
of the pupil last for several days when the lens becomes ilbsorbed.

Often the cure is spontaneous, but part male remain belind, berome opsepue, and require operation. Iritis and graucoma are not uncommon. Treat on general lines indicated.

\section{B. (4) Cataracts of Eye Complications.}

The following conditions ma! lead 10 calaracts:-

Iridocyclitis, choroidits, severe comeal ulcer, glaucoma, retinitis pigmentosal, and retinal detaclment.

Such often begin in the posterior part of the lens and tend to desenerate. Treat the causative factors.

\section{B. (5) After Cataract.}

This is due to the opaque remains of the lens capsule, proliferation of subscapular epithelium or new connective tisste after ordinary calaract operation.

l'ratere discission five weelis after extraction, bu will a rery sharp knife and not an ordinary discission necelle. An iridocysteciomy may be necessary.

\section{OCLI.AR MANIPESTMUNS OF GEXERAL DISEASES.}

These will be dealt with briefly seriatim.

Pernicious andemia causes hamorrhages and retinitis.

llamophilia ma! resule in profuse laemorthage after injury.

leukiemia causes retinal hamorrhages and a distinct retinitis.

Purpura causes subconjunctival hemorrhage, retinal hemorrhage. Endocarditis causes embolism of the central artery.

Arric aneury'sm causes modriasis and exophthalmos or miosis. and Areriosclerosis results in marlied changes in the exophloalmos fundus.

Oral sepsis to a quiet cyclitis, iritis and heratitis.

Dyspepsia 10 irdocyclitis, choroiditis and retinitis.

Rectal lenesmus lo subconjunctival, retinal and vitreous hamorrhage.

Acromegal! 10 bitemporal hemianopsia, reduced acuteness of rision. optic neuritis, optic atroplyg, some ocular paralysis, exophthalmos, (p)phorota and eye pain.

Exophthahmic soine in exophlhalmos and corneal ulcers, ocular pilresis.

Cerebrospinal meningitis to conjunctival adema, ocular paresis, strabismus, pasis, mọtagmus, lieratilis, optic neuritis and atrophy.

Diphlheria to paralysis of extrinsic muscles and optic neuritis. 
Erysipelas to orbital cellulitis, \&c.

Influenza : asthenopia, corneal ulcer, paresis of ocular muscles.

Pneumonia : corneal herpes and ulceration.

Septicæmia and pyemia: retinal hæmorrhage, emboli, purulent choroiditis.

Syphilis : iritis (25 per cent. of all iritis cases), choroiditis, chorioretinitis, optic neuritis and atrophy, gummata of the iris, ciliary body and periosteum, interstitial keratitis, paralysis and paresis.

Tuberculosis: deposits in the iris, choroid, sclera, conjunctiva and lids.

Variola: pustules of the lids, conjunctive, keratitis and ulceration, complete destruction of the globe, ectropion, entropion and trichiasis.

Nephritis: odema, albuminuric retinitis and amblyopia.

Diabetes: cataract, retinal hæmorrhage, retinitis, optic neuritis, retrobulbar neuritis, pareses, paralyses and myopia.

Gout : episcleritis and scleritis, dry catarrh, marginal ulcer of the cornea, glaucoma and hamorrhagic retinitis.

Rickets: congenital zonular cataract, interstitial keratitis, phlyctenular keratoconjunctivitis.

Scurvy : hamorrhages of any part, temporary night blindness, apoplexy and retinal hamorrhage.

Friedreich's disease: nystagmus, ocular paralyses, optic neuritis.

G.P.I.: irregular pupils, miosis, mydriasis, Argyll-Robertson pupil, impaired or loss of accommodation, optic atrophy, paralysis of the third, fourth and sixth nerves leading to diplopia, strabismus and ptosis.

Coma: choked disc, mydriasis (organic brain disease), miosis, inequality of pupils, conjugate deviation (cerebral hremorrhage), a!buminuric retinitis (uræmia), dilated pupils and pareses (alcohol), extreme miosis (opium).

Epilepsy: transient flashes of light, coloured sensations, hemianopia, complete loss of vision, muscular spasm, conjugate deviation, distension after narrowing of the retinal arteries.

Poisonings: retrobulbar neuritis, optic atrophy (tobacco and iodoform, lead, arsenic, e.g., atoxyl in trypanosomiasis, carbonbisulphide and nitrobenzol).

Heat-stroke: amblyopia and optic neuritis.

Typhoid fever: convergence, impaired accommodation and mydriasis.

Relapsing fever : iridocyclitis and iritis.

Cholera: focal necrosis of cornea and conjunctiva with ulceration, dry catarrh, enophthalmos from loss of fluid, subconjunctival hemorrhage. 
Dysentery : keratilis, irisis, iridocyclitis.

Beri-beri: adema of lids, Iymphatic spaces in optic nerve and retina distended, defective central vision, loss of central colour vision. axial retrobulbar reluilis, spasm and paralysis of ocular muscles.

Kala-ayar : retinal h:emorrhages with deposits of pigment emboli.

Snake bite : iridocyclitis, intruncular hamorthages.

Oriental sore: cicatricial contraction, ectropion, epiphora, spasm of the orbicularis.

Vellow fever : amblyopia, hyperamia of conjuncliva.

Trypanosomiasis: iritis, lieratitis and retinal changes in untreated cases; callaract and optic atrophy in treated cases.

Plague: photophobia, lachrymation, conjunctivitis, deepened anterior chamber, punctate keratitis, iritis, contracted pupil, serous exudate, later plastic, prosterior synechia, increased tension of eyeball, later diminished tension, shallow anterior chamber, vitreous exudates, retinal hamorrhages, optic neuritis, optic atrophy and panophthalmitis as in order given.

Leprosy : eyelashes lost early, fromalis muscle paralysed, nodulal lepromata and ulceration of eyelids with destruction later, conjunctivitis leading to lagopthahmits, ectropion, xerophthalmia, infiltrated ejisclera, whitish grey masses spreading round cornea, keratitis punctata, leprosa, anterior and posterior weitis, nodules in ciliary body or in spaces of Fontana causing iridocyclitis and chronic glaucoma.

The optic nerve, relina, lens and vitreous humour are rarely attacked. A plastic exudate ma! cause posterior șnechia, i.e., adhesions between the iris and lens and caluse occlusion of the pupil and conseguent blindness. This may be hroken down in early cases by an effective mydratic. Cyclitis causes impaired accommodation and diminished vision. Cleemation and perforation of the anterior chamber are not uncommon in bad cases. Yision will be affected by the scar if in the pupillary area.

Hypopyon is not an uncommon sequel, i.e., pus, fibrin and leucocytes wilh pus organisms in the anterior chamber. In cases of perforation the pyogenic organisms enter the eychall and cause total destruction by panophthalmitis.

See article on l.eprosy.

Malaria : eye troubles are due to circulatory disturbances more than to infammatory conditions. There are conjunctival congestion, photophobia and lachrymation.

Keratitis dendritica, a herpes cornea \%oster, giving rise sometimes le ulcers, branch-shaped wibl linobs on them: nine out of ten are or malarial origin.

Keratitis profunda, a central infiltration of the cornea which is 
brought on by a chronic malarial cachexia. These are opaque greyish dots.

It abates in from four to eight weeks.

Vitreous opacities are due to hamorrhages and serous effusion.

Amaurosis is due to atrophy of the optic nerve.

In retinochoroiditis the papillae are obscured and the retina hazy. The condition is due to the parasites in the vessels. A pigmented retina is left. If there is embolism of the central artery, less rare, there is sudden loss of vision. Optic neuritis and oedema may be caused by the blockage.

Paralysis of the accommodation is not uncommon in malarial conditions, but secondary iritis is rare.

Supraorbital and ciliary neuralgia are common.

Amblyopia is less frequent and may be caused by the disease or the treatment of it, i.e., malaria or quinine. The points of differentiation are set forth in the following table:-

\section{QUININE AND MALARIAL AMBLYOPIA. (After Manson.)}

QUININE

\section{History-}

Quinine must have been taken in large doses, not less than thirty grains.

\section{Onset-}

Sudden, accompanied by deafness ; both eyes are affected

\section{Pupils}

Widely dilated, and whilst loss of vision continues they do not react to light

Vision-

Completely lost for a time

\section{Ophthalmoscopic appearances-}

A white haze over fundus; cherry-red spot at macular; optic disc pale; retinal vessels markedly constricted

\section{Termination-}

Usually some permanent defect in the field of vision or in colour vision. Central vision recovers first; optic disc is unusually white, and retinal vessels small

\section{Treatment-}

Stop quinine. Amyl nitrite has been recommended to induce dilatation of retinal vessels
MALARIA

Quinine may have been taken but not necessarily in large doses

Not usually sudden, but it may be so if hæmorrhage has occurred in the macular region. There is no deafness, and both eyes are not necessarily affected

\section{React to light}

Never completely lost

There is optic neuritis; optic disc is of characteristic greyish - red colour; $1 \mathrm{e}$ tinal hamorrhages and sometimes vitreous opacities

Some cases recover completely; in others greater or less permanent defect of vision remains

Give quinine

Quinine causes buzzing in the ears with partial or complete deafness. It is usually temporary, but is sometimes permanent. 
Persums comtemplating residence in malatrial tropics should undergo a course of healy duses of quinine in lintupe first.

Persons umable la take llee drug well should not be perminted to reside in the erepies. Sime are susceptible to the drug in small doses,

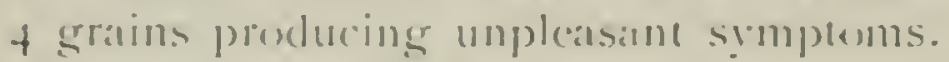

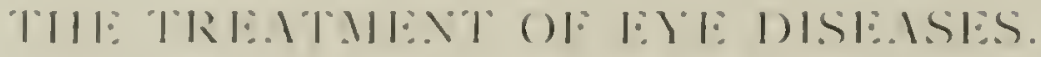

The treatment of eye diseases is lexal and general, but we are only concerned lirere whih local remedies, which will be dealt with on general lines.

(1) Eye Rest.

Correce corors of refraction by suitable lenses.

Give temperary cessation from all work involving ege-strain. Paralyse the accommodalion with atropine, homatropine or hyoscyamine.

Prescribe protective goggles or glasses, epe-shades or bandages. Green srlasses cul out the ultra-riolet rays.

Smolied ,, $\quad$, glare of light.

Blue .. $\quad$, heat rays.

\section{(2) Cleansing and Antiseptic Solutions.}

All sulutions must be warm.

For washing the eyeball and remoring discharges use :-

Sterilized warm water.

Saturated solution of boric acid.

Phrsiological sălinte.

Mercuric chloride, a in 10,000 on $1 \mathrm{in} 6,000$ (one grain to a pint), formalin, I in 8,000 (one minim to a pint).

Cundy's fuid pure, I in 2,000 (four grains to a pint).

Carbolic acid, o*io per cent.

(3) Stimulating and Astringent Remedies.

For conjunctivitis :-

lR Zinci sulph. $\ldots \quad \ldots \quad \ldots \quad$ 2 granns

Aquate destill... $\quad \ldots \quad \ldots$ i ounce

Give two drops twice daily.

l1 Acidi tamnici... $\quad \ldots \quad \ldots \quad \pm$ grain

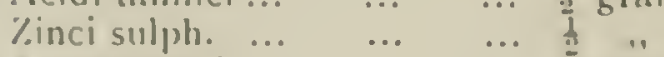

Aquate destull.... $\quad \ldots \quad \ldots$ i ounce

l. Zinci sulph. $\quad \ldots \quad \ldots \quad \ldots \quad \ldots$ grain

Sodii biboras ... $\quad \ldots \quad \ldots \quad$ j grains

Aquax destill. $\quad \ldots \quad \ldots \quad$ I ounce

18 Acid. tannic.... ... ... t grain

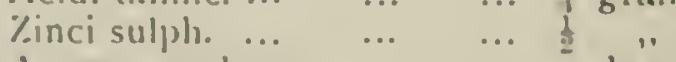

Aque camphoric $\quad \ldots \quad \ldots .2$ drächms

iquae destill.

... $\quad \ldots$ 
Silver nitrate can be used, grain $\frac{I}{4}$ to $I$ to one drachm. This can be painted on the lids in chronic cases, or stronger, grains I to 5 to one drachm. Wash well later with saline solution. Keep solution in a stoppered bottle and in the dark.

Protargol is commonly used, io to 25 per cent.

Copper sulphate, grains $\stackrel{7}{:}$ to one drachm. It is usually used as a pencil in trachoma.

In blepharitis, conjunctival interstitial phlyctenular keratitis use yellow oxide of mercury.

For persistent ulcerative blepharitis use :-

$\begin{array}{lllll}\mathrm{R} & \begin{array}{l}\text { Ichthyol } \\ \text { Ung. zinc. ox. }\end{array} & \ldots & \ldots & \text { ro grains } \\ & \ldots & 2 & \text { drachms }\end{array}$

When vasoconstrictors are required for the absorption of exudates use :-

\begin{tabular}{|c|c|c|c|}
\hline & ( & $(1: 1 \cdot 000)$ & \\
\hline & A & & $\mathrm{I}$ ou \\
\hline & $i(\ddot{1}: 1 \cdot 000)$ & $\begin{array}{l}\ldots \\
\ldots\end{array}$ & \\
\hline & ph. & $\ldots$ & $\frac{1}{3}$ ounce \\
\hline & till... & $\ldots$ & I \\
\hline
\end{tabular}

Both these are contra-indicated in corneal ulcer.

In iritis, keratitis, glaucoma and corneal ulcers use:-

Dionin, a derivative of morphine, 2 to 4 per cent. solution, two drops every two minutes for ten drops. It relieves pain, induces local anzesthesia, is a vasodilator and a lymphagogue; it is a counter-irritant and will assist in dilating the pupil and breaking down the posterior synechia caused by the iritis which so often ends in blindness. This drug loses its effect in a few days, so that it cannot be used orer long periods.

\section{(4) Disinfectants (so-called).}

These are for restricted use only and required to be followed by excess of bland solutions to remove any disinfectant left behind.

In cases of purulent conjunctivitis and corneal ulcers use :-

Mercuric chloride I : 5,000. I : 5, ooo has been used upon the conjunctiva after trachoma.

Chlorine water diluted with Io to 20 parts of water, freshly made.

Carbolic acid, 3 per cent. and pure carbolic to infected corneal ulcers. Formalin, I $:$ I, 000 to I $: 2,000$. I $: 500$ to infected ulcers.

Tincture of iodine for infected ulcers. 
Silver nitratre, 1 lo 2 per come.

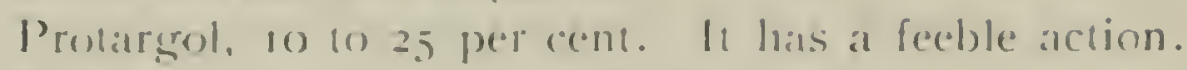

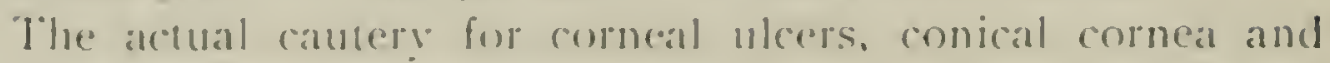
cpitheliomat of the lid.

\section{(5) Mydriatics and Cycloplegics.}

Phese are 10 dilate lhe pupil and paralyse the accommodation. Indicalions.

To dilate the pupil for aphthalmic examination.

To prevent adhesion in iritis.

For central ulcer of the cornea.

To paralyse the acommodation in order 10 estimate the refraction.

For lamellar and nuclear calaract.

Ifer some operations.

In certain diseases of the corncal and derper struclures.

Remedies.

Alropine in solution or ointment, I per cent. The effect lasts for a week or more. It may precipiate an attack of glaucoma in a predisposed eye. Some patients are susceptible to its use and shom loxic symptoms. Give morphine as an antidote.

Homalropino hydrobromate is milder and almost as useful. The effect lasts one day unly or a little more. Ĺse 2 per cent. solution, one drop every live minutes for three in four doses.

Euphlhalmin for cammination purposes, 5 to in per cent. solution: wo drops only. The effect passes off in a few hours.

Cocuine hydrochlorale. one or wo dreps of a + per cent. solution. The effert passes off in aboul one hour. It will usually diminish intracular teision.

(6) Myotics.

To contract the pupil and diminish intrancular tension.

liseful in glaucoma and peripheral corneal ulecrs.

Escrinc salicylate $\frac{1}{N}$ to ? per cent. It may cause irritation. Pilocapine nitrate (muriate) ? 10 l per cent. is milder.

$$
\begin{array}{llll}
\text { li } \begin{array}{l}
\text { Eserine sulph. } \\
\text { Cocaine mur. }
\end{array} & \ldots & \ldots & 1-1 \text { grain } \\
\text { Ayure destill. ad } & \ldots & \ldots & \text { i ounce }
\end{array}
$$

(7) Anæsthetics.

Gineral. Chloruform for children; ether for adults if possible.

Cocanc hydrochlorate, $210+$ per cent. It assists the action of mydriatics and miatics. One drop is sufficient for the removal of a foreign body. 
Holocain hydrochlorate, I per cent. It acts quickly and penetrates deeply. It keeps well.

Eucaine B. This is best used hypodermically, 5 per cent. solution is not toxic.

Novocain, 3 per cent. in adrenalin chloride, $1: 1$, ooo for hypodermic use.

(8) Yarious.

Hot compresses, frequently renewed for affections of the cornea, iris and ciliary body are useful.

Cold compresses in conjunctival inflammation. Lint laid on ice, then to the closed eyelid, frequently renewed is soothing. Electricity. Distorted eyelashes are removed by electrolysis. Ionization is employed for corneal ulcers, interstitial lieratitis, trachoma, rodent ulcer, \&c. It is painful and the results are not very encouraging.

Blood letting by leeches in cases of iritis and deeper affections is a favourite remedy with some. Apply two to four to the temple.

Massage in cases of interstitial keratitis, glaucoma, corneal opacities, \&c., has done good. Place a small portion of ointment in the conjunctival sac, close the lid and massage.

\section{(9) Native Treatment.}

Fumigation is done for inflammatory conditions. Wheat grains are also inserted under the deltoid muscle for these affections.

Powders are commonly used made up of sugar, alum and lime which, when mixed, are blown into the eye. In Arabia they give a "drop of mother's milk."

Ectropion is treated by removing or pinching up the skin and retaining it with matches. It sloughs and a cicatrix results Some cannot close the eyelids at all afterwards.

Black antimony is used to beautify the eyelashes. This is inserted with a feather, which latter is a suitable vehicle for carrying the gonococcus from one to the other.

Cataract is treated by the dislocation of the lens or couching. A needle or thorn is passed through the sclera to the pupil and the lens is pressed down; the patient jumps slightly and the lens falls. Some recover and nthers become septic. There is much iridocyclitis, loss of vision and secondary glaucoma. 


\section{SECTION VIII.}

\section{TROPICAL HYGIENE, SANITATION, ETC.}

IIIGIENE.

CIIMATE.

TEMPERATURE.

RAINFALL.

Soll.

Constructionai Mrgiese.

Physiological, EfFects ol Hot Cimates on Europeans.

PATIOIOCICAI FHFCTS OF HOT CIIMATES ON EUROPEANS.

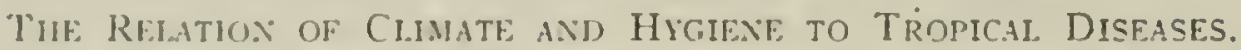

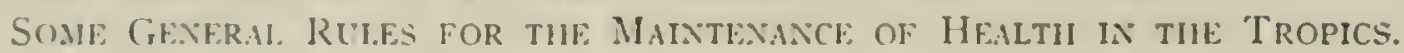

EPIDEMiologr.

WATER.

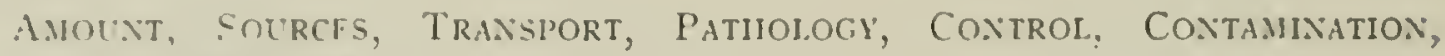
PURIFICATION.

FOOI).

Nitrition.

ADULTERATIO: OF FOODS.

SOMF, MORHD Disturbancfes CaUSFd Bi FoOD.

Fiamination of Meats.

ExAMINATION OF LIVING ANIMALS.

MEAT P'RESFRTATION.

VEGFTARIANISM.

INFANT FERING IN TIE, TrOPICS.

CONCENTRATFD FOODS.

DRINKS.

S.1.ITATION.

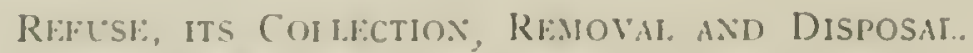

DISINRECTION.

QUARANTINE.

IMMUITY, VACCIIES AND SERA.

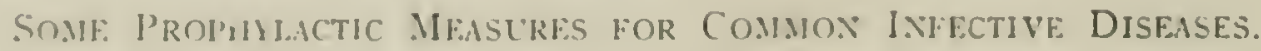




\title{
TROPICAL HYGIENE, SANITATION, ETC.
}

\author{
HYGIENE.
}

\section{CLIMATE.}

Castellani defines climate as "the combined effects of the sun, the atmosphere, and the earth at any one place of the earth's surface."

The sun exercises its influence by its heat, light and chemical rays.

The atmosphere by its chemical composition, temperature, diathermacy, humidity, pressure, motion (winds) and electric conditions.

The earth by its latitude, relative proportion of land and water, ocean currents, soil vegetation, configuration of the surface and altitude.

\section{TEMPERATURE.}

Climates known as tropical and subtropical lie between $35^{\circ} \mathrm{N}$. and $35^{\circ} \mathrm{S}$. (approximately) of the equator, the northern parallel being the Tropic of Cancer and the southern being the Tropic of Capricorn.

The sun is always vertical over some part of this broad belt, the average mean temperature of which does not go below $68^{\circ} \mathrm{F}$. $\left(30^{\circ} \mathrm{C}\right.$.).

When the sun's rays are vertical, so per cent. of the sun's heat that enters the atmosphere reaches the earth. As the sun's rars become more oblique less heat reaches the earth until probably none reaches it, when the sun's rays are horizontal to it.

The highest temperatures are reached over the two parallels mentioned above, because the sun remains vertical for the longest periods over those parallels.

The superficial land temperature may rise to $200^{\circ} \mathrm{F} .\left(93^{\circ} 3^{\circ} \mathrm{C}\right.$.), while superficial water temperature rarely exceeds $90^{\circ} \mathrm{F} .\left(32^{\circ} 2^{\circ} \mathrm{C}\right.$.).

The mean temperature of the earth increases with its depth, about $I^{\circ} \mathrm{C}$. per 28 metres. Thus mines in temperate zones may become tropical or subtropical, and consequently tropical diseases, such as ankylostomiasis, may flourish there under suitable conditions, e.g., the tin mines of Cornwall.

A daily range of temperature of more than about $20^{\circ} \mathrm{F}$. ( $\mathrm{II}^{\circ} \mathrm{C}$.) is apt to be dangerous if precautions are not taken. Short journeys 


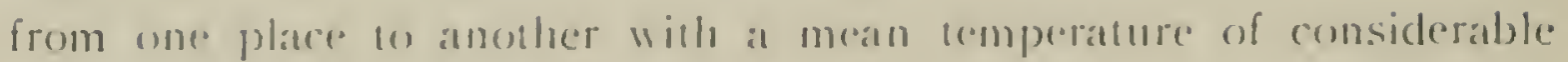
variation muss be dome alt intervals.

Ileath resolts can be found in the mopies al high altitudes.

It is ustoful u linuw that appruximately the tempreature decreases

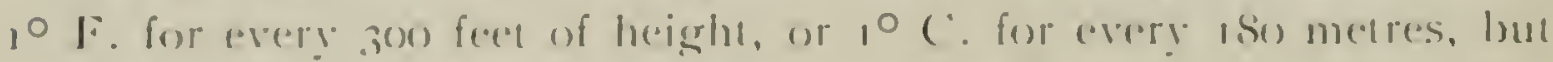
here again if the mean arerage temperature is much lower that that of a previous residence, sick people must approbch it gradually, in stages, mo matter how beneficial that altiude maly be. This hats been manifested very foreibly during the late war in emmertion with avation. When aviators move very rapidly through varying atmospheric pressures there are such results produced upon the vilat organs of the body that only selected persons can become aviaters.

Atmospheric pressure is much less on the expator than about the parallels of Cancer and Capricorn, hecause the constant heating of the air, more or less loaded with aqueous vipour, ascends (1) high altitudes with considerable force. This expansion and rising of air is followed by an inrush of cool air frem the north and soum fo fill the space, hence if heat were universally uniform there would not be any winds. The currents of air on either side of the equator, not acruss the oreatn, meet at the equator and produce calms or " doldrums."

The casterly direction of winds is due to the rotation of the earth.

The air over the land is heated more rapidly than that neer the sea, bence the land air rising is replaced by the coolet moist sea air, and is known as the "sea breeres." At night the land, having given oft its heat reiry rapidly is conler than the seat, henee the conditions are reversed and we gee "land breezes," when wind comes from the land to the sea. Hence the presence of large tracts of water have a cooling influence wer the adjacent. land. The land breeze, being dry, is apt 10 extract 100 much moisture abruptly, and auses a cooling process which ma! catuse serious chills and so reduce one's resistance to discase.

The X.L. and S. IT. monsoons of India are explained by Dove as follows: Owing to the heating of the great plains of Asia. where the air ascends during the months from May in . Mugust, the south-east trade wind, wheh is blowing south from he equator, is drawn northwards, at the simme lime being deflected to the west. Hus forming the S. WT. monsoun. Compersely, when he plains conl from Xovember 10 March, hlore is a breege from the noth-east inwards the equator which, lhough known as the X.le. momsonn, is really a lrade wind. \%he S.IV. monsonn is laden with moisture. I failure of the ratis from this monsonn means a serions Indian famine accompanied by lons of thumsands of deaths. 


\section{RAINFALL.}

Rain is always cooling, and may be abundant in tropical countries.

Tropical rains are usually very heavy, so that they predispose to floods and the formation of swamps. In India the prevailing winds regulate the rainy seasons. Generally there are two rainy seasons each vear between $5^{\circ}$ and $10^{\circ}$ from the equator, one when the sun is on the zenith and going away from the equator, and the other when it is returning. Otherwise there is but one rainy season during the year. The violence of tropical rains is caused by the additional amount of water that warm air can take up, thus at:-

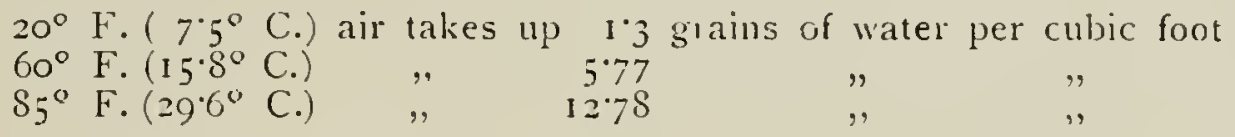

When saturated air at $85^{\circ} \mathrm{F} .\left(29^{\circ} 6 \mathrm{C}\right.$.) is cooled to $60^{\circ} \mathrm{F}$. $\left(15^{\circ} 8^{\circ} \mathrm{C}\right.$.) every cubic foot will yield seven grains of water (Hill).

Owing to the excessive heat and moisture in the tropics, putrefaction and fermentation are very rapid and often dangerous to man.

\section{SOIL.}

Soil affects the temperature by its power of absorption and radiation and its vegetation. Sandy soils are hottest and emit a glare which is very trying to the eyes. Clayey soils are coolest because of the water they hold. They cause a high atmospheric humidity, retard evaporation and radiation. The climate in consequence is made more equable, but more oppressive and predisposing to rheumatic troubles and chest diseases.

Soil temperature varies but slightly at a depth of $S$ feet below the surface, and is not affected at all at a depth of 40 feet (Galton). Trees and shrubs intercept the sun's rays and check evaporation from the soil and thus render the ground cooler.

Evaporation, however, is great and takes much moisture from the soil.

In Algeria the Eucalyptus globulus absorbed and evaporated twelve times the rainfall. Grass renders soil cooler, drier, and of more equable temperature.

When soil is permanently damp it is injurious to health. In order to keep the subsoil water at a low level subsoil drains must be put in, periodically cleaned and relaid.

A permanent dampness may be due to:-

(I) Clayey soils that do not drain themselves readily, thus forming swampy and mosquito breeding grounds.

(2) By an impervious layer approaching the surface at a given place and bringing the subsoil water-level higher than it otherwise would be. This level should always be 5 feet below the surface. 


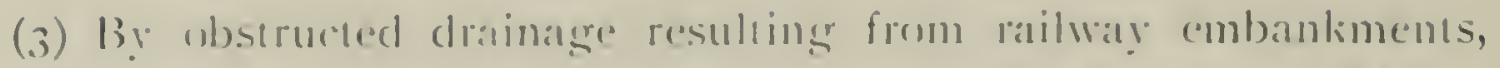
excatrattions, de. resulting from such constructional worlis. Also by sand pits and rlay loules.

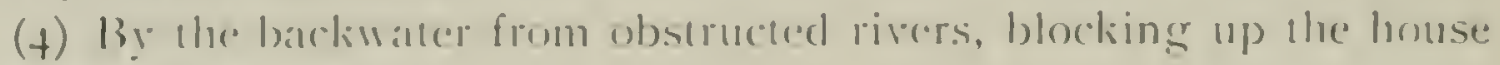
drains by its rofuse.

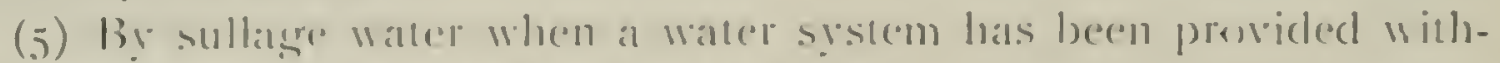

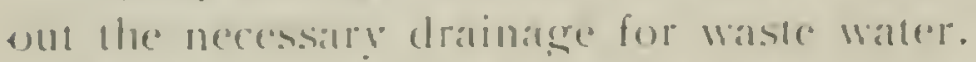

(6) De ut neatl the irregularities of the earth's surface, ats alt the fout of hills and low-lying coundry. Such places are often flouded for months after the rains.

(7) By He effects of large-scale irrigations, unacompanied by efficient drainage. To correct this latter fault the Indian Sanitary powers have lavel down the following resulations:-

(1) The irrigation canals w be constructed along the line of the watlershed.

(2) The smaller camals lo be so constructed as not to be carried across the natural lines of drainage.

(.) Dranage cuts to be constructed along the natural lines of outfall.

(t) The supply of water to be limited to the amount required. and to the particulat lime needed to secure the success of the crops. The Govermment of the Soudan have introduced the following regulations in regard lo irrigation:-

(1) Irrigation chamnels should be comstructed on a higher level than the surrounding (round, so that when :he fow of water in them ceases liney ma! drain drỵ.

(2) They should be constructed of such material, and in such a manner ats in prevent leakliage.

(3) Their bankis and beds should be kept in good repair and the breds even to prevent lace formation of ponls.

(4) "Drad ands" of irrigation rhannels should be reduced to :he smallest sime rompantble with efficieney, so that water will not slagmate in them).

(5) Vergelation should be periodically cleared out of the channels.

(6) Shluices should be comstructed sis bhat there is mo leabage to form slangmant puddles.

(7) Ilhere possible lish should be introduced. and licpt in the main chammels en destros lalrie.

(S) Lands where water is apt on stand should have proper surface drains.

(9) (reps, such as sugar rame, rice, or others, which require to stand in water, should not be grown wibhin a mile of an! town or villaige. 
(ro) In the event of an engine or pump breaking down, particular care should be taken to deal with stagnant pools, and petroleum should be used where necessary.

(i ) Malarial fever and excessive numbers of mosquitoes should be notified to the Governor of the province by the manager of the concession.

Rice-bearing ground must be closely watched and well controlled to avoid mosquito breeding, otherwise cultivation decreases the healthiness of the soil.

Ground air must be cut off from tropical dwellings by having a basement or foundations in cement or other impermeable material.

Loose sand may contain $5^{\circ}$ per cent. of ground air.

Ground air contains moisture, organic matter of animal origin, sometimes of vegetable origin, also, in consequence, ammonia, hydrogen sulphide, marsh gas and carbon dioxide in excess.

The ground air is in constant movement as a result of the wind, percolation of the rain, varieties of temperature and barometri: pressure, also by the rise and fall of the ground water level.

Organisms exist in the soil in abundance, nitroso bacteria (nitrous organisms), nitro bacteria (nitric organisms). These are found within $I \frac{1}{2}$ inclies of the surface. B. tetanus is world-wide. B. antluracis, bacillus of malignant aedema and B. typhosus may survive twenty weeks in the soil. The $B$. coli exists in the soil, but for shorter periods.

\section{CONSTRUCTIONAL HYGIENE.}

\section{Building Site.}

In selecting a site for a house, choose the side of a hill away from the native quarters and secure natural drainage.

Tablelands are always good. Avoid all depressions, feet of hills, ravines and narrow valleys. Avoid a luxuriant fertile soil which means dense vegetation. What is good for vegetation is bad for man. Select a dry site, granite if possible; "build your house upon a rock."

Good water should be accessible.

Clear all bush for 200 metres from the nearest dwelling. Leave high trees. Plant Bahama or Kasai grass, but keep it cut short or it will harbour mosquitoes.

There should be a slope from the house in all directions if possible, with small, shallow, open drains to carry off the water.

\section{The House.}

Mosquito-proofed brick houses, raised from the ground, will a broad verandah are most suitable for the tropics.

The rooms and verandalis should be ceiled to form a ventilated air space under the roof. The rooms should be lofty and boarded. 
If there is any glass in the windows it should be shaded from the direct sunlight by screens. Through ventilation is essential in all good houses. There must be a very good damp course in cement around all the walls. It should be remembered that an ordinary brick holds 16 ounces of water.

One must insist on the instant removal of every impurity and houschold refuse by burial or fire. One should aroid windowless cubicles and narrow passages. The kitchen should be built apart.

\section{Villages, Camps and Stations.}

The houses should be built along the lines indicated as far as possible. Always plan and plot as for a larger settlement.

Construction must be controlled or every cluster of dwellings will become a death-trap. Make all streets of one-storeyed houses 30 feet wide (10 metres), and those for double-storeyed houses 50 feet wide (is metres). The principal streets should be 60 to roo feet wide (22 to 36 metres). All streets should be parallel to or at right angles with each other and shaded by suitable trees.

All ordinary houses should be in alignment with one of the streets. If a township is anticipated plan for a large population, and allow for upen spaces, gardens, recreation grounds, schools, hospitals, cemeteries, latrines, wash-houses, \&c. All proposed buildings should be submitted by plan to the responsible authorities before their construction. Consider the possible demands for tram lines, rail roads, \&c. Provide a lane I 5 to 20 feet (5 to 7 metres) wide for sanitary purposes at the back of each row of houses. This will also serve for drainage, scavenging, air space, \&c. It will also prevent encroachments and will facilitate alignment.

Native rillages should be on a healthy site, well away from any European drellings. The huts should be of brick, daub or ant-hill clay with a grass roof. Such are cool, but the usual iron buildings are insufferably hot, even to a native. There should be permanent openings below the wall-plates for ventilation. The houses should not be more than $\mathrm{I} 6$ feet in height $(5$ metres) and built along a roadway, 30 feet (Io metres wide). Nine feet ( 3 metres) should be allowed between detached huts. They should be properly constructed with a latrine for each, and a back lane, parallel with the road, 15 feet (5 metres) wide.

\section{Jails.}

Formerly they were the endemic centres of bacillary dysentery.

At the present time much improved buildings are being erected.

The site area should be $5^{\circ}$ square yards ( $5^{\circ}$ square metres) for each prisoner. The dormitories should be 20 feet $(6$ metres $)$ wide and I 2 feet (4 metres) high with two rows of beds, one on either side and 
a good passage between them. Each bed should have 36 square feet (4 square metres), for the hospital $5+$ square feet (6 square metres) each (Bengal Jail Code). Solitary cells should have 75 superficial feet (S metres) and a cubic space of $\mathrm{I}$, ooo feet.

Drinking water must be sterile. Latrines must be well controlled.

If a prisoner loses more than four pounds in weight in two weeks lie should be sent to the Medical Officer.

\section{PHYSIOLOGICAL EFFECTS OF HOT CLIMATES ON EUROPEANS.}

The effects of hot climates upon individuals vary according to the humidity, altitude, sedentary occupation, town or country life, habits, \&c. The climate has been blamed for much that has been due to insanitary conditions, alcoholism and other excesses, but when all pathological conditions are ruled out the following generally hold good until acclimatization has taken place, a process requiring two to four years :-

(1) There is a greater supply of blood to the surface of the body accompanied by excess of slin functions, e.g., pigmentation and increased perspiration. Persons who cannot perspire should not reside in tropical countries, as perspiration is the chief means of maintaining a normal body temperature.

(2) The body temperature of an European in the tropics is slightly above the normal until thoroughly acclimatized. It is increased $0^{\circ} 05^{\circ} \mathrm{F}$. for every $1^{\circ} \mathrm{F}$. increase above the average normal temperature. Exercise, tight-fitting and unsuitable clothing will raise the body temperature.

(3) Owing to the modified distribution of the blood, the capacity of the lungs increases, more blood is drawn out and more air enters, thus reducing the reight of the lungs. As respirations are decreased in number the lungs actualiy do less work than in temperate countries. One must also remember that tropical air is rarefied and contains less oxygen than cold air, consequently less carbon dioxide is taken off in a given time, and there is a tendency to retain carbonaceous matter in the blood.

Nine per cent. less oxygen in inspired at $\mathrm{SO}^{\circ} \mathrm{F} .\left(27^{\circ} \mathrm{C}\right.$.) than at $32^{\circ} \mathrm{F} .\left(\mathrm{O}^{\circ} \mathrm{C}.\right)$.

(4) As the pulse is slowed and respiration slightly impaired the heart's action is weakened and the rital organs are less well nourished; hence there are diminished rigour, general debility, anamia, irritability, nervous depression, sleeplessness and irritability (the (ropical fury of Phehn).

(5) O'Connell in rgog sought to show that exposure to a damp tropical climate could cause an intermittent fever with hamolysis, 
poikilocytosis, melanzemia and enlarged spleen. This is not commonly held.

(6) The urine is diminished in quantity and in solids.

(7) The generative organs act more vigorously.

(S) Menstruation begins one year earlier with European girls living in the tropics.

(9) The children of European parents residing in the tropics during the active stages of growth are generally weaker than their brothers and sisters of temperate climes.

(Io) The effects of the sunlight are dealt with elsewhere. During the two to four years of acclimatization tropical residents are especially liable to disease from diminished resistance of both body and mind. Acclimatization for permanent residence of Europeans in the tropics does not take place.

Anderson's views upon this subject are as follows:-

(I) When a white species is well adapted to the conditions which environ it, it flourishes; when imperfectly adapted, it decars; when ill adapted, it becomes extinct.

(2) When a white man, a native of the temperate zone, goes to the tropics, there occurs a biological reaction of his system to the new environment, and a readjustment of co-ordination between his vital processes.

(3) In the tropics the white man, individually, can exist; racially, he cannot exist.

(4) Acclimatization is not possible.

(5) No superior race can successfully govern an inferior race superior in numbers with equality before the law.

(6) Only by partial enslavement of the coloured natives, superior in numbers, can the white man rule and govern the tropics, and it is only by relays of fresh representatives that he can continue his sovereignty.

(7) No colony of northern origin has ever been able to lead a permanent and independent existence in the tropics.

To obtain the best results all excesses must be aroided, while daily baths and exercise should be carried out religiously.

\section{PATHOLOGICAL EFFECTS OF HOT CLIMATES ON EUROPEANS.}

Those effects not due to parasites are of congestive or nervous origin. The abstraction of heat by the cool evening breezes often causes congestion leading to chills and predisposing to disease. At such times the blood leaves the surface of the body and concentrates in the viscera, such as the spleen and liver, causing congestion in them. Excessive exercise and alcohol will produce the same effects. 
The sun's rays will cause congestion of the skin, followed by increased pigmentation, sumburn, dermatitis, and probabl! xeroderma pigmentation.

Heat exhaustion is perhaps due directly to meteorological conditions, but sunstroke and siriasis are not so easy to explain. For details of these two disorders see separate section.

Neurasthenia is almost universal.

Loss of memory is common, especially on the IVest Coast of Africa, where it is known as "Coast Memory."

\section{THE RELATION OF CLIMATE AND HYGIENE TO TROPICAL DISEASES.}

A few of the outstanding features only will here be dealt with as these diseases are described elsewhere.

\section{Asiatic Cholera.}

This disease often subsides or disappears in the winter and in temperate climates, but it reappears with the warm weather without fresh introduction; hence heat is an important predisposing cause of the disease. Heat is not all, however, for if the temperature is rising, as in Calcutta during April and May, and the rainy season sets in, cholera is diminished, but when the rains cease in Norember it again increases, for at this time the temperature again falls. A moist soil, prolonged heat and drought are the best conditions for the spread of the disease.

It is a water-borne disease; hence no water should be allowed to become polluted, and all water should be sterilized before drinking.

Insanitary conditions of air, water, food, soil, drainage and housing determine the point of attack.

\section{Yellow Fever.}

This disease is endemic only where the mean temperature reaches $68^{\circ} \mathrm{F} .\left(20^{\circ} \mathrm{C}.\right)$. Frost ahways arrests it. [nless the temperature is very low, atmospheric humidity and rainfall favour it.

It is limited to the sea coasts and the mouths of great rivers.

It is seldom found over 700 feet high.

The disease commences usually in the most crowded and filthiest quarter of the town or ship if similar conditions prevail.

As the ultra-microscopic virus is mosquito-borne (Stegomria calopus), all conditions farourable for mosquito breeding are farourable for the spread of the disease, e.g., no mosquitoes, no yellow ferer.

\section{Plague.}

This is an infectious disease of filth that has robbed India alone of more than $7,000,000$ of its inhabitants. It is fostered by filh, orercrowding, lack of rentiation and destitution. 


\section{Malarial Diseases.}

Given the parasite and the mosquito, malaria tends to increase with the temperature. Atmospheric humidity favours it by encouraging mosquito breeding and by predisposing to chills in European residents. Clay soil is bad on account of the high subsoil water which encourages mosquito breeding. Complete temporary flooding or good draining will reduce its incidence anywhere. Its prevalence decreases with altitude, other things being equal.

\section{Relapsing Fever.}

Neither soil nor climate affect this disease.

Destitution, filth and overcrowding are powerful predisposing causes. These conditions favour the presence of the soft ticks, e.g., Ornithodorus moubata and Argas persicus, which are blamed as carriers of the disease.

\section{Leprosy.}

Climate and tropical conditions exercise but little influence upon this disease. Sanitary improvements in habits and surroundings will certainly assist in suppressing it. It has been called a disease of semi-civilization.

\section{Undulant Fever.}

This disease is carried largely by the infected milk of infected goats. It is also carried by water.

\section{Pellagra.}

The eruption recurs each spring and autumn. Exposure to the sunlight is a strong predisposing factor of the eruption.

The symptoms abate during the summer and almost disappear in winter. Agricultural classes are most affected.

It is almost world-wide in its distribution.

\section{Trypanosomiasis.}

Insanitary conditions favour the breeding of the Glossina which are necessary for its propagation. Destitution predisposes to the disease and raises the case incidence and mortality.

\section{Dysentery.}

Personal cleanliness is absolutely essential on the part of the patients and carriers. Excreta are the chief factors of its spread. Poverty, overcrowding and debility give good ground for bacterial dysentery.

\section{Beriberi.}

This is a deficiency disease in all probability. The best food under the best hrgienic conditions will prevent its onset and spread. 


\section{Dengue.}

Its prevalence is greatest in the hot season. It affects maritime towns and seldom spreads inland. Low-lying, filthy and overcrowded quarters are the first attacked.

\section{SOME GENERAL RULES FOR THE MAINTENANCE OF HEALTH IN THE TROPICS.}

All defective teeth should be corrected by a qualified dentist before the traveller leaves Europe.

It is preferable to abstain absolutely from alcohol.

If alcohol is taken it should be in great moderation after sunset.

Take a daily tepid bath, preferably at sundown, before food.

Cold baths are unsafe for all who have any bowel weaknesses.

Judicious exercise is of great value, taken in the early morning or the late afternoon, such as golf, cricket, croquet, polo and tennis.

Chills afterwards should be avoided.

The great temptation to lead a sedentary life must be overcome.

A muslin mosquito net, twenty strands to the inch, tucked under the mattress one hour before sundown, and kept in good repair, is essential.

High boots, leggings, or puttees are of great value for all travelling through bush for the protection of the legs from snake and insect bites.

Light khaki clothes with spine pads for the bush and white drill for the stations and evenings are best. Wool is irritable to the skin.

A mixture of wool-silk is comfortable, e.g., Anglo-Indian gauze.

Silk next to the skin may be necessary for those whose skins are irritable as a result of perspiration. \&c.

All personal servants should take a daily bath and be free from infectious disease.

All water should be boiled and filtered. It is a good plan to drink weak tea or coffee instead of water, as this ensures the water having been boiled. If a filter is not obtainable a little alum added to the vater is useful. Boil it after standing.

Keep all food supplies away from flies in a gauze receptacle.

All fresh milk must be boiled.

Take quinine; hydrochloride is better than the sulphate, 5 grains daily, except Sundays, when take 2 grains of calomel, followed by half an ounce of Epsom salts.

Jaeger sleeping sacks are useful for those sleeping out.

Always dress according to the temperature, and not according to appearances and the "climate."

After perspiring change clothes frequently. 
Take an hour's rest during the hottest part of the day.

Food should be well cooked, excess of meat and fat avoided, fresh fruits, salads and regetables should be sought after and substituted for the salted meat and rum of olden days. The effects from over indulgence of alcohol are marked.

If possible, it is advisable to spend the hottest months at a greater elevation. The next best thing is to use fans and punkahs to avoid heat debility.

Cholera belts are not necessary for healthy individuals, and to such are often a source of discomfort when worn.

It is absolutely essential to protect oneself from the sun's rays.

The measures to be adopted are as follows:-

Wear a light, well-ventilated pith or rubber helmet or topee with a broad brim and a prolongation for the neck. A white covering is best as it reflects the heat, but does not absorb it. The helmet may transmit chemical rays, and hence it is best that it should be lined with coloured material.

For those stooping at their work spine-pads are essential.

Broad rerandahs should be added to all buildings.

Reflection of the sun's ray's from the floors must be avoided.

There should be ventilated ceilings to all dwellings.

The roads should be shaded with trees having spreading branches.

Plots of green grass will prevent the glare from the sun.

Alcohol is the most predisposing cause of sunstroke.

Smoked glasses are very comforting from the glare. They will often prevent the onset of troublesome eye symptoms.

\section{EPIDEMIOLOGY.}

Communicable diseases may manifest themselves in one of four ways when one of the following terms is applied to it:--

\section{(1) Sporadic.}

A disease is sporadic when cases occur singly or in small groups in scattered communities, e.g., typhoid ferer, undulant fever, \&c. Such sporadic cases may precede endemic or epidemic manifestations, and should always be closely watched and controlled.

A sporadic disease may at certain times and places become endemic, epidemic and sometimes pandemic, such as undulant and typhoid fever. The divisions are purely arbitrary.

\section{(2) Endemic.}

A disease is endemic when the cases attach themselves more or less to a certain district or locality, e.g., cholera in the delta of the Ganges; leprosy in parts of Norway, \&c. 
These are often considered by those residing in the endemic area to be some of the unaroidable ills to which man is heir.

\section{(3) Epidemic.}

A disease is said to be epidemic when the cases are widely prevalent orer a larger or smaller area. Epidemic diseases, as a rule, tend to spread rapidly and to attack fresh communities.

Examples are small-pox, yellow fever, cholera, plague, dengue, trypanosomiasis, relapsing fever, anthrax, \&c., though some of these are limited in their distribution by the prevalence of their carriers, e.g., the mosquito, glossince, rat and rat flea, bugs, \&c.

\section{(E) Pandemis.}

A disease is said to be pandemic when the cases become diffused over most parts of the world, as influenza from Russia and plague from India.

The study of the modes of the transmission of communicable disfases increases much with the opening of new means of intercourse between countries and continents, e.g., the Suez Canal, the TransSiberian Railway, the Panama Canal, \&c. For example, plague may be brought from Guayaquil on the Pacific Coast, where it is endemic, and introduced via the l'anama Canal into countries where it has been obliterated, e.g., England. The arrival of one plague-infected rat in Liverpool from Japan caused a consternation in that city only a few months ago. There were eight cases of plague brought io London in October, 1919 .

When studying the question one has to consider the carriers and conditions under which the disease thrives. For example, if there are no mosquitoes (Stegomyia calopus) in the country of arrival, one need not be over-anxious about importing a case of rellow ferer, just as one does not hesitate to send to Europe cases of trypanosomiasis from infected areas to places where there are not any Glossina.

There are many other factors to be considered as locality, climate, season, surroundings, age and sex of those exposed.

The spread of ankylostomiasis needs heat and moisture; cold rill cut off the epidemic at once and define its margins. Likewise, bilharzial disease requires molluses; no molluses, no bilharzial disease. The old theories of air dissemination are in the main exploded, they being but a cloak for our ignorance, as the old term "eczema" was for skin diseases in general.

The whole facts affecting the spread of these diseases are not ret well understood even in adranced countries. We do not know why scarlet fever should tend to become epidemic at intervals of five rears in temperate climates where it has becone established.

The same applies to measles, which tends to be epidemic every two 
years. Whooping-cough of uncertain causation shows no marked periodicity, but tends to become epidemic every two years on an average.

\section{IVATER.}

\section{WATER-AMOUNT.}

One should estimate for about 15 gallons per head per diem for personal and domestic use, made up as follows :-

\begin{tabular}{lcccccc}
\multicolumn{2}{l}{ Domestic washing ... } & $\ldots$ & $\ldots$ & $\ldots$ & 4 gallons \\
Ablutions $\ldots$ & $\ldots$ & $\ldots$ & $\ldots$ & $\ldots$ & 3 &, \\
General baths & $\ldots$ & $\ldots$ & $\ldots$ & $\ldots$ & 4 &, \\
W.c. $\quad \ldots$ & $\ldots$ & $\ldots$ & $\ldots$ & $\ldots$ & 4 &,
\end{tabular}

One should allow for a little waste in excess of this.

In towns ro gallons per head should be allowed daily for municipal purposes, and if trade processes are at all numerous another 10 gallons should be added.

Hence, roughly, one may say provide :-

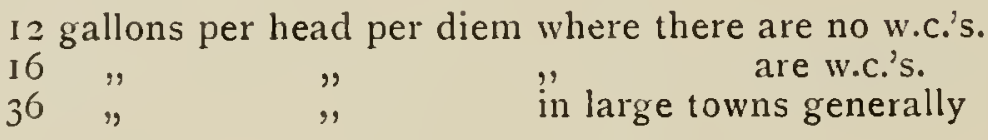

\section{WATER_SOURCES.}

All natural waters, springs, rivers, lakes, \&c., are derived ultimately from rainfall, which is water taken up from lumid surfaces of the earth, distilled under the influence of the sun's rays and distributed to the earth afresh.

Part of the rainfall is quickly evaporated. Some of it flowing above an impervious layer finds its way into shallow springs, streams and lakes. The remainder sinks below the surface below an impervious layer, when it may form deep springs.

Bodies of water may lie in large "pockets" either just above or below an impervious stratum; in the former case surface wells can be dug, and in the latter deep wells will be required in order to obtain it. A deep well, however, may not be so deep as a shallow well, the difference being according to the nearness to the surface of the impervious layer.

Ninety per cent. of rain water sinks below the soil surface in sandy and gravel districts, while a clayey soil permits of an exceedingly small filtration.

In places where rainfall is scanty, as at Aden, or only salt water is available, as on the ocean and on salt lakes, drinking water is obtained by the distillation of the salt water.

This process is carried out for the European residents and troops at Aden, for the miners on some Australian mines and on board war and other vessels. 
Distilled water can be aerated at the same time by a Normandy's condenser. On British warships five gallons per man per diem is distilled and aerated.

In land districts where there are no rivers, wells, or storage tanks for the rainfall, boring should be carried out. This should be done in a hilly country for preference. The required depth will depend upon the distance from the surface of the impervious layer.

Select the following places for experimental borings:-

(I) Under dry river beds.

(2) At the junction of ravines and valleys.

(3) At the foot of hills.

(4) In a thickly wooded country. When a country is cleared of vegetation and timber, the rain water flows away very rapidly and permanent streams are few.

(5) Examine along the line of any geological fault in the part.

(6) Test localized areas over which fogs or swarms of insects are noticed in the early morning.

(7) Limestone and sandstone districts are good for deep wells, but unfortunately there are large inland areas where such do not exist.

We will now deal with the subject under three heads, viz.: Rain Water; Surface Water; Subterranean Water.

\section{Rain Water.}

Rain water is soft, well aerated and pure, apart from the impurities taken up by it from the atmosphere. It also absorbs atmospheric gases, such as carbon dioxide, oxygen and nitrogen, the last two in the proportion of $S$ and $16^{\circ} 5$ per litre.

Tropical rain water varies much in quantity, from 600 inches per annum at Cherrapunji, in India, to rainless desert tracts. At Aden rain may not fall for several years.

Rain water may be colfected from the roofs and stored in tanks, or from large surface areas made impermeable by cement, \&c., and stored as at Gibraltar. When collected from iron roofs the latter should not be painted, as the paint, containing lead, may lead to lead poisoning.

In some parts, as in the Choco, Colombia, there is nearly one inch of rainfall every night all the year round, so that permanent storage is not required and fresh water is always available.

Birds should always be kept off collecting roofs as they pollute the water. Wires should be placed above and along the ridges and the guttering to keep them off.

In rainy districts pollution from the dust and decayed regetation does not arise.

To determine the rain water available it is necessary to know two things :- 
The annual rainfall in inches as ascertained by a rain gauge.

The square inches of sectional area of the building (not the slant surface of the roof).

The annual rainfall in inches multiplied by the square inches of sectional catchment areas equals the cubic inches of water per annum. This multiplied again by $0^{\circ} 0036$ gives the number of gallons per annum.

The storage of rain water is often a problem. In Venice the system is good, e.g., the soil is excavated, and the reservoir thus formed is lined with brick and puddled clay. In the centre of this a brick well is built, with openings in the sides towards the bottom of the shaft. The remaining part of the reservoir is filled with sand, into which the rain water first flows. In other countries there are underground tanks (Buenos Aires), stone vaults (Jerusalem), or large galvanized vats on pillars (Demerara and South Africa). Probably the best is a tank of galvanized jron or in brick and cement, raised from the ground, protected by a roof and made mosquito proof. All such, whatever their construction, must be under the constant observation of a Medical Officer of Health to control fly breeding, \&c.

When preparing storage tanks the longest duration of the dry season must be known with the accurate rain returns and the amount of water required daily. The average during twenty years, less onethird, is about the rainfall in the driest year, and plus one-third in the wettest year.

Only a little more than one-half of the rainfall should be considered available for storage.

First washings from the roof should be rejected. This should be by a small receiving tank with a float and plunger which, when the tank is full, diverts the flow into the storage tank. A simple method is to have the elbow of the tank supply pipe half-way between the roof and the waste gully at the bottom. When the rain has flushed the roof, plug the bottom of the pipe, the water then rises up to the elbow, when it passes down into the tank. Some suggest that it is always better to pass the water through a sand filter before running it into the storage tank.

All pipes should be protected by mosquito copper gauze.

To ascertain the dimensions of supply pipes needed and the possible discharge of cubic feet in a given time through a given pipe is often troublesome. The following formula will be a guide:-

If water with a head of $I I$ feet flows through $L$ feet of pipe $D$ inches in diameter, the discharge $W$ will be in cubic feet per minute:-

$$
\mathrm{W},=4.72, \frac{/ \overline{\mathrm{I} \times \mathrm{D}^{2}}}{\mathrm{~L}} \text { hence } \mathrm{D}=.538^{5}, \frac{\mathrm{I} \times \mathrm{W}^{2}}{\mathrm{H}}
$$




\section{Surface Water.}

We have stated that rain water flows in part over the surface of the ground into natural drainage channels, such as rivers and lakes. The water becomes polluted according to the media through which it passes.

Lakes. These when natural or artificial form the purest supply when containing upland waters. Their purity is the result of :-

(1) The action of light, destroying surface bacteria.

(2) The subsidence of suspended matters entangling bacteria.

(3) The draining of underground waters which have already undergone filtration discharge into it.

(4) Its free oxidation.

Algae are apt to form in all standing waters and should be removed by screening and filtration. It is better to store underground water in covered reservoirs, 12 feet deep at least, with perpendicular sides to diminish the growth of alga when this is troublesome. Copper sulphate can be added also in the strength of $\mathrm{I}$ in I, 000,000.

The catchment area should be cultivated and be as free as possible from dwellings of man or animals.

Open conduits for water converance should be immediately condemned.

There should not be any peat in the catchment area, for not only does it make the water brackish but it is particularly solvent of lead. If the presence of peat cannot be avoided, add lime to the water.

Rivers. Tropical rivers are usually of greater volume and are less polluted than rivers in Europe, hence the water is purer.

The fouling of the water by dead bodies in India and Africa is becoming a thing of the past.

The purity of these waters is due to:-

(I) The great dilution of any pollution that reaches them.

(2) The great volumes of filtered water that reach them.

(3) The gradual subsidence of organic matter to the bottom and sides where it undergoes disintegration.

(4) The oxidation and bactericidal influence of sun and light. There is no river long enough in the Lnited Kingdom to become pure by oxidation alone.

The water from small shallow rivers is always dangerous and should be aroided. In any case a pipe to draw river water should have its intake as near to the middle of the bed of the river as possible and above any villages that may be on or near its banks.

The water should always be boiled and filtered.

Tanks and Ponds. Water from surface drainage is often directed into tanks and ponds as is the case at Calcutta, but they are always 
subjected to much pollution. They should not be encouraged especially amongst the native peoples of a country. They form at good growing ground for all kinds of parasites, worms, cyclops, cholera and typhoid bacillus, mosquito larræ, \&c., \&c.

\section{Subterranean Water.}

When rain water falls upon permeable soil much of it percolates through the first impermeable stratum, the depth of whicil may vary much. The water flows on the surface of this layer in the direction of its fall and may :-

(I) Become collected in the indentations of its surface and form shallow wells,

(2) Form a reservoir at the foot of the fall,

(3) Find a place where the impermeable layer comes to the surface when the water passes beneath it, forming deep reservoirs of water, deep springs, constant or intermittent or when tapped deep wells are formed. This deep water may also pass into a river, lake, or into the sea, its movement being fairly constant but slow. The level of this underground water varies much according to the rainfall, adjacent springs, river level, \&c.

Subterranean water is then classified according to its relation to the first impermeable stratum thus:-

(A) Ground Water above the impermeable layer forming :-

(1) Shallow wells,

(2) Surface springs.

(B) Decp-seated Water beneath the impermeable layer forming:-

(1) Deep wells.

(2) Nearly all springs,

(3) Artesian wells; in these the water must be under sufficient hydrostatic pressure to force itself to the surface when the well is bored.

\section{Shallow Wells and Springs.}

The depth varies from 2 to 5 o feet according to the depth of the upper impervious stratum and the depth of the water lying upon it.

The area drained and affected by pumping from it varies according to the nature and lay of the surrounding soil, but it may be from 20 ta 200 times the area of the depression.

The water may be pure while the drainage area is free from contamination as was the case in the South African War before men and horses were picketed upon it, and as at Maidstone before the hop-pickers squatted upon it, and as in certain districts of India prior to their occupation by pilgrims. 
As the water percolates through the soil it becomes charged with carbon dioxide from the air interstices of the soil, after which it may dissolve out lime, mineral salts and organic matter. The soil tends to filter and remove this while the oxygen present in the soil may oxidize it with the formation of nitrates.

The water may be contaminated by: Cesspools, privies, manure heaps, sullage water, stables, burial grounds, latrines, and as the result of men and animals residing on the area. The contamination

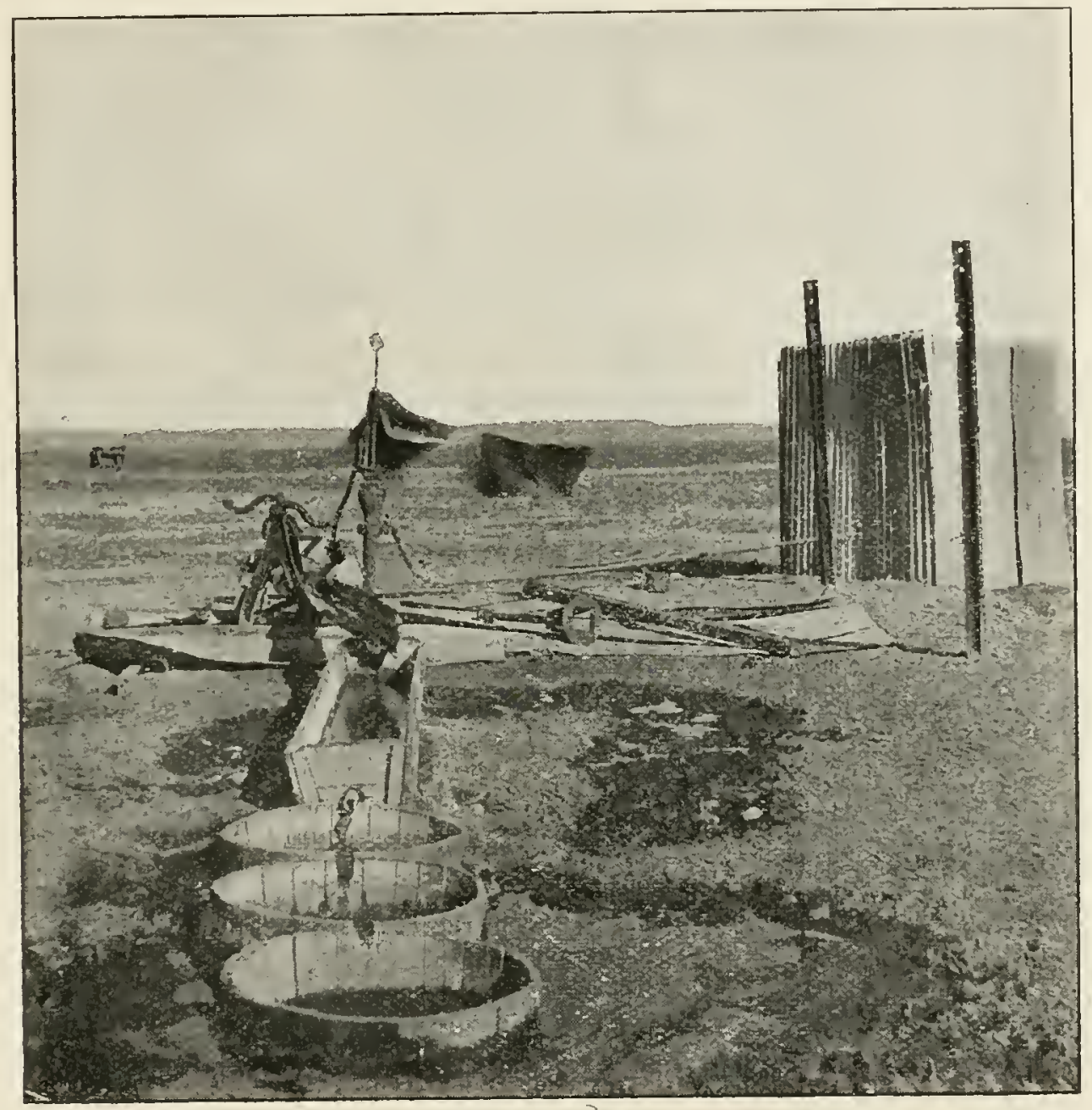

Well with bath and washhouse on one side. These are covered with old galvanized iron, old rail, \&c. Tubs where horses and callle stand and drink. The drainage runs back into the well.

may not be obvious until the rains have washed the filth into the water or until the water has risen in level until it reaches the filth, or carries water with the pollution over eminences of the impervious layer contaminating the wells and springs beyond.

In India and China infected natives often wash themselves neas to a well or spring, so that the ablution water will clrain back again into the well or spring in a polluted condition.

A native will bathe thrice daily, yet he will drink water unhesitatingly which is obviously filthy。 
A large proportion of typhoid epidemics is due to the use of shallow wells.

Any well should have the brick-work run up to a height of several

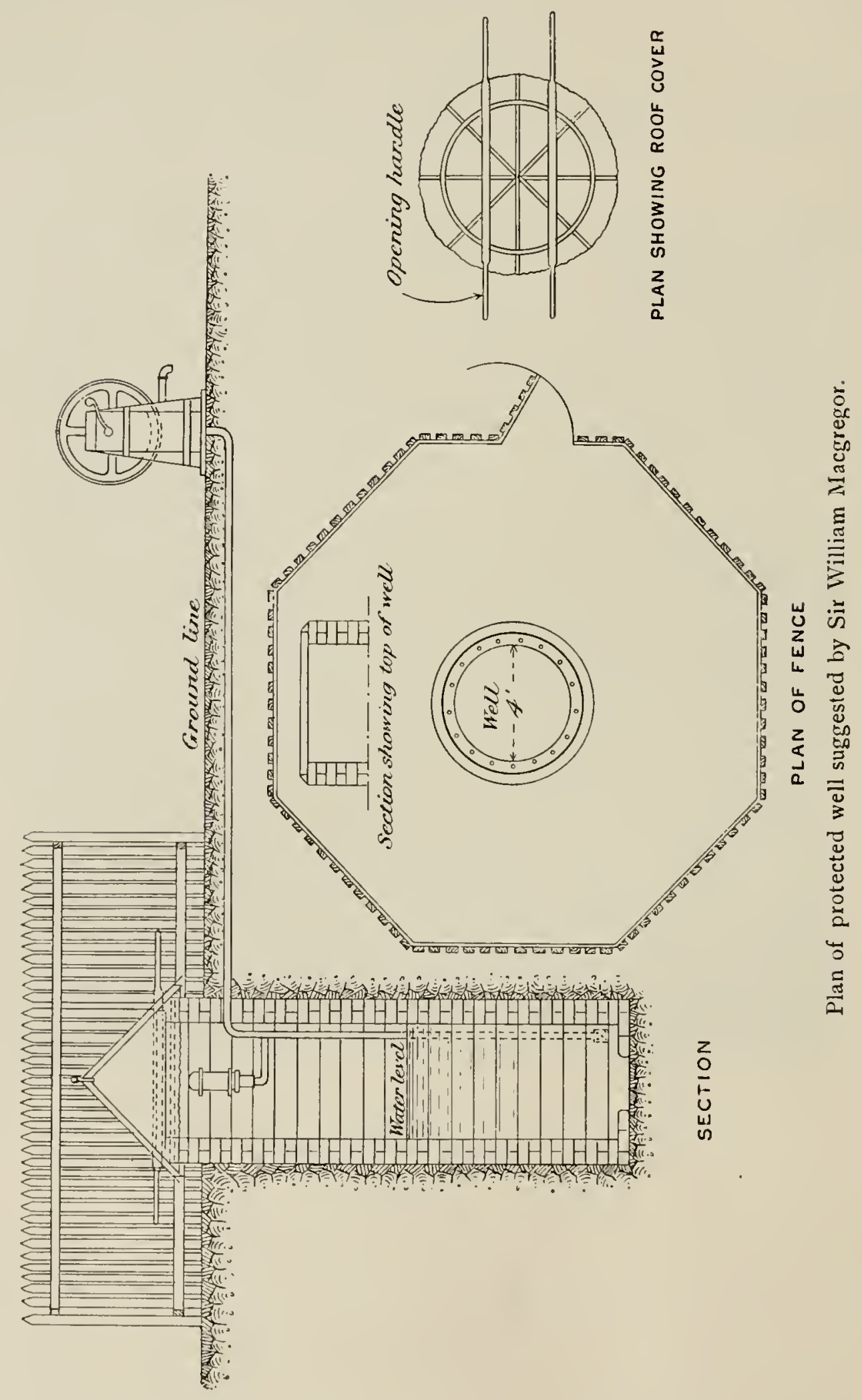

feet above the surface of the ground, with a dome-shaped cover over the top, well cemented, and the sicles cemented down to the impervious layer or to below the water level. Around the well for a distance of I 2 feet should be concreted, sloping off from the well so that drainage away from the well will be facilitated. 
A tube or Abyssinian well for shallow water in the tropics is often a temporary necessity. The water is usually within 25 feet of the surface. This tube-well cannot penetrate rock.

It has the advantage that the tube, $I \frac{1}{4}$ to 4 inches in diameter, may be driven beneath the upper impervious layer and so reach deep water. A pump is attached to the upper length of piping which is embedded in cement at the top. Water is yielded according to the diameter of the pipe, the supply being constant. Thus:-

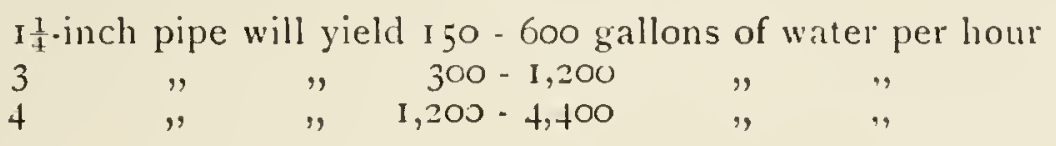

The Sullivan's diamond drill produces a form of bore well that can penetrate rock and produce large supplies of water. The tube can be sunk at an average rate of 28 feet in twenty-four hours.

Such a bore-hole will produce much more water than a well of 6 feet diameter. The Chinese are credited with boring by means of a rope and chisel down to a depth of 1,000 feet in the olden days.

An ordinary well can be converted into a tube-well by inserting a tube and filling the well with sand and pebbles.

The pump can be erected some distance away from the well.

\section{Deep Wells.}

This water supply is derived from a wide catchment area or sometimes from distant hills. As a rule the filtration has removed all organic matter but the surface and surroundings of the well must be protected.

Artesian (from Artois in France) wells are artificial springs.

The water is pure if it does not contain an excess of mineral matter. A great advantage of this kind is the depth to which they can be sunk, I, 500 to 4,000 feet. In this way cholera can be avoided as at Pondicherry.

Water can be found in districts formerly considered waterless, by these means as at Queensland, and pure water has been obtained on the sea shore as at Java.

The water from these wells is sometimes brackish though it is pure and its temperature sometimes raised.

\section{WATER_-TRANSPORT.}

House to house distribution by means of pipes is a rare luxury in the tropics. A supply in each street is not as common as it should be. In India the Mahommedan water-carrier serves each house from his mashak of goat or calf skin by means of which he can generally infect water that is pure prior to its being put into the receptacle. 
The metal vessels used by the Hindus for water transport are certainly an improvement on the mashak, especially as the metal tends to sterilize the water. They can also be better cleaned.

A pipe supply from a reservoir is preferable. For a reservoir a valley could have a concrete or clay puddle and a dressed stone, water-tight division thrown across it. Or an excavation could be made. An overflow weir and an emptying pipe are essential.

The points to watch before construction are: Suitability of site, sufficiency of water supply, amount in gallons to be supplied per head per diem, size of reservoir, number of days' supply to be stored, the primary use of the reservoir, i.e., is it to be a quiescent settling basin or preferably a continuous-flow settling basin?

Conveyance by means of pipes, galvanized and lined with pitch or other anticorrosive substance is to be preferred to the open channels.

Air vents will be needed at the summit, debris sluices below and cleansing sections at intervals.

Iron pipes become diminished in diameter owing to :-

(I) Incrustations from oxidation which can only be prevented by several more layers of pitch on the inner side.

(2) Ferruginous slime in acid waters formed by germs and young chlamydothrix which become attached to and grow rapidly upon the inner linings. Alkaline waters and those containing carbon dioxide are free from them. The Clarke softening process is necessary, followed by efficient filtration.

(3) Debris in the irregularities of the water-main which can be corrected by brushing and rapid sluicing.

\section{WATER_PATHOLOGY.}

A pure soft water acts upon zinc, e.g., on galvanized kettles, \&c., as a solvent so as to make the water injurious to health. Lead is also dissolved by soft waters. Such water should be hardened with lime.

There is no evidence that hard water is injurious. It does not bring on old age neither does it cause gout, rheumatism, \&c.

Waters that are peaty owing to their acidity often dissolve lead from the service pipes as lead carbonate. On standing or boiling the lead carbonate is thrown out with calcium carbonate.

Vegetable matter in solution or suspension, from any source, may, if in sufficiency, cause diarrhoea.

Animal matter in solution or suspension is always dangerous to health, causing reduction in general bodily resistance, acute alimentary disturbance, epidemic diarrhœa, dysentery, cholera, parasitic diseases and diseases of the typhoid group. 
Mineral or chemical constituents may cause epidemic soitre, plumbism, lead colic, urinary calculi (?), cretinism, \&c.

Brackish water often causes diarrhaca in those newly coming into the district.

Excess of alkaline carbonates causes constipation.

A large number of non-pathogenic bacteria will cause mucoenteritis.

Suspended clay, mica, quantities of sodium, calcium and magnesium salts will cause intestinal disorders.

Putrefying carcases of horses, cattle and humans will lead to diarrhoea and dysentery.

Dysentery is nearly always due to a contamination by excrementitious matter, both bacillary and amoebic, but urine is another factor in typhoid cases.

The ova and embryos of entozoa and leeches pass to the body in impure water.

\section{WATER_CONTROL.}

A suitable qualified Medical Officer must control the water supplies on behalf of the Company or Sanitary Authority.

His supervision must include:-

A knowledge of the "life-history" of the water.

The systematic inspection of the source, gathering ground, reservoirs, means of conveyance and the control of the storage tanks, filter beds, \&c.

The physical, chemical and bacterial examination of samples from various points of the supply.

\section{WATER-SOURCE OF CONTAMINATION.}

Elementary tests are very useful to ascertain the source of contamination and often of its nature. This preliminary being necessary previous to the adoption of purifying measures.

Where apparatus and chemical reagents are not to hand obvious contaminations should be dealt with at once without awaiting analysis.

To ascertain source of contamination test by putting chemicals in the water and searching for them later elsewhere, e.g.,

(1) Sodium chloride is traced by the silver nitrate test.

(2) Lithium chloride is traced by the evaporation of quantities of the water, the residue gives a crimson colour. One part in one million can be recognized. The test is expensive.

(3) Ammonium chloride can be traced in one million gallons of water if seven pounds of the salt are used. 
(4) Fluorescence or resorcin thallin is traced by its colour, one in one hundred million parts of water. Six ounces of the substance should be dissolved and distributed on the refuse suspected of carrying the contamination. This will show itself in the drains, wells, \&c., in a short time if such contaminated water drains into them. Should the water happen to be acid there will be no colour, but one must add some caustic potash when making the solution. Pour plenty of water on the refuse heap after the fluorescence has been distributed. Bromine will remove the colour when the test is finished.

One must always exclude the presence of the testing salts in the water before making the test.

\section{WATER - PURIFICATION.}

The purification of all tropical waters is essential.

For small quantities the apparatus must be small and the process simple, rapid and efficacious.

For large quantities there will be a permanent apparatus and the process will be elaborate, slow and also efficacious.

The ways and means will be also controlled by the peculiar contamination to be removed as alga, lead, iron, \&c.

There are many methods, selected according to each particular case, two or more of which may be used for the same water.

For the personal use of travellers the best means are :-

(I) Boiling.

(2) Berkefeld's pump or ordinary filter.

(3) Chemical soloids of calcium hypochloride, \&c., such as are supplied by B. W. and Co.

Such methods can also be used for larger quantities.

The principal means in general for water purification are mechanical, chemical and filtering effected by means of storage, precipitation, chemicals, heat, electricity, filtration and oxidation.

\section{(1) Storage.}

This does not sterilize water.

In water stored for one week, 99 per cent. of cholera organisms will be killed. In that stored for three weeks none remained.

In water stored for five to eight weeks the typhoid bacillus will die, hence storage is a great help towards sterilization.

Storage for one month with a series of reservoirs and a continuous flow to avoid the development of algre, is said to be almost an absolute protection against epidemic infections. Not only are the bacteria enormously reduced but other unpalatable conditions and injurious 
ingredients as suspended matter, ammoniacal nitrogen and also hardness are removed.

By storage the bacteria become entangled in the precipitate and are carried down.

\section{(2) Precipitation.}

To assist in this process various substances are added such as:-

Vegetable Juices containing tannin, e.g., tea, kino, \&c., which coagulate the organic matter. Cold tea is therefore an excellent drink for travellers because :-

It ensures the water having been boiled.

The tea strains suspended matters.

The tannin coagulates and precipitates organic matter.

Chemicals are also used, as alum 6 grains $\left(0^{\circ}+\mathrm{grm}.\right)$ per gallon ( 45 litres) followed by 5 grains $\left(0_{3} 3 \mathrm{grm}.\right)$ of precipitated lime per gallon. Alum also decreases the number of micro-organisms present but not those of the typhoid and cholera groups.

\section{Chemicals.}

Chemicals are used for softening and purifying water, but all such methods should be followed by filtering.

The alum, lime and soda compound method of Maignen's process (anti-calcaire) is well known. All similar processes contain lime which combines with the carbon dioxide and forms a deposit of calcium carbonate carrying down with it the organic matter.

Clarke's process for softening water is popular. Use freshly burned lime, 6 pounds $(2 \cdot 5 \mathrm{k}$.) per 100,000 gallons of water, for each degree of hardness.

The Porter-Clarke process is a modification of this. The former slow process is hastened by filtration under pressure through cloth.

Chloride of lime is also used, i to 6 parts of available chloride per million is sufficient. Filtration and passing the water through carbon will remove the excess of chlorine.

If the water is free from suspended matter, calcium hypochlorite is useful.

Use three parts per million = one part of available chlorine, and remove the excess a little later with sodium bisulphate (Thresh). This more or less sterilizes such water at a cost of $\mathrm{Id}$. per 25,000 gallons. The only apparatus required is that of ordinary tanks.

Six hundred American cities now use this process.

In smaller quantities of water use a teacupful of calcium hypochlorite and three teacupfuls of water. Of this mixture use one teaspoonful to two gallons (nine litres) of water. Allow it to rest for fifteen minutes. 
To those accompanying expeditions, \&c., the following facts by Dr. Thresh may be useful :-

(I) Obtain a supply of high quality chlorinated lime in quarterpound hermetically sealed tins. (From the United Alkali Co. Liverpool.)

(2) Obtain a corresponding number of half-pound tins of sodium hyposulphite. This salt, known more commonly as hyposulphite of soda used in photography, is nearly tasteless and combines with all the available chlorine in about half its weight of chlorinated lime. From these the following stock can be prepared.

(3) Add the contents of a tin of No. I to a gallon of water and shake until uniformly mixed.

(4) Add one packet of thiosulphite to one gallon of water and shake until dissolved.

One gallon of solution No. 3 will sterilize 8,ooo gallons of any ordinary clear well or river water in fifteen minutes, and if at the expiration of that time the one gallon of thiosulphite solution No. 4 be added to the water any excess of chlorine will be eliminated. One fluid ounce of No. 3 is sufficient for 50 gallons of water. In no case should less than I part of chlorine be added to $\mathrm{I}, 000,000$ parts of water, and if the water is known to be foul, 2, 3, or even more parts should be used.

This process can be clepended upon to remove any typhoid or cholera organisms which may be present in the water, and the same applies to the species of bacilli which are the probable cause of dysentery.

Iron is removed by adding lime and then filtering through sand.

For Anderson's process iron is required. The water passes through revolving drums and is subjected to showers of metallic iron. The excess of the latter is removed by oxidation, precipitation and filtration. It is not applicable to peaty waters.

Ozone is now becoming better known. The water falls from a tower through rough stones and ozone, which latter is admitted at the bottom of the tower. The ozone rises, meets the descending vater, and sterilizes it as it descends. The ozone is prepared by passing dried air through ozonizers supplied with electricity.

These machines are expensive, but the method is very efficacious.

Each ozonizer of one horse power and of 8,000 volts yields 13.5 to 27 grams of ozone in twenty-four hours and sterilizes 26,400 to 52,800 gallons per diem. The excess of ozone is lost by passing the water over cascades at the bottom of the tower.

Water containing iron would still require filtering. 
A portable petroleum-driven motor and apparatus is on the market which is capable of sterilizing foo to 600 gallons per hour equivalent to 3 cubic metres. This is very suitable for troops.

Like most processes it has its disadvantages, e.g.,

(1) The water often acquires a disagreeable taste.

(2) Nitrous acids are often produced along with the ozones.

(3) The initial cost is considerable.

(4) Skilled supervision is necessary.

Permanganate of potash, I in 4 ,ooo or 4 ounces ( $113 \mathrm{grm}$.) for an ordinary well is sufficient. It is useful for cholera-infected wells.

The water should remain pink for half-an-hour afterwards.

Alum will remore the excess of colour. It will also remove the odour of offensive-smelling waters. For smaller quantities, boil the water and then add the permanganate until a pink tinge remains for thirty minutes.

The Compound Tablet is now in use and has proved its worth.

The ingredients of these tablets vary but that of Vaillard is a good preparation :-

(1) Blue tablet contains: Pot. iodide, O' I grm., sod. iodide, 0.0156 gim., and of methriene blue, a sufficiency.

(2) Red tablet contains: Tartaric acid, 0*100 grm., fuchsin, a sufficiency.

(3) White tablet contains: Sodium hyposulphite, 0*1 I6 grm.

To one litre of water add one tablet of Nos. I and 2. As a result of this 0.06 grm. of free iodine is liberated which sterilizes the water in fifteen minutes. Then add a white tablet which neutralizes the free iodine. The resulting water is colourless and odourless.

Ora and probably parasites are not destroyed by it.

Liquid chlorine has been used for the troops in the field.

I' 50 grm. of chlorine for 50 gallons of water.

Then sodium sulphite, $36 \mathrm{grm}$. are added to neutralize the chlorine.

The gas disappears spontaneously in twenty-four hours, after which period no sodium sulphite is required. The cost is low, only id. per 500 gallons.

Copper sulphate is used, I in $\mathrm{r}, 000,000$ to :--

(I) Clear rivers and lakes of alga.

(2) Kill mosquito larve in water tanks.

(3) To rid waters of odours arising from micro-organisms and alga.

Fishes are killed by it.

Chalk, finely ground, is used, one grain ( $0^{\circ} \circ 6$ grm.) per gallor.

It prevents plumbo-solvency where this may take place. The vater must then be filtered and lime water added. 
Peaty waters containing acids from moorlands cause plumbosolvency.

Lacmoid will become reddenea in such waters, thus indicating their presence. Manganese oxide on the filter beds is efficacious.

\section{Thermal.}

Boiling destroys all living organisms and reduces hardness but the difficulty is with cooling afterwards. Earthernware vessels placed in an air current are useful for this purpose. The flat taste can be removed by oxidation. Weak tea is advisable for travellers for reasons previously described.

Heat has been used to sterilize water under pressure so that no gases are lost; the cool water passing in being used to cool the water passing out, but the process is expensive when used on a large scale. The pipes are readily occluded when the water is hard. A number of inventions are on the market for this purpose.

The Maiche Automatic Sterilizer is serviceable. It cost (pre-war) $f_{56}$, uses 5 gallons of kerosene weekly, cost of maintenance is gd. daily, and it produces soo to I, ooo gallons of sterilized water per diem.

The oil lamp is filled twice daily, otherwise it is left to work automatically.

Steam pipes conveying steam to water tanks will sterilize the water, after which the water can be left to cool.

Distillation is of universal application and is essential for all important ships as sea water can be utilized br these means.

The flat taste is removed by the water falling through a sieve or by charging it with carbon dioxide under pressure.

\section{Electrical.}

Ultra-riolet rays, generated by a quartz mercury vapour lamp, are now used as sterilizing agents. Courmont and Nogier found that when a Kromayer lamp was immersed in water containing $1,000,000$ bacteria and 100,000 B. coli per c.c. that it was completely sterilized in one minute. Previous filtration is almost obligatory as suspended and colloidal matter nullifies the action.

Thresh and Beale found that a small Cooper Hewitt quartz lamp would purify 50 to 200 gallons per hour. At Luneville in France, there was an installation with a ten-lamp canal which has practically banished typhoid from the city.

The energy required is from 50 to 130 units per million gallons of water. A portable apparatus is now on the market.

\section{Filtration.}

\section{A. Domestic Filtration.}

The Pasteur-Chamberland porcelain filter and the Berkefeld filter of diatomaceous earth are the best. 
Unfortunately the mud and slime of tropical rivers rapidly stops the action of the best filters so that constant cleansing is necessary. They should be scrubbed and boiled once weekly.

The candles are very frail and readily break. A large stock of them must be kept.

Other filters are not considered to be as efficacious.

\section{A.-Domestic Filtration.}

A cemented reservoir is prepared, 5 to 6 feet deep ( 1 to 2 metres) and made up in layers of broken stones and pebbles, coarse gravel, coarse sand and fine sand. Mater should not flow through the filter at more than 4 inches $(100 \mathrm{~mm}$.) an hour, and not more than 200 , 000 gallons per acre per hour. The finer the sand and the thicker the layer the more efficacious it becomes.

A sand filter affords :-

(1) Mechanical obstruction to coarse particles.

(2) Oxidation of organic matter.

(3) Nitrification by micro-organisms on the surface layer.

(4) Removal of turbidity.

(5) Reduction of hardness and removal of iron.

There is a slimy layer on the surface of all large filters containing a gelatinous mass of intercepted bacilli, streptococci, micrococci, alga and other bodies, while immediately below this film is a layer of nitrifying organisms. By this film the pathogenic micro-organisms are intercepted and destroyed, the organic matter broken up into carbonic acid and ammonia, the ammonia being converted into nitrous and nitric acids by the nitrifying organisms. When the slimy layer becomes so thick that it prevents the water from passing through, half an inch should be scraped off the surface and the under layer distributed by means of a rake. When the sand layer is only one inch thick, the bed should be made up again. The first water which passes through before the slimy layer is formed (during the first twenty-four hours) may be impure and should be rejected.

Allow three feet (one metre) of water to stand in the filter for twenty-four hours.

There should not be more than roo micro-organisms per c.c. in the filtered water. 98 to 99 per cent. of those in the original water should have been removed by the filter.

Too much exposure to the sun favours the growth of alga, hence filter beds should be covered over. By this means dust and mosquito breeding will be avoided.

Mrater should pass through a settling or storage tank before and after filtration. The processes of filtration and sedimentation should always be combined. 
Mechanical sand filters are as the above but smaller and constructed of iron or steel, with pressure to hasten the process. Sulphate of alumina, $2 \mathrm{gr}$. per gallon ( $0^{\circ} \mathrm{I} 2 \mathrm{grm}$. per 4.5 litres) is added to form an artificial film on the surface.

The pressure is by compressed air, the pressure being reversed and often steam applied for the cleansing process.

The rate of filtration is about fifty times greater than the slow

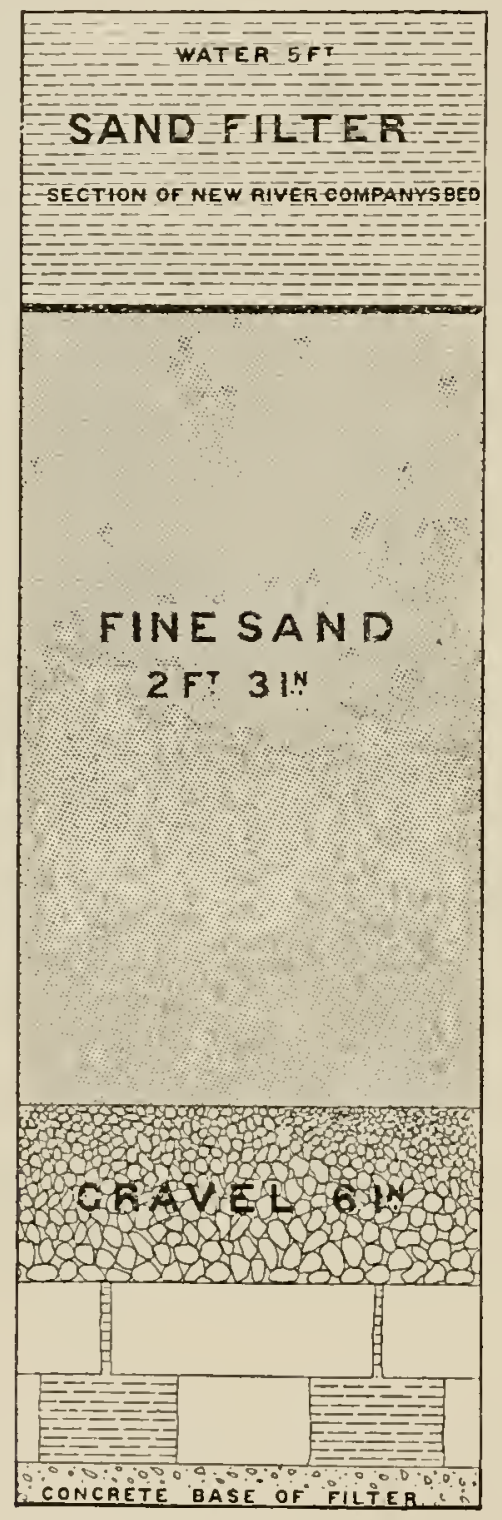

Diagram of a section of a sand filter.

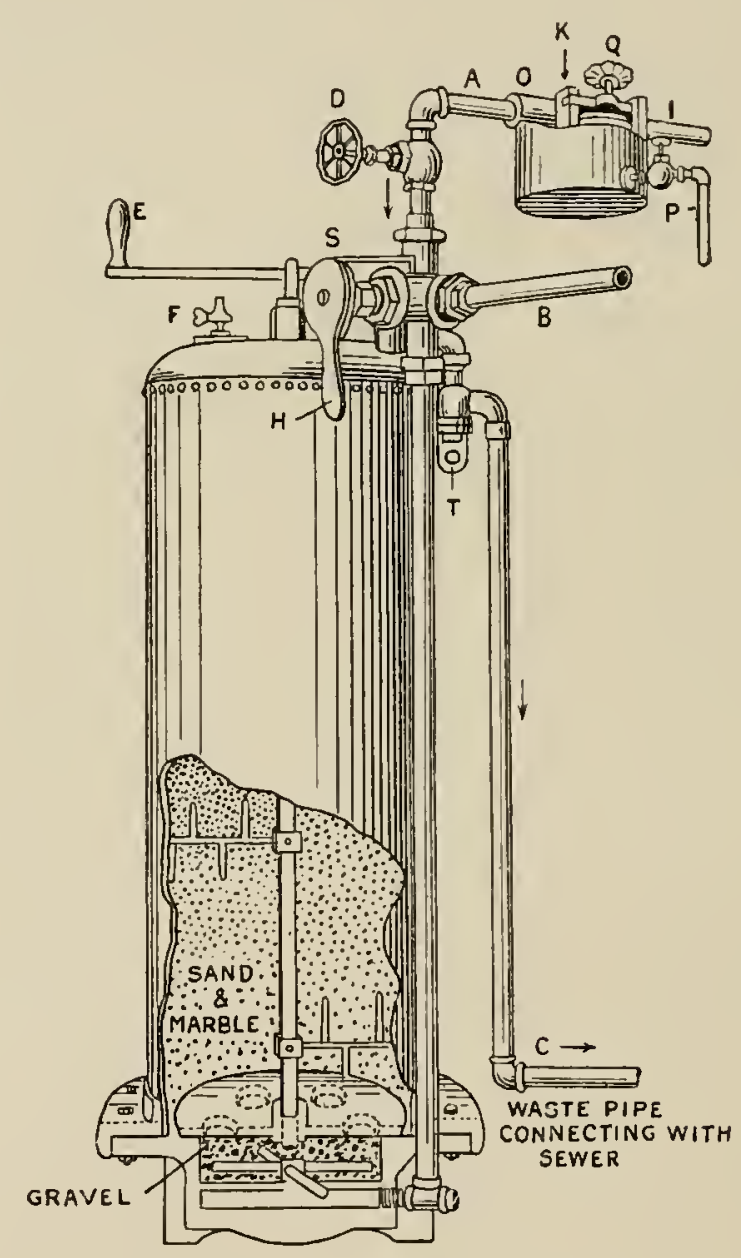

Diagram of a mechanical filter.

sand filter. Such filters lave proved useful at Alexandria and Mrsore, where the water is turbid. They are largely used in the States of America where the river water is heavily charged with suspended matter.

It is often necessary to cleanse washing water as for troops in the field. The following method as practised by Norman Goode and Basil Hughes has proved useful to such an extent that the same water can be used an unlimited number of times.

The soapy water runs from the bath house into a rinixing tank. 
In this tank slaked lime is placed and the whole well mixed. The lime throws down the soap as insoluble calcium stearates. It also brings down all the dirt and impurities.

The effuent flows through a series of three up-and-down settling tanks connected together, the scum is stopped by canvas screens placed vertically reaching two-hirds of the distance down from the top and up from the bottom alternately.

All the calcium stearates setile in the first two tanks.

The third tank is used for precipitating the lime and remoring the soapy oils. Washing soda is run into this tank which produces insoluble calcium carbonates which sink to the botiom. Consequently the soapy oils separate out, float and are collected on canras.

The canvas is changed daily.

The water then flows into a charcoal filter, sis feet square by eighteen inches deep with four inches of charcoal between two lavers. of sacking in a perforated zinc tank.

It passes from this into a concreted well.

The water is pumped from this well into an elevated tank for repeated use.

The mixing tank is cleaned out daily and the sludge is buried.

The first precipitating tank is cleaned out every third day.

The water thus treated is free from dirt, soap, lime, soapy oils and readily lathers with soap.

4.000 gallons pass through daily and 2,000 men use the same water daily.

\section{Oxidation.}

The "fishy", waters of Mauritius were made potable by means of a special oxidation process. The water was run over perforated trays, allowing it to drop 4 to 6 feet, finely divided, during which time the water became almost saturated with oxygen.

Oxidation is also carried out to some extent during intermittent filtration. The process can be used to get rid of iron.

This process :-

(I) Oxidizes the ferrous compounds.

(2) Oxidizes the ferric hydroxide, in both instances precipitating organic matters.

(3) Facilitates the destruction of residual organic matter.

(4) Aerates and hence makes potable certain drinking waters.

All water should be passed through a filter afterwards.

Boiled and clistilled water could be aerated in this way.

\section{Filterable Viruses.}

This term is applied to micro-organisms so small that ther wilf pass through an ordinary filter which will keep back ordinary bacteria. 
The first was demonstrated in 1892 by Iwanowski, the mosaic disease of the tobacco plant.

In man we find the viruses of :-

Yellow fever, which is destroyed at $53^{\circ} \mathrm{C}$. in ten minutes.

Dengue fever, Three days fever, Pappataci fever, Molluscum contagiosum, Poliomyelitis, Measles, Scarlatina, Verruca vulgaris, Trachoma, Typhus, which is destroyed by a temperature of $55^{\circ} \mathrm{C}$.

Variola and Vaccinia are both destroyed at $5^{\circ} \mathrm{C}$. in fifteen minutes.

In nearly every case the immunity produced by the disease is complete.

Secondary infections may give rise to many symptoms which may be confused with the true symptoms of the original disease.

It should be remembered that filtration of water will not aid much in the prophylaxis of the above diseases.

In advising as to water purification, all the facts of the particular case must be taken into consideration. Thus if the water is derived from a source free from human pollution and the ingredients to be removed are suspended particles, colouring matter and iron in solution, the mechanical filter will be sufficient.

But if the water is or may be polluted, some process of storage, sterilization, and slow sand filtration are necessary.

Mechanical filters combined with storage will give a satisfactory water supply in the majority of instances, but some waters will always require an adequate sterilization process as in some parts of India where pilgrimages are common.

For important notes on the Bacteriology of Tropical Waters, see p. 6fo, Secion IX, Laboratory Hints.

\section{FOOD.}

\section{NUTRITION.}

The essential constituents of food are:-

( I) Nitrogenous, animal and vegetable albuminoids (proteins) and gelatin.

(2) Fatty, animal and vegetable fats and oils.

(3) Carbohydrates, starches and sugars.

(4) Salts, organic and inorganic.

(5) Mater and vitamines.

Nitrogenous foods are necessary for growth, maintenance, repair, functional activity and in some measure the production of heat and force for the body.

Assimilation of excess of nitrogen, in the absence of exercise, leads 
to imperfect oxidation with the formation of urates and uric acid followed by congestion of the liver, looseness of the bowels, slight pyrexia and general debility.

Deficiency of nitrogen is followed by anamia, wasting, reduction of energy and resistance.

Gelatin is able to replace the circulatory albumin but does not form tissue albumin or repair its waste. Its nutritive value is 24 per cent. that of albumin.

Fats and carbohydrates provide material for oxidation which process produces heat and force. As a result of oxidation carbon dioxide and water are formed which in turn assist in nutrition and the removal of waste material.

Ingestion of excess results in lessened oxidation of nitrogenous matter and the storage of fat in the tissue.

Deficiency causes increased metabolism of circulating albumin, loss of reight and the impairment of nutrition.

Fatty tissue is a good lubricant, a non-conductor of heat, a protection for the deeper structures, and affords rotundity for the body.

An excess of fatty food may lead to inconveniences as in obesity, or to dangers as in fatty infiltration of the heart.

Carbohydrates are readily assimilated but are less nutritive than fats by to per cent.

Salts are used up in the body as follows:-

(I) For bone formation, e.g., lime, potash, soda and magnesia.

(2) The nutrition of the firmer tissues, e.g., potash.

(3) The maintenance of the fluid tissues, e.g., soda, chlorides, phosphates.

(4) The maintenance of alkaline fluids, e.g., organic acids as. lactates, tartrates, citrates, \&c.

(5) To increase oxygen-carrying capacity, e.g., iron salts.

Vegetable products are rich in potassium salts while animal foods are poor in them.

Trater. This is our greatest solvent and makes up two-thirds of oul body weight. $\mathrm{B} y$ it :-

(1) Food is dissolved and carried to the tissues.

(2) Chemical changes are made possible.

(3) The body temperature is regulated.

(4) Waste products are removed and excreted.

Food-adjuncts are non-essential foods used as a habit, to produce variety, stimulate appetite and to modify taste, e.g., alcoholic liquors, rolatile or essential oils and aromatic compounds found in condiments and spices. One may also include regetable acids and alkaloids as theine in tea, caffeine in coffee and theobromine in cocoa. 
The following remarks may prove useful: An average diet to nourish an adult male not engaged in hard work should be:-

\author{
Protein, 70 to $80 \mathrm{grm}$. per diem \\ Carbohydrate, 300 to $400 \mathrm{grm}$. per diens \\ Fats, 25 to $50 \mathrm{grm}$. per diem \\ Salts and vitamines
}

Variety and taste are not here considered.

A menu could be made up as follows :-

\begin{tabular}{|c|c|c|c|c|}
\hline White bread & $\ldots$ & $\ldots$ & 400 & grm. \\
\hline Butter or margarine & $\ldots$ & $\ldots$ & 30 & \\
\hline Bacon ... & $\ldots$ & $\ldots$ & 50 & ," \\
\hline Sugar $\quad .$. & .. & $\ldots$ & $5^{\circ}$ & $"$ \\
\hline Janı or conserves & $\ldots$ & $\ldots$ & 30 & \\
\hline Quaker oats ... & $\ldots$ & $\ldots$ & 30 & , \\
\hline Condensed mill & $\ldots \quad \ldots$ & $\cdots$ & 30 & , \\
\hline
\end{tabular}

The total average weight of solid food is about 40 to $5^{\circ}$ ounces daily.

To this is added water 50 to so ounces daily.

A man consumes daily $\frac{1}{10} \overline{0}$ of his weight of dry solid food and $\frac{3}{1} \overline{0}$ of his weight as water.

Women require 10 per cent. less than men, children of ten years of age half as mucl as a woman.

During a fair day's work the energy expended equals one-fiftl mechanical labour and four-fiftlis heat.

Deatl ensues when the tissue loss is about 40 per cent. of the normal body weight. This is from siarvation only.

The common ground nut (Arachis hypogea) would be a good food if one could digest all one could consume, as it contains :-

Of proteins, 245 per cent.; of fatty oil 50 per cent.; of other nitrogenous matter II 7 per cent.; salts I $^{\circ} 8$ per cent.; water 7.5 per cent.; fibre +5 per cent.

The soy bean (Glycine soja) contains in comparison proteins $35^{\circ} 3$ per cent.; fatty oil is.9 per cent.; other nitrogenous matter 26 per cent.; salts $4^{\circ} 6$ per cent.; water I I per cent.; fibre $4^{\circ} 2$ per cent.

The cassava plant is a common food in the tropics. Of it there are two varieties, viz.: the sweet (Manihot aipi) and the bitter (Manihot utilissima). The latter contains a small amount of prussic acid in its milky juice, which is dissolved out when meal or tapioca is made from it. The latter (tapioca) contains 86 per cent. starch.

The chief native foods are cereals, e.g., rice, maize, millet, $\&$ c., all deficient in fat and protein which is somewhat compensated for. by the pulses and the beans.

Dry rice when boiled absorbs four times its weiglit of water.

Its progress through the stomach is slow, the latter being somewhat distended by it for several hours. Its starch is well digested in 
the intestines. The best rice is lacking in phosphoric acid, lime and other mineral and nitrogenous matters.

Eggs, oil, beans, \&c., should be taken to compensate for this.

To calculate the caloric value of any food remember that :-

$\begin{array}{cccc}\text { One gramme of protein equals } & 4^{\circ} \text { I } & \text { large calories } \\ ,, & \text { fat } & 9^{\circ} 3 & , \\ ,, & \text { carbohydrates } & 4^{\circ} \text { I } & ,,\end{array}$

Voit's standard diet is :-

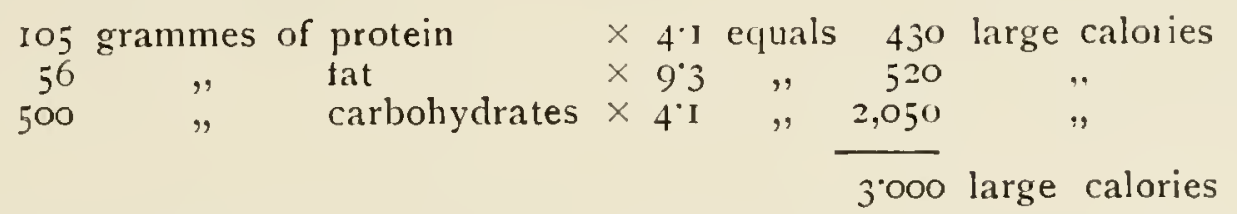

One large calorie when converted into mechanical force is equal to 425 kilogram metres or $\mathrm{I}^{\prime} 32$ foot tons, hence the available energy in the 3 , ooo calories would be 3 , ,oo $\times 425$ equals 1,275 , ooo kilogram metres or 3,960 foot tons.

An average man of II stone (7o kilos) has an average daily waste of nitrogen $20 \mathrm{grm}$. and carbon $320 \mathrm{grm}$. , hence his food must replace this waste and also about 3 ,ooo calories for mechanical energy.

Remember that to obtain $16 \mathrm{grm}$. of nitrogen, Ioo grm. of nitrogen must be ingested.

An average ratio of daily food is, according to Hutchison :-

Protein 125, fat 50, carbohydates 500, total $676 \mathrm{grm}$.

Calories 3,027. Nutrient value I : 5 .

$676 \mathrm{grm}$. is $2 \mathrm{I}^{\circ} 7$ ounces (one ounce equals $3^{\circ} \mathrm{IO}+\mathrm{grm}$. .).

Or $9^{\circ} 6 \mathrm{grm}$. per kilogram of body weight.

Or $0^{\circ}$ I 4 I ounces per pound of body weight.

Thus taking Voit's standard diet as above it would be classified thus :-

\begin{tabular}{|c|c|c|c|c|c|c|c|c|}
\hline \multirow{5}{*}{$\begin{array}{l}\text { Protein } \\
\text { Fat ... } \\
\text { Carbohydrates }\end{array}$} & \multicolumn{7}{|c|}{ In 24 hours per kilogramme of body weight } & \multirow{3}{*}{ Total ounces } \\
\hline & & Grm. & & Grm & & Ounces & & \\
\hline & $\ldots$ & 118 & $\ldots$ & $I^{\circ} 7$ & $\ldots$ & 0.025 & $\ldots$ & \\
\hline & $\ldots$ & 56 & $\ldots$ & 0.8 & $\ldots$ & 0.012 & $\ldots$ & $1 \cdot 8$ \\
\hline & $\ldots$ & 500 & $\ldots$ & $7^{\cdot 1}$ & $\ldots$ & 0.110 & $\ldots$ & $160^{\circ}$ \\
\hline & & 674 & & $9^{\circ} 6$ & & 0.147 & & $21 \cdot 6$ \\
\hline
\end{tabular}

One may consider generally that an average male requires daily :-

For bare sustenance $12 \cdot 14$ ounces, equals $375-435$ grammes

For moderate work 20-23 ", , 622.715 ,

For hard work $21-25 \quad, ", \quad 653-777$,

I native's food consisting of :-

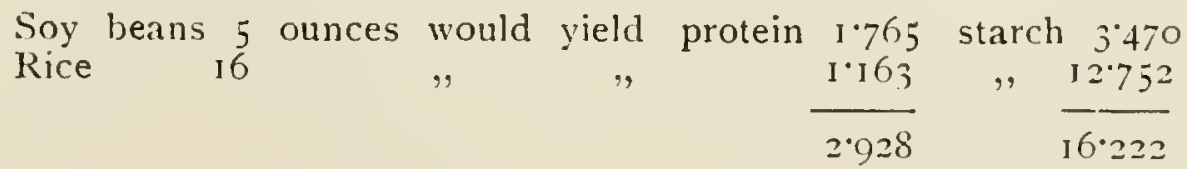

The beans are also rich in oil, the above containing about $3 \mathrm{I} g \mathrm{~m}$. 
or an ounce. There should be about $1 \frac{1}{2}$ ounces in his diet. This would be a fair native diet but some variety is essential.

Meat proteins are more easily assimilated than those of vegetable products and repair the tissues more rapidly in the proportion of 97 to 80 .

Only 50 per cent. of protein should be procured from meats, the remainder from vegetables and bread. Quantities above this place too much strain on the kidneys and liver. Over-eating is common in the tropics, which is so often accompanied by a sedentary life.

Hindus take no animal food except that in milk and ghee, but they are neither robust nor active.

There must be a necessary modification of diet to suit the climate.

The Hindu can live and fare well on rice principally while the Esquimaux require large quantities of fat to maintain their body heat.

Salted rations only will produce disease, as happened in the first Burmese Mar and in the Cameroons. On the other hand excess of fresh meat will cause intestinal disorders as happened in the French campaign in Algeria and in the American Civil War, when the enemy's cattle were captured and eaten.

The Aryan races were meat eaters when they invaded India, but now they are practically vegetarians.

Meat extracts are not a concentrated form of nourishment, such exist in name only. There is no true meat extract. About four-fifths of the nutritive constituents of meat existing in the muscle fibre are insoluble in either hot or cold water, but they are rendered soluble when acted on by the pancreatic and gastric juices, hence nutritive constituents cannot be present in water preparations.

Some of the connective tissue can be dissolved out as gelatine but its nutritive powers are very low and even then it is not a true tissue former. Small quantities of albumin which are soluble in cold water are insoluble after being coagulated in hot water. This, however, will yield to the digestive juices.

The meat bases, e.g., creatinine, zanthine, \&c., and half the salts are dissolved out by water, the former being nerve stimulants like the caffeine of coffee and are not nutrients.

When the above nutrients have been peptonized they can be taker in medicinal doses only, as increased quantities cause purging and hence cannot be foods. One pound of beef steak, about $45^{\circ} \mathrm{grm}$., is about all the flesh former a man of eleven stone requires in twenty-four hours, which in ex-war times costs about two shillings. The approximate equivalent in meat extract would be six bottles of 4 ounces each, costing 1os. 6d, as against is. for the real thing. In addition to that its consumption would be unwise because it would contain a dangerous amount of nerve excitant and $3 \frac{1}{2}$ ounces of common salt. How can such preparations be called "meat extracts" ? 
When salt in such quantities is ingested it requires about roo parts to one of water to correct it and hold it in solution in the body. Such weight of water should not be mistaken for healthy increase, it is a serious symptom.

Such preparations, however, when taken in small quantities have a limited use.

Sausages. These, as commercially made, are not as nutritive as they could be. Only too often there have been discovered amongst the ingredients such materials as: boiled rice, soaked bread, steamed potatoes, sawdust and pulped wood. Preservatives and blood colouring matters are only too frequently used. The tropical resident would do well to avoid all tinned sausages.

Cercal breakfast foods, such as Quaker Oats, \&c., are always good, but the endless variety of proprietary breakfast foods cannot improve upon the real thing with regard to nutrition. Some of them may be partly predigested, but, apart from invalids, this is not an advantage.

Whole milk cheese is made from coagulum formed from milk, treated with rennet or acid or allowed to sour spontaneously. It is then known as cream cheese, otherwise it is drained, salted and left to ferment. A good variety contains:-

Water, 34 to 40 parts; protein, 20 to 30 ; fat, 25 to 35 ; mill sugar, 1 to 3 ; mineral salts, 3 to 6 parts.

Ripening is caused by yeasts, moulds and lactic acid bacteria, causing decomposition of the sugar principally, the by-products being very numerous. The micro-organisms number from +50 , 000,000 to 450,000,000, ooo per pound. Nevertheless, whole mills cheese is one of the most nutritious of foods. One pound contains about as much protein as $1 \frac{1}{2}$ pounds of meat.

Proprictary protein foods are prepared from milk casein, waste meat fibre, vegetable gluten and a mixture of these treated chemically.

Some of them contain large quantities of protein and may in selected cases be taken. Their constant consumption is not advised.

Milk when pure is an excellent food. It should be sipped, as Nature intended and not swallowed like a glass of water. Curds in the stomach may cause a disagreeable train of symptoms. One must remember that milk contains solids to the extent of $1 \mathrm{in} \&$.

Milk is both a food and a beverage.

It is best to boil all milk in the tropics to aroid infection by the urganisms of undulant fever and other diseases.

When heating milk remember that:-

The rennin enzyme is destroyed at $\quad \ldots \quad \ldots 60^{\circ} \mathrm{C}-140^{\circ} \mathrm{F}$.

All micro-organisms are destroyed, not spores at $\ldots 68^{\circ} \mathrm{C}-154^{\circ} \mathrm{F}$.

The Pasteurization temperature is $\quad \ldots \quad \ldots \quad \ldots \quad 75^{\circ} \mathrm{C}-167^{\circ} \mathrm{F}$.

The boiling temperature is $\quad \ldots \quad \ldots \quad \ldots \quad \ldots$ IO1 $^{\circ} \mathrm{C}-213^{\circ} 5^{\circ} \mathrm{F}$.

All germs are destroyed in one hour if under pressure at $105^{\circ} \mathrm{C}-22 \mathrm{I}^{\circ} \mathrm{F}$. 
Homogenised milk has the fat globules broken up by powerful mechanical means and is consequently more easily digested.

skimmed milk may be :-

Iland skimmed, that is deprived of its cream after standing.

Nachine skimmed, better known as separated milk.

The latter is less nutritious than the former, separated milk only containing fat $0^{\circ} 10$ to $0^{\circ}+5$ per cent. as against $0^{\circ} 75$ to a per cent. in hand skimmed. Skimmed milk should contain at least 9 per cent. of non-fatty solids.

Condensed milk is made by concentration in vacuum pans at a low temperature, $50^{\circ} \mathrm{C}$. or $132^{\circ} \mathrm{F}$. and then sugar added.

There are several varieties as:-

Whole milk, sweetened and unsweetened.

Skimmed milk, sweetened and unsweetened.

The volume is reduced to about one third of the original quantity.

The amount of sugar added is from 30 to 50 per cent. of the finished product. It is never sterile, but pathogenic germs are almost invariably absent.

Dricd milk is used more in the confectionery trades than in ordinary consumption. It can be taken in six and a half parts of water, when it equals cow's milk in its concentration.

Dried skimmed milk should not be used for infants. It may be used in cooking, 1 in ro of water.

Butter milk is similar to skimmed milk, but is soured by lactic acid fermentation. It is not very palatable. The flavour disappears in the cooking.

ITh' $y$ is produced in cheese making. When rennet is added coagulation takes place; casein is thrown down with the fat forming the curd, the remaining fluid being known as sweet-whey; but when it is allowed to sour spontaneously this fluid is known as sour-whey.

It is useful for fever cases, but it is less nourishing than milk.

Buffalo's milk is twice as rich in fat as cow's milk, and contains a larger amount of total solids.

Ghee can be prepared from the mills of the cow or buffalo.

The milk is curdled with dobi or sour milk, churned, water added, upon the top of which the butter floats; the latter is collected, washed, placed over a fire in an iron pan; the butter melts, casein and water fall to the bottom, which is collected and called Kutcha ghee, or if the heat is increased so as to evaporate the water and slightly char the casein, it is called "pucka" or Valo ghee.

The former is used in the preparation of sweetmeats and food-stuffs ; the latter is often mixed with rice or spread on bread. It is also used for religious purposes.

Goat's milk cream is in finer globules than that of cow's, and does 
not so readily separate out. It should always be tried when cow's milk is not available. It is not sufficiently appreciated in some parts of the tropics.

\section{Yitamines.}

Vitamines are essential food substances without which some deficiency disease soon sets in. They are contained in rice polishings and reast, hence the former should not be rejected. They prevent polyneuritis. Vitamines (CO $(\mathrm{NH})_{2} \quad \mathrm{C}_{16} \mathrm{H}_{18} \mathrm{O}_{6}$ ?) belong to the Pyramidine group, and may be a constituent of nucleic acid. They are nitrogenous bodies which do not contain phosphorus or fat. They are soluble in alcohol and water. They are crystallizable and not destroyed by boiling water. They are unimpaired when milk is sterilized or pasteurized. They are found in all food-stuffs in a minute quantity, but they are more plentiful in commercial lecithin, milk, ox brain and brewer's yeast. They are probably the mother substances of ferments and hormones. They are destroyed at $120^{\circ}-130^{\circ} \mathrm{C}$. in twenty ninutes, and also by extreme dryness.

\section{ADULTERATION OF FOODS.}

Adulterants in milk are common in the tropics, such as :-

(1) Sewage from wells, tanks and ponds.

(2) Molasses or potassa to raise the specific gravity after adding water.

(3) Arrowroot and pulped banana. Cream is usually removed from milk samples where these adulterants are used.

(4) Preservatives are not so commonly used.

Test for nitrites in milk.

Coagulate a little milk in a test-tube with acetic acid.

Filter, add to the filtrate a few c.c. of sulphanilic acid and naphthylamine sulphate, equal parts; a rose-red colour is positive for nitrites.

Test for molasses and botassa.

To one test-tube containing io c.c. of milk add 5 grm. of ammonium molybdate and 10 c.c. of dilute alcohol ( $\mathrm{I}$ in $\mathrm{IO}$ ).

To a second test-tube place 6 per cent. solution of lactose and treat as above.

Place both test-tubes in a water bath and raise the temperature gradually to $80^{\circ} \mathrm{C}$.

A blue coloration in the tube is positive for cane sugar, while lactose is unaltered.

This test will detect $o^{\circ}$ I per cent. of cane sugar.

Another test.

To 1 o c.c. of milk add $\mathrm{O}^{\circ} \mathrm{I}$ grm. of resorcin and a few drops of $\mathrm{HCl}$ Boil for a few minutes. 
A rose-red colour is positive for cane sugar.

Any deposit should be examined with a microscope for starch, pus, and dirt which can be readily detected.

Milk with a specific gravity below I.028 contains an exceptional amount of cream or has been adulterated. It is normally I'02S-I'035. If there is less than 3 per cent. of fat it has usually been adulterated.

Bacterially milk should be examined for the tubercle bacillus and the cholera vibrio. The tubercle bacillus is rarely found in India.

For T.B.

Centrifuge a small quantity of milk.

Take the deposited portion and inoculate two guinea-pigs.

If $\mathrm{T} . \mathrm{B}$. is present the animals will show it in three to six wceks.

The tuberculin reaction is useful for cors.

For cholera vibrio.

Isolate in Durham's peptone solution with 1 c.c. of milk and plate out. Identify the organisms. For more details, see special section on Laboratory Hints.

Milk may be manipulated as follows :-

(New South Wales Report of the Government Bureau, 1909).

(I) Mechanical contrivances.

Centrifugalization.

Filtering.

(2) The application of heat.

Sterilization.

Boiling.

Pasteurization.

Concentration.

Desiccation.

Thermophore treatment, similar to thermos treatment, to prevent excessive growth of bacteria by continuous warming.

(3) The application of cold.

Freezing.

Chilling.

Cooling.

(4) The addition of antiseptics.

Sodium bicarbonate.

Boric acid, borax or mixtures.

Formalin.

Salicylic acid.

Peroxide of hydrogen.

(5) The inducement of alcoholic fermentation.

Koumiss.

Kephir, \&c. 
Butter.

Butter may be adulterated by: adding an excessive amount of water, reducing its fat content, adding milk or milk powder.

Artificial colouring is allowed, as also are some preservatives.

Excess of water is commonest, and may be present as the result of :-

Carelessness in making, by having too high a temperature when churning or to deliberate intention.

I2-I 4 per cent. is not excessive. I6 per cent. should constitute grounds for prosecution.

If there is more than I per cent. of curd suspect added milk, and test for milk sugar.

Foreign fats are frequently added. Those usually employed are lard, beef fat, cotton-seed oil and cocoanut oil, or a combination of these. Artificial preparations, containing tributyrin or triacetin in alcoholic solution, are even added to make up for the increase in volatile fatty acids caused by the use of animal fats.

Special works must be consulted for their detection.

For butter analysis, see Laboratory Hints.

For preservatives and their excesses, see p. 574 .

\section{SOME MORBID CONDITIONS CAUSED BY FOOD.}

Scurvy.-This disease is probably due to a deficiency of fresh vegetables and fruits; it is known as a deficiency disease.

Partial decomposition of preserved foods and a damp, low-lying locality may also be predisposing factors.

The symptoms are due to blood changes causing: marked debility, sallow complexion, swollen, spongy and ulcerated gums, effusion and hæmorrhage into the joints, accompanied by foul ulcers and dysentery. The condition should be corrected by giving fresh lemon or lime juice, fresh vegetables, fruits, potatoes, yams, green vegetables and yeast.

Rickets.-This is probably due to a deficiency of fat and protein with excess of starches in children's diet, and is hence known as a deficiency disease.

Artificial starchy foods cannot be digested by infants.

Intestinal Disorders. - These are caused by decomposed and mouldy grains. Such should be always condemned as unfit for human consumption.

Beriberi.-This is in all probability a deficiency disease usually found amongst rice-eating peoples. The essential vitamines are contained in the rice polishings which are so often rejected.

For details, see article on Beriberi. 
Pellagra.-In all probability another deficiency disease about the atiology of which there is still much disagreement. See article on same for details.

Lathyrism is caused by eating the Lathyrus sativus, a vetch used as a grain at famine times by the poor of India. The toxin affects the prramidal tracts of the cord.

Ergotisn is produced by the mycelial fungus, Claviceps purpurea, in rye. It causes sclerosis of the posterior columns of the cord and stimulation of the vasomotor centre.

Beriberi, Pellagra, Lathyrism and Ergotism are somewhat allied in symptomatology, all producing nervous affections, the three latter affecting the spinal cord.

The subject may also be considered from the point of view of the causative agent thus:-

Milk, when decomposed, forms lacto-toxin or tyro-toxin, producing outbrealss of nausea, vomiting, dry mouth and fauces, diarrhoea, cramps and collapse.

Infected milk disseminates Undulant fever, Cholera, Typhoid fever, Dysentery and Tuberculosis.

Meat when decomposed and infected produces a diarrhoea of a choleraic nature with vomiting, cramps, headache, prostration, \&c., due to organisms of the Gaertner group, principally B. enteritidis, B. botulinus, and B. enteritidis sporogenes.

Infective conditions are produced when :-

Tenice are found in beef, mutton, pork and dog meat.

Trichince in "measly" pork.

Strongylus filaria in the lungs of sheep.

Distoma hepaticum in the liver of sheep and cattle.

Conurus cerebralis in the brain of sheep and cattle.

Watercress may contain the encysted forms of trematodes, nva and bacteria of Enteric, Cholera and Dysentery. This is particularly so when the garden has been tended by a Chinaman.

Fresh fish and shell fish may acquire poisonous properties when spawning, while other fish in the tropical seas are unsafe owing to their feeding on poisonous meduse and corals.

Oysters have been responsible for Cholera in India and Typhoid fever in many parts.

Canned fresh herrings and sardines have been found to rield a metallic tin content in the fish of from 2-5 grains per pound (Everitt Atkinson).

\section{THE EXAMINATION OF MEATS.}

Good fresh meat should be firm, elastic, not tough, should not pit on pressure. Thin red meat juice should exude from it on standing.

It should be red throughout, not pale, purple, or green in parts. 
It should be free from purulent or gelatinous fluids in the septa between muscular bands.

It should be fresh and pleasant to the smell, after the insertion of a skewer or cutting it with a knife.

The reaction should be slightly acid. Alkalinity denotes decomposition. It should be free from "spots" which are cysticerci.

The fat should be free from hamorrhages, firm, white, seldom yellow, and never gelatinous.

Goat flesh has less fat than mutton and is somewhat darker.

Carcases of sheep should have the feet attached to distinguish them from goat meat.

Good prescroed meat in tins should have the ends of the tins slightly concave. All tins that show signs of bulging should be condemned as the tin has not been rendered sterile, the retained microorganisms producing the gas which distends the tin.

Tins sounding hollow when struck with a wooden mallet should be examined more critically.

Frozen meat should be refused when the pleura have been stripped from the carcases.

Prolonged refrigeration for several weeks is fatal to cysticerci.

Partially decomposed meat is neutral or alkaline in reaction, easily tears, pits on pressure, and usually has an offensive odour.

\section{THE EXAMINATION OF LIVING ANIMALS.}

Healthy live animals should appear well nourished, able to walk with ease, bright eyed, breathing quietly and without offensive odour, mouth and nostrils cold, moist and without discharge, coat smooth and free from eruption, not rough, no shivering, diarrhoea, \&c.

All organs should be healthy when killed.

When the animal is slaughtered one should examine the chest wall, pleura, diaphragm and lymphatic glands. One should look for dropsical fluids in the connective tissue in the flanks, under the shoulders and armpits, beneath the diaphragm, above and behind the kidneys.

Examine for glistening spots or bladder-like bodies, and confirm with a microscope in doubtful cases.

When the carcase is ill-bred, bile-stained, with patches of extravasations, the viscera congested and dark-coloured, suspect infectious disease as Anthrax and Piroplasmosis.

Exclude the following diseases by the microscope when possible:-

Anthrax by absence of the anthrax bacillus in the blood.

Piroplasmosis by absence of Piroplasma bigeminum in the blood.

Pasteurellosis by absence of small encapsulated diplococcus in the gland juice, \&c. 
Swine fever, Swine typhus, Pneumo-enteritis and Hog cholera by the examination of the lungs and intestine after death.

Rinderpest by absence of abrasions of the mouth and tongue, congestion with cheesy deposits on the throat and nostrils, crackling on pressure in adranced stages.

Pleuro-pneumonia by absence of hepatization of the lungs after death.

Tuberculosis by absence of small tubercles or caseous masses in the lungs, on the pleura, peritoneum, liver, kidneys, and without the enlargement of the lymphatic glands of the carcase.

Actinomycosis by the absence of the ray fungus (Streptothrix actinomyces) about the upper and lower jaws and the nasal cavities. There should be no interstitial inflammation of the tongue (woody tongue).

Taniasis by absence of cysticerca amongst the muscle fibres of the carcase.

Trichinosis by the absence of Trichina spiralis in the flesh of animals that destroy rats, such as pigs, \&c. Each capsule ingested gives rise to a brood of one or several hundred young trichina.

Coccidiosis of rabbits, cattle and sheep by the absence of the psorosperms. The chief ones are Coccidium oviforme. They are found about the bile ducts and intestines of carcases.

Tuberculosis.-With regard to this vexed question the recomnendations of the Royal Commission on Human and Animal Tuberculosis are :-

The carcase, if otherwise healthy, shall not be condemned, but every part of it containing tuberculous lesions shall be seized when-

(r) The lesions are confined to the lungs and the thoracic lymphatio glands.

(2) The lesions are confined to the liver.

(3) The lesions are confined to the pharyngeal lymphatic glands.

(4) The lesions are confined to any combination of the foregoing, but are collectively small in extent.

The entire carcase and all the organs may be seized when-

(r) There is miliary tuberculosis of both lungs.

(2) Tuberculous lesions are present on the pleura or peritoneum.

(3) Tuberculous lesions are present in the muscular system, or in the lymphatic glands embedded in or between the muscles.

(4) Tuberculous lesions exist in any part of an emaciated carcase.

\section{MEAT PRESERYATION.}

From ancient times certain methods and means have been adopted for the preservation of meats, especially where all that in hand could not be consumed within a reasonable time. 
Primitive peoples adopt such methods as smoking, salting or pickling.

Modern peoples, on the other hand, use such chemical materials as sodium benzoate, salicylic acid, \&c., which when present in food in sufficient quantity to preserve it are tasteless, odourless, and can only be known by the label or chemical analysis.

The old-fashioned methods of salt and smoke had much in their favour.

It should not be forgotten that fresh meat can be kept for four to six days as a result of boiling it slightly each morning.

Meat preservation is all-important for the tropics and ocean steamers. Some method of preservation is essential.

The methods usually employed are (after Whitelegge and Newman) :-

(I) Exclusion of air.

The meat may be dipped into boiling water, when the external coagulated albumin forms an impervious layer, or it may be coated by paraffin or fat.

(2) Preservative Injections.

Inject the blood-vessels with water, then with a solution of common salt, or alum with aluminum chloride.

(3) External Preservatives as:-

Salt or brine, sugar, boric acid, weak carbolic acid, \&c.

(4) Pickles.

Salt and a little potassium nitrate are mixed together and well rubbed in.

(5) Drying after exposure to smoke from a wood fire.

This is the favourite method of natives.

(6) Refrigeration.

The application of cold, just above freezing point, must be continuous. Decomposition is rapid when the cold is withdrawn if rigor mortis had set in before the freezing.

(-) Sterilized Enclosures.

Hermetically sealed in vessels in vacuo or in sterilized air.

The air is expelled previous to sealing by boiling, steam, chemicals, e.g., sodium sulphite, \&c.

Imperfections in sterilization result in partial decomposition of the contents and when ingested of toxic symptoms in the consumer.

The dangers from tinned foods are from :-

(I) Added preservatives in excess.

(2) Colouring agents.

(3) Salts of tin, lead and zinc from chemical reaction in the tin.

(4) Absence of vitamines. 
The most common chemical preservatives are:-

Formaldehyde and Borax to milk.

Salicylic acid to fruit juices.

Sodium bensoate to ketchup, jams and sauces.

Sulphites to dried fruits, gelatin and chopped meat.

Fluorides to beer.

Some of these, such as salicylic acid, borax, benzoic acids, and fluorides are normally present in certain products, hence their mere presence cannot always mean that such have been added.

Minute amounts of preservatives of a certain specified kind are essential in most cases but there is not the slightest doubt that the opportunity to add preservatives by unscrupulous manufacturers is a constant temptation to put on the market food unsuitable, and partly decomposed. When preservatives are added to arrest decomposition the quantity must be sufficient to interfere with the normal digestion of the consumer.

The following notes may be of interest.

The nutritive value of meat after refrigeration is unaltered.

Salt in butter causes mycelial fungi to disappear and reduces the vigorous multiplication of micro-organisms.

Formaldelyde in small quantities is apt to cause headache; nausea, romiting and intestinal cramps.

Boric acid rubbed on hams penetrates the substance deeply and its consumption may derange the digestive tract.

Boric acid, 20 grains to the pound, was found in certain sausages.

This inhibits putrefaction but does not appreciably inhibit the growth of the Coli group of organisms, hence stale meat can be used for sausages (Bernstein).

Certain preserved tinned lobsters contained from $0^{\circ}$ So to $4^{\circ}+7$ grains per pound of dissolved metal in their contents. More than $\mathrm{I}$ to 8 grains has caused poisoning. As much as 13 grains per pound has been found (Wiler).

Copper sulphate, added to make vegetables a nice green colour, is unnecessary, unwholesome and unadvisable.

The black spots sometimes seen on chilled beef are due to a mycelium, Oidium carnis, and though unsightly are harmless.

The following organisms have been found in preserved fish, sardines, \&c., B. enteritidis (Gaertner), B. prodigiosus, B. botulinus, Penicillium glaucum, Aspergillus glaucus and others.

Daries proposes:-

All preservatives in milk should be forbidden.

Borax preservative in hams, bacon and sausages should be allowed.

Salicylic acid in wines and jams to be allowed. 
Nil else should be permitted without permission from the Board of Agriculture.

Good firms turn out canned products which are a great boon to most tropical residents.

For qualitative and quantitative tests for preservatives a larger work should be consulted. We venture to give here one simple qualitative method for each principal preservative.

Formaldehyde.

Put 5 c.c. of milk in a test tube.

Add to this 5 c.c. of the liquid to be tested.

Pour carefully down the side 5 c.c. of concentrated sulphuric acid so that it shall not mix with the diluted milk.

$A$ violet ring at the junction of the two liquids is positive for formaldehyde. The delicacy of the test is about I part in 200,00o.

Note.-Pure sulphuric acid does not give the test. In commercial acid some ferric salts are present which produce the test. Hence if only pure sulphuric acid is to hand add a little ferric chloride.

Benzoic Acid and Sodium Benzoate.

This salt is very widely used in many varieties of foods.

If the material is a liquid, acidify 25 c.c. with 5 c.c. of sulphuric acid and shake with 25 c.c. of ether in a separatory funnel.

If the substance is not fluid, dilute it or macerate it and obtain a watery solution of it, strained and treated as above.

The mixing must be thorough but not violent as emulsions may be formed.

Separate the other layer and evaporate it at a low temperature.

If the original material contained much fat shake the ether with ammonia, evaporate and test the residue.

Crystalline scales may be seen in the residue which give off a characteristic odour when heated if much benzoic acid was present. The residue must be tested as follows :-

Add to the residue 2 to 3 c.c. of concentrated sulphuric acid.

Heat until the acid gives off fumes strongly.

The benzoic acid is changed to sulpho-benzoic acid, in the charring.

Add ammonia nitrate in small portions while it is still fuming.

The organic matter is oxidized and m-dinitrobenzoic acid formed.

Let it cool.

Add water and ammonia in excess, transfer to test tube.

Pour down side of test tube a little freshly prepared colourless ammonium sulphide. It must not be mixed.

A red ring at the junction of the two fluids is positive for benzoic acid and is due to ammonium m-diamidobenzoate. 
Add a few drops of ammonium sulphide to the remainder in the casserole and a red colour will form in clouds on the surface.

Salicylic Acid.

Prepare a solution as for benzoic acid test, but $5^{\circ} \mathrm{grm}$. only.

Divide solution into two portions as benzoic acid and salicylic acid are often present together in the same supply.

Irash twice with one-tenth of its volume of water when one has prepared the other extract as for benzoic test.

The washing is to get rid of the mineral acid as the test is much more delicate in its absence.

Evaporate ether in porcelain dish.

To residue add two drops of dilute ( $\mathrm{r}$ per cent.) ferric chloride solution.

Or better, three to four drops of ferric alum solution.

Rub it around with a glass rod.

A violet colour is positive for salicylic acid.

If positive oonfirm by another test as several other organic substances give the coloration.

To the solution to be tested add four to five drops of a ro per cent. solution of potassium or sodium nitrite.

Add four to five drops of acetic acid.

Add one drop of ro per cent. solution of copper sulphate.

Heat to boiling.

A reddish tinge is positive for salicylic acid.

There may be sufficient to give a blood red.

Phenol gives the same reaction but benzoic acid does not.

As salicylic acid occurs naturally in some fruits not more than $50 \mathrm{grm}$. of the substance to be examined should be taken.

This is known as the Jorissen test.

Boric Acid and Borax.

The turmeric paper test is useful.

Take $25 \mathrm{grm}$. of the sample.

Make distinctly alkaline with sodium hydroxide.

Evaporate to dryness in a porcelain dish.

Boil with 15 c.c. of water.

Add hydrochloric acid until acid to litmus paper, then add ten drops more acid.

Filter and evaporate to dryness on the water bath with a piece of turmeric paper partly immersed in the fluid.

Cherry red colour of the paper is positive for boric acid and borax.

The colour changes to a bluish green by a drop of ammonia.

A quantitative test should be carried out because boric acid occurs in apples and other fruits and vegetables in nature in minute quantities. It occurs in greater quantities in common salt. 
Sulphurous Acids and Sulphites.

The preservative may be present as acid sulphites of sodium or calcium or as sulphur dioxide gas itself. This is the case especially in wines or molasses where the acids are present probably partly in combination with aldehydes and sugars.

Weigh $50 \mathrm{grm}$. of sample into a 500 c.c. flask.

If a solid, add recently boiled water.

Through the separatory funnel of the flask add 5 to ro c.c. of a 20 per cent. solution of phosphoric acid.

Distil by steam in a current of carbon dioxide, collecting the distillate in 25 c.c. of saturated bromine water.

Distil about $5^{\circ}$ c.c. adding a little more bromine water if necessary.

After distillation is complete boil off the excess of bromine.

Dilute the solution to 250 c.c.

Add 5 c.c. of hydrochloric acid.

Heat to boiling.

Add very slowly, with constant stirring, a hot ro per cent. Solution of barium chloride, until in slight excess.

Allow to stand for an hour, filter, wash with hot water, ignite and weigh as barium sulphate.

A "blank" experiment should be done without any of the sample in the flask and any barium sulphate obtained subtracted from the main amount.

A special work should be consulted for this more technical test for which special apparatus is necessary.

Fluorides.

The fluorides of sodium and ammonia have been used more especially in beer and malt extracts.

The etching of glass method is the best for detection.

See a special work for this more technical test.

\section{Saccharin.}

It has some antiseptic power but it is generally employed as a sweetener. The continued use of small quantities, say of $0^{\circ} 3 \mathrm{grm}$. per diem, is sufficient to impair the digestion.

Extract with ether as for benzoic acid.

Evaporate spontaneously and taste the residue.

A sweet taste is positive for saccharin to $20 \mathrm{mg}$. per litre.

If present a more complicated test should be carried out to get rid of any "false saccharin" which may be present.

Copper Sulphate.

Make an ordinary watery solution.

Add ammonium sulphide.

A black colour is positive for copper sulphate (and lead).

The colour is not discharged by acids. 


\section{VEGETARIANISM.}

Nature never intended that man should be strictly regetarian.

In herbivorous animals the cacum is very much larger in proportion than man's, in order to accommodate and digest the bulk of vegetable matter necessary. In man, this organ, not being so necessary, has atrophied, the often troublesome appendix being a portion of the atrophied organ.

The gastric and intestinal juices of man are intended for the digestion of meat foods.

Peas, beans and lentils are amongst the most nutritive of regetable foods, but much of their protein is undigestible. The cellulose wall of most regetable cells containing about 20 per cent. of protein cannot be digested in man and forms the large residue of the stools of regetable feeders.

Nuts are only to be consumed in very limited quantities.

Milk, eggs and cheese are certainly good and rich in protein but they belong to the animal kingdom.

It is true that less meat and more regetable foods should be consumed by Europeans residing in the tropics.

\section{INFANT FEEDING IN THE TROPICS.}

When Nature's provision is sufficient, infant feeding is simplicity itself. It is with the artificial feeding of white babies that we are here concerned and that in countries where good cow's milk is rarely available.

IV. E. Deeks has studied this subject at Panama and gives us the results of his experience as follows.

Table for Infant Feeding.

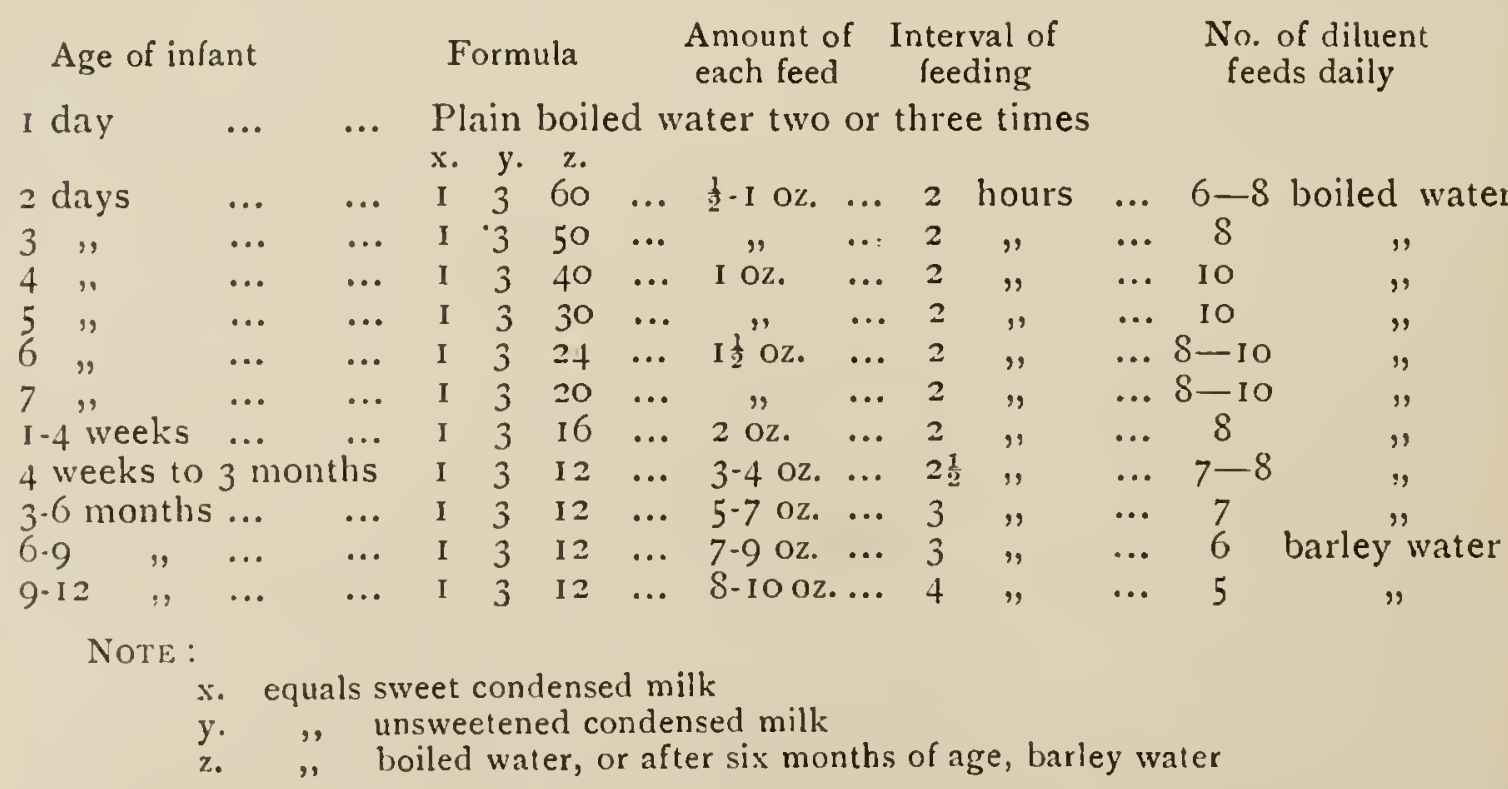

The contents of every bottle should be tasted first by the mother to see that no alteration has talien place in the contents. 
A proper tubeless feeding bo:tle, liept scrupulously clean, is essential.

To make the barley water: take one tablespoonful of pearl barley and wash it.

Add one pint of cold water.

Soak for ten to twelve hours and strain.

Boil slowly for two hours.

Add water from time to time so as 10 have one pint of the finished product.

Season with half a teaspoonful of salt and strain through muslin.

To make oatmeal jelly :-

This food is useful after the child is nine months old when there is a tendency to constipation.

Take one cupful of rolled oats, soak in two cupfuls of water for ten to twelve hours, and strain through muslin.

Add one cupful of cold water.

Boil for two hours and stir frequently.

Add half a teaspoonful of salt, when cool a jelly is formed.

Add wo to four teaspoonfuls to each bottle.

When the child is one year old give one hen's egg daily in milk or boiling water when it jellies. Afterwards they may be lightly boiled and given with a rusk or toasted bread and butter.

The child should not have more than five feeds daily.

After fifteen months old, give two eggs daily, and four feeds in all.

After eighteen months old, give a light general diet with a little green vegetable.

After twenty-four months old, feed thrice daily and give water only between meals.

From age three months the child may have fresh unsweetened fruit juices.

Sugar is usually given to babies in excess and should be but minute in amount.

The majority of prepared infant foods contain a large carbohydrate content, e.g., powdered biscuit, \&c. No child's digestive juices can deal with it adequately until more than one year old. If the child takes too much of it there will be fatness, inactivity, slow dentition, constipation and occasional diarrhoea. Generally such foods should be aroided. Probably the most useful of all are Allenbury's.

The better nourished the baby the less disturbance may one expect during teething.

If there is vomiting, absolute anorexia, high fever and sometimes diarrhoea, in all probability milk poisoning is the cause.

Examine and jealously guard the milk supply. 
Excess of sugar and other carbohydrates may cause :-

Fever, enteritis, entero-colitis, eczema, enuresis and rheumatisms, recurring bronchitis, pustular dermatitis and dry lustreless hair.

Curds in the stools (fatty acid soaps) are not important.

Banana flour is a useful food. Add one ounce (3I grm.) to one pint of water. Boil for five minutes.

The methods here adrised are cheap, sterile and efficacious (W. E. Deeks).

\section{CONCENTRATED TABLET FOODS.}

The food tablet will never become universally used. Science will never request that we should sit down to a lozenge lunch or tablet tea.

Sugar is almost essential as a food but it cannot be compressed.

The solids of our normal diet, daily, when all gases and water have been driven off, will occupy three-quarters of a pint.

If we take this in 5 -grain tablets we should require about 1,700 daily. But these we could not digest without water and six pints would be required for these tablets only and water cannot be compressed. The question of taste, smell, \&c., are all important to stimulate the digestive juices to flow.

There would have to be some definite way of distinguishing the tablets to know when one was about to partake of the meat or pudding course.

Some dried foods can be prepared and are useful, as evaporated milk, casein, \&c.

\section{DRINKS.}

\section{Water.}

There is nothing to equal Nature's first thirst quencher, viz. : cool, pure water. Mankind, however, requires variety.

\section{Tea.}

Tea (Thea sinensis, \&c.) has an odour due io essential oil, a nerve stimulant due to caffeine, 2 to 5 per cent., and an astringent due to tannic acid, 7 to i 5 per cent.

Indian and Ceylon teas contain about twice as much tannin as the China teas, and tannin is prejudicial to digesion, forming with proteins a leathery substance which is insoluble.

Strong tea or an excess of weak tea induces indigestion, nervousness and irritability. The nervous breakdown of tea-tasters is due to this. Tea should be drunk within ten minutes of its being made.

\section{Coffee.}

Coffea arabica contains half to three-quarters as much caffeine as tea.

Black coffee as a common and frequent indulgence produces a 
deplorably neurotic state. In moderation it is a good muscular and mental tonic. Dyspeptic persons should not take coffee after dinner.

Chicory, previously considered harmless, is now said to have an unfarourable effect upon growth and development.

\section{Cocoa.}

Cocoa (Theobroma cacao) powder, commonly known as cocoa " essence," is pure cocoa deprived of part of its fat, which originally amounts to $5^{\circ}$ per cent. or more of the whole. This fat is not extracted when the cocoa powder is to be used for chocolate. For this latter sugar and flavourings are added, and the whole is ground in a hot mill. Cocoa powder contains 27-33 per cent. of fat, I2-I 5 per cent. of proteins, of which 60 per cent. is not assimilable. There are also traces of caffeine. In pure cocoa nibs there is $0^{\circ} 9-2 \cdot 7$ per cent. theobromine.

Cocoa powder forms a pleasing, mildly stimulating beverage, but is not a food. When starches are added cocoa is more assimilable.

Cocoa contains too many solids for it to be a good solvent of other foods when they are taken at the same time. It is estimated that 8o-go per cent. of the cocoa bean is insoluble in water. The so-called soluble cocoas are not soluble, but potassium carbonate is added, which saponifies some of the fat and keeps the particles longer in suspension. This chemical does not aid the digestion, especially of invalids and children.

\section{Alcohol.}

Alcohol is made from malted and unmalted grain, potatoes, maize, rye, grape, beetroot, molasses, palm tree sap, coal and coke gases, \&c.

The total effect of small quantities is to favour gastric digestion, but excess is a common cause of acute and chronic dyspepsia and many other ailments. Alcohol is a source of heat and energy, but it reduces the activity of metabolism and stimulates, but later depresses the circulation and the nervous system independently of its action on tissue changes.

It is not a food because, while it is oxidizable, it acts detrimentally on the nervous tissues.

Men soon get thirsty in the tropics, and excesses seem to be the rule. Alcohol is not generally necessary and strenuous work can be done without it. Taken medicinally it is of great value.

The alcoholic average contents of common drinks are :-

\begin{tabular}{|c|c|c|c|c|c|c|c|}
\hline Whiskies & .. & ... & $50-60$ & French wines & • & ․ & $8-11$ \\
\hline Brandies & $\ldots$ & .. & $45-65$ & British wines & $\ldots$ & $\ldots$ & $20-25$ \\
\hline Gin & $\ldots$ & . & $35-45$ & Champagne & $\cdots$ & $\ldots$ & $10-12$ \\
\hline Rum & $\ldots$ & $\ldots$ & $35-70$ & Beer & $\ldots$ & $\ldots$ & $3-5$ \\
\hline
\end{tabular}

Koumiss $\mathrm{I}-3$ per cent. alcohol is made from mare's or cow's milk. The body manufactures about two ounces $(62 \mathrm{grm}$.$) of alcohol$ 
daily, or an amount equal to that contained in about four ounces of whisky or brandy.

\section{Mineral Waters.}

Soda water contains little or no soda at all, but carbon dioxide gas. It is useful for some invalids.

Lemonade from fresh limes, not the chemically made article, is a refreshing beverage.

Commercial articles contain saccharin which has a sweet taste, but no nutritive value. It is a drug and not a food, and is forbidden in the United States except for medicinal purposes. Sugar is not desirable as a substitute in these instances because it would ferment.

Saponin is a hamolytic poison which is used in drinks and should be prohibited. This produces the "head" so well known in beers.

Gum arabic could be substituted, but why should a "head" be required at all?

Coal tar colouring matters are sometimes used, some of which are poisonous.

\section{SANITATION.}

\section{REFUSE, ITS COLLECTION, REMOYAL AND DISPOSAL.}

An outline only of this subject is attempted here.

The systems to be adopted vary according to the population, the nature of the surrounding country and the local tribes for which provision is to be made.

A medical officer is often placed where there is no legal enforcement of hygienic measures, and he must adopt that system, most hygienic, which the particular people with whom he has to deal are prepared to use.

All waste products and refuse decompose much more rapidly in the tropics, and must be removed and disposed of daily. This consists of domestic refuse, human excreta, solid and liquid, slop water, animal excreta and waste.

In large towns sewers are in existence for dealing with human excreta, but in villages and small towns manual labour is required for its removal and disposal.

The amount to be removed is one and a half times as much for tropical natives as for Europeans, the latter being largely meat eaters and the former vegetable feeders. For each vegetable feeder one could estimate as a maximum 8 ounces $(225 \mathrm{grm}$.) of solids and 40 ounces ( $I$ ' 2 litres) of liquid excreta per man per diem, or if ablution water is to be included So ounces $(2 \cdot 25$ litres $)$ of liquids. Hence for $\mathrm{I}$, ooo people there rould be $500 \mathrm{lb} .(226 \mathrm{k}$.) of solids and 500 gallons $(2 \cdot 268$ litres $)$ of liquids to be removed daily. 
Now I, ooo ounces of solids equal I cubic foot, and 6.25 gallons of fluids equal I cubic foot.

One adult yields I + cubic feet of solids and liquids per annum without ablution water. If the latter is added it is best to double the amount given above, then one can ascertain the amount of land necessary for its disposal.

For its removal use bucliets of I cubic foot capacity and carts of 12 cubic feet capacity.

Now I, ooo persons yield per diem $500 \mathrm{lb}$. of solids or $\frac{500 \times 16}{1.000}$ or 8 cubic feet of solids and also 500 gallons or $\frac{500}{6.25}$ or 80 cubic feet. This equals eighty-eight buckets daily. Now each scavenger carries two buckets and goes twice daily to the trenching ground, hence to remove the total excreta of $\mathrm{r}$, ooo persons there would be required twenty-two men with forty-four buckets of I cubic fnot capacity each. Each man to make two trips daily.

\section{LATRINES.}

\section{(A) For Natives.}

(I) The Bush.-All primitive natives in small communities use the bush. If a small hole is made in the ground and the excreta covered, the method would be hygienic for districts where there are small communities and extensive bush abounds.

(2) Pits.-Some natives dig pits about 2-6 metres deep and 2-4 metres in diameter; the sizes are by no means constant. These holes are covered over with sticks and clay, leaving a small aperture at the top. When one is nearly full it is covered with earth and another prepared. It is surprising what a minimum amount of nuisance such pits cause.

(3) Shallow Trenches.-These are useful in certain small bush communities where there is ample supervision. An area is screened off and divided for the sexes. The trenches are half a metre deep and a quarter of a metre wide. They are made as long as required. The native squats astride. Earth is thrown on daily, and the whole trench is filled in when it is within a short distance from the top. A fresh trench is then prepared in front of the old one, and so on until the whole screened area is exhausted. The screens are then removed to another part. In some instances the excreta is mixed with earth and removed daily. 
(4) Deep Trenches. - These are often used, but are very unsatisfactory and should be condemned. They are 3-4 metres deep, I-2 metres wide, with sticks thrown across at intervals. The sides are nearly always fouled.

(5) Prepared Trenches.-These are useful in certain instances close to works for the use of the native workmen. The floor of the latrine is covered over with dry earth, the excreta covered with another layer immediately, and the whole removed daily. Constant attendants are required.

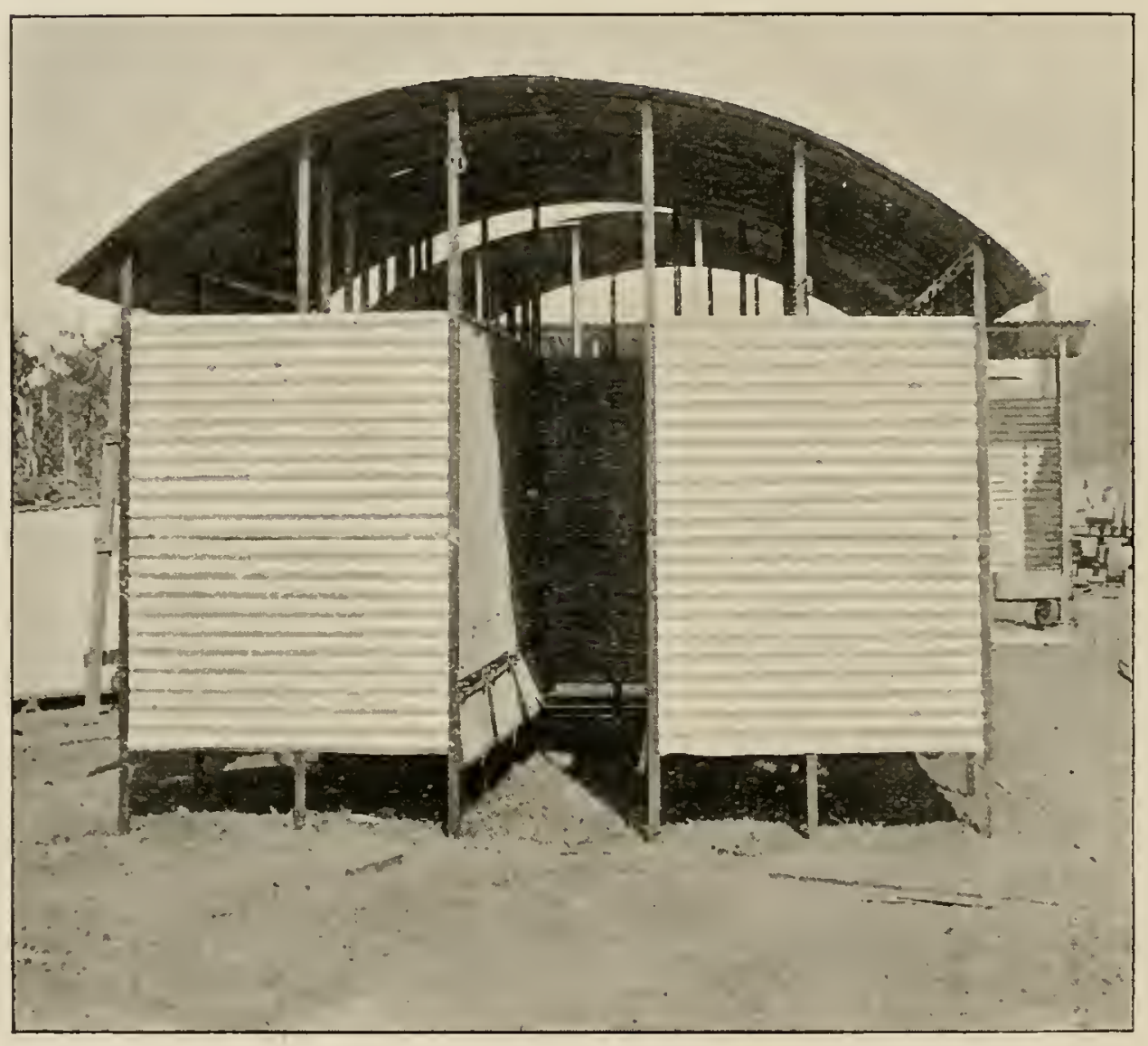

A double public latrine.

Exposure to the sun is advantageous, but rain and flood water must be excluded in the rainy season.

(6) River Latrines.-These are often used by companies for their employees, but the practice fouls the river banks and the river bed at low water, and in any case pollutes the water for the villages downstream. The practice should be condemned.

(7) Deep Pit Latrines.-For better class natives one has found useful a latrine as follows: A hole is dug about 6 metres deep and 0.5 by 0.75 metres in diameter. A box seat is fixed over it with a lid to make it fly-proof. A small house with a locked door is built over this, say of corrugated iron and made movable. 
A small portion of earth is thrown down daily from a box of loam kept in the little house for the purpose. A latrine like this may last a family for a year or more, after which time it is filled in, another hole dug, and the house removed over it. This method can only be adopted where there are small communities, and where there can be no possibility of drainage affecting the water supply. Flies do not descend deeply to breed. There is no nuisance with latrines of this kind when kept properly.

(8) Chinesc Latrines.-Men use public latrines; the women and children use rases and pots in the house, which are emptied daily. The public latrines may be platforms over fish ponds or raised masonry reservoirs with holes over which the user squats. The use of petroleum will remove all objections to these masonry latrines, and they are useful.

These can be made elaborate and the system applied to private dwellings. It is always much better to use pails beneath holes so that the excreta can be removed daily from outside the house. The floor and sides of the recess for the pail should be cemented. The buckets should have a special lid to prevent splashing.

A double number of pails is required to replace those removed. The pails should be washed out with cyllin before being replaced.

Some of these latrines are modified so as to separate the solid from the fluid excreta. The former can be applied to the land, and is of more value than the fluids. When they are not separated decomposition sets in more rapidly and gives rise to liberated ammonia, carburetted hydrogen and foetid organic gases. The solid matter is mixed with earth, the urine passing down an inclined plane in front of it and treated separately, but in the same way.

The solids are removed, deposited in pits on the farms, covered with earth, kept three to six months, and then used upon the land.

The urine is used for manuring soon after collection.

The Chinese are the most careful people in the world in the conservation and profitable disposal of servage, and the most careless with regard to personal cleanliness.

Solid excreta is often used in the production of silkworms by applying it to the mulberry trees and also for breeding maggots for the fattening of ducks. 
(9) Indian Latrines.-The separation system is commonly used. The urine and washings are led by a pipe to a special receptacle and are not immediately mixed with earth. There are many modifications of platforms both with regard to material and pattern. They are made in iron, stonework, glazed ware, \&c., and may be so constructed as to avoid splashing. Instead of a seat there are the necessary foot rests for squatters such as are employed in all oriental latrines. Pails for the removal of the excreta should have a tight, well-fitting lid. They are taken away in carts or carried by coolies. Their contents should not be emptied into carts near to dwellings.

\section{(B) European.}

(I) Deep Pit Latrines.-These are useful for small communities, with a box seat over a pit, and a small movable house over the whole as previously described.

(2) The Bucket System.- This is good when the natives attending to them are reliable and the supervision sufficient. Loam should be used and the bucket emptied daily and washed out with cyllin. No nuisance need arise. The receptacle for the bucket should be so constructed that it is not necessary for the scavengers to enter the dwellings.

(3) Water Closets.-These are the best of all where a water supply is laid on and the drainage is good. They should be outside the house, as in the tropics the evaporation of water allows the best of traps to leak sewer gas.

(4) A Chemical Method.-For troops in the field the system suggested by Myer Coplans and James Menzies has found favour with the War Office, and is useful for the purpose for which it is intended. The writers describe it as follows :-

"The apparatus consists of :-

"(I) A partitioned chamber for reception of excreta,

"(2) A condenser,

"(3) A receptacle for the condensed products.

"The tank into which the excreta, mixed urine and faeces are introduced is cylindrical in shape and divided into several sections by horizontal partitions. The sewage is poured in through a hopper and broken up by a revolving comb; it passes on from above downwards through the successive partitions, being exposed all the time to a high temperature. As a result there ensues complete disintegration. The solid residue comes out at 
the bottom carbonized into charcoal; the rolatilized products are led through a condenser and received into a chamber, where they settle out into:-

"(I) A heary ammoniacal liquor",

"(2) A layer of oil,

"(3) Gaseous products.

"These last are piped off to a buner, where their combustion provides the heat required to produce the disintegrating action on the serage that takes place in the partitioned chamber.

"The ammoniacal liquor forming the lowest layer in the receiver contains pyridine bases, which are of value as an insecticide and as a bactericide. The layer of oil is collected and used for working the motor that actuates the revolving comb or scraper in the cylinder first mentioned. The solid carbonized residue is practically pure carbon, which may be used as fuel or for many other purposes. Hence these excretory products can be rendered innocuous and disposed of at a very minimum of cost, or even at no cost at all; it is quite possible that the residue charcoal may come to be a source of profit. At any rate, it seems proved that the working expenses are covered. The fact of the apparatus being self-contained and self-supporting-viz.: (1) That the heat required for the destructive distillation of the excreta is provided by the gas which is set free; (2) that the petroleum oil for running the motor is also obtained from the results of the same distillation-distinguishes it from all other chemical processes or methods of disinfection depending on heat.

"It is stated that no fuel is required at all except for the commencement of the working. That an insecticide and a bactericide should be obtainable from the condensation liquor is also much in favour of this method, if in practice it can be recovered without difficulty. In camp life such a substance would be most useful for the destruction of flies, fleas and vermin generally, as well as for its more important disinfectant applications to contagious pathogenic material."

\section{Cesspools.}

Cesspools or receptive tanks for houses or blocks of buildings are generally difficult to keep clean and to empty.

To correct these nuisances several methods of emptying cesspools 
have been adopted, the most recent being the "Tonne Vidangeuse Pneumatique," but it is a costly method.

No town with a population of over 15 , ooo can deal efficiently with its excreta other than by a pipe-water system.

\section{The Disposal of Excreta.}

Apart from what has already been said generally on this subject one may include the following remarks:-

(1) Excreta may be collected into cesspools and pits and then used for agricultural purposes on farms as previously stated.

(2) It may be carried to an incinerator and destroyed completely, but such is regarded generally as being a needless waste.

(3) It can be carried by a water-carriage system in the case of larger towns and discharged into large rivers, or by a pipe out to sea. The failings of the latter system are waste, offensive odours and troubles from the ebb tide.

(4) After a water-carriage system it can be treated by precipitation with or without filtration, intermittent filtration through land, broad irrigation, or bacterial treatment. Special works should be consulted for technical details. The principles have been dealt with under Water Purification.

(5) Chemical processes are used apart from the one mentioned above proposed by Coplane and Menzies.

The "Poudrette" method is fairly well known.

After fermentation by urea, volatile ammonium carbonate is formed which is usually fixed with calcium sulphate. The sewage is then desiccated by evaporation in numerous shallow pans.

The resulting powder is known as "Poudrette," and is sold to farmers for manure. The process is costly and the demand is usually small. Two tons of excreta yield about two hundredweight of poudrette. The system is unsuitable where there is much rain. There are various modifications of this system.

(6) Trenching and cultivation are perhaps the methods most commonly employed in hot climates. The trenching ground must be high, well drained, with a loamy soil, to the leeward of the village or town, and well screened from the public view.

The area should be large enough to deal with all excreta from the population and divided into twelve monthly plots for trenching. The trenches should be 0.6 metre wide and $0_{4} 4$ metre deep. They should be $0^{\prime} 3$ metre apart. Each trench should receive 0.2 metre of excreta. The area required for a population of $\mathrm{I}$, ooo persons would be I4 square metres 
per diem, or for the same number 5, r io square metres per annum. This affords twelve months' rest for each trench. Where the soil and all conditions are particularly favourable

Disposal of Excrement as Manure. Chinese Method.

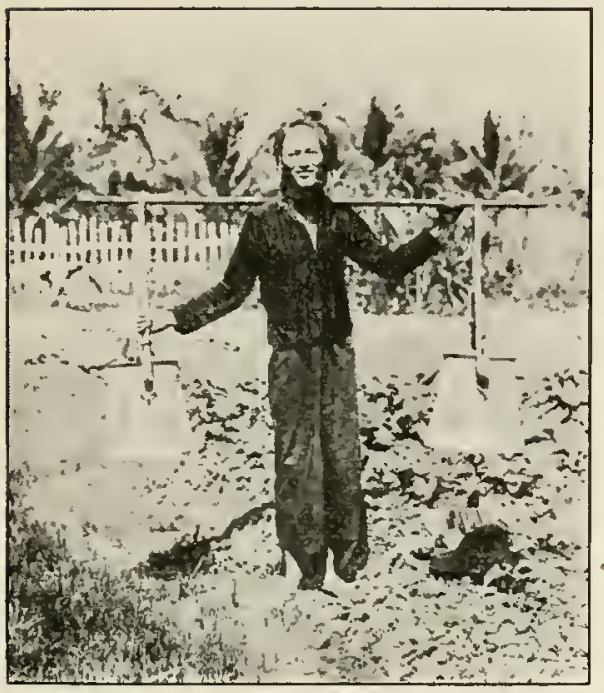

(I) Chinaman taking from public latrines in a Government receptacle fresh human ordure.

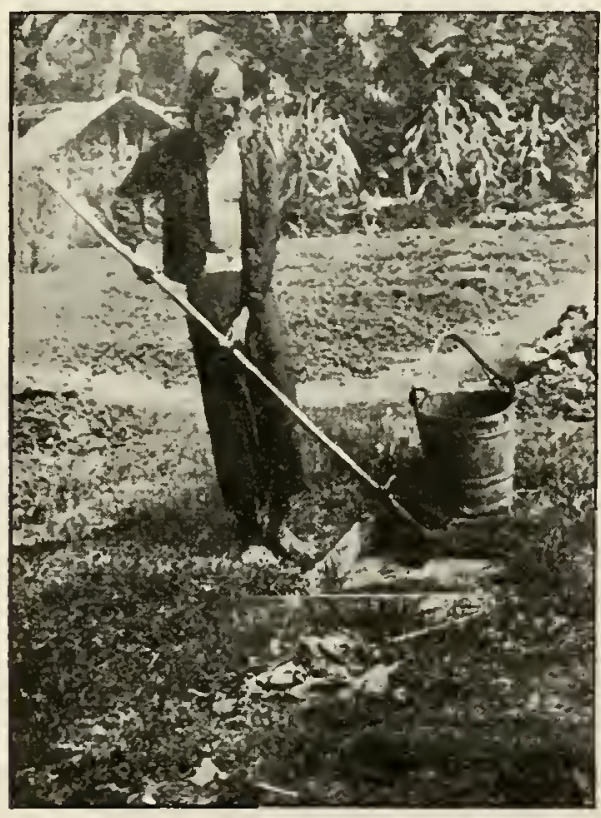

(3) Filling his private bucket with the contents of manure pit, consisting in part of fres kept sufficiently long for eggs to be destroyed, but in part sufficiently fresh for eggs to retain their vitality. To the contents of this bucket water is usually added.

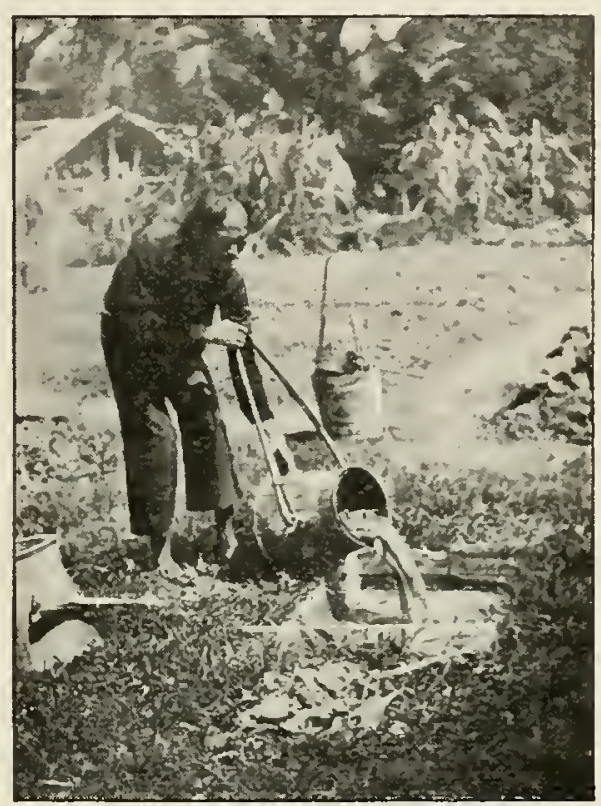

(2) Decanting into his manure pit where, if kept long enough, the eggs of most entozoa will be destroyed. It is rarely kept long enough.

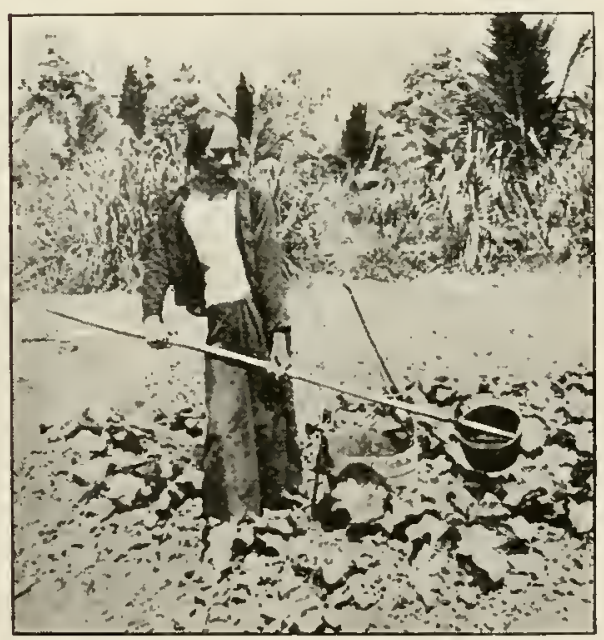

(4) Watering vegetables, including those used for salads or eaten raw, with the contents of this bucket. The great prevalence of "flukes" (trematoda) amongst the Chinese is probably due to the general use of some such method.

the land can be retrenched in six months. It is always best to arrange it so that one trench takes one day's supply, thirtyone trenches for a month's supply. The earth should be raised dome-shaped when covering them in to allow for sinking. 
After three months plough up each plot and sow with rye grass, sugar cane, or tobacco. After the first crop, vegetables can be grown and sent to the market for sale and consumption.

Swarms of flies about a trenching ground indicate improper management.

Such trenches could be dug at the base of cocoanut or palm trees, a safe, effective and profitable means of disposal.

Sullage water should be taken to trenching grounds in special slop carts where there are no good drains.

Storm water requires surface drainage.

Wells must be protected and sewage controlled.

Dry Refuse.-This always exists in large quantities.

In Calcutta, with its population of three-quarters of a million, there are nearly 1,500 tons daily. There are 900 carts employed making three trips daily.

For disposal incineration is always the best.

Failing this, hollows can be filled up, covered with soil and planted, but the system is not to be recommended. It should not be thrown into ponds or marshes. The putrefaction, fermentation and formation of gases thus produced will go on for years and may escape, proving deadly to man and beast alike, even though the refuse may be covered with several feet of earth.

\section{DISINFECTION.}

Disinfection is the destruction of the specific virus and is equivalent to a bactericide. The term should not be applied in a general manner to antiseptics which arrest or impede the growth of organisms. Disinfection means destruction of the agents of infection.

Deodorants, of course, only oxidize and destroy or mask the effluvia.

Much money is wasted in the purchase of disinfectants, while the inefficient application of them engenders a false feeling of security and often prevents simple means of purification.

Whenever possible one should employ a simple and useful method, namely, of exposing articles to direct sunlight, or rather, to the ultraviolet rays of white sunlight. This is sufficient to destroy ultimately most pathogenic organisms. Desiccation only will kill cholera vibrios.

Disinfection by heat or chemicals should always be preceded by thorough cleansing, e.g., stripping the paper from the walls, scraping and washing the walls and floors, allowing plenty of air and as much sun as possible to enter the apartments, washing, brushing, beating and suspending all garments, blankets, \&c.

The chief means of disinfection are heat, hot air, steam and chemicals. 


\section{Heat.}

This is the most efficient means of disinfection, but unfortunately it is not of universal application. For example, infected garments, articles of small value, native huts, \&c., should be destroyed completely by fire, and in the latter case killing all the rats as they attempt to escape.

\section{Hot Air.}

This is sometimes used for clothes and bedding, but it is slow and not very efficacious owing to the poor penetrating power of dry heat. Some articles may be scorched. It is useful for books and leather goods. The air temperature should be $230^{\circ}-250^{\circ} \mathrm{C}$. for four to eight hours, but steam should be substituted wherever possible.

\section{Steam.}

Steam is more rapid and efficacious because of its increased penetrating power. The essentials of its application are that the steam under pressure shall permeate the whole article to be disinfected. The steam temperature must be $221^{\circ}$ to $270^{\circ} \mathrm{C}$. After the steaming, hot dry air should be passed through the articles for drying them.

Steam is inadmissible for leather objects, such being destroyed by it.

All fusible substances such as glue and sealing wax are ruined by steam in any form.

The Current Steam Disinfector is the best. The machine is light, has no heavy boiler, is of easy construction, the cost is low and the management of it is not expensive.

The Wrashington Lyon apparatus by Manlove and Alliott is good. It has doors at both ends. The steam in the jacket is at a pressure of $30 \mathrm{lb}$. to the square inch and in the chambers at $20 \mathrm{lb}$. It is not superheated.

Elliott and Paton's vacuum apparatus is an improvement owing to the greater penetrating power and its increased efficiency and rapidity. Ten to twelve minutes will suffice for the disinfection. The articles are dried in a current of hot air.

Thresh's Disinfector has been extremely useful in the colonies and the tropics.

\section{Chemical.}

The chemical disinfectants used are legion. The most common are: Corrosive sublimate, 1 in I, ooo; Cyllin, I in 200; Izal, I in 100; Carbolic acid, I in 20 ; Potassium permanganate and Lysol.

A good chemical disinfectant should have the following characters:

(I) Germicidal within a reasonable time.

(2) Its chemical properties should not unfit it for ordinary use. 
(3) It should be soluble and emulsible in water.

(4) It should not produce injurious effects on human tissue.

(5) It should not be too costly in proportion to its germicidal value.

(6) Heating should not affect it.

Corrosive sublimate is poisonous, acts on metals, is thrown out of solution by alkalies and organic matter, is acted on by oxides, sulphur and sulphuretted hydrogen, loses its efficiency when mixed with sputum, fieces, \&c., because a harmless albuminate of mercury is formed. Its use is therefore limited. It is usually mixed with other chemicals to overcome some of these difficulties.

Carbolic acid is not so widely used as in former times because its bactericidal power is sixteen times less than cyllin and kindred preparations.

Cyllin, lysol and similar preparations have the advantages of forming a permanent emulsion with water, they do not act on metals, they are less toxic than carbolic acid and are sixteen times stronger than carbolic acid. They are compatible with soap. They should not be mixed with anything markedly acid.

Lysol and cyllin are the routine disinfectants used by the author.

The former gives a clear solution for instruments, \&c., for operations, the latter being used for general sanitary purposes.

For general use one may use the following:-

For washing drains, courtyards, \&c., pot. perman., I in 250 ; cyllin, I in 200 .

For disinfecting walls, floors, furniture and clothing, cyllin, $1 \frac{1}{2} \mathrm{oz}$. in a three-gallon bucket, or lysol, $\mathrm{I}$ in 40.

For cowshed floors, dung-heaps, privies, cesspools, dust-bins, use the above but double strength.

For solid and liquid excreta use as above or chloride of lime, 4 per cent.

For oxidizing organic matter in water, pot. perman. until a pink colour remains.

For douches, $\frac{7}{2}$ per cent. lysol.

For hand lotions, 1 per cent. solution of lysol.

For surgical instruments, 1 per cent. lysol.

For Insecticides Castellani recommends:-

For body lice, kerosene, vaseline, guaiacol, anise preparations, iodoform, lysol, cyllin, carbol solution, naphthaline, camphor in the order mentioned for efficiency. Sulphur, corrosive sublimate and zinc sulphate are useless.

For bed bugs, kerosene, guaiacol and pyrethrum.

For moths, naphthalene, menthol powder. The latter is repellent to lice, fleas and mosquitoes.

For patients entering the hospital with lice, \&c. : strip off all clothes, 
sterilize clothes, shave patient completely, bath in soft soap each night for three nights, put powdered naphthaline down the shirt collar next to the skin. The leat at night will dissolve it and kill any remaining bugs.

For houses and closed spaces fumigation is usually resorted to.

The means used are usually sulphur dioxide and sulphurous acid gas, formaldehyde, chlorine gas, bromine gas and nitrous acid.

(I) Sulphur Anhydride and Sulphurous Acid Gas.

This is generated by burning rolls of sulphur in an iron vessel with a little spirit to commence the burning.

Three pounds of sulphur are used for each 1, ooo cubic feet of closed air-space rielding theoretically $1^{\circ}$ I to $3^{\circ} 3$ per cent. of sulphurous acid gas in the space.

The Clayton apparatus is in common use for extensive fumigations with this gas. As in other methods the essential factors are to stop up completely all the air holes in the place to be disinfected. It is useful for large rooms, stores, ships, \&c.

Three per cent. of this gas for two hours will kill any pathogenic bacteria in exposed spaces, also rats, mice, insects, weevils, beetles, cockroaches, lice, bugs, mosquitoes, the eggs and the larva of insects. Anthrax spores are not destroyed.

Air saturated with from 6 to 8 per cent. of the gas for six hours destroys :-

The bacillus of plague, diphtheria, cholera, typhoid bacillus, tubercle bacillus, the smallpox infective agent and vaccines.

For a loaded ship's hold use 3 per cent. for eight to twelve hours and leave closed until next day. $0^{\circ} 5$ per cent. will kill rats and insects in two hours but the 3 per cent. is needed for the penetration.

The gas destroys fruits and potatoes. It injures wheat in bags but not in bulk, neither maize, rice, nor other grains in bulk. It will kill the weevils in these.

The method is more applicable for houses than any other because the articles need not be removed from the house. It is easier to take a Clayton apparatus to the house than to take the articles to the disinfector. Everything is disinfected in situ.

(2) Formic Aldehyde.

Formic aldehyde vapour by the alformant lamp is good. It burns 30 tablets per I, ooo cubic feet. The exposure is for five to ten hours.

Trillat's autoclave for formalin is more efficient but much more costly.

Singer's apparatus uses formalin 30 per cent. with glycerine ro per cent. This is raporized and is ejected as a fine spray. One needs 20 ounces of formalin for each r, ono cubic feet. I per cent. of vapour or $0^{\circ} 5$ per cent. solution will kill most organisms. It is more rapid, 
penetrating and diffusible than chlorine. It is harmless to colour but iron is affected.

Other means of using formalin are as follows :-

Mix $142 \frac{1}{6}$ grammes of permanganate

$$
285 \text {, formalin }
$$

Put it in a metal tray 7 inches square and $3 \cdot 4$ inches deep for the combustion

The Formanganate process consists of :-

$16 \mathrm{oz}$. of 40 per cent. formaldehyde solution

240 grammes of permanganate for each $I, 000$ cubic feet

Warmth to $63^{\circ} \mathrm{F}$., and moisture to $60-65$ per cent. humidity are essential.

A "homely" method is to use $80 z$. of permanganate for each pint of formaldehyde for every 1,000 cubic feet of air space. Suspend wet sheets in the roon. All fires should be extinguished as the gas is slightly inflanmable.

(3) Chlorine.

Chlorine is produced by adding crude hydrochloric acid to calcium chloride, $1 \frac{1}{2}$ to 2 pints of the acid to each pound of lime. Two pounds of calcium chloride with three pounds of the acid for each I, ooo cubic feet are generally used.

Nitrous fumes are generated by adding copper filings to nitric acid.

Some medical officers have found useful the following for the destruction of mosquitoes :-

Crenyl or creolin vaporized by a spirit lamp.

Use 600 c.c. for a room of 100 cubic metres with 270 c.c. of alcohol.

It is not necessary to paste up all the cracks.

It should be left for eight hours.

It is cheap, there is no smoke, it does not damage objects and does not have the bleaching effect of sulphurous acid.

In Great Britian rats do an enormous amount of damage reaching the sum of $E_{\mathrm{i}}$,000,o0o per annum.

For rat extermination viruses are sometimes used, e.g., the Live:pool virus, the Danysz virus and Ratin at a comparative cost of $\mathrm{I}, \mathrm{I} \frac{\mathrm{l}}{2}$ and $2 \frac{1}{4}$, but they rapidly lose their virulence in the tropics.

Barium carbonate, $I$ in 4 of meal on fish or bread, is effective against rats and mice.

Warehouses and stores should be disinfected with Clayton's apparatus every two months.

\section{QUARANTINE.}

Land quarantine was abolished in Europe some time ago but the governments have the right to close the frontiers if necessary.

The International Regulations of the Paris Convention of Ig0.3 were formed to prevent the importation of cholera, plague and yellow fever.

These are the only diseases as yet under international control. 
For large seaports the Convention recommends as follows :-

(I) A properly organized port medical service and permanent medical supervision of the health of the crews and of the population of the port.

(2) Suitable accommodation for the isolation of the sick and for keeping suspected persons under observation.

(3) A bacterial laboratory and buildings with the necessary plant for efficient disinfection.

A supply of drinking water of quality above suspicion at the disposal of the port authorities and a system of scavenging that offers sufficient guarantee for the removal and disposal of excrement and refuse.

All arriving ships are classified as:-

(I) Infected when there is a case of plague or cholera on board or has been on board within seven days of arrival in port.

The sick are immediately disembarked and isolated. The other persons disembarked, and if cholera they are kept under observation either on a ship or at a sanitary station before being allowed "free pratique" ; or they may be placed under "surveillance" for five days after arrival in port. Such as have "free pratique" are watched by the local authorities in the districts where they go. WVith regard to the ship the bilge water and drinking water are replaced.

If it is a case of plague observation may be followed by surveillance for five days. The soiled linen, wearing apparel and personal effects of crew and passengers are disinfected by the sanitary authorities.

Parts of the ship are also disinfected. All rats must be destroyed within forty-eight hours.

(2) Suspected when there has been a case of plague or cholera on board at the time of departure from port or during the royage, but when there has not been a new case within seven days of arrival in port.

Passengers and crews of suspected ships are subject to surveillance for more than five days after arrival of the ship in port.

Soiled linen and personal effects are disinfected and such parts of the ship as have been occupied by persons infected are also disinfected. For plague, all rats are destroyed and for cholera the bilge water is removed and good drinking water substituted for that on board.

(3) Healthy when there has been no case on board of plague or cholera either before or during the voyage, nor on arrival in port.

The passengers and crews of healthy ships from an infected port are subjected to five days' surveillance after cleparture of the ship from the infected port. The authorities may require the disinfection of clothes, the destruction of rats or the complete emptying of the bilge water. 
In tropical countries it is more difficult and costly to carry out these measures althougli the dangers are greater.

At ports where large numbers of coolies or immigrants are arriving, there is usually a strict quarantine for fourteen days at a quarantine station.

This is followed by the disinfection of the clothes and the bathing of the bodies of natives.

For English Regulations in detail see the Local Government Board Order of September 9th, I907.

\section{IMIMUNITY, VACCINES AND SERA.}

\section{SOME PROPHYLACTIC MEASURES FOR COMMON INFECTIYE DISEÁSES.}

Vaccines and sera are administered in order to produce partial or complete immunity against given diseases.

The fundamental principle of vaccine therapy is "to exploit in the interest of the infected tissues the unexercised immunizing capacities of the uninfected tissues" (Wright).

Immunity depends upon some property in the living blood serum which opposes or annuls, in part or wholly, the products and action of the infecting organisms (Whitelegge and Newman).

Natural Immunity denotes natural resistance to some given disease, e.g., white rats are immune to anthrax; the lower animals are immune to cholera and typhus fever; man is immune to swine fever.

Acquired Immunity may be acquired as the result of a natural attack of a disease, e.g., smallpox, or it may be after an artificial attack of the disease, e.g., inoculation.

Active Acquired Immunity is produced as the result of inoculating attenuated bacteria into the tissues, e.g., as for rabies.

Passive Acquired Immunity is produced by inoculation of antitoxins into the tissues, e.g., diphtheria.

Yaccination is an inoculation by an attenuated virus.

Vaccines are prepared from laboratory bacteria or bacteria from the patients about to be treated. These latter are known as autogenous vaccines. The bacteria are isolated, cultivated, killed by heat or antiseptics, tested for sterility, counted and preserved in antiseptics.

Imported vaccines do not keep at all well in the tropics. To preserve them for the longest time possible cut a portion of banana stem, clear out the central core, insert the vaccines, plug up the ends of the banana stem with a piece of the same material and keep in a cool place. The internal temperature of a banana stem is $23^{\circ}$ to $25^{\circ} \mathrm{C}$., and is fairly constant for some days. When becoming dry make another. The interior can be cooled daily by sprinkling it and its contents with ethyl chloride. 
Some medical officers use a tube of asbestos cloth surrounded by cotton silicate, the whole placed within a wooden or bamboo tube. Others have found thermos flasks useful.

Tabite of Vaccines and Thetr DOSES.

\section{Vacine}

Acne bacillus

Acne bacillus with staphylococcus

Bacillus septus

Cerebro-spinal meningitis

Cholera

Coli bacil'us

Diphtheria

Friedländer's bacillus

Gonococcus

Micrococcus melitensis

Pneumococcus

Staphylococcus

Streptococcus

Typhoid

Dysentely

Mallein

Plague
Approximate Dose

5 million initial, to 20 million

5 million with roo million increased

50-500 million

5-10 million initial

No. I followed by $\mathrm{No} 2$

10-25 initial up to 500 million

ro-roo million

50 million initial to $\mathrm{I}, \mathrm{OoO}$ million

25-50 million to $\mathrm{I}, 000$ million

25-250 million

25-500 initial to 500 million

I00-5,000 million

Up to 500 million

500-1,000 million. (Broth cultures)

In four strengths, for immunizing and curative in chronic conditions

(IO-I 5 minims in humans

11 c.c. for diagnusis in animals 75-8o million or I c.c.

Vaccines are :-

(1) Killed or attenuated organisms with or without toxins. Such are known as Bacterial Emulsions.

(2) The toxins only as in Mallein.

(3) The fluids of animals suffering from an infectious disease, e.g., calf lymph.

(4) Sensitized raccines.

Antitoxic Sera are usually obtained from the horse. A healthy one is reared, tested for tuberculosis and glanders, inoculated with attenuated serum, later with increasing doses of virulent toxin until a high degree of tolerance has been obtained. The horse rests a few days, 16 to 20 pints of blood are then drawn off from the jugular vein into sterile flasks, the serum is decanted after coagulation and mixed with antiseptic, it is tested for sterility and toxicity and then standardized. A standard dose is sufficient to neutralize a given minimal lethal dose in an animal of known weight.

A unit dose of antitoxin will exactly neutralize roo minimal lethal doses. In diphtheria a unit dose of toxin will kill a $250 \mathrm{grm}$. guineapig in four dars; this is considered as a minimum lethal dose. The former or unit dose of antitoxin will neutralize the latter or animal lethal dose and prevent symptoms.

Sera have been used for diphtheria, tetanus and bacterial dysentery, with high value, and in snake bite and plague with some value, ir. cholera with little value, and in tuberculosis and leprosy with doubtful value. 
The Protection afforded by these sera and vaccines is accounted for along the lines of five principal hypotheses as follows :-

(1) The Exhaustion (Pabulum) Theory.

During the first attack the invading organisms remove certain chemical substances necessary for their own growth and in consequence the same species cannot later reinvade the tissues with success owing to shortage of essential chemical food. This view is not widely held.

If correct, each specific disease must have a specific chemical pabulum as one disease is only protective against itself.

(2) The Antidote or Retention Theory.

It is assumed that after a bacterial atack some product is left behind which inhibits any further multiplication of the organism. This theory preceded the antitoxin and antibacterial theories. There is experimental evidence in support of it. Some of the bodies being formed are known as Agglutinins, which agglutinate bacteria or corpuscles, Cytolysins which destroy living cells and Precipitins which precipitate the albumin of the serum.

They have been summed up as follows :-

\begin{tabular}{|c|c|c|}
\hline Antibody & Antigen & Action \\
\hline Antitoxin & Toxin & Neutralization \\
\hline Precipitin & Coagulable protein & Precipitation \\
\hline Agglutinin & Cells, bacteria, \&c. & Clumping \\
\hline $\begin{array}{l}\text { Cytolysin, including bacte- } \\
\text { riolysin and hæmolysin }\end{array}$ & Cells, bacteria, \&c. & $\begin{array}{l}\text { Prepares cells, bacteria, \&c., } \\
\text { for solution by complement }\end{array}$ \\
\hline Opsonin & Cells, bacteria, \&c. & $\begin{array}{l}\text { Prepares cells, bacteria, Sc., } \\
\text { for ingestion by leucocytes }\end{array}$ \\
\hline
\end{tabular}

Some authorities state that opsonin should not be classed as an antibody. Possibly all antibodies may play the part of an opsonin.

(3) The Acclimatisation Theory.

This is a condition of increased cellular resistance following a bacterial attack. The cells acquire a tolerance of the poison so that ordinary symptoms do not manifest themselves.

(4) The Phagocytic Theory.

The phagocytic cells, polymorphonuclear leucocytes, mononuclears, \&c., absorb and destroy the invading bacteria. The inoculation of an attenuated virus is said to stimulate the cellular activity in this direction. Metchnikoff's views were based on this theory.

Opsonin (opsono = I cater for) is a chemical substance in the blood stimulating the leucocytes to ingest bacteria. The extent of its action, known as the opsonic index, is a valuable test in estimating the trend of immunization. This opsonic index is estimated by comparing the number of bacteria ingested by a given number of leucocytes in a normal serum and in a "stimulated" serum. 
(5) Ehrlich's Side-chain Theory.

Antitoxins and antimicrobic substances are assumed to be normally present in the blood, which when stimulated by the introduction of toxin become increased. A central or mother cell is imagined which throws off two varieties of small cells. These are receptor cells capable of combining with food-stuffs for the nourishment of the mother cell. One variety links up with simple food substances, the other breaks down compound bodies preparatory for assimilation. Toxins also have two varieties known as haptophores and toxophores. One becomes fixed to one variety of the receptor cells and the other remaining free, and if in sufficient numbers, produces toxic changes.

If the dose of toxin injected is small the mother cells throws off the receptor cell with the toxin attached, such passing free, and now being without harmful effect because it is "locked up" by the attached receptor. The mother cell produces more receptors so as to exhaus! the toxins if these latter are not too excessive. The mother cell their continues to throw off receptor cells into the circulation unfixed, seeking toxin cells to fix, but if these latter are exhausted the excess of receptor cells constitutes antitoxin molecules which are ready to render innocuous or non-toxic any new toxic substances of that specific nature which may enter the system. Hence immunity is established, partially or completely, for that specific disease. The toxins may be of the nature of albumoses, and the antitoxins are probably of the nature of globulins.

The immunity afforded may be feeble and of short durationweeks, months, or years. In small-pox seven years or thereabouts.

After an inoculation there is an increased susceptibility for several days, a condition known as anaphylaxis or a negative phase, consequently just after an inoculation there is increased susceptibility lowards the disease. Because of this negative stage inoculations should not be given within seven to ten days of the former one.

Inoculations should be given before the disease is established, during the prodromal period if it is known, or antisera may be given therapeutically during the disease.

As to plague vaccine. IIaffkine claims that the case mortality is reduced to less than one-half after inoculation.

Cholera vaccine gives a marlied protection against an attack, but the case mortality amongst the inoculated is not much diminished.

Antityphoid vaccine reduces the case incidence and case mortality as confirmed in the Great European War. It was only on its trial during the South African War. The protection is not permanent and lasts but from six to twelve months. One could not expect an inoculation to be permanent, really knowing that all the body cells are renewed entirely every seven years or so. 
Bacterial dysentery vaccines for chronic cases are most useful where there are epidemics of chronic dysentery. The sera are more useful in the acute stage.

Combined raccines are used and recommended by Castellani. One vaccine made and used by him was for Cholera, Plague, Typhoid. Paratyphoid $A$ and $B$ and I Indulant Fever together. The inoculation was harmless. Time and experiments alone will indicate their therapeutic and prophylactic value. 


\section{SECTION IX.}

\section{LABORATORY HINTS.}

THE EXANINATION OF THE BLOOD, INCLUDING:-

The Preparation of Slides.

THE CHOICE OF STAINS.

THE FIXING OF FiLMis.

A Difherential Leucocite Count.

ABNORMAL BLOOD-CELLS.

The Estimation of Red BloOd-cells aNd Hamogiloein.

To Denonstrate Living Malarial Parasites.

To Stain Malarial Parasites. Their Varieties.

TO DEMONSTRATE MICRO-FILARIA.

THE WASSERIIANA AND WIDAL REACTIONS.

To Estimate tile Specific Gravity of the Blood, Spectroscopic ExaminaTION, AND THE, TONICITY OF THE BLOOD.

SPECTROSCOPICAL EXAMINATION.

'THE TONICITY OF THE BLOOD.

THE EXAMINATION OF MICRO-ORGANISAS :-

STAINING METHODS.

Diagrostic Features of MiCro-organisus.

THE Preparation of Cultures axd CUiture Meids.

THE PREPARATION AND STAINING OF SECTIONS.

MALARIAL PIGMENT, WHERE IT IS AND HOW TO FIND IT.

THE TRICHOMYCETES.

THE HPHOMTCETES (MOULDS).

THE BLASTOMIYETES (IEASTS).

THE EXAMAATION OF F.AEES.

Macroscopical, Microscopical, including Ova.

DIAGNOSTIC FEATURES OF OVA.

THE DISSECTION OF MOSQUITOES.

THE EAAMINATION OF WATER.

Biological, Bacterial, Phiysical, Chemical.

THE EXAMINATION OF SEWAGE.

THE ANALYSIS OF MILK. 


\section{LABORATORY HINTS.}

\section{THE EXAMINATION OF THE BLOOD.}

\section{To Clean Slides.}

Slides should be of the medium, not the best, quality for the tropics.

Boil in strong soda water for five minutes.

Wash well in running water.

Place in 50 per cent. $\mathrm{I}_{2} \mathrm{~S}()_{4}$ for five minutes.

Wash thoroughly to remove all acid.

Drain and place in methylated spirit.

\section{To Clean Cover Slips.}

These should be of the best quality.

Boil for ten minutes in bichromate solution with a low flame.

Wash well and remove all traces of yellow colour.

Drain and place in methylated spirit.

\section{CHOICE OF STAINS.}

Basic stains do well for newly growing celis, but more mature and permanent ones as red cells take acid stains better.

Hamatoxylin and eosin are good in the tropics and do not fade.

Leishman's stain is better at first but fades later.

Hamatoxylin should be matured in quality by light and air.

Leave the bottle in the sun with the cork loose.

To stain for blood changes, use hamatoxylin and eosin, but use I.eishman's for unfixed blood films.

To stain for bacteria, use Loeffler's methylene blue or carbol fuchsin (dilute, four minutes).

To stain for animal and vegetable parasites, use carbol thionin or carbol fuchsin.

Another film must be used for the acid-fast test.

To stain for tissue changes in sections, use hamatorylin two and a half minutes, ensin ten seconds.

If for protozoal parasites use :-

Hamatoxylin, fifteen minutes, acid alcohol ten seconds, or

Carbol thionin or

Van Gieson stain. 


\section{TO FIX BLOOD FILMS.}

Agents.-Time, heat, IIg perchloride, formalin, osmic acid, absolute alcohol.

The best is absolute alcohol and ether, equal parts.

Place blood films in it from ten minutes to ad lib.

This solution does not lose its fixative power.

Blood films to be stained with Leishman's need no fixing at all.

\section{A NORMAL DIFFERENTIAL BLOOD COUNT WILL SHOW :-}

Some "blood dust" which must not be taken for parasites, though Brownian movement may be present.

Léncocyles.-6,000-8,o00 per c.mm.

Lymphocyles.-- r5-25 per cent. They have a large deep staining nucleus with little protoplasm.

Large Mononuclears.-5-10 per cent. These are larger cells with a larger and paler nucleus with more protoplasm.

Polymorphonuclears.-65-75 per cent. There are several nuclei which are connected by nuclear filaments.

Eosinophiles.-I-3 per cent. The granules show an affinity for acid stains.

Transitional.-Few. These have a horseshoe-shaped nucleus.

\section{Remember that:-}

(1) The lymphocytes are increased during digestion, scurvy (6o per cent. are lymphocrtes), lymphatic leukamia (-7o-\$5 per cent. are lymphocytes), enteric and Malta fever, but these latter are not constant.

(2) The large mononuclears are increased during malarial fever (15-20 per cent.). This is not reduced after taking quinine. Quinine may have cleared up the parasites, but the above condition will assist the diagnosis.

(3) The polymorphonuclears are increased physiologically during youth and pregnancy, and pathologically during suppuration and pneumonia.

(4) Eosinophiles are increased during worm infections, e.g., trichiasis, bilharziasis, filariasis, \&c. Trypanosomiasis, verminous conditions, asthma and bronchitis, urticaria, pemphigus, and after dietetic errors, such as "high" cheese, venison, \&c.

(5) The total leucocytes are diminished in trypanosomiasis and kala-azar $(3,000$ or less).

\section{ABNORMAL BLOOD CELLS.}

\section{White Cells.}

Mast cells are degenerated leucocytes of the polynuclear type.

The nucleus stains poorly. The granules are coarse and are called meta-chromatic granules, because they neither take hrematoxylin nor eosin well, but stain better with a combination of the two. 
Leishman's is good and gives them a brownish tint. They are sometimes found in normal blood. Their significance is not known.

In lymphatic leukremia the leucocytes are irregular in size, shape and staining qualities. They are increased to 1 , ooo per c.mm.

From to to 98 per cent. are lymphocytes. The red cells are diminished in number and hb.

In spleno-medullary leuliamia, myelocytes are found. The cell may be very large or smaller than normal, the nucleus stains poorly, and it is sometimes sudded over with eosinophilic granules. These cells are pathognomonic of this disease. As a rule they are more numerous and larger than any other leucocyte. The nucleus is never horseshoe-shaped. The blood looks milky. The red cells are reduced to 700,000 per $\mathrm{c} . \mathrm{mm}$., some are nucleated. There are also polychromatic and mast cells.

\section{Red Cells.}

Variation in colour usually indicates the amount of hb. A rough indication of anemia is thus given.

Poikilocytosis as seen in pernicious anemia may be imitated artificially in making the film by drawing the cells down one slide with another, but the elongation is in the same direction in all the cells. To avoid it in making a blood film with two slides, the drop of blood should be drawn away from the operator and not pushed away with the second slide.

Nucleated red cells may appear in normal blood, primary and secondary anamia. The nucleus may be divided, or it may appear as two rouncled bodies.

Polychromatic cells are not uncommon in the anamias, especially in malaria. They are degenerated red cells and take up both eosin and methylene blue.

Some red cells are basophilic; they show stippled dots peripherally which may be polychromatic. They indicate a degenerative process. They are very common in lead poisoning. They do not indicate latent malaria as Plehn supposed.

\section{COUNTING BLOOD CELLS AND ESTIMATING HB.}

\section{To Count Red Cells.}

Use Gowers's hamacriometer.

Draw into the pipette blood from a small needle wound in the lobe of the ear or finger-tip up to the mark on the stem near the bulb.

Fill to mark above the bulb with Hayem's solution.

Shake well, then place one drop on special slide provided.

Place on this a cover slip. The drop should cover the central disc, but should not run over this so as to come between the cover slip and 
the main slide. Practice will soon enable one to estimate the size of drop required.

Use $\frac{1}{6}$-in. lens and No. + eye-piece and focus so that a set of sixteen squares is seen.

Count the red cells in each square and on two sides of it.

Count up four sets or more of sixteen squares each and divide the sum by the number in each.

Thus sixty-four squares contain 768 red cells $=$ I 2 in each square. The content of each square is $\frac{1}{20} \times \frac{1}{20} \times \frac{1}{10}=\frac{1}{4} \overline{0} \bar{c} \mathrm{cmm}$.

Hence one c.mm. of the solution contains कोo $\times 12$ red cells.

But the solution was diluted roo times with the sodium sulphate solution. cells.

Hence I c.mm. of blood contains $\frac{100}{4 \cos \times 1_{2}}=4,800,000$ red blood

\section{For White Cells.}

l'se I per cent. acetic solution and a drop of methylene blue. A separate pipette giving a ro per cent. dilution is better.

\section{In estimating the $\mathrm{Hb}$. in the Tropics.}

A book with stained papers and corresponding blank blotting papers is used.

A little of the latter is touched with the blood, and then is matched with the stained paper, which gives the percentage of hb.

A small book costing 5s. will last five years.

\section{TO DEMONSTRATE LIVING MALARIAL PARASITES.}

Take a small drop of blood on centre of cover slip.

Edge the cover slip with vaseline and place it on a slide.

Examine middle zone with a $\frac{1}{6}$-in. objective.

In the outer zone are rouleaux.

In the middle zone are single red cells.

In the central zone very few red cells.

A dull light is required as corpuscles are colourless.

The parasite will be seen in a red cell and pigment in the parasite.

If the field is flooded with light the pigment is still clearly seen, but the granular leucocyte is not clearly seen.

The parasite shows amoboid morement; the males will throw out long pseudopodia which lash the red cells, and eventually become free to enter the female cell. The actual entry has not been witnessed.

\section{TO STAIN THE MALARIAL PARASITE IN THE BLOOD.}

(1) Leishman's stain is the best. It is polychrome. No fixing is required.

The eosin stains the red ceils. 
The red methylene blue stains the chromatin.

The blue methylene blue stains the nucleus.

The soloids are dissolved in pure methyl-alcohol and put up in phials.

The film should be fresh and unfixed.

Stain half to one minute.

Add distilled water in drops as 2 to 1 of stain and move about gently live to seven minutes. One can see under $\frac{l}{6}$ in. when it is stained sufficiently.

Flush off with distilled water.

Dry and mount with Canada balsam and cover slip.

Unmounted blood films will keep a long time.

Other methods are :-

(2) Borax methylene blue.

Fix in alcohol and ether, ten minutes.

Dry and stain with borax methylene blue, thirty seconds.

Wash well, dry and mount.

This will not differentiate the chromatin.

It is not so good for the white cells.

It is better than Leishman's for old films.

(3) Hæmatoxylin and eosin.

Fix in alcohol and ether, ten minutes or more, and dry.

Stain with hæmatoxylin, seven minutes.

Flush off with tap water for five minutes.

Stain with eosin, thirty seconds.

Wash, dry in air, mount.

(4) Carbol-thionin.

Fix in alcohol and ether, ten minutes.

Dry and stain with dilute carbol thionin, ten minutes.

Wash, dry and mount.

Strong solutions are used for sections. This will keep.

This is diluted with three parts of water for blood films.

This soon degenerates-twenty-four hours.

One cannot over-stain with it.

The older the film the less time required for staining.

(5) Eosin-azur. No fixing required.

This stain shows up the crescents well.

Stain as with Leishman's; only use three times the distilled water from twelve to fifteen minutes.

Dissolve one soloid in ro c.c. of methyl alcohol.

It is twice the price of Leishman's stain.

This is good for the trypanosome and halteridium. 


\section{THE VARIETIES OF MALARIAL PARASITES.}

There are three to look for:-

The benign tertian parasite fully grown in forty-eight hours.

The subtertian, astivo-autumnal or malignant parasite, fully grown in thirty-four to forty-eight hours.

The quartan parasite fully grown in seventy-two hours.

There may be several "crops" of one parasite, so that the same slide may show two or three groups at different ages.

There mav be a multiple infection, two varieties of parasites in the same film.

One family of parasites may be so few in number that there will be no corresponding rise of temperature.

If there are two infections of the same variety the rises of temperature are seldoni equal.

In benign tertian the red corpuscle is enlarged and pale. Schüfner's dots may be seen, which consist of peculiar granular degeneration staining brick red and diagnostic of benign tertian. Parasites are plentiful in the peripheral blood, but tend to sporulate in the deeper organs; the gametocyte is rounded or ring-shaped, fifteen to twenty-five spores, yellow brown, pigment coarse. Active amoboid morement. Life-crcle forty-eight hours.

In the subtertian the red cell is smaller, no Schuiffner's dots. loung ring forms are seen in the peripheral blood, but not always; it matures in the deeper organs. The sporulating form found in the spleen may be seen in the peripheral blood just before death. The corpuscle becomes yellow- "brassy body." The gametocyte is crescentic in shape, pigment black and fine. It is rery amoboid. Life-cycle is variable, perhaps thirty-four to forty-eight hours.

Spores, 7-8, rarely 20. Very active, but small processes.

The male and female cells can be distinguished.

Male, has pigment and chromatin more scattered.

Female, one central mass of chromatin with the granules arranged around it. There may be two or three or more small, clean-cut ring forms in one cell which is rare in benign tertian or quartan.

Some subtertian parasites may be seen that have not penetrated the red cell which is uncommon in benign tertian.

The chromatin is more flattened, and not knob-shaped as in benign tertian.

A second slide taken twenty-four hours later will show parasites enlarged if benign, but they will be little altered or gone to the deeper organs to sporulate if subtertian.

In benign tertian and quartan there are usually a few early or late sporulating parasites which would not be seen in subtertian. 
The finding of crescents establishes the diagnosis.

A sportulating subtertian fills about one-third of the red cell.

A sporulating quartan fills the cell.

The crescents or subtertian gametocytes are especially interesting.

The sexes are differentiated. They do not produce fever. They undergo no cliange in man's blood. They appear one week after the fever has commenced and persist for six weeks after quinine has been given. Although the patient has no ferer, he is infective as long as these crescents remain in the blood.

In the cuartan parasites the red cells are never enlarged. The affected cell is darker than the normal ones, it requires longer to develop, seventy-two hours; it has fewer spores, five to twelve, than benign tertian, but they are the largest of all. Sporulates in the peripheral blood. Corpuscle becomes smaller and darker. Gamete rounded, the pigment is black, and granules coarse. There is little amoboid morement. The parasite may be stretched out across the cell "cigar-shaped," when it is called an "equatorial" parasit".

The pigment of the male dances about, throws off flagella which are very active. In ten to fifteen minutes they break off and swim freely in the blood until they pierce a female cell. The female cell is quiescent after the polar bodies have been extruded. The fertilized female or zygote becomes oval "oökinet," and later when it moves it is known as a travelling vermicule. The leucocytes gather around the male cell when the flagella have been thrown off and remove them. This process can be seen under the microscope in specimens of fresh blood.

\section{TO EXAMINE FRESH BLOOD FOR MICROFILARIÆ.}

Make a thick film of several drops of blood and let it dry.

Place it inverted in a watch-glass of water so as to remore hb. from the blood.

Fix it in alcohol and ether.

Stain with hot hematoxylin five minutes, then flush with water.

Examine while wet. Repeat stain if not well blue.

Dry and mount.

\section{THE WASSERMANN REACTION (FLEMING'S MODIFICATION).}

This important diagnostic reaction of the blood depends upon the principle of hamolysis. If the case for diagnosis be really a case of syphilis, then no hamolysis will occur. A control experiment is carried nut at the same time, using normal serum.

The substances required are:-

(1) An alcoholic extract of heart muscle. It is made from I grm. of heart muscle (guinea-pig) rubbed in a mortar with 25 c.c. absolute alcohol, heated to $60^{\circ} \mathrm{C}$. for an hour and filtered. 
(2) Sheep's corpuscles washed and diluted with normal saline.

(3) Serum in an ordinary blood tube.

Use a throttle pipette with a long stem and rubber teat.

Half an inch from the capillary end mark with a grease pencil.

This equals one volume. Permit a bubble of air and draw up another volume, and so on until all required are drawn up into the pipette. Prepare the following:-

(1) Extract of heart muscle . .. ... \& volumes $\begin{array}{llllll}\text { Syphilitic serum } \quad \ldots & \ldots & \ldots & 1 & ,\end{array}$

(2) Heart muscle ... $\ldots \quad \ldots \quad \ldots 4^{\prime}$, Normal serum $\quad \ldots \quad \ldots \quad \ldots \quad$ I $\quad$,

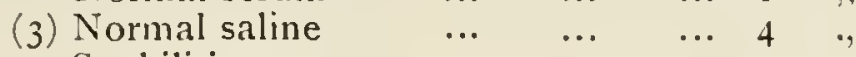
$\begin{array}{llllll}\text { Syphilitic serum } \quad \ldots & \ldots & \ldots & 1\end{array}$

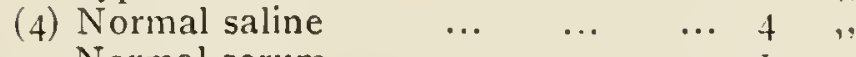

Normal serum $\quad \ldots \quad \ldots \quad \ldots \quad$ I $\quad$,

(5) Heart muscle $\ldots \quad \ldots \quad \ldots \quad \ldots \quad 4 \quad$,

Incubate all these for one hour at $37^{\circ} \mathrm{C}$. Add then to each tube one volume of sheep's corpuscles. Incubate again for one-and-a-half to two hours. The results should now be :-

No hrmolysis in tubes (1) and (5)

Hæmolysis in all the others.

The heart contains a syphilitic antigen which serves the same purpose as a syphilitic foctal liver. To discuss the whole biochemical process is not our purpose, but the explanation of the reactions in each lube is here given in brief.

(I) Syphilitic antigen in extract + syphilitic serum (syphilitic amboceptor + complement) + sheep's corpuscles.

The complement is fixed, therefore there is no hamolysis.

(2) Syphilitic antigen + normal serum (non-specific amboceptor + complement) + sheep's corpuscles.

The complement is not fixed, therefore hamolysis.

(3) Normal saline + syphilitic serum (amboceptor + complement) + sheep's corpuscles.

The complement is not fixed, therefore hamolysis.

(4) Normal saline + normal serum (amboceptor + complement) + sheep's corpuscles.

The complement is not fixed, therefore hamolysis.

(5) Antigen + sheep's corpuscles (no amboceptor, no complement).

Therefore there is no hamolysis.

\section{THE WIDAL REACTION.}

An important diagnostic blood reaction for typhoid and allied fevers, depending upon the agglutination of the bacteria.

Clean the lobe of the ear with ether.

Collect a few drops of blood in a sterile capillary tube.

Seal the ends in a flame with blood in the central part. 
Centrifuge or let it stand several hours, break off end, and blow out serum when required.

Lse a pipette as for the $\mathrm{M}^{2}$ assermann reaction.

Draw seven rolumes of normal saline into pipette.

Express one volume on each of seren clean slides or one large stide.

Take one volume of serum to be tested and place on a clean slide.

Take another volume of serum to be tested and place on a slide with one volume of saline.

This serum is now diluted $\mathrm{I}$ in 2.

Take one volume of this latter, mis with another slide with one volume of saline.

This is 1 in 3 dilution.

Repeat this process through the series excepting the seventh slide, as this last is of normal saline for a control.

Take a thick emulsion of typhoid bacilli from an agar culture.

Add one volume of this to each of the slides and mix well.

Examine under a $\frac{1}{6}$-in. objective.

The reaction is positive if complete agglutination occurs immediately in the 1 in 2 dilution and within half an hour in the $I$ in 6 dilution.

These can be examined by drawing them up into a long pipette separated by an air bubble, or separate fluid drops on one large slide or on separate slides.

\section{THE SPECIFIC GRAVITY OF THE BLOOD.}

From 1035 to I068.

To estimate it prepare a fluid of glycerine and water or chloroform and benzol in known proportion about the normal specific gravity of the blood.

Place in it one drop of blood, and alter the specific gravity of the fluid by adding glycerine and water or chloroform and benzol until the diop neither floats nor sinks. The specific gravity of the fluid is then that of the blood.

\section{THE SPECTROSCOPIC EXAMINATION.}

A small direct vision spectroscope is the most useful.

Dilute the blood with distilled water.

Focus the spectrum, closing the slit as much as possible so as to bring out Fraunhöfer's lines distinctly.

Oxyhamoglobin and reduced hamoglobin can be obtained from the same specimen by shaking it up with air to oxidize it and adding ammonium sulphide to reduce it.

(Syhamoglobin shows two narrow unequal dark bands between the lines I) and $E$. 
Reduced hamoglobin shows one broad band between lines D and $\mathrm{E}$.

Methamoglobin shows wo equal bands between D and I: and one between ( and $\mathrm{I}$ ). The urine is brown. It is often seen in mild cases of blackwater fever.

Urobilin shows one broad band between $\mathrm{E}$ and $\mathrm{F}$.

\section{THE TONICITY OF THE BLOOD.}

Some specimens of corpuscles lave the power of retaining hb. better than others. Distilled water will remove the hb. but saline solution over a certain strength will not remove it. The resistance or tonicity can be measured by the strength of that saline solution which is just sufficient to prevent the solution of the lib. This solution is said to be "isotonic." Normal blood is hypertonic.

Normal saline solution, 75 per cent., prevents the solution of the hb.

Make a series of weaker solutions differing $0^{\circ} \mathrm{O} 2$ per cent.

Place one drop of blood in this, shake and allow to stand.

The weakes: saline solution that does not cause solution of the hb. is the index of the tonicity of the blood used. The normal is $0{ }^{*} 6$ to $0{ }^{4} \mathrm{~S}$ per cent.

A decrease of tonicity often precedes a hamolytic attack.

Persons of a normally low tonicity should not be allowed to live where blackwater fever is endemic.

\section{THE EXAMINATIOX OF MICRO-ORGANISMS.}

\section{TO STAIN MICRO-ORGANISMS.}

The Gram method is important.

\section{To Stain Smears.}

Stain with aniline gentian violet, five minutes.

Pour off, treat with Gram's iodine solution, two minutes.

Pour off, treat with alcoholic eosin until the colour ceases to come ou: freely.

Wash, dry and mount.

Gram's iodine is I part of potassium iodine and 300 parts of water.

\section{To Stain Sections.}

Stain with aniline and gentian violet, ten minutes.

Stain with Gram's iodine, two minutes.

Pour off and blot.

Stain with alcoholic eosin, thirty seconds.

Oil of cloves and xylol. 
Important Differentiations.

Gram-positinle

Staphylococcus, all varieties

Streptococcus pyogenes

Micrococcus tetragenus

Fraenkel's bacillus

Bacillus acne

," anthracis

, botulinus

, diphtheria

- enteritidis (Klein)

, Oppler-loas

" pseudo-diphtheriae

" xerosis

, tuberculosis

, smeginie?

" leprae

" subtilis

" welchii

," telani

Aspergillus

Sarcina, all varieties

Yeasts (blastonıycetes)

Ringworm fungi

Streptothrix of actinomycosis $"$

madura disease

\section{Gram-nesation}

liacillus mallei

" coli communis

, coli dysenteriae

, enteritidis (Gäıtner)

,. pestis

" pyocyaneus

, influenze

, Friedländer's pneumo

,, malignant cedema

, prodigiosus

, proteus vulgaris

$"$ fluorescens

,, smegma

" soft sore

Diplococcus intracellularis meningitidis Diplococcus catarhalis

Gonococcus

Spirillum choleræe asiaticre

Spirochates of syphilis, relapsing fever. Vincent's augina, and other parasitic protozoa

Micrococcus nelitensis

Most organisms when stained with fuchsin are readily decolorized by weak solutions of mineral acids. The exceptions are: Tubercle bacillus, bacillus lepre, smegma bacillus, the bacillus of timothy grass, the dung bacillus and others less important.

If an organism retains its stain after treatment by 25 per cent. $\mathrm{H}_{2} \mathrm{SO}_{4}$ or 30 per cent. nitric it is acid-fast.

\section{The Acid-Fast Method.}

Stain with warm carbol fuchsin, five minutes. (To warm, heat a penny over a Bunsen flame and place slide upon it. Repeat the process during five minutes.)

Decolorize with 20 per cent. strong $\mathrm{H}_{2} \mathrm{SO}_{1}, \mathrm{H}_{\mathrm{NO}}$ or $\mathrm{HICl}$.

Wash well, dry and mount if a film; dehydrate, clear and mount if a section.

Some organisms are so rapidly decolorized that they are not seen, as the pneumo-bacillus. For these use aniline oil instead of acid alcohol.

\section{Weigert's Method.}

Aniline gentian violet, five minutes.

Gram's iodine, two minutes.

Pour off, treat with aniline nil, then stop its action with water.

Counterstain other Grams with Bismarck brown one minute, or aqueous eosin 20 seconds.

Wash, dry and mount. 


\section{To Find the Diplococcus pneumoniæ stain thus :-}

Aqua eosin, twenty seconds.

Wash, treat with aniline gentian violet, ten minutes.

Pour off, treat with Gram's iodine, two minutes.

Pour off, blot, and treat with aniline oil until colour ceases to come out freely.

Treat with xylol to stop the action of aniline oil.

\section{To Stain Spores.}

Hot carbol fuchsin, five minutes.

Wash, treat with 20 per cent. acid, two dashes.

Wash well to get rid of the acid.

Stain with Loeffler's blue, four to five minutes.

Wash, dry and mount.

In examining a micro-organism ascertain its :-

Motility, morphology, acid-fast or not, Gram-positive or not, does it form spores or not.

Remember that no acid-fast organism is motile.

no coccus is acid-fast or forms spores,

all acid-fast organisms are Gram-positive,

Gram-negative organisms are non-sporing except the bacilli of malignant cedema.

Thus a cholera vibrio would be motile, hence it would not be acid-fast nor would it form spores.

Motility depends upon flagella but the extent of movement does not depend upon the number of flagella. These latter are difficult to stain and readily break off.

Brownian movement can be performed by inanimate matter as well as by some micro-organisms.

\section{TO DIAGNOSE MICRO-ORGANISMS.}

One must know their morphology.

The chief ones from the acid-fast group are :-

\section{(1) The Tubercle Bacillus.}

A non-motile slender rod with rounded ends, often slightly curved, 2.5 to $5 \mu$ long. Some portions do not stain well and hence it often presents a "beaded" appearance.

It is acid-fast.

The culture is aerobic and facultatively anaerobic.

Grows slowly at blood heat.

In glycerine broth it develops as a floating pellicle.

On glycerine agar it gives a creamish, dry and wrinkled film.

It does not liquefy gelatin. 
Pure cultures can be formed by inoculating a guinea-pig from tubercular sputum.

\section{(2) The Bacillus pseudo-tuberculosis of Pfeiffer.}

It grows rapidly and readily forms a creamy growth on agar very different from that of tubercle. On gelatin a whitish growth without liquefaction. It is not acid-fast. Is Gram-negative. Found in milk and sewage. Pathogenic to guinea-pigs and rabbits.

(3) The Bacillus lepræ.

A long slender straight rod, with somewhat pointed extremities, non-motile, does not form spores. Gram-positive. Acid-fast.

Stains more rapidly and decolorizes more readily than the tubercle bacillus. Attempts to grow cultures have not been very successful.

\section{(4.) The Smegma Bacillus.}

Shorter and flatter than the tubercle bacillus in cultures.

Grows readily, acid-fast.

Non-patlogenic in small infections.

\section{(5) The Bacillus of Timothy Grass.}

Acid-fast. Resembles tubercle bacilli, but is harmless.

It grows readily in any ordinary medium.

It is found in fodder about cow-sheds, and when in milk may be taken for the tubercle bacillus.

The chief ones from the spore-forming groups are:-

\section{(1) The Anthrax Bacillus.}

It is aerobic and facultatively anaerobic. 5 to $6 \mu$ long.

Non-motile, square ends, occurs in short chains and singly in blood.

Some segmentation may be seen on staining. It is surrounded by a sheath in which it divides; this process may continue, forming chains.

In the presence of much oxygen and a temperature of $20^{\circ}$ to $38^{\circ}$ C., spores are developed, but only after death when the blood discharges come into contact with the air. In cultures it liquefies gelatin in two days.

In a stab, fine branching filaments grow outwards from the needle track called the " inverted fir tree growth." Iiquefication begins at the top.

In both a focculent growth forms at the bottom.

On agar a thick grey-white sticky growth.

The bacilli are Gram-positive.

To stain spores heat them for twenty minutes in warm carbol fuchsin. 
(Heat a penny over the flame, remove it, place upon it the slide with spores and stain. Repeat several times.)

\section{(2) The Tetanus Bacillus.}

Sliort straight slender rod with rounded ends, slightly motile with many flagella, forms spherical spores at one end, giving the "drumstick" appearance. Gram-positive. Not acid-fast.

A strict anaerobe. Liquefies gelatin. In glucose-agar stab, a feathery radiated growth with slight gas formation. Spores very resistant. Nearly pure cultures obtained by heating ordinary earth to $\mathrm{So}^{\circ} \mathrm{C}$. on two to three successive days and then preparing agar shake cultures.

Produces two alkaloidal toxins, tetanotoxine and spasmotoxine, which both produce convulsions. Readily destroyed by heat and light.

Natives bury carcases in order to produce this toxin for arrow poison.

(3) The Bacillus œdematis maligni (Bacillus septicus).

A motile slender rod ( $4 \mu$ long), with several flagellae. Gramnegative.

Stains readily. Forms spores in a central position. Tends to grow in long filaments. A strict anaerobe. Gelatin liquefied with production of foul smelling gas. Blood serum liquefied.

\section{(4) The Bacillus botulinus.}

Large (4 to $6 \mu$ ), with rounded ends, slight motility, four to eight flagella, forms terminal spores, an obligatory anaerobe. Grampositive. Does not curdle milk, ferments glucose with production of acid and gas, lactose not fermented. In glucose gelatin yellowishbrown translucent colonies are formed surrounded by a liquefied zone. Causes botulism.

\section{(5) The Bacillus welchii.}

A long ( 3 to $6 \mu$ ), thick bacillus in ones, chains and clumps, often has a capsule, forms spores only in serum cultures, strictly anaerobic, Gram-positive. Non-motile. Liquefies gelatin slowly, produces gas in dextrose and lactose, a honey-combed curd in milk with gas in twenty-four hours. Cultures are strongly acid. Found in septicamic and pycemic infections of the gastro-intestinal tract.

\section{The important ones from the Coli Group are:-}

(1) Bacillus coli.

(2) The capsulated bacilli.

(3) The bacillus enteritidis group.

(4) Bacillus typhosus.

(5) Bacillus dysenteriz.

All are as a rule short, stout with rounded ends, form no spores. 
Gram-negative. Do not liquefy gelatine. On gelatin plates form thin, irregular, notched colonies (see table for differentiation).

\begin{tabular}{|c|c|c|c|c|c|c|}
\hline Class & Motility & Glucose & Lactose & Litmus paper & $\begin{array}{c}\text { Indol } \\
\text { Production }\end{array}$ & Neutral red \\
\hline $\begin{array}{l}\text { Typhoid ba- } \\
\text { cillus }\end{array}$ & $\begin{array}{l}\text { Actively mo- } \\
\text { tile ; } 8 \text { to I } 2 \\
\text { flagella }\end{array}$ & $\begin{array}{c}\text { Acid formed; } \\
\text { no gas }\end{array}$ & No action & $\begin{array}{l}\text { Permanent } \\
\text { acidity; no } \\
\text { other change }\end{array}$ & No indol & No change \\
\hline $\begin{array}{l}\text { Intermediate } \\
\text { or Gärtner } \\
\text { group }\end{array}$ & Ditto & $\begin{array}{l}\text { Acid and } \\
\text { gas formed }\end{array}$ & No action & $\begin{array}{l}\text { First acid, } \\
\text { then alkaline, } \\
\text { no other } \\
\text { change }\end{array}$ & $\begin{array}{l}\text { No indol } \\
\text { as a rule }\end{array}$ & $\begin{array}{l}\text { Becomes } \\
\text { yellow }\end{array}$ \\
\hline Colon group & $\begin{array}{l}\text { Feebly mo- } \\
\text { tile; } 3 \text { to } 4 \\
\text { flagella }\end{array}$ & Ditto & $\begin{array}{c}\text { Acid and gas } \\
\text { formed }\end{array}$ & $\begin{array}{l}\text { Permanent } \\
\text { acidity with } \\
\text { curdling }\end{array}$ & $\begin{array}{l}\text { Indol } \\
\text { formed }\end{array}$ & Ditto \\
\hline $\begin{array}{c}\text { Dysentery } \\
\text { bacillus }\end{array}$ & $\begin{array}{l}\text { Non-motile } \\
\text { no flagella }\end{array}$ & $\begin{array}{c}\text { Acid formed; } \\
\text { no gas }\end{array}$ & No action & $\begin{array}{l}\text { First acid, } \\
\text { then usually } \\
\text { becoming } \\
\text { alkaline }\end{array}$ & $\begin{array}{l}\text { Sometimes } \\
\text { formed }\end{array}$ & No change \\
\hline
\end{tabular}

\section{(1) The Bacillus coli.}

Short $(2$ to $+\mu)$ rounded ends, sometimes almost oval, three to four flagella, feebly motile, no spores. Gram-negative. It never liquefies gelatin, produces permanent acidity in milk which is curdled within seven days at $37^{\circ} \mathrm{C}$. Ferments glucose and lactose with acid and gas, forms indol, a thick vellow-brown growth on potato, reduces nitrates.

\section{(2) The Capsulated Bacilli.}

Bacillus pneumonia, or the pneumo-bacillus of Friedländer.

A short rod ( 1 to $2 \mu$ ) with rounded ends, encapsulated, frequently in pairs, non-motile, non-sporing, aerobic and facultative anaerobic. Gram-negative. (The pneumococcus is Gram-positive.) It loses its capsule when cultivated, a nail-shaped growth in stab gelatin, milk slowly coagulated.

Bacillus lactis aerogenes, non-motile, does not ferment dulcitol, milk rapidly curdled with formation of capsules, forms " nail-head" growth in gelatin.

\section{(3) The Enteritidis Group.}

The enteritidis group includes such as Gärtner's bacillus which causes meat poisoning epidemics.

The pneumonic group such as bacillus psittacosis causing epidemic pneumonia in parrots and a fatal broncho-pneumonia in man.

The paratyphoid group causing paratyphoid fever.

The non-pathogenic group (to man), as the bacillus suicholeræ or hog cholera bacillus and the Danysz's bacillus for exterminating rats.

They all resemble the typhoid bacillus, morphologically being Gram-negative, actively motile, multi-flagellate, non-sporing, but they 
differ in cultures. They reduce neutral red, do not curdle milk, produce acid and gas from glucose, do not attack lactose.

Litmus milk is first acid then alkaline.

The agglutination test is often necessary to differentiate them.

\section{(4) Bacillus typhosus.}

Short ( 2 to $4 \mu$ ), thick, rounded ends, no spores but granules and vacuoles are sometimes seen. Involution forms $10:$ to $30 \mu$ on repeated sub-culture are characteristic. Aerobic and facultative anaerobic, Gram-negative. Not killed by drying. Direct sunlight kills in five hours. On agar a thick greyish-creamy growth, on gelatin a thin white growth without liquefaction, turbidity in broth with some deposit, milk made slightly acid, no curdling, ferments glucose with acid but no gas, lactose not affected, neutral red not affected, forms little or no indol. Infects humans through water, shell-fish, dust and air, flies, vegetables, soil, milk (dirty), filters, contact with carriers.

\section{(5) The Dysentery Bacillus.}

Fifteen types are said to exist. The Flexner and the Shiga-Kruse varieties are the best known.

All resemble Bacillus coli and typhoid bacilli in morphology and staining reactions. Generally said to be non-motile. Grow well in milk without clotting, with acid then alkaline reaction. In a few days ferments glucose with acid but no gas. Agglutination identifies the variety

The Flexner type ferments mannitol with acid but no gas.

The Shiga-Kruse bacillus has no action on this polyhydric alcohol.

Other important micro-organisms are:-

\section{The Bacillus diphtheriæ.}

The Klebs-Löffler bacillus is a slender ( 3 to $5 \mu$ ) non-motile, nonsporing rod with rounded ends. A parallel arrangement is common and club-shaped forms are frequent. Not killed by drying.

Aerobic and facultative anaerobic. Shows segmentation on staining with Löfler's blue. Gram-positive. Shows growth on gelatin without liquefaction, rapid growth on agar and blood serum at blood heat, does not coagulate milk, forms acid therein but no gas.

\section{The Bacillus pestis.}

A short, thick rod, usually linked in pairs, like a cocco-bacillus, Gram-negative, non-motile, no spores, bipolar staining. Grows best from $25^{\circ}$ to $20^{\circ} \mathrm{C}$. Easily killed by disinfectants, cultures lose their virulence quickly. In broth with a little butter flocculent tapering masses of growth are seen depending upon the oil droplets floating on the surface-Haffkine's stalactite growth.

On gelatin a thick whitish punctate growth without liquefaction.

It does not coagulate milk. 
The Staphylococcus pyogenes aureus.

Occurs as a diplococcus in grape-like masses, non-motile, no spores. Gram-positive. General turbidity in broth, liquefies gelatin with an orange-yellow sediment, on agar and blood serum a pale, then later a golden streak is formed. Staphylococcus pyogenes albus produces a white and Staphylococcus pyogenes citreus a lemon-yellow growth on agar and blood serum.

\section{The Bacillus pyocyaneus.}

A small bacillus found in blue-green pus, very motile. Nonsporing. Creamy growth on agar with greenish fluorescence, liquefies gelatin rapidly. Gram-negative. The pigments, pyocyanin and pyoxanthin, can be extracted with chloroform.

\section{The Streptococcus pyogenes.}

In chains, ten to fifteen bacteria in pus, thirty to forty bacteria in broth cultures. Gram-positive. Grows well with or without oxygen. Produces turbidity in broth, but growth ceases after four days owing to the production of an inhibitory metabolic substance. Slow growth on gelatin without liquefaction. Coley's fluid is from Streptococcus pyogenes and Bacillus prodigiosus, grown together for two weeks and sterilized by heat $65^{\circ} \mathrm{C}$.

\section{The Gonococcus.}

Small coffee-bean in shape, usually in pairs, a strict parasite.

Does not grow on ordinary media, but can be cultivated on blood or blood serum agar. A pure culture has a raised grevish-white mulberry appearance.

\section{The Meningococcus.}

The Diplococcus intracellularis meningitidis is Gram-positive.

Grows on blood agar at blood heat, occurs within the pus cells as it diplococcus, produces epidemic cerebrospinal meningitis (or spotted fever).

\section{The Diplococcus pneumoniæ.}

Usually seen as a diplococcus, but sometimes in chains of pus.

The cocci are oval or lance-shaped in a gelatinous capsule.

Gram-positive. Grows well on blood serum, but not readily seen. Does not grow well on gelatin. In cultivation the capsule is lost. No growth on potato. Produces curdling and acid with milk.

\section{The Micrococcus melitensis.}

Small, in pairs or singly or short chains with active Brownian movement. Slow growth, in three to four days small semi-transparent droplets on agar, which later become yellowish-orange. On gelatin a dirty white streak without liquefaction. Gram-negative. It occurs in the blood and milk of goats. 


\section{The Glanders Bacillus.}

The Bacillus mallei is a short rod $(2-5 \mu)$ with rounded ends.

Bipolar or beaded in stained preparations, no spores.

Non-motile, but active Brownian movement. A creamy growth on glycerine agar, does not liquefy gelatin. On potato, four days, looks like drops of honey, but later darkens to chocolate colour, but the potato remains unstained. Gram-negative. Does not readily stain at all; methylene blue, and then + to 5 per cent. acetic for ten seconds is the best stain.

\section{The Spirillum choleræ asiaticæ.}

Koch's comma bacillus is a curved $\operatorname{rod}(2-3, \mu)$ S-shaped in liquid media, actively motile, one flagellum, no spores, desiccation and sunlight rapidly fatal. Gram-negative. Readily stained with aniline dyes, grows readily on most media, but a slight alkaline reaction is necessary. Forms indol rapidly, creamy growths on agar.

General turbidity in broth and $\mathrm{H}_{2} \mathrm{~S}$ in both. Occurs in flakes in a "fish in stream" position. For bacterial diagnosis, see Cholera.

The Finkler-Prior spirillum may be confounded with it. These are thicker and longer than the Koch's comma bacillus, and it can be differentiated by the cultural reactions:-

Cultural Characters

Gelatin stab culture Potato at $37^{\circ} \mathrm{C}$. ... Peptone water
Koch's Comma Bacillus

... Slow liquefaction ...

... Slow light greyish brown growth $\ldots$... $\quad \ldots$

... Indol reaction marked within twelve hours ...
Vibrio Proteus

(Finkler-Prior Spirillum)

Rapid liquefaction

Rapid slimy yellow growth

Indol reaction feeble or nil in three days

\section{TO PREPARE CULTURES.}

For nutrient media, test-tubes, Petri dishes and other vessels are used.

Wash all with 25 per cent. IICl, rinse well with water and drain, rinse with alcohol and dry, plug test-tubes with cottonwool, sterilize at $140^{\circ} \mathrm{C}$.

The common media are :-

\section{Beef Broth.}

Use I lb. of beef steak, free from fat and connective tissue, mince, add I, ooo c.c. of water, boil and stir well for thirty minutes. Strain through muslin, add water up to s,ooo c.c., add sodium chloride, $5 \mathrm{grm}$. , and peptone, $10 \mathrm{grm}$., boil for five minutes, carefully neutralize with sodium hydrate solution, make it slightly alkaline to litmus, then boil for ten minutes, shaking well, filter it into flasks plugged with sterile cottonwool. 


\section{Glucose and Lactose Broth.}

To each $100 \mathrm{c} . c$. of above 1 to $2 \mathrm{grm}$. of fine glucose or lactose.

This is used for anaerobic bacteria.

\section{Nutrient Gelatin.}

To one litre of acid beef broth add Ioo grm. of gelatin, Io grm. of peptone, and $5 \mathrm{grm}$. of salt. Place in water bath until solution is clear, make faintly alkaline with $\mathrm{Na}_{2} \mathrm{OH}$, cool to $50^{\circ} \mathrm{C}$, add the white of an egg, after stirring steam for an hour, filter, and run into testtubes. Sterilize for fifteen minutes on three successive days.

\section{Agar-agar.}

I $5 \mathrm{grm}$. of powdered agar-agar well boiled with one litre of nutrient broth for two hours until dissolved, replace the water lost, see that it

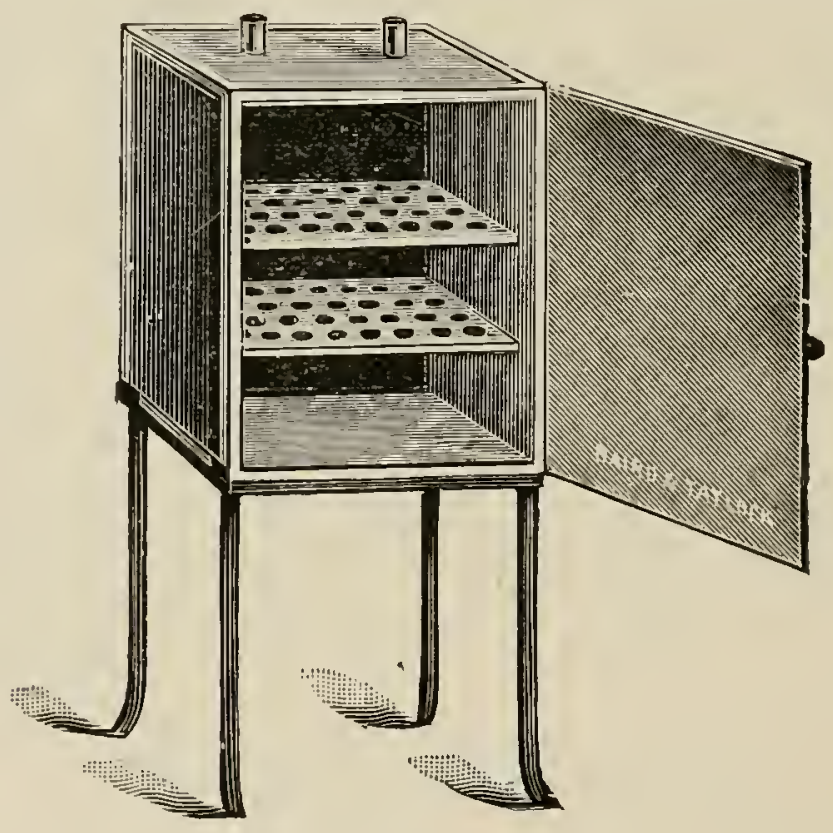

Hot-air sterilizer.

is faintly alkaline, clear witl egg-albumen, filter through a "Chardin " filter paper. It remains solid at $40^{\circ} \mathrm{C}$., and only melts completely at $90^{\circ} \mathrm{C}$.

\section{Glucose and Lactose Peptone Waters.}

$10 \mathrm{grm}$. of peptone and $5 \mathrm{grm}$ of salt dissolved in $\mathrm{s}, \mathrm{ooO}$ c.c. of distilled water, boil well, neutralize in the usual way, boil and filter, run into tubes and sterilize thrice.

Add I to 2 per cent. of glucose or lactose to this, tinge it with litmus solution to indicate production of acid and alkaline.

It is a useful media for determining the fermentative power of organisms. This medium is best placed in Durham's miniature test- 
tubes, which will become filled during the sterilization. During fermentation these become gas-holders.

\section{Blood Serum.}

Take blood from the jugular vein, operation wound, or placenta with aseptic precautions, let it stand, draw off with large pipette into test-tubes set on a slant.

The serum sets after heating in hot-air sterilizer to $65^{\circ} \mathrm{C}$.

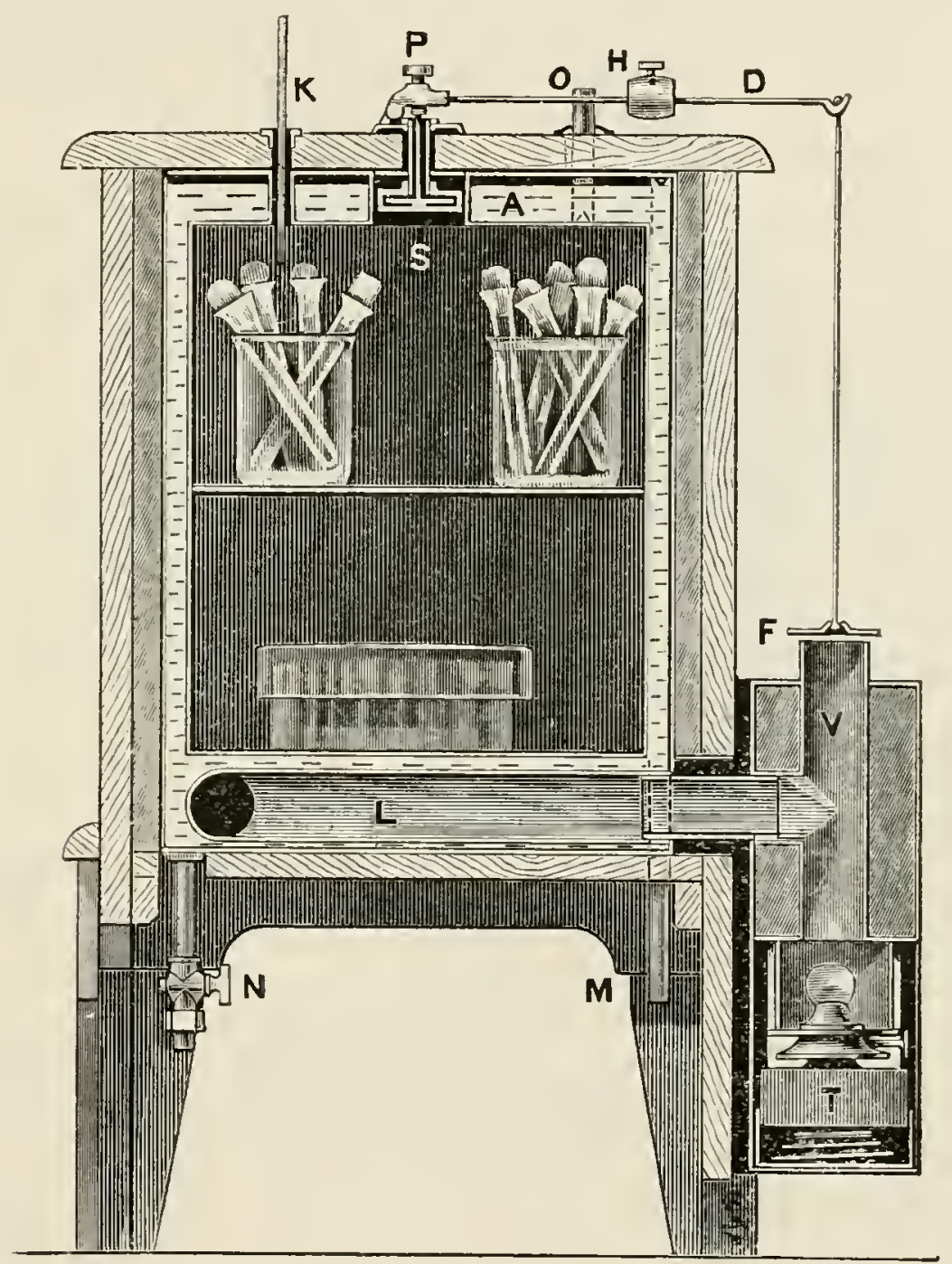

Hearson's incubator, working with petroleum lamp.

\section{Gelatin Plate Cultures.}

Place three test-tubes containing nutrient gelatin in boiling water until liquid, then cool to $40^{\circ} \mathrm{C}$. Introduce into tube a sterilized platinum loopful, a mere trace, of a culture of the organisms to be examined, and mix well. Sterilize the loop, and put one loopful from tube I to tube 2 and shake, put two loopfuls into tube 3 from tube 2 . Pour the contents of each into Petri dishes. In these the colonies may be seen and counted without removing the lid.

Agar plates are prepared in the same way, but it is necessary to 
work more quickly as the agar soon solidifies and the organisms are not evenly distributed. When the agar has set invert dishes to prevent water of condensation spoiling colonies.

\section{"Streak" Cultures.}

An oblique slope of agar or nutrient gelatin in a test-tube is taken, and with a sterilized platinum loop a trace from the culture is drawn up the slope.

\section{"Stab" Cultures.}

A test-tube of solid media is taken, and the straight platinum wire with the growth on the tip is introduced down the centre of the medium.

\section{"Shake" Cultures.}

Liquefy the gelatin in a test-tube by putting it in a beaker of water at $40^{\circ} \mathrm{C}$, and inoculate the medium with the organism. Gently mix to distribute the organisms; gas producing organisms form bubbles.

\section{"Anaerobic" Cultures.}

Glucose agar or gelatin is most frequently used. A tube threequarters full is kept in boiling water for five minutes to soften the medium and expel the oxygen. After the stab is made, warm the top of the medium to seal the needle track. For fluid media place thereon a layer of olive oil, and then inoculate with a sterile pipette.

\section{In differentiating growth note:-}

If the colonies are separate, rounded, minute or large indefinable masses. Plague bacteria grows in small rounded masses, but Coli form one creamy mass. Notice if any colour is produced and if the medium is stained as with Bacillus pyocyaneus.

Does the growth spread away from the line of inoculation, and if so, what kind of an edge does it form? Is gelatin liquefied, broth rendered turbid? Does a scum form on the surface? Is the deposit at the bottom? Does it produce acid or gas or both in a sugar medium? Is milk clotted or acid formed in it? Will it grow on potato, and if so, is any colour produced?

\section{THE PREPARATION OF SECTIONS.}

\section{(1) Fixing the Tissues.}

Place the piece of tissue in ro per cent. formalin, twelve to twenty-four hours.

Wash in running water and then proceed to harden it.

After it is hardened the tissue must be prepared for section cutting.

This is by freezing or embedding in paraffin. 


\section{(2) Hardening of Tissues.}

Place the tissue in three strengths of alcohol successively, viz.: 5o per cent., 75 per cent., and absolute, the tissue remaining in each twenty-four to forty-eight hours.

If one desires to keep the tissue, place in 70 per cent. alcohol.

Cut from the tissue small blocks 10 to $20 \mathrm{~mm}$. square and immerse in alcohol.

(3) Freezing of Tissues.

Place the blocks of tissue in an open vessel and allow water

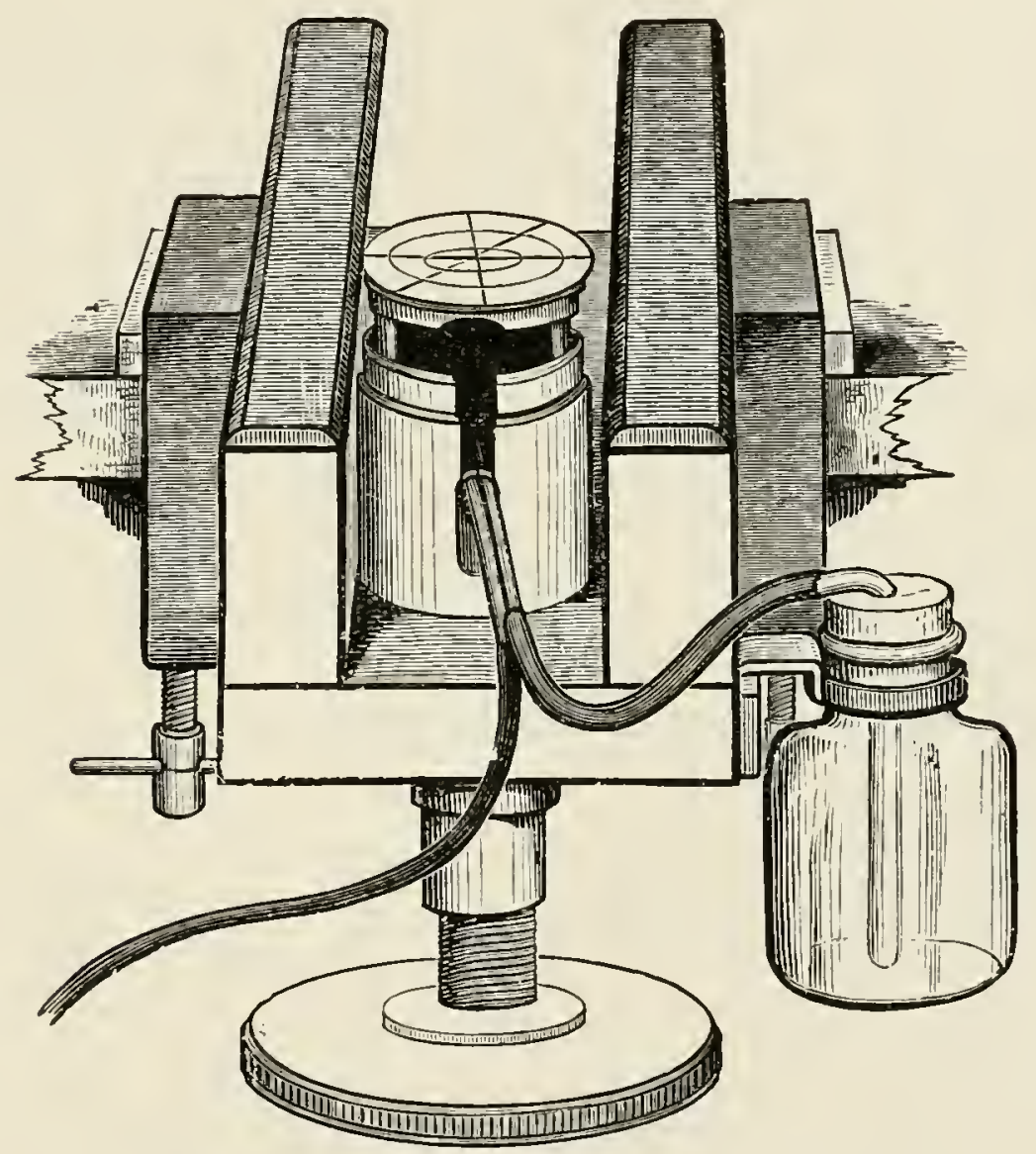

Microtome, Cathcart's, with spray bellows.

to run into it for two hours to remove the alcohol, then soak in a syrupy mucilage of gum acacia with a little carbolic acid. Place it on the plate of the freezing microtome, a little mucilage is added and the whole frozen. The sections are cut by the linife moistened with water and brushed into tepid water with a camel-hair brush.

These can now be stained or preserved in to per cent. alcohol.

\section{(4) Embedding of Tissues.}

After hardening place in absolute alcohol twelve to twentyfour hours, then in pure xylol for the same time, then in a 
bath of melted paraffin wax six to eighteen hours; the paraffin must be kept melted. The paraffin that is most useful for all purposes is that having a melting point of $50^{\circ}$ to $60^{\circ} \mathrm{C}$.

Skin tissues become friable if kept long in the melted paraffin.

After impregnation of the tissue it must be embedded.

Pour a little paraffin wax into a small (pill) box, place the block of tissue in the centre and fill the box with wax. When set remove the box and attach to the plate of the microtome.

\section{(5) Mounting Sections on Slides.}

Place section in a dish of warm water so that the paraffin is softened but not melted, then the sections become flat.

Introduce a slide into the water under the section and raise it out of the water, adjusting it with a needle.

Dry in warm incubator for two hours.

If the sections are thick it is preferable to smear the slide first with egg albumen mixture to make the section adhere sufficiently for staining.

For individual sections place a few drops of water on the slide, float section in it, and warm over a flame until it straightens out.

Pour off the water and the section will adhere.

\section{To Stain Sections.}

Remove paraffin with xylol and the xylol with spirit and the spirit with water. This is unnecessary if it is not a paraffin section.

Stain with hæmatoxylin two and a half minutes, and flush with water until blue.

Stain with eosin ten seconds and wash with water.

Treat with spirit to remove water, then with clove oil.

Treat with xylol and mount with Canada balsam.

Never let the section dry.

To tell if completely dehydrated when using clove oil place on a dark slab, when it will appear milky as long as water remains in the section.

\section{To show Parasites and Bacteria.}

Remove the paraffin with xylol, spirit and water as above.

Stain with strong carbol-thionin ten minutes.

Pour off and blot lightly.

Treat with spirit rapidly, two dashes only.

Treat with oil of cloves until transparent.

Treat with xylol and mount with Canada balsam. 


\section{Yan Gieson's Stain.}

Treat as above to remove paraffin.

Stain with hamatoxylin fifteen to twenty minutes and blue well in tap water.

Stain with van Gieson half to one minute (picric acid $1 \frac{1}{2}$ per cent. of acid fuchsin).

Treat with spirit, oil of cloves, xylol and mount.

The hæmatoxylin stains the nuclei and parasites blue.

The picric acid stains the protoplasm of the cells yellow.

The fuchsin stains the fibrous tissue red.

\section{To show Malarial Parasites in the Brain.}

Remove the paraffin. Stain with hrematoxylin twenty minutes and blue well in tap water.

Treat with acid alcohol one to four seconds, wash well.

Treat with spirit, oil of cloves, xylol and mount.

\section{To Embed in Celluloidin.}

Celluloidin is a product of gun cotton and looks like glass cotton.

It must be packed in water for transit.

It is a gummy transparent substance, and is useful for holding the brain tissue and the malarial parasites together in subtertian cases.

It must be dried before using.

Place the section in spirit twenty-four hours, in another solution of spirit for the same period, in absolute alcohol twenty-four hours, in alcohol and ether for twenty-four hours, in thin celluloidin twentyfour hours.

Pour some thick celluloidin on a wooden block and place the tissue on it; leave it in the air for a few minutes.

Place in 60 per cent. spirit to harden it until it is cut.

\section{To mount and stain the Section.}

Place in a watery hæmatoxylin stain twelve minutes. (Watch glasses are the best for these processes.)

Wash and blue well in tap water.

Treat, with acid alcohol until red, five seconds.

Wash well and stain with alcoholic eosin three to four minutes.

Pass through three baths of spirit.

Clear in carbol xylol and mount.

A low power shows the brain capillaries plainly because of the malarial pigment present in subtertian cases. 


\section{MALARIAL PIGMENT: WHERE IT IS AND HOW TO LOOK FOR IT.}

This pigment is found in the parasites, rarely in the polymorphonuclears and in large mononuclears. When in the latter it is pathognomonic of malaria. The pigment is carried by the cells to the liver and spleen. The endothelial cells of the capillary also take it up and may then become detached. If pigment is found elsewhere it is not malaria. The pigment is nearly always intracellular.

The mononuclears soon give it up again, so that to find these cells carrying it indicates that malaria was progressing at the time.

In the liver it is found in the small cells of the connective tissue type with a dark staining nucleus which penetrates between and into the liver lobules. The containing cell becomes degenerate, the nucleus stains faintly, and the pigment appears in larger clumps.

Later, the pigment alone remains, lying in the connective tissue.

This latter stage is reached from the twentieth to the thirty-second day, so that from the examination of the liver one can tell how long the malarial process has been going on, and it indicates that the liver was active at the time of death.

In the spleen pigment is always present, chiefly in the macrophages, which may give a dark appearance to the organ on section according to the number of malarial attacks and the amount of pigment deposited.

A slaty-black colour is almost diagnostic.

Malarial pigment consists of :-

(I) Melanin, which is diagnostic.

(2) Hremosiderin, not diagnostic.

(3) Iron-bearing granules, not diagnostic.

Melanin contains iron, but in such firm combination that it does not give the iron reaction. It is insoluble in acids, soluble in alkalies, insoluble in alcohol.

Hamosiderin is insoluble in acids and alkalies, but soluble in alcohol.

It does not require staining to be seen. It is said not to contain iron. Carbol thionin stains it violet.

The iron-free granules contain iron in inorganic combination, so will give the reaction for free iron. The more acute the hæmolytic process, the more numerous the iron granules; the more chronic the process, the more numerous are the yellow granules.

In blackwater fever there is much blue pigment; and in ankylostomiasis much yellow pigment. The hæmosiderin and the ironfree granules are seen in any disease causing hæmolysis, as pernicious 
anæmia, blackwater, \&c. They are found in the true secreting cells lining the first portion of the convoluted tubules of the kidney.

Rarely the three kinds may be found in the same cell.

\section{To show the three Pigments:-}

Stain with carmine ten minutes.

Stain with acid alcohol three minutes.

Stain with potassium ferrocyanide five minutes.

Stain with acid alcohol until blue.

Treat with spirits, oil of cloves, xylol and mount.

This is a chemical process, and Prussian blue is formed.

The potassium ferrocyanide and acid alcohol can be repeated several times until the part is sufficiently blue.

The pigment in the lung is chiefly carbon; treat the section with an alkali if suspicious, carbon will not be dissolved, but melanin will.

The pigment in the skin of the dark races is not like that of malarial pigment. It contains no iron whatever, and is dissolved in weak acids, which distinguishes it from true melanin. Tattoo pigment is deposited very irregularly, and can be diagnosed at once under the microscope.

Between bacteria proper (Schizomycetes) and the moulds (Hyphomycetes) comes a group known as-

\section{THE TRICHOMYCETES.}

This group is divided into:-

The leptothrix, no branching.

The cladothrix, false branching.

The streptothrix, true branching.

\section{The Actinomyces.}

This ray fungus is a streptothrix consisting of filaments, cocci and clubs. The filaments are long and thin, interlacing in the centre of the colony forming a network. In older filaments the protoplasm is broken up into coccoid bodies which may break out from the sheath. The clubs are involution forms perhaps produced by resistance of the tissues.

In actinomyces from human tissues:-

The threads are Gram-positive, the clubs Gram-negative, but from cattle the threads are Gram-negative and the clubs are Gram-positive.

Man may be injected with the bovine type. In artificial media the clubs are not found. It grows well on potato and glycerine agar.

On potato it forms a thick, grey, raised, wrinkled growth of sulphur yellow or light chocolate colour.

It causes actinomycosis in man and cattle. 


\section{Streptothrix madura.}

This fungus produces in Cyprus, India, South America and Egypt the white form of madura disease or mycetoma, which is much more common than the black or red varieties. It does not form yellow or black pigments in cultures, and does not liquefy gelatin. It is not infective for rabbits. The clubs do not stain by Gram.

Stain with hæmatoxylin and acid or carbol thionin.

\section{THE HYPHOMYCETES.}

The moulds or mycelial fungi are multicellular organisms composed of filaments which interlace, forming a mass of mycelium.

\section{(1) Mucorinal.}

The end of a filament or hypha swells into a knob around which a spherical seed capsule forms. When ripe, the spores burst the enclosing membrane and thus become free. It occurs in any decomposing material and in man may occur in the ear, old abscesses and bronchiectatic cavities.

\section{(2) Aspergillinæ.}

The heads of the filaments are covered with a number of spore carriers (sterigmata), each sterigmata bears a chain of spores.

They may invade the lung, blocking up the acini, giving rise to symptoms much like phthisis.

\section{(3) Penicilliaceæ.}

The filaments are branched, from these basidia arise the sterigmata from which in turn chains of spores arise. They will soon overrun any exposed agar plate.

Penicillium glaucum is the commonest mould and is seen on moist bread, damp boots, \&c., as a bluish-green fur, giving off a musty odour.

To mount moulds remove the thin layer of fat that normally covers them, with alcohol and a little ammonia. Then mount in glycerine.

They can be stained with Löfler's methylene blue, which stains the mycelium and hyphe, the spores remaining unchanged.

Another method of showing moulds is to :-

Stain with aniline gentian violet, five minutes.

Pour off, blot, treat with Gram's iodine, two minutes.

Treat with aniline oil and iodine until differentiation is complete, about fifteen minutes.

Treat with xylol and mount in Canada balsam.

\section{Ringworm.}

This is a kind of mould. It has the power of living on the keratinized products of skin which it breaks up and digests. 
Most forms disappear before the human host has reached twent? years of age.

(1) Microsporon andouini.

Net with in children. Never attacks the scalp of adults. Never affects the beard and nails. Very intractable. It occurs as a whitish sheath around the stumps of broken hairs. They are round or ovoid spores, 3 to $+\mu$.

(2) Trichophyton megalosporon.

The endothrix variety is exclusively of human origin : the spores are seen in the interior of the affected hairs.

The ectothrix variety which affects the beard and nails is derived from animals, the spores lying on the exterior of the hair. The spores are large, + to $12 \mu$. To facilitate examination soak the hair in 10 per cent. caustic potash and then alcohol and ether as for moulds.

\section{THE BLASTOMYCETES.}

These yeasts are round or oral unicellular organisms having hyaline protoplasm in healthy, and granular protoplasm in old cells, surrounded by a wall of cellulose.

The torula multiply by budding.

The saccharomycetes by budding and spore formation.

Spores are formed when the food materials are exhausted.

They must have moisture and plenty of fresh air, temperature above $25^{\circ} \mathrm{C}$. is best while fermenting nutrient liquids facilitate the formation of spores.

\section{S. cerevisiæ.}

There are two typical bremery yeasts, the typical English, a " top " fermentation variety, and the reast of the Continental lager beer, a "bottom" fermentation. The "top " and "bottom " are also known as the "high" and "low" as they develop in high and low temperatures respectively. They are $S$ to $9 \mu$ in diameter. Three to four spores form in one mother cell.

There are "wild yeasts" as :-

S. pastorianus. There are three varieties with peculiarly shaped cells and having two to four spores in each mother cell

" No. $\mathrm{I}$ is a bottom form and causes a disagreeable smell and a strong bitter taste in beer.

, No. 2 is a feeble top form of little importance.

, No. 3 is a clangerous "top" form causing turbidity and disease.

Bulgarian sour milk is used for therapeutic purposes. 
A yeast attacks the milk sugar after lactic acid has been formed, inhibits the growth of pathogenic and putrefactive bacteria and improves the taste. The reast may get the upper hand and carry the fermentation too far.

Pathogenic yeusts cause blastomycetic dermatitis as sometimes seen in man.

They have also been recovered from tumours, lung diseases, sprue and dysentery cases.

\section{To Stain Yeasts.}

Use Gram or any simple stain.

Remember that they stain mahogany brown and not gentian violet with Gram.

\section{TIIE EXAMINATION OF FECES.}

\section{MACROSCOPIC.}

This is a very necessary routine practice in the tropics.

Note the number, bulk, colour, odour, consistency and the reaction of the stools.

Note the presence or absence of gaseous fermentation.

The presence or absence of blood, mucus, pus and their degree of admixture with the stool.

Any visible animal parasites, undigested food materials, \&c.

Remember that mucus from the small intestine is stained with bile and is not frequently seen, but mucus is common from the lower bowel and may be caused by anything that sets up inflammation in it such as bilharzia, chronic ulcerations as from ulcerated hamorrhoids, malignant and granulomatous growths and chronic dysentery.

In acute dysentery the mucus is usually clear, not mixed with facal matter, and may be streaked with bright red blood.

If it comes away in condensed hard masses with much débris and epithelial tissue with pus the affection is usually of the rectum.

If burel casts are passed (membranous colitis) they may be twisted, these should be floated out in water as they may resemble worms.

If bright red blood is passed it does not necessarily mean that it has come from the rectum, such may come from the small intestines.

If the stools are numerous, pale, frothy, it is suspicious of sprue.

Remember that in the tropics the crecum and upper part of the culon may be ulcerated acutely and extensively without passage of either mucus or blood or with tenesmus. Such may be rapidly fatal.

Remember that rice and plantain eating peoples pass much larger stools normally than do meat eating peoples. The people of the East eat excess of carbohydrate food to obtain sufficient protein: 
Europeans in the tropics give an average stool per diem of $1,30,51 m$.

Natives give an average stool of $233 \mathrm{grm}$. per diem.

The reaction of a normal stool is nearly neutral to litmus, acid on fasting, faintly alkaline when taking milk. In most cases of diarrhoea they are decidedly alkaline. All stools should be examined as soon as possible after being passed.

Macroscopic mucus usually means catarh in the large intestine.

Microscopic mucus usually means calarh in the small intestine.

Earth eaters pass pale stools which may in this respect resemble obstructive jaundice. Those who eat coal-clust and take iron or bismuth have blackish stools which may resemble melana. The stools are dark blue after taking methylene blue and brick rellow after ipecacuanha.

Bile acids are recognized by Pettenkofer's reaction.

Mix a little faecal matter with a little sugar on a white dish, add a little $\mathrm{H}_{2} \mathrm{SO}_{1}$. I crimson colour indicates the presence of bile acids.

\section{To Estimate Nitrogen.}

Weigh a portion of facal material.

Add 20 c.c. of $\frac{\mathrm{N}}{10} \mathrm{H}_{2} \mathrm{SO}_{4}$ to prevent loss of ammonia.

Dry in a water bath.

Complete desiccation in a drying chamber at $10^{\circ} \mathrm{C}$. orer $\mathrm{I}_{2} \mathrm{SO}$. Weigh out I grm.

Mix with 2.5 c.c. of strong $\mathrm{II}_{2} \mathrm{SO}_{4}$ and 1 grm. of sodium hypophospliate.

Permit to stand four hours, then boil cautiously.

The nitrogen now is converted and ammonimm sulphate is formed.

Allow to cool.

Add 600 c.c. of water.

Add sodium hydrate solution till strongly alkaline.

Add a few pieces of granulated zinc to prevent bumping.

Distill this, passing it into $\underset{7}{\mathrm{~N}} \mathrm{H}_{2} \mathrm{SO}_{4}$.

Some of the acid is neutralized by the ammonia.

Titrate the $\mathrm{II}_{2} \mathrm{SO}_{4}$ to estimate the amount of the ammonia distilled over.

\section{To Estimate Fat.}

Dry faces over $\mathrm{H}_{2} \mathrm{SO}_{4}$.

Treat with I per cent. of $\mathrm{HCl}$ to split up the soaps.

Add ether to dissolve fat.

Add more ether and pour off and add to former.

For bile pigments, bilirubin and biliverdin employ :-

(i) Schmidt's reaction.

Add to faeces a saturated solution of $\mathrm{Hg}$ perchloride.

A bright green indicates their presence. 
(2) Gmelin's reaction.

Add a few drops of yellow nitric acid to fæces.

A display of colours including green is positive.

(3) Huppert's test.

Add slaked lime suspended in water to the fæeces.

Filter, wash and dry the precipitate.

Make an extract with hot alcohol and a little $\mathrm{H}_{2} \mathrm{SO}_{4}$.

Bile pigment gives a green colour.

A complete absence of red or green colouring shows absence of bile when icterus is present. When the latter is not present it indicates temporary suspension of bile secretions. In fresh brown stools giving no red coloration there is usually intestinal decomposition.

When green colouring is macroscopic it indicates too rapid a passage through the colon.

Urobilin is always present in small quantities 0.03 to $0^{\circ} 06 \mathrm{grm}$. per diem. Any hemolytic disease increases the amount, especially malaria. In subtertian it may go up to $1^{\circ} 72$ grm. or more.

It is one of the final products of destroyed hb. The smell is due chiefly to indole and skatol. Food materials are absorbed principally in the latter half of the small intestine. In the event of increased peristalsis or catarrh of this part the contents are hurried through without benefiting the patient. Water is absorbed from the large gut, increased peristalsis or inflammation hastens the contents through and gives loose stools, while diminished peristalsis or constipation from any cause means hard faces due to their remaining too long in the colon and tou much water being absorbed.

The water in an average stool is 75 per cent. Weigh before and after drying.

Dry over $\mathrm{H}_{2} \mathrm{SO}_{4}$ as volatile substances are present and do not heat over $60^{\circ} \mathrm{C}$.

Evaporate ether from bath.

Weigh residue $=$ total fats.

\section{To Examine for Macroscopic Parasites.}

Place the stool in a muslin or fine wire gauze strainer, add water and stir well.

Repeat with a finer mesh each time and use fresh water.

The entozoa are readily found.

For diagnosing helminths and amobe see section on : "Diseases due to Helminths" and "Amœbic Dýsentery."

\section{MICROSCOPIC.}

Use water with firm stools, but as little water as possible. Cover with a cover-slip and spread out the material by pressure on the slide.

To distinguish mucus from connective tissue add a drop of acetic acid : fibrous tissue is dissolved and mucus is more distinct. 
Muscle fibres looking like brown splinters of wood will indicate the powers of protein digestion in the small intestines.

Connective tissue indicates gastric indigestion and that organ should be minutely examined.

A normal stool contains 23 per cent. of fat of the dry substance, slight variations from this are unimportant; a morbid increase gives a clay coloured sour copious stool, and the sebacic acid flocculi in a cold acetic acid preparation are much increased as are also neutral fat drops, salts of lime and soap crystals. If bile is absent and fat increased the disease is in all probability in the biliary duct system. If with excess of fat there are muscle fibres and hydrobilirubin present and mucus is absent the pancreas is the seat of the disease. When biliary and pancreatic disorders are excluded the excess of fat may be due to severo intestinal disease such as tuberculosis, amyloid disease or tabes mesenterica.

Errors in the examination of faeces are not uncommon.

Banana fibres may be mistaken for tapeworms, especially T. nana and $T$. diminuta, owing to the fact that they are made up of segments resembling tapeworm strobila.

Orange débris resembles certain trematodes, liver flukes, \&c.

Celery shreds may be mistaken for hookworms.

Oatmeal may simulate the segments of worms.

Undigested pollen grains might be mistaken for eggs.

The ascus of a mould mistaken for an egg-shell.

The oncospheres for a dividing ovum.

Always consider the patient's regetable diet first.

\section{Bacteria, \&c.}

The microscope will demonstrate mild infections due to staphylococci from the more dangerous streptococcal infections. Typhoid, cholera and dysentery bacilli can be detected by culture. These are dealt with under their respective headings. The amoba can be seen in fresh stools and when the slide is liept warm their movements can be recognized. Water kills them and should not be added in examining them.

Free moring larvat in fresh stonls are never ankylostomes but probably. Strongvlus stercoralis.

\section{Blood.}

Blood in small quantities and altered blood can be recognized by Weber's test :-

Extract the fat with etler.

Rub the stool up with water.

Add one-third bulk of acetic acid and shake up the whole with ether. 

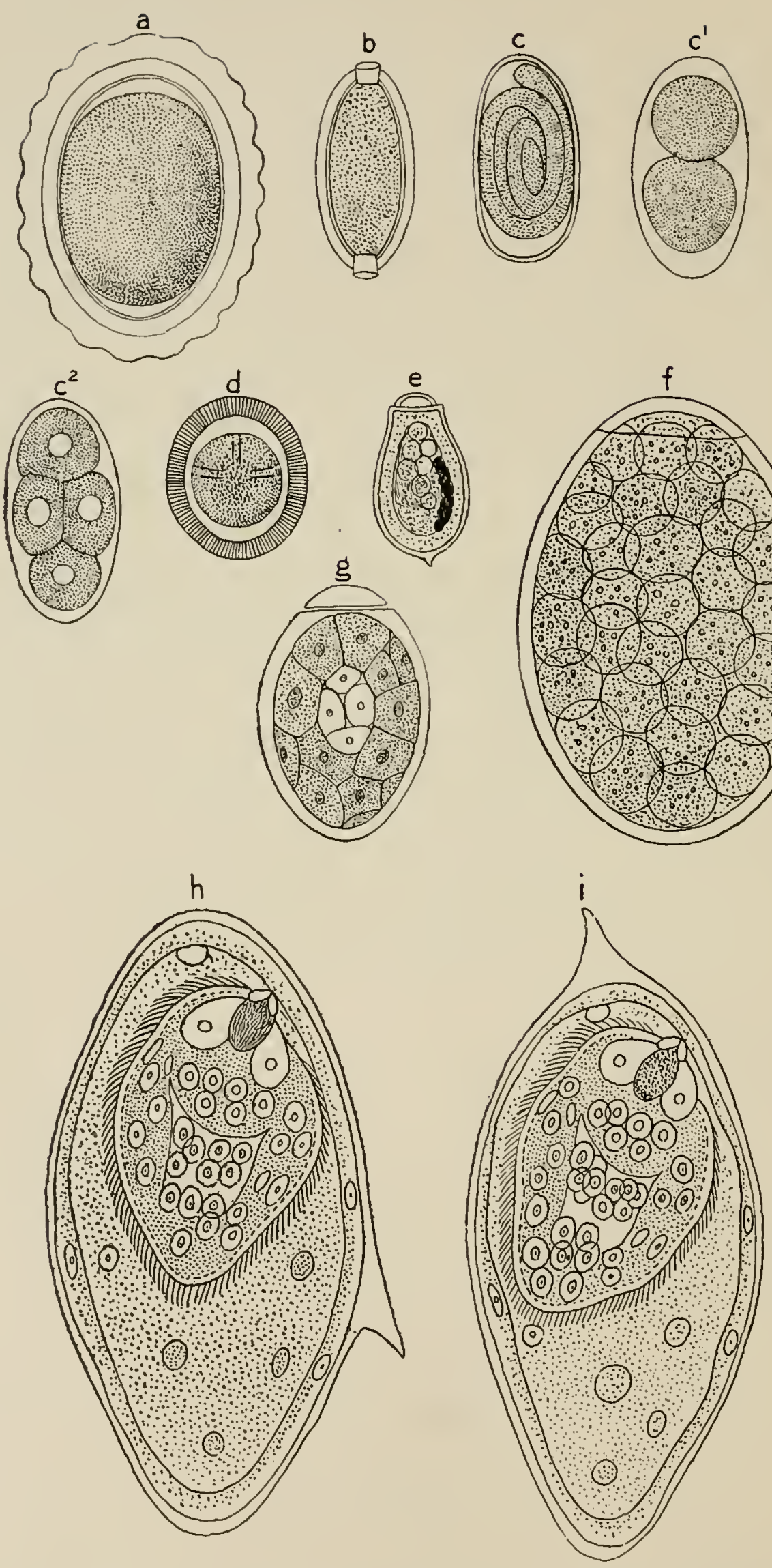

a, Ascaris lumbricoides; b, Trichocephalus disfar; c, Oxyuris vermicularis; $c^{1}, c^{2}$, Ankylostomum duodenale; d, oncosphere of Cestode; e, f, $g$, various Fasciolide; $h$, Schistosomum (?) hamatobium ifrom freces); $i$, Schistosomum hamatobium (from urine). 
An ethereal solution of acid hamatin is mbtained.

If altered blood is present it is seen by means of the spectroscope.

\section{THE DETECTION OF OYA.}

This is all-important. Their appearance is described in the section on "Diseases due to Helminths," but to facilitate laboratory work a concise description will be given here.

A moist stool needs no water; to a dry one add a litule water and place a small portion on the slide, and press it out gently with a cover glass. As eggs are heavier than water more will be at the bottom of a water stool.

Many eggs of Ascaris matter little as a rule, and those of Trichocephalus dispar less, but a few ankylostomes are of considerable importance.

A $\frac{2}{3}$ objective should diagnose all.

(I) Ascaris lumbricoides.-Ovoid or round with a double contour. Thick clear yellow shell. May or may not be surrounded by an irregular protective coat of stained albumin, granular yellow contents not completely filling interior. The shell resists formalin.

(2) Trichocephalus dispar.-Small oval yellow eggs, double contour, a distinct "plug" of mucus at each end, a granular interior and a very thin unstained inner capsule.

(3) Oxyuris vermicularis. - Not unlike the ankylostome egg, but somewhat flattened, and contains a well-formed embryo when freshly passed.

(4) Ankylostomum duodenale.-Unstained, a single thin transparent capsule, segmented nuclear matter into two to fortyeight cells, does not fill cavity, an embryo in about forty-eight hours.

(5) Trematodes.-These are yellow, small, and have a lid or operculum, which may be split off. Their size differentiates them.

(6) Chlonorchis sinensis.-This has a "jug" shape, with a distinct lid above and a small spine below.

(7) Schistosoma hrmatobium (from urine usually).-Large terminal sharp spike, thick shell containing embryo. Add a little water to the slide and the embryo will come out.

Schistosoma mansoni as above with a lateral spine.

Schistosoma japonicum has a knob laterally, no spine and no operculum.

(8) Oncosphere of Cestode.-A radical striation of the thick capsule with three pairs of hooklets in the central embryonic matter readily distinguishable from other ova. 
(9) Bothriocephalus.-The only egg outside the trematode eggs with a lid. Oval, cellular contents, but little stained, otherwise much as trematode eggs. Later it becomes encysted, and ciliated for swimming about in water.

\section{THE DISSECTION OF MOSQUITOES.}

To kill the mosquito, if alive, put it in a test-tube and tap the tube on your arm.

Remove legs, mount and dry, fixing cover slip with gummed paper having a window in it. Study well the complete structure.

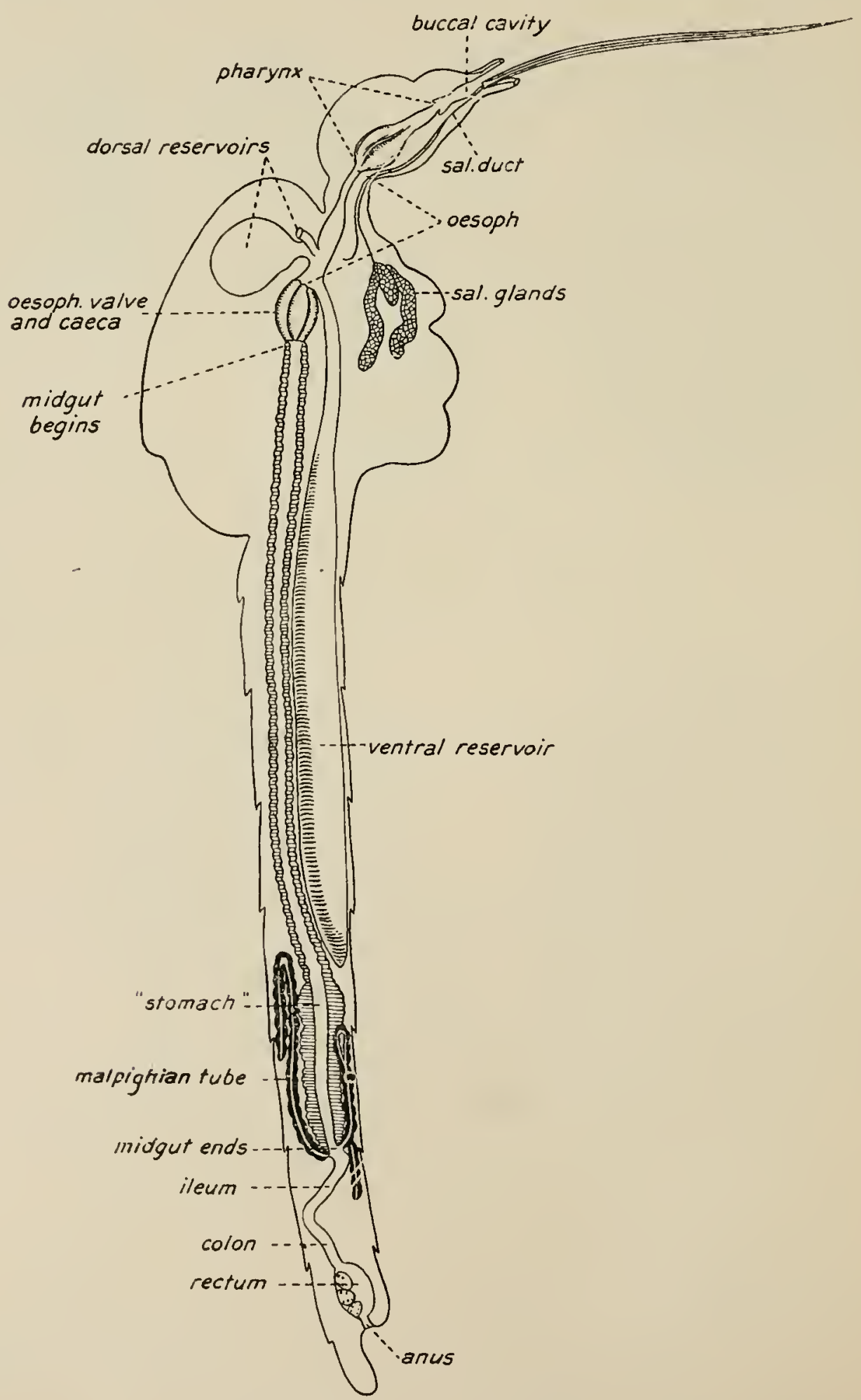

Internal anatomy of the mosquito 
Remore wings at right angles to the body and mount likewise.

Transfix the thorax with a fine needle and remove the head by pulling it away from and backwards off the thorax; in this way the salivary glands may be withdrawn from the thorax, and they can be examined under the microscope for parasites.

Then take the remainder, bruise the abdomen across the last segment, and draw it away with another ncedle; in this way the internal viscera will be drawn out also, and if one is fortunate the salivary glands, if not already removed, will be withdrawn.

The stomach, rectal glands, spermatheca, \&c., will be well shown.

Examine the gastric contents for malarial parasites.

If the salivary glands are not removed, tease the thorax into very small portions in a drop of saline and examine. There is much débris resulting from this methed, and the glands may be lost.

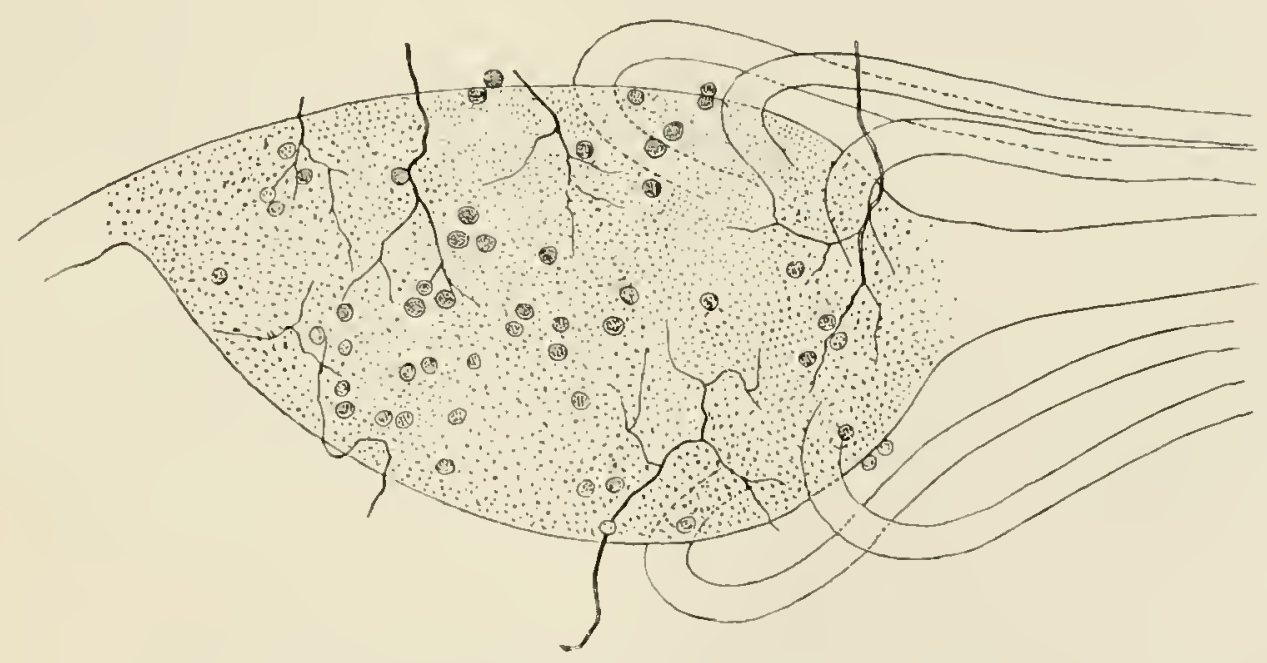

Stomach of mosquito showing malarial parasites.

The parts not being examined should be kept in saline, or better, liquor potassa in a watch-glass.

Press the proboscis out under a cover slip and examine; several of the elements may be seen in each specimen thus treated. Examine the base of the proboscis and the thoracic muscle for filarial embryos.

\section{THL EXAMIXITON OF WATER.}

Good waters are spring, deep well, and upland service waters.

Suspicious waters are stored rain and surface water from cultivated land.

Dangerous waters are shallow well and river water to which sewage has access.

There are four examinations :-

Biological, Physical, Bacterial, Chemical. 


\section{(1) THE BIOLOGICAL EXAMINATION.}

This enables us to determine the absence or presence of lowly organisms, bacteria, protozoa, larve, \&c.

The following are common and should be looked for:-

\begin{tabular}{ll}
\multicolumn{2}{c}{ In imfure water } \\
Vorticella & Rotifera \\
Crenothrix & Ulothrix \\
Asterionella & Algæ \\
Diatoma & Sprrilla \\
Actinophrys & Spirogyra \\
Protococcus & Gammarus pulex \\
Euglena & Cyclops \\
Oscillaria & Water bear \\
Desmids & Bomina longitirastis
\end{tabular}

Carchesium

Oscillatoria nigra

Leptomitus

Sphærotilus natans

Beggiatia

Ulva latissina

Enteromorpha

Infusoria

\section{(2) THE PHYSICAL EXAMINATION.}

The reaction should be neutral.

Add a little neutral litmus to the sample.

$\mathrm{CO}_{2}$ will turn it red.

Remember that acid waters attack lead and iron pipes.

The colour should be a pale blue or green.

Half fill a two-feet tube and lay it upon the table.

Look through the upper half which is colourless.

Look through the lower half which assumes the colour of the water.

It should be odourless. To test it warm to about $37^{\circ} \mathrm{C}$.

Certain mineral waters contain $\mathrm{H}_{2} \mathrm{~S}$, also given off $b y$ beggiatoa and crenothrix.

Volvox and uroglena give a fishy odour.

Anabana and rivularia give a grassy odour.

Asterionella and diatoma give an aromatic odour.

There should be no sediment. If there is such it should be examined under the microscope.

\section{(3) THE BACTERIOLOGICAL EXAMINATION.}

This indicates the presence or absence of pathogenic and other bacteria and the number thereof. These are:-

(I) Normal inhabitant bacteria.

(2) Accidental soil bacteria.

(3) Intestinal bacteria.

Sunlight reduces their number if the water is shallow and the exposure prolonged.

Cold inhibits their growth and warmth stimulates it.

Presence of food materials stimulates their growth.

Most bacteria tend to settle at the bottom and so thus assist in self-purification of the water.

Typhoid and cholera organisms die out if stored long, about twelve weeks. 


\section{To Collect the Sample.}

Use sterile glass-stoppered bottles and immerse in the water $12 \mathrm{in}$. below the surface before remoring the stopper.

If from a lap, let it run for five minutes before taking sample.

Remember that a local cistern contamination gives a fallacy not to be applied to the rater supply in general.

For deep wells Sclaver's "smash" bottles should be used.

These are small vacuum bulbs, let down by a string and mechanically broken when under water.

Examine the sample within three hours or pack it in ice.

\section{To Examine the Sample.}

Lntil recently bacteriological examinations of water supplies in tropical countries have been few and far between, owing to the lack of well equipped laboratories. When sufficient statistics are to hand we shall be able to appreciate the difference of the bacterial content of tropical waters and to modify existing standards of bacterial impurity.

To one already acquainted with the subject in a temperate climate there will appear difficulties innumerable. He will find that standards of impurity cannot be applied generally and that the local water conditions vary enormously.

For example in the tropics:-

(1) The rains are torrential and periodic, so that the bacterial flora of districts where vegetation is rank will be brought down. The surface organisms of sandy districts, where much vegetation is absent, may give an altogether different flora from the above.

(2) The sun and soil kill off all the B. coli communis, where it has existed, but other resistant ypes persist, new probably to the sanitary worker. Their significance as infectious and dangerous agents in water supplies has to be ascertained.

(3) A clay subsoil, instead of acting as a liter in purifying the water, in some measure may provide a culture media for its increased pollution, owing to the organisms being held up, cultured, and swept away into surface waters during the first shower.

(4) The sanitarian will be frequently called upon to deal with the bacteria persisting after the action of sun and soil, which residual bacteria may not include the notorious $B$. coli communis, the presence of which forms a basis for a standard of impurity in temperate countries.

(5) Animals residing in a catchment area will give a very distinct bacterial flora from that in temperate climates where the pollution is largely from luman facal bacteria from the large towns on the river banks. 
(6) The bacterial flora of human origin is very different to that of animal origin and reasoning based upon the former and applied to the latter in tropical climates may often lead one into very serious error.

(7) The worker will find that high winds laden with dust will gain access to his plates and colonies and repeatedly ruin his works.

Each district water supply must be judged on its merits.

Data from many different parts of the tropics must be provided before standards of impurity can be generally recommended and adopted.

The first data came from :-

Daniels in Malay.

Balfour and Archibald in the Soudan.

Clemesha in India.

(A) Daniels working in the Federated Malay States (IgoS) proposes the following as a basis:-

(1) The number of organisms (exclusive of known, easily recognized, non-pathogenic organisms, e.g., B. subtilis, B. megaterium, 13. violaceus, and some of the organisms which form characteristic yellow colonies) which have been proved to be non-pathogenic to lower animals.

(2) The amount of the water required to react, forming acid and gas, with MacConkey's medium, from $0^{\circ} 2$ to 2 c.c. being employed in the tests.

(3) Indol formation in forty-eight hours in peptone water with Io c.c., 5 c.c. and 1 c.c. of the water to be tested.

Is a standard Daniels and Finlayson suggest that :-

(1) Not more than 100 organisms, exclusive of those mentioned, should be present in I c.c. of the water.

(2) That no acid and gas should be formed in twenty-four hours in MacConkey's medium at $37^{\circ} \mathrm{C}$. with 2 c.c. of the water.

(3) That no indol should be formed in peptone water in forty-eight hours with 5 c.c. of the water added.

They remark that the standard is not a high one but it is exceptional to find a natural water that will pass all three tests.

In unprotected shallow wells liable to be polluted by surface washings, they found the average bacterial count to vary between 300 to 900 per c.c, while in twenty-three out of twenty-seven wells examined, acid and gas forming organisms were present in 3 c.c. and usually in 4 c.c. of the water, and indol formers in 5 c.c. in the case of ten of the wells. B. pyocaneus was found in one instance, and in two cases organisms indistinguishable from B. coli communis.

(B) Balfour and Archibald have done good work in the Soudan in connection with river and shallow well water in Khartoum. 
Dealing with river water, B. coli was found present in $0^{\circ} 5$ to 1 c.c. of the water taken near the bank but only in 5 c.c. taken from miclstream.

In shallow well water B. pyocyaneus was found and dysentery was observed to follow the use of this water.

Deep well water was examined by Captain Archibald with the following results :-

\section{(1) The colony count in I c.c.}

This was always carried out with agar plates as the heat prevented the use of gelatin. The percentages of the various constituents of the agar was the same throughout all the tests. The acidity was plus ro to phenol-phthalein. One c.c., $0^{\circ}$ c.c., and $0^{\circ}$ c.c. of the water sample is taken and run into melted agar at a temperature of $4 \mathrm{r}^{\circ} \mathrm{C}$. This was then placed out in Petri dishes, incubated at $37^{\circ} \mathrm{C}$. for forty-eight hours, and all colonies visible to the unaided eye counted.

\section{(2) Sporogenes Test.}

Twenty c.c. of the sample of water are pui into sterile milk conlained in tubes. The milk is then heated up to $\mathrm{SO}^{\circ} \mathrm{C}$. for twenty minutes, and a layer of hot vaseline is run on to the surface of the milk so as to render the latter anaerobic. The milk tubes are then incubated at $37^{\circ} \mathrm{C}$. for forty-eight hours.

Evidence of the presence of the spores of B. enteritidis sporogenes is shown by the clotting of the milk.

(3) The test for the presence of the lactose-fermenting organisms and their subsequent isolation.

Lactose bile-salt-neutral-red-broth is used and, as varying quantities of water are inoculated into tubes containing this medium, the latter is made up in single, double, and triple strength.

One tube is inoculated with 20 c.c. of the water, two tubes with lo c.c., four tubes with 5 c.c., five tubes with I c.c., and five tubes with $0^{\circ}$ or c.c. All are incubated at $37^{\circ} \mathrm{C}$, only tubes that give both acid and gas being counted as having given the reaction.

If only one tube in the first seven of these gives a reaction, then facal bacilli are present in 60 c.c., and if three out of the five I c.c. tubes gives a positive reaction, then facal bacilli are considered to be present in 1 c.c. The same applies, more or less, to the $o^{\circ}$ I c.c. and the $0^{\circ}$ or c.c. groups. The next step in the procedure is to isolate the various facal organisms present; and in order to to this satisfactorily, the tube containing so c.c. of water or more, that has given a positive reaction in forty-eight hours, is taken. Of this, three to five platinum loopfuls are removed and inoculated into 10 c.c. of sterile water. The latter is then vigorously shaken, and one loopful of this 
mixture is then plated out on lactose bile-salt-neutral-red agar, which is incubated at $37^{\circ} \mathrm{C}$. for forty-eight hours.

At the end of that time fifteen to twenty colonies are found to be present on the plate. Two colonies are in any case picked off, and each single colony is inoculated into a tube containing a few drops of sterile water. From these tubes further inoculations are made into broth, glucose-peptone, and four different special sugars are incubated at $37^{\circ} \mathrm{C}$. The inoculated broth tube is examined at the end of eighteen hours for motility and the production of indol.

The glucose-peptone is inoculated for forty-eight hours and at the end of that time, if a strong growth is present, a few drops of a very concentrated solution of caustic potash are added for the purpose of finding if the Voges-Proskauer reaction is given. If this is present a bright orange-red colour is produced. It may take twentyfour hours before this colour is apparent. The four sugars that are inoculated are saccharose, sulcite, adonit and inulin.

In these a positive reaction is represented at the end of forty-eight hours' incubation at $37^{\circ} \mathrm{C}$. by the production of acid and gas. It was assumed that the same species of bacillus always fermented the same sugar. In connection with the indol test the paradimethyl benzoldehyde reaction was always used, as it seems to be a very delicate and reliable test. With regard to the Voges-Prosliauer reaction considerable difference of opinion exists, but there can be but little doubt that it appears a good test for the B. cloaca, B. lactis aerogenes, and Oxytocus perniciosus group.

The term B. coli was only applied to Escherich's organisms.

Experiment (I).

Sample taken from the Blue Nile, two metres from the bank.

Local rainfall nil. River steadily rising for three weeks.

Temperature of water, $27^{\circ} \mathrm{C}$.

Total colonies on agar at $37^{\circ} \mathrm{C}$. were 2 is per c.c.

Facal bacilli present in $0^{\circ} \mathrm{x}$ c.c.

Siporogenes test plus in 20 c.c.

Experiment (2).

Sample from same place. Local rainfall nil. River steadily rising for two months. Temperature of water, $245^{\circ} \mathrm{C}$.

Total colony count in agar at $37^{\circ} \mathrm{C}$. was 700 per c.c.

Frecal bacilli present in $o^{\circ}$ I per c.c.

Sporogenes test plus in $20 \mathrm{c.c}$.

Experiment (3).

Sample from shallow well siluated in a native yard about a quarter of a mile from the Blue Nile.

The water was at a depth of in metres from the surface.

The water temperature was $30^{\circ} \mathrm{C}$. 
Total colony count in agar at $37^{\circ} \mathrm{C}$. Was 700 per c.c.

Facal bacilli present in o०or c.c.

Sporogenes milk test plus in 20 c.c.

Exporiment (4).

Sample from well No. 7 of the Khartoum water supply.

Gas was present in MacConker's medium when the latter was inoculated with $5^{\circ}$ c.c. of the sample.

Sporogenes milk test negative in 20 c.c.

Pemarks on these and other Tests.

The observations have been too few to justify any definite conclusions being arrived at with regard to the position that the $B$. coli occupies in this country as an indication of recent pollution.

Experiments should be carried out every month of the year to determine the effects of external natural forces, such as sunshine and rain on all organisms of facal origin.

There was more facal pollution present in a "falling" than in a "rising" river, although the colony count in the latter was nearly four times as great as in the former.

Experiments have demonstrated that there are different types of lactose fermenters present in human faces in different months of the vear.

Experiments also go to prove that B. coli communis is extremely susceptible to the bactericidal effects of sunlight, and that B. coscoroba, 13. schaefferi, B. neapolitanus, and B. vesiculosis are extremely resistant.

B. coli wert not found in trenches seven days old, hence soil destroys them.

The chief bacilli found in the faeces of animals were :-

B. coli, B. clacac, B. neapolitanus and others in goat faeces.

B. schaefferi and B. neapolitanus in cow faeces.

(C) Probably the best methods and the best data yet given come from Major Clemesha of the Madras Presidency.

He states that there must be considerable doubt as to whether the standards of purity in common use amongst sanitarians in England were suitable to India.

Most Indian waters are Inaded with facal contamination that no analyst in England would dream of passing as fit for human consumption. And yet it is remarkable that the evil effects of using such water as drinking-water are not always apparent.

The resulting diseases are not in proportion to the cause at all times.

In England a cautious Medical Officer would condemn water for drinking which contained true coli in I c.c., but in India the B. coli 
communis is a very rare organism in its waters, while other organisms are particularly common.

Clemesha comes to these conclusions: :-

(I) That standards in use in cold climates are useless, and worse than useless, in tropical climates.

(2) That it is necessary to separate the individual species of bacilli by well-established tests and to study their characteristics and their position in nature.

(3) That it is advisable to classify all lactose fermenting organisms according to their ability to resist the action of sunlight, and on this to base the standard of bacterial purity.

The general conclusions of his experiments are :-

The Bacteriology of Earth.

(1) B. cloace, B. grunthal, B. No. 75, and to a less extent B. coscoroba, are capable under favourable conditions of remaining alive in the soil for a considerable length of time, probably up to three to four years.

(2) Facal organisms of any kind do not appear to exist in large numbers after they have been in the earth for longer than one to two years; night soil buried in the ground, even in large quantities, loses most of its organisms in this period.

(3) Sporogenes enteritidis spores are capable of remaining alive in the ground for a period of something between three to four years.

(4) B. coli communis has never been isolated from the trenching ground samples, hence it is probable, though not certain, that this organism does not remain alive in the ground as long as the more resistant organisms like cloaca, \&c. It has been proved in one experiment to exist in the ground for a period of ninety-seven days.

The Bacteriology of Water and Feces during Wonsoon Weather.

(1) The conditions obtaining during a heavy monsoon when fresh faces may find their way easily into water, the whole country being submerged, is in some way connected with the appearance of a set of rare micro-organisms in all the water supplies over a wide area. This fact has not been noticed with previous and subsequent heary rain.

(2) These and allied bacteria may suddenly become extremely common in the faces of animals and man. The cause of this increased prevalence is unknown. 
(3) The flood conditions having passed away, the bacilli very rapidly disappear from all waters. Consequently this supports the suggestion that these organisms are especially susceptible to natural forces inimical to bacteria.

The Bacteriology of Water from a Shallow Well.

(1) In common with most other water supplies, including rivers, lakes, springs, \&c., B. cloaca would appear to be the predominating organism in a well after prolonged drought.

(2) Even a small downpour of rain, after a prolonged spell of dry weather, has a very marked influence in increasing the number of facal bacilli in wells situated in porous soils.

(3) Early showers, following on a long period of hot weather, have caused an increase in the number of Coli communis in this well. It is not by any means certain where these coli come from, but it is unlikely that they come from the surface of the ground, and probable that they remained alive in the lower layers of the soil.

(4) After heavy downpours of rain, in consequence of which it is certain that surface contamination has penetrated deep into the layers of the soil, a mixture of frecal organisms is usually found in the water obtained from wells. Coli communis appears to be present after early rains, but disappears as the rains become more plentiful.

(5) Plentiful rains improve the quality of the subsoil water after the contaminated surface water has run off the land.

(6) Shallow wells are a most unsatisfactory source of water supply, especially if they are situated in a highly porous soil, and unless the greatest possible care is exercised in protecting a large space of ground in the immediate vicinity from all chances of pollution.

The Bacteriology of Human and Animal Faces.

(1) The flora of the intestinal tract of men and animals are subject to very considerable changes due to influences which are at present unknown. These influences have been proved to operate over very wide areas.

(2) Within certain very wide limits, these forces appear to affect man and animals equally, both as regards number and kind of micro-organisms.

(3) Under well-defined conditions, such as heary rainfall, the water supplies contain the same organisms as the faces of man and animals at that particular time, but this similarity of bacterial flora is also noted occasionally when rain is absent, and there is no apparent cause for it. The explanation of this occurrence is at present unknown. 
(4) Having regard to the variation in the bacteria in feces, both in quantity and kind, no constant approximate composition can be arrived at. Even in the large groups, suggested by MacConkey, variation in percentage composition in the same animal is considerable.

(5) No lactose-fermenting organism has been isolated by us that has been proved to be the inhabitant of the intestinal tract of cattle or man only.

(6) The numerical relation of the organisms constituting MacConkey's groups in the intestines of cattle in India is entirely different from that in England, while in the intestines of man it appears to be very similar in the two countries.

(7) A study of the organisms present in faces at different times of the year is necessary for the proper interpretation of the results obtained from water analyses.

The Bacteriology of Lake Water.

(I) The action of the sun is powerful in destroying all frecal organisms in water, particularly where they are "naked" and not surrounded by mucus derived from the intestine.

(2) All facal organisms do not possess the power of resisting the action of sunlight to an equal degree.

(3) It is possible to divide fæcal organisms, with a reasonable degree of accuracy, into the following classes:-

(a) The delicate organisms or those that are very susceptible to the action of sunlight.

(b) An intermediate class containing a very large number of organisms which occupy an intermediate position between the two extremes, and

(c) The resistant organisms or those capable of resisting the sunlight for a considerable length of time.

(4) The surface layers of any large volume of water are in consequence purer than the deeper ones.

(5) Consequently the outlet from the reservoir should be arranged as near the surface as possible.

(6) In the Red Itills lake, B. cloace is by far the commonest faecal organism isolated from the bottom.

(7) Coli communis even in large numbers, in a huge volume of water, disappears with great rapidity. The organisms never survived longer than six days.

(8) There is some evidence that the middle layers of water in the lake of over $\mathrm{I} 2 \mathrm{ft}$. deep contain more water organisms and more frecal bacilli than the bottom or the surface. When taking samples the depth should be stated from which they are taken. 
(9) The B. cloacce and B. grunthal will persist in water exposed to the action of sun and storage for many months, and may therefore be looked upon as resistant to these forces.

(10) The rareness of such organisms as Coli communis in the samples analysed demonstrates the fact that organisms very susceptible to the action to sunlight do exist, Coli communis being the commonest of these.

(I I) Storage in the tropics is capable of rendering a highly contaminated water extremely pure, and of killing a large number of the facal organisms present after gross pollution.

In establishing bacterial standards, for drinking-water in India, a preliminary grouping is given to us thus:-

Class (1).- - See (3), (a) above.

Those waters containing Coli communis and the members of the susceptible group.

The contamination is recent and dangerous.

These are not commonly found.

Class (2).-See (3), (c) above.

Those waters containing such resistant organisms as B. grunthal, or B. cloacie or both.

These represent a pollution months old from a water supply becoming exhausted.

Class (3).

Those waters containing both the above organisms.

This condition is to be expected in rainy weather and flooded rivers.

In general be guided by:-

The number of facal organisms present.

The number of different species present in the ten colonies isolated.

To which group the organisms tend to belong most.

In fresh samples of facal contamination the Lac:is aerogenes is not often present, hence if it is found it may be considered as evidence that a considerable amount of purification of the water has taken place.

Clemesha proposes to classify river waters thus:-

(1) Good river water.

This should not contain more than Ioo colonies on agar at $37^{\circ} \mathrm{C}$.

Fiecal organisms should not exceed one in Io c.c.

No organisms in Class ( 1 ) should be present in 50 c.c.

Facal organisms present should belong to either Class (3) or the more resistant group of Class (2).

(2) Fair or usable river water.

This should not contain more than 300 colonies on agar at $37^{\circ} \mathrm{C}$. 
It should not contain more facal organisms than one in I c.c.

There should not be any organisms of Class (I) in less than 29 c.c.

The facal organisms present should consist mainly of mixtures of Class (3) and Class (2), and there should be a tendency for one organism to preponderate.

(3) Bad river waters to be condemned.

When the total colonies are more than 800 on agar at $37^{\circ} \mathrm{C}$.

When lactose fermenters are present in number of 10 to 100 per c.c.

When organisms belonging to Class ( $\mathrm{I}$ ) exceed one in 5 c.c.

When the facal organisms isolated (Class ( 1 ) being absent) are rich in varieties such as occur in an emulsion of faces.

For Well and Spring Waters.

A good water should contain no fæcal bacilli in 20 c.c.

No Class (1) in 100 c.c.

Total colonies under fifty per c.c.

For the details of this excellent work one must see "A Study of the Bacteriology of Drinking Water Supplies in Tropical Climates" (1909), by W. W. Clemesha.

Sanitarians are recommended to adopt similar methods.

Unfortunately he has not told us anything of deep wells.

It seems to be agreed that if experiments are carried out along the lines adopted by Clemesha, in most tropical countries, sufficient data will be available for suggesting some standard for a tropical water supply fit for drinking purposes.

We venture to give, however, for comparison, the standards as adopted by British sanitarians.

(a) Enumerate the bacteria capable of growth at room temperature ( $18-22^{\circ}$ C.).

(b) Identify and enumerate the bacilli coli present.

(c) Enumerate bacteria capable of growth at blood heat $\left(36^{\circ}\right.$ to $38^{\circ} \mathrm{C}$.).

(d) Enumerate the streptococci present.

(e) Detect the typhoid bacillus.

(f) Detect the cholera vibrio.

(a) To enumerate bacteria grown at room temperature.

Nutrient gelatin is most often the medium used.

(In countries with a high temperature nutrient agar should be used, but it is not as useful as nutrient gelatin in showing the presence of liquef ying organisms (Simpson).)

Professor Hewlett prefers beef broth.

The percentage of gelatin should be increased with the temperature. 
Melt the gelatin in three tubes in a water bath or incubator at blood heat.

Inoculate three sterile Petri dishes with $0^{\circ} 5,0^{\circ} 3,0^{\circ} 2$ c.c. of water well shaken.

Pour the contents of a gelatin tube into each Petri dish.

Move quietly to mix the water and gelatin.

Allow to solidify on a flat surface.

Count the colonies at the end of seventy-two hours, but inspect daily.

1, ooo organisms can be counted on a $10 \mathrm{~cm}$. dish, but 200 colonies should be the maximum.

In pure water less than ro per cent. will liquefy gelatin.

In the purest upland streams I c.c. contains often under ion bacteria, while in town sewage the same quantity contains millions.

In deep well water over roo organisms per c.c. is conclusive evidence of contamination. 500 per c.c. and above is suspicious water, 1,000 or more means condemnation of the water for drinking purposes.

(b) To enumerate bacteria grown at blood heat.

Inoculate two sterile Petri dishes with 1 and $0^{*} 1$ c.c. of the sample.

Melt two tubes of nutrient agar and allow to cool to $45^{\circ}$ or $40^{\circ} \mathrm{C}$. and pour into the dishes. Mix quickly and place to cool.

Count them at the end of forty-eight hours.

(c) To enumerate Bacillus coli present. The most important datum of bacterial examination.

The sample should be 50 c.c. of shallow well and surface water.

The sample should be 100 c.c. of deep well water.

Add a medium that contains a substance fermented by the Bacillus coli, and also a substance to inhibit the growth of other water organisms as Pake's method, glucose-formate broth.

Savage's method, neutral red glucose broth.

MacConkey's method, bile-salt broth, generally used.

In this is lactose, the Bacillus coli ferments giving acid gas.

\begin{tabular}{|c|c|c|c|c|c|c|c|}
\hline \multicolumn{6}{|c|}{ Sodium taurocholate... $\quad \ldots \quad \ldots \quad \ldots \quad \ldots 0.5$ per cent } & \multicolumn{2}{|c|}{0.5 per cent. } \\
\hline Wittes'p & ne & & 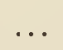 & & 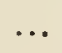 & 2.0 & , \\
\hline Lactose & .. & $\ldots$ & & . & & 1.0 & , \\
\hline Agar ... & $\ldots$ & ... & $\ldots$ & ... & $\ldots$ & $2 \cdot 0$ & ", \\
\hline
\end{tabular}

Made with tap water and I per cent. solution of neutral red added. A double strength is also made.

Bacillus coli gives bright red colonies, and Bacillus typhosus white colonies without fermentation.

Inoculate four tubes containing 5, IO, I5, 20 c.c. of this double strength medium with 5 , ro, $\mathbf{1 5}, 20$ c.c. of the water sample.

Incubate at $+2^{\circ} \mathrm{C}$. for twenty-four to forty-eight hours. 
If acid and gas is produced it is presumptive evidence of Bacillus coli.

Take a loopful of liquid from the tube with the smallest amount of water, and add it to a test-tube of io c.c. of sterile water. Shake and inoculate a similar tube with one loopful of this.

Repeat from the second to a third tube.

Smear one loopful of each dilution over a gelatin or agar slope.

One of these is sure to give discrete colonies.

Subculture these into tubes of nutrient broth, and later examine for motile organisms and do a Gram stain.

From the broth, culture inoculations are made into the various media necessary to identify the organism and the attributes of the organism thus collected.

If typical Bacillus coli have been found in the tube to which Io c.c. of the water had been added and not in that of 5 c.c., it would be stated that Bacillus coli was present in 10 c.c., but absent in 5 c.c.

Those waters showing no Bacillus coli in $5^{\circ}$ c.c. are of a high degree of purity.

Spring waters should not show them in 50 c.c.

In upland surface waters their presence in ro or 40 c.c. means contamination, but not necessarily dangerous, as it may be from animals grazing, and not pathogenic human excreta.

If there are more Bacillus coli than 5oo per litre the water is suspicious.

(d) To enumerate streptococci.

As above, but use glucose broth or glucose neutral red broth, and incubate at $37^{\circ} \mathrm{C}$. for four hours, and then make a hanging drop preparation.

The primary Bacillus coli tubes can, of course, be examined for them.

Streptococci do not multiply after sampling, but Bacillus coli do. Their numbers should not exceed that permissible for Bacillus coli.

(e) To detect the typhoid bacillus.

This is difficult. Remember that as the incubation of typhoid fever is twelve to fourteen days, the organisms may have disappeared from the water before the examination is started. Water containing typhoid bacilli will contain large numbers of Bacillus coli also. Other organisms may overgrow those of the typhoid bacilli.

There are many ways of concentrating the agar in the water, such as filtering through a porcelain filter, adding a little alum, entangling them in the aluminium hydrate, centrifuging, \&c. 
To isolate the organism from the concentrated deposit :-

Plate in Conradi-Drigalski agar or litmus lactose bile-salt agar.

Subculture the colonies into broth and work out their attributes in other media.

Try agglutinating (Widal) reactions in each subculture.

Do a Pfeiffer's test, depending upon bacteriolysis to clinch a diagnosis.

(f) To detect the cholera vibrio. (See "Cholera.")

\section{(II) THE CHEMICAL EXAMINATION OF WATER (after Stewart).}

The results of water analyses are expressed in parts per 100 , ooo or as grains per gallon = parts per 70,000 , since there are 70,000 gr. per gallon. Ioo c.c. is a convenient quantity to work with for certain estimates. When one is using standard solutions of a strength I c.c. = o.ool grm., the number of c.c. used in the titration gives the result in parts per 100,000 . Likewise, if to c.c. are taken of the sample, the actual titration gives the result in grains per gallon. This much facilitates the work as is demonstrated in a simple example thus :-

In estimating chlorine in a water sample, $3^{\circ} 4$ c.c. of standard silver nitrate solution ( 1 c.c. $=0^{\circ} 001$ grm. of chlorine) were required to reduce it. Hence there were $3+$ parts of chlorine in 100, ooo parts of the water because :-

$$
\frac{3+}{\times 0.001} \times \frac{100,000}{100}-\frac{34}{1}
$$

To convert grains per gallon to parts per roo,ooo multiply the result by 10 and divide by 7 , and conversely to multiply by 7 and divide by ro gives grains per gallon.

One is required to ascertain the
(1) Total solids
(2) The degree of hardness
(3) The metals present
(4) Chlorine
(5) Nitrites
(6) Nitrates
(7) Oxidizable organic matter
(8) Saline ammonia
(9) Albuminoid ammonia
(Io) Degree of aeration

in the ordinary drinking-water and sewage.

These will be dealt with seriatim, applied to drinking-water first.

\section{(1) To Estimate the Total Solids.}

Weigh a small porcelain dish.

Take 200 c.c. of the water sample.

Evaporate to drymess in dish over a water bath, pouring water in as it evaporates until 200 c.c. have been poured in.

Dry the dish in a water oven for an hour.

Cool, weigh the dish and residue. 
Deduct the weight of dish, remainder equals total solids in 200 c.c. Simply calculate how much in roo, ooo c.c.

Example:-

(2) To Estimate the Hardness.

$$
\begin{aligned}
\text { Dish }+ \text { total solids } & =27.848 \mathrm{grm} . \\
\text { Dish alone } & =27.797, \\
\text { Weight of residue } & =0.05 \mathrm{~g} \mathrm{grm} . \\
& =\frac{100,000}{200}=\begin{array}{l}
25.5 \mathrm{parts} \\
\text { per } 100,000
\end{array}
\end{aligned}
$$

The hardness of water is largely due to the presence of bicarbonates, sulphates and chlorides of calcium and magnesium.

(I) Total Hardness.

In a stoppered bottle of $\mathrm{I}_{5} \mathrm{O}$ c.c. capacity place I00 c.c. of sample.

Run in from a burette standard soap solution ( $\mathrm{c}$ c.c. $=0^{\circ}$ oo I $\mathrm{grm}$. $\mathrm{CaCO}_{3}$ ), shaking vigorously until a permanent lather of $\frac{1}{4} \mathrm{in}$. is obtained.

\section{Example.}

I 3.8 c.c. of soap solution is required.

Hence $13^{\circ} 8 \times 0^{\circ}$.0OI is contained in 100 c.c. of water.

$$
\frac{13.8 \times 001 \times 100,000}{10}
$$

If Ioo c.c. are used this latter calculation is avoided as the c.c. used, $13^{\circ} 8$ in this case, indicates the parts per 100,000 .

(2) Temporary Hardness.

This can be removed by boiling.

It is due to the bicarbonates of lime and magnesia.

On boiling the $\mathrm{CO}_{2}$ is driven off and the carbonates are precipitated. Commercially it is removed by adding lime.

The degree of temporary hardness is calculated by subtracting the permanent from the total hardness.

(3) Permanent Hardness.

This is due to the sulphates and chlorides of calcium and magnesium.

They are soluble in water and can not be precipitated by boiling.

To estimate them :-

Boil 100 c.c. in a beaker for fifteen minutes.

Filter when cold and make up the filtrate with distilled water to ioo c.c. Shake well.

Run in soap solution as described.

Example: t² $^{*}$ c.c. required.

Hence $4^{\circ} 2$ parts per Ioo,ooo.

But the total hardness was $13 . \mathrm{S}$

The permanent $, \quad, \quad \overline{42}=9^{\circ} 6$ parts per 100,000 temporary hardness. 


\section{(3) Detection and Estimation of Poisonous Metals.}

To 100 c.c. of the water add a few drops of acetic acid.

Pass in $\mathrm{H}_{2} \mathrm{~S}$.

A light clear brown deposit $=$ copper.

A dirty granular deposit = lead.

Copper.

To another 100 c.c. add a little acetic acid.

To this add 2 c.c. of fresh solution of potassium ferrocyanide.

To estimate the amount of copper present:-

Match this chestnut brown colour by a standard copper solution.

Copper solution ( 1 c.c. $=0^{\circ} 0001$ grm. ( Cu) as is described in Nesslerizing the ammonia solutions.

Lead.

To Ioo c.c. of water add a little acetic acid.

To this add a few drops of potassium chromate and stir well.

A yellow turbidity (of lead chromate) indicates lead.

If lead is in excess a yellow deposit will be formed.

Pass $\mathrm{H}_{2} \mathrm{~S}$ in 100 c.c. of the sample.

Match the brown coloration with a standard lead solution (i c.c. $=0$ oooo g grm. Pb) to estimate lead.

Example, I 2 c.c. of the standard solution are necessary to match the colour in the given sample.

Hence there is $0^{\circ} 000 \mathrm{I} \times \mathbf{I} 2 \mathrm{grm}$. of lead in the I oo c.c. of the sample, there is $\frac{0.000 \mathrm{I} \times 12 \times 1 \mathrm{co}, 0}{1 \mathrm{CO}} !^{\circ} 2 \mathrm{grm} . \mathrm{Pb}$ in I00,000 parts.

Iron.

If neither copper nor lead is present test for iron.

Take roo c.c. of the sample.

Add 5 c.c. of nitric acid, $I$ in 5 .

Then 5 c.c. of io per cent. potassium sulphocyanide.

A red coloration indicates iron.

Match this colour with a standard iron solution (I c.c. $=0$ oooor c.c. grm. Fe) to estimate the amount.

If the colour is too intense to be matched conveniently take a smaller amount and dilute to roo c.c. with distilled water.

(4) To Detect and Estimate Chlorine.

This is present in most waters as sodium, potassium or calcium chloride.

Its presence may be due to:-

(1) The water passing through saline compounds in the strata, e.g., sandstone, greensand.

(2) Pollution by organic matter, especially urine.

(3) Deep wells near the sea coast.

(4) Tidal rivers. 
Place 100 c.c. of the water in a large porcelain dish.

Add a few drops of 5 per cent. potassium chromate.

Run in from a burette standard $\mathrm{AgNO}_{3}$ solution ( 1 c.c. $=0^{\circ} \mathrm{oor} \mathrm{grm}$. of $\mathrm{Cl}$ ) until a reddish tint remains.

Silver chromate is formed which is red.

If say $I^{\prime} \gamma$ c.c. are required then these are the parts per 100,000 .

Since $1.7 \times 0.001 \times 100.100,000=1 \%$

To obtain the results in terms of sodium chloride multiply the result by $1 \cdot 647$ since $\mathrm{Cl} \times{ }_{1} \cdot 647=\mathrm{NaCl}$.

This factor is obtained from the ratio $\frac{\mathrm{NaCl}}{\mathrm{Cl}}=\frac{58 \cdot 5}{355}=1 \cdot 647$.

\section{(5) To Detect and Estimate Nitrites.}

These generally indicate recent organic pollution.

Nitrites are eventually oxidized to nitrates.

Sometimes nitrates are reduced to nitrites by ferrous salts or in deep wells due to the metal of the bore pipes, but these are the exceptions.

Nitrites are usually found in sewage efluent.

Test (I).

Place 100 c.c. of the water in a Nessler cylinder.

Add 6 drops of dilute $\mathrm{HCl}$.

Add 6 drops of naphthylamine hydrochloride.

Add 6 drops of sulphanilic acid.

Stir with a glass rod.

A pink colour indicates nitrites.

If no coloration in fifteen minutes nitrites are absent.

Test (2).

Place 100 c.c. of water in a Nessler cylinder.

Add a few drops of $\mathrm{H}_{2} \mathrm{SO}_{4}$.

Add ' 1 crystal of KI and mix well.

Add 5 c.c. of fresh starch solution. A blue colour indicates nitrites. Test (3).

To roo c.c. of water add a few drops of diluted $\mathrm{HCl}$.

Add 5 c.c. of metaphenylene-diamine solution.

Mix and allow to stand.

A yellowish-orange colour indicates nitrites.

If the reagent is not colourless, shake it with a little animal charcoal and filter.

To estimate the amount match this colour by using a standard sodium nitrite solution ( 1 c.c. $=0^{\circ} 001 \mathrm{grm}$.).

(6) To Detect and Estimate Nitrates.

These are usually present.

Strata containing red sandstone, \&c., impart them to the water. 
They are also the final products of completely oxidized organic nitrogenous matter.

Test (I).

Fill a large test-tube with the sample.

Add a few c.c. of diphenylamine solution.

Pour concentrated $\left.\mathrm{H}_{2} \mathrm{SO}\right)_{4}$ carefully down the side of the tube.

A blue ring at the junction of the two liquids = nitrates.

Test (2)

Repeat the above using a solution of brucine in $\mathrm{H}_{2} \mathrm{SO}_{4}$.

A red ring is formed which turns rellow, then brown.

Both these tests are given with nitrites also, hence nitrites should be excluded or Test (3) applied, which does not react with nitrites.

Test (3).

Evaporate 50 c.c. of the water to dryness in a water bath.

Add I c.c. of phenolsulphonic acid.

Mix well with a glass rod.

Heat on a water bath for one minute.

Transfer the fluid to a roo c.c. Nessler glass, washing the basin and rod with water, adding same to the glass.

Add ro c.c. of concentrated ammonia.

Stir and make up to 100 c.c. with distilled water and shake.

A yellow coloration denotes nitrates.

By this method the nitrates are converted into picric acid.

The ammonia forms ammonium picrate which has an intense yellow colour.

To Estimate.

\section{Wethod (I).}

Retain the above yellow fluid and match it.

Take 5 c.c. of the standard $\mathrm{KNO}_{3}$ solution ( 1 c.c. $=0$ oooo grm. N).

Evaporate to dryness and treat it exactly as the above solution.

There are now two cylinders of yellow fluid that are not of similar tints.

Suppose the Sample is the darker of the two Solutions.

Pour out some of the darker, say is c.c., and make up the

$S_{5}$ c.c. to 100 c.c. with distilled water.

If now too dark pour out more, say io c.c., and make up the

90 c.c. to roo c.c. with distilled water.

Suppose the tints now coincide we get :-

$\underbrace{100 \times 100 \times 0.0005 \times 100,000}_{85 \times 90 \times 50}=1.3$ parts $N$ as nitrate per Ioo,oon. 
Suppose the Water Sample is the lighter of the two.

Pour out some of the darker, say first is c.c., then io c.c. as above, then the amount of the original solution remaining in the standard cylinder is $\frac{85 \times 90}{100 \times 100}$

Therefore in the sample we have:-

$\frac{85 \times 90 \times 00005 \times 100,000}{160 \times 100 \times 50}=0^{\circ} 766$ parts $N$ as nitrate per 100,000 parts.

Method (2).

Dissolve $2 \mathrm{grm}$. of sodium hydrate in 100 c.c. of distilled water.

This is a pure solution of caustic soda free from nitrates.

Add roo c.c. of the sample water and a piece of aluminium foil.

Permit to stand for twenty-four hours in a dark place.

Then all the oxidized nitrogen is converted into ammonia.

Distil this solution and Nesslerize as above.

Before distilling test for nitrites to see that all have been reduced to nitrates.

Method (3).

Coat some zinc foil with copper by exposing it to copper sulphate until it becomes black.

Put zinc foil copper-coated into a bottle with 250 c.c. of the sample water, add $0.5 \mathrm{grm}$. oxalic acid and leave for twentyfour hours.

All oxidized nitrogen is converted into ammonia.

Distil and Nesslerize.

\section{(7) To Estimate the Oxidizable Organic Matter in Water (Oxygen absorbed).}

This can be done in three hours at $37^{\circ} \mathrm{C}$, or in four hours at $80^{\circ} \mathrm{F}$.

The putrescent organic matter is chiefly oxidized in the first thirty minutes.

Oxygen may be absorbed by nitrites, $\mathrm{H}_{2} \mathrm{~S}$, ferrous salts, \&c.

In a stoppered bottle of 400 c.c. capacity place 200 c.c. of sample and add Io c.c. of $\mathrm{KMnO}_{4}$ solution ( $\mathrm{c}$ c.c. $=0^{\circ} \mathrm{ooO} 1 \mathrm{grm}$. of available $\left.\mathrm{O}_{2}\right)$.

Add io c.c. of $\mathrm{H}_{2} \mathrm{SO}_{4}$ ( 1 in 4).

Place in incubation at $37^{\circ} \mathrm{C}$. for three hours.

If the pink colour quickly fades add more $\mathrm{KMnO}_{4}$ always counting the c.c. used.

At the end of three hours at $37^{\circ} \mathrm{C}$. a pink colour should be present.

Cool under the tap.

Add 5 c.c. of KI 10 per cent. solution.

Run in from burette sodium thiosulphate solution ( $\mathrm{g} \mathrm{grm}$. in I, ooo c.c.) until the yellow colour has nearly disappeared. 
Add 5 c.c. of fresh starch solution.

Add more thiosulphate until the blue colour has disappeared, shaking constantly.

We will suppose that i5.5 c.c. were necessary.

Wre must now find out the strength of the thiosulphate in terms of $\mathrm{O}_{2}$.

For this we do a blank experiment.

Fill a similar bottle to the above with 200 c.c. of distilled water.

Add at once 5 c.c. of io per cent. $\mathrm{KI}$, io c.c. of $\mathrm{H}_{2} \mathrm{SO}_{4}$ ( $\mathrm{I}$ in 4 ) and ro c.c. of $\mathrm{KMnO}_{4}$ as above.

Run in the thiosulphate solution from the burette until the yellow colour has nearly disappeared.

Then add 5 c.c. of fresh starch solution and add more thiosulphate until the blue colour has disappeared.

Suppose $20^{\circ} 5$ c.c. are necessary.

Thus blank experiment requires $20^{\circ} 5$ c.c. of thiosulphate.

Water sample requires $15^{\circ} 5$ c.c. of thiosulphate.

Therefore the difference is 5 c.c. of thiosulphate.

Now $20^{\circ} 5$ c.c. of thiosulphate $=10$ c.c. of $\mathrm{K}^{\mathrm{M}} \mathrm{InO}_{4}$ or $0^{\circ} \mathrm{OO} \mathrm{g} \mathrm{grm}$. of $\mathrm{O}_{2}$.

Hence 5 c.c. of thiosulpliate $\frac{0^{\circ 00 \mathrm{r} \times 5^{\circ} \mathrm{J}}}{2 \mathrm{u}_{5}}=0^{\circ} 00026$ of $\mathrm{O}_{2}$.

In 200 parts of sample water $0: 00026$ parts of $\mathrm{O}_{2}$ are absorbed.

$\therefore$ in 100,000 parts of sample water $0^{\circ} 13$ parts of $\mathrm{O}_{2}$ are absorbed.

Water cannot be judged on this experiment alone as peaty waters absorb much oxygen and they are not as detrimental as waters absorbing the same amount due to the presence of nitrites.

Those not exceeding $0^{\circ} \mathrm{J}=$ good organic purity.

Those not exceeding $0^{\circ} 3$ medium organic purity.

Those not exceeding $0^{\circ}+$ doubtful organic purity.

Those more than $0^{\circ}$ - polluted organic purity.

If other than upland surface waters are examined use half the amount for the experiment.

\section{(8) To Estimate Free and Saline Āmmonia.}

During decomposition of organic matter, $N$ combines with $\mathrm{H}$ and forms ammonia. This may combine with the saline constituents of the water forming saline ammonia or remain free.

As this ammonia-the product of decomposing animal matter-is very small, all vessels, \&c., used in the experiment must be ammonia free.

Place 300 c.c. of distilled water into a flask and distil it over.

Test the distillate with 2 c.c. of Nessler's solution until it does not give a yellow colour. Then the vessel is ammonia free.

(Nessler's solution is caustic potash with potassio-mercurio iodide.) 
In the emptied flask place 500 c.c. of the water sample.

Add a pinch of calcined sodium carbonate.

The ammonium carbonate thus formed yields ammonia readily on boiling.

Distil and collect the distillate in 50 c.c. Nessler's glasses successively.

Add 2 c.c. of Nessler's solution to the first cylinder, stir and let it stand three minutes.

Match this tint with a standard solution of ammonium chloride ( 1 c.c. $=0$ o000oI grm. of $\mathrm{NH}_{3}$ ).

In a Nessler's glass place 50 c.c. of distilled water.

Add 2 c.c. of Nessler's solution.

To this add $0^{*} 5$, or 1 , or 2 c.c. of the standard ammonium chloride until the tint is the same as the first cylinder distilled.

If the tint does not match make a fresh standard or have, say, six cylinders containing known and varying amounts for matching.

Treat the other cylinders the same as they are distilled over.

Two or three are usually sufficient and contain all the ammonia. Thus the first cylinder requires $3^{\circ}$ o c.c. of ammonia chloride solution.

And the and cylinder requires 0.8 ,

And the $3^{\text {rd }}$ cylinder requires nil.

$$
3.8 \text { c.c. }
$$

Hence amount of $\mathrm{NH}_{3}$ in 500 c.c. of water is $3^{\cdot} 8 \times 0^{\circ} 00001$.

Hence amount of $\mathrm{NH}_{3}$ in 100,000 c.c. of water

$$
\begin{aligned}
& 3.8 \times 0.0000 \mathrm{I} \times \mathrm{ICO}, 000 \\
& 500 \\
& =0^{\circ} 0076 \text { parts per 100,000. }
\end{aligned}
$$

Save the resulting solution for estimating albuminoid ammonia.

Good water does not usually contain more than o'or parts per 100,000 .

\section{(9) To Estimate Albuminoid Ammonia.}

When nitrogenous organic matter is heated with an alkaline solution of $\mathrm{KMnO}_{1}$, it is oxidized and part of the $\mathrm{O}_{2}$ present is converted into ammonia.

If the albuminoid ammonia exceeds the saline ammonia it indicates vegetable pollution.

If the saline ammonia is high and the albuminoid ammonia above 0.005 with high figures for saline ammonia it indicates animal pollution. There may be free ammonia in deep well water, the metal of the bore pipes reducing the nitrates.

Boil in a beaker fifteen minutes $5^{\circ}$ c.c. of alkaline permanganate solution with 100 c.c. of water. 
Pour this into the flask with its contents left from the above experiment.

Add a fer pieces of pipe siem to prevent bumping.

Distil over about four 50-c.c. Nessler glasses.

Nesslerize and estimate as for saline ammonia.

The amount in good water does not exceed 0.01 part per roo,oco.

(10) To Estimate the Aeration of Waters $\left(\mathrm{O}_{2}\right.$ dissolved).

Measure exactly the rolume of a stoppered bottle about 250 c.c.

Fill completely with water sample, aroiding air bubbles.

By means of a long pipette, the rod inserted close to the bottom of the bottle:-

Add l c.c. of to per cent. manganese chloride.

Add 3 c.c. of ( 50 per cent $\mathrm{NaOH}$ and 10 per cent. KI) solution.

Insert stopper, avoiding air bubbles, shake.

Subtract + c.c. from volume of bottle for reagents used.

Allow to stand ten minutes.

Run in 5 c.c. of concentrated $\mathrm{IICl}$, holding pipette just above the precipitate.

Insert stopper and shake.

Free iodine is liberated and a clear reddish brown fluid obtained.

Transfer this fluid to a flask.

Rinse out old flask with Io c.c. of distilled water and add to new flask.

Run in this from a burette sodium thiosulphate solution $(4 \mathrm{grm}$. per $I$, ooo c.c.) until a faint yellow colour remains.

Add 5 c.c. of fresh starch solution.

Continue with thiosulphate until a blue colour is discharged.

Now do a blank experiment :-

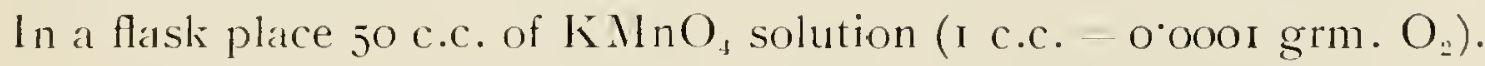

20 c.c. of $\mathrm{H}_{2} \mathrm{SO}_{4}$ solution ( $\mathrm{I}$ in 4 ).

IO C.c. of KI solution (io per cent.).

Titrate the liberated iodine as above.

Suppose the blank required 25 c.c. of thiosulphate.

Suppose the sample required is c.c. of thiosulphate.

$5^{\circ}$ c.c. KMnO $\mathrm{H}_{4} \mathrm{O} \times 0^{\circ} 0001=0.005 \mathrm{grm}$. oxygen.

Hence 25 c.c. thiosulphate solution 0.005 grm. oxygen.

Hence 15 c.c. thiosulphate solution $\frac{0.05}{25} \times 15=\mathrm{OCO}_{3}$

The volume of the bottle $-(22+-+ \text { c.c. })^{2} 220$.

In 220 parts of the sample there are grm. 0.003 of $\mathrm{O}_{2}$.

$\therefore$ In I00,000 parts of the sample there are $\frac{0.003 \times 100,000}{220}=I^{3} 3^{6}$ "xygen absorbed.

A good water should have about I'I parts per Ioo,000. 
Certificate of Analysis of a Sample of Water.

Date received:

From :

Particulars on label: London tap water. Taken at Laboratory. Physical characters :

Colour in $2 \mathrm{ft}$. stratum : clear pale blue.

Suspended matters: nil.

Taste.

Odour when warmed to $37^{\circ} \mathrm{C}$. : nil.

Reaction : alkaline.

Chemical characters :-

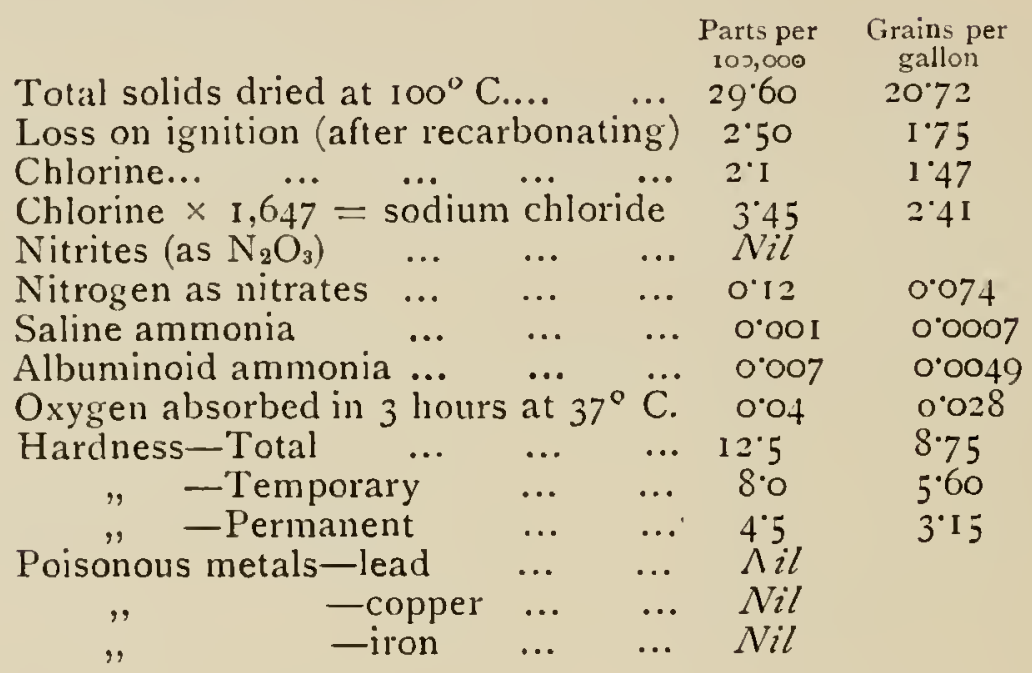

\section{TO EXAMINE SEWAGE AND SEWAGE EFFLUENTS.}

The same processes as for water with a few modifications.

\section{The Ammonias.}

Dilute sewage five times when estimating the ammonias.

Make roo c.c. up to 500 c.c. with ammonia-free water.

When distilling collect 200 c.c., mix well, Nesslerize, and multiply the result by 4 .

It is best to collect 50 c.c. more to see that no more ammonia remains.

\section{The Solids in Suspension.}

The estimation of these is required.

Filter I,O0O c.c. of the effluent through a weighed filter paper.

Wash and dry the residue and weigh again.

\section{The Rate of $\mathrm{O}_{2}$ Absorption after Filtration.}

Do this as for estimating the aeration of water, p. 66r, but tested at different times:-

$\mathrm{O}_{2}$ absorbed in four hours at $60^{\circ} \mathrm{F}$.

$\mathrm{O}_{2}$ absorbed in twenty-four hours at $60^{\circ} \mathrm{F}$.

$\mathrm{O}_{2}$ absorbed in forty-eight hours at $60^{\circ} \mathrm{F}$.

$\mathrm{O}_{2}$ absorbed in five days. 
The Royal Commission on Sewage Disposal recommended that an effluent "should not contain more than 3 parts per 100,000 of suspended matter; after being filtered it should not absorb more than

0.5 by weight of $\mathrm{O}_{2}$ per roo, 000 in twenty-four hours.

I'o by weight of $\mathrm{O}_{2}$ per 100,000 in forty-eight hours.

I'5 by weight of $\mathrm{O}_{2}$ per 100,000 in five days."

Chlorine should not exceed ro parts per ioo,ooo.

$\mathrm{O}_{2}$ absorbed in four hours at $60^{\circ} \mathrm{F}$. (or two hours at $S 0^{\circ} \mathrm{F}$.) and the saline ammonia should not exceed I's per 100,000 .

The albuminoid ammonia not more than $0^{\circ} \mathrm{I} 5$ part.

Incubation of final effluent when at $37^{\circ} \mathrm{C}$., and for five days should be odourless.

Generally high nitrates are a good feature.

\section{THE ANALYSIS OF MILK.}

\section{The Average Composition of Cow's Milk is:-}

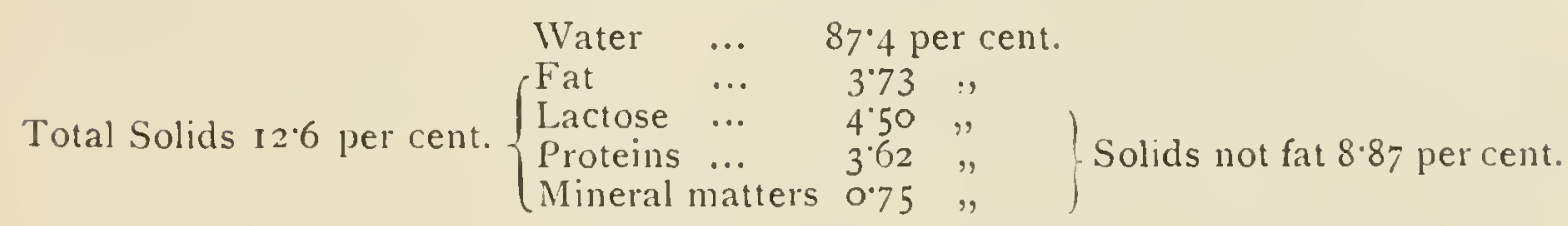

The specific gravity is about 1032 at $15^{\circ} \mathrm{C}$.

\section{(1) To Estimate the Total Solids.}

Weigh a small porcelain dish.

Put therein io c.c. of milk and reweigh.

Evaporate to dryness on a water bath for one hour.

Dry in an oven for one hour.

Cool in a desiccator and weigh.

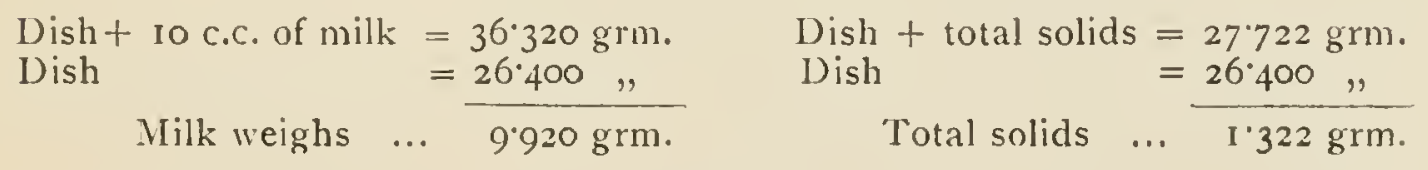

Hence roo grm. of milk will contain :-

$$
\frac{1.322 \times 100}{9^{\circ} 920}=13.32 \text { per cent. of total solids }
$$

\section{(2) To Estimate the Ash or Mineral Matter.}

Ileat the residue from the above to a white ash on a porcelain triangle.

Stir with a glass rod meanwhile.

Cool in the desiccator and weigh. Suppose ash is $0.0 ; 6$.

Ioo grm. of milk contains about $0^{\circ} 76$ per cent.

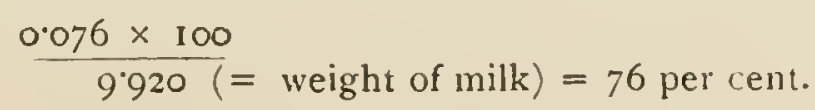




\section{(3) To Estimate Fat.}

Weigh ro c.c. of mills.

l'lace it into a Stoker's graduated tube.

Add ro c.c. concentrated $\mathrm{ICl}$ and heat cautiously till brown.

Cool and fill up to the 5o c.c. mark with ether.

Shake well and allow ether to settle out.

Read off the volume of ether, say 26 c.c.

Pipette out Io c.c. of ether solution into a small weighed glass flask.

Evaporate ether, dry hask in oven for one hour.

Cool and weigh.

If a Stokes tube is not available heat the milk in a large test-tube, and transfer when cool to a graduated roo c.c. cylinder. Wash out the tube with ether and place same in the cylinder, and the volume is now made up to 50 c.c., and the process continued as described above.

Example:-

Flask fat 23.57 I

Flask 23.421

0.150

In Io c.c. of ether there is 0.15 of fat

In 26 c.c. of ether there is $0.15 \times 26$

$$
\frac{10}{\text { IO }}=0.39
$$

The amount of fat in $10^{\circ} 213 \mathrm{grm}$. of milk (10 c.c.) $=0.39$

'The amount of fat in $100 \mathrm{grm}$. of milk $0.39 \times 0.100$

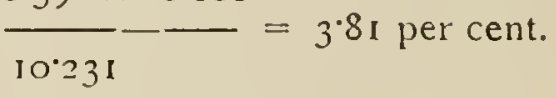

\section{(4) To Estimate Solids not Fat.}

Subtract the fat from the total solids.

Example: $13^{\circ} 32-3.81=9^{\circ} 5^{1}$ per cent of solids, not fat.

(5) To Estimate Lactose (Fehling's method).

Pipette 20 c.c. milk into a 100 c.c. graduated flask.

Add a few drops of acetic acid.

Mix well, place on a water bath for a few minutes.

Cool and make up to roo c.c. with distilled water, shake and filter. Fill a burette with this solution.

In a porcelain basin place ıo c.c. of Fehling's solution.

Add to c.c. of water and boil.

Run in milk solution from the burette slowly.

Continually try a few drops of liquid from the dish with a starch and KI paper.

When it ceases to give a blue colour stop burette flow.

Keep the liquid gently boiling during the process.

For the indicator it is handy to have a series of test-tubes.

In each place 2 c.c. of the indicator and one drop of acetic acid. 
To make the indicator:-

Dissolve $0.5 \mathrm{grm}$. of starch by boiling in water.

Dissolve Io grm. of $\mathrm{KI}$ in water.

Mix and make up to roo c.c.

E.xample: $7 \cdot 2$ c.c. of diluted mills were required

But 7.2 c.c. of diluted milk $=0^{\circ} 0678 \mathrm{grm}$. lactose

Since Io c.c. Fehling's solution $=0.0678 \mathrm{grm}$. laciose

Hence $\mathrm{I}$ oo c.c. of diluted milk $=0.678 \times \mathrm{I} 00 \mathrm{grm}$. lactose

$7 \cdot 2$

But this is in $20 \mathrm{c}^{\circ} \mathrm{c}$. sample of milk

$\therefore$ In 100 c.c. sample of milk $=\frac{0.0678 \times 100 \times 100}{7.2 \times 20}=4.7$ per cent. lactose

(6) To Estimate the Specific Gravity.

This can be estimated by Westphal's balance, the Sprengel tube lactometer, or the specific gravity bottle. The lactometer is best for tropical use.

It is usually between IO2S and 1034 .

\section{(7) To Estimate Amount of Added Water and Deficiency of Fat.}

These are obtained from two simple formula.

(A) $8.5-\mathrm{S.N.H} \times 100$

$\frac{5}{8 \cdot 5}=$ per cent. added water

S.N.F. solids not fat found in sample, e.g., $2 \cdot 7$

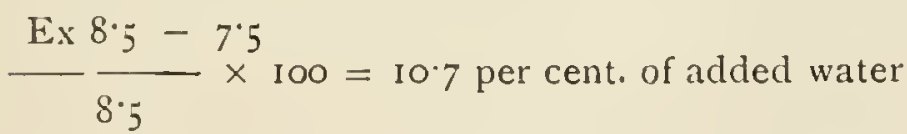

(B) $\underline{3-\mathrm{F}} \times 100=$ per cent. deficient in fat. $\mathrm{F}$. = fat in sample, e.g., $2 \cdot 7$

Ex. $\underline{3-27} \times 100=10$ per cent. deficient in fat

3

(8) Common Preservatives are:-

(1) Boric Acid.

Acidify 3 c.c. of milk in a test-tube with distilled $\mathrm{HCl}$.

Mix well and place a drop on a turmeric paper.

Dry same on watch glass in oven.

A red spot indicates that boric acid is present.

This turns green instead of black on adding caustic potash.

(2) Formaldehyde.

In a test-tube place io c.c. of milk.

Add Io c.c. of concentrated $\mathrm{HCl}$ and one drop of ferric chloride.

Stir well, heat to boiling.

A violet colour indicates formaldehyde. 


\section{Sale of Milk Regulations.}

The solids not fat must not be less than 8.5 per cent.

The milk fat must not be less than 3 per cent.

No preservative is allowed in milk of any kind.

\section{To Analyse Condensed Milk.}

Employ the methods already mentioned with a few modifications. Use a ro per cent. solution of the condensed milk.

For the total solids and proteins take 15 c.c. of this solution.

For the fat take ro c.c. of this solution.

The fat, ash, proteins and lactose subtracted from the total solids give the cane sugar.

An average analysis should yield :-

$$
\begin{aligned}
& \text { Water } \ldots \quad \ldots \quad 25^{\circ} 2 \text { per cent. } \\
& \text { Fat } \ldots \text {... II } 0 \text {, } \\
& \begin{array}{lll}
\text { Proteins ... } & \ldots & 9.07 \quad ",
\end{array} \\
& \begin{array}{llll}
\text { Ash } \quad \ldots & \ldots & \text { I.90 }
\end{array} \\
& \text { Lactose ... } \quad . .613 .40 \text {, } \\
& \text { Cane sugar } \quad \cdots \quad \underline{39^{\circ} 43} \quad \text { " } \\
& 100^{\circ} 00
\end{aligned}
$$




\section{APPENDIX.}

FALLACIES IN BLOOD EXAMINATION,

THE GENERAL TREATMENT OF FEVER.

EMBALMING.

SOME HINTS ON TROPICAL SURGERY.

COMPARATIVE NORMAL TEMPERATURES.

PRINCIPAL REFERENCES. 


\section{APPENDIX.}

\section{FALLACIES IN BLOOD EXAMINATION.}

Conditions leading to fallacies may be dealt with in two classes:-

\section{(1) ExternaI.}

Insect hairs may simulate filariae. Low and Stiles took this view of the F. gigas described by Prout.

Cotton fibres once led to a wholesale diagnosis of filarice cases.

Care and cleanliness should exclude these.

Films sent from the tropics may contain yeast cells or spores which may cause considerable confusion.

Less troublesome are pollen grains, animal and vegetable débris found in the dust fouling the specimen.

Films made from the blood of shot or slanghtered beasts or birds may contain parasites carried from the gastro-intestinal tract, skin, hairy hides or feathers of the cadaver.

A piece of blotting paper may be used to dry one slide in staining and in blotting a second with the same paper some of the parasites from the first may be carried to the second slide.

Flaws and cracks in the glass should always be anticipated, especially as "seconds" are better than "firsts" for tropical work, as the best quality slides "fog" readily.

(2) Internal.

\section{Fresh Blood.}

Crenations of red cells are readily differentiated from parasites as a rule.

Vacuoles are a little more troublesome. They are highly refractive, never show granules in their interior and exhibit no movements.

Splits and cracks in red cells have received many names and as they appear more frequently in pathological specimens they may be more readily taken for hematozoa. They have no amœboid movement but the contraction and the coagulation of the protoplasm may cause them to change their position slightly. They have probably been mistaken for bodies occurring in pernicious anæmia, leukxmia and lead poisoning. The so-called Cropper's bodies may be of a similar nature.

Cranules from broken-down leucocytes may overlie red cells and cause trouble. They are easily differentiated by focusing.

A raised temperature may cause a peculiar degeneration of red cells that must be watched. They are most often seen when one is working with a hemostat.

A blood platelet lying on a red cell may simulate a malarial plasmodium. These can soon be diagnosed by altering the light and by focusing.

Blood dust may be confused with granular débris from leucocytes 
or with micrococci. They sometimes appear like minute fat droplets, which may be correct, as such do appear after a meal.

Under artificial conditions lymphocytes and myelocytes may become flagellates, induced by excitants such as atropine, methylene blue and a cancerous plasma.

Normal blood has shown bodies with pseudopodia supposed at one time by Koch and Kliene to be associated with piroplasmosis. They are only altered blood cells.

Bodies like vesicles in red cells have been mistaken for a special form of intra-corpuscular spirochretal inclusion.

Stained Blood.

Blood platelets on red cells must be differentiated from the malarial parasite, and a mass of platelets from a crescent or a clump of spores or Leishman-Donovan bodies.

The corps en pessaire and corps en annean of French writers should be differentiated from pathological findings in malarial cachectics. In such patients anticipate endoglobular and anæmic degeneration of the red cells.

The degeneration and vacuolation of leucocytes may make the student almost unaware of their origin. Experience alone will aid in the process of differentiation.

Oval and round rings occur in the cytoplasm of lymphocytes, probably under the influence of alkaline stains, \&c.

When animal blood conditions are considered the subject becomes much more comprehensive.

For details upon this subject the student is recommended to: "Fallacies and Puzzles in Blood Examination," by Andrew Balfour. M.D., in the Fourth Report of the Wellcome Tropical Research Laboratory, Vol. A. Medical, of which the above is an abstract. That article will be found sufficient for general purposes.

\section{THE GENERAL TREATMENT OF FEVER.}

In this outline of treatment the specific cause of the fever is not dealt with. This should be known and treated accordingly, but in all fevers there are so many things in common that the general treatment may be along similar lines for a!l.

When death takes place probably the chief source of weakness is the heart, hence one must consider those factors telling against the circulation as : -

Under and improper feeding.

Insufficient water.

Vomiting because it withdraws food and water from the patient, is exhausting and prevents rest and sleep.

Diarthoe for the same reasons.

The breathing of foul air.

Insufficient oxygen.

The interference of oxygenation due to lung troubles.

The impairment of the renal secretion.

The loss of sleep and rest due to noises, bright light, pain, toxæmia and pyrexia.

The elevation of the head. 
The main things one must watch and deal with are diet, vomiting, diarrhoea, constipation, pure air, the maintenance of oxygen for the blood, sweating, renal secretion, rest and sleep, the control of pain, prrexia, elevation of the head, sustenance of the heart and care during convalescence. These will now be dealt with seriatim.

\section{(1) Diet.}

It should consist of carbohydrates, proteins, fats, water, sodium chloride and regetable salts, in a word it must be complete. The feeding must be adapted to the condition of the patient thus:-

The patient may not be able to take food by the mouth or rectum owing to diarrhuea and vomiting, hence one must feed by giving salines intravenously or subcutaneously at a temperature of $100^{\circ} \mathrm{F}$. in the funnel. Give at least one pint in twelve hours. This is, of course, not a complete food or anything like it, but it is the best that can be done for the time.

The patient may not be able to take food by the mouth owing to vomiting but can take it by the rectum. Then give peptonized milk, IOO c.c. for an infant, I25 c.c. for a child and 250 c.c. for an adult every six hours. If rejected give a suppository and repeat the food. Raise the buttocks to assist by gravity the entrance of the food.

Rectal feeds vary enormously as regards constituents and each will use that which in his experience has proved most useful.

There is much controversy as to the absorption of foods per rectum but in such cases as those mentioned it is best to give them.

When the patient can take some food by the mouth it must be an incomplete food at first, such as junket, raw meat juice, white of egg. Beef teas and broths are not good foods.

A little alcohol in special cases, albumen water and whey.

When this can be retained begin with complete foods as follows:-

No. I Diet.

Milk about four pints in twenty-four hours.

For infants during the first six weeks of life 2 water and I milk.

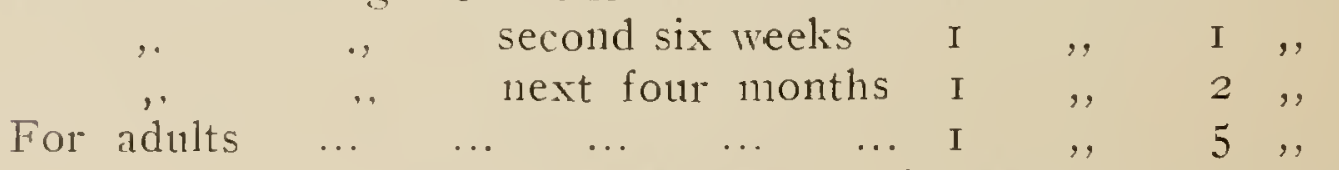

If curds are formed add barley water, rice water, arrowroot water, aerated water or lime water. Add sodium bicarbonate, ro grains $(0.65$ grm. $)$ to the pint of mill.

The milk may be boiled or prepeptonized.

Allenbury's food may be given. Vegetable juices, as lemon squash, lime juice, \&c.

N'asal feeding may be required in some cases.

No. 2 Dict.

Milk, two pints daily with arrowroot, cornflour, rice, tapioca and bread. Egy albumen, cream and butter. Stewed apples, fresh fruit, tea and weak coffee, pounded raw meat. Beef tea and soups. 
Vo. 3 Diet.

No. 2 diet plus fish, vegetables, raw oysters, sweetbread, tripe.

Vo. 4 Diet.

No. 3 diet plus meat, as pigeon, chicken, mutton, beef, and rarely pork.

\section{Yomiting.}

Provide digestible food. Reduce the quantity. Feed per rectum. Apply a mustard poultice over the stomach. Wash the stomach with stomach tube and sodium bicarbonate.

\section{Diarrhœa.}

Examine the stools for foulness, undigested food, excessive putrifaction. worms' eggs, parasites and treat accordingly. Salol is always good.

\section{Constipation.}

Give a mild laxative.

Enemata are useful.

Petroleum jelly is good.

Give--

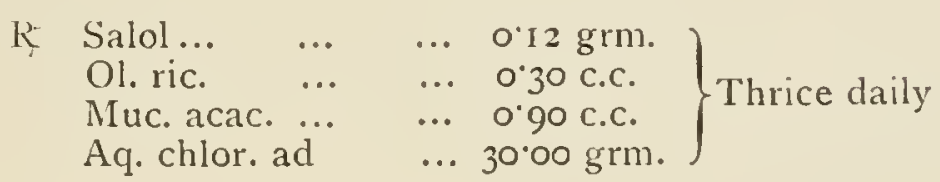

\section{Pure Air.}

Open air treatment is always good.

Prevent draughts.

Skilful nursing is required.

\section{Maintenance of Oxygen for the Blood.}

\section{Keep the air passages open.}

Use the steam jet when there is laryngeal obstruction.

A cold douche or bath is good with pulmonary collapse with children. Tracheotomy may be necessary.

\section{Sweating.}

This is of minor importance, but the patient should be washed down with tepid water when the perspiration is finished and chills prevented by suitable clothing.

When there is a persistent dry skin with a high temperature then induce sweating by hot drinks, blankets, hot bottles, \&c.

\section{Renal Secretion.}

Apply large poultices over the loins every four hours.

Dry cupping can be tried but it is less effective.

Give fluids in sufficiency. Salines per rectum, I to 2 pints each twelve hours. If children, seat them in a hot bath. If adults. foment the abdomen. Test the bladder with a catheter.

For Hæmoglobinuria, see p. 380. 


\section{Rest and Sleep.}

Exclude noise. Have a dull light in the evening.

For insomnia give phenacetin $0^{\circ} 30$ to 0.60 grm. at eight o'clock, or

$$
\begin{array}{lllll}
\text { R Pot. brom. ... } & \ldots & \text { I } & \text { grm. } \\
\text { Chlor. hydrat. } & \ldots & \text { I } & " \\
\text { Aq. ment. pip. ad } & \ldots & 30 & , "
\end{array}
$$

For a child, is to $30 \mathrm{grm}$; for an adult, 30 to $60 \mathrm{grm}$. at eight o'clock.

Trional is useful, I to I.5 grm. at eight o'clock.

Morphia may be necessary at times but it is often followed by depression.

\section{Control Pain.}

Give aspirin, 030 to I grm. every six hours; or opium, $0^{\circ} 02$ grm. every six hours.

\section{Pyrexia.}

If the temperature is above $103^{\circ} \mathrm{F}$. reduce it.

If it is above $106^{\circ} \mathrm{F}$. reduce it immediately.

Keep the wards cool for typhoid, warm for measles, diphtheria and whooping-cough.

Give-

$$
\begin{gathered}
\text { R7 Quin. hydrochlor. } \ldots .0 \% 30-\text { I.00 grm. } \\
\text { Phenacetin .. } \\
\text { Thrice daily. }
\end{gathered}
$$

Hydrotherapy.

Sponge the whole body with hot water in four minutes to dilate the blood-ressels.

Or a tepid sponge in fifteen minutes.

Or a cold pack twenty minutes. This is more active and is used at once in high temperatures or when sponging is useless.

Or a continnons pack. The sheet is kept moistened with water at $90^{\circ} \mathrm{F}$. for several hours daily.

Mackintosh baths can be given. Ice bags can be applied.

Children and the aged do not stand baths well owing to the tendency to cardiac weakness.

As a last resort some give pilocarpine nitrate subcutaneously.

\section{Depress the Head.}

Avoid the elevation of the head. It not only causes muscular stress but as the brain is not well supplied with blood owing to grarity and heart weakness, depression, fainting and syncope may ensue.

\section{Sustain the Heart.}

This should be done with digitalis, strophanthus, strychnine, camphor and alcohol.

Raise the foot of the bed. Give salines. Hot bottles.

Some onit digitalis and strophanthus.

\section{Watch the Heart during Convalescence.}

After hamoglobinuria, beriberi, typhoid, diphtheria, \&c., sudden heart failure may occur. 
Watch the rate and rhythm, a weak first sound at the apex and an accentuated second sound in the aortic area.

Exercises should be very gradual. The blood count must be over $3: \frac{1}{2}$ millions per c.mm.

\section{EMBATMING.}

In order to preserve bodies for autopsy inject 300 c.c. of 5 per cent. solution of formalin. The fluid can be injected into the large arteries. e.g.. the femoral and brachial towards the extremities, the common carotid in both directions and the cavities and organs by trocar and cannula.

A more elaborate method is as follows:-

Prepare io litres of a io per cent. solution of mercuric chloride. Expose the abdominal aorta and hoth iliac arteries.

Incise the aorta and tie a large cannula and syringe in the opening. Inject 3 litres into the upper part, slowly and resting when the pressure is great. Ligature both sides of the opening.

Inject I litre into each leg through the common iliac artery.

Ligature the mesentery and intestines from the jejunum to the sigmoid, divide and remove them. Open and wash then. Put into them a I per cent. solution of mercuric perchloride, replace them wrapped in sublimate wool, or better, cremate the lot.

Wash the stomach, duodentum and rectum in the same way and pack with sublimate wool. Treat in the same way the bladder. vagina, external ear, nostrils, \&c. Clean out the abdominal carity, dry it and sew up the incision.

Wipe the surface of the body with the same solution and dry it (the hair excepted).

Some also inject into the axillary and carotid arteries.

\section{SOME IIINTS ON TROPICAL SURGERY'.}

All surgical instruments and appliances must be of the best quality obtainable. People at lome sometimes send out those goods not readily disposed of at home, much to the chagrin of the tropical surgeon abroad. It is awkward indeed, if when attempting to remove a stone from the bladder, one of the jaws of the instrument breaks off or bends considerably.

Vulcanite and gum-elastic goods should be avoided. Rubber rapidly perishes, but if kept in water in a dark place it will last longer. Rubber gloves can be kept in a glass jar, in powder, well stoppered. Catheters should be rubber or metal, preferably the latter. Rubber tourniquets are often useless when most urgently needed. Good metal ones are now made.

Syringes slould be all metal or all glass. If they are made of two or more kinds of material, the unequal alternate contraction and expansion will cause fracture of the instrument. All needles should be of irido-platinum. They keep their points well and do not become oxidized.

Microscopic slides should be of second-class quality, as the best quickly "fog" and are useless. The cover-slips should be of the best quality.

Take duplicates of fragile instruments likely to be used by native 
assistants, as these latter are usually careless, and fresh supplies of instruments may require months to obtain.

All metal goods should be well plated, vaselined, and well packed in a tin-lined soldered case for dispatch. The Customs anthorities usually open them and leave them open, but protection has been afforded for the ocean passagc. "Air-tight" boxes are not usually air-tight, air escapes about the fastenings owing to expansion from heat, and is ustrally reabsorbed in a moist condition when the atmosphere becomes cool. All cases should be tin-lined and well soldered.

T'elvet-lined cases of instruments are only welcomed by cockroaches and other insects.

The anæsthetic of choice is chloroform. Although given by native assistants over thousands of administrations very few accidents occur. Ether is nsed at the Ancon Hospital, Panama, a fresh small tin being opened for each case. Tropical workers as a rule find that ether evaporates too quickly. Chloroform should be dispatched in glass bottles. with glass stoppers well sealed. Cork perishes, and unsealed glass stoppers become loose in transit.

Intraspinal anasthesia is coming to the fore. Boyd and Young report on over 6.000 cases at the Santo Tomas Hospital. Panama Canal Zone. It was used for all operations below the umbilicus and sometimes for the upper abdomen. The mixture was Stovaine 0I grm., Sodium chloride, O. I grm. in I c.c. of distilled water. Five c.c. were usually given for operations of half an hour or less. Ansesthesia is complete in three and a half minutes and lasts fifty to sixty minutes. It was preceded by an injection of morphia. Cases showing a low blood-pressure from shock following loss of blood were excluded.

The sepsis of wounds is much more prevalent in the Tropics than in Europe. as conditions are much less perfect and operations are carried out in theatres open to air currents.

The treatment of fractures is difficult. The patients are usually restless, the skin is moist, and may be shed in cases requiring prolonged extension. Adhesive plaster is seldom adhesive, and plaster-of-Paris scldom hardens sufficiently.

One is often consulted as to when the spleen should be removed. The writer follows Cantlie's list:-

(I) Rupture of the spleen.

(2) Primary hydatids.

(3) ,, malignant growth

(4) ., abscess.

(5) $\because$ massive tuberculosis of the spleen.

(6) Chronic splenic anæmia.

( $\mathrm{T}$ ) .. hamolytic (splenic) jaundice.

(S) Banti's disease.

SOME COMIARATIVE NORMAT TEMPER.ITLRES.

\begin{tabular}{|c|c|c|c|c|}
\hline Man & & $37^{\circ} 0^{\circ} \mathrm{C}$. & Covv & $39^{\circ} 5^{\circ} \mathrm{C}$ \\
\hline Cat & $\ldots$ & $38 \cdot 80 \mathrm{C}$. & Sheep .. & ‥ $39^{\circ} 60^{\circ} \mathrm{C}$ \\
\hline Dog & $\ldots$ & $39^{\circ} 2^{\circ} \mathrm{C}$. & Pigeon... & $42.00 \mathrm{C}$ \\
\hline Goat & & $39^{\circ} 3^{\circ} \mathrm{C}$. & Duck & $42 \cdot 2^{\circ} \mathrm{C}$ \\
\hline abbit & 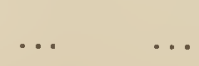 & $39^{\circ} 5^{\circ} \mathrm{C}$. & Chicken & $42^{\circ} 5^{\circ}$ \\
\hline
\end{tabular}




\section{PRINCIPAL REFERENCES.}

"A Manual of Tropical Medicine" ...

"Tropical Diseases" ... ... ...

"Tropical Medicine and Hygiene" ...

"The Principles of Hygiene"

" Laboratory Studies in Tropical Medicine,

"Yellow Fever and its Prevention"

"Sleeping Sickness in the Island of Principe",

"Trypanosomes and Trypanosomiasis" ...

"Venoms, Venomous Animals, \&c."

"Medical Entomology"

"Recent Advances in Tropical Medicine" Balfour and Archibald.

"Review of Recent Advances in Tropical $\begin{array}{llllll}\text { Medicine } " & \ldots & \ldots & \ldots & \ldots & \ldots\end{array}$

"Diseases of China", $\ldots \quad \ldots \quad \ldots \quad \ldots \quad \ldots$

"Amœbic Dysentery" ... $\quad \ldots \quad \ldots \quad \ldots$

"Sprue and its Treatment" $\ldots \quad \ldots \quad \ldots$

"Beriberi" ".. .. $\quad$ ". ..

"Human Intestinal Protozoa" $\quad \ldots \quad \ldots$ Wenyon and O'Connor.

"Researches on Egyptian Bilharziasis" ... Leiper.

"The Animal Parasites of Man" ... ... Fantham, Theobald.

"Gnats or Mosquitoes" $\quad \ldots \quad \ldots \quad \ldots$ Giles.

"Hygiene Coloniale" ... $\quad . . \quad \ldots \quad \ldots$ Alliott, Clarac, \&c.

"The Etiology of Relapse in Malarial Fever" James.

"Notice sur les Glossines ou Tsetses" ... Hegh.

"Infant Feeding in the Tropics" ... ... Deeks.

"Manual of Bacteriology" ... $\quad . . \quad$... Hewlett.

"Hygiene and Public Health" $\quad \ldots \quad \ldots$ Whitelegge and Newman.

"Diseases of the Skin" $\quad \ldots \quad \ldots \quad \ldots$ Morris.

$\begin{array}{lllllll}\text { "Nutrition " } & \ldots & \ldots & \ldots & \ldots & \ldots & \text { Sohn. }\end{array}$

"Diseases of the Eye" $\quad \ldots \quad \ldots \quad \ldots$ May and Worth.

"The Extra-Pharmacopœia" $\quad \ldots \quad \ldots$ Westcott.

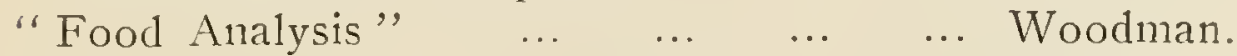

"Food and Hygiene" $\ldots \quad \ldots \quad \ldots \quad \ldots$ Tibbles.

"Reports of the Sleeping Sickness Com-

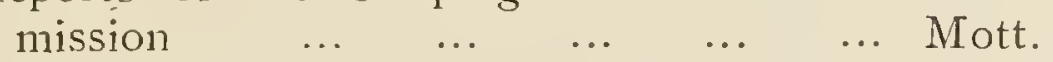

"The Classification and Pathology of Beri-

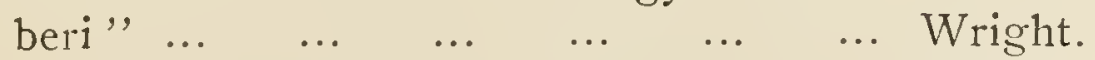

"Album of Photographs." B.M.A. Indian Section. Annals of Tropical Medicine and Parasitology.

Tropical Diseases Bullctin.

Transactions of the Society of Tropical Medicine and Hygienc. Journal of Tropical Medicine and Hygiene. 


\section{NDEX.}

ABDomes in typhoid fever in the tropics, 217

Abscess, evacuation, in filarial lymphangitis, $28 \mathrm{I}$

Abscesses, filarial, 282 site of, 282

Abyssinian well for shallow water in tropics, $55^{1}$

Acanthokeratodermia, 450

Acariasis, infected barley causing, 459

- simptoms, 450

Acarina, order of, ticks, largest specimens of, ro

Acclimatization theory of protection formed by vaccines and sera, 600

Acid-fast method for staining microorganisms, 614

Ackec poisoning, ætiology, 455

- nigh mortality in, 456

pathology, $45^{6}$

prophylaxis, 456

symptomatology, 455

(vomiting sickness of Jamaica), 455

Acne-like cruption, produced by sparganum, 261

Acnitis, treatment, 460

Acrodermatitis chronica atrophicans, 460 vesiculosa tropica, 460

Acrodermatosis, 460

Actinomyces, diagnosis of, 629

Arlenalin in sprue, 360

injections in snake bite, 445

Aeration of waters, nethods of estimating, 661

Africa, poisonous snakes in, 431

West, mortality from yellow fever iil, 370

- lirest Coast of, loss of memory in, 539

After cataract, discission for, 522

Agar-agar, preparation of, 622

Agarsidre (tick), spirochates of Miana fever, \&c., transmitted by, 19

Agglutinins, action of, 600

- in snake venom, 440

Aglypha, 423

Ainhum, definition, 412

pathology, 412

symptomatology, +13

Air, hot, as neans of disinfection, 593

"Alastrim" and chicken-pox, differentiation between, 401

- symptomatology, 403

Albinism, 461

Alcohol, effect of, in Tropics, 450 medicinal value of, 583

Alcoholic drinks, avoidance in sprue, 359 stimulants, strong, aroidance in vellow fever, 37 I
Algre, growth of, in standing water, method of removal, 547

Alimentary tract of fleas, 178

Alkalies, effect in sprue, 360

Alopecia, 469 treatment, 470

- - by external irritants, 470 by nourishing ointment, 470

Alum, rectal injections in oxyuriasis, 313 - stick in trachoma, 515

Amblyopia, quinine and malarial, differentiation between, 525

- toxic, 5 Io

Ammonia, albuminoid, in water, method of estimating, 660

— and ether in snake bite, $4+5$

free and saline, in water, method of estimating, 659

Ammonias in sewage, detection of, method, 662

Ammonium chloride in treatment of tapeworms, 260

Amocbiasis, urinary, t1 $^{2}$

Amok, 407

- exciting causes, 407

Amputation in treatment of Madura foot, 482

Anamia and intestinal irritation in Hymenolepis nana infection, 263

- grave, in blackwater fever, 387

in African trypanosomiasis, 142

in verruca peruana (severe type), 364

severe, with fever produced by Dibothriocephalus latus, 260

Anaerobic cultures, 624

Anresthetics in eye diseases, 528

Anderson, effects of hot climates on white men, 538

Anderson's process, sterilization of water by, 556

Anemones, venomous, 448

— sting from, symptoms, 449

- - treatment, 449

Aneurism, late manifestation of yaws, 25

Angio-fibroma contagiosum tropicum, 461

Angiomata, 502

Angio-sarcoma, 502

Animal matter in water, danger from, $55^{2}$

Animals, epidemic gangrenous rectitis in, 408

- living, examination of, 573

Ankylostoma duodenale, points of difference between, and the Necator americanus, 320

Ankylostomes, 3 I 4

Ankylostomiasis, blood examination in, $317-8$ 
Ankylostomiasis, complications, 3 I life-history, 316 prophrlaxis, 318 spread of, factors necessary for, 543 symptomatology, 317 treatment, 318-9

Ankylostomum duodenale, 314-5

- ova of, 637

Anopheles maculipennis, larva of, 295

Anthrax bacillus, diagnosis of, 616

Anti-bacterial action, how produced, 164

Antibactericidal substances, probability. of, in snake venom, 441

Antibodies, 600

Antidote, or retention theory of protection afforded by sera and vaccines, 600

Antidysenteric serum, summarizing of work by Flexner, 201

Antifibrin ferment in snake venom, ffl

Antigens, 600

Antihamolysins in snake venom, 441

Antimony jn kala-azar, 61

- preparations in treatment of African tripanosomiasis, i $4 \mathrm{~S}$

Antitoric sera, 599

Antitoxins, 163

Antityphoid vaccine, effect on case incidence and case mortality of typhoid fever, 601

of protection afforded by, length

Antivenene in snake bite, 446

Ants, venom of, simptoms, 452

Anuria in yellow fever, treatment, 371

Appetite perverted in cerebral stage of African trypanosomiasis, 145

Archibald's fever, definition, 397

_ symptomatology, 397

Archibald and Balfour, bacteriological examination of river and well water in Khartoum, 6.42, 643

Archoplasm in protozoa, 3

Argas persicus (tick) attacks poultry and humans, 2 I

Arm, elephantiasis of, 293

Arrow poisons, treatment, 457

Arsenic emetic in treatment of African trypanosomiasis, 149

— in afebrile splenomegaly, 417

in lichen planus, 475

in onyalai, 417

in psoriasis, 487

Arsenical poisoning in atiology of beriberi, 337

- preparations in treatment of African trypanosomiasis, 148

treatment in rat-bite fever, 398

Artesian wells, 551

Arran races, vegetarianism of, 566

Ascariasis, definition, 309 geographical distribution, 309 parasite causing, 309

symptomatology, 3 I I treatment, $31 \mathrm{I}$

Ascaris lumbricoides, examination of frces for eggs of, 3 I I

\footnotetext{
- life-history, 309, $31 \mathrm{I}$

— ova of, 637
}

Ascirasite causing ascariasis, 300

Ascites, 'chylosus, 284

- in filariasis, 279
Asia, poisonous snakes in, 428

Aspergillina, diagnosis of, 630

Aspergillosis, 461

- ocular, due to fungi, 511

Aspirin in filarial lymphangitis, 28 I

- in phlcbotomus fever, 36 I in yellow fever, 371

Asthenia in African trypanosomiasis, I42

Asthenopsia, sponge-fishers', 509

Atoryl in pellagra, 336

- in treatment of African trypanosomiasis, $1+8$

Atripliasmus, 454

- symptoms, 454

Antigens, 600

Auchmeromyia luteola, larvæ of, causing myiasis, $4 S_{3}$

Auto-intoxication in actiology of beriberi, 340

Aviation, dangers of atmospheric changes in, 532

BABESIA hominis, 6

Bacilli, 162

- capsulated, 6 is

characters of, 162

Bacillus asiaticus fever, symptomatology,

397

- - botulinus, diagnosis of, 617

coli, diagnosis of, 618 in water, quantity of, testify-

ing to measure of purity of, 652

Brition by method

of British sanitarians, 65 I

diphtheriae, diagnosis of, 619

leprae, 180

- diagnosis of, 616

mallei, diagnosis of, 62 I

odematis maligni (Bacillus septi-

cus), diagnosis of, 617.

of timothy grass, diagnosis of, 616

paratyphosus A, geographical distri-

bution, 215

baratyphosus B, geographical distri-

bution, 2 It

- pestis, behaviour of, 168

- carried by fleas, 177

- characters, 168 cultures of, 170 diagnosis of, 6 in habitat, 168

pseudo-tuberculosis of Pfeiffer, dia-

gnosis of, 616

- pyocyaneus, diagnosis of, 620

typhosus, diagnosis of, 619

vibrio in flies, transmission of

cholera by, 206

welchii, diagnosis of, 617

Bacteria, death of, how produced, 163 demonstration of, in laboratory

work, 626

detection in faces, 635

diseases due to, 161, 162

distribution, 164

entrance into tissues, 165

general features of, 162

in aetiology of pellagra, 331

in waters, examination by methods

of British sanitarians, $650,65 \mathrm{I}$

infection by, conditions necessary for, 164

life of, conditons necessary for, 163 
Bacteria, mellods of locomotion, 162 non-pathogenic, in water cause of muco-enteritis, 553

- physiology, 162, 163

- reproduction by simple fission, 162 by sporc formation, 162

resistant to effects of sunlight, 645

shape of, 162

staining for, 604

susceptible to effects of sunlight, 645

Bacterial activity, products of, 163 cmulsions, 599

Bacteriolysins, 164

Balantidium coli, $7,40,45,40$

- _ parasite of ciliary dysentery, 44 minutum, 7, 46

Balfour and Archibald, bacteriological examination of river and well water in Khartoum, 642, 643

Balkan War (1913), vaccination against cholera during, results, 2 I 3

Bamana flour in infant feeding, 582

Bandaging in elephantiasis of legs, 286

Barcoo rot, 461

Basic stains, 604

Beans causing favism, 389

Becf broth as nutrient medium for cultures, preparation of, 621

Bees and wasps, sting of, symptoms, 452

- - - treatment, $45^{2}$

Beetles, venom of, 45

Belladonna, application in climatic bubo, 411

Benzoic acid and sodium benzoate as preservative, test for, 577

Beriberi, xtiology, 337

- atrophic, dry or paralytic variety,

343,344

deficiency disease, 337

definition, 336

diagnosis, 347

dietetic causes of, 571

geographical distribution, 336-7

history, 337

hypertrophic, wet or odematous

varicty, $345^{-6}$

- infantile varicty, 347

inixed variety, 346-7

odematous variety, 344

pathology, 34 I

post-mortem examination, 34 I, 343

prognosis, 349

resemblances to and differences from

epidemic dropsy, 348-9

- spread and onset of, prevention, 540

trcatment, 349-50

varieties, 343

wet, acute, 340

Berkefeld filter, 558

Beta-naphthol treatment of ankylostomiasis, 310

l3everages, kinds of, causing pain in sprue, 357

13 ig heel, course of, 412 geograplical distribution, 412

Bileatment, 412

Bile, leaking tumour discharging fluid giving reaction of, 501
Bilharzia Mission (1915), conclusions as to prevention and treatment of schistosomiasis based oll, 249, 250

Bilharziasis, transmission of molluscs esscntial for, 543

Binary fission, asexual method of reproduction in protozoa, 3

Biotripsis (life-wear), $46 \mathrm{I}$

Bismuth-milk treatment in amœbic dysentery, 43

iodide (emetine) in amabic dyscntery, 43

Black tongue, 476

Blackwater fever, blue pigment in, 628 from, 370

- - presumed cause of, 6

- sce also Hemoglobinuric fever

Bladdcr, post-mortem appearance in schistosomiasis, 245,246

stricture in schistosomiasis, 248

Blastomycetes, diagnosis of, 63I

Blastomycosis, diagnosis, 462 ocular, due to fungi, 5 I I

symptomatology, 461

treatment, 462

Blindness, 508

causes classified, 508

due to lightning, 510

Blood, acidity of, in cerebral stage of

African trypanosomiasis, I 45

cells, abnormal, 605

_- red, abnormal, 606

- counting of, 606

- - hamophilic, 600

nucleated, 606

transitional, $60_{5}^{5}$ whitc, abnormal, 605

- - counting of, $60 \%$

changes in, 173

- staining for, 604

condition in verruga peruviana, 364

condition in yellow fever, 367

- count, differential normal, results

of, 605

counting of, 606,607

600,610

detection in faces, 635

dust, 605

effects of tropical climates on, 537

examination of, 604

in ankylostomiasis, 3 I 7

films, fixation of, 605

fresh, examination of, for micro-

filarix, 610

- in blackwater fever, 387.

malaria-infected, injections of, re-

sult, 72

malarial parasites in, 72, 73

serum, as nutrient medium, prepara-

spccific gravity of, method of

estimating, 6r2

spectroscopic examination of, 612

state of, in sprue, 357

- tonicity of, 6I3

tion of, 623

Boiling in sterilization of water, 558

Boils, treatment, 462

- tropical, 462 
Bone-marrow in blackwater fever, 386 - microscopical appearance in African trypanosomiasis, I39

Bone sarcoma, cystic (myeloid?), of lower jaw, 499

Bones and joints, how affected in chappa, 413

Boomerang les, atiology, 415 geographical distribution, $4 \mathbf{I}$ j symptomatology, 415

Borax methylene blue for staining malarial parasite in blood, 608

Bore well, drill for producing, $55 \mathrm{I}$

Boric acid and borax as preservative, test for, 578

Bothriocephalus, ova of, $63 \mathrm{~S}$

Botromycosis hominis, 462

Boyces summary of prophylactic measures against yellow fever, 372

Brackish water, ill-effects of, 553

Brain, amnebic suppuration of, 40

- malarial parasites in, manmer of demonstrating in laboratory work, 627 - pernicious manifestations of malaria in, 74

Breast, elephantiasis of, 29r, 293

British sanitarians, methods of examination of bacteria in waters, 651

Bromiclosis, 462

Bronchocele, fibroid, 498

Bubo, climatic, atiology, 410

- bacillus isolated from, 410

- definition, 410

- - geographical distribution, 410

_- pathology, 4 I I

- symptomatology, 4II

—- treatment, 4 I 1 see also Plague bubo

Bucket system and European latrines, $58 S$

Bugrs, venom of 45 I

Building sites, suitable in tropical districts, 535

Burns and scalds of eye, 509

Bush, the, as latrines for natives, 585

Butter, adulteration of, $57 \mathbf{I}$

Butter-milk, 568

Buttock, elephantoid tumour of, $2 \$ 7$

CAcostomy in amobic dysentery, 43

Crecum, section from, in case of amœlic drsentery, 33

Caffeine injections in snake bite, 445

Calabar swellings, definition, 290 geographical distribution, 200

symptomatology, 299

- - S treatment, 300

Calcium carbonate in sprue, 360 - hypochlorite, use in purification of water, 555

- lactate for viper venom, 445

Calomel in oxyuriasis, 3 I 3

Calorie value of foods, 565

Canities, 470

- in young persons, treatment, prescription for, 470

Cantlie, Sir James, nature of climatic bubo, 4 Io

Cantlies treatment of sprue, 359

Carbohydrates, food value of, 563
Carbol fuchsin, use of, as stain, 604

Carbol-thionin for staming malarial parasite in blood, 608

Carbolic acid as disinfectant, 504

Carbon dioxide poisoning in atiology of beriberi, 337

Carbonates, alkaline, excess in water, effects of, 553

Carcinoma, melanotic, 495

- of cervix, 502

- of ovary, 502

of pylorus, 502

Cardiac and rascular tonic, probability of in snake venom, $44 \mathrm{I}$

- insufficiency in yellow fever, treatment, $37 \mathrm{r}$

- symptoms in South American trypanosomiasis, 157

Carrion's fever, see Verruga permiana (severe type)

Cassava plant, varicties of, 564

Castor oil in amœbic dysentery, 42

- in ciliar dysentery (Balantidium coli), 46

C. - seeds, poisoning by, $45+$

Cataract, 509,510

classification, 517

congenital and juvenile, completc, $52 \mathrm{I}$

521

extraction of, complications, 52 I

- operation, 520

in India, 510

lamellar or zonular, 5 is

nomenclature, 517

polar, anterior, or pyramidal, 5 I 8

- posterior, 5 IS

senile, 5 is

- stages in growth of, 519

— tests for time of operation, 519

— treatment by cxtraction, 5I9

traumatic, 521

- uncommon forms of, $; 1 S$

Cataracts, eye complications leading to, 522

Catarrh, intestinal, resulting from Strongyloides stercoralis infection, $27 \mathrm{I}$

Catchment areas for water supply, 547

Cate avoidance of peat in, 547

Caterpillars, venom of, symptoms, 452

Caute- treatment, 452

Cauterization of tropical ulcers. 505

Celluloidin, embedding in, of section of malarial parasites in brain, 627

Centipedes, venom of, mortality in children, $45 \mathrm{I}$

- - - symptoms, 451

Centreatment, $45 \mathrm{I}$

Centrosomes, in protozoa, 3

Ceratophyllus fasciatus, transmission of plague by", 171

Cercomonas hominis, habitat, 5

- vaginalis, habitat, 5

Cerebral stage in African trypanosomiasis, $144-6$

Cerebrospinal system, microscopical appearance in African trypanosomiasis, $13 \mathrm{~S}$

Cesspools in tropics, 589 
Cestoda, classification, $2-8$

life-luistory, 258

morphology, 257

pathogenic to man (human tape-

worms), 257

Cestode, onchosphere of, 637

Cestodes, preservation of, 323

Chappa, pathology, +13

- treatment, +13

Chaulmoorra oil in leprosy, is

Chaulmoogric acid in leprosy, $18 S$

Cheek, epipthelioma of, fungating, papil-

lomatous, 500

- horny growth from, 504

Cheese, whole milk, most nutritious of all foods, 567

Cheilitis exfoliativa, +76

- glandularis, 476

Cheiropompholys, 463

- treatment, 403

Cheloid, 502

- causes of, 502

Chemicals used for testing water suspected of contamination, 553, 554

Chicken-pox common in India, tou

Children, Davainea madagascarensis infection in, 263

- intestinal ulceration and anæemia resulting from infection by, in children, $26_{3}$

- men and women, food necessary for, compared, 564

- of natives, epidemic gangrenous rectitis, in, 408

- very young, alone affected by ponos, 413

fever in, 368

Chlamydophrys stercorea, habitat, 5

Chloasma, causative agents of, 463

_- varieties of, 463

Chloride, cold, in snalie bite, $\$+6$

Chlorine as disinfectant for horses, 590

- in water, detection and estimation

of, method, 655

- sterilization of water by, 557

Cholera, xtiology of, 205

- carriers of, 213

_ _ transmission of cholera by, 206

collapse of, treatment, 210,211

complications of, 208

definition of, 203

development of, climatic conditions

best suitable for, $\$ 39$

- diagnosis of, $208-9$

- - bacteriological, 200

epidemics in Europe, 203-4

- relation of, to wars, 204-j

geographical distribution of, 203

modes of infection, 205

or plaguc on infected ships, quaran-

tine measures respecting, 507

- organisms, removal from water,

chemical process for, 556

- pathology of, 206

- post-mortem appearances, 206-7

- prophylaxis against, 212

spirilla, 205

symptomatology of, 207-S

treatment, 200,210

-.. _ by Rogers' method, 210
Cholera, treatment, indications for, 209 old and new methods compared, 211

- vaccination against, results, 212, 213 vibrio in milk, tests for, 5\%o vomiting in, treatment, 210

Chondroma, 505

Chondrosarcoma of humerus, 498

Chromidiosomes in protozoa, 3

Chrysarobin ointment, application in various forms of tinea (ringworm), 490 , $49 \mathrm{I}, 493,494$

Chrysomyia macellaria (screw-worm), larva of, causing myiasis, 482

Chrysops dimidiata and silacea, carrier of Loa loa,

Chyle, double conjunctivitis discharging, associated with filaria infection, $29+$

Chylocele, $2 S_{t}$

Chylous extravasations, 282,283

- in filariasis, 279

Chyluria and lymphuria, symptomatology, $2 S_{3}$

- - treatment, $2 \delta_{t}$

in filariasis, 279

Ciliata, 5,7 (infusoria), 5

Cimex lectularius (bed-bug), carrier of parasite of European relapsing fever, 10

Cirrhosis, biliary, pathology, flo

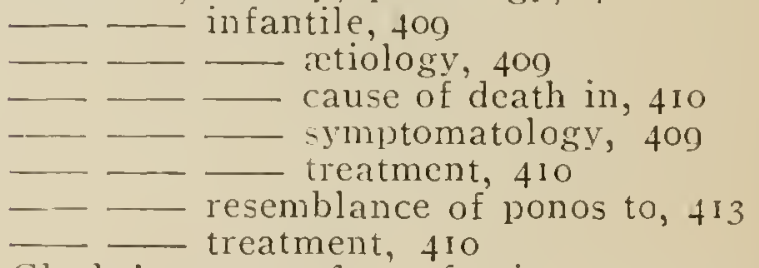

Clarke's process for softening water, 555

Clayton apparatus, use of, for extensive fumigations with sulplur anhydride and sulphurous acid gas, 595

Clemesha, Major, methods of bacteriological examination of waters in India, 645,646

- classification of river waters for drinking purposes, 649, 650

Climate, definition, 531 effect of, on skin, $4=8$

- relation to tropical diseases, 339

Clonorchis simensis, ova of, 637

Coast memory, 539

Cobra seizing prey, photograph of, 424

Cocci, sliape of, 162

Coccidium oviformis, diseases caused by, 6

- liabitat, 6

Cocoa, qualities of, $5 s_{3}$

( od-liver oil in onyalai, 417

Coffee, qualities of, $582-3$

Collargol in five-day fever, 398 - solutions of, in trcatment of African trypanosomiasis, I 49

Colon, dysenteric ulceration of, healing, 35

Colubridx, 423 orders of 423

Colubrine venom (snake bite), postmortem, $4+2$

Coma in ackee poisoning, 456 
Comma bacillus, cause of cholera, 205 Koch's, 621 differentiation from Fink-

ler-Prior spirillum (Vibrio proteus), 62 I

Compound tablet, sterilization of water by, 557 .

Conjunctivitis astivalis (spring catarrh),

516 and keratitis, phlyctenular, 516

- - treatment, 516

astringent remedies for, 526, 527

catarrhalis, 511

double, discharging chyle, associated

with filaria infection, 294

gonorrhoeal, 5 I0, 512

- - resulting from unclcan habits, 510

Connective tissue tumours, benign, 502

Conalignant, 502

Continents, new means of intercourse between, opening of, increasing study of transmission of communicable diseases, $5+3$

Convulsions in ackee poisoning, 456

Coolie itch, treatment, 403

Copper in water, method of estimating, 655

- sulphate, test for, 570

- _ pencil in trachoma, 5 I4

Copra itch, 463

Corals, venomous, $4+8$

sting from, symptoms, $4+0$

Cordylobia anthropluaga, larva of, causing myiasis, 482

Cordylobiasis, 464

Cormea, abrasions of, $; 00$

Countries, new means of intercourse between, opening of, increasing study of transmission of communicable diseases, 543

Cover slips, method of cleaning, 6ot

Cox's method of treatment of cholera, 211

Craw craw (nodular dermatitis), treatment, 464

Creeping disease or cruption, difficulty of treatment, 464

Crocidura cocrulea (Indian musk rat), resistent to plague, $17 \mathrm{I}$

Crotalina, $426,+31$

in America, 435

Crotaline venom (snake bite), symptoms, $-43$

Crotalus confluentus (Pacific or mottled rattlesnake), 429

- terrificus (dog-faced rattlesnake), 428

Culex fatigans, breeding places, 295

_ _ carrier of filaria, 294

_- Larva and pupae of, 294, 295

- morphology, 204

- - transmission of dengue by, 376

Cultures, differentiating growth, 624

preparation of, 621

Current steam disinfector, 593

Cycloplegics in cye diseases, 528

Cyclops quadricornis, intermediate host of Dracunculus medinemsis (guineaworm), 301

Cyllin as disinfectant, 594

Cytolysins, action of, 600

in snake venom, four groups of, 440
DANIELS, diagrosis of microfilaria, 277

tests for bacteriological examination of water, $6 t_{2}$

Darling, discovery of Histoplasma capsulatum by, 6

Davainea madagascaricnsis, infection in children, 263

Dengue, atiology, 377

- definition, 376

diagnosis, 378

_ _ of phlebotomus fever from, 36 I

— of yellow fever from, 370

geographical distribution, 370

incubation period, 377

insects transmitting, 376 .

Ieucopenia in, 377

seasonal prevalence and reneral

habitat of, $54^{1}$

sequelee and complications, 3-8

symptomatology, 377

treatment, 378

Dermatitis bullosa plantaris, 464

_- difficulty in treatment, $46_{5}$

cupoliformis, difficulty of treatment,

405

405 exfoliativa, local applications in,

465 (pityriasis rubra), treatment,

- macrogyrata, 460

nodosa rubra, treatment, 406

papillaris capillitii, 470

rimosa of the toes, treatment, 460

solaris, 460

venenata, classification, 460

- treatment, 460

vesicular, 505

Dermatobia cyaniventris, larva of, causing myiasis, 482

Dermatophiliasis, 466

symptoms, 407

treatment, 467

Diamond drill, Sullivan's, for producing bore well, 551

Diarrhoa, chylous and Iymph, 284

- dysenteric, infantile, symptomato-

logy of, 199

__ hill, retiology, 35 I

- - definition, $35 \mathrm{I}$

- diagnosis of sprue from, 358

- pathology, $35 \mathrm{I}$

- symptoms, 352 theories of, $35 \mathrm{I}$ treatment, 352

infantile, treatment of, 202

Dibothriocephalus latus (human tapeworm), 260

- - morphology, 266, $26 \mathrm{I}$

fever, 260

Diet, articles of, causing pain in sprue, 357

average, for male not engaged in

hard work, 564

in pellagra, 336

in sprue, 358,350

- articles to be avoided, 359

in typhoid fever in the tropics,

222

in yellow ferer, 37 r

of natives, 565 
Diet of natives of India and of Europeans contrasted, in relation to incidence of typhoid fcver, 215 Voit's standard, 565

- classification, 505

Digestion in cerebral stage of African trypanosomiasis, $1+5$

Digitalis, tincture of, in heat stroke, $39+$

Dionin in iritis, keratitis, glaucoma and corneal ulcers, 527

Diphtheria, antitoxic serum in, value, 599

Diplococci, arrangement, 162

Diplococus preumonix, diagnosis of, 620

- stain, method of finding, 615

Dipylidium caninum, parasite found in dogs and cats, 262

Diseases, general, ocular manifestations of, 522

Disinfectants, chemical, 595

- characters of, 503-t

- in eye diseases, 527

Disinfection, 502

- chief means of, 592

Distillation of water, 558

Dog, Schistosoma japonicum from, 251

Dogs and cats, infection from, to man of Dipylidium caninum, 263

Double continued fever, low mortality in, 390

- symptonatology, 390

Dover's powder in dengue, 378

Dracontiasis, geographical distribution, 301

parasite causing, 301

— pathological lesions, 304

-.. prophylaxis against, 305

—_ symptomatology, 304

treatment, $304-5$

Dracunculus medinensis (guinea-rorm), host and intermediate host of, 301

- life-history, 301, 304

— morphology, 301, 302, 303

- parasite causing clracontiasis,

301

Drewer's ointment, in psoriasis, formula for, 487

Drinking water, boiling and filtration, in prophylaxis against dracontiasis, 305

- distillation from salt water, 544 649

Drinks in tropics, 522

Dropsy, epidemic, atiology, 350

- definition, 350

— diagnosis, 351

- geographical distribution, 350

from beriberi, 348-9

- - symptoms, 350-1

D- treatment, 35 I

Drug-taliers, Whitmore's disease common in, 415

Drugs in typhoid fever in the tropics, 222 - useless in hyperpyrexial fever, 395

Dusting porder, antiseptic, application, in filarial lymphangiectasis, 282

Dysenteries (the), causes, 20

classification, 20

history, 27

- of Ancon hospital, classification, 29

- protozoal, 29
Disenterjes, pscudo-dysenteries, most common in temperate climater, 29

- verminous, 20

Dysentery, amobic, 146

— - acute, symptomatology, 36

- chronic, 30

complication of African try-

panosomiasis, I 46

- complications in, $\mathrm{fI}^{\mathrm{I}}$

definition, 30

- diagnosis, 42 geographical distribution, 30

$3+, 35$

- 2 lesions in great intestine, 36

liver abscess in, 50

method of search for cysts in

stools of patient suffering from, 4 I

parasite of, 30

- - pathology, 32 macroscopic, 34

- - pathology, 32 macroscopic, 34

- section from intestine in case

of, showing ulcer of Harris, 38

treatment, 42

antitoxic serum in, value, 599

bacillary, 29

— - acute, symptomatology of, 198

_ and amobic, differentiation be-

tween, 42

- — atiology of, 196

- chronic attacks, treatment of,

202

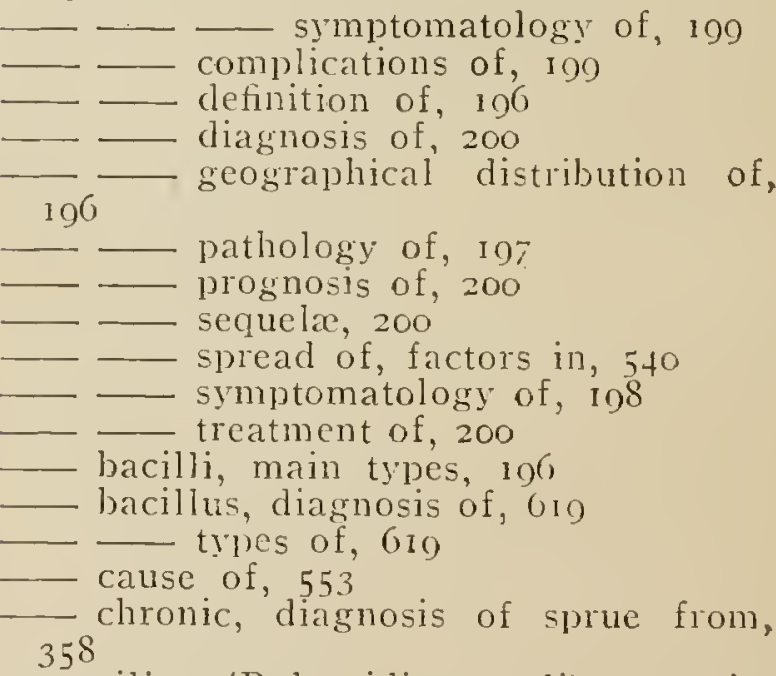

of, 44

ment, 46

- mortality from, 44

— (Tetramitus mesnili), 47

spread of, factors in, 5 to

vaccines, bacterial, 602

Dysidrosis exfoliativa, 467

EARTH, bacteriology of, 640

Echinococcus granulosus causing cystic

form of hydatid disease, 207

—- dangers of, to man, 268

- - life-cycle, 267

- morphology, 267

pathogenicity, 268 
Echinococcus multilocularis, geographical distribution, 268

— morphology, 268

hydatid disease, 268

Eclipse blindness, 500

Eczema, definition, 467

- symptomatology, 467

treatment, 468

- varieties, 468

Egypt, percentage of population infected with Schistosoma hamatobium, 242

Ehrlich's side-chain theory of protection afforded by sera and vaccines, 601

Elapinze, $423,428,43 \mathrm{I}$

- in America, 434

in Australia, 433

Elapine venom (snake-bite), symptoms, 442

Elaps fulvius (harlequin or coral snake), 427

Elastorrhexis or pseudo-exanthoma, 502

Electrical methods of sterilizing water, 558

Electricity and iodides in dengue, 379

Electrolysis in hirsuties, 47 I

Elephantiasis, $28_{4}$

arabum, reasons why due to Filaria bancrofti, $2 S 7,2 S S$

- causes of, 279,280

of arm, 293

of breast, 291, 293

of legs, $2 S_{4}-287$

- after operation 286

- before operation, 285

- symptomatology, 2S $4-2 S_{7}, 293$

- treatment, 286

of localized areas, 293

of prepuce and legs, 288

of scalp, 293

of scrotum, 289,290

tion, 292

- - operation for removal of mass,

291

- symptomatology, 290

- ireatment, 291

of vulva, 292

post-mortem findings, 280

Elephantoid tumour of buttock, 287

Emaciation in cerebral stage of African trypanosomiasis, 145

Emetine in amobic dysentery, 43

coli), 46

in liver abscess, 54

- in sprue, 360

Endemic diseases, 543

Endo-ectothrix tinea, 489

Endothrix tinea, 489 .

histolytica in tissues, 37

- tetragena of amœbic dysentery, lifehistory, 3 I

binary fission, 3 I

gemmation, 3 I

spore formation, 3 I reproduction by reproduction by reproduction by parasite of amobic dysentery.
Enteritidis group of bacilli, diagnosis of, 6 I 8

Eosin-azur for staining malarial parasite in blood, 608

use of, as stain, 604

Eosinophiles, discases during which in creased, 605

- percentage in normal blood count, 605

Epidemic diseases, sporadic, $5+3$

Epileptiform, a complication of African trypanosomiasis, $1+6$

Epinys norvegicus (Norway rat), plague in, 170

- rattus (brown rat), plague in, i 70

Epithelial tumours, benign, 495

- malignant, 495

Epithelioma of check, fungating papillomatolss, 500

Epitheliosis desquamativa (Samoan eye disease), 516

Ergotism, 453

causes of, 572

Erysipelas, treatment, 468

Erythasma, 468

Erythema of verruca peruviana, 364 solare, 468

Erythematous ermptions in African trypanosomiasis, 1 to

Erythropsia, 500

Espundia, 68, 504. See also Leishmaniasis, naso-oral and oro-pharyngeal

Eucalyptus treatment of ankylostomiasis, 3 I9

Europe, cholera epidemics, 203-4

Europeans first entering Tropics, liability to typhoid fever, 215

- in India, diet of, in relation to incidence of typhoid fever, 2 I5

- pathological cffects of hot climates on, 538

physiological cfiects of hot climates on, 537

Excreta, chemical method in disposal of, 588,590

- disposal of, 500

Exhaustion (pabulum) theory of protection afforded by sera and vaccines, 600

Eye conditions, local, resulting in blindness, 5 II

contusions and concussion injuries of, 500

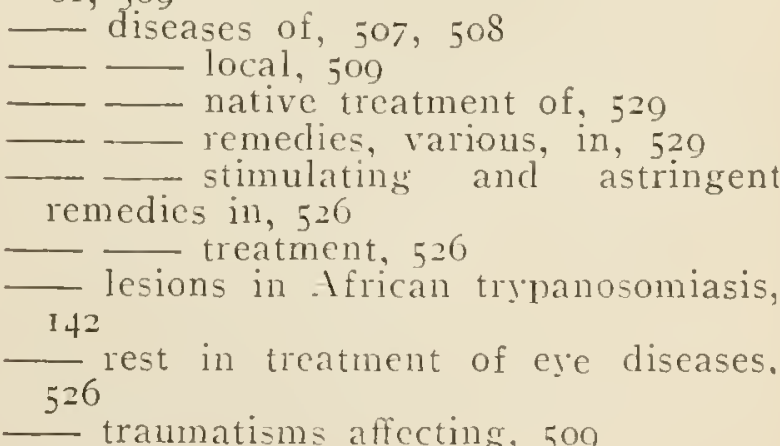

F ECAL carricrs of typhoid fever, 2 I 4 excessive, 645

Freces and water, bacteriology during monsoon weather, $0_{4} 6$ 
Frecs examination for eggs of Ascaris lumbricoides, 311

—_ diagnosis of diseases from, $0_{32}$ macroscopic, 632

- microscopic, 634

- helmintl ova in, prescrvation of, 323 648

- in sprue, 357

- paleness of, causes, 358

Fat, deficient, in actiology of beriberi, 339

- estimation of, in freces, process, 633

Fats, food value of, 563

$\mathrm{F}$ avism, atiology, 389

- definition, 389

_- distribution, 389

_ symptomatology, 380

- treatment, 389

Febrile or glandular stage in African trypanosomiasis, I 40

Fever, daily, in cerebral stage of African trypanosomiasis, 145

in African trypanosomiasis, 140

Fibrin ferment in snake venom, $44 \mathrm{I}$

Fibrolipoma, pendulous, of back of neck, 497

Fibrolysin injections in elephantiasis of legs, 286

Fibroma molluscum, 502

- multiplc, 499

of neck, 495

pendulum, 502

Fibromatous condition of skin, curious example of, 496

Field rat, reservoir host for spirochates, 17

Filaria bancrofti, adult, 273

— carriers of , 278

- cause of elephantiasis arabum, reasons explained, 287,288

- embryo, 274. Sec also Micro-

filaria nocturna

$$
\text { life-history, } 273
$$

morplolog!, 272

- ovum, 273

carriers of, 294

- infections associated with double

conjunctivitis, discharging chyle, 294

Filariasis, clinical manifestations, 279

_ Geographical distribution, 278

- historical notes, 271

pathology, 278,270

Filarida, discases caused by, 271

Filtration of water, 558,559

Finkler-Prior sujirlum, 621

Fish venom, effects of, 447

-1 high mortality from, 448

- symptoms, 448

Fishes, venomous and poisonous, 447

- which poison after their consumption as food, 448

- - - - - symptoms, 448

wounds of +48

pines, mortality

from

from, $4+8$

Five-day fever (Volhynia fever), 398
Flea, species infesting rats and conveying plague, 171

Fleas, alimentary tract of, i 8

- carriers of plague bacillus, 177

- destruction of, 179

- external appearance, 157

families of, 179

larvac of, I 79

- reproduction, method of, iz 8

Fleming's modification of Trassermann reaction, 610, 611

Flexner type of dysentery bacillus, 6ra

Flexner's process of preparing antidysenteric scrum, 201

Flies and disease, 223

- danger of, to health, manner of infection by, 223

-_transmission of cholera by, 206

- ways in which dangerous to health, 223

Fluorides, as preservative test for food, 579

Fly, biting, in actiology of pellagra, 332

carriers of typhoid fever, 2 It

Folliculitis decalvans, 47 1

- - treatment, 471

Fomites, transmission of cholera by, $20^{\circ}$

Food adjuncts, 503

- deficiency in xtiology of beriberi, $338-9$

__ in atiology of pellagra, 329-30 morbid conditions caused by, 571

poisoning in atiology of beriberi, 338

Foods, adulteration of, 560 calorie value of, 565

essential consituents of, 562

tinued, dangers from, 575

Foot, amputation of, for perforating ulcer in lepers, 185

Fordyce's discasc, 477

Foreign bodies in cre, 509

Formaldehyde, as preservative test for food, 577

Formalin in spirit, application in trichomycosis, 495

- solutions of, in treatment of African trypanosomiasis, 140

Formic aldelyde for disinfection of houses, 595

Frambosia tropica (yaws), diagnosis, 26

— contagious discase due to Tre-

ponema pertenuis, 21

- histopathology, 22
incubation, 23
infection of by direct contact,

25

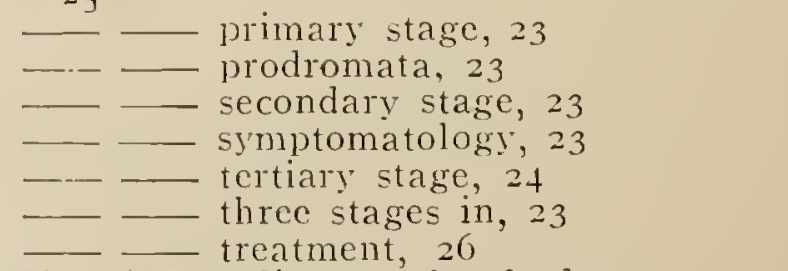

Fungi, eye diseases, local, due to, 511 in actiology of pellagra, 331 ocular aspergillosis due to, 511 blastomycosis due to, 5 I I gonosporosis due to, 5 I I moniliasis due to, 5 I I 
Fungi, ocular nocardiasis due to, 5 I sporotrichosis due to, 511 tinea flue to, 511

producing madura foot, 478

Funiculitis, endemic, definition, to 7 geographical distribution, 407 - patholog!, 407

symptomatologi, fos

treatment, 408

_- must be surgical, tos

Galre in frambosia tropica, 27 in treatment of Ifrican trypanosomiasis, 148

Gangosa, definition, 404

diagnosis, 405

distribution, 404

late manifestation of yaws, 25

(rhino-pharyngitis mutilans), tot

symptomatology, fot

treatnicit, to5

Gastro-enteritis, hamorrhagic febrile, of children, prognosis grave, 403

Gelatin, nutrient, preparation of, 622 plate cultures, 623

Gemmation, asexual method of reproduction in protozoa, 3

Genosporosis, ocular, due to fungi, $j 11$

Ghee, 568

Glanders, 504

Glanders bacillus, see Bacillus mallei

Glossina bocayei, 151

— fusca, i 5 I

longipalpis, 151

longipennis, 151

morsitans, $1 ; 1$

_- carriers of Trypanosoma

pecorum, I 35 food of, 152

- habits of, 152

reproduction of, 152

pallicera, I 5 I

pallidipes, 151

palpalis, $15 \mathrm{I}$

- _ - food of, 152

- habits of, I53

- - reproduction of, 152

tachinoides, 151

Glossince, notes on, 1;1-3

Glossitis areata exfoliativa, 477

Glottis, odema of, complication of

African trypanosomiasis, 146

Glucose and lactose broth, preparation of, 622

$-\frac{22}{62}$ peptone waters, preparation of,

Glycerine of tannin in trachoma, 515

Gnathostoma spinigerum rarely found in man, 27 I

Goitre in the Tropics, +10

Fonococcus, diagnosis of, 620

Goode, Norman, method of cleansing washing water, 560, 56 I

Goundou, definition, $41 \mathrm{I}$

_ geographical distribution, +I1

- pathology, 4I I

- symptomatology, 4 I 2

C. treatment, 4 I 2

Grain itch, 460

__ treatment, formula for, 469
Gram-negative and Gram-positive organisms, important differentiations in staining, $6 r t$

Granules, metachromatic, volutine, and metaplasmic, in protozoa, 3

Granuloma inguinale, treatment, 460

ulcerating, 503, 505

Green, protective colour in prevention of sunstroke, 392

Grooved tongue, 477

Ground-nut, common (Arachis hypogcea). nutritive value of, 564

Ground squirrel, spread of plague by, I7 I

Guinea-worm, see Dracunculus medinen. sis

Gỵnocardic acid in leprosy, 189

HaMADIPSA in atiology of hirudiniasis, 324

Hamato-chyluria, 283 in filariasis, 279 lympluria, $28_{3}$

Hrematoxylin and eosin for staining malarial parasites in blood, 608

- use of, as stain, 604

Hamoglobin, estimation of, in Tropics, 607

- reduced, demonstration by spectroscope, 612, 613

Hremoglobinuric (blackwater) fever, atiology, 381

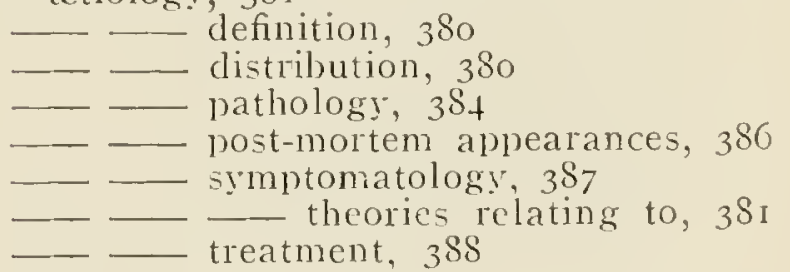

Hremolysins in snake venom, $4+0$

Hrmolysis due to parasites of malaria,

Hämoptysis, endemic, see Paragonominsis

Hamorrhage in yellow fever, treatment, 371

Hamorhages, rectal, causing sudden death in spirochatosis ictero-hamorrhagica, is

Hamorrhagin in suake venom, 441

Haffkine's propbylaxis against plague, 175, I 76

- vaccine in prophylaxis of plague, 176

Hair affections, 469

colour of, changes in, causes of, 470

poor development of, in natives of Tropics, $45 \mathrm{~S}$

Hansen's Bacillus leprae, iso

Hardness, total, of water, method of estimating, $65 t$

permanent, of water, method of estimating, 65.t

- temporary, of water, method of estimating, 654

Harris, ulcer of, section of intestine showing in case of amobic dysentery, 38

Hay fever, tropical (rhinitis plastica vasomotoria), tot 
Hay fever, tropical, treatment, 404

Health, maintenance of, in Tropics, general rules for, $54 \mathrm{I}$

Heart failure cause of sudden death in

Asiatic relapsing fever, 15

- treatment of, in typhus, 380

Heat as means of disinfection, 593

- exhaustion, 530

stroke (sunstroke), ætiology, 39 I

- and heat syncope, differences

between, 390

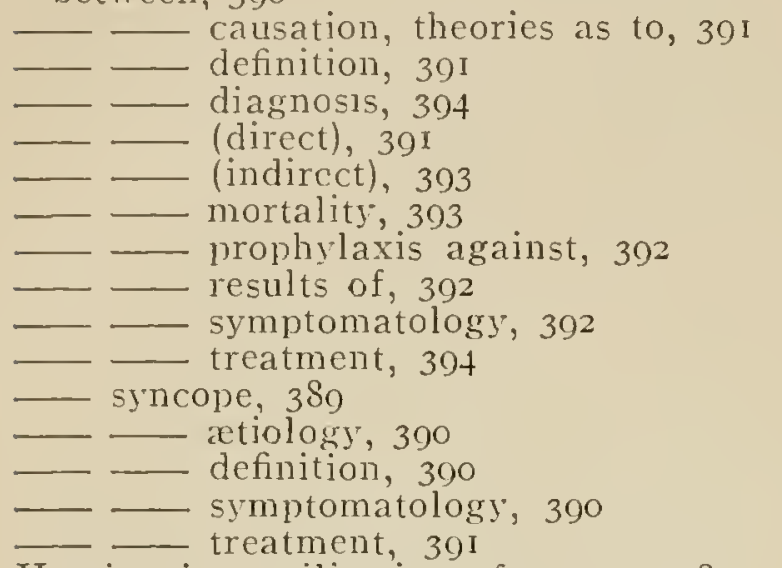

Heating in sterilization of water, 558

Helminth ova in freces, preservation of, 323

Helminthiasis, complication of African trypanosomiasis, 146

Helminths, diseases due to, 226

- distribution, 228 factors controlling, 228

grouping of, 231

- mode of infection by, 320

- nomenclature, 230

- pathogenic action, variation of, 229

32 I -2 preservation and examination of,

- toxic, 449

Herpetomonas, 5

Hindu water-carriers, vessels used by, 552 Hirsuties, $47 \mathrm{r}$

- treatment, $47 \mathrm{I}$

Hirudiniasis, atiology, 324

— definition, 323

- symptomatology, 327

treatment, 324

Histoplasma capsulatum, 6

— date of discovery, 6

- diseases caused by, 6

- habitat, 6

Horny growth from cheek, 504

Horse, preparation of antitoxic sera from, 599

- scrum, immune in spirochatosis icterohamorthagica, 19

Horsley, Sir Victor", death of, from heat stroke, 393

Hot-air sterilizer, 622

Hot climates, pathological effects on Europeans, 5.38

- physiological effects on Europeans, 5.37

House-fly (Musca domestica), encmies of, 225

habits, \&c., of, 224

how man is infected by, 225

ways of suppression of, 225
Ilouses, disinfection of, fumigation as means of, 595

- suitable, construction in tropical districts, 535,536

Hughes, Basil, method of cleansing washing water, $560,56 \mathrm{I}$

Humerus, chondrosarcoma of, 498

Hydatid disease, cystic form of, due to infection by Echinococcus granulosus, 267

duce multilocular form of, pro268

Hydrocele, see Orchitis and hydrocele, filarial

IJydrochloric acid in sprue, 360

Hydrochloride (emetine) in amobic dysentery, 42

Hydrophinze, 425

- genera of, 426 in Australia, 433

Hydrophine venom (snake bite), symptoms, 442

Hydrophis coronatus, photograph of, 424

Hygiene, constitutional, 535

- relation to tropical diseases, 539

Hymenolepis diminuta common to rats and mice, 263

— nana, dwarf tapeworm of man, 263 - - intestinal irritation and anxmia resulting from infection by, in children, 263

Iyperzesthesia, deep, in African trypanosomiasis, 142

- in cercbral stage of African trypanosomiasis, 1.45

Hyperkeratosis, 495

IIperpyrexial fever, high mortality in, 396

Hyphomycetes, divisions of, 630

ICHTHYOL in acrodermatitis vesiculosis tropica, 460

— in climatic bubo, $4 \mathrm{II}$

— in filarial lymphangitis, $28 \mathrm{I}$

- ointment, application to Calabar swellings, 300

Ich thyosis glossae, 477

- hystrix, 473

- - treatment, 473

Immunity, acquired, definition, 598

- active, 165

natural, 165

—_ definition, 598

- passive, 165

- thcories of, 163

- to diseases afforded by raccines and sera, 60 I

Impetigo, 473

- treatment, 473

Incubation of trypanosomiasis, African incubation, 139

India, bacterial standards for purity of drinking water in, 649

- deaths from snake bite annually in, 419

- diet of natives and Europeans contrasted in relation to incidence of typhoid fever, 215 
India, standard for bacteriological cxamination of water in, 645,646

- waters of, faccal contamination excessive, 645

Infant feeding in Tropics, 580

L table for, 580

foods, prepared, dangers of, $58 \mathrm{r}$

Infants, biliary cirrhosis in, 400

Infective diseases, common, some proplyylactic measures for, $; 08$

Infectivity theory in atiology of pellagra, 331

Influenza, diagnosis of phlebotomus fever from, 361

Injections, local, in snalie bite, 446

- prescrvative, for preservation of meat, 555

Insecticides, 594

Insects, eye diseases (local), due to, 510

- how infected with animal parasites, 7

Intertrigo saccharomycetica, 473

- - treatment, 473

Intestinal disorders, dietetic causes of, 57 I

- symptoms and lesions of ascariasis. 311

Intestine, elimination of toxins by, in yellow fever, $37 \mathrm{I}$

great, lesions of amobic dysenter! in, 36

- irrigation of, in intestinal schistosomiasis, 256

_- with sodium hypochloride or tannic acid in schistosomiasis, 256

- sections from, in case of amœbic dysentery, showing ulcer of Harris, 38

Intestines, post-mortem appearances of, in schistosomiasis infected with $S$. japonicum, 252, 253

Iodides in granuloma inguinale, 460 in sporotrichosis, 488

Iodine, tincture of, application in different forms of tinea (ringworm), 490 , $491,493,494$

Iodoform injections in leprosy, 180

- suppositories in oxvuriasis, 313

Ipecacuanha in ciliar dysentery (Balantidium coli), 46

- in amobic dysentery, +3

- in hepatitis in liver abscess, 55

in sprue, 360

Irido-cyclitis in cerebral stage of African trypanosomiasis, 146

Iritis, complication of African trypanosomiasis, $1+6$

Iron in afebrile splenomegaly, 4i in water, method of estimating, 655 water pipes, diminution in diameter, 552

Irrigation, regulations regarding, in the Soudan, 534

Ixodidx (tick), spirochates of, African tick fever transmitted by, in

JAIL, planning and construction, in tropical districts, 536

Japanese river fever, atiology, 394
Japanese river fever and spotted fever of Rocky Mountains, similarity between, $3 ; 6$

$\frac{-}{-}-$ distribution, 394 ('Tsutsusamushi disease),

-—— - mortality, 395

Jaundice not treatment, 395

due to increased formation rluagica, is

Jaw, lower, cystic bone, sarcoma (myeloid?) of, 499

- sarcoma of (? myeloid), 497

Jelly fish, stimg from, symptoms, $4+9$

- venomous, t+s

Jiggers, complication of African trypanosomiasis, 146

KALA-AZAR, atiology, 56

_ and oriental sore, differentiation be-

twcen, 66-6S

- blood in, 60

definition, $; 6$

diagnosis, 60

geographical distribution, 56

history, 56

incubation, 58

infantile, atiology, 62

- antimony, 63

- complications, 62

- definition, $6 r$

- distribution, $6 \mathbf{I}$

— - symptomatology, 62

- - sinonyms, 61

—_ morbid anatomy, 60

_- prognosis, 60

- symptoms, 59

termination, 59

- transmission, mode of, $S$ treatmest, $6 \mathbf{i}$

- prescription in, 61

- see also Pseudn-kalitazar

Kaposi's disease (Nerodermia pigmentosum), 47!

Kerandal's cure for African trypanosomiasis, I 50

$-\operatorname{sign}$ in Alrican trypanosomiasis, 142 Keratoma plantare sulcatum, $47 t$

Keratomalacia, 510

Keratosis pilaris, treatment, 473

Th - (xerodermia), 473

Khartoum, bacteriological examination of river and well water in, 642,643

Fidney, most venom excreted by, in snake bite, $4+5$

Kidneys, elimimation of toxins by, in yellow fever, 37 I

- in blackwater fever, 386

Klebs-Löffer bacillus. See Bacillus diphtherive

Flein's protective inoculation prepared from organs of animals dead of plague, I77

- - advantages, I77

Finee, acute symovitis of, associated with filaria infection, 294 
Koch's comma bacillus, 621 cause of cholera, 205

Fraurosis vulva, 477

Kreotoxismus, 455

treatment, 455

LABOR.ATORl, hints for work in, 603

Lacertilia, $\$ 23$

Lactose fermenting organisms in water, test for $6+3$

Lake water, bacteriology of, 648

Lakes, purity of, $5+7$

water supplies from, $5+7$

Lamblia intestinalis, 5,47

- parasite of ciliar dysentery, 46

Lamus megistus as agent in spreading South Anerican trypanosomiasis, 154

Lanoline, application in ichthyosis hystrix, 473

- ointment in filarial lymphangitis, $2 S_{1}$

Laryngtis, complication of African trypanosomiasis, 146

Lâtah, geographical distribution, 406

- symptomatolog!, 406, 407

Lathyrismus, 453

- causes of, 572

_- symptoms, 453,454

- treatment, 454

- when endemic and when epidemic, $+53$

Latrines, Chinese, 587

European, 588

for troops in the field, chemical

method, 588

- in tropics, 585

_- Indian, 588

native, $5 S_{5}$

- separation sistem in, $5 \delta 7,58 \delta$

- river, practice of, insanitary, 586

Lead and opium lotion in filarial lymphangitis, 28 :

- in filarial orchitis and

hydrocele, 281

dissolution from water pipes by

peaty water, 552

-in water, method of estimating, 655 4 I 1

Lecches, land and water, in atiology of

Hirudiniasis, $32 t$

Leris, elephantiasis of, 28t-2 $\delta_{7}, 203$

Leishman-Donovan parasite of kala-azat, 57

Leishmania, 6

Leishmaniases, 55

Leishmaniasis, canine, 68

- acute and chronic, 69

dermal (oriental sore), 63

xtiolowy, 63

definition, $6_{3}$

diaguosis, 65

- points to

distribution, 63

histopathology, 63

prognosis, 65

symptomatology; 64

treatment, 66

naso-oral and oro-pharyngeal, 68

simptoms, 68

treatment, 68
Leishman's stain, 604

for staining malarial parasite

in blood, 608

Leproline in leprosy, 189

Leprosy, asthetic, macula in, 187 cleformities in, $18+$ varicty, 186

antitoxic serum in, 599

bacillus, i 80

definition, 179

geographical distribution, 180

history, 179

how spread, 181

little influenced by climatic and

tropical conditions, 540

- inixed variety, 188

nodular, in Hindoo woman, $18_{3}$

pathology, 18 I

post-mortem appearances, i 82

- prognosis, $1 S S$

symptomatology, $182-3$

- treatment, 188

tubercular variety, ISt, 186

Leptothrix, 47 i

- treatment, $47 \mathrm{I}$

Leucocytes in lymphatic leukamia, 606 605

total discases during which

diminished, 605

Leucocytosis in blackwater fever, 387

- in verruga peruviana, 364

Leucocytozoida, 5

Leucoderma, 474

- treatment, 474

Leucolysins in snake venom, $44 \mathrm{I}$

Leucopenia in dengue, 377

- in sprue, 357

Leukamia, lymphatic, leucocytes in, 606 606

Leukoplakia of tongue, 477

- varietics, 477

Lice, venom of, $45 \mathrm{I}$

Lichen convex, 474

- treatment, 474

planus, 475

- treatment, 475

— - cxternal, formula for, 475

- formula for, 475

Ligature in snake bite, $4+5$

Lightning causing blindness, 510

Lime, chloride of, use in purification of water, 555

- chlorinated, for purification of water, 556

Limnatis in atiology of Hirudiniasis, 324

Liponatosis, nodular, 483

Liquor arsenicalis in pellagra, 336

- plumbi application to Calabar swellings, 300

Liver abscess, causation, 48

- - liagnosis, 52

- in amobic dysentery, 50

_- incidence, $4 \$$

54

4 operation for, after-treatment, method of, 53 pathology, 50 
Liver abscess, position, is

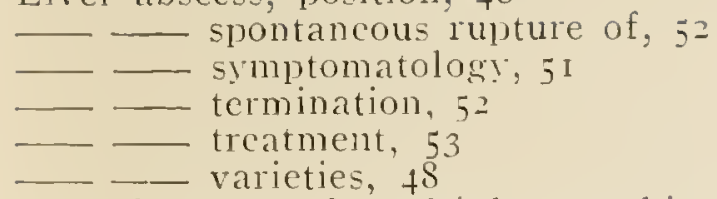

abscesses of, multiple, amobic, 49 condition in vellow fever, 367

_- in blackwater fever, 386 microscopical appearance in Lfrican

trypanosomiasis, 139

- post-mortem appearances of, in schistosomiasis infected with $S$. japonicum, 252

- tropical, 4It

- parasite of dermal leish-

maniasis, 63

- - symptomatolog!, 4 It

- treatment, $55,+1+$

- - prescription for,$+1 t$

Lizards, venomous, $4 ; 3$

Loa loa, carrier of, 298
296 - causative parasite of loasis,
- habits, $29 S$
- - illustrations of, 296, 297, 208
- life-listory, $29 S$
- morphologi, 206, 207, 298
Loasis, causative parasite, $29^{6}$
- definition, 295
— geographical distribution, 295
— symptomatology, 299
treatment, 299

Lolismus, 454

Loos hypothesis of schistosomiasis, conclusions as to prevention and treatment based on, 249-250

- theory of, relating to $\mathrm{S}$. mansoni, 255

Lotions, application to tropical ulcerations, 505

I_ow intermittent non-malarial fever, long course of, 396

$-\div-$ symptomatology, 396

Lungs, microscopical appearance in

African trypanosomiasis, 139

Lupus erythematosus, differentiation from lupus vulgaris, 475

- treatment, 476

Lustig's and Galeotti's serum in prophylaxis of plague, 176

Lymphangiectasis, filarial, definition, 28 I — - symptomatologs, $2 S_{1}$

L treatment, 282

Lymphangiomata, 502

Limphangitis, filarial, definition, 280

— post-mortem findings, 280

— - symptomatology, $2 \mathrm{SI}_{1}$

- treatment, $2 S_{I}$

Limphatic glands, fibrosis, in filariasis, 279

- in African trypanosomiasis, $1+2$ in cerebral stage of African trypanosomiasis, $1+5$

- microscopical appearance in

African trypanosomiasis, 139

- varicose, 282

- - treatment, $2 \delta_{2}$

tissue, bacilli of typhoid fever chiefly invading, 216
Lymphacele, 284

Lymphocytes, diseases in which increased, 605

- percentage in normal blood count, 605

Limphoderma perniciosum, 502

Limphuria. Sce Chyluria and lymphuria

Lịsol as disinfectant, 594

MACGREGor, Sir William, plan of protected well suggested by, 550

Maculax in asthetic leprosi, 187

Macular fever of Tunisia, definition, 397

____ - simptomatolog!, 397

Mahommedan water-carriers, infection of water by , 551

Maiche automatic sterilizer, 558

Maignen's process for softening water, 555

Maize theory in actiology of pellagra, $330-1$

Malaria, 60

— atiology, exciting causes, 7I, 72

- - predi-posing causes, to

carricrs of, 69

complication of African trepano-

somiasis, I 46

- cure of, possible future method, 74 definition, 60

- diagnosis of phlebotomus fever

from, 361

- cradication of, by quinine in pre-

vention of hamoglobinuria fever, 384

history of, 69 , 70

parasites of, $69,71,72$

behaviour in blood, 72, 73

hemolytic action, it

- infection by, mode of, 8

- intracorporcal or human cycle,

74

- life-cycle, is

life-history, 72,73

pernicious manifestations of, in

brain and pancreas, $7 t$

- preceding typhoid fever in the tropics, 217

_ prevalcnce of, conditions farouring, $5+0$

- - recurrent attacks of, followed by

blackwater ferer, 38 ;

- subtertian and blackwater fever, relation between, $3 S_{4}$

- - diagnosis of yellow fever from, 370

- symptoms, general, on

Malarial fever, factors necessary for an attack of, 72

- large mononuclears increased during, 605

- phases of related to phases of

life-cricle of parasite, 72

- quartan, parasite causing, 73

tertian, parasite causing, 73

infection, active, theory that hamo-

globinuric feycr is manifestation of, $38 \mathrm{r}$ - previous theory that hamoglobinuric fever is condition brought about by, $38_{3}$

pigment, constituents of, 628 
Malarial pigment, where it is and how to look for it, 628

- pigments, manmer of showing in laboratory work, 629

Male, adult. not cngaged in hard work, average dict for, 504

fern in hymenolepis nana, 263

___ in intestinal schistosomiasis, 256

— in schistosomiasis, 248,256

- in tapeworms, 269

Malignant connective tissue tumours, 502

Malta fever, diagnosis of phlebotomus fever from, $36 \mathrm{I}$

\section{_ _ see also Undulant fever}

Manchineel tree fruit, poisoning by, $45+$

Manchuria, pneumonic plague in, how started, $17 \mathbf{I}$

Mania in cerebral stage of African trypanosomiasis, $\quad+5$

Manson, Sir Patrick, reasons for elephantiasis arabum being clue to Filariabancrofti, $2 S 7,2 S 8$

Massage in dengue, 370

__ in elephantiasis of legs, 286

of scrotum, $20 \mathrm{I}$

Mast cells, 605

- staining of 605

Mastigophora (flagellata), $t$

Measles in tropical coumtries, for

Meat, decomposed, results from consumption of, $5 \% 2$

diet in spruc, 350

extracts, limited use of, 507

566

frozen, means of testing, 573

inspection and high cooking in, pro-

phylaxis against trichiniasis, 309

preservation of, 574

methods, 575

— native method, 575

- preserved, means of testing, 573

Meats, examination of, $5 \% 2$

Medical officer, control of water by, 553

Memory, loss of, in tropical regions, 539

Men, women and clildren, food necessary for, compared, 564

Meningitis, cerebro-spinal, a complication of African trypanosomiasis, 146

- sunstroke, a form of, 393

Meningococcus, diagnosis of, 620

Menstruation in cercbral stage of African trypanosomiasis, 145

Mercurial ointment in oxyuriasis, 313

Mercury, atoxylate of, in treatment of African trypanosomiasis, 148

_ - solutions of, in treatincnt of African tryounosomiasis, $1+9$

Metals, moisonous, in water, detection and estimation of, 655

Metchnikoff, phagocrtic theory of, 600

Methaemoglobin, demonstration by spectroscope, 6I 3

Methylene blue in ciliar dysentery ( $\bar{B}$ alantidium coli), 46

Loeffler"s, use of, as stain, 604

Mucorinal, diagnosis, 630

Micrococcus melitensis, cause of undulant fercr, I $100-1$
Micrococcus melitensis, diagnosis of, 620

Microfilaria demarquayi, film preparation, 276 diagnosis of, points of value in, 277 infection of man, dangerous elements in, 276

- loa, film preparation, 275 nocturna, 274 habits in man, 274 - habits in mosquito, 275 perstans, film preparation, 275 various species of, differentiation, 277

Microfilarize, examination of fresh blood for, 610

Micro-organisms, diagnosis of, 615 cxamination of, 613

staining of, 613

Microsporon audouini (ringworm), 63 I

Microsporons, +89

- and trichopytons, differences between, 480

Nilk, adulteration of, common occurrence in the tropics, 560

- bufialoes, 568

condensed, 568

decomposed, results from consumption of, 572

diet in sprue, 358

dried, 568

food value of, 567

goats', 568

heating of, $; 67$

homogenized, 568

infected, results from consumption

of, 572

manipulations of, 5 ;o

skimmed, 568

- dried, 568

sour Bulgarian, use of, for therapeutic purposes, 631

- transmission of, cholera by, 206

Mineral acidosis, theory in atiology of pellagra, 332-3

or chemical constituents of water, ill-effects of, 553

- waters, 584

Molasses and botassa in milk, tests for, 569

Moles, cpithelial, 495

Molluscs cssential for transmission of bilharzia, $5+3$

Nolluscum contagiosum, 495

Mongolian spots, +76

Monilia vaccines in sprue, 360

Moniliasis, ocular, due to fungi, 5 I I

Monilethrix, 47

Mononuclears, large, jncreased during malarial fever, 605

- percentage in normal blood count, 605

Monsoon weather, bacteriology of water and faeces during, 646

Montana, spotted fever of Rocky Moumtains in, mortality and prophylaxis a gainst, $3-6$

Morphia for relicf in griping in amobic dy"sentery, 42

- in spruc, 360

Morphine injector's septicamia, 4I5, fi6. See also IThitmore's disease 
Mosquito destruction as prophylactic measure against yellow fever, 372 habits of, microfilaria in, 275

Mosquitoes, destruction of, 596 disscction of ${ }^{6} \mathrm{~S}$

time of clay at which biting, 7 I venom of, 453

Muco-cnteritis, from drinking water containing non-pathogenic bacteria, 553

Mlucorinal, diagnosis of, 630

Mucons membrane, pathological skin conditions affecting, 476

Mucus in faces, examination of, 632

Murzena (fish poisomous by bite), 447

Musca domestica, 224. See also House-fly

Miscles, calcification of Trichinella spiralis in, 308

Mussurana, enemy of venomous snakes, 447

Mycetoma (Madura foot), 478

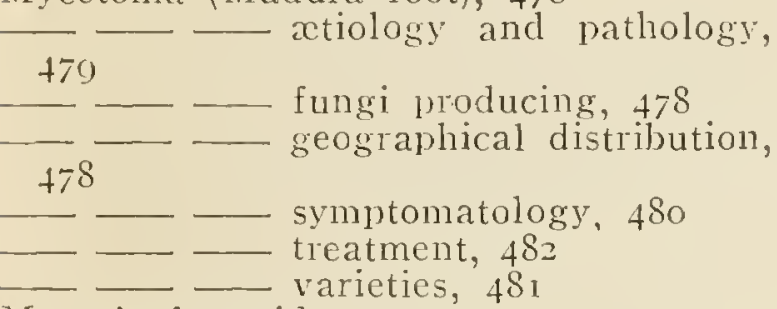

Mycosis fungoides, 502

Mydriatics and cycloplegics in eyc diseases, 52

Myelocene, inunctions in keratosis pilaris, 473

Myelocytes in spleno-medullary leulicemia, 606

Myiasis, 482,483

- kinds of flies causing, 482,483

Myocarditis considerable in typhoid fever in the tropics, 218

- European, fatal complication of European relapsing fever. I I

Myocardium, section from cases of beriberi, 339, 340, $34 \mathrm{I}$.

Myomata, 502

Myotics in eye diseases, 528

YajA, species of, in Africa, 43I, 432 bungarus (king cobra), mortality caused by, 429

- fasciatus, characteristics of, 428,429

tripudians (cobra-di-capello), photograph of, 425

Naphthalin suppositories in oxyuriasis, 313

Nasha fever, symptomatology, $39^{\text {r, }}$

- treatment, 396

"Nastin" for leprosy, 188

Vecator americanus, 314-5

points of clifference between

the Ankylostome duodenale and, 320

Neck, fibroma of, 496

- pendulous fibroma of back of, 497

- sarcoma of, 500

Negraes, inmunity of, from sunstroke causes, 39 I

Nemathelmia (group of helminths), 23 I

Nematodes, morphology, 270 pathogenic to man, 270

- preservation and examination of, $270,32 \mathrm{I}-2$
Neosalvarsan in framboesia tropica; injection, intramuscular, 27

- _ - intravenous, 26

in sranuloma inguinale, 460

in treatment of African trypano-

somiasis, 148

Ncosporidia, 5, 6

Nerve symptoms in typhus, dlugs in, 380

Nervous symptoms in South American trypanosomiasis, 157

- system, disturbances of, in tropics, 405

Neurasthenia, tropical, 405

Neuritis, peripheral, endemic, 406

Neurofibrosis of Recklinghausen, 502

Neurotoxins, in snake venom, importance of, 440

Night blindness, 509

Nitrates in water, detection of, tests, 656 , 657

657,658 estimation of, methods, 656 , in milk, test for, 560

Nitrogen, deficient, in atiology of beriberi, $338-9$.

— estimation of, in freces, process, 633 ingestion of, 565

Nitrogenous foods, necessity for, 562,563

Nocardiasis, ocular, due to fungi, $5 \mathbf{I} 1$

Nodes, juxta-articular, 473

Nomenclature, international rules of, 230

- language, rule of, 230

- rule of, appropriateness, 231

Nourishment, priority, 231

Tucleus of , average, for man, 565

Nutnegroa, 3

Nutmegs poisonous in large doses, 454

Nutrient media, vessels used for, 621

Nutrition, 562

$\mathrm{O} \dot{2}$, absorption in sewage, rate of, after filtration, 662

Oatmeal jelly in infant feeding, 5S I

Edema in ccrebral stage of African trypanosomiasis, 146

- localized, in African trypanosomiasis, 142

Ointments, application in different forms of tinea (ringworm), 490-494

- nourishing application in alopecia, 470

Oleate of iodine, hot fomentations on, for tropical fever, 55

Oleum chenopodii treatment of ankylostomiasis, 319-20

Onchocera (filaria) volvulus, life history, 300

$\square-$ morphology, 300

Onyalai, atiology, 417

definition, 416

- geographical distribution, 417

—_ symptomatology, 417

treatment, 417

Ophidia, 423

Ophthalmic irritation, 510

wind and dust, 510

Opisthoglypha, 423

Opium contra-indicated in yellow fever, 371 
Opium, tincture of, in amobic dysentery, 43

Opsonic index, 164,600

Opsonin, 600

Orchitis and hydrocelc, filarial, symptomatolog! , 281

Oriental sore, 504

Ornithodorus moubata (tick) found throughout tropical Africa, 19

Orpiment in treatment of African trypanosomiasis, $1+8$

Ova, detection in faeces, 637

Ovari, carcilloma of, 502

Oxalate poisoning in retiology of beriberi, 337

Oxidation, water rendered potable by, 561

Oxidizable organic matter in water, method of estimating, $6=8$

Oxyhæmoglobin, demonstration by spectroscope, 612

Oxyuriasis, 312

- parasite causing, 312

- symptomatology, 313

treatment, 313

vermicularis, morphology and life-

history, 312

- ova of, 637

- parasite causing, 312

Oysters, cholera and typhoid fever resulting from eating, 572

Ozone, sterilization of water by, 556

PAGET's disease of nipple, 495

Paints, application to mouth in sprue, 360

Pancreas, pernicious manifestation of malaria in, 74

Pancreatin in sprue, 360

Pancreatitis, chronic, diagnosis of sprue from, 358

Pandemic diseases, $5+3$

Papalismus, 454

Papular fever, symptomatoloss, 403

- treatment, 379 .

Paragonimiasis (endemic hamoptysis), definition, 240

- diagnosis, 242

- distribution, 240

morphology, 241

- parasite of, 240

pathology, 241

symptomatology, 242

treatment, 209,242

Paralysis, general, late manifestation of yaws, 25

Parasites, animal and vegetable, staining for, 604

- - cye diseascs, local, due to, 5 II - in atiology of pellagra, 331

cfferted, 7 infection of insects with, how 626 demon-tration in laboratory work,

in hamoglobinuric fever, question of, 382

- macroscopic examination of faces for, 634

- malarial, benign tertian, 609

— in blood, staining of, 607,608

- in brain, mounting and stain-

ing section, 627
Parasites, malarial, in brain, manner of showing in laboratory work, 627

- L living, demonstration of, $60 \%$ quartan, 600

- subtcrtian, restivo-autumnal or malignant, 609

- varieties of, 600

331

Parasitic causes in retiology of beriberi, 340

- theory in atiology of pellagra, 33 I

Paris Convention (1003), International regulations to prevent importation of cholera, plague and yellow fever, $596-98$

l'arotid tumour, mised, 501

Parrots, epizootic fatal among, 189 . See also Psittacosis

Parthogenesis in protozoa, 4

Pasteur-Chamberland filter, 558

$36 I$

Peat, avoidance of, in catchment arcas for water supplies, 547

- in water, effect on leaden pipes, 552

Pediculosis, 483

- pathology, 483

- treatment, 484

Pellagra, æetiology, 320

- deficiency discase, 572

- definition, 329

diaguosis, 335

- eruption of, predisposing factor, 540 geographical distribution, 329

pathology, 333

prognosis, $335-6$

- seasonal prevalence, $5+0$

svmptomatology, 333-5

—— trcatment, 336

Penphigus contagiosus, $48+$

- treatment, $4 \delta_{4}$

Penicilliacea, diagnosis of, 630

Pcnicilliosis, 485

Peptone waters, glucose and lactose, preparation of, 622

Perforation in typhoid fever in the tropics, signs of, 219

Peritonitis, fatal in ascariasis, 3 I I

- in severe trichiniasis, 308

Perlèche, 478

Permanganate pills in cholera, 209, 2 Io

Perspiration, importance of, in tropical climates, 537

Phagedxina (tropical sloughing), 485

Phagocytes, 164 treatment, 485

Phagocytic theory of protection formed by vaccines and sera, 600

Phagocytosis, 164

Phenacetin in ycllow fever, 371

Phlebectasis, filarial, 282

Phlebotomus fever, xtiology, 361

- carrier of, 361,362

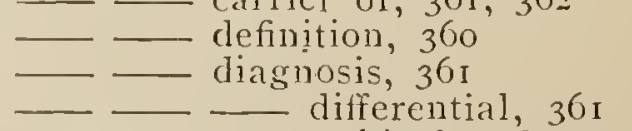

- seograplical and scasonal dis-

tribution, 360, $36 \mathrm{I}$

- symptoms, $36 \mathrm{I}$ 
Phleljotomus fever, treatment, 361

- papatasii, carrier of phlcbotonus fever, 302

- characters, life-bistory and

habits, 362

Phosphorus, in food, deficient, in atiolog! of beriberi, 339

Photodynamic theory in xtiology of pellagra, 330-I

Pickles for meservation of meat, 575

Piedra, $47 \mathrm{I}$

- treatment, 485

Pigmentary fever, 395

Pigmentation of viscera in malaria, how explaincd, 72

Pinguecula resulting from exposure to wind and dust, 510

Pinta (carate), 485

- - symptoms, 485

Preatment, 486

Pits as latrines for natives, 585

- deep, as latrincs for Europeans, 588

- - for natives, 586

Pityriasis rubra; see Dermatitis erfoliativa

- versiculor; see Tinca atersicolor

Plague, 16 ;

- abortion in, 173

— ambulatory, 174

_- antitoxic serum in, value, 599

- bacillus causing, i68

__ blood changes in, 173

- bubo, inguinal, external appearance, 173

\footnotetext{
- bubonic, 167, 174

of, I I I transmission to man, mode

of, carricr of, Is I

- cause of death in, 173

- historical memoranda, 166

- in rats, acutc, 169,170

incubation period, I 72
}

introduction into countries where

obliterated, 543

- mode of onset, I73

mortality, 174

or cholera on infected ships, quaran-

tine measures respecting, 597

pathology, 171, I 72

pneumonic, 167

- in Manchuria, how started, I 7 I

- primary infection in, 172

- primary and secondary, i 74

- transmission, mode of, $17 \mathrm{I}$

post-mortem appearances, 172

prodromal symptoms, 172

prophylaxis against, private, 176

- public, It5

septicamic, general features, I74

species of rat principally infected

with, I 70

- resistant to, I> I

spread of, by ground squiriel, i 7 I

by rats, 167

conditions favouring, 539

symptomatology, 172,173

transmission from one specics of rat

to another, I 70

601
Plague, varietics of, general characteristics, $17 t$

Plasmodium malariac, cause of quartan malarial fever, 73

- vivas, cause of tertian malarial fever, 73

Platyhelmia (group of helminths), 23 I

Pneumonia a complication of African trypanosomiasis, I 46

- lobular and lobar, in pneumonic plague, 172

Podophyllin in treatment of tapeworms, 269

Poikiloxytosis, 606

Poison ordeals, 456

Poisons for animals, 457

for fish, 457

talien accidentally by man, 453

Polychromatic cells, 606

Polymorphonuclears, vercentage in normal blood count, 605

when increased physiologically and when pathologically, 605

Polyneuritis, sciatic nerve of forl suffering from, $3+2$

Ponos, limited to young children, ti3

- resemblance to biliary cirrosis, 43

- Symptomatology, 414

Popliteal nerve in case of acute beriberi, 342

Pork, inspection in prophylaxis against trichiniasis, 309

Porocephalosis, gcographical distribution, 325

- pathology, 325

- symptomatologr, 325

Porter-Clarke process for softening water, 555

Potash, permanganate of, stcrilization of water by, 557

Potassium iodides in blastonycosis, 462 - in frambosia tropica, 27. salts, vegetable products rich in, 563

"Poudrette" method in disposal of excreta, 590

Precipitins, action of, 600

Prepuce and legs, elephantiasis of, 288

Preservatives, chemical, $;-6$

cxternal, for preservation of meat, 575

Prickly heat, 486 atiology, theories as to, 486

$\longrightarrow$ treatment, 486,487

Protargol in amobic dysentery, 43

- ointment for ulcerative lesions in frambcesia tropica, 27

Protein deficiency theory in actiology of pellagra, 331 567

Proteolytic ferments, $44 \mathrm{I}$

$44, \overline{441}$

Proteroglypha, 423

Protozoa as factors in causation of

tropical diseases, 2 classification, 4

definition, 2

diseases due to, I

life-history, 3

morpholog!, 3 
Protozoa, of pathogenic significance in man, 5

origin of infection by, 2

reproduction asexual, 3

- methods of, 3

transmission of, 2

Protozoal discases, modes of transmission, 7,8

Protozoology, introduction to, 2

Pseudo-Banti's discase, 41\%. See also Splenomegaly, afebrile

Pseudo-kala-azar, definition, 69

- geographical distribution, 6

_ prognosis, 60

- symptoms, 69

Pseudo-myxœedenatous symptoms in chronic stage of South American trypanosomiasis, 156

Pseudopelade, $47 \mathrm{I}$

Pscudoxanthoma or elastorhexis, 502

Psittacosis, xtiology of, I 99

- definition, 189

pathology, igo

— sypmtomatolog:, 190

- treatment, 190

Psoriasis, 487

- treatment, $4 S_{7}$

Pterygium, 510

- resulting from exposure to wind and dust, 510

Pulex irritans, transmission of plague by, 171

Pulse in typhoid fever in the tropics,

Pus, spontaneous evacuation of, in liver abscess, positions of, dangers, 52

Pylorus, carcinoma of, 502

Pyosis Corletti (1915), 484

- - treatment, 485

- palmaris, 487

Pyrethrum powder as protection against Ornithodorus moubata, 2 I

Pyrexia, charts of, in undulant fever, $192-3$

QUARANTINE, 596

Quartz lamp in sterilization of water, 558

Quassia, rectal injections in oxyuriasis, 313

Quinine bibydrochloride in chronic stages of amobic dysentery, 43

in chyluria and lymphuria, 284

- intestinal irrigation with, in ciliar dysentery (Balantidium coli), 46 poisoning, theory that hamoglobinuric fever is result of, $38 \mathrm{I}$

RADIOTHERAPY in granuloma inguinale, 460

Radium, in varicose, lymphatic glands, $2 \mathrm{~S}_{2}$

Rainfall, 533

- annual, estimation of, 546

- as predisposing cause of malaria, 7

Rain-water, storage of, 546

collection of, $5+5$

rejection of roof-rashings, 546

Rash in typhoid fever in the tropics, 217

Rat-bite fever, atiology, 398

- - definition, $30 \mathrm{~S}$ gegraphical distribution, $39 \mathrm{~S}$
Rat-bite fever, mortality in, $30 \mathrm{~s}$

_- symptomatology, 398

Rats, destruction in prophylaxis of trichiniasis, 309

_-_ extermination of, 596

plague in, 169, I70

specics principally infected with plague, I 70 .

- - resistant to plague, 171

spread of plague by, 167

transmission of plague from one species to another, 170

Trichinella spiralis parasitic in, 308

Recklinghausen, neurofibrosis of, 502

Rectitis, gangrenous epidemic, cause of death in, 409

- 1409 definition, 408

dren of natives and animais, 408

_- symptomatology, 408

_- treatment for animals and children, 409

Refrigeration for preservation of meat,

Refuse, collection, removal and disposal of, in tropics, 584

dry, disposal of, in tropics, 592

Relapsing fever, American, 13

- Asiatic, 13

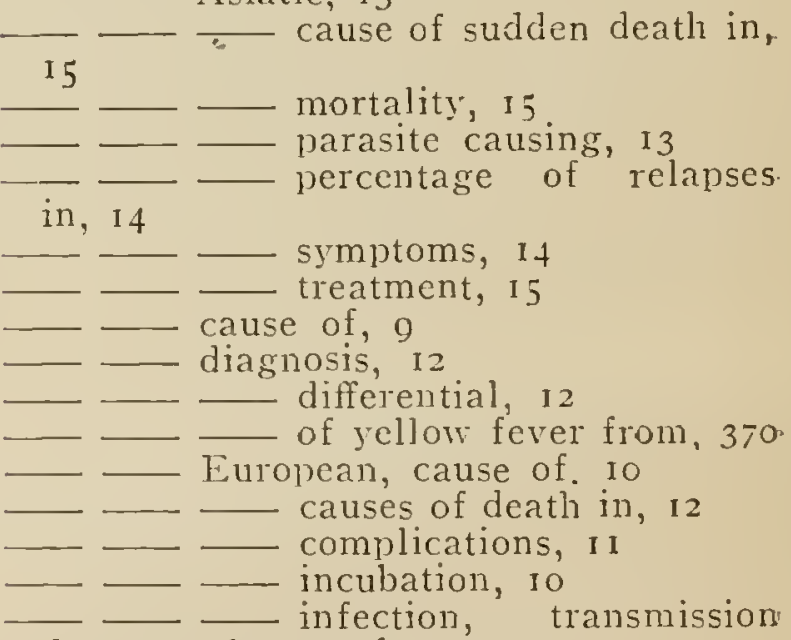

from mother to fotus, is

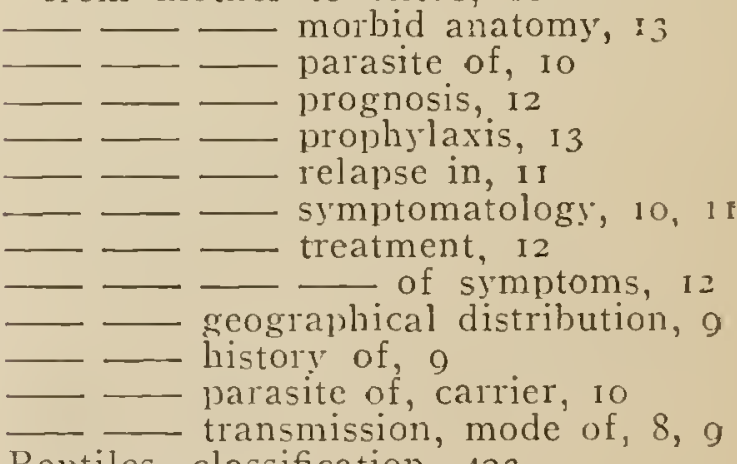

Reptiles, classification, 423

_ cranial slieletons of, 422

general features, 4ig

geographical distribution and pecu-

liarities, $427,42 \mathrm{~S}$

- heads of, 420,421

- poisonous, manner of striking prey, 436

- venom apparatus of, 435

— yield of venom of various

species, $436,437,438$ 
Reservoirs, construction of, $55^{2}$

- points to be watched before, 552

filtration of water in, method, 550 pipe-supply of water from, 552

Resorcin and salicylic acid, spirit lotion of, for removal of horny masses in elephantiasis of legs, 286

- application in different forms of tinca (ringworns), 491, 492, 493, 494

- ointment, application in xanthoma areatum, 506

Respiration, effects of tropical climates on, 537

Rhinitis spastica vasomotoria. See Hay ferer, tropical

Rhino-pharygutis mutilans. See Ganuns 2

Rlinosporidiosis, geographical distribution, 7

- treatment, 7

Rhinosporidium seeleri, habitat, 7

Rhizoplast in protozoa, 3

Rice, nutritive value of, 564,565

Rickets, dietetic causes of, 571

Ringworm, diagnosis of, 630 fungi, families of, 480

(timea), appearance of lesion, 480 - varieties of, $490-494$

- - - lesions and regions of body attacked, 490-494

\section{See also Tinea}

River water in Khartoun, bacteriological examination, 642

River waters, classification for drinking purposes, 649, 650

— — tropical, purity of, 547

Rivers, shallow, avoidance for water sulpply, 547

Rocky Mountains, spotted ferer of, $37 t$

Rodent ulcer, 495.

Rogers' method in treatment of paragonimiasis, 209

- in treatment of cholera, 209 210

Russell's viper, 431

fatality of bite of 442

SACCHARIN, test for, 50

Saccharomyces cerevisiae, 631

pastorianus (wild yeast), 631

Salamanders, venomous, toxicity of 448

Salicylic acid, application in jchthyosis hystrix, 473

- - as preservative, test for, 578

Saline purgatires in Nasha fever, 396 solution, intravenous injection in cholera, 210

—- isotonic, 613

results, 211

Salines in ciliar dysentery (Balantidium coli), 46

in snake bite, 445

normal, in blackiwater fever, 388

Salisbury cure, in sprue, 359

Salol in chrluria and limphuria, 284

Salt solution, rertal injections in oxvuriasis, 3 I 3
Salt water, distillation of, to oblain

drinking water, 544

Salts, uses of, in body, 563

Salvarsan in chappa, 413

___ in framboesia tropica, 26

- in treatment of African trypanosomiasis, 148

Sambon, theory of, relating to $S$. mansolli, 254

Sand filters, mechanical, 560

Sanitation in tropics, 584

Santonin in ascariasis, 311

- in oxyuriasis, 313 in sprue, 350,360

Saponin, dangers of, in mineral waters, 584

Sarcina, arrangement, 162

Sarcocystis lindemanni, 6

- muris, 6 tenella buboli, habitat, 0

Sarcodina (Rhizopoda) 4, 5

Sarcoma (myeloid?) of lower jaw, 49\%

- - of neck, 500

- - sec also Bone sarcoma

Sarcomatosis, multiple, 502

Sarcosporidiosis, 7

Sardines, dangers of, 572

Sausages, nutrition in, 567

Scabies, 487

- treatment, 487

Scalp, elepliantiasis of, 293

Scarlet fever, no spread of, in tropics, 401

Schistosoma hrematobium, infection in schistosomiasis, incubation time of, 248,249

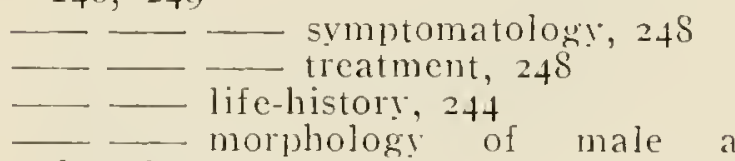

female, 243

and

- ova of, 243, 637

- percentage of population of

Egypt infected with, 242

japonicum from dog, 25 I

—_ infection in schistosomiasis, 25

- diagnosis, 254

geographical distribution, 251

ova of, 637

l)athology, 252

prognosis bad, 254

_- schistosomiasis spread by, 251

__ symptomatolog!, 254

- - treatment, $25+$

mansoni, infection in schistoso

miasis, theory of Loos, 255

- - theory of Sambon

254

- - ova of, 637

Schistosomiasis, causative parasites, 242 geographical distribution, 242

infected with Schistosoma hrmato-

bium, low mortality in, 249

Schistosoma japonicum, 251

_- post-mortem appearances,

252,253

intestinal, geographical distribu-

tion, 255

_- - patholog!, 250

prognosis, 256

treatment, 256 
Schistosomiasis, patholog!, 245

- post-mortem appearances of bladder in, 245,240

- prevention, conclusions as to based on Loos' hypothesis and on Bilharzia

Mission (1915), contrasted, 249, 250

- symptomatology, 256

- treatment, $25^{6}$

- conclusions as to, based on

Loos' hypothesis and Bilharzia Mission

(ig) 5), contrasted, 249,250

Schüfner's dots in benign tertian parasite, 600

Sciatic nerve of fowl suffering from polyneuritis, 342

Scorpions, venomous, mortality in childreis from, 449

— stings from, mortality, 449

- — - symptoms, 449

- - treatment, 450

Scrotum, elephantiasis of, 289,200

Scurvy, dietetic causes of, 57 I

Seaports, large, recommendations of Paris Convention regarding, 597

Sea serpents, 425. See also Hydrophina Sebaceous glands, high development in natives of Tropics, 458

Seborrhœa, 488

treatment, 488

Sections, mounting of, on slides, 626

- preparation of, 624

- staining of , 613, 626

Septicamia, development in plague, 1 ;

- morphine injections, 4, I5, 416. See also II'hitmore's discase

Sera, antitoxic, 599

—_ comparative value, in treat-

meul of various diseases, 599 preparation from horse, 599

- protection afforded by, hypotheses accounting for, 600

Serotherapy in typhoid fever in the Tropics, 222

Serum in prevention of snake-bite, 444

Lustig's and Galeotti's, in prophylaxis of plague, 176

- Jersin's, in prophylaxis of plague, 176

Sewage, disposal of, care of Chinese in, 587

effluents, examination of, 662

Sexual desire in cerebral stage of African trypanosomiasis, 145

Shake" cultures, 624

Shiga-Kruse type of dysentery bacillus, 6 ig

Ships, healthy, quarantine measures respecting, 597

- infected, quarantine measures respecting, 597

- with yellow fever, distance of anchorage from shore, 372

- suspected, quarantine measures respecting, 597

Side-chain theory, Ehrlich's, of protectection afforded by sra and vaccines, 601

Simaruba officinalis in amobic dysentery, prescriptions, 44
Simpson, W. J., results of inoculation of living vaccines against cholera, 212 - summary of cholera epidemics in Europe, 203-4

simulidxe in atiolog! of pellagra, 332

Siriasis, 539

Sistrurus catenatus (Prairie rattlesnake), 430

Sitotoxismus, 453

Skin, condition of, in cerebral stage of African trypanosomiasis, 145 diseases of, 458

elimination of toxins by, in yellow fever, 371

- fibromatous condition of, curious example, 496

- in typhoid fever in the Tropics, 217

_-_pigments, 488

Sleeping sickness stage in African trypanosomiasis, $144-6$

Slides, method of cleaning, 604

Smears, manner of staining, 6r3

Smegma bacillus, (10

Smoking, avoidance in sprue, 359

Snake-bite, antitoxic serum in, value of, 599

- deaths per annum due to, in India, 419

- immunity from, acquired and

natural, 444

- maintenance of strength of patient

in, 445

prevention of secondary infection

in, 445

- prophylactic measures against, 440

- symptomatic treatment in, 445

symjtoms, 44I

treatment, 443

Snake venom, aid in execretion of, in treatment of snake-bite, $\$ 45$

435

- characters of, 438

_- manner of analysing, 440

varieties of, 438

Snakes, cranial skeletons of, 422

heads of, 420,421

Soamin in treatment of African trypanosomiasis, 148

Sodium antimonyl. in kala-azar, $\sigma_{1}$

- bicarbonate in onyalai, 417

bisulphate, tabloids of, in schisto-

somiasis, 250

- hyposulphite, for purification of water, 556

Soil, 53.3

disturbance of, predisposing cause

of malaria, 7 I

- permanent dampness of, injurious to health, 5.33

Solids in suspension in sewage, method of estimating, 662

- lotal, in water, method of estimating, 653

Solutions, cleansing and antiseptic, 526

Soya bean (glycine soja), nutritive value of, 504

Sparganum (human tapeworm), 261

producing acne-like eruption, 261

Spectro-copic examination of blood, 612 
Spiders, remonnous, +40

effect of venom, 450

stings from, mortality, +50

symptoms, +50

treatment, 450

Spirillum choleræ asiatice, diagnosis of, 621

- Finkler-Prior, differentiation from Koch's comma bacillus, 62 I (vibrio-proteus), 621

Spirit lotion, application to skin in prickly heat, formula for, 487

Spirochata carteri cause of Asiatic relapsing fever, 13

duttoni, 10

- recurrentis, 10

Spirochates causing relapsing fever, ()

— field rat reservoir host for, it

Spirochatosis icterohamorrhagica (Weil's

disease), retiology, I7

-__ definition, i7

_... diagnosis, Is

— - geographical distribution, i

—_ history, I7

- prognosis, is

—— - symptomatology, 17

- treatment, 10

Spleen, enlargement in relapsing fever, I 3

- microscopical appearance in African

trypanosomiasis, I 30

Splenomegaly, a febrile, definition, 417

—- Ecographical distribution, +it

_- _ symptomatology, +17

- - treatment, 417

Sponge-fishers' asthenousia, 509

Sporadic diseases, $5+2$

Spore formation in protozoa, 4

Spores, method of staining, 615

Spurogenes test for bacteriological ex-

amination of water, 643

Sporotrichosis, 488

ocular, due to fungi, 5 I 1

treatment, 488

Sporozoa, 4

Spotted fever of Rocky Mountains, retiology, $37+$

$-\longrightarrow$ complications, 375

definition, 374

diagnosis, 375,376 differential, 375,376 geographical distribution

and seasonal incidence, $37 t$

- in Montana, mortality.

$3 \%($

376 prophylaxis against,

\section{—_... incubation period, 375 \\ - — pathology, 374 \\ - - prognosis, 376 \\ —_ symptomatology, 375}

Spring catarih (conjunctivitis restivalis). 516

spring "water, 548

Springs, contamination of, $5+9$

Sprue (psilosis), ætiology, 352-6

causes of death in, 357

condition of tongue in, 357

definition, 352

diagnosic, 359
Sinruc, diannosis, difterential, $358^{\circ}$ geographical distribution, 352 pathology, 356

post-mortem examination, 356

state of blood in, 337

treatment by Cantlie's method, $3 ; 0$ by drugs, 360

- by vaccines, 360 dietetic, 358, 359

local, 360

symptomatology, 357

- reast infection in, 35 ?

"Stab" cultures, 624

Stains, choice of, 604

Staphylococci, arrangement, 162

Staphylocuccus progenes aureus, dia gnosis of, 620

Starvation, death from, 564

Steam as means of disinfection, 503

- pipes in sterilization of water, 558

Steromyia calopus carrier of virus of rellow fever, $365,366,367$

_- (fasciata), adult female, 373

-_ - eggs and larve, 373

- - - habits, 373

- - notes on, 373

by, 376

Sterilized enclosures for preservation of meat, 575

Stermberg's treatment in yellow fever, formula for, 371

Stomatitis, diagnosis of sprue from, 358

Stools in typhoid fever in the Tropics, 217

Storage tanks for rain water, ; fo

"Streak" cultures, $62+$

Streptobacilli, 162

Streptococci, arrangement, 162 $65_{2}$

Streptococcus pyogenes, diagnosis of, 620

Streptothrix madura, diagnosis of, 630

Strongyloides stercoralis, 270

- intestinal catarrh produced by,

271

Sublimate, corrosive, as disinfectant, 594

Sullivan's diamond drill for producing bore well, 551

Sulphates in amobic dysentery, 44

Sulphur anhydride and sulphurous acid gas as disinfectant for houses, 595

- ointment, application in different forms of tinea (ringworm), 490, 493, 494

\section{- - in trichomycosis, 495 \\ in scabies, 487 in seborrha'a, 488}

Sulphurous acids and sulphites as pre servative, test for, 579

Sun, rays of, in Tropics, effect of on skin, 450

Sunlight, bacteria susceptible to, and resistant to effects of, 645

- - direct, simple method of disinfection, 592

- pathological effects, 530 predisposing factor in eruption of

ucllagra, 540

Sunstroke, 530

- see also Heat stroke 
Sillace water, 547

- catchment aleals, site of, $5+7$

Sweat, secretion of, in excess in natives of Tropics, $45^{8}$

Symovitis, acute, of knee associated witl filaria infection, 294

Syphilis, $48 S$.

- diagnosis b! Massemnann reaction, 610, 611

- medisposing factor in hamoglo-

binuric fever, $3 S_{4}$

sipluilitic ulcers, 504

TABE, late manifestations of yams, 25

Tablet foods, concentrated, 582

Trenia saginata affecting muscles of tongue and of mastication of cattle, 260 - _ largest human tapeworn, 265 solium, 264

Tannic acid in amobic dysentery, 43

- intestinal irrigation with, in

ciliar dysentery (Balantidium coli), 40

lapeworms found in man, list of, 260

- treatment, preliminary, 209

- prescription for, 269

- - prophylactic, 270

-_. thorough, 260

- See also Cestoda pathogenic to man

Tanioca, 564

climination of poisonous elements

from, 454

Tarabagan (Arctomys bolxe), spread of pmeumonic plague in Manchuria by, 171

Tartar cmetic in granuloma inguinale, 460

- in treatment of African trypanosomiasis, 148

- method of administration in lreatment of African trypanosomiasis, 149

Tattooing, 488

- risks of, 488

- treatment, 480

Tea, cold, why a suitable drink for travellers, 555

- qualities of, 582

Telangiectasis, multiple, 502

Telosporidia, 4,6 .

Temperature of air in tropics, 53 I

"letanus, antitoxic serum in, value, 599

- bacillus, diagnosis of, 617

leeracocci, arrangement, 162

Tetramitus mesnili, ;

- - - warasite of ciliar dysentery, 47

Fetrodin in poisonous fish, 448

Tetrodonic acid in poisonous fish, 448

Theft, propensity to, in cerebral stage of

African trypanosomiasis, 145

Thermal methods of sterilizing water, $5,5 \mathrm{~S}$

Thiosinamin injection in elcphantiasis of legs, 286

Thiosulphite for murifation of water, 550

Thoracic duct, blocking of, in filariasis,

Three-day fever. See Phlebotomus ferer

Thresh, J. C., method of purifying water, $55^{\circ}$
Thresh's disinfector, 505

Thymol treatment of anliylostomiasis, 3 I $8-9$

Tick fever, African, mode of transmission, 8

licks, largest specimens of order of Acarina, 19

- manner of proparation, 19

venom of, infection by, symptoms, 450,451

$\frac{450}{\text { Tinea alba, } 400}-$ treatment, $45 \mathrm{I}$

- lesions and regions attacked,

490

- parasite, 490
- regions attaclied, 400
- - treatment, 490
- albigena, 490
- parasite, 490
- barba (sycosis), 490
$-\quad-$ lesions and regions at

tacked, 400

- - - parasite, 490

treatment, 490

capitis (tonsurans), lesions and regions attaclicd, 490

- _ - parasite, $49 \mathrm{I}$

- Cincinata tringworm of the body),

lesions and regions attacked, to I

$-\frac{-}{-}-$ parasite, $49 \mathrm{I}$

itch), lesions and regions attacked, f(g) $-\div-$ parasite, 491

decalvans, 492

flava, lesions and regions attaclied,

492

- parasite, 492

- treatment, 492

- imbricata, lesions and regions of

body attaclied, 492

- parasite, 492

intersecta, lesions and regions of

body attacked, 493

- narasite, 493

- treatment, 493

nigra, 493

- lesion and legions of body:

attacked, 493

_- varasite, 493

nigro-cincinata, lesion and regions

of body attacked, 403

- - varasite, 493

treatment, 493

nodosa, 47 I, 493

treatment, 471

ocular, due to fungi, 511

palpebralis, 494

(ringwolm), 480

Sabouraudi tropicalis, lesions and

region attacked, 494

- - parasite, 494

- - treatment, $4(9)$

tolssuralss, 489,490

ungium, lesions, 494

parasite, 494

- treatment, f94 
Tinea versiculor (Pityriasis versicolor), lesions and regions attacked, 494

- _- parasite, 494

Tisse cheatment, 494

Tissue changes in sections, staining for, 604

Tissucs, embedding of, in preparation of tissues, 625

624

fixing of, in preparation of sections,

- hardening of, in preparation of sections, 625

'Toads, venomous, slightly' toxic to man, $4+8$

Tongue and mastication, muscles of, in cattle affected by taenia saginata, 260

- condition of, in sprue, 357.

denuded epithelium of, diseases in which seen, 358

- leukoplakia of, $47 \mathrm{t}$

Townships, planning and construction in tropical districts, 536

Toxicity theory in xetiology of pellagra, $33 i$

Toxins, elimination in yellow fever, 370 , 371

Trachoma, xtiology, 513

- complications and sequelæ, 573

definition, 513

(granular conjunctivitis), 512

surgical remedies for, 515

simptomatology, 513

treatment, 514

Trematoda pathogenic to man, classifica tion, 232, 236

$-\ldots$ habitat, 235

- life-history, 235

- - morphology, 234

- - treatment, 230

Trematodes, ova of, 637

pathogenic, varieties, 236

- preservation and examination of, 322

Trench fever, atiology, 400

- definition, too

- - symptomatology, 400

Treatment, 401

'Trenches, deep, unsatisfactory as latrines for natives, $; 86$

prepared as latrines for natives, 586 shallow, as latrines for natives, 585

frenching and cultivation in disposal of excreta, 590-592

'Treponema pertenuis, frambosia tropica (raws) due to, 2 I

Trichinella spiralis, calcification in muscles, 308

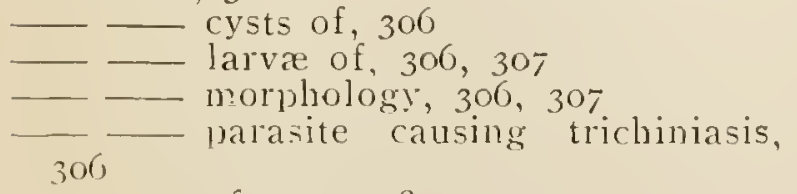

- - of rats, 308

Trichiniasis, definition, 306 geographical distribution, 306

parasite of, 300

prophylaxis against, 300

symptomatology, 308

treatment, $30 \mathrm{~s}$

'Trichocephalus dispar, ova of, 637
Trichocephalus trichiuris (whipworm), morplology, 305

- - parasite causing trichocephaliasis, 305

T- pathogenicity, 300

Trichocephaliasis, definition, 305

parasile of, 305

treatment, 306

Tricliononas hominis, 5

- intestinalis, characters, 5

- varasite of ciliar dysentery, 47 vaginalis, 5

- - geograuhical distribution, 5

Trichomycetes, divisions of, 629

Trichomycosis, 494 capillitii, 472

\section{treatment, 495}

Trichonocardiasis (nodules on the hairs), 405

Trichophyton megalosporon (ringworm), 631

Trichophytons, 489

and microsporons, difference between, +80

Trichorrhexis nodosa, 472

treatment, 472

Tropical diseases, protozoa as factors in causation of, 2

— liver, Europeans attacked by, 55

- internal medications in, 55

Trypanosoma brucei (rhodesiense), I27

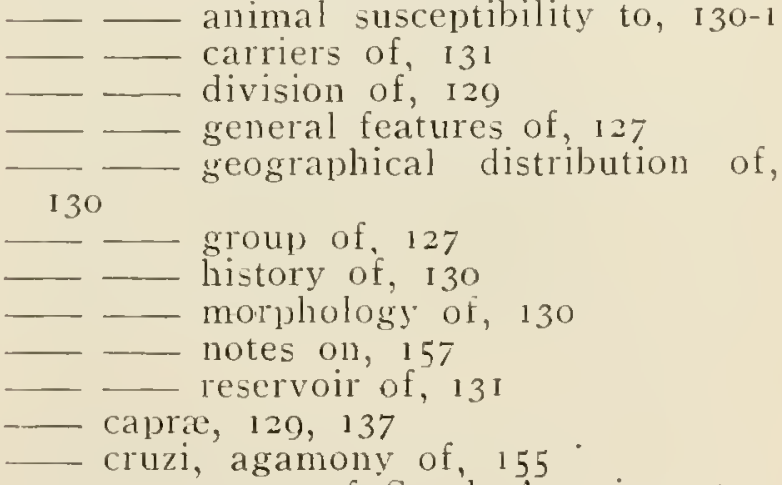

cruzi, agamony of, 155 cause of Soutl American try-

panosomiasis, I 54

- … microscopical examination of, 156

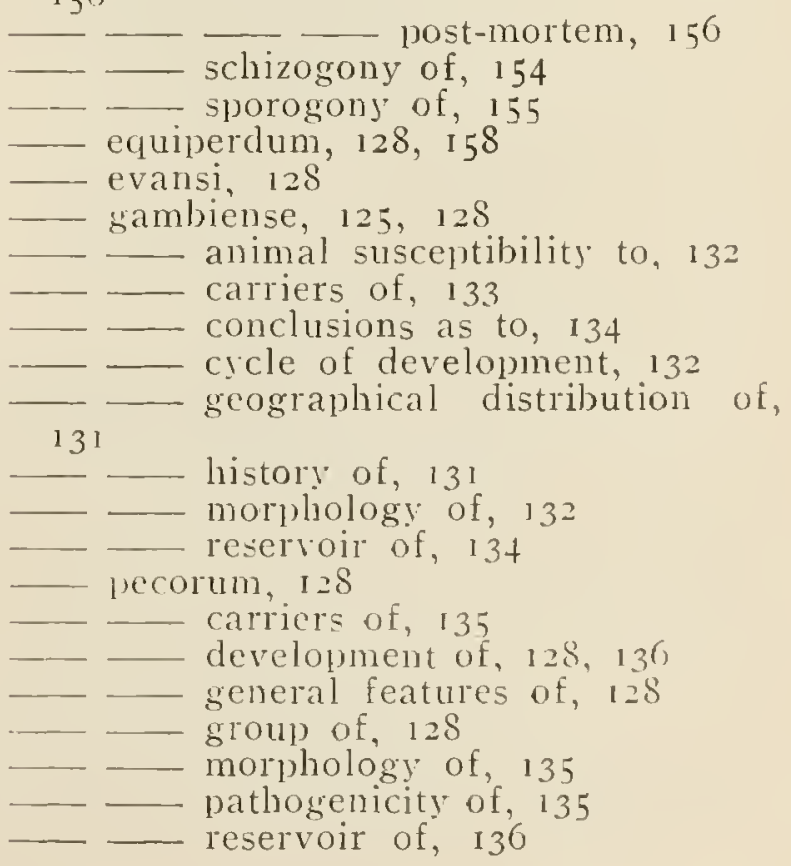


Trypanosona simia, 128,136 uniforme, $129,13^{6}$

vivax, 129 , I 36 development of, 129 general features of, 120

- general features

Trypanosome cazalboui, notes on, I57, 159

- dimorphon, motes on, I60

equinum, notes on, 158

evansi, notes on, 157

- hippicum, notes on, I 59

Soudanese, notes on, I 59

Tripanosomes, classification of, $126-T$

- habitat of, 126

methods of searching for, $1+7$

pathogenic, Central African, 127

ters, $127 \cdot 137$

Trypanosomiasis, African, atiology of, $124-5$

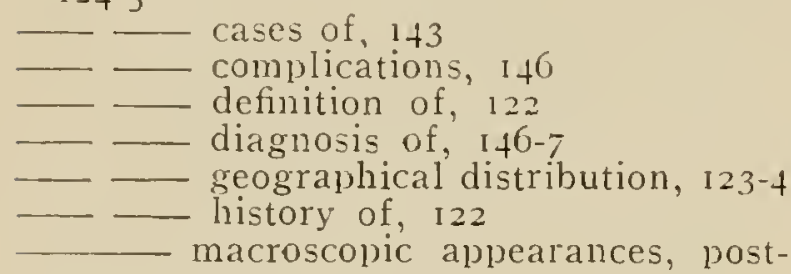
mortem, I 37

___ microscopic appearances, postmortem, I 38

- - pathology of, 137

- prognosis of, 147

- prophylaxis against, 150

- symptoms, summary of, I 40 sy"mptomatology of, 139

treatment of, $i 47-8$

- development, conditions favourable

for, 540

_ mammalian, pathological, notes on, 157

\section{_ parasite of, 125}

South American, atiology of, I 54

- chronic, with acute and

subacute exacerbations, I57

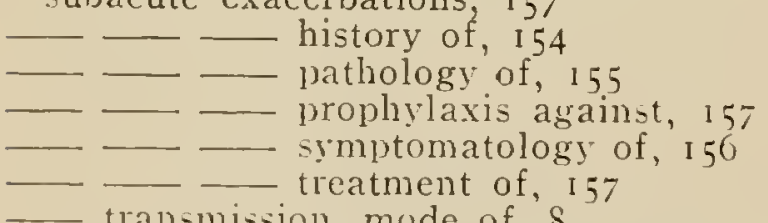

- transmission, mude of, $\delta$

Trypanosomide, $;$

Tube well for shallow water in tropics, 551

Pubercle bacillus, diagnosis of, 615

in milk, test for, 570

Tubercular ulcers, 504

'luberculosis, antitoxic serum in, 599

- in animals, recommendations of

Royal Commission on Human and

Animal Tuberculosis, 574

Tumoul, Ieaking, discharging fluid giv-

ing reaction of bile, $50 \mathrm{I}$

"limours, 495

Tumentine, application in tinea flava. 492

- oil, application in tinea capitis, 49

'lyphoid bacilli, removal from water" chemical process for, 556

- bacillus, destruction by storage of water, 554
Typhoid bacillus, in water, difticulties of detection, 652

- — metlod of detection of, 652

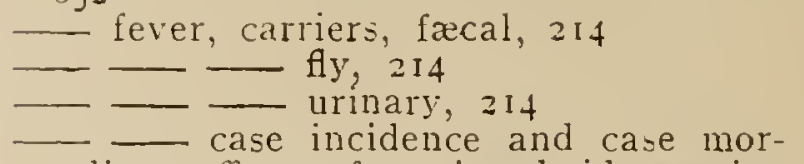
tality, affect of anti-typhoid raccine on, 601

- diagnosis by Widal reaction, 611

Mountains from, $37 ;$

- - epidemic following use of contaminated shallow wells, 550

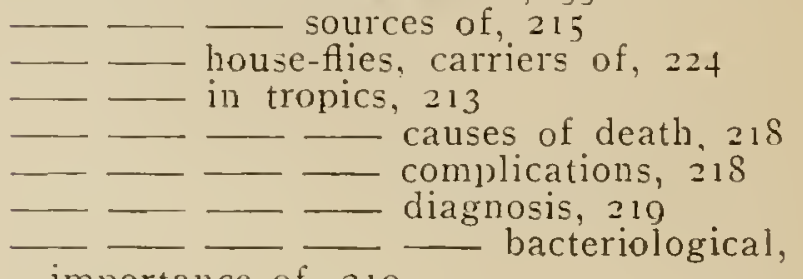
importance of, 219

- - liability of freshly

arrived Europeans to, 215

$\because-\frac{\text { pathology, } 2 \text { I5 }}{-}-$ symptomatology, 217

ptoms, 223

1 - sources of infection, 214

'Typhus fever, retiology, 213,379

- and spotted fever of Rocky

Mountains, similarity between, 376

- complications in, 380

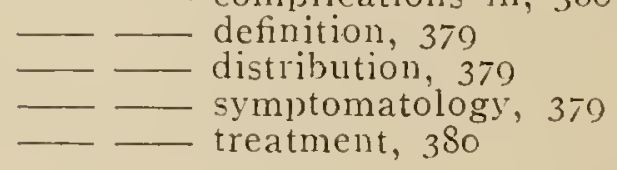

ULCER, perforating, amputation for, in the foot of lepers, i 85

Ulcerations, tropical, 502

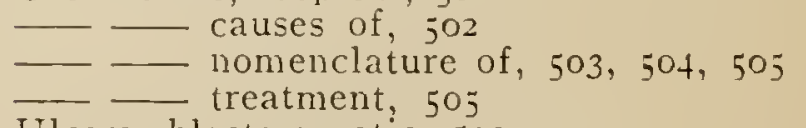

Ulcers, blastomycotic

- cancerous, 503

- diphtheroid, 503

_- frambosial, 504

_.. infantile, 504

— interdigital, 504

leprotic, 504

- - or' elephantoid, 503, 504

- septic, 504

- syphilitic, 504

- tubercular, 504

varicose, 503, 505

Ultra-violet rays in sterilization of water, 558

Undulant fever, atiology of, I9o

- complications of, I05

- definition of, Igo

diagnostic features, I05

100 geographical distribution of,

- - modes of infection, 191 pathology of, 191 post-mortem appearances, roI prophylaxis against, I05 
[indulant fever, symptomatology of, 191 , $104-5$

- - - transmission, modes of, 540 treatment of, 195

Linited States, mortality from vellow fever in, 370

Urinary calriers of typhoid fever, 214

Urine in blackwater fever, 387

in chyluria and lymphuria, 283

Trobilin, inclease of, in faces, hamolytic diseases, 634

Urotropine in chyluria and lymphuria, $28_{4}$

Uterus, cervix, carcinoma, 502

VACCINATION, definition, 598

Vaccine, Haffkine"s, in prophylaxis of plague, 1 ; 6

dosage, 176

_- inorulation against cholera, 212, 213

Saccines, altogenous, 508

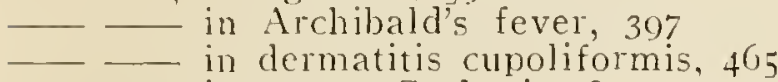

- in pyosis Corletti, 485

classification of, 590

compound, 602

imported, manner of preservation, 598

- living and devitalized, inoculation against cholera, 2 I3

- protection afforded by, hypotheses accounting for, 600

- sensitized, 599

-_ sireptococcal in sprue, 360

rable of, with doses, 599

Tacuoli in protozoa, 3

Vacuum apparatus (Elliott and Paton's) for steam disinfection, 595

Van fieson's stain in staining sections, 627

Tuse of $60 t$

Variola in India and Africa, 401

Vegetable matter in water, ill-effects of, 552

- products, rich in potassium salts, 563

Tegetables, deficient, in retiology of beriberi, 339

- green, transmission of cholera by, 200

Vegetarianism, 580

Teldt sore, 503, 505

Venom, absorption of, in snake bite, pre vention of 443

- - manner of neutralizing, 444

_- See also Snake venom

V'enoms, diseases due to, 418, 410

Verrucx, 495

l'erruga peruviana, retiology, 363

atypical, 365

blood condition in, 364

definition, 363

erythema of, 364

geographical and seasonal dis-

tribution, 363

- histopathology, $3^{6} .3$

history, 363

mild type, 364

pathology, 363

prognosis, 365

- regional distribution, with various resulting lesions and symptoms, 365
Verruga peruviana, sovere tipe (Carrion's fever), 364

__ - symptomatology, 364

- ireatment, 365

Vertigo, paralytic, cndemic, for

Vesicular fever, diagnosis, 403

Vibrio-proteus. See Spirillum. Finkle' Prior

Tillages, native, planning and construc tion in tropical districts, 536

Vipera russelii (daboia, or Russell's viper), 431

Viperidæe, 426

Viperina, 426,430

in Africa, 432

Viperine venom (snake bite), post-mortem, 443

Ti symptoms, $4+2$

Viruses, filterable, 561,562

Vitamines, 560

Voit's standard diet, 565

Tolvulosis, 300

- geographical distribution, 300

Vomit of yellow fever, 368

Vomiting in ackee poisoning, 455 - - in blackwater fever, drugs in cure of, 388

- in cholera, treatment, 210

rulva, elephantiasis of, 292

ITARS, relation of cholera epidemics to, 204,205

IVarts, 405

Washing water, cleansing of, method described, 560, 561

$\mathrm{NT}^{\top}$ ashington-Lyon apparatus for disinfection by steam, 595

ITasps. See Bees

Wassermann reaction (Fleming"s modification), diagnosis of syphilis by, 610 , 611

$\frac{61}{61}, 610,611-$ substances required

Watel and faeces, bacteriology during monsoon weather, 646

- amount required daily for personal and domestic use, $5+4$

Gonalysis of sample of, certificate, 002

- - as thirst quencher, 582

bacteria in, enumeration by British methods, 650, 651

- boring for, $5+5$

carriers. Hindu, vessels used by, $55^{2}$

Mahommedan, infection of

water by, 551

chemical examination of, 653

closets in tropics, 588

containing peat, effect on leaden water-pipes, 552

- contamination, source of, methods

of tracing, 553

- control of, by medical officer, 553

distilled, aeration of, $\mathbf{5 4 5}$

examination, 639 bacteriological, 640, 64I

$6+5,646-$ in India, standard for, tests for, $642,643-646$ 
11 ater cxamination, biological, 640 physical, 640

- filtration of, $55 \mathrm{~S}$

hard, not injurious to health. 552

- pathology of, 552, 553

- murification, 554

- - by jecilitation, 555

- by storage, 554 chemicals used for, 555

___ rendering potable by oxidation, 561 soft, ill-effects of, 5,2

softening of, chemicals used for, 555

solvent uses of, in diet, 563

sources of, 544

sterilization aided by storage, 554 by electrical methods, 558

- by thermal methods, 558

subterranean, 548

classification of, 548

transmission of cholera by, 205

transport of, 551

- unfiltered, spread of schistosomiasis

by, 250

Whatercress, dangers of, 572

IVater-pipes, iron, diminution in diameter, $55^{2}$

VIater supply pipes, dimensions of, method of ascertaining, 546

Veigert's method for staining microorganisms, 6rt

IVeil's disease. See Spirochatosis icterohamorrhagica

IVell and spring waters, classification for drinking purposes, 650

- protected, plan of, 550

- shallow, bacteriolog! of water from, 647

- water in Khartoum, bacteriological examination, 643

IVells, deep, 551 shallow, 548 contaminated, use of, followed

by typhoid fever epidemics, 550

Whey, 568

WThipworm. See Trichocephalus trichiurus

White men, racial existence in tropics impossible, 538

Whitmore's disease, xtiology, 415

—_ common in drug-takers, 415

- - definition, 415

$\log 5,415,416$

Widal reactions in diagnosis of typhoid fever, 219 , 6ri

- method of performance, 611, 612

IVind and dust, exposure to, causing pinguecula pterygium and ophthalmic irritation, 510

Women, men and cliildren, food necessar. for, compared, 564
XaNTHOMA areatum, 506 treatment, 506

diabeticorum, 502

planum, 502

tuberosum, 502

Tenopsylla cheopis (rat-flea), transmis sion of plague by, $17 \mathrm{I}$

Nerosis, 510

among debilitated children, 510

X-ray treatment of tropical ulcers, 505

Yaws. See Frambesia tropica

Jeast, infection in sprue, 357

Yeasts, staining of, 632

Yellow fever, blood condition in, 367

- course, crisis and termination, 369,370

- -370 definition, 365

- - diagnosis, 370

- - differential, 370

370

disinfectant measures against,

372 endemic centres of, 366 cndemicity of, mean tempera-

ture necessary for, 539

_- geographical distribution, 366 immunity to, 368

children, 368 natural, in very young

- _ importation of cases into regions free from Stegomvia callopus not dangerous, 543

__ _ incubation period, 360

- mortality, 370

- pathology, 367

__ post-mortem findings in bodily

regions and organs, 368

—_ prognosis, 370

- - prophylaxis against, 371,372

372

- spread of, dependent on presence of mosquitoes, 539

_ _ factors necessary for, 371

- symptomatology, 369
- transmission, mode of, 8
- treatment, 370

ptoms, 371

bỵ elimination of toxins,

370,371

- dietetic, 371 indications for, 370

- virus of, 366,367

romit of, 368

Yersin's serum in prophylaxis of plague, 1,6 




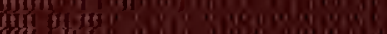

